\title{
INTEC SBW Solid Sludge Surrogate Recipe and Validation
}

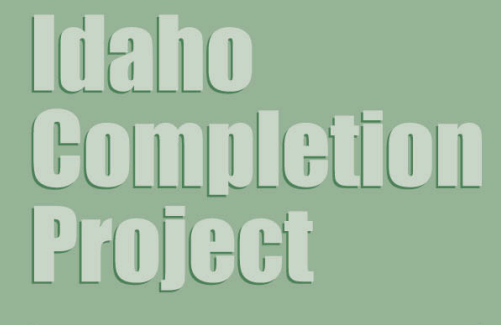

Bechtel BWXT ldaho, LLC

June 2004 
ICP/EXT-04-00415

Revision 0

Project No. 22681

\section{INTEC SBW Solid Sludge Surrogate Recipe and Validation}

June 2004

Idaho Completion Project

Idaho Falls, Idaho 83415

Prepared for the

U.S. Department of Energy

Assistant Secretary for Environmental Management

Under DOE Idaho Operations Office

Contract DE-AC07-99ID13727 


\begin{abstract}
A nonhazardous INTEC tank farm sludge surrogate that incorporated metathesis reactions to generate solids from solutions of known elements present in the radioactive INTEC tank farm sodium-bearing waste sludges was formulated. Elemental analyses, physical property analyses, and filtration testing were performed on waste surrogate and tank farm waste samples, and the results were compared. For testing physical systems associated with moving the tank farm solids, the surrogate described in this report is the best currently available choice. No other available surrogate exhibits the noted similarities in behavior to the sludges. The chemical morphology, particle size distribution, and settling and flow characteristics of the surrogate were similar to those exhibited by the waste sludges. Nonetheless, there is a difference in chemical makeup of the surrogate and the tank farm waste. If a chemical treatment process were to be evaluated for final treatment and disposition of the waste sludges, the surrogate synthesis process would likely require modification to yield a surrogate with a closer matching chemical composition.
\end{abstract}




\section{CONTENTS}

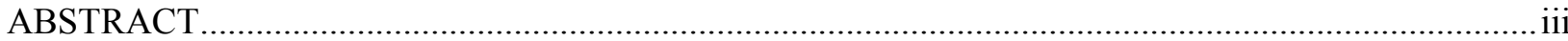

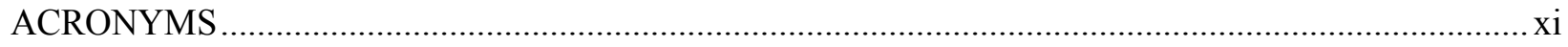

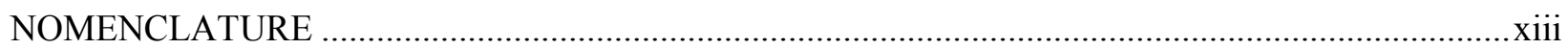

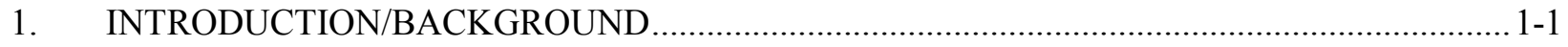

2. SURROGATE DEVELOPMENT AND PREPARATION .....................................................2-1

$2.1 \quad$ Rationale for Surrogate Development …................................................................... 2-1

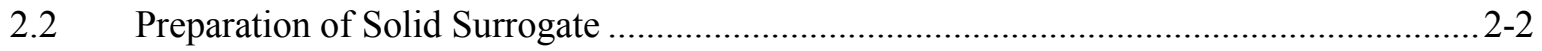

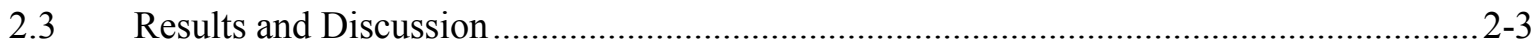

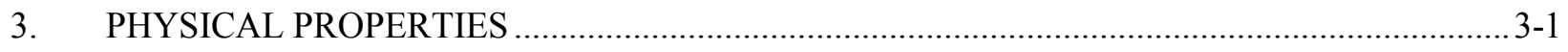

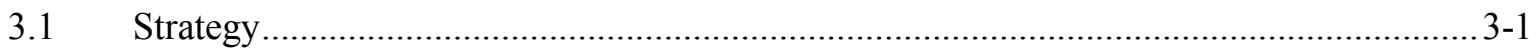

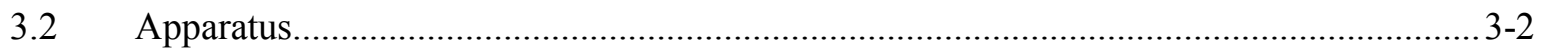

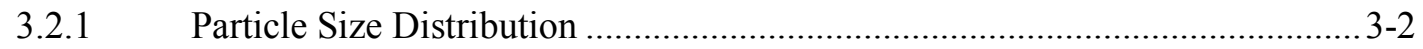

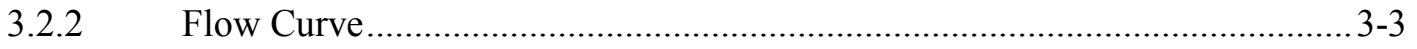

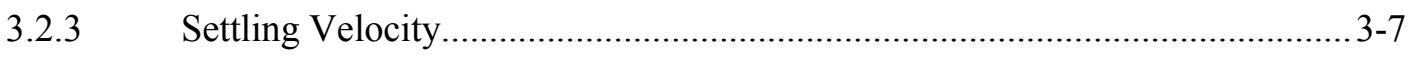

3.2.4 Solids Weight Percentage................................................................. 3-8

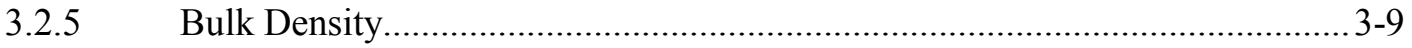

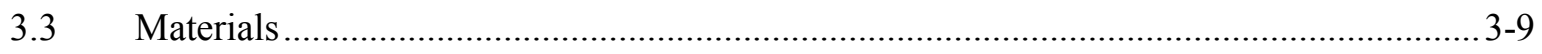

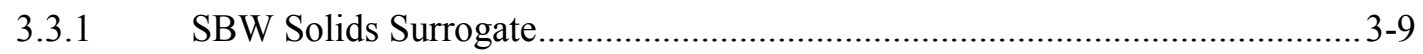

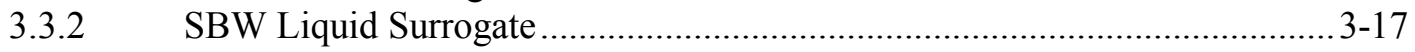

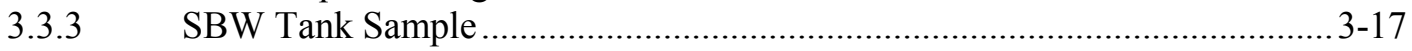

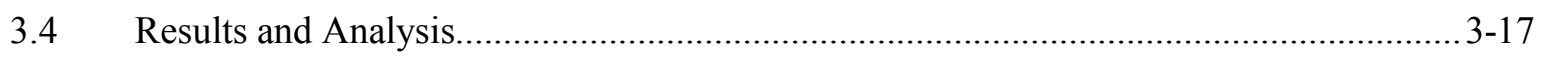

3.4.1 Particle Size Distribution ................................................................ 3-18

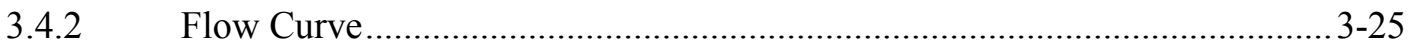

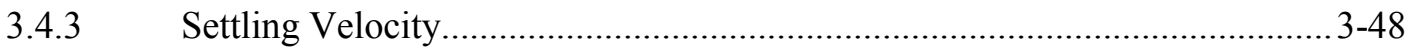

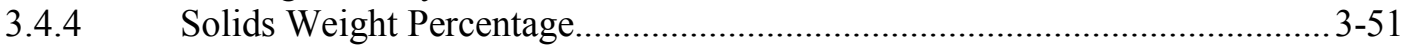

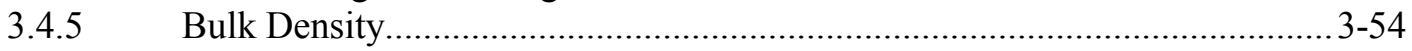

4. CAKE FILTRATION PERFORMANCE ........................................................................

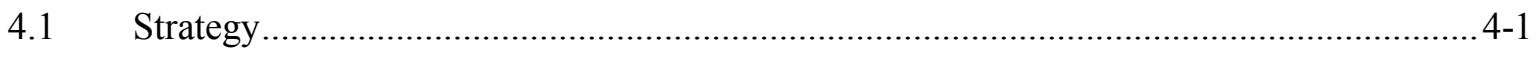

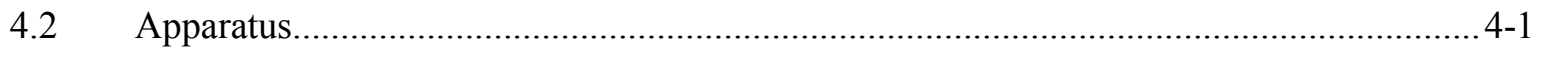




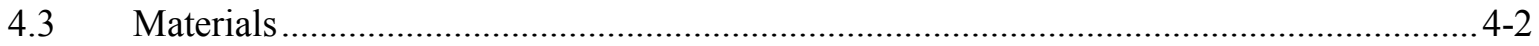

4.3.1 SBW Slurry Surrogate \#1 .................................................................... 4-2

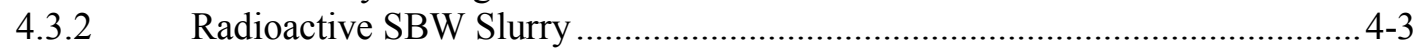

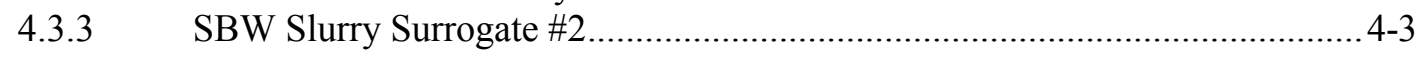

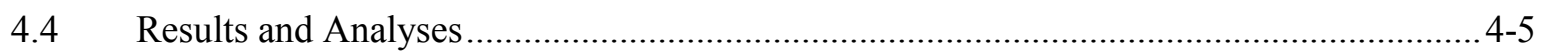

4.4.1 SBW Slurry Surrogate \#1 Test Results ..................................................... $4-5$

4.4.2 Radioactive SBW Slurry Test Results .................................................... 4-8

4.4.3 SBW Slurry Surrogate \#2 Test Results ..................................................... 4-8

4.4.4 Comparison of SRF $(\alpha)$ and Cake Compressibility ....................................... 4-9

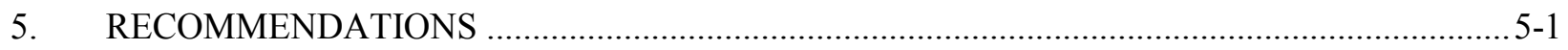

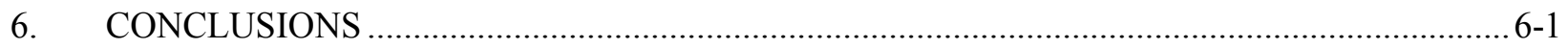

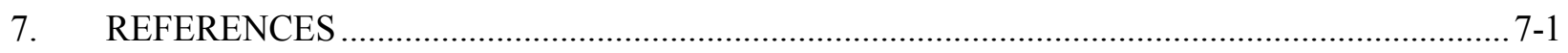

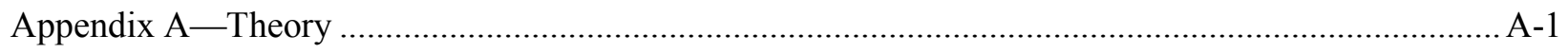

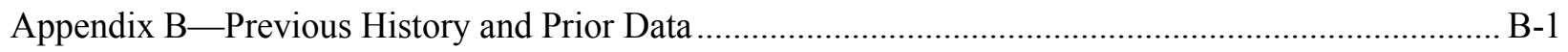

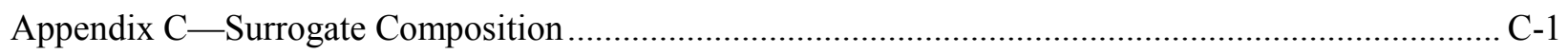

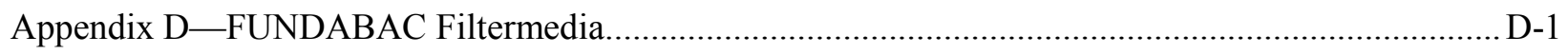

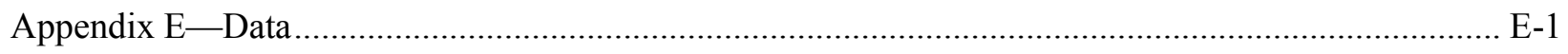

\section{FIGURES}

3-1. Fisher Scientific Model 500 sonic dismembrator, soundproof enclosure, stir plate, and scissor lift

3-2. Microtrac FRA particle size distribution analyzer ....................................................................

3-3. R/S rheometer, FTKY water jacket, MB-45/48F measuring chamber, and CC-45 spindle...........3-4

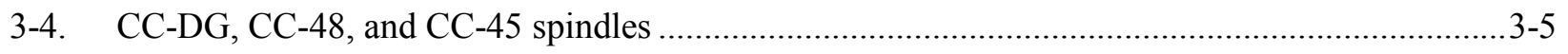

3-5. MB-45/48F and MB-DGF measuring chambers..............................................................

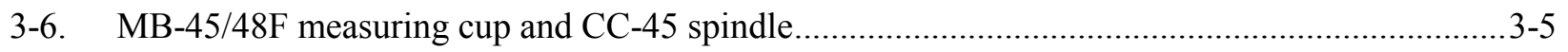

3-7. MB-DGF measuring system and CC-DG spindle ..................................................................

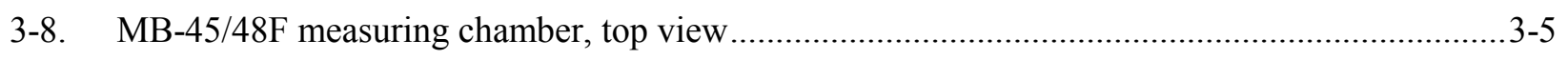

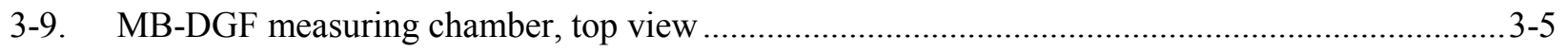




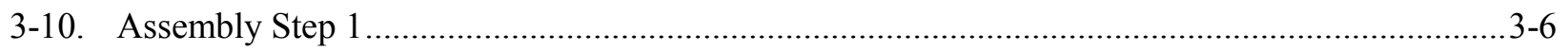

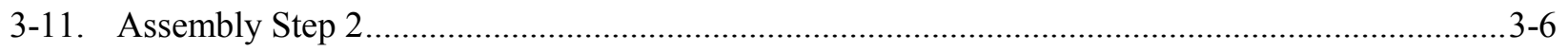

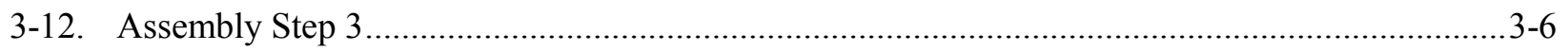

3-13. MB-45/48F machine shop hot cell modification top view ........................................................

3-14. MB-DGF machine shop hot cell modification top view ............................................................

3-15. Adapter collar machine shop hot cell modifications .........................................................

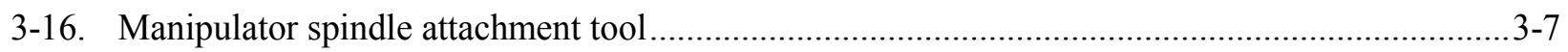

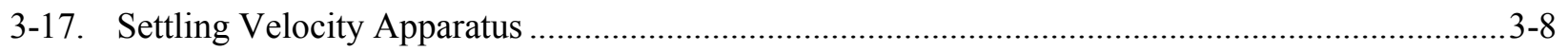

3-18. $187 / 5 \mathrm{wt} \% / \mathrm{D} / \mathrm{U}$ and $187 / 20 \mathrm{wt} \% / \mathrm{D} / \mathrm{U}$ particle size distributions ...................................................

3-19. Comparison of WM-189, WM-180, WM-182, and WM-183 solids particle size distribution analysis under nonsonicated condition...................................................................................

3-20. $187 / 5 \mathrm{wt} \% / \mathrm{D} / \mathrm{S}$ and 187/20wt $\% / \mathrm{D} / \mathrm{S}$ particle size distributions ..............................................13

3-21. 180/5wt $\% / \mathrm{U} / \mathrm{N}, 189 / 5 \mathrm{wt} \% / \mathrm{U} / \mathrm{N}$, and META/5wt\%/U/N sample particle size distributions ........3-14

3-22. META/5wt\%/U/N, META/10wt\%/U/N, and META/20wt\%/U/N sample particle size

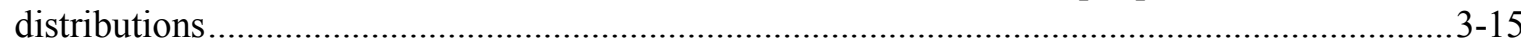

3-23. 180/5wt\%/U/S, 189/5wt\%/U/S, and META/5wt\%/U/S sample particle size distributions .......... 3-16

3-24. META/5wt\%/U/S, META/10wt\%/U/S, META/15wt\%/U/S, and META/20wt\%/U/S sample particle size distributions .......................................................................................

3-25. Nonsonicated SBW surrogate sample particle size distributions ............................................ 3-20

3-26. Sonicated SBW surrogate sample particle size distributions ............................................... $3-20$

3-27. SBW surrogate sample mean particle diameter before and after sonication.............................. 3-21

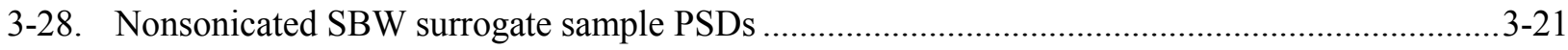

3-29. Nonsonicated FY-03 SBW surrogate sample PSDs...........................................................

3-30. Nonsonicated, as-received FY-04 SBW surrogate sample PSD ............................................. 3-22

3-31. Solids weight percentage adjusted, nonsonicated FY-04 SBW surrogate sample PSDs .............3-23

3-32. A comparison of the particle size distributions for various tank farm solid waste samples.........3-24

3-33. WM-187 supernate, metathesis mother liquor surrogate, and 187 liquid surrogate flow curve comparison . 
3-34. WM-187 supernate and metathesis surrogate mother liquor viscosity comparison

3-35. WM-187 as received and META/5wt\%/U/S surrogate data regression.....................................3-30

3-36. WM-187 as received and META/5wt \%/U/S surrogate viscosity comparison...........................3-30

3-37. WM-187 gravity settled and META/10wt $\% / \mathrm{U} / \mathrm{S}$ surrogate data regression ..............................3-33

3-38. WM-187 gravity settled and META/10wt\%/U/S surrogate viscosity comparison .....................3-33

3-39. WM-187 centrifuged and META/20wt\%/U/S surrogate data regression ................................3-36

3-40. WM-187 centrifuged and META/20wt\%/U/S surrogate viscosity comparison..........................3-36

3-41. WM-187 centrifuged and 187/20wt $\% / \mathrm{D} / \mathrm{S}$ surrogate data comparison ..........................................

3-42. WM-187 centrifuged and 187/20wt \%/D/S surrogate viscosity comparison .............................. -40

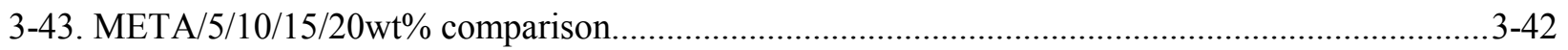

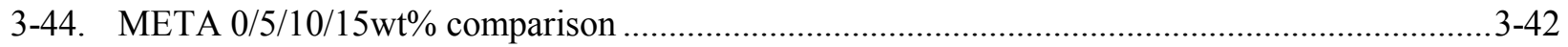

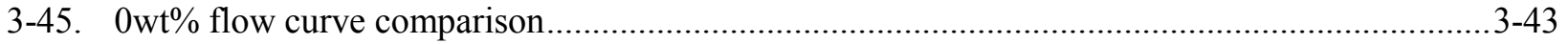

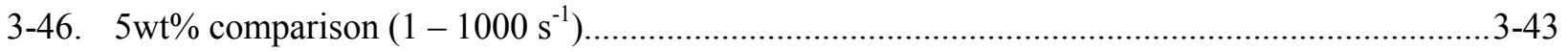

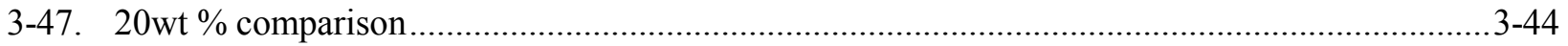

3-48. META/5wt\%/U/S versus META/5wt \%/U/N flow curve comparison ........................................45

3-49. META/10wt\%/U/S versus META/10wt\%/U/N flow curve comparison .................................... -45

3-50. META/15wt\%/U/S versus META/15wt\%/U/N flow curve comparison .................................... $3-45$

3-51. META/20wt $\% / \mathrm{U} / \mathrm{S}$ versus META/20wt $\% / \mathrm{U} / \mathrm{N}$ flow curve comparison .....................................

3-52. 180/5wt \%/U/S versus 180/5wt\%/U/N flow curve comparison............................................. $3-46$

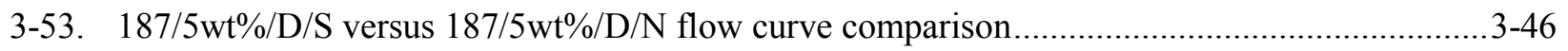

3-54. 189/5wt\%/U/S versus $189 / 5 \mathrm{wt} \% / \mathrm{U} / \mathrm{N}$ flow curve comparison.................................................

3-55. 187/20wt $\% / \mathrm{D} / \mathrm{S}$ versus $187 / 20 \mathrm{wt} \% / \mathrm{D} / \mathrm{N}$ flow curve comparison............................................ $3-46$

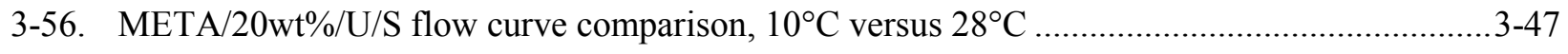

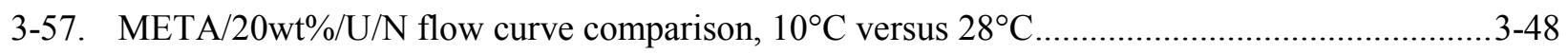

3-58. META/5wt\%/U/S and META/10wt $\% / \mathrm{U} / \mathrm{S}$ interface velocity in $100 \mathrm{~mL}$ settling chamber ........3-50

3-59. WM-187 sample interface velocity in $100-\mathrm{mL}$ settling chamber........................................... 3-51 


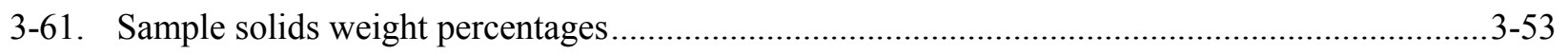

4-1. Bench scale constant pressure filtration apparatus...................................................................4-2

4-2. $\quad$ PSD comparison of SBW slurry surrogates and the modified SBWs slurry ...............................4-4

4-3. Determination of cake filtration resistance in a constant pressure filtration run.......................... 4-7

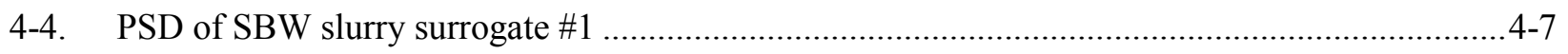

4-5. Comparison of cake compressibility (Model I) .................................................................. $4-10$

\section{TABLES}

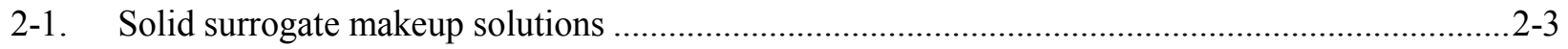

2-2. Analytical results for the surrogate and two samples of tank farm sludges ..............................2-4

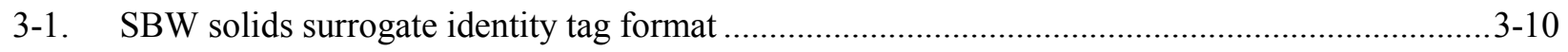

3-2. Dried metathesis-prepared SBW solids surrogate samples and associated mean particle size....3-11

3-3. Undried metathesis-prepared SBW solids surrogate samples and associated mean particle size

3-4. Summary of important 180, 187, and 189 liquid surrogate components ................................ 3-17

3-5. Nonsonicated and sonicated SBW surrogate sample mean particle diameter........................... 3-23

3-6. Nonsonicated and sonicated WM-187 sample mean particle diameter......................................24

3-7. Range of shear rates of some familiar materials and processes .......................................... $3-25$

3-8. Tank farm waste sample and SBW surrogate supernate flow curve data regression..................3-29

3-9. Tank farm waste sample and SBW surrogate suspended slurry flow curve data regression ........3-32

3-10. Tank farm waste sample and SBW surrogate gravity settled slurry flow curve data regression

3-11. Tank farm waste sample and SBW surrogate centrifuged slurry flow curve data regression.......3-38

3-12. Tank farm waste sample and SBW surrogate centrifuged slurry flow curve data regression......3-39

3-13. Settling velocity for META/5wt $\% / \mathrm{U} / \mathrm{S}$ and META/10wt $\% / \mathrm{U} / \mathrm{S}$ samples ................................. 3-49

3-14. Settling velocity for META/5wt $\% / \mathrm{U} / \mathrm{S}$ and META/10wt $\% / \mathrm{U} / \mathrm{S}$ samples ................................. $3-50$

3-15. WM-187 tank farm waste sample solids weight percentages ............................................... 3-52 


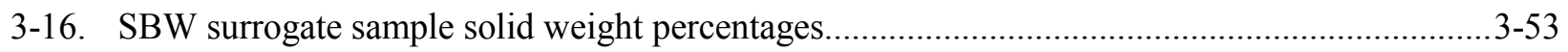

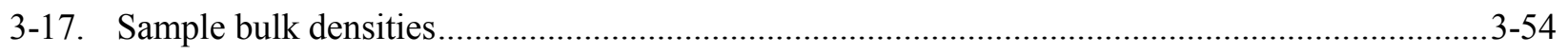

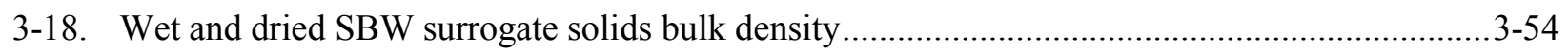

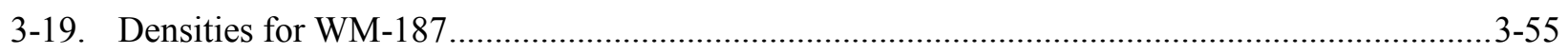

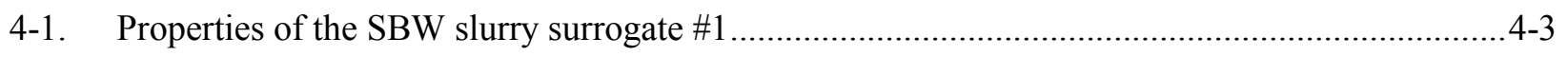

4-2. $\quad$ Properties of the modified radioactive SBW slurry .......................................................... $4-3$

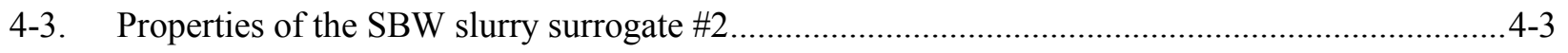

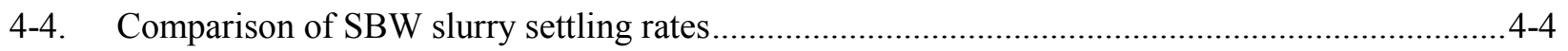

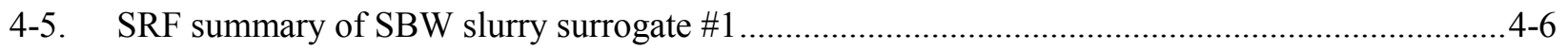

4-6. SRFsSummary of radioactive SBW slurry ...........................................................................

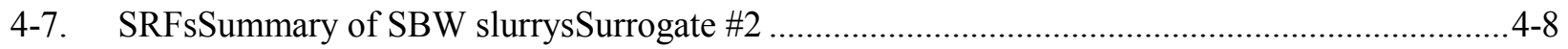

4-8. Comparison of cake compressibility coefficients .................................................................. $4-10$ 


\section{ACRONYMS}

ACMM Analytical Chemistry Methods Manual

BBWI Bechtel BWXT Idaho, LLC

$\mathrm{CH} \quad$ contact-handled

CsIX Cesium Ion Exchange

DOE Department of Energy

EDF Engineering Design File

FY fiscal year

IHR Independent Hazard Review

INEEL Idaho National Engineering and Environmental Laboratory

INTEC Idaho Nuclear Technology and Engineering Center

IRC INEEL Research Center

LDUA Light-Duty Utility Arm

NYC North Yellowstone Complex

OD outside diameter

PPS polypropylene sulfide

PSD particle size distribution

PVDF polyvinylidene fluoride

RAL Remote Analytical Laboratory

RCRA Resource Conservation and Recovery Act

RH remote-handled

S\&CL Standards and Calibration Laboratory

SBW sodium-bearing waste

SRF specific resistance off filtration

TDS total dissolved solids

TRU transuranic 
UDS undissolved solids

WIPP Waste Isolation Pilot Plant 


\section{NOMENCLATURE}

\section{Physical Properties Nomenclature}

$\begin{array}{ll}\text { a } & \text { acceleration } \\ \text { A } & \text { area } \\ \text { c } & \text { constant of proportionality, } \mathrm{cm}^{-1} \mathrm{~s}^{-1} \\ \mathrm{C} & \text { Corey shape factor } \\ \mathrm{C}_{\mathrm{D}} & \text { drag coefficient } \\ \mathrm{D} & \text { diameter } \\ \mathrm{D} & \text { shear rate, } \mathrm{sec}^{-1} \\ \bar{D} & \text { mean value of the major and minor axis of particle } \\ \mathrm{d} & \text { fall distance } \\ \mathrm{D}_{\mathrm{a}} & \text { major axis of particle } \\ \mathrm{D}_{\mathrm{b}} & \text { minor axis of particle } \\ \mathrm{D}_{\mathrm{c}} & \text { intermediate axis of particle } \\ \mathrm{E} & \text { energy } \\ \mathrm{F} & \text { force } \\ \mathrm{F}^{\prime} & \text { shear stress, dynes/cm } \\ \mathrm{f}^{\prime} & \text { yield value } \\ \mathrm{F}_{\mathrm{D}} & \text { drag force } \\ \mathrm{g} & \text { acceleration due to gravity } \\ \mathrm{I} & \text { immersed weight of particle } \\ \mathrm{k} & \text { settling coefficient } \\ \mathrm{m} & \text { mass } \\ \mathrm{r} & \text { radius } \\ \mathrm{Re} & \text { Reynold's number } \\ \mathrm{U} & \text { fluid upthrust } \\ \mathrm{V} & \text { Plate velocity } \\ & \end{array}$

\section{Cake Filtration Nomenclature}

A surface area of the filter medium $\left(\mathrm{m}^{2}\right)$

C mass of dry cake per unit volume of filtrate $\left(\mathrm{kg} / \mathrm{m}^{3}\right)$

$k \quad$ cake permeability $\left(\mathrm{m}^{2}\right)$

L filter cake depth (m)

n cake compressibility

$p \quad$ pressure drop across the collected filter cake and filter medium (psi)

q volumetric flow rate of the filtrate

$q \quad$ instantaneous rate, $\mathrm{d} v / \mathrm{d} t(\mathrm{~m} / \mathrm{sec})$

$q_{\mathrm{av}} \quad$ average rate, $v / t(\mathrm{~m} / \mathrm{sec})$

$\mathrm{R}$ total resistance in filtration $(1 / \mathrm{m})$

$\mathrm{R}_{\mathrm{c}} \quad$ cake resistance $(1 / \mathrm{m})$

$\mathrm{R}_{\mathrm{m}} \quad$ medium resistance $(1 / \mathrm{m})$

$t \quad$ the time since the start of filtration (sec)

$v \quad$ filtrate volume collected at time $t$ per unit area of filtration $\left(\mathrm{m}^{3} / \mathrm{m}^{2}\right)$

$\mathrm{V}$ total liquid filtrate volume collected at time $t\left(\mathrm{~m}^{3}\right)$

$\mathrm{w}_{\mathrm{c}} \quad$ total mass of dry cake solids per unit area of filter surface $\left(\mathrm{kg} / \mathrm{m}^{2}\right)$ 


\section{Physical Property Greek Letters}

$\tau \quad$ shear stress, $\mathrm{Pa}$

$\mu \quad$ viscosity, poise

$\rho_{1} \quad$ density of fluid (liquid)

$\rho_{\mathrm{s}} \quad$ density of particle (solid)

$\omega$ fall velocity

$\omega_{\mathrm{n}} \quad$ natural grains settling velocity

$\omega_{\mathrm{s}} \quad$ disc settling velocity

\section{Cake Filtration Greek Letters}

$\alpha \quad$ specific resistance of filtration $(\mathrm{m} / \mathrm{kg})$

$\alpha_{0} \quad$ initial specific resistance of filtration $(\mathrm{m} / \mathrm{kg})$

$\alpha_{\mathrm{av}} \quad$ average specific resistance of filtration $(\mathrm{m} / \mathrm{kg})$

$\mu \quad$ liquid filtrate viscosity $(\mathrm{kg} / \mathrm{m} \cdot \mathrm{sec})$ 


\section{INTEC SBW Solid Sludge Surrogate Recipe and Validation}

\section{INTRODUCTION/BACKGROUND}

As a consequence of reprocessing spent U.S. Government nuclear reactor fuel for over 40 years, The Department of Energy's (DOE's) Idaho National Engineering and Environmental Laboratory (INEEL) has generated a legacy of $\sim 1,000,000$ gallons of radioactive sodium-bearing waste (SBW). The waste is presently stored in three underground storage tanks at INEEL's Idaho Nuclear Technology and Engineering Center (INTEC). As a result of the tanks' noncompliance to the Environmental Protection Agency's Resource Conservation and Recovery Act (RCRA) requirements for compatible secondary containment, as well as the location of the tank waste above the Idaho Snake River Aquifer, various state and national regulatory bodies have negotiated with DOE to remove and successfully treat and dispose of this high profile waste inventory by 2012 . Collectively, this milestone, as well as other critical legacy INEEL waste treatment and disposal milestones, is enforceable under a legally binding $1996 \mathrm{DOE} / \mathrm{State}$ of Idaho agreement.

Over the past ten years, DOE and its various contractors have investigated, tested, and evaluated a variety of treatment options (i.e., vitrification, calcination, direct solidification, actinide and fission product separation) for removing, immobilizing, and dispositioning the SBW. Current approaches include classifying this waste as "transuranic" (TRU) as opposed to "high level", and various legal and regulatory actions are ongoing by DOE to secure this TRU classification.

A determination that the SBW is transuranic would allow the waste to be disposed of at the DOE's Waste Isolation Pilot Plant (WIPP) site near Carlsbad, New Mexico. In anticipation of this classification, DOE's current contractor at the INEEL, Bechtel BWXT Idaho, LLC (BBWI), has conducted preliminary tests, systems engineering evaluations, and down-selection exercises to limit the pursued options to four treatment technologies. The final four technologies are presently as follows:

1. Use of an existing calciner to denitrite, volumetrically reduce, and solidify the waste, followed by the direct placement of the calcined solids in canisters for shipment to, and disposal at, WIPP as a remote-handled (RH)-TRU waste

2. Development, design, construction, and deployment of a new steam reformer to denitrite, volumetrically reduce, and solidify the waste, followed by the direct placement of the reformed solids in canisters for shipment to, and disposal at, WIPP as an RH-TRU waste

3. Development, design, construction, and deployment of a nonthermal, benign processing method using filtration and Cesium Ion Exchange (CsIX) to partition the SBW into a large volume of solidified, contact-handled (CH)-TRU waste and a small volume of RH-TRU waste. The large amount of $\mathrm{CH}$ waste and the smaller amount of $\mathrm{RH}$ waste would be packaged in canisters and shipped to WIPP for disposal.

4. Development, design, construction, and deployment of a direct evaporation method to substantially reduce the waste volume and inherently solidify, upon cooling, the evaporator residue for shipment to, and disposal at, WIPP as a RH-TRU waste.

To enable a final DOE decision, as well as support feasibility studies and conceptual design, BBWI developed a technology development plan outlining the risk-based issues associated with each of the four options as well as a development and testing strategy for addressing the risks. 
All of the four candidate technologies will require an accurate and working knowledge of the SBW tank farm heel solids, since these solids make up approximately $2 \%$ of the total waste volume. The majority of the solids are concentrated in tank WM-187, where the solids volume percentage is approximately 5\%. A high process failure risk has been associated with these solids, since they have been hard to simulate in the past and a precise and accurate solids surrogate is needed for development of many aspects of each of the four technologies.

This report describes collection of physical property data of an SBW tank farm waste sample and a metathesis-prepared SBW solids surrogate based on a formulation developed in fiscal year (FY)-03 (Janikowski 2003). Comparison of actual and simulated solids physical properties will provide a quantitative measure of how accurately the solids surrogate represents actual SBW tank waste. Following verification of similar surrogate and actual waste physical properties, the surrogate will be made available for use in associated heel solids transferring, mixing, and processing tests. 


\section{SURROGATE DEVELOPMENT AND PREPARATION}

\subsection{Rationale for Surrogate Development}

The INTEC tank farm sludges are complex mixtures of unknowns. The sludges consist of small particles. The conditions under which the sludges were formed varied from tank to tank and there is a large variety of elements present in different concentrations between the tanks. Those conditions made duplicating the sludge a difficult and uncertain task. Early attempts to make surrogate sludges used the conventional method of mixing together commercially available solid chemicals and minerals so the final elemental composition would be similar to the waste sludges. The analytical results (elemental analysis, anion analysis, and particle size) from WM-182 and WM-183 were averaged to generate a target set of values for the surrogate. This "first generation" surrogate had a reasonably close elemental balance and particle size, but did not exhibit many of the other physical properties needed to adequately test control technologies that would be used later to move and treat the waste sludges.

A "second generation" surrogate was formulated that incorporated metathesis reactions to generate solids from solutions of known elements present in the waste sludges. Early analyses of the tank sludge did not identify any of the mineral matter, nor did they reveal the true nature of the particles. Subsequent analyses revealed some of the mineral matter and the nature of the particles, making it desirable to modify the solid surrogate formulation and method of preparation. At the time of this undertaking, only one sample of a waste sludge was available for analysis and comparison testing, that from WM- 186 . Analytical data collected from that sample was subsequently used to formulate a new surrogate.

One option for preparing a new surrogate was to use metathesis reactions in the laboratory, but under conditions similar to those in the tank farm tanks. Metathesis reactions in this scenario are those reactions that occur in aqueous solution between cations and anions, which result in the formation of solids (precipitates and suspended solids). The overall scheme was to (1) prepare an aqueous acidic solution of soluble compounds, and (2) to subsequently add solutions containing ions and compounds that when mixed with the first solution would cause solids to form. This approach was used to produce solid surrogate for several tests and was modified slightly between subsequent preparations.

Chemicals used in the preparation of the solid surrogate were chosen to:

1. Be representative of those present in the tank farm wastes

2. Contain no hazardous elements or compounds

3. Be nonhazardous in their final form

4. Be removed with rinsing prior to scale-up testing.

These conditions could not be met rigorously, since hazards are defined to minimal levels. However, the hazards were minimized as much as possible. As such, no hazardous metals were used, and the acids could be washed away after the solid surrogate was formed. There are hazards associated with some of the aluminum compounds formed, and the fine particle size of the surrogate presents a respiratory hazard when the material is dry. These hazards were unavoidable; the tank farm wastes have these chemicals/properties and they contribute significantly to the chemical and physical behavior of the wastes, as they similarly contribute to the chemical and physical properties of the surrogate. 


\subsection{Preparation of Solid Surrogate}

The synthetic scheme to make a surrogate was based on the early analysis of a sample of WM-186 sludge and several assumptions, listed below.

1. WM-186 sludge would be representative of all the tank farm sludges. The analysis of the WM-186 sample would be representative of the entire contents of the tank and, thus, all the waste sludges from the tank farm tanks. Representative in this sense is a loosely defined term. The elemental composition of this sludge is not the same throughout the tank nor as any other sludge. Neither does it compare closely to a calculated average composition for all of the sludges. Rather, a surrogate made to approximate the composition of the WM-186 sludge using metathesis reactions would yield a product that would behave chemically and physically similar to any of the waste sludges under the performance tests that were to be conducted.

2. All of the major mineral components associated with the elements of interest (i.e., those contributing to the chemical and physical properties) in the waste sludges could be made by metathesis reactions in the lab; further, they would precipitate in a form comparable to the actual waste sludges. This is a very crude assumption in that the majority of elements present in the waste sludges were not incorporated into the surrogate, and the time of formation was short in comparison to the $30+$ years the waste sludges were stored. Regardless, the assumption was that the major types of species would be made (e.g., silica in many of its hydrated forms would be made) and their behavior would be sufficiently similar to the waste sludges to allow for meaningful comparisons.

3. Most of the metals and non-nitrate anions would form insoluble mineral phases on a short time scale. Solubility products for the anticipated reactions were known in neutral aqueous solutions, but not in highly acidic solutions. This made the results quite tentative.

4. The resulting surrogate would have similar chemical formulation, chemical properties, and physical properties to the tank farm sludges. Despite the shortcomings of the assumptions, this approach offered a potentially better synthetic route to a surrogate than previous methods.

Technical and reagent grade chemicals were used to prepare the solid surrogate. They are shown in Table 2-1 with the approximate amount used to generate $2 \mathrm{~kg}$ of solid surrogate.

Three solutions were prepared with water added as necessary to dissolve the compounds. Solution 1 was mixed in a 12-L 3-neck round-bottom flask and heated to $50{ }^{\circ} \mathrm{C}$. Solutions 2 and 3 were simultaneously added to the round-bottom flask containing Solution 1, over a period ranging from $1 / 2$ hour to 1 hour. The solution was stirred constantly using a magnetic stirrer while the additions were made.

Precipitates formed immediately, and stirring was discontinued upon complete addition of all chemicals to the round-bottom flask. The mixture was allowed to stand over night; after which, it was filtered under vacuum in a Buchner funnel using Whatman No. 42 filter paper. Portions of the solid surrogate were washed with a water rinse, or other solution, for subsequent analyses. The bulk of the material was not rinsed and was later used in the various physical tests reported herein. 
Table 2-1. Solid surrogate makeup solutions.

\begin{tabular}{|c|c|c|c|}
\hline \multirow[t]{11}{*}{ Solution 1} & Chemical Added & Chemical Supplier & $\begin{array}{l}\text { Amount added to make } 2 \mathrm{~kg} \\
\text { solid surrogate (dry weight } \\
\text { of surrogate basis) }\end{array}$ \\
\hline & $\mathrm{Al}\left(\mathrm{NO}_{3}\right)_{3} \cdot 9 \mathrm{H}_{2} \mathrm{O}$ & $\begin{array}{l}\text { Sigma-Aldrich } 98+\% \text { ACS } \\
\text { Reagent }\end{array}$ & $1504 \mathrm{~g}$ \\
\hline & $\mathrm{ZrO}\left(\mathrm{NO}_{3}\right)_{2} \cdot \mathrm{H}_{2} \mathrm{O}$ & Aldrich & $243 \mathrm{~g}$ \\
\hline & $\mathrm{Ca}\left(\mathrm{NO}_{3}\right)_{2} \cdot 4 \mathrm{H}_{2} \mathrm{O}$ & $\begin{array}{l}\text { Sigma-Aldrich } 99 \% \text { ACS } \\
\text { Reagent }\end{array}$ & $141 \mathrm{~g}$ \\
\hline & $\mathrm{Fe}\left(\mathrm{NO}_{3}\right)_{2} \cdot 9 \mathrm{H}_{2} \mathrm{O}$ & Aldrich $98+\%$ ACS Reagent & $141 \mathrm{~g}$ \\
\hline & $\mathrm{Mn}\left(\mathrm{NO}_{3}\right)_{2} \cdot \mathrm{H}_{2} \mathrm{O}$ & Aldrich 98\% & $32 \mathrm{~g}$ \\
\hline & $\mathrm{Mg}\left(\mathrm{NO}_{3}\right)_{2} \cdot 6 \mathrm{H}_{2} \mathrm{O}$ & Aldrich 99\% ACS Reagent & $97 \mathrm{~g}$ \\
\hline & $\mathrm{SnCl}_{2} \cdot 2 \mathrm{H}_{2} \mathrm{O}$ & Fisher Certified ACS & $97 \mathrm{~g}$ \\
\hline & $\mathrm{NaF}$ & C. P. Baker's Analyzed & $10 \mathrm{~g}$ \\
\hline & $\mathrm{HNO}_{3}(70 \%)$ & Fisher ACS & $825 \mathrm{~mL}$ \\
\hline & Water & Tap water & up to ca. $3.5 \mathrm{~L}$ \\
\hline Solution 2 & $27 \% \mathrm{SiO}_{2}$ in $14 \% \mathrm{NaOH}$ & Aldrich & $68 \mathrm{~mL}$ \\
\hline \multirow[t]{3}{*}{ Solution 3} & $\mathrm{H}_{2} \mathrm{SO}_{4}\left(95-98 w \mathrm{w}^{\%} \%\right)$ & Fisher Certified ACS Plus & $2.4 \mathrm{~mL}$ \\
\hline & $\mathrm{H}_{3} \mathrm{PO}_{4}(85 \mathrm{wt} \%)$ & Aldrich ACS Reagent & $10.4 \mathrm{~mL}$ \\
\hline & Water & Tap water & $50 \mathrm{~mL}$ \\
\hline
\end{tabular}

\subsection{Results and Discussion}

The assumptions for making the surrogate described earlier were met to a limited degree individually, and to a greater degree overall. Table 2-2 shows the highest concentration element in the surrogate to be silicon. Further analyses revealed that it existed as silica. The washed sample from WM-186 had equal amounts of silicon and zirconium with an appreciable amount of aluminum. The WM-187 sample had primarily zirconium compounds with appreciable amounts of tin and titanium.

The metathesis reactions were largely incomplete under the acidic conditions. Silica was produced at a significantly higher concentration; the results of which are skewed due to the incomplete metathesis formation of other solids and, hence, less solid formation overall. Iron, tin, and to a lesser degree zirconium precipitated in relatively close concentration to the initial target. The aluminum, calcium, magnesium, and manganese precipitated in vastly lower concentrations than expected. These findings appear to exert little influence on the behavior of the surrogate and the formulation was not changed. However, there are several ways the surrogate synthesis could be changed to yield a surrogate with a chemical composition closer to the waste sludge, if a closer representation was required.

The composition of the surrogate could be changed by conducting the synthesis in slightly acidic or neutral solutions. Under these conditions, the metals would precipitate with behavior more characteristic of the known solubility products. The $\mathrm{pH}$ could be lowered later to influence mineral 


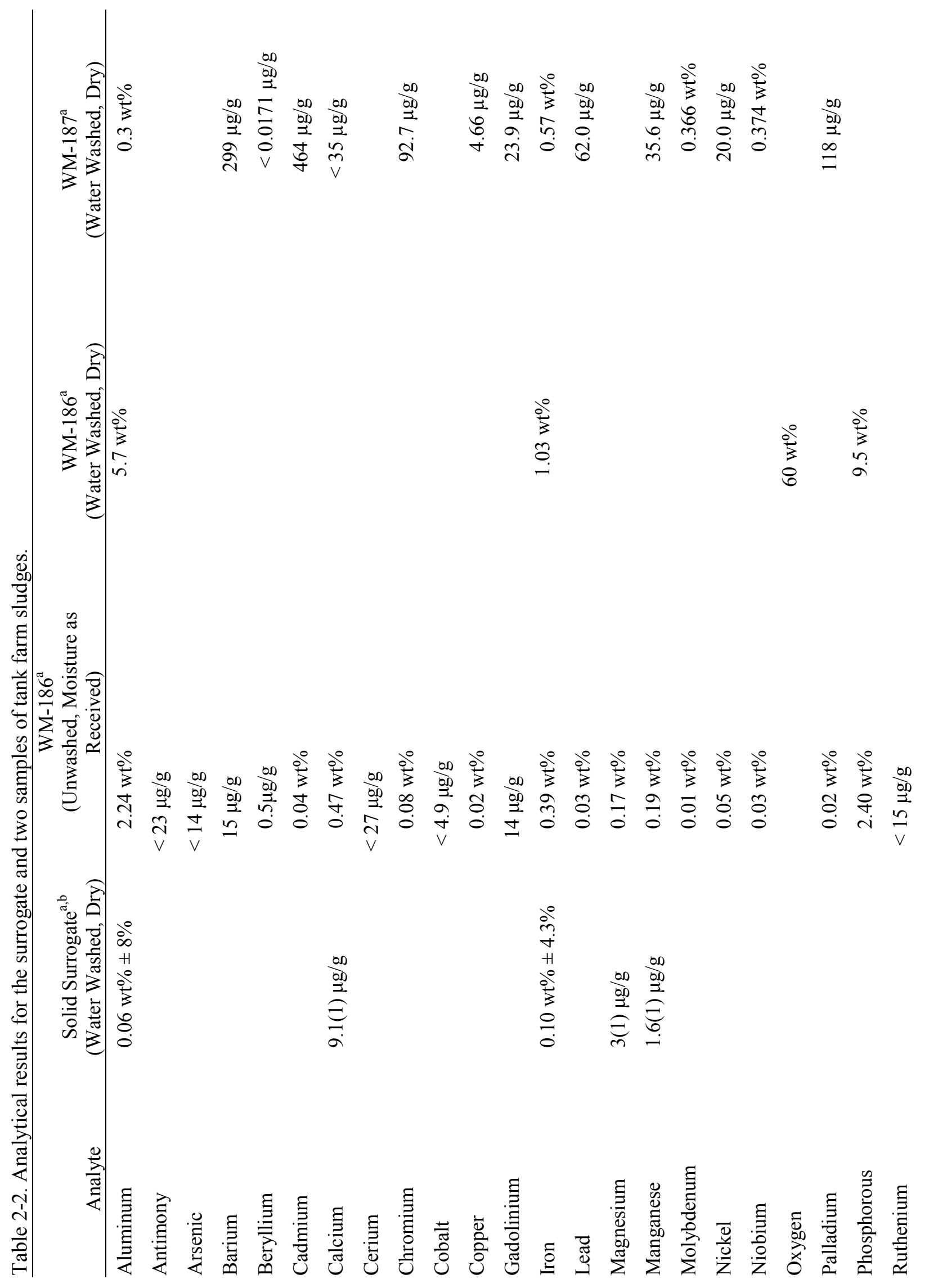




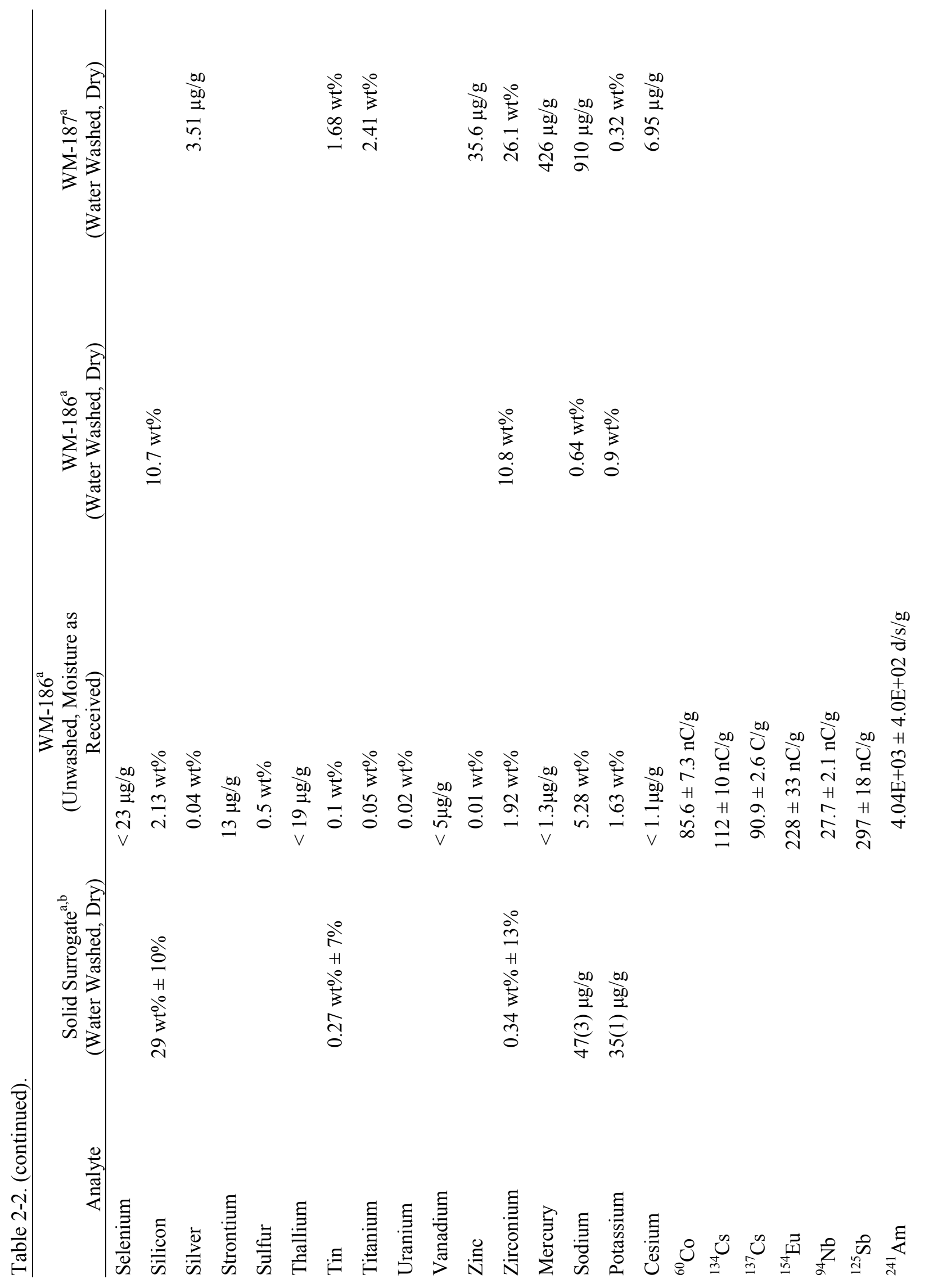




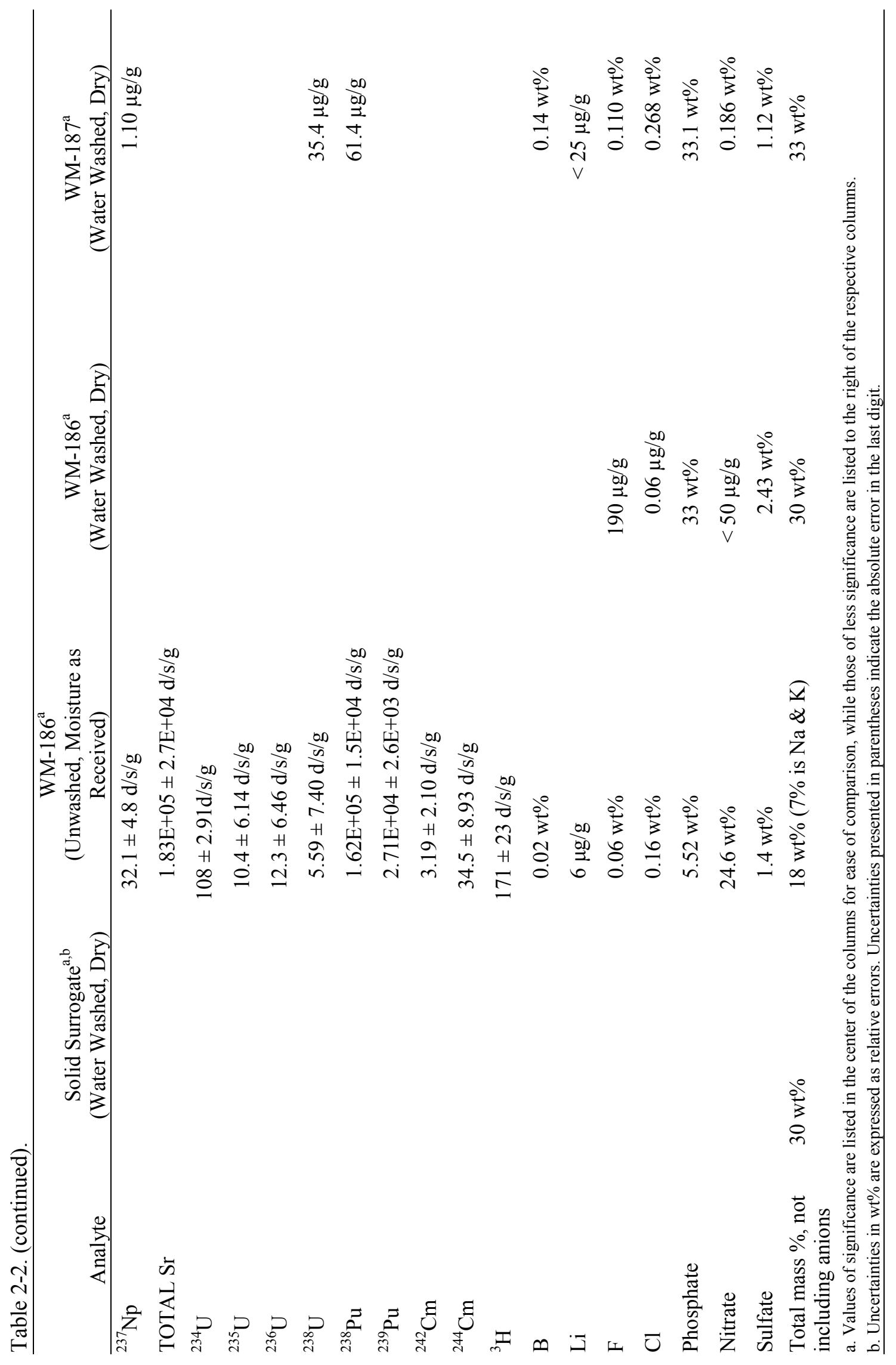


formation characteristic of the highly acidic waste sludges. This is a plausible synthesis scheme and reflects the actual historic conditions of the tanks. Many acidic solutions were discharged directly into the tanks, but other solutions of near-neutral $\mathrm{pH}$ were pumped into the tanks during cleanup activities, where large relative volumes of rinse water accompanied the contaminants. Subsequent

evaporation/concentration of the supernatant resulted in the characteristic highly acidic solutions that the sludges sat in for years. 


\section{PHYSICAL PROPERTIES}

\subsection{Strategy}

The objective of the testing described in this report is to provide measurement of physical properties of the metathesis-prepared SBW surrogate and the solid waste heel sample collected from INTEC tank WM-187 for purposes of comparison. In this document, slurry is defined as a mixture comprised of a solid and a liquid phase. The liquid phase may contain suspended or dissolved solids. Solid phase material with only interstitial liquid present (no free liquid) is referred to as sludge.

According to Heywood (Heywood 1999), the first stage in developing a slurry handling system is to measure the relevant physical properties of the slurry. Variables Heywood recommends measuring at the laboratory scale include:

- $\quad$ Flow curve (viscosity as function of shear rate)

- $\quad$ Slurry density

- $\quad$ Slurry solids content

- $\quad$ Particle size/size distribution

- $\quad$ Settling rates.

Heywood recommends performing these analyses on the best available representative sample of the slurry as well as on samples with varying formulations (higher than average solids concentration, shifts in particle size distribution, etc.) to provide data needed to design the slurry handling system (transfer, mixing, etc.) such that it can manage unexpected changes in process feed or process operating conditions.

Selected physical property measurements suggested by Heywood were performed on the waste surrogate and WM-187 waste samples. In addition to the slurry flow curve, flow curves for the liquid and sludge phases were obtained. The bulk density of the liquid, wet sludge (sludge with interstitial liquid), and dried sludge (interstitial liquid evaporated) was measured. The moisture percentage of the settled sludge phase was determined. Additionally, waste surrogate and waste sample $\mathrm{pH}$ analyses were performed. Methods and equipment used in these analyses are described in Section 3.2.

Only one rheometer was available for waste surrogate and WM-187 waste sample flow curve analysis. In order to collect WM-187 waste sample flow curve data, the viscometer had to be transferred to the INTEC Remote Analytical Laboratory (RAL). Since equipment placed in the RAL is immediately contaminated, all waste surrogate flow curve measurements had to be completed prior to performing the first WM-187 waste sample analysis in the RAL. In order to increase the probability of finding a waste surrogate with similar flow curve properties to the WM-187 waste sample, a range of differently prepared surrogates was tested prior to recording any WM-187 waste sample flow curve data. Waste surrogate and WM-187 waste sample flow curves with similar profiles are compared in Section 3.4.2.1. Flow curve measurements for each sample were repeated until the flow curve profile ceased changing to ensure data integrity and complete thixotropy measurement. Complete flow curve data is included in Appendix E of this report (grayed-out flow curve data in Appendix E represent data outside of the operating tolerances of the rheometer and are included for reference only). 


\subsection{Apparatus}

\subsubsection{Particle Size Distribution}

3.2.1.1 SBW Surrogate. SBW solids surrogate sample particle size distribution analysis was performed using a Microtrac FRA particle size distribution (PSD) analyzer. Samples for select physical property analyses (flow curve, settling velocity, PSD) were sonicated with a Fisher Scientific Model 500 sonic dismembrator probe prior to analysis. The samples were circulated through the sonication chamber and an external cold water bath using a Cole Parmer Masterflex Model L/S peristaltic pump to prevent excessive sample heating during sonication.

Figure 3-1 is a photograph of the Fisher Scientific Model 500 sonic dismembrator. The sonication probe protrudes through the top of the soundproof enclosure. The stir plate and scissor lift in the bottom of the soundproof enclosure were used to stir the sample to ensure uniform particle sonication and elevate the sample to a level where it was in contact with the sonication probe, respectively.

Figure 3-2 is a photograph of the Microtrac FRA particle size distribution analyzer.

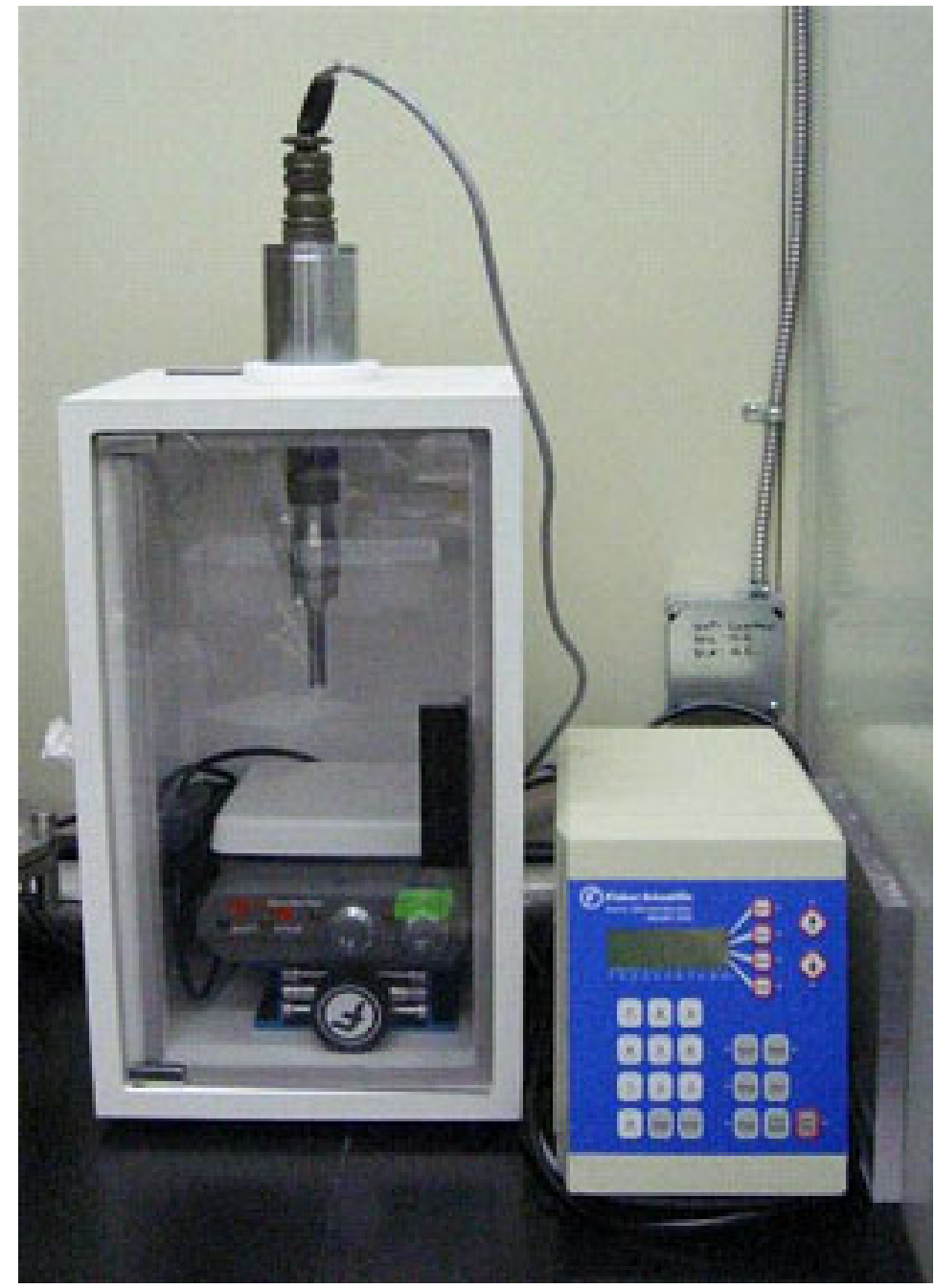

Figure 3-1. Fisher Scientific Model 500 sonic dismembrator, soundproof enclosure, stir plate, and scissor lift. 


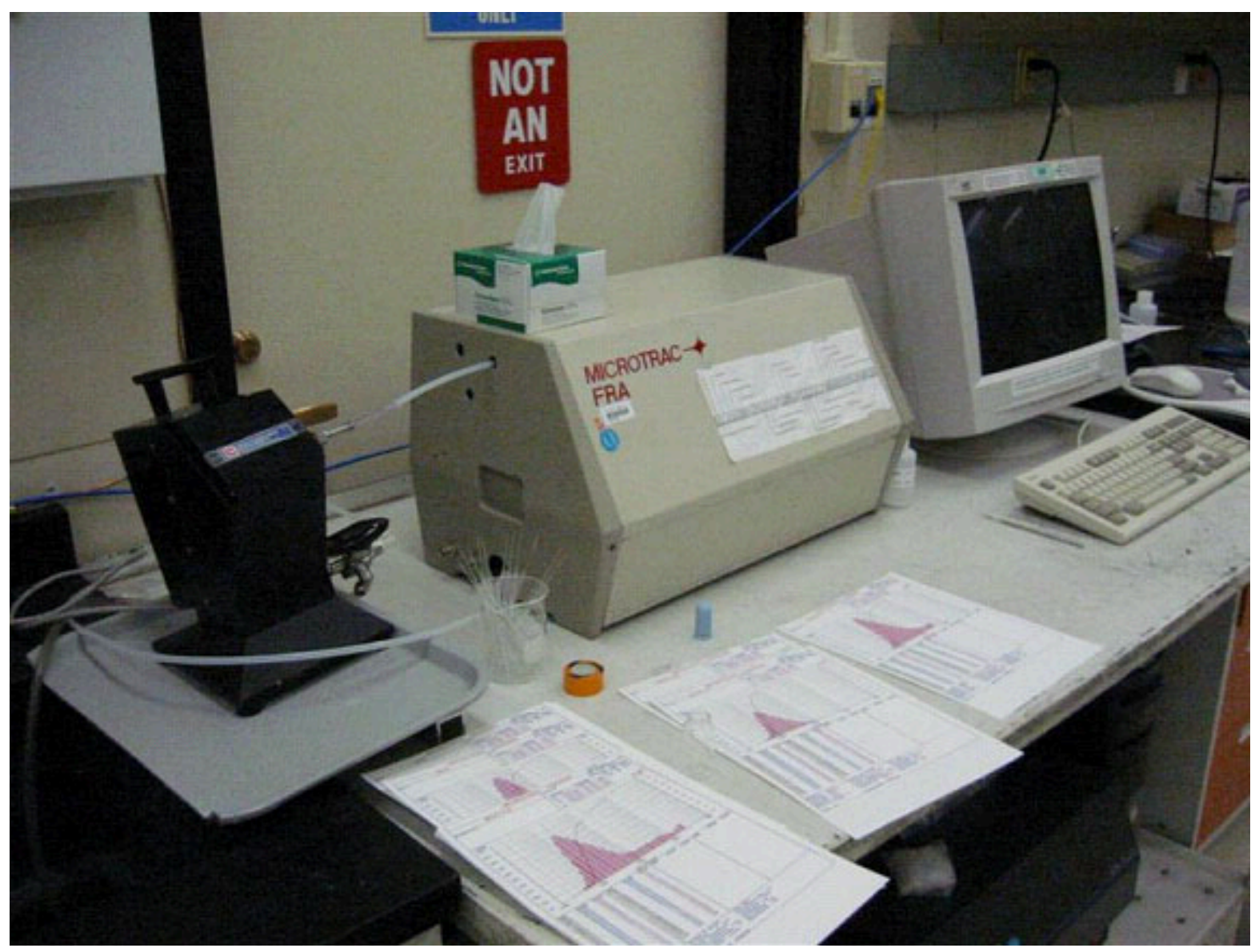

Figure 3-2. Microtrac FRA particle size distribution analyzer.

3.2.1.2 Tank Farm Sample. The first WM-187 sample (containing WM-182 and WM-183 solids) was analyzed with a Horiba LA-300 particle size analyzer. This was done in the RAL hot cell. Both nonsonicated and sonicated results were obtained.

\subsubsection{Flow Curve}

3.2.2.1 SBW Surrogate. A Brookfield Engineering R/S rheometer (INEEL Standards and Calibration Laboratory (S\&CL) ID\# 721776, expiration 01/20/05) with a Brookfield Engineering FTKY water jacket and PT-100 temperature probe was used to record sample flow curves. A Julabo F25 circulating constant temperature bath with a Julabo MD control unit was plumbed to the rheometer water jacket and used to control the sample temperature. The rheometer and circulating constant temperature bath were placed on one of the stainless steel workbenches located in the North Yellowstone Complex (NYC) Laboratory secondary containment area. A localized ventilation system air evacuation port was placed in close proximity to the rheometer sample measuring chamber to vent fumes emitted by the surrogate samples. A computer workstation with Brookfield RHEO 2000 control and data analysis software was positioned immediately outside the spill containment area. Communications between the $\mathrm{R} / \mathrm{S}$ rheometer and computer workstation were transmitted via a vendor-supplied RS-232 data cable.

The R/S rheometer is depicted in Figure 3-3 with the FTKY water jacket, MB-45/48F measuring chamber, and CC-45 spindle installed. Note also the temperature probe and heat transfer fluid tubing (bottom port is heat transfer fluid inlet, top port is heat transfer fluid outlet) connected to the right-hand side of the water jacket. 


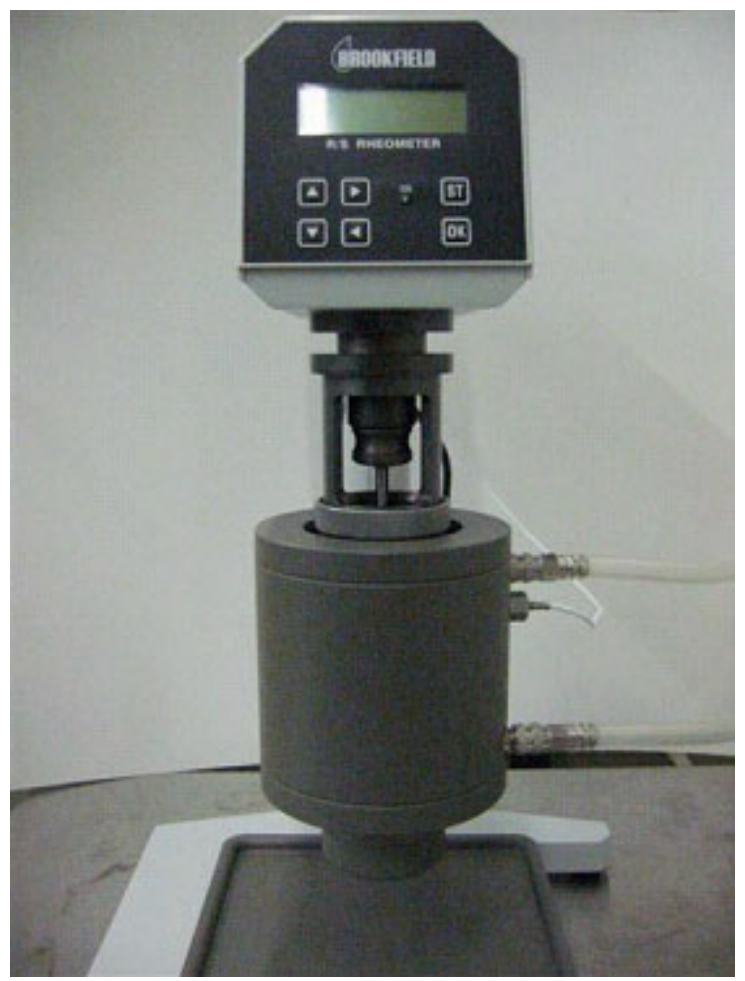

Figure 3-3. R/S rheometer, FTKY water jacket, MB-45/48F measuring chamber, and CC-45 spindle.

Detailed views of the R/S rheometer hardware accessories are shown in Figure 3-4 through Figure 3-9. Figure 3-4 is a photograph of the CC-DG ("Double Gap"), CC-48 (48 mm spindle outside diameter [OD]), and CC-45 (45 mm spindle OD) spindles. The CC-DG spindle is hollow and open on the bottom end while the CC-48 and CC-45 spindles are solid. The CC-48 and CC-45 spindles are used with the MB-45/48F measuring chamber and the CC-DG spindle is used with the MB-DGF measuring chamber. The MB-45/48F and MB-DGF measuring chambers are shown in Figure 3-5. When using the MB-45/48F measuring chamber, the sample is placed into the chamber and the spindle is immersed in the sample. When using the MB-DGF measuring chamber, the sample is placed in the annular gap between the measuring chamber outer and inner concentric cylinders and the hollow, cylindrical CC-DG spindle is then inserted into the annular gap with the sample.

Figure 3-6 and Figure 3-7 are photographs of the MB-45/48F measuring cup and CC-45 spindle pairing, and MB-DGF and CC-DG spindle pairing, respectively. The two fittings at the base of the MBDGF measuring chamber are ports through which heat transfer fluid can circulate (through the inner concentric cylinder of the measuring chamber to provide sample temperature control). Figure 3-8 and Figure 3-9 are top views showing the volume occupied by the sample in the MB-45/48F and MB-DGF measuring chambers, respectively. In the MB-45/48F measuring chamber, the sample occupies the full cylindrical volume inside the measuring chamber while in the MB-DGF measuring chamber, the sample occupies only the annular gap between the inner and outer concentric cylinders. 

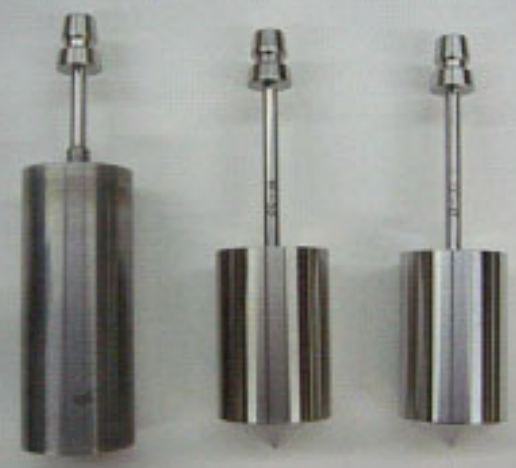

Figure 3-4. CC-DG, CC-48, and CC-45 spindles.

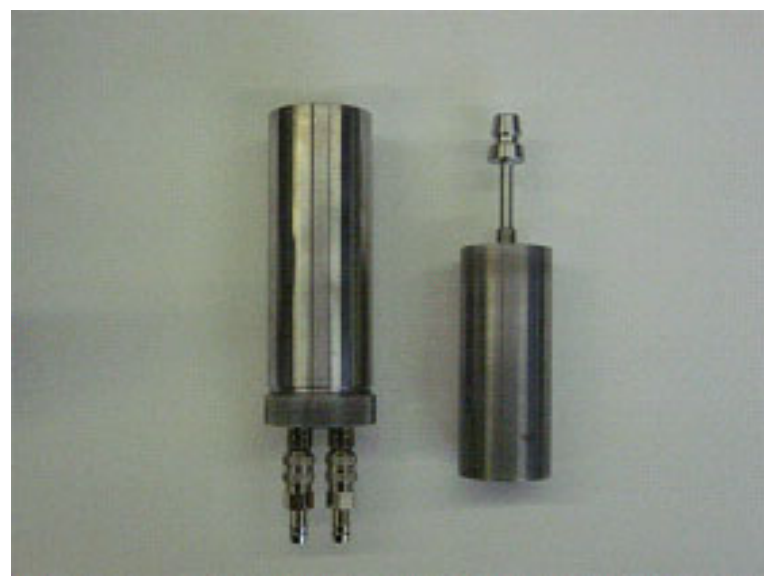

Figure 3-6. MB-45/48F measuring cup and CC-45 spindle.

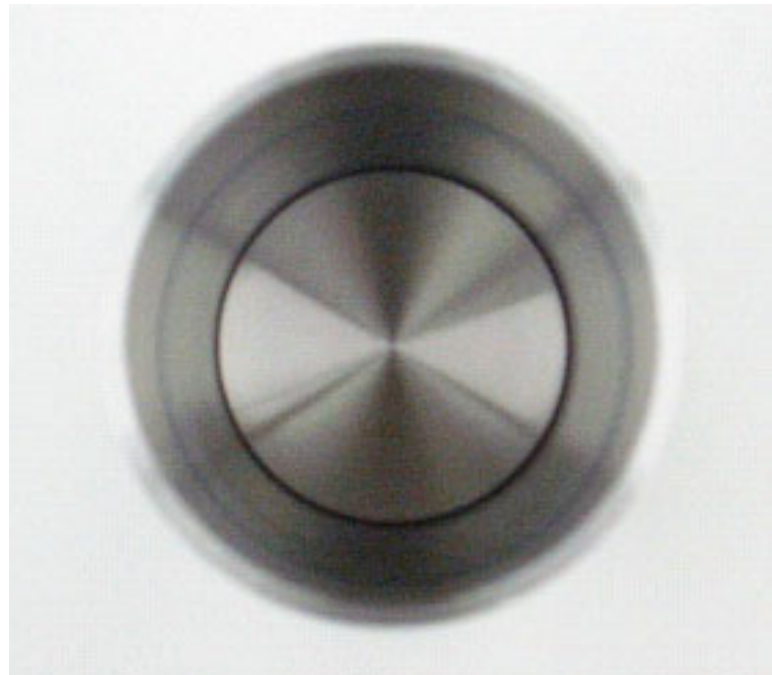

Figure 3-8. MB-45/48F measuring chamber, top view.

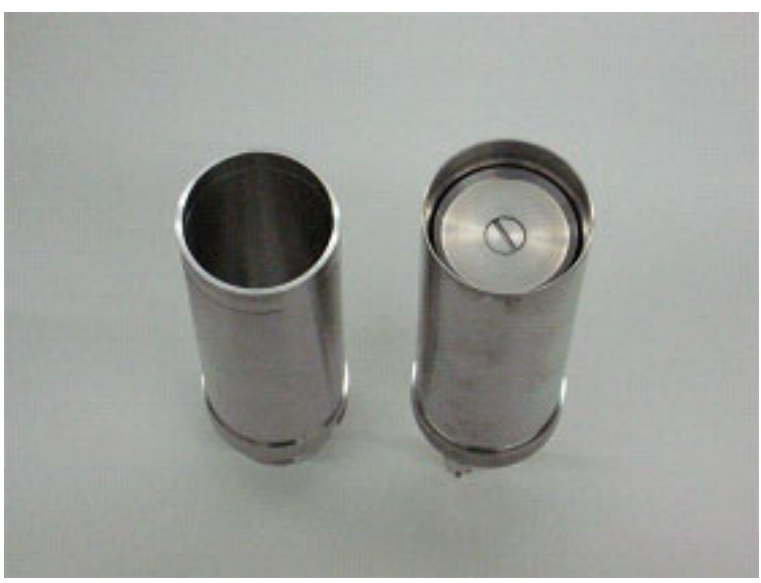

Figure 3-5. MB-45/48F and MB-DGF measuring chambers.

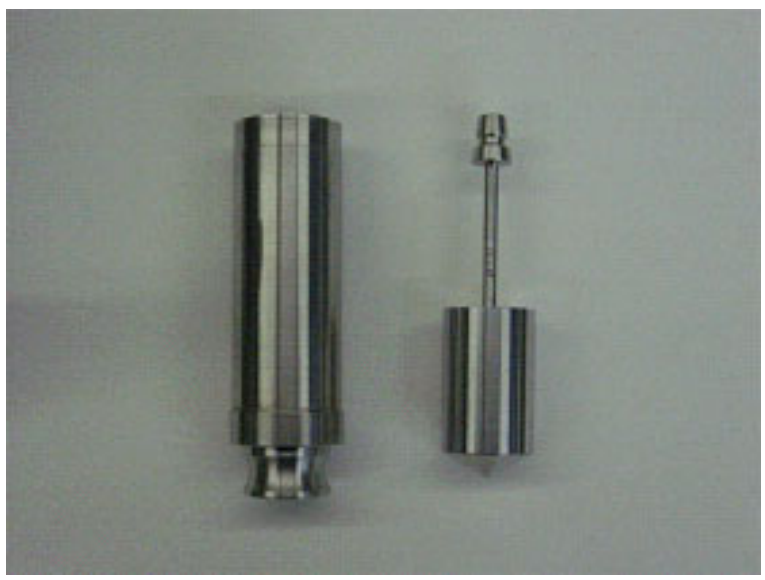

Figure 3-7. MB-DGF measuring system and CC-DG spindle.

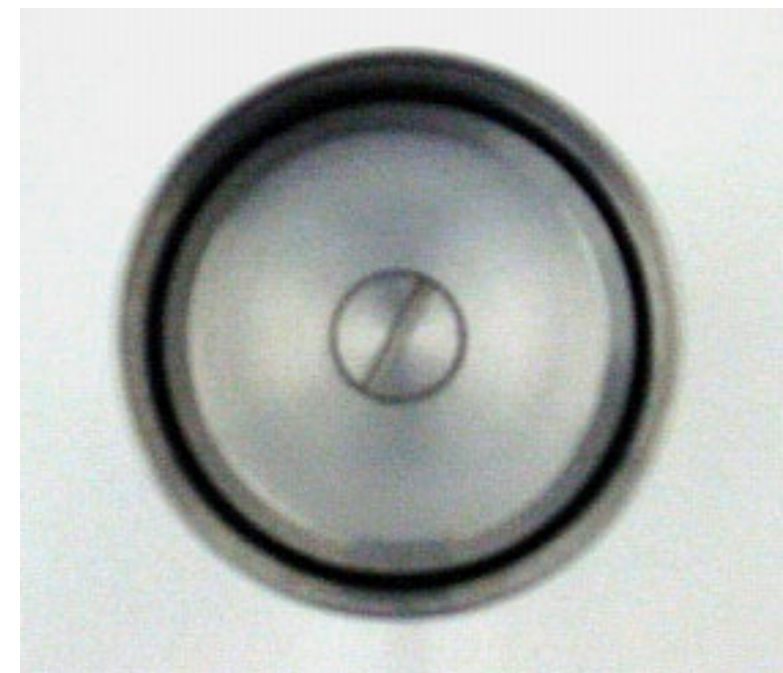

Figure 3-9. MB-DGF measuring chamber, top view. 
Figure 3-10 through Figure 3-12 illustrate the steps required to properly assemble the water jacket, measuring chamber, and spindle onto the R/S rheometer base unit. In Figure 3-10, the spindle attachment fitting collar is raised to the "open" position to allow insertion of the spindle base (note the red painted ring around the rheometer hub). The measuring chamber is inserted into the base of the water jacket and secured with a threaded plastic support (step not shown). The required volume of the sample to be analyzed is then added to the measuring chamber (step not shown). Once the sample has been added to the measuring chamber, the spindle is carefully immersed in the sample (step not shown). The water jacket, measuring chamber, spindle, and sample assembly are secured to the $\mathrm{R} / \mathrm{S}$ rheometer by threading the connecting collar on the water jacket to the threaded support structure on the rheometer base as shown in Figure 3-11. The spindle is secured by inserting the spindle base into the rheometer hub and sliding the spindle attachment fitting collar down into the "closed" position as shown in Figure 3-12.

3.2.2.2 Tank Farm SBW Sample. Prior to placing the Brookfield R/S rheometer into the INTEC RAL hot cell, several modifications to the rheometer hardware accessories were required to allow operating of the instrument with the hot cell mechanical manipulators. No circulating constant temperature bath is available in the hot cell, so inclusion of the FTKY water jacket in the experimental setup would have been superfluous. In addition, the FTKY water jacket was too large in diameter for the hot cell mechanical manipulators to grip. Modifications enabling direct connection of the MB- $45 / 48 \mathrm{~F}$ and MB-DGF measuring chambers to the R/S rheometer threaded support structure were completed. The modifications consisted of adding exterior support pins to the MB-45/48F and MB-DGF measuring chambers (see Figure 3-13 and Figure 3-14) and constructing an adapter (see Figure 3-15) to connect the measuring chambers to the rheometer threaded support structure. Additionally, fabrication of a spindle attachment collar tool (see Figure 3-16) was required to allow raising and lowering the collar using the mechanical manipulators. The R/S rheometer was connected to a computer workstation with the RHEO 2000 measuring/analysis software located outside of the hot cell using a RS-232 data cable that passed through the hot cell wall.

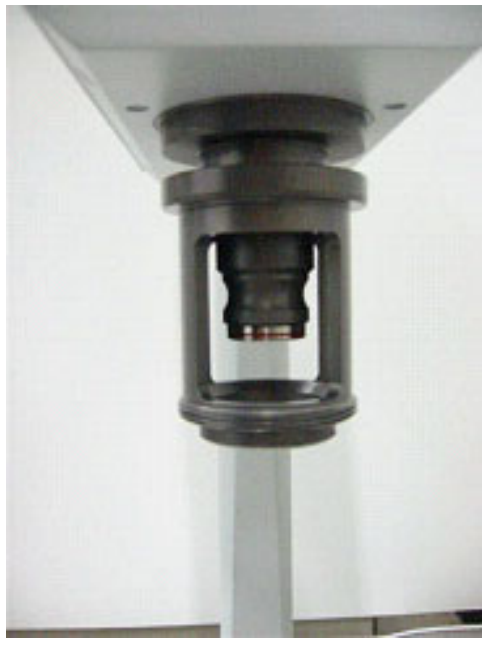

Figure 3-10. Assembly Step 1.

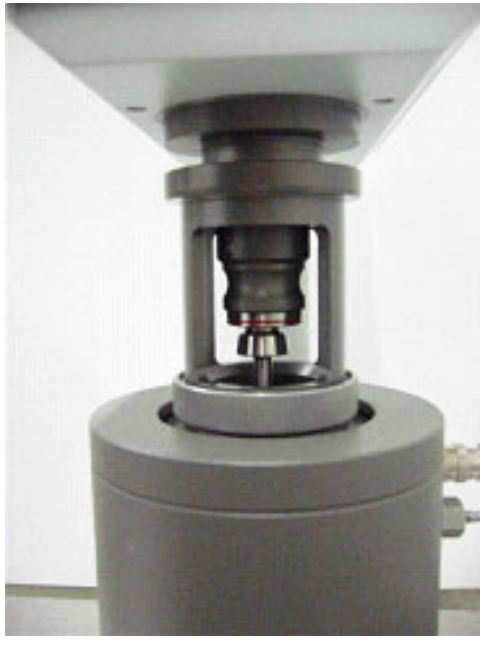

Figure 3-11. Assembly Step 2.

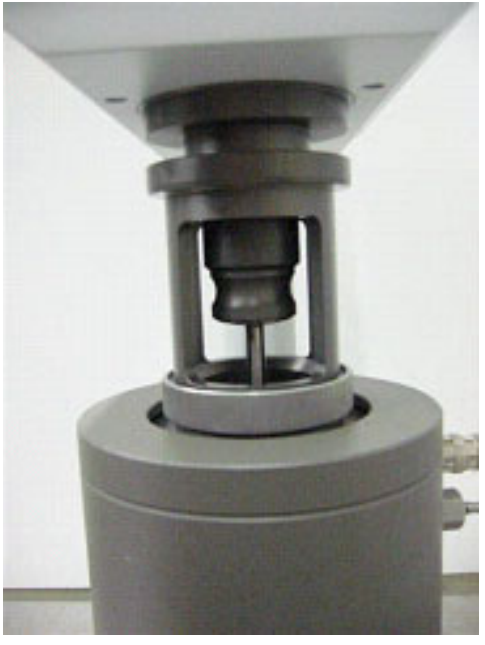

Figure 3-12. Assembly Step 3. 


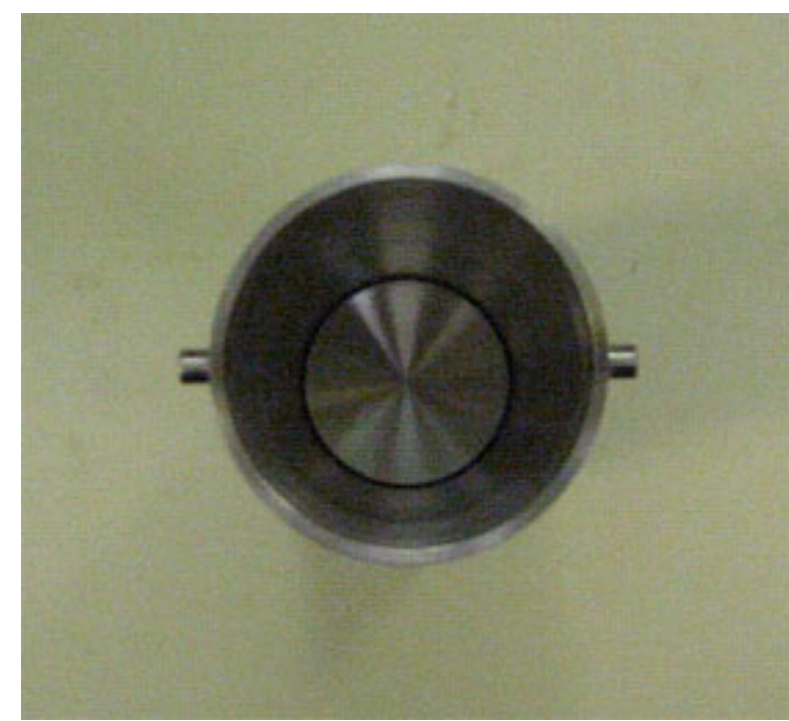

Figure 3-13. MB-45/48F machine shop hot cell modification top view.

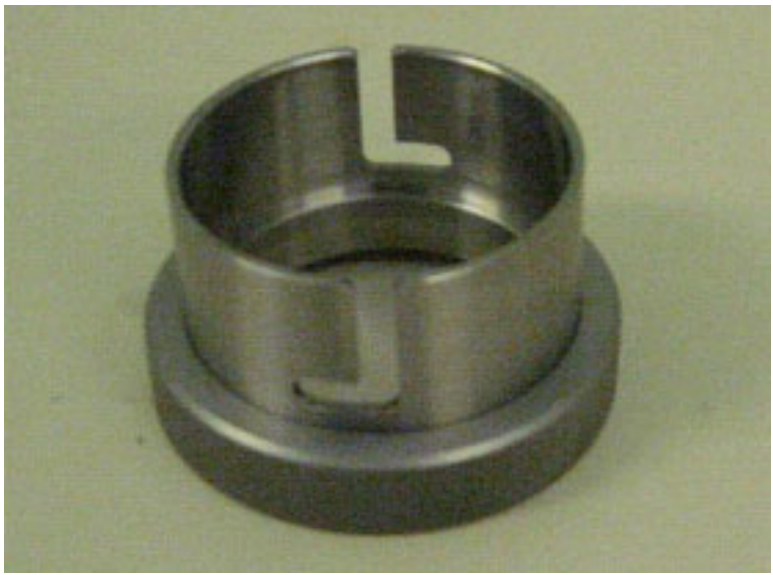

Figure 3-15. Adapter collar machine shop hot cell modifications.

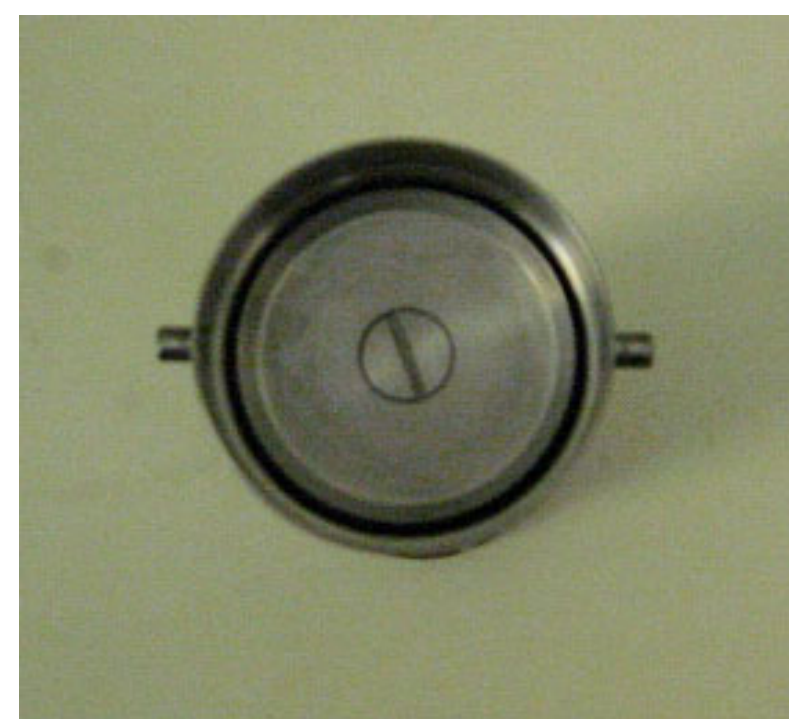

Figure 3-14. MB-DGF machine shop hot cell modification top view.

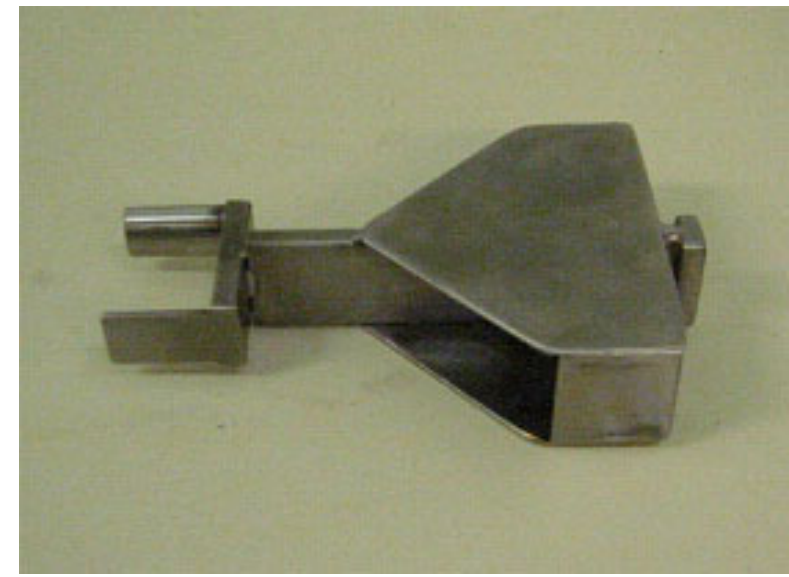

Figure 3-16. Manipulator spindle attachment tool.

Each rheometer measuring chamber and spindle pairing requires the adding of a specific volume of sample to the measuring chamber to bring the sample level to the measuring chamber fill line. Once the correct sample volume has been added to the measuring chamber, the spindle and measuring chamber were sequentially connected to the rheometer.

\subsubsection{Settling Velocity}

3.2.3.1 SBW Surrogate. Settling velocity was determined by measuring the solid-liquid interface position of a constant volume slurry sample in a glass settling chamber as a function of time. Glass settling chambers with two diameters were used. The first settling chamber was a $100-\mathrm{mL}$ graduated glass burette with a 14.3-mm inner diameter modified by the INEEL glass shop to have a sealed, flat bottom rather than a stopcock. A stand capable of holding two of the 100-mL graduate glass burettes was constructed. The second settling chamber was a $250-\mathrm{mL}$ glass graduated cylinder with a $31.8 \mathrm{~mm}$ inner 
diameter. Each of the settling chambers had a stopper that was used to seal the vessel during the experimental phase to prevent evaporation of the slurry sample.

Figure 3-17 is a photograph of the settling velocity apparatus. The settling chambers of the same volume contain different slurry samples in these photographs. Note the settled solids and liquid supernate interface in each settling chamber and the difference in interface position between the two $100-\mathrm{mL}$ settling chambers and the two $250-\mathrm{mL}$ settling chambers.

3.2.3.2 Tank Farm Sample. A small apparatus similar to that shown in Figure 3-17 was used to determine settling velocity for the WM-187 samples. A $250-\mathrm{mL}$ graduated cylinder was also used to determine the settling velocity. An "as received" tank farm waste sample was mixed and poured into the settling apparatus and allowed to settle. Times were noted as settling occurred.

\subsubsection{Solids Weight Percentage}

3.2.4.1 SBW Surrogate. Surrogate solids weight percentage was determined by drying slurry surrogate samples to constant mass. 120-mL evaporating dishes were weighed using a Mettler Toledo AB204-S analytical balance (INEEL S\&CL ID\# 716971, expiration 01/26/05) with four-place decimal accuracy. Approximately $60 \mathrm{~mL}$ of sample was placed in a dry evaporating dish and weighed. The samples were then placed in a Fisher Scientific Isotemp 500 Series drying oven set at approximately $105^{\circ} \mathrm{C}$ and weighed on a daily basis. When the sample weight ceased changing between weight measurements, the drying operation was stopped and the results were recorded.

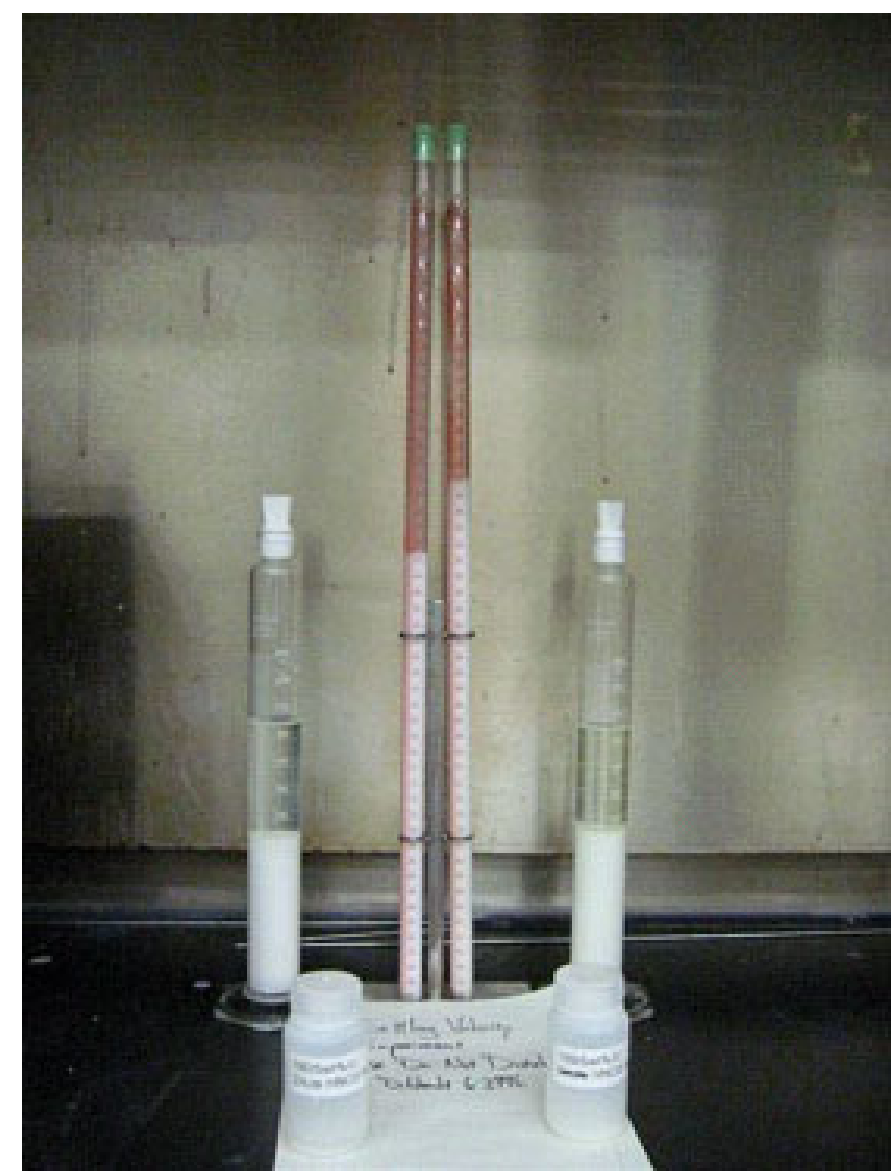

Figure 3-17. Settling Velocity Apparatus. 
Tank Farm SBW Sample. Tank farm waste sample solids weight percentage was determined using the same sample drying method used for determining surrogate solids weight percentage. The tank farm sample weight percentage analysis was performed at the INTEC RAL hot cell. Evaporating dishes were fabricated by the INEEL glass shop by cutting the base off $125-\mathrm{mL}$ glass Erlenmeyer flasks. The tare mass of each glass evaporating dish was recorded and written on each dish. The evaporating dishes were then placed in the RAL hot cell, filled with approximately $20 \mathrm{~mL}$ of sample, and the wet mass was recorded using a Mettler Toledo balance (INEEL S\&CL ID\# 714070, expiration 03/08/05). The samples were then placed in a drying oven set at approximately $100^{\circ} \mathrm{C}$ and dried for several days. When the sample mass stabilized between readings, the drying operation was stopped and the results were recorded.

\subsubsection{Bulk Density}

3.2.5.1 SBW Surrogate. Bulk density was determined by measuring the mass and volume of the various slurry samples. Sludge, liquid, and slurry samples were placed in a graduated cylinder of a known mass and the sample mass and volume were recorded. The graduated cylinders and samples were weighed using a Mettler Toledo AB204-S analytical balance (INEEL S\&CL ID\# 716971, expiration 01/26/05) with four place decimal accuracy. The graduated cylinders used in the analysis had 25 and $50 \mathrm{~mL}$ capacities.

3.2.5.2 Tank Farm SBW Sample. The sample used for bulk density was the third sample from the WM-187 tank. This is the first sample taken after solids from the following tanks were added to WM187: WM-181, WM-182, WM-183, WM-184, WM-185, and WM-186. These sampled solids are dark gray to black in color. They suspend easily when stirred or shaken. Drying and then resuspending the sampled solids? in water took a significant amount of shaking and mixing. Bulk density was determined by measuring the mass and volume of a washed and dried slurry sample. The bulk density analysis was performed in the RAL hot cell. An "as received" sample was placed in a 40-mL centrifuge tube and allowed to settle over night. As much of the liquid as possible was removed with a plastic transfer pipette after a day of settling. The solids were then washed twice with water and centrifuged after each wash. The remaining solids were then air dried. A mass was obtained using a Mettler PM1200 balance in the hot cell. A volume was read off the centrifuge tube and the density was calculated.

\subsection{Materials}

\subsubsection{SBW Solids Surrogate}

The SBW solids surrogate was prepared by INEEL chemist S. K. Janikowski using a metathesis formulation procedure. Two separate batches of the SBW solids surrogate were used in physical properties testing. The first batch consisted of surrogate prepared in FY-03 for testing in the Fundabac filtration process. A significant mass of the FY-03 SBW solids surrogate remained following completion of Fundabac filtration process testing. The unused FY-03 SBW solids surrogate was acquired for physical property analysis. The second batch of SBW solids surrogate was prepared in FY-04 specifically for physical and chemical properties analysis. The two surrogate batches differed in preparation in that the FY-03 batch was dried and mixed with WM-187 liquid surrogate in a 10 solids wt \% solution in preparation for Fundabac filtration testing. The FY-04 SBW solids surrogate batch was not dried and, following formulation, was retained in the metathesis mother liquor. Details of sample preparation activities are discussed in the following sections. 
Metathesis-prepared SBW solids surrogate samples were given sample identity tags. The tags, which were placed on each sample bottle immediately after sample collection, specify the sample liquid surrogate, solids weight percentage, metathesis-prepared SBW solids origination batch, and sonication history. The tag format is as follows:

[liquid] / [solids wt \%] / [solids batch] / [sonication].

Table 3-1 provides a complete listing of the sample composition/preparation classification categories in each unit of the sample identity tag.

Table 3-1. SBW solids surrogate identity tag format.

\begin{tabular}{clcl}
\hline [Liquid] & [Solids Wt \%] & [Solids Batch] & [Sonication] \\
\hline 180 & $0 \mathrm{wt} \%$ & $\mathrm{D}=$ dried & $\mathrm{N}=$ nonsonicated \\
187 & $5 \mathrm{wt} \%$ & $\mathrm{U}=$ undried & $\mathrm{S}=$ sonicated \\
189 & $10 \mathrm{wt} \%$ & & \\
META & $15 \mathrm{wt} \%$ & & \\
& $20 \mathrm{wt} \%$ & & \\
& $\mathrm{AR}=$ as received & & \\
& &
\end{tabular}

3.3.1.1 Dried Metathesis-Prepared SBW Solid Surrogate Preparation. "Dried metathesisprepared SBW solid surrogate" refers to the SBW solids surrogate initially used in FY-03 Fundabac filtration process testing. The FY-03 SBW solids surrogate was formulated using the metathesis preparation method of Janikowski. A 10 solids wt \% SBW -surrogate slurry was required for Fundabac filtration process testing. The solids produced by the metathesis preparation procedure were blended with liquid to the required slurry solids weight percentage via vacuum filtration and oven drying of the solids, followed by mixing with the requisite mass of WM-187 liquid surrogate. Approximately $12 \mathrm{~L}$ of 10 solids wt \% FY-03 SBW surrogate remained following completion of the Fundabac filtration process testing. The SBW surrogate slurry was allowed to settle for approximately six weeks before samples were collected for physical and chemical property analysis.

It was determined that Fundabac filtration testing may have altered the slurry solids wt $\%$ from the original 10 solids weight percentage mix. Additionally, physical property testing required slurries ranging from 5 to 20 solids wt \%. Therefore, the FY-03 dried metathesis-prepared SBW solid surrogate slurry was separated into liquid and sludge fractions, each fraction was analyzed for solids weight percentage, and the fractions were recombined into 5 and 20 -wt \% slurries.

The liquid fraction of the residual, settled filtration surrogate was collected from the 20-L storage carboy via decanting the clear WM-187 liquid surrogate into 4-L Nalgene sample bottles. Approximately $8 \mathrm{~L}$ of WM-187 liquid surrogate were collected. The solids remaining in the bottom of the 20 -L storage carboy had formed an agglomerated layer that would not pour from the carboy. Relatively vigorous shaking and pounding against the carboy walls was required to loosen the solids cake. After the solids were broken apart and freed from the carboy interior walls, they were scooped into a 4-L Nalgene sample bottle.

The wet solids were then filtered to remove any free liquid and stored in an airtight 4-L Nalgene bottle to prevent additional drying. During filtration, it was observed that the FY-03 SBW solids surrogate settled at a rapid rate and the solids were primarily in the form of coarse, brittle agglomerates. A sample 
of the wet solids was placed in a drying oven and allowed to dry at $110^{\circ} \mathrm{C}$ for several days until no further weight loss was recorded between sample weight measurements. The moisture content of the wet solids, determined by weight loss of the sample during the drying operation, was determined to be $43 \mathrm{wt} \%$ liquid (57 solids wt \%).

The mass of WM-187 liquid surrogate and 57 solids wt \% FY-03 SBW solids surrogate required to make 5 and 20 solids wt \% slurries was determined. Seven 100-mL samples of 5 solids wt \% slurry and five $100-\mathrm{mL}$ samples of 20 solids wt \% slurry were then prepared from the WM-187 liquid surrogate and wet FY-03 SBW solids surrogate. Additionally, three 120-mL samples of WM-187 liquid surrogate were collected.

Particle size distributions of the $187 / 5 \mathrm{wt} \% / \mathrm{D} / \mathrm{N}$ and $187 / 20 \mathrm{wt} \% / \mathrm{D} / \mathrm{N}$ samples were measured. A PSD plot of the nonsonicated samples is presented in Figure 3-18. A summary of the dried metathesisprepared SBW solids surrogate nonsonicated sample mean particle sizes is provided in Table 3-2.

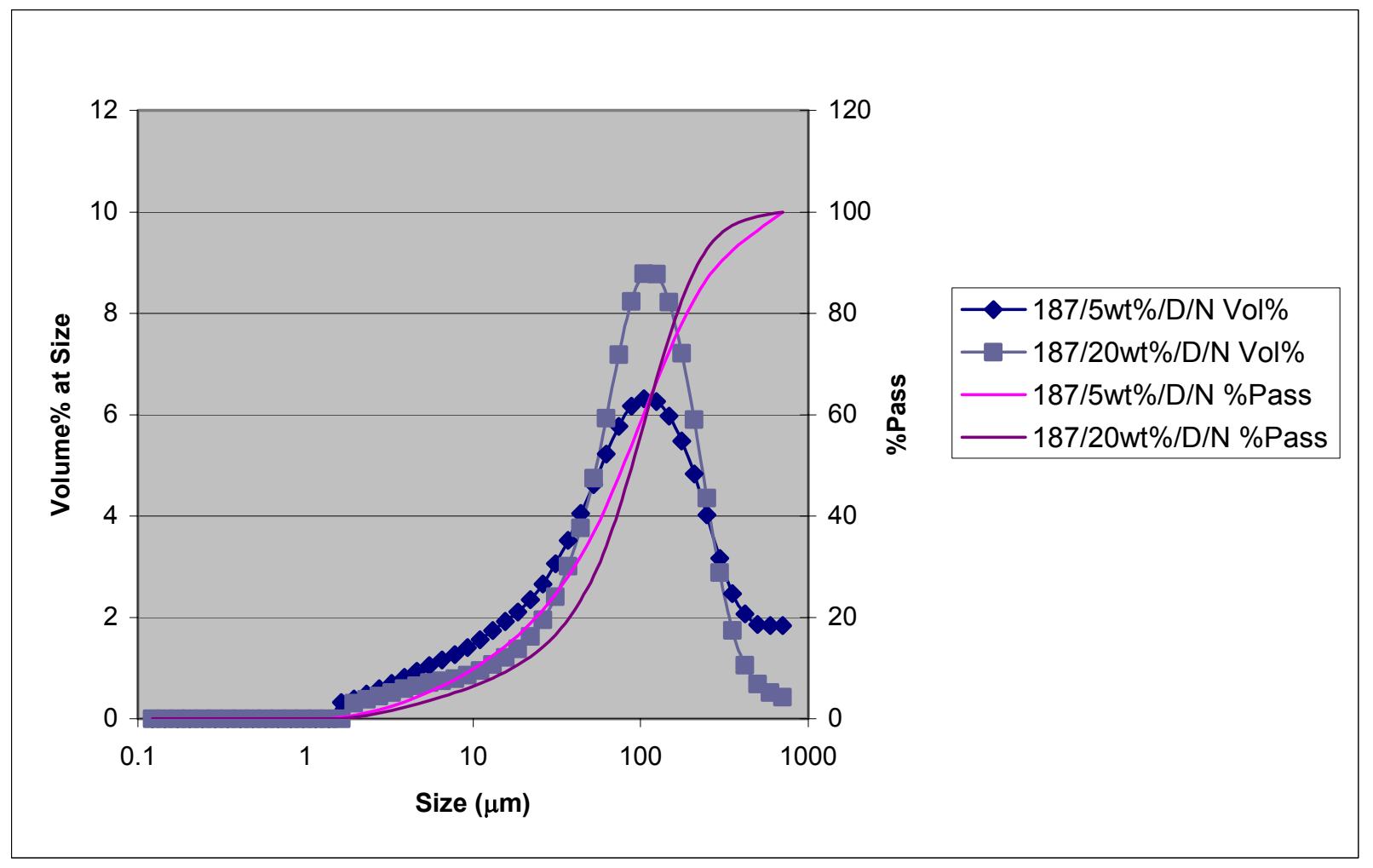

Figure $3-18.187 / 5 \mathrm{wt} \% / \mathrm{D} / \mathrm{U}$ and $187 / 20 \mathrm{wt} \% / \mathrm{D} / \mathrm{U}$ particle size distributions.

Table 3-2. Dried metathesis-prepared SBW solids surrogate samples and associated mean particle size.

\begin{tabular}{|c|c|c|}
\hline Sample Identity Tag & $\begin{array}{c}\text { Nonsonicated Sample Mean } \\
\text { Particle Size }(\mu \mathrm{m})\end{array}$ & $\begin{array}{c}\text { Sonicated Sample Mean Particle } \\
\text { Size }(\mu \mathrm{m})\end{array}$ \\
\hline 187 / 5wt\% / D & 123.8 & 21.5 \\
\hline $187 / 20 \mathrm{wt} \% / \mathrm{D}$ & 110.7 & 21.7 \\
\hline
\end{tabular}


The dried metathesis-prepared SBW solids surrogate samples were sonicated to decrease the particle size distributions in the samples and make the sample PSDs more closely resemble the PSDs of the actual SBW tank farm waste samples. The particle size distribution data presented in "Feed Composition for the Sodium-Bearing Waste Treatment Process, Rev. 2" (Barnes and Millet 2003) indicates that previous tank farm samples have PSD peak values ranging from approximately 10 to 50 microns (see Figure 3-19). The metathesis-prepared SBW solids surrogate samples were sonicated until a particle size distribution with a mean value in the range of 15 to 20 microns was achieved. A plot of the sonicated sample PSDs is shown in Figure 3-20. A summary of the dried metathesis-prepared SBW solids surrogate sonicated sample mean particle sizes is provided in Table 3-2.

3.3.1.2 Undried Metathesis-Prepared SBW Solid Surrogate Preparation. "Undried metathesis-prepared SBW solid surrogate" refers to the SBW solids surrogate prepared for FY-04 surrogate physical properties analysis. The FY-04 SBW solids surrogate was formulated using the metathesis preparation method of INEEL chemist S. K. Janikowski. The quantity of chemicals used for synthesis of the FY-04 SBW solids surrogate was selected to yield a mass of two kilograms of SBW solids surrogate.

Following SBW solids surrogate formulation, the surrogate solids and mother liquor were transferred to a 20-L tank. A Cole-Parmer Stir Pak variable speed mixer with 3-in. propeller was used to suspend the precipitated SBW solids surrogate in the mother liquor so that the solids and liquid were evenly distributed throughout the tank. Seven "as received" surrogate samples, designated META/AR/U/N, were drawn from a spigot in the bottom of the 20-L tank.

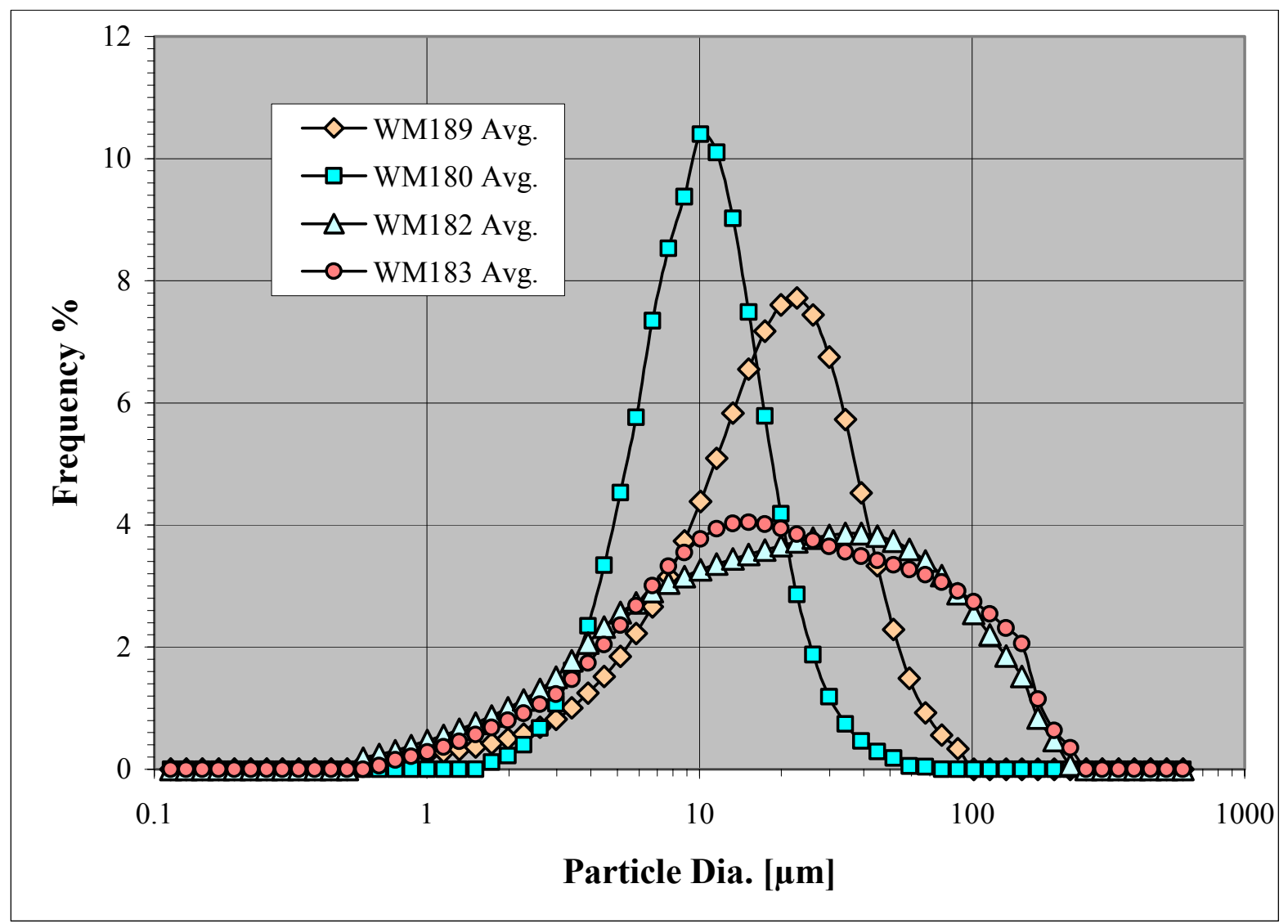

Figure 3-19. Comparison of WM-189, WM-180, WM-182, and WM-183 solids particle size distribution analysis under nonsonicated condition. 


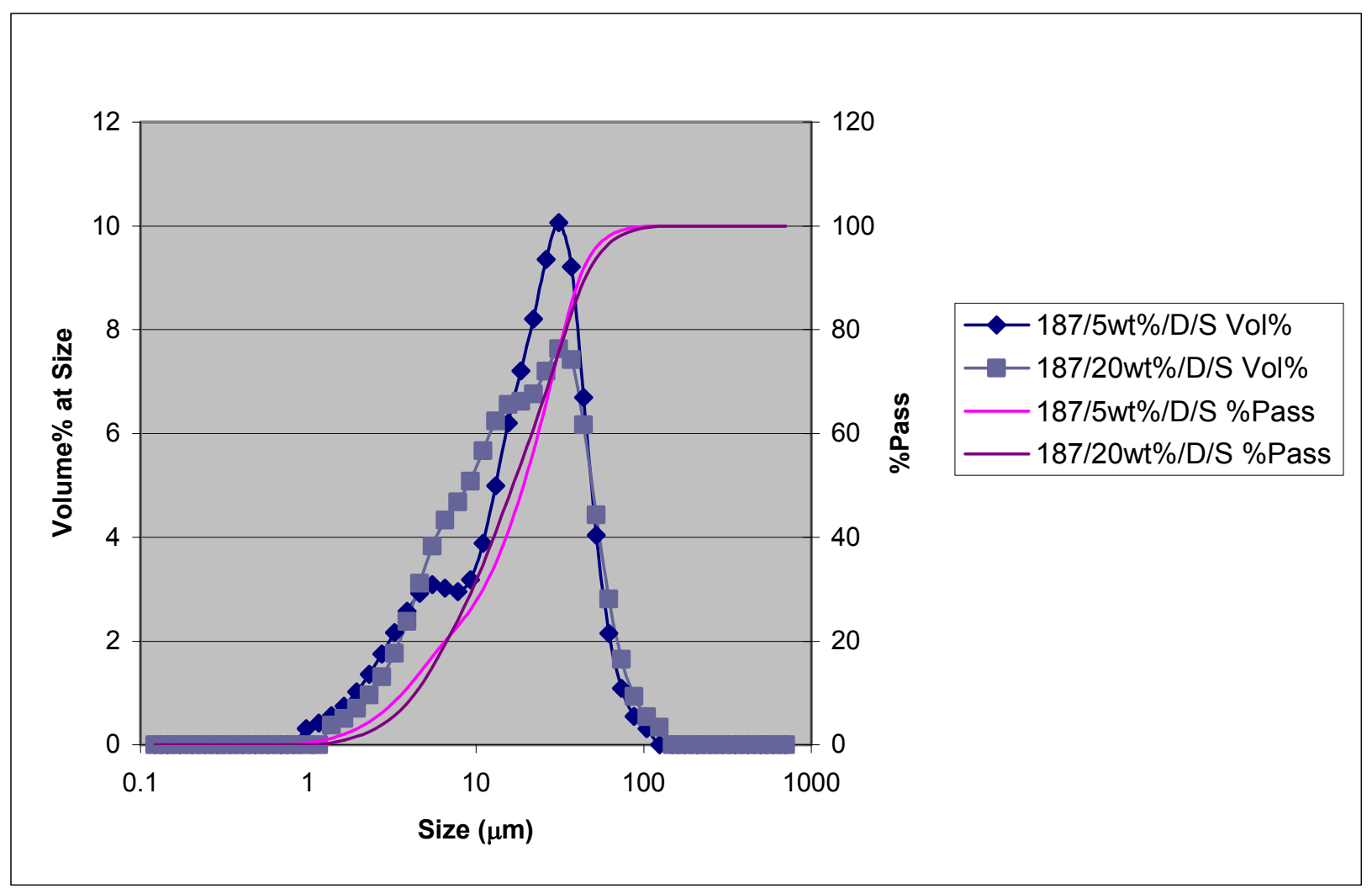

Figure 3-20. 187/5wt\%/D/S and 187/20wt\%/D/S particle size distributions.

Following collection of the "as received" SBW solids surrogate samples, the FY-04 solids were centrifuged to separate the solids from the mother liquor supernate. The centrifugation was performed at 5,000 RPM for 5 minutes per batch in a Du Pont Instruments Sorvall RC-5B centrifuge with a 3-L-capacity Sorvall SLA-3000 Super-lite rotor. Centrifugation yielded approximately 2 L of wet FY-04 SBW solids surrogate. The wet solids had a granular texture with a pasty consistency following centrifugation. The solids did not flow from the centrifuge tubes under the force of gravity. Samples of the liquid, designated META/0wt\%, were collected for analysis. The remaining liquid was set aside for use in mixing various solids weight percentage slurries at a later point in time.

A sample of the wet FY-04 SBW solids was placed in a drying oven operating at approximately $110^{\circ} \mathrm{C}$ and allowed to dry for several days until no further weight loss was recorded between sample weight measurements. The moisture content of the wet solids, determined by weight loss of the sample during the drying operation, was determined to be $77 \mathrm{wt} \%$, or 23 solids wt \%.

Nonhazardous WM-180 and WM-189 liquid surrogates were prepared for use in mixing slurries with different solids weight percentages and liquid phase chemical compositions. The WM-180 and WM189 liquid surrogates were prepared according to the "Procedure for Preparing NonHazardous SodiumBearing Waste Surrogates" included in Appendix C.

The mass of wet FY-04 SBW solids surrogate and the various liquid surrogates required to make 5, 10,15 , and 20 solids weight percentage slurries was determined. The metathesis mother liquor, WM-180, and WM-189 liquid surrogates were mixed with the mass of wet FY-04 SBW solids surrogate calculated to produce the 5 solids weight percent slurry samples META $/ 5 \mathrm{wt} \% / \mathrm{U} / \mathrm{N}, 180 / 5 \mathrm{wt} \% / \mathrm{U} / \mathrm{N}$, and $189 / 5 \mathrm{wt} \% / \mathrm{U} / \mathrm{N}$, respectively. The metathesis mother liquor was mixed with the mass of wet FY-04 SBW 
solids surrogate calculated to produce the 10 and 15 weight percentage slurry samples META/10wt $\% / \mathrm{U} / \mathrm{N}$ and META/15wt\%/U/N, respectively. Samples of the wet (23 solids weight percentage) FY-04 SBW solids surrogate were collected without addition of any of the liquid surrogates to produce the META/20wt $\% / \mathrm{U} / \mathrm{N}$ samples.

Particle size distribution plots for the nonsonicated 5 solids weight percentage slurries are presented in Figure 3-21. Particle size distributions for the nonsonicated 5, 10, and 20 solids weight percentage metathesis mother liquor slurries are presented in Figure 3-22. A summary of the undried metathesis-prepared SBW solids surrogate nonsonicated sample mean particle sizes is provided in Table 3-3.

As in the case of the dried metathesis-prepared SBW solids surrogate samples, the undried metathesis-prepared SBW solids samples were sonicated to decrease the particle size distributions and make the sample PSDs more closely resemble the PSDs of the actual SBW tank farm waste samples. The metathesis-prepared SBW solids surrogate samples were sonicated until particle size distributions with mean values in the range of 15 to 20 microns were achieved. This particle size mean value range was established to emulate the tank farm SBW sample PSD data presented in Barnes and Millet, 2003 (see Figure 3-19).

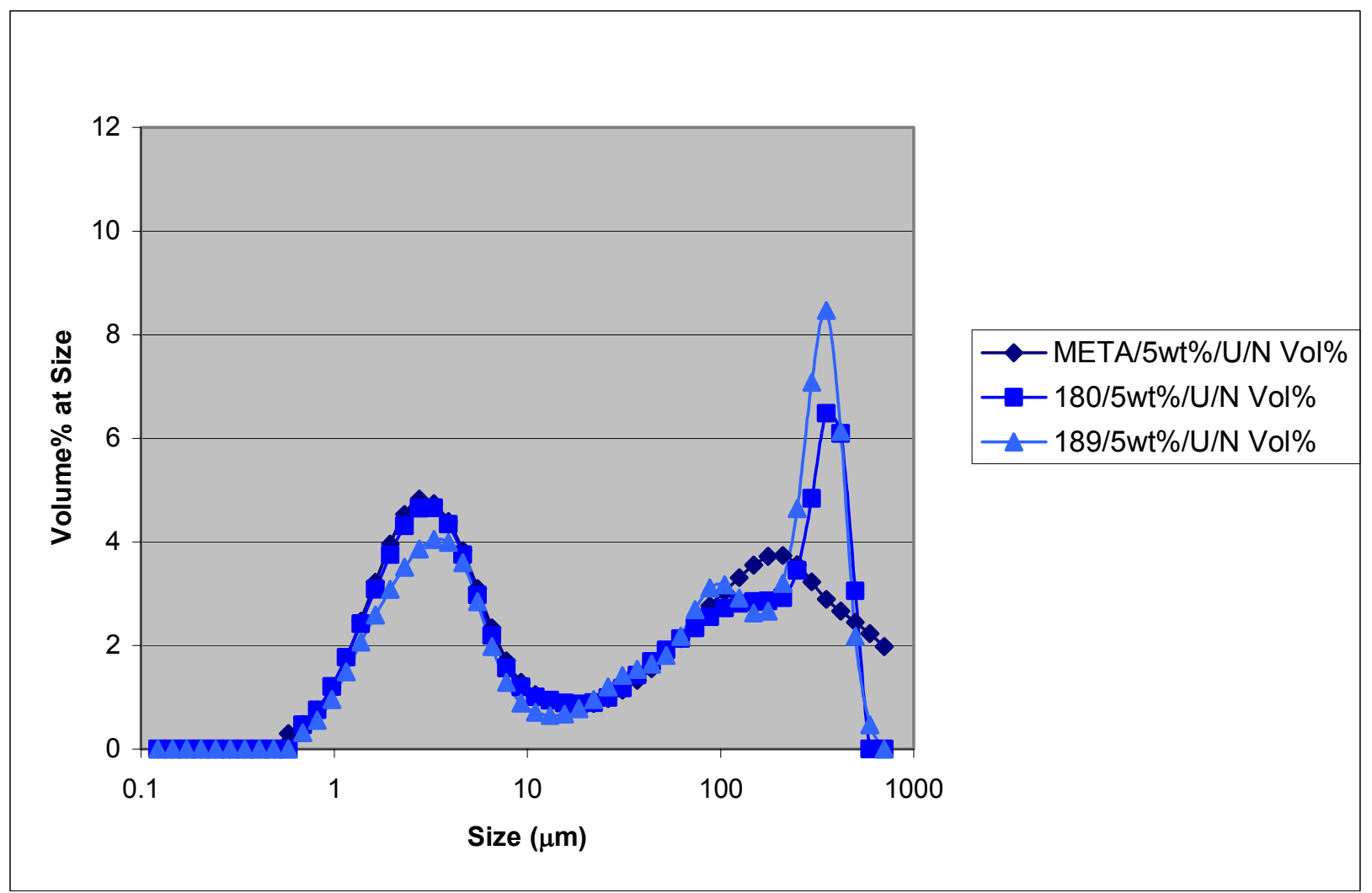

Figure 3-21. 180/5wt\%/U/N, 189/5wt\%/U/N, and META/5wt $\% / \mathrm{U} / \mathrm{N}$ sample particle size distributions. 


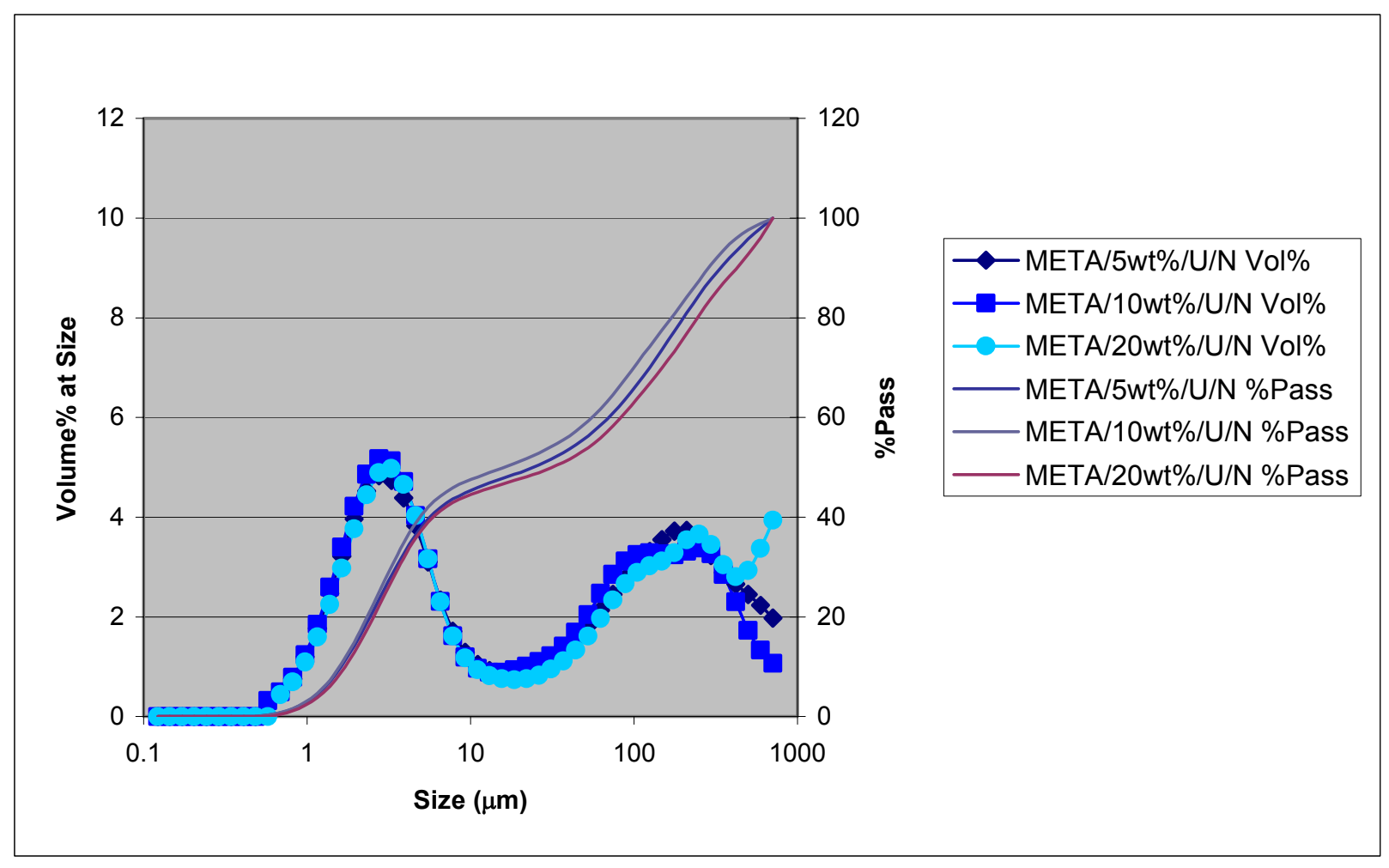

Figure 3-22. META/5wt $\% / \mathrm{U} / \mathrm{N}, \mathrm{META} / 10 \mathrm{wt} \% / \mathrm{U} / \mathrm{N}$, and META/20wt $\% / \mathrm{U} / \mathrm{N}$ sample particle size distributions

Table 3-3. Undried metathesis-prepared SBW solids surrogate samples and associated mean particle size.

Sample Identity Tag

Nonsonicated Sample Mean Particle Size $(\mu \mathrm{m})$
Sonicated Sample Mean Particle Size $(\mu \mathrm{m})$

\begin{tabular}{lcc}
\hline META / AR / U & 85.2 & 19.7 \\
META / 5wt\% / U & 106.8 & 19.4 \\
180 / 5wt\% / U & 108.6 & 15.0 \\
189 / 5wt\% / U & 123.5 & 16.1 \\
META / 10wt\% / U & 90.2 & 21.1 \\
META / 15wt\% / U & not available & 19.5 \\
META / 20wt\% / U & 127.0 & 21.0 \\
\hline
\end{tabular}

Particle size distribution plots for the sonicated 5 solids weight percentage slurries are presented in Figure 3-23. Particle size distributions for the sonicated 5, 10, 15, and 20 solids weight percentage metathesis mother liquor slurries are presented in Figure 3-24. A summary of the undried metathesis-prepared SBW solids surrogate sonicated sample mean particle sizes is provided in Table 3-3. 


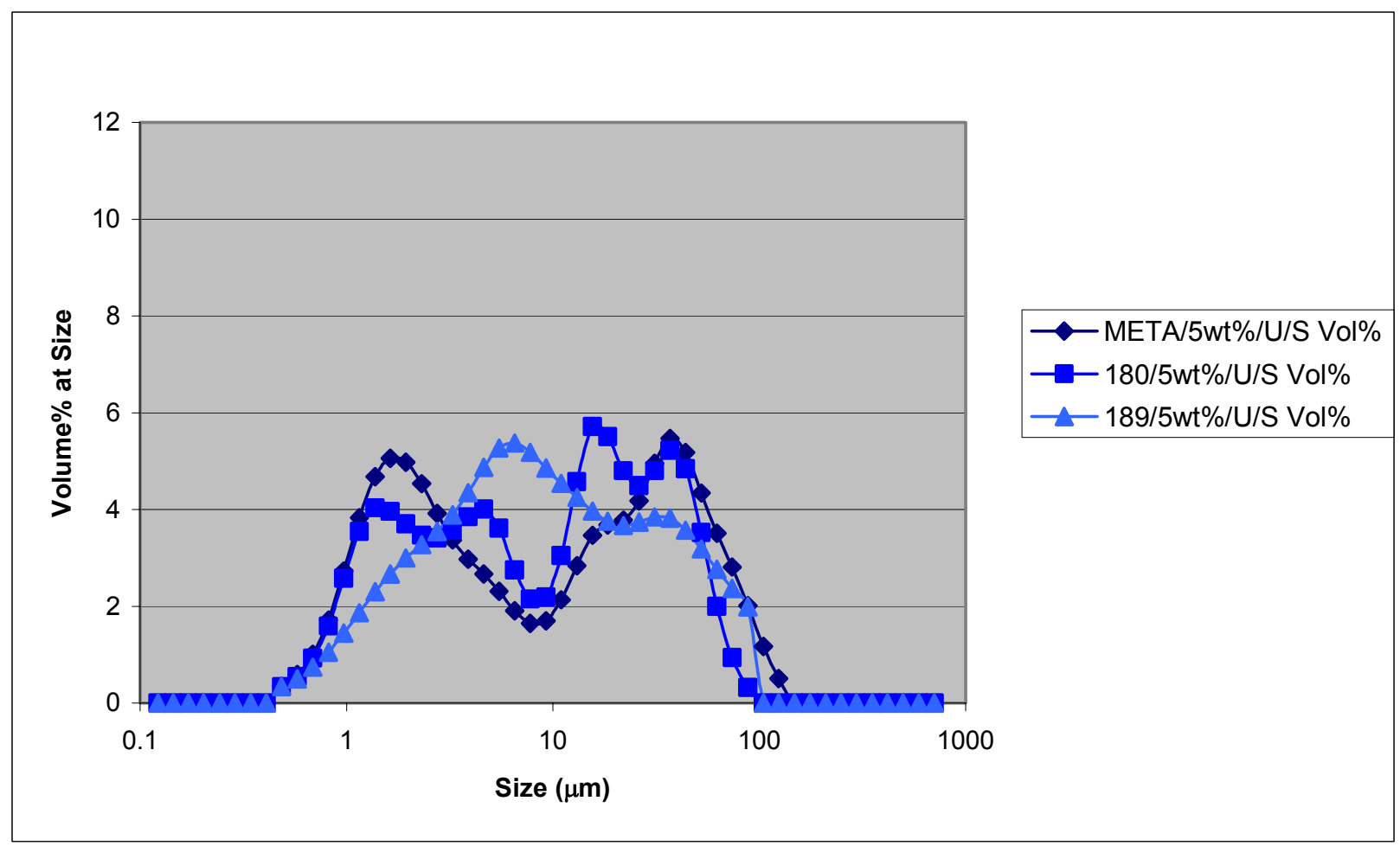

Figure 3-23. 180/5wt\%/U/S, 189/5wt\%/U/S, and META/5wt\%/U/S sample particle size distributions.

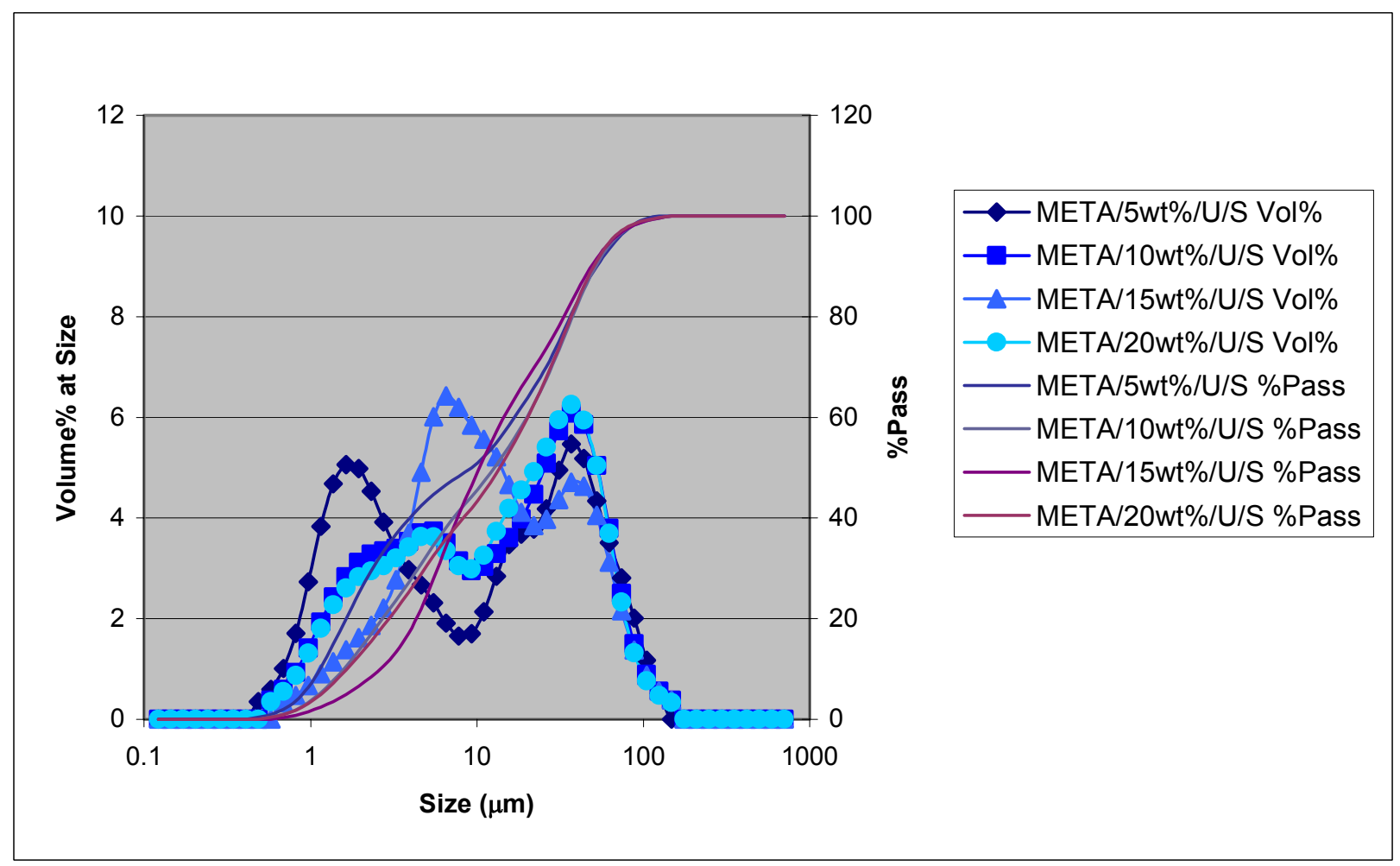

Figure 3-24. META/5wt \%/U/S, META/10wt $\% / \mathrm{U} / \mathrm{S}$, META/15wt $\% / \mathrm{U} / \mathrm{S}$, and META/20wt\%/U/S sample particle size distributions. 


\subsubsection{SBW Liquid Surrogate}

Table 3-4 is a summary of the major components in the 180,187 , and 189 liquid surrogates. The complete compositions as well as preparation procedures can be found in Appendix C.

Table 3-4. Summary of important 180, 187, and 189 liquid surrogate components.

\begin{tabular}{lccc}
\hline & 180 liquid surrogate & $\begin{array}{c}187 \text { liquid } \\
\text { surrogate }\end{array}$ & $\begin{array}{c}189 \text { liquid } \\
\text { surrogate }\end{array}$ \\
\hline $\mathrm{H}^{+}$concentration, mol/L & $1.01 \mathrm{E}+00$ & $2.29 \mathrm{E}-01$ & $2.86 \mathrm{E}+00$ \\
$\mathrm{Al}^{3+}$ concentration, mol/L & $6.63 \mathrm{E}-01$ & $3.58 \mathrm{E}-02$ & $7.11 \mathrm{E}-01$ \\
$\mathrm{Na}^{+}$concentration, mol/L & $2.06 \mathrm{E}+00$ & $1.13 \mathrm{E}-01$ & $2.04 \mathrm{E}+00$ \\
$\mathrm{NO}_{3}{ }^{-}$concentration, mol/L & $5.16 \mathrm{E}+00$ & $4.54 \mathrm{E}-01$ & $7.27 \mathrm{E}+00$ \\
total dissolved solids content & $25.0 \%$ & $4.7 \%$ & $22.4 \%$ \\
\hline
\end{tabular}

\subsubsection{SBW Tank Sample}

In February 2004, approximately 2 L of WM-187 tank farm waste samples were obtained for flow curve analysis. At the time of sampling, tank WM-187 contained the waste previously in tanks WM-182, WM-183, WM-184, WM-185, WM-186, and part of WM-181. Solution including solids was pumped from WM-187 using a steam jet to tank NCC-102. Tank NCC-102 is continuously air sparged to obtain representative samples. Samples were obtained from NCC-102 in 50-mL glass bottles and transported to the RAL through the Pneumatic Transfer System . Three different samplings produced $60+$ bottles for analytical log number 0402101. The tank farm waste sample was separated into four fractions representative of waste in various positions inside the waste tanks. These four fractions were the "as-received" sample, liquid supernate, gravity-settled solids, and centrifuged solids. The "as-received" sample was collected by agitating the full tank farm waste sample to suspend any settled particles and distribute the solids evenly throughout the sample volume. An aliquot of the "a- received" sample was then collected and set aside for flow curve analysis.

The liquid supernate and gravity settled solids fractions were obtained by allowing the tank farm sample to settle and decanting off the liquid supernate, leaving behind the gravity settled slurry with no free liquids. The liquid supernate and gravity-settled solids fractions were collected and set aside for flow curve analysis.

The centrifuged solids fraction, which was prepared to emulate settled and compacted solids at the bottom of the tank farm tanks, was prepared by centrifuging the gravity-settled solids fraction and collecting the concentrated solids phase. The centrifuged solids fraction was collected and set aside for flow curve analysis.

\subsection{Results and Analysis}

Physical properties of SBW surrogate and tank farm slurry samples were compared. In general, the surrogate provided a very good representation of the tank farm waste. The following sections provide the results and analyses of various quantitative physical property comparisons performed on the surrogate and tank farm waste. Prior to discussion of the quantitative physical property analyses, it should be noted that 
several qualitative differences between the SBW surrogate and tank farm waste were observed while handling the respective samples.

The first obvious difference between the samples was the color. The tank farm waste samples were very dark, with a color that could be described as blackish brown (when viewed through the thick radiation-absorbing windows of the INTEC RAL hot cell). The SBW surrogate solids samples were white in color, with some of the sonicated samples taking on a slightly grayish tone, presumably due to retaining fine metallic particles worn off the sonication probe during the sonication operation.

Another noticeable difference between the tank farm waste and surrogate samples was the difference in the consistency of slurry samples aged for a period of several hours. Surrogate samples with appreciable solids concentrations (approximately 10 solids wt \% and greater) allowed to sit undisturbed overnight developed a coagulated solids phase. In many cases the coagulated solids phase would be cohesive enough that it would remain motionless in the bottom of an inverted sample bottle. The coagulated solids phase would break apart, suspend in the liquid phase, and revert to fluid-like slurry properties upon agitation. The amount of agitation required to break apart the coagulated solids depended on the solids concentration of the sample and the amount of time the sample had been undisturbed. In comparison with the surrogate, the tank farm solids displayed very little or no tendency to coagulate after a period with no disturbances. Settled solids in both tank farm waste and surrogate slurry sample bottles were suspended (via shaking) successfully after sitting undisturbed for periods of up to approximately two and six weeks, respectively.

The surrogate samples also displayed qualitative physical property differences between batches and samples prepared in different manners. SBW surrogate slurries prepared with the dried FY-03 solids surrogate were different in appearance from samples with the same solids weight percentage prepared with the undried FY-04 solids surrogate. Samples prepared with the dried FY-03 solids surrogate had a noticeably lower solids volume percentage than samples prepared with the undried FY-04 solids surrogate, indicating that the undried FY-04 SBW solids were relatively more dispersed than the dried FY-03 solids. The solids in the samples prepared using the dried FY-03 solids surrogate tended to have more granular, brittle characteristics and settle at a faster rate than the solids in samples prepared from the undried FY-04 solids surrogate. Sonication of the surrogate slurry samples prepared with the dried FY-03 solids and undried FY-04 solids decreased, but did not completely eliminate, the differences in physical properties of the solids. Sonication significantly increased the solids volume percentage of samples prepared with the dried FY-03 solids surrogate. Sonication also increased the solids volume percentage of samples prepared with the undried FY-04 solids surrogate, but not to the extent observed with the dried FY-03 solids surrogate. In addition to increasing the relative level of solids dispersion within the surrogate samples, sonication appeared to universally decrease the settling rate of solids particles in the surrogate samples. The differences between the surrogate batches and preparation methods provide information that may be used to prepare a surrogate with physical properties similar to those desired for representation of the tank farm waste.

The following sections describe the results of quantitative analyses performed on the SBW surrogate and tank farm waste samples. Although there were differences in the results of the surrogate and waste physical property analyses, the surrogate generally provided a good representation of the waste physical properties.

\subsubsection{Particle Size Distribution}

Particle size distribution analyses were performed on SBW surrogate samples as described in Section 3.2.1. Sample PSD analyses were performed on nonsonicated samples to determine the particle size distribution following sample formulation/weight percentage adjustment. Samples were 
then sonicated in preparation for flow curve, settling velocity, bulk density, solids weight percentage, etc., analyses. Samples were sonicated until the sample mean particle diameter was in the range of 15-20 microns. This particle size range was selected to produce surrogate samples with a PSD comparable to that reported for previous tank farm samples (see the comparison of WM-189, WM-180, WM-182, and WM-183 solids PSD analyses under nonsonicated conditions provided in Batcheller, Taylor, and Johnson 1999). The results of the particle size distribution analyses are presented below. Figure 3-25 is a plot of all nonsonicated SBW surrogate sample particle size distributions following sample synthesis and mixing with the various liquid surrogates to obtain slurries with the specified solids weight percentage loadings. Figure 3-26 is a particle size distribution plot of the SBW surrogate samples following sonication. The decrease in sample mean particle diameter following sonication is apparent upon comparison of Figure 3-25 and Figure 3-26. Figure 3-27 provides a comparison of the mean particle diameter for each of the SBW surrogate slurries before and after sonication.

Several distinct particle size distribution profiles can be identified in Figure 3-28, which is identical to Figure 3-25, the plot of the nonsonicated SBW surrogate sample PSDs. Figure 3-29 through Figure 3-31 are plots of the similar PSD profiles that can be extracted from Figure 3-28.

Figure 3-29 is a plot of the nonsonicated FY-03 SBW surrogate sample particle size distributions. These samples were formulated from the same batch of SBW solids surrogate (FY-03 batch) and were mixed with WM-187 liquid surrogate in 5 and 20 solids weight percentage quantities. Adjustment of the solids weight percentage did not have an appreciable affect on the sample mean particle diameter; the $187 / 5 \mathrm{wt} \% / \mathrm{D} / \mathrm{N}$ sample had a mean particle diameter of $123.8 \mu \mathrm{m}$ and the $187 / 20 \mathrm{wt} \% / \mathrm{D} / \mathrm{N}$ sample had a mean particle diameter of $110.7 \mu \mathrm{m}$.

Figure 3-30 is a plot of the nonsonicated, as-received FY-04 SBW surrogate sample particle size distribution. The nonsonicated, as-received FY-04 SBW surrogate sample (META/AR/U/N) varies in particle size distribution profile and mean particle size diameter from the solids weight percentage adjusted, nonsonicated FY-04 samples shown in Figure 3-31. The solids weight percentage-adjusted sample preparation procedure altered the sample PSD properties. The solids weight percentage-adjusted samples were formulated from WM-180, WM-189, and metathesis mother liquor liquid surrogates and wet FY-04 SBW solids surrogate (free liquids removed via centrifugation) to form slurries with the specified solids weight percentages. The solids centrifugation step likely changed the sample PSDs by retaining the larger solids surrogate particles while allowing the smaller particle fines to be decanted from the concentrated wet solids. Figure 3-31 also illustrates that use of the WM-180 and WM-189 liquid surrogates resulted in a higher concentration of particles of approximately 350 microns in diameter. It is presumed that this is due to the WM-180 and WM-189 liquid surrogates changing the zeta potential (particle charge) of the SBW solids surrogate particles, increasing surrogate particle agglomeration, and skewing the particle size distribution upward. 


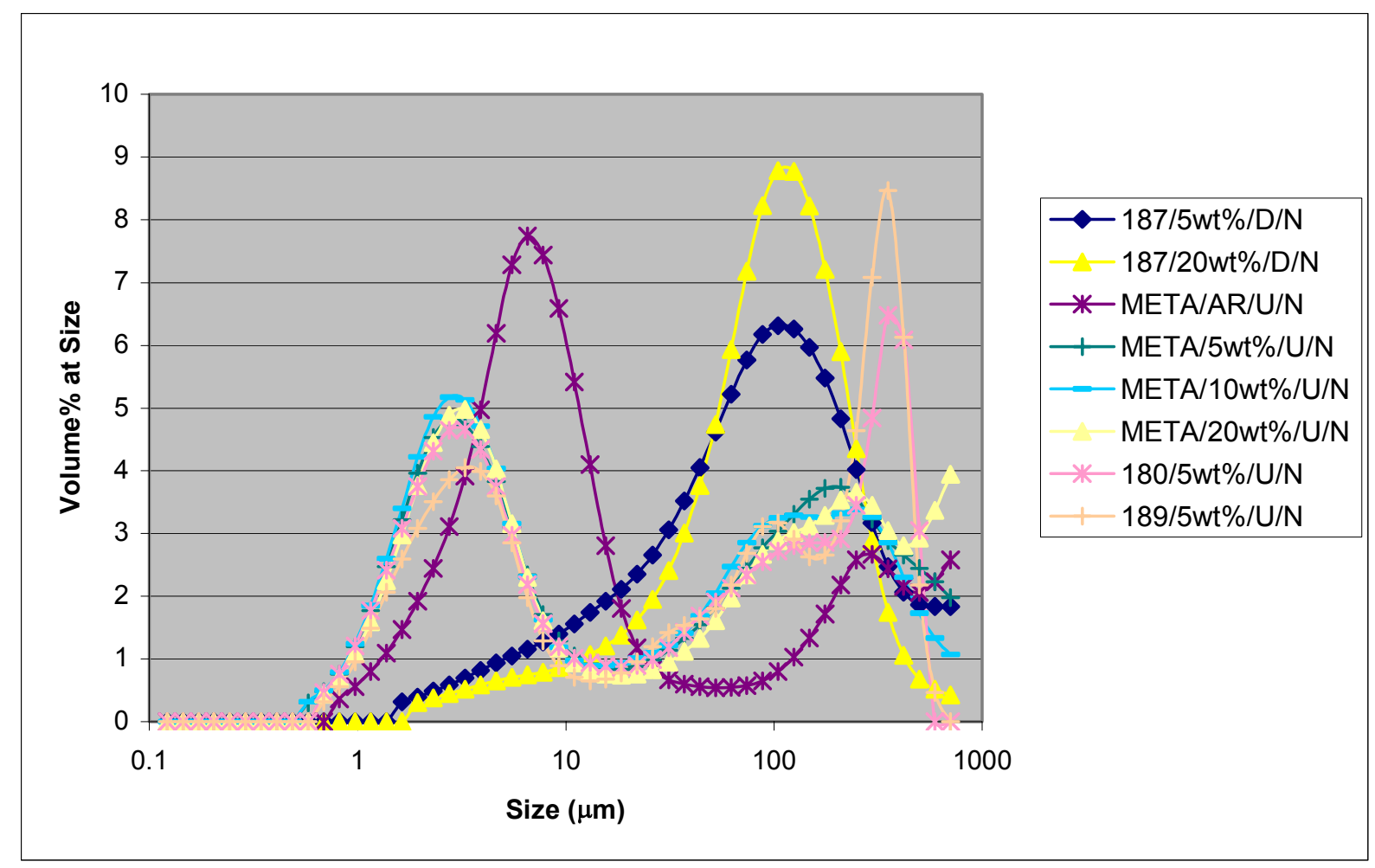

Figure 3-25. Nonsonicated SBW surrogate sample particle size distributions.

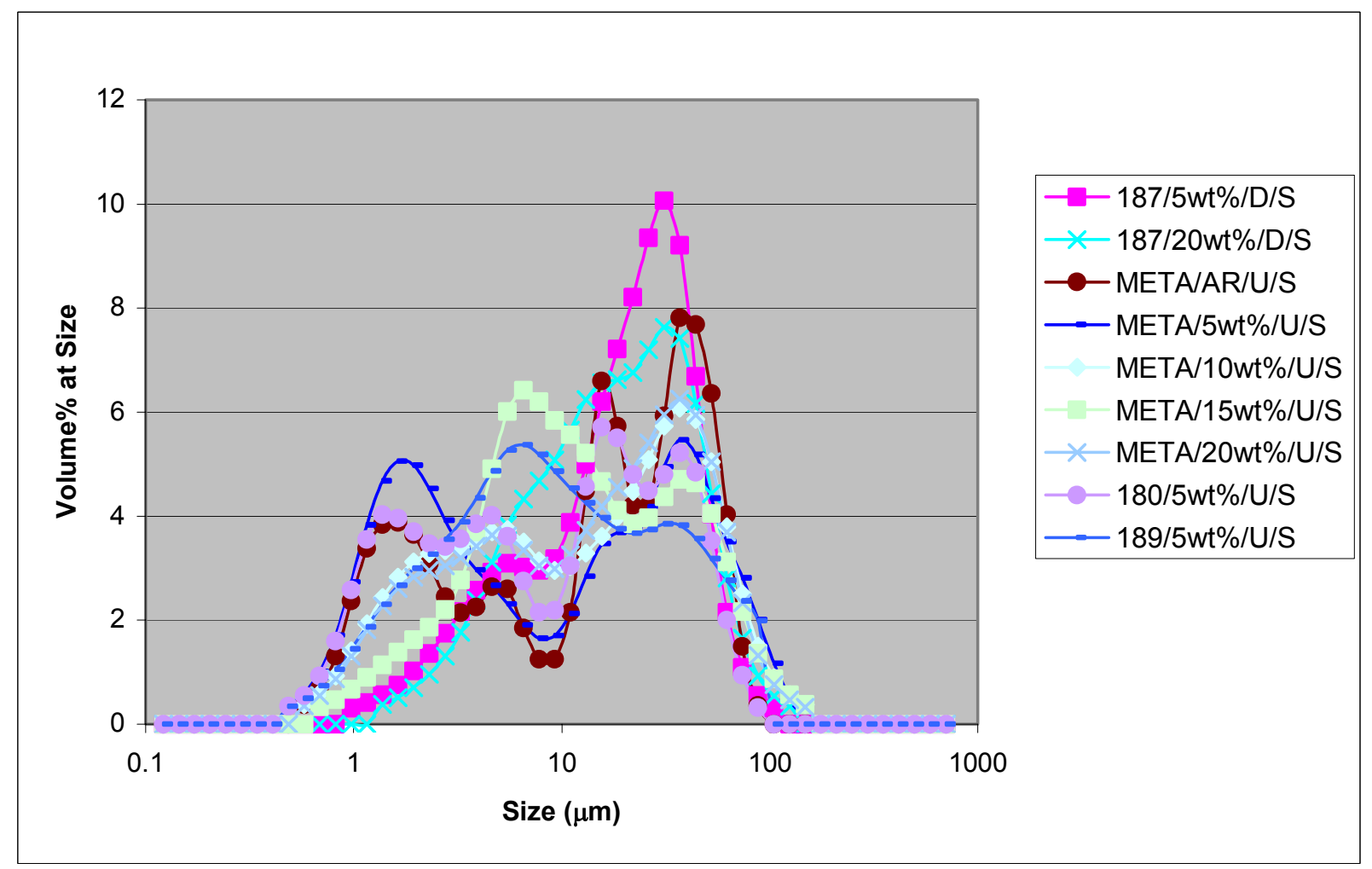

Figure 3-26. Sonicated SBW surrogate sample particle size distributions. 


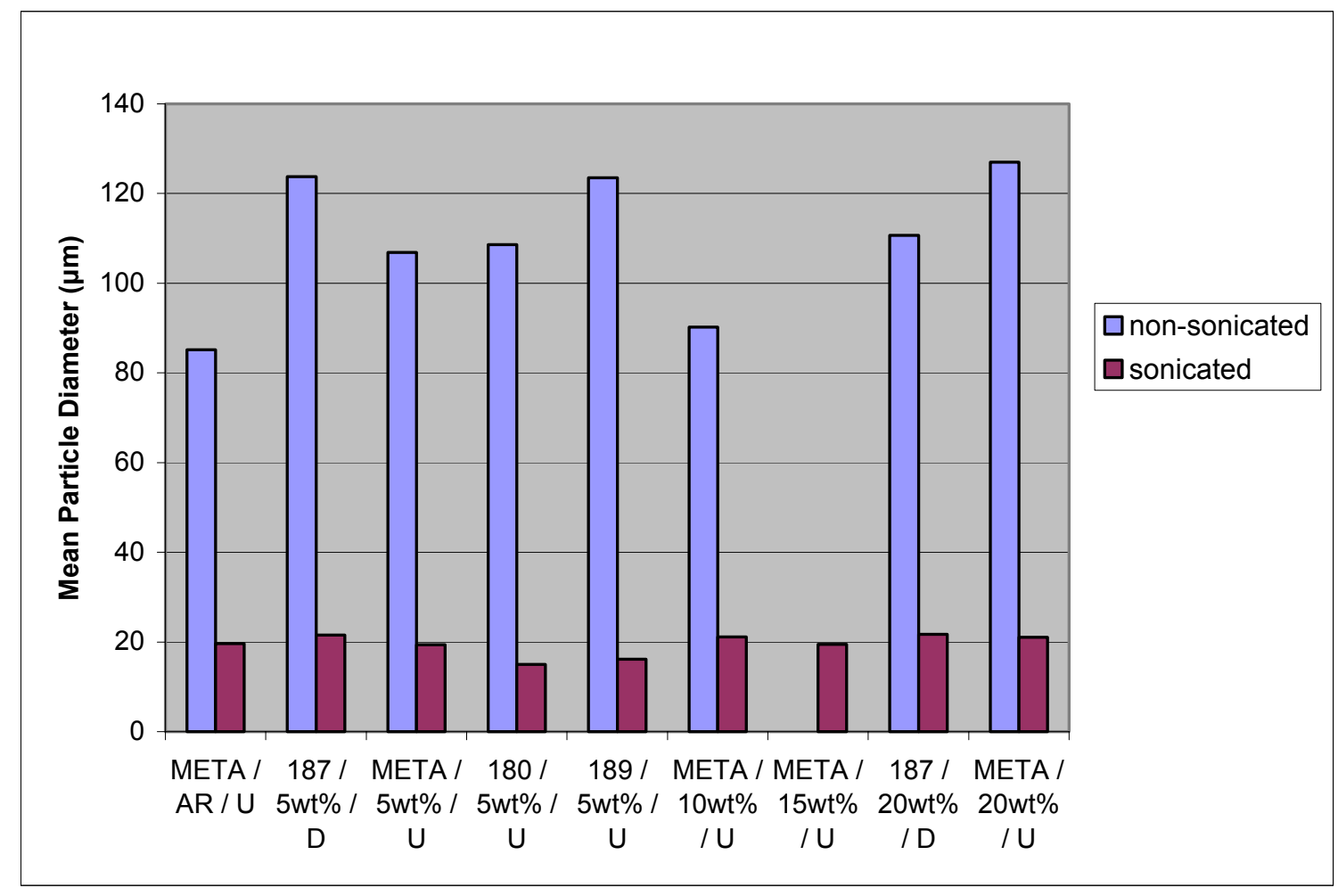

Figure 3-27. SBW surrogate sample mean particle diameter before and after sonication.

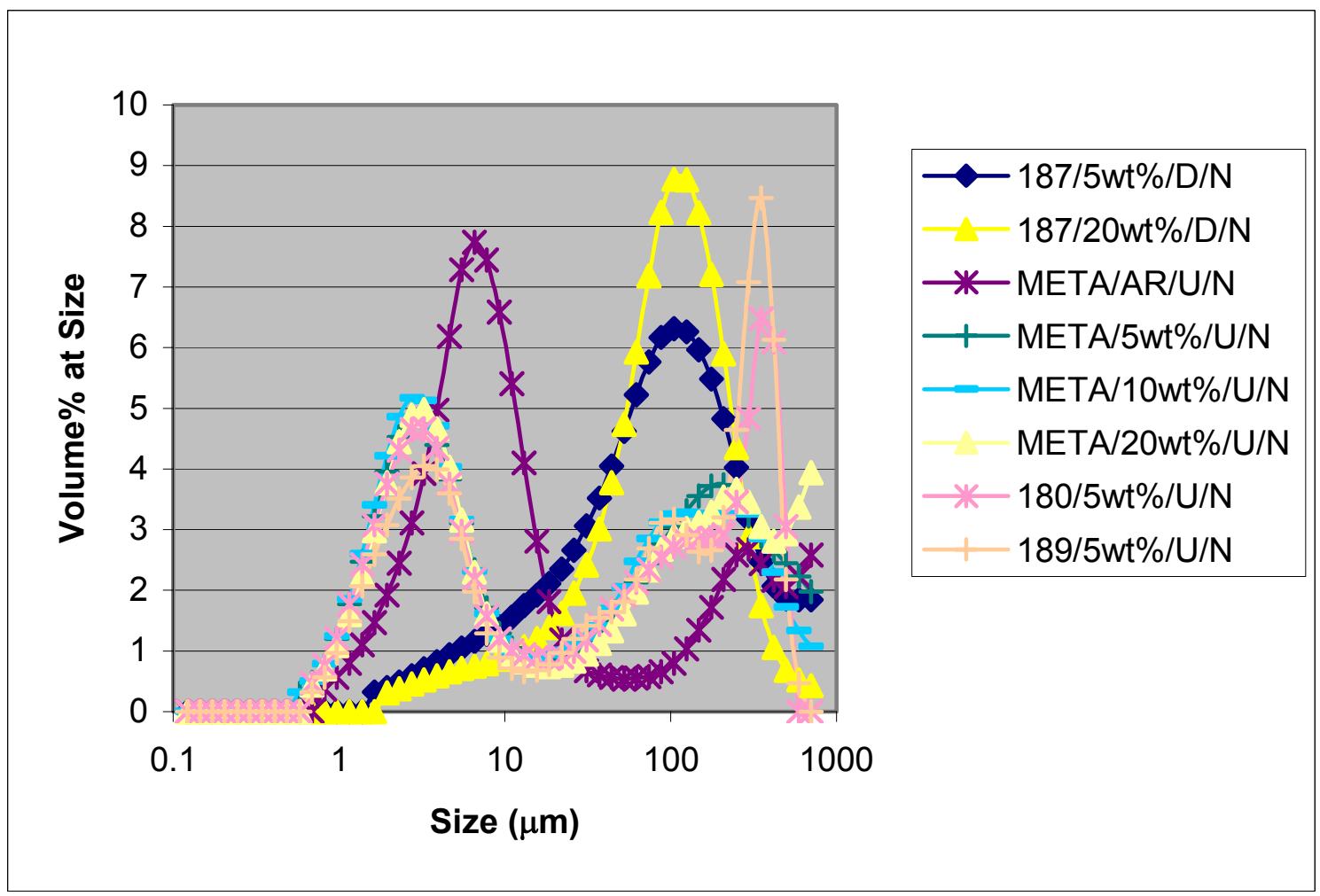

Figure 3-28. Nonsonicated SBW surrogate sample PSDs. 


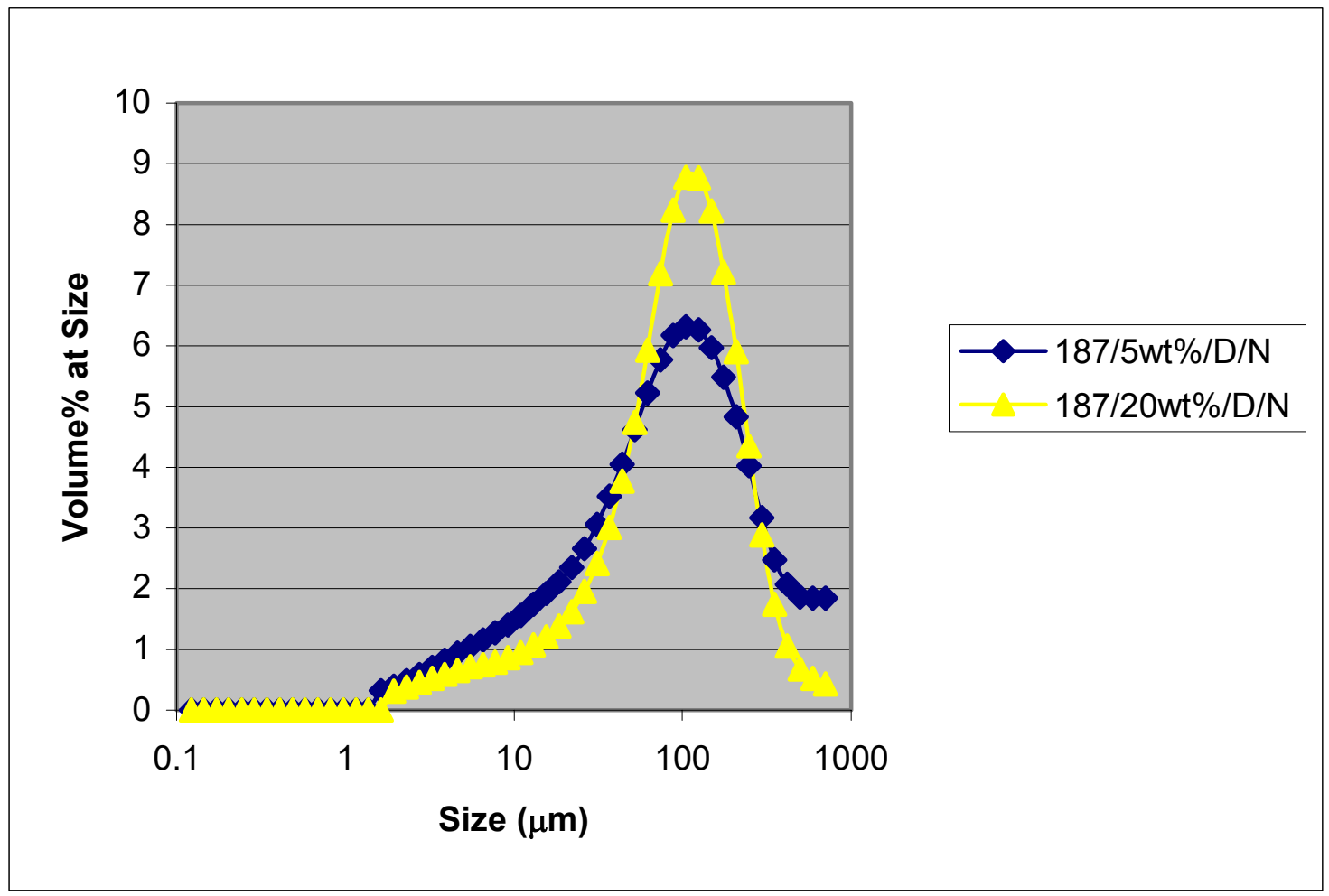

Figure 3-29. Nonsonicated FY-03 SBW surrogate sample PSDs.

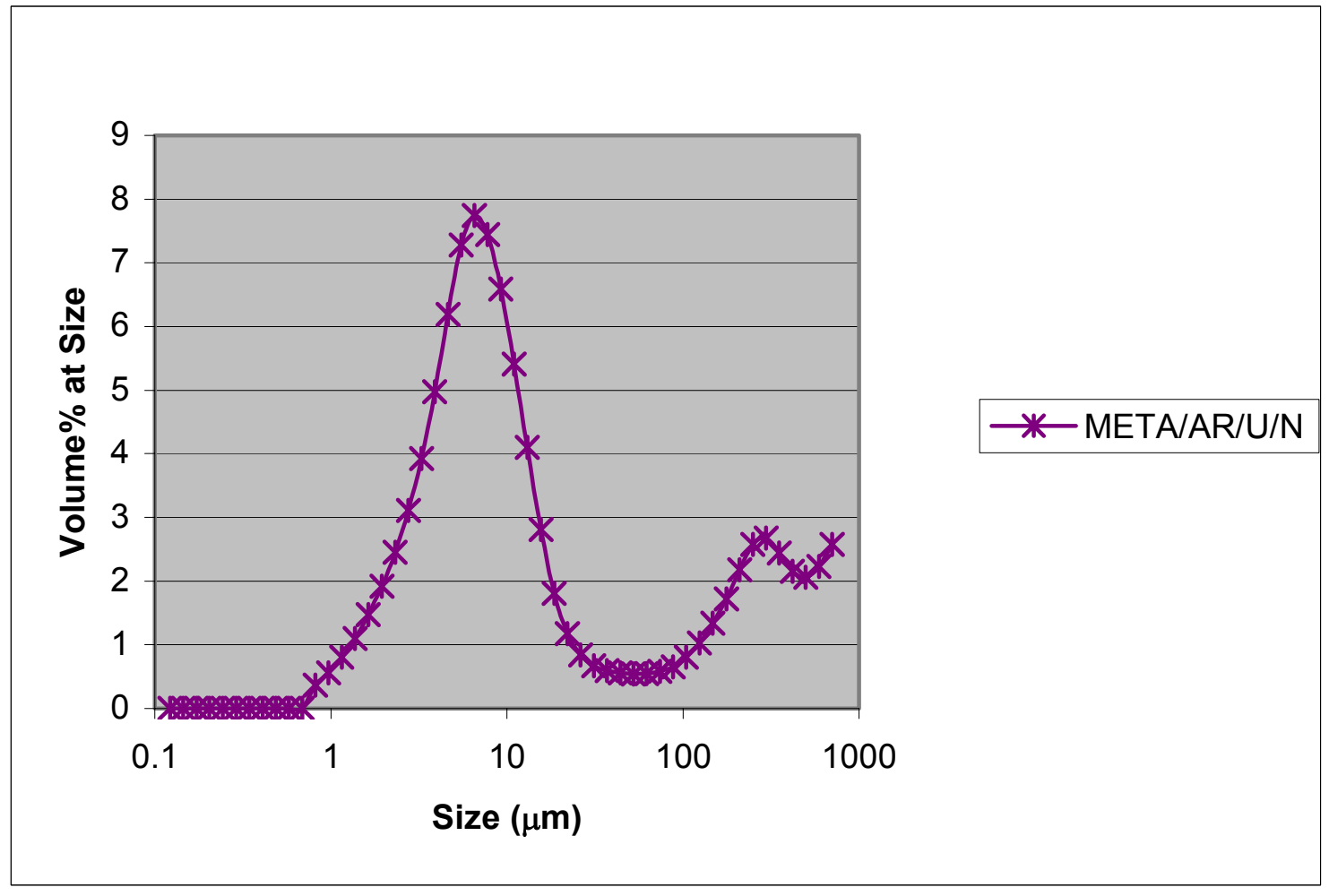

Figure 3-30. Nonsonicated, as-received FY-04 SBW surrogate sample PSD. 


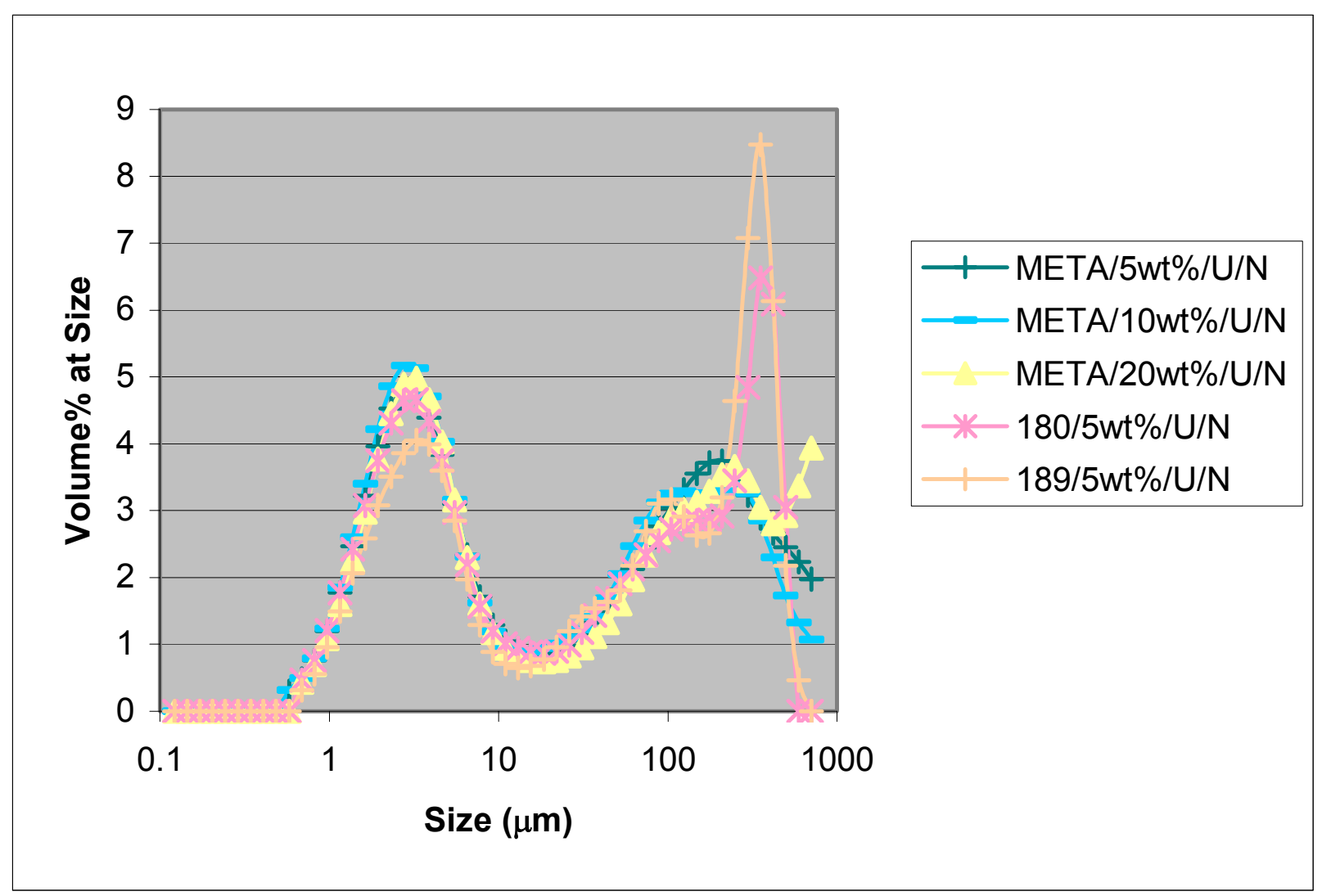

Figure 3-31. Solids weight percentage adjusted, nonsonicated FY-04 SBW surrogate sample PSDs.

Table 3-5 provides mean particle diameter data for nonsonicated and sonicated SBW surrogate slurry samples.

Table 3-5. Nonsonicated and sonicated SBW surrogate sample mean particle diameter (microns).

\begin{tabular}{lcc}
\hline & (N)onsonicated & (S)onicated \\
\hline META / AR / U & 85.2 & 19.7 \\
187 / 5 wt \% / D & 123.8 & 21.5 \\
META / 5 w t \% / U & 106.8 & 19.4 \\
180 / 5 wt \% / U & 108.6 & 15.0 \\
189 / 5 wt \% / U & 123.5 & 16.1 \\
META / $10 \mathrm{wt} \%$ / U & 90.2 & 21.1 \\
META / 15 wt \% / U & N/A & 19.5 \\
187 / 20 wt \% / D & 110.7 & 21.7 \\
META / 20 wt \% / U & 127.0 & 21.0 \\
\hline
\end{tabular}


Three different aliquots of the first WM-187 SBW sample (analytical log number 0308181) were analyzed using the remote Horiba LA-300 particle size analyzer. Other samples were not analyzed as above because the remoted Horiba LA-300 instrument failed and is not repairable. The results are presented in Table 3-6 below. For the sonicated value in Table 8 the samples were sonicated for only about 10 seconds.

The WM-187 sample mean particle diameter was lower than the historical tank farm sample mean particle diameter. This comparison is shown in Figure 3-32. The WM-187 PSD is similar to that of the WM-188 sample, but higher in magnitude and the WM-187 doesn't have as big of a range. The WM-187 is very close to the WM-181, which were the last solids washed to the WM-187 tank. One hypothesized explanation is that the fine particulates from the WM-181, WM-182, WM-183, WM-184, WM-185, and WM-186 tank waste added to tank WM-187 migrated to the sample collection point prior to sampling, decreasing the mean particle size of the WM-187 sample.

Table 3-6. Nonsonicated and sonicated WM-187 sample mean particle diameter (microns).

\begin{tabular}{lcc}
\hline & Nonsonicated & Sonicated \\
\hline WM-187 aliquot \#1 & 4.2 & 4.2 \\
WM-187 aliquot \#2 & 4.2 & 4.2 \\
WM-187 aliquot \#3 & 4.2 & 4.2 \\
Average & 4.2 & 4.2 \\
\hline
\end{tabular}

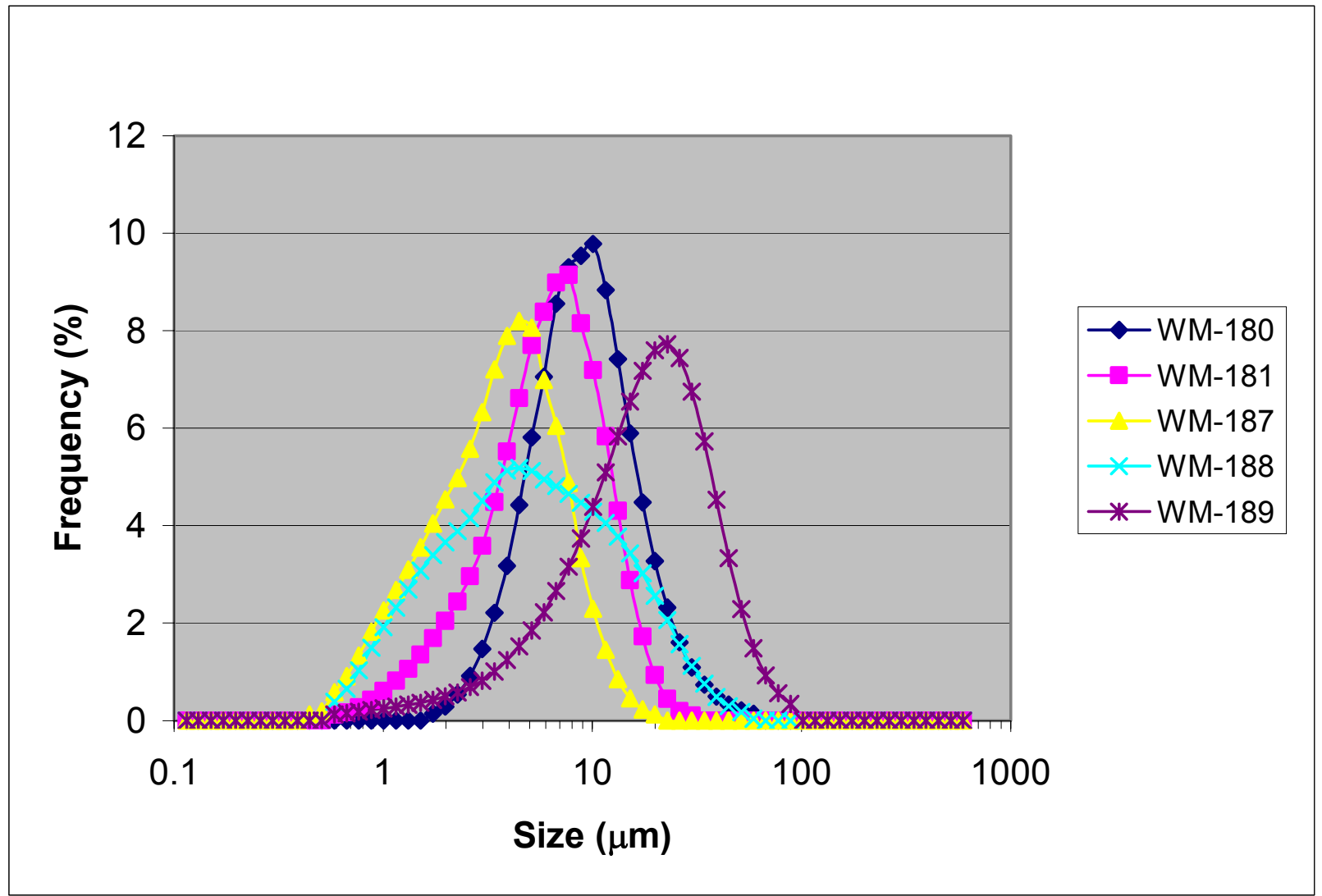

Figure 3-32. A comparison of the particle size distributions for various tank farm solid waste samples. 


\subsubsection{Flow Curve}

Flow curve analyses were performed on SBW surrogate and tank farm waste samples as described in Section 3.2.2. Analysis of the SBW surrogate samples was completed prior to analysis of tank farm waste samples. The Brookfield R/S rheometer unit was used to record all flow curves. The SBW surrogate samples had to be analyzed before the rheometer was used to analyze the tank farm waste samples, since the tank farm waste samples would contaminate the rheometer and render it unsuitable for use outside of the INTEC RAL.

A wide range of SBW surrogate samples were tested prior to placing the Brookfield R/S rheometer in the INTEC RAL hot cell. SBW surrogate sample variables tested included liquid surrogate composition, sample solids weight percentage, sample solids surrogate batch, sample particle size distribution, and sample temperature.

The tank farm waste sample was divided into four fractions. A flow curve was recorded for each fraction of the tank farm sample using the Brookfield R/S rheometer in the INTEC RAL hot cell. The four sample fractions included the "as-received" tank farm waste sample, liquid supernate, gravity settled slurry, and centrifuged slurry.

Sample flow curves were recorded using controlled shear rate mode, with shear rates ranging from $0.1 \mathrm{~s}^{-1}$ to $4000 \mathrm{~s}^{-1}$, depending on the sample viscosity. Lower viscosity sample flow curves were recorded over a shear rate range of $500-4000 \mathrm{~s}^{-1}$. Higher viscosity sample flow curves were recorded over a shear rate range of $0.1-1000 \mathrm{~s}^{-1}$.

Table 3-7 provides a summary of typical shear rates experienced by materials in a variety of applications. The shear rates at which the SBW surrogate and tank farm waste samples were tested correspond to processes ranging from draining under gravity $\left(0.1 \mathrm{sec}^{-1}\right)$ to mixing, stirring, and pipe flow $\left(1000 \mathrm{sec}^{-1}\right)$.

Table 3-7. Range of shear rates of some familiar materials and processes [Barnes et al., 1989].

\begin{tabular}{lll}
\multicolumn{1}{c}{ Process } & $\begin{array}{c}\text { Range of Shear } \\
\text { Rates }\left(\mathrm{s}^{-1}\right)\end{array}$ & \multicolumn{1}{c}{ Application } \\
\hline $\begin{array}{l}\text { Sedimentation of fine powders } \\
\text { in a suspending liquid }\end{array}$ & $10^{-6}-10^{-4}$ & Medicines, paints \\
Leveling due to surface tension & $10^{-2}-10^{-1}$ & Paints, printing inks \\
Draining under gravity & $10^{-1}-10^{1}$ & Painting, coating \\
Screw extruders & $10^{0}-10^{2}$ & Polymer melts, dough \\
Chewing and swallowing & $10^{1}-10^{2}$ & Foods \\
Dip coating & $10^{1}-10^{2}$ & Paints, confectionery \\
Mixing and stirring & $10^{1}-10^{3}$ & Manufacturing liquids \\
Pipe flow & $10^{0}-10^{3}$ & Pumping, blood flow \\
Spraying and brushing & $10^{3}-10^{4}$ & Fuel atomization, painting \\
Rubbing & $10^{4}-10^{5}$ & Application of creams and lotions to the skin \\
Injection mold gate & $10^{4}-10^{5}$ & Polymer melts \\
Milling pigments in fluid bases & $10^{3}-10^{5}$ & Paints, printing inks \\
Blade coating & $10^{5}-10^{6}$ & Paper \\
Lubrication & $10^{3}-10^{7}$ & Gasoline engines \\
\hline
\end{tabular}


Flow curve analyses included both increasing shear rate analysis steps as well as decreasing shear rate analysis steps. Comparison of the increasing and decreasing shear rate curves provided information about the sample thixotropy, or the property of various materials of becoming fluid when disturbed (as by shaking).

3.4.2.1 Surrogate Sample vs. Tank Farm Sample Flow Curves. SBW surrogate samples with similar physical properties (solids weight percentage, free liquid content, particle size distribution, etc.) to those of the four tank farm waste sample fractions were identified. Flow curves for these SBW surrogate samples were then compared with those of the tank farm waste samples. SBW surrogate samples were considered qualified to represent the tank farm samples if (a) the surrogate flow curve had the same general shape as the tank farm waste flow curve, (b) the surrogate flow curve data values were close in absolute value to the tank farm waste flow curve data values, and (c) the surrogate flow curve provided a conservative representation of the tank farm waste flow curve. A conservative representation requires the surrogate flow curve to have shear stress data values higher than tank farm waste flow curve shear stress data values throughout the majority of the shear rate range.

A SBW surrogate sample would not have been classified as the best representation of a tank farm sample if the surrogate had the closest flow curve data values but had other physical properties substantially different from those of the tank farm waste sample. The surrogate samples with the best flow curve characteristics had physical properties very similar to those of the tank farm waste samples.

The following sections contain SBW surrogate and tank farm waste sample flow curve plots and data regression correlations. All data included in this report were filtered by the Brookfield Engineering RHEO 2000 control and analysis software to eliminate data below the R/S rheometer torque threshold level required to ensure data accuracy.

The Brookfield Engineering RHEO 2000 software includes flow curve regression calculation capabilities. The RHEO 2000 software calculates Newton, Ostwald, Bingham, Casson, Steiger/Ory, and Herschel/Bulkley regressions for flow curve data (shear stress, $\tau[\mathrm{Pa}]$ vs. shear rate, $\mathrm{D}[1 / \mathrm{s}]$ ) recorded using the R/S rheometer. These six regression formulas are provided below. All six flow curve regression correlations were calculated for each step of each flow curve analysis. The regression correlation with the highest correlation coefficient was selected to represent the experimental flow curve data in the flow curve plots found in the following sections. The correlations may also be used for determining equipment and energy requirements in future SBW treatment process design studies.

\section{Newton}

$\tau=k_{1} D$

$\mathrm{k}_{1}=$ dynamic viscosity in Pa·s

\section{Ostwald}

$\tau=k_{2} D^{k_{3}}$

$\mathrm{k}_{2}=$ Ostwald consistence factor

$\mathrm{k}_{3}=$ Ostwald flow exponent

$$
\begin{aligned}
& \text { Bingham } \\
& \tau=k_{1}+k_{2} D \\
& \mathrm{k}_{1}=\text { Bingham yielding point in } \mathrm{Pa} \\
& \mathrm{k}_{2}=\text { Bingham viscosity in } \mathrm{Pa} \cdot \mathrm{s}
\end{aligned}
$$

\section{Casson}

$\sqrt{\tau}=\sqrt{k_{1}}+\sqrt{k_{2} D}$

$\mathrm{k}_{1}=$ Casson yielding point in $\mathrm{Pa}$

$\mathrm{k}_{2}=$ Casson viscosity in Pa $\cdot \mathrm{s}$

$$
\begin{aligned}
& \text { Steiger/Ory } \\
& D=k_{1} \tau+k_{2} \tau^{3}
\end{aligned}
$$

$$
\begin{aligned}
& \text { Herschel/Bulkley } \\
& \tau=k_{1}+k_{2} D^{k_{3}} \\
& \mathrm{k}_{1}=\text { yielding point in } \mathrm{Pa} \\
& \mathrm{k}_{2}=\text { plastic viscosity in } \mathrm{Pa} \cdot \mathrm{s} \\
& \mathrm{k}_{3}=\text { flow exponent }
\end{aligned}
$$


3.4.2.1.1 Supernate—The metathesis mother liquor liquid surrogate provides a conservative representation of the tank farm waste sample supernate. The WM-187 liquid surrogate provides a flow curve essentially indistinguishable from that of the WM-187 tank farm waste supernate sample. Physical properties of the metathesis mother liquor surrogate (META/0wt\%) and WM-187 liquid surrogate $(187 / 0 \mathrm{wt} \%)$ are similar to those of the tank farm waste sample supernate. Each liquid contained no visible undissolved solids. The tank farm waste supernate sample had a density of $1.029 \mathrm{~g} / \mathrm{mL}$ and a dissolved solids weight percentage of $4.7 \%$. The META/0wt $\%$ liquid surrogate had a density of 1.12 $\mathrm{g} / \mathrm{mL}$ and a dissolved solids weight percentage of $8.7 \%$. The $187 / 0 \mathrm{wt} \%$ liquid surrogate had a density of $1.05 \mathrm{~g} / \mathrm{mL}$ and a dissolved solids weight percentage of $4.7 \%$.

The WM-187 tank farm sample liquid supernate, SBW metathesis mother liquor supernate, and WM-187 liquid surrogate flow curves are presented in Figure 3-33. A plot of the viscosity vs. shear rate for these samples is presented in Figure 3-34. The tank farm waste shear stress was $\leq 15 \%$ less than the surrogate shear stress throughout the increasing and decreasing shear rate ranges tested. The WM-187 liquid surrogate flow curve shear stress data points overlap the tank farm waste supernate flow curve data points throughout the shear rate range tested. Tank farm waste and surrogate sample shear stress shear rate data regression correlations were calculated for increasing shear rate over the interval 500 $4000 \mathrm{sec}^{-1}$ and decreasing shear rate over the interval $4000-500 \mathrm{sec}^{-1}$. The shear stress - shear rate data regression correlations are presented in Figure 3-33. The solid lines in Figure 3-33 represent the data regression expressions for each increasing and decreasing shear rate step presented in Table 3-8, while the marker symbols represent experimental flow curve data.

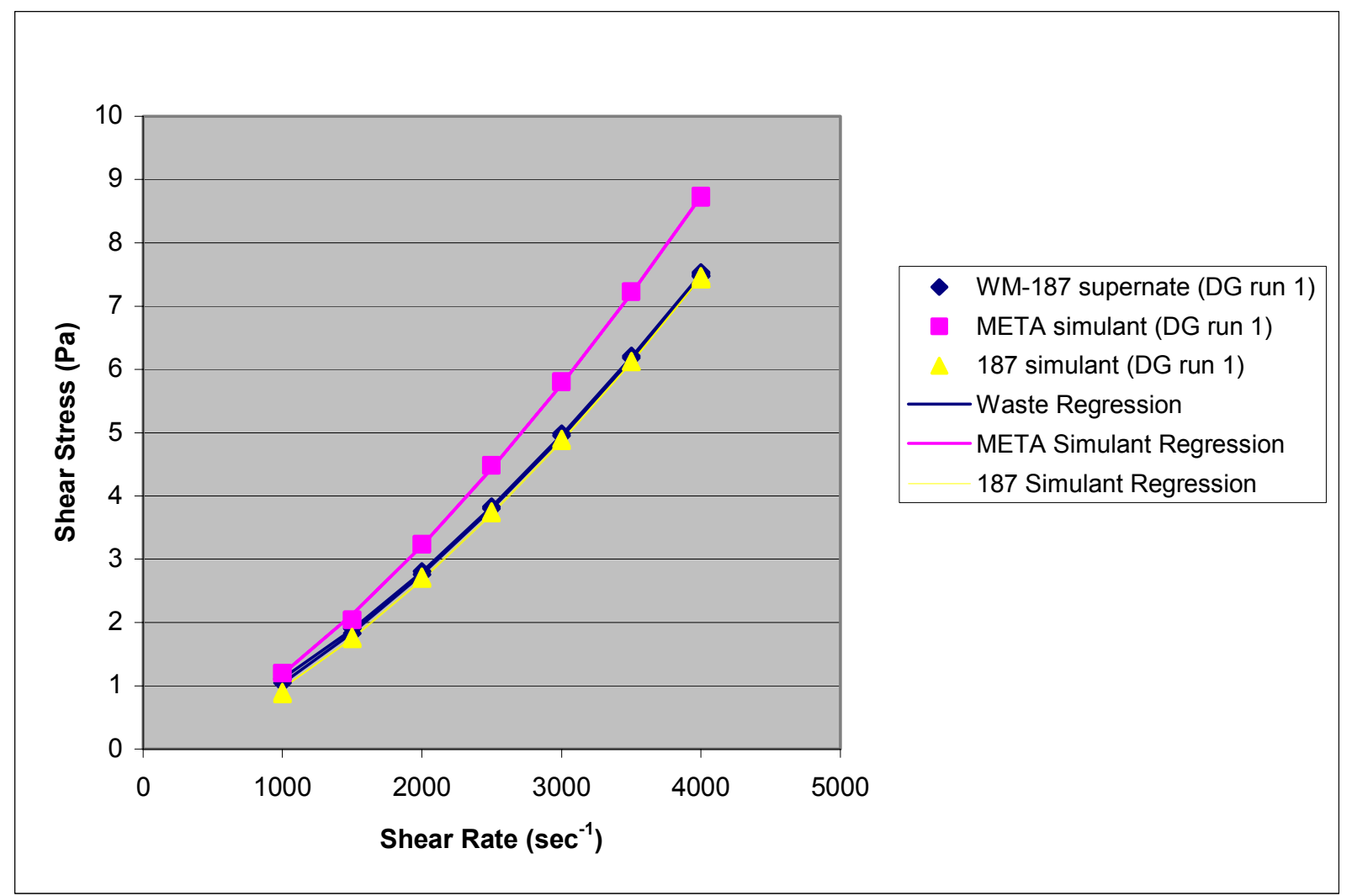

Figure 3-33. WM-187 supernate, metathesis mother liquor surrogate, and 187 liquid surrogate flow curve comparison. 


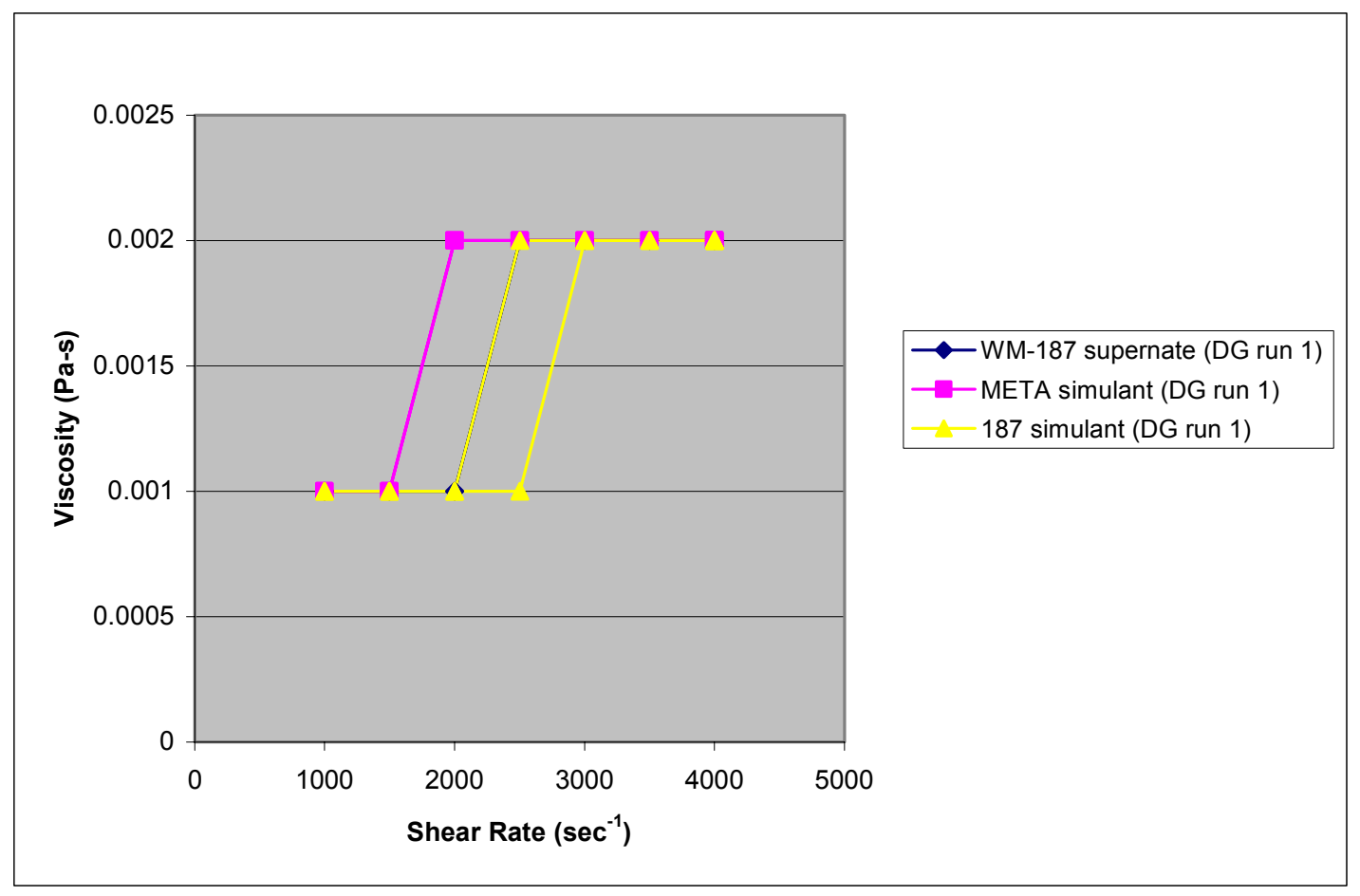

Figure 3-34. WM-187 supernate and metathesis surrogate mother liquor viscosity comparison.

3.4.2.1.2 Suspended Slurry ("As-Received")—The META/5wt\%/U/S surrogate (5 wt \% sonicated FY-04 undried SBW solids surrogate in metathesis mother liquor liquid surrogate) provides a conservative representation of the tank farm waste "as-received" slurry sample. Physical properties of the META $/ 5 \mathrm{wt} \% / \mathrm{U} / \mathrm{S}$ surrogate are similar to those of the tank farm waste "as-received" sample. Both samples contained approximately 90 volume percent visible undissolved solids, which were readily suspended when agitated. Following gravity settling, the "as-received" tank farm waste and META $/ 5 \mathrm{wt} \% / \mathrm{U} / \mathrm{S}$ SBW surrogate samples formed solids layers with sharp, distinct liquid interfaces. In each case, the liquid supernate contained no visible suspended solids. The tank farm waste "as-received" sample had a density of $1.066 \mathrm{~g} / \mathrm{mL}$ and a solids weight percentage of $7.1 \%$. The META $/ 5 \mathrm{wt} \% / \mathrm{U} / \mathrm{S}$ surrogate had a density of $1.13 \mathrm{~g} / \mathrm{mL}$ and a solids weight percentage of $11.3 \%$.

The WM-187 tank farm "as-received" slurry sample and META/5wt\%/U/S SBW slurry surrogate flow curves are presented in Figure 3-35. A plot of the viscosity vs. shear rate for these samples is presented in Figure 3-36. The tank farm waste shear stress was $\leq 33 \%$ less than the surrogate shear stress throughout the increasing and decreasing shear rate ranges tested.

The thixotropy of the META/5 wt \%/U/S SBW slurry surrogate exceeded that of the tank farm waste "as-received" sample. Multiple flow curve analysis runs were preformed to obtain a stabilized surrogate flow curve loop with minimal thixotropic hysteresis. Flow curve data for repeat analysis of the META $/ 5 \mathrm{wt} \% / \mathrm{U} / \mathrm{S}$ slurry surrogate is included in Figure 3-35. The META/5 wt \%/U/S flow curve did not change visibly in the additional analyses performed.

A number of surrogate formulation and conditioning measures could be taken to reduce the particle agglomeration and thixotropy of the surrogate slurries. The bulk of the SBW solids surrogate is composed of silica and alumina. The silica is largely in the form of silica gels, which contribute to the agglomeration and high thixotropy. Although the silica composition is similar to that of the tank farm waste, the tank 


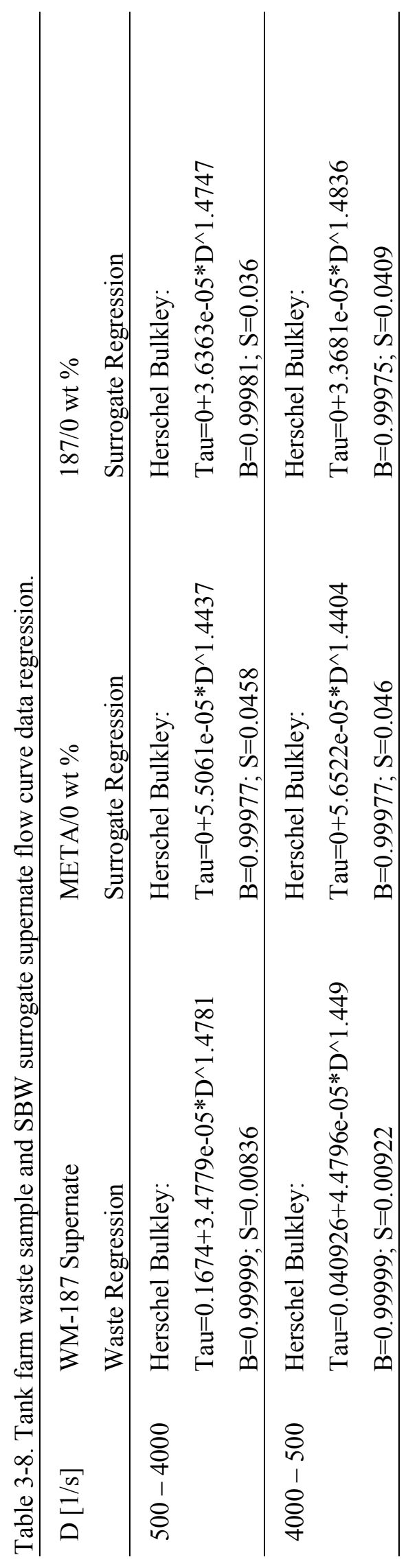




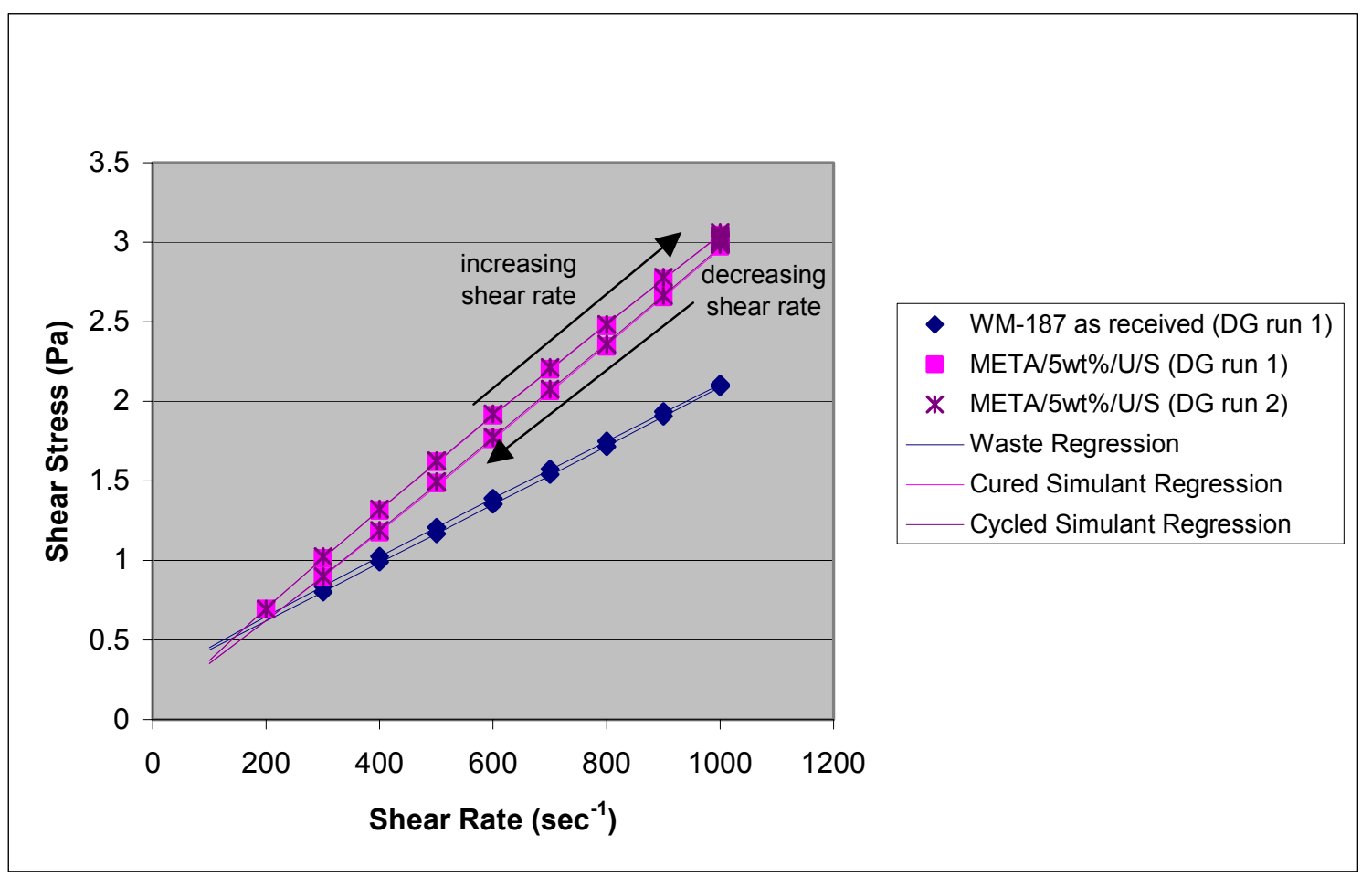

Figure 3-35. WM-187 as received and META/5wt\%/U/S surrogate data regression.

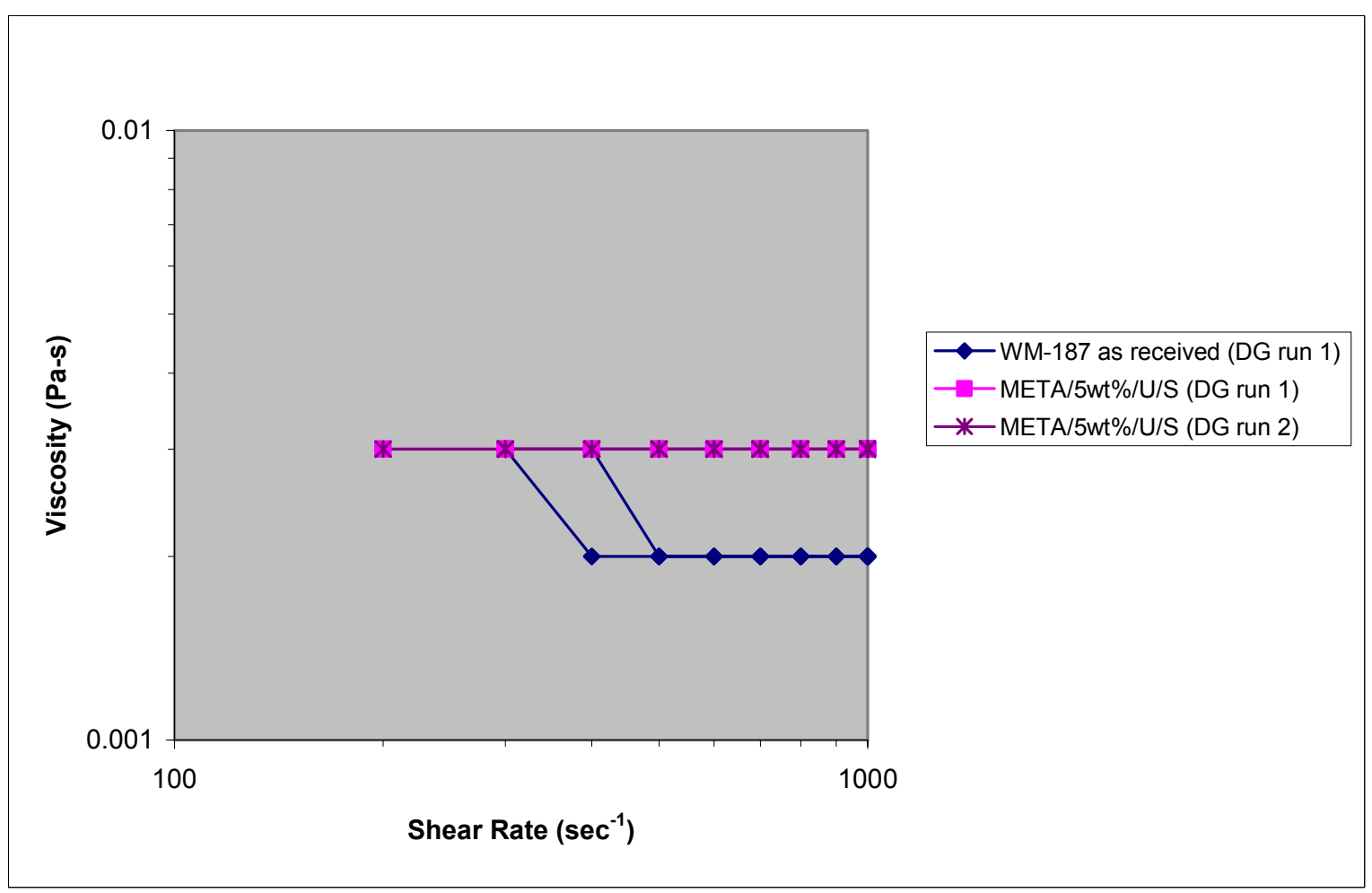

Figure 3-36. WM-187 as received and META/5wt\%/U/S surrogate viscosity comparison. 
farm waste has had years of aging in a highly radioactive environment that have propagated silica gel dewatering reactions. The surrogate could be artificially aged by thermal conditioning. Alternatively, the composition of the solids surrogate could be altered by precipitating a higher quantity of metal oxides from the metathesis mother liquor. It is expected that either modification would reduce the tendency of the SBW solids surrogate particles toward agglomeration, thereby reducing the surrogate thixotropy.

Tank farm waste and surrogate sample shear stress - shear rate data regression correlations were calculated for increasing shear rate over the interval $100-1000 \mathrm{sec}^{-1}$ and decreasing shear rate over the interval $1000-100 \mathrm{sec}^{-1}$. The shear stress - shear rate data regression correlations are presented in Table 3-9. The solid lines in Figure 3-35 represent the data regression expressions for each increasing and decreasing shear rate step presented in Table 3-9, while the marker symbols represented experimental flow curve data.

3.4.2.1.3 Gravity-Settled Slurry-The META/10wt $\% / \mathrm{U} / \mathrm{S}$ surrogate (10 wt $\%$ sonicated FY-04 undried SBW solids surrogate in metathesis mother liquor liquid surrogate) provides a conservative representation of the tank farm waste gravity settled slurry sample. Physical properties of the META/10wt\%/U/S surrogate are similar to those of the tank farm waste gravity-settled sample. Both samples contained approximately 95 volume percent visible undissolved solids, which were readily suspended when agitated. The tank farm waste gravity-settled sample had a density of $1.035 \mathrm{~g} / \mathrm{mL}$ and a solids weight percentage of $10 \%$. The META $/ 10 \mathrm{wt} \% / \mathrm{U} / \mathrm{S}$ surrogate had a density of $1.16 \mathrm{~g} / \mathrm{mL}$ and a solids weight percentage of $15.1 \%$.

The WM-187 tank farm gravity-settled slurry sample and META/10wt\%/U/S SBW slurry surrogate flow curves are presented in Figure 3-37. A plot of the viscosity vs. shear rate for these samples is presented in Figure 3-38.

The Run \#1 surrogate flow curve analysis (following overnight aging) shear stress was several times greater than the Run \#1 tank farm waste shear stress for the increasing shear rate steps below $100 \mathrm{sec}^{-1}$. The tank farm waste shear stress was $\leq 40 \%$ less than the surrogate shear stress for the increasing shear rate steps in the range of 100 to $1000 \mathrm{sec}^{-1}$. The tank farm waste shear stress was $\leq 20 \%$ less than the surrogate shear stress for the decreasing shear rate steps in the range of 1000 to $500 \mathrm{sec}^{-1}$. The surrogate shear stress was less than the tank farm waste shear stress for the decreasing shear rate steps below $500 \mathrm{sec}^{-1}$.

The Run \#8 surrogate flow curve analysis (stabilized surrogate flow curve) shear stress was less than the Run \#2 tank farm waste shear stress for the increasing shear rate steps below $400 \mathrm{sec}^{-1}$. The tank farm waste shear stress was $\leq 10 \%$ less than the surrogate shear stress for the increasing shear rate steps in the range of 400 to $1000 \mathrm{sec}^{-1}$. The tank farm waste shear stress was $\leq 10 \%$ less than the surrogate shear stress for the decreasing shear rate steps in the range of 1000 to $900 \mathrm{sec}^{-1}$. The surrogate shear stress was less than the tank farm waste shear stress for the decreasing shear rate steps below $900 \mathrm{sec}^{-1}$.

The differences between the META/10 wt \%/U/S Run \#1 and Run \#8 flow curves are indicative of the thixotropic nature of the surrogate. The tank farm waste gravity-settled sample displayed a much lower level of thixotropy than the META/10wt\%/U/S SBW slurry surrogate sample. The META $/ 10 \mathrm{wt} \% / \mathrm{U} / \mathrm{S} \mathrm{SBW}$ surrogate sample thixotropy may render the surrogate conservative for activities involving simulation of gravity-settled SBW handling operations. However, the META/10wt\%/U/S surrogate thixotropy could result in a unconservative estimate of gravity-settled SBW slurry flow properties for extended pumping/agitation operations. 


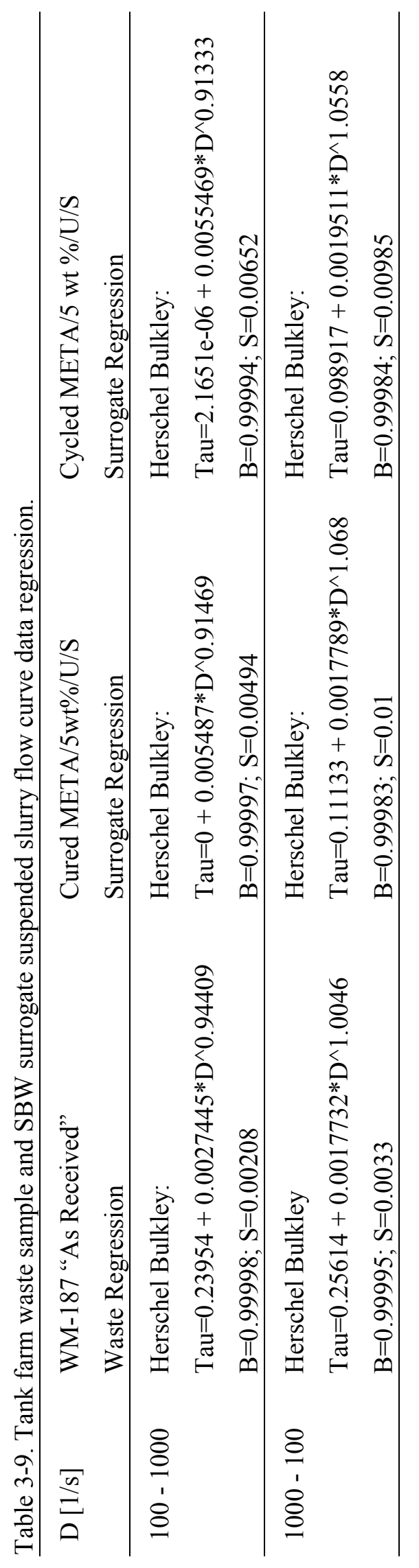




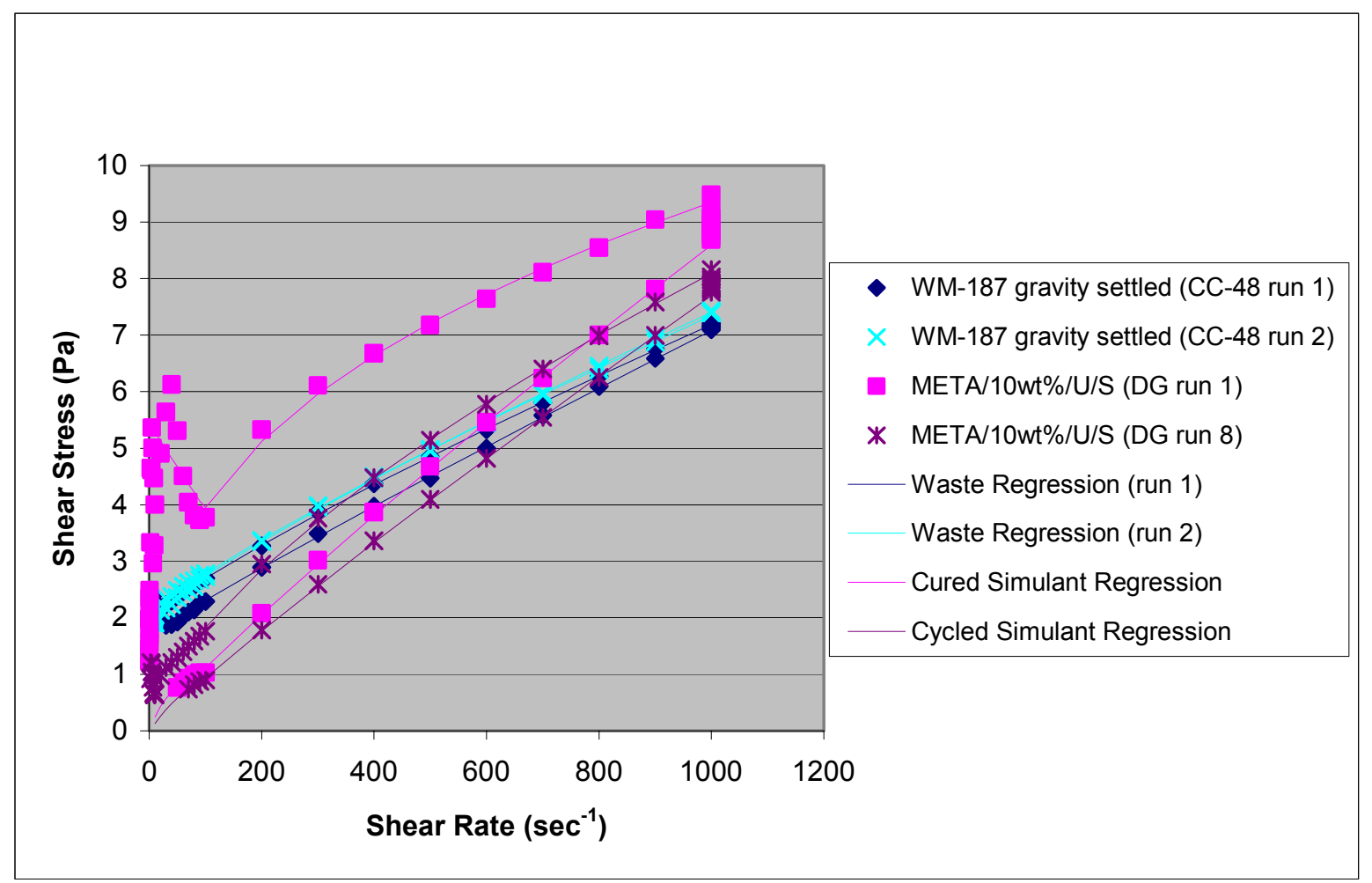

Figure 3-37. WM-187 gravity settled and META/10wt\%/U/S surrogate data regression.

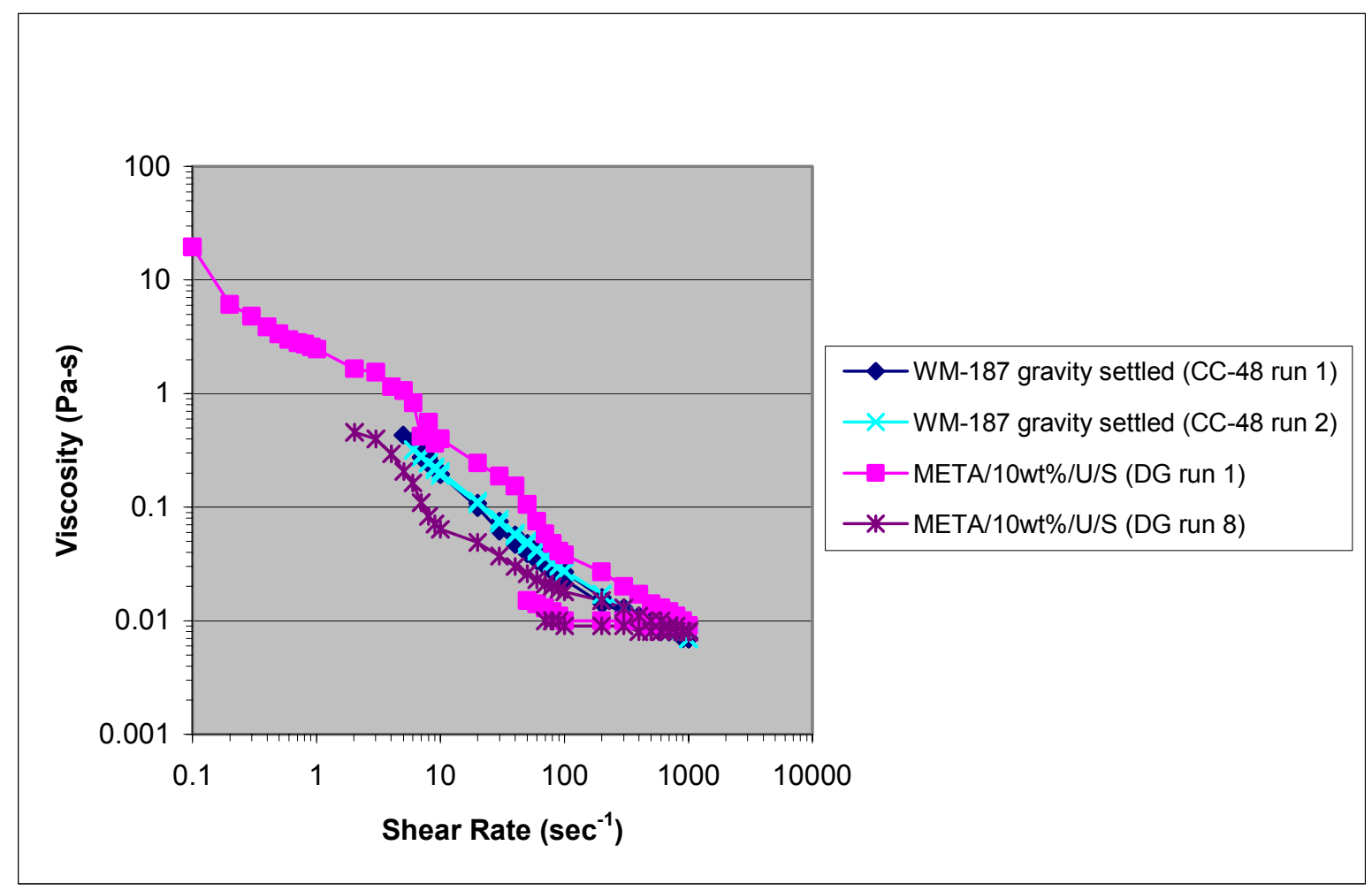

Figure 3-38. WM-187 gravity settled and META/10wt\%/U/S surrogate viscosity comparison. 
The META/10 wt \%/U/S surrogate and gravity-settled tank farm waste are pseudoplastic fluids. Pseudoplastic fluids display a decreasing viscosity with an increasing shear rate, as shown in Figure 3-38. Pseudoplastic flow behavior is sometimes referred to as "shear thinning."

A number of surrogate formulation and conditioning measures could be taken to reduce the particle agglomeration and thixotropy of the surrogate slurries. The bulk of the SBW solids surrogate is composed of silica and alumina. The silica is largely in the form of silica gels, which contribute to the agglomeration and high thixotropy. Although the silica composition is similar to that of the tank farm waste, the tank farm waste has had years of aging in a highly radioactive environment that have propagated silica gel dewatering reactions. The surrogate could be artificially aged by thermal conditioning. Alternatively, the composition of the solids surrogate could be altered by precipitating a higher quantity of metal oxides from the metathesis mother liquor. It is expected that either modification would reduce the tendency of the SBW solids surrogate particles toward agglomeration, thereby reducing the surrogate thixotropy.

Tank farm waste and surrogate sample shear stress - shear rate data regression correlations were calculated for increasing shear rate over the interval $100-1000 \mathrm{sec}^{-1}$ and decreasing shear rate over the interval $1000-100 \mathrm{sec}^{-1}$. The shear stress - shear rate data regression correlations are presented in Table 3-10. The solid lines in Figure 3-37 represent the data regression expressions for each increasing and decreasing shear rate step presented in Table 3-10, while the marker symbols represent experimental flow curve data.

3.4.2.1.4 Centrifuged Slurry-The META/20wt $\% / \mathrm{U} / \mathrm{S}$ surrogate $(20 \mathrm{wt} \%$ sonicated FY-04 undried SBW solids surrogate in metathesis mother liquor liquid surrogate) provides a conservative representation of the tank farm waste centrifuged slurry sample. Physical properties of the META $/ 20 \mathrm{wt} \% / \mathrm{U} / \mathrm{S}$ surrogate are similar to those of the tank farm waste centrifuged sample. Neither sample included any appreciable quantity of free liquid. The tank farm waste centrifuged sample had a solids weight percentage of $11.5 \%$ while the META/20 wt \%/U/S surrogate had a solids weight percentage of $26.5 \%$.

The WM-187 tank farm centrifuged slurry sample and META/20wt\%/U/S SBW slurry surrogate flow curves are presented in Figure 3-39. A plot of the viscosity vs. shear rate for these samples is presented in Figure 3-40.

The Run \#1 surrogate flow curve analysis (following overnight aging) shear stress was several times greater than the Run \#1 tank farm waste shear stress for the increasing shear rate steps below $100 \mathrm{sec}^{-1}$. The tank farm waste shear stress was $\leq 45 \%$ less than the surrogate shear stress for the increasing shear rate steps in the range of 100 to $1000 \mathrm{sec}^{-1}$. The tank farm waste shear stress was $\leq 30 \%$ less than the surrogate shear stress for the decreasing shear rate steps in the range of 1000 to $400 \mathrm{sec}^{-1}$. The surrogate shear stress was less than the tank farm waste shear stress for the decreasing shear rate steps below $400 \mathrm{sec}^{-1}$.

The Run \#8 surrogate flow curve analysis (stabilized surrogate flow curve) shear stress was several times greater than the Run \#2 tank farm waste shear stress for the increasing shear rate steps below $100 \mathrm{sec}^{-1}$. The tank farm waste shear stress was $\leq 60 \%$ less than the surrogate shear stress for the increasing shear rate steps in the range of 100 to $1000 \mathrm{sec}^{-1}$. The tank farm waste shear stress was $\leq 30 \%$ less than the surrogate shear stress for the decreasing shear rate steps in the range of 1000 to $500 \mathrm{sec}^{-1}$. The surrogate shear stress was less than the tank farm waste shear stress for the decreasing shear rate steps below $500 \mathrm{sec}^{-1}$. 


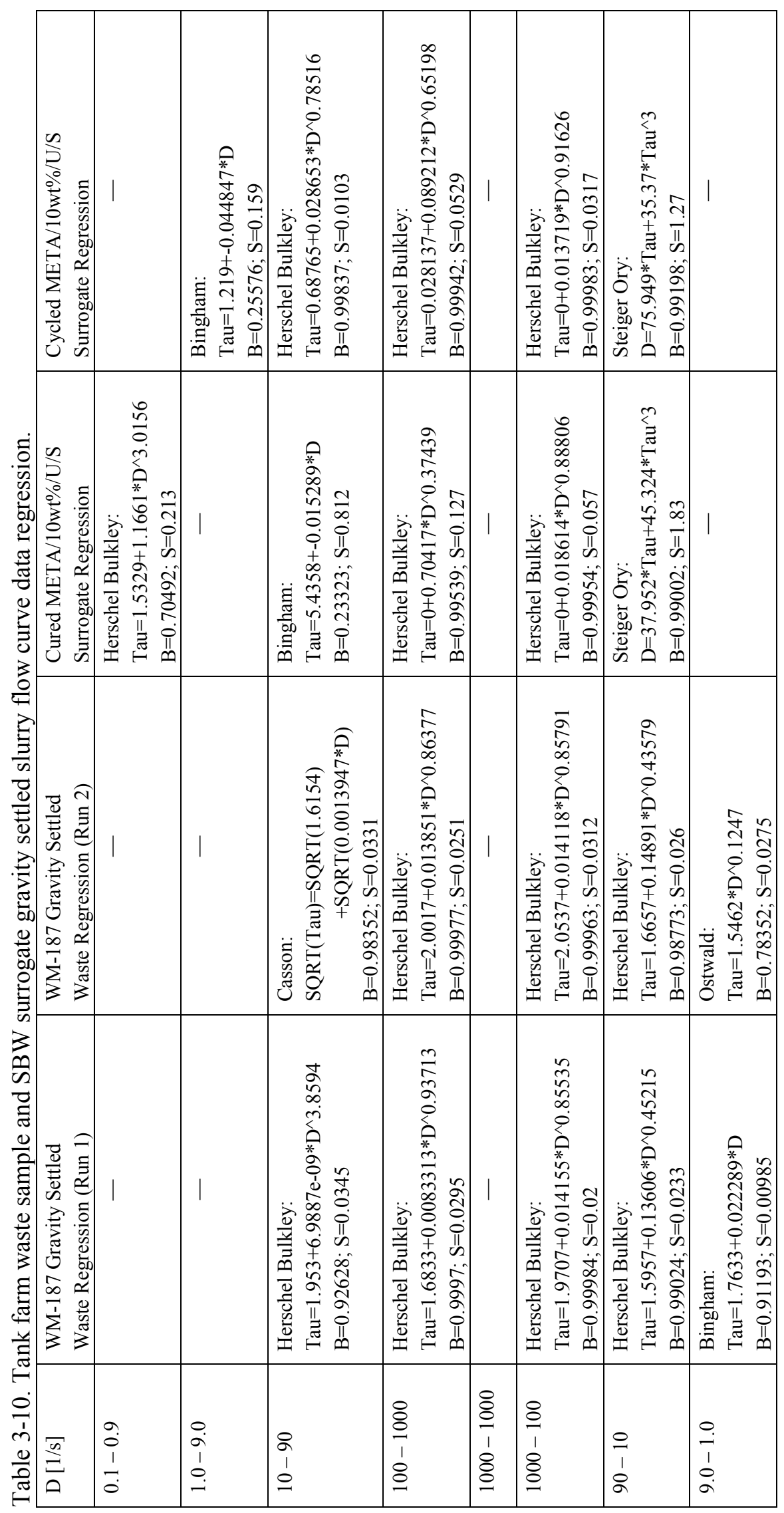




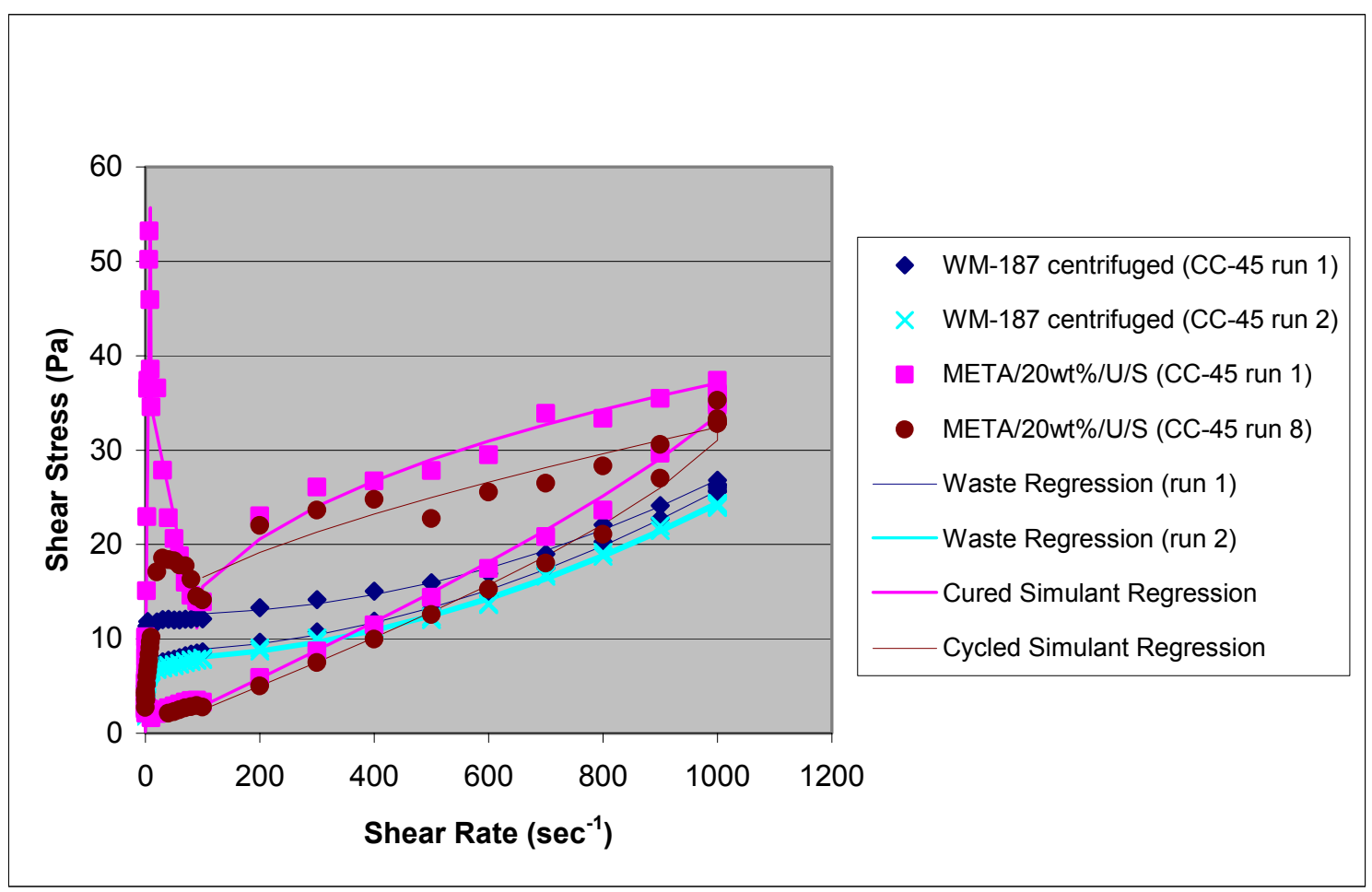

Figure 3-39. WM-187 centrifuged and META/20wt $\% / \mathrm{U} / \mathrm{S}$ surrogate data regression.

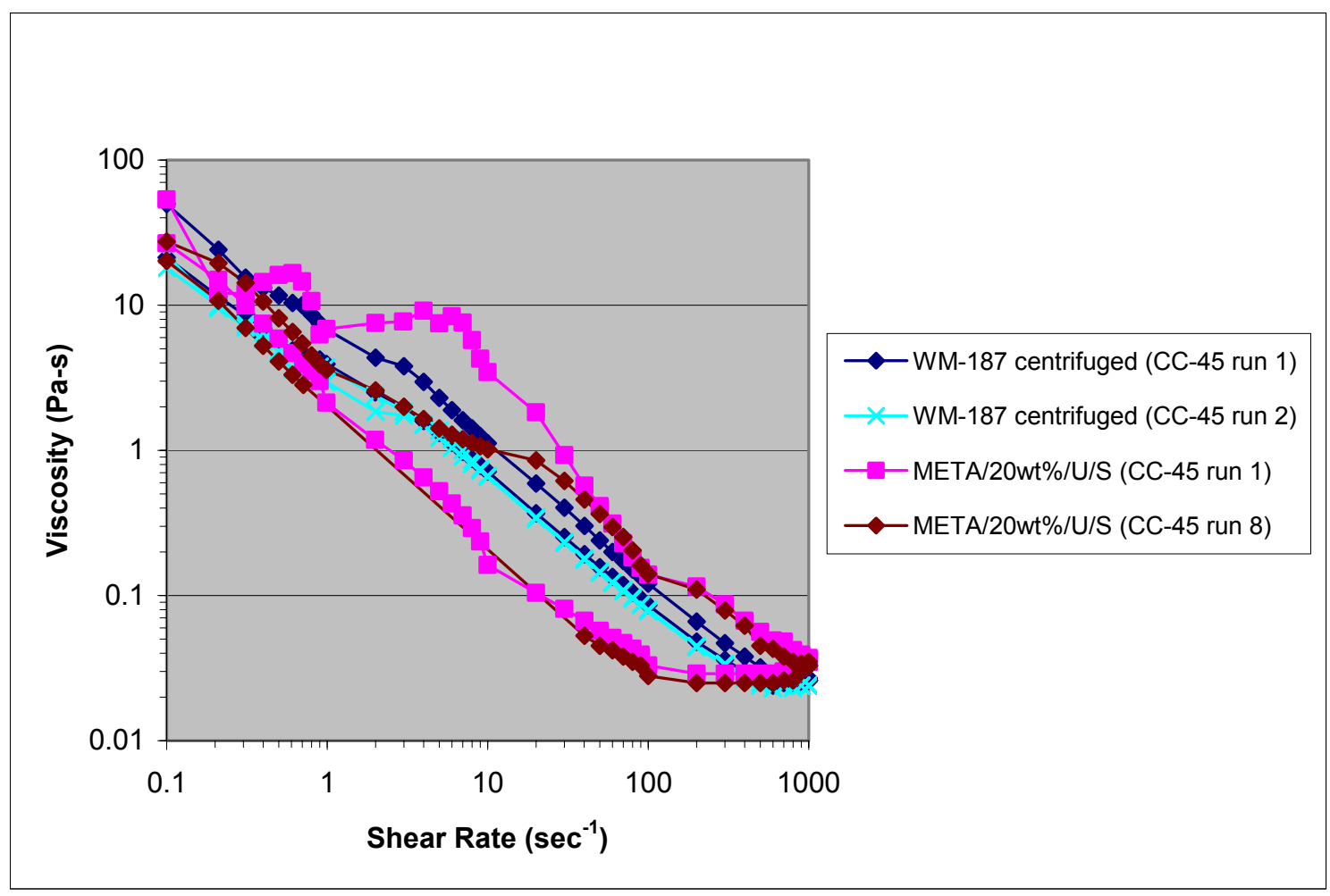

Figure 3-40. WM-187 centrifuged and META/20wt $\% / \mathrm{U} / \mathrm{S}$ surrogate viscosity comparison. 
The differences between the META/20wt\%/U/S Run \#1 and Run \#8 flow curves are indicative of the thixotropic nature of the surrogate. The tank farm waste centrifuged slurry sample displayed a much lower level of thixotropy than the META/20wt $\% / \mathrm{U} / \mathrm{S}$ SBW slurry surrogate sample. The META $/ 20 \mathrm{wt} \% / \mathrm{U} / \mathrm{S} \mathrm{SBW}$ surrogate sample thixotropy may render the surrogate conservative for activities involving simulation of settled SBW sludge handling operations. However, the META/20wt\%/U/S surrogate thixotropy could result in a unconservative estimate of SBW sludge flow properties for extended pumping/agitation operations.

The META/20wt $\% / \mathrm{U} / \mathrm{S}$ surrogate and tank farm waste centrifuged slurry are pseudoplastic fluids. Pseudoplastic fluids display a decreasing viscosity with an increasing shear rate, as shown in Figure 3-40. Pseudoplastic flow behavior is sometimes referred to as "shear thinning."

A number of surrogate formulation and conditioning measures could be taken to reduce the particle agglomeration and thixotropy of the surrogate slurries. The bulk of the SBW solids surrogate is composed of silica and alumina. The silica is largely in the form of silica gels, which contribute to the agglomeration and high thixotropy. Although the silica composition is similar to that of the tank farm waste, the tank farm waste has had years of aging in a highly radioactive environment that have propagated silica gel dewatering reactions. The surrogate could be artificially aged by thermal conditioning. Alternatively, the composition of the solids surrogate could be altered by precipitating a higher quantity of metal oxides from the metathesis mother liquor. It is expected that either modification would reduce the tendency of the SBW solids surrogate particles toward agglomeration, thereby reducing the surrogate thixotropy.

Tank farm waste and surrogate sample shear stress - shear rate data regression correlations were calculated for increasing shear rate over the intervals of $0.1-0.9,1.0-9.0,10-90$, and $100-1000 \mathrm{sec}^{-1}$ and decreasing shear rate over the intervals of $1000-100,90-10,9.0-1.0$, and $0.9-0.1 \mathrm{sec}^{-1}$. The shear stress - shear rate data regression correlations are presented in Table 3-11. The solid lines in Figure 3-39 represent the data regression expressions for each increasing and decreasing shear rate step presented in Table 3-11, while the marker symbols represent experimental flow curve data.

Figure 3-41 indicates that the shape of the $187 / 20 \mathrm{wt} \% / \mathrm{D} / \mathrm{S}$ sample flow curve bears a significant resemblance to that of the centrifuged WM-187 tank farm waste sample flow curve. Although the $187 / 20 \mathrm{wt} \% / \mathrm{D} / \mathrm{S}$ flow curve shear stress is lower than that of the centrifuged WM-187 tank farm waste sample, the thixotropy of the $187 / 20 \mathrm{wt} \% / \mathrm{D} / \mathrm{S}$ sample is more similar to that of the centrifuged WM-187 tank farm waste sample than the META/20 wt \%/U/S sample. One notable difference between the $187 / 20 \mathrm{wt} \% / \mathrm{D} / \mathrm{S}$ and centrifuged WM-187 tank farm waste sample was the solids volume percentage. The solids volume percentage of the $187 / 20 \mathrm{wt} \% / \mathrm{D} / \mathrm{S}$ sample ( $\sim 95$ solids volume percentage $)$ was less than that of the centrifuged WM-187 tank farm waste sample ( $\sim 100$ solids volume percentage). If the solids volume percentage of the $187 / 20 \mathrm{wt} \% / \mathrm{D} / \mathrm{S}$ sample was increased to the level of the centrifuged WM-187 tank farm waste sample, or approximately 100 solids volume percent, there is a high probability that the $187 / 20 \mathrm{wt} \% / \mathrm{D} / \mathrm{S}$ surrogate sample could provide a conservative representation of the centrifuged tank farm solids. A comparison of the $187 / 20 \mathrm{wt} \% / \mathrm{D} / \mathrm{S}$ and centrifuged WM-187 tank farm waste viscosity versus shear rate plots is provided in Figure 3-42.

A WM-187 centrifuged tank farm waste sample was allowed to sit overnight and the flow curve analysis was repeated. The flow curve was essentially identical to the WM-187 centrifuged sample flow curve shown in Figure 3-39. The WM-187 centrifuged tank farm waste sample was then allowed to sit for an additional six days and the flow curve analysis was repeated with substantially different results. It is suspected that during the six day period the sample dried out to the extent that the flow curve was no longer representative of a wet tank farm sample. The six day sample is, therefore, not included in this report. The data and flow curve plot are, however, included in Appendix E. 


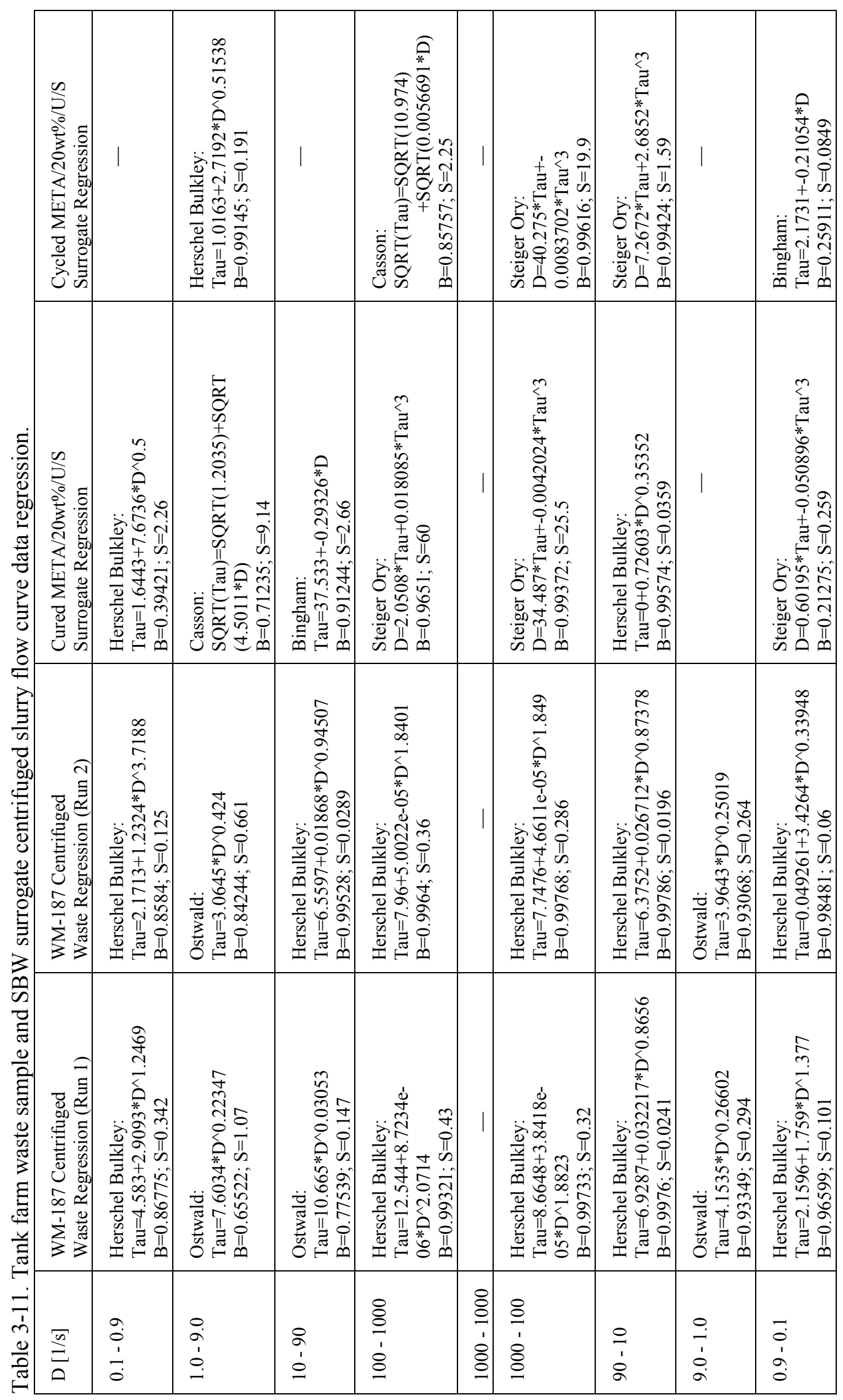




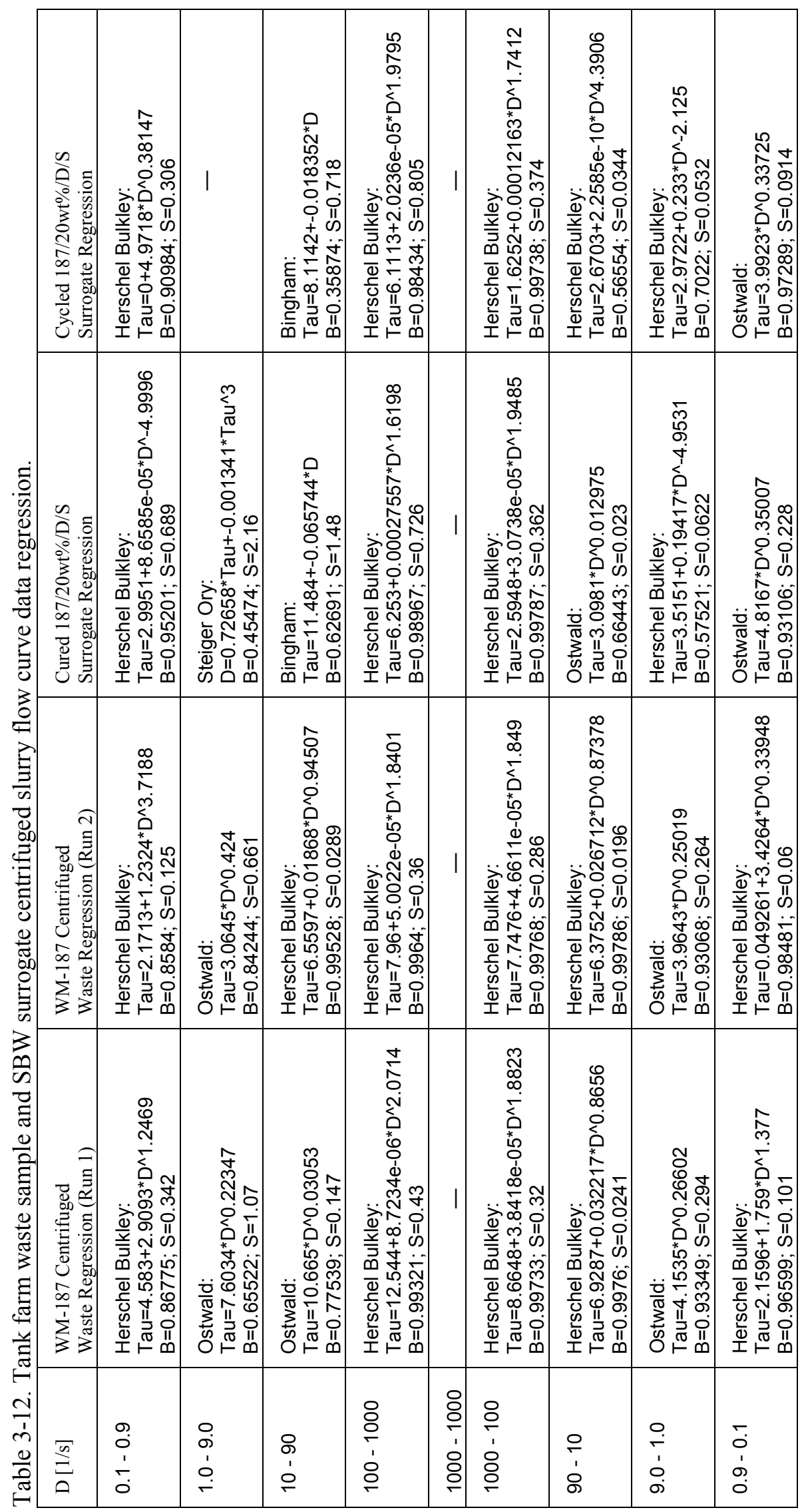




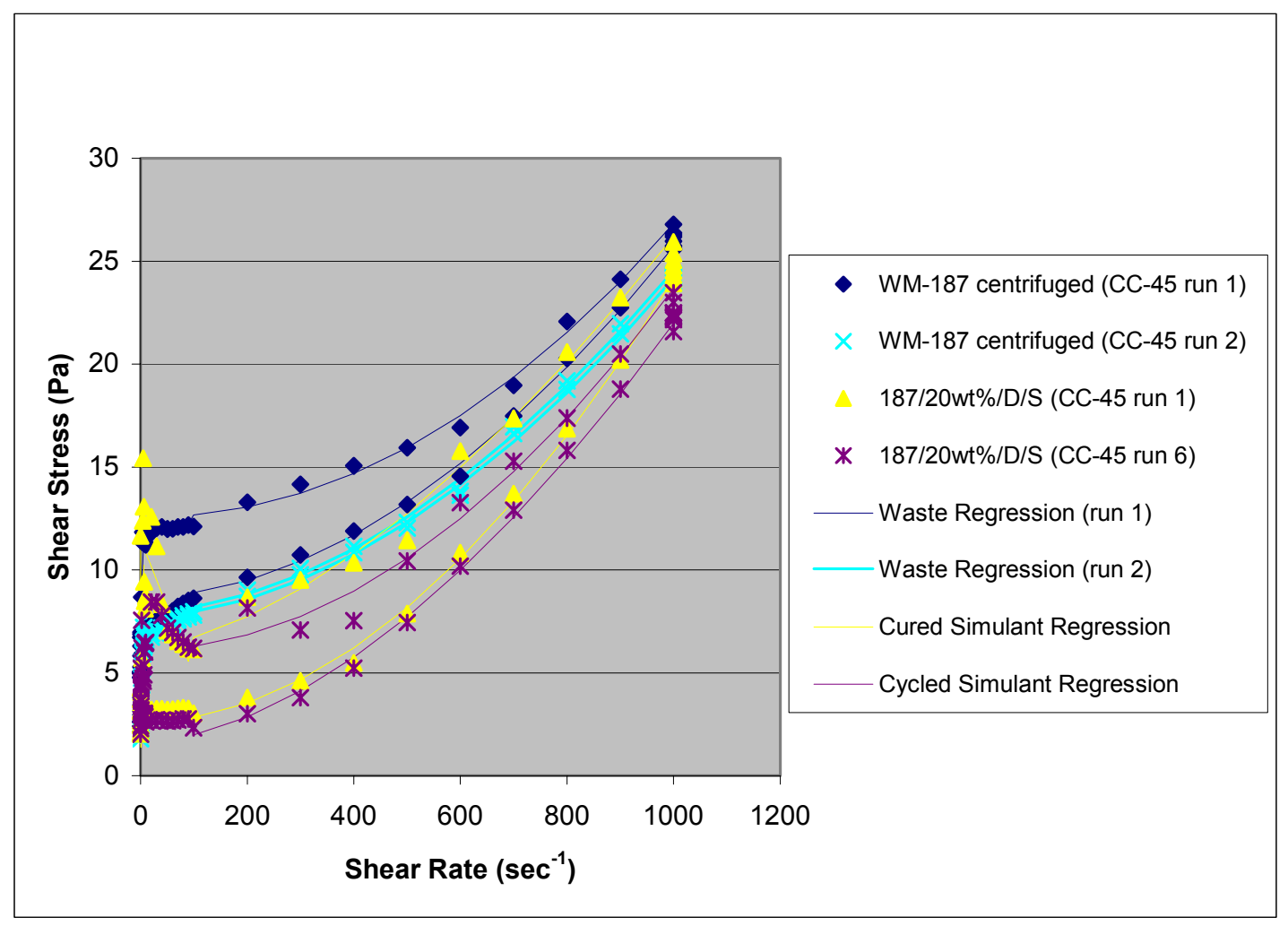

Figure 3-41. WM-187 centrifuged and 187/20wt\%/D/S surrogate data comparison.

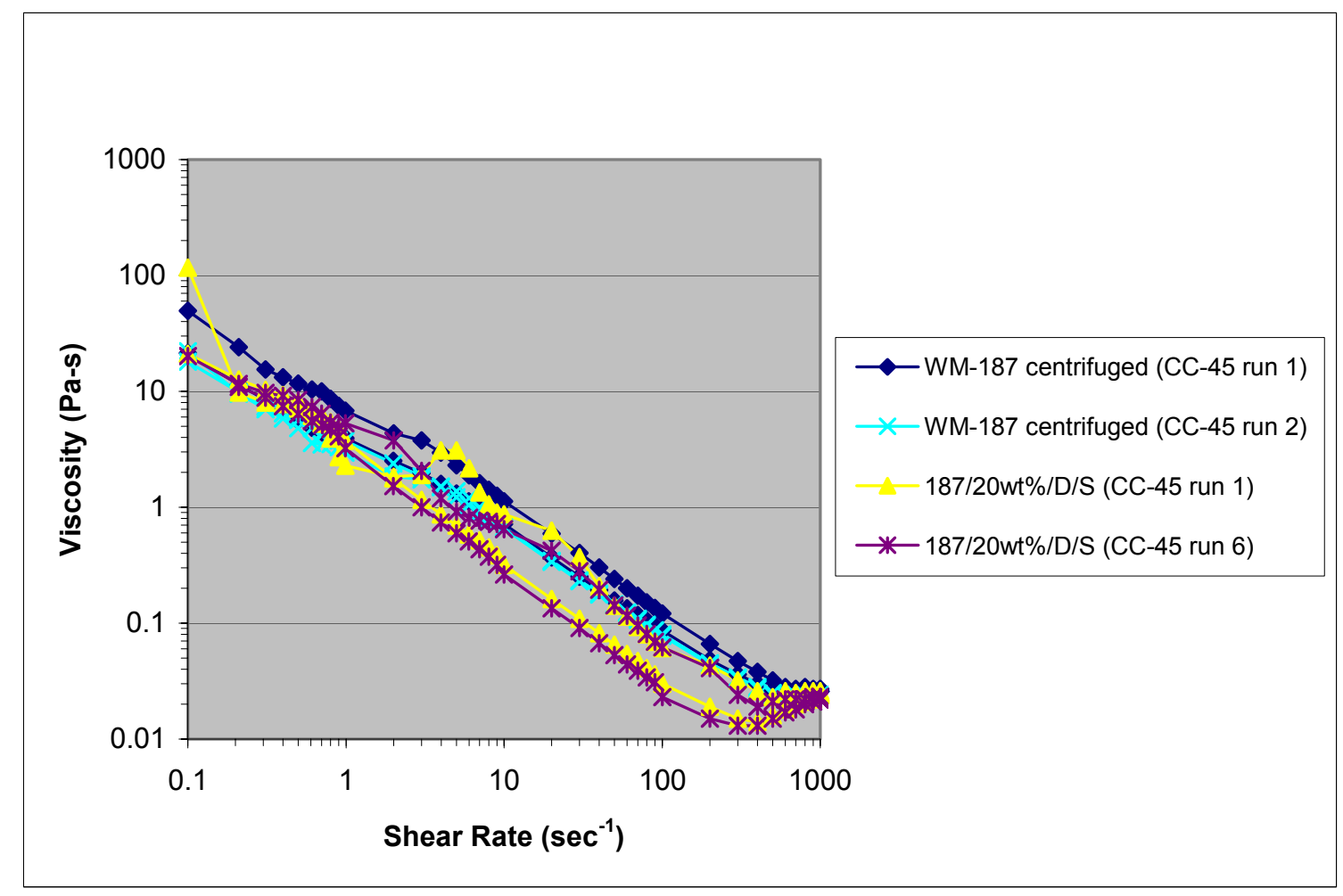

Figure 3-42. WM-187 centrifuged and $187 / 20 \mathrm{wt} \% / \mathrm{D} / \mathrm{S}$ surrogate viscosity comparison. 
3.4.2.2 Flow Curves for Various Surrogate Samples. Several SBW surrogate preparation parameters were varied to produce surrogates with a range of compositions and physical properties. The sample preparation parameters that were varied were solids weight percentage, liquid surrogate composition, solids surrogate batch (i.e., FY-03 or FY-04 solids), and particle size distribution. A flow curve analysis was performed on each of the differently prepared samples. The sample preparation parameters were varied to increase the probability of testing a range of surrogates with physical properties similar to the tank farm waste samples analyzed after the SBW surrogates. Analysis of the differently prepared samples provided insight into how the sample flow curve profile and rheological properties responded to changes in each of the sample preparation parameters. In addition to variation of sample preparation parameters, flow curve analyses were performed at different temperatures. These data are useful because they provide insight into how the tank farm waste may react to similar changes.

3.4.2.2.1 Solids Weight Percentage-Physical property analyses were performed on SBW surrogate samples with solids weight percentages ranging from 0 to $20 \mathrm{wt} \%$. The analyses performed on each SBW surrogate sample included a shear stress versus shear rate flow curve.

The flow curves of samples prepared using 0 to 20 weight percentage sonicated FY-04 undried SBW solids surrogate in metathesis mother liquor liquid surrogate were compared. The lower viscosity limit of the Brookfield R/S rheometer prevented the zero solids weight percent surrogate from being tested in the CC-45 measuring system geometry in which the $5-20$ solids weight percentage surrogates were analyzed at shear rates from 0.1 to $1000 \mathrm{sec}^{-1}$. Conversely, the 20 solids weight percent surrogate was not tested in the DG measuring system in which the 0 to 15 solids weight percentage surrogates were analyzed at shear rates from $500-4000 \mathrm{sec}^{-1}$ due to solids loading concerns. Therefore, two flow curve comparison plots were constructed, the first with 5 to 20 solids weight percentage surrogates analyzed at shear rates ranging from 0.1 to $1000 \mathrm{sec}^{-1}$, and a second with 0 to 15 solids weight percentage surrogates analyzed at shear rates ranging from 500 to $4000 \mathrm{sec}^{-1}$. Both flow curve comparison plots illustrated a trend of increasing shear stress with increasing sample solids weight percentage for a given shear rate. Figure 3-43 is a plot of the 5 to 20 solids weight percentage sample flow curves with a shear rate range of 0.1 to $1000 \mathrm{sec}^{-1}$. The shear stress is greatest for the samples of the highest solids weight percentage, 20 $\mathrm{wt} \%>15 \mathrm{wt} \%>10 \mathrm{wt} \%>5 \mathrm{wt} \%$. Figure 3-44 is a plot of the 0 to 15 solids weight percentage sample flow curves with a shear rate range of 500 to $4000 \mathrm{sec}^{-1}$. Again, the shear stress is greatest for the samples of the highest solids weight percentage, $15 \mathrm{wt} \%>10 \mathrm{wt} \%>5 \mathrm{wt} \%>0 \mathrm{wt} \%$.

\subsection{Liquid Surrogate and Solids Surrogate Batch-Physical property} analyses were performed on SBW surrogate samples formulated using different liquid surrogates. Liquid surrogates used in SBW surrogate sample formulation included WM-180, WM-187, and WM-189 liquid surrogates as well as the metathesis mother liquor. A shear rate versus shear stress flow curve analysis was performed on several samples formulated with each of the four liquid surrogates.

The flow curves of samples prepared using the various liquid surrogates were compared. Samples with 0,5 , and 20 solids weight percentage sonicated FY-04 undried SBW solids surrogate were compared. Flow curve comparison plots were constructed for samples that had identical solids weight percentages prepared using different liquid surrogates. The samples compared in these plots had similar particle size distributions and the solid surrogates were from the same batch where possible (the WM-187 liquid surrogate was only used with FY-03 solids surrogate, and the FY-03 solids surrogate was only used with the WM-187 liquid surrogate).

Figure 3-45 is a plot of the various 0 solids weight percent surrogate flow curves at shear stresses ranging from 500 to $4000 \mathrm{sec}^{-1}$. Figure 3-46 and Figure 3-47 are plots of the 5 and 20 weight percent surrogate flow curves, respectively, at shear stresses ranging from 0.1 to $1000 \mathrm{sec}^{-1}$. In each of the plots, the shear stress profiles for the four liquid surrogates follow the pattern WM-189 > WM-180 > META > WM-187. 


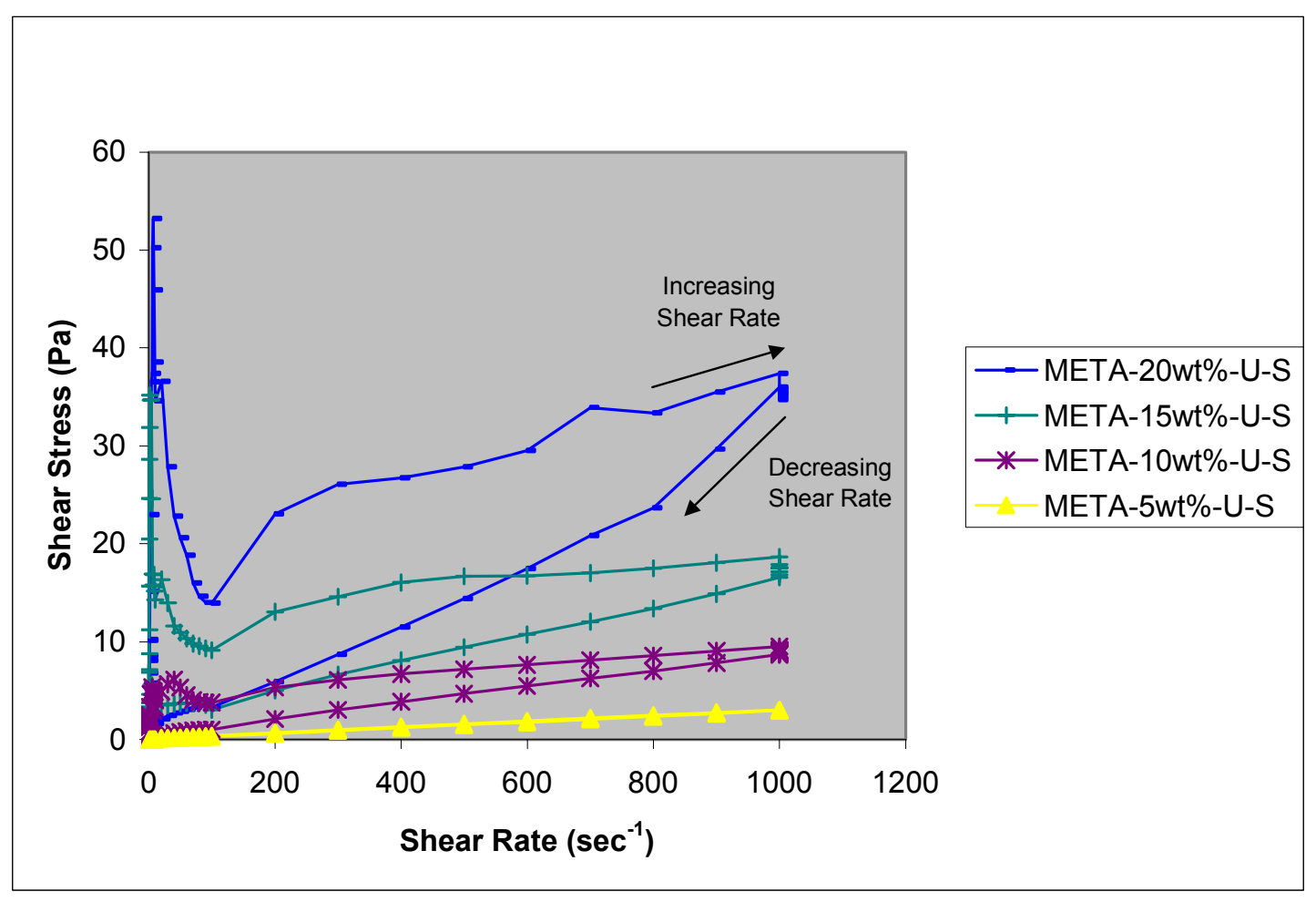

Figure 3-43. META/5/10/15/20 wt $\%$ comparison.

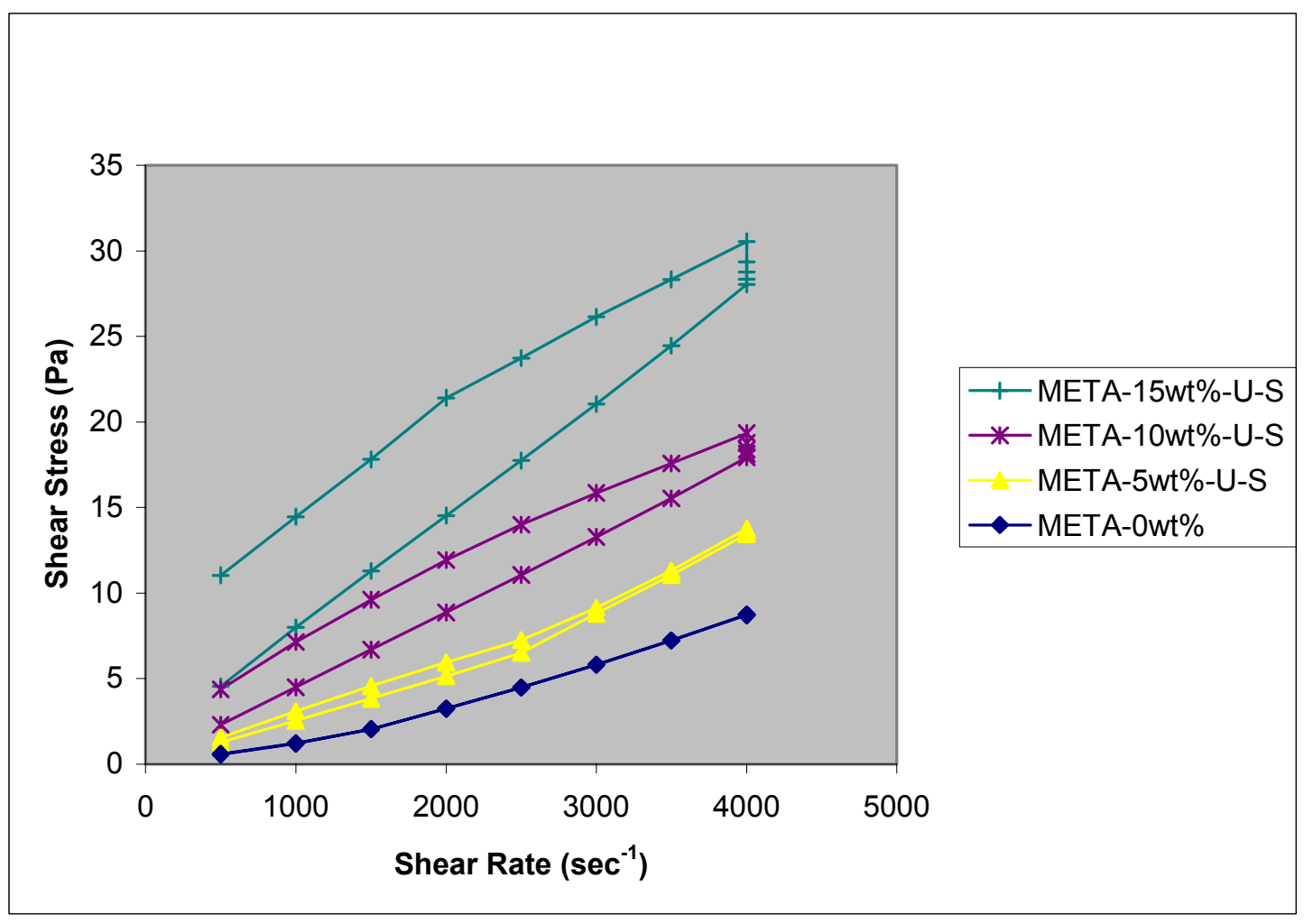

Figure 3-44. META 0/5/10/15 wt\% comparison. 


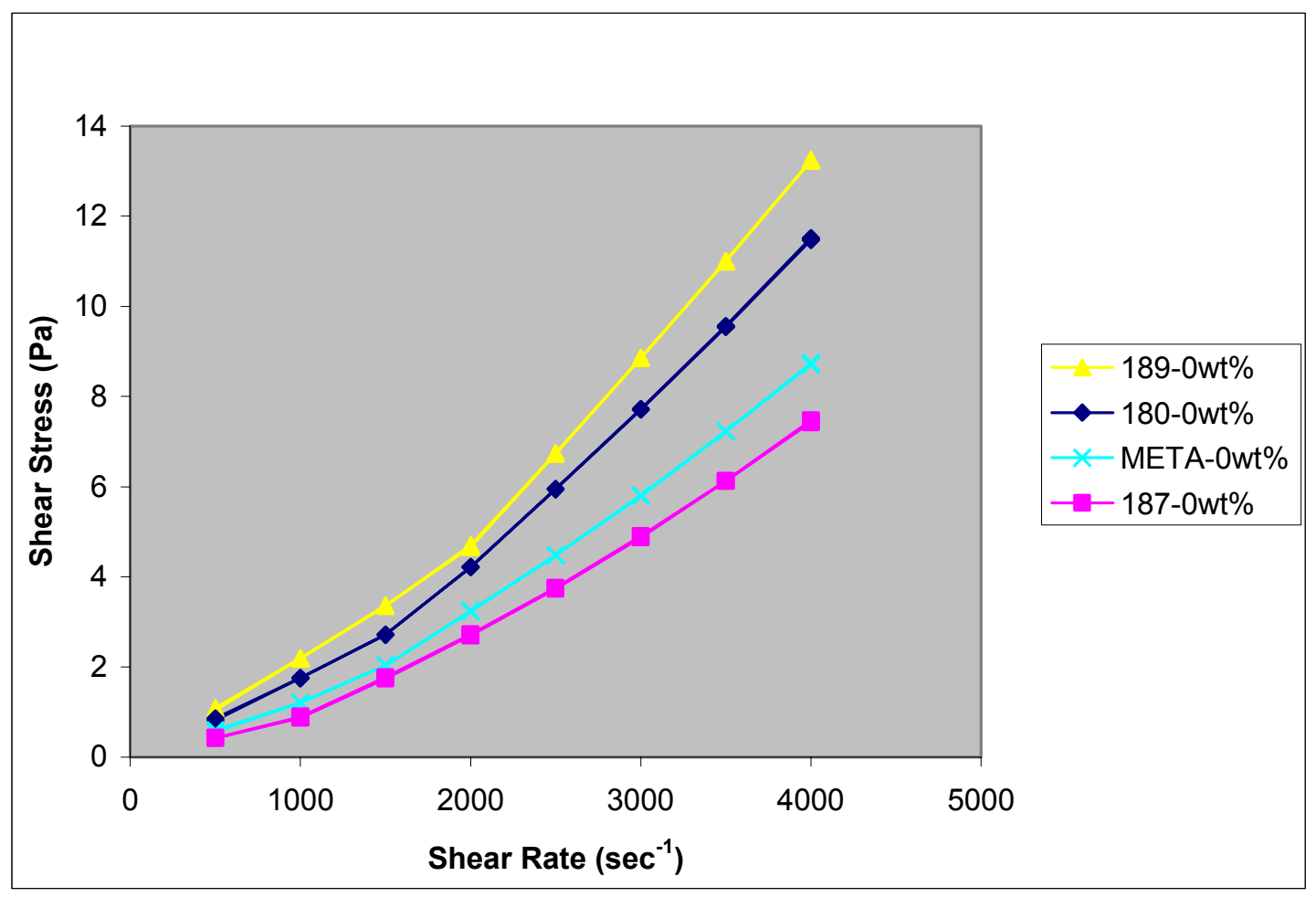

Figure 3-45. $0 \mathrm{wt} \%$ flow curve comparison.

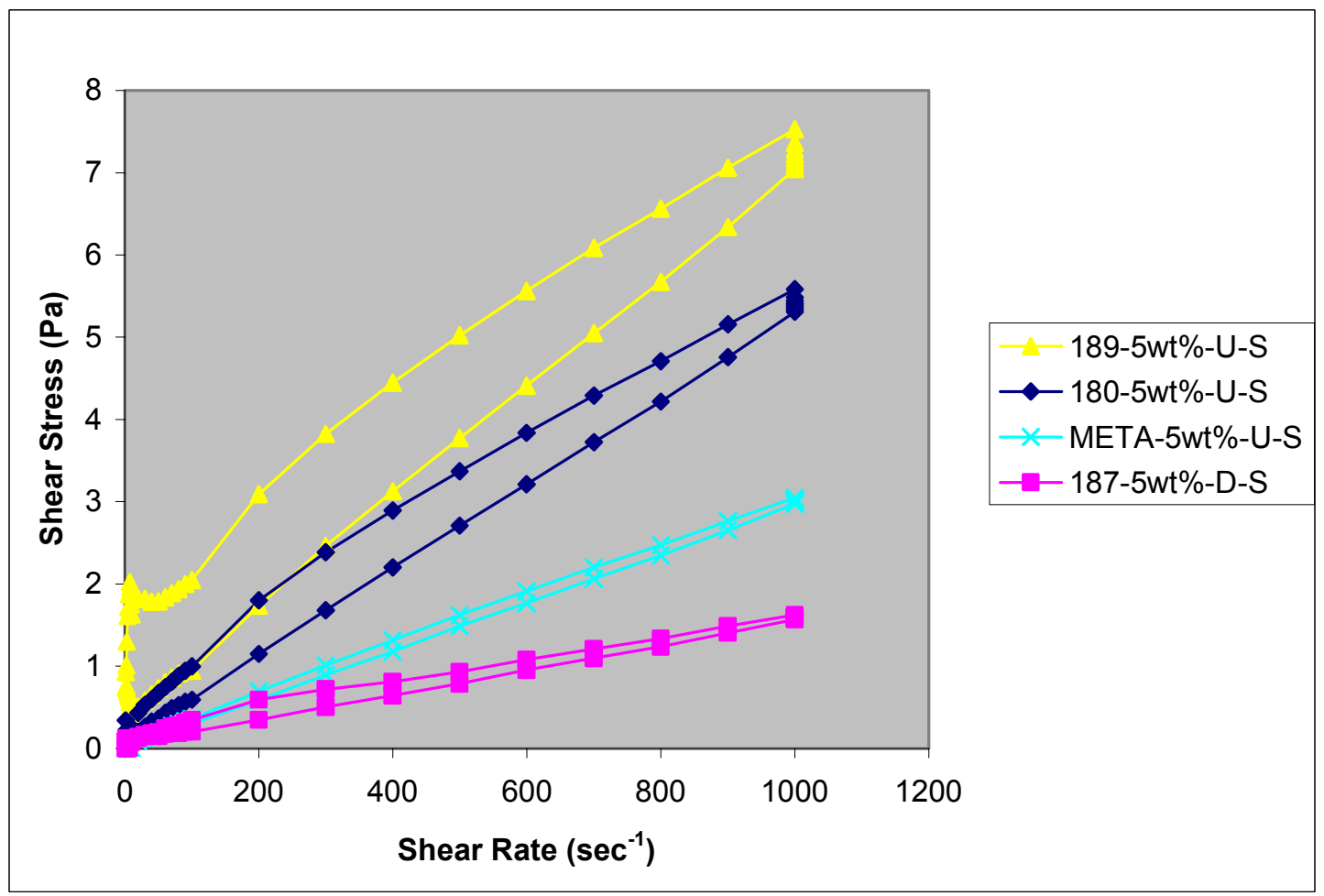

Figure 3-46. 5wt \% comparison $\left(1-1000 \mathrm{~s}^{-1}\right)$. 


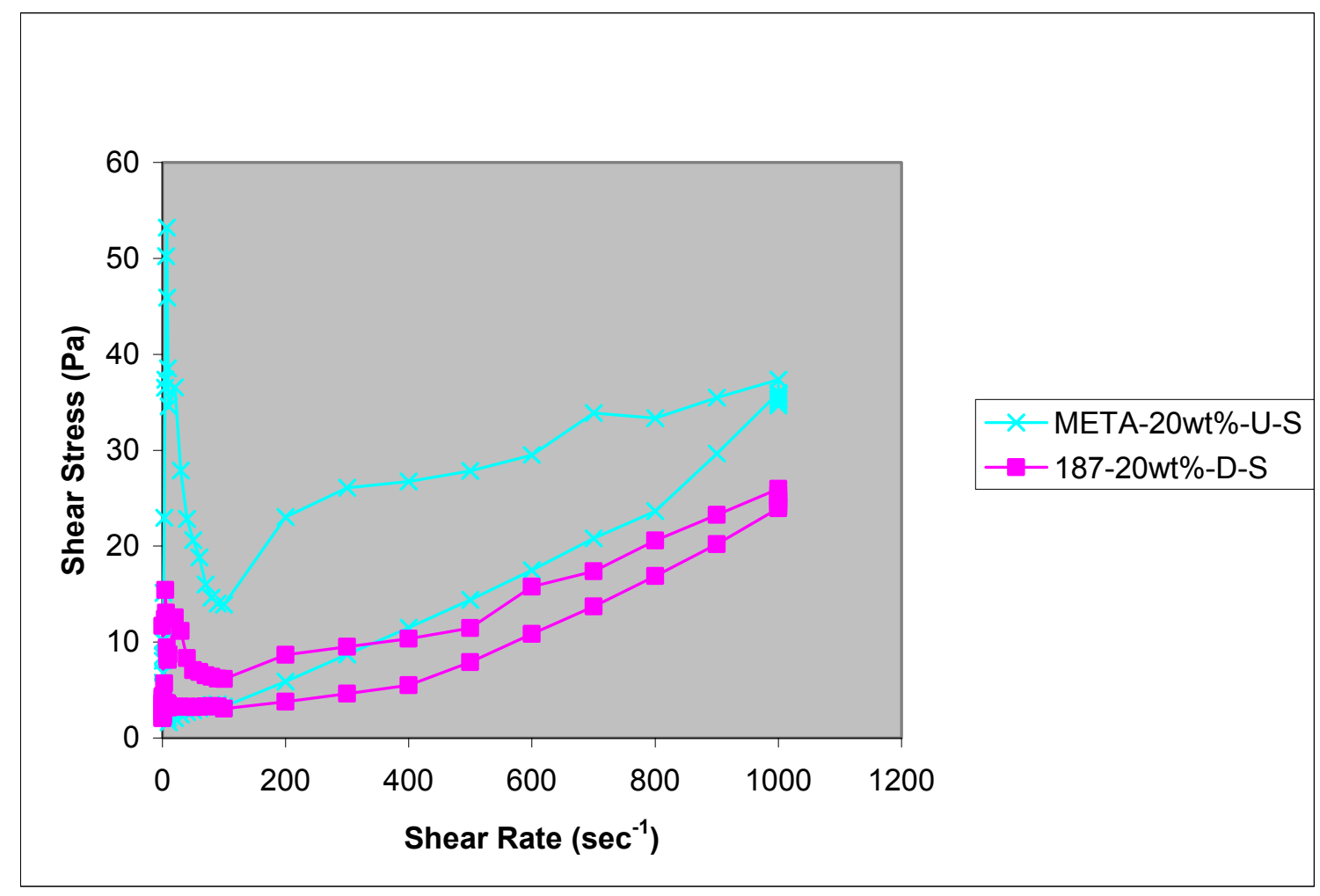

Figure 3-47. $20 \mathrm{wt} \%$ comparison.

3.4.2.2.3 Particle Size Distribution-Physical property analyses were performed on SBW surrogate samples with nonsonicated and sonicated SBW solids surrogate. Sonication of the SBW surrogate broke apart surrogate particle agglomerates and decreased the particle size distribution to result in a smaller mean particle diameter of the surrogate particles. A shear rate versus shear stress flow curve analysis was performed on several samples in each sonication state.

Flow curve comparison plots were constructed for samples that had identical surrogate compositions but different particle size distributions arising from the sample sonication state. The samples compared in these plots were formulated with the same solids weight percentage from the same solids and liquid surrogate batches. Each flow curve analysis was performed on a sample of the nonsonicated surrogate prior to sonicating the sample and repeating the flow curve analysis.

Figure 3-48 through Figure 3-55 are plots of flow curves for various nonsonicated versus sonicated SBW surrogates. All plots provide flow curve data at shear rates ranging from 0.1 to $1000 \mathrm{sec}^{-1}$.

For six of the eight samples compared, the sonicated sample flow curve had a higher shear stress profile than the nonsonicated sample. The remaining two samples, the META/20 $\mathrm{wt} \% / \mathrm{U}$ and $187 / 5 \mathrm{wt} \% / \mathrm{D}$ samples, displayed the opposite trend and had a nonsonicated sample flow curve with a higher shear stress profile than the sonicated sample. It is unknown why this anomaly occurred, but is suspected that the FY-04 (undried) solids surrogate, which was in general more gelatinous than the FY-03 (dried) solids surrogate, used in the META/20wt $\% / \mathrm{U}$ sample attained a higher shear stress when the nonsonicated solids loading was at the highest level. The sonicated samples may have flowed more readily at the higher solids loading. It is suspected that the FY-03 (dried) solids surrogate behaved in the opposite manner. More specifically, the granular FY-03 solids surrogate in the low solids loading $187 / 5 \mathrm{wt} \% / \mathrm{D}$ samples flowed less readily in nonsonicated form when the larger particles provided more 

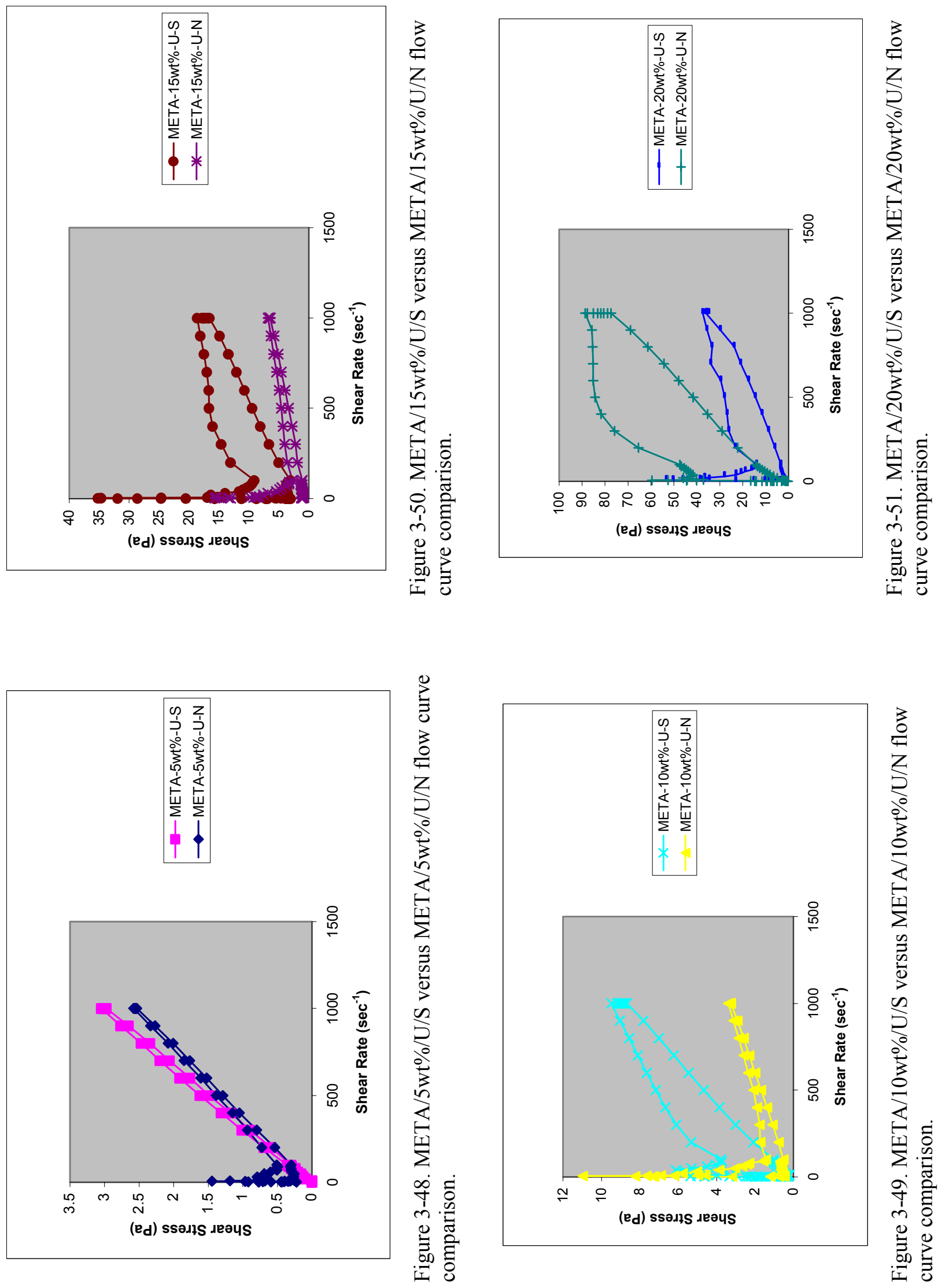

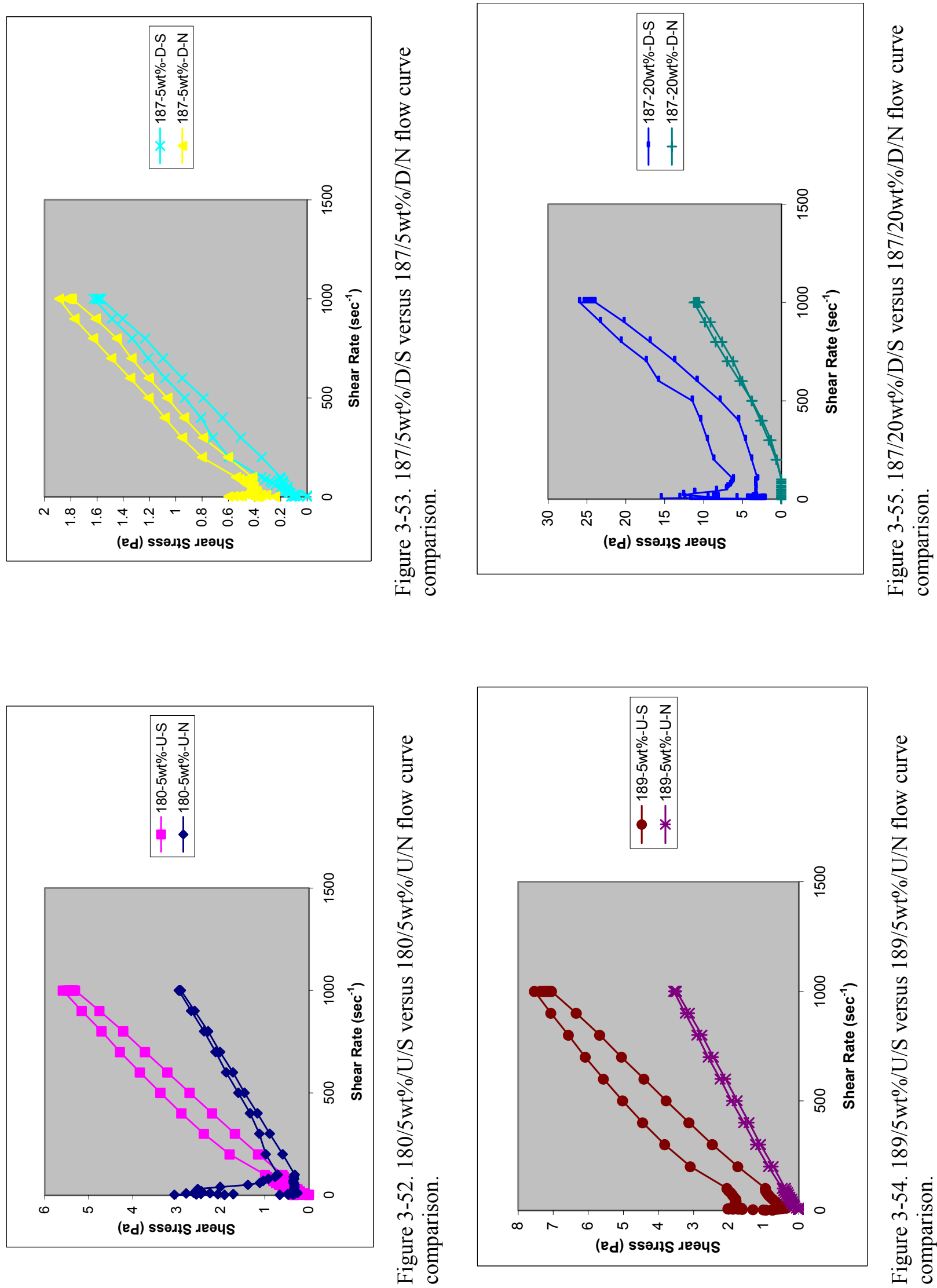
"structural" flow resistance. Sonication of the 187/5 wt \%/D samples allowed them to flow around each other more readily, thus decreasing the shear stress recorded in the flow curve analysis.

The flow curve analyses were repeated in the $500-4000 \mathrm{sec}^{-1}$ shear rate range with the same general results found for the 0.1 to $1000 \mathrm{sec}^{-1}$ shear rate plots. One exception was the $187 / 5 \mathrm{wt} \% / \mathrm{D} / \mathrm{N}$ and $187 / 5 \mathrm{wt} \% / \mathrm{D} / \mathrm{S}$ samples, where the sonicated sample shear stress surpassed the nonsonicated sample shear stress at a shear rate of approximately $2500 \mathrm{sec}^{-1}$.

3.4.2.2.4 Temperature—Flow curve analyses were performed on identically prepared SBW surrogate samples at two different temperatures. The two temperature ranges were selected to represent two temperatures at which waste analyses/processing operations would likely take place. The first temperature selected, $28^{\circ} \mathrm{C}$, was selected to provide a close match to the INTEC RAL hot cell internal temperature so that SBW surrogate sample flow curves could be compared with as few discrepancies as possible. The second temperature selected, $10^{\circ} \mathrm{C}$, was selected to provide information on how the sample flow properties would change at decreased temperatures, such as those likely to be encountered when working with SBW stored in the INTEC underground tank farm.

The flow curves of META $/ 20 \mathrm{wt} \% / \mathrm{U} / \mathrm{S}$ and META/20wt $\% / \mathrm{U} / \mathrm{N}$ samples at 10 and $28^{\circ} \mathrm{C}$ were compared at shear rates ranging from 0.1 to $1000 \mathrm{sec}^{-1}$. Figure 3-56 is a plot of the META/20 wt \%/U/S samples and Figure 3-57 is a plot of the META/20wt $\% / \mathrm{U} / \mathrm{N}$ samples. In each plot, the shear stress profile of the $10^{\circ} \mathrm{C}$ sample exceeds that of the $28^{\circ} \mathrm{C}$ sample. Since viscosity is generally a decreasing function of temperature, this is as expected.

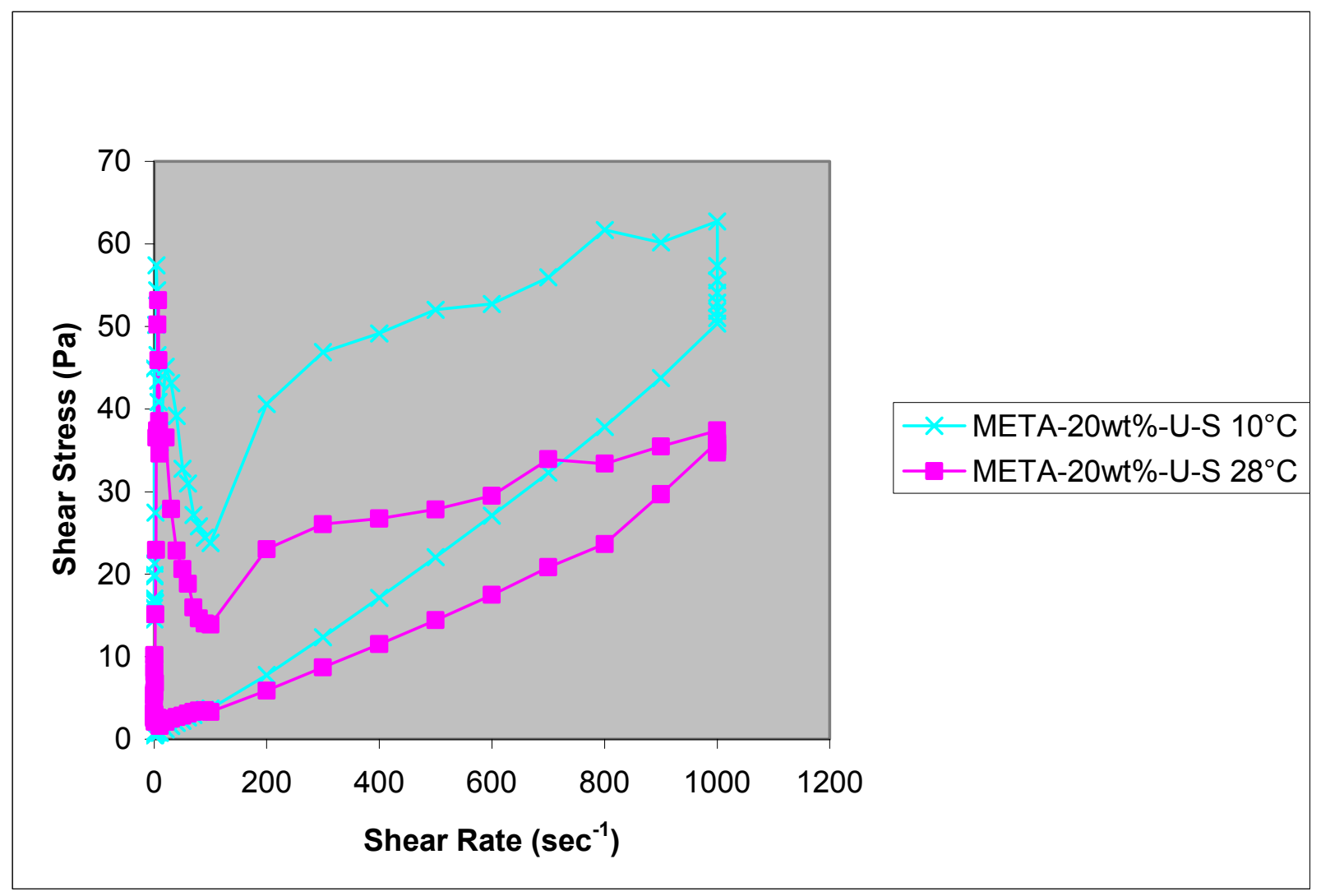

Figure 3-56. META/20wt\%/U/S flow curve comparison, $10^{\circ} \mathrm{C}$ versus $28^{\circ} \mathrm{C}$. 


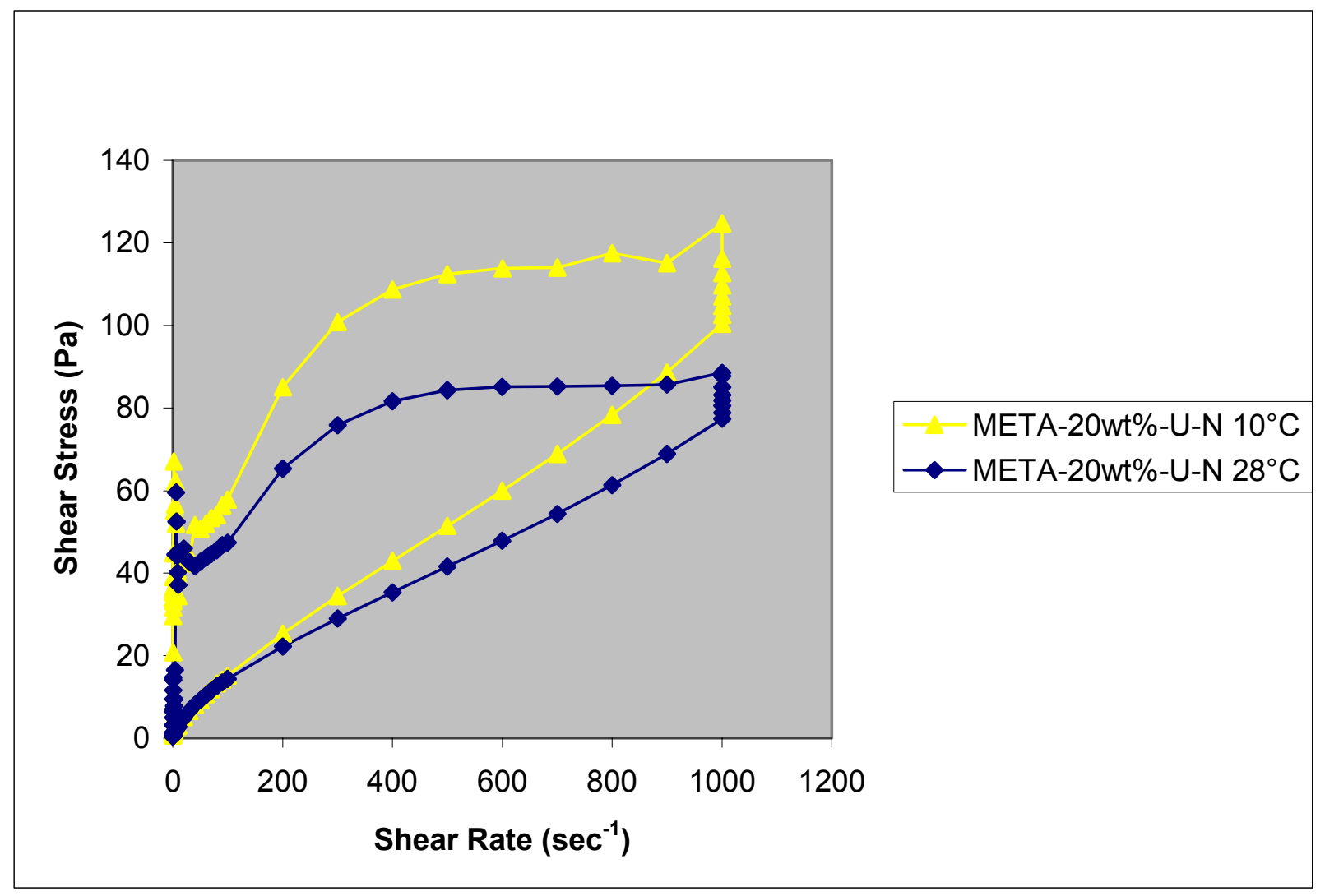

Figure 3-57. META/20wt $\% / \mathrm{U} / \mathrm{N}$ flow curve comparison, $10^{\circ} \mathrm{C}$ versus $28^{\circ} \mathrm{C}$.

\subsubsection{Settling Velocity}

Settling velocity measurements were performed as described in Section 3.2.3. Stoke's terminal particle settling velocities were calculated using the physical properties of the SBW solids and liquid surrogates for comparison with the experimental settling velocity results. A derivation of Stoke's settling velocity equations is provided in Appendix A. The settling velocity experiments measured the settling velocity of the collection of particles in various SBW surrogate slurries, rather than the settling velocity of a single particle in a uniform liquid phase. Settling velocity calculations were, therefore, performed using the physical properties (density, viscosity) of the liquid surrogate and the surrogate slurry for comparison purposes. The expression for Stoke's terminal settling velocity is:

$u_{t}=\frac{g D_{p}^{2}\left(\rho_{p}-\rho\right)}{18 \mu}$

where $u_{t}=$ Stoke's terminal settling velocity

$$
\begin{aligned}
& g=\text { gravitational constant } \\
& D_{p}=\text { particle diameter } \\
& \rho_{p}=\text { particle density }
\end{aligned}
$$




$$
\begin{aligned}
& \rho=\text { fluid density } \\
& \mu=\text { fluid viscosity. }
\end{aligned}
$$

Physical properties of the solids surrogate for use in the Stoke's terminal settling velocity are:

SBW surrogate particle density, $\rho_{\mathrm{p}}, 2051 \mathrm{~kg} / \mathrm{m}^{3}$.

The metathesis mother liquor liquid supernate was the liquid surrogate used in the settling velocity experiments (liquid surrogates with flow curve profiles similar to those of the tank farm sample). The physical properties of the META/0 wt \% liquid surrogate are:

liquid density, $\rho, 1120 \mathrm{~kg} / \mathrm{m}^{3}$

liquid viscosity, $\mu, 0.001 \mathrm{~Pa} \cdot \mathrm{s} @ 28^{\circ} \mathrm{C}$

Table 3-13 provides a comparison of the settling velocities calculated using the META/0 $\mathrm{wt} \%$ liquid surrogate physical properties and the minimum, mean, and maximum particle diameters of the META $/ 5 \mathrm{wt} \% / \mathrm{U} / \mathrm{S}$ and META/10wt $\% / \mathrm{U} / \mathrm{S}$ samples.

The META $/ 5 \mathrm{wt} \% / \mathrm{U} / \mathrm{S}$ and META/10wt $\% / \mathrm{U} / \mathrm{S}$ surrogates were used in the settling velocity experiments since the flow curve profiles were most similar to those of the tank farm "as-received" and "gravity-settled" samples. The META/0wt \% and META/20wt\%/U/S samples were not analyzed because no settling occurred in these samples. The META/0wt\% samples had no undissolved solids and the META $/ 20 \mathrm{wt} \% / \mathrm{U} / \mathrm{S}$ sample had no free liquid. The physical properties of the META $/ 5 \mathrm{wt} \% / \mathrm{U} / \mathrm{S}$ and META/10wt\%/U/S surrogate slurries are:

META/5 wt \%/U/S slurry density, $\rho, 1130 \mathrm{~kg} / \mathrm{m}^{3}$

META/5 wt \%/U/S slurry viscosity, $\mu, 0.006 \mathrm{~Pa} \cdot \mathrm{s} @ 28^{\circ} \mathrm{C}$

META $/ 10$ wt \%/U/S slurry density, $\rho, 1160 \mathrm{~kg} / \mathrm{m}^{3}$

META $/ 10$ wt \%/U/S slurry viscosity, $\mu, 2.2 \mathrm{~Pa} \cdot \mathrm{s} @ 28^{\circ} \mathrm{C}$.

The META/5 wt \%/U/S and META/10 wt \%/U/S slurry viscosity readings were taken after the flow curve analysis readings had stabilized and displayed no further thixotropic effects. This condition was selected to simulate the well-mixed conditions under which the settling velocity samples were loaded into the settling chambers. The viscosity readings were taken at the lowest shear rate the viscometer was able to record for each sample, $20 \mathrm{sec}^{-1}$ for the META/5wt $\% / \mathrm{U} / \mathrm{S}$ sample and $0.1 \mathrm{sec}^{-1}$ for the META/10wt $\% / \mathrm{U} / \mathrm{S}$ sample.

Table 3-13. Settling velocity for META/5wt\%/U/S and META/10wt $\% / \mathrm{U} / \mathrm{S}$ samples (using liquid density and viscosity in settling velocity calculations).

\begin{tabular}{cccc}
\hline & $\begin{array}{c}\text { Minimum Particle } \\
\text { Diameter Settling } \\
\text { Velocity [mm/hr] }\end{array}$ & $\begin{array}{c}\text { Mean Particle Diameter } \\
\text { Settling Velocity [mm/hr] }\end{array}$ & $\begin{array}{c}\text { Maximum Particle } \\
\text { Diameter Settling } \\
\text { Velocity [mm/hr] }\end{array}$ \\
\hline META/5wt\%/U/S & $0.4 \mathrm{~mm} / \mathrm{hr}(0.49 \mu \mathrm{m})$ & $690 \mathrm{~mm} / \mathrm{hr}(19.4 \mu \mathrm{m})$ & $28000 \mathrm{~mm} / \mathrm{hr}(125 \mu \mathrm{m})$ \\
META/10wt\%/U/S & $0.6 \mathrm{~mm} / \mathrm{hr}(0.58 \mu \mathrm{m})$ & $810 \mathrm{~mm} / \mathrm{hr}(21.1 \mu \mathrm{m})$ & $40000 \mathrm{~mm} / \mathrm{hr}(148 \mu \mathrm{m})$ \\
\hline
\end{tabular}


Table 3-14 provides a comparison of the settling velocities calculated using the META/5 wt \%U/S and META/10 wt \%/U/S slurry physical properties and the minimum, mean, and maximum particle diameters of the META/5 wt \%/U/S and META/10wt\%/U/S samples.

Table 3-14. Settling velocity for META/5wt $\% / \mathrm{U} / \mathrm{S}$ and META/10wt $\% / \mathrm{U} / \mathrm{S}$ samples (using slurry density and viscosity in settling velocity calculations).

\begin{tabular}{|c|c|c|c|}
\hline Sample & $\begin{array}{c}\text { Minimum Particle } \\
\text { Diameter Settling velocity } \\
{[\mathrm{mm} / \mathrm{hr}]}\end{array}$ & $\begin{array}{c}\text { Mean Particle Diameter } \\
\text { Settling Velocity }[\mathrm{mm} / \mathrm{hr}]\end{array}$ & $\begin{array}{c}\text { MAXIMUM PARTICLE } \\
\text { DIAMETER SETTLING } \\
\text { VELOCITY }[\mathrm{mm} / \mathrm{hr}]\end{array}$ \\
\hline META $/ 5$ wt \%/U/S & $0.07 \mathrm{~mm} / \mathrm{hr}(0.49 \mu \mathrm{m})$ & $110 \mathrm{~mm} / \mathrm{hr}(19.4 \mu \mathrm{m})$ & $4600 \mathrm{~mm} / \mathrm{hr}(125 \mu \mathrm{m})$ \\
\hline META $/ 10 \mathrm{wt} \% / \mathrm{U} / \mathrm{S}$ & $0.0003 \mathrm{~mm} / \mathrm{hr}(0.58 \mu \mathrm{m})$ & $0.35 \mathrm{~mm} / \mathrm{hr}(21.1 \mu \mathrm{m})$ & $17 \mathrm{~mm} / \mathrm{hr}(148 \mu \mathrm{m})$ \\
\hline
\end{tabular}

Figure 3-58 is a plot of the META/5wt $\% / \mathrm{U} / \mathrm{S}$ and META/10 wt \%/U/S settling velocity measurements. The settling type was identified as compression settling (see Appendix A for a discussion of compression settling versus particulate settling). The settling velocity readings for the

META $/ 5 \mathrm{wt} \% / \mathrm{U} / \mathrm{S}$ sample ranged from 0.04 to $0.25 \mathrm{~mm} / \mathrm{hr}$. The settling velocity readings for the META $/ 10 \mathrm{wt} \% / \mathrm{U} / \mathrm{S}$ sample ranged from 0.01 to $0.12 \mathrm{~mm} / \mathrm{hr}$. The minimum observed settling velocity readings are comparable to the settling velocity values calculated using the slurry density and viscosity physical properties. The maximum observed settling velocity readings are comparable to the settling velocity values calculated using the liquid surrogate density and viscosity. In each case, the calculated settling velocity values are slightly greater than the observed values. This may be due to electrical charges on the individual slurry particles altering the settling velocity. Particle charges are not accounted for in Stoke's terminal velocity expression. An attempt was made to quantify the electrical charge, or zeta potential, of the SBW surrogate solid particles in the surrogate slurry samples, but the $\mathrm{pH}$ of the samples was below the $\mathrm{pH}$ range acceptable to the zeta potentiometer. Creation of a zeta potential versus dilution ratio curve to extrapolate the zeta potential at low $\mathrm{pH}$ was attempted unsuccessfully.

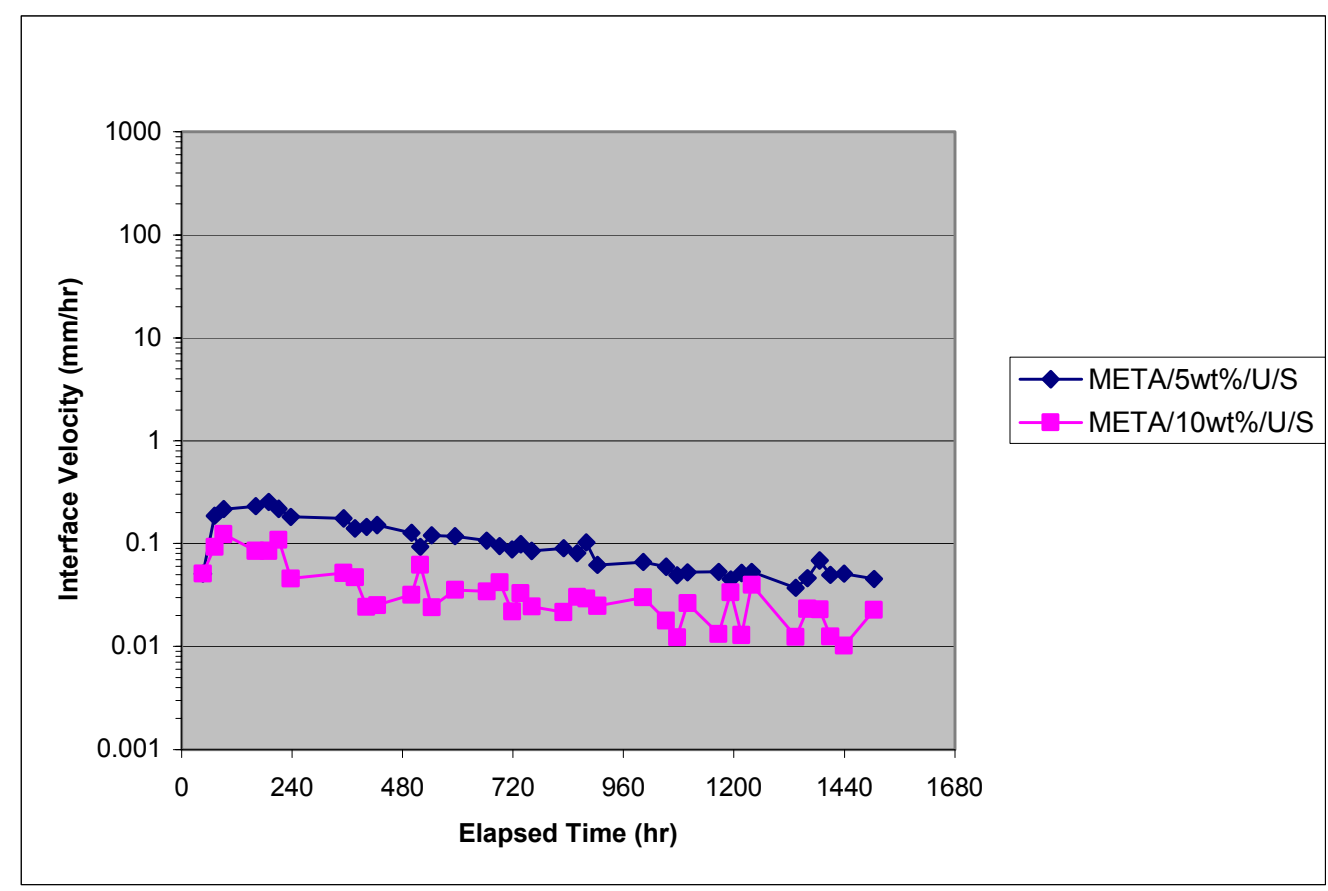

Figure 3-58. META/5wt\%/U/S and META/10wt\%/U/S interface velocity in $100 \mathrm{~mL}$ settling chamber. 
Settling of the WM-187 in the $100-\mathrm{mL}$ tubes resulted in a $74.4 \mathrm{~mL}$ of as-received sample volume settling to $45.2 \mathrm{~mL}$ of solids in the first tube in 15 days. The second tube resulted in a $90.6 \mathrm{~mL}$ of asreceived sample volume settling to $53.0 \mathrm{~mL}$ of solids in 15 days. The $250-\mathrm{mL}$ graduated cylinder started with $88 \mathrm{~mL}$ of as-received sample and settled to $55 \mathrm{~mL}$ in 8 days. Figure 3-59 is a comparison of the settling velocities of the three WM-187 samples allowed to settle in 100-mL settling chambers. Sample WM-187-1 had tank solids from WM-187, WM-182, and WM-183 in WM-187. Sample WM-187-2 had tank solids from WM-187, WM-182, WM-183, WM-184, WM-185, and WM-186 in WM-187. Sample WM-187-3 had tank solids from WM-187, WM-181, WM-182, WM-183, WM-184, WM-185, and WM-186 in WM-187.

\subsubsection{Solids Weight Percentage}

Solids weight percentage analysis was performed as described in Section 3.2.4. SBW surrogate and tank farm waste samples were analyzed for solids weight percentage.

Nonsonicated and sonicated surrogate samples displayed very different characteristics following drying. Nonsonicated samples displayed a tendency to wither and discolor upon drying. The dried nonsonicated surrogate samples tended to be crumbly and were readily broken apart. The sonicated samples displayed a tendency to crack upon drying, while continuing to conform to the general shape of the sample evaporating dish. The dried sonicated surrogate samples tended to be hard and brittle and were difficult to break into smaller pieces. Breaking the dried sonicated surrogate samples produced very fine dry particulates, which were difficult to prevent from becoming airborne upon disruption. Figure 3-60 is a photograph of nonsonicated and sonicated surrogate samples following drying.

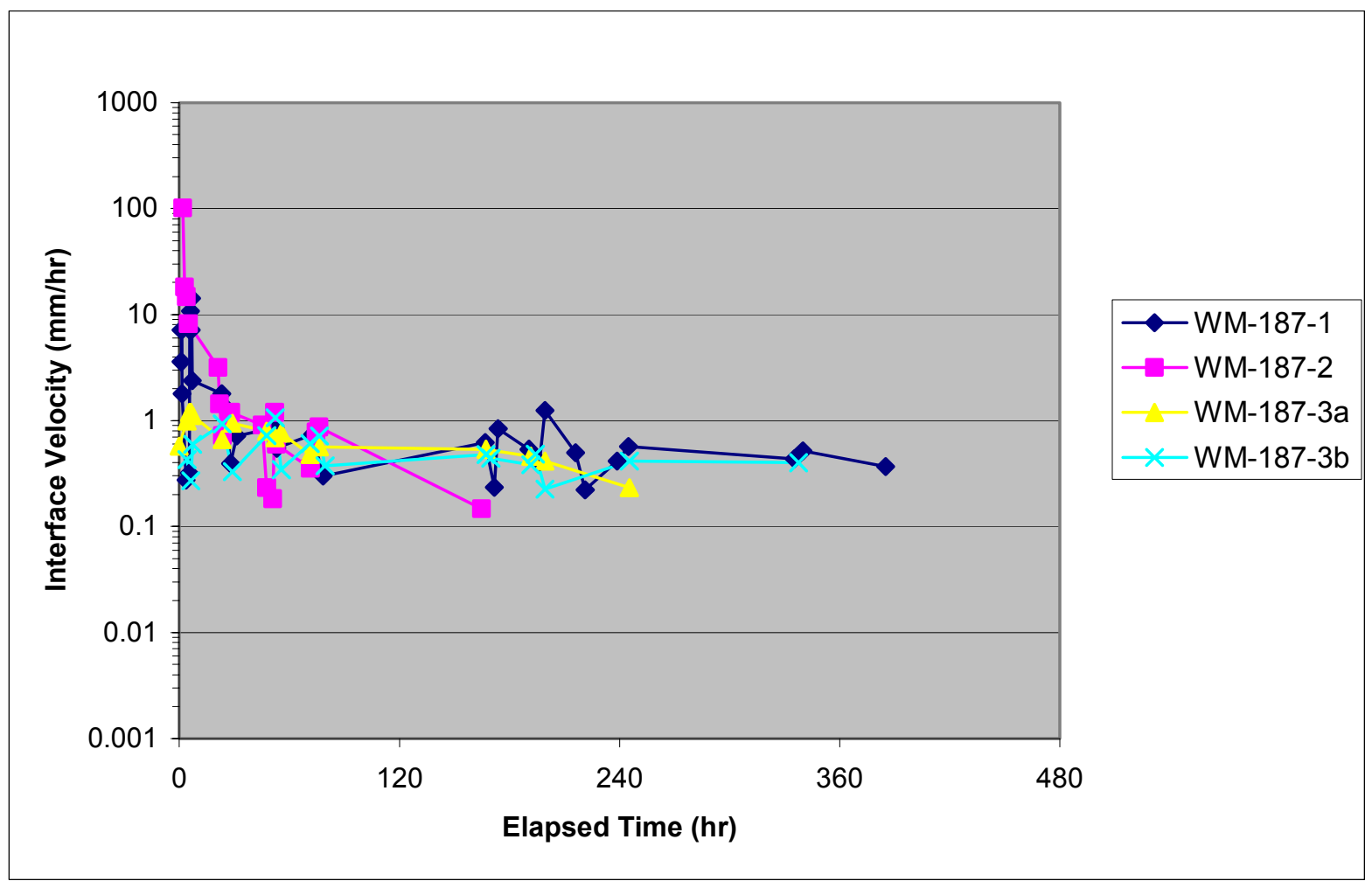

Figure 3-59. WM-187 sample interface velocity in 100-mL settling chamber. 


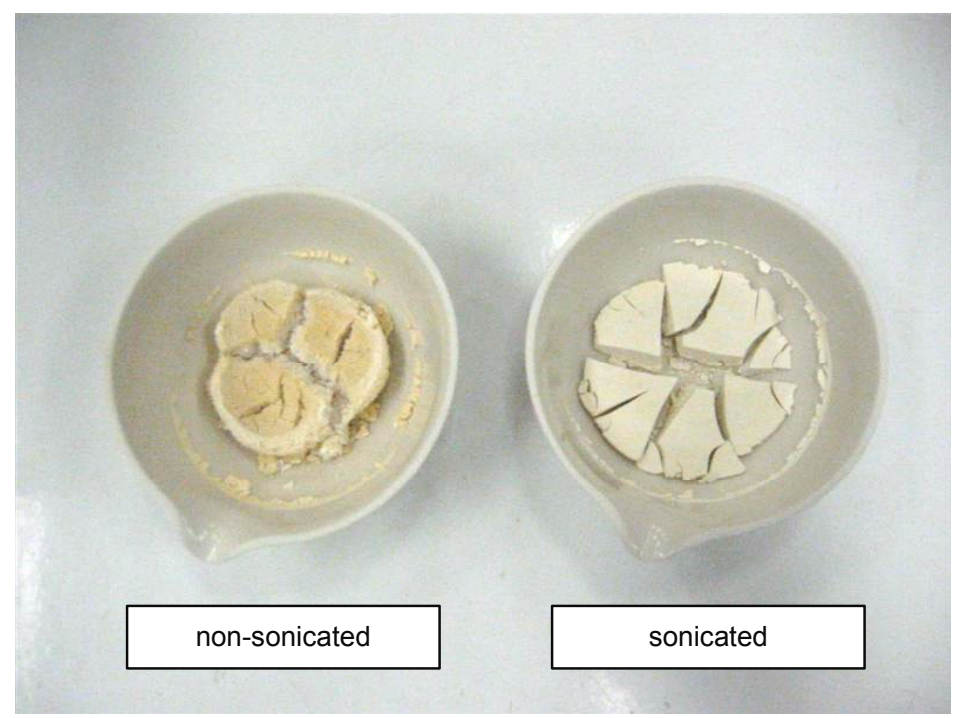

Figure 3-60. META/20wt\%/U/N and META/20wt\%/U/S Solids Weight Percent Analysis.

The solids weight percentages of the various WM-187 tank farm waste sample fractions are provided in Table 3-15. The SBW surrogate sample solids weight percentages are provided in Table 3-16. The solids weight percentage values reported include both dissolved and undissolved solids content. The 180,189 , and META liquid surrogates contain more dissolved solids than the WM-187 tank farm waste sample supernate. It should be noted that tank WM-187, when sampled, was dilute in dissolved solids in comparison with the other tanks. During surrogate slurry sample preparation, the quantity of dissolved solids in the liquid surrogate solutions was not accounted for, resulting in SBW surrogate slurries with higher solids weight percentages than targeted.

A comparison of the solids weight percentages for the four surrogate and tank farm waste sample fractions with similar rheological properties is provided in Figure 3-61. Surrogate samples with rheological properties similar to those of the tank farm waste were identified in Section 3.4.2.1. The SBW surrogate samples have consistently higher solids weight percentages than the tank farm waste samples for several reasons: (1) as described above, the metathesis mother liquor SBW liquid surrogate has a higher dissolved solids content than the WM-187 sample supernate; and (2) the tank farm waste solids are relatively more dispersed than the surrogate solids. In comparing the centrifuged waste and surrogate samples, which have very little free liquid and thus very similar solids volume percentages, it can be seen that the solids weight percentage of the surrogate exceeds that of the waste. Therefore, SBW surrogate samples with very similar solids volume percentages and flow curve properties have consistently higher solids weight percentages than the corresponding tank farm samples.

Table 3-15. WM-187 tank farm waste sample solids weight percentages.

\begin{tabular}{|c|c|}
\hline Sample & Solids Weight Percentage \\
\hline WM-187 supernate & $4.7 \%$ \\
\hline WM-187 as received & $7.1 \%$ \\
\hline WM-187 gravity settled sludge & $10.0 \%$ \\
\hline WM-187 centrifuged sludge & $11.5 \%$ \\
\hline
\end{tabular}


Table 3-16. SBW surrogate sample solid weight percentages.

\begin{tabular}{|c|c|}
\hline Sample & Solids Weight Percentage \\
\hline $180 / 0 w t \%$ & $25.0 \%$ \\
\hline $187 / 0 w t \%$ & $4.7 \%$ \\
\hline $187 / 5 \mathrm{wt} \% / \mathrm{D} / \mathrm{N}$ & $8.6 \%$ \\
\hline $187 / 5 \mathrm{wt} \% / \mathrm{D} / \mathrm{S}$ & $8.3 \%$ \\
\hline $187 / 20 \mathrm{wt} \% / \mathrm{D} / \mathrm{N}$ & $21.0 \%$ \\
\hline $187 / 20 \mathrm{wt} \% / \mathrm{D} / \mathrm{S}$ & $25.5 \%$ \\
\hline $189 / 0 w t \%$ & $22.4 \%$ \\
\hline META/0wt $\%$ & $8.7 \%$ \\
\hline META/AR/U/N & $9.5 \%$ \\
\hline META/5wt $\% / \mathrm{U} / \mathrm{N}$ & $10.8 \%$ \\
\hline META/5wt $\% / \mathrm{U} / \mathrm{S}$ & $11.3 \%$ \\
\hline META/10wt $\% / U / N$ & $13.1 \%$ \\
\hline META/10wt $\% / U / S$ & $15.1 \%$ \\
\hline META/15wt $\% / \mathrm{U} / \mathrm{N}$ & not available \\
\hline META/15wt $\% / U / S$ & $17.6 \%$ \\
\hline META $/ 20 w t \% / U / N$ & $22.7 \%$ \\
\hline META $/ 20 w t \% / U / S$ & $26.5 \%$ \\
\hline
\end{tabular}

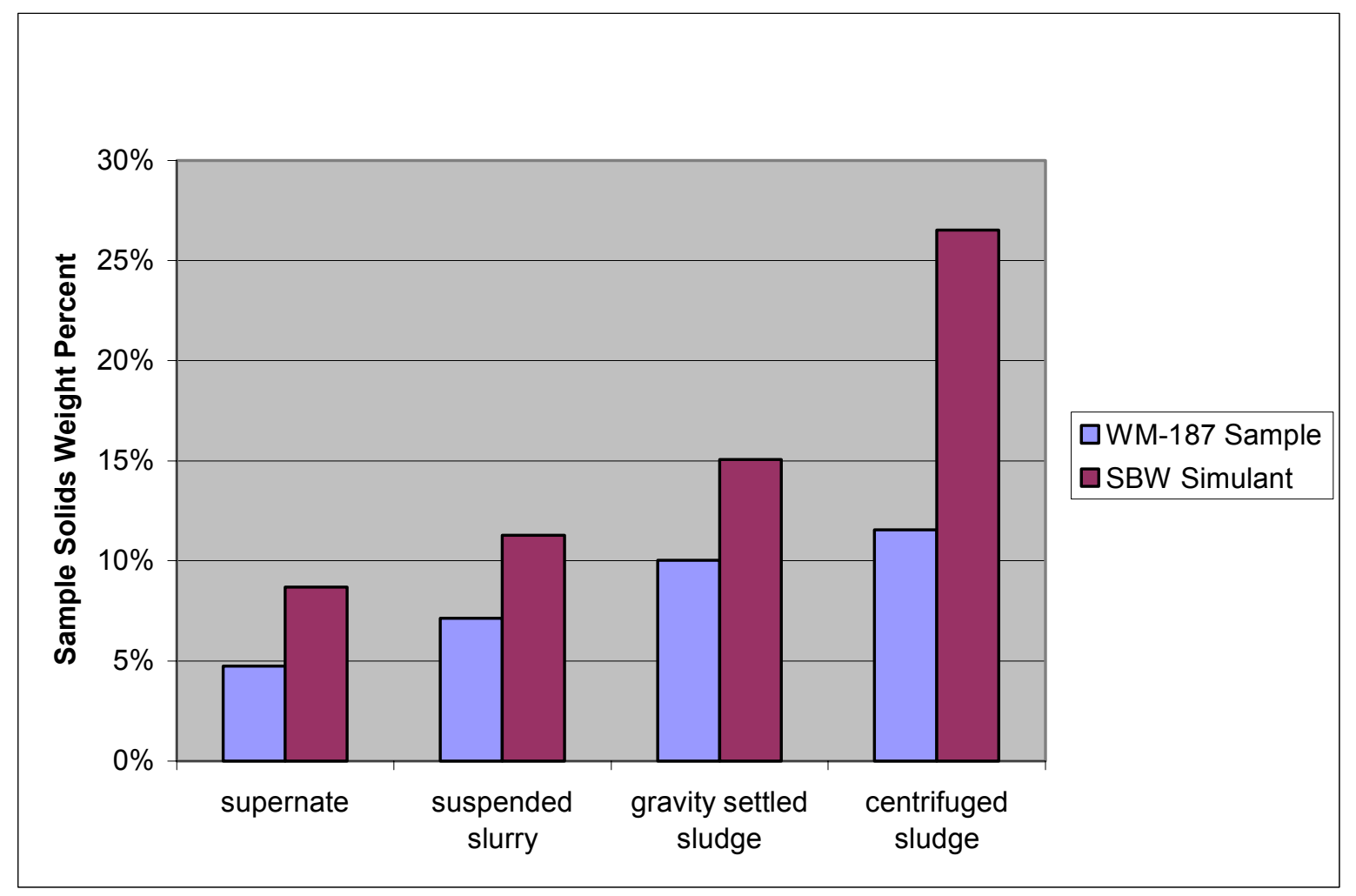

Figure 3-61. Sample solids weight percentages. 


\subsubsection{Bulk Density}

Bulk density analyses were performed as described in Section 3.2.5. The bulk densities of the various SBW surrogate samples are provided in Table 3-17. Table 3-18 provides wet (FY-03 and FY-04 SBW solids surrogates saturated with WM-187 and metathesis mother liquor liquid surrogates, respectively) and dry (SBW solids surrogates dried to constant mass in drying oven) bulk densities of the FY-03 and FY-04 SBW solids surrogates.

Table 3-17. Sample bulk densities.

\begin{tabular}{|c|c|}
\hline Sample & Bulk Density $(\mathrm{g} / \mathrm{mL})$ \\
\hline META / AR / U / N & 1.12 \\
\hline 187 / 5wt $\%$ / D / N & 1.07 \\
\hline 187 / 5wt $\%$ / D / S & 1.07 \\
\hline META / 5wt $\%$ / U / N & 1.13 \\
\hline META / 5wt\% / U / S & 1.13 \\
\hline $180 / 5 w t \% / U / N$ & 1.23 \\
\hline 189 / 5wt\% / U / N & 1.27 \\
\hline META / 10wt $\%$ / U / N & 1.15 \\
\hline META / 10wt $\%$ / U / S & 1.16 \\
\hline META / 15wt $\%$ / U / S & 1.17 \\
\hline $187 / 20 w t \% / D / N$ & 1.17 \\
\hline $187 / 20 \mathrm{wt} \% / \mathrm{D} / \mathrm{S}$ & 1.20 \\
\hline META / 20wt $\%$ / U / N & 1.23 \\
\hline META / 20wt $\%$ / U / S & 1.24 \\
\hline META / 0wt $\%$ & 1.12 \\
\hline 187 / 0wt $\%$ & 1.05 \\
\hline $180 / 0 w t \%$ & 1.25 \\
\hline $189 / 0 w t \%$ & 1.31 \\
\hline
\end{tabular}

Table 3-18. Wet and dried SBW surrogate solids bulk density.

\begin{tabular}{lcc}
\hline & $\begin{array}{c}\text { Wet Solids Bulk } \\
\text { Density }(\mathrm{g} / \mathrm{mL})\end{array}$ & $\begin{array}{c}\text { Dried Solids Bulk } \\
\text { Density }(\mathrm{g} / \mathrm{mL})\end{array}$ \\
\hline FY-03 filtered solids & 1.08 & 0.70 \\
FY-03 sonicated filtered solids & 1.20 & 0.75 \\
FY-04 centrifuged solids & 1.18 & 1.08 \\
FY-04 sonicated centrifuged solids & 1.24 & 0.72 \\
\hline
\end{tabular}


The dried solids bulk density for the third WM-187 sample (0402101) was $0.421 \mathrm{~g} / \mathrm{mL}$. This is lower than the other bulk densities that have been run but within $0.1 \mathrm{~g} / \mathrm{mL}$ of the first WM-187 sample $(0.50 \mathrm{~g} / \mathrm{mL})$. The analysis started with $22 \mathrm{~mL}$ of as-received sample weighing $23.454 \mathrm{~g}$. The sample was allowed to settle overnight and the supernate removed with a plastic transfer pipette. The solids portion of the sample was washed with deionized water and then centrifuged. The supernate was removed with a plastic transfer pipette. This wash was repeated two more times. The solids portion of the sample was air dried, the volume read, and weighed. Table 3-19 provides densities of the various WM-187 sample fractions. Wet solids are the solids with the original interstitial fluid still between the solids. That is, the original liquid in between the solids has not been removed. It has only been removed above the settled solids.

Table 3-19. Densities for WM-187 (0402101).

\section{Sample WM-187}

\section{As Received Sample}

Liquid

Wet Solids Density $(\mathrm{g} / \mathrm{mL})$

Dried Solids Bulk Density $(\mathrm{g} / \mathrm{mL})$ 0.421 


\section{CAKE FILTRATION PERFORMANCE}

\subsection{Strategy}

The objective of the testing described in this report is to provide an experimental approach to compare cake filtration properties of the metathesis-prepared SBW slurry surrogate and the actual radioactive slurry sample collected from INTEC tank WM-187. Constant pressure filtration was adapted because it is the most common method for collecting experimental filtration data in the laboratory; also, because of its simplicity.

The bench scale cake filtration apparatus was designed so that the test system can be operated either manually in a laboratory fume hood or remotely in the INTEC RAL. Cake filtration tests were performed to collect data for characterizing the SBW slurry and supporting solid/liquid separation technology for treating SBW slurry. Because of the radioactive nature and the limited quantities of the actual SBW slurry, cost, and difficulty of remote "hot cell" experiments; it is desirable to use nonradioactive surrogate, where possible, when conducting process design experiments. To ensure a high quality surrogate that accurately represents the unique properties of the actual waste, slurry surrogate was prepared using the metathesis method, which emulates the conditions that formed the actual waste slurry. The surrogate filtered cake properties were determined and compared with the cake formed by the radioactive SBW slurry as a means to optimize the processing conditions.

\subsection{Apparatus}

The test apparatus used for specific resistance of filtration (SRF) experiments, illustrated in Figure 4-1, is comprised of a 600-mL clear filtration cylinder, a filtrate collection vessel, a pressurized air supply, an air heater, a pressure regulator, and a mass flow meter. The upper part of the filtration cylinder consists of double-wall plastic pipe, $20 \mathrm{~cm}$ high, with an inside diameter of $6.4 \mathrm{~cm}$. The filter cup is $5 \mathrm{~cm}$ high, has an inside diameter that gradually decreases from 6.4 to $3.2 \mathrm{~cm}$ and a cylinder height above the filtration area of $1.2 \mathrm{~cm}$. A filter cup housed the filter solids, the filter medium, and the filter medium support, which was fabricated for these tests.

The filter cup is sandwiched between the upper cylinder section and the lower receiving part by a screw connection. The lower receiving part of the filtration cylinder was used to collect and release filtrate. Connections between the upper cylinder, the filter cup, and the receiving part of were sealed with Viton O-rings to prevent leakage. A 50-psig air supply capable of temperatures of up to $80^{\circ} \mathrm{C}$, as well as feed and wash water inlets were connected to the top of the filtration cylinder. Filter medium cloths made of polypropylene sulfide (PPS) - Ryton, polyvinylidene fluoride (PVDF) - Kynar, were used and they contained mean pore sizes of 2.9, 5.5, and $11.5 \mu \mathrm{m}$ (Appendix D). The filtration cylinder assembly was designed to be remotely assembled and disassembled (and was for the test involving SBW solids), the entire test apparatus can be operated with hot cell manipulators. Two identical test systems were constructed for surrogate and SBW slurries, respectively, to avoid contamination.

Compressed air was introduced into the filter test vessel immediately after a predetermined amount of either surrogate or SBW slurry was added to the filtration vessel, and each experiment was carried out at a constant pressure. Filtrate was collected in a receiver equipped with an electronic balance. The filtration time and mass of the filtrate were continuously recorded by a data acquisition system until filtration was complete. When the filtrate flow ceased, air pressure was stopped and the filter cup with cake was removed and weighed. The mass of filtered solids was measured after oven drying at $\sim 100$ to $105^{\circ} \mathrm{C}$. 


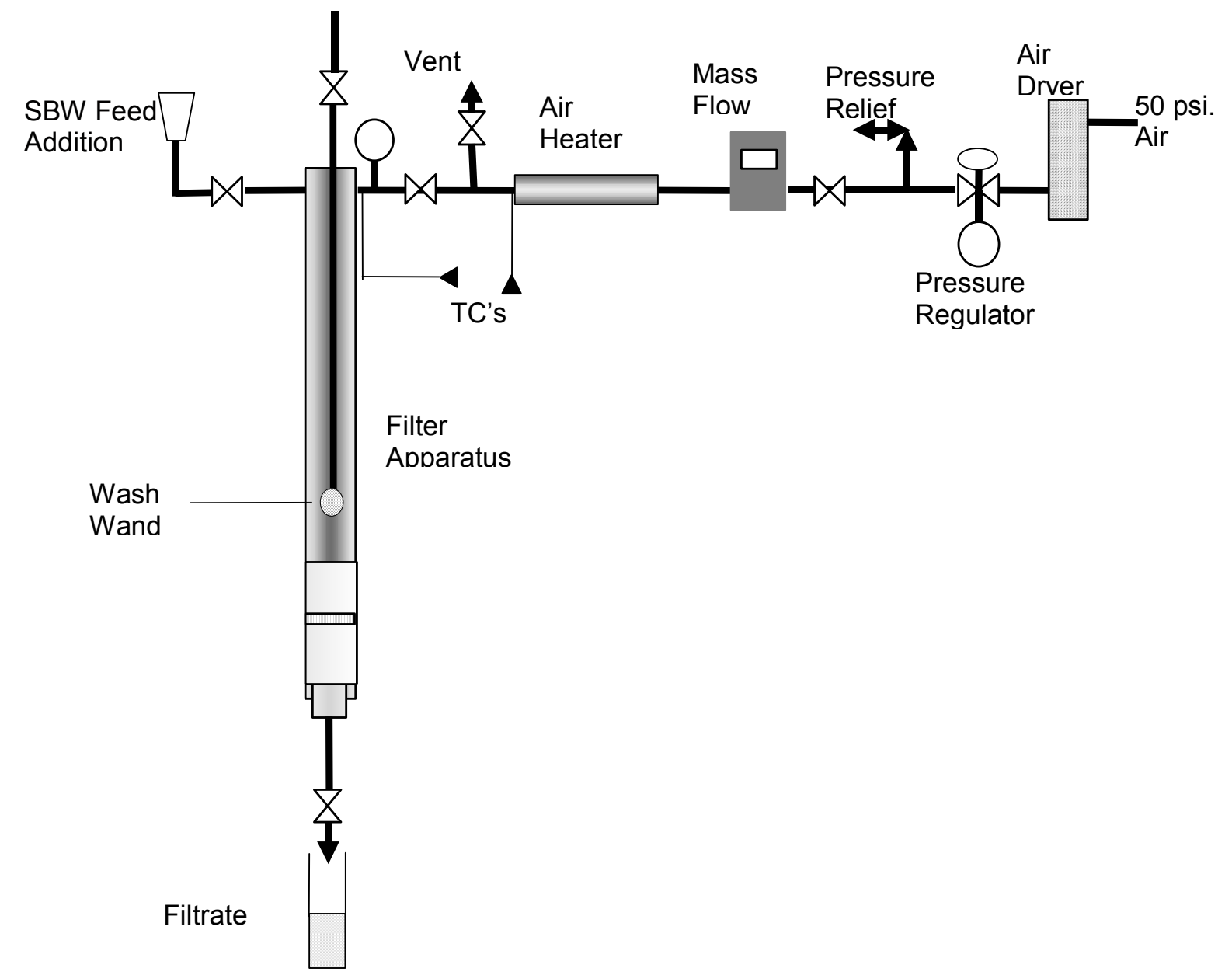

Figure 4-1. Bench scale constant pressure filtration apparatus.

\subsection{Materials}

Constant pressure filtration experiments using the surrogate suspension were conducted to determine the filtered cake properties (e.g., specific resistance of filtration, $\alpha$, cake compressibility, $n$ ) of the surrogate solids and compare them with the corresponding properties of the cake formed by the radioactive SBW slurry. Because of limited quantities of the actual SBW slurry, cost and difficulty of conducting the remote "hot cell" experiments, surrogate was used to test processing conditions.

\subsubsection{SBW Slurry Surrogate \#1}

To prepare SBW slurry surrogate for this study, a high-solids content slurry surrogate was prepared via a metathesis process (Janikowski 2003), sonicated to break up the agglomerated solids and mixed with a diluted SBW liquid surrogate (Christian 2001; Pao and Lewis 2003). Detailed chemical composition is not reported here, however, some of its properties are listed in Table 4-1. This mixture is referred as SBW slurry surrogate \#1. 
Table 4-1. Properties of the SBW slurry surrogate \#1.

\begin{tabular}{ccccccc}
\hline PH & $\begin{array}{c}\text { Settled Solid } \\
\text { Volume } \%\end{array}$ & $\begin{array}{c}\text { Particle Density } \\
(\mathrm{g} / \mathrm{mL})\end{array}$ & $\begin{array}{c}\text { Slurry UDS } \\
(\mathrm{g} / \text { liter })\end{array}$ & $\begin{array}{c}\text { Liquid TDS } \\
(\mathrm{g} / \text { liter })\end{array}$ \\
\hline $0.2 \sim 0.7$ & 35 & 2.13 & $\sim 27.5$ & 98 \\
\hline \multicolumn{6}{c}{ Filtrate Density $\left((\mathrm{g} / \mathrm{mL}) /{ }^{\circ} \mathrm{C}\right)$} \\
\hline $1.09 / 17$ & $1.08 / 20$ & $1.075 / 22$ & $1.07 / 23$ & $1.32 / 17$ & \multicolumn{4}{c}{ Filtrate Viscosity $\left(\mathrm{cp} /{ }^{\circ} \mathrm{C}\right)$} \\
\hline
\end{tabular}

\subsubsection{Radioactive SBW Slurry}

The radioactive SBW sludge samples were collected and transferred to the RAL at INTEC. Because the received actual sludge samples have relatively high acid content $(2.39 \mathrm{M})$ and high liquid density $\left(1.28 \mathrm{~g} / \mathrm{mL} @ 33^{\circ} \mathrm{C}\right)$, each of the samples for filtration testing were modified by mixing $30 \mathrm{~mL}$ of the actual SBW slurry with $45 \mathrm{~mL}$ of water. The mixed samples were allowed to stabilize for a minimum of two days before testing. At this time the settled SBW slurry layer constituted approximately $50 \%$ of the sample volume in the sample bottle. Approximately $35 \mathrm{~mL}$ of supernate was then removed and $40-\mathrm{mL}$ samples were used to minimize the sample size for the filtration test. Properties of the modified SBW slurry (post decant) are listed in Table 4-2.

Table 4-2. Properties of the modified radioactive SBW slurry (decant).

\begin{tabular}{|c|c|c|c|c|c|c|}
\hline $\mathrm{pH}$ & & $\begin{array}{l}\text { Settled Solid } \\
\text { Volume \% }\end{array}$ & $\begin{array}{l}\text { Particle Density } \\
(\mathrm{g} / \mathrm{mL})\end{array}$ & $\begin{array}{c}\text { Slurry UDS } \\
\text { (g/liter) }\end{array}$ & & $\begin{array}{l}\text { iquid TDS } \\
\text { (g/liter) }\end{array}$ \\
\hline$\sim 0$ & & 85 & N/A & 30 & & 105 \\
\hline \multicolumn{4}{|c|}{ Filtrate Density $\left((\mathrm{g} / \mathrm{mL}) /{ }^{\circ} \mathrm{C}\right)$} & \multicolumn{3}{|c|}{ Filtrate Viscosity $\left(\mathrm{cp} /{ }^{\circ} \mathrm{C}\right)$} \\
\hline $1.122 / 31.7$ & $1.12 / 32.7$ & $1.11 / 33.7$ & $1.01 / 31.7$ & $0.987 / 32.7$ & $0.96 / 33.7$ & $0.947 / 34.2$ \\
\hline
\end{tabular}

\subsubsection{SBW Slurry Surrogate \#2}

Because some differences were observed from the filtration test results of the surrogate \#1 and the actual radioactive SBW slurry samples (Maio et al. 2004), the SBW slurry surrogate \#2 was prepared in order to reduce the difference of the PSD of the solids material in the actual SBW and the surrogate mixture. To prepare the surrogate \#2, slurry products from the metathesis process were first sonicated, and the large size solids were removed via settling. The rest of small particles were collected via centrifugation, decanting free liquid, and adding an appropriate amount of the diluted SBW liquid surrogate. Properties of the SBW slurry surrogate \#2 are listed in Table 4-3.

Table 4-3. Properties of the SBW slurry surrogate \#2.

\begin{tabular}{ccccc}
\hline pH & $\begin{array}{c}\text { Settled Solid } \\
\text { Volume } \%\end{array}$ & $\begin{array}{c}\text { Particle Density } \\
(\mathrm{g} / \mathrm{mL})\end{array}$ & $\begin{array}{c}\text { Slurry UDS } \\
(\mathrm{g} / \text { liter })\end{array}$ & $\begin{array}{c}\text { Liquid TDS } \\
(\mathrm{g} / \text { liter })\end{array}$ \\
\hline$\sim 0.1$ & 40 & $2.13^{\mathrm{a}}$ & 28 & 78 \\
\hline \multicolumn{2}{c}{ Filtrate Density $\left((\mathrm{g} / \mathrm{mL}) /{ }^{\circ} \mathrm{C}\right)$} & & \multicolumn{2}{c}{ Filtrate Viscosity $\left(\mathrm{cp} /{ }^{\circ} \mathrm{C}\right)$} \\
\hline $1.078 / 20$ & $1.077 / 22$ & $1.076 / 24$ & $1.3 / 20$ & \multicolumn{2}{c}{$1.17 / 22$} & $1.1 / 24$ \\
\hline
\end{tabular}

a. Assumed the particle density is the same as surrogate \#1. 
A Horiba Model LA-300 was utilized to measure the particle size distribution on the surrogate solids and the modified radioactive SBW solids, the optics of the Horiba Model LA-300 are designed to measure particles with diameters ranging from $0.1 \mu \mathrm{m}$ to $600 \mu \mathrm{m}$. The measured PSD of all three slurry samples is shown in Figure 4-2. For solids material in the SBW slurry surrogate, the average initial diameter was $7.95 \mu \mathrm{m}(\mathrm{SD}=+/-10.7 \mu \mathrm{m})$ for surrogate $\# 1$ and $5.12 \mu \mathrm{m}(\mathrm{SD}=+/-2.52 \mu \mathrm{m})$ for surrogate \#2. The average diameter of the modified radioactive SBW slurry was $7.68 \mu \mathrm{m}$ $(\mathrm{SD}=+/-5.25 \mu \mathrm{m})$.

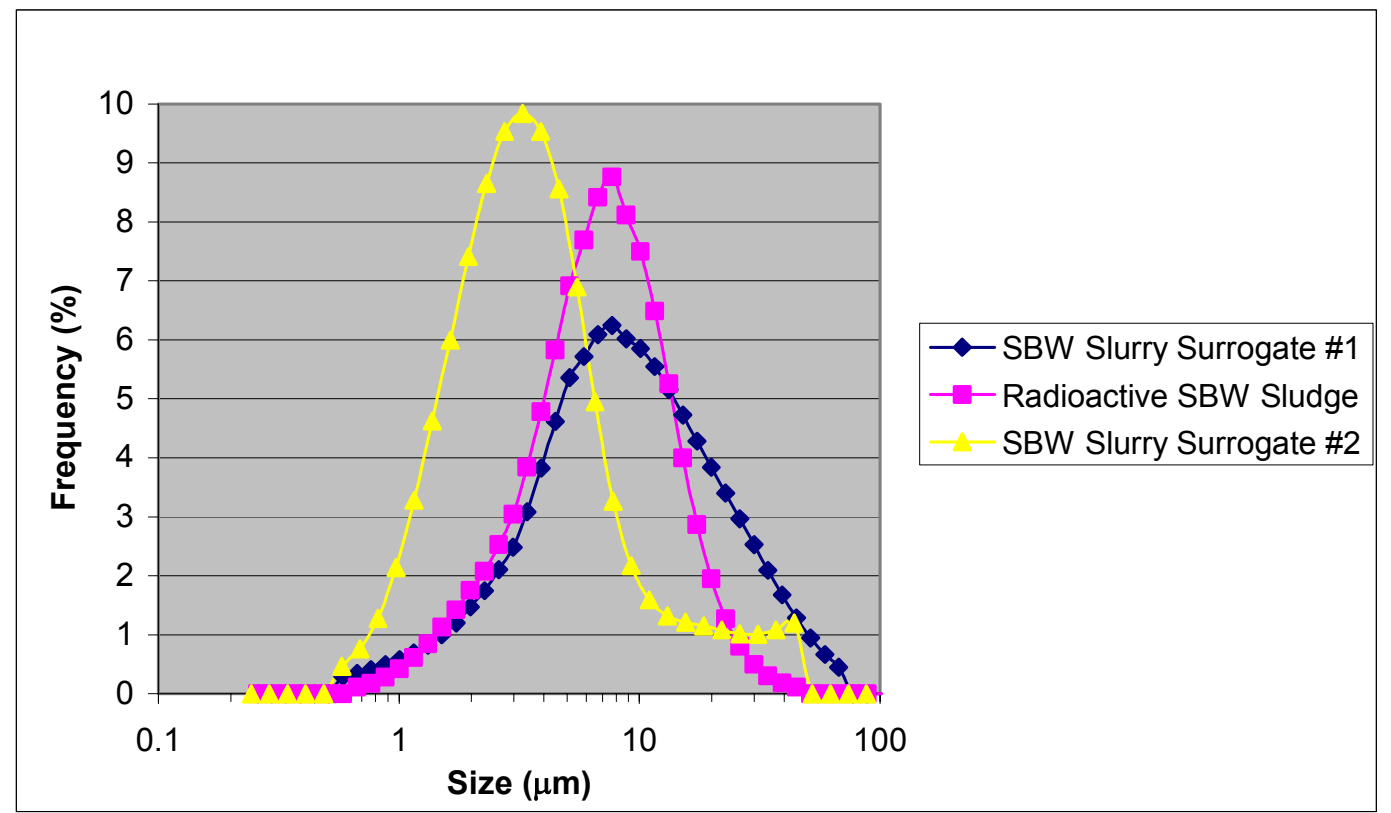

Figure 4-2. PSD comparison of SBW slurry surrogates and the modified SBWs slurry.

The settling rate of a solid is usually a function of concentration. To obtain settling rates, a sample of the surrogate and SBW slurry were shaken in a $12 \mathrm{~mm}$ diameter glass column and the height of the solids layer was monitored over time. For radioactive SBW slurry, the solids settled very slowly, and the undecanted modified actual SBW slurry was used for the measurement. Table 4-4 provides the calculated values of the liquid-solid interface velocity for all three slurries. For both surrogate \#1 and \#2, it took approximately two days to settle; however, it took more than 5 days for the modified SBW slurry to settle completely.

Table 4-4. Comparison of SBW slurry settling rates.

\begin{tabular}{cccccc}
\hline Surrogate \#1 & $\begin{array}{c}\text { Interface } \\
\text { Velocity } \\
\text { Time }(\mathrm{mr} / \mathrm{hr})\end{array}$ & Time $(\mathrm{hr})$ & $\begin{array}{c}\text { Modified Hot SBW Slurry } \\
\text { Interface } \\
\text { Velocity } \\
(\mathrm{mm} / \mathrm{hr})\end{array}$ & Time (hr) & $\begin{array}{c}\text { Surrogate \#2 } \\
\text { Velocity } \\
(\mathrm{mm} / \mathrm{hr})\end{array}$ \\
\hline 1.4 & - & 2.5 & - & 1.5 & - \\
2.1 & 48 & 5 & 2.26 & 2.5 & 57 \\
5.4 & 9.5 & 21.25 & 0.35 & 4 & 5.7 \\
9.8 & 5 & 25.5 & 0.68 & 8 & 1.9 \\
25 & 3.4 & 29.25 & 0.75 & 22 & 0.81 \\
47 & 1.4 & 117 & 0.32 & 32 & 0.38 \\
\hline
\end{tabular}




\subsection{Results and Analyses}

For constant pressure filtration studies reported in the literature, filtrate volume (or mass) vs. time are the data obtained (Christensen and Dick 1985). Many slurry filtration data display anomalies associated with the initial stages of filtration, even though their overall character is parabolic. Anomalies in the initial stages of filtration may be the result of a slow buildup of pressure drop across the cake, or error in determining the initial start time and corresponding filtrate volume (Christensen and Dick 1985; Tiller 1983). The formation of the first particle bridge is a delicate process. A collapse of these structures induced by a high-pressure load at the beginning of cake formation might cause severe medium clogging and blinding. However, a slowly increasing pressure might permit the formation of stable structures. Once the solids bridge the pores of the filter medium, pressure drop across the cake begins to increase concomitantly with cake development.

For data that fit both constant pressure filtration models I and II (equations (6)-(7) in Appendix A.4), initial timing of the filtrate mass (or volume) reading does not affect, theoretically, the slope of the standard plot or the calculated $\alpha$. In order to obtain consistent results, data collection may be delayed for 10 seconds or longer, as long as the system is under constant pressure, and the time and filtrate mass (volume) readings are matched pairs (Christensen and Dick 1985; Tiller 1983).

\subsubsection{SBW Slurry Surrogate \#1 Test Results}

Laboratory filtration tests usually yield the $\alpha$ (or SRF) data via the slope of the line generated when plotting the reciprocal instantaneous rate, $1 / q$, versus the total mass of dry cake solids per unit area of filter surface, $\mathrm{w}_{\mathrm{c}}$, which are then usable in process design and scale-up calculations, and surrogate validation.

Table 4-5 summarizes the filtration results obtained from 28 constant pressure filtration runs on three filter mediums, with various amounts of SBW slurry surrogate (Pao and Lewis, 2003; Maio et al 2004). SRFs were calculated using both instantaneous rate (model I) and average rate (model II) over the entire filtration cycle. An example of filtration data (at $49 \mathrm{psi}$ ) that fit the standard models is presented in Figure 4-3, and as evident by the figure, the data fit both models well. As indicated by the theory-based equations, the slope of the $p t / \mu v$ vs. $\mathrm{w}_{\mathrm{c}}$ line is approximately half of the slope of the $p \mathrm{~d} t / \mu \mathrm{d} v$ (or $p / \mu q$ ) line. The wet cake thicknesses were approximately $3.0,3.5$ and $5.5 \mathrm{~mm}$ for 30,40 and $60-\mathrm{mL}$ samples; and the equivalent total dry cake mass (undissolved solids [UDS] + total dissolved solids [TDS]) was approximately $0.7,1.04$ and $1.77 \mathrm{~g}$, respectively. After washing the filter cake to remove the dissolved solids, the amount of UDS in each of the dried filter cakes was estimated to be approximately $81 \%$ of the original cake mass. Because of sample variation, each sample cake was collected and its mass verified individually.

The initial $\alpha$ determined for the fresh SBW slurry surrogate \#1 ranged from $1.00 \times 10^{13}$ to $1.17 \times$ $10^{13} \mathrm{~m} / \mathrm{kg}$ at $30 \mathrm{psig}$ and $49 \mathrm{psig}$ (Test Set 1 ). However, the SRF measurements of the same slurry surrogate gradually decreased to between $7.18 \times 10^{12}$ and $8.42 \times 10^{12} \mathrm{~m} / \mathrm{kg}$ (Test Set 6 ) under identical test pressure, two weeks later. This observed decrease of $\alpha$ was probably due to sample aging, i.e., the fine particles agglomerated over the duration (see Figure 4-4). 
Table 4-5. SRF summary of SBW slurry surrogate \#1.

Test Set: 1

Sample Size: $30 \mathrm{~mL}$

Filter Medium: PVDF, Mean Pore Size: $5.5 \mu \mathrm{m}$

\begin{tabular}{llllrll}
\multicolumn{1}{c}{ Model } & \multicolumn{1}{c}{ I } & \multicolumn{2}{c}{ II } \\
\hline Pressure (psi) & 30 & 40 & 49 & 30 & 40 & 49 \\
$\alpha_{\text {av }}(\mathrm{m} / \mathrm{kg}$ )*E-12 & 10.2 & 10 & 11.7 & 8.98 & 11.36 & 11.4 \\
& 10.6 & 11.6 & & 10.58 & 10.28 & \\
\hline
\end{tabular}

Test Set: 2

Sample Size: $30 \mathrm{~mL} \quad$ Filter Medium: PPS, Mean Pore Size: $2.9 \mu \mathrm{m}$

\begin{tabular}{lcccccc}
\multicolumn{1}{c}{ Model } & \multicolumn{5}{c}{ I } \\
\hline Pressure (psi) & 30 & 40 & 49 & 30 & 40 & 49 \\
$\alpha_{\text {av }}(\mathrm{m} / \mathrm{kg}) * \mathrm{E}-12$ & 8.86 & 10.7 & 11.3 & 9.28 & 9.86 & 10.2 \\
& 8.87 & 10. & 10.8 & 8.86 & 10.3 & 10.94 \\
\hline
\end{tabular}

Test Set: 3

Sample Size: $40 \mathrm{~mL} \quad$ Filter Medium: PVDF, Mean Pore Size: $11.5 \mu \mathrm{m}$

\begin{tabular}{lcccccl}
\multicolumn{1}{c}{ Model } & \multicolumn{1}{c}{ I } \\
\hline Pressure (psi) & 30 & 40 & 49 & 30 & 40 & 49 \\
$\alpha_{\text {av }}(\mathrm{m} / \mathrm{kg}) * \mathrm{E}-12$ & 9.56 & 10.5 & 11.2 & 9.64 & 10.24 & 10.38 \\
& 8.86 & 10.4 & 11.7 & 8.52 & 10.24 & 10.8 \\
\hline
\end{tabular}

Test Set: 4

Sample Size: $40 \mathrm{~mL}$ Filter Medium: PVDF, Mean Pore Size: $5.5 \mu \mathrm{m}$

\begin{tabular}{lcccccc}
\multicolumn{1}{c}{ Model } & I & \multicolumn{3}{c}{ II } \\
\hline Pressure (psi) & 30 & 40 & 49 & 30 & 40 & 49 \\
$\alpha_{\text {av }}(\mathrm{m} / \mathrm{kg}) * \mathrm{E}-12$ & 9.77 & 10.4 & 11.2 & 9.28 & 10.14 & 10.82 \\
& & 9.36 & 10.7 & & 9.16 & 10.78 \\
\hline
\end{tabular}

Test Set: 5

Sample Size: $40 \mathrm{~mL} \quad$ Filter Medium: PPS, Mean Pore Size: $2.9 \mu \mathrm{m}$

\begin{tabular}{lcccccc}
\multicolumn{1}{c}{ Model } & I & \multicolumn{3}{c}{ II } \\
\hline Pressure (psig) & 30 & 40 & 49 & 30 & 40 & 49 \\
$\alpha_{\mathrm{av}}(\mathrm{m} / \mathrm{kg}) * \mathrm{E}-12$ & 7.94 & 8.92 & 9.23 & 8.1 & 8.6 & 9.12 \\
\hline
\end{tabular}

Test Set: 6

Sample Size: $60 \mathrm{~mL} \quad$ Filter Medium: PPS, Mean Pore Size: $2.9 \mu \mathrm{m}$

\begin{tabular}{lcccccc}
\multicolumn{1}{c}{ Model } & I & \multicolumn{4}{c}{ II } \\
\hline Pressure (psig) & 30 & 40 & 49 & 30 & 40 & 49 \\
$\alpha_{\mathrm{av}}(\mathrm{m} / \mathrm{kg}) * \mathrm{E}-12$ & 7.18 & 8.15 & 8.42 & 7.16 & 8.04 & 8.16 \\
\hline
\end{tabular}




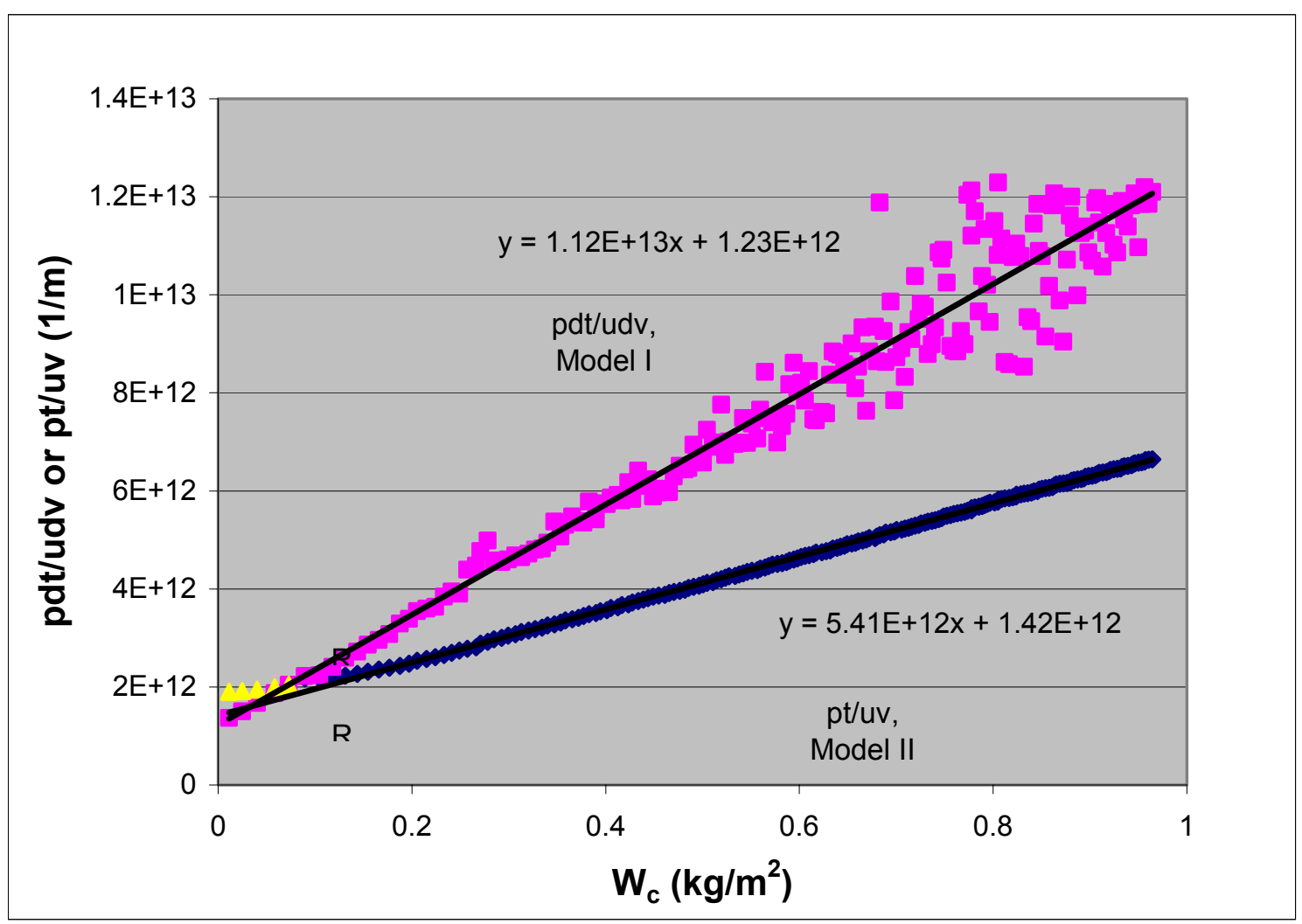

Figure 4-3. Determination of cake filtration resistance in a constant pressure filtration run.

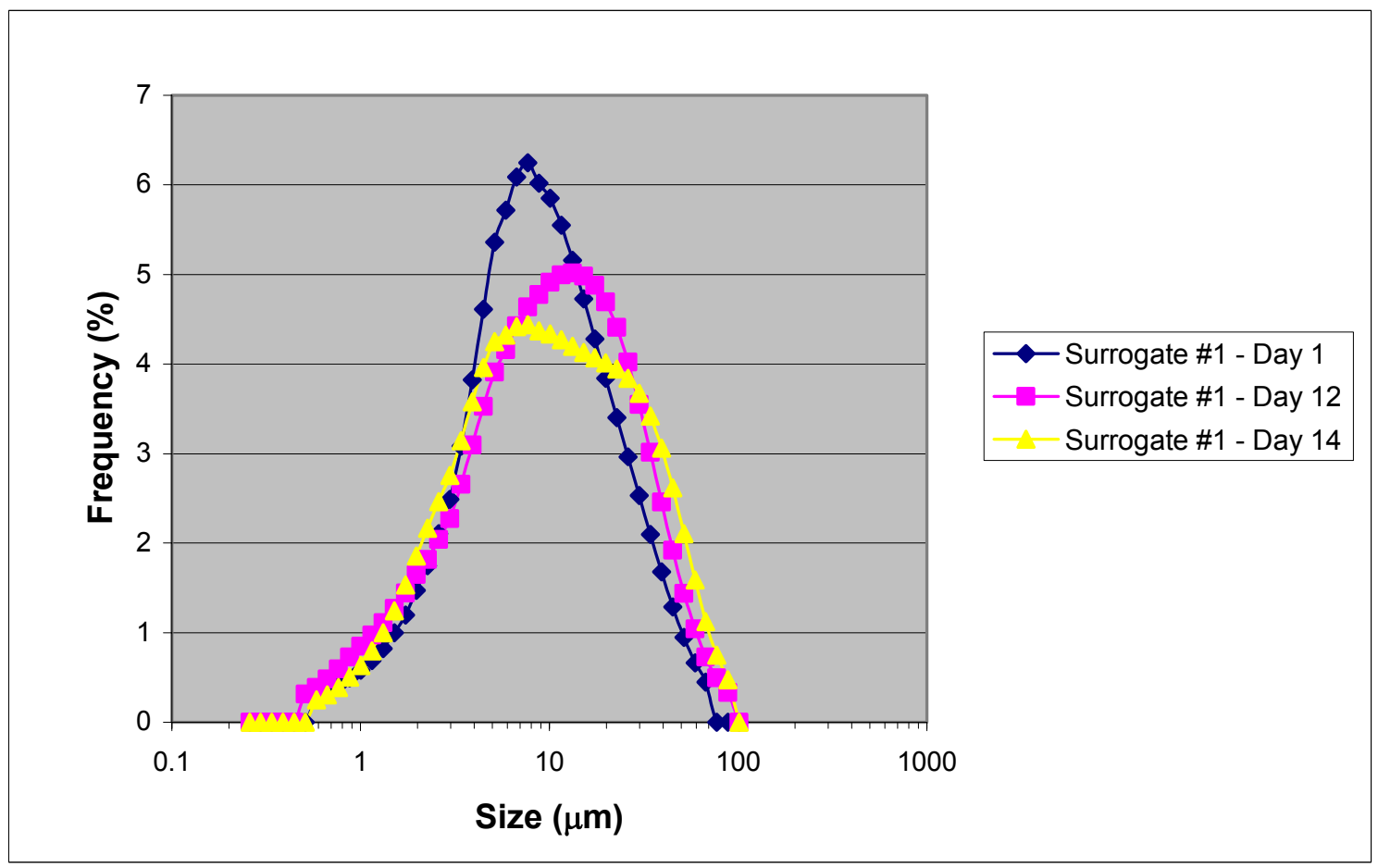

Figure 4-4. PSD of SBW slurry surrogate \#1. 


\subsubsection{Radioactive SBW Slurry Test Results}

A total of 18 filtration tests were performed at the RAL at INTEC. All tests were conducted by using the filter medium made of PVDF; at mean pore size of $5.5 \mu \mathrm{m}$. Filtration tests were performed within four days after the fresh sample was prepared, and analyses of the control sample showed that the difference of PSD is insignificant, over the duration (Maio et al. 2004).

Specific filtrate volume $\left(\mathrm{m}^{3} / \mathrm{m}^{2}\right)$ was calculated by dividing the filtrate volume by the filter area. Table 4-6 shows the derived values of the SRF for the SBW slurry using Models I and II. The wet cake thickness ranged between $8-10 \mathrm{~mm}$ for the $40-\mathrm{mL}$ sample, and the equivalent total dry cake mass (UDS+TDS) was determined at 1.4-1.8 g. The total UDS was estimated at approximately $75 \%$ of the dry cake mass.

Table 4-6. SRF summary of radioactive SBW slurry.

\begin{tabular}{|c|c|c|c|c|c|c|}
\hline Model & & I & & & II & \\
\hline \multirow[t]{4}{*}{ Pressure (psig) } & 30 & 40 & 50 & 30 & 40 & 50 \\
\hline & 22.7 & 28.6 & 37.1 & 22.0 & 27.2 & 38.4 \\
\hline & 24.4 & 27.1 & 33.0 & 24.2 & 26.8 & 32.2 \\
\hline & 21.4 & 28.3 & 30.4 & 20.8 & 27.8 & 30.4 \\
\hline \multirow[t]{4}{*}{$\alpha_{\mathrm{av}}(\mathrm{m} / \mathrm{kg}) * \mathrm{E}-12$} & 22.4 & 26.6 & 31.7 & 21.0 & 26.8 & 29.8 \\
\hline & 19.8 & 23.7 & 29.8 & 20.8 & 22.6 & 30.4 \\
\hline & 20.5 & & 27.4 & 17.9 & & 28.0 \\
\hline & & & 31.1 & & & 29.0 \\
\hline
\end{tabular}

\subsubsection{SBW Slurry Surrogate \#2 Test Results}

Table 4-7 summarizes the filtration results obtained from 14 filtration tests, all tests were conducted by using the filter medium made of PVDF; at a mean pore size of $5.5 \mu \mathrm{m}$. Tests were performed within seven days after the fresh samples were prepared, and the analyses of sample PSD showed a minimum change over the period. The wet cake thicknesses were at approximately $7 \mathrm{~mm}$ of the 40-mL sample, and the equivalent total dry cake mass (UDS+TDS) was determined at 1.2-1.4 g. The total UDS was estimated at approximately $85 \%$ of the dry cake mass.

Table 4-7. SRF summary of SBW slurry surrogate \#2.

\begin{tabular}{ccccccc}
\hline Model & \multicolumn{3}{c}{ I } & \multicolumn{3}{c}{ II } \\
\hline Pressure (psig) & 30 & 40 & 49 & 30 & 40 & 49 \\
& 6.27 & 7.41 & 8.52 & 6.06 & 7.36 & 8.3 \\
& 5.63 & 7.63 & 10.1 & 5.84 & 7.1 & 9.18 \\
$\alpha_{\text {av }}(\mathrm{m} / \mathrm{kg}) * \mathrm{E}-12$ & 6.86 & 7.66 & 8.38 & 6.64 & 7.16 & 8.06 \\
& 6.28 & 7.41 & 9.29 & 5.94 & 7.0 & 9.14 \\
& & 7.91 & 9.24 & & 7.78 & 8.76 \\
\hline
\end{tabular}




\subsubsection{Comparison of SRF ( $\alpha)$ and Cake Compressibility}

Following the cake filtration tests carried out at different pressures (30 to $50 \mathrm{psig}$ ), the calculated parameter " $\alpha$ " was plotted as a function of the applied pressure " $p$ ". A useful expression for this purpose is only valid over a specified range of pressures and takes the form of equation (10) in Appendix A.4. The results of the $\alpha$ vs. $p$ relationship for both surrogates and the radioactive SBW slurry are shown in Figure 4-5. The data illustrate increased cake permeability for the surrogate samples when compared to the SBW slurry sample. The increase of surrogate cake permeability is likely a consequence of less efficient particle packing.

The SRF value for the SBW sample is higher than the value for both surrogates \#1 and \#2. Surrogate $\# 1$ has a greater mean particle size distribution and is relatively quite broad $(0.1 \sim 70 \mu \mathrm{m})$ while surrogate \#2 has a smaller mean particle size and a narrower distribution. Under the acidic sample conditions, the particles should be dispersed (i.e., they have a strong positive charge), but a high dissolved solid contents can also lead to a compressed double layer charges. Settling behavior seems to support the case for dispersed particles, i.e., settling takes place over a period of time greater than two hours. So, for the case of surrogate $\# 2$, it seems reasonable to conclude that the increased cake permeability relative to surrogate \#1 is due to reduced particle packing efficiency. But when comparing the radioactive SBW slurry to surrogate \#1, another important element of the experiment that can be used to explain the observed behavior is the settling data. The radioactive SBW sample took much longer to settle, implying a reduced density of the solid and/or a significant fraction of small particles that would be able to infiltrate the cake and create a low permeability filter cake.

In addition, the permeability (flow rate) similarly decreases when the particles are better dispersed; SBW slurry (radioactive) with slower sedimentation velocity would increase SRF. Over the pressure range of this study, the SRF for the radioactive SBW slurry was within experimental error of that determined for the surrogates. Regardless, filtering the actual SBW slurries took approximately 2-3 times longer under the given conditions to filter than the surrogates. The filter cake collected from the SBW slurry surrogate \#1 had an average cake compressibility of " 0.33 ," a low-to-moderate compressibility filter cake. In contrast, the cake collected from SBW radioactive slurry and slurry surrogate \#2, had an average cake compressibility of " 0.71 " and " 0.76 ", respectively, a moderate-to-high compressibility filter cake (Table 4-8).

Particle interactions play a very important role in fine-particle filtration, because ionic strength and zeta potential in the mixture would affect the aggregate size in the suspension (Wakeman et al. 1991; Koenders and Wakeman 1997), and the extent of aggregation in suspensions determines the degree of cake compressibility. Compressibility depends on particle size distribution, particle shape, and degree of aggregation (Perry and Chilton 1997; Rushton et al. 1996; Wakeman et al. 1989; Wakeman et al. 1991). While there exist many factors influencing the filtration behaviors, the underlying phenomena are currently not well understood. In this study, the radioactive SBW slurry was not settled readily, and the suspension is said to be very stable. The particles in these dispersions maintain their resistance to aggregation through interactions at their surfaces, and it may be necessary to apply high pressure in order to squeeze the liquid from the stable SBW slurry dispersions. On the contrary, the filtration results of surrogate \#2 showed that the filter cake has high permeability (or lower SRF), even though the surrogate \#2 solids have a smaller PSD. This difference is probably due to the fact that the surrogate is relatively unstable versus the SBW slurry. However, surrogate \#2 has showed that it has a cake compressibility that is similar to that of the radioactive SBW slurry. 


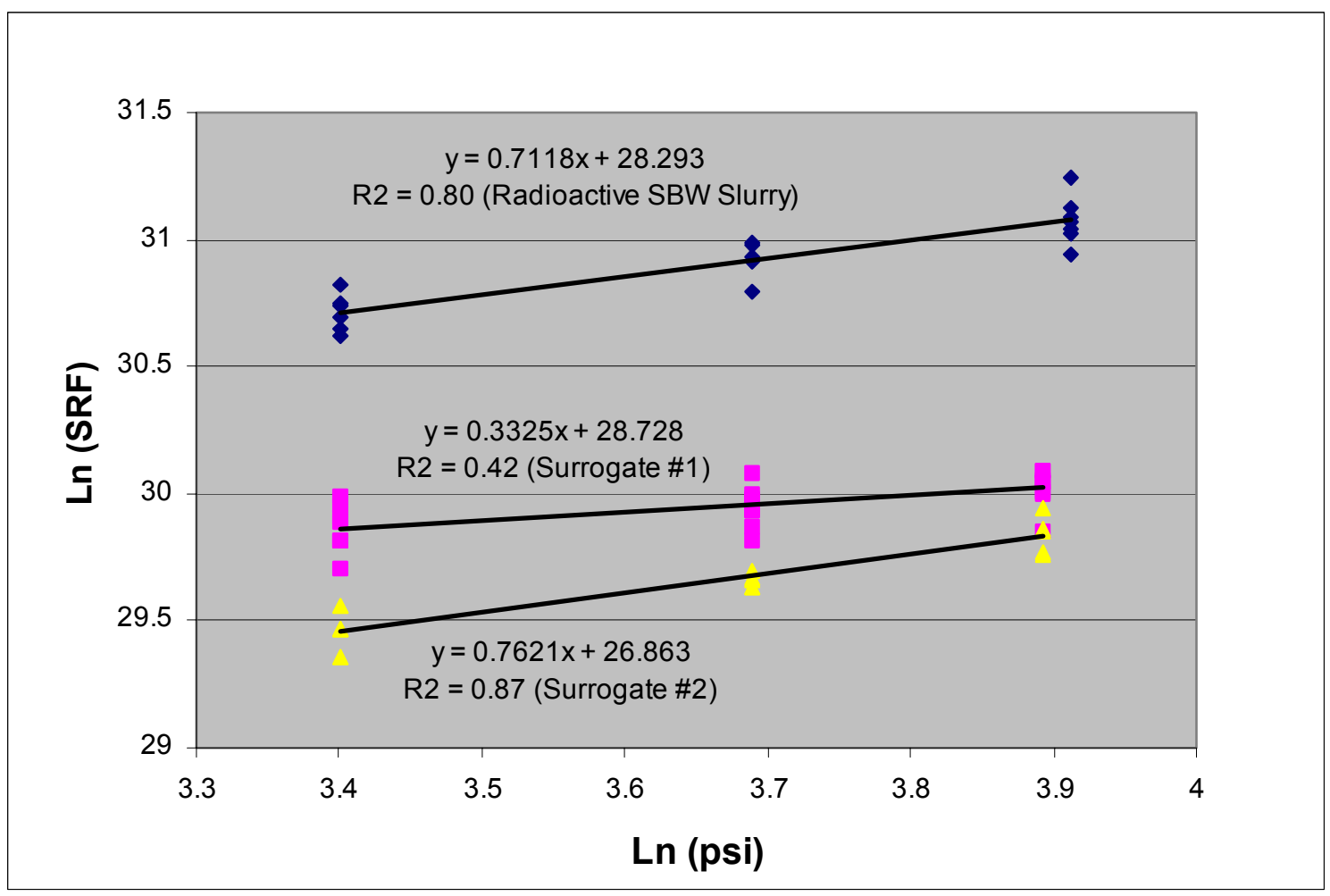

Figure 4-5. Comparison of cake compressibility (Model I).

Table 4-8. Comparison of cake compressibility coefficients.

Model

I

II

Radioactive

Radioactive

Sample Stream Surrogate \#1

SBW Slurry Surrogate \#2 Surrogate \#1

SBW Slurry

Surrogate \#2

Mean

0.33

0.71

0.76

0.29

0.76

0.71

n Upper $95 \%$

0.50

0.90

0.95

0.45

0.98

0.87

Lower 95\%

0.16

0.53

0.58

0.14

0.54

0.51 


\section{RECOMMENDATIONS}

Prior to using the metathesis-prepared SBW surrogate in waste processing design and mockup studies, it is recommended that additional research be performed in several areas. First, it is recommended that the SBW tanks be sampled in multiple locations to provide bounding SBW solids characterization data.

Any future surrogate development should be designed to emulate the expected wastes that will result from the planned tank farm consolidation. The decision to consolidate WM-180 waste into WM187 changes data needed for tank slurries. Surrogate required for testing coprocessing scenarios must be representative of the tank farm solids in concentrated SBW. The uncertainty in the average undissolved solids weight percentage in the tanks could be between 2 and $4 \%$. Surrogate required for testing the CsIX process must be representative of the high solids ( $\sim \mathrm{wt} \%$ undissolved solids), concentrated SBW in WM-187. Testing of heel processing requires a surrogate with properties representative of wash water slurry with low solids content.

It is recommended that a surrogate with similar thixotropy to that of the tank farm waste be developed. This surrogate would be used to emulate the properties of the tank farm waste during extended waste handling operations. The time-dependent shear thinning characteristic of the undried FY-04 metathesis-prepared SBW surrogate may render it nonconservative for applications that occur over a significant period of time (with or without applied shear stresses). Surrogate preparation steps suggested to reduce thixotropy differences between the surrogate and tank farm waste include thermal treatment of the surrogate and reformulation of the surrogate to include more metal oxides and less silica (to reduce the amount of silica gels in the surrogate). Flow curve analysis of dried FY-03 metathesis-prepared SBW surrogate samples with a lower solids volume percentage than the centrifuged WM-187 tank farm waste indicated that the samples had similar thixotropy, but that the shear stress curve of the surrogate was lower than the shear stress curve of the waste. It is recommended that flow curve analyses be performed on dried metathesis-prepared SBW surrogate and tank farm waste samples with similar solids volume percentages as part of the effort to identify a surrogate with similar rheology to the tank farm waste.

Although every effort was made to collect and prepare waste and surrogate samples as consistently as possible, variation between the various samples of each surrogate preparation inevitably existed. Sample collection methods were devised to minimize these differences as much as possible. Nonetheless, small variations in solids volume percentage and solids characteristics were present in otherwise similar samples. Repetition of physical property analyses would provide additional certainty that the surrogate provides a good representation of the tank farm waste physical properties. 


\section{CONCLUSIONS}

Slurries formed from undried, sonicated FY-04 metathesis-prepared SBW solids surrogate and metathesis mother liquor had flow curve profiles similar to tank farm waste sample slurries of comparable solids volume and weight percentages. The slurries formed from undried, sonicated FY-04 metathesis-prepared SBW solids surrogate and metathesis mother liquor had flow curve profiles that approximated the flow curve profiles of each of the tank farm waste sample fractions tested ("as-received," gravity-settled, and centrifuged sludge) more accurately than waste surrogate slurries formed from any other combination of solid and liquid surrogates tested. The waste surrogate slurry formed from the FY-04 SBW solids surrogate and metathesis mother liquor liquid surrogate provided better representations of the tank farm waste sample fractions than waste surrogate slurries formed from FY-04 SBW solids surrogate and WM-180 or WM-189 liquid surrogates.

Waste surrogate slurry formed from dried, sonicated FY-03 SBW solids surrogate and WM-187 liquid surrogate $(187 / 20 \mathrm{wt} \% / \mathrm{D} / \mathrm{S})$ had very similar thixotropy to that of the centrifuged tank farm waste sample. Although the thixotropy of the $187 / 20 \mathrm{wt} \% / \mathrm{D} / \mathrm{S}$ surrogate was similar to that of the centrifuged tank farm waste sample, its shear stress profile fell below that of the centrifuged tank farm waste sample, rendering it nonconservative for tank farm solids transportation operations simulation. Nevertheless, the flow curve data collected from this surrogate (and all other FY-03 SBW solids surrogate and WM-187 liquid surrogate slurries) provide evidence that drying (or other thermal treatment) of the SBW solids surrogate prior to final surrogate slurry sample preparation may produce surrogates with thixotropy more similar to the WM-187 tank farm waste sample.

Flow curve analyses performed on waste surrogate samples with different chemical and physical makeups (liquid surrogate, solids surrogate batch, solids weight percentage, and particle size distribution) and at varying test temperatures show several trends in response to changes in these parameters (assuming no change in other variables). Understanding these trends will help in the subsequent development of more accurate waste surrogates and may provide insight into the character of the tank farm waste. The shear stress profile universally increased with increasing solids weight percent for all surrogate samples tested. The shear stress profiles for samples prepared with the four different liquid surrogates follow the pattern WM-189 > WM-180 > META > WM-187. The shear stress profile universally increased with decreasing temperature. No definitive trend could be concluded for changes to sample particle size distribution. Additional data and analyses are needed to further investigate the effect of changes in the sample particle size distribution on the flow curve shear stress profile.

One important difference between the waste surrogate and the tank farm waste were the properties of samples allowed to sit undisturbed for extended periods. Surrogate slurry samples became noticeably coagulated after sitting undisturbed for periods on the order of $24 \mathrm{hrs}$. The surrogate slurry samples continued to coagulate and form cohesive sludge phases the longer they were allowed to sit undisturbed. Slurries prepared with dried FY-03 solids surrogate formed relatively "brittle" sludge phases after sitting undisturbed, while slurries prepared with undried FY-04 solids surrogate formed relatively "gelatinous" sludge phases after sitting undisturbed. Coagulation was not observed in tank farm waste slurry samples allowed to sit undisturbed for several weeks.

For testing physical systems associated with moving the tank farm solids, the surrogate described in this report is the best currently available choice. No other available surrogate exhibits the noted similarities in behavior to the tank farm waste sludges. Nonetheless, one or more additional waste surrogate development and analysis iterations would allow the surrogate to be prepared so as to target the tank farm waste sample properties more accurately. An additional waste surrogate development and analysis iteration would require synthesis of another batch of waste surrogate, procurement of a new viscometer, and completion of another flow curve data collection campaign. Synthesis and preparation of 
another batch of waste surrogate (with knowledge of the similarities and differences between the waste surrogate and tank farm waste sample gleaned from the testing described in this report) may lead to the development of a surrogate with properties increasingly similar to those of the tank farm waste.

Confidence that the waste surrogate is an accurate representation of the tank farm waste would be further elevated by this effort. Since there is a difference in chemical makeup of the surrogate and the tank farm waste, if a chemical treatment process were to be evaluated for final treatment and disposition of the waste sludges, the surrogate synthesis process would likely require modification to yield a surrogate with a closer matching chemical composition.

The development of realistic surrogates that simulate actual hazardous and/or radioactive materials, such as waste streams from the DOE nuclear industry, is paramount if appropriate treatment technologies are to be successfully developed and tested within an acceptable safety and economical envelope. However, the degree of precision required for simulating a toxic waste, albeit one of a solid or sludge nature, depends on the type of technological processes and unit operations that will be developed and optimized with the waste surrogate. Large volumes of liquid surrogates that contain the major chemical constituents, dissolved salts, and acidity level of the actual waste are less arduous to prepare and validate, especially if they are to be used in the development of nonphysical processes. These liquid waste surrogates are simply established with rudimentary aqueous chemical mixing techniques as long as statistically-accurate analysis data on representative samples of the real waste are available.

Solids and slurries, on the other hand, require a more thorough, broad, and rigorous surrogate approach, since their resulting surrogate is usually utilized to develop, test, and identify the full-scale parameter ranges for the more temperamental waste physical processes (e.g., filtration, drying, centrifuging, sizing, transport, mixing, and pumping). Consequently, hazardous and/or radioactive waste solids/sludge surrogates must be prepared in a method reflective of their actual production (e.g., metathesis synthesis) and not by simply adding together raw solid chemicals to mimic the actual solid composition.

Whereas a simple chemical constituent addition technique validated with a corresponding concentration comparison may be adequate for validating a liquid waste surrogate, more complex data are necessary for a solid/slurry waste surrogate. The extra surrogate data for solids and sludges are a manifestation of their ubiquitous, yet unique, physical properties (such as coagulation, cohesiveness, friability, compressibility, and caking resistivity). Collectively, these traits acquire a determination of not only the solids simple chemical makeup, but also their deeper chemical structure, mineralogy/morphology, and even more important, their dynamic physical properties. Physical properties include those presented in this paper: particle size distribution, slurry rheology, settling velocities, solids weight percentage, bulk density, the filtration properties of cake compressibility, and cake resistance. As indicated by the preliminary results presented in this paper, comparison of the metathesis-prepared solids surrogate and actual INTEC tank radioactive slurry wastes under constant pressure filtration test conditions, provides an early indication of the iterative nature of the process for aligning the properties of the surrogate with those of the actual solids. Even though use of this approach for all the chemical and physical properties defined in this paper, in addition to a statistical comparison between the surrogate and actual solids, may be costly and time-consuming, it is a more economically viable option than integrated testing with actual wastes. Above all, the rigorous surrogate method outlined will ensure reliable development data for a successful waste technology deployment. Conversely, poorly-assumed and simple solids surrogate representation in the cold development phase will inevitably result in inadequate full-scale design and operation, along with cost-prohibitive retrofits and reworks. As proven by documented waste processing case histories, such financial setbacks are not an option available to project engineering managers responsible for successfully meeting high profile waste treatment and disposal milestones across the DOE complex. 


\section{REFERENCES}

Alles, Carina Maria, Harald Anlauf, and Werner Stahl, 1998, "Compressible Cake Filtration Under Variable Pressure," Advances in Filtration and Separation Technology, Vol. 12, p. 628, America, Filtration \& Separation Society, Northport, Alabama.

Analytical Chemistry Methods Manual, Volume I, "Bulk Density," Idaho National Engineering and Environmental Laboratory, ACMM-7007, Rev. 0, August 6, 2003.

Analytical Chemistry Methods Manual, Volume I "Determination of Total Solids in Aqueous Samples," Idaho National Engineering and Environmental Laboratory, ACMM-7975, Rev. 5, September 28, 2000.

Barnes, C. M. and C. B. Millet, 2003,. Feed Composition for the Sodium-Bearing Waste Treatment Process, INEEL/EXT-2000-01378, Rev. 2, January 2003.

Barnes H. A., J. F. Hutton, and K. Walters, 1989, An Introduction to Rheology, Amsterdam: Elsevier.

Batcheller, T. A., D. D. Taylor, and V. J. Johnson, 2002,, Characterization of Tank WM-189 SodiumBearing Waste at the Idaho Nuclear Technology and Engineering Center, INEEL/EXT-02-01171, Rev. 1, (draft), December 2002.

Brookfield Engineering, Inc. More Solutions to Sticky Problems: A Guide to Getting More from Your Brookfield Viscometer, http://www.brookfieldengineering.com/download/files/more solutions.pdf, Web page visited on October 20, 2003.

Christensen, G. L. and Richard I. Dick, 1985, "Specific Resistance Measurement: Non-parabolic Data," Journal of Environmental Engineering, Vol. 111, No. 3, p. 243.

Christian, J. D, 2001, Composition and Simulation of Tank WM-180 SBW at the Idaho Nuclear Technology and Engineering Center, INEEL/EXT-2001-00600.

Darcy, J. P. G., 1856, Les Fontaines Publiques de la Ville De Dijon, Paris: Victor Dalmont.

Derivation of the sediment settling velocity equations,

http://freespace.virgin.net/mark.davidson3/sediment/stokes/SETTLEV.html, Web page visited on October 23, 2003.

EDF-TST-001, 2001, “Solids Characterization,” Poloski, A. and M. Wilcox, February 10, 2001.

EDF-1914, 2001, "Rheology of the INTEC Tank Farm Closure Surrogate and WM-182 LDUA Sample/Dissolution of the WM-182 LDUA Sample in 6M Nitric Acid," Poloski, A. P., February 19, 2001.

EDF-15722-040, 2000, "INTEC Tank Farm Sludge Density Measurements/Calculations," Poloski, A. P., July 7, 2000.

EDF-15722-041, 2000, "Surrogate Sludge for Tank Farm Closure Mockups," Poloski, A. and M. Wilcox, October 30, 2000.

Geankoplis, C. J., 1978, Transport Processes and Unit Operation, Allyn and Bacon. 
Heywood, N. I., 1999, "Stop Your Slurries From Stirring Up Trouble,” Chemical Engineering Progress, September 1999.

Heywood, N. I. and N. J. Alderman, 2003, "Developments in Slurry Pipeline Technologies,” Chemical Engineering Progress, April 2003.

Janikowski, S., 2003, Sodium Bearing Waste: Solids Characterization and Solids Simulant Development, FY-03 Year End Report, Doc ID 1398707, September 16, 2003.

Janikowski, S. K, 2003, Metathesis Formation of Tank Farm Solid Simulant, INEEL-IHR \#1013-03-IRC.

Koenders, M. A. and R. J. Wakeman, 1997, "Initial Deposition of Interacting Particles by Filtration of Dilute Suspensions," AICHE Journal, 43, p. 946-958.

Maio, V., et al., 2004, "Sludge Surrogate Development and Validation using Caking Filtration Parameters for a Radioactive Sodium Bearing Waste Derived Sludge at the Idaho National Engineering and Environmental Laboratory," Waste Management Conference, Tucson Arizona, February 2004.

Pao, Jenn-Hai, and R. R. Lewis, 2003, Experimental Study of Filtration and Solids' Post-Treatment from Simulated SBW Derived Slurry, INEEL/EXT-03-01106, September 2003.

Perry and Green, 1984, Perry's Chemical Engineers' Handbook, New York: McGraw Hill, $6^{\text {th }}$ edition.

Perry and Green, 1997, Perry's Chemical Engineers' Handbook, New York: McGraw-Hill, $7^{\text {th }}$ edition.

Pierre, A. C. and K. Ma, 1999, "DLVO Theory and Clay Aggregate Architectures formed with $\mathrm{AlCl}_{3}$," Journal of the European Ceramic Society, 19:1615-1622.

Rushton, A., A. S. Ward, and R. G. Holdich, 1996, "Solid-Liquid Filtration and Separation Technology," VCH Verlagsgesellschaft mbH, Weinhein, Federal Republic of Germany, 1996.

Sperry, D. R, 1916, "Principles of Filtration, II," Chem. Met. Eng, 19, p. 161.

Tien, Chi., Renbi Bai, and B. V. Ramarao, 1997, "Analysis of Cake Growth in Cake Filtration: Effect of Fine Particle Retention,” AICHE Journal, Vol. 43, No. 1, January 1997, p. 33.

Tiller, F. M., 1990, “Tutorial: Interpretation of Filtration Data, I," Fluid/Particle Separation Journal, Vol. 3, p. 85.

Tiller, F. M. and J. R. Crump, 1997, "Solid-Liquid Separation: An Overview,” Chemical Engineering Progress, October 1997, p. 65.

Tiller, F. M. and W. Leu, 1983, "Experimental Study of the Mechanism of Constant Pressure Cake Filtration: Clogging of Filter Media," Separation Science and Technology, Vol. 18, \#12/13, p. 1351.

Wakeman, R. J., M. N. Sabri, and E. S. Tarleton, 1991, "Factors Affecting the formation and Properties of Wet Compacts," Powder Technology, 65, pp. 283-292. 
Wakeman, R. J., S. T. Thuraisingham, and E. S. Tarleton, 1989, "Colloid Science in Solid-Liquid Separation Technology," Filtration \& Separation, July/August 1989, pp. 277-283.

Water Environment Research Foundation, 1993, Guidance Manual for Polymer Selection in Wastewater Treatment, Project 91-ISP-5. 
7-4 
Appendix A

Theory 


\section{A-1 VISCOSITY}

The following viscosity theory discussion was adapted from Brookfield Engineering's Support web page (http://www.brookfieldengineering.com).

Viscosity is the measure of the internal friction of a fluid. This friction becomes apparent when a layer of fluid is made to move in relation to another layer. The greater the friction, the greater the amount of force required to cause this movement, which is called "shear." Shearing occurs whenever the fluid is physically moved or distributed, as in pouring, spreading, spraying, mixing, etc. Highly viscous fluids, therefore, require more force to move than less viscous materials.

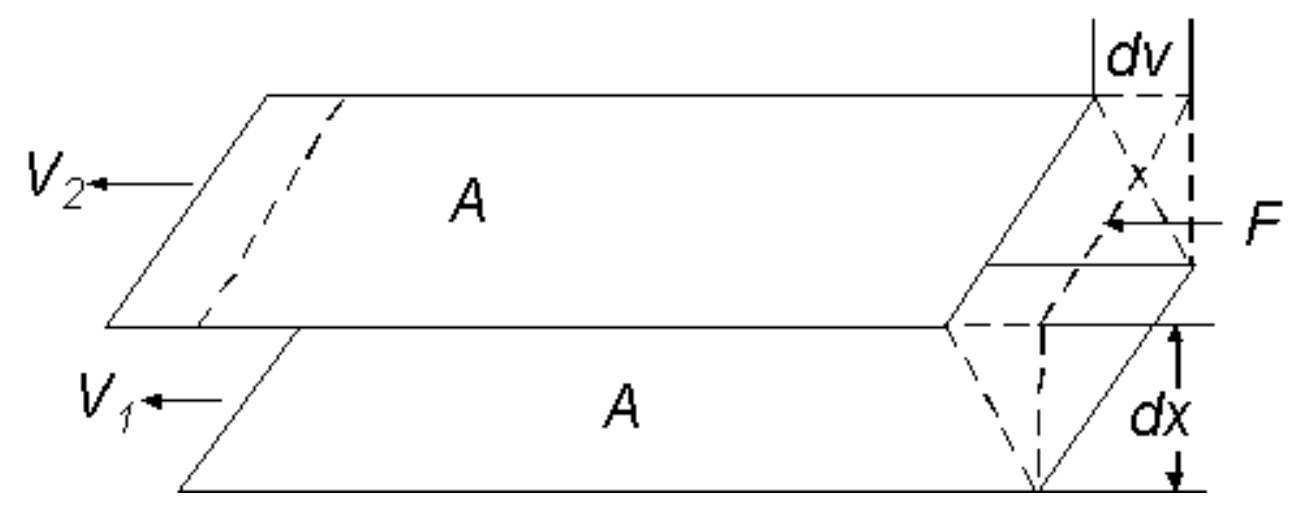

Isaac Newton defined viscosity by considering the model represented in the figure above. Two parallel planes of fluid of equal area "A" are separated by a distance " $\mathrm{dx}$ " and are moving in the same direction at different velocities " $\mathrm{V}_{1}$ " and " $\mathrm{V}_{2}$." Newton assumed that the force required to maintain this difference in speed was proportional to the difference in speed through the liquid, or the velocity gradient. To express this, Newton wrote:

$$
\frac{F}{A}=\mu \frac{d v}{d x}
$$

where $\mu$ is a constant for a given material and is called its "viscosity."

The velocity gradient, $\mathrm{dv} / \mathrm{dx}$, is a measure of the change in speed at which the intermediate layers move with respect to each other. It describes the shearing the liquid experiences and is thus called "shear rate." This will be symbolized as " $\mathrm{S}$ " in subsequent discussions. Its unit of measure is called the "reciprocal second" $\left(\sec ^{-1}\right)$.

The term F/A indicates the force per unit area required to produce the shearing action. It is referred to as "shear stress" and will be symbolized by " $F$ '." Its unit of measurement is "dynes per square centimeter" (dynes $\left./ \mathrm{cm}^{2}\right)$.

Using these simplified terms, viscosity may be defined mathematically by this formula:

$$
\mu=\text { viscosity }=\frac{\mathrm{F}^{\prime}}{\mathrm{S}}=\frac{\text { shear stress }}{\text { shear rate }}
$$


The fundamental unit of viscosity measurement is the "poise." A material requiring a shear stress of one dyne per square centimeter to produce a shear rate of one reciprocal second has a viscosity of one poise, or 100 centipoise. Other units of viscosity include "Pascal-seconds" (Pa's) and "milli-Pascalseconds" ( $\mathrm{mPa} \cdot \mathrm{s})$; these are units of the International System and are sometimes used in preference to the Metric designations. One Pascal-second is equal to ten poise; one milli-Pascal-second is equal to one centipoise.

\section{A-1.1 Newtonian Fluids}

A Newtonian fluid is represented graphically in the figure below. Graph A shows that the relationship between shear stress $\left(\mathrm{F}^{\prime}\right)$ and shear rate $(\mathrm{S})$ is a straight line. Graph B shows that the fluid's viscosity remains constant as the shear rate is varied. Typical Newtonian fluids include water and thin motor oils.
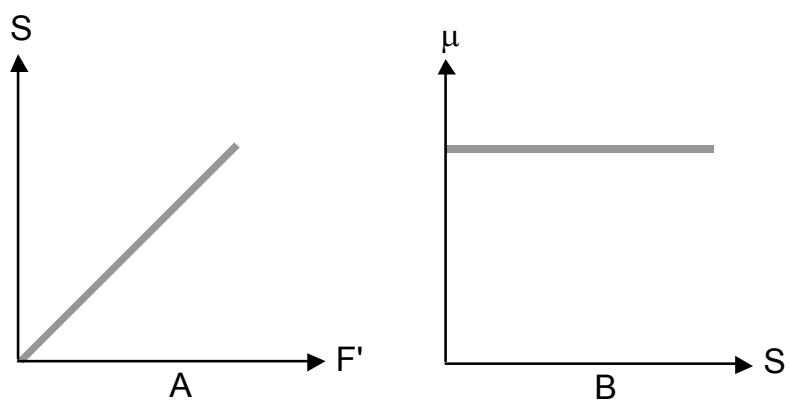

\section{A-1.2 Non-Newtonian Fluids}

A non-Newtonian fluid is broadly defined as one for which the relationship $\mathrm{F}^{\prime} / \mathrm{S}$ is not a constant. In other words, when the shear rate is varied, the shear stress doesn't vary in the same proportion (or even necessarily in the same direction). The viscosity of such fluids will therefore change as the shear rate is varied. Thus, the experimental parameters of viscometer model, spindle and speed all have an effect on the measured viscosity of a non-Newtonian fluid. This measured viscosity is called the "apparent viscosity" of the fluid and is accurate only when explicit experimental parameters are defined and adhered to.

Non-Newtonian flow can be envisioned by thinking of any fluid as a mixture of molecules with different shapes and sizes. As they pass by each other, as happens during flow, their size, shape, and cohesiveness will determine how much force is required to move them. At each specific rate of shear, the alignment may be different and more or less force may be required to maintain motion.

There are several types of non-Newtonian flow behavior, characterized by the way a fluid's viscosity changes in response to variations in shear rate. The most common types of non-Newtonian fluids that may be encountered include:

Psuedoplastic: This type of fluid will display a decreasing viscosity with an increasing shear rate, as shown in the figure below. Probably the most common of the non-Newtonian fluids, pseudo-plastics include paints, emulsions, and dispersions of many types. This type of flow behavior is sometimes called "shear-thinning." 

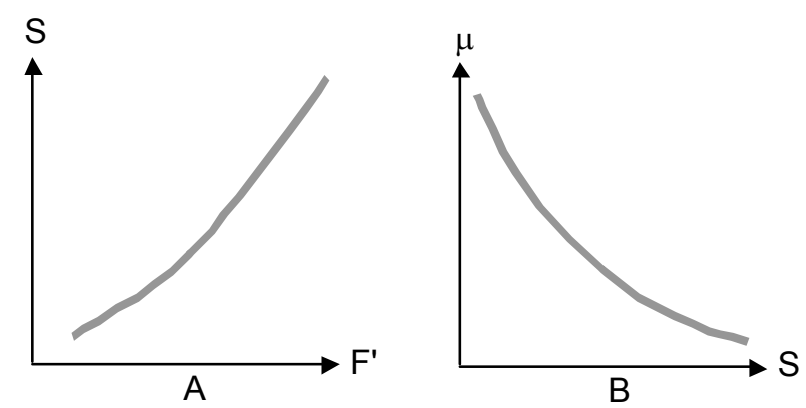

Dilatant: Increasing viscosity with an increase in shear rate characterizes the dilatant fluid; see the figure below. Although rarer than pseudoplasticity, dilatancy is frequently observed in fluids containing high levels of deflocculated solids, such as clay slurries, candy compounds, corn starch in water, and sand/water mixtures. Dilatancy is also referred to as "shear-thickening" flow behavior.
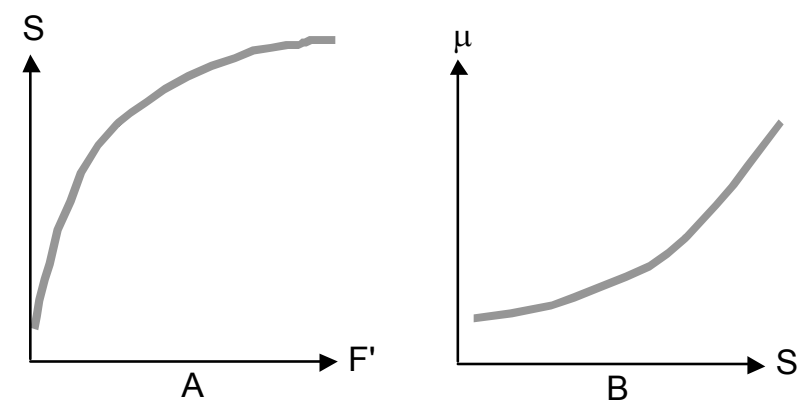

Plastic: This type of fluid will behave as a solid under static conditions. A certain amount of force must be applied to the fluid before any flow is induced; this force is called the "yield value." Tomato catsup is a good example of this type fluid; its yield value will often make it refuse to pour from the bottle until the bottle is shaken or struck, allowing the catsup to gush freely. Once the yield value is exceeded and flow begins, plastic fluids may display Newtonian, pseudoplastic, or dilatant flow characteristics. See the following figure.
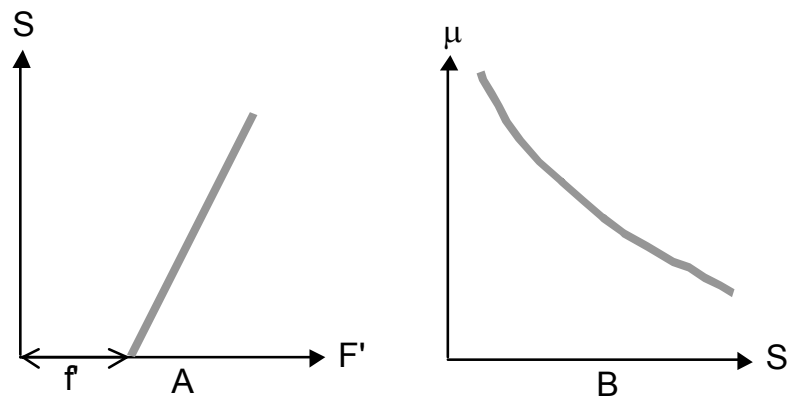


\section{A-1.3 Thixotropy and Rheopexy}

Some fluids display a change in viscosity with time under conditions of constant shear rate. There are two categories to consider:

Thixotropy: As shown in the figure below, a thixotropic fluid undergoes a decrease in viscosity with time as it is sheared at a constant rate.

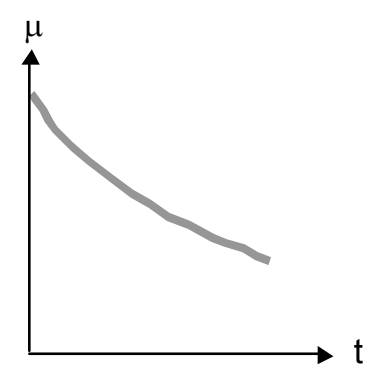

Rheopexy: This is essentially the opposite of thixotropic behavior, in that the fluid's viscosity increases with time as it is sheared at a constant rate. See the figure below.

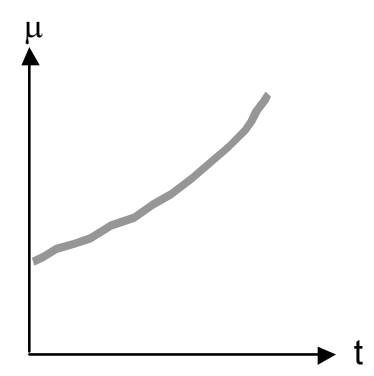

Both thixotropy and rheopexy may occur in combination with any of the previously discussed flow behaviors, or only at certain shear rates. The time element is extremely variable; under conditions of constant shear, some fluids will reach their final viscosity value in a few seconds, while others may take up to several days.

Rheopectic fluids are rarely encountered. Thixotropy, however, is frequently observed in materials such as greases, heavy printing inks, and paints.

When subjected to varying rates of shear, a thixotropic fluid will react as illustrated in the following figure. A plot of shear stress versus shear rate was made as the shear rate was increased to a certain value, then immediately decreased to the starting point. Note that the "up" and "down" curves do not coincide. This "hysteresis loop" is caused by the decrease in the fluid's viscosity with increasing time of shearing. Such effects may or may not be reversible; some thixotropic fluids, if allowed to stand undisturbed for a while, will regain their initial viscosity, while others never will. 


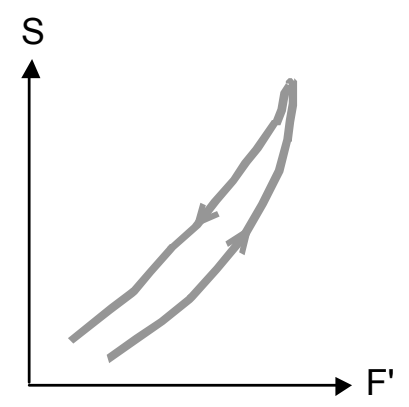

\section{A-1.4 Laminar and Turbulent Flow}

The very definition of viscosity implies the existence of what is called "laminar flow": the movement of one layer of fluid past another with no transfer of matter from one layer to the other. Viscosity is the friction between these layers.

Depending on a number of factors, there is a certain maximum speed at which one layer of fluid can move with relation to another, beyond which an actual transfer of mass occurs. This is called "turbulence." Molecules or larger particles jump from one layer to another and dissipate a substantial amount of energy in the process. The net result is that a larger energy input is required to maintain turbulent flow than laminar flow at a given velocity.

The increased energy input is manifested as an apparently greater shear stress than would be observed under laminar flow conditions at the same shear rate. This results in an erroneously high viscosity reading.

The point at which laminar flow evolves into turbulent flow depends on other factors besides the velocity at which the layers move. A material's viscosity and specific gravity as well as the geometry of the Viscometer spindle and sample container all influence the point at which the laminar to turbulent transition occurs.

Care should be taken to distinguish between turbulent flow conditions and dilatant flow behavior. In general, dilatant materials will show a steadily increasing viscosity with increasing shear rate; turbulent flow is characterized by a relatively sudden and substantial increase in viscosity above a certain shear rate. The material's flow behavior may be Newtonian or non-Newtonian below this point.

\section{A-1.5 What Affects the Rheological Property?}

Viscosity data often functions as a "window" through which other characteristics of a material may be observed. Viscosity is more easily measured than some of the properties that affect it, making it a valuable tool for material characterization.

\section{A-1.5.1 Temperature}

One of the most obvious factors that can have an effect on the rheological behavior of a material is temperature. Some materials are quite sensitive to temperature, and a relatively small variation will result in a significant change in viscosity. Others are relatively insensitive. Consideration of the effect of temperature on viscosity is essential in the evaluation of materials that will be subjected to temperature variations in use or processing, such as motor oils, greases, and hot-melt adhesives. 


\section{A-1.5.2 Shear Rate}

Non-Newtonian fluids tend to be the rule rather than the exception in the real world, making an appreciation of the effects of shear rate a necessity for anyone engaged in the practical application of rheological data. It would, for example, be disastrous to try to pump a dilatant fluid through a system, only to have it go solid inside the pump, bringing the whole process to an abrupt halt. While this is an extreme example, the importance of shear rate effects should not be underestimated.

When a material is to be subjected to a variety of shear rates in processing or use, it is essential to know its viscosity at the projected shear rates. If these are not known, an estimate should be made. Viscosity measurements should then be made at shear rates as close as possible to the estimated values.

\section{A-2 SETTLING TYPE AND RATE}

Perry's Chemical Engineers' Handbook (Perry and Green 1984) has a general discussion of various settling regimes that will be applicable to WM-187 solids/slurries included in this study:

At low concentrations, the type of settling encountered is called particulate settling. Regardless of their nature, particles are sufficiently far apart to settle freely. Faster settling particles may collide with slower settling ones and, if they do not cohere, continue downward at their own specific rate. Those that do cohere will form floccules of a larger diameter that will settle at a rate greater than that of individual particles.

There is a gradual transition from particulate settling into the zone-settling regime, where the particles are constrained to settle as a mass. The principal characteristic of this zone is that the settling rate of the mass, as observed in batch tests, will be a function of the solids concentration (for any particular condition of flocculation, particle density, etc.).

The solids concentration ultimately will reach a level at which particle descent is restrained not only by the hydrodynamic forces but partially by mechanical support from the particles below; therefore, the weight of the particles in mutual contact can influence the rate of sedimentation of those at lower levels. This compression, as it is termed, will result in further solids concentration because of compaction of the individual floccules and partial filling of the interfloc voids by the deformed floccules.

Accordingly, the rate of sedimentation in the compression regime is a function of both the solids concentration and the depth of pulp in this particular zone. As indicated in Figure A-1, granular, nonflocculent particles may reach their ultimate solids concentration without passing through this regime.

These types of settling are further discussed by Pierre and Ma (Pierre and Ma 1999). In this paper, Pierre and Ma describe two types of settling, accumulation sedimentation (which is analogous to particulate settling) and flocculation sedimentation (which is analogous to zone/compression settling). These types of sedimentation are illustrated in Figure A-2. 


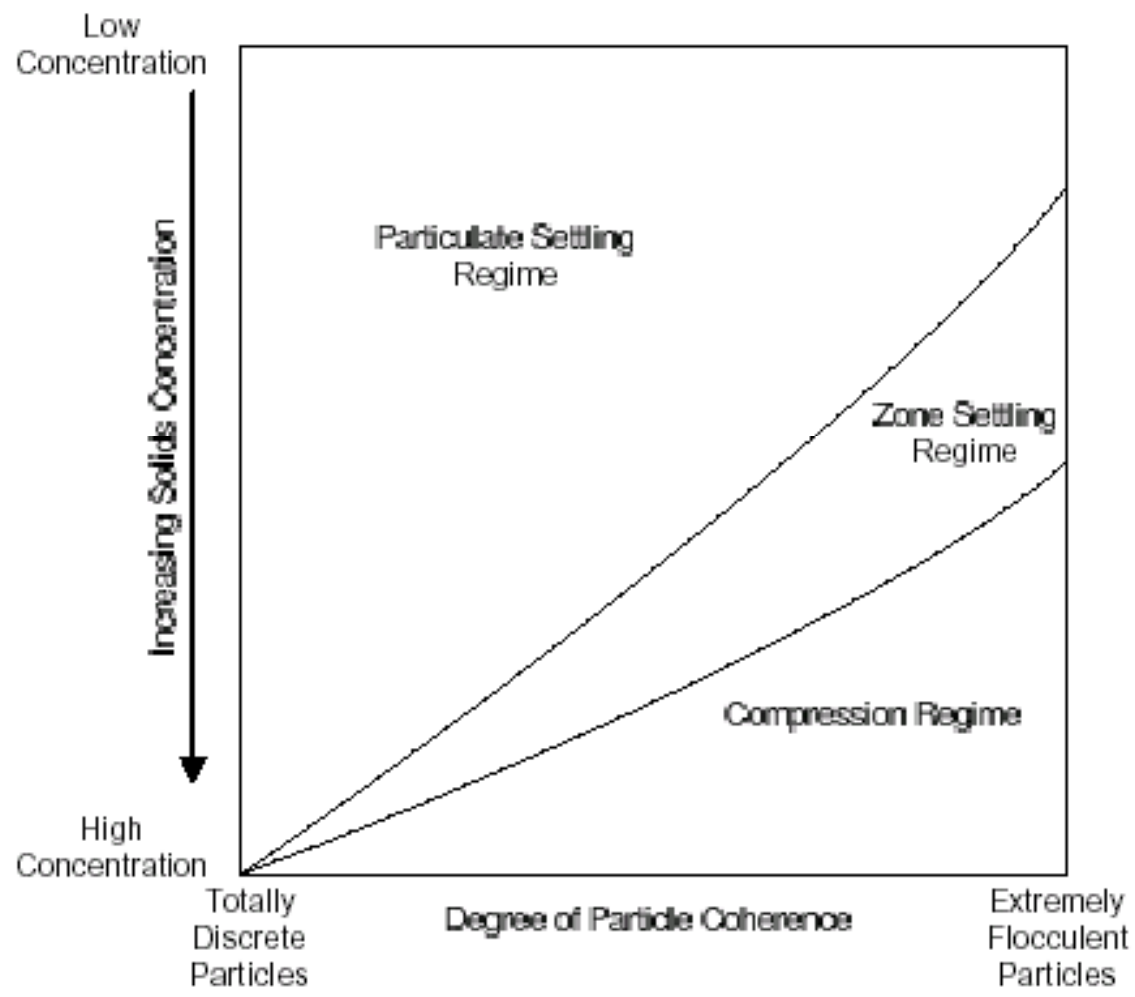

Figure A-1. Settling regimes.

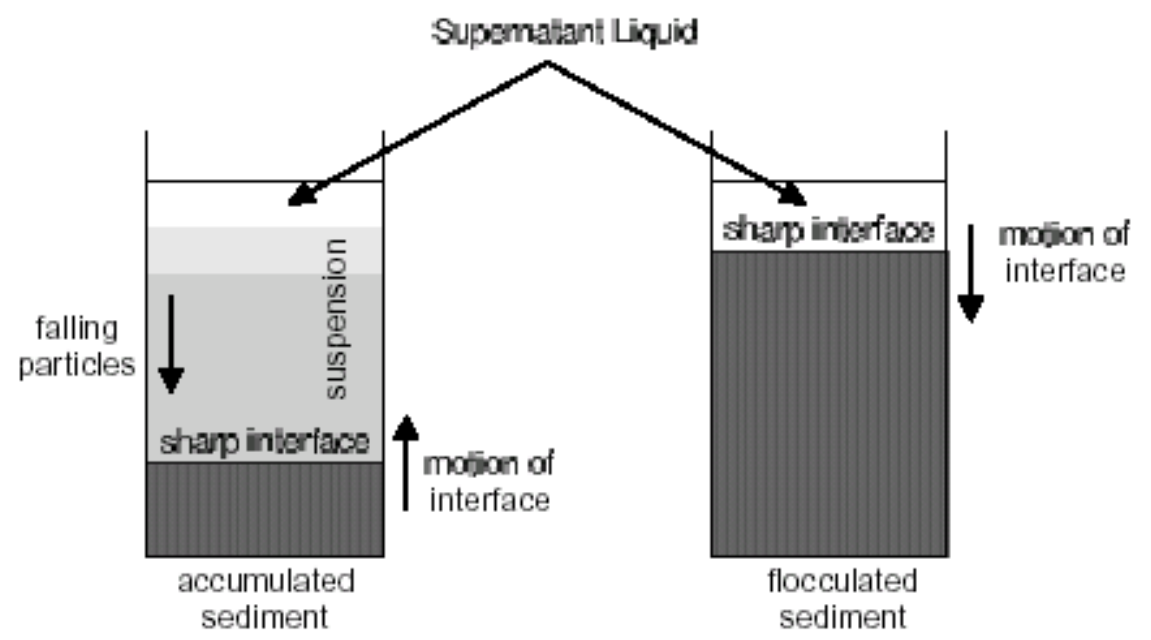

Figure A-2. Accumulation sedimentation and flocculation sedimentation.

\section{A-3 PARTICLE SETTLING VELOCITY}

The "Derivation of the Sediment Settling Velocity Equations" web page

(http://freespace.virgin.net/mark.davidson3/sediment/stokes/SETTLEV.html) provides a derivation of the settling velocity equations for a particle settling in the particulate settling regime. 


\section{A-3.1 Spherical particles}

Consider a simple spherical grain falling through a motionless fluid. The particle accelerates through the fluid until the immersed weight of the particle is balanced by the drag forces. At this point the particle maintains a constant velocity called the terminal velocity.

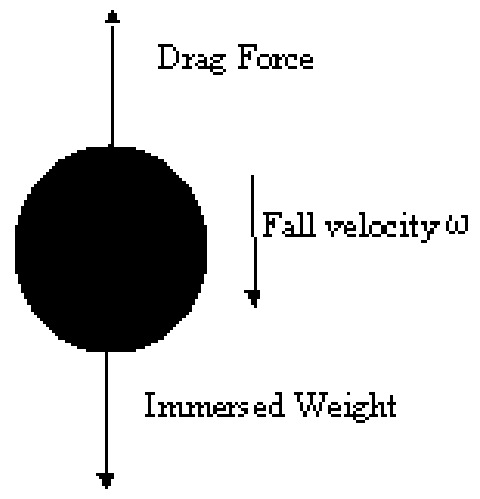

In cross-section the spherical grain is circular with a radius $r$ (or diameter $D$ ). If the particle falls a distance $d$ through the water column, it displaces a cylindrical volume of water.

The kinetic energy required to displace this volume of water is given by:

$E=\frac{1}{2} m \varpi^{2}$

From Figure 2, the mass of water displaced is given by:

$m=\frac{d \pi D^{2}}{4} \rho_{l}$

where $\rho_{1}$ is the density of the fluid. Substituting equation (1) into equation (2):

$E=\frac{1}{2} \frac{d \pi D^{2}}{4} \rho_{l} \varpi^{2}$.

Energy is defined as a force times a distance $(E=F d)$. Therefore, we can write the drag force as:

$E=\frac{1}{2} \frac{d \pi D^{2}}{4} \rho_{l} \varpi^{2}=F_{D} d \quad$ Thus: $F_{D}=\frac{\pi D^{2} \rho_{l} \varpi^{2}}{8}$.

Equation (4) is the theoretical drag force on a sphere. However, this equation does not take into account the viscosity of the fluid or the dynamics of the flow around the sphere. So for a more practical solution, which includes these factors, we must introduce a drag coefficient $C_{D}$. Thus, allowing for fluid viscosity and the flow dynamics equation (4) becomes:

$F_{D}=\frac{C_{D} \pi D^{2} \rho_{l} \varpi^{2}}{8}$ 
The flow regime around the object is best described in terms of the dimensionless Reynold's number where:

$\mathrm{Re}=\frac{\varpi D \rho_{l}}{\mu}$.

Here $\mu$ is the coefficient of molecular viscosity. For $R e<1$ there is laminar flow around the sphere. This is called the viscous regime. Here the drag coefficient is inversely related to the Reynold's number where:

$C_{D}=\frac{24}{\mathrm{Re}}$

Substituting equation (7) into (5) gives:

$F_{D}=\frac{3 \pi D^{2} \rho_{l} \varpi^{2}}{\operatorname{Re}}=3 \mu \pi D \varpi$

When the particle reaches its terminal velocity, this drag force is balanced by the immersed weight of the particle. The immersed weight is given by:

$I=$ weight of particle $(W)$ - fluid upthrust $(U)$.

The weight of the particle is the product of its mass and the acceleration due to gravity $(g)$ (Newton's second law of motion, $F=m a$ ), and the mass is equal to the volume times the density of the particle, therefore:

$$
\begin{aligned}
& m=\frac{4}{3} \pi r^{3} \rho_{s}=\frac{4}{3} \pi \frac{D^{3}}{8} \rho_{s} \\
& W=\frac{4}{3} \pi \frac{D^{3}}{8} \rho_{s} g .
\end{aligned}
$$

Here $\rho_{\mathrm{s}}$ is the density of the particle.

The upthrust is equal to the weight of water that the particle displaces (Archimedes' Law). Thus;

$U=\frac{4}{3} \pi \frac{D^{3}}{8} \rho_{l} g$

Combining equations (9), (11), and (12) gives the immersed weight of the particle as:

$I=\frac{4}{3} \pi \frac{D^{3}}{8} g\left(\rho_{s}-\rho_{l}\right)$ 
Within the viscous regime $(\boldsymbol{R} \boldsymbol{e}<1)$, the terminal fall velocity of the particle may be found by combining equations $(8)$ and (13) $\left\{\right.$ drag force $\left(F_{D}\right)=$ immersed weight $\left.(I)\right\}$ :

$3 \mu \pi D \varpi=\frac{4}{3} \pi \frac{D^{3}}{8} g\left(\rho_{s}-\rho_{l}\right)$

Rearranging the above in terms of the fall velocity gives Stoke's Law:

$\varpi=\frac{D^{2} g\left(\rho_{s}-\rho_{l}\right)}{18 \mu}$.

For a given fluid density, particle density, and fluid viscosity equation (15) simplifies to:

$\omega=c D^{2}$

where $\mathrm{c}$ is a constant of proportionality. For quartz particles in water, $\mathrm{c}$ has the following values for a salinity of 35 ppt:

\begin{tabular}{|l|c|}
\hline $\begin{array}{c}\text { Temperature } \\
{ }^{\circ} \mathrm{C}\end{array}$ & $\begin{array}{c}\mathrm{c} \\
\left(\mathrm{cm}^{-1} \mathrm{~s}^{-1}\right)\end{array}$ \\
\hline 20 & 8975 \\
\hline 10 & 6880 \\
\hline 5 & 5920 \\
\hline
\end{tabular}

At higher Reynold's numbers $(>1)$, flow separation occurs, turbulent eddies are formed (the turbulent regime) and the drag coefficient deviates from equation (7). For Reynold's number above $10^{3}$, the drag coefficient reaches a constant value (approx. 0.4 for spheres). Equations (5) and (13) imply that if $C_{D}$ is constant then $\varpi \propto \sqrt{D}$. This is known as the Impact Law.

\section{A-3.2 Nonspherical Particles}

The drag coefficient will vary significantly with the shape of the particle. The shape of an irregular, nonspherical particle is often characterized by the Corey shape factor;

$$
C=\frac{D_{c}}{\sqrt{D_{a} D_{b}}}
$$

where $D_{a}, D_{b}$ and $D_{c}$ are the major, minor, and intermediate axes of the particle. The theoretical settling velocity for the settling of discs (similar to clay minerals) within the viscous regime $(R e<1)$ is:

$\omega_{s}=\frac{1}{2 k \mu} \frac{\bar{D}}{D_{c}}\left(\rho_{s}-\rho_{l}\right) g \bar{D}^{2}$

where

$\bar{D}=\frac{1}{2}\left(D_{a}+D_{b}\right)$ 
The coefficient $k$ has a theoretical value of 5.1 for broadside settling of infinitely thin particles. The best fit to natural data; however, gives $k=9$. Thus, for spherical particles $\left(\bar{D}=D_{c}\right)$, equation (18) is equivalent to equation (15). For turbulent conditions, the particle will oscillate while falling and even falls along a zigzag path under extreme conditions.

Due to the complex effects of the flow field around the particle, particle shape, and the irregular trajectories of the particles, empirical relationships often are used to describe the fall velocity of particles such as the tank farm facility solids/sludge pertinent to this study. Baba and Komar (1981) found a good empirical fit to their data for sand grains with different shape factors. A good fit to their data was given by:

$\omega_{n}=0.977 \omega_{s}^{0.913}$

where $\omega_{n}$ is settling velocity of the natural grains and $\omega_{s}$ is given by equation (15) taking $D=D_{b}$.

\section{A-4 CAKE FILTRATION}

\section{A-4.1 Cake Filtration Description}

Cake (or dead-end) filtration is a process in which suspended solids are separated a suspension via the permeation of suspending medium through porous membrane. Permeation of the filtrate is driven by gravity or by the application of a vacuum, or pneumatic or hydrostatic pressure. As permeation of the filtrate takes place, a filter cake builds up on the porous membrane. The structure/permeability of the filter cake is often the rate limiting factor for permeate flux in the process.

Cake filtration, as a solid/liquid separation process, is widely used in both the chemical and process industries. Despite its simplicity and long history of development, filtration is not easily described like some other transport unit operations, such as heat transfer, mass transfer, fluid mixing, and fluid transport. For these operations, properties are well defined and predictable. However, in the case of filtration, solids can have widely varying properties in addition to size distribution, that depend on conditioning and processing. For example, particle size and shape can change with treatment, aging, flocculation, $\mathrm{pH}$, and pumping. For these reasons, filtration and other solid/sludge waste processing technologies are difficult to understand, and are very underrated engineering disciplines.

Filtration performance (or permeate flux) is affected by a series of parameters, some of which are related to the suspended solid or flocculent properties of the sludge feed. An optimization of the filtration operation implies a systematic analysis of each important parameter. Noted "Father of filtration," F. M. Tiller of the University of Houston, stated that: "Experiment is a necessary part of any filtration design procedure, and average filtration resistances are noticeably affected by sludge concentration, rate of change of applied stresses, and internal shear forces. Even under carefully controlled conditions, it is difficult to measure resistance within $\pm 10 \%$. Caution and judgment are essential to interpret and make use of filtration data correctly" (Tiller and Crump 1997).

The most commonly used analysis for measuring the de-liquoring rates of sludges is the specific resistance to filtration (SRF) test (Water Environmental Research Foundation 1993; Geankoplis 1978; Perry and Chilton 1997; Christensen and Dick 1985). The SRF test is a laboratory procedure that measures the rates at which sludges will de-liquor under pressure or vacuum. The test is based on an analysis of pressure drop for flow through a porous medium (i.e., the filter cake). The theoretical description of filtration identifies the SRF as the proportionality factor between the amount of cake solids 
deposited in the cake and the total flow resistance of the cake. As expected the SRF, as briefly derived below, is related to cake permeability.

In cake filtration, the sludge to be treated is dispersed in a liquid medium, whereafter it is brought into contact with a filter medium with openings smaller than the diameters of most of the particles present in the sludge. The cake filtration behaviors are strongly affected by the properties of the filter cake formed by the accumulation of the particles and/or macromolecules on the surface of the filter medium. A cake is formed above the filter medium, which subsequently provides filtering, and the cake thickness increases with time. The cake structure may undergo changes as a result of cake compression caused by the sludge flow. In turn, this change in cake structure may dynamically affect filtration performance (Alles et al. 1998).

If the cake from the sludge filtration contains a wide range of particle sizes there will be a tendency for the cake to behave like one composed of its finer particles rather than one composed of its coarser particles, because the small particles migrate into the cake creating a denser cake. Sludges containing fine particles are extremely difficult to separate because they form highly compressible cakes. While the majority of the particles are retained to form a cake, a small amount of finer ones may penetrate into the cake. The permeability of a cake depends on the extent of the compression to which it is subjected, as well as the amount of fines retained within the cake. Fine particle retention can contribute significantly to the decrease of cake permeability and may significant effect the performance of cake filtration even if the amounts of fines are small (Tien et al. 1997).

\section{A-4.2 Cake Filtration Theory}

Liquid flow through porous media is the common characteristic of filtration process. As the waste slurry liquid passes around the surface of the solid particles trapped on a porous medium (a frit or the filter cake), friction between the liquid and the solid particles being collected on the filtration medium creates a pressure drop over the length of the medium, resulting in a reduction in flow through the medium. A basic flow equation - Darcy's Law (Darcy 1856) - provides a fundamental relation between this pressure drop and the liquid flow through both the cake and the filter medium.

$$
\mathrm{q}=\mathrm{dV} / \mathrm{d} t=k(\mathrm{~A} p) /(\mu L)
$$

or $\quad q=\mathrm{d} v / \mathrm{d} t=p /(\mu \mathrm{R})$

where $\mathrm{q}$ is the volumetric flow rate of the filtrate; $\mathrm{V}$, the total liquid filtrate volume collected at time, $t ; t$, the time since the start of filtration; $k$, the cake permeability (assumed constant here, but not so in reality); A, the cross-sectional area of the collected solid (also equal to the surface area of the filter medium); $p$, the pressure drop across the collected cake and the filter medium; $L$, the porous cake depth; $\mu$, the liquid filtrate viscosity; $R$, the sum of the resistance of the cake, $R_{c}$, and the resistance of the filter medium, $R_{m}$, ( also equal to $L / k$ ); $v$, the filtrate volume collected at time, $\mathrm{t}$ (i.e., $\mathrm{V}$ ) per unit area of filtration (i.e., A).

Both the solid filter cake resting on a filter medium and the medium itself contribute to the various resistances during constant pressure filtration (Figure A-3). Starting with equation (2) and using the defined resistance identities of equations (4) and (5) below, the following resistance model of cake filtration is obtainable:

$$
\begin{aligned}
& \mathrm{dv} / \mathrm{dt}=\mathrm{p} / \mu(\alpha \mathrm{CV} / \mathrm{A}+\mathrm{Rm})=\mathrm{p} / \mu(\mathrm{Rc}+\mathrm{Rm}) \\
& \text { where } \mathrm{R}=\mathrm{Rc}+\mathrm{Rm} \text { and } \\
& \mathrm{R}_{\mathrm{c}}=(\alpha \mathrm{C} \mathrm{V}) / \mathrm{A}=\alpha_{\mathrm{av}} \mathrm{C} v=\alpha_{\mathrm{av}} \mathrm{W}_{\mathrm{c}}
\end{aligned}
$$




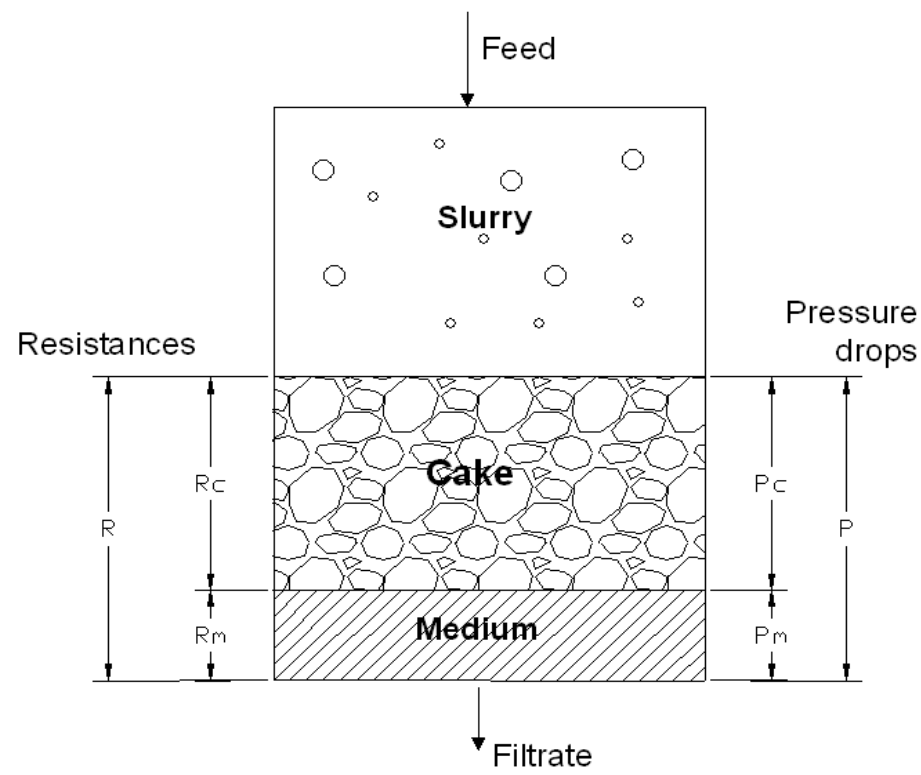

Figure A-3. Relationship between the applied filter pressure and the filter cake/medium resistance.

$\alpha$ is the specific resistance of filtration (SRF) for the filter cake in units of $\mathrm{m} / \mathrm{kg}$; $\mathrm{C}$, the slurry concentration expressed as the mass of dry cake per unit volume of filtrate $\left(\mathrm{kg} / \mathrm{m}^{3}\right) ; \mathrm{R}_{\mathrm{c}}$ and $\mathrm{R}_{\mathrm{m}}$ are the cake and filter medium resistance, respectively $(1 / \mathrm{m}) ; \mathrm{w}_{\mathrm{c}}=\mathrm{C} v$, the total mass of dry cake solids per unit area of filter surface $(\mathrm{kg} / \mathrm{m} 2)$.

Inspection of equation (3) reveals a linear relationship between the rate of filtration and the total volume of filtrate collected. In order to obtain this $v$ vs. $t$ relationship for constant pressure filtration, Tiller (Tiller 1990; Tiller 1983) recommends using the following equations, as derived from equations (3):

$$
\begin{aligned}
& \mathrm{pdt} / \mu \mathrm{dv}=\mathrm{p} /(\mu \mathrm{q})=\alpha \mathrm{av} \mathrm{C} \mathrm{v}+\mathrm{Rm} \\
& \mathrm{pt} / \mu \mathrm{v}=\mathrm{p} /(\mu \mathrm{qav})=(\alpha \mathrm{av} / 2) \mathrm{C} \mathrm{v}+\mathrm{Rm}
\end{aligned}
$$

since $\mathrm{R}_{\mathrm{c}}=\alpha_{\mathrm{av}} \mathrm{C} v$

$$
\begin{aligned}
& \mathrm{R}=\mathrm{p} / \mu \mathrm{q}=\mathrm{Rc}+\mathrm{Rm} \\
& \mathrm{p} /(\mu \mathrm{qav})=1 / 2 \mathrm{Rc}+\mathrm{Rm}
\end{aligned}
$$

where $q=\mathrm{d} v / \mathrm{d} t$, the instantaneous rate, and $q_{\mathrm{av}}=v / t$, the average rate over the entire filtration cycle. Since equations (6) and (7) are of linear form, a plot of $1 / q$ versus $v$ provides a curve whose slope is related directly proportional to the particular cake SRF (or $\alpha_{\mathrm{av}}$ ), as well as a Y intercept that is representative of the resistance of the filter medium. As will be discussed in detail later, graphical interpretations of equations (6) and (7), designated as filtration models I and II by Teller, are provided in Figure A-4. However, experimental data are frequently not precise, and undoubtedly any points on both the $p / \mu q$ and $p / \mu q_{\text {av }}$ plots will probably deviate from straight lines. Since the slope of the equation (6) is twice that of the equation (7), Tiller (Tiller 1990; Tiller 1983) advised plotting both lines to reach a compromise on both the slopes and intercepts. 


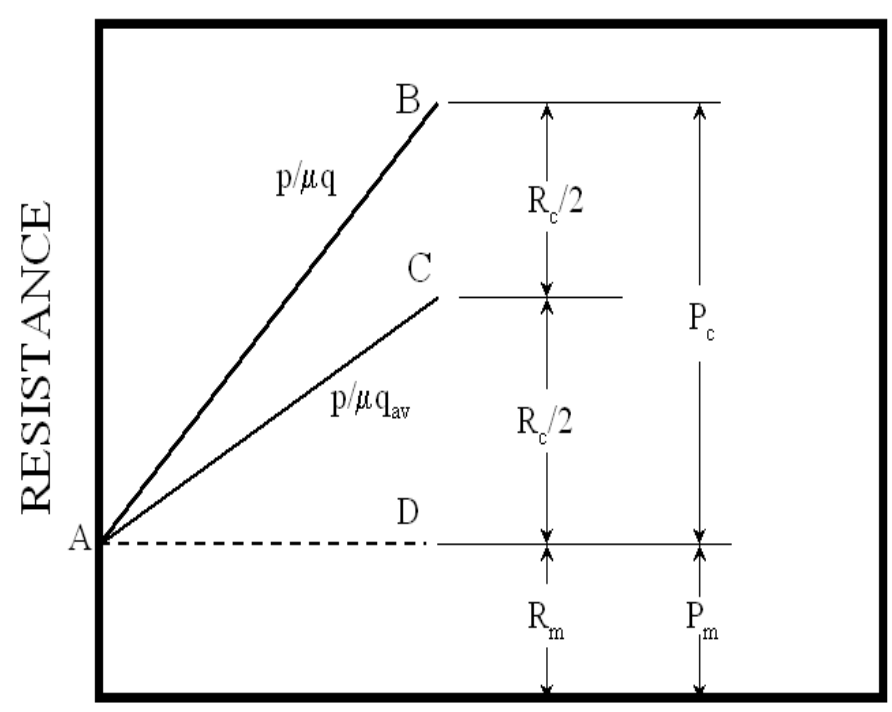

$\mathrm{w}_{\mathrm{c}}(\mathrm{Cv})$

Figure A-4. Overall filtration resistance plot.

It should be noted that the filtration resistance $\alpha$ (or SRF) is a measure of the filterability of the suspensions. The greater the $\alpha$, the greater the time required for filtration (smaller filtration rate) for a given pressure drop across the filter. To determine the effect of a change in the applied filtration pressure for a particular sludge, at least three different constant pressure tests are to be conducted, and for each case, $\alpha$ was determined. Based on reference (Sperry 1916), plotting the natural $\log$ of $\alpha(\operatorname{Ln} \alpha)$ versus $\operatorname{Ln} p$ also results in an approximate straight line. The straight line indicates that equation (10) as proposed by Sperry, and given below, is consistent with numerous documented experiments with sludge cake filtration. Sperry's results indicate that $\alpha_{0}$ and $\mathrm{n}$ are empirical constants for a particular sludge, and the slope of the line, as generated by the Ln-Ln plot described above, is the compressibility of the cake, $n$. This value varies from 0 for a rigid, incompressible cake, to $n>1$ for super-compactable cake. Values of $n$ used to classify the cake compressibility are listed in Table A-1.

$\alpha_{\mathrm{av}}=\alpha_{0} *(p)^{\mathrm{n}}$

Table A-1. Cake compressibility classifications.

\begin{tabular}{|l|l|}
\hline Incompressible & $\mathrm{n}=0$ \\
\hline Moderately compressible & $\mathrm{n} \sim 0.5-0.6$ \\
\hline Highly compressible & $\mathrm{n} \sim 0.7-0.8$ \\
\hline Super compressible & $\mathrm{n}>1$ \\
\hline
\end{tabular}

Cake filtration and cake compressibility are influenced by numerous factors such as the particle size, particle size distribution, the particle shape, and their aggregation (Perry and Chilton 1997; Rushton et al. 1996; Wakeman et al. 1989; Wakeman et al. 1991). In the case of low or moderate cake 
compressibility, higher filtration pressure leads to higher filtration rates - a desired result. On the other hand, highly compressible cakes can lead to greatly increased filtration times as a consequence of pressure induced pore constriction. Generally speaking, for the very compressible filter cake, high pressures and long filtration times are often necessary. 
A-18 


\section{Appendix B \\ Previous History and Prior Data}


B-2 


\section{B-1 PREVIOUS HISTORY AND PRIOR DATA}

SBW solids samples and previous solids surrogates have been compared in the past. In 2000, physical property data was collected on the 1999 Light- Duty Utility Arm (LDUA) WM-182 sample. Physical property data collected included sludge density measurements, sludge viscosity measurements, solids settling type and rate, and solids particle size distribution. The WM-182 sludge physical property data collected was used to select an appropriate sludge surrogate for the FY-2000 Tank Farm Closure "Wash and Pump" and "Slurry Pipe Flow" mock-up tests. The waste surrogate selected was kaolin clay (pigmented with iron-oxide) flocculated by the addition of aluminum sulfate. In addition, calcium sulfate dihyrdate (hydrated gypsum) provides effective adjustment of settling rate and particle size of the waste surrogate (Poloski and Wilcox 2000). A summary of the WM-182 sludge and surrogate sludge physical property data collected is summarized in the following sections.

\section{B-2 SLUDGE DENSITY MEASUREMENTS}

The average density of the 1999 LDUA WM-182 sludge sample was reported as $1.25 \mathrm{~g} / \mathrm{mL}$ by Poloski (Poloski 2001). The kaolin clay/aluminum sulfate surrogate described in "Surrogate Sludge for Tank Farm Closure Mockups" (Poloski and Wilcox 2000) had an expected sludge density in the range of $1.16-1.48 \mathrm{~g} / \mathrm{mL}$, which encompasses the tank farm sludge density of $1.25 \mathrm{~g} / \mathrm{mL}$.

\section{B-3 SLUDGE VISCOSITY MEASUREMENTS}

Poloski reported the viscosity of the 1999 LDUA WM-182 sludge sample at shear rates ranging from 0 to $50 \mathrm{~s}^{-1}$ (Poloski and Wilcox 2001). The viscosity of undiluted WM-182 sludge sample was approximately $200 \mathrm{cP}$ at a shear rate of $35 \mathrm{~s}^{-1}$ and the viscosity of diluted WM-182 sludge sample was approximately $50 \mathrm{cP}$ at a shear rate of $35 \mathrm{~s}^{-1}$. The kaolin clay/aluminum sulfate surrogate described in "Surrogate Sludge for Tank Farm Closure Mockups" (Poloski and Wilcox 2000) had a viscosity higher than that of the actual waste, approximately $1600 \mathrm{cP}$ at a shear rate of $35 \mathrm{~s}^{-1}$ (see Figure B-1).

\section{B-4 SETTLING TYPE AND RATE}

Poloski reported the settling type of the 1999 LDUA WM-182 sludge sample as slow flocculation sedimentation. Figure B-2 is a plot of the WM-182 solids settling velocity data collected (Poloski and Wilcox 2001). Figure B-3 illustrates the kaolin/alum waste surrogate settling rate in comparison to the WM-182 sludge settling rate (Poloski and Wilcox 2000). The kaolin/alum surrogate displayed a slower settling rate than the WM-182 sludge. In the tank farm closure mockup, a slower waste surrogate settling rate would result in a greater amount of solids being transferred from the mockup than in actual tank farm closure operations. Therefore, various quantities of hydrated gypsum, which acts as a settling agent, were added to the kaolin/alum waste surrogate to increase the waste surrogate settling rate. Figure B-4 illustrates the kaolin/alum/gypsum waste surrogate settling rate in comparison to the WM-182 sludge settling rate (Poloski and Wilcox 2000). 


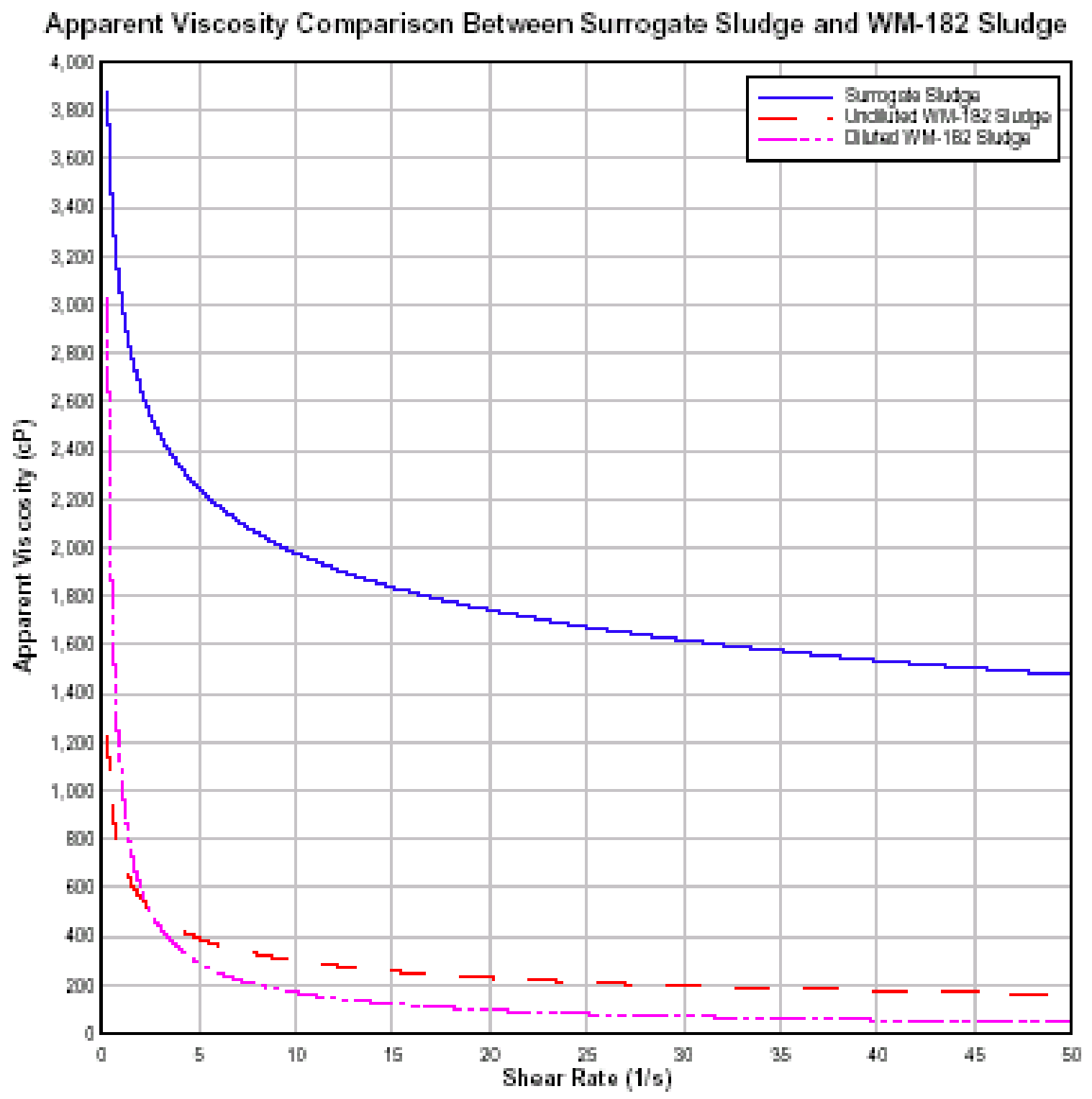

Figure B-1. Apparent viscosity comparison between surrogate sludge developed in 2000 and actual WM-182 sludge. 


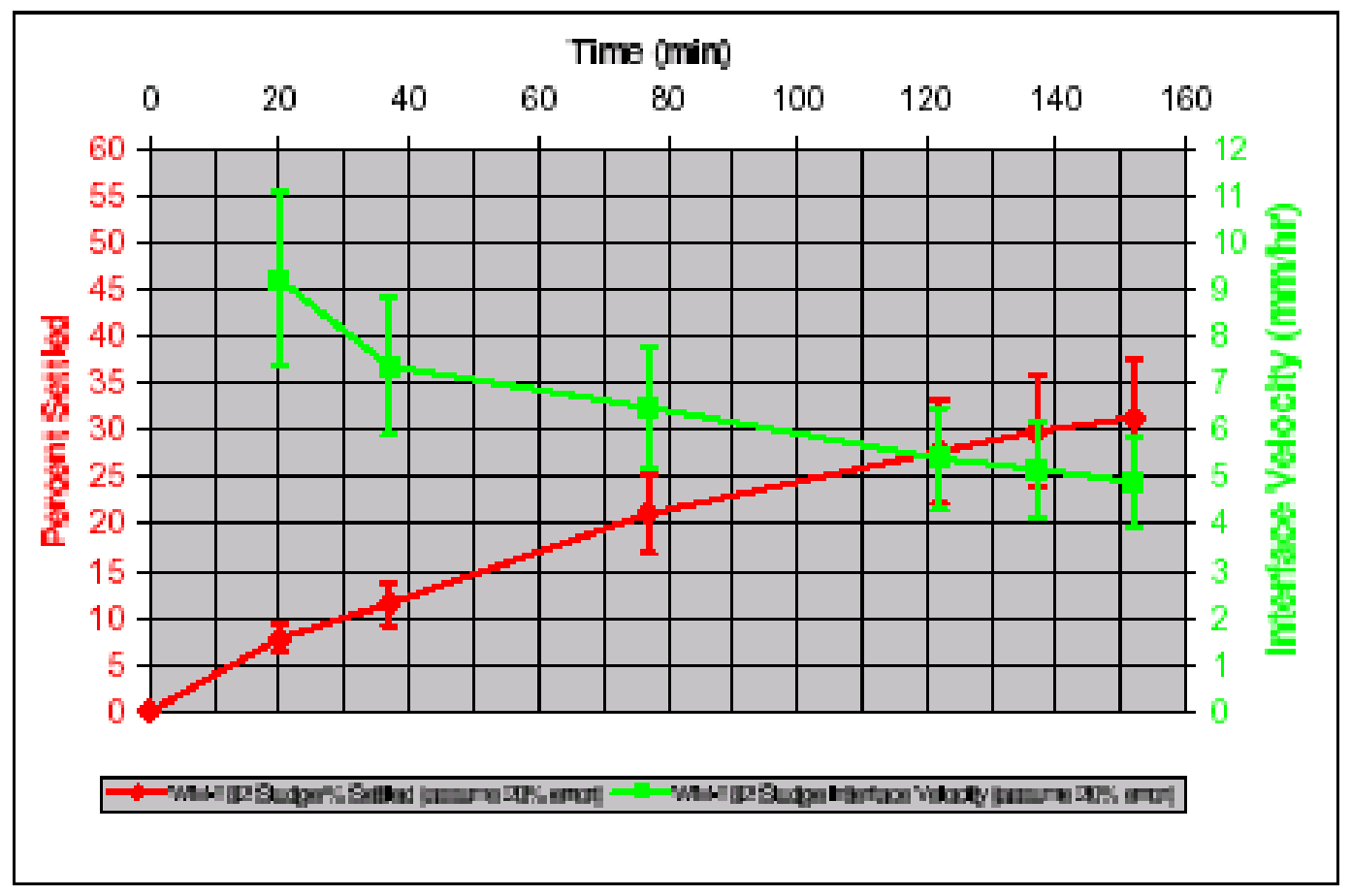

Figure B-2. WM-182 sludge compression settling rate.

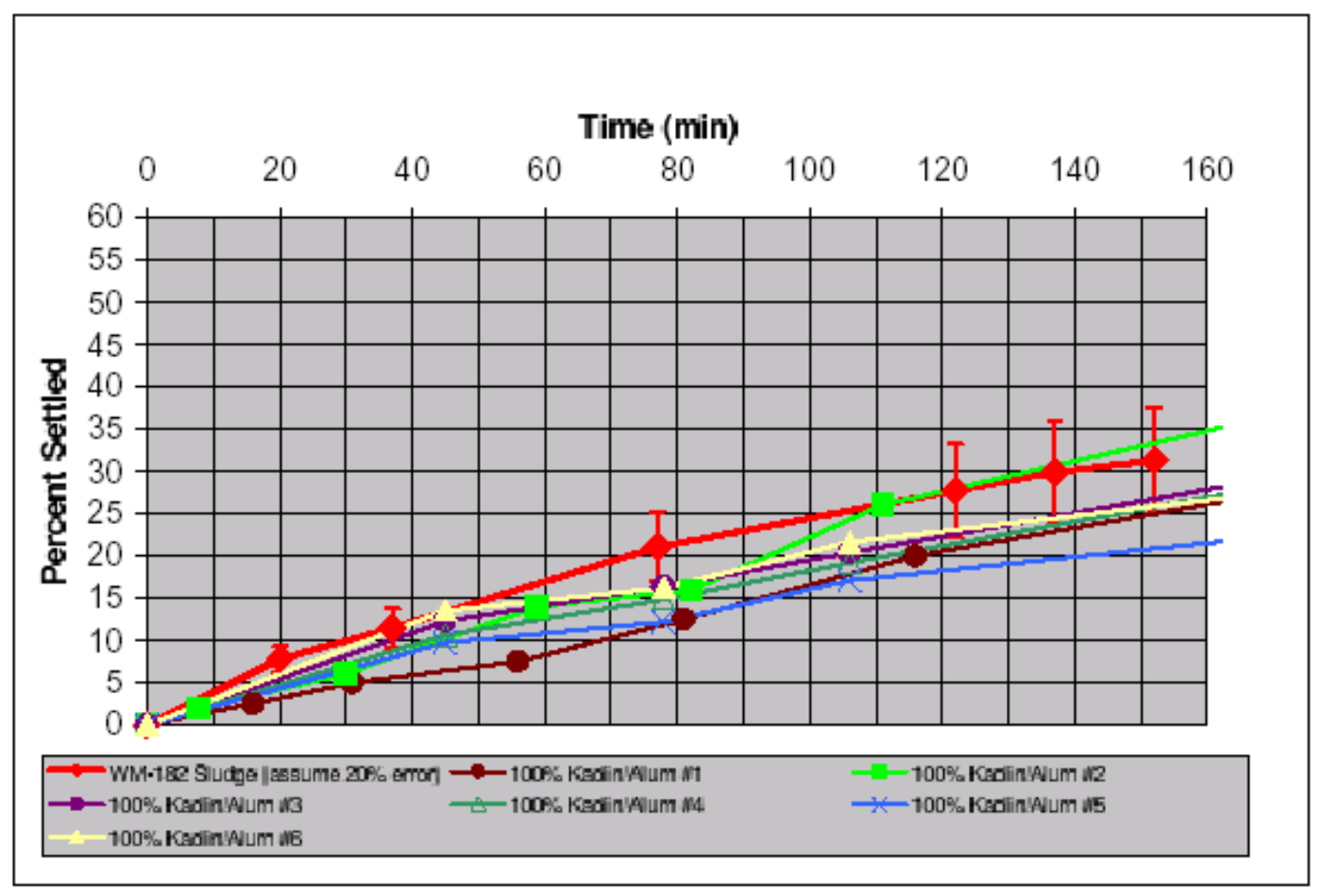

Figure B-3. Kaolin/alum compression settling rate. 


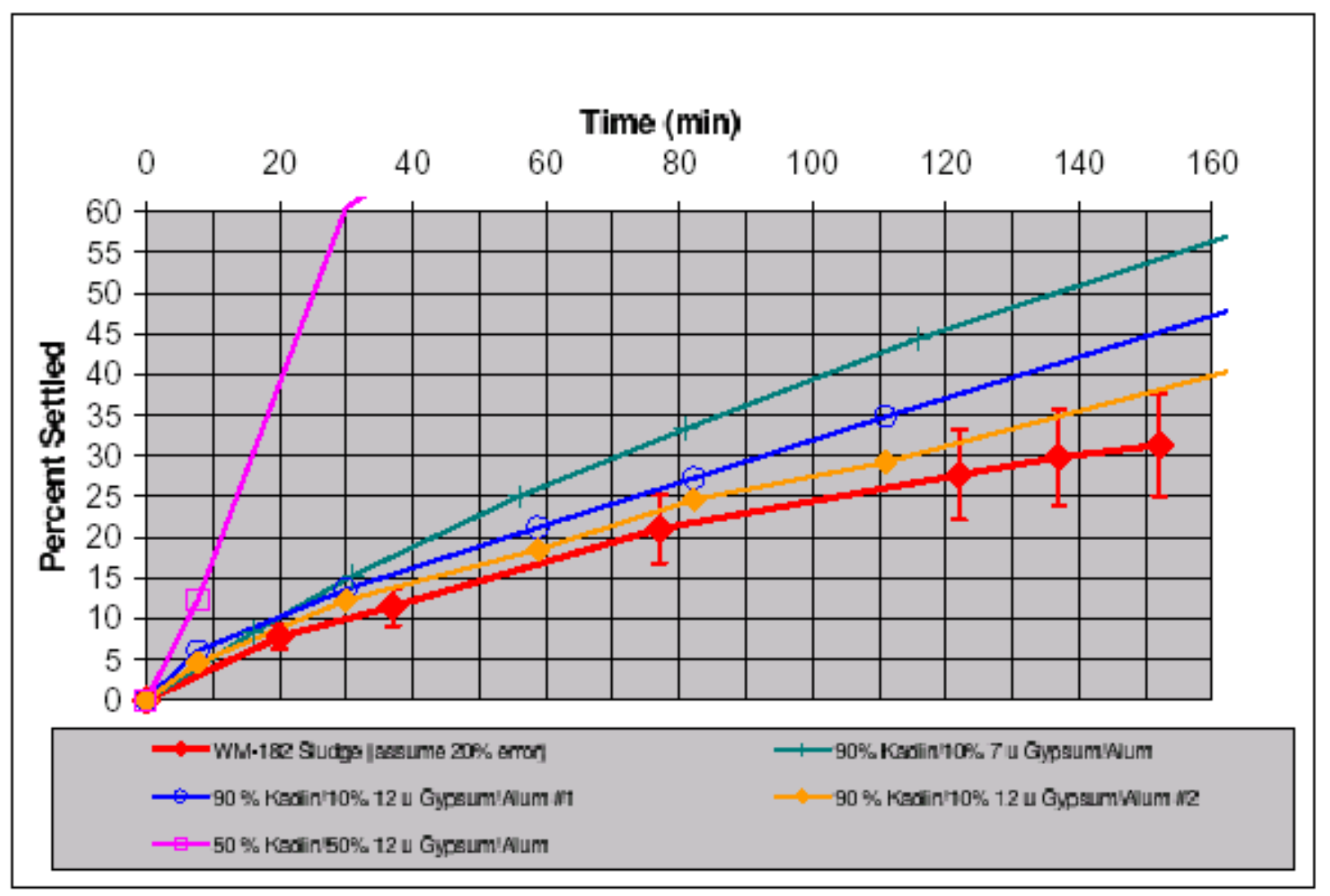

Figure B-4. Kaolin/alum/gypsum compression settling rate.

\section{B-5 PARTICLE SIZE DISTRIBUTION}

Sonicated and unsonicated WM-182 and WM-183 sludge particle size distributions were reported by Poloski and Wilcox. Figure B-5 summarizes the WM-182 and WM-183 particle size distribution data (Poloski and Wilcox 2000). Figure B-6 is a particle size distribution comparison of sonicated WM-182 and WM-183 samples with the kaolin/alum/iron oxide surrogate sludge (Poloski and Wilcox 2000). 


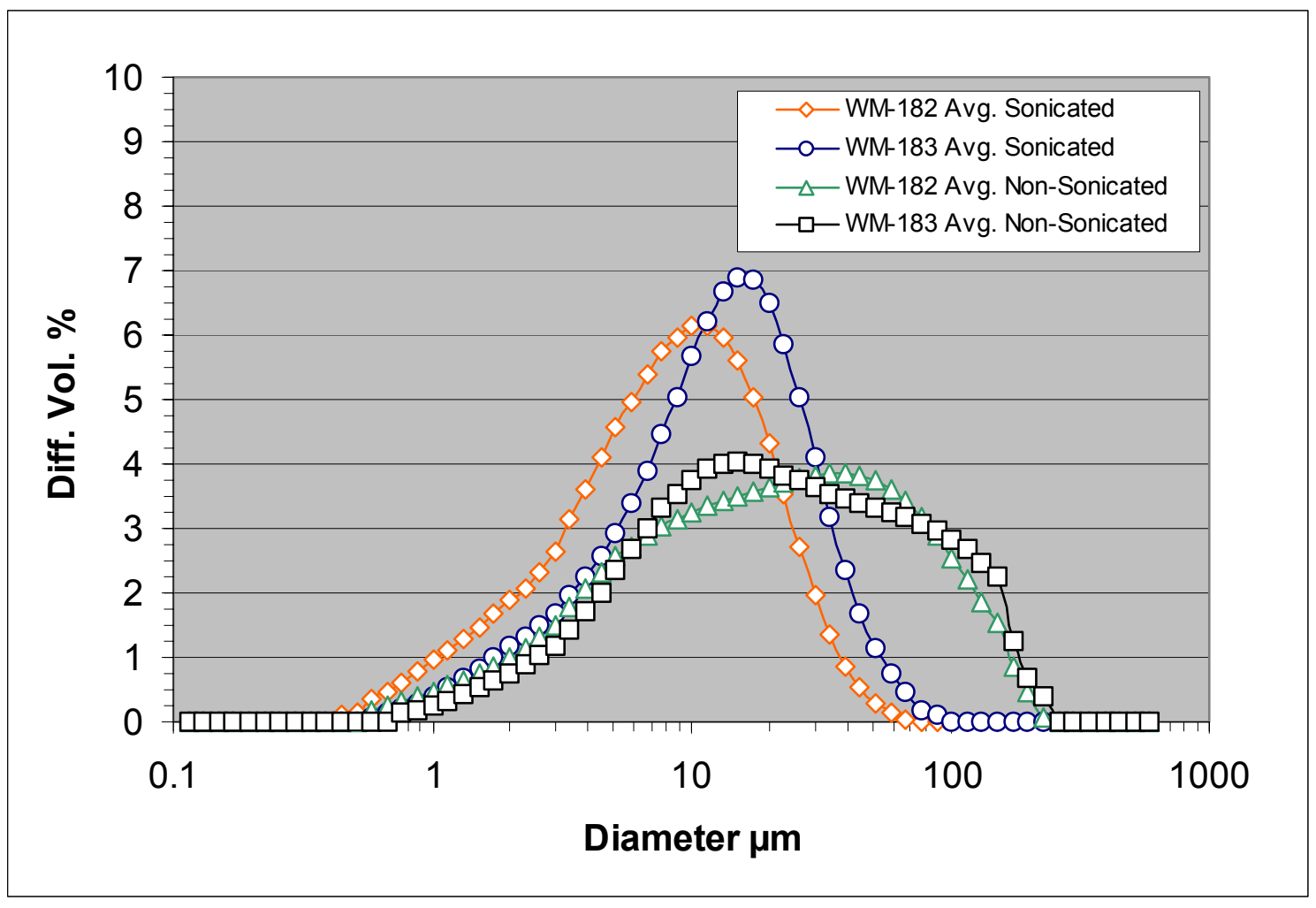

Figure B-5. WM-182 and WM-183 particle size distributions.

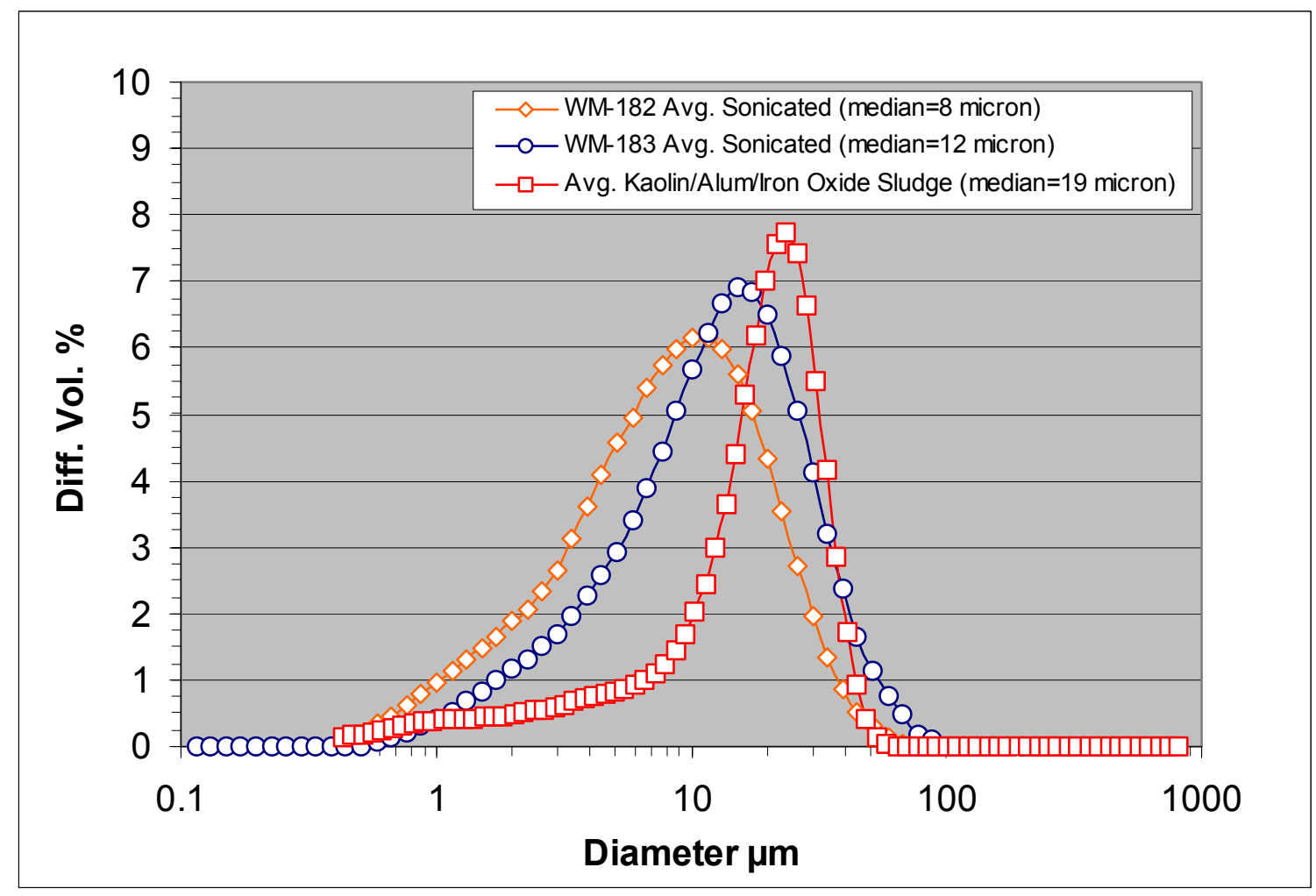

Figure B-6. WM-182, WM-183, and kaolin/alum/iron oxide surrogate sludge PSD comparison. 
B-8 


\section{Appendix C}

Surrogate Composition 


$$
\text { C-2 }
$$




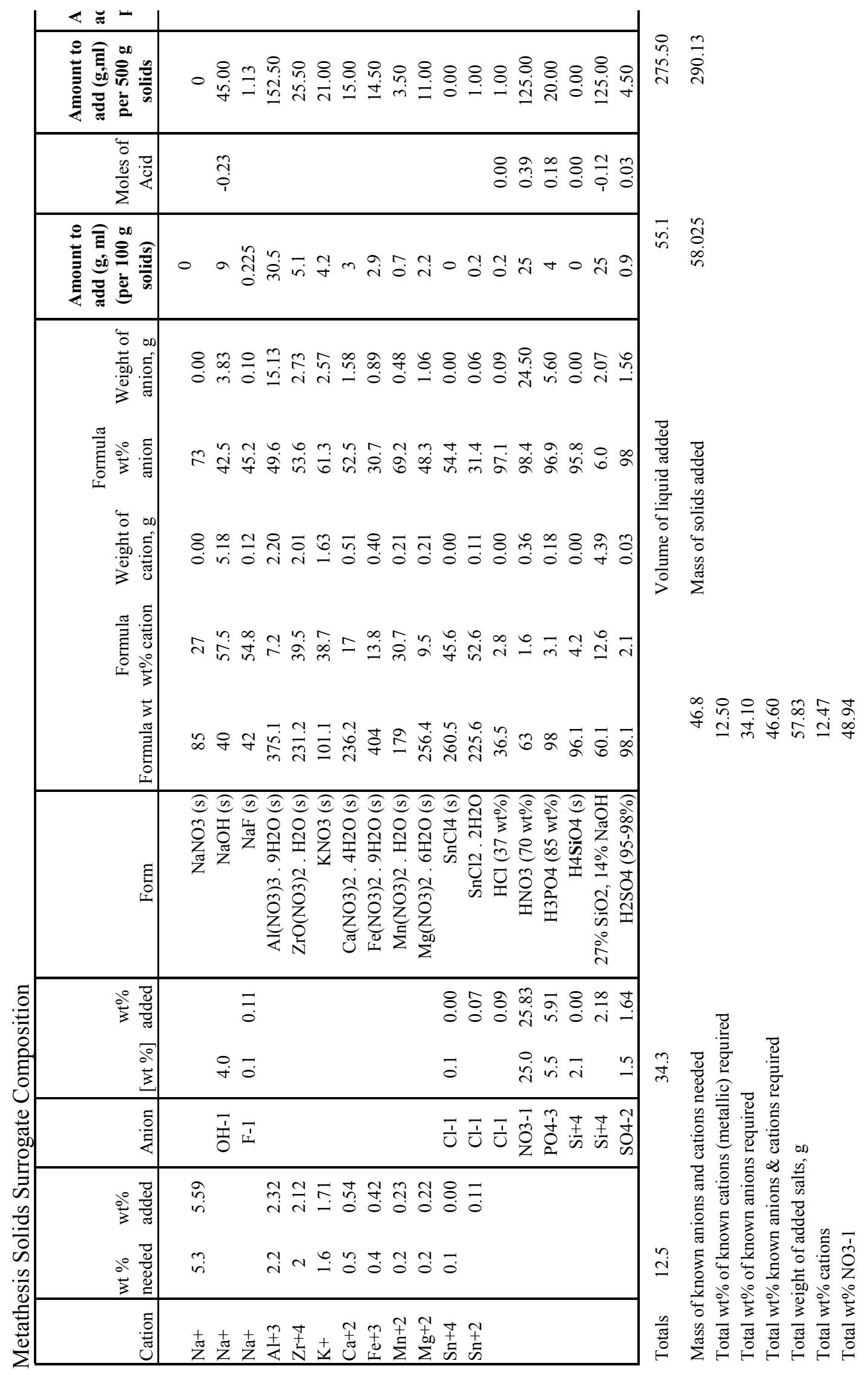




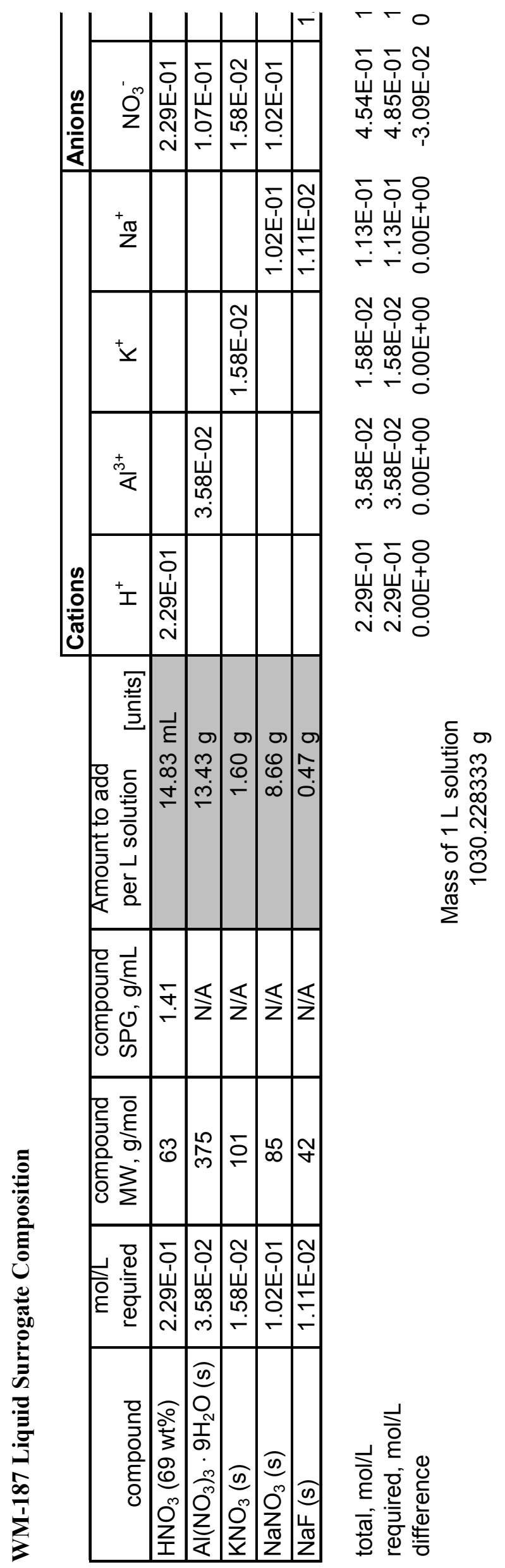




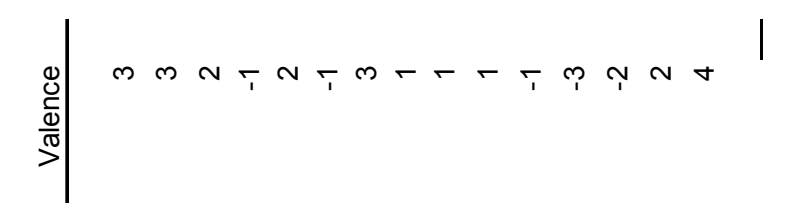

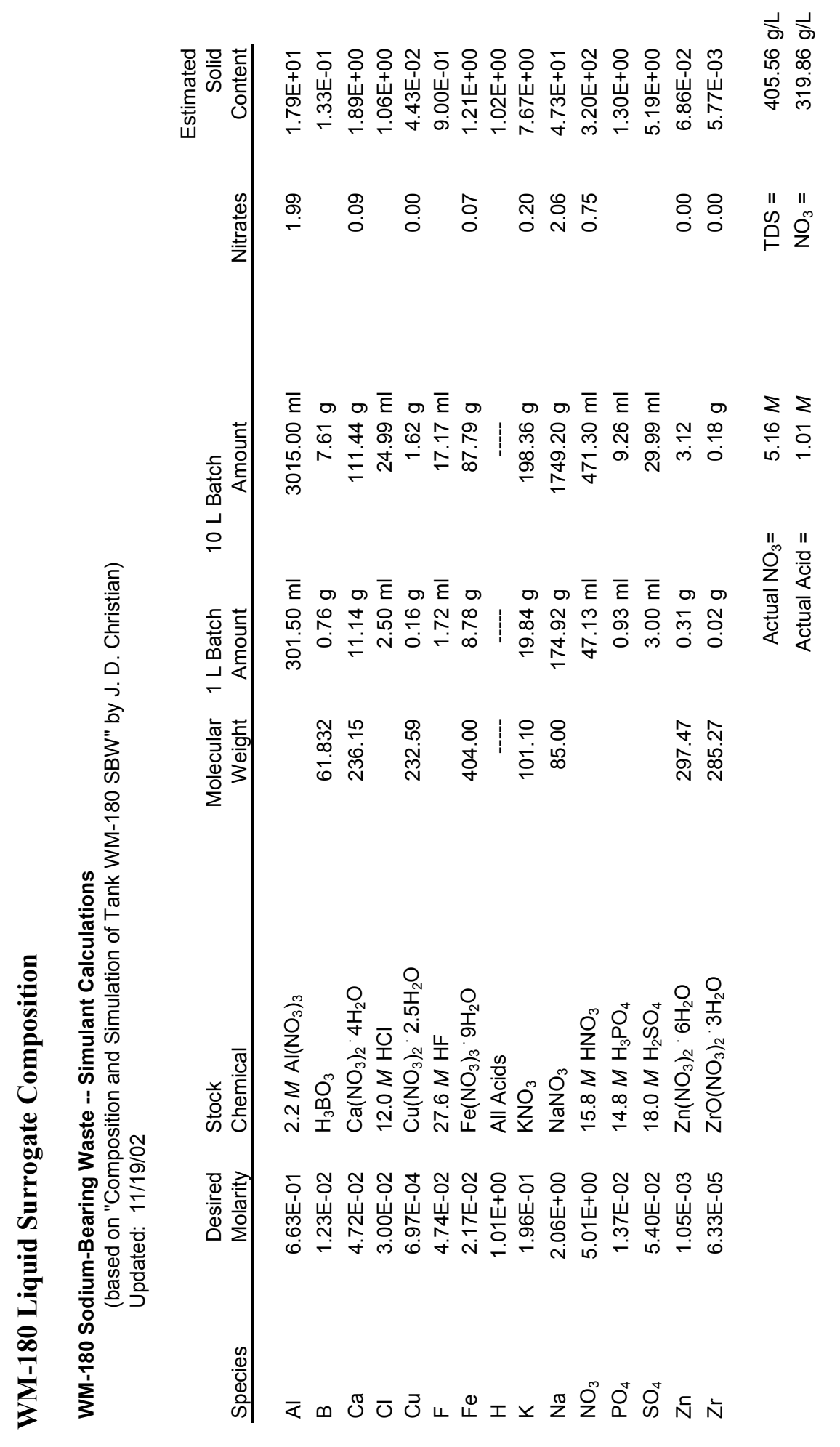


|

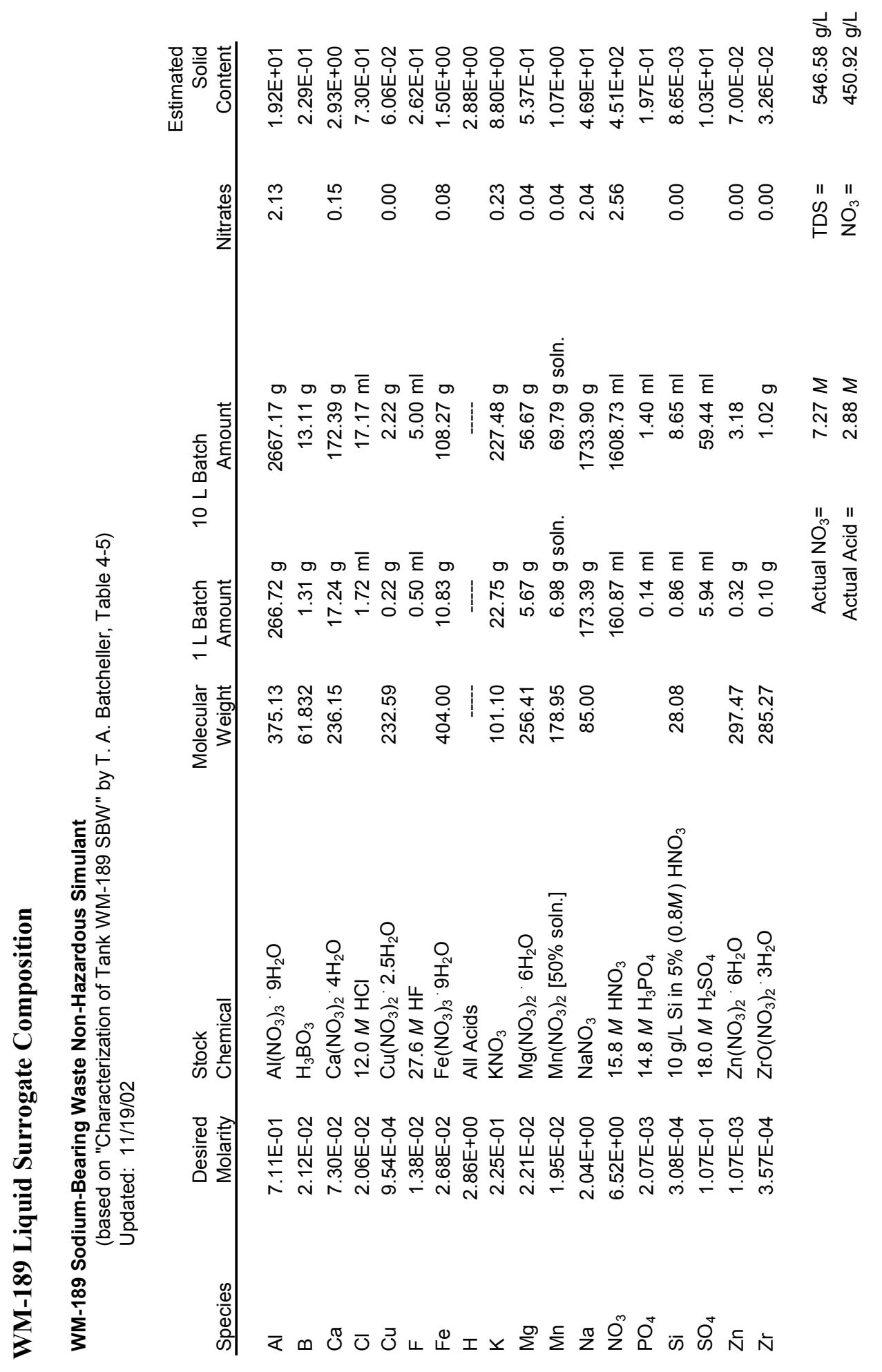


Procedure for Preparing Nonhazardous SBW Surrogates

1. Add the following salts to a 2-L glass beaker in the amounts listed in composition tables: $\mathrm{NaNO}_{3}$, $\mathrm{KNO}_{3}, \mathrm{Ca}\left(\mathrm{NO}_{3}\right)_{2}, \mathrm{Cu}\left(\mathrm{NO}_{3}\right)_{2}, \mathrm{Fe}\left(\mathrm{NO}_{3}\right)_{3}, \mathrm{Zn}\left(\mathrm{NO}_{3}\right)_{2}$, and $\mathrm{Mg}\left(\mathrm{NO}_{3}\right)_{2}$ (WM-189 only). Add just enough demineralized water to dissolve $100 \%$ of the salts. Stir and warm as needed.

2. To the above solution, add the $\mathrm{Al}\left(\mathrm{NO}_{3}\right)_{3}$ and the $50 \%$ solution of $\mathrm{Mn}\left(\mathrm{NO}_{3}\right)_{2}$ (WM-189 only). Continue heating and stirring while performing the following steps.

3. In a separate glass beaker, dissolve as much of the $\mathrm{H}_{3} \mathrm{BO}_{3}$ as possible in approximately $40 \mathrm{~mL}$ of water. Heat and stir as necessary. It may not be possible to dissolve all of the $\mathrm{H}_{3} \mathrm{BO}_{3}$ in the water alone. Discontinue heating and add the HF. The HF will immediately cause any undissolved $\mathrm{H}_{3} \mathrm{BO}_{3}$ to go into solution. Stir well and combine with the solution from Steps 1 and 2 while stirring vigorously.

4. Add the $\mathrm{H}_{2} \mathrm{SO}_{4}$ to the solution generated in Steps 1-3 and mix thoroughly.

5. Add demineralized water to the solution prepared in Steps 1-4 bringing the total solution volume to about $750 \mathrm{~mL}$.

6. Slowly add the remaining components $\left(\mathrm{HCl}, \mathrm{H}_{3} \mathrm{PO}_{4}\right.$, and $\left.\mathrm{HNO}_{3}\right)$ to the solution.

7. Add demineralized water (approximately $50 \mathrm{~mL}$ ) to the mixture solution, bringing the final volume to $1 \mathrm{~L}$.

NOTE: This procedure and composition spreadsheet target the acid content while the nitrate content is allowed to float. In these cases, the nitrate composition comes in slightly higher than the desired molarity. Since the nitrate content is very large, the small variation is not significant to SBW experiments. The acid content is more important. 
Appendix D

FUNDABAC Filtermedia 
D-2 
FUNDABAC Filtermedia

\begin{tabular}{|c|c|c|c|c|c|c|c|}
\hline \multirow{2}{*}{$\frac{\text { Type }}{\text { B31 MU-100 }}$} & \multirow{2}{*}{\begin{tabular}{l}
\multicolumn{1}{c}{ Warp } \\
PVDF \\
Monofilament
\end{tabular}} & \multirow{2}{*}{$\begin{array}{l}\quad \text { Weft } \\
\text { PVDF } \\
\text { Multifilament }\end{array}$} & \multirow{2}{*}{$\begin{array}{c}\text { Air } \\
\text { permeability } \\
\text { L/dm2 } 2 \text { min } \\
50-100\end{array}$} & \multicolumn{3}{|c|}{$\begin{array}{l}\text { Porometer value in } \\
\text { microns }^{\mathrm{a}} \\
\times 10 \times 50 \times 90\end{array}$} & \multirow{2}{*}{$\begin{array}{c}\begin{array}{c}\text { Max. Operating } \\
\text { Temperature }{ }^{\circ} \mathrm{C}\end{array} \\
130\end{array}$} \\
\hline & & & & 24 & 11.5 & 4.2 & \\
\hline B31 U-005 & $\begin{array}{l}\text { PVDF } \\
\text { Multifilament }\end{array}$ & $\begin{array}{l}\text { PVDF } \\
\text { Multifilament }\end{array}$ & $3-6$ & 1.35 & 5.5 & 14.5 & 130 \\
\hline B46 U-010 & $\begin{array}{l}\text { PPS } \\
\text { Multifilament }\end{array}$ & $\begin{array}{l}\text { PPS } \\
\text { Multifilament }\end{array}$ & $6-10$ & 6.4 & 2.9 & 1.7 & 180 \\
\hline
\end{tabular}

a. The indicated porometer values are based on the bubble point method and indicate the percentage of pore bigger than the size indicated in micrometers. For example, a value of 5.0 in the $\times 10$ column means that $10 \%$ of the opening are bigger than 5 microns and $90 \%$ are smaller than 5 microns etc. 
Appendix E

Data 
E-2 


\section{E-1 PARTICLE SIZE DISTRIBUTION \\ E-1.1 SBW Surrogate Samples}

$187 / 5 w t \% / D / N$

Summary Data

MV

$\mathrm{MN}$

MA

CS

SD

SampleLD

Dist

Prog

0 min sonication

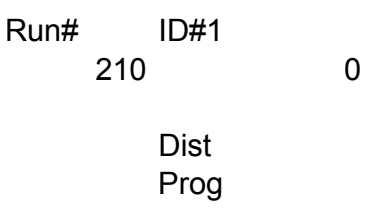

0.242791

101.240437

0.196

0

Percentile Percentile Data

$\begin{array}{lr}10 & 10.214151 \\ 20 & 23.840677 \\ 30 & 40.339854 \\ 40 & 58.481072 \\ 50 & 79.006476 \\ 60 & 104.158986 \\ 70 & 137.787312 \\ 80 & 189.221174 \\ 90 & 297.559629 \\ 95 & 439.744205\end{array}$

Number of Channels

50

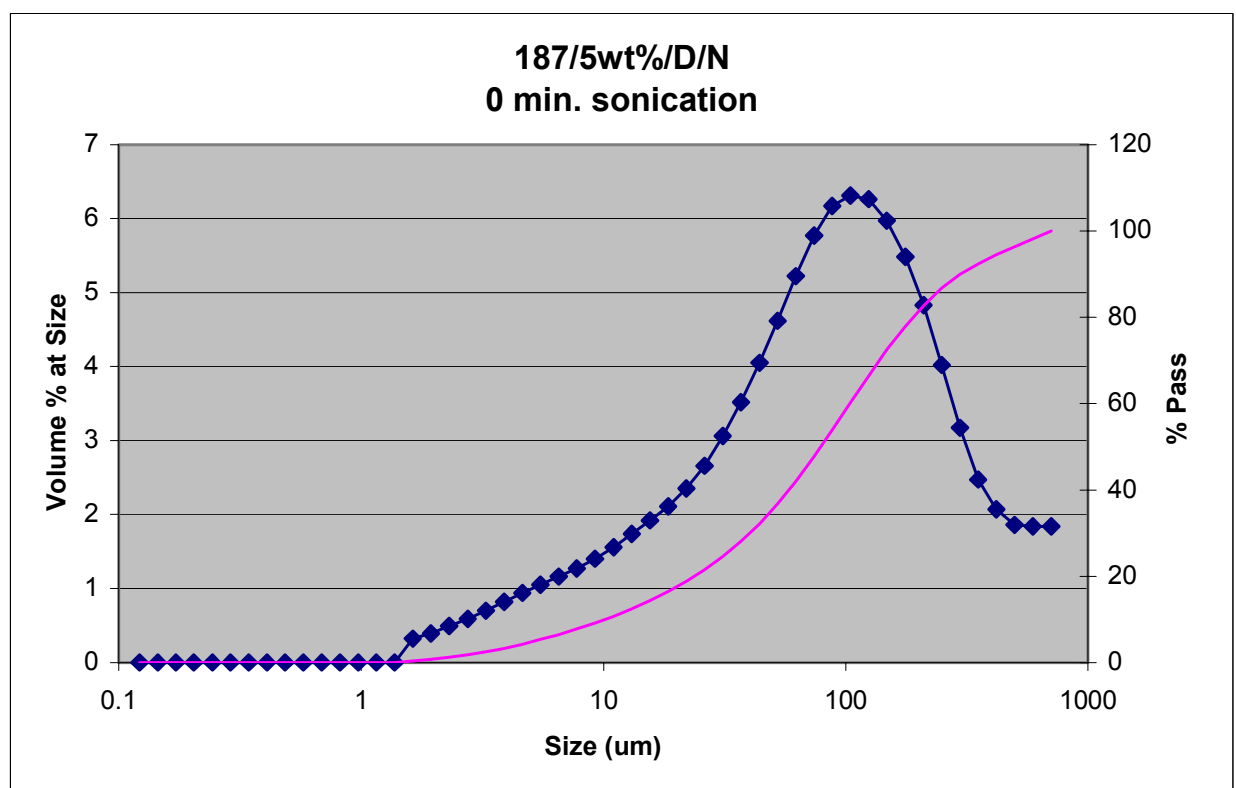

E-3 


\begin{tabular}{rrrr} 
187/5wt\%/D/N & \multicolumn{2}{c}{ Selected } \\
Channel & \multicolumn{2}{c}{ Selected ElVolume } & \multicolumn{1}{c}{ Cummulative } \\
1 & 704 & 1.84 & 100 \\
2 & 592 & 1.84 & 98.16 \\
3 & 497.8 & 1.86 & 96.32 \\
4 & 418.6 & 2.07 & 94.46 \\
5 & 352 & 2.47 & 92.39 \\
6 & 296 & 3.17 & 89.92 \\
7 & 248.9 & 4.02 & 86.75 \\
8 & 209.3 & 4.83 & 82.73 \\
9 & 176 & 5.48 & 77.9 \\
10 & 148 & 5.97 & 72.42 \\
11 & 124.5 & 6.26 & 66.45 \\
12 & 104.7 & 6.31 & 60.19 \\
13 & 88 & 6.17 & 53.88 \\
14 & 74 & 5.77 & 47.71 \\
15 & 62.23 & 5.22 & 41.94 \\
16 & 52.33 & 4.62 & 36.72 \\
17 & 44 & 4.05 & 32.1 \\
18 & 37 & 3.52 & 28.05 \\
19 & 31.11 & 3.06 & 24.53 \\
20 & 26.16 & 2.66 & 21.47 \\
21 & 22 & 2.35 & 18.81 \\
22 & 18.5 & 2.11 & 16.46 \\
23 & 15.56 & 1.92 & 14.35 \\
24 & 13.08 & 1.74 & 12.43 \\
25 & 11 & 1.56 & 10.69 \\
26 & 9.25 & 1.4 & 9.13 \\
27 & 7.778 & 1.27 & 7.73 \\
28 & 6.541 & 1.16 & 6.46 \\
29 & 5.5 & 1.05 & 5.3 \\
30 & 4.625 & 0.94 & 4.25 \\
31 & 3.889 & 0.82 & 3.31 \\
32 & 3.27 & 0.7 & 2.49 \\
33 & 2.75 & 0.59 & 1.79 \\
34 & 2.312 & 0.49 & 1.2 \\
35 & 1.945 & 0.39 & 0.71 \\
36 & 1.635 & 0.32 & 0.32 \\
37 & 1.375 & 0 & $1.05471 \mathrm{E}-15$ \\
38 & 1.156 & 0 & $1.05471 \mathrm{E}-15$ \\
39 & 0.972 & 0 & $1.05471 \mathrm{E}-15$ \\
40 & 0.818 & 0 & $1.05471 \mathrm{E}-15$ \\
41 & 0.688 & 0 & $1.05471 \mathrm{E}-15$ \\
42 & 0.578 & 0 & $1.05471 \mathrm{E}-15$ \\
43 & 0.486 & 0 & $1.05471 \mathrm{E}-15$ \\
44 & 0.409 & 0 & $1.05471 \mathrm{E}-15$ \\
45 & 0.344 & 0 & $1.05471 \mathrm{E}-15$ \\
46 & 0.289 & 0 & $1.05471 \mathrm{E}-15$ \\
47 & 0.243 & 0 & $1.05471 \mathrm{E}-15$ \\
48 & 0.204 & 0 & $1.05471 \mathrm{E}-15$ \\
49 & 0.172 & 0 & $1.05471 \mathrm{E}-15$ \\
50 & 0.145 & 0 & $1.05471 \mathrm{E}-15$ \\
51 & 0.122 & 0 & $1.05471 \mathrm{E}-15$
\end{tabular}


187/5wt\%/D/S

Summary Data

MV

MN

MA

CS

SD

SampleLD

Dist

Prog
210 min. sonication

$\begin{array}{lc}\text { Run\# } & \text { ID\#1 } \\ & 216 \text { P-004? } \\ & \text { Dist } \\ & \text { Prog } \\ & \\ & \\ & \end{array}$

Percentile Percentile Data

$\begin{array}{rrr}10 & 3.670706 & 10 \\ 20 & 6.568494 & 3.670706 \\ 30 & 11.02589 & \\ 40 & 15.108638 & \\ 50 & 19.197204 & \\ 60 & 23.50631 & \\ 70 & 28.086258 & \\ 80 & 33.450741 & \\ 90 & 41.629979 & \\ 95 & 49.882973 & \end{array}$

Number of Channels

50

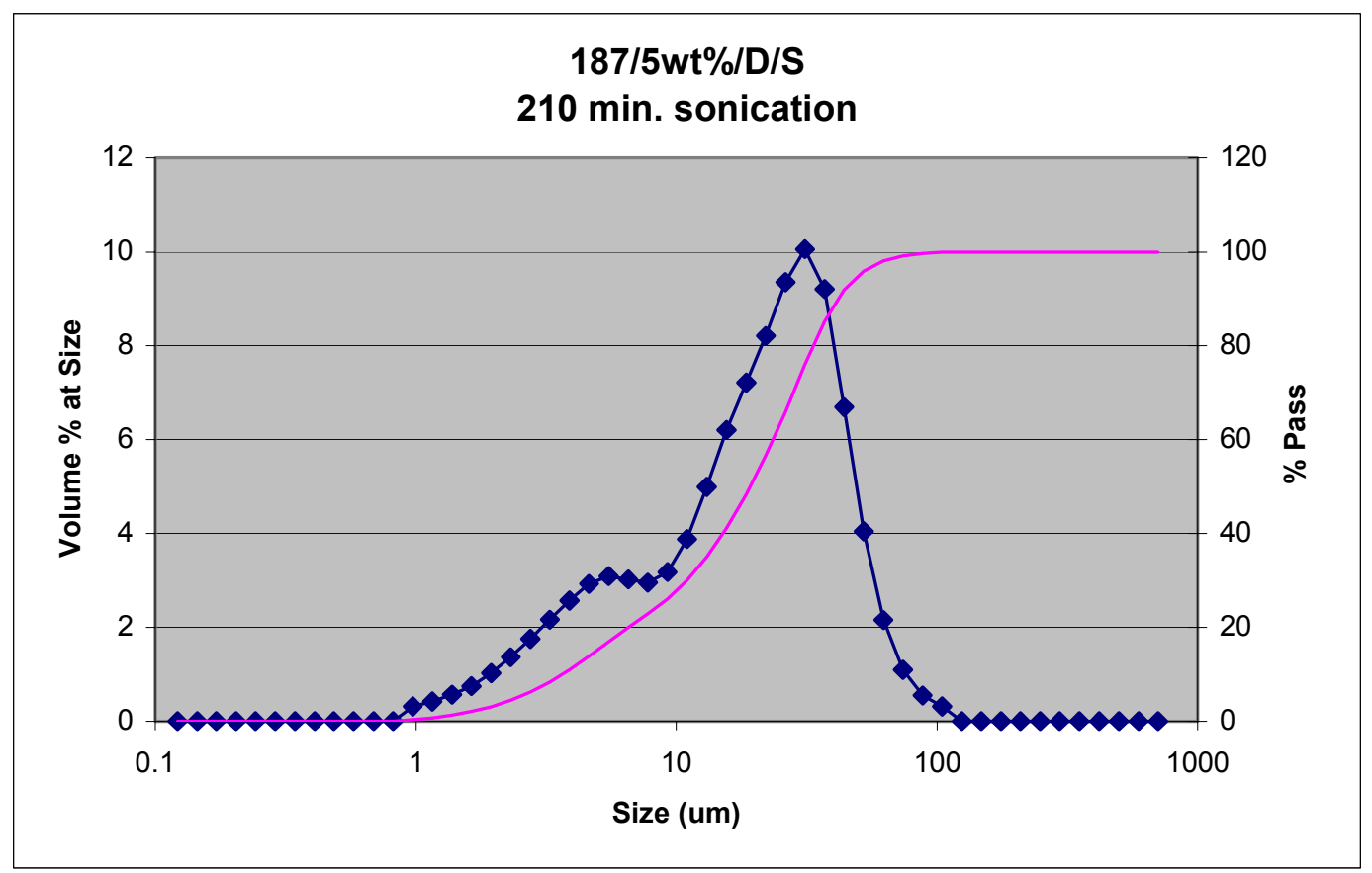

E-5 


\begin{tabular}{|c|c|c|c|}
\hline \multicolumn{4}{|c|}{ 187/5wt\%/D/S } \\
\hline \multirow{2}{*}{ Channel } & Selected E & ume & Cummulative \\
\hline & 704 & 0 & 100 \\
\hline 2 & 592 & 0 & 100 \\
\hline 3 & 497.8 & 0 & 100 \\
\hline 4 & 418.6 & 0 & 100 \\
\hline 5 & 352 & 0 & 100 \\
\hline 6 & 296 & 0 & 100 \\
\hline 7 & 248.9 & 0 & 100 \\
\hline 8 & 209.3 & 0 & 100 \\
\hline 9 & 176 & 0 & 100 \\
\hline 10 & 148 & 0 & 100 \\
\hline 11 & 124.5 & 0 & 100 \\
\hline 12 & 104.7 & 0.31 & 100 \\
\hline 13 & 88 & 0.55 & 99.69 \\
\hline 14 & 74 & 1.09 & 99.14 \\
\hline 15 & 62.23 & 2.15 & 98.05 \\
\hline 16 & 52.33 & 4.04 & 95.9 \\
\hline 17 & 44 & 6.69 & 91.86 \\
\hline 18 & 37 & 9.21 & 85.17 \\
\hline 19 & 31.11 & 10.06 & 75.96 \\
\hline 20 & 26.16 & 9.35 & 65.9 \\
\hline 21 & 22 & 8.21 & 56.55 \\
\hline 22 & 18.5 & 7.21 & 48.34 \\
\hline 23 & 15.56 & 6.2 & 41.13 \\
\hline 24 & 13.08 & 4.99 & 34.93 \\
\hline 25 & 11 & 3.88 & 29.94 \\
\hline 26 & 9.25 & 3.18 & 26.06 \\
\hline 27 & 7.778 & 2.95 & 22.88 \\
\hline 28 & 6.541 & 3.02 & 19.93 \\
\hline 29 & 5.5 & 3.09 & 16.91 \\
\hline 30 & 4.625 & 2.92 & 13.82 \\
\hline 31 & 3.889 & 2.57 & 10.9 \\
\hline 32 & 3.27 & 2.16 & 8.33 \\
\hline 33 & 2.75 & 1.75 & 6.17 \\
\hline 34 & 2.312 & 1.36 & 4.42 \\
\hline 35 & 1.945 & 1.02 & 3.06 \\
\hline 36 & 1.635 & 0.75 & 2.04 \\
\hline 37 & 1.375 & 0.56 & 1.29 \\
\hline 38 & 1.156 & 0.42 & 0.73 \\
\hline 39 & 0.972 & 0.31 & 0.31 \\
\hline 40 & 0.818 & 0 & 0 \\
\hline 41 & 0.688 & 0 & 0 \\
\hline 42 & 0.578 & 0 & 0 \\
\hline 43 & 0.486 & 0 & 0 \\
\hline 44 & 0.409 & 0 & 0 \\
\hline 45 & 0.344 & 0 & 0 \\
\hline 46 & 0.289 & 0 & 0 \\
\hline 47 & 0.243 & 0 & 0 \\
\hline 48 & 0.204 & 0 & 0 \\
\hline 49 & 0.172 & 0 & 0 \\
\hline 50 & 0.145 & 0 & 0 \\
\hline 51 & 0.122 & 0 & 0 \\
\hline
\end{tabular}


Summary Data

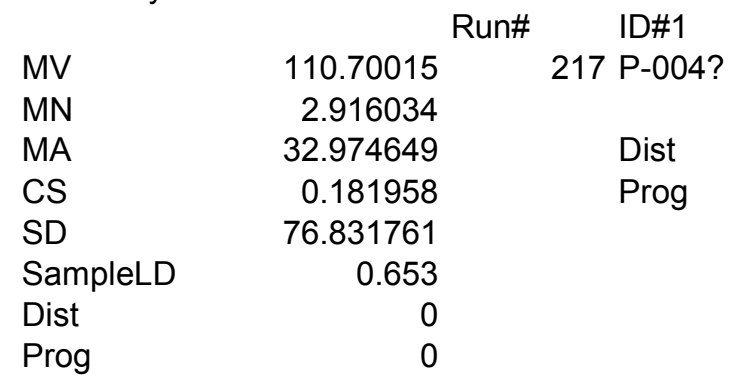

Percentile Percentile Data

$10 \quad 17.165946$

$20 \quad 37.740502$

$\begin{array}{rr}30 & 55.50203 \\ 40 & 71.972762\end{array}$

10

40
50

$60 \quad 108.354964$

$70 \quad 132.363106$

$80 \quad 165.487664$

$90 \quad 222.206521$

$95 \quad 284.003078$

Number of Channels

50

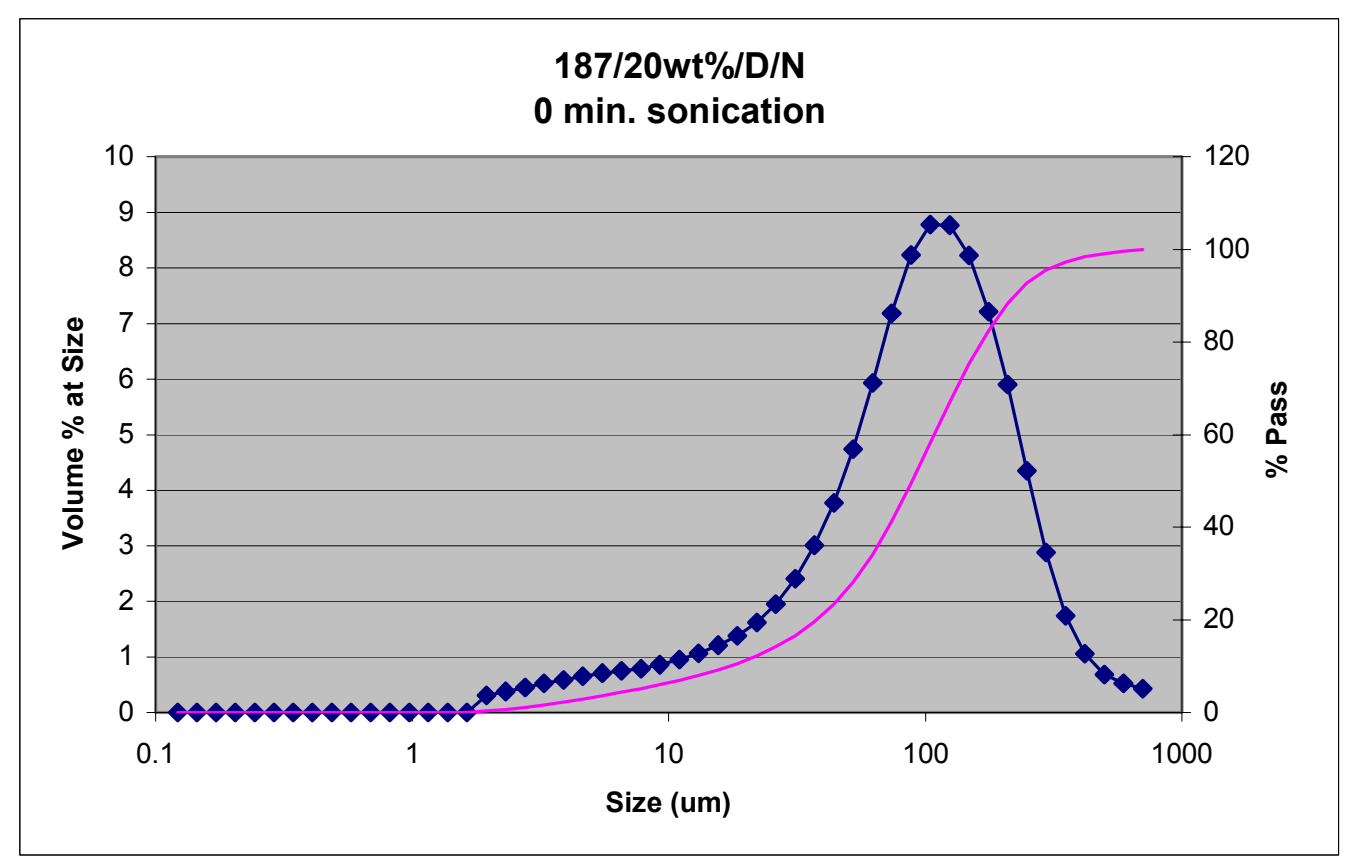




\begin{tabular}{|c|c|c|c|}
\hline \multicolumn{4}{|c|}{ 187/20wt\%/D/N } \\
\hline Channel & Selected E & & Cummulative \\
\hline 1 & 704 & 0.43 & 100 \\
\hline 2 & 592 & 0.52 & 99.57 \\
\hline 3 & 497.8 & 0.68 & 99.05 \\
\hline 4 & 418.6 & 1.06 & 98.37 \\
\hline 5 & 352 & 1.74 & 97.31 \\
\hline 6 & 296 & 2.88 & 95.57 \\
\hline 7 & 248.9 & 4.35 & 92.69 \\
\hline 8 & 209.3 & 5.9 & 88.34 \\
\hline 9 & 176 & 7.21 & 82.44 \\
\hline 10 & 148 & 8.22 & 75.23 \\
\hline 11 & 124.5 & 8.77 & 67.01 \\
\hline 12 & 104.7 & 8.78 & 58.24 \\
\hline 13 & 88 & 8.23 & 49.46 \\
\hline 14 & 74 & 7.18 & 41.23 \\
\hline 15 & 62.23 & 5.93 & 34.05 \\
\hline 16 & 52.33 & 4.74 & 28.12 \\
\hline 17 & 44 & 3.77 & 23.38 \\
\hline 18 & 37 & 3.01 & 19.61 \\
\hline 19 & 31.11 & 2.41 & 16.6 \\
\hline 20 & 26.16 & 1.95 & 14.19 \\
\hline 21 & 22 & 1.62 & 12.24 \\
\hline 22 & 18.5 & 1.38 & 10.62 \\
\hline 23 & 15.56 & 1.21 & 9.24 \\
\hline 24 & 13.08 & 1.07 & 8.03 \\
\hline 25 & 11 & 0.95 & 6.96 \\
\hline 26 & 9.25 & 0.86 & 6.01 \\
\hline 27 & 7.778 & 0.79 & 5.15 \\
\hline 28 & 6.541 & 0.75 & 4.36 \\
\hline 29 & 5.5 & 0.71 & 3.61 \\
\hline 30 & 4.625 & 0.65 & 2.9 \\
\hline 31 & 3.889 & 0.59 & 2.25 \\
\hline 32 & 3.27 & 0.52 & 1.66 \\
\hline 33 & 2.75 & 0.45 & 1.14 \\
\hline 34 & 2.312 & 0.38 & 0.69 \\
\hline 35 & 1.945 & 0.31 & 0.31 \\
\hline 36 & 1.635 & 0 & 0 \\
\hline 37 & 1.375 & 0 & 0 \\
\hline 38 & 1.156 & 0 & 0 \\
\hline 39 & 0.972 & 0 & 0 \\
\hline 40 & 0.818 & 0 & 0 \\
\hline 41 & 0.688 & 0 & 0 \\
\hline 42 & 0.578 & 0 & 0 \\
\hline 43 & 0.486 & 0 & 0 \\
\hline 44 & 0.409 & 0 & 0 \\
\hline 45 & 0.344 & 0 & 0 \\
\hline 46 & 0.289 & 0 & 0 \\
\hline 47 & 0.243 & 0 & 0 \\
\hline 48 & 0.204 & 0 & 0 \\
\hline 49 & 0.172 & 0 & 0 \\
\hline 50 & 0.145 & 0 & 0 \\
\hline 51 & 0.122 & 0 & 0 \\
\hline
\end{tabular}




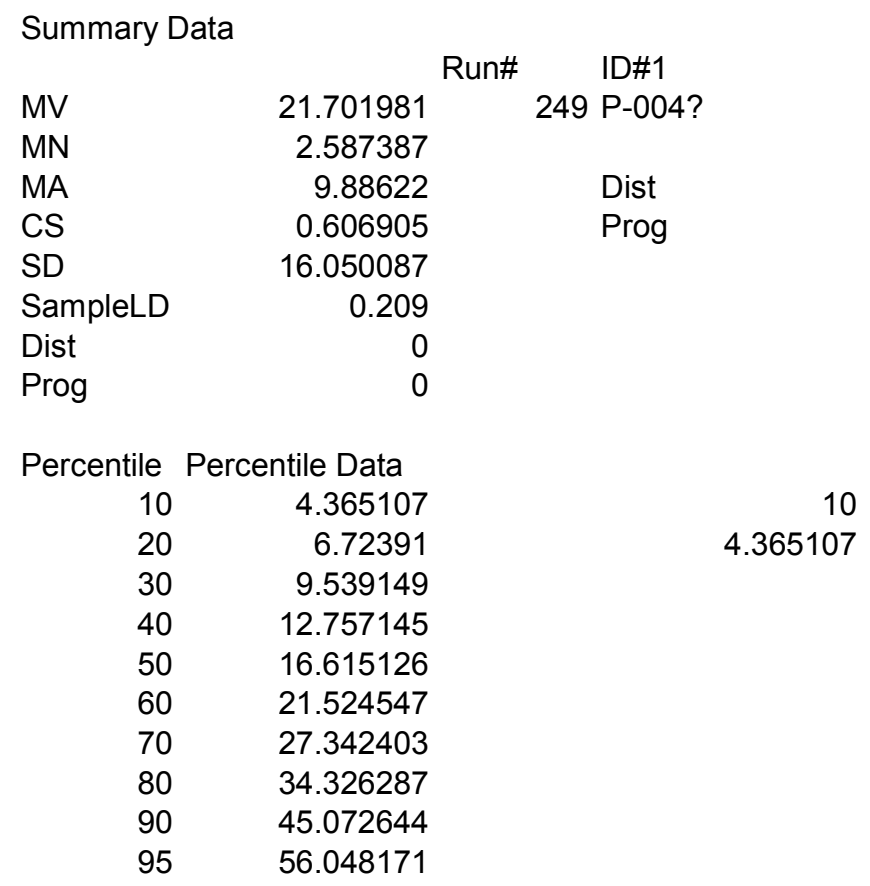

Number of Channels

50

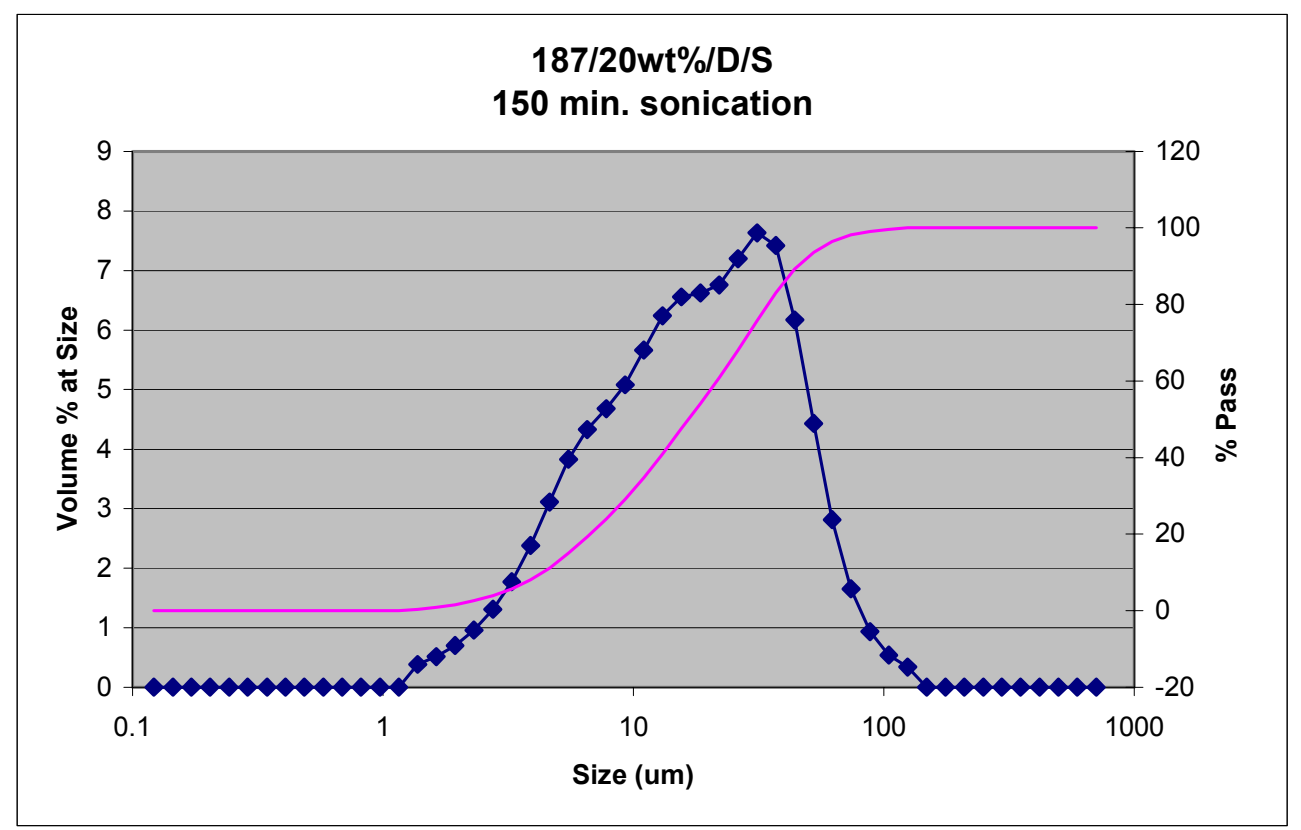


187/20wt\%/D/S

\begin{tabular}{|c|c|c|c|}
\hline \multirow[b]{2}{*}{ Channel } & \multicolumn{2}{|c|}{ Selected } & 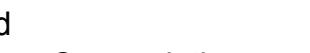 \\
\hline & Selected E & me & mmulative \\
\hline 1 & 704 & 0 & 100 \\
\hline 2 & 592 & 0 & 100 \\
\hline 3 & 497.8 & 0 & 100 \\
\hline 4 & 418.6 & 0 & 100 \\
\hline 5 & 352 & 0 & 100 \\
\hline 6 & 296 & 0 & 100 \\
\hline 7 & 248.9 & 0 & 100 \\
\hline 8 & 209.3 & 0 & 100 \\
\hline 9 & 176 & 0 & 100 \\
\hline 10 & 148 & 0 & 100 \\
\hline 11 & 124.5 & 0.34 & 100 \\
\hline 12 & 104.7 & 0.54 & 99.66 \\
\hline 13 & 88 & 0.93 & 99.12 \\
\hline 14 & 74 & 1.65 & 98.19 \\
\hline 15 & 62.23 & 2.81 & 96.54 \\
\hline 16 & 52.33 & 4.43 & 93.73 \\
\hline 17 & 44 & 6.17 & 89.3 \\
\hline 18 & 37 & 7.42 & 83.13 \\
\hline 19 & 31.11 & 7.63 & 75.71 \\
\hline 20 & 26.16 & 7.2 & 68.08 \\
\hline 21 & 22 & 6.76 & 60.88 \\
\hline 22 & 18.5 & 6.62 & 54.12 \\
\hline 23 & 15.56 & 6.56 & 47.5 \\
\hline 24 & 13.08 & 6.24 & 40.94 \\
\hline 25 & 11 & 5.66 & 34.7 \\
\hline 26 & 9.25 & 5.08 & 29.04 \\
\hline 27 & 7.778 & 4.68 & 23.96 \\
\hline 28 & 6.541 & 4.33 & 19.28 \\
\hline 29 & 5.5 & 3.83 & 14.95 \\
\hline 30 & 4.625 & 3.11 & 11.12 \\
\hline 31 & 3.889 & 2.38 & 8.01 \\
\hline 32 & 3.27 & 1.77 & 5.63 \\
\hline 33 & 2.75 & 1.31 & 3.86 \\
\hline 34 & 2.312 & 0.96 & 2.55 \\
\hline 35 & 1.945 & 0.7 & 1.59 \\
\hline 36 & 1.635 & 0.51 & 0.89 \\
\hline 37 & 1.375 & 0.38 & 0.38 \\
\hline 38 & 1.156 & 0 & $-1.96509 \mathrm{E}-14$ \\
\hline 39 & 0.972 & 0 & $-1.96509 \mathrm{E}-14$ \\
\hline 40 & 0.818 & 0 & $-1.96509 \mathrm{E}-14$ \\
\hline 41 & 0.688 & 0 & $-1.96509 \mathrm{E}-14$ \\
\hline 42 & 0.578 & 0 & $-1.96509 \mathrm{E}-14$ \\
\hline 43 & 0.486 & 0 & $-1.96509 \mathrm{E}-14$ \\
\hline 44 & 0.409 & 0 & $-1.96509 \mathrm{E}-14$ \\
\hline 45 & 0.344 & 0 & $-1.96509 \mathrm{E}-14$ \\
\hline 46 & 0.289 & 0 & $-1.96509 \mathrm{E}-14$ \\
\hline 47 & 0.243 & 0 & $-1.96509 \mathrm{E}-14$ \\
\hline 48 & 0.204 & 0 & $-1.96509 \mathrm{E}-14$ \\
\hline 49 & 0.172 & 0 & $-1.96509 \mathrm{E}-14$ \\
\hline 50 & 0.145 & 0 & $-1.96509 \mathrm{E}-14$ \\
\hline 51 & 0.122 & 0 & $-1.96509 \mathrm{E}-14$ \\
\hline
\end{tabular}

E-10 


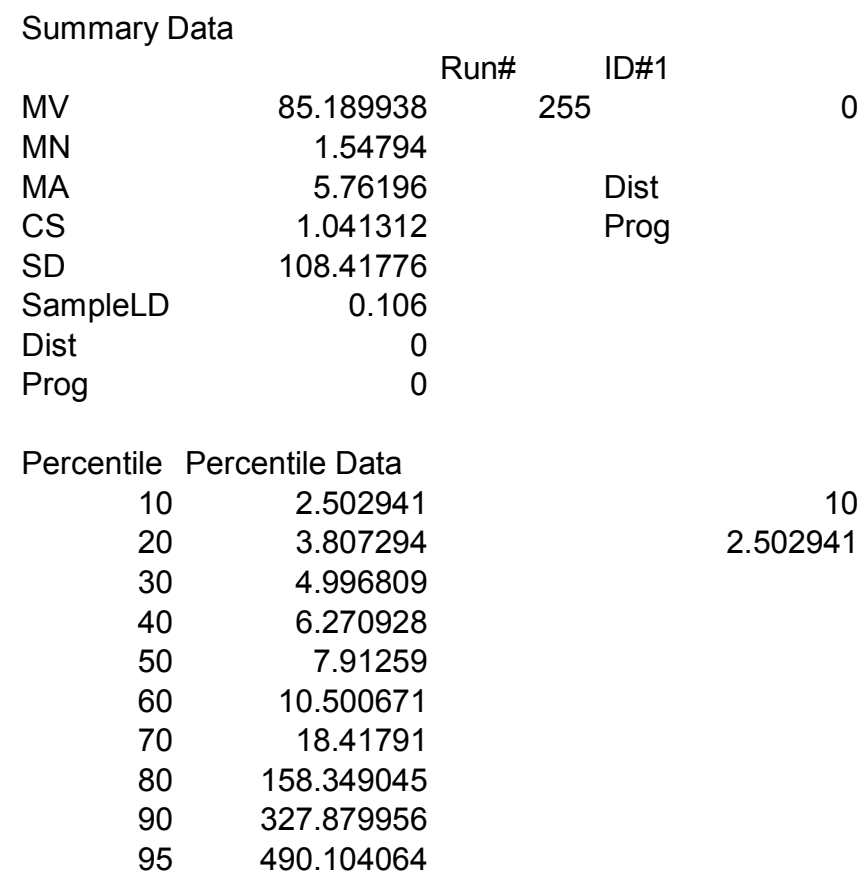

Number of Channels 50

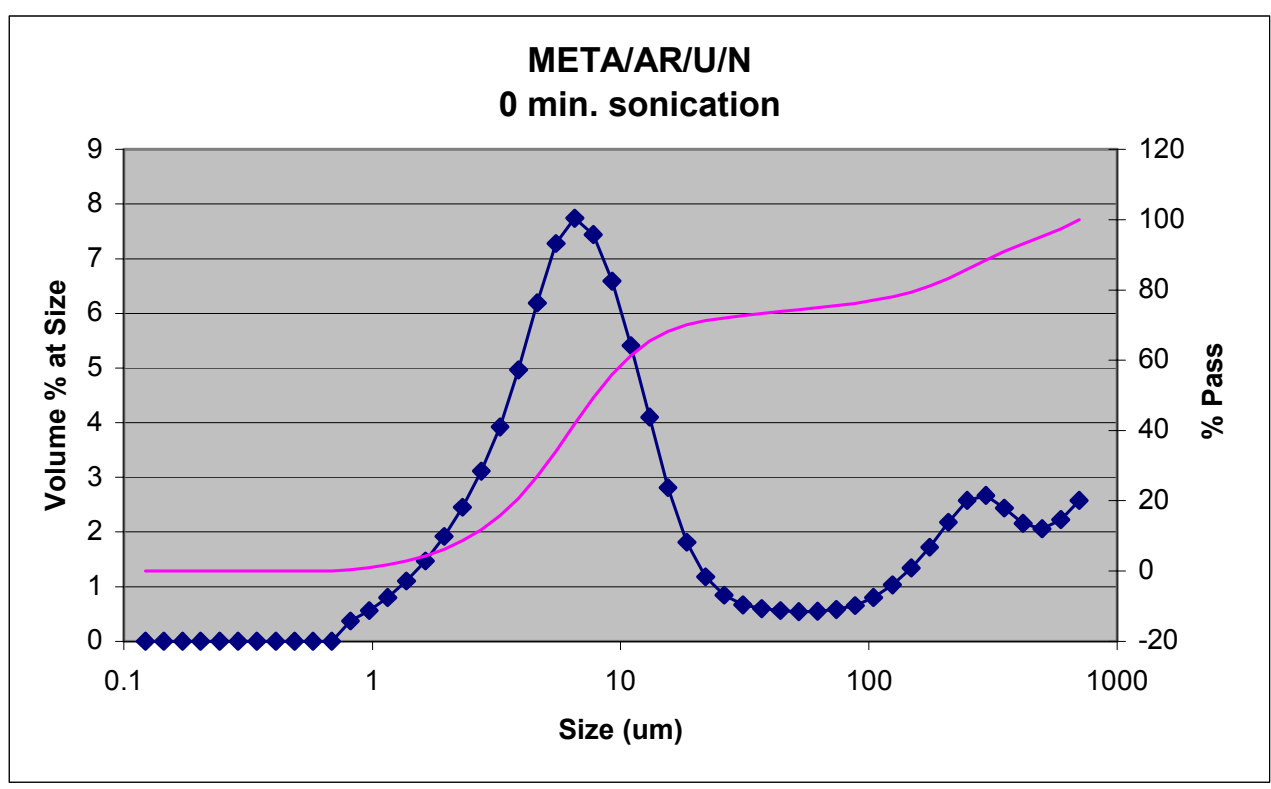




\begin{tabular}{|c|c|c|c|}
\hline \multicolumn{4}{|c|}{ META/AR/U/N } \\
\hline \multicolumn{4}{|c|}{ Selected } \\
\hline Channel & Selected E & me & Cummulative \\
\hline 1 & 704 & 2.58 & 100 \\
\hline 2 & 592 & 2.23 & 97.42 \\
\hline 3 & 497.8 & 2.06 & 95.19 \\
\hline 4 & 418.6 & 2.16 & 93.13 \\
\hline 5 & 352 & 2.44 & 90.97 \\
\hline 6 & 296 & 2.67 & 88.53 \\
\hline 7 & 248.9 & 2.58 & 85.86 \\
\hline 8 & 209.3 & 2.18 & 83.28 \\
\hline 9 & 176 & 1.72 & 81.1 \\
\hline 10 & 148 & 1.34 & 79.38 \\
\hline 11 & 124.5 & 1.03 & 78.04 \\
\hline 12 & 104.7 & 0.8 & 77.01 \\
\hline 13 & 88 & 0.65 & 76.21 \\
\hline 14 & 74 & 0.58 & 75.56 \\
\hline 15 & 62.23 & 0.55 & 74.98 \\
\hline 16 & 52.33 & 0.54 & 74.43 \\
\hline 17 & 44 & 0.56 & 73.89 \\
\hline 18 & 37 & 0.6 & 73.33 \\
\hline 19 & 31.11 & 0.67 & 72.73 \\
\hline 20 & 26.16 & 0.84 & 72.06 \\
\hline 21 & 22 & 1.18 & 71.22 \\
\hline 22 & 18.5 & 1.81 & 70.04 \\
\hline 23 & 15.56 & 2.81 & 68.23 \\
\hline 24 & 13.08 & 4.1 & 65.42 \\
\hline 25 & 11 & 5.41 & 61.32 \\
\hline 26 & 9.25 & 6.59 & 55.91 \\
\hline 27 & 7.778 & 7.44 & 49.32 \\
\hline 28 & 6.541 & 7.74 & 41.88 \\
\hline 29 & 5.5 & 7.28 & 34.14 \\
\hline 30 & 4.625 & 6.19 & 26.86 \\
\hline 31 & 3.889 & 4.97 & 20.67 \\
\hline 32 & 3.27 & 3.92 & 15.7 \\
\hline 33 & 2.75 & 3.11 & 11.78 \\
\hline 34 & 2.312 & 2.45 & 8.67 \\
\hline 35 & 1.945 & 1.92 & 6.22 \\
\hline 36 & 1.635 & 1.47 & 4.3 \\
\hline 37 & 1.375 & 1.1 & 2.83 \\
\hline 38 & 1.156 & 0.8 & 1.73 \\
\hline 39 & 0.972 & 0.56 & 0.93 \\
\hline 40 & 0.818 & 0.37 & 0.37 \\
\hline 41 & 0.688 & 0 & $-3.56382 E-14$ \\
\hline 42 & 0.578 & 0 & $-3.56382 \mathrm{E}-14$ \\
\hline 43 & 0.486 & 0 & $-3.56382 \mathrm{E}-14$ \\
\hline 44 & 0.409 & 0 & $-3.56382 \mathrm{E}-14$ \\
\hline 45 & 0.344 & 0 & $-3.56382 E-14$ \\
\hline 46 & 0.289 & 0 & $-3.56382 \mathrm{E}-14$ \\
\hline 47 & 0.243 & 0 & $-3.56382 E-14$ \\
\hline 48 & 0.204 & 0 & $-3.56382 E-14$ \\
\hline 49 & 0.172 & 0 & $-3.56382 \mathrm{E}-14$ \\
\hline 50 & 0.145 & 0 & $-3.56382 \mathrm{E}-14$ \\
\hline 51 & 0.122 & 0 & $-3.56382 E-14$ \\
\hline
\end{tabular}

E-12 


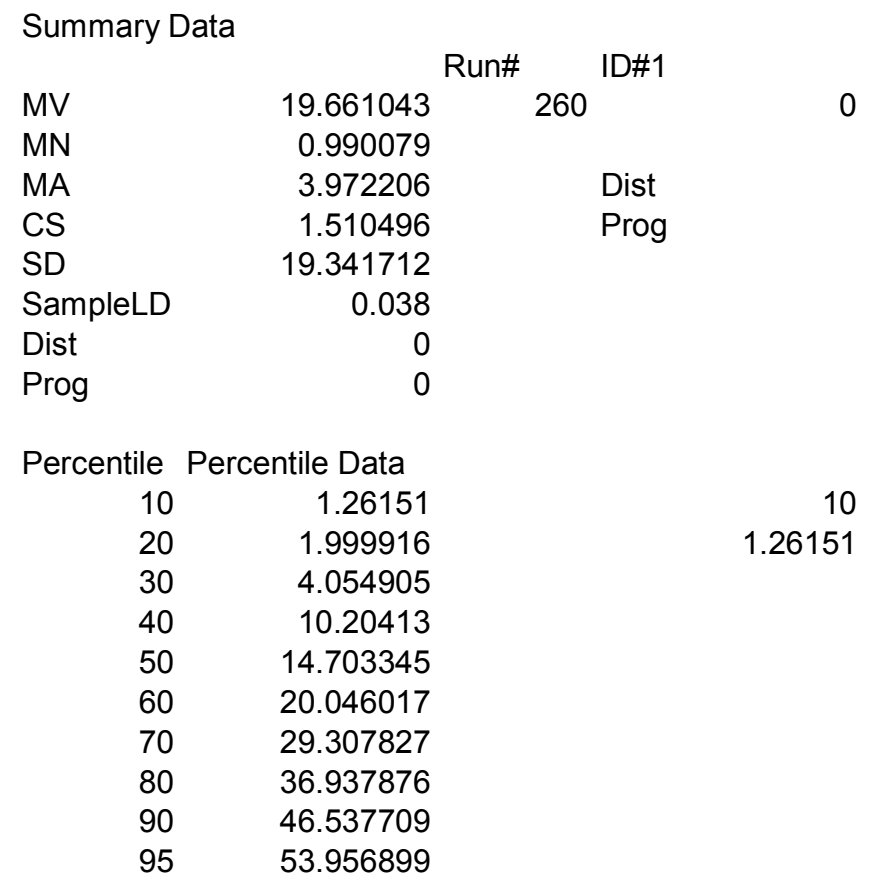

Number of Channels

50

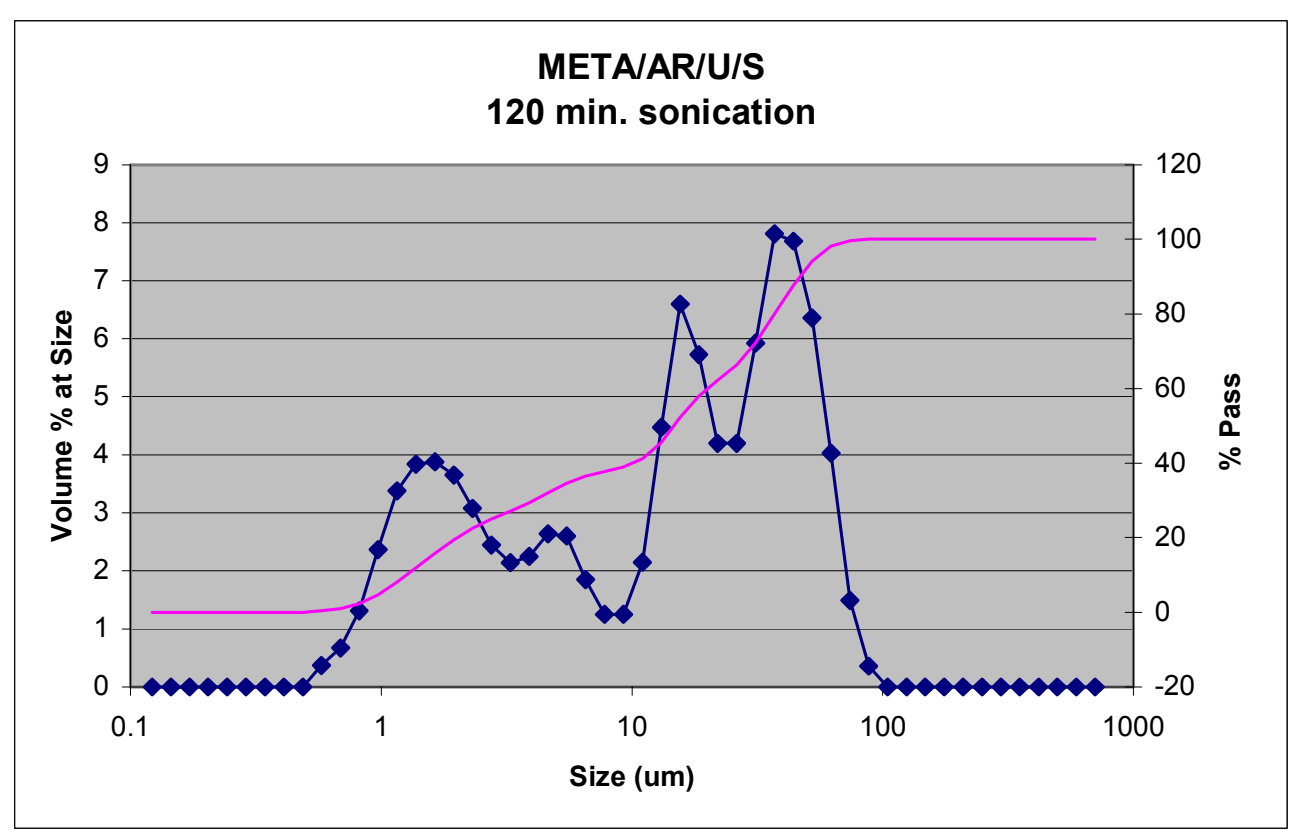




\begin{tabular}{|c|c|c|c|}
\hline \multicolumn{4}{|c|}{ META/AR/U/S } \\
\hline Channel & Selected E & & mmulative \\
\hline 1 & 704 & 0 & 100 \\
\hline 2 & 592 & 0 & 100 \\
\hline 3 & 497.8 & 0 & 100 \\
\hline 4 & 418.6 & 0 & 100 \\
\hline 5 & 352 & 0 & 100 \\
\hline 6 & 296 & 0 & 100 \\
\hline 7 & 248.9 & 0 & 100 \\
\hline 8 & 209.3 & 0 & 100 \\
\hline 9 & 176 & 0 & 100 \\
\hline 10 & 148 & 0 & 100 \\
\hline 11 & 124.5 & 0 & 100 \\
\hline 12 & 104.7 & 0 & 100 \\
\hline 13 & 88 & 0.36 & 100 \\
\hline 14 & 74 & 1.49 & 99.64 \\
\hline 15 & 62.23 & 4.03 & 98.15 \\
\hline 16 & 52.33 & 6.36 & 94.12 \\
\hline 17 & 44 & 7.68 & 87.76 \\
\hline 18 & 37 & 7.81 & 80.08 \\
\hline 19 & 31.11 & 5.93 & 72.27 \\
\hline 20 & 26.16 & 4.2 & 66.34 \\
\hline 21 & 22 & 4.2 & 62.14 \\
\hline 22 & 18.5 & 5.73 & 57.94 \\
\hline 23 & 15.56 & 6.6 & 52.21 \\
\hline 24 & 13.08 & 4.48 & 45.61 \\
\hline 25 & 11 & 2.15 & 41.13 \\
\hline 26 & 9.25 & 1.25 & 38.98 \\
\hline 27 & 7.778 & 1.25 & 37.73 \\
\hline 28 & 6.541 & 1.85 & 36.48 \\
\hline 29 & 5.5 & 2.6 & 34.63 \\
\hline 30 & 4.625 & 2.64 & 32.03 \\
\hline 31 & 3.889 & 2.25 & 29.39 \\
\hline 32 & 3.27 & 2.14 & 27.14 \\
\hline 33 & 2.75 & 2.45 & 25 \\
\hline 34 & 2.312 & 3.08 & 22.55 \\
\hline 35 & 1.945 & 3.65 & 19.47 \\
\hline 36 & 1.635 & 3.88 & 15.82 \\
\hline 37 & 1.375 & 3.84 & 11.94 \\
\hline 38 & 1.156 & 3.38 & 8.1 \\
\hline 39 & 0.972 & 2.37 & 4.72 \\
\hline 40 & 0.818 & 1.31 & 2.35 \\
\hline 41 & 0.688 & 0.67 & 1.04 \\
\hline 42 & 0.578 & 0.37 & 0.37 \\
\hline 43 & 0.486 & 0 & $-9.32587 \mathrm{E}-15$ \\
\hline 44 & 0.409 & 0 & $-9.32587 \mathrm{E}-15$ \\
\hline 45 & 0.344 & 0 & $-9.32587 \mathrm{E}-15$ \\
\hline 46 & 0.289 & 0 & $-9.32587 \mathrm{E}-15$ \\
\hline 47 & 0.243 & 0 & $-9.32587 E-15$ \\
\hline 48 & 0.204 & 0 & $-9.32587 E-15$ \\
\hline 49 & 0.172 & 0 & $-9.32587 \mathrm{E}-15$ \\
\hline 50 & 0.145 & 0 & $-9.32587 \mathrm{E}-15$ \\
\hline 51 & 0.122 & 0 & $-9.32587 E-15$ \\
\hline
\end{tabular}

E-14 


\section{Summary Data}

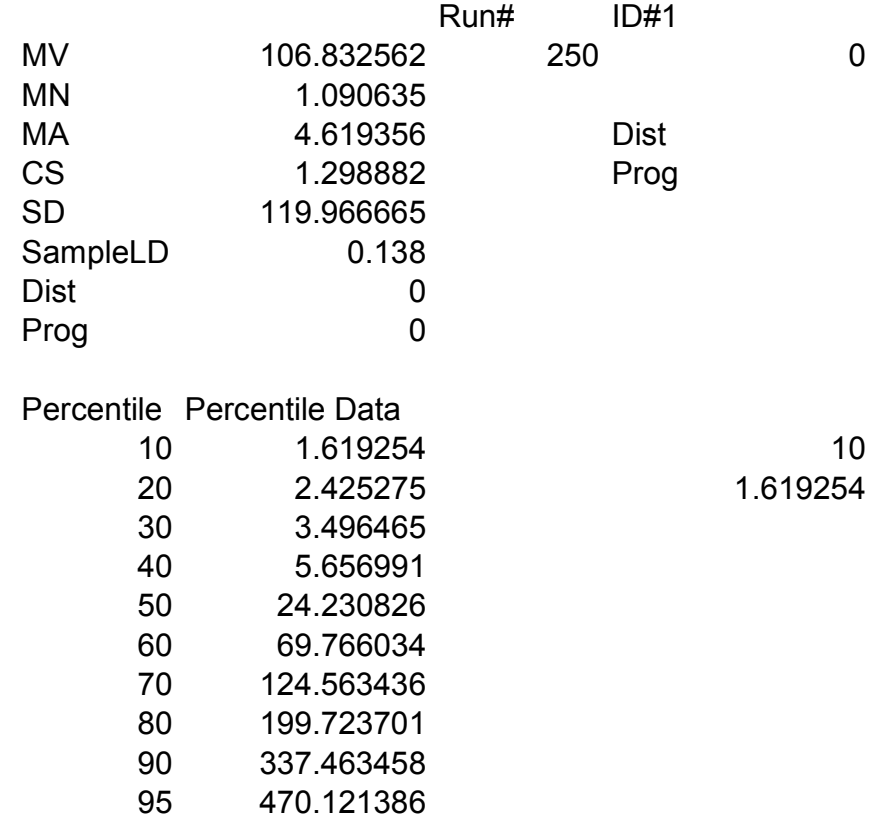

Number of Channels

50

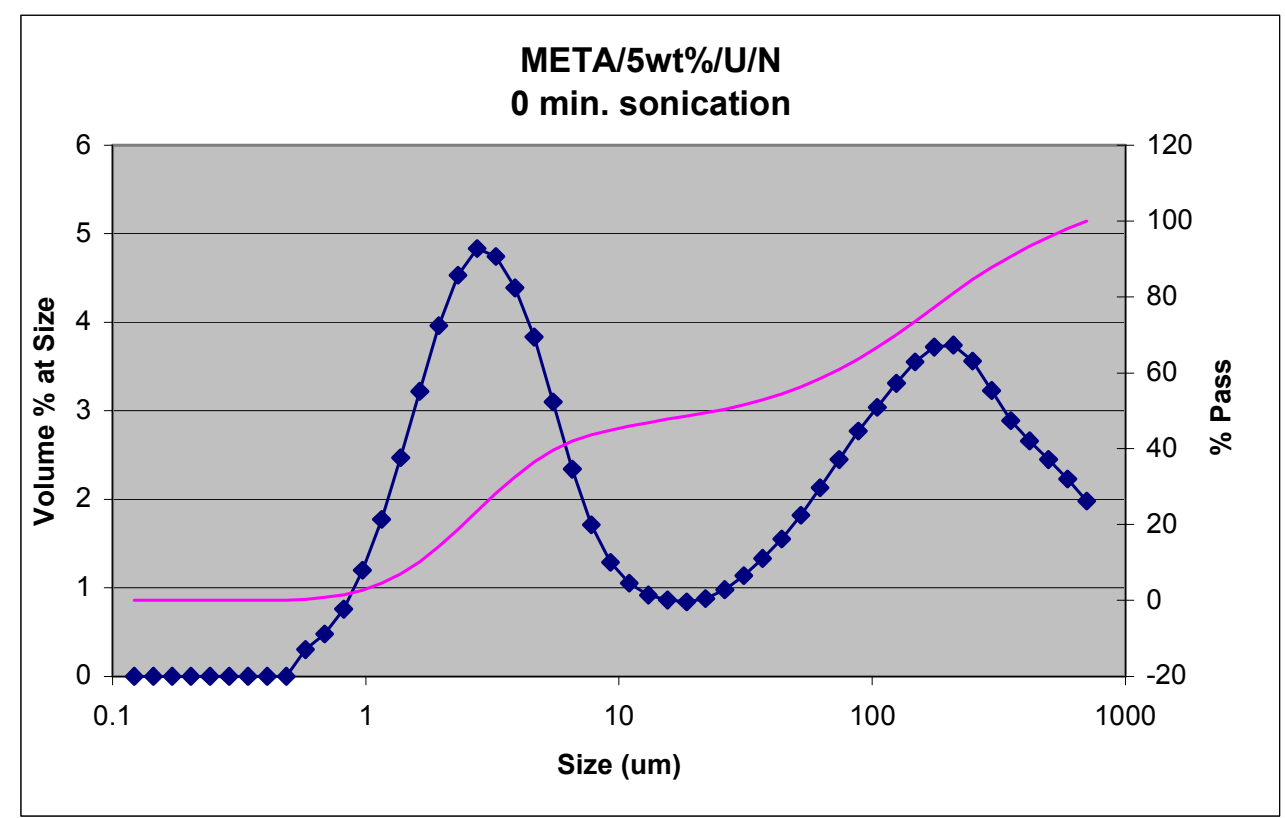




\begin{tabular}{|c|c|c|c|}
\hline \multicolumn{4}{|c|}{ META/5wt\%/U/N } \\
\hline \multicolumn{4}{|c|}{ Selected } \\
\hline Channel & Selected E & me & Cummulative \\
\hline 1 & 704 & 1.98 & 100 \\
\hline 2 & 592 & 2.23 & 98.02 \\
\hline 3 & 497.8 & 2.45 & 95.79 \\
\hline 4 & 418.6 & 2.66 & 93.34 \\
\hline 5 & 352 & 2.89 & 90.68 \\
\hline 6 & 296 & 3.23 & 87.79 \\
\hline 7 & 248.9 & 3.56 & 84.56 \\
\hline 8 & 209.3 & 3.74 & 81 \\
\hline 9 & 176 & 3.72 & 77.26 \\
\hline 10 & 148 & 3.55 & 73.54 \\
\hline 11 & 124.5 & 3.31 & 69.99 \\
\hline 12 & 104.7 & 3.04 & 66.68 \\
\hline 13 & 88 & 2.77 & 63.64 \\
\hline 14 & 74 & 2.45 & 60.87 \\
\hline 15 & 62.23 & 2.13 & 58.42 \\
\hline 16 & 52.33 & 1.82 & 56.29 \\
\hline 17 & 44 & 1.55 & 54.47 \\
\hline 18 & 37 & 1.33 & 52.92 \\
\hline 19 & 31.11 & 1.14 & 51.59 \\
\hline 20 & 26.16 & 0.98 & 50.45 \\
\hline 21 & 22 & 0.88 & 49.47 \\
\hline 22 & 18.5 & 0.84 & 48.59 \\
\hline 23 & 15.56 & 0.86 & 47.75 \\
\hline 24 & 13.08 & 0.92 & 46.89 \\
\hline 25 & 11 & 1.05 & 45.97 \\
\hline 26 & 9.25 & 1.29 & 44.92 \\
\hline 27 & 7.778 & 1.71 & 43.63 \\
\hline 28 & 6.541 & 2.34 & 41.92 \\
\hline 29 & 5.5 & 3.1 & 39.58 \\
\hline 30 & 4.625 & 3.83 & 36.48 \\
\hline 31 & 3.889 & 4.39 & 32.65 \\
\hline 32 & 3.27 & 4.74 & 28.26 \\
\hline 33 & 2.75 & 4.83 & 23.52 \\
\hline 34 & 2.312 & 4.53 & 18.69 \\
\hline 35 & 1.945 & 3.96 & 14.16 \\
\hline 36 & 1.635 & 3.22 & 10.2 \\
\hline 37 & 1.375 & 2.47 & 6.98 \\
\hline 38 & 1.156 & 1.77 & 4.51 \\
\hline 39 & 0.972 & 1.2 & 2.74 \\
\hline 40 & 0.818 & 0.76 & 1.54 \\
\hline 41 & 0.688 & 0.48 & 0.78 \\
\hline 42 & 0.578 & 0.3 & 0.3 \\
\hline 43 & 0.486 & 0 & $-1.97065 \mathrm{E}-14$ \\
\hline 44 & 0.409 & 0 & $-1.97065 \mathrm{E}-14$ \\
\hline 45 & 0.344 & 0 & $-1.97065 E-14$ \\
\hline 46 & 0.289 & 0 & $-1.97065 \mathrm{E}-14$ \\
\hline 47 & 0.243 & 0 & $-1.97065 \mathrm{E}-14$ \\
\hline 48 & 0.204 & 0 & $-1.97065 \mathrm{E}-14$ \\
\hline 49 & 0.172 & 0 & $-1.97065 \mathrm{E}-14$ \\
\hline 50 & 0.145 & 0 & $-1.97065 \mathrm{E}-14$ \\
\hline 51 & 0.122 & 0 & $-1.97065 \mathrm{E}-14$ \\
\hline
\end{tabular}

E-16 


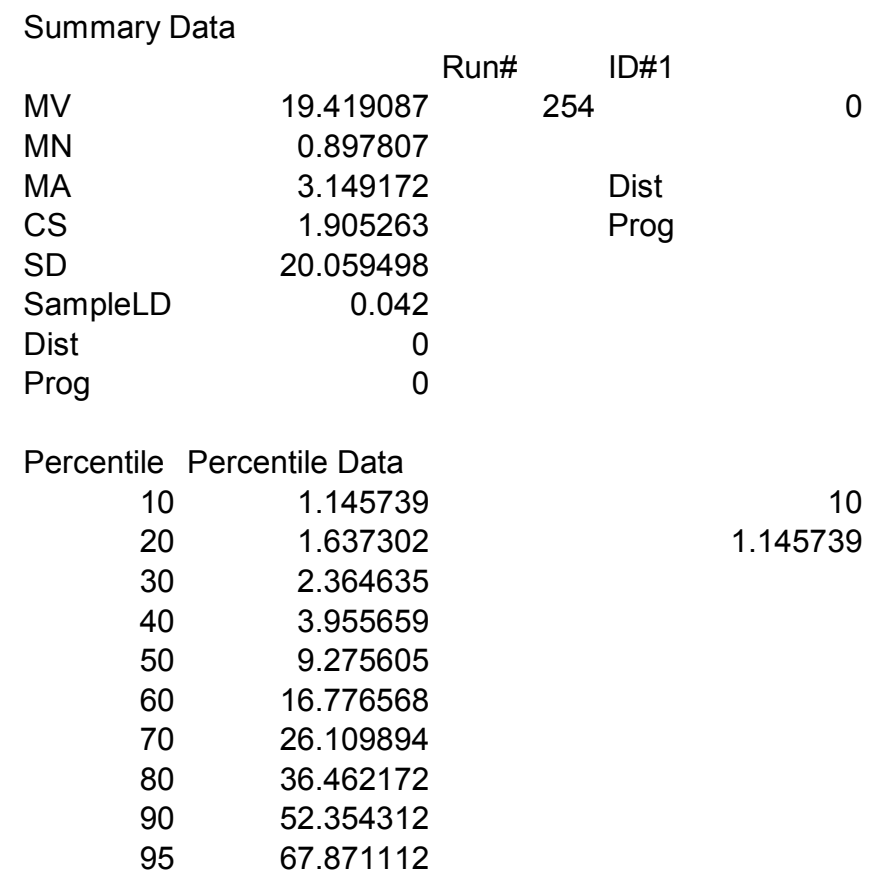

Number of Channels 50

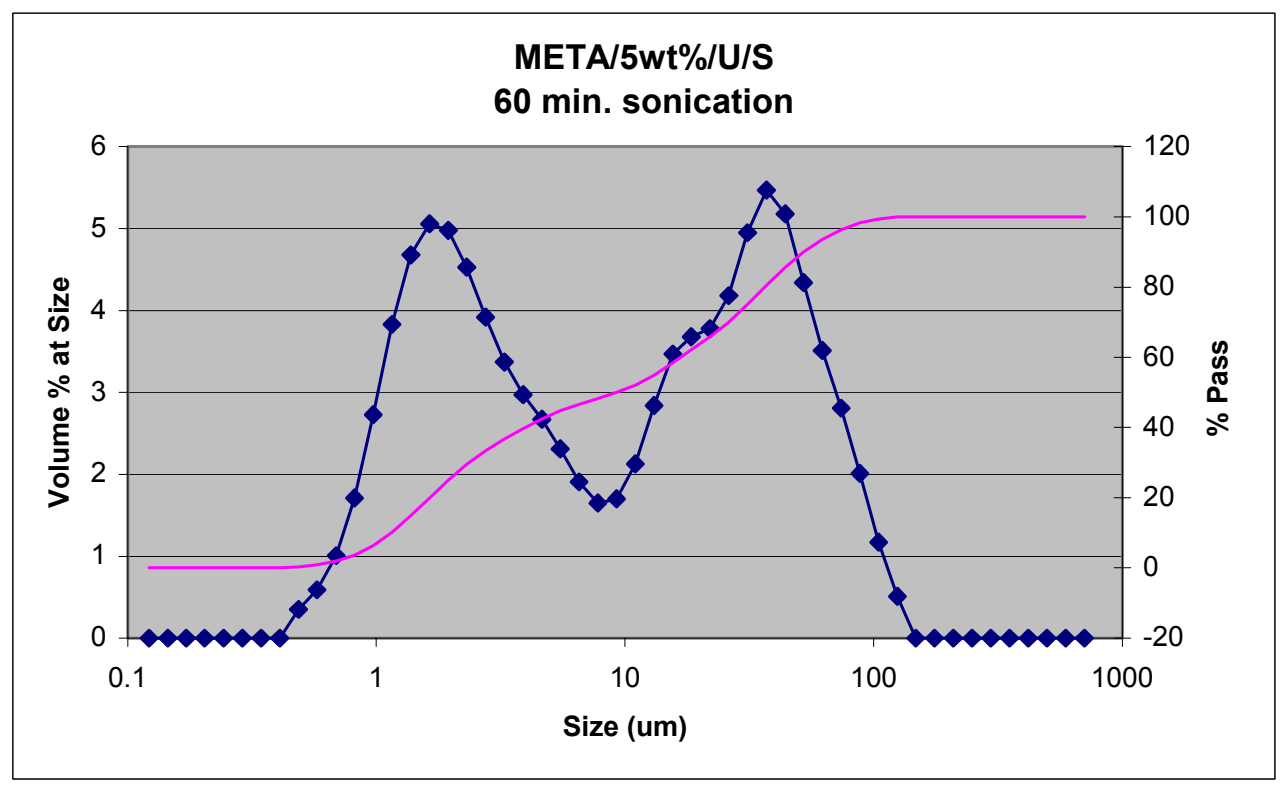




\begin{tabular}{|c|c|c|c|}
\hline \multicolumn{4}{|c|}{ META/5wt\%/U/S } \\
\hline Channel & Selected E & & mmulative \\
\hline 1 & 704 & 0 & 100 \\
\hline 2 & 592 & 0 & 100 \\
\hline 3 & 497.8 & 0 & 100 \\
\hline 4 & 418.6 & 0 & 100 \\
\hline 5 & 352 & 0 & 100 \\
\hline 6 & 296 & 0 & 100 \\
\hline 7 & 248.9 & 0 & 100 \\
\hline 8 & 209.3 & 0 & 100 \\
\hline 9 & 176 & 0 & 100 \\
\hline 10 & 148 & 0 & 100 \\
\hline 11 & 124.5 & 0.51 & 100 \\
\hline 12 & 104.7 & 1.17 & 99.49 \\
\hline 13 & 88 & 2.01 & 98.32 \\
\hline 14 & 74 & 2.81 & 96.31 \\
\hline 15 & 62.23 & 3.51 & 93.5 \\
\hline 16 & 52.33 & 4.34 & 89.99 \\
\hline 17 & 44 & 5.18 & 85.65 \\
\hline 18 & 37 & 5.47 & 80.47 \\
\hline 19 & 31.11 & 4.95 & 75 \\
\hline 20 & 26.16 & 4.18 & 70.05 \\
\hline 21 & 22 & 3.78 & 65.87 \\
\hline 22 & 18.5 & 3.68 & 62.09 \\
\hline 23 & 15.56 & 3.47 & 58.41 \\
\hline 24 & 13.08 & 2.84 & 54.94 \\
\hline 25 & 11 & 2.13 & 52.1 \\
\hline 26 & 9.25 & 1.7 & 49.97 \\
\hline 27 & 7.778 & 1.65 & 48.27 \\
\hline 28 & 6.541 & 1.91 & 46.62 \\
\hline 29 & 5.5 & 2.31 & 44.71 \\
\hline 30 & 4.625 & 2.67 & 42.4 \\
\hline 31 & 3.889 & 2.97 & 39.73 \\
\hline 32 & 3.27 & 3.37 & 36.76 \\
\hline 33 & 2.75 & 3.92 & 33.39 \\
\hline 34 & 2.312 & 4.53 & 29.47 \\
\hline 35 & 1.945 & 4.98 & 24.94 \\
\hline 36 & 1.635 & 5.06 & 19.96 \\
\hline 37 & 1.375 & 4.68 & 14.9 \\
\hline 38 & 1.156 & 3.83 & 10.22 \\
\hline 39 & 0.972 & 2.73 & 6.39 \\
\hline 40 & 0.818 & 1.71 & 3.66 \\
\hline 41 & 0.688 & 1.01 & 1.95 \\
\hline 42 & 0.578 & 0.59 & 0.94 \\
\hline 43 & 0.486 & 0.35 & 0.35 \\
\hline 44 & 0.409 & 0 & $-2.24265 \mathrm{E}-14$ \\
\hline 45 & 0.344 & 0 & $-2.24265 \mathrm{E}-14$ \\
\hline 46 & 0.289 & 0 & $-2.24265 E-14$ \\
\hline 47 & 0.243 & 0 & $-2.24265 \mathrm{E}-14$ \\
\hline 48 & 0.204 & 0 & $-2.24265 \mathrm{E}-14$ \\
\hline 49 & 0.172 & 0 & $-2.24265 \mathrm{E}-14$ \\
\hline 50 & 0.145 & 0 & $-2.24265 \mathrm{E}-14$ \\
\hline 51 & 0.122 & 0 & $-2.24265 \mathrm{E}-14$ \\
\hline
\end{tabular}

E-18 
as received, batch 1

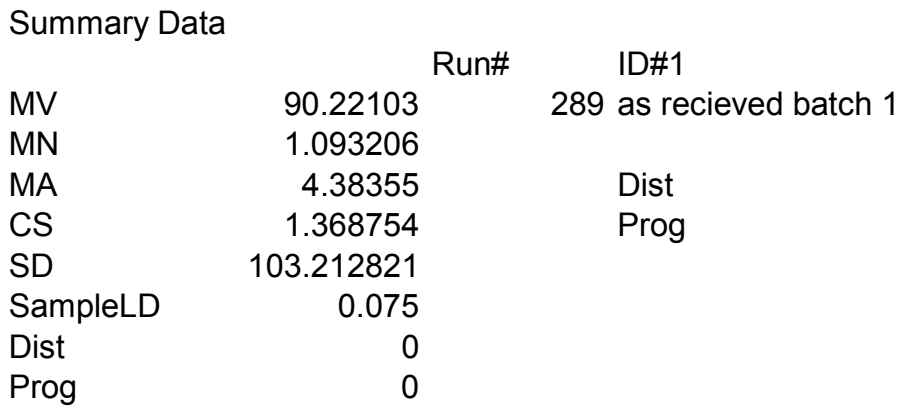

Percentile Percentile Data

$\begin{array}{rrr}10 & 1.582949 & 10 \\ 20 & 2.329749 & 1.582949 \\ 30 & 3.261217 & \\ 40 & 4.910314 & \\ 50 & 16.011441 & \\ 60 & 55.246235 & \\ 70 & 99.468926 & \\ 80 & 169.018888 & \\ 90 & 284.47071 & \\ 95 & 390.2206 & \end{array}$

Number of Channels

50

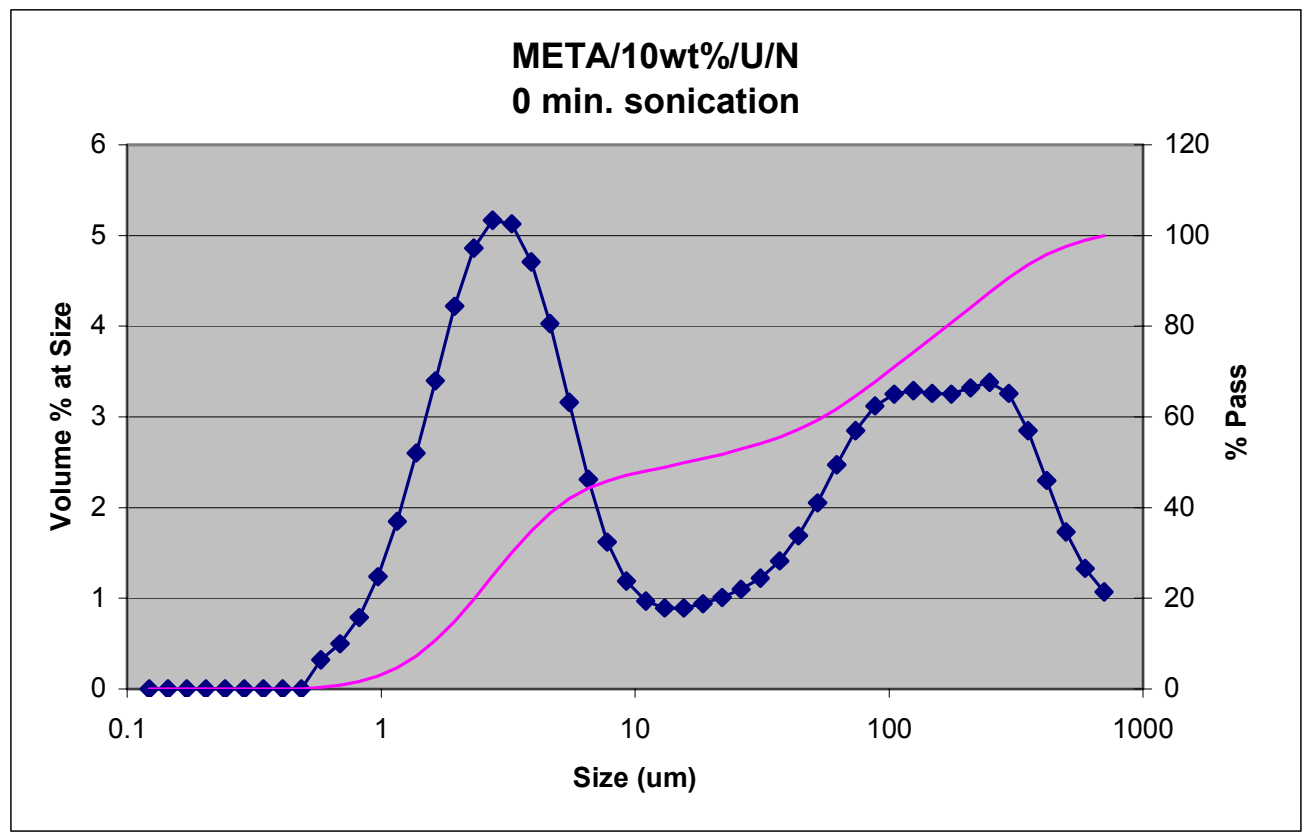




\begin{tabular}{|c|c|c|c|}
\hline \multicolumn{4}{|c|}{ META/10wt\%/U/N } \\
\hline Channel & Selected E & & Cummulative \\
\hline 1 & 704 & 1.07 & 100 \\
\hline 2 & 592 & 1.33 & 98.93 \\
\hline 3 & 497.8 & 1.73 & 97.6 \\
\hline 4 & 418.6 & 2.3 & 95.87 \\
\hline 5 & 352 & 2.85 & 93.57 \\
\hline 6 & 296 & 3.26 & 90.72 \\
\hline 7 & 248.9 & 3.38 & 87.46 \\
\hline 8 & 209.3 & 3.32 & 84.08 \\
\hline 9 & 176 & 3.25 & 80.76 \\
\hline 10 & 148 & 3.26 & 77.51 \\
\hline 11 & 124.5 & 3.29 & 74.25 \\
\hline 12 & 104.7 & 3.25 & 70.96 \\
\hline 13 & 88 & 3.12 & 67.71 \\
\hline 14 & 74 & 2.85 & 64.59 \\
\hline 15 & 62.23 & 2.47 & 61.74 \\
\hline 16 & 52.33 & 2.05 & 59.27 \\
\hline 17 & 44 & 1.69 & 57.22 \\
\hline 18 & 37 & 1.41 & 55.53 \\
\hline 19 & 31.11 & 1.22 & 54.12 \\
\hline 20 & 26.16 & 1.1 & 52.9 \\
\hline 21 & 22 & 1.01 & 51.8 \\
\hline 22 & 18.5 & 0.94 & 50.79 \\
\hline 23 & 15.56 & 0.89 & 49.85 \\
\hline 24 & 13.08 & 0.89 & 48.96 \\
\hline 25 & 11 & 0.97 & 48.07 \\
\hline 26 & 9.25 & 1.19 & 47.1 \\
\hline 27 & 7.778 & 1.62 & 45.91 \\
\hline 28 & 6.541 & 2.31 & 44.29 \\
\hline 29 & 5.5 & 3.16 & 41.98 \\
\hline 30 & 4.625 & 4.03 & 38.82 \\
\hline 31 & 3.889 & 4.71 & 34.79 \\
\hline 32 & 3.27 & 5.13 & 30.08 \\
\hline 33 & 2.75 & 5.17 & 24.95 \\
\hline 34 & 2.312 & 4.86 & 19.78 \\
\hline 35 & 1.945 & 4.22 & 14.92 \\
\hline 36 & 1.635 & 3.4 & 10.7 \\
\hline 37 & 1.375 & 2.6 & 7.3 \\
\hline 38 & 1.156 & 1.85 & 4.7 \\
\hline 39 & 0.972 & 1.24 & 2.85 \\
\hline 40 & 0.818 & 0.79 & 1.61 \\
\hline 41 & 0.688 & 0.5 & 0.82 \\
\hline 42 & 0.578 & 0.32 & 0.32 \\
\hline 43 & 0.486 & 0 & $2.40363 \mathrm{E}-14$ \\
\hline 44 & 0.409 & 0 & 2.40363E-14 \\
\hline 45 & 0.344 & 0 & $2.40363 \mathrm{E}-14$ \\
\hline 46 & 0.289 & 0 & $2.40363 \mathrm{E}-14$ \\
\hline 47 & 0.243 & 0 & $2.40363 \mathrm{E}-14$ \\
\hline 48 & 0.204 & 0 & 2.40363E-14 \\
\hline 49 & 0.172 & 0 & $2.40363 \mathrm{E}-14$ \\
\hline 50 & 0.145 & 0 & 2.40363E-14 \\
\hline 51 & 0.122 & 0 & $2.40363 \mathrm{E}-14$ \\
\hline
\end{tabular}




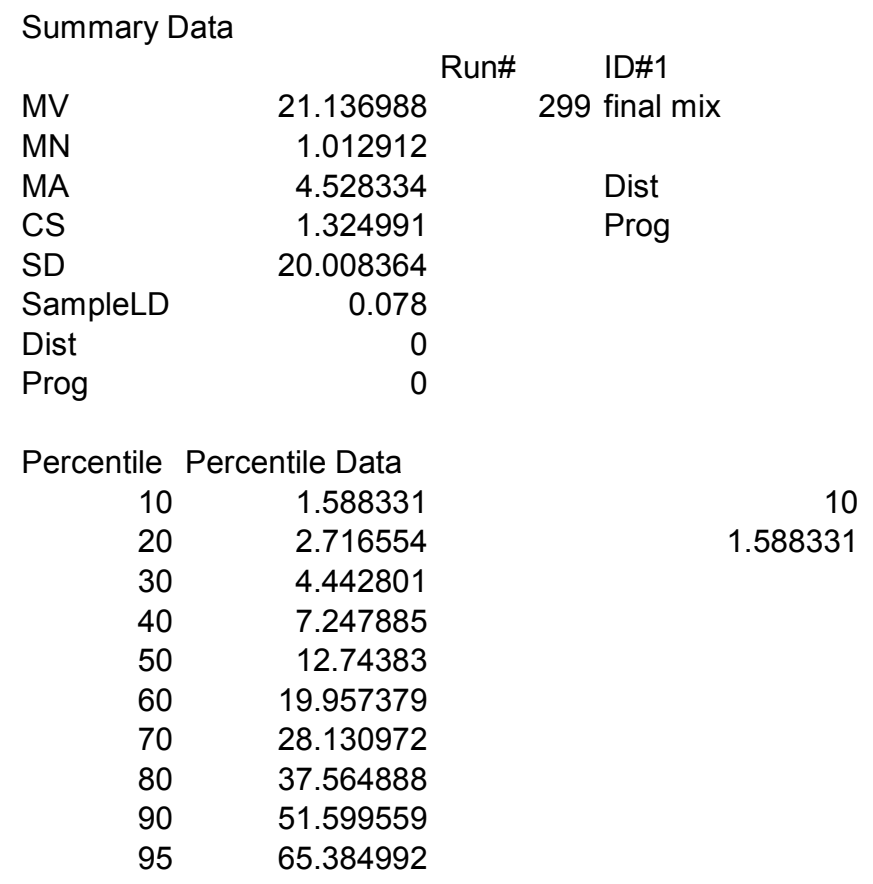

Number of Channels

50

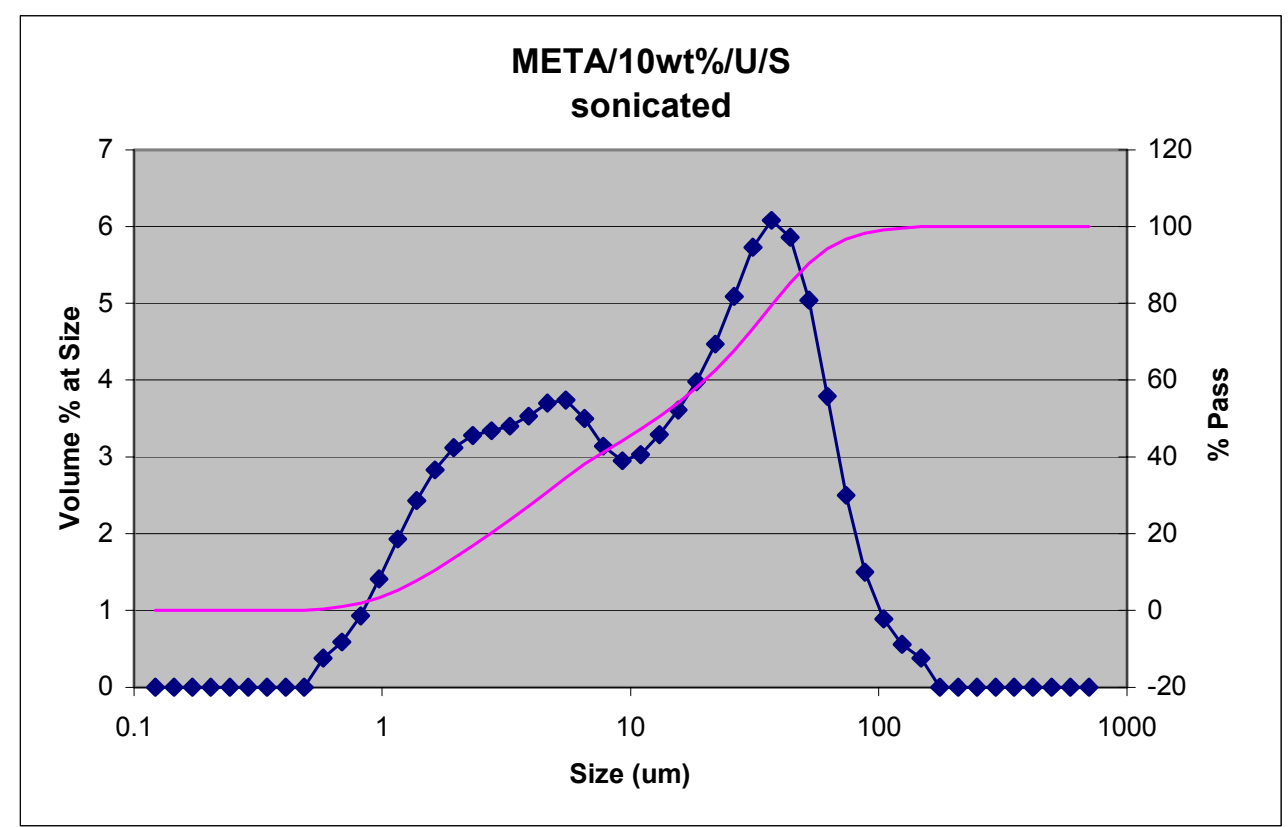




\begin{tabular}{|c|c|c|c|}
\hline \multicolumn{4}{|c|}{ META/10wt\%/U/S } \\
\hline Channel & Selected E & & mmulative \\
\hline 1 & 704 & 0 & 100 \\
\hline 2 & 592 & 0 & 100 \\
\hline 3 & 497.8 & 0 & 100 \\
\hline 4 & 418.6 & 0 & 100 \\
\hline 5 & 352 & 0 & 100 \\
\hline 6 & 296 & 0 & 100 \\
\hline 7 & 248.9 & 0 & 100 \\
\hline 8 & 209.3 & 0 & 100 \\
\hline 9 & 176 & 0 & 100 \\
\hline 10 & 148 & 0.38 & 100 \\
\hline 11 & 124.5 & 0.56 & 99.62 \\
\hline 12 & 104.7 & 0.89 & 99.06 \\
\hline 13 & 88 & 1.5 & 98.17 \\
\hline 14 & 74 & 2.5 & 96.67 \\
\hline 15 & 62.23 & 3.79 & 94.17 \\
\hline 16 & 52.33 & 5.04 & 90.38 \\
\hline 17 & 44 & 5.86 & 85.34 \\
\hline 18 & 37 & 6.08 & 79.48 \\
\hline 19 & 31.11 & 5.73 & 73.4 \\
\hline 20 & 26.16 & 5.09 & 67.67 \\
\hline 21 & 22 & 4.47 & 62.58 \\
\hline 22 & 18.5 & 3.98 & 58.11 \\
\hline 23 & 15.56 & 3.61 & 54.13 \\
\hline 24 & 13.08 & 3.29 & 50.52 \\
\hline 25 & 11 & 3.03 & 47.23 \\
\hline 26 & 9.25 & 2.95 & 44.2 \\
\hline 27 & 7.778 & 3.14 & 41.25 \\
\hline 28 & 6.541 & 3.5 & 38.11 \\
\hline 29 & 5.5 & 3.74 & 34.61 \\
\hline 30 & 4.625 & 3.7 & 30.87 \\
\hline 31 & 3.889 & 3.53 & 27.17 \\
\hline 32 & 3.27 & 3.4 & 23.64 \\
\hline 33 & 2.75 & 3.34 & 20.24 \\
\hline 34 & 2.312 & 3.28 & 16.9 \\
\hline 35 & 1.945 & 3.12 & 13.62 \\
\hline 36 & 1.635 & 2.83 & 10.5 \\
\hline 37 & 1.375 & 2.43 & 7.67 \\
\hline 38 & 1.156 & 1.93 & 5.24 \\
\hline 39 & 0.972 & 1.41 & 3.31 \\
\hline 40 & 0.818 & 0.93 & 1.9 \\
\hline 41 & 0.688 & 0.59 & 0.97 \\
\hline 42 & 0.578 & 0.38 & 0.38 \\
\hline 43 & 0.486 & 0 & $-1.36557 \mathrm{E}-14$ \\
\hline 44 & 0.409 & 0 & $-1.36557 \mathrm{E}-14$ \\
\hline 45 & 0.344 & 0 & $-1.36557 \mathrm{E}-14$ \\
\hline 46 & 0.289 & 0 & $-1.36557 \mathrm{E}-14$ \\
\hline 47 & 0.243 & 0 & $-1.36557 \mathrm{E}-14$ \\
\hline 48 & 0.204 & 0 & $-1.36557 \mathrm{E}-14$ \\
\hline 49 & 0.172 & 0 & $-1.36557 \mathrm{E}-14$ \\
\hline 50 & 0.145 & 0 & $-1.36557 \mathrm{E}-14$ \\
\hline 51 & 0.122 & 0 & $-1.36557 \mathrm{E}-14$ \\
\hline
\end{tabular}


Summary Data

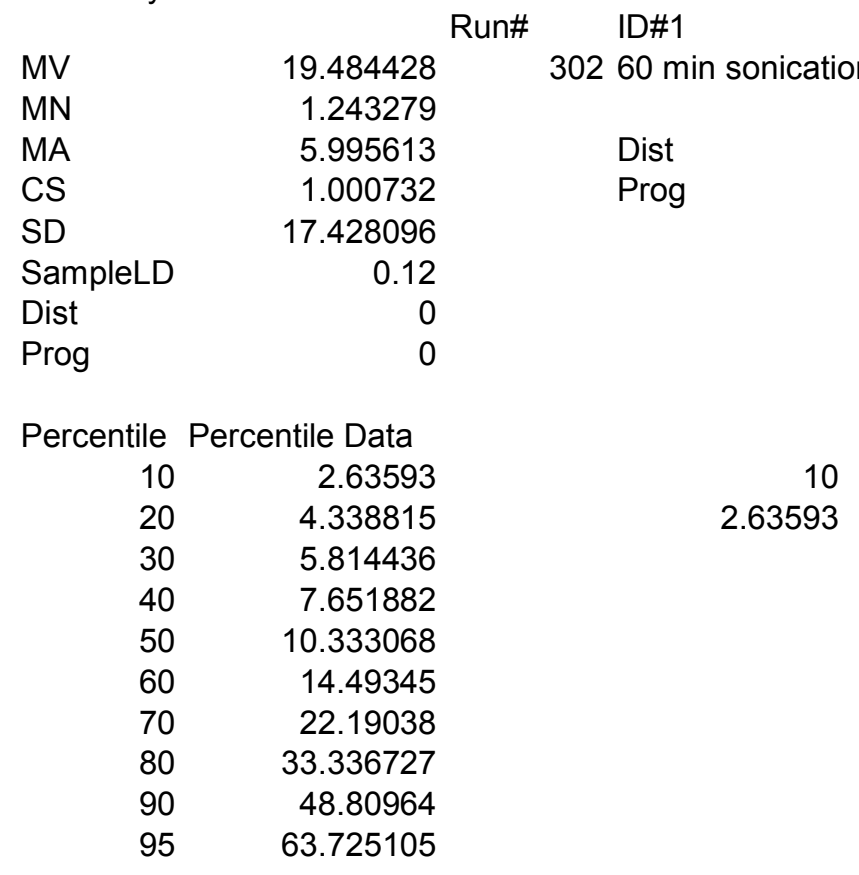

Number of Channels

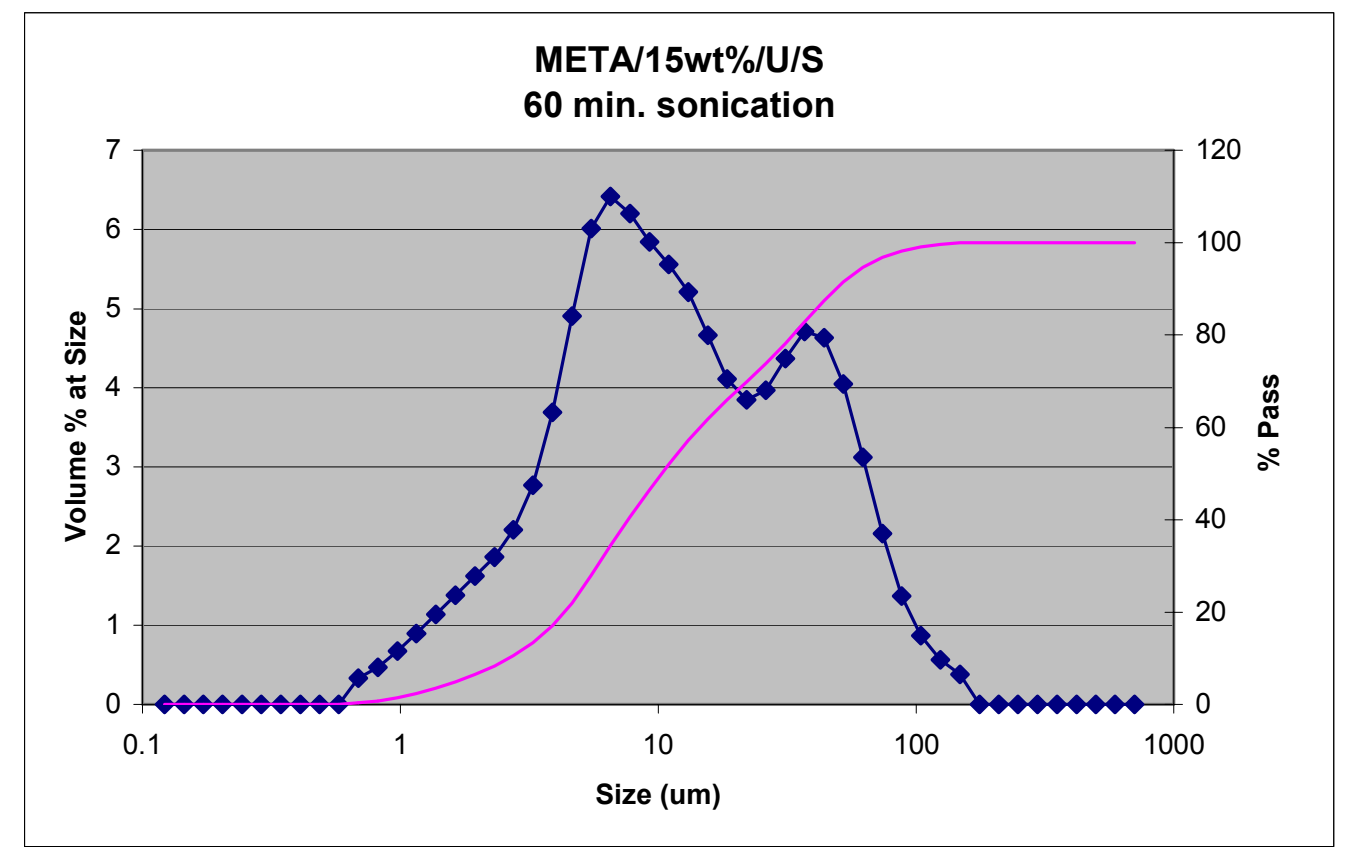




\begin{tabular}{|c|c|c|c|}
\hline \multicolumn{4}{|c|}{ META/15wt\%/U/S } \\
\hline Channel & Selected E & me & Immulative \\
\hline 1 & 704 & 0 & 100 \\
\hline 2 & 592 & 0 & 100 \\
\hline 3 & 497.8 & 0 & 100 \\
\hline 4 & 418.6 & 0 & 100 \\
\hline 5 & 352 & 0 & 100 \\
\hline 6 & 296 & 0 & 100 \\
\hline 7 & 248.9 & 0 & 100 \\
\hline 8 & 209.3 & 0 & 100 \\
\hline 9 & 176 & 0 & 100 \\
\hline 10 & 148 & 0.38 & 100 \\
\hline 11 & 124.5 & 0.56 & 99.62 \\
\hline 12 & 104.7 & 0.87 & 99.06 \\
\hline 13 & 88 & 1.37 & 98.19 \\
\hline 14 & 74 & 2.16 & 96.82 \\
\hline 15 & 62.23 & 3.12 & 94.66 \\
\hline 16 & 52.33 & 4.05 & 91.54 \\
\hline 17 & 44 & 4.63 & 87.49 \\
\hline 18 & 37 & 4.71 & 82.86 \\
\hline 19 & 31.11 & 4.37 & 78.15 \\
\hline 20 & 26.16 & 3.97 & 73.78 \\
\hline 21 & 22 & 3.85 & 69.81 \\
\hline 22 & 18.5 & 4.11 & 65.96 \\
\hline 23 & 15.56 & 4.66 & 61.85 \\
\hline 24 & 13.08 & 5.21 & 57.19 \\
\hline 25 & 11 & 5.56 & 51.98 \\
\hline 26 & 9.25 & 5.84 & 46.42 \\
\hline 27 & 7.778 & 6.2 & 40.58 \\
\hline 28 & 6.541 & 6.42 & 34.38 \\
\hline 29 & 5.5 & 6.01 & 27.96 \\
\hline 30 & 4.625 & 4.91 & 21.95 \\
\hline 31 & 3.889 & 3.69 & 17.04 \\
\hline 32 & 3.27 & 2.77 & 13.35 \\
\hline 33 & 2.75 & 2.21 & 10.58 \\
\hline 34 & 2.312 & 1.86 & 8.37 \\
\hline 35 & 1.945 & 1.62 & 6.51 \\
\hline 36 & 1.635 & 1.38 & 4.89 \\
\hline 37 & 1.375 & 1.14 & 3.51 \\
\hline 38 & 1.156 & 0.9 & 2.37 \\
\hline 39 & 0.972 & 0.67 & 1.47 \\
\hline 40 & 0.818 & 0.47 & 0.8 \\
\hline 41 & 0.688 & 0.33 & 0.33 \\
\hline 42 & 0.578 & 0 & $1.17129 \mathrm{E}-14$ \\
\hline 43 & 0.486 & 0 & $1.17129 \mathrm{E}-14$ \\
\hline 44 & 0.409 & 0 & $1.17129 \mathrm{E}-14$ \\
\hline 45 & 0.344 & 0 & $1.17129 \mathrm{E}-14$ \\
\hline 46 & 0.289 & 0 & $1.17129 \mathrm{E}-14$ \\
\hline 47 & 0.243 & 0 & $1.17129 \mathrm{E}-14$ \\
\hline 48 & 0.204 & 0 & $1.17129 \mathrm{E}-14$ \\
\hline 49 & 0.172 & 0 & $1.17129 \mathrm{E}-14$ \\
\hline 50 & 0.145 & 0 & $1.17129 \mathrm{E}-14$ \\
\hline 51 & 0.122 & 0 & $1.17129 \mathrm{E}-14$ \\
\hline
\end{tabular}


Summary Data

\begin{tabular}{|c|c|c|}
\hline MV & 127.026598 & $2680 \mathrm{~min}$ sonicatic \\
\hline $\mathrm{MN}$ & 1.212747 & \\
\hline MA & 4.924112 & Dist \\
\hline CS & 1.218494 & Prog \\
\hline SD & 147.718885 & \\
\hline SampleLD & 0.107 & \\
\hline Dist & 0 & \\
\hline Prog & 0 & \\
\hline Percentile & Percentile Data & \\
\hline 10 & 1.713664 & 10 \\
\hline 20 & 2.549863 & 1.713664 \\
\hline 30 & 3.628936 & \\
\hline 40 & 5.892355 & \\
\hline 50 & 31.688157 & \\
\hline 60 & 83.018655 & \\
\hline 70 & 148.328903 & \\
\hline 80 & 243.645189 & \\
\hline 90 & 424.874129 & \\
\hline 95 & 563.29269 & \\
\hline
\end{tabular}

Number of Channels

50

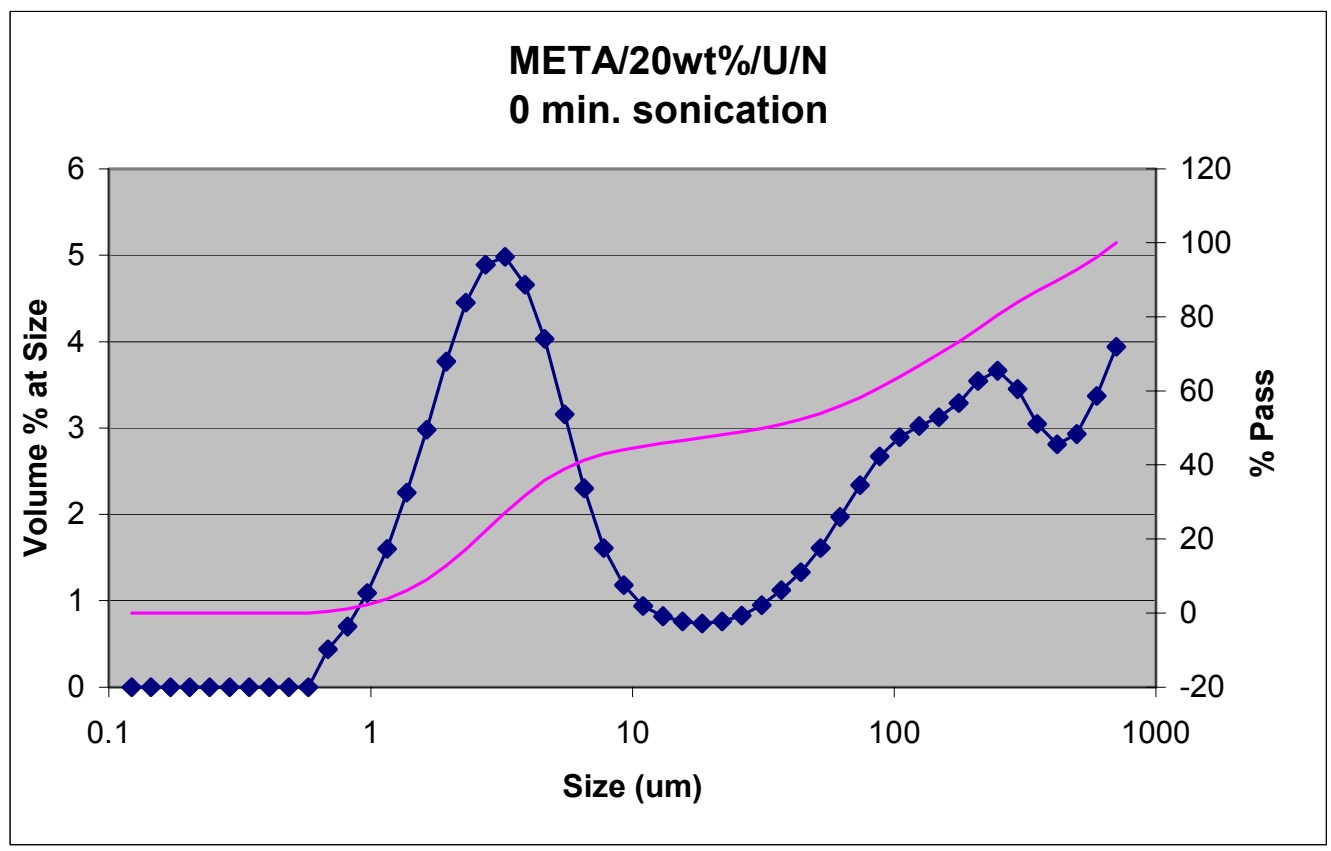




\begin{tabular}{|c|c|c|c|}
\hline \multicolumn{4}{|c|}{ META/20wt\%/U/N } \\
\hline Channel & Selected E & & Cummulative \\
\hline 1 & 704 & 3.94 & 100 \\
\hline 2 & 592 & 3.37 & 96.06 \\
\hline 3 & 497.8 & 2.93 & 92.69 \\
\hline 4 & 418.6 & 2.81 & 89.76 \\
\hline 5 & 352 & 3.05 & 86.95 \\
\hline 6 & 296 & 3.45 & 83.9 \\
\hline 7 & 248.9 & 3.66 & 80.45 \\
\hline 8 & 209.3 & 3.54 & 76.79 \\
\hline 9 & 176 & 3.29 & 73.25 \\
\hline 10 & 148 & 3.12 & 69.96 \\
\hline 11 & 124.5 & 3.02 & 66.84 \\
\hline 12 & 104.7 & 2.89 & 63.82 \\
\hline 13 & 88 & 2.67 & 60.93 \\
\hline 14 & 74 & 2.34 & 58.26 \\
\hline 15 & 62.23 & 1.97 & 55.92 \\
\hline 16 & 52.33 & 1.61 & 53.95 \\
\hline 17 & 44 & 1.33 & 52.34 \\
\hline 18 & 37 & 1.12 & 51.01 \\
\hline 19 & 31.11 & 0.95 & 49.89 \\
\hline 20 & 26.16 & 0.83 & 48.94 \\
\hline 21 & 22 & 0.76 & 48.11 \\
\hline 22 & 18.5 & 0.74 & 47.35 \\
\hline 23 & 15.56 & 0.76 & 46.61 \\
\hline 24 & 13.08 & 0.82 & 45.85 \\
\hline 25 & 11 & 0.94 & 45.03 \\
\hline 26 & 9.25 & 1.18 & 44.09 \\
\hline 27 & 7.778 & 1.61 & 42.91 \\
\hline 28 & 6.541 & 2.3 & 41.3 \\
\hline 29 & 5.5 & 3.16 & 39 \\
\hline 30 & 4.625 & 4.03 & 35.84 \\
\hline 31 & 3.889 & 4.66 & 31.81 \\
\hline 32 & 3.27 & 4.98 & 27.15 \\
\hline 33 & 2.75 & 4.89 & 22.17 \\
\hline 34 & 2.312 & 4.45 & 17.28 \\
\hline 35 & 1.945 & 3.77 & 12.83 \\
\hline 36 & 1.635 & 2.98 & 9.06 \\
\hline 37 & 1.375 & 2.25 & 6.08 \\
\hline 38 & 1.156 & 1.6 & 3.83 \\
\hline 39 & 0.972 & 1.09 & 2.23 \\
\hline 40 & 0.818 & 0.7 & 1.14 \\
\hline 41 & 0.688 & 0.44 & 0.44 \\
\hline 42 & 0.578 & 0 & $-1.249 \mathrm{E}-14$ \\
\hline 43 & 0.486 & 0 & $-1.249 \mathrm{E}-14$ \\
\hline 44 & 0.409 & 0 & $-1.249 \mathrm{E}-14$ \\
\hline 45 & 0.344 & 0 & $-1.249 \mathrm{E}-14$ \\
\hline 46 & 0.289 & 0 & $-1.249 \mathrm{E}-14$ \\
\hline 47 & 0.243 & 0 & $-1.249 E-14$ \\
\hline 48 & 0.204 & 0 & $-1.249 \mathrm{E}-14$ \\
\hline 49 & 0.172 & 0 & $-1.249 \mathrm{E}-14$ \\
\hline 50 & 0.145 & 0 & $-1.249 \mathrm{E}-14$ \\
\hline 51 & 0.122 & 0 & $-1.249 E-14$ \\
\hline
\end{tabular}




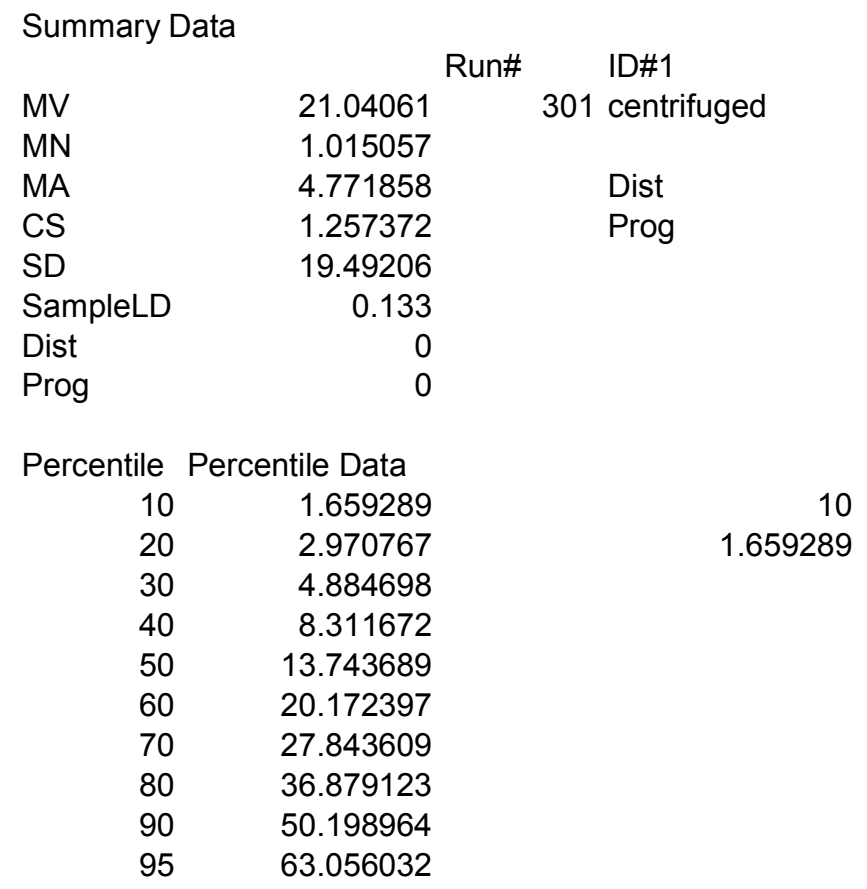

Number of Channels

50

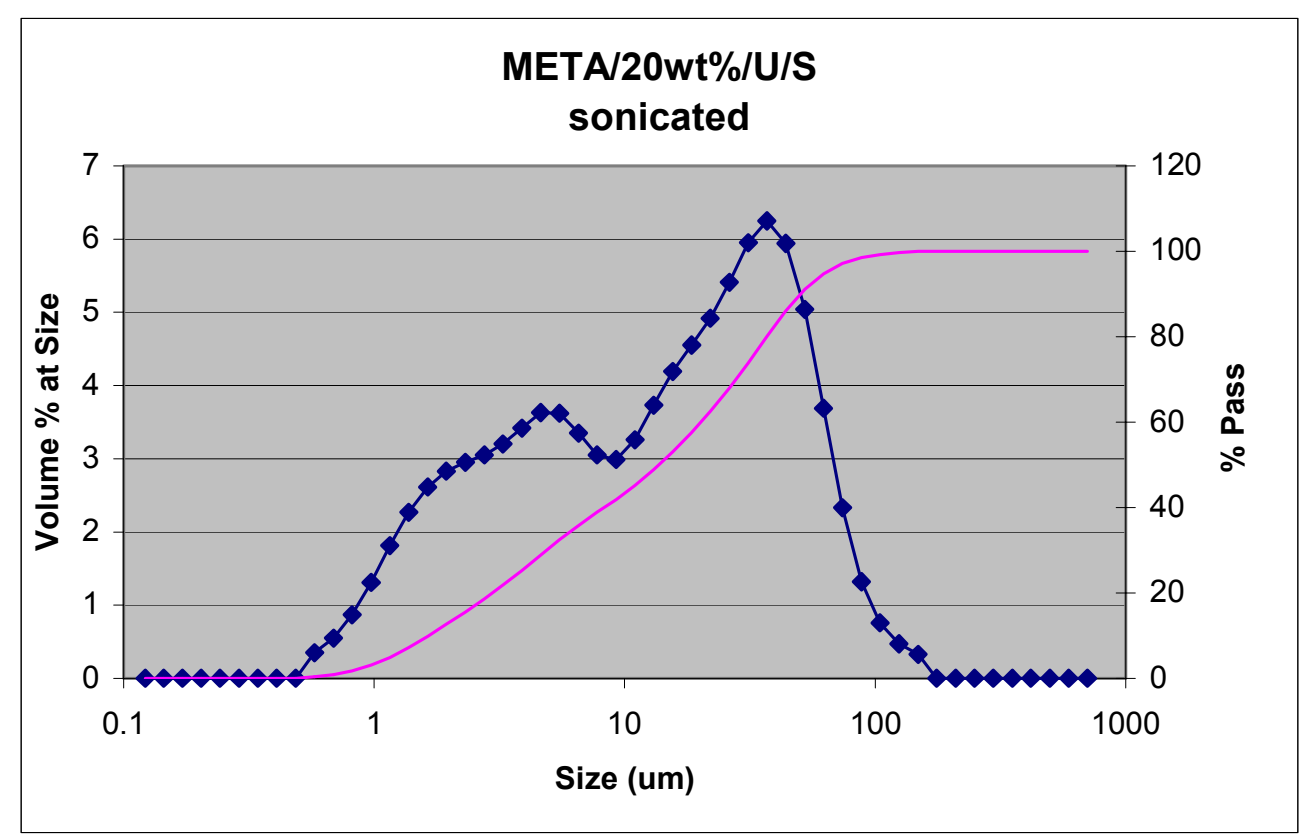




\begin{tabular}{|c|c|c|c|}
\hline \multicolumn{4}{|c|}{ META/20wt\%/U/S } \\
\hline Channel & Selected E & me & mmulative \\
\hline 1 & 704 & 0 & 100 \\
\hline 2 & 592 & 0 & 100 \\
\hline 3 & 497.8 & 0 & 100 \\
\hline 4 & 418.6 & 0 & 100 \\
\hline 5 & 352 & 0 & 100 \\
\hline 6 & 296 & 0 & 100 \\
\hline 7 & 248.9 & 0 & 100 \\
\hline 8 & 209.3 & 0 & 100 \\
\hline 9 & 176 & 0 & 100 \\
\hline 10 & 148 & 0.33 & 100 \\
\hline 11 & 124.5 & 0.47 & 99.67 \\
\hline 12 & 104.7 & 0.76 & 99.2 \\
\hline 13 & 88 & 1.32 & 98.44 \\
\hline 14 & 74 & 2.33 & 97.12 \\
\hline 15 & 62.23 & 3.69 & 94.79 \\
\hline 16 & 52.33 & 5.04 & 91.1 \\
\hline 17 & 44 & 5.94 & 86.06 \\
\hline 18 & 37 & 6.25 & 80.12 \\
\hline 19 & 31.11 & 5.95 & 73.87 \\
\hline 20 & 26.16 & 5.41 & 67.92 \\
\hline 21 & 22 & 4.92 & 62.51 \\
\hline 22 & 18.5 & 4.55 & 57.59 \\
\hline 23 & 15.56 & 4.19 & 53.04 \\
\hline 24 & 13.08 & 3.73 & 48.85 \\
\hline 25 & 11 & 3.26 & 45.12 \\
\hline 26 & 9.25 & 2.99 & 41.86 \\
\hline 27 & 7.778 & 3.05 & 38.87 \\
\hline 28 & 6.541 & 3.35 & 35.82 \\
\hline 29 & 5.5 & 3.62 & 32.47 \\
\hline 30 & 4.625 & 3.63 & 28.85 \\
\hline 31 & 3.889 & 3.42 & 25.22 \\
\hline 32 & 3.27 & 3.2 & 21.8 \\
\hline 33 & 2.75 & 3.05 & 18.6 \\
\hline 34 & 2.312 & 2.95 & 15.55 \\
\hline 35 & 1.945 & 2.83 & 12.6 \\
\hline 36 & 1.635 & 2.61 & 9.77 \\
\hline 37 & 1.375 & 2.27 & 7.16 \\
\hline 38 & 1.156 & 1.81 & 4.89 \\
\hline 39 & 0.972 & 1.31 & 3.08 \\
\hline 40 & 0.818 & 0.87 & 1.77 \\
\hline 41 & 0.688 & 0.55 & 0.9 \\
\hline 42 & 0.578 & 0.35 & 0.35 \\
\hline 43 & 0.486 & 0 & $1.28786 \mathrm{E}-14$ \\
\hline 44 & 0.409 & 0 & $1.28786 \mathrm{E}-14$ \\
\hline 45 & 0.344 & 0 & $1.28786 \mathrm{E}-14$ \\
\hline 46 & 0.289 & 0 & $1.28786 \mathrm{E}-14$ \\
\hline 47 & 0.243 & 0 & $1.28786 \mathrm{E}-14$ \\
\hline 48 & 0.204 & 0 & 1.28786E-14 \\
\hline 49 & 0.172 & 0 & $1.28786 \mathrm{E}-14$ \\
\hline 50 & 0.145 & 0 & $1.28786 \mathrm{E}-14$ \\
\hline 51 & 0.122 & 0 & $1.28786 \mathrm{E}-14$ \\
\hline
\end{tabular}




\section{Summary Data}

\begin{tabular}{lrrl} 
& \multicolumn{2}{c}{ Run\# } & ID\#1 \\
MV & 108.62403 & 261 & \\
MN & 1.18615 & & \\
MA & 4.873517 & & Dist \\
CS & 1.231144 & & Prog \\
SD & 145.180602 & & \\
SampleLD & 0.057 & & \\
Dist & 0 & \\
Prog & 0 &
\end{tabular}

Percentile Percentile Data

$\begin{array}{rrr}10 & 1.659129 & 10 \\ 20 & 2.515352 & 1.659129 \\ 30 & 3.667892 & \\ 40 & 6.350615 & \\ 50 & 31.607274 & \\ 60 & 77.286328 & \\ 70 & 145.263893 & \\ 80 & 253.692604 & \\ 90 & 344.367142 & \\ 95 & 393.656746 & \end{array}$

Number of Channels

50

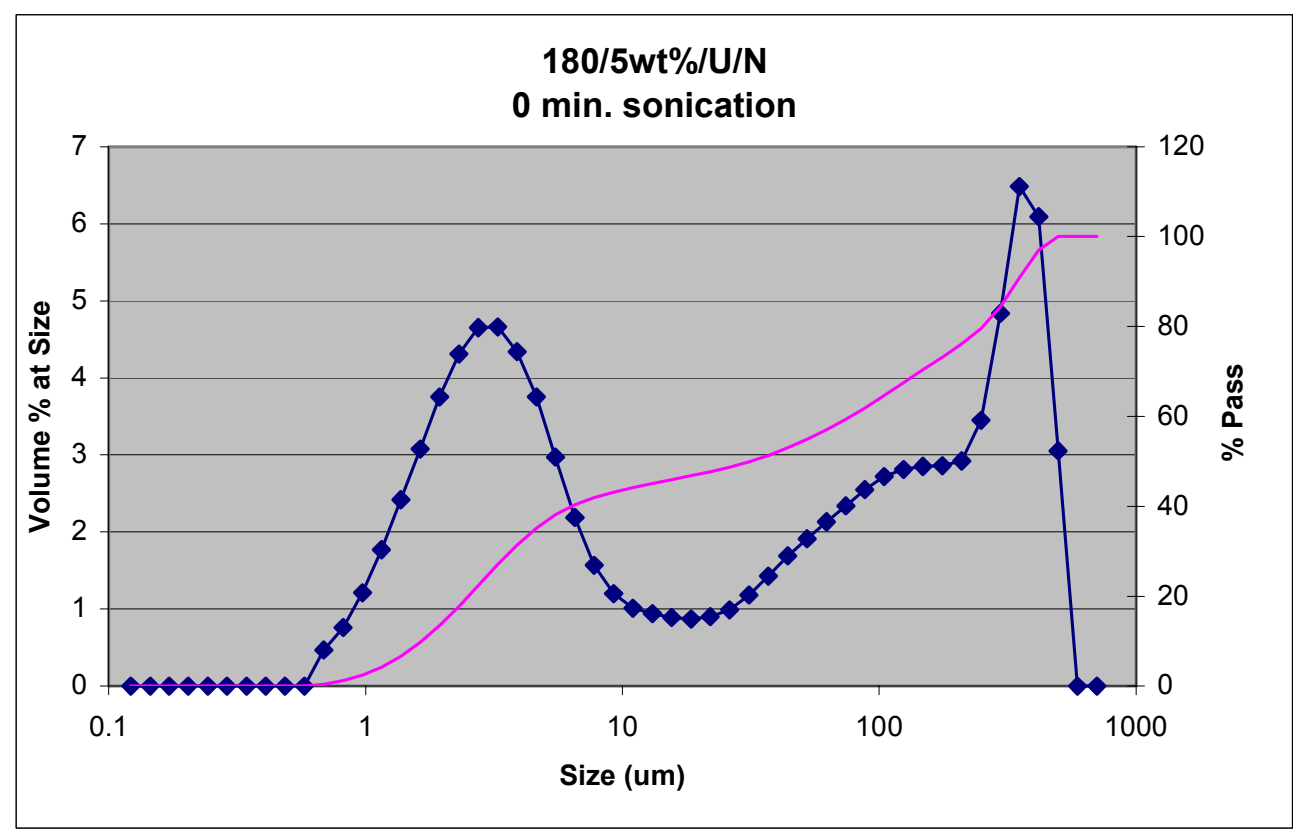




\begin{tabular}{rrrr} 
180/5wt\%/U/N & \multicolumn{2}{c}{ Selected } \\
Channel & \multicolumn{2}{c}{ Selected E Volume } & \multicolumn{1}{c}{ Cummulative } \\
1 & 704 & \multicolumn{1}{c}{0} & 100 \\
2 & 592 & 0 & 100 \\
3 & 497.8 & 3.05 & 100 \\
4 & 418.6 & 6.09 & 96.95 \\
5 & 352 & 6.48 & 90.86 \\
6 & 296 & 4.84 & 84.38 \\
7 & 248.9 & 3.45 & 79.54 \\
8 & 209.3 & 2.92 & 76.09 \\
9 & 176 & 2.86 & 73.17 \\
10 & 148 & 2.85 & 70.31 \\
11 & 124.5 & 2.81 & 67.46 \\
12 & 104.7 & 2.72 & 64.65 \\
13 & 88 & 2.55 & 61.93 \\
14 & 74 & 2.34 & 59.38 \\
15 & 62.23 & 2.13 & 57.04 \\
16 & 52.33 & 1.91 & 54.91 \\
17 & 44 & 1.69 & 53 \\
18 & 37 & 1.43 & 51.31 \\
19 & 31.11 & 1.18 & 49.88 \\
20 & 26.16 & 0.99 & 48.7 \\
21 & 22 & 0.9 & 47.71 \\
22 & 18.5 & 0.87 & 46.81 \\
23 & 15.56 & 0.89 & 45.94 \\
24 & 13.08 & 0.94 & 45.05 \\
25 & 11 & 1.01 & 44.11 \\
26 & 9.25 & 1.2 & 43.1 \\
27 & 7.778 & 1.57 & 41.9 \\
28 & 6.541 & 2.19 & 40.33 \\
29 & 5.5 & 2.97 & 38.14 \\
30 & 4.625 & 3.75 & 35.17 \\
31 & 3.889 & 4.34 & 31.42 \\
32 & 3.27 & 4.66 & 27.08 \\
33 & 2.75 & 4.65 & 22.42 \\
34 & 2.312 & 4.31 & 17.77 \\
35 & 1.945 & 3.75 & 13.46 \\
36 & 1.635 & 3.08 & 9.71 \\
37 & 1.375 & 2.42 & 6.63 \\
38 & 1.156 & 1.77 & 4.21 \\
39 & 0.972 & 1.21 & 2.44 \\
40 & 0.818 & 0.76 & 0.47 \\
41 & 0.688 & 0.47 & 0 \\
42 & 0.578 & 0 & $4.44089 \mathrm{E}-15$ \\
43 & 0.486 & 0 & $4.44089 \mathrm{E}-15$ \\
44 & 0.409 & 0 & $4.44089 \mathrm{E}-15$ \\
45 & 0.344 & 0 & $4.44089 \mathrm{E}-15$ \\
46 & 0.289 & 0 & $4.44089 \mathrm{E}-15$ \\
47 & 0.243 & 0 & $4.44089 \mathrm{E}-15$ \\
48 & 0.204 & 0 & $4.44089 \mathrm{E}-15$ \\
49 & 0.172 & 0 & $4.44089 \mathrm{E}-15$ \\
50 & 0.145 & 0 & $4.44089 \mathrm{E}-15$ \\
51 & 0.122 & 0 & $4.44089 \mathrm{E}-15$ \\
& & &
\end{tabular}


$180 / 5 w t \% / U / S$

Summary Data

$\begin{array}{lrc}\text { MV } & 14.97223 & 267 \text { 150 min sonicatin } \\ \text { MN } & 0.888923 & \\ \text { MA } & 3.349096 & \text { Dist } \\ \text { CS } & 1.791528 & \text { Prog } \\ \text { SD } & 15.241099 & \\ \text { SampleLD } & 0.039 & \\ \text { Dist } & 0 & \\ \text { Prog } & 0 & \end{array}$

Percentile Percentile Data

$10 \quad 1.179823$

$20 \quad 1.833546$

$30 \quad 3.019057$

$40 \quad 4.722987$

$50 \quad 9.092152$

$60 \quad 13.990122$

$70 \quad 19.105722$

$80 \quad 27.81263$

$90 \quad 39.09908$

$95 \quad 47.607196$
150 min sonication, after transfer

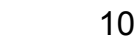

1.179823

Number of Channels

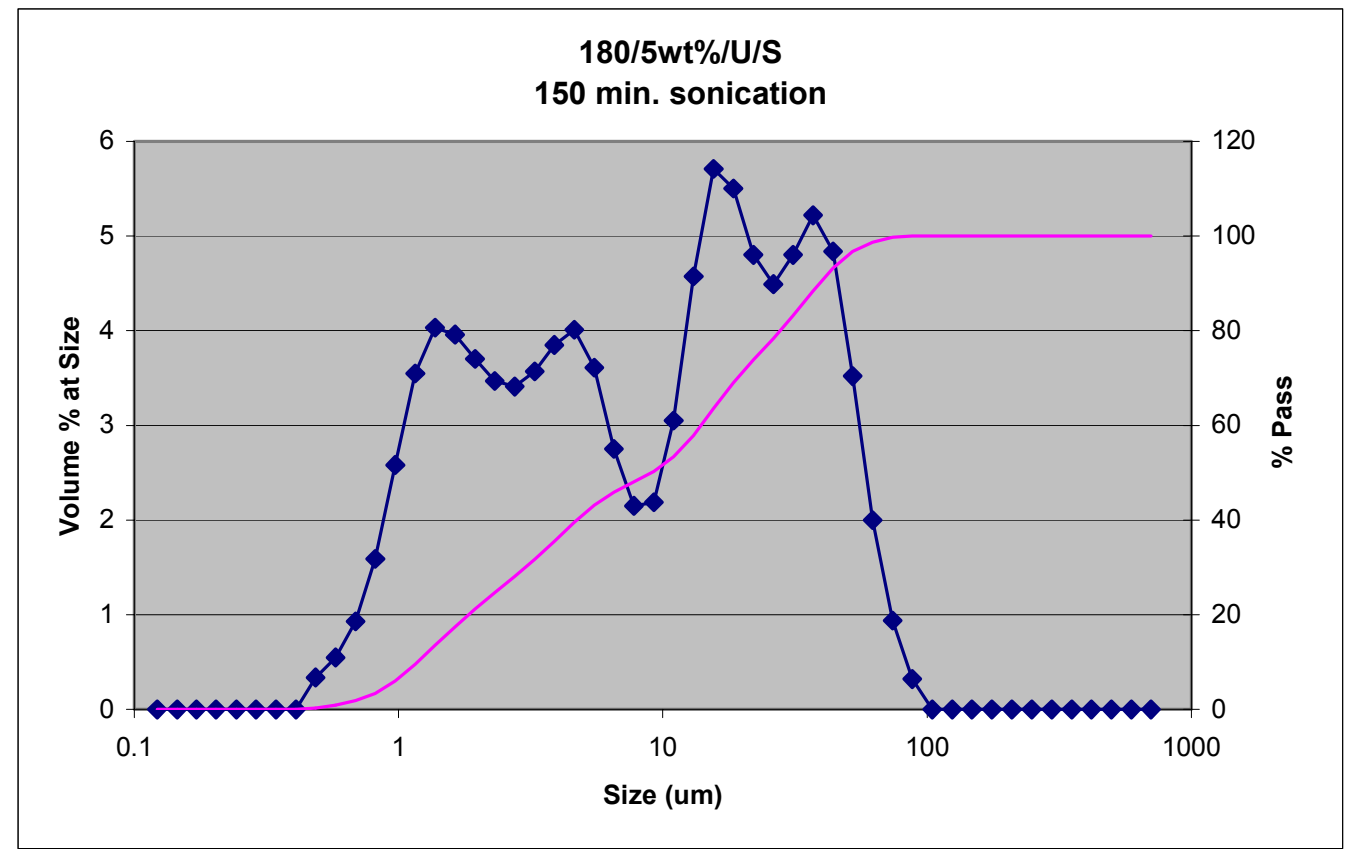




\begin{tabular}{|c|c|c|c|}
\hline \multirow{3}{*}{ Channel } & \multicolumn{3}{|c|}{ Selected } \\
\hline & Selected E & me & mmulative \\
\hline & 704 & 0 & 100 \\
\hline 2 & 592 & 0 & 100 \\
\hline 3 & 497.8 & 0 & 100 \\
\hline 4 & 418.6 & 0 & 100 \\
\hline 5 & 352 & 0 & 100 \\
\hline 6 & 296 & 0 & 100 \\
\hline 7 & 248.9 & 0 & 100 \\
\hline 8 & 209.3 & 0 & 100 \\
\hline 9 & 176 & 0 & 100 \\
\hline 10 & 148 & 0 & 100 \\
\hline 11 & 124.5 & 0 & 100 \\
\hline 12 & 104.7 & 0 & 100 \\
\hline 13 & 88 & 0.32 & 100 \\
\hline 14 & 74 & 0.94 & 99.68 \\
\hline 15 & 62.23 & 2 & 98.74 \\
\hline 16 & 52.33 & 3.52 & 96.74 \\
\hline 17 & 44 & 4.84 & 93.22 \\
\hline 18 & 37 & 5.22 & 88.38 \\
\hline 19 & 31.11 & 4.8 & 83.16 \\
\hline 20 & 26.16 & 4.49 & 78.36 \\
\hline 21 & 22 & 4.8 & 73.87 \\
\hline 22 & 18.5 & 5.5 & 69.07 \\
\hline 23 & 15.56 & 5.71 & 63.57 \\
\hline 24 & 13.08 & 4.57 & 57.86 \\
\hline 25 & 11 & 3.05 & 53.29 \\
\hline 26 & 9.25 & 2.19 & 50.24 \\
\hline 27 & 7.778 & 2.15 & 48.05 \\
\hline 28 & 6.541 & 2.75 & 45.9 \\
\hline 29 & 5.5 & 3.61 & 43.15 \\
\hline 30 & 4.625 & 4.01 & 39.54 \\
\hline 31 & 3.889 & 3.85 & 35.53 \\
\hline 32 & 3.27 & 3.57 & 31.68 \\
\hline 33 & 2.75 & 3.41 & 28.11 \\
\hline 34 & 2.312 & 3.47 & 24.7 \\
\hline 35 & 1.945 & 3.7 & 21.23 \\
\hline 36 & 1.635 & 3.96 & 17.53 \\
\hline 37 & 1.375 & 4.03 & 13.57 \\
\hline 38 & 1.156 & 3.55 & 9.54 \\
\hline 39 & 0.972 & 2.58 & 5.99 \\
\hline 40 & 0.818 & 1.59 & 3.41 \\
\hline 41 & 0.688 & 0.93 & 1.82 \\
\hline 42 & 0.578 & 0.55 & 0.89 \\
\hline 43 & 0.486 & 0.34 & 0.34 \\
\hline 44 & 0.409 & 0 & $2.7478 \mathrm{E}-14$ \\
\hline 45 & 0.344 & 0 & $2.7478 \mathrm{E}-14$ \\
\hline 46 & 0.289 & 0 & $2.7478 \mathrm{E}-14$ \\
\hline 47 & 0.243 & 0 & $2.7478 \mathrm{E}-14$ \\
\hline 48 & 0.204 & 0 & $2.7478 \mathrm{E}-14$ \\
\hline 49 & 0.172 & 0 & $2.7478 \mathrm{E}-14$ \\
\hline 50 & 0.145 & 0 & $2.7478 \mathrm{E}-14$ \\
\hline 51 & 0.122 & 0 & $2.7478 \mathrm{E}-14$ \\
\hline
\end{tabular}

E-32 
189/5wt\%/U/N

Summary Data

$\begin{array}{lr}\text { MV } & 123.493506 \\ \text { MN } & 1.222786 \\ \text { MA } & 5.776366 \\ \text { CS } & 1.038715 \\ \text { SD } & 150.631633 \\ \text { SampleLD } & 0.078 \\ \text { Dist } & 0 \\ \text { Prog } & 0\end{array}$

Percentile Percentile Data

$\begin{array}{rrr}10 & 1.836136 & 10 \\ 20 & 2.941866 & 1.836136 \\ 30 & 4.606364 & \\ 40 & 18.834953 & \\ 50 & 59.270366 & \\ 60 & 106.949017 & \\ 70 & 199.015953 & \\ 80 & 278.369574 & \\ 90 & 343.062138 & \\ 95 & 386.988817 & \end{array}$

Number of Channels

50

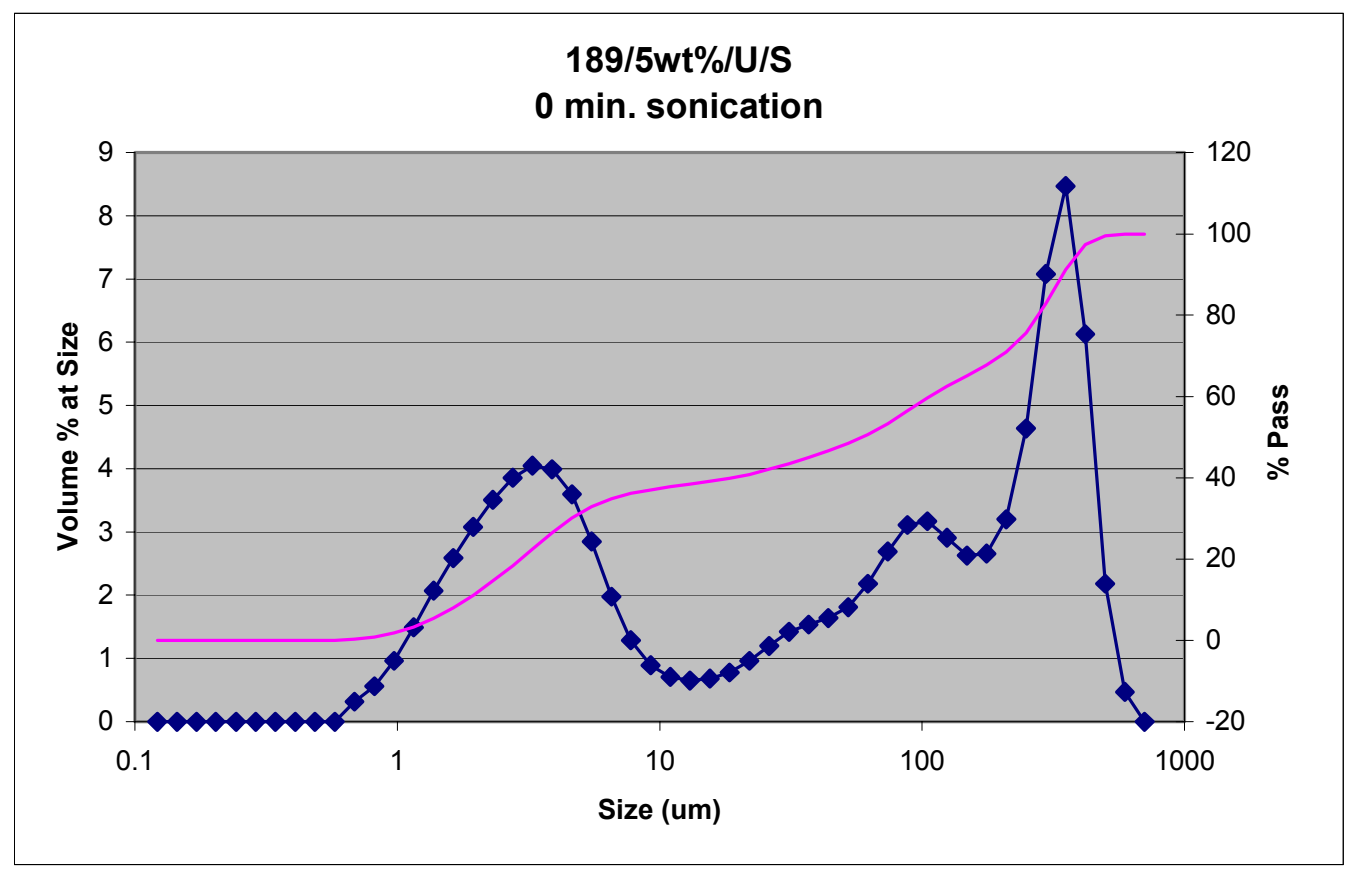

E-33 


\begin{tabular}{|c|c|c|c|}
\hline \multirow{3}{*}{$\begin{array}{r}\text { Channel } \\
1\end{array}$} & \multicolumn{2}{|c|}{ Selected } & \multirow[b]{2}{*}{ Cummulative } \\
\hline & Selected E & me & \\
\hline & 704 & 0 & 100 \\
\hline 2 & 592 & 0.47 & 100 \\
\hline 3 & 497.8 & 2.18 & 99.53 \\
\hline 4 & 418.6 & 6.13 & 97.35 \\
\hline 5 & 352 & 8.47 & 91.22 \\
\hline 6 & 296 & 7.08 & 82.75 \\
\hline 7 & 248.9 & 4.64 & 75.67 \\
\hline 8 & 209.3 & 3.2 & 71.03 \\
\hline 9 & 176 & 2.66 & 67.83 \\
\hline 10 & 148 & 2.63 & 65.17 \\
\hline 11 & 124.5 & 2.91 & 62.54 \\
\hline 12 & 104.7 & 3.17 & 59.63 \\
\hline 13 & 88 & 3.11 & 56.46 \\
\hline 14 & 74 & 2.69 & 53.35 \\
\hline 15 & 62.23 & 2.18 & 50.66 \\
\hline 16 & 52.33 & 1.81 & 48.48 \\
\hline 17 & 44 & 1.64 & 46.67 \\
\hline 18 & 37 & 1.54 & 45.03 \\
\hline 19 & 31.11 & 1.42 & 43.49 \\
\hline 20 & 26.16 & 1.2 & 42.07 \\
\hline 21 & 22 & 0.96 & 40.87 \\
\hline 22 & 18.5 & 0.78 & 39.91 \\
\hline 23 & 15.56 & 0.68 & 39.13 \\
\hline 24 & 13.08 & 0.65 & 38.45 \\
\hline 25 & 11 & 0.71 & 37.8 \\
\hline 26 & 9.25 & 0.89 & 37.09 \\
\hline 27 & 7.778 & 1.29 & 36.2 \\
\hline 28 & 6.541 & 1.98 & 34.91 \\
\hline 29 & 5.5 & 2.85 & 32.93 \\
\hline 30 & 4.625 & 3.6 & 30.08 \\
\hline 31 & 3.889 & 3.99 & 26.48 \\
\hline 32 & 3.27 & 4.05 & 22.49 \\
\hline 33 & 2.75 & 3.86 & 18.44 \\
\hline 34 & 2.312 & 3.51 & 14.58 \\
\hline 35 & 1.945 & 3.08 & 11.07 \\
\hline 36 & 1.635 & 2.59 & 7.99 \\
\hline 37 & 1.375 & 2.07 & 5.4 \\
\hline 38 & 1.156 & 1.49 & 3.33 \\
\hline 39 & 0.972 & 0.96 & 1.84 \\
\hline 40 & 0.818 & 0.56 & 0.88 \\
\hline 41 & 0.688 & 0.32 & 0.32 \\
\hline 42 & 0.578 & 0 & $-1.19349 \mathrm{E}-14$ \\
\hline 43 & 0.486 & 0 & $-1.19349 \mathrm{E}-14$ \\
\hline 44 & 0.409 & 0 & $-1.19349 \mathrm{E}-14$ \\
\hline 45 & 0.344 & 0 & $-1.19349 \mathrm{E}-14$ \\
\hline 46 & 0.289 & 0 & $-1.19349 \mathrm{E}-14$ \\
\hline 47 & 0.243 & 0 & $-1.19349 E-14$ \\
\hline 48 & 0.204 & 0 & $-1.19349 E-14$ \\
\hline 49 & 0.172 & 0 & $-1.19349 E-14$ \\
\hline 50 & 0.145 & 0 & $-1.19349 \mathrm{E}-14$ \\
\hline 51 & 0.122 & 0 & $-1.19349 \mathrm{E}-14$ \\
\hline
\end{tabular}


$189 / 5 w t \% / U / S$

Summary Data

MV

$\mathrm{MN}$

MA

CS

SD

SampleLD

Dist

Prog
150 min sonication, after transfer

Run\# ID\#1

$16.130435 \quad 276150 \mathrm{~min}$ sonication

0.879804

4.009774

1.496344

15.699682

0.047

0

Percentile Percentile Data

10

1.544291

2.655508

4.038821

5.659618

7.869351

11.41831

17.619385

28.055016

44.701454

59.639753
Dist

Prog

Number of Channels

50

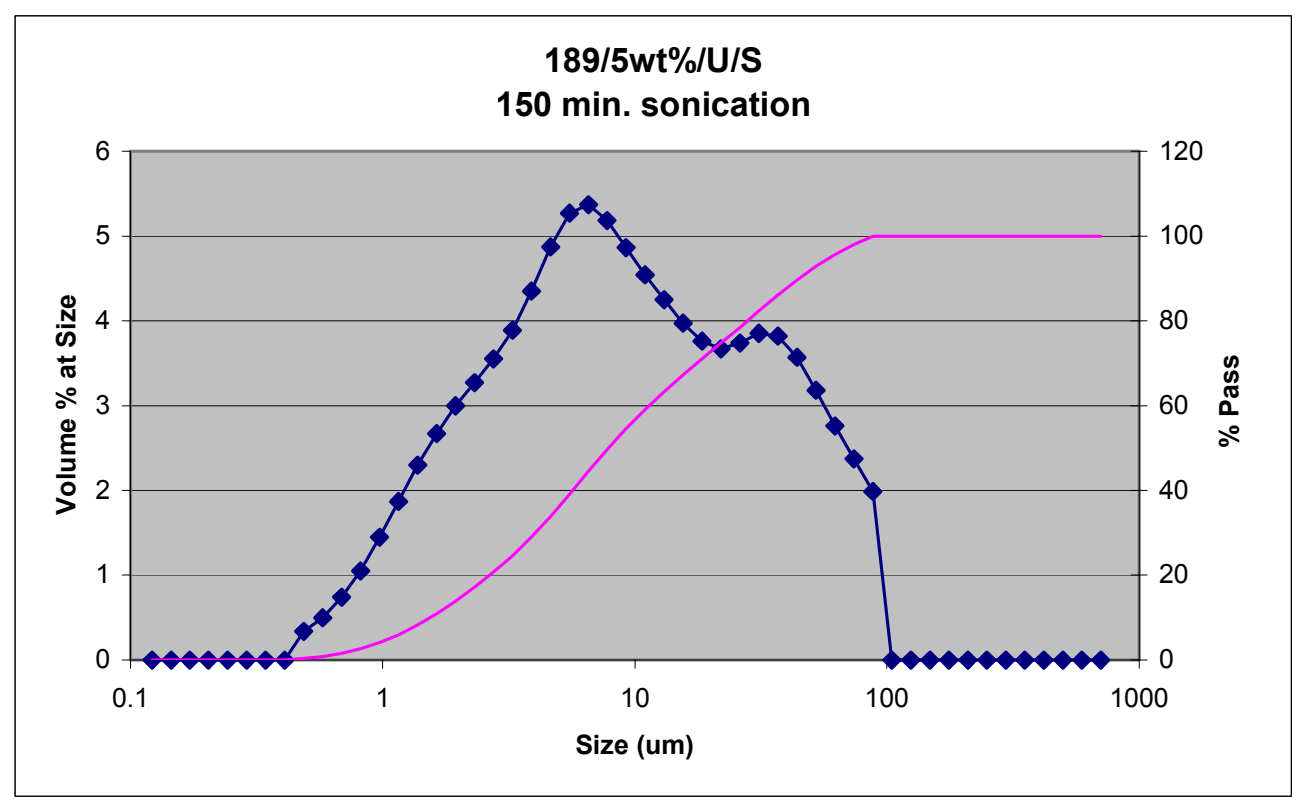




\begin{tabular}{|c|c|c|c|}
\hline \multicolumn{4}{|c|}{$189 / 5 w t \% / U / S \quad$ Selected } \\
\hline Channel & Selected E & me & Cummulative \\
\hline 1 & 704 & 0 & 100 \\
\hline 2 & 592 & 0 & 100 \\
\hline 3 & 497.8 & 0 & 100 \\
\hline 4 & 418.6 & 0 & 100 \\
\hline 5 & 352 & 0 & 100 \\
\hline 6 & 296 & 0 & 100 \\
\hline 7 & 248.9 & 0 & 100 \\
\hline 8 & 209.3 & 0 & 100 \\
\hline 9 & 176 & 0 & 100 \\
\hline 10 & 148 & 0 & 100 \\
\hline 11 & 124.5 & 0 & 100 \\
\hline 12 & 104.7 & 0 & 100 \\
\hline 13 & 88 & 1.99 & 100 \\
\hline 14 & 74 & 2.37 & 98.01 \\
\hline 15 & 62.23 & 2.76 & 95.64 \\
\hline 16 & 52.33 & 3.18 & 92.88 \\
\hline 17 & 44 & 3.57 & 89.7 \\
\hline 18 & 37 & 3.82 & 86.13 \\
\hline 19 & 31.11 & 3.85 & 82.31 \\
\hline 20 & 26.16 & 3.74 & 78.46 \\
\hline 21 & 22 & 3.67 & 74.72 \\
\hline 22 & 18.5 & 3.76 & 71.05 \\
\hline 23 & 15.56 & 3.97 & 67.29 \\
\hline 24 & 13.08 & 4.25 & 63.32 \\
\hline 25 & 11 & 4.54 & 59.07 \\
\hline 26 & 9.25 & 4.86 & 54.53 \\
\hline 27 & 7.778 & 5.18 & 49.67 \\
\hline 28 & 6.541 & 5.37 & 44.49 \\
\hline 29 & 5.5 & 5.27 & 39.12 \\
\hline 30 & 4.625 & 4.87 & 33.85 \\
\hline 31 & 3.889 & 4.35 & 28.98 \\
\hline 32 & 3.27 & 3.89 & 24.63 \\
\hline 33 & 2.75 & 3.55 & 20.74 \\
\hline 34 & 2.312 & 3.27 & 17.19 \\
\hline 35 & 1.945 & 3 & 13.92 \\
\hline 36 & 1.635 & 2.67 & 10.92 \\
\hline 37 & 1.375 & 2.3 & 8.25 \\
\hline 38 & 1.156 & 1.87 & 5.95 \\
\hline 39 & 0.972 & 1.45 & 4.08 \\
\hline 40 & 0.818 & 1.05 & 2.63 \\
\hline 41 & 0.688 & 0.74 & 1.58 \\
\hline 42 & 0.578 & 0.5 & 0.84 \\
\hline 43 & 0.486 & 0.34 & 0.34 \\
\hline 44 & 0.409 & 0 & $8.72 \mathrm{E}-15$ \\
\hline 45 & 0.344 & 0 & $8.72 \mathrm{E}-15$ \\
\hline 46 & 0.289 & 0 & $8.72 \mathrm{E}-15$ \\
\hline 47 & 0.243 & 0 & $8.72 \mathrm{E}-15$ \\
\hline 48 & 0.204 & 0 & 8.72E-15 \\
\hline 49 & 0.172 & 0 & $8.72 \mathrm{E}-15$ \\
\hline 50 & 0.145 & 0 & $8.72 \mathrm{E}-15$ \\
\hline 51 & 0.122 & 0 & 8.72E-15 \\
\hline
\end{tabular}




\section{E-1.2 Tank Farm Waste Samples}

\begin{tabular}{|c|c|c|c|}
\hline & WM-187 \#1 & WM-187 \#2 & WM-187 \#3 \\
\hline Diameter & Frequency (\%) & Frequency (\%) & Frequency (\%) \\
\hline 0.115 & 0 & 0 & 0 \\
\hline 0.131 & 0 & 0 & 0 \\
\hline 0.15 & 0 & 0 & 0 \\
\hline 0.172 & 0 & 0 & 0 \\
\hline 0.197 & 0 & 0 & 0 \\
\hline 0.226 & 0 & 0 & 0 \\
\hline 0.259 & 0 & 0 & 0 \\
\hline 0.296 & 0 & 0 & 0 \\
\hline 0.339 & 0 & 0 & 0 \\
\hline 0.389 & 0 & 0 & 0 \\
\hline 0.445 & 0 & 0.381 & 0 \\
\hline 0.51 & 0 & 0.562 & 0 \\
\hline 0.584 & 0.467 & 0.811 & 0.477 \\
\hline 0.669 & 0.793 & 1.132 & 0.803 \\
\hline 0.766 & 1.237 & 1.519 & 1.241 \\
\hline 0.877 & 1.769 & 1.955 & 1.762 \\
\hline 1.005 & 2.245 & 2.318 & 2.219 \\
\hline 1.151 & 2.703 & 2.686 & 2.655 \\
\hline 1.318 & 3.15 & 3.067 & 3.083 \\
\hline 1.51 & 3.652 & 3.447 & 3.567 \\
\hline 1.729 & 4.154 & 3.911 & 4.062 \\
\hline 1.981 & 4.641 & 4.423 & 4.548 \\
\hline 2.269 & 5.079 & 4.864 & 4.982 \\
\hline 2.599 & 5.659 & 5.489 & 5.571 \\
\hline 2.976 & 6.399 & 6.256 & 6.322 \\
\hline 3.409 & 7.241 & 7.172 & 7.195 \\
\hline 3.905 & 7.887 & 7.91 & 7.889 \\
\hline 4.472 & 8.137 & 8.25 & 8.196 \\
\hline 5.122 & 7.968 & 8.139 & 8.08 \\
\hline 5.867 & 6.91 & 7.01 & 7.042 \\
\hline 6.72 & 5.99 & 6.027 & 6.129 \\
\hline 7.697 & 4.857 & 4.801 & 4.981 \\
\hline 8.816 & 3.371 & 3.19 & 3.448 \\
\hline 10.097 & 2.356 & 2.132 & 2.402 \\
\hline 11.565 & 1.523 & 1.298 & 1.544 \\
\hline 13.246 & 0.912 & 0.719 & 0.916 \\
\hline 15.172 & 0.507 & 0.363 & 0.504 \\
\hline 17.377 & 0.264 & 0.168 & 0.258 \\
\hline 19.904 & 0.13 & 0 & 0.125 \\
\hline 22.797 & 0 & 0 & 0 \\
\hline 26.111 & 0 & 0 & 0 \\
\hline 29.907 & 0 & 0 & 0 \\
\hline 34.255 & 0 & 0 & 0 \\
\hline 39.234 & 0 & 0 & 0 \\
\hline 44.938 & 0 & 0 & 0 \\
\hline 51.471 & 0 & 0 & 0 \\
\hline 58.953 & 0 & 0 & 0 \\
\hline 67.523 & 0 & 0 & 0 \\
\hline 77.339 & 0 & 0 & 0 \\
\hline 88.583 & 0 & 0 & 0 \\
\hline 101.46 & 0 & 0 & 0 \\
\hline 116.21 & 0 & 0 & 0 \\
\hline 133.103 & 0 & 0 & 0 \\
\hline 152.453 & 0 & 0 & 0 \\
\hline 174.616 & 0 & 0 & 0 \\
\hline 200 & 0 & 0 & 0 \\
\hline 229.075 & 0 & 0 & 0 \\
\hline 262.376 & 0 & 0 & 0 \\
\hline 300.518 & 0 & 0 & 0 \\
\hline 344.206 & 0 & 0 & 0 \\
\hline 394.244 & 0 & 0 & 0 \\
\hline 451.556 & 0 & 0 & 0 \\
\hline 517.2 & 0 & 0 & 0 \\
\hline 592.387 & 0 & 0 & 0 \\
\hline
\end{tabular}




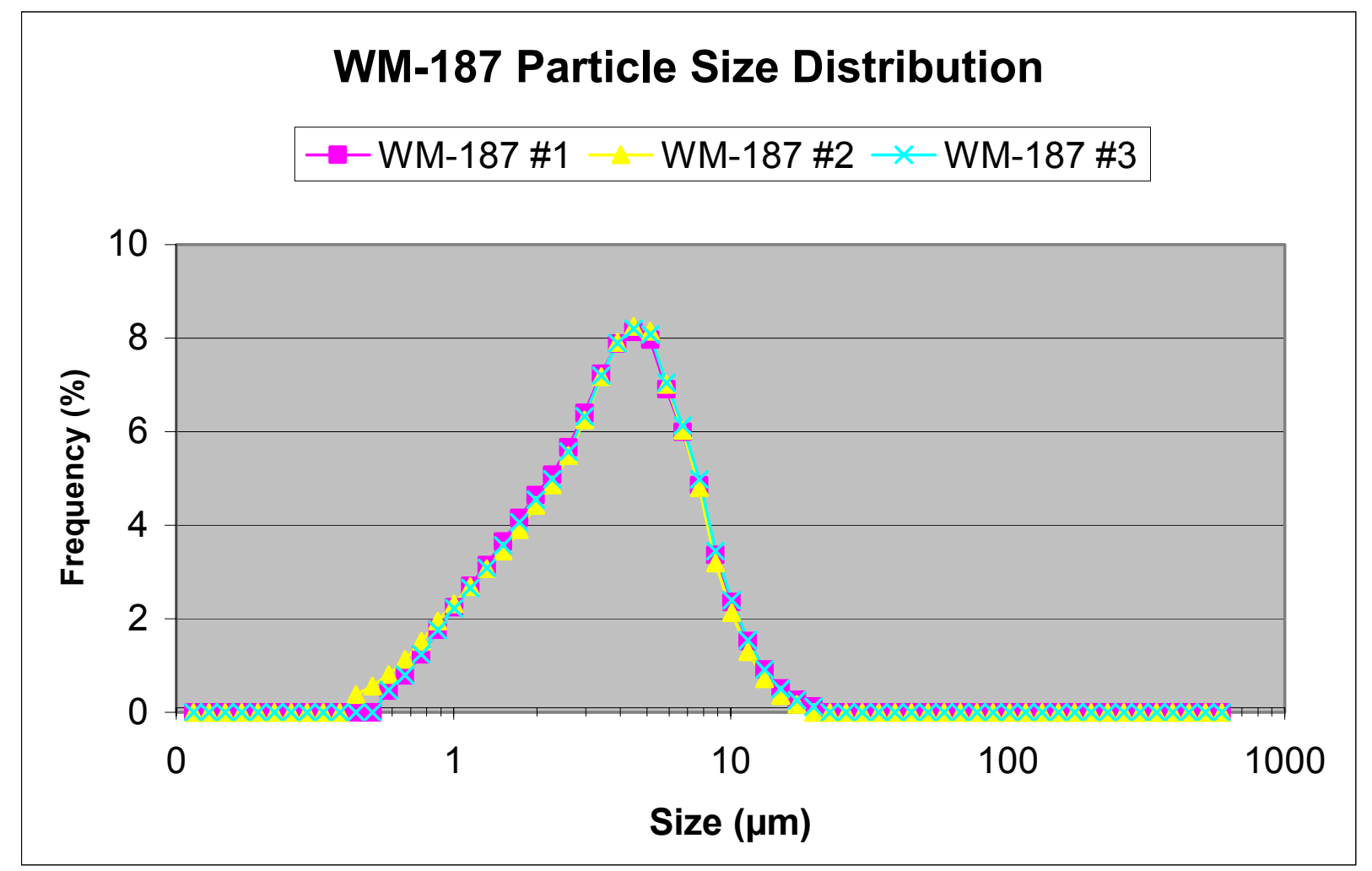


WM-187 \#1 Ultra WM-187 \#2 Ultra WM-187 \#3 Ultra

\begin{tabular}{|c|c|c|c|}
\hline Diameter & Frequency (\%) & Frequency (\%) & Frequency (\%) \\
\hline 0.115 & - the & the & ) \\
\hline 0.131 & 0 & 0 & 0 \\
\hline 0.15 & 0 & 0 & 0 \\
\hline 0.172 & 0 & 0 & 0 \\
\hline 0.197 & 0 & 0 & 0 \\
\hline 0.226 & 0 & 0 & 0 \\
\hline 0.259 & 0 & 0 & 0 \\
\hline 0.296 & 0 & 0 & 0 \\
\hline 0.339 & 0 & 0 & 0 \\
\hline 0.389 & 0 & 0 & 0 \\
\hline 0.445 & 0 & 0 & 0 \\
\hline 0.51 & 0 & 0.333 & 0 \\
\hline 0.584 & 0.491 & 0.543 & 0.51 \\
\hline 0.669 & 0.83 & 0.842 & 0.854 \\
\hline 0.766 & 1.291 & 1.226 & 1.316 \\
\hline 0.877 & 1.839 & 1.672 & 1.86 \\
\hline 1.005 & 2.321 & 2.053 & 2.331 \\
\hline 1.151 & 2.775 & 2.422 & 2.772 \\
\hline 1.318 & 3.212 & 2.788 & 3.199 \\
\hline 1.51 & 3.701 & 3.187 & 3.679 \\
\hline 1.729 & 4.183 & 3.606 & 4.159 \\
\hline 1.981 & 4.649 & 4.023 & 4.623 \\
\hline 2.269 & 5.06 & 4.38 & 5.026 \\
\hline 2.599 & 5.62 & 4.836 & 5.579 \\
\hline 2.976 & 6.336 & 5.42 & 6.288 \\
\hline 3.409 & 7.162 & 6.042 & 7.112 \\
\hline 3.905 & 7.8 & 6.472 & 7.761 \\
\hline 4.472 & 8.056 & 6.562 & 8.039 \\
\hline 5.122 & 7.907 & 6.33 & 7.919 \\
\hline 5.867 & 6.888 & 5.505 & 6.928 \\
\hline 6.72 & 5.99 & 4.832 & 6.044 \\
\hline 7.697 & 4.871 & 4.053 & 4.927 \\
\hline 8.816 & 3.38 & 3.125 & 3.416 \\
\hline 10.097 & 2.358 & 2.452 & 2.38 \\
\hline 11.565 & 1.517 & 1.872 & 1.526 \\
\hline 13.246 & 0.9 & 1.407 & 0.9 \\
\hline 15.172 & 0.493 & 1.054 & 0.489 \\
\hline 17.377 & 0.251 & 0.799 & 0.247 \\
\hline 19.904 & 0.12 & 0.622 & 0.117 \\
\hline 22.797 & 0 & 0.504 & 0 \\
\hline 26.111 & 0 & 0.431 & 0 \\
\hline 29.907 & 0 & 0.394 & 0 \\
\hline 34.255 & 0 & 0.387 & 0 \\
\hline 39.234 & 0 & 0.408 & 0 \\
\hline 44.938 & 0 & 0.46 & 0 \\
\hline 51.471 & 0 & 0.546 & 0 \\
\hline 58.953 & 0 & 0.668 & 0 \\
\hline 67.523 & 0 & 0.82 & 0 \\
\hline 77.339 & 0 & 0.986 & 0 \\
\hline 88.583 & 0 & 1.129 & 0 \\
\hline 101.46 & 0 & 1.206 & 0 \\
\hline 116.21 & 0 & 1.184 & 0 \\
\hline 133.103 & 0 & 1.062 & 0 \\
\hline 152.453 & 0 & 0.874 & 0 \\
\hline 174.616 & 0 & 0.486 & 0 \\
\hline 200 & 0 & 0 & 0 \\
\hline 229.075 & 0 & 0 & 0 \\
\hline 262.376 & 0 & 0 & 0 \\
\hline 300.518 & 0 & 0 & 0 \\
\hline 344.206 & 0 & 0 & 0 \\
\hline 394.244 & 0 & 0 & 0 \\
\hline 451.556 & 0 & 0 & 0 \\
\hline 517.2 & 0 & 0 & 0 \\
\hline 592.387 & 0 & 0 & 0 \\
\hline
\end{tabular}




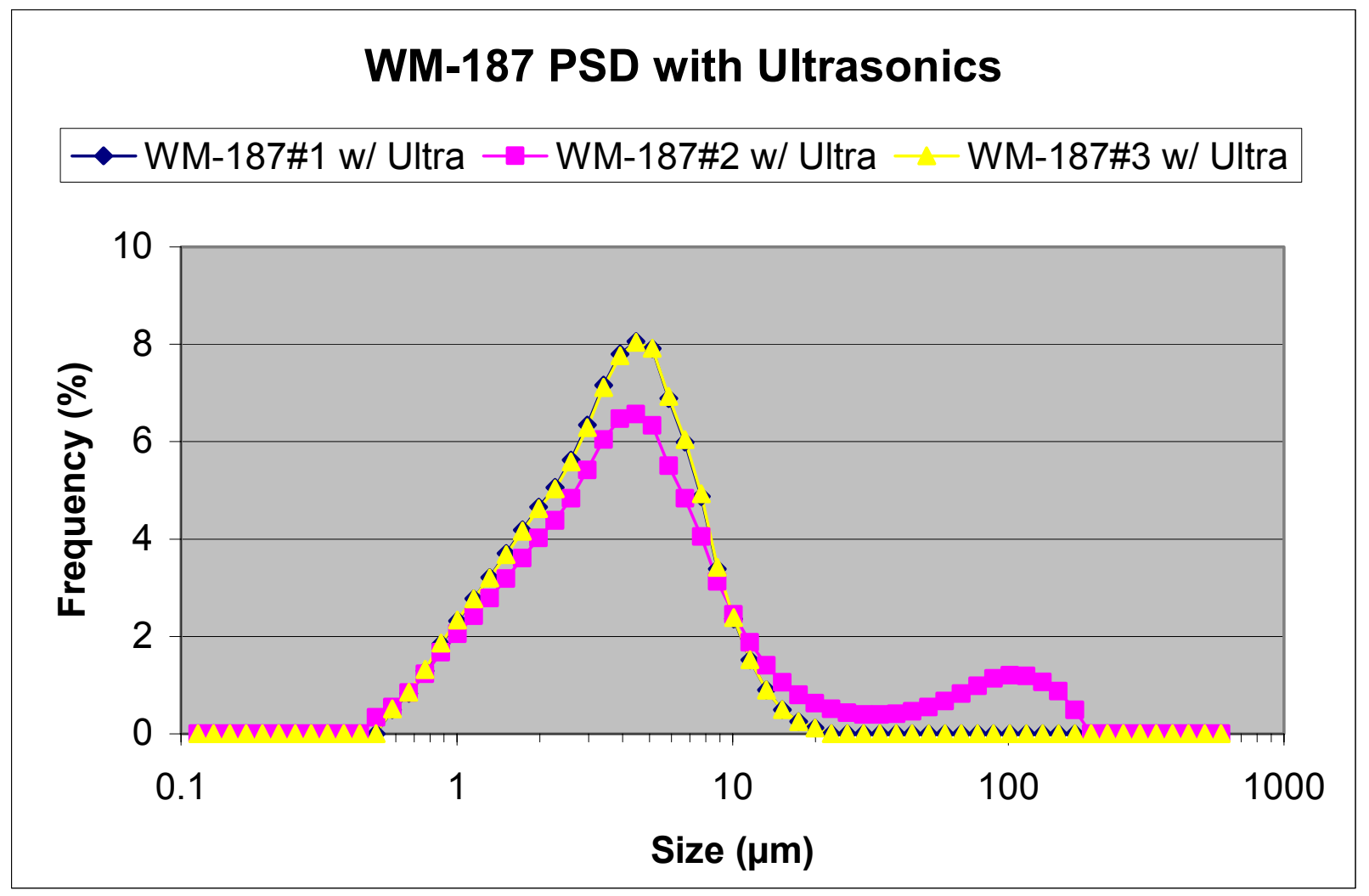


WM-180 Average WM-181 Average WM-187 Average WM-188 Average WM-189 Average

\begin{tabular}{|c|c|c|c|c|c|}
\hline Diameter & Frequency (\%) & Frequency (\%) & Frequency (\%) & Frequency (\%) & Frequency (\%) \\
\hline 0.115 & ; & - & ( & ) & 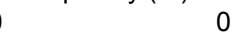 \\
\hline 0.131 & 0 & 0 & 0 & 0 & 0 \\
\hline 0.15 & 0 & 0 & 0 & 0 & 0 \\
\hline 0.172 & 0 & 0 & 0 & 0 & 0 \\
\hline 0.197 & 0 & 0 & 0 & 0 & 0 \\
\hline 0.226 & 0 & 0 & 0 & 0 & 0 \\
\hline 0.259 & 0 & 0 & 0 & 0 & 0 \\
\hline 0.296 & 0 & 0 & 0 & 0 & 0 \\
\hline 0.339 & 0 & 0 & 0 & 0 & 0 \\
\hline 0.389 & 0 & 0 & 0 & 0 & 0 \\
\hline 0.445 & 0 & 0 & 0.127 & 0 & 0 \\
\hline 0.51 & 0 & 0 & 0.187 & 0 & 0 \\
\hline 0.584 & 0 & 0.122 & 0.585 & 0.383 & 0.131 \\
\hline 0.669 & 0 & 0.166 & 0.909 & 0.655 & 0.152 \\
\hline 0.766 & 0 & 0.276 & 1.332 & 1.034 & 0.185 \\
\hline 0.877 & 0 & 0.426 & 1.829 & 1.496 & 0.218 \\
\hline 1.005 & 0 & 0.603 & 2.261 & 1.917 & 0.249 \\
\hline 1.151 & 0 & 0.817 & 2.681 & 2.322 & 0.285 \\
\hline 1.318 & 0 & 1.063 & 3.1 & 2.691 & 0.325 \\
\hline 1.51 & 0 & 1.355 & 3.555 & 3.075 & 0.365 \\
\hline 1.729 & 0.142 & 1.688 & 4.042 & 3.394 & 0.425 \\
\hline 1.981 & 0.288 & 2.048 & 4.537 & 3.66 & 0.499 \\
\hline 2.269 & 0.535 & 2.439 & 4.975 & 3.888 & 0.577 \\
\hline 2.599 & 0.917 & 2.96 & 5.573 & 4.153 & 0.69 \\
\hline 2.976 & 1.467 & 3.58 & 6.326 & 4.51 & 0.815 \\
\hline 3.409 & 2.213 & 4.483 & 7.203 & 4.888 & 1.009 \\
\hline 3.905 & 3.174 & 5.52 & 7.895 & 5.127 & 1.245 \\
\hline 4.472 & 4.421 & 6.612 & 8.194 & 5.184 & 1.521 \\
\hline 5.122 & 5.813 & 7.702 & 8.062 & 5.118 & 1.846 \\
\hline 5.867 & 7.056 & 8.386 & 6.987 & 4.945 & 2.223 \\
\hline 6.72 & 8.553 & 8.987 & 6.049 & 4.812 & 2.659 \\
\hline 7.697 & 9.304 & 9.144 & 4.88 & 4.651 & 3.157 \\
\hline 8.816 & 9.535 & 8.155 & 3.336 & 4.473 & 3.735 \\
\hline 10.097 & 9.778 & 7.193 & 2.297 & 4.285 & 4.387 \\
\hline 11.565 & 8.835 & 5.831 & 1.455 & 4.055 & 5.094 \\
\hline 13.246 & 7.418 & 4.306 & 0.849 & 3.773 & 5.831 \\
\hline 15.172 & 5.898 & 2.876 & 0.458 & 3.43 & 6.549 \\
\hline 17.377 & 4.48 & 1.728 & 0.23 & 3.022 & 7.174 \\
\hline 19.904 & 3.27 & 0.931 & 0.128 & 2.558 & 7.602 \\
\hline 22.797 & 2.316 & 0.45 & 0 & 2.06 & 7.718 \\
\hline 26.111 & 1.605 & 0.196 & 0 & 1.568 & 7.439 \\
\hline 29.907 & 1.093 & 0.109 & 0 & 1.124 & 6.751 \\
\hline 34.255 & 0.736 & 0 & 0 & 0.758 & 5.729 \\
\hline 39.234 & 0.49 & 0 & 0 & 0.484 & 4.527 \\
\hline 44.938 & 0.323 & 0 & 0 & 0.295 & 3.331 \\
\hline 51.471 & 0.211 & 0 & 0 & 0.174 & 2.291 \\
\hline 58.953 & 0.137 & 0 & 0 & 0.039 & 1.487 \\
\hline 67.523 & 0 & 0 & 0 & 0 & 0.924 \\
\hline 77.339 & 0 & 0 & 0 & 0 & 0.56 \\
\hline 88.583 & 0 & 0 & 0 & 0 & 0.338 \\
\hline 101.46 & 0 & 0 & 0 & 0 & 0 \\
\hline 116.21 & 0 & 0 & 0 & 0 & 0 \\
\hline 133.103 & 0 & 0 & 0 & 0 & 0 \\
\hline 152.453 & 0 & 0 & 0 & 0 & 0 \\
\hline 174.616 & 0 & 0 & 0 & 0 & 0 \\
\hline 200 & 0 & 0 & 0 & 0 & 0 \\
\hline 229.075 & 0 & 0 & 0 & 0 & 0 \\
\hline 262.376 & 0 & 0 & 0 & 0 & 0 \\
\hline 300.518 & 0 & 0 & 0 & 0 & 0 \\
\hline 344.206 & 0 & 0 & 0 & 0 & 0 \\
\hline 394.244 & 0 & 0 & 0 & 0 & 0 \\
\hline 451.556 & 0 & 0 & 0 & 0 & 0 \\
\hline 517.2 & 0 & 0 & 0 & 0 & 0 \\
\hline 592.387 & 0 & 0 & 0 & 0 & 0 \\
\hline
\end{tabular}




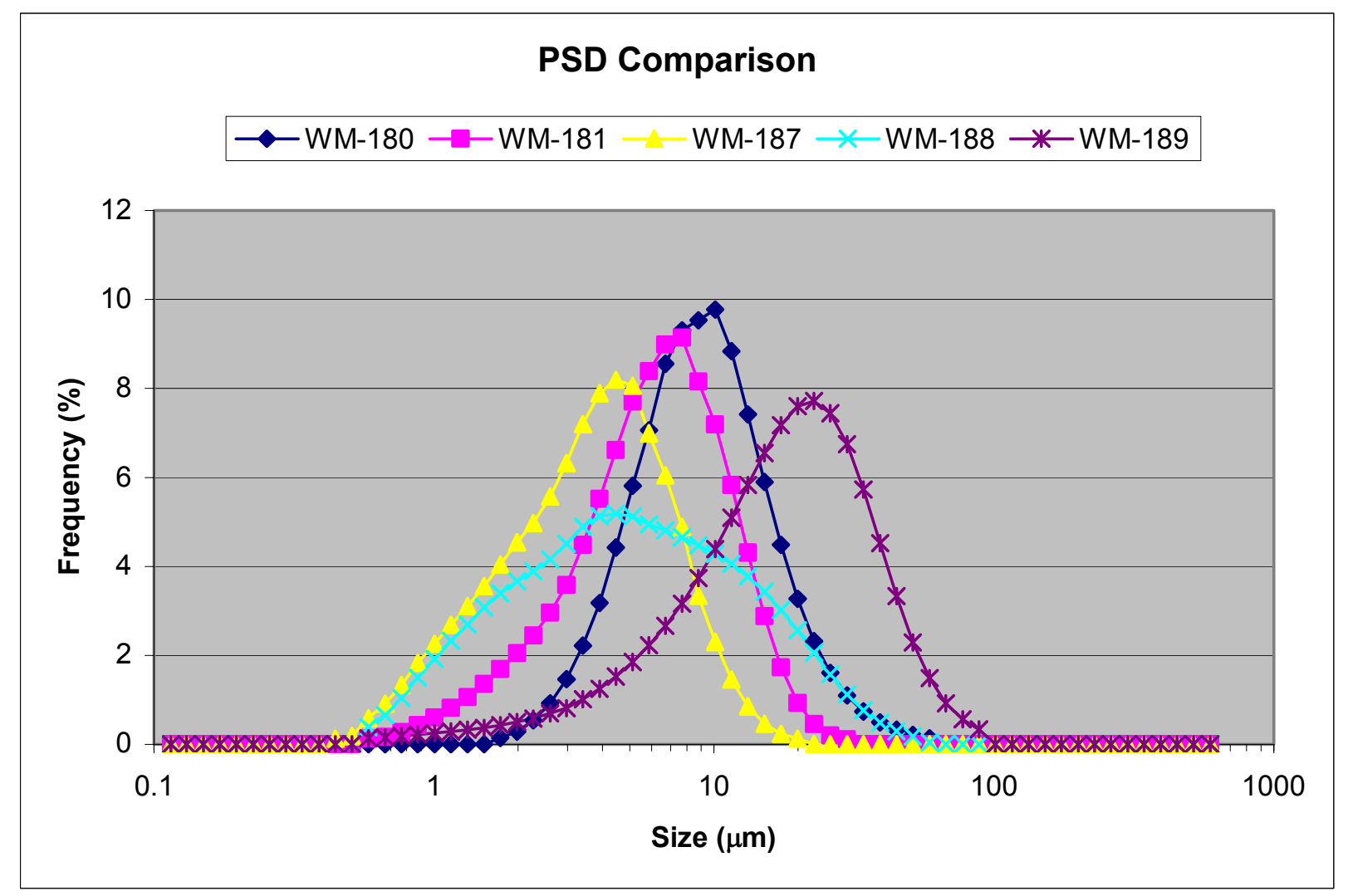

\section{E-2 FLOW CURVE}

Data in gray text was below the $10 \%$ (permil scale range $0-1,000 \%$ ) torque threshold limit of the Brookfield R/S rheometer. The grayed-out data are included to provide a complete data set and are for reference only.

\section{E-2.1 SBW Surrogate Samples}

\section{E-2.1.1 180/0wt\%}

2004.01.22 180-0 wt\% 500-4000 s-1 DG run 1.txt

Mode Step t[s] $\mathrm{n}[\mathrm{rpm}] \quad \mathrm{M}[\%$.] $\quad \mathrm{Phi}[\mathrm{rad}]$

$\begin{array}{rrrrrrr}20 & 99.23 & 12.2 & 207.56895 & 0 & 0 & 28 \\ 40 & 198.46 & 26.22 & 621.50049 & 0 & 0 & 28 \\ 60 & 297.68 & 40.48 & 1243.71805 & 0 & 0 & 28 \\ 80 & 396.91 & 63.05 & 2074.01271 & 0 & 0 & 28 \\ 100 & 496.13 & 88.76 & 3110.20029 & 0 & 0 & 28 \\ 120 & 595.36 & 115.26 & 4355.76638 & 0 & 0 & 28 \\ 140 & 694.58 & 142.83 & 5809.46219 & 0 & 0 & 28 \\ 160 & 793.81 & 171.85 & 7470.66099 & 0 & 0 & 28 \\ 180 & 793.81 & 171.53 & 9151.29447 & 0 & 0 & 28 \\ 200 & 793.81 & 171.43 & 10814.26041 & 0 & 0 & 28 \\ 220 & 793.81 & 171.29 & 12476.81323 & 0 & 0 & 28 \\ 240 & 793.81 & 171.27 & 14156.81996 & 0 & 0 & 28\end{array}$

$\begin{array}{rcccccc}\mathrm{D}[1 / \mathrm{s}] & \mathrm{Tau}[\mathrm{Pa}] & \mathrm{Eta}[\mathrm{Pas}] & \mathrm{MP} \# & \text { Block } & \mathrm{M}[\mathrm{mNm}] & \mathrm{G}[\mathrm{Pa}] \\ 500.02 & 0.818 & 0.002 & 1 & 1 & 0.6102 & 0 \\ 1000.04 & 1.756 & 0.002 & 2 & 1 & 1.3108 & 0 \\ 1500.01 & 2.712 & 0.002 & 3 & 1 & 2.02395 & 0 \\ 2000.03 & 4.224 & 0.002 & 4 & 1 & 3.1525 & 0 \\ 2500 & 5.947 & 0.002 & 5 & 1 & 4.4378 & 0 \\ 3000.02 & 7.723 & 0.003 & 6 & 1 & 5.76315 & 0 \\ 3499.99 & 9.569 & 0.003 & 7 & 1 & 7.1414 & 0 \\ 4000.01 & 11.514 & 0.003 & 8 & 1 & 8.59245 & 0 \\ 4000.01 & 11.493 & 0.003 & 9 & 1 & 8.5766 & 0 \\ 4000.01 & 11.486 & 0.003 & 10 & 1 & 8.57135 & 0 \\ 4000.01 & 11.476 & 0.003 & 11 & 1 & 8.56425 & 0 \\ 4000.01 & 11.475 & 0.003 & 12 & 1 & 8.5637 & 0\end{array}$




\begin{tabular}{|c|c|c|c|c|c|c|c|c|c|c|c|c|c|}
\hline 3 & 260 & 694.58 & 142.43 & 15613.89299 & 0 & 0 & 28 & 3499.99 & 9.543 & 0.003 & 13 & 1 & 7.12155 \\
\hline 3 & 280 & 595.36 & 115.1 & 16861.84983 & 0 & 0 & 28 & 3000.02 & 7.711 & 0.003 & 14 & 1 & 5.75475 \\
\hline 3 & 300 & 496.13 & 88.62 & 17902.16467 & 0 & 0 & 28 & 2500 & 5.938 & 0.002 & 15 & 1 & 4.4311 \\
\hline 3 & 320 & 396.91 & 62.89 & 18735.35510 & & 0 & 28 & 2000.03 & 4.214 & 0.002 & 16 & 1 & 3.1445 \\
\hline 3 & 340 & 297.68 & 40.52 & 19360.72054 & 0 & 0 & 28 & 1500.01 & 2.715 & 0.002 & 17 & 1 & 2.0261 \\
\hline 3 & 360 & 198.46 & 26.14 & 19778.04578 & 0 & 0 & 28 & 1000.04 & 1.751 & 0.002 & 18 & 1 & 1.30695 \\
\hline 3 & 380 & 99.23 & 12.8 & 19987.63477 & 0 & 0 & 28 & 500.02 & 0.857 & 0.002 & 19 & 1 & 0.63975 \\
\hline
\end{tabular}

2004.01.22 180-0 wt\% 500-4000 s-1 DG run 2.txt

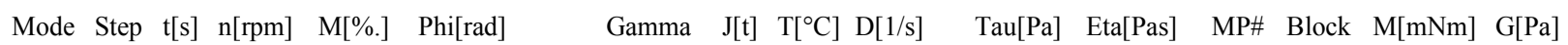

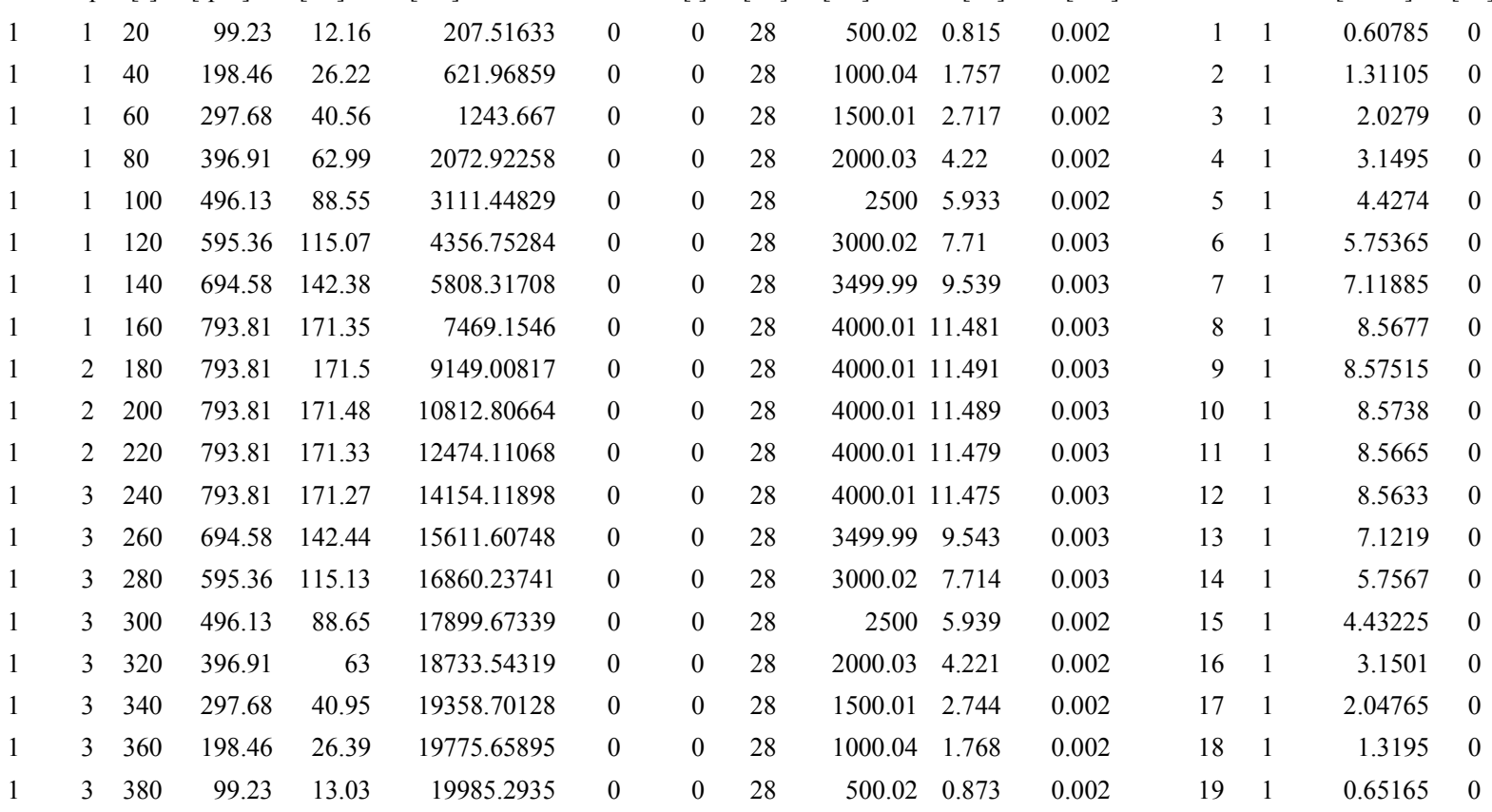



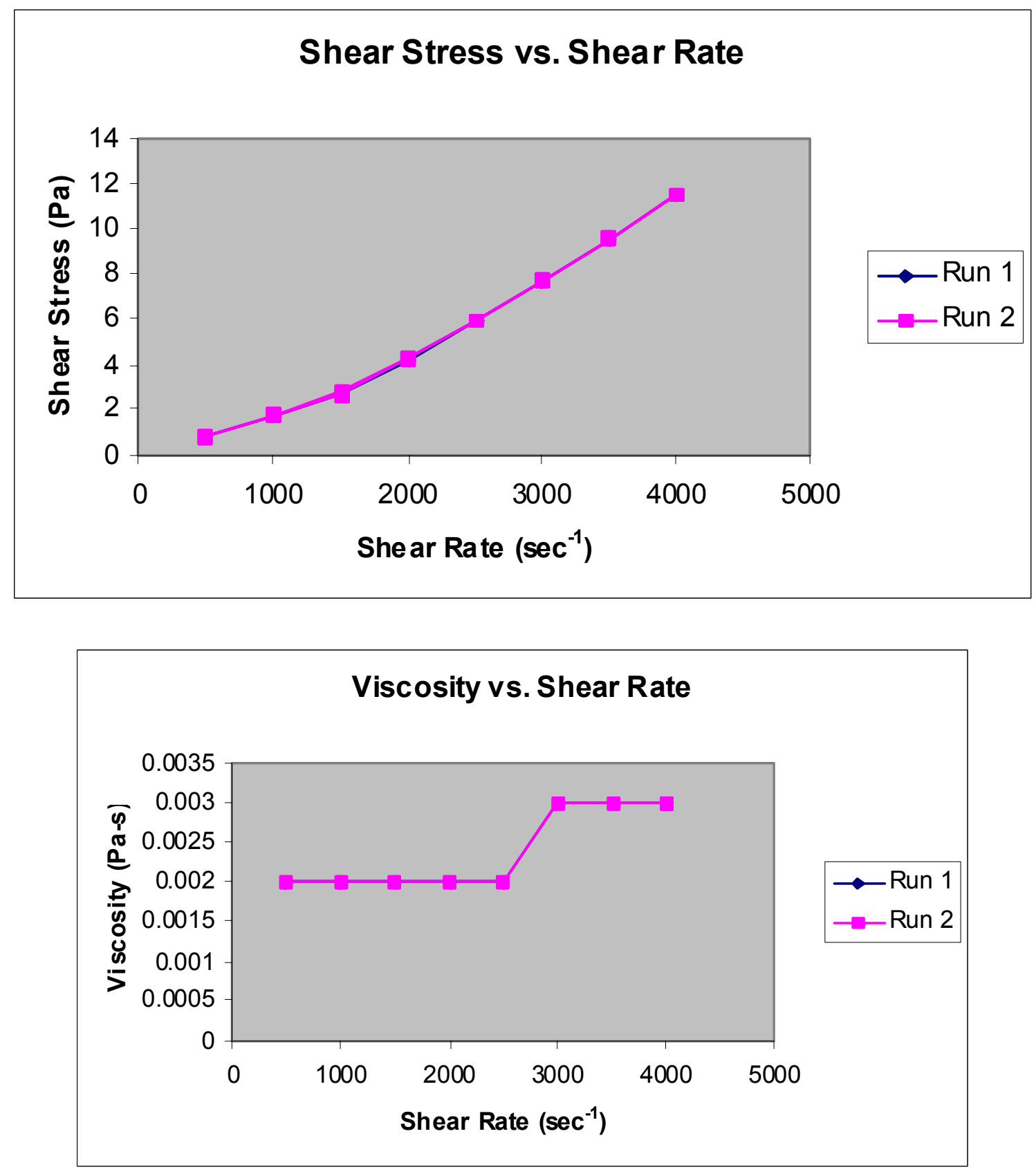

\section{E-2.1.2 187/0wt\%}

2004.01.22 187-0 wt\% 500-4000 s-1 DG run 1.txt Mode Step t[s] $\mathrm{n}[\mathrm{rpm}] \quad \mathrm{M}[\%$.]

$\begin{array}{rrr}20 & 99.27 & 6.31 \\ 40 & 198.46 & 13.41 \\ 60 & 297.68 & 26.39 \\ 80 & 396.91 & 40.65 \\ 100 & 496.13 & 56.01 \\ 120 & 595.36 & 73.17 \\ 140 & 694.58 & 91.5\end{array}$

Phi[rad]

207.72917

621.76674

1243.31043

2073.1904

3111.4585

4356.09153

5809.16217

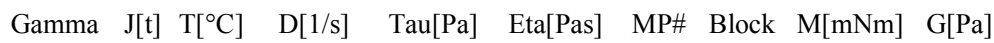

$0.898 \quad 0.001$

$1.768 \quad 0.001$

$2.723 \quad 0.001$

$3.753 \quad 0.002$

$4.903 \quad 0.002$

$6.13 \quad 0.002$ $\begin{array}{llll}1 & 1 & 0.31545 & 0\end{array}$

$\begin{array}{llll}2 & 1 & 0.67035 & 0\end{array}$

$\begin{array}{llll}3 & 1 & 1.31955 & 0\end{array}$

$\begin{array}{llll}4 & 1 & 2.0323 & 0\end{array}$

$\begin{array}{llll}5 & 1 & 2.80045 & 0\end{array}$

$\begin{array}{llll}6 & 1 & 3.6587 & 0\end{array}$

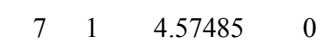




$\begin{array}{rrrrrrrrrrrrrrrrr}1 & 1 & 160 & 793.81 & 111.53 & 7470.00047 & 0 & 0 & 28 & 4000.01 & 7.473 & 0.002 & 8 & 1 & 5.5767 & 0 \\ 1 & 2 & 180 & 793.81 & 111.46 & 9149.83284 & 0 & 0 & 28 & 4000.01 & 7.468 & 0.002 & 9 & 1 & 5.5729 & 0 \\ 1 & 2 & 200 & 793.81 & 110.97 & 10813.63052 & 0 & 0 & 28 & 4000.01 & 7.435 & 0.002 & 10 & 1 & 5.5486 & 0 \\ 1 & 2 & 220 & 793.81 & 110.84 & 12476.1802 & 0 & 0 & 28 & 4000.01 & 7.426 & 0.002 & 11 & 1 & 5.54185 & 0 \\ 1 & 3 & 240 & 793.81 & 110.9 & 14156.19007 & 0 & 0 & 28 & 4000.01 & 7.43 & 0.002 & 12 & 1 & 5.545 & 0 \\ 1 & 3 & 260 & 694.58 & 91.29 & 15613.74848 & 0 & 0 & 28 & 3499.99 & 6.116 & 0.002 & 13 & 1 & 4.5643 & 0 \\ 1 & 3 & 280 & 595.36 & 72.75 & 16861.99356 & 0 & 0 & 28 & 3000.02 & 4.874 & 0.002 & 14 & 1 & 3.63755 & 0 \\ 1 & 3 & 300 & 496.13 & 55.66 & 17903.07338 & 0 & 0 & 28 & 2500 & 3.729 & 0.001 & 15 & 1 & 2.7831 & 0 \\ 1 & 3 & 320 & 396.91 & 40.24 & 18735.42422 & 0 & 0 & 28 & 2000.03 & 2.696 & 0.001 & 16 & 1 & 2.01175 & 0 \\ 1 & 3 & 340 & 297.68 & 25.95 & 19361.13208 & 0 & 0 & 28 & 1500.01 & 1.738 & 0.001 & 17 & 1 & 1.29735 & 0 \\ 1 & 3 & 360 & 198.46 & 13.02 & 19778.16359 & 0 & 0 & 28 & 1000.04 & 0.873 & 0.001 & 18 & 1 & 0.65115 & 0 \\ 1 & 3 & 380 & 99.23 & 6.37 & 19987.81384 & 0 & 0 & 28 & 500.02 & 0.427 & 0.001 & 19 & 1 & 0.3185 & 0\end{array}$

2004.01.22 187-0 wt\% 500-4000 s-1 DG run 2.txt

Mode Step t[s] n[rpm] M[\%.] Phi[rad]

$\begin{array}{rrrrrr}1 & 1 & 20 & 99.31 & 5.84 & 207.8352 \\ 1 & 1 & 40 & 198.46 & 12.99 & 621.61202 \\ 1 & 1 & 60 & 297.68 & 25.82 & 1243.46672 \\ 1 & 1 & 80 & 396.91 & 39.98 & 2073.19118 \\ 1 & 1 & 100 & 496.13 & 55.46 & 3110.68017 \\ 1 & 1 & 120 & 595.36 & 72.73 & 4355.67449 \\ 1 & 1 & 140694.58 & 91.21 & 5808.69722 \\ 1 & 1 & 160793.81 & 111.27 & 7470.36804 \\ 1 & 2 & 180793.81 & 111.32 & 9150.19648 \\ 1 & 2 & 200793.81 & 111.07 & 10813.99652 \\ 1 & 2 & 220793.81 & 111.07 & 12475.30213 \\ 1 & 3 & 240793.81 & 110.95 & 14155.31199 \\ 1 & 3 & 260694.58 & 91.25 & 15612.07715 \\ 1 & 3 & 280595.36 & 72.67 & 16859.76303 \\ 1 & 3 & 300496.13 & 55.45 & 17900.83971 \\ 1 & 3 & 320396.91 & 40.15 & 18734.13852 \\ 1 & 3 & 340297.68 & 25.84 & 19359.84246 \\ 1 & 3 & 360198.46 & 12.93 & 19776.97685 \\ 1 & 3 & 380 & 99.23 & 6.39 & 19986.57763\end{array}$

Gamma $\quad \mathrm{J}[\mathrm{t}] \quad \mathrm{T}\left[{ }^{\circ} \mathrm{C}\right] \quad \mathrm{D}[1 / \mathrm{s}]$

0
0
0
0
0
0
0
0
0
0
0
0
0
0
0
0
0
0
0

$\begin{array}{llll}0 & 28 & 500.42 & 0.391 \\ 0 & 28 & 1000.04 & 0.871 \\ 0 & 27.9 & 1500.01 & 1.73 \\ 0 & 28 & 2000.03 & 2.679 \\ 0 & 28 & 2500 & 3.716 \\ 0 & 28 & 3000.02 & 4.873 \\ 0 & 28 & 3499.99 & 6.111 \\ 0 & 28 & 4000.01 & 7.455 \\ 0 & 28 & 4000.01 & 7.458 \\ 0 & 28 & 4000.01 & 7.441 \\ 0 & 28 & 4000.01 & 7.442 \\ 0 & 28 & 4000.01 & 7.434 \\ 0 & 28 & 3499.99 & 6.113 \\ 0 & 28 & 3000.02 & 4.869 \\ 0 & 28 & 2500 & 3.715 \\ 0 & 28 & 2000.03 & 2.69 \\ 0 & 28 & 1500.01 & 1.731 \\ 0 & 28 & 1000.04 & 0.867 \\ 0 & 28 & 500.02 & 0.428 \\ 0 & & & \end{array}$

Eta[Pas] MP\# Block M[mNm] G[Pa]

$\begin{array}{lllll}0.001 & 1 & 1 & 0.292 & 0 \\ 0.001 & 2 & 1 & 0.64965 & 0 \\ 0.001 & 3 & 1 & 1.2908 & 0 \\ 0.001 & 4 & 1 & 1.9989 & 0 \\ 0.001 & 5 & 1 & 2.77295 & 0 \\ 0.002 & 6 & 1 & 3.63645 & 0 \\ 0.002 & 7 & 1 & 4.56065 & 0 \\ 0.002 & 8 & 1 & 5.56325 & 0 \\ 0.002 & 9 & 1 & 5.566 & 0 \\ 0.002 & 10 & 1 & 5.5533 & 0 \\ 0.002 & 11 & 1 & 5.55355 & 0 \\ 0.002 & 12 & 1 & 5.5476 & 0 \\ 0.002 & 13 & 1 & 4.56225 & 0 \\ 0.002 & 14 & 1 & 3.6333 & 0 \\ 0.001 & 15 & 1 & 2.77265 & 0 \\ 0.001 & 16 & 1 & 2.00765 & 0 \\ 0.001 & 17 & 1 & 1.29185 & 0 \\ 0.001 & 18 & 1 & 0.6467 & 0 \\ 0.001 & 19 & 1 & 0.31955 & 0\end{array}$



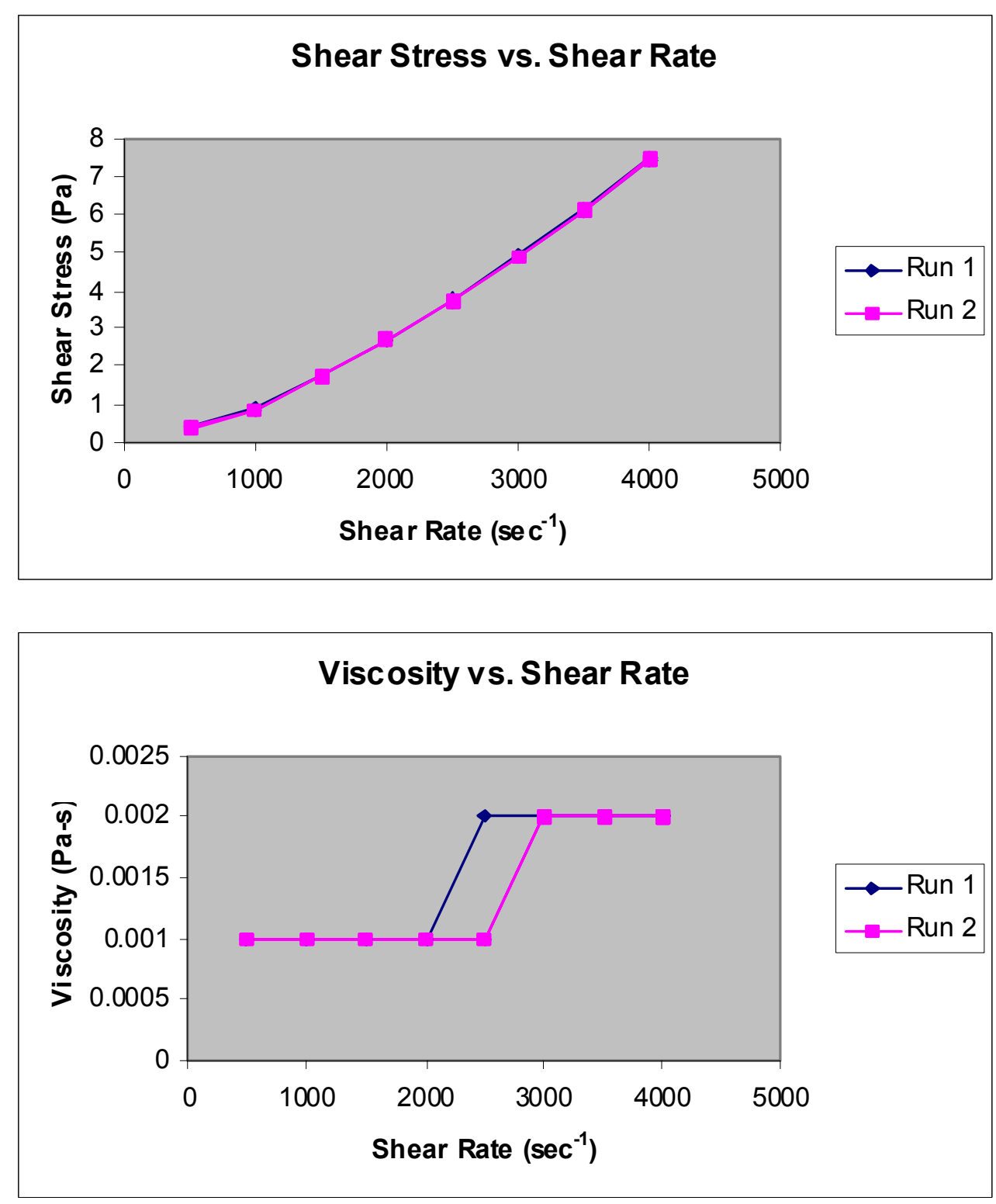

\section{E-2.1.3 189/0wt\%}

2004.01.22 189-0 wt\% 500-4000 s-1 DG run 1.txt Mode Step t[s] $\mathrm{n}[\mathrm{rpm}] \quad \mathrm{M}[\%$.] Phi[rad]

$\begin{array}{rrrrrr}1 & 1 & 20 & 99.23 & 15.63 & 207.67184 \\ 1 & 1 & 40 & 198.46 & 32.62 & 621.39525 \\ 1 & 1 & 60 & 297.68 & 49.95 & 1243.24995 \\ 1 & 1 & 80 & 396.91 & 70.13 & 2073.18097 \\ 1 & 1 & 100 & 496.13 & 100.72 & 3111.13413 \\ 1 & 1 & 120 & 595.36 & 132.27 & 4356.07425 \\ 1 & 1 & 140 & 694.58 & 164.07 & 5809.81955 \\ 1 & 1 & 160 & 793.81 & 197.58 & 7470.18975 \\ 1 & 2 & 180 & 793.81 & 197.76 & 9151.7013 \\ 1 & 2 & 200 & 793.81 & 197.79 & 10813.84179\end{array}$

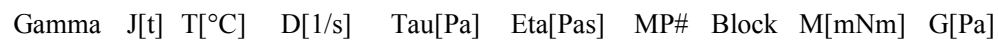

$\begin{array}{llllllllll}0 & 0 & 28 & 500.02 & 1.047 & 0.002 & 1 & 1 & 0.7814 & 0 \\ 0 & 0 & 28 & 1000.04 & 2.186 & 0.002 & 2 & 1 & 1.63115 & 0 \\ 0 & 0 & 28 & 1500.01 & 3.347 & 0.002 & 3 & 1 & 2.49755 & 0 \\ 0 & 0 & 28 & 2000.03 & 4.699 & 0.002 & 4 & 1 & 3.5067 & 0 \\ 0 & 0 & 28 & 2500 & 6.748 & 0.003 & 5 & 1 & 5.03585 & 0 \\ 0 & 0 & 28 & 3000.02 & 8.862 & 0.003 & 6 & 1 & 6.6134 & 0 \\ 0 & 0 & 28 & 3499.99 & 10.993 & 0.003 & 7 & 1 & 8.20345 & 0 \\ 0 & 0 & 28 & 4000.01 & 13.238 & 0.003 & 8 & 1 & 9.8791 & 0 \\ 0 & 0 & 28 & 4000.01 & 13.25 & 0.003 & 9 & 1 & 9.88805 & 0 \\ 0 & 0 & 28 & 4000.01 & 13.252 & 0.003 & 10 & 1 & 9.8896 & 0\end{array}$




$\begin{array}{rrrrrl}1 & 2 & 220 & 793.81 & 197.81 & 12476.39069 \\ 1 & 3 & 240 & 793.81 & 197.61 & 14155.98665 \\ 1 & 3 & 260 & 694.58 & 164.33 & 15612.74709 \\ 1 & 3 & 280 & 595.36 & 132.23 & 16862.26138 \\ 1 & 3 & 300 & 496.13 & 100.42 & 17903.07417 \\ 1 & 3 & 320 & 396.91 & 69.61 & 18735.4415 \\ 1 & 3 & 340 & 297.68 & 50.27 & 19360.27679 \\ 1 & 3 & 360 & 198.46 & 32.67 & 19777.99708 \\ 1 & 3 & 380 & 99.23 & 16.2 & 19987.67483\end{array}$

2004.01.22 189-0 wt\% 500-4000 s-1 DG run 2.txt

Mode Step t[s] n[rpm] M[\%.] Phi[rad]

$\begin{array}{rrrrrl}1 & 1 & 20 & 99.23 & 15.53 & 207.62 \\ 1 & 1 & 40 & 198.46 & 32.57 & 621.3442 \\ 1 & 1 & 60 & 297.68 & 50.25 & 1243.71648 \\ 1 & 1 & 80 & 396.91 & 69.5 & 2072.76393 \\ 1 & 1 & 100 & 496.13 & 100.67 & 3110.24977 \\ 1 & 1 & 120 & 595.36 & 132.64 & 4356.75127 \\ 1 & 1 & 140 & 694.58 & 164.91 & 5808.26211 \\ 1 & 1 & 160 & 793.81 & 198.36 & 7469.46169 \\ 1 & 2 & 180 & 793.81 & 198.23 & 9148.89586 \\ 1 & 2 & 200 & 793.81 & 198.06 & 10811.86652 \\ 1 & 2 & 220 & 793.81 & 198.22 & 12475.25029 \\ 1 & 3 & 240 & 793.81 & 198.15 & 14155.25545 \\ 1 & 3 & 260 & 694.58 & 165.1 & 15612.38267 \\ 1 & 3 & 280 & 595.36 & 133.05 & 16860.39292 \\ 1 & 3 & 300 & 496.13 & 100.86 & 17901.20492 \\ 1 & 3 & 320 & 396.91 & 69.65 & 18734.81239 \\ 1 & 3 & 340 & 297.68 & 50.59 & 19359.22592 \\ 1 & 3 & 360 & 198.46 & 33.09 & 19776.94308 \\ 1 & 3 & 380 & 99.23 & 16.39 & 19986.41584\end{array}$

$\begin{array}{llllllllll}0 & 0 & 28 & 4000.01 & 13.254 & 0.003 & 11 & 1 & 9.8907 & 0 \\ 0 & 0 & 28 & 4000.01 & 13.24 & 0.003 & 12 & 1 & 9.8806 & 0 \\ 0 & 0 & 28 & 3499.99 & 11.01 & 0.003 & 13 & 1 & 8.21625 & 0 \\ 0 & 0 & 28 & 3000.02 & 8.86 & 0.003 & 14 & 1 & 6.6116 & 0 \\ 0 & 0 & 28 & 2500 & 6.728 & 0.003 & 15 & 1 & 5.02115 & 0 \\ 0 & 0 & 28 & 2000.03 & 4.664 & 0.002 & 16 & 1 & 3.4806 & 0 \\ 0 & 0 & 28 & 1500.01 & 3.368 & 0.002 & 17 & 1 & 2.5133 & 0 \\ 0 & 0 & 28 & 1000.04 & 2.189 & 0.002 & 18 & 1 & 1.6335 & 0 \\ 0 & 0 & 28 & 500.02 & 1.086 & 0.002 & 19 & 1 & 0.81025 & 0\end{array}$

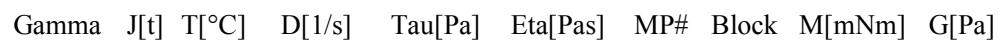

$\begin{array}{llllllllll}0 & 0 & 28 & 500.02 & 1.04 & 0.002 & 1 & 1 & 0.77625 & 0 \\ 0 & 0 & 28 & 1000.04 & 2.182 & 0.002 & 2 & 1 & 1.6283 & 0 \\ 0 & 0 & 28 & 1500.01 & 3.367 & 0.002 & 3 & 1 & 2.5125 & 0 \\ 0 & 0 & 28 & 2000.03 & 4.656 & 0.002 & 4 & 1 & 3.47475 & 0 \\ 0 & 0 & 28 & 2500 & 6.745 & 0.003 & 5 & 1 & 5.0337 & 0 \\ 0 & 0 & 28 & 3000.02 & 8.887 & 0.003 & 6 & 1 & 6.6322 & 0 \\ 0 & 0 & 28 & 3499.99 & 11.049 & 0.003 & 7 & 1 & 8.2455 & 0 \\ 0 & 0 & 28 & 4000.01 & 13.29 & 0.003 & 8 & 1 & 9.9181 & 0 \\ 0 & 0 & 28 & 4000.01 & 13.281 & 0.003 & 9 & 1 & 9.91145 & 0 \\ 0 & 0 & 28 & 4000.01 & 13.27 & 0.003 & 10 & 1 & 9.9029 & 0 \\ 0 & 0 & 28 & 4000.01 & 13.281 & 0.003 & 11 & 1 & 9.9109 & 0 \\ 0 & 0 & 28 & 4000.01 & 13.276 & 0.003 & 12 & 1 & 9.90735 & 0 \\ 0 & 0 & 28 & 3499.99 & 11.062 & 0.003 & 13 & 1 & 8.25495 & 0 \\ 0 & 0 & 28 & 3000.02 & 8.914 & 0.003 & 14 & 1 & 6.6525 & 0 \\ 0 & 0 & 28 & 2500 & 6.758 & 0.003 & 15 & 1 & 5.0432 & 0 \\ 0 & 0 & 28 & 2000.03 & 4.667 & 0.002 & 16 & 1 & 3.4825 & 0 \\ 0 & 0 & 28 & 1500.01 & 3.389 & 0.002 & 17 & 1 & 2.5294 & 0 \\ 0 & 0 & 28 & 1000.04 & 2.217 & 0.002 & 18 & 1 & 1.65465 & 0 \\ 0 & 0 & 28 & 500.02 & 1.098 & 0.002 & 19 & 1 & 0.8196 & 0\end{array}$

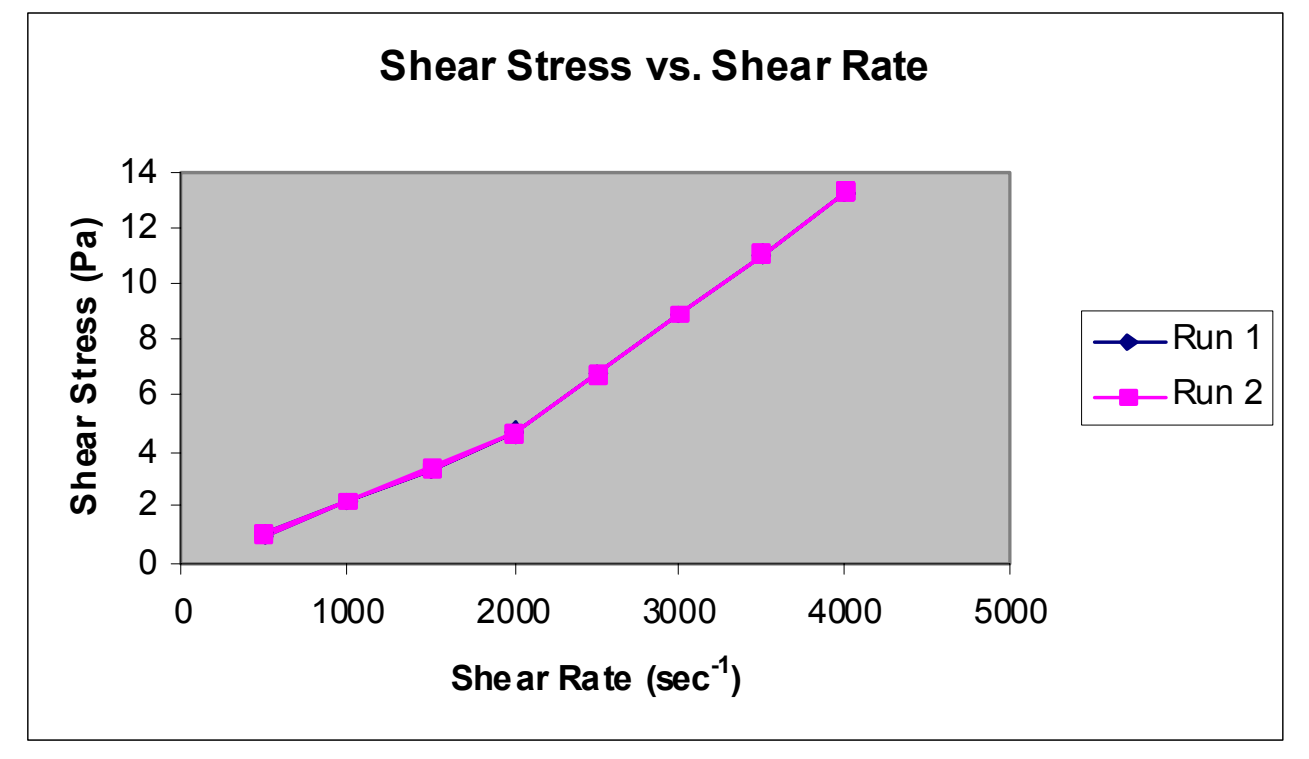




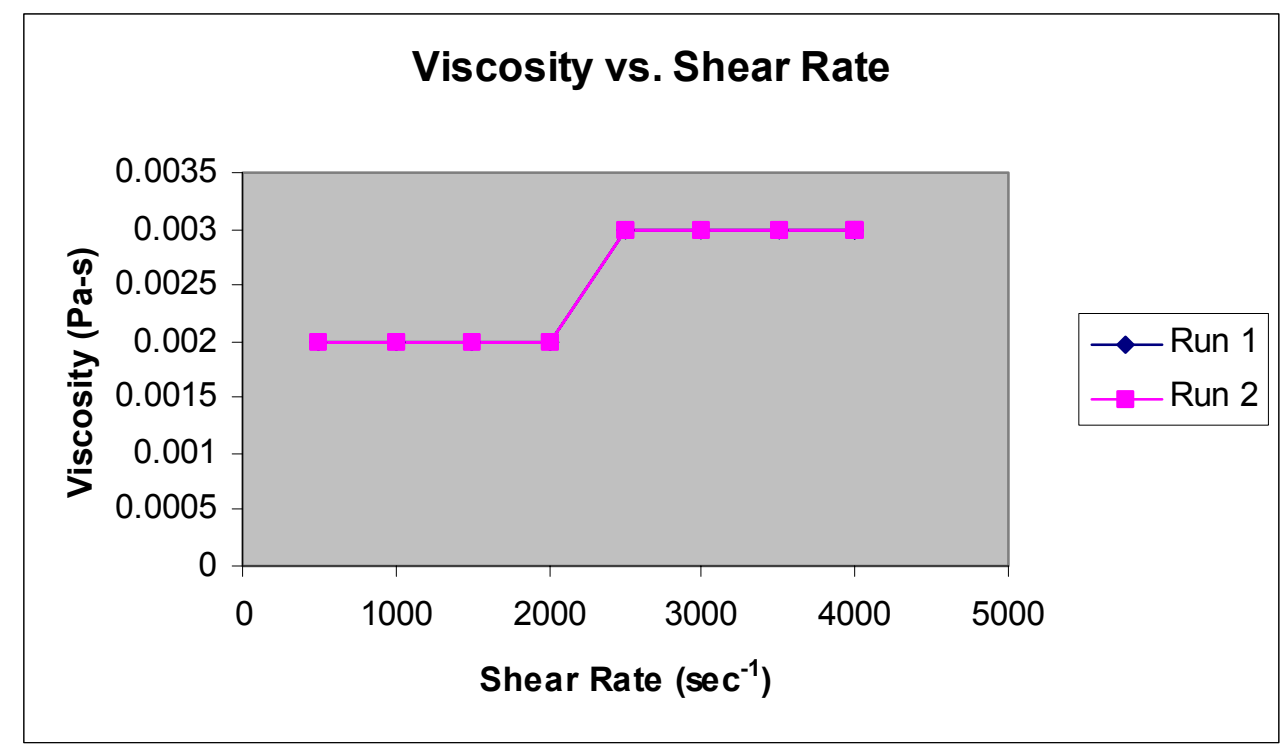

\section{E-2.1.4 META/0wt\%}

2004.01.22 META-0 wt\% 500-4000 s-1 DG run 1.txt Mode Step $\mathrm{t}[\mathrm{s}] \quad \mathrm{n}[\mathrm{rpm}] \quad \mathrm{M}[\%$.] $\quad \mathrm{Phi}[\mathrm{rad}]$

$\begin{array}{rrrrrr}1 & 1 & 20 & 99.23 & 8.34 & 207.56895 \\ 1 & 1 & 40 & 198.46 & 17.96 & 621.91832 \\ 1 & 1 & 60 & 297.68 & 30.45 & 1243.61673 \\ 1 & 1 & 80 & 396.91 & 48.23 & 2073.28779 \\ 1 & 1 & 100 & 496.13 & 66.88 & 3110.72493 \\ 1 & 1 & 120 & 595.36 & 86.69 & 4355.98079 \\ 1 & 1 & 140 & 694.58 & 107.85 & 5808.32572 \\ 1 & 1 & 160 & 793.81 & 130.37 & 7469.16402 \\ 1 & 2 & 180 & 793.81 & 130.46 & 9149.03802 \\ 1 & 2 & 200 & 793.81 & 130.1 & 10812.0071 \\ 1 & 2 & 220 & 793.81 & 130.16 & 12474.14288 \\ 1 & 3 & 240 & 793.81 & 130.06 & 14153.73727 \\ 1 & 3 & 260 & 694.58 & 107.78 & 15610.49614 \\ 1 & 3 & 280 & 595.36 & 86.48 & 16858.47497 \\ 1 & 3 & 300 & 496.13 & 66.8 & 17899.01287 \\ 1 & 3 & 320 & 396.91 & 48.36 & 18732.02737 \\ 1 & 3 & 340 & 297.68 & 30.46 & 19358.03133 \\ 1 & 3 & 360 & 198.46 & 17.97 & 19774.99686 \\ 1 & 3 & 380 & 99.23 & 8.91 & 19984.7987\end{array}$

2004.01.22 META-0 wt\% 500-4000 s-1 DG run 2.txt Mode Step $\mathrm{t}[\mathrm{s}] \quad \mathrm{n}[\mathrm{rpm}] \quad \mathrm{M}[\%$.] $\quad \mathrm{Phi}[\mathrm{rad}]$

$\begin{array}{rrrrrr}1 & 1 & 20 & 99.23 & 8.24 & 207.62158 \\ 1 & 1 & 40 & 198.46 & 18.07 & 621.50206 \\ 1 & 1 & 60 & 297.68 & 30.51 & 1244.03142 \\ 1 & 1 & 80 & 396.91 & 48.42 & 2073.54776 \\ 1 & 1 & 100 & 496.13 & 66.94 & 3110.77677 \\ 1 & 1 & 120 & 595.36 & 86.74 & 4356.03184\end{array}$

$\begin{array}{ccccccccccc}\text { Gamma } & \mathrm{J}[\mathrm{t}] & \mathrm{T}\left[{ }^{\circ} \mathrm{C}\right] & \mathrm{D}[1 / \mathrm{s}] & \mathrm{Tau}[\mathrm{Pa}] & \mathrm{Eta}[\mathrm{Pas}] & \mathrm{MP} \# & \text { Block } & \mathrm{M}[\mathrm{mNm}] & \mathrm{G}[\mathrm{Pa}] \\ 0 & 0 & 28 & 500.02 & 0.559 & 0.001 & 1 & 1 & 0.4168 & 0 \\ 0 & 0 & 28 & 1000.04 & 1.203 & 0.001 & 2 & 1 & 0.89785 & 0 \\ 0 & 0 & 28 & 1500.01 & 2.04 & 0.001 & 3 & 1 & 1.5225 & 0 \\ 0 & 0 & 28 & 2000.03 & 3.232 & 0.002 & 4 & 1 & 2.4117 & 0 \\ 0 & 0 & 28 & 2500 & 4.481 & 0.002 & 5 & 1 & 3.34375 & 0 \\ 0 & 0 & 28 & 3000.02 & 5.808 & 0.002 & 6 & 1 & 4.33445 & 0 \\ 0 & 0 & 28 & 3499.99 & 7.226 & 0.002 & 7 & 1 & 5.39235 & 0 \\ 0 & 0 & 28 & 4000.01 & 8.735 & 0.002 & 8 & 1 & 6.51855 & 0 \\ 0 & 0 & 28 & 4000.01 & 8.741 & 0.002 & 9 & 1 & 6.5229 & 0 \\ 0 & 0 & 28 & 4000.01 & 8.716 & 0.002 & 10 & 1 & 6.50485 & 0 \\ 0 & 0 & 28 & 4000.01 & 8.721 & 0.002 & 11 & 1 & 6.50785 & 0 \\ 0 & 0 & 28 & 4000.01 & 8.714 & 0.002 & 12 & 1 & 6.5031 & 0 \\ 0 & 0 & 28 & 3499.99 & 7.221 & 0.002 & 13 & 1 & 5.3888 & 0 \\ 0 & 0 & 28 & 3000.02 & 5.794 & 0.002 & 14 & 1 & 4.3239 & 0 & \\ 0 & 0 & 28 & 2500 & 4.476 & 0.002 & 15 & 1 & 3.34015 & 0 & \\ 0 & 0 & 28 & 2000.03 & 3.24 & 0.002 & 16 & 1 & 2.41775 & 0 & \\ 0 & 0 & 28 & 1500.01 & 2.041 & 0.001 & 17 & 1 & 1.52315 & 0 & \\ 0 & 0 & 28 & 1000.04 & 1.204 & 0.001 & 18 & 1 & 0.89835 & 0 & \\ 0 & 0 & 28 & 500.02 & 0.597 & 0.001 & 19 & 1 & 0.44555 & 0\end{array}$

Gamma $J[t] \mathrm{T}\left[{ }^{\circ} \mathrm{C}\right] \quad \mathrm{D}[1 / \mathrm{s}] \quad \mathrm{Tau}[\mathrm{Pa}] \quad$ Eta[Pas $] \quad$ MP\# Block $\mathrm{M}[\mathrm{mNm}] \quad \mathrm{G}[\mathrm{Pa}]$

$\begin{array}{llllllllll}0 & 0 & 28 & 500.02 & 0.552 & 0.001 & 1 & 1 & 0.4122 & 0 \\ 0 & 0 & 28 & 1000.04 & 1.211 & 0.001 & 2 & 1 & 0.9036 & 0 \\ 0 & 0 & 28 & 1500.01 & 2.044 & 0.001 & 3 & 1 & 1.52525 & 0 \\ 0 & 0 & 28 & 2000.03 & 3.244 & 0.002 & 4 & 1 & 2.4211 & 0 \\ 0 & 0 & 28 & 2500 & 4.485 & 0.002 & 5 & 1 & 3.34725 & 0 \\ 0 & 0 & 28 & 3000.02 & 5.811 & 0.002 & 6 & 1 & 4.3369 & 0\end{array}$




$\begin{array}{rrrrr}1 & 140 & 694.58 & 108.05 & 5808.37835 \\ 1 & 160 & 793.81 & 130.76 & 7469.63055 \\ 2 & 180 & 793.81 & 130.97 & 9148.60683 \\ 2 & 200 & 793.81 & 130.86 & 10811.16201 \\ 2 & 220 & 793.81 & 130.89 & 12474.54422 \\ 3 & 240 & 793.81 & 130.89 & 14153.72235 \\ 3 & 260 & 694.58 & 108.39 & 15610.89748 \\ 3 & 280 & 595.36 & 87.21 & 16859.23524 \\ 3 & 300 & 496.13 & 67.19 & 17900.81222 \\ 3 & 320 & 396.91 & 48.5 & 18733.83379 \\ 3 & 340 & 297.68 & 30.62 & 19358.70128 \\ 3 & 360 & 198.46 & 18.14 & 19776.1404 \\ 3 & 380 & 99.23 & 8.99 & 19985.68306\end{array}$

$\begin{array}{llllllllll}0 & 0 & 28 & 3499.99 & 7.239 & 0.002 & 7 & 1 & 5.40225 & 0 \\ 0 & 0 & 28.1 & 4000.01 & 8.761 & 0.002 & 8 & 1 & 6.53795 & 0 \\ 0 & 0 & 28 & 4000.01 & 8.775 & 0.002 & 9 & 1 & 6.54825 & 0 \\ 0 & 0 & 28 & 4000.01 & 8.768 & 0.002 & 10 & 1 & 6.54305 & 0 \\ 0 & 0 & 28 & 4000.01 & 8.77 & 0.002 & 11 & 1 & 6.5446 & 0 \\ 0 & 0 & 28 & 4000.01 & 8.77 & 0.002 & 12 & 1 & 6.5447 & 0 \\ 0 & 0 & 28 & 3499.99 & 7.262 & 0.002 & 13 & 1 & 5.4195 & 0 \\ 0 & 0 & 28 & 3000.02 & 5.843 & 0.002 & 14 & 1 & 4.3603 & 0 \\ 0 & 0 & 28.1 & 2500 & 4.502 & 0.002 & 15 & 1 & 3.35945 & 0 \\ 0 & 0 & 28 & 2000.03 & 3.25 & 0.002 & 16 & 1 & 2.42505 & 0 \\ 0 & 0 & 28.1 & 1500.01 & 2.052 & 0.001 & 17 & 1 & 1.5312 & 0 \\ 0 & 0 & 28.1 & 1000.04 & 1.215 & 0.001 & 18 & 1 & 0.907 & 0 \\ 0 & 0 & 28 & 500.02 & 0.603 & 0.001 & 19 & 1 & 0.44975 & 0\end{array}$
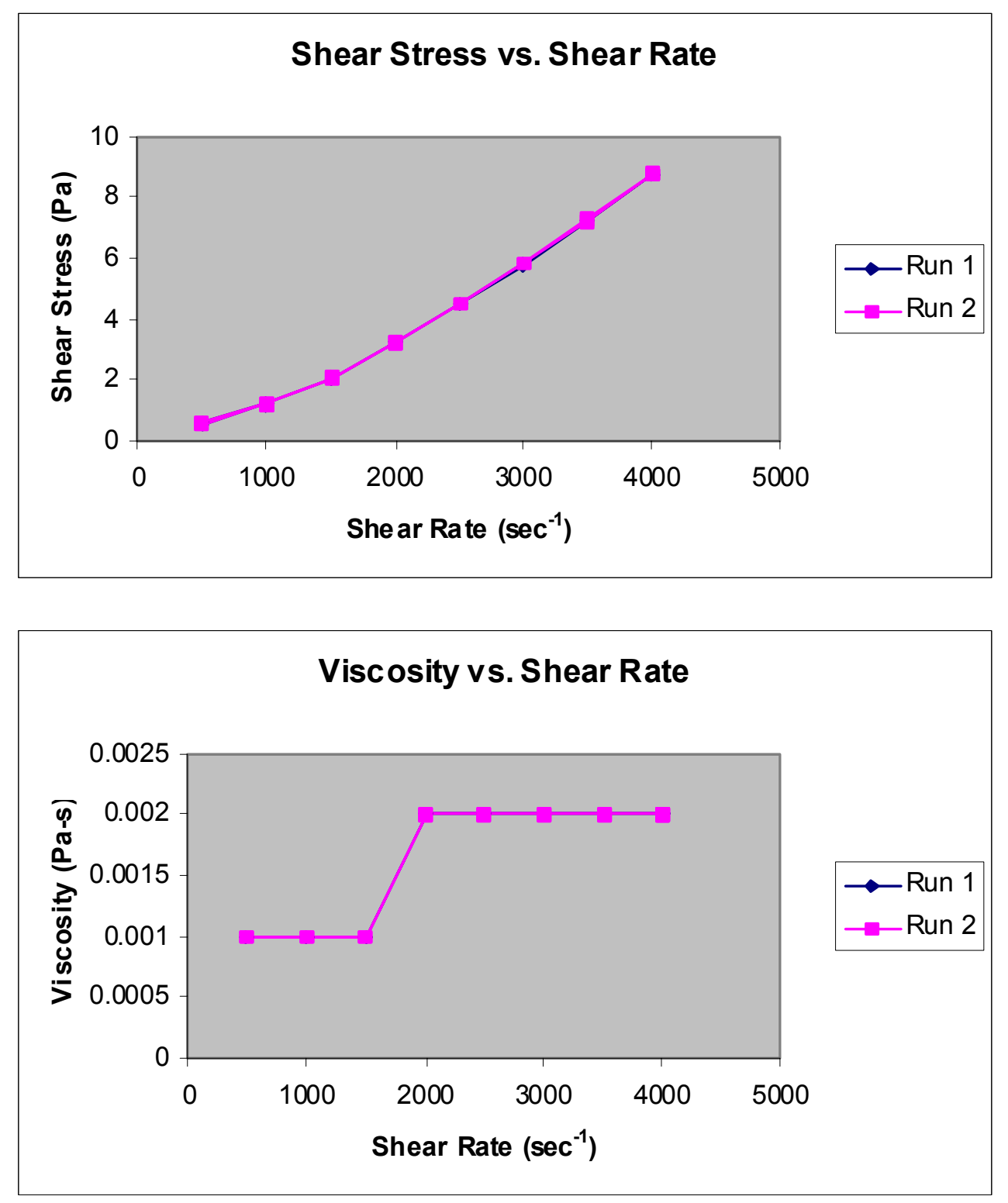


\section{E-2.1.5 META/AR/U/N}

2004.02.06 META-AR-U DG 1-1000 s-1 run 1.txt

\begin{tabular}{|c|c|c|c|c|c|c|c|c|c|c|c|c|c|c|}
\hline Mode & Step & $\mathrm{t}[\mathrm{s}]$ & $\mathrm{n}[\mathrm{rpm}]$ & $\mathrm{M}[\%]$. & Phi[rad] & Gamma & $J[t]$ & $\mathrm{T}\left[{ }^{\circ} \mathrm{C}\right]$ & $\mathrm{D}[1 / \mathrm{s}]$ & $\mathrm{Tau}[\mathrm{Pa}]$ & Eta[Pas] & MP\# & Block & $\mathrm{M}[\mathrm{mNm}]$ \\
\hline 1 & 1 & 20 & 0.2 & 0 & 0.41705 & 0 & 0 & 28 & 1.01 & 0 & 0 & 1 & 1 & 0 \\
\hline 1 & 1 & 40 & 0.4 & 0 & 1.248 & 0 & 0 & 28 & 2.02 & 0 & 0 & 2 & 1 & 0 \\
\hline 1 & 1 & 60 & 0.6 & 0 & 2.49835 & 0 & 0 & 28 & 3.02 & 0 & 0 & 3 & 1 & 0 \\
\hline 1 & 1 & 80 & 0.8 & 0 & 4.16261 & 0 & 0 & 28 & 4.03 & 0 & 0 & 4 & 1 & 0 \\
\hline 1 & 1 & 100 & 1 & 0 & 6.24392 & 0 & 0 & 28 & 5.04 & 0 & 0 & 5 & 1 & 0 \\
\hline 1 & 1 & 120 & 1.19 & 0 & 8.7407 & 0 & 0 & 28 & 6 & 0 & 0 & 6 & 1 & 0 \\
\hline 1 & 1 & 140 & 1.39 & 0 & 11.65531 & 0 & 0 & 28 & 7 & 0 & 0 & 7 & 1 & 0 \\
\hline 1 & 1 & 160 & 1.59 & 0 & 14.9854 & 0 & 0 & 28 & 8.01 & 0 & 0 & 8 & 1 & 0 \\
\hline 1 & 1 & 180 & 1.79 & 0 & 18.73096 & 0 & 0 & 28 & 9.02 & 0 & 0 & 9 & 1 & 0 \\
\hline 1 & 2 & 200 & 1.98 & 0 & 22.90614 & 0 & 0 & 28 & 9.98 & 0 & 0 & 10 & 1 & 0 \\
\hline 1 & 2 & 220 & 3.97 & 1.93 & 31.18423 & 0 & 0 & 28 & 20 & 0.129 & 0.006 & 11 & 1 & 0.0966 \\
\hline 1 & 2 & 240 & 5.95 & 2.57 & 43.62023 & 0 & 0 & 28 & 29.98 & 0.172 & 0.006 & 12 & 1 & 0.12835 \\
\hline 1 & 2 & 260 & 7.94 & 3.13 & 60.21412 & 0 & 0 & 28 & 40.01 & 0.21 & 0.005 & 13 & 1 & 0.1565 \\
\hline 1 & 2 & 280 & 9.92 & 3.51 & 80.95884 & 0 & 0 & 28 & 49.99 & 0.235 & 0.005 & 14 & 1 & 0.1756 \\
\hline 1 & 2 & 300 & 11.91 & 4.31 & 105.85204 & 0 & 0 & 28 & 60.01 & 0.288 & 0.005 & 15 & 1 & 0.21525 \\
\hline 1 & 2 & 320 & 13.89 & 4.85 & 134.94319 & 0 & 0 & 28 & 69.99 & 0.325 & 0.005 & 16 & 1 & 0.2426 \\
\hline 1 & 2 & 340 & 15.88 & 5.17 & 168.16239 & 0 & 0 & 28 & 80.02 & 0.346 & 0.004 & 17 & 1 & 0.2585 \\
\hline 1 & 2 & 360 & 17.86 & 5.81 & 205.50257 & 0 & 0 & 28 & 90 & 0.389 & 0.004 & 18 & 1 & 0.29025 \\
\hline 1 & 3 & 380 & 19.85 & 6.38 & 247.40985 & 0 & 0 & 28 & 100.02 & 0.427 & 0.004 & 19 & 1 & 0.31885 \\
\hline 1 & 3 & 400 & 39.69 & 11.53 & 330.28585 & 0 & 0 & 28 & 200 & 0.772 & 0.004 & 20 & 1 & 0.5763 \\
\hline 1 & 3 & 420 & 59.54 & 16.02 & 454.69291 & 0 & 0 & 28 & 300.02 & 1.073 & 0.004 & 21 & 1 & 0.80095 \\
\hline 1 & 3 & 440 & 79.38 & 20.11 & 620.80777 & 0 & 0 & 28 & 400 & 1.347 & 0.003 & 22 & 1 & 1.0053 \\
\hline 1 & 3 & 460 & 99.23 & 24.25 & 828.21493 & 0 & 0 & 28 & 500.02 & 1.625 & 0.003 & 23 & 1 & 1.21255 \\
\hline 1 & 3 & 480 & 119.07 & 28.18 & 1077.29767 & 0 & 0 & 28 & 599.99 & 1.888 & 0.003 & 24 & 1 & 1.409 \\
\hline 1 & 3 & 500 & 138.92 & 32.3 & 1367.7764 & 0 & 0 & 28 & 700.02 & 2.164 & 0.003 & 25 & 1 & 1.61515 \\
\hline 1 & 3 & 520 & 158.76 & 36.19 & 1700.22288 & 0 & 0 & 28 & 799.99 & 2.424 & 0.003 & 26 & 1 & 1.80925 \\
\hline 1 & 3 & 540 & 178.61 & 40.34 & 2073.59724 & 0 & 0 & 28 & 900.02 & 2.703 & 0.003 & 27 & 1 & 2.0169 \\
\hline 1 & 3 & 560 & 198.45 & 44.44 & 2489.31398 & 0 & 0 & 28 & 999.99 & 2.978 & 0.003 & 28 & 1 & 2.2221 \\
\hline 1 & 4 & 570 & 198.45 & 44.12 & 2701.21362 & 0 & 0 & 28 & 999.99 & 2.956 & 0.003 & 29 & 1 & 2.206 \\
\hline 1 & 4 & 580 & 198.45 & 44.05 & 2908.82184 & 0 & 0 & 28 & 999.99 & 2.951 & 0.003 & 30 & 1 & 2.20245 \\
\hline 1 & 4 & 590 & 198.45 & 43.94 & 3117.15813 & 0 & 0 & 28 & 999.99 & 2.944 & 0.003 & 31 & 1 & 2.19685 \\
\hline 1 & 4 & 600 & 198.45 & 43.82 & 3324.76635 & 0 & 0 & 28 & 999.99 & 2.936 & 0.003 & 32 & 1 & 2.1912 \\
\hline 1 & 4 & 610 & 198.45 & 43.71 & 3532.68717 & 0 & 0 & 28 & 999.99 & 2.929 & 0.003 & 33 & 1 & 2.1855 \\
\hline 1 & 4 & 620 & 198.45 & 43.62 & 3740.60719 & 0 & 0 & 28 & 999.99 & 2.922 & 0.003 & 34 & 1 & 2.18095 \\
\hline 1 & 5 & 640 & 198.45 & 43.51 & 4160.60357 & 0 & 0 & 28 & 999.99 & 2.915 & 0.003 & 35 & 1 & 2.1754 \\
\hline 1 & 5 & 660 & 178.61 & 39.03 & 4535.36023 & 0 & 0 & 28 & 900.02 & 2.615 & 0.003 & 36 & 1 & 1.95125 \\
\hline 1 & 5 & 680 & 158.76 & 34.54 & 4867.9308 & 0 & 0 & 28 & 799.99 & 2.314 & 0.003 & 37 & 1 & 1.7268 \\
\hline 1 & 5 & 700 & 138.92 & 30.51 & 5159.40698 & 0 & 0 & 28 & 700.02 & 2.044 & 0.003 & 38 & 1 & 1.52555 \\
\hline 1 & 5 & 720 & 119.07 & 26.3 & 5409.04029 & 0 & 0 & 28 & 599.99 & 1.762 & 0.003 & 39 & 1 & 1.31515 \\
\hline 1 & 5 & 740 & 99.23 & 22.32 & 5617.12211 & 0 & 0 & 28 & 500.02 & 1.495 & 0.003 & 40 & 1 & 1.1159 \\
\hline 1 & 5 & 760 & 79.38 & 18.06 & 5783.73569 & 0 & 0 & 28 & 400 & 1.21 & 0.003 & 41 & 1 & 0.90305 \\
\hline 1 & 5 & 780 & 59.54 & 13.88 & 5908.74516 & 0 & 0 & 28 & 300.02 & 0.93 & 0.003 & 42 & 1 & 0.694 \\
\hline 1 & 5 & 800 & 39.69 & 9.51 & 5992.18115 & 0 & 0 & 28 & 200 & 0.637 & 0.003 & 43 & 1 & 0.47545 \\
\hline 1 & 5 & 820 & 19.85 & 5.27 & 6034.05701 & 0 & 0 & 28 & 100.02 & 0.353 & 0.004 & 44 & 1 & 0.2634 \\
\hline 1 & 6 & 840 & 17.86 & 4.95 & 6071.71057 & 0 & 0 & 28 & 90 & 0.332 & 0.004 & 45 & 1 & 0.24765 \\
\hline 1 & 6 & 860 & 15.88 & 4.4 & 6105.0036 & 0 & 0 & 28 & 80.02 & 0.295 & 0.004 & 46 & 1 & 0.2202 \\
\hline
\end{tabular}




\begin{tabular}{|c|c|c|c|c|c|c|c|c|c|c|c|c|c|c|}
\hline 1 & 6 & 880 & 13.89 & 4.13 & 6134.14501 & 0 & 0 & 28 & 69.99 & 0.277 & 0.004 & 47 & 1 & 0.2065 \\
\hline & 6 & 900 & 11.9 & 3.7 & 6159.10653 & 0 & 0 & 28 & 59.96 & 0.248 & 0.004 & 48 & 1 & 0.185 \\
\hline & 6 & 920 & 9.92 & 2.93 & 6179.91095 & 0 & 0 & 28 & 49.99 & 0.196 & 0.004 & 49 & 1 & 0.14645 \\
\hline & 6 & 940 & 7.93 & 2.64 & 6196.54097 & 0 & 0 & 28 & 39.96 & 0.177 & 0.004 & 50 & 1 & 0.132 \\
\hline & 6 & 960 & 5.95 & 2.25 & 6209.03194 & 0 & 0 & 28 & 29.98 & 0.151 & 0.005 & 51 & 1 & 0.1124 \\
\hline & 6 & 980 & 3.96 & 1.76 & 6217.36658 & 0 & 0 & 28 & 19.95 & 0.118 & 0.006 & 52 & 1 & 0.0882 \\
\hline & 6 & 1000 & 1.98 & 0 & 6221.5449 & 0 & 0 & 28 & 9.98 & 0 & 0 & 53 & 1 & 0 \\
\hline & 7 & 1020 & 1.79 & 0 & 6225.31324 & 0 & 0 & 28 & 9.02 & 0 & 0 & 54 & 1 & 0 \\
\hline & 7 & 1040 & 1.59 & 0 & 6228.64883 & 0 & 0 & 28 & 8.01 & 0 & 0 & 55 & 1 & 0 \\
\hline & 7 & 1060 & 1.39 & 0 & 6231.56894 & 0 & 0 & 28 & 7 & 0 & 0 & 56 & 1 & 0 \\
\hline & 7 & 1080 & 1.19 & 0 & 6234.07122 & 0 & 0 & 28 & 6 & 0 & 0 & 57 & 1 & 0 \\
\hline & 7 & 1100 & 0.99 & 0 & 6236.15802 & 0 & 0 & 28 & 4.99 & 0 & 0 & 58 & 1 & 0 \\
\hline & 7 & 1120 & 0.8 & 0 & 6237.82778 & 0 & 0 & 28 & 4.03 & 0 & 0 & 59 & 1 & 0 \\
\hline & 7 & 1140 & 0.6 & 0 & 6239.08284 & 0 & 0 & 28 & 3.02 & 0 & 0 & 60 & 1 & 0 \\
\hline & 7 & 1160 & 0.4 & 0 & 6239.92008 & 0 & 0 & 28 & 2.02 & 0 & 0 & 61 & 1 & 0 \\
\hline & 7 & 1180 & 0.2 & 0 & 6240.34262 & 0 & 0 & 28 & 1.01 & 0 & 0 & 62 & 1 & 0 \\
\hline
\end{tabular}

2004.02.06 META-AR-U DG 1-1000 s-1 run 2.txt

Mode Step $\mathrm{t}[\mathrm{s}] \quad \mathrm{n}[\mathrm{rpm}] \quad \mathrm{M}[\%$.$] \quad Phi[rad]$

\begin{tabular}{|c|c|c|c|c|}
\hline 1 & 1 & 20 & 0.2 & 0 \\
\hline 1 & 1 & 40 & 0.4 & 0 \\
\hline 1 & 1 & 60 & 0.6 & 0 \\
\hline 1 & 1 & 80 & 0.8 & 0 \\
\hline 1 & 1 & 100 & 1 & 0 \\
\hline 1 & 1 & 120 & 1.19 & 0 \\
\hline 1 & 1 & 140 & 1.39 & 0 \\
\hline 1 & 1 & 160 & 1.59 & 0 \\
\hline 1 & 1 & 180 & 1.79 & 0 \\
\hline 1 & 2 & 200 & 1.98 & 0 \\
\hline 1 & 2 & 220 & 3.97 & 2.19 \\
\hline 1 & 2 & 240 & 5.95 & 2.61 \\
\hline 1 & 2 & 260 & 7.94 & 3.05 \\
\hline 1 & 2 & 280 & 9.92 & 3.53 \\
\hline 1 & 2 & 300 & 11.91 & 4.24 \\
\hline 1 & 2 & 320 & 13.89 & 4.72 \\
\hline 1 & 2 & 340 & 15.88 & 5.01 \\
\hline 1 & 2 & 360 & 17.86 & 5.55 \\
\hline 1 & 3 & 380 & 19.85 & 5.97 \\
\hline 1 & 3 & 400 & 39.69 & 10.32 \\
\hline 1 & 3 & 420 & 59.54 & 14.52 \\
\hline 1 & 3 & 440 & 79.38 & 18.61 \\
\hline 1 & 3 & 460 & 99.23 & 22.9 \\
\hline 1 & 3 & 480 & 119.07 & 26.84 \\
\hline 1 & 3 & 500 & 138.92 & 30.99 \\
\hline 1 & 3 & 520 & 158.76 & 34.86 \\
\hline 1 & 3 & 540 & 178.61 & 39.08 \\
\hline 1 & 3 & 560 & 198.45 & 43.24 \\
\hline 1 & 4 & 570 & 198.45 & 43.04 \\
\hline 1 & 4 & 580 & 198.45 & 42.94 \\
\hline 1 & 4 & 590 & 198.45 & 42.85 \\
\hline
\end{tabular}

0.41626

1.24878

2.49678

4.16182

6.24392

8.74148

11.65531

14.98383

18.72939

22.903

31.17559

43.6108

60.20627

80.96041

105.88502

134.91884

168.14668

205.52378

247.45461

330.31962

454.57118

620.73787

828.04057

1077.30082

1367.77954

1699.96605

2073.90197

2489.10821

2701.20577

2908.81478

3116.52746

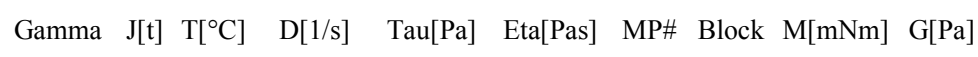

$\begin{array}{llllllllll}0 & 0 & 28 & 1.01 & 0 & 0 & 1 & 1 & 0 & 0 \\ 0 & 0 & 28 & 2.02 & 0 & 0 & 2 & 1 & 0 & 0 \\ 0 & 0 & 28 & 3.02 & 0 & 0 & 3 & 1 & 0 & 0 \\ 0 & 0 & 28 & 4.03 & 0 & 0 & 4 & 1 & 0 & 0 \\ 0 & 0 & 28 & 5.04 & 0 & 0 & 5 & 1 & 0 & 0 \\ 0 & 0 & 28 & 6 & 0 & 0 & 6 & 1 & 0 & 0 \\ 0 & 0 & 28 & 7 & 0 & 0 & 7 & 1 & 0 & 0 \\ 0 & 0 & 28 & 8.01 & 0 & 0 & 8 & 1 & 0 & 0 \\ 0 & 0 & 28 & 9.02 & 0 & 0 & 9 & 1 & 0 & 0 \\ 0 & 0 & 28 & 9.98 & 0 & 0 & 10 & 1 & 0 & 0 \\ 0 & 0 & 28 & 20 & 0.146 & 0.007 & 11 & 1 & 0.1093 & 0 \\ 0 & 0 & 28 & 29.98 & 0.175 & 0.006 & 12 & 1 & 0.13055 & 0 \\ 0 & 0 & 28 & 40.01 & 0.204 & 0.005 & 13 & 1 & 0.1526 & 0 \\ 0 & 0 & 28 & 49.99 & 0.236 & 0.005 & 14 & 1 & 0.17645 & 0 \\ 0 & 0 & 28 & 60.01 & 0.284 & 0.005 & 15 & 1 & 0.21195 & 0 \\ 0 & 0 & 28 & 69.99 & 0.316 & 0.005 & 16 & 1 & 0.23605 & 0 \\ 0 & 0 & 28 & 80.02 & 0.336 & 0.004 & 17 & 1 & 0.25075 & 0 \\ 0 & 0 & 28 & 90 & 0.372 & 0.004 & 18 & 1 & 0.2774 & 0 \\ 0 & 0 & 28 & 100.02 & 0.4 & 0.004 & 19 & 1 & 0.2987 & 0 \\ 0 & 0 & 28 & 200 & 0.691 & 0.003 & 20 & 1 & 0.51575 & 0 \\ 0 & 0 & 28 & 300.02 & 0.973 & 0.003 & 21 & 1 & 0.72595 & 0 \\ 0 & 0 & 28 & 400 & 1.247 & 0.003 & 22 & 1 & 0.93035 & 0 \\ 0 & 0 & 28 & 500.02 & 1.534 & 0.003 & 23 & 1 & 1.1448 & 0 \\ 0 & 0 & 28 & 599.99 & 1.798 & 0.003 & 24 & 1 & 1.3419 & 0 \\ 0 & 0 & 28 & 700.02 & 2.076 & 0.003 & 25 & 1 & 1.5493 & 0 \\ 0 & 0 & 28 & 799.99 & 2.335 & 0.003 & 26 & 1 & 1.7428 & 0 \\ 0 & 0 & 28 & 900.02 & 2.618 & 0.003 & 27 & 1 & 1.95385 & 0 \\ 0 & 0 & 28 & 999.99 & 2.897 & 0.003 & 28 & 1 & 2.1618 & 0 \\ 0 & 0 & 28 & 999.99 & 2.884 & 0.003 & 29 & 1 & 2.1521 & 0 \\ 0 & 0 & 28 & 999.99 & 2.877 & 0.003 & 30 & 1 & 2.14675 & 0 \\ 0 & 0 & 27.9 & 999.99 & 2.871 & 0.003 & 31 & 1 & 2.1423 & 0\end{array}$




\begin{tabular}{|c|c|c|c|c|c|c|c|c|c|c|c|c|c|c|}
\hline 1 & 4 & 600 & 198.45 & 42.81 & 3324.96663 & 0 & 0 & 28 & 999.99 & 2.868 & 0.003 & 32 & 1 & 2.14055 \\
\hline 1 & 4 & 610 & 198.45 & 42.7 & 3532.16016 & 0 & 0 & 28 & 999.99 & 2.861 & 0.003 & 33 & 1 & 2.1351 \\
\hline 1 & 4 & 620 & 198.45 & 42.66 & 3739.97573 & 0 & 0 & 28 & 999.99 & 2.858 & 0.003 & 34 & 1 & 2.1332 \\
\hline 1 & 5 & 640 & 198.45 & 42.56 & 4159.9729 & 0 & 0 & 28 & 999.99 & 2.852 & 0.003 & 35 & 1 & 2.12805 \\
\hline 1 & 5 & 660 & 178.61 & 38.16 & 4534.45938 & 0 & 0 & 28 & 900.02 & 2.557 & 0.003 & 36 & 1 & 1.9079 \\
\hline 1 & 5 & 680 & 158.76 & 33.81 & 4867.28913 & 0 & 0 & 28 & 799.99 & 2.265 & 0.003 & 37 & 1 & 1.6905 \\
\hline 1 & 5 & 700 & 138.92 & 29.89 & 5158.77631 & 0 & 0 & 28 & 700.02 & 2.003 & 0.003 & 38 & 1 & 1.49455 \\
\hline 1 & 5 & 720 & 119.07 & 25.79 & 5408.49208 & 0 & 0 & 28 & 599.99 & 1.728 & 0.003 & 39 & 1 & 1.2894 \\
\hline 1 & 5 & 740 & 99.23 & 21.95 & 5616.63674 & 0 & 0 & 28 & 500.02 & 1.471 & 0.003 & 40 & 1 & 1.0975 \\
\hline 1 & 5 & 760 & 79.38 & 17.66 & 5783.03198 & 0 & 0 & 28 & 400 & 1.183 & 0.003 & 41 & 1 & 0.88305 \\
\hline 1 & 5 & 780 & 59.54 & 13.6 & 5908.13491 & 0 & 0 & 28 & 300.02 & 0.911 & 0.003 & 42 & 1 & 0.67985 \\
\hline 1 & 5 & 800 & 39.69 & 9.19 & 5991.50885 & 0 & 0 & 28 & 200 & 0.615 & 0.003 & 43 & 1 & 0.4593 \\
\hline 1 & 5 & 820 & 19.85 & 4.93 & 6033.42555 & 0 & 0 & 27.9 & 100.02 & 0.331 & 0.003 & 44 & 1 & 0.24675 \\
\hline 1 & 6 & 840 & 17.86 & 4.64 & 6071.08775 & 0 & 0 & 27.9 & 90 & 0.311 & 0.003 & 45 & 1 & 0.232 \\
\hline 1 & 6 & 860 & 15.88 & 4.07 & 6104.39884 & 0 & 0 & 27.9 & 80.02 & 0.273 & 0.003 & 46 & 1 & 0.2034 \\
\hline 1 & 6 & 880 & 13.89 & 3.84 & 6133.51041 & 0 & 0 & 27.9 & 69.99 & 0.257 & 0.004 & 47 & 1 & 0.19195 \\
\hline 1 & 6 & 900 & 11.9 & 3.39 & 6158.45544 & 0 & 0 & 27.9 & 59.96 & 0.227 & 0.004 & 48 & 1 & 0.1695 \\
\hline 1 & 6 & 920 & 9.92 & 2.7 & 6179.27556 & 0 & 0 & 27.9 & 49.99 & 0.181 & 0.004 & 49 & 1 & 0.1348 \\
\hline 1 & 6 & 940 & 7.93 & 2.41 & 6195.915 & 0 & 0 & 27.9 & 39.96 & 0.161 & 0.004 & 50 & 1 & 0.12045 \\
\hline 1 & 6 & 960 & 5.95 & 2.06 & 6208.41462 & 0 & 0 & 27.9 & 29.98 & 0.138 & 0.005 & 51 & 1 & 0.103 \\
\hline 1 & 6 & 980 & 3.96 & 1.61 & 6216.74219 & 0 & 0 & 28 & 19.95 & 0.108 & 0.005 & 52 & 1 & 0.0805 \\
\hline 1 & 6 & 1000 & 1.98 & 0 & 6220.91894 & 0 & 0 & 28 & 9.98 & 0 & 0 & 53 & 1 & 0 \\
\hline 1 & 7 & 1020 & 1.79 & 0 & 6224.68885 & 0 & 0 & 28 & 9.02 & 0 & 0 & 54 & 1 & 0 \\
\hline 1 & 7 & 1040 & 1.59 & 0 & 6228.02444 & 0 & 0 & 28 & 8.01 & 0 & 0 & 55 & 1 & 0 \\
\hline 1 & 7 & 1060 & 1.39 & 0 & 6230.94455 & 0 & 0 & 28 & 7 & 0 & 0 & 56 & 1 & 0 \\
\hline 1 & 7 & 1080 & 1.19 & 0 & 6233.4484 & 0 & 0 & 28 & 6 & 0 & 0 & 57 & 1 & 0 \\
\hline 1 & 7 & 1100 & 0.99 & 0 & 6235.53442 & 0 & 0 & 27.9 & 4.99 & 0 & 0 & 58 & 1 & 0 \\
\hline 1 & 7 & 1120 & 0.8 & 0 & 6237.20496 & 0 & 0 & 27.9 & 4.03 & 0 & 0 & 59 & 1 & 0 \\
\hline 1 & 7 & 1140 & 0.6 & 0 & 6238.45924 & 0 & 0 & 27.9 & 3.02 & 0 & 0 & 60 & 1 & 0 \\
\hline 1 & 7 & 1160 & 0.4 & 0 & 6239.29726 & 0 & 0 & 27.9 & 2.02 & 0 & 0 & 61 & 1 & 0 \\
\hline 1 & 7 & 1180 & 0.2 & 0 & 6239.71902 & 0 & 0 & 27.9 & 1.01 & 0 & 0 & 62 & 1 & 0 \\
\hline
\end{tabular}

2004.02.06 META-AR-U DG 500-4000 s-1 run 1.txt

Mode Step t[s] n[rpm] M[\%.]

$\begin{array}{rrrrrr}1 & 1 & 20 & 99.27 & 21.64 & 207.56189 \\ 1 & 1 & 40198.46 & 42.61 & 622.01335 \\ 1 & 1 & 60297.68 & 63.7 & 1243.29393 \\ 1 & 1 & 80396.91 & 84.46 & 2073.38047 \\ 1 & 1 & 100496.13 & 103.81 & 3111.39017 \\ 1 & 1 & 120595.36 & 125.98 & 4356.69629 \\ 1 & 1 & 140694.58 & 161.67 & 5810.02454 \\ 1 & 1 & 160793.81 & 197.89 & 7469.61091 \\ 1 & 2 & 180793.81 & 201.09 & 9151.12875 \\ 1 & 2 & 200793.81 & 203.42 & 10813.26217 \\ 1 & 2 & 220793.81 & 203.58 & 12475.81578 \\ 1 & 3 & 240793.81 & 202.59 & 14155.40624 \\ 1 & 3 & 260694.58 & 165.61 & 15611.85802 \\ 1 & 3 & 280595.36 & 129.23 & 16860.75263 \\ 1 & 3 & 300496.13 & 98.88 & 17901.98168 \\ 1 & 3 & 320396.91 & 79 & 18734.11653\end{array}$

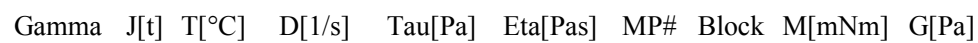

$\begin{array}{cccccccccc}0 & 0 & 28 & 500.22 & 1.45 & 0.003 & 1 & 1 & 1.08175 & 0 \\ 0 & 0 & 28 & 1000.04 & 2.855 & 0.003 & 2 & 1 & 2.1303 & 0 \\ 0 & 0 & 28 & 1500.01 & 4.268 & 0.003 & 3 & 1 & 3.1848 & 0 \\ 0 & 0 & 28 & 2000.03 & 5.659 & 0.003 & 4 & 1 & 4.22315 & 0 \\ 0 & 0 & 28 & 2500 & 6.955 & 0.003 & 5 & 1 & 5.1905 & 0 \\ 0 & 0 & 28 & 3000.02 & 8.44 & 0.003 & 6 & 1 & 6.29885 & 0 \\ 0 & 0 & 27.9 & 3499.99 & 10.832 & 0.003 & 7 & 1 & 8.08365 & 0 \\ 0 & 0 & 28 & 4000.01 & 13.259 & 0.003 & 8 & 1 & 9.8946 & 0 \\ 0 & 0 & 27.9 & 4000.01 & 13.473 & 0.003 & 9 & 1 & 10.0547 & 0 \\ 0 & 0 & 27.9 & 4000.01 & 13.629 & 0.003 & 10 & 1 & 10.1712 & 0 \\ 0 & 0 & 27.9 & 4000.01 & 13.64 & 0.003 & 11 & 1 & 10.179 & 0 \\ 0 & 0 & 28 & 4000.01 & 13.573 & 0.003 & 12 & 1 & 10.1293 & 0 \\ 0 & 0 & 28 & 3499.99 & 11.096 & 0.003 & 13 & 1 & 8.28055 & 0 \\ 0 & 0 & 28 & 3000.02 & 8.658 & 0.003 & 14 & 1 & 6.4615 & 0 \\ 0 & 0 & 28 & 2500 & 6.625 & 0.003 & 15 & 1 & 4.9438 & 0 \\ 0 & 0 & 28 & 2000.03 & 5.293 & 0.003 & 16 & 1 & 3.9502 & 0\end{array}$




\begin{tabular}{|c|c|c|c|c|c|c|c|c|c|c|c|c|c|}
\hline 1 & 3 & 340297.68 & 59.5 & 19359.87388 & 0 & 0 & 28 & 1500.01 & 3.986 & 0.003 & 17 & 1 & 2.9748 \\
\hline 1 & 3 & 360198.46 & 39.92 & 19777.37897 & 0 & 0 & 27.9 & 1000.04 & 2.675 & 0.003 & 18 & 1 & 1.9959 \\
\hline 1 & 3 & $\begin{array}{ll}380 & 99.23\end{array}$ & 20.9 & 19986.89493 & 0 & 0 & 28 & 500.02 & 1.4 & 0.003 & 19 & 1 & 1.04475 \\
\hline
\end{tabular}

2004.02.06 META-AR-U DG 500-4000 s-1 run 2.txt

$\begin{array}{cccccc}\text { Mode } & \text { Step } & \mathrm{t}[\mathrm{s}] & \mathrm{n}[\mathrm{rpm}] & \mathrm{M}[\% .] & \mathrm{Phi}[\mathrm{rad}] \\ 1 & 1 & 20 & 99.3 & 20.52 & 207.81792 \\ 1 & 1 & 40198.46 & 40.3 & 621.74946 \\ 1 & 1 & 60297.68 & 60.06 & 1243.81072 \\ 1 & 1 & 80396.91 & 79.62 & 2073.5344 \\ 1 & 1 & 100496.13 & 99.09 & 3111.23073 \\ 1 & 1 & 120595.36 & 130.84 & 4355.49699 \\ 1 & 1 & 140694.58 & 164.43 & 5807.78616 \\ 1 & 1 & 160793.81 & 199.83 & 7469.86852 \\ 1 & 2 & 180793.81 & 199.98 & 9150.96774 \\ 1 & 2 & 200793.81 & 199.79 & 10813.10509 \\ 1 & 2 & 220793.81 & 199.62 & 12475.65556 \\ 1 & 3 & 240793.81 & 199.5 & 14154.83212 \\ 1 & 3 & 260694.58 & 164.14 & 15613.10288 \\ 1 & 3 & 280595.36 & 129.69 & 16861.68175 \\ 1 & 3 & 300496.13 & 96.4 & 17902.49611 \\ 1 & 3 & 320396.91 & 76.92 & 18734.43383 \\ 1 & 3 & 340297.68 & 57.84 & 19360.35611 \\ 1 & 3 & 360198.46 & 38.69 & 19777.34363 \\ 1 & 3 & 380 & 99.23 & 20.04 & 19987.06143\end{array}$

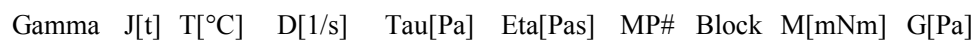

$\begin{array}{lcccccccll}0 & 0 & 28 & 500.37 & 1.375 & 0.003 & 1 & 1 & 1.02585 & 0 \\ 0 & 0 & 28 & 1000.04 & 2.7 & 0.003 & 2 & 1 & 2.0148 & 0 \\ 0 & 0 & 28 & 1500.01 & 4.024 & 0.003 & 3 & 1 & 3.0028 & 0 \\ 0 & 0 & 28 & 2000.03 & 5.335 & 0.003 & 4 & 1 & 3.9811 & 0 \\ 0 & 0 & 28 & 2500 & 6.639 & 0.003 & 5 & 1 & 4.95435 & 0 \\ 0 & 0 & 28 & 3000.02 & 8.766 & 0.003 & 6 & 1 & 6.5418 & 0 \\ 0 & 0 & 28 & 3499.99 & 11.017 & 0.003 & 7 & 1 & 8.2215 & 0 \\ 0 & 0 & 28 & 4000.01 & 13.388 & 0.003 & 8 & 1 & 9.9913 & 0 \\ 0 & 0 & 28 & 4000.01 & 13.399 & 0.003 & 9 & 1 & 9.9992 & 0 \\ 0 & 0 & 27.9 & 4000.01 & 13.386 & 0.003 & 10 & 1 & 9.9897 & 0 \\ 0 & 0 & 27.9 & 4000.01 & 13.375 & 0.003 & 11 & 1 & 9.98105 & 0 \\ 0 & 0 & 27.9 & 4000.01 & 13.366 & 0.003 & 12 & 1 & 9.97485 & 0 \\ 0 & 0 & 27.9 & 3499.99 & 10.997 & 0.003 & 13 & 1 & 8.20705 & 0 \\ 0 & 0 & 27.9 & 3000.02 & 8.689 & 0.003 & 14 & 1 & 6.48455 & 0 \\ 0 & 0 & 27.9 & 2500 & 6.459 & 0.003 & 15 & 1 & 4.8202 & 0 \\ 0 & 0 & 27.9 & 2000.03 & 5.153 & 0.003 & 16 & 1 & 3.84585 & 0 \\ 0 & 0 & 28 & 1500.01 & 3.875 & 0.003 & 17 & 1 & 2.8919 & 0 \\ 0 & 0 & 28 & 1000.04 & 2.592 & 0.003 & 18 & 1 & 1.9345 & 0 \\ 0 & 0 & 28 & 500.02 & 1.342 & 0.003 & 19 & 1 & 1.00175 & 0\end{array}$

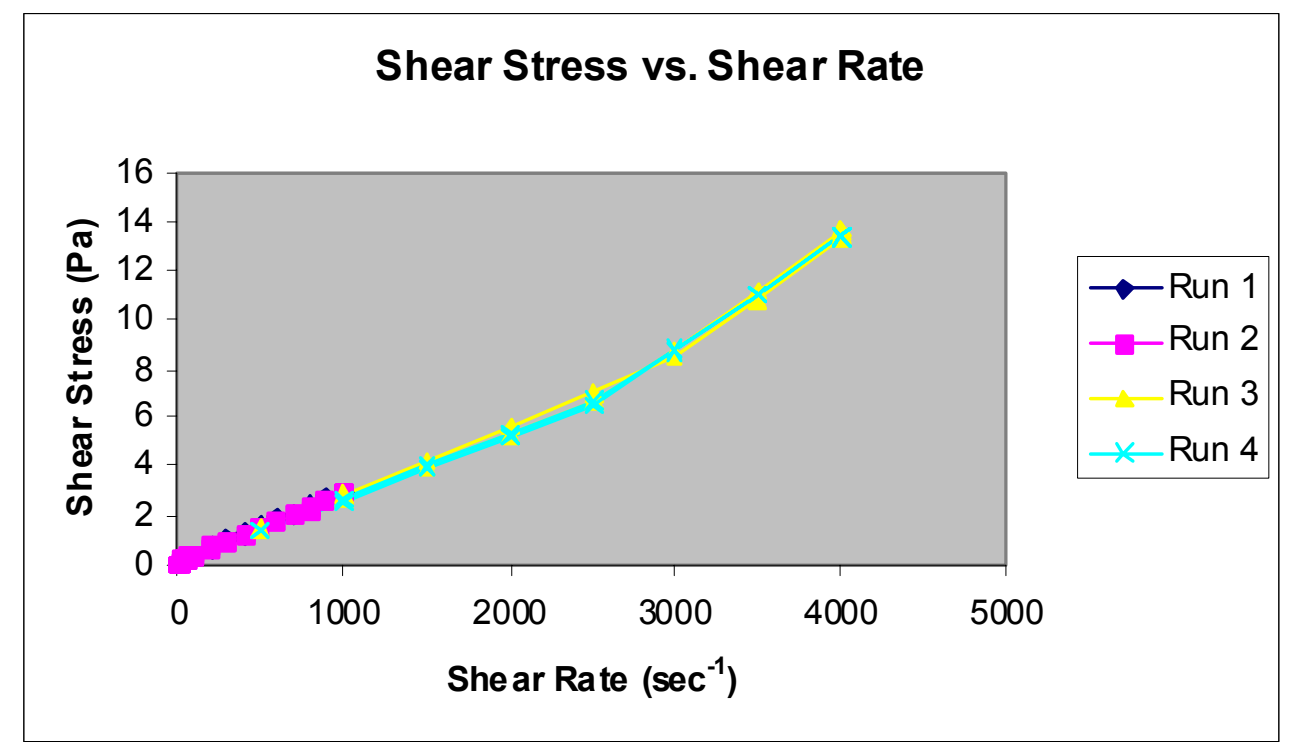




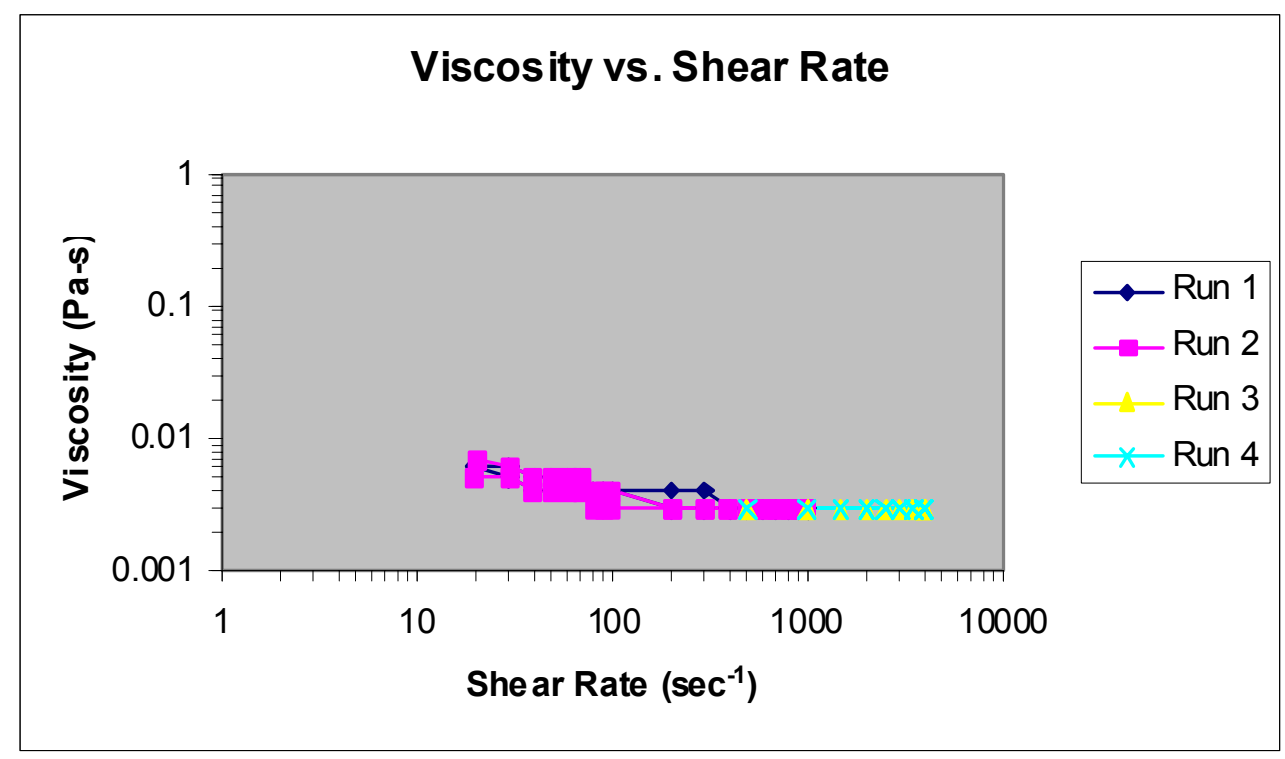

\section{E-2.1.6 META/AR/U/S}

2004.02.04 META-AR-U [sonicated] DG 1-1000 s-1 run 1.txt

\begin{tabular}{|c|c|c|c|c|c|c|c|c|c|c|c|c|c|c|c|}
\hline Mode & Step & $\mathrm{t}[\mathrm{s}]$ & $\mathrm{n}[\mathrm{rpm}]$ & $\mathrm{M}[\%]$. & Phi[rad] & Gamma & $J[t]$ & $\mathrm{T}\left[{ }^{\circ} \mathrm{C}\right]$ & $\mathrm{D}[1 / \mathrm{s}]$ & $\mathrm{Tau}[\mathrm{Pa}]$ & $\mathrm{Eta}[\mathrm{Pas}]$ & MP\# & Block & $\mathrm{M}[\mathrm{mNm}]$ & $\mathrm{G}[\mathrm{Pa}]$ \\
\hline 1 & 1 & 20 & 0.2 & 16.54 & 0.40998 & 0 & 0 & 28 & 1.01 & 1.108 & 1.097 & 1 & 1 & 0.82715 & 0 \\
\hline 1 & 1 & 40 & 0.4 & 22.59 & 1.23779 & 0 & 0 & 28 & 2.02 & 1.513 & 0.749 & 2 & 1 & 1.12945 & 0 \\
\hline 1 & 1 & 60 & 0.6 & 36.64 & 2.48107 & 0 & 0 & 28 & 3.02 & 2.455 & 0.813 & 3 & 1 & 1.8319 & 0 \\
\hline 1 & 1 & 80 & 0.8 & 56.19 & 4.1414 & 0 & 0 & 28 & 4.03 & 3.765 & 0.934 & 4 & 1 & 2.80965 & 0 \\
\hline 1 & 1 & 100 & 1 & 14.3 & 6.24234 & 0 & 0 & 28 & 5.04 & 0.958 & 0.19 & 5 & 1 & 0.71495 & 0 \\
\hline 1 & 1 & 120 & 1.19 & 0 & 8.74148 & 0 & 0 & 28 & 6 & 0 & 0 & 6 & 1 & 0 & 0 \\
\hline 1 & 1 & 140 & 1.39 & 0 & 11.65295 & 0 & 0 & 28 & 7 & 0 & 0 & 7 & 1 & 0 & 0 \\
\hline 1 & 1 & 160 & 1.59 & 0 & 14.98383 & 0 & 0 & 28 & 8.01 & 0 & 0 & 8 & 1 & 0 & 0 \\
\hline 1 & 1 & 180 & 1.78 & 14.15 & 18.71525 & 0 & 0 & 28 & 8.97 & 0.948 & 0.106 & 9 & 1 & 0.70745 & 0 \\
\hline 1 & 2 & 200 & 1.99 & 48.08 & 22.903 & 0 & 0 & 28 & 10.03 & 3.222 & 0.321 & 10 & 1 & 2.40415 & 0 \\
\hline 1 & 2 & 220 & 3.96 & 7.07 & 31.16774 & 0 & 0 & 28 & 19.95 & 0.474 & 0.024 & 11 & 1 & 0.35345 & 0 \\
\hline 1 & 2 & 240 & 5.94 & 37.83 & 43.60845 & 0 & 0 & 28 & 29.93 & 2.535 & 0.085 & 12 & 1 & 1.8915 & 0 \\
\hline 1 & 2 & 260 & 7.94 & 7.57 & 60.20234 & 0 & 0 & 28 & 40.01 & 0.507 & 0.013 & 13 & 1 & 0.37855 & 0 \\
\hline 1 & 2 & 280 & 9.92 & 11.18 & 80.9612 & 0 & 0 & 28 & 49.99 & 0.749 & 0.015 & 14 & 1 & 0.55895 & 0 \\
\hline 1 & 2 & 300 & 11.91 & 22.74 & 105.86539 & 0 & 0 & 28 & 60.01 & 1.524 & 0.025 & 15 & 1 & 1.137 & 0 \\
\hline 1 & 2 & 320 & 13.89 & 10.36 & 134.91648 & 0 & 0 & 28 & 69.99 & 0.694 & 0.01 & 16 & 1 & 0.51775 & 0 \\
\hline 1 & 2 & 340 & 15.88 & 13.63 & 168.16317 & 0 & 0 & 28 & 80.02 & 0.913 & 0.011 & 17 & 1 & 0.68165 & 0 \\
\hline 1 & 2 & 360 & 17.86 & 16.58 & 205.49079 & 0 & 0 & 28 & 90 & 1.111 & 0.012 & 18 & 1 & 0.829 & 0 \\
\hline 1 & 3 & 380 & 19.85 & 21.11 & 247.40985 & 0 & 0 & 28 & 100.02 & 1.415 & 0.014 & 19 & 1 & 1.0556 & 0 \\
\hline 1 & 3 & 400 & 39.69 & 26.44 & 330.28663 & 0 & 0 & 28 & 200 & 1.772 & 0.009 & 20 & 1 & 1.3222 & 0 \\
\hline 1 & 3 & 420 & 59.54 & 27.99 & 454.68113 & 0 & 0 & 28 & 300.02 & 1.876 & 0.006 & 21 & 1 & 1.3997 & 0 \\
\hline 1 & 3 & 440 & 79.38 & 25.71 & 620.71431 & 0 & 0 & 28 & 400 & 1.723 & 0.004 & 22 & 1 & 1.2856 & 0 \\
\hline 1 & 3 & 460 & 99.23 & 26.27 & 828.22435 & 0 & 0 & 28 & 500.02 & 1.76 & 0.004 & 23 & 1 & 1.3136 & 0 \\
\hline 1 & 3 & 480 & 119.07 & 30.72 & 1076.99608 & 0 & 0 & 28 & 599.99 & 2.058 & 0.003 & 24 & 1 & 1.53605 & 0 \\
\hline 1 & 3 & 500 & 138.92 & 35.28 & 1367.69315 & 0 & 0 & 28 & 700.02 & 2.363 & 0.003 & 25 & 1 & 1.7638 & 0 \\
\hline 1 & 3 & 520 & 158.76 & 39.48 & 1700.12863 & 0 & 0 & 28 & 799.99 & 2.645 & 0.003 & 26 & 1 & 1.9739 & 0 \\
\hline 1 & 3 & 540 & 178.61 & 44.04 & 2073.60745 & 0 & 0 & 28 & 900.02 & 2.951 & 0.003 & 27 & 1 & 2.2021 & 0 \\
\hline 1 & 3 & 560 & 198.45 & 48.45 & 2488.91736 & 0 & 0 & 28 & 999.99 & 3.246 & 0.003 & 28 & 1 & 2.4223 & 0 \\
\hline
\end{tabular}




\begin{tabular}{|c|c|c|c|c|c|c|c|c|c|c|c|c|c|c|}
\hline 1 & 4 & 5701 & 198.45 & 47.98 & 2701.3275 & 0 & 0 & 28 & 999.99 & 3.214 & 0.003 & 29 & 1 & 2.39875 \\
\hline 1 & 4 & 5801 & 198.45 & 47.81 & 2908.83205 & 0 & 0 & 28 & 999.99 & 3.203 & 0.003 & 30 & 1 & 2.3903 \\
\hline 1 & 4 & 5901 & 198.45 & 47.67 & 3116.95943 & 0 & 0 & 28 & 999.99 & 3.194 & 0.003 & 31 & 1 & 2.3837 \\
\hline 1 & 4 & 6001 & 198.45 & 47.53 & 3324.3603 & 0 & 0 & 28 & 999.99 & 3.185 & 0.003 & 32 & 1 & 2.3767 \\
\hline 1 & 4 & 6101 & 198.45 & 47.41 & 3531.96931 & 0 & 0 & 28 & 999.99 & 3.177 & 0.003 & 33 & 1 & 2.37055 \\
\hline 1 & 4 & 6201 & 198.45 & 47.34 & 3740.40849 & 0 & 0 & 28 & 999.99 & 3.172 & 0.003 & 34 & 1 & 2.3668 \\
\hline 1 & 5 & 6401 & 198.45 & 47.22 & 4160.71824 & 0 & 0 & 28 & 999.99 & 3.164 & 0.003 & 35 & 1 & 2.36105 \\
\hline 1 & 5 & 6601 & 178.61 & 42.26 & 4535.11048 & 0 & 0 & 28 & 900.02 & 2.832 & 0.003 & 36 & 1 & 2.11315 \\
\hline 1 & 5 & 6801 & 158.76 & 37.38 & 4867.9308 & 0 & 0 & 28 & 799.99 & 2.505 & 0.003 & 37 & 1 & 1.86905 \\
\hline 1 & 5 & 7001 & 138.92 & 32.94 & 5159.26169 & 0 & 0 & 28 & 700.02 & 2.207 & 0.003 & 38 & 1 & 1.64705 \\
\hline 1 & 5 & 7201 & 119.07 & 28.25 & 5408.78111 & 0 & 0 & 28 & 599.99 & 1.893 & 0.003 & 39 & 1 & 1.41265 \\
\hline 1 & 5 & 740 & 99.23 & 23.79 & 5616.97681 & 0 & 0 & 28 & 500.02 & 1.594 & 0.003 & 40 & 1 & 1.1894 \\
\hline 1 & 5 & 760 & 79.38 & 18.99 & 5783.45531 & 0 & 0 & 28 & 400 & 1.272 & 0.003 & 41 & 1 & 0.9496 \\
\hline 1 & 5 & 780 & 59.54 & 14.37 & 5908.59908 & 0 & 0 & 27.9 & 300.02 & 0.962 & 0.003 & 42 & 1 & 0.71825 \\
\hline 1 & 5 & 800 & 39.69 & 9.46 & 5992.03664 & 0 & 0 & 27.9 & 200 & 0.634 & 0.003 & 43 & 1 & 0.47295 \\
\hline 1 & 5 & 820 & 19.85 & 4.86 & 6033.87008 & 0 & 0 & 27.9 & 100.02 & 0.325 & 0.003 & 44 & 1 & 0.24275 \\
\hline 1 & 6 & 840 & 17.86 & 4.6 & 6071.53307 & 0 & 0 & 28 & 90 & 0.308 & 0.003 & 45 & 1 & 0.2302 \\
\hline 1 & 6 & 860 & 15.88 & 4.07 & 6104.81746 & 0 & 0 & 28 & 80.02 & 0.273 & 0.003 & 46 & 1 & 0.20355 \\
\hline 1 & 6 & 880 & 13.89 & 3.82 & 6133.9518 & 0 & 0 & 27.9 & 69.99 & 0.256 & 0.004 & 47 & 1 & 0.19075 \\
\hline 1 & 6 & 900 & 11.9 & 3.39 & 6158.92668 & 0 & 0 & 27.8 & 59.96 & 0.227 & 0.004 & 48 & 1 & 0.16965 \\
\hline 1 & 6 & 920 & 9.92 & 2.58 & 6179.73188 & 0 & 0 & 27.8 & 49.99 & 0.173 & 0.003 & 49 & 1 & 0.1292 \\
\hline & 6 & 940 & 7.93 & 2.29 & 6196.35797 & 0 & 0 & 27.8 & 39.96 & 0.153 & 0.004 & 50 & 1 & 0.1143 \\
\hline & 6 & 960 & 5.95 & 1.92 & 6208.85051 & 0 & 0 & 27.8 & 29.98 & 0.129 & 0.004 & 51 & 1 & 0.096 \\
\hline & 6 & 980 & 3.96 & 1.62 & 6217.18908 & 0 & 0 & 27.8 & 19.95 & 0.109 & 0.005 & 52 & 1 & 0.0811 \\
\hline & 6 & 1000 & 1.98 & 0 & 6221.36348 & 0 & 0 & 27.8 & 9.98 & 0 & 0 & 53 & 1 & 0 \\
\hline & 7 & 1020 & 1.79 & 0 & 6225.13182 & 0 & 0 & 27.8 & 9.02 & 0 & 0 & 54 & 1 & 0 \\
\hline 1 & 7 & 1040 & 1.59 & 0 & 6228.46819 & 0 & 0 & 27.9 & 8.01 & 0 & 0 & 55 & 1 & 0 \\
\hline & 7 & 1060 & 1.39 & 0 & 6231.38594 & 0 & 0 & 27.9 & 7 & 0 & 0 & 56 & 1 & 0 \\
\hline & 7 & 1080 & 1.19 & 0 & 6233.89058 & 0 & 0 & 27.9 & 6 & 0 & 0 & 57 & 1 & 0 \\
\hline 1 & 7 & 1100 & 0.99 & 0 & 6235.97738 & 0 & 0 & 27.9 & 4.99 & 0 & 0 & 58 & 1 & 0 \\
\hline , & 7 & 1120 & 0.8 & 0 & 6237.64792 & 0 & 0 & 27.9 & 4.03 & 0 & 0 & 59 & 1 & 0 \\
\hline 4 & 7 & 1140 & 0.6 & 0 & 6238.90142 & 0 & 0 & 27.9 & 3.02 & 0 & 0 & 60 & 1 & 0 \\
\hline & 7 & 1160 & 0.4 & 0 & 6239.73944 & 0 & 0 & 28 & 2.02 & 0 & 0 & 61 & 1 & 0 \\
\hline & 7 & 1180 & 0.2 & 0 & 6240.16198 & 0 & 0 & 28 & 1.01 & 0 & 0 & 62 & 1 & 0 \\
\hline
\end{tabular}

2004.02.04 META-AR-U [sonicated] DG 1-1000 s-1 run 2.txt

Mode Step $\mathrm{t}[\mathrm{s}] \quad \mathrm{n}[\mathrm{rpm}] \quad \mathrm{M}[\%$.]
Phi[rad]

$\begin{array}{llll}0.41626 & 0 & 0 & 28 \\ 1.24957 & 0 & 0 & 28 \\ 2.49678 & 0 & 0 & 28 \\ 4.16261 & 0 & 0 & 28 \\ 6.2447 & 0 & 0 & 28 \\ 8.74227 & 0 & 0 & 28 \\ 11.65452 & 0 & 0 & 28 \\ 14.98461 & 0 & 0 & 28 \\ 18.73018 & 0 & 0 & 28 \\ 22.903 & 0 & 0 & 28 \\ 31.18031 & 0 & 0 & 28 \\ 43.62808 & 0 & 0 & 28 \\ 60.21648 & 0 & 0 & 28\end{array}$

$\mathrm{D}[1 / \mathrm{s}] \quad \mathrm{Tau}[\mathrm{Pa}] \quad$ Eta[Pas $]$ MP\# Block M[mNm] G[Pa]

$\begin{array}{lllllll}1.01 & 0 & 0 & 1 & 1 & 0 & 0 \\ 2.02 & 0 & 0 & 2 & 1 & 0 & 0 \\ 3.02 & 0 & 0 & 3 & 1 & 0 & 0 \\ 4.03 & 0 & 0 & 4 & 1 & 0 & 0 \\ 5.04 & 0 & 0 & 5 & 1 & 0 & 0 \\ 6 & 0 & 0 & 6 & 1 & 0 & 0 \\ 7 & 0 & 0 & 7 & 1 & 0 & 0 \\ 8.01 & 0 & 0 & 8 & 1 & 0 & 0 \\ 9.02 & 0 & 0 & 9 & 1 & 0 & 0 \\ 9.98 & 0 & 0 & 10 & 1 & 0 & 0 \\ 20 & 0.123 & 0.006 & 11 & 1 & 0.0921 & 0 \\ 29.98 & 0.157 & 0.005 & 12 & 1 & 0.1174 & 0 \\ 40.01 & 0.186 & 0.005 & 13 & 1 & 0.1388 & 0\end{array}$




\begin{tabular}{|c|c|c|c|c|c|c|c|c|c|c|c|c|c|c|}
\hline 1 & 2 & 280 & 9.92 & 3.15 & 80.94785 & 0 & 0 & 28 & 49.99 & 0.211 & 0.004 & 14 & 1 & 0.15765 \\
\hline 1 & 2 & 300 & 11.91 & 3.94 & 105.86539 & 0 & 0 & 28 & 60.01 & 0.264 & 0.004 & 15 & 1 & 0.1969 \\
\hline 1 & 2 & 320 & 13.89 & 4.41 & 134.92748 & 0 & 0 & 28 & 69.99 & 0.296 & 0.004 & 16 & 1 & 0.22055 \\
\hline & 2 & 340 & 15.88 & 4.85 & 168.15532 & 0 & 0 & 28 & 80.02 & 0.325 & 0.004 & 17 & 1 & 0.2423 \\
\hline & 2 & 360 & 17.86 & 5.4 & 205.50493 & 0 & 0 & 28 & 90 & 0.362 & 0.004 & 18 & 1 & 0.27005 \\
\hline & 3 & 380 & 19.85 & 5.85 & 247.40278 & 0 & 0 & 28 & 100.02 & 0.392 & 0.004 & 19 & 1 & 0.2924 \\
\hline & 3 & 400 & 39.69 & 11.36 & 330.35182 & 0 & 0 & 28 & 200 & 0.761 & 0.004 & 20 & 1 & 0.5678 \\
\hline & 3 & 420 & 59.54 & 16.51 & 454.65443 & 0 & 0 & 28 & 300.02 & 1.106 & 0.004 & 21 & 1 & 0.8253 \\
\hline & 3 & 440 & 79.38 & 21.28 & 620.69624 & 0 & 0 & 28 & 400 & 1.426 & 0.004 & 22 & 1 & 1.06395 \\
\hline 1 & 3 & 460 & 99.23 & 26.12 & 828.04136 & 0 & 0 & 28 & 500.02 & 1.75 & 0.003 & 23 & 1 & 1.3062 \\
\hline 1 & 3 & 480 & 119.07 & 30.75 & 1077.22777 & 0 & 0 & 28 & 599.99 & 2.06 & 0.003 & 24 & 1 & 1.5376 \\
\hline 1 & 3 & 500 & 138.92 & 35.41 & 1367.86279 & 0 & 0 & 28 & 700.02 & 2.372 & 0.003 & 25 & 1 & 1.7703 \\
\hline 1 & 3 & 520 & 158.76 & 39.66 & 1699.81054 & 0 & 0 & 28 & 799.99 & 2.657 & 0.003 & 26 & 1 & 1.98305 \\
\hline 1 & 3 & 540 & 178.61 & 44.22 & 2073.93182 & 0 & 0 & 28 & 900.02 & 2.963 & 0.003 & 27 & 1 & 2.2109 \\
\hline 1 & 3 & 560 & 198.45 & 48.71 & 2489.2433 & 0 & 0 & 28 & 999.99 & 3.263 & 0.003 & 28 & 1 & 2.43535 \\
\hline 1 & 4 & 570 & 198.45 & 48.31 & 2701.02905 & 0 & 0 & 28 & 999.99 & 3.237 & 0.003 & 29 & 1 & 2.4156 \\
\hline 1 & 4 & 580 & 198.45 & 48.16 & 2909.15721 & 0 & 0 & 28 & 999.99 & 3.227 & 0.003 & 30 & 1 & 2.40815 \\
\hline 1 & 4 & 590 & 198.45 & 48.09 & 3116.66097 & 0 & 0 & 28 & 999.99 & 3.222 & 0.003 & 31 & 1 & 2.40435 \\
\hline 1 & 4 & 600 & 198.45 & 47.94 & 3324.68624 & 0 & 0 & 28 & 999.99 & 3.212 & 0.003 & 32 & 1 & 2.3972 \\
\hline 1 & 4 & 610 & 198.45 & 47.82 & 3532.29447 & 0 & 0 & 28 & 999.99 & 3.204 & 0.003 & 33 & 1 & 2.39075 \\
\hline 1 & 4 & 620 & 198.45 & 47.72 & 3740.00636 & 0 & 0 & 28 & 999.99 & 3.197 & 0.003 & 34 & 1 & 2.38585 \\
\hline 1 & 5 & 640 & 198.45 & 47.63 & 4160.52346 & 0 & 0 & 28 & 999.99 & 3.191 & 0.003 & 35 & 1 & 2.38125 \\
\hline 1 & 5 & 660 & 178.61 & 42.63 & 4534.53164 & 0 & 0 & 28 & 900.02 & 2.856 & 0.003 & 36 & 1 & 2.1317 \\
\hline 1 & 5 & 680 & 158.76 & 37.72 & 4867.37239 & 0 & 0 & 28 & 799.99 & 2.527 & 0.003 & 37 & 1 & 1.88615 \\
\hline 1 & 5 & 700 & 138.92 & 33.24 & 5158.85878 & 0 & 0 & 28 & 700.02 & 2.227 & 0.003 & 38 & 1 & 1.6621 \\
\hline 1 & 5 & 720 & 119.07 & 28.54 & 5408.50308 & 0 & 0 & 28 & 599.99 & 1.912 & 0.003 & 39 & 1 & 1.427 \\
\hline 1 & 5 & 740 & 99.23 & 23.98 & 5616.63674 & 0 & 0 & 28 & 500.02 & 1.606 & 0.003 & 40 & 1 & 1.19875 \\
\hline 1 & 5 & 760 & 79.38 & 19.16 & 5783.16706 & 0 & 0 & 28 & 400 & 1.284 & 0.003 & 41 & 1 & 0.9582 \\
\hline 1 & 5 & 780 & 59.54 & 14.58 & 5908.15533 & 0 & 0 & 28 & 300.02 & 0.977 & 0.003 & 42 & 1 & 0.7291 \\
\hline 1 & 5 & 800 & 39.69 & 9.63 & 5991.65493 & 0 & 0 & 28 & 200 & 0.645 & 0.003 & 43 & 1 & 0.4816 \\
\hline 1 & 5 & 820 & 19.85 & 4.92 & 6033.53079 & 0 & 0 & 28 & 100.02 & 0.329 & 0.003 & 44 & 1 & 0.24585 \\
\hline 1 & 6 & 840 & 17.86 & 4.54 & 6071.19299 & 0 & 0 & 28 & 90 & 0.304 & 0.003 & 45 & 1 & 0.22705 \\
\hline 1 & 6 & 860 & 15.88 & 3.98 & 6104.48602 & 0 & 0 & 28 & 80.02 & 0.267 & 0.003 & 46 & 1 & 0.19915 \\
\hline 1 & 6 & 880 & 13.89 & 3.75 & 6133.61408 & 0 & 0 & 28 & 69.99 & 0.251 & 0.004 & 47 & 1 & 0.18745 \\
\hline 1 & 6 & 900 & 11.9 & 3.31 & 6158.55754 & 0 & 0 & 28 & 59.96 & 0.222 & 0.004 & 48 & 1 & 0.1654 \\
\hline 1 & 6 & 920 & 9.92 & 2.67 & 6179.37766 & 0 & 0 & 28 & 49.99 & 0.179 & 0.004 & 49 & 1 & 0.13365 \\
\hline 1 & 6 & 940 & 7.93 & 2.24 & 6196.0336 & 0 & 0 & 28 & 39.96 & 0.15 & 0.004 & 50 & 1 & 0.11205 \\
\hline 1 & 6 & 960 & 5.95 & 1.89 & 6208.51436 & 0 & 0 & 28 & 29.98 & 0.126 & 0.004 & 51 & 1 & 0.09435 \\
\hline 1 & 6 & 980 & 3.96 & 1.54 & 6216.84979 & 0 & 0 & 28 & 19.95 & 0.103 & 0.005 & 52 & 1 & 0.07695 \\
\hline 1 & 6 & 1000 & 1.98 & 0 & 6221.02575 & 0 & 0 & 28 & 9.98 & 0 & 0 & 53 & 1 & 0 \\
\hline 1 & 7 & 1020 & 1.79 & 0 & 6224.79802 & 0 & 0 & 28 & 9.02 & 0 & 0 & 54 & 1 & 0 \\
\hline 1 & 7 & 1040 & 1.59 & 0 & 6228.13125 & 0 & 0 & 28 & 8.01 & 0 & 0 & 55 & 1 & 0 \\
\hline 1 & 7 & 1060 & 1.39 & 0 & 6231.05215 & 0 & 0 & 28 & 7 & 0 & 0 & 56 & 1 & 0 \\
\hline 1 & 7. & 1080 & 1.19 & 0 & 6233.55364 & 0 & 0 & 28 & 6 & 0 & 0 & 57 & 1 & 0 \\
\hline 1 & 7 & 1100 & 0.99 & 0 & 6235.64044 & 0 & 0 & 28 & 4.99 & 0 & 0 & 58 & 1 & 0 \\
\hline 1 & 7. & 1120 & 0.8 & 0 & 6237.3102 & 0 & 0 & 28 & 4.03 & 0 & 0 & 59 & 1 & 0 \\
\hline 1 & 7 & 1140 & 0.6 & 0 & 6238.56448 & 0 & 0 & 28 & 3.02 & 0 & 0 & 60 & 1 & 0 \\
\hline 1 & 7 & 1160 & 0.4 & 0 & 6239.4025 & 0 & 0 & 28 & 2.02 & 0 & 0 & 61 & 1 & 0 \\
\hline 1 & 7 & 1180 & 0.2 & 0 & 6239.82347 & 0 & 0 & 28 & 1.01 & 0 & 0 & 62 & 1 & 0 \\
\hline
\end{tabular}


2004.02.04 META-AR-U [sonicated] DG 500-4000 s-1 run 1.txt

\begin{tabular}{|c|c|c|c|c|c|c|c|c|c|c|c|c|c|c|c|}
\hline Mode & Step & $\mathrm{t}[\mathrm{s}]$ & $\mathrm{n}[\mathrm{rpm}]$ & $\mathrm{M}[\%]$. & Phi[rad] & Gamma & $J[t]$ & $\mathrm{T}\left[{ }^{\circ} \mathrm{C}\right]$ & $\mathrm{D}[1 / \mathrm{s}]$ & $\mathrm{Tau}[\mathrm{Pa}]$ & $\mathrm{Eta}[\mathrm{Pas}]$ & MP\# & Block & $\mathrm{M}[\mathrm{mNm}]$ & $\mathrm{G}[\mathrm{Pa}]$ \\
\hline 1 & 1 & 20 & 99.28 & 25.14 & 207.73781 & 0 & 0 & 28 & 500.27 & 1.684 & 0.003 & 1 & 1 & 1.257 & 0 \\
\hline 1 & 1 & 40 & 198.46 & 49.56 & 621.66778 & 0 & 0 & 28 & 1000.04 & 3.321 & 0.003 & 2 & 1 & 2.4782 & 0 \\
\hline 1 & 1 & 60 & 297.68 & 72.25 & 1243.52091 & 0 & 0 & 28 & 1500.01 & 4.841 & 0.003 & 3 & 1 & 3.6127 & 0 \\
\hline 1 & 1 & 80 & 396.91 & 93.59 & 2073.39932 & 0 & 0 & 28 & 2000.03 & 6.271 & 0.003 & 4 & 1 & 4.6795 & 0 \\
\hline 1 & 1 & 100 & 496.13 & 113.51 & 3111.40666 & 0 & 0 & 28 & 2500 & 7.605 & 0.003 & 5 & 1 & 5.6755 & 0 \\
\hline 1 & 1 & 120 & 595.36 & 139.43 & 4356.34836 & 0 & 0 & 28 & 3000.02 & 9.342 & 0.003 & 6 & 1 & 6.97155 & 0 \\
\hline 1 & 1 & 140 & 694.58 & 173.24 & 5808.27624 & 0 & 0 & 28 & 3499.99 & 11.607 & 0.003 & 7 & 1 & 8.6619 & , \\
\hline 1 & 1 & 160 & 793.81 & 208.6 & 7469.52923 & 0 & 0 & 28 & 4000.01 & 13.976 & 0.003 & 8 & 1 & 10.4299 & 0 \\
\hline 1 & 2 & 180 & 793.81 & 206.92 & 9148.96419 & 0 & 0 & 28 & 4000.01 & 13.864 & 0.003 & 9 & 1 & 10.34615 & P \\
\hline 1 & 2 & 200 & 793.81 & 205.64 & 10811.93406 & 0 & 0 & 28 & 4000.01 & 13.778 & 0.003 & 10 & 1 & 10.2821 & 0 \\
\hline 1 & 2 & 220 & 793.81 & 204.72 & 12474.48296 & 0 & 0 & 28 & 4000.01 & 13.716 & 0.003 & 11 & 1 & 10.23585 & 0 \\
\hline 1 & 3 & 240 & 793.81 & 204.17 & 14154.4944 & 0 & 0 & 28 & 4000.01 & 13.679 & 0.003 & 12 & 1 & 10.20855 & 0 \\
\hline 1 & 3 & 260 & 694.58 & 167.04 & 15610.52756 & 0 & 0 & 28 & 3499.99 & 11.192 & 0.003 & 13 & 1 & 8.352 & 0 \\
\hline 1 & 3 & 280 & 595.36 & 132.51 & 16859.10643 & 0 & 0 & 28 & 3000.02 & 8.878 & 0.003 & 14 & 1 & 6.6254 & 0 \\
\hline 1 & 3 & 300 & 496.13 & 98.09 & 17900.39046 & 0 & 0 & 28 & 2500 & 6.572 & 0.003 & 15 & 1 & 4.9044 & 0 \\
\hline 1 & 3 & 320 & 396.91 & 78.12 & 18733.73168 & 0 & 0 & 28 & 2000.03 & 5.234 & 0.003 & 16 & 1 & 3.9061 & 0 \\
\hline 1 & 3 & 340 & 297.68 & 58.73 & 19358.76411 & 0 & 0 & 28 & 1500.01 & 3.935 & 0.003 & 17 & 1 & 2.9363 & 0 \\
\hline 1 & 3 & 360 & 198.46 & 38.83 & 19776.11213 & 0 & 0 & 28 & 1000.04 & 2.601 & 0.003 & 18 & 1 & 1.9413 & 0 \\
\hline 1 & 3 & 380 & 99.23 & 19.63 & 19985.62494 & 0 & 0 & 28 & 500.02 & 1.315 & 0.003 & 19 & 1 & 0.98125 & 0 \\
\hline
\end{tabular}

2004.02.04 META-AR-U [sonicated] DG 500-4000 s-1 run 2.txt

$\begin{array}{cccccccccccccccc}\text { Mode } & \text { Step } & \mathrm{t}[\mathrm{s}] & \mathrm{n}[\mathrm{rpm}] & \mathrm{M}[\% .] & \mathrm{Phi}[\mathrm{rad}] & \mathrm{Gamma} & \mathrm{J}[\mathrm{t}] & \mathrm{T}\left[{ }^{\circ} \mathrm{C}\right] & \mathrm{D}[1 / \mathrm{s}] & \mathrm{Tau}[\mathrm{Pa}] & \mathrm{Eta}[\mathrm{Pas}] & \mathrm{MP \#} & \mathrm{Block} & \mathrm{M}[\mathrm{mNm}] & \mathrm{G}[\mathrm{Pa}] \\ 1 & 1 & 20 & 99.27 & 19.24 & 207.52183 & 0 & 0 & 28 & 500.22 & 1.289 & 0.003 & 1 & 1 & 0.96215 & 0 \\ 1 & 1 & 40 & 198.46 & 39.35 & 621.86884 & 0 & 0 & 28 & 1000.04 & 2.637 & 0.003 & 2 & 1 & 1.96765 & 0 \\ 1 & 1 & 60 & 297.68 & 59.62 & 1243.72119 & 0 & 0 & 28 & 1500.01 & 3.995 & 0.003 & 3 & 1 & 2.9812 & 0 \\ 1 & 1 & 80 & 396.91 & 79.54 & 2073.1849 & 0 & 0 & 28 & 2000.03 & 5.329 & 0.003 & 4 & 1 & 3.977 & 0 \\ 1 & 1 & 100 & 496.13 & 99.62 & 3110.3613 & 0 & 0 & 28 & 2500 & 6.675 & 0.003 & 5 & 1 & 4.981 & 0 \\ 1 & 1 & 120 & 595.36 & 131.65 & 4356.86122 & 0 & 0 & 28 & 3000.02 & 8.821 & 0.003 & 6 & 1 & 6.5826 & 0 \\ 1 & 1 & 140 & 694.58 & 164.86 & 5810.29707 & 0 & 0 & 28 & 3499.99 & 11.045 & 0.003 & 7 & 1 & 8.24285 & 0 \\ 1 & 1 & 160 & 793.81 & 201.51 & 7469.05328 & 0 & 0 & 28 & 4000.01 & 13.501 & 0.003 & 8 & 1 & 10.07565 & 0 \\ 1 & 2 & 180 & 793.81 & 201.18 & 9149.73467 & 0 & 0 & 28 & 4000.01 & 13.479 & 0.003 & 9 & 1 & 10.0588 & 0 \\ 1 & 2 & 200 & 793.81 & 200.72 & 10812.70375 & 0 & 0 & 28 & 4000.01 & 13.448 & 0.003 & 10 & 1 & 10.03605 & 0 \\ 1 & 2 & 220 & 793.81 & 200.48 & 12475.25579 & 0 & 0 & 28 & 4000.01 & 13.432 & 0.003 & 11 & 1 & 10.02395 & 0 \\ 1 & 3 & 240 & 793.81 & 200.3 & 14154.84861 & 0 & 0 & 28 & 4000.01 & 13.42 & 0.003 & 12 & 1 & 10.01495 & 0 \\ 1 & 3 & 260 & 694.58 & 163.9 & 15610.93675 & 0 & 0 & 28 & 3499.99 & 10.981 & 0.003 & 13 & 1 & 8.19505 & 0 \\ 1 & 3 & 280 & 595.36 & 130.2 & 16860.45104 & 0 & 0 & 28 & 3000.02 & 8.723 & 0.003 & 14 & 1 & 6.51005 & 0 \\ 1 & 3 & 300 & 496.13 & 95.62 & 17901.00386 & 0 & 0 & 28 & 2500 & 6.406 & 0.003 & 15 & 1 & 4.7809 & 0 \\ 1 & 3 & 320 & 396.91 & 76.04 & 18734.04741 & 0 & 0 & 28 & 2000.03 & 5.095 & 0.003 & 16 & 1 & 3.8022 & 0 \\ 1 & 3 & 340 & 297.68 & 57.21 & 19359.03193 & 0 & 0 & 28 & 1500.01 & 3.833 & 0.003 & 17 & 1 & 2.8607 & 0 \\ 1 & 3 & 360 & 198.46 & 37.75 & 19776.33125 & 0 & 0 & 28 & 1000.04 & 2.529 & 0.003 & 18 & 1 & 1.8875 & 0 \\ 1 & 3 & 380 & 99.23 & 19.07 & 19985.90454 & 0 & 0 & 28 & 500.02 & 1.278 & 0.003 & 19 & 1 & 0.95365 & 0\end{array}$



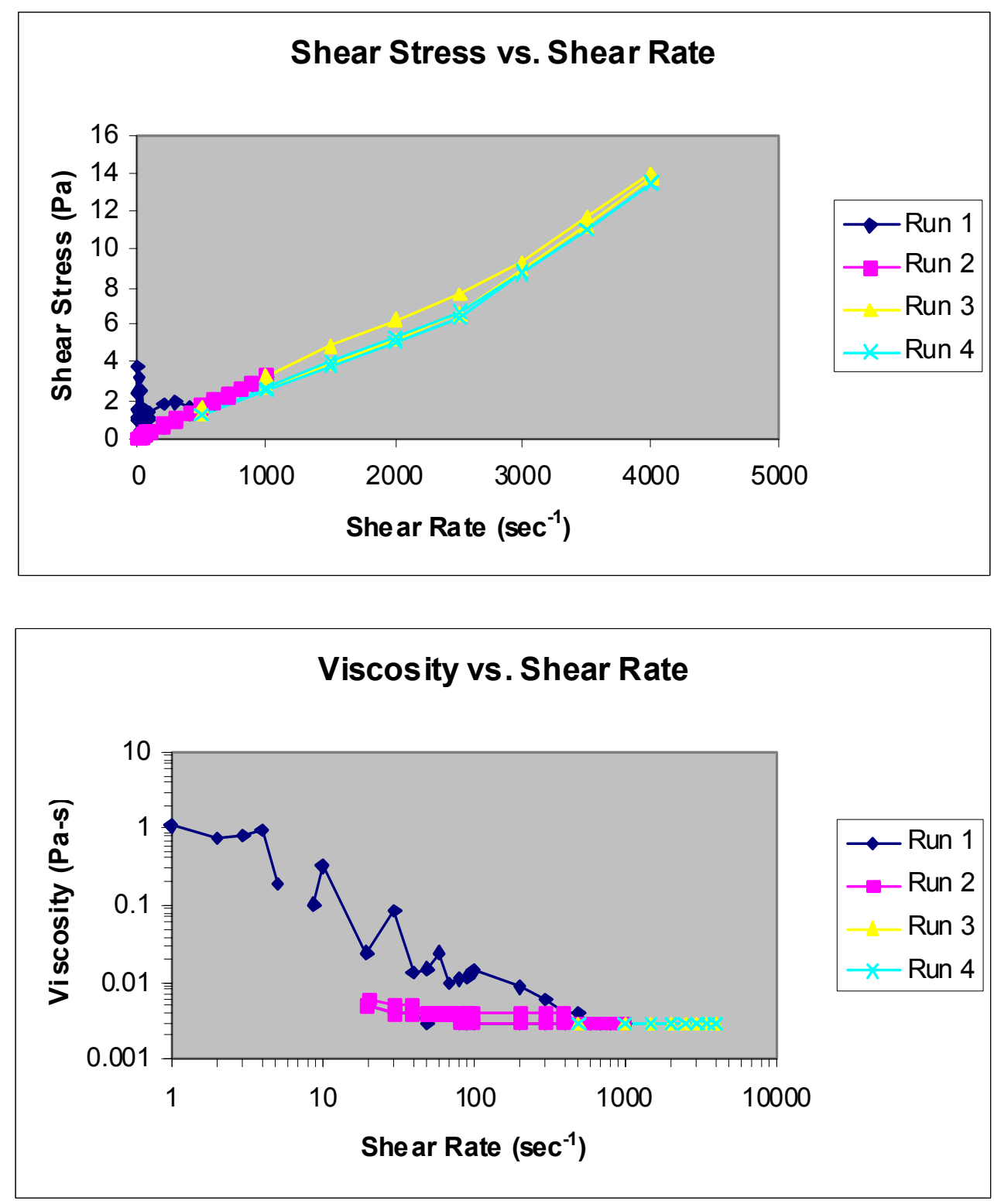

\section{E-2.1.7 187/5wt\%/D/N}

2004.02.06 187-5 wt\%-D DG 1-1000 s-1 run 1.txt Mode Step $\quad \mathrm{t}[\mathrm{s}] \quad \mathrm{n}[\mathrm{rpm}] \quad \mathrm{M}[\%$.] $\quad$ Phi[rad]

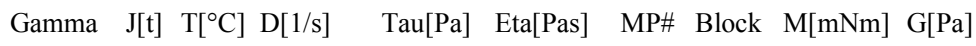

$\begin{array}{rlllllllllllll}20 & 0.2 & 3.69 & 0.41312 & 0 & 0 & 28 & 1.01 & 0.247 & 0.245 & 1 & 1 & 0.1847 & 0 \\ 40 & 0.4 & 8.95 & 1.24486 & 0 & 0 & 28 & 2.02 & 0.6 & 0.297 & 2 & 1 & 0.4475 & 0 \\ 60 & 0.6 & 8.35 & 2.49521 & 0 & 0 & 28 & 3.02 & 0.56 & 0.185 & 3 & 1 & 0.4177 & 0 \\ 80 & 0.8 & 7.8 & 4.16025 & 0 & 0 & 28 & 4.03 & 0.522 & 0.13 & 4 & 1 & 0.3898 & 0 \\ 100 & 1 & 6.15 & 6.24313 & 0 & 0 & 28 & 5.04 & 0.412 & 0.082 & 5 & 1 & 0.3077 & 0 \\ 120 & 1.19 & 5.99 & 8.73991 & 0 & 0 & 28 & 6 & 0.401 & 0.067 & 6 & 1 & 0.2995 & 0 \\ 140 & 1.39 & 6.66 & 11.65295 & 0 & 0 & 28 & 7 & 0.446 & 0.064 & 7 & 1 & 0.333 & 0 \\ 160 & 1.59 & 5.22 & 14.98226 & 0 & 0 & 28 & 8.01 & 0.35 & 0.044 & 8 & 1 & 0.261 & 0 \\ 180 & 1.79 & 5.42 & 18.73018 & 0 & 0 & 28 & 9.02 & 0.363 & 0.04 & 9 & 1 & 0.2711 & 0 \\ 200 & 1.98 & 5.23 & 22.90614 & 0 & 0 & 28 & 9.98 & 0.35 & 0.035 & 10 & 1 & 0.2614 & 0\end{array}$




\begin{tabular}{|c|c|c|c|c|c|c|c|c|c|c|c|c|c|}
\hline 2 & 220 & 3.97 & 6.47 & 31.17952 & 0 & 0 & 28 & 20 & 0.433 & 0.022 & 11 & 1 & 0.3234 \\
\hline 2 & 240 & 5.95 & 6.34 & 43.62808 & 0 & 0 & 28 & 29.98 & 0.425 & 0.014 & 12 & 1 & 0.3172 \\
\hline 2 & 260 & 7.94 & 6.3 & 60.21098 & 0 & 0 & 28 & 40.01 & 0.422 & 0.011 & 13 & 1 & 0.3149 \\
\hline 2 & 280 & 9.92 & 6.1 & 80.95413 & 0 & 0 & 28 & 49.99 & 0.409 & 0.008 & 14 & 1 & 0.3052 \\
\hline 2 & 300 & 11.91 & 6.62 & 105.85439 & 0 & 0 & 28 & 60.01 & 0.443 & 0.007 & 15 & 1 & 0.331 \\
\hline 2 & 320 & 13.89 & 7.04 & 134.91648 & 0 & 0 & 28 & 69.99 & 0.472 & 0.007 & 16 & 1 & 0.3521 \\
\hline 2 & 340 & 15.88 & 7.35 & 168.13647 & 0 & 0 & 28 & 80.02 & 0.492 & 0.006 & 17 & 1 & 0.3673 \\
\hline 2 & 360 & 17.86 & 7.7 & 205.54106 & 0 & 0 & 28 & 90 & 0.516 & 0.006 & 18 & 1 & 0.385 \\
\hline 3 & 380 & 19.85 & 8.08 & 247.48053 & 0 & 0 & 28 & 100 & 0.542 & 0.005 & 19 & 1 & 0.4042 \\
\hline 3 & 400 & 39.69 & 11.97 & 330.25364 & 0 & 0 & 28 & 200 & 0.802 & 0.004 & 20 & 1 & 0.5985 \\
\hline 3 & 420 & 59.54 & 14.22 & 454.75418 & 0 & 0 & 28 & 300 & 0.953 & 0.003 & 21 & 1 & 0.7111 \\
\hline 3 & 440 & 79.38 & 16.19 & 620.70331 & 0 & 0 & 28 & 400 & 1.085 & 0.003 & 22 & 1 & 0.8094 \\
\hline 3 & 460 & 99.23 & 18.05 & 828.17252 & 0 & 0 & 28 & 500 & 1.209 & 0.002 & 23 & 1 & 0.9024 \\
\hline 3 & 480 & 119.1 & 20.1 & 1077.3809 & 0 & 0 & 28 & 600 & 1.347 & 0.002 & 24 & 1 & 1.0049 \\
\hline 3 & 500 & 138.9 & 22.27 & 1368.0882 & 0 & 0 & 28 & 700 & 1.492 & 0.002 & 25 & 1 & 1.1134 \\
\hline 3 & 520 & 158.8 & 24.36 & 1700.109 & 0 & 0 & 28 & 800 & 1.632 & 0.002 & 26 & 1 & 1.218 \\
\hline 3 & 540 & 178.6 & 26.45 & 2073.8486 & 0 & 0 & 28 & 900 & 1.772 & 0.002 & 27 & 1 & 1.3223 \\
\hline 3 & 560 & 198.5 & 28.2 & 2489.2637 & 0 & 0 & 28 & 1000 & 1.889 & 0.002 & 28 & 1 & 1.4099 \\
\hline 4 & 570 & 198.5 & 27.32 & 2701.267 & 0 & 0 & 28 & 1000 & 1.831 & 0.002 & 29 & 1 & 1.3662 \\
\hline 4 & 580 & 198.5 & 27.15 & 2909.4996 & 0 & 0 & 28 & 1000 & 1.819 & 0.002 & 30 & 1 & 1.3575 \\
\hline 4 & 590 & 198.5 & 27.05 & 3117.2123 & 0 & 0 & 28 & 1000 & 1.812 & 0.002 & 31 & 1 & 1.3525 \\
\hline 4 & 600 & 198.5 & 26.94 & 3324.7169 & 0 & 0 & 28 & 1000 & 1.805 & 0.002 & 32 & 1 & 1.3472 \\
\hline 4 & 610 & 198.5 & 26.89 & 3532.5332 & 0 & 0 & 28 & 1000 & 1.801 & 0.002 & 33 & 1 & 1.3444 \\
\hline 4 & 620 & 198.5 & 26.81 & 3740.2459 & 0 & 0 & 28 & 1000 & 1.796 & 0.002 & 34 & 1 & 1.3406 \\
\hline 5 & 640 & 198.5 & 26.73 & 4160.3468 & 0 & 0 & 28 & 1000 & 1.791 & 0.002 & 35 & 1 & 1.3367 \\
\hline 5 & 660 & 178.6 & 24.03 & 4534.8215 & 0 & 0 & 28 & 900 & 1.61 & 0.002 & 36 & 1 & 1.2016 \\
\hline 5 & 680 & 158.8 & 21.65 & 4867.3912 & 0 & 0 & 28 & 800 & 1.451 & 0.002 & 37 & 1 & 1.0825 \\
\hline 5 & 700 & 138.9 & 19.97 & 5159.0237 & 0 & 0 & 28 & 700 & 1.338 & 0.002 & 38 & 1 & 0.9986 \\
\hline 5 & 720 & 119.1 & 18 & 5408.6044 & 0 & 0 & 28 & 600 & 1.206 & 0.002 & 39 & 1 & 0.8998 \\
\hline 5 & 740 & 99.23 & 15.89 & 5616.7475 & 0 & 0 & 28 & 500 & 1.065 & 0.002 & 40 & 1 & 0.7945 \\
\hline 5 & 760 & 79.38 & 14 & 5783.3713 & 0 & 0 & 28 & 400 & 0.938 & 0.002 & 41 & 1 & 0.7001 \\
\hline 5 & 780 & 59.54 & 11.86 & 5908.3281 & 0 & 0 & 28 & 300 & 0.794 & 0.003 & 42 & 1 & 0.5928 \\
\hline 5 & 800 & 39.69 & 8.98 & 5991.8065 & 0 & 0 & 28 & 200 & 0.602 & 0.003 & 43 & 1 & 0.4489 \\
\hline 5 & 820 & 19.85 & 6.25 & 6033.6494 & 0 & 0 & 28 & 100 & 0.419 & 0.004 & 44 & 1 & 0.3127 \\
\hline 6 & 840 & 17.86 & 6.93 & 6071.3477 & 0 & 0 & 28 & 90 & 0.464 & 0.005 & 45 & 1 & 0.3464 \\
\hline 6 & 860 & 15.88 & 7 & 6104.5921 & 0 & 0 & 28 & 80.02 & 0.469 & 0.006 & 46 & 1 & 0.3499 \\
\hline 6 & 880 & 13.89 & 7.02 & 6133.7193 & 0 & 0 & 28 & 69.99 & 0.47 & 0.007 & 47 & 1 & 0.3511 \\
\hline 6 & 900 & 11.9 & 6.78 & 6158.706 & 0 & 0 & 28 & 59.96 & 0.454 & 0.008 & 48 & 1 & 0.339 \\
\hline 6 & 920 & 9.92 & 6.37 & 6179.5002 & 0 & 0 & 28 & 49.99 & 0.426 & 0.009 & 49 & 1 & 0.3183 \\
\hline 6 & 940 & 7.93 & 6.18 & 6196.1428 & 0 & 0 & 28 & 39.96 & 0.414 & 0.01 & 50 & 1 & 0.3089 \\
\hline 6 & 960 & 5.95 & 5.77 & 6208.6377 & 0 & 0 & 28 & 29.98 & 0.387 & 0.013 & 51 & 1 & 0.2887 \\
\hline 6 & 980 & 3.96 & 5.7 & 6216.9692 & 0 & 0 & 28 & 19.95 & 0.382 & 0.019 & 52 & 1 & 0.2848 \\
\hline 6 & 1000 & 1.98 & 4.69 & 6221.1436 & 0 & 0 & 28 & 9.98 & 0.314 & 0.031 & 53 & 1 & 0.2346 \\
\hline 7 & 1020 & 1.79 & 5.89 & 6224.9111 & 0 & 0 & 28 & 9.02 & 0.395 & 0.044 & 54 & 1 & 0.2946 \\
\hline 7 & 1040 & 1.59 & 5.66 & 6228.2459 & 0 & 0 & 28 & 8.01 & 0.379 & 0.047 & 55 & 1 & 0.2828 \\
\hline 7 & 1060 & 1.39 & 6.34 & 6231.1676 & 0 & 0 & 28 & 7 & 0.425 & 0.061 & 56 & 1 & 0.3169 \\
\hline 7 & 1080 & 1.19 & 5.7 & 6233.6683 & 0 & 0 & 28 & 6 & 0.382 & 0.064 & 57 & 1 & 0.285 \\
\hline 7 & 1100 & 0.99 & 5.81 & 6235.7543 & 0 & 0 & 28 & 4.99 & 0.389 & 0.078 & 58 & 1 & 0.2904 \\
\hline 7 & 1120 & 0.8 & 7.41 & 6237.4264 & 0 & 0 & 28 & 4.03 & 0.497 & 0.123 & 59 & 1 & 0.3706 \\
\hline 7 & 1140 & 0.6 & 6.33 & 6238.6799 & 0 & 0 & 28 & 3.02 & 0.424 & 0.14 & 60 & 1 & 0.3166 \\
\hline 7 & 1160 & 0.4 & 5.51 & 6239.518 & 0 & 0 & 28 & 2.02 & 0.369 & 0.183 & 61 & 1 & 0.2757 \\
\hline 7 & 1180 & 0.2 & 5.69 & 6239.9389 & 0 & 0 & 28 & 1.01 & 0.381 & 0.377 & 62 & 1 & 0.2845 \\
\hline
\end{tabular}


2004.02.06 187-5 wt\%-D DG 1-1000 s-

1 run 2.txt

Mode Step $\quad \mathrm{t}[\mathrm{s}] \mathrm{n}[\mathrm{rpm}] \quad \mathrm{M}[\%$. $] \quad$ Phi[rad

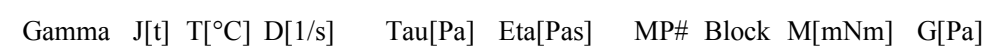

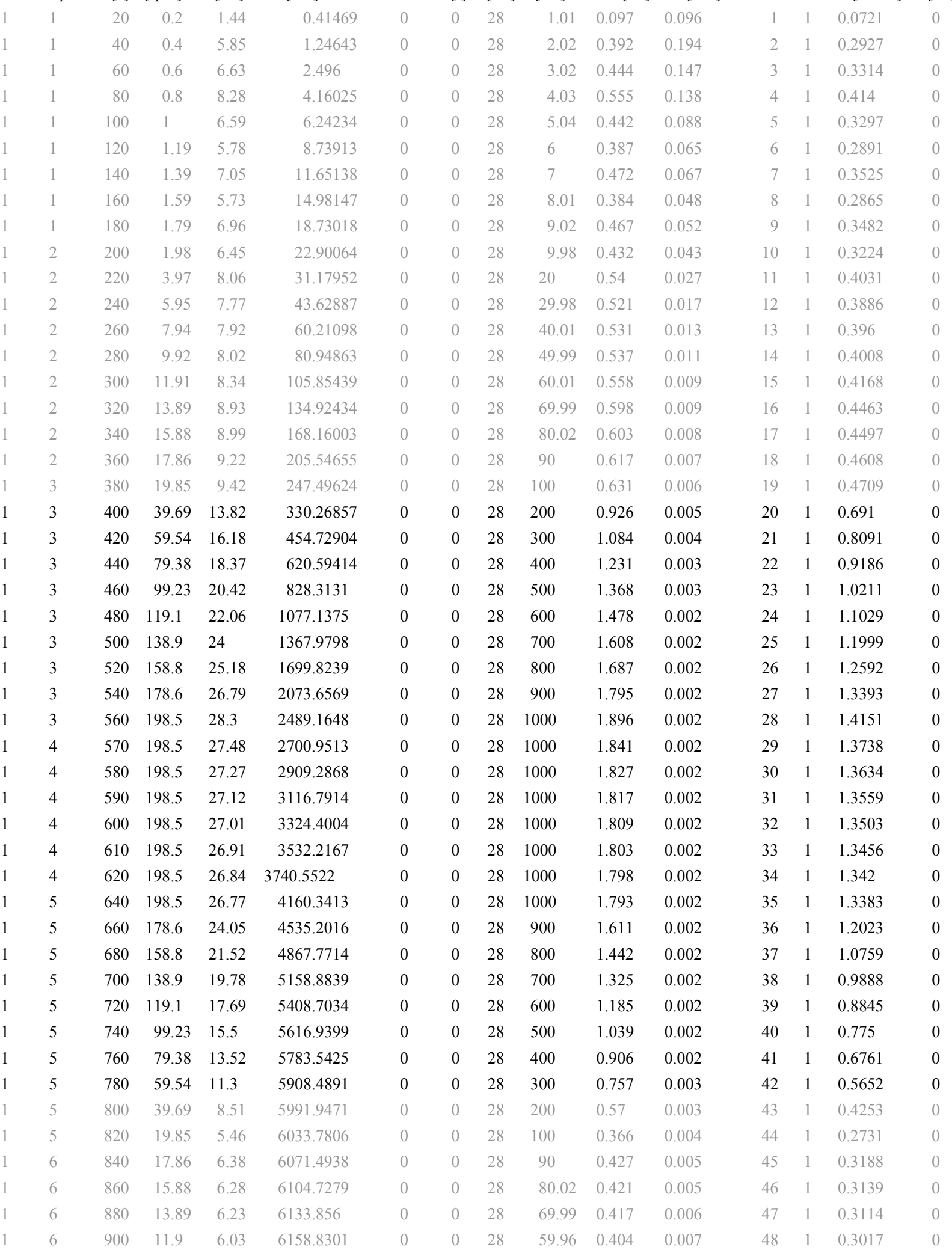




$\begin{array}{rrrrrrrrrrrrrrrrr}1 & 6 & 920 & 9.92 & 5.47 & 6179.6353 & 0 & 0 & 28 & 49.99 & 0.366 & 0.007 & 49 & 1 & 0.2735 & 0 \\ 1 & 6 & 940 & 7.93 & 5.17 & 6196.2912 & 0 & 0 & 28 & 39.96 & 0.346 & 0.009 & 50 & 1 & 0.2586 & 0 \\ 1 & 6 & 960 & 5.95 & 4.89 & 6208.7728 & 0 & 0 & 28 & 29.98 & 0.328 & 0.011 & 51 & 1 & 0.2448 & 0 \\ 1 & 6 & 980 & 3.96 & 4.67 & 6217.1074 & 0 & 0 & 28 & 19.95 & 0.313 & 0.016 & 52 & 1 & 0.2335 & 0 \\ 1 & 6 & 1000 & 1.98 & 3.55 & 6221.2826 & 0 & 0 & 28 & 9.98 & 0.238 & 0.024 & 53 & 1 & 0.1777 & 0 \\ 1 & 7 & 1020 & 1.79 & 4.85 & 6225.0517 & 0 & 0 & 28 & 9.02 & 0.325 & 0.036 & 54 & 1 & 0.2425 & 0 \\ 1 & 7 & 1040 & 1.59 & 4.36 & 6228.3889 & 0 & 0 & 28 & 8.01 & 0.292 & 0.036 & 55 & 1 & 0.218 \\ 1 & 7 & 1060 & 1.39 & 5.06 & 6231.3058 & 0 & 0 & 28 & 7 & 0.339 & 0.048 & 0 \\ 1 & 7 & 1080 & 1.19 & 4.46 & 6233.8089 & 0 & 0 & 28 & 6 & 0.299 & 0.05 & 1 & 0.2532 & 0 \\ 1 & 7 & 1100 & 0.99 & 4.66 & 6235.8965 & 0 & 0 & 28 & 4.99 & 0.312 & 0.063 & 58 & 1 & 0.2329 & 0 \\ 1 & 7 & 1120 & 0.8 & 5.03 & 6237.567 & 0 & 0 & 28 & 4.03 & 0.337 & 0.084 & 59 & 1 & 0.2515 & 0 \\ 1 & 7 & 1140 & 0.6 & 4.66 & 6238.8213 & 0 & 0 & 28 & 3.02 & 0.312 & 0.103 & 60 & 1 & 0.233 & 0 \\ 1 & 7 & 1160 & 0.4 & 4.25 & 6239.6585 & 0 & 0 & 28 & 2.02 & 0.285 & 0.141 & 61 & 1 & 0.2124 & 0 \\ 1 & 7 & 1180 & 0.2 & 4.48 & 6240.0803 & 0 & 0 & 28 & 1.01 & 0.3 & 0.297 & 62 & 1 & 0.2241 & 0\end{array}$

2004.02.06 187-5 wt\%-D DG 500-4000 s-1 run 1.txt

Mode Step $\quad \mathrm{t}[\mathrm{s}] \mathrm{n}[\mathrm{rpm}] \quad \mathrm{M}[\%$.] Phi[rad]

$\begin{array}{rrrrrr}1 & 1 & 20 & 99.24 & 25.85 & 207.67106 \\ 1 & 1 & 40 & 198.5 & 34.5 & 621.44787 \\ 1 & 1 & 60 & 297.7 & 47.7 & 1243.8225 \\ 1 & 1 & 80 & 396.9 & 59.37 & 2072.6634 \\ 1 & 1 & 100 & 496.1 & 69.83 & 3110.6739 \\ 1 & 1 & 120 & 595.4 & 82.82 & 4355.9297 \\ 1 & 1 & 140 & 694.6 & 103.9 & 5809.3656 \\ 1 & 1 & 160 & 793.8 & 123.2 & 7470.983 \\ 1 & 2 & 180 & 793.8 & 122.2 & 9150.3811 \\ 1 & 2 & 200 & 793.8 & 121.9 & 10813.762 \\ 1 & 2 & 220 & 793.8 & 121.9 & 12475.484 \\ 1 & 3 & 240 & 793.8 & 121.8 & 14154.659 \\ 1 & 3 & 260 & 694.6 & 101.2 & 15612.568 \\ 1 & 3 & 280 & 595.4 & 81.77 & 16860.595 \\ 1 & 3 & 300 & 496.1 & 63.27 & 17901.616 \\ 1 & 3 & 320 & 396.9 & 46.04 & 18734.061 \\ 1 & 3 & 340 & 297.7 & 29.66 & 19359.909 \\ 1 & 3 & 360 & 198.5 & 16.68 & 19776.989 \\ 1 & 3 & 380 & 99.23 & 8.86 & 19986.493\end{array}$

Gamma J[t] $\mathrm{T}\left[{ }^{\circ} \mathrm{C}\right] \mathrm{D}[1 / \mathrm{s}] \quad \mathrm{Tau}[\mathrm{Pa}]$ Eta[Pas $] \quad$ MP\# Block M[mNm] G[Pa

$\begin{array}{llllllllll}0 & 0 & 28 & 500.1 & 1.732 & 0.003 & 1 & 1 & 1.2923 & 0 \\ 0 & 0 & 28 & 1000 & 2.312 & 0.002 & 2 & 1 & 1.7252 & 0 \\ 0 & 0 & 28 & 1500 & 3.196 & 0.002 & 3 & 1 & 2.3849 & 0 \\ 0 & 0 & 28 & 2000 & 3.978 & 0.002 & 4 & 1 & 2.9687 & 0 \\ 0 & 0 & 28 & 2500 & 4.678 & 0.002 & 5 & 1 & 3.4913 & 0 \\ 0 & 0 & 28 & 3000 & 5.549 & 0.002 & 6 & 1 & 4.1408 & 0 \\ 0 & 0 & 28 & 3500 & 6.964 & 0.002 & 7 & 1 & 5.1971 & 0 \\ 0 & 0 & 28 & 4000 & 8.257 & 0.002 & 8 & 1 & 6.1621 & 0 \\ 0 & 0 & 28 & 4000 & 8.184 & 0.002 & 9 & 1 & 6.1075 & 0 \\ 0 & 0 & 28 & 4000 & 8.169 & 0.002 & 10 & 1 & 6.0961 & 0 \\ 0 & 0 & 28 & 4000 & 8.165 & 0.002 & 11 & 1 & 6.093 & 0 \\ 0 & 0 & 28 & 4000 & 8.159 & 0.002 & 12 & 1 & 6.0886 & 0 \\ 0 & 0 & 28 & 3500 & 6.778 & 0.002 & 13 & 1 & 5.0585 & 0 \\ 0 & 0 & 28 & 3000 & 5.479 & 0.002 & 14 & 1 & 4.0886 & 0 \\ 0 & 0 & 28 & 2500 & 4.239 & 0.002 & 15 & 1 & 3.1637 & 0 \\ 0 & 0 & 28 & 2000 & 3.085 & 0.002 & 16 & 1 & 2.3022 & 0 \\ 0 & 0 & 28 & 1500 & 1.987 & 0.001 & 17 & 1 & 1.4831 & 0 \\ 0 & 0 & 28 & 1000 & 1.118 & 0.001 & 18 & 1 & 0.8342 & 0 \\ 0 & 0 & 28 & 500 & 0.593 & 0.001 & 19 & 1 & 0.4428 & 0\end{array}$

2004.02.06 187-5 wt\%-D DG 500-4000

s-1 run 2.txt

\begin{tabular}{|c|c|c|c|c|c|c|c|c|c|c|c|c|c|c|c|}
\hline Mode & Step & $\mathrm{t}[\mathrm{s}]$ & $\mathrm{n}[\mathrm{rpm}]$ & $\mathrm{M}[\%]$. & Phi[rad] & Gamma & $J[t]$ & $\mathrm{T}\left[{ }^{\circ} \mathrm{C}\right]$ & $\mathrm{D}[1 / \mathrm{s}]$ & $\mathrm{Tau}[\mathrm{Pa}]$ & Eta[Pas] & MP\# & Block & $\mathrm{M}[\mathrm{mNm}]$ & $\mathrm{G}[\mathrm{Pa}]$ \\
\hline 1 & 1 & 20 & 99.32 & 14.37 & 207.67969 & 0 & 0 & 28 & 500.5 & 0.963 & 0.002 & 1 & 1 & 0.7184 & 0 \\
\hline 1 & 1 & 40 & 198.5 & 25.72 & 621.50913 & 0 & 0 & 28 & 1000 & 1.724 & 0.002 & 2 & 1 & 1.2862 & 0 \\
\hline 1 & 1 & 60 & 297.7 & 41.4 & 1243.8304 & 0 & 0 & 28 & 1500 & 2.774 & 0.002 & 3 & 1 & 2.0702 & 0 \\
\hline 1 & 1 & 80 & 396.9 & 57.38 & 2073.3467 & 0 & 0 & 28 & 2000 & 3.845 & 0.002 & 4 & 1 & 2.8691 & 0 \\
\hline 1 & 1 & 100 & 496.1 & 72.68 & 3111.0454 & 0 & 0 & 28 & 2500 & 4.87 & 0.002 & 5 & 1 & 3.6342 & 0 \\
\hline 1 & 1 & 120 & 595.4 & 84.55 & 4356.5604 & 0 & 0 & 28 & 3000 & 5.665 & 0.002 & 6 & 1 & 4.2276 & 0 \\
\hline 1 & 1 & 140 & 694.6 & 102.5 & 5808.1255 & 0 & 0 & 28 & 3500 & 6.865 & 0.002 & 7 & 1 & 5.1231 & 0 \\
\hline 1 & 1 & 160 & 793.8 & 122.7 & 7468.9645 & 0 & 0 & 28 & 4000 & 8.222 & 0.002 & 8 & 1 & 6.1356 & 0 \\
\hline 1 & 2 & 180 & 793.8 & 121.6 & 9150.5248 & 0 & 0 & 28 & 4000 & 8.145 & 0.002 & 9 & 1 & 6.0784 & 0 \\
\hline 1 & 2 & 200 & 793.8 & 121.8 & 10811.413 & 0 & 0 & 28 & 4000 & 8.158 & 0.002 & 10 & 1 & 6.088 & 0 \\
\hline 1 & 2 & 220 & 793.8 & 121.7 & 12474.796 & 0 & 0 & 28 & 4000 & 8.154 & 0.002 & 11 & 1 & 6.085 & 0 \\
\hline 1 & 3 & 240 & 793.8 & 121.5 & 14155.636 & 0 & 0 & 28 & 4000 & 8.143 & 0.002 & 12 & 1 & 6.0769 & 0 \\
\hline
\end{tabular}




$\begin{array}{llllllllllllll}260 & 694.6 & 101 & 15612.41 & 0 & 0 & 28 & 3500 & 6.768 & 0.002 & 13 & 1 & 5.0506 & 0 \\ 280 & 595.4 & 81.77 & 16861.069 & 0 & 0 & 28 & 3000 & 5.478 & 0.002 & 14 & 1 & 4.0884 & 0 \\ 300 & 496.1 & 63.25 & 17901.159 & 0 & 0 & 28 & 2500 & 4.238 & 0.002 & 15 & 1 & 3.1627 & 0 \\ 320 & 396.9 & 46.07 & 18734.644 & 0 & 0 & 28 & 2000 & 3.086 & 0.002 & 16 & 1 & 2.3033 & 0 \\ 340 & 297.7 & 29.77 & 19359.413 & 0 & 0 & 28 & 1500 & 1.995 & 0.001 & 17 & 1 & 1.4886 & 0 \\ 360 & 198.5 & 16.8 & 19776.91 & 0 & 0 & 28 & 1000 & 1.126 & 0.001 & 18 & 1 & 0.8402 & 0 \\ 380 & 99.23 & 9.02 & 19986.509 & 0 & 0 & 28 & 500 & 0.605 & 0.001 & 19 & 1 & 0.4512 & 0\end{array}$
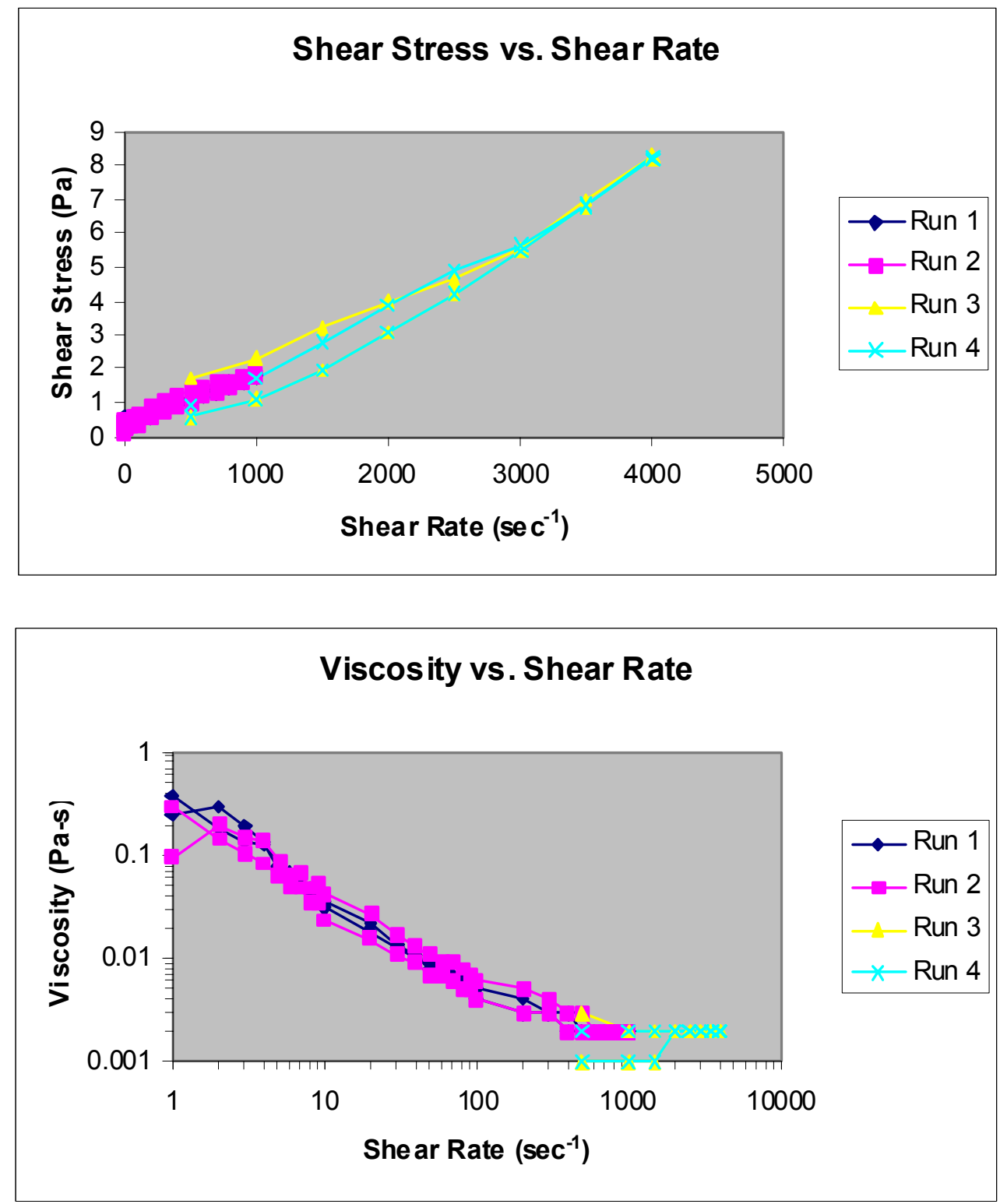

\section{E-2.1.8 187/5wt $\% / D / S$}

2004.01.30 187-5 wt\%-D [sonicated] DG 1-1000 s-1 run 1.txt

Mode Step $\mathrm{t}[\mathrm{s}] \quad \mathrm{n}[\mathrm{rpm}] \quad \mathrm{M}[\%$.$] \quad Phi[rad]$

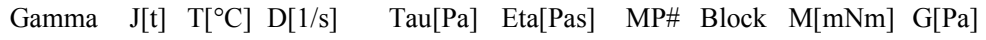
0.41626 


\begin{tabular}{|c|c|c|c|c|c|c|c|c|c|c|c|c|c|}
\hline 1 & 60 & 0.6 & 0 & 2.49757 & 0 & 0 & 28 & 3.02 & 0 & 0 & 3 & 1 & 0 \\
\hline 1 & 80 & 0.8 & 0 & 4.16182 & 0 & 0 & 28 & 4.03 & 0 & 0 & 4 & 1 & 0 \\
\hline 1 & 100 & 1 & 0 & 6.24392 & 0 & 0 & 28 & 5.04 & 0 & 0 & 5 & 1 & 0 \\
\hline 1 & 120 & 1.19 & 0 & 8.74227 & 0 & 0 & 28 & 6 & 0 & 0 & 6 & 1 & 0 \\
\hline 1 & 140 & 1.39 & 1.06 & 11.65531 & 0 & 0 & 28 & 7 & 0.071 & 0.01 & 7 & 1 & 0.0529 \\
\hline 1 & 160 & 1.59 & 1.15 & 14.98383 & 0 & 0 & 28 & 8.01 & 0.077 & 0.01 & 8 & 1 & 0.05745 \\
\hline 1 & 180 & 1.79 & 1.13 & 18.73018 & 0 & 0 & 28 & 9.02 & 0.076 & 0.008 & 9 & 1 & 0.05635 \\
\hline 2 & 200 & 1.98 & 1.16 & 22.90535 & 0 & 0 & 28 & 9.98 & 0.078 & 0.008 & 10 & 1 & 0.0582 \\
\hline 2 & 220 & 3.97 & 2.09 & 31.17874 & 0 & 0 & 28 & 20 & 0.14 & 0.007 & 11 & 1 & 0.10425 \\
\hline 2 & 240 & 5.95 & 2.43 & 43.62494 & 0 & 0 & 28 & 29.98 & 0.163 & 0.005 & 12 & 1 & 0.12155 \\
\hline 2 & 260 & 7.94 & 2.73 & 60.21962 & 0 & 0 & 28 & 40.01 & 0.183 & 0.005 & 13 & 1 & 0.13625 \\
\hline 2 & 280 & 9.92 & 2.97 & 80.95806 & 0 & 0 & 28 & 49.99 & 0.199 & 0.004 & 14 & 1 & 0.14855 \\
\hline 2 & 300 & 11.91 & 3.59 & 105.84968 & 0 & 0 & 28 & 60.01 & 0.241 & 0.004 & 15 & 1 & 0.1796 \\
\hline 2 & 320 & 13.89 & 3.95 & 134.92748 & 0 & 0 & 28 & 69.99 & 0.264 & 0.004 & 16 & 1 & 0.19725 \\
\hline 2 & 340 & 15.88 & 4.16 & 168.15532 & 0 & 0 & 28 & 80.02 & 0.279 & 0.003 & 17 & 1 & 0.20805 \\
\hline 2 & 360 & 17.86 & 4.71 & 205.52378 & 0 & 0 & 28 & 90 & 0.315 & 0.004 & 18 & 1 & 0.23525 \\
\hline 3 & 380 & 19.85 & 5.16 & 247.43184 & 0 & 0 & 28 & 100.02 & 0.346 & 0.003 & 19 & 1 & 0.2579 \\
\hline 3 & 400 & 39.69 & 8.88 & 330.29841 & 0 & 0 & 28 & 200 & 0.595 & 0.003 & 20 & 1 & 0.44395 \\
\hline 3 & 420 & 59.54 & 10.71 & 454.7369 & 0 & 0 & 28 & 300.02 & 0.718 & 0.002 & 21 & 1 & 0.5357 \\
\hline 3 & 440 & 79.38 & 12.06 & 620.57215 & 0 & 0 & 28 & 400 & 0.808 & 0.002 & 22 & 1 & 0.60315 \\
\hline 3 & 460 & 99.23 & 13.89 & 828.1356 & 0 & 0 & 28 & 500.02 & 0.931 & 0.002 & 23 & 1 & 0.6947 \\
\hline 3 & 480 & 119.07 & 16.13 & 1077.4469 & 0 & 0 & 28 & 599.99 & 1.08 & 0.002 & 24 & 1 & 0.80625 \\
\hline 3 & 500 & 138.92 & 18.06 & 1367.92641 & 0 & 0 & 28 & 700.02 & 1.21 & 0.002 & 25 & 1 & 0.903 \\
\hline 3 & 520 & 158.76 & 19.88 & 1700.03124 & 0 & 0 & 28 & 799.99 & 1.332 & 0.002 & 26 & 1 & 0.99405 \\
\hline 3 & 540 & 178.61 & 22.16 & 2073.60195 & 0 & 0 & 28 & 900.02 & 1.485 & 0.002 & 27 & 1 & 1.1079 \\
\hline 3 & 560 & 198.45 & 24.23 & 2489.12156 & 0 & 0 & 28 & 999.99 & 1.623 & 0.002 & 28 & 1 & 1.2114 \\
\hline 4 & 570 & 198.45 & 23.89 & 2700.9081 & 0 & 0 & 28 & 999.99 & 1.6 & 0.002 & 29 & 1 & 1.1943 \\
\hline 4 & 580 & 198.45 & 23.81 & 2909.03547 & 0 & 0 & 28 & 999.99 & 1.595 & 0.002 & 30 & 1 & 1.1903 \\
\hline 4 & 590 & 198.45 & 23.68 & 3117.16363 & 0 & 0 & 28 & 999.99 & 1.586 & 0.002 & 31 & 1 & 1.1838 \\
\hline 4 & 600 & 198.45 & 23.56 & 3324.46083 & 0 & 0 & 28 & 999.99 & 1.579 & 0.002 & 32 & 1 & 1.1782 \\
\hline 4 & 610 & 198.45 & 23.53 & 3532.69266 & 0 & 0 & 28 & 999.99 & 1.577 & 0.002 & 33 & 1 & 1.17665 \\
\hline 4 & 620 & 198.45 & 23.49 & 3740.30089 & 0 & 0 & 28 & 999.99 & 1.574 & 0.002 & 34 & 1 & 1.1745 \\
\hline 5 & 640 & 198.45 & 23.42 & 4160.5054 & 0 & 0 & 28 & 999.99 & 1.569 & 0.002 & 35 & 1 & 1.17075 \\
\hline 5 & 660 & 178.61 & 20.95 & 4535.1686 & 0 & 0 & 28 & 900.02 & 1.404 & 0.002 & 36 & 1 & 1.0475 \\
\hline 5 & 680 & 158.76 & 18.42 & 4867.65513 & 0 & 0 & 28 & 799.99 & 1.234 & 0.002 & 37 & 1 & 0.9212 \\
\hline 5 & 700 & 138.92 & 16.36 & 5158.92318 & 0 & 0 & 28 & 700.02 & 1.096 & 0.002 & 38 & 1 & 0.8182 \\
\hline 5 & 720 & 119.07 & 14.2 & 5408.75362 & 0 & 0 & 28 & 599.99 & 0.951 & 0.002 & 39 & 1 & 0.70995 \\
\hline 5 & 740 & 99.23 & 11.76 & 5616.79303 & 0 & 0 & 28 & 500.02 & 0.788 & 0.002 & 40 & 1 & 0.5879 \\
\hline 5 & 760 & 79.38 & 9.61 & 5783.45845 & 0 & 0 & 28 & 400 & 0.644 & 0.002 & 41 & 1 & 0.4804 \\
\hline 5 & 780 & 59.54 & 7.54 & 5908.48834 & 0 & 0 & 28 & 300.02 & 0.505 & 0.002 & 42 & 1 & 0.37685 \\
\hline 5 & 800 & 39.69 & 5.17 & 5991.85207 & 0 & 0 & 28 & 200 & 0.346 & 0.002 & 43 & 1 & 0.25835 \\
\hline 5 & 820 & 19.85 & 3.05 & 6033.74756 & 0 & 0 & 28 & 100.02 & 0.204 & 0.002 & 44 & 1 & 0.1524 \\
\hline 6 & 840 & 17.86 & 3.01 & 6071.41997 & 0 & 0 & 28 & 90 & 0.202 & 0.002 & 45 & 1 & 0.1506 \\
\hline 6 & 860 & 15.88 & 2.72 & 6104.69651 & 0 & 0 & 28 & 80.02 & 0.182 & 0.002 & 46 & 1 & 0.1361 \\
\hline 6 & 880 & 13.89 & 2.73 & 6133.81828 & 0 & 0 & 28 & 69.99 & 0.183 & 0.003 & 47 & 1 & 0.1366 \\
\hline 6 & 900 & 11.9 & 2.59 & 6158.79238 & 0 & 0 & 28 & 59.96 & 0.173 & 0.003 & 48 & 1 & 0.12945 \\
\hline 6 & 920 & 9.92 & 2.19 & 6179.57558 & 0 & 0 & 28 & 49.99 & 0.147 & 0.003 & 49 & 1 & 0.10955 \\
\hline 6 & 940 & 7.93 & 2.3 & 6196.23545 & 0 & 0 & 28 & 39.96 & 0.154 & 0.004 & 50 & 1 & 0.1152 \\
\hline 6 & 960 & 5.95 & 2.17 & 6208.72485 & 0 & 0 & 28 & 29.98 & 0.146 & 0.005 & 51 & 1 & 0.1087 \\
\hline 6 & 980 & 3.96 & 1.91 & 6217.05949 & 0 & 0 & 28 & 19.95 & 0.128 & 0.006 & 52 & 1 & 0.09545 \\
\hline
\end{tabular}




$\begin{array}{rrrrrrrrrrrrrrrrrrr}1 & 6 & 1000 & 1.98 & 1.4 & 6221.2331 & 0 & 0 & 28 & 9.98 & 0.094 & 0.009 & 53 & 1 & 0.0701 & 0 \\ 1 & 7 & 1020 & 1.79 & 1.33 & 6225.00615 & 0 & 0 & 28 & 9.02 & 0.089 & 0.01 & 54 & 1 & 0.0667 & 0 \\ 1 & 7 & 1040 & 1.59 & 1.4 & 6228.34174 & 0 & 0 & 28 & 8.01 & 0.094 & 0.012 & 55 & 1 & 0.06995 & 0 \\ 1 & 7 & 7 & 1060 & 1.39 & 1.39 & 6231.25949 & 0 & 0 & 28 & 7 & 0.093 & 0.013 & 56 & 1 & 0.0696 & 0 \\ 1 & 7 & 7 & 1080 & 1.19 & 1.53 & 6233.76334 & 0 & 0 & 28 & 6 & 0.102 & 0.017 & 57 & 1 & 0.0764 & 0 \\ 1 & 7 & 7100 & 0.99 & 1.45 & 6235.84936 & 0 & 0 & 28 & 4.99 & 0.097 & 0.019 & 58 & 1 & 0.0724 & 0 \\ 1 & 7 & 7120 & 0.8 & 1.69 & 6237.5199 & 0 & 0 & 28 & 4.03 & 0.113 & 0.028 & 59 & 1 & 0.0846 & 0 \\ 1 & 7 & 7140 & 0.6 & 1.75 & 6238.77418 & 0 & 0 & 28 & 3.02 & 0.117 & 0.039 & 60 & 1 & 0.08765 & 0 \\ 1 & 7 & 7160 & 0.4 & 1.82 & 6239.61142 & 0 & 0 & 28 & 2.02 & 0.122 & 0.06 & 61 & 1 & 0.09075 & 0 \\ 1 & 7 & 1180 & 0.2 & 1.37 & 6240.03396 & 0 & 0 & 28 & 1.01 & 0.092 & 0.091 & 62 & 1 & 0.0684 & 0\end{array}$

2004.01.30 187-5 wt\%-D [sonicated] DG 1-1000 s-1 run 2.txt

Mode Step $\mathrm{t}[\mathrm{s}] \quad \mathrm{n}[\mathrm{rpm}] \quad \mathrm{M}[\%$.] $\quad$ Phi[rad]

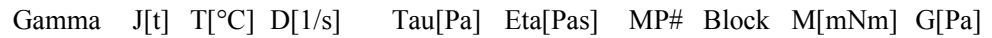

\begin{tabular}{|c|c|c|c|c|c|c|c|c|c|c|c|c|c|}
\hline 1 & 20 & 0.2 & 6.04 & 0.41469 & 0 & 0 & 28 & 1.01 & 0.404 & 0.4 & 1 & 1 & 0.3018 \\
\hline 1 & 40 & 0.4 & 4.4 & 1.248 & 0 & 0 & 28 & 2.02 & 0.295 & 0.146 & 2 & 1 & 0.22 \\
\hline 1 & 60 & 0.6 & 3.79 & 2.49678 & 0 & 0 & 28 & 3.02 & 0.254 & 0.084 & 3 & 1 & 0.18925 \\
\hline 1 & 80 & 0.8 & 3.07 & 4.16104 & 0 & 0 & 28 & 4.03 & 0.205 & 0.051 & 4 & 1 & 0.1533 \\
\hline 1 & 100 & 1 & 2.85 & 6.24392 & 0 & 0 & 28 & 5.04 & 0.191 & 0.038 & 5 & 1 & 0.14225 \\
\hline 1 & 120 & 1.19 & 3.04 & 8.73913 & 0 & 0 & 28 & 6 & 0.204 & 0.034 & 6 & 1 & 0.152 \\
\hline 1 & 140 & 1.39 & 2.78 & 11.65374 & 0 & 0 & 28 & 7 & 0.186 & 0.027 & 7 & 1 & 0.1389 \\
\hline 1 & 160 & 1.59 & 3.05 & 14.98461 & 0 & 0 & 28 & 8.01 & 0.205 & 0.026 & 8 & 1 & 0.15265 \\
\hline 1 & 180 & 1.79 & 2.99 & 18.73175 & 0 & 0 & 28 & 9.02 & 0.2 & 0.022 & 9 & 1 & 0.1496 \\
\hline 1 & 200 & 1.98 & 2.78 & 22.90378 & 0 & 0 & 28 & 9.98 & 0.186 & 0.019 & 10 & 1 & 0.13885 \\
\hline 1 & 220 & 3.97 & 4.4 & 31.18423 & 0 & 0 & 28 & 20 & 0.295 & 0.015 & 11 & 1 & 0.22005 \\
\hline 1 & 240 & 5.95 & 4.74 & 43.61787 & 0 & 0 & 28 & 29.98 & 0.317 & 0.011 & 12 & 1 & 0.2368 \\
\hline 1 & 260 & 7.94 & 4.94 & 60.22197 & 0 & 0 & 28 & 40.01 & 0.331 & 0.008 & 13 & 1 & 0.24695 \\
\hline 1 & 280 & 9.92 & 5.08 & 80.95413 & 0 & 0 & 28 & 49.99 & 0.34 & 0.007 & 14 & 1 & 0.254 \\
\hline 1 & 300 & 11.91 & 5.63 & 105.85911 & 0 & 0 & 28 & 60.01 & 0.377 & 0.006 & 15 & 1 & 0.2814 \\
\hline 1 & 320 & 13.89 & 6.02 & 134.94319 & 0 & 0 & 28 & 69.99 & 0.403 & 0.006 & 16 & 1 & 0.3009 \\
\hline 1 & 340 & 15.88 & 6.18 & 168.14668 & 0 & 0 & 28 & 80.02 & 0.414 & 0.005 & 17 & 1 & 0.30885 \\
\hline 1 & 360 & 17.86 & 6.54 & 205.52378 & 0 & 0 & 28 & 90 & 0.438 & 0.005 & 18 & 1 & 0.32695 \\
\hline 1 & 380 & 19.85 & 6.76 & 247.44205 & 0 & 0 & 28 & 100.02 & 0.453 & 0.005 & 19 & 1 & 0.33805 \\
\hline 1 & 400 & 39.69 & 11.47 & 330.31883 & 0 & 0 & 28 & 200 & 0.768 & 0.004 & 20 & 1 & 0.5734 \\
\hline 1 & 420 & 59.54 & 14.71 & 454.61202 & 0 & 0 & 28 & 300.02 & 0.986 & 0.003 & 21 & 1 & 0.7355 \\
\hline 1 & 440 & 79.38 & 17.33 & 620.73708 & 0 & 0 & 28 & 400 & 1.161 & 0.003 & 22 & 1 & 0.86655 \\
\hline 1 & 460 & 99.23 & 18.17 & 828.29033 & 0 & 0 & 28 & 500.02 & 1.217 & 0.002 & 23 & 1 & 0.90855 \\
\hline 1 & 480 & 119.07 & 17.43 & 1077.17672 & 0 & 0 & 28 & 599.99 & 1.168 & 0.002 & 24 & 1 & 0.87155 \\
\hline 1 & 500 & 138.92 & 18.74 & 1367.72849 & 0 & 0 & 28 & 700.02 & 1.255 & 0.002 & 25 & 1 & 0.93675 \\
\hline 1 & 520 & 158.76 & 20.36 & 1699.91578 & 0 & 0 & 28 & 799.99 & 1.364 & 0.002 & 26 & 1 & 1.01775 \\
\hline 1 & 540 & 178.61 & 22.65 & 2073.94517 & 0 & 0 & 28 & 900.02 & 1.518 & 0.002 & 27 & 1 & 1.1326 \\
\hline 1 & 560 & 198.45 & 24.73 & 2488.94406 & 0 & 0 & 28 & 999.99 & 1.657 & 0.002 & 28 & 1 & 1.2365 \\
\hline 1 & 570 & 198.45 & 24.43 & 2700.84448 & 0 & 0 & 28 & 999.99 & 1.637 & 0.002 & 29 & 1 & 1.22155 \\
\hline 1 & 580 & 198.45 & 24.34 & 2909.07631 & 0 & 0 & 28 & 999.99 & 1.631 & 0.002 & 30 & 1 & 1.21695 \\
\hline 1 & 590 & 198.45 & 24.25 & 3116.47719 & 0 & 0 & 28 & 999.99 & 1.625 & 0.002 & 31 & 1 & 1.2126 \\
\hline 1 & 600 & 198.45 & 24.16 & 3324.60535 & 0 & 0 & 28 & 999.99 & 1.619 & 0.002 & 32 & 1 & 1.2081 \\
\hline 1 & 610 & 198.45 & 24.08 & 3532.21357 & 0 & 0 & 28 & 999.99 & 1.613 & 0.002 & 33 & 1 & 1.20385 \\
\hline 1 & 620 & 198.45 & 23.99 & 3739.92625 & 0 & 0 & 28 & 999.99 & 1.607 & 0.002 & 34 & 1 & 1.1994 \\
\hline 1 & 640 & 198.45 & 23.92 & 4160.23444 & 0 & 0 & 28 & 999.99 & 1.602 & 0.002 & 35 & 1 & 1.19585 \\
\hline 1 & 660 & 178.61 & 21.46 & 4534.62667 & 0 & 0 & 28 & 900.02 & 1.438 & 0.002 & 36 & 1 & 1.0732 \\
\hline 1 & 680 & 158.76 & 18.83 & 4867.37396 & 0 & 0 & 28 & 799.99 & 1.261 & 0.002 & 37 & 1 & 0.9413 \\
\hline
\end{tabular}




\begin{tabular}{|c|c|c|c|c|c|c|c|c|c|c|c|c|c|c|}
\hline 1 & 5 & 700 & 138.92 & 16.67 & 5158.72526 & 0 & 0 & 28 & 700.02 & 1.117 & 0.002 & 38 & 1 & 0.8336 \\
\hline 1 & 5 & 720 & 119.07 & 14.42 & 5408.36878 & 0 & 0 & 28 & 599.99 & 0.966 & 0.002 & 39 & 1 & 0.7209 \\
\hline 1 & 5 & 740 & 99.23 & 12 & 5616.6061 & 0 & 0 & 28 & 500.02 & 0.804 & 0.002 & 40 & 1 & 0.5998 \\
\hline 1 & 5 & 760 & 79.38 & 9.81 & 5783.04219 & 0 & 0 & 28 & 400 & 0.657 & 0.002 & 41 & 1 & 0.49025 \\
\hline 1 & 5 & 780 & 59.54 & 7.65 & 5908.02024 & 0 & 0 & 28 & 300.02 & 0.512 & 0.002 & 42 & 1 & 0.38235 \\
\hline 1 & 5 & 800 & 39.69 & 5.2 & 5991.45623 & 0 & 0 & 28 & 200 & 0.348 & 0.002 & 43 & 1 & 0.25985 \\
\hline 1 & 5 & 820 & 19.85 & 2.99 & 6033.3313 & 0 & 0 & 28 & 100.02 & 0.2 & 0.002 & 44 & 1 & 0.1495 \\
\hline 1 & 6 & 840 & 17.86 & 2.96 & 6071.01235 & 0 & 0 & 28 & 90 & 0.198 & 0.002 & 45 & 1 & 0.148 \\
\hline 1 & 6 & 860 & 15.88 & 2.54 & 6104.30616 & 0 & 0 & 28 & 80.02 & 0.17 & 0.002 & 46 & 1 & 0.1272 \\
\hline 1 & 6 & 880 & 13.89 & 2.52 & 6133.40988 & 0 & 0 & 28 & 69.99 & 0.169 & 0.002 & 47 & 1 & 0.1262 \\
\hline 1 & 6 & 900 & 11.9 & 2.31 & 6158.37847 & 0 & 0 & 28 & 59.96 & 0.155 & 0.003 & 48 & 1 & 0.11555 \\
\hline 1 & 6 & 920 & 9.92 & 1.98 & 6179.18759 & 0 & 0 & 28 & 49.99 & 0.133 & 0.003 & 49 & 1 & 0.09895 \\
\hline 1 & 6 & 940 & 7.93 & 1.97 & 6195.82233 & 0 & 0 & 28 & 39.96 & 0.132 & 0.003 & 50 & 1 & 0.09865 \\
\hline 1 & 6 & 960 & 5.95 & 1.85 & 6208.32273 & 0 & 0 & 28 & 29.98 & 0.124 & 0.004 & 51 & 1 & 0.09265 \\
\hline 1 & 6 & 980 & 3.96 & 1.72 & 6216.65344 & 0 & 0 & 28 & 19.95 & 0.115 & 0.006 & 52 & 1 & 0.08615 \\
\hline 1 & 6 & 1000 & 1.98 & 1.01 & 6220.82862 & 0 & 0 & 28 & 9.98 & 0.068 & 0.007 & 53 & 1 & 0.05065 \\
\hline 1 & 7 & 1020 & 1.79 & 0 & 6224.59932 & 0 & 0 & 28 & 9.02 & 0 & 0 & 54 & 1 & 0 \\
\hline 1 & 7 & 1040 & 1.59 & 1.25 & 6227.9349 & 0 & 0 & 28 & 8.01 & 0.084 & 0.01 & 55 & 1 & 0.06275 \\
\hline 1 & 7 & 1060 & 1.39 & 1.26 & 6230.85344 & 0 & 0 & 28 & 7 & 0.084 & 0.012 & 56 & 1 & 0.0628 \\
\hline 1 & 7 & 1080 & 1.19 & 1.35 & 6233.35808 & 0 & 0 & 28 & 6 & 0.09 & 0.015 & 57 & 1 & 0.0674 \\
\hline 1 & 7 & 1100 & 0.99 & 1.07 & 6235.44409 & 0 & 0 & 28 & 4.99 & 0.072 & 0.014 & 58 & 1 & 0.0536 \\
\hline 1 & 7 & 1120 & 0.8 & 1.17 & 6237.11464 & 0 & 0 & 28 & 4.03 & 0.079 & 0.02 & 59 & 1 & 0.0587 \\
\hline 1 & 7 & 1140 & 0.6 & 1.45 & 6238.36813 & 0 & 0 & 28 & 3.02 & 0.097 & 0.032 & 60 & 1 & 0.07245 \\
\hline 1 & 7 & 1160 & 0.4 & 1.5 & 6239.20694 & 0 & 0 & 28 & 2.02 & 0.101 & 0.05 & 61 & 1 & 0.07515 \\
\hline & 7 & 1180 & 0.2 & 1.12 & 6239.62791 & 0 & 0 & 28 & 1.01 & 0.075 & 0.074 & 62 & 1 & 0.0558 \\
\hline
\end{tabular}

2004.01.30 187-5 wt\%-D [sonicated] DG 500-4000 s-1 run 1.txt

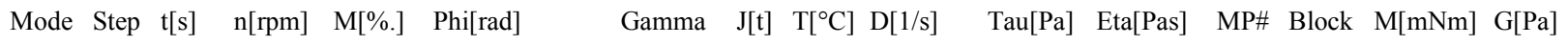

$\begin{array}{rrrrrrrrrrrrrrrr}1 & 1 & 20 & 99.31 & 27.28 & 207.40245 & 0 & 0 & 28 & 500.42 & 1.828 & 0.004 & 1 & 1 & 1.3641 & 0 \\ 1 & 1 & 40 & 198.46 & 35.09 & 621.80444 & 0 & 0 & 28 & 1000.04 & 2.351 & 0.002 & 2 & 1 & 1.75465 & 0 \\ 1 & 1 & 60 & 297.68 & 39.26 & 1243.50285 & 0 & 0 & 28 & 1500.01 & 2.63 & 0.002 & 3 & 1 & 1.96285 & 0 \\ 1 & 1 & 80 & 396.91 & 56.22 & 2072.96656 & 0 & 0 & 28 & 2000.03 & 3.767 & 0.002 & 4 & 1 & 2.811 & 0 \\ 1 & 1 & 100 & 496.13 & 74.21 & 3110.97548 & 0 & 0 & 28 & 2500 & 4.972 & 0.002 & 5 & 1 & 3.71025 & 0 \\ 1 & 1 & 120 & 595.36 & 96.07 & 4355.86691 & 0 & 0 & 28 & 3000.02 & 6.437 & 0.002 & 6 & 1 & 4.8036 & 0 \\ 1 & 1 & 140 & 694.58 & 115.09 & 5808.88807 & 0 & 0 & 28 & 3499.99 & 7.711 & 0.002 & 7 & 1 & 5.7545 & 0 \\ 1 & 1 & 160 & 793.81 & 136.84 & 7469.7248 & 0 & 0 & 28 & 4000.01 & 9.168 & 0.002 & 8 & 1 & 6.842 & 0 \\ 1 & 2 & 180 & 793.81 & 135.72 & 9149.17939 & 0 & 0 & 28 & 4000.01 & 9.093 & 0.002 & 9 & 1 & 6.7858 & 0 \\ 1 & 2 & 200 & 793.81 & 135.21 & 10811.733 & 0 & 0 & 28 & 4000.01 & 9.059 & 0.002 & 10 & 1 & 6.7603 & 0 \\ 1 & 2 & 220 & 793.81 & 134.51 & 12474.69973 & 0 & 0 & 28 & 4000.01 & 9.013 & 0.002 & 11 & 1 & 6.72575 & 0 \\ 1 & 3 & 240 & 793.81 & 134.19 & 14155.12271 & 0 & 0 & 28 & 4000.01 & 8.991 & 0.002 & 12 & 1 & 6.70935 & 0 \\ 1 & 3 & 260 & 694.58 & 111.18 & 15611.15745 & 0 & 0 & 28 & 3499.99 & 7.449 & 0.002 & 13 & 1 & 5.55875 & 0 \\ 1 & 3 & 280 & 595.36 & 89.57 & 16860.64189 & 0 & 0 & 28 & 3000.02 & 6.001 & 0.002 & 14 & 1 & 4.4783 & 0 \\ 1 & 3 & 300 & 496.13 & 69.32 & 17900.19725 & 0 & 0 & 28 & 2500 & 4.645 & 0.002 & 15 & 1 & 3.46615 & 0 \\ 1 & 3 & 320 & 396.91 & 50.46 & 18733.21803 & 0 & 0 & 28 & 2000.03 & 3.381 & 0.002 & 16 & 1 & 2.52285 & 0 \\ 1 & 3 & 340 & 297.68 & 32.26 & 19358.86071 & 0 & 0 & 28 & 1500.01 & 2.161 & 0.001 & 17 & 1 & 1.6128 & 0 \\ 1 & 3 & 360 & 198.46 & 20.01 & 19775.88122 & 0 & 0 & 28 & 1000.04 & 1.341 & 0.001 & 18 & 1 & 1.0005 & 0 \\ 1 & 3 & 380 & 99.23 & 10.21 & 19985.73411 & 0 & 0 & 28 & 500.02 & 0.684 & 0.001 & 19 & 1 & 0.51045 & 0\end{array}$

2004.01.30 187-5 wt\%-D [sonicated] DG 500-4000 s-1 run 2.txt

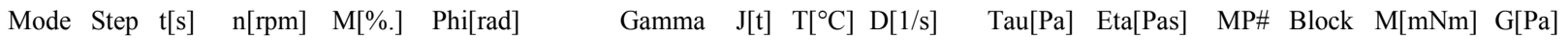




$\begin{array}{rrrrrrrrrrrrrrrr}1 & 1 & 20 & 99.23 & 9.85 & 207.56895 & 0 & 0 & 28 & 500.02 & 0.66 & 0.001 & 1 & 1 & 0.49225 & 0 \\ 1 & 1 & 40 & 198.46 & 21.01 & 621.97016 & 0 & 0 & 28 & 1000.04 & 1.408 & 0.001 & 2 & 1 & 1.05055 & 0 \\ 1 & 1 & 60 & 297.68 & 33.52 & 1243.71962 & 0 & 0 & 28 & 1500.01 & 2.246 & 0.001 & 3 & 1 & 1.6758 & 0 \\ 1 & 1 & 80 & 396.91 & 51.23 & 2073.23595 & 0 & 0 & 28 & 2000.03 & 3.432 & 0.002 & 4 & 1 & 2.56125 & 0 \\ 1 & 1 & 100 & 496.13 & 70.79 & 3110.6731 & 0 & 0 & 28 & 2500 & 4.743 & 0.002 & 5 & 1 & 3.53935 & 0 \\ 1 & 1 & 120 & 595.36 & 91.76 & 4355.92817 & 0 & 0 & 28 & 3000.02 & 6.148 & 0.002 & 6 & 1 & 4.5882 & 0 \\ 1 & 1 & 140 & 694.58 & 110.04 & 5808.27467 & 0 & 0 & 28 & 3499.99 & 7.373 & 0.002 & 7 & 1 & 5.50195 & 0 \\ 1 & 1 & 160 & 793.81 & 134.54 & 7471.13616 & 0 & 0 & 28 & 4000.01 & 9.014 & 0.002 & 8 & 1 & 6.727 & 0 \\ 1 & 2 & 180 & 793.81 & 133.64 & 9151.84582 & 0 & 0 & 28 & 4000.01 & 8.954 & 0.002 & 9 & 1 & 6.6822 & 0 \\ 1 & 2 & 200 & 793.81 & 133.54 & 10815.22881 & 0 & 0 & 28 & 4000.01 & 8.947 & 0.002 & 10 & 1 & 6.67705 & 0 \\ 1 & 2 & 220 & 793.81 & 133.04 & 12476.94754 & 0 & 0 & 28 & 4000.01 & 8.914 & 0.002 & 11 & 1 & 6.65215 & 0 \\ 1 & 3 & 240 & 793.81 & 132.98 & 14157.37288 & 0 & 0 & 28 & 4000.01 & 8.909 & 0.002 & 12 & 1 & 6.6488 & 0 \\ 1 & 3 & 260 & 694.58 & 110.2 & 15613.04083 & 0 & 0 & 28 & 3499.99 & 7.383 & 0.002 & 13 & 1 & 5.5098 & 0 \\ 1 & 3 & 280 & 595.36 & 88.77 & 16862.92897 & 0 & 0 & 28 & 3000.02 & 5.947 & 0.002 & 14 & 1 & 4.43825 & 0 \\ 1 & 3 & 300 & 496.13 & 68.73 & 17902.64691 & 0 & 0 & 28 & 2500 & 4.605 & 0.002 & 15 & 1 & 3.4363 & 0 \\ 1 & 3 & 320 & 396.91 & 49.98 & 18735.91352 & 0 & 0 & 28 & 2000.03 & 3.349 & 0.002 & 16 & 1 & 2.49895 & 0 \\ 1 & 3 & 340 & 297.68 & 31.95 & 19360.98679 & 0 & 0 & 28 & 1500.01 & 2.141 & 0.001 & 17 & 1 & 1.5974 & 0 \\ 1 & 3 & 360 & 198.46 & 19.67 & 19778.6898 & 0 & 0 & 28 & 1000.04 & 1.318 & 0.001 & 18 & 1 & 0.9836 & 0 \\ 1 & 3 & 380 & 99.23 & 10.01 & 19988.23717 & 0 & 0 & 28 & 500.02 & 0.671 & 0.001 & 19 & 1 & 0.50055 & 0\end{array}$

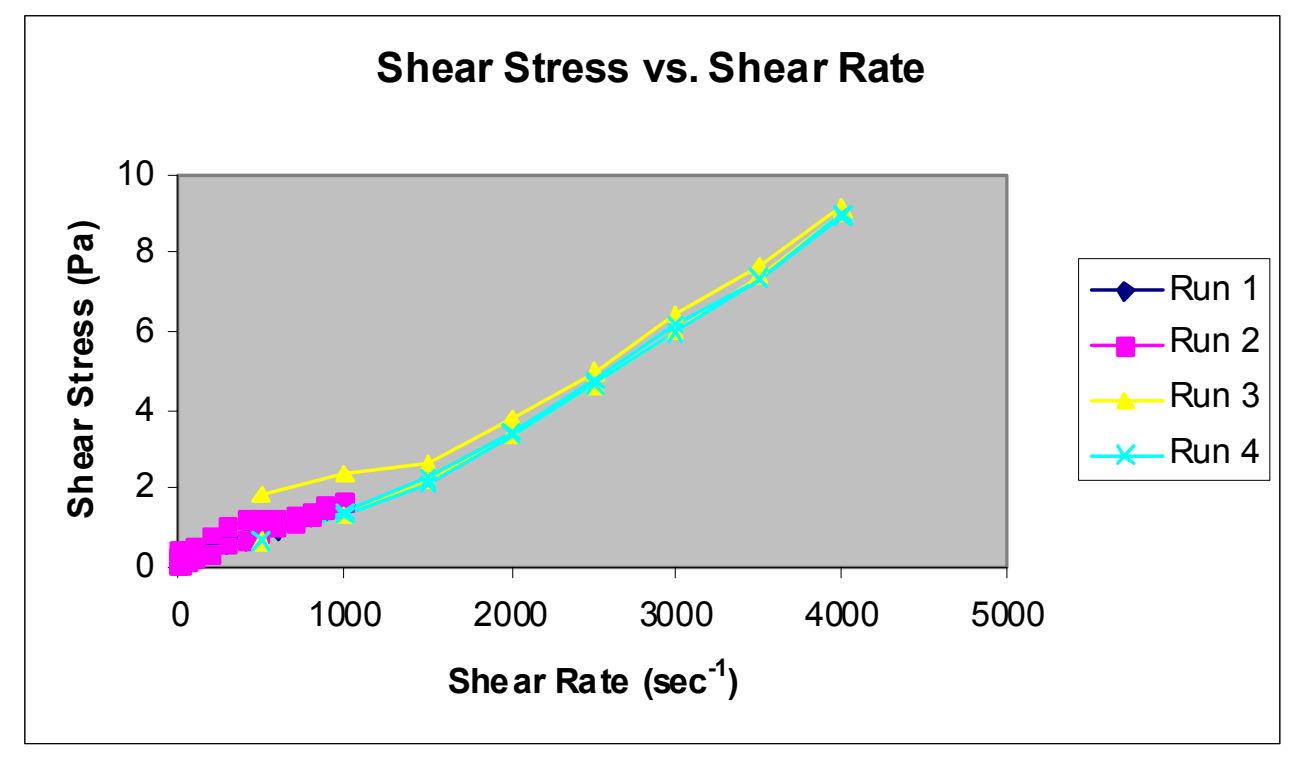




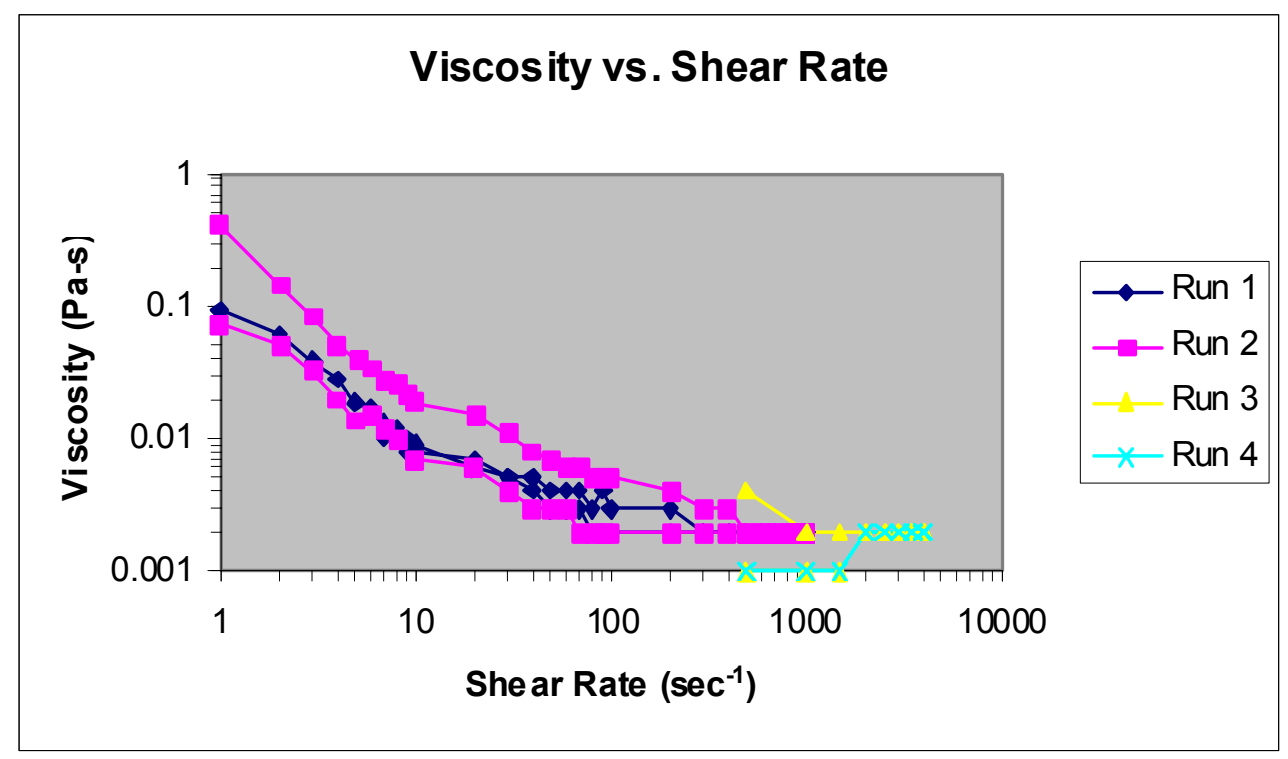

\section{E-2.1.9 180/5wt $\% / U / N$}

2004.02.06 180-5 wt\%-U DG 1-1000 s-1 run 1.txt

Mode Step t[s] n[rpm] M[\%.] Phi[rad]

Gamma J[t $\quad \mathrm{T}\left[{ }^{\circ} \mathrm{C}\right] \quad \mathrm{D}[1 / \mathrm{s}] \quad \mathrm{Tau}[\mathrm{Pa}] \quad \mathrm{Eta}[\mathrm{Pas}] \quad \mathrm{MP} \#$ Block $\mathrm{M}[\mathrm{mNm}] \quad \mathrm{G}[\mathrm{Pa}]$

\begin{tabular}{|c|c|c|c|c|c|c|c|c|c|c|c|c|c|c|}
\hline 1 & 1 & 20 & 0.19 & 28.66 & 0.40369 & 0 & 0 & 28 & 0.96 & 1.92 & 2 & 1 & 1 & 1.4328 \\
\hline 1 & 1 & 40 & 0.4 & 45.57 & 1.24014 & 0 & 0 & 28 & 2.02 & 3.053 & 1.511 & 2 & 1 & 2.27835 \\
\hline 1 & 1 & 60 & 0.6 & 38.19 & 2.48657 & 0 & 0 & 28 & 3.02 & 2.559 & 0.847 & 3 & 1 & 1.90945 \\
\hline 1 & 1 & 80 & 0.8 & 30.86 & 4.1579 & 0 & 0 & 28 & 4.03 & 2.067 & 0.513 & 4 & 1 & 1.5429 \\
\hline 1 & 1 & 100 & 1 & 25.61 & 6.23371 & 0 & 0 & 28 & 5.04 & 1.716 & 0.34 & 5 & 1 & 1.2806 \\
\hline 1 & 1 & 120 & 1.19 & 36.58 & 8.71871 & 0 & 0 & 28 & 6 & 2.451 & 0.409 & 6 & 1 & 1.82895 \\
\hline 1 & 1 & 140 & 1.39 & 38.06 & 11.63882 & 0 & 0 & 28 & 7 & 2.55 & 0.364 & 7 & 1 & 1.90295 \\
\hline 1 & 1 & 160 & 1.59 & 41.65 & 14.97283 & 0 & 0 & 28 & 8.01 & 2.79 & 0.348 & 8 & 1 & 2.08245 \\
\hline 1 & 1 & 180 & 1.8 & 33.5 & 18.72232 & 0 & 0 & 28 & 9.07 & 2.244 & 0.247 & 9 & 1 & 1.67495 \\
\hline 1 & 2 & 200 & 1.99 & 38.98 & 22.88807 & 0 & 0 & 28 & 10.03 & 2.611 & 0.26 & 10 & 1 & 1.94875 \\
\hline 1 & 2 & 220 & 3.97 & 36.48 & 31.16303 & 0 & 0 & 28 & 20 & 2.444 & 0.122 & 11 & 1 & 1.82415 \\
\hline 1 & 2 & 240 & 5.95 & 37.66 & 43.60295 & 0 & 0 & 28 & 29.98 & 2.523 & 0.084 & 12 & 1 & 1.88295 \\
\hline 1 & 2 & 260 & 7.94 & 30.06 & 60.19527 & 0 & 0 & 28 & 40.01 & 2.014 & 0.05 & 13 & 1 & 1.50275 \\
\hline 1 & 2 & 280 & 9.92 & 20.66 & 80.96513 & 0 & 0 & 28 & 49.99 & 1.384 & 0.028 & 14 & 1 & 1.0331 \\
\hline 1 & 2 & 300 & 11.91 & 16.65 & 105.86382 & 0 & 0 & 28 & 60.01 & 1.116 & 0.019 & 15 & 1 & 0.8326 \\
\hline 1 & 2 & 320 & 13.89 & 15.47 & 134.91805 & 0 & 0 & 28 & 69.99 & 1.037 & 0.015 & 16 & 1 & 0.77355 \\
\hline 1 & 2 & 340 & 15.88 & 13.71 & 168.15296 & 0 & 0 & 28 & 80.02 & 0.918 & 0.011 & 17 & 1 & 0.68535 \\
\hline 1 & 2 & 360 & 17.86 & 11.48 & 205.51985 & 0 & 0 & 28 & 90 & 0.769 & 0.009 & 18 & 1 & 0.5738 \\
\hline 1 & 3 & 380 & 19.85 & 10.53 & 247.44912 & 0 & 0 & 28 & 100.02 & 0.706 & 0.007 & 19 & 1 & 0.5265 \\
\hline 1 & 3 & 400 & 39.69 & 14.66 & 330.3047 & 0 & 0 & 28 & 200 & 0.982 & 0.005 & 20 & 1 & 0.7329 \\
\hline 1 & 3 & 420 & 59.54 & 16.76 & 454.58767 & 0 & 0 & 28 & 300.02 & 1.123 & 0.004 & 21 & 1 & 0.83805 \\
\hline 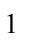 & 3 & 440 & 79.38 & 19.94 & 620.74415 & 0 & 0 & 28 & 400 & 1.336 & 0.003 & 22 & 1 & 0.9969 \\
\hline 1 & 3 & 460 & 99.23 & 23.9 & 828.09869 & 0 & 0 & 28 & 500.02 & 1.601 & 0.003 & 23 & 1 & 1.1951 \\
\hline 1 & 3 & 480 & 119.07 & 28.1 & 1077.10918 & 0 & 0 & 28 & 599.99 & 1.882 & 0.003 & 24 & 1 & 1.40475 \\
\hline 1 & 3 & 500 & 138.92 & 31.65 & 1367.95233 & 0 & 0 & 28 & 700.02 & 2.121 & 0.003 & 25 & 1 & 1.5826 \\
\hline 1 & 3 & 520 & 158.76 & 35.53 & 1699.79483 & 0 & 0 & 28 & 799.99 & 2.38 & 0.003 & 26 & 1 & 1.77645 \\
\hline 1 & 3 & 540 & 178.61 & 39.88 & 2073.54383 & 0 & 0 & 28 & 900.02 & 2.672 & 0.003 & 27 & 1 & 1.99385 \\
\hline 1 & 3 & 560 & 198.45 & 44.16 & 2489.27078 & 0 & 0 & 28 & 999.99 & 2.958 & 0.003 & 28 & 1 & 2.20775 \\
\hline
\end{tabular}




\begin{tabular}{|c|c|c|c|c|c|c|c|c|c|c|c|c|c|c|}
\hline 1 & 4 & 570 & 198.45 & 43.92 & 2701.26467 & 0 & 0 & 28 & 999.99 & 2.942 & 0.003 & 29 & 1 & 2.19575 \\
\hline 1 & 4 & 580 & 198.45 & 43.73 & 2908.97657 & 0 & 0 & 28 & 999.99 & 2.93 & 0.003 & 30 & 1 & 2.1866 \\
\hline 1 & 4 & 590 & 198.45 & 43.69 & 3116.68846 & 0 & 0 & 28 & 999.99 & 2.927 & 0.003 & 31 & 1 & 2.18435 \\
\hline 1 & 4 & 600 & 198.45 & 43.58 & 3324.92108 & 0 & 0 & 28 & 999.99 & 2.92 & 0.003 & 32 & 1 & 2.1791 \\
\hline & 4 & 610 & 198.45 & 43.53 & 3532.63297 & 0 & 0 & 28 & 999.99 & 2.916 & 0.003 & 33 & 1 & 2.17625 \\
\hline & 4 & 620 & 198.45 & 43.47 & 3740.55379 & 0 & 0 & 28 & 999.99 & 2.913 & 0.003 & 34 & 1 & 2.1736 \\
\hline & 5 & 640 & 198.45 & 43.4 & 4160.34282 & 0 & 0 & 28 & 999.99 & 2.908 & 0.003 & 35 & 1 & 2.1701 \\
\hline & 5 & 660 & 178.61 & 38.75 & 4534.73506 & 0 & 0 & 28 & 900.02 & 2.596 & 0.003 & 36 & 1 & 1.93755 \\
\hline & 5 & 680 & 158.76 & 34.18 & 4867.64885 & 0 & 0 & 28 & 799.99 & 2.29 & 0.003 & 37 & 1 & 1.7088 \\
\hline & 5 & 700 & 138.92 & 30.1 & 5159.12503 & 0 & 0 & 28 & 700.02 & 2.017 & 0.003 & 38 & 1 & 1.5052 \\
\hline & 5 & 720 & 119.07 & 25.76 & 5408.75833 & 0 & 0 & 28 & 599.99 & 1.726 & 0.003 & 39 & 1 & 1.2882 \\
\hline & 5 & 740 & 99.23 & 21.81 & 5616.84015 & 0 & 0 & 28 & 500.02 & 1.461 & 0.003 & 40 & 1 & 1.0906 \\
\hline & 5 & 760 & 79.38 & 17.46 & 5783.49458 & 0 & 0 & 28 & 400 & 1.17 & 0.003 & 41 & 1 & 0.87285 \\
\hline & 5 & 780 & 59.54 & 13.29 & 5908.43179 & 0 & 0 & 28 & 300.02 & 0.89 & 0.003 & 42 & 1 & 0.6645 \\
\hline & 5 & 800 & 39.69 & 8.88 & 5991.9416 & 0 & 0 & 28 & 200 & 0.595 & 0.003 & 43 & 1 & 0.44405 \\
\hline & 5 & 820 & 19.85 & 4.84 & 6033.8261 & 0 & 0 & 28 & 100.02 & 0.324 & 0.003 & 44 & 1 & 0.24205 \\
\hline 1 & 6 & 840 & 17.86 & 5.13 & 6071.50479 & 0 & 0 & 28 & 90 & 0.344 & 0.004 & 45 & 1 & 0.2565 \\
\hline 1 & 6 & 860 & 15.88 & 5 & 6104.79704 & 0 & 0 & 28 & 80.02 & 0.335 & 0.004 & 46 & 1 & 0.2499 \\
\hline 1 & 6 & 880 & 13.89 & 5.31 & 6133.89525 & 0 & 0 & 28 & 69.99 & 0.356 & 0.005 & 47 & 1 & 0.2655 \\
\hline 1 & 6 & 900 & 11.9 & 5.13 & 6158.85835 & 0 & 0 & 28 & 59.96 & 0.344 & 0.006 & 48 & 1 & 0.25645 \\
\hline 1 & 6 & 920 & 9.92 & 4.91 & 6179.67925 & 0 & 0 & 28 & 49.99 & 0.329 & 0.007 & 49 & 1 & 0.24525 \\
\hline 1 & 6 & 940 & 7.93 & 4.86 & 6196.32184 & 0 & 0 & 28 & 39.96 & 0.326 & 0.008 & 50 & 1 & 0.243 \\
\hline 1 & 6 & 960 & 5.95 & 5.04 & 6208.81203 & 0 & 0 & 28 & 29.98 & 0.337 & 0.011 & 51 & 1 & 0.25175 \\
\hline 1 & 6 & 980 & 3.96 & 5.53 & 6217.13882 & 0 & 0 & 28 & 19.95 & 0.371 & 0.019 & 52 & 1 & 0.27665 \\
\hline 1 & 6 & 1000 & 1.98 & 3.77 & 6221.314 & 0 & 0 & 28 & 9.98 & 0.253 & 0.025 & 53 & 1 & 0.18845 \\
\hline 1 & 7 & 1020 & 1.79 & 5.14 & 6225.08626 & 0 & 0 & 28.1 & 9.02 & 0.344 & 0.038 & 54 & 1 & 0.25675 \\
\hline 1 & 7 & 1040 & 1.59 & 5.35 & 6228.42263 & 0 & 0 & 28 & 8.01 & 0.358 & 0.045 & 55 & 1 & 0.26745 \\
\hline 1 & 7 & 1060 & 1.39 & 5.03 & 6231.34275 & 0 & 0 & 28 & 7 & 0.337 & 0.048 & 56 & 1 & 0.25165 \\
\hline 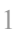 & 7 & 1080 & 1.19 & 4.84 & 6233.84581 & 0 & 0 & 28 & 6 & 0.324 & 0.054 & 57 & 1 & 0.24195 \\
\hline 1 & 7 & 1100 & 0.99 & 7.16 & 6235.92869 & 0 & 0 & 28 & 4.99 & 0.48 & 0.096 & 58 & 1 & 0.3582 \\
\hline 1 & 7 & 1120 & 0.8 & 5.52 & 6237.60158 & 0 & 0 & 28 & 4.03 & 0.37 & 0.092 & 59 & 1 & 0.2759 \\
\hline 1 & 7 & 1140 & 0.6 & 5.23 & 6238.85429 & 0 & 0 & 28 & 3.02 & 0.351 & 0.116 & 60 & 1 & 0.26165 \\
\hline & 7 & 1160 & 0.4 & 7.04 & 6239.69074 & 0 & 0 & 28 & 2.02 & 0.472 & 0.234 & 61 & 1 & 0.352 \\
\hline & 7 & 1180 & 0.2 & 9.87 & 6240.11093 & 0 & 0 & 28 & 1.01 & 0.661 & 0.654 & 62 & 1 & 0.49325 \\
\hline
\end{tabular}

2004.02.06 180-5 wt\%-U DG 1-1000 s-1 run 2.txt

Mode Step $\mathrm{t}[\mathrm{s}] \quad \mathrm{n}[\mathrm{rpm}] \quad \mathrm{M}[\%$.] $\quad \mathrm{Phi}[\mathrm{rad}]$

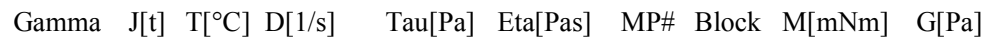

$\begin{array}{rrrrrrrrrrrrrrrr}1 & 1 & 20 & 0.2 & 11.97 & 0.4139 & 0 & 0 & 28.1 & 1.01 & 0.802 & 0.794 & 1 & 1 & 0.59865 & 0 \\ 1 & 1 & 40 & 0.4 & 10.23 & 1.24486 & 0 & 0 & 28 & 2.02 & 0.685 & 0.339 & 2 & 1 & 0.51135 & 0 \\ 1 & 1 & 60 & 0.6 & 9.53 & 2.48814 & 0 & 0 & 28 & 3.02 & 0.638 & 0.211 & 3 & 1 & 0.47635 & 0 \\ 1 & 1 & 80 & 0.8 & 5.8 & 4.16104 & 0 & 0 & 28 & 4.03 & 0.388 & 0.096 & 4 & 1 & 0.2898 & 0 \\ 1 & 1 & 100 & 1 & 6.67 & 6.24077 & 0 & 0 & 28 & 5.04 & 0.447 & 0.089 & 5 & 1 & 0.3334 & 0 \\ 1 & 1 & 120 & 1.19 & 8.34 & 8.7407 & 0 & 0 & 28 & 6 & 0.559 & 0.093 & 6 & 1 & 0.4172 & 0 \\ 1 & 1 & 140 & 1.39 & 5.64 & 11.65374 & 0 & 0 & 28 & 7 & 0.378 & 0.054 & 7 & 1 & 0.28195 & 0 \\ 1 & 1 & 160 & 1.59 & 7.62 & 14.98304 & 0 & 0 & 28 & 8.01 & 0.51 & 0.064 & 8 & 1 & 0.38075 & 0 \\ 1 & 1 & 180 & 1.79 & 5.18 & 18.72703 & 0 & 0 & 28 & 9.02 & 0.347 & 0.038 & 9 & 1 & 0.25915 & 0 \\ 1 & 2 & 200 & 1.98 & 6.87 & 22.90143 & 0 & 0 & 28.1 & 9.98 & 0.46 & 0.046 & 10 & 1 & 0.3436 & 0 \\ 1 & 2 & 220 & 3.97 & 6.07 & 31.18031 & 0 & 0 & 28 & 20 & 0.407 & 0.02 & 11 & 1 & 0.3035 & 0 \\ 1 & 2 & 240 & 5.95 & 6.23 & 43.62337 & 0 & 0 & 28 & 29.98 & 0.418 & 0.014 & 12 & 1 & 0.31165 & 0 \\ 1 & 2 & 260 & 7.94 & 5.91 & 60.20548 & 0 & 0 & 28 & 40.01 & 0.396 & 0.01 & 13 & 1 & 0.2953 & 0\end{array}$




\begin{tabular}{|c|c|c|c|c|c|c|c|c|c|c|c|c|c|c|}
\hline 1 & 2 & 280 & 9.92 & 5.56 & 80.94471 & 0 & 0 & 28 & 49.99 & 0.373 & 0.007 & 14 & 1 & 0.27805 \\
\hline 1 & 2 & 300 & 11.91 & 5.96 & 105.86932 & 0 & 0 & 28 & 60.01 & 0.4 & 0.007 & 15 & 1 & 0.29815 \\
\hline 1 & 2 & 320 & 13.89 & 5.93 & 134.94633 & 0 & 0 & 28 & 69.99 & 0.397 & 0.006 & 16 & 1 & 0.29645 \\
\hline 1 & 2 & 340 & 15.88 & 5.99 & 168.1404 & 0 & 0 & 28 & 80.02 & 0.401 & 0.005 & 17 & 1 & 0.2995 \\
\hline 1 & 2 & 360 & 17.86 & 6.29 & 205.52692 & 0 & 0 & 28 & 90 & 0.421 & 0.005 & 18 & 1 & 0.31455 \\
\hline 1 & 3 & 380 & 19.85 & 6.65 & 247.45697 & 0 & 0 & 28 & 100.02 & 0.446 & 0.004 & 19 & 1 & 0.3325 \\
\hline 1 & 3 & 400 & 39.69 & 11.3 & 330.23951 & 0 & 0 & 28 & 200 & 0.757 & 0.004 & 20 & 1 & 0.56475 \\
\hline 1 & 3 & 420 & 59.54 & 15.03 & 454.70941 & 0 & 0 & 28 & 300.02 & 1.007 & 0.003 & 21 & 1 & 0.75155 \\
\hline 1 & 3 & 440 & 79.38 & 18.69 & 620.65776 & 0 & 0 & 28.1 & 400 & 1.252 & 0.003 & 22 & 1 & 0.9347 \\
\hline 1 & 3 & 460 & 99.23 & 22.74 & 828.07513 & 0 & 0 & 28.1 & 500.02 & 1.524 & 0.003 & 23 & 1 & 1.13715 \\
\hline 1 & 3 & 480 & 119.07 & 26.55 & 1077.33459 & 0 & 0 & 28.1 & 599.99 & 1.779 & 0.003 & 24 & 1 & 1.32745 \\
\hline 1 & 3 & 500 & 138.92 & 30.72 & 1367.67901 & 0 & 0 & 28.1 & 700.02 & 2.058 & 0.003 & 25 & 1 & 1.53615 \\
\hline 1 & 3 & 520 & 158.76 & 34.63 & 1700.19774 & 0 & 0 & 28.1 & 799.99 & 2.32 & 0.003 & 26 & 1 & 1.73155 \\
\hline 1 & 3 & 540 & 178.61 & 39.08 & 2073.58231 & 0 & 0 & 28 & 900.02 & 2.618 & 0.003 & 27 & 1 & 1.95375 \\
\hline 1 & 3 & 560 & 198.45 & 43.4 & 2489.30848 & 0 & 0 & 28 & 999.99 & 2.908 & 0.003 & 28 & 1 & 2.1702 \\
\hline 1 & 4 & 570 & 198.45 & 43.22 & 2701.1987 & 0 & 0 & 28 & 999.99 & 2.895 & 0.003 & 29 & 1 & 2.1608 \\
\hline 1 & 4 & 580 & 198.45 & 43.11 & 2908.91138 & 0 & 0 & 28.1 & 999.99 & 2.888 & 0.003 & 30 & 1 & 2.15555 \\
\hline 1 & 4 & 590 & 198.45 & 43.16 & 3117.2461 & 0 & 0 & 28.1 & 999.99 & 2.892 & 0.003 & 31 & 1 & 2.158 \\
\hline 1 & 4 & 600 & 198.45 & 43.07 & 3324.75143 & 0 & 0 & 28.1 & 999.99 & 2.885 & 0.003 & 32 & 1 & 2.15325 \\
\hline 1 & 4 & 610 & 198.45 & 42.97 & 3532.46411 & 0 & 0 & 28.1 & 999.99 & 2.879 & 0.003 & 33 & 1 & 2.1485 \\
\hline 1 & 4 & 620 & 198.45 & 42.94 & 3740.28047 & 0 & 0 & 28.1 & 999.99 & 2.877 & 0.003 & 34 & 1 & 2.1469 \\
\hline 1 & 5 & 640 & 198.45 & 42.93 & 4160.17318 & 0 & 0 & 28.1 & 999.99 & 2.877 & 0.003 & 35 & 1 & 2.14665 \\
\hline 1 & 5 & 660 & 178.61 & 38.41 & 4534.76333 & 0 & 0 & 28.1 & 900.02 & 2.573 & 0.003 & 36 & 1 & 1.9205 \\
\hline 1 & 5 & 680 & 158.76 & 33.89 & 4867.59387 & 0 & 0 & 28.1 & 799.99 & 2.27 & 0.003 & 37 & 1 & 1.6944 \\
\hline 1 & 5 & 700 & 138.92 & 29.84 & 5159.07005 & 0 & 0 & 28.1 & 700.02 & 1.999 & 0.003 & 38 & 1 & 1.4919 \\
\hline 1 & 5 & 720 & 119.07 & 25.53 & 5408.57848 & 0 & 0 & 28 & 599.99 & 1.71 & 0.003 & 39 & 1 & 1.2764 \\
\hline 1 & 5 & 740 & 99.23 & 21.49 & 5616.61867 & 0 & 0 & 28 & 500.02 & 1.44 & 0.003 & 40 & 1 & 1.0744 \\
\hline 1 & 5 & 760 & 79.38 & 17.19 & 5783.38776 & 0 & 0 & 28 & 400 & 1.152 & 0.003 & 41 & 1 & 0.8595 \\
\hline 1 & 5 & 780 & 59.54 & 13.11 & 5908.27314 & 0 & 0 & 28 & 300.02 & 0.878 & 0.003 & 42 & 1 & 0.6554 \\
\hline 1 & 5 & 800 & 39.69 & 8.76 & 5991.75075 & 0 & 0 & 28.1 & 200 & 0.587 & 0.003 & 43 & 1 & 0.43805 \\
\hline 1 & 5 & 820 & 19.85 & 4.75 & 6033.63604 & 0 & 0 & 28.1 & 100.02 & 0.319 & 0.003 & 44 & 1 & 0.2377 \\
\hline 1 & 6 & 840 & 17.86 & 4.76 & 6071.29745 & 0 & 0 & 28 & 90 & 0.319 & 0.004 & 45 & 1 & 0.23815 \\
\hline 1 & 6 & 860 & 15.88 & 4.57 & 6104.59205 & 0 & 0 & 28 & 80.02 & 0.306 & 0.004 & 46 & 1 & 0.22865 \\
\hline 1 & 6 & 880 & 13.89 & 4.61 & 6133.71932 & 0 & 0 & 28 & 69.99 & 0.309 & 0.004 & 47 & 1 & 0.2304 \\
\hline 1 & 6 & 900 & 11.9 & 4.45 & 6158.67614 & 0 & 0 & 28 & 59.96 & 0.298 & 0.005 & 48 & 1 & 0.2225 \\
\hline 1 & 6 & 920 & 9.92 & 3.98 & 6179.49154 & 0 & 0 & 28 & 49.99 & 0.267 & 0.005 & 49 & 1 & 0.19895 \\
\hline 1 & 6 & 940 & 7.93 & 3.77 & 6196.13963 & 0 & 0 & 28 & 39.96 & 0.253 & 0.006 & 50 & 1 & 0.1886 \\
\hline 1 & 6 & 960 & 5.95 & 3.73 & 6208.6306 & 0 & 0 & 28 & 29.98 & 0.25 & 0.008 & 51 & 1 & 0.18635 \\
\hline 1 & 6 & 980 & 3.96 & 3.62 & 6216.95425 & 0 & 0 & 28 & 19.95 & 0.242 & 0.012 & 52 & 1 & 0.1808 \\
\hline 1 & 6 & 1000 & 1.98 & 2.76 & 6221.13178 & 0 & 0 & 28.1 & 9.98 & 0.185 & 0.019 & 53 & 1 & 0.13785 \\
\hline 1 & 7 & 1020 & 1.79 & 3.14 & 6224.90327 & 0 & 0 & 28 & 9.02 & 0.21 & 0.023 & 54 & 1 & 0.15695 \\
\hline 1 & 7 & 1040 & 1.59 & 3.64 & 6228.23728 & 0 & 0 & 28 & 8.01 & 0.244 & 0.03 & 55 & 1 & 0.182 \\
\hline 1 & 7 & 1060 & 1.39 & 2.91 & 6231.15739 & 0 & 0 & 28 & 7 & 0.195 & 0.028 & 56 & 1 & 0.1455 \\
\hline 1 & 7 & 1080 & 1.19 & 3.76 & 6233.66046 & 0 & 0 & 28 & 6 & 0.252 & 0.042 & 57 & 1 & 0.18805 \\
\hline 1 & 7 & 1100 & 0.99 & 4.93 & 6235.74726 & 0 & 0 & 28 & 4.99 & 0.331 & 0.066 & 58 & 1 & 0.2467 \\
\hline 1 & 7 & 1120 & 0.8 & 2.8 & 6237.41623 & 0 & 0 & 28 & 4.03 & 0.188 & 0.047 & 59 & 1 & 0.13995 \\
\hline 1 & 7 & 1140 & 0.6 & 3.89 & 6238.67051 & 0 & 0 & 28 & 3.02 & 0.26 & 0.086 & 60 & 1 & 0.19425 \\
\hline 1 & 7 & 1160 & 0.4 & 5.56 & 6239.50774 & 0 & 0 & 28 & 2.02 & 0.373 & 0.185 & 61 & 1 & 0.2782 \\
\hline 1 & 7 & 1180 & 0.2 & 7.77 & 6239.92793 & 0 & 0 & 28 & 1.01 & 0.52 & 0.515 & 62 & 1 & 0.38825 \\
\hline
\end{tabular}


2004.02.06 180-5 wt\%-U DG 500-4000 s-1 run 1.txt Mode Step t[s] n[rpm] M[\%.] Phi[rad]

Gamma $\mathrm{J}[\mathrm{t}] \quad \mathrm{T}\left[{ }^{\circ} \mathrm{C}\right] \quad \mathrm{D}[1 / \mathrm{s}] \quad \mathrm{Tau}[\mathrm{Pa}] \quad$ Eta[Pas $] \quad$ MP\# Block $\mathrm{M}[\mathrm{mNm}] \quad \mathrm{G}[\mathrm{Pa}]$

$\begin{array}{rrrrrrrrrrrrrrrr}1 & 1 & 20 & 99.3 & 25.14 & 207.65456 & 0 & 0 & 28 & 500.37 & 1.684 & 0.003 & 1 & 1 & 1.25685 & 0 \\ 1 & 1 & 40 & 198.46 & 44.64 & 621.53348 & 0 & 0 & 28 & 1000.04 & 2.991 & 0.003 & 2 & 1 & 2.2322 & 0 \\ 1 & 1 & 60 & 297.68 & 66.16 & 1243.59395 & 0 & 0 & 28 & 1500.01 & 4.432 & 0.003 & 3 & 1 & 3.3078 & 0 \\ 1 & 1 & 80 & 396.91 & 87.79 & 2073.88783 & 0 & 0 & 28 & 2000.03 & 5.882 & 0.003 & 4 & 1 & 4.38965 & 0 \\ 1 & 1 & 100 & 496.13 & 111.56 & 3110.54429 & 0 & 0 & 28 & 2500 & 7.475 & 0.003 & 5 & 1 & 5.578 & 0 \\ 1 & 1 & 120 & 595.36 & 145.01 & 4356.10803 & 0 & 0 & 28 & 3000.02 & 9.716 & 0.003 & 6 & 1 & 7.2504 & 0 \\ 1 & 1 & 140 & 694.58 & 180.1 & 5808.39877 & 0 & 0 & 28 & 3499.99 & 12.067 & 0.003 & 7 & 1 & 9.00495 & 0 \\ 1 & 1 & 160 & 793.81 & 219.69 & 7470.06566 & 0 & 0 & 28 & 4000.01 & 14.719 & 0.004 & 8 & 1 & 10.9845 & 0 \\ 1 & 2 & 180 & 793.81 & 219.57 & 9149.91609 & 0 & 0 & 28 & 4000.01 & 14.711 & 0.004 & 9 & 1 & 10.97865 & 0 \\ 1 & 2 & 200 & 793.81 & 216.99 & 10812.47127 & 0 & 0 & 28 & 4000.01 & 14.539 & 0.004 & 10 & 1 & 10.8497 & 0 \\ 1 & 2 & 220 & 793.81 & 216.56 & 12474.60548 & 0 & 0 & 28 & 4000.01 & 14.51 & 0.004 & 11 & 1 & 10.82805 & 0 \\ 1 & 3 & 240 & 793.81 & 216.9 & 14153.7844 & 0 & 0 & 28 & 4000.01 & 14.533 & 0.004 & 12 & 1 & 10.8452 & 0 \\ 1 & 3 & 260 & 694.58 & 179.28 & 15610.18198 & 0 & 0 & 28 & 3499.99 & 12.012 & 0.003 & 13 & 1 & 8.96405 & 0 \\ 1 & 3 & 280 & 595.36 & 141.11 & 16858.76164 & 0 & 0 & 28 & 3000.02 & 9.454 & 0.003 & 14 & 1 & 7.05555 & 0 \\ 1 & 3 & 300 & 496.13 & 102.6 & 17900.87741 & 0 & 0 & 28 & 2500 & 6.874 & 0.003 & 15 & 1 & 5.13005 & 0 \\ 1 & 3 & 320 & 396.91 & 80.16 & 18732.86853 & 0 & 0 & 28 & 2000.03 & 5.371 & 0.003 & 16 & 1 & 4.0081 & 0 \\ 1 & 3 & 340 & 297.68 & 59.57 & 19358.06118 & 0 & 0 & 28 & 1500.01 & 3.991 & 0.003 & 17 & 1 & 2.97855 & 0 \\ 1 & 3 & 360 & 198.46 & 39.17 & 19775.41548 & 0 & 0 & 28 & 1000.04 & 2.624 & 0.003 & 18 & 1 & 1.9583 & 0 \\ 1 & 3 & 380 & 99.23 & 19.68 & 19985.13878 & 0 & 0 & 28 & 500.02 & 1.318 & 0.003 & 19 & 1 & 0.98375 & 0\end{array}$

2004.02.06 180-5 wt\%-U DG 500-4000 s-1 run 2.txt

Mode Step t[s] n[rpm] M[\%.] Phi[rad]

$\begin{array}{lllllll}\text { Gamma } & J[\mathrm{t}] & \mathrm{T}\left[{ }^{\circ} \mathrm{C}\right] & \mathrm{D}[1 / \mathrm{s}] \quad \mathrm{Tau}[\mathrm{Pa}] & \mathrm{Eta}[\mathrm{Pas}] & \mathrm{MP} \# \text { Block } & \mathrm{M}[\mathrm{mNm}] \quad \mathrm{G}[\mathrm{Pa}]\end{array}$

$\begin{array}{rrrrrrrrrrrrrrrr}1 & 1 & 20 & 99.3 & 19.44 & 207.61058 & 0 & 0 & 28 & 500.37 & 1.302 & 0.003 & 1 & 1 & 0.97185 & 0 \\ 1 & 1 & 40 & 198.46 & 39.95 & 622.06126 & 0 & 0 & 28 & 1000.04 & 2.677 & 0.003 & 2 & 1 & 1.9974 & 0 \\ 1 & 1 & 60 & 297.68 & 60.71 & 1244.12174 & 0 & 0 & 28 & 1500.01 & 4.067 & 0.003 & 3 & 1 & 3.03535 & 0 \\ 1 & 1 & 80 & 396.91 & 81.61 & 2072.80634 & 0 & 0 & 28 & 2000.03 & 5.468 & 0.003 & 4 & 1 & 4.08035 & 0 \\ 1 & 1 & 100 & 496.13 & 105.03 & 3110.55293 & 0 & 0 & 28 & 2500 & 7.037 & 0.003 & 5 & 1 & 5.25165 & 0 \\ 1 & 1 & 120 & 595.36 & 142.28 & 4356.37585 & 0 & 0 & 28 & 3000.02 & 9.532 & 0.003 & 6 & 1 & 7.11375 & 0 \\ 1 & 1 & 140 & 694.58 & 179.35 & 5808.66659 & 0 & 0 & 28 & 3499.99 & 12.016 & 0.003 & 7 & 1 & 8.96725 & 0 \\ 1 & 1 & 160 & 793.81 & 217.67 & 7470.74817 & 0 & 0 & 28 & 4000.01 & 14.584 & 0.004 & 8 & 1 & 10.88345 & 0 \\ 1 & 2 & 180 & 793.81 & 217.43 & 9151.84817 & 0 & 0 & 28 & 4000.01 & 14.568 & 0.004 & 9 & 1 & 10.8716 & 0 \\ 1 & 2 & 200 & 793.81 & 215.63 & 10814.39942 & 0 & 0 & 28 & 4000.01 & 14.447 & 0.004 & 10 & 1 & 10.78145 & 0 \\ 1 & 2 & 220 & 793.81 & 215.05 & 12476.95303 & 0 & 0 & 28 & 4000.01 & 14.408 & 0.004 & 11 & 1 & 10.75245 & 0 \\ 1 & 3 & 240 & 793.81 & 215.3 & 14156.12881 & 0 & 0 & 28 & 4000.01 & 14.425 & 0.004 & 12 & 1 & 10.7652 & 0 \\ 1 & 3 & 260 & 694.58 & 178.32 & 15613.98174 & 0 & 0 & 28 & 3499.99 & 11.948 & 0.003 & 13 & 1 & 8.9162 & 0 \\ 1 & 3 & 280 & 595.36 & 140.65 & 16861.05736 & 0 & 0 & 28 & 3000.02 & 9.423 & 0.003 & 14 & 1 & 7.03245 & 0 \\ 1 & 3 & 300 & 496.13 & 102.69 & 17902.39087 & 0 & 0 & 28 & 2500 & 6.88 & 0.003 & 15 & 1 & 5.1345 & 0 \\ 1 & 3 & 320 & 396.91 & 79.6 & 18735.83891 & 0 & 0 & 28 & 2000.03 & 5.333 & 0.003 & 16 & 1 & 3.97975 & 0 \\ 1 & 3 & 340 & 297.68 & 59.17 & 19361.02606 & 0 & 0 & 28 & 1500.01 & 3.965 & 0.003 & 17 & 1 & 2.9587 & 0 \\ 1 & 3 & 360 & 198.46 & 38.94 & 19778.01593 & 0 & 0 & 28 & 1000.04 & 2.609 & 0.003 & 18 & 1 & 1.947 & 0 \\ 1 & 3 & 380 & 99.23 & 19.58 & 19987.73766 & 0 & 0 & 28 & 500.02 & 1.312 & 0.003 & 19 & 1 & 0.9789 & 0\end{array}$



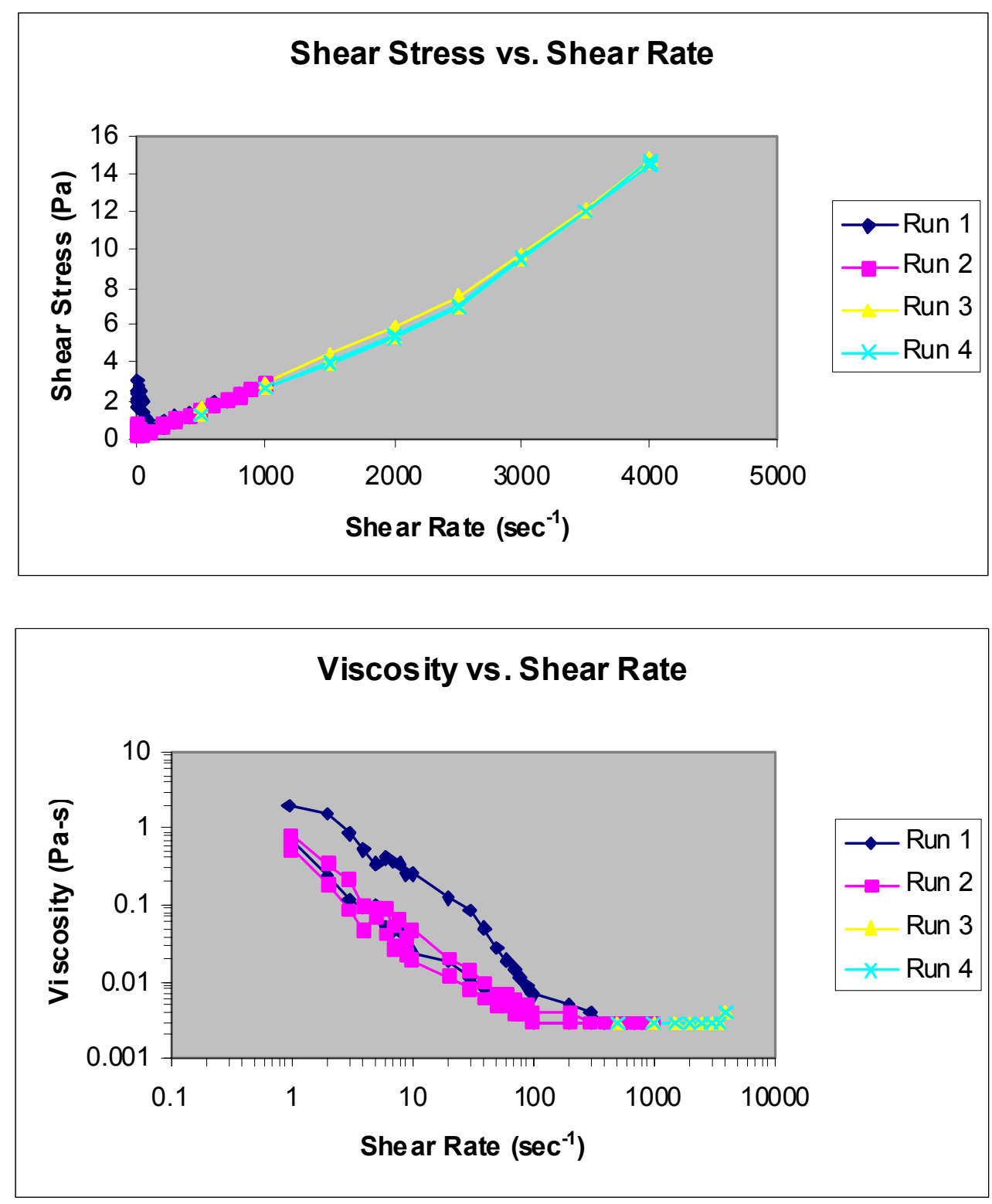

\section{E-2.1.10 180/5wt $\% / \mathrm{U} / \mathrm{S}$}

2004.02.05 180-5 wt\%-U [sonicated] DG 1-1000 s-1 run 1.txt

Mode Step t[s] n[rpm] M[\%.] Phi[rad]

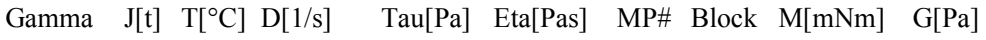

$\begin{array}{rrrrrrrrrrrrrrr}20 & 0.2 & 5.08 & 0.41548 & 0 & 0 & 28 & 1.01 & 0.34 & 0.337 & 1 & 1 & 0.25385 & 0 \\ 40 & 0.4 & 3.29 & 1.248 & 0 & 0 & 28 & 2.02 & 0.22 & 0.109 & 2 & 1 & 0.1645 & 0 \\ 60 & 0.6 & 3.23 & 2.496 & 0 & 0 & 28 & 3.02 & 0.216 & 0.072 & 3 & 1 & 0.1615 & 0 \\ 80 & 0.8 & 3.42 & 4.16104 & 0 & 0 & 28 & 4.03 & 0.229 & 0.057 & 4 & 1 & 0.1708 & 0 \\ 100 & 1 & 3.34 & 6.24234 & 0 & 0 & 28 & 5.04 & 0.224 & 0.044 & 5 & 1 & 0.1671 & 0 \\ 120 & 1.19 & 3.44 & 8.73991 & 0 & 0 & 28 & 6 & 0.231 & 0.039 & 6 & 1 & 0.1722 & 0 \\ 140 & 1.39 & 3.43 & 11.65374 & 0 & 0 & 28 & 7 & 0.229 & 0.033 & 7 & 1 & 0.17125 & 0 \\ 160 & 1.59 & 3.4 & 14.9854 & 0 & 0 & 28 & 8.01 & 0.228 & 0.028 & 8 & 1 & 0.17 & 0 \\ 180 & 1.79 & 3.54 & 18.73096 & 0 & 0 & 28 & 9.02 & 0.237 & 0.026 & 9 & 1 & 0.1772 & 0 \\ 200 & 1.98 & 3.75 & 22.90692 & 0 & 0 & 28 & 9.98 & 0.251 & 0.025 & 10 & 1 & 0.18735 & 0\end{array}$




\begin{tabular}{|c|c|c|c|c|c|c|c|c|c|c|c|c|c|}
\hline 1 & 220 & 3.97 & 6.47 & 31.18345 & 0 & 0 & 28 & 20 & 0.433 & 0.022 & 11 & 1 & 0.32335 \\
\hline 1 & 240 & 5.95 & 7.93 & 43.6218 & 0 & 0 & 28 & 29.98 & 0.532 & 0.018 & 12 & 1 & 0.39665 \\
\hline 1 & 260 & 7.94 & 8.93 & 60.19998 & 0 & 0 & 28 & 40.01 & 0.598 & 0.015 & 13 & 1 & 0.4464 \\
\hline 1 & 280 & 9.92 & 10.05 & 80.95884 & 0 & 0 & 28 & 49.99 & 0.673 & 0.013 & 14 & 1 & 0.5023 \\
\hline 1 & 300 & 11.91 & 11.11 & 105.88345 & 0 & 0 & 28 & 60.01 & 0.744 & 0.012 & 15 & 1 & 0.5554 \\
\hline 1 & 320 & 13.89 & 12.06 & 134.94554 & 0 & 0 & 28 & 69.99 & 0.808 & 0.012 & 16 & 1 & 0.60305 \\
\hline 1 & 340 & 15.88 & 13.17 & 168.12155 & 0 & 0 & 28 & 80.02 & 0.883 & 0.011 & 17 & 1 & 0.6587 \\
\hline 1 & 360 & 17.86 & 14.06 & 205.51671 & 0 & 0 & 28 & 90 & 0.942 & 0.01 & 18 & 1 & 0.70315 \\
\hline 1 & 380 & 19.85 & 14.88 & 247.45461 & 0 & 0 & 28 & 100.02 & 0.997 & 0.01 & 19 & 1 & 0.744 \\
\hline 1 & 400 & 39.69 & 26.85 & 330.23637 & 0 & 0 & 28 & 200 & 1.799 & 0.009 & 20 & 1 & 1.34235 \\
\hline 1 & 420 & 59.54 & 35.58 & 454.75653 & 0 & 0 & 28 & 300.02 & 2.384 & 0.008 & 21 & 1 & 1.77895 \\
\hline 1 & 440 & 79.38 & 43.17 & 620.75672 & 0 & 0 & 28 & 400 & 2.892 & 0.007 & 22 & 1 & 2.15845 \\
\hline 1 & 460 & 99.23 & 50.29 & 828.22514 & 0 & 0 & 28 & 500.02 & 3.369 & 0.007 & 23 & 1 & 2.5145 \\
\hline 1 & 480 & 119.07 & 57.26 & 1077.24427 & 0 & 0 & 28 & 599.99 & 3.836 & 0.006 & 24 & 1 & 2.86295 \\
\hline 1 & 500 & 138.92 & 64.03 & 1367.67194 & 0 & 0 & 28 & 700.02 & 4.29 & 0.006 & 25 & 1 & 3.2016 \\
\hline 1 & 520 & 158.76 & 70.29 & 1700.02338 & 0 & 0 & 28 & 799.99 & 4.709 & 0.006 & 26 & 1 & 3.51435 \\
\hline 1 & 540 & 178.61 & 76.99 & 2073.77238 & 0 & 0 & 28 & 900.02 & 5.158 & 0.006 & 27 & 1 & 3.8494 \\
\hline 1 & 560 & 198.45 & 83.36 & 2489.18596 & 0 & 0 & 28 & 999.99 & 5.585 & 0.006 & 28 & 1 & 4.168 \\
\hline 1 & 570 & 198.45 & 81.87 & 2701.17985 & 0 & 0 & 28 & 999.99 & 5.485 & 0.005 & 29 & 1 & 4.0935 \\
\hline 1 & 580 & 198.45 & 81.25 & 2908.9962 & 0 & 0 & 28 & 999.99 & 5.444 & 0.005 & 30 & 1 & 4.0627 \\
\hline 1 & 590 & 198.45 & 80.75 & 3116.91623 & 0 & 0 & 28 & 999.99 & 5.41 & 0.005 & 31 & 1 & 4.03725 \\
\hline 1 & 600 & 198.45 & 80.33 & 3324.73258 & 0 & 0 & 28 & 999.99 & 5.382 & 0.005 & 32 & 1 & 4.01675 \\
\hline 1 & 610 & 198.45 & 79.98 & 3532.75628 & 0 & 0 & 28 & 999.99 & 5.358 & 0.005 & 33 & 1 & 3.99885 \\
\hline 1 & 620 & 198.45 & 79.65 & 3740.05349 & 0 & 0 & 28 & 999.99 & 5.336 & 0.005 & 34 & 1 & 3.9824 \\
\hline 1 & 640 & 198.45 & 79.23 & 4160.05065 & 0 & 0 & 28 & 999.99 & 5.309 & 0.005 & 35 & 1 & 3.96165 \\
\hline 1 & 660 & 178.61 & 71 & 4534.53792 & 0 & 0 & 28 & 900.02 & 4.757 & 0.005 & 36 & 1 & 3.5499 \\
\hline 1 & 680 & 158.76 & 62.96 & 4867.61821 & 0 & 0 & 28 & 799.99 & 4.218 & 0.005 & 37 & 1 & 3.14795 \\
\hline 1 & 700 & 138.92 & 55.57 & 5158.9491 & 0 & 0 & 28 & 700.02 & 3.723 & 0.005 & 38 & 1 & 2.77825 \\
\hline 1 & 720 & 119.07 & 47.93 & 5408.6044 & 0 & 0 & 28 & 599.99 & 3.211 & 0.005 & 39 & 1 & 2.39625 \\
\hline 1 & 740 & 99.23 & 40.43 & 5616.63516 & 0 & 0 & 28 & 500.02 & 2.709 & 0.005 & 40 & 1 & 2.02165 \\
\hline 1 & 760 & 79.38 & 32.83 & 5783.12387 & 0 & 0 & 28 & 400 & 2.199 & 0.005 & 41 & 1 & 1.64135 \\
\hline 1 & 780 & 59.54 & 25.08 & 5908.27 & 0 & 0 & 28 & 300.02 & 1.68 & 0.006 & 42 & 1 & 1.2538 \\
\hline 1 & 800 & 39.69 & 17.13 & 5991.66514 & 0 & 0 & 28 & 200 & 1.148 & 0.006 & 43 & 1 & 0.8564 \\
\hline 1 & 820 & 19.85 & 8.89 & 6033.53079 & 0 & 0 & 28 & 100.02 & 0.595 & 0.006 & 44 & 1 & 0.44435 \\
\hline 1 & 840 & 17.86 & 8.48 & 6071.22362 & 0 & 0 & 28 & 90 & 0.568 & 0.006 & 45 & 1 & 0.42375 \\
\hline 1 & 860 & 15.88 & 7.82 & 6104.50958 & 0 & 0 & 28 & 80.02 & 0.524 & 0.007 & 46 & 1 & 0.39085 \\
\hline 1 & 880 & 13.89 & 7.31 & 6133.60701 & 0 & 0 & 28 & 69.99 & 0.49 & 0.007 & 47 & 1 & 0.3657 \\
\hline 1 & 900 & 11.9 & 6.45 & 6158.58817 & 0 & 0 & 28 & 59.96 & 0.432 & 0.007 & 48 & 1 & 0.32255 \\
\hline 1 & 920 & 9.92 & 5.47 & 6179.37845 & 0 & 0 & 28 & 49.99 & 0.366 & 0.007 & 49 & 1 & 0.27335 \\
\hline 1 & 940 & 7.93 & 4.87 & 6196.02967 & 0 & 0 & 28 & 39.96 & 0.326 & 0.008 & 50 & 1 & 0.24345 \\
\hline 1 & 960 & 5.95 & 3.9 & 6208.51907 & 0 & 0 & 28 & 29.98 & 0.262 & 0.009 & 51 & 1 & 0.19515 \\
\hline 1 & 980 & 3.96 & 3.04 & 6216.84901 & 0 & 0 & 28 & 19.95 & 0.204 & 0.01 & 52 & 1 & 0.1519 \\
\hline 1 & $6 \quad 1000$ & 1.98 & 1.4 & 6221.02575 & 0 & 0 & 28 & 9.98 & 0.094 & 0.009 & 53 & 1 & 0.0701 \\
\hline 1 & $7 \quad 1020$ & 1.79 & 1.51 & 6224.79331 & 0 & 0 & 28 & 9.02 & 0.101 & 0.011 & 54 & 1 & 0.07565 \\
\hline 1 & $7 \quad 1040$ & 1.59 & 1.45 & 6228.13047 & 0 & 0 & 28 & 8.01 & 0.097 & 0.012 & 55 & 1 & 0.07235 \\
\hline 1 & $7 \quad 1060$ & 1.39 & 1.45 & 6231.04979 & 0 & 0 & 28 & 7 & 0.097 & 0.014 & 56 & 1 & 0.0725 \\
\hline 1 & $7 \quad 1080$ & 1.19 & 1.32 & 6233.55128 & 0 & 0 & 28 & 6 & 0.088 & 0.015 & 57 & 1 & 0.0658 \\
\hline 1 & 71100 & 0.99 & 1.2 & 6235.63887 & 0 & 0 & 28 & 4.99 & 0.081 & 0.016 & 58 & 1 & 0.06015 \\
\hline 1 & $7 \quad 1120$ & 0.8 & 1.16 & 6237.30942 & 0 & 0 & 28 & 4.03 & 0.078 & 0.019 & 59 & 1 & 0.05785 \\
\hline 1 & $\begin{array}{ll}7 & 1140\end{array}$ & 0.6 & 0 & 6238.56291 & 0 & 0 & 28 & 3.02 & 0 & 0 & 60 & 1 & 0 \\
\hline
\end{tabular}




$\begin{array}{lllllllllllllll}1 & 7 & 1160 & 0.4 & 0 & 6239.40093 & 0 & 0 & 28 & 2.02 & 0 & 0 & 61 & 1 & 0 \\ 1 & 7 & 1180 & 0.2 & 0 & 6239.82347 & 0 & 0 & 28 & 1.01 & 0 & 0 & 62 & 1 & 0\end{array}$

2004.02.05 180-5 wt\%-U [sonicated] DG 1-1000 s-1 run 2.txt

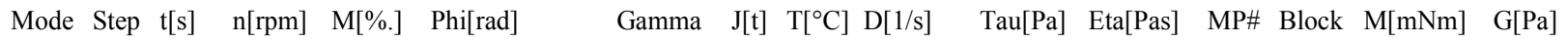

\begin{tabular}{|c|c|c|c|c|c|c|c|c|c|c|c|c|c|c|}
\hline 1 & 1 & 20 & 0.2 & 1.87 & 0.41626 & 0 & 0 & 28 & 1.01 & 0.125 & 0.124 & 1 & 1 & 0.09345 \\
\hline 1 & 1 & 40 & 0.4 & 1.98 & 1.24878 & 0 & 0 & 28 & 2.02 & 0.133 & 0.066 & 2 & 1 & 0.099 \\
\hline 1 & 1 & 60 & 0.6 & 2.21 & 2.49678 & 0 & 0 & 28 & 3.02 & 0.148 & 0.049 & 3 & 1 & 0.11055 \\
\hline 1 & 1 & 80 & 0.8 & 2.26 & 4.16182 & 0 & 0 & 28 & 4.03 & 0.152 & 0.038 & 4 & 1 & 0.11315 \\
\hline 1 & 1 & 100 & 1 & 2.33 & 6.24156 & 0 & 0 & 28 & 5.04 & 0.156 & 0.031 & 5 & 1 & 0.1165 \\
\hline & 1 & 120 & 1.19 & 2.62 & 8.7407 & 0 & 0 & 28 & 6 & 0.176 & 0.029 & 6 & 1 & 0.1312 \\
\hline & 1 & 140 & 1.39 & 2.76 & 11.65295 & 0 & 0 & 28 & 7 & 0.185 & 0.026 & 7 & 1 & 0.1378 \\
\hline & 1 & 160 & 1.59 & 3 & 14.98304 & 0 & 0 & 28 & 8.01 & 0.201 & 0.025 & 8 & 1 & 0.1498 \\
\hline & 1 & 180 & 1.79 & 3.17 & 18.72939 & 0 & 0 & 28 & 9.02 & 0.212 & 0.024 & 9 & 1 & 0.15845 \\
\hline & 2 & 200 & 1.98 & 3.42 & 22.90221 & 0 & 0 & 28 & 9.98 & 0.229 & 0.023 & 10 & 1 & 0.1712 \\
\hline & 2 & 220 & 3.97 & 5.95 & 31.17952 & 0 & 0 & 28 & 20 & 0.399 & 0.02 & 11 & 1 & 0.2976 \\
\hline & 2 & 240 & 5.95 & 7.36 & 43.61395 & 0 & 0 & 28 & 29.98 & 0.493 & 0.016 & 12 & 1 & 0.3679 \\
\hline & 2 & 260 & 7.94 & 8.26 & 60.22512 & 0 & 0 & 28 & 40.01 & 0.553 & 0.014 & 13 & 1 & 0.41305 \\
\hline & 2 & 280 & 9.92 & 9.31 & 80.96827 & 0 & 0 & 28 & 49.99 & 0.624 & 0.012 & 14 & 1 & 0.46535 \\
\hline & 2 & 300 & 11.91 & 10.43 & 105.8489 & 0 & 0 & 28 & 60.01 & 0.698 & 0.012 & 15 & 1 & 0.52125 \\
\hline & 2 & 320 & 13.89 & 11.36 & 134.9102 & 0 & 0 & 28 & 69.99 & 0.761 & 0.011 & 16 & 1 & 0.56805 \\
\hline & 2 & 340 & 15.88 & 12.43 & 168.16239 & 0 & 0 & 28 & 80.02 & 0.833 & 0.01 & 17 & 1 & 0.6215 \\
\hline & 2 & 360 & 17.86 & 13.28 & 205.51985 & 0 & 0 & 28 & 90 & 0.89 & 0.01 & 18 & 1 & 0.66415 \\
\hline & 3 & 380 & 19.85 & 14.23 & 247.47896 & 0 & 0 & 28 & 100.02 & 0.953 & 0.01 & 19 & 1 & 0.7114 \\
\hline & 3 & 400 & 39.69 & 25.95 & 330.21987 & 0 & 0 & 28 & 200 & 1.739 & 0.009 & 20 & 1 & 1.29745 \\
\hline & 3 & 420 & 59.54 & 34.74 & 454.72983 & 0 & 0 & 28 & 300.02 & 2.328 & 0.008 & 21 & 1 & 1.73715 \\
\hline & 3 & 440 & 79.38 & 42.23 & 620.51089 & 0 & 0 & 28 & 400 & 2.829 & 0.007 & 22 & 1 & 2.1114 \\
\hline & 3 & 460 & 99.23 & 49.55 & 828.08298 & 0 & 0 & 28 & 500.02 & 3.32 & 0.007 & 23 & 1 & 2.47755 \\
\hline & 3 & 480 & 119.07 & 56.62 & 1077.16573 & 0 & 0 & 28 & 599.99 & 3.793 & 0.006 & 24 & 1 & 2.83095 \\
\hline & 3 & 500 & 138.92 & 63.61 & 1367.7277 & 0 & 0 & 28 & 700.02 & 4.262 & 0.006 & 25 & 1 & 3.1806 \\
\hline & 3 & 520 & 158.76 & 70.17 & 1700.24644 & 0 & 0 & 28 & 799.99 & 4.702 & 0.006 & 26 & 1 & 3.5086 \\
\hline & 3 & 540 & 178.61 & 76.97 & 2074.08732 & 0 & 0 & 28 & 900.02 & 5.157 & 0.006 & 27 & 1 & 3.84855 \\
\hline 1 & 3 & 560 & 198.45 & 83.5 & 2489.08543 & 0 & 0 & 28 & 999.99 & 5.595 & 0.006 & 28 & 1 & 4.1752 \\
\hline & 4 & 570 & 198.45 & 82.12 & 2701.19399 & 0 & 0 & 27.9 & 999.99 & 5.502 & 0.006 & 29 & 1 & 4.10605 \\
\hline & 4 & 580 & 198.45 & 81.61 & 2908.90667 & 0 & 0 & 28 & 999.99 & 5.468 & 0.005 & 30 & 1 & 4.0806 \\
\hline 1 & 4 & 590 & 198.45 & 81.15 & 3116.93037 & 0 & 0 & 28 & 999.99 & 5.437 & 0.005 & 31 & 1 & 4.0575 \\
\hline 1 & 4 & 600 & 198.45 & 80.75 & 3324.74672 & 0 & 0 & 28 & 999.99 & 5.411 & 0.005 & 32 & 1 & 4.0377 \\
\hline & 4 & 610 & 198.45 & 80.43 & 3532.77042 & 0 & 0 & 28 & 999.99 & 5.389 & 0.005 & 33 & 1 & 4.0213 \\
\hline & 4 & 620 & 198.45 & 80.1 & 3740.06841 & 0 & 0 & 28 & 999.99 & 5.367 & 0.005 & 34 & 1 & 4.00495 \\
\hline & 5 & 640 & 198.45 & 79.77 & 4159.96112 & 0 & 0 & 28 & 999.99 & 5.345 & 0.005 & 35 & 1 & 3.9885 \\
\hline & 5 & 660 & 178.61 & 71.42 & 4534.72877 & 0 & 0 & 28 & 900.02 & 4.785 & 0.005 & 36 & 1 & 3.57105 \\
\hline & 5 & 680 & 158.76 & 63.34 & 4867.64335 & 0 & 0 & 28 & 799.99 & 4.244 & 0.005 & 37 & 1 & 3.167 \\
\hline 1 & 5 & 700 & 138.92 & 55.89 & 5158.68285 & 0 & 0 & 28 & 700.02 & 3.744 & 0.005 & 38 & 1 & 2.7943 \\
\hline & 5 & 720 & 119.07 & 48.19 & 5408.26589 & 0 & 0 & 28 & 599.99 & 3.229 & 0.005 & 39 & 1 & 2.4096 \\
\hline & 5 & 740 & 99.23 & 40.66 & 5616.66972 & 0 & 0 & 28 & 500.02 & 2.724 & 0.005 & 40 & 1 & 2.0331 \\
\hline 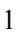 & 5 & 760 & 79.38 & 32.94 & 5783.15921 & 0 & 0 & 27.9 & 400 & 2.207 & 0.006 & 41 & 1 & 1.64705 \\
\hline 1 & 5 & 780 & 59.54 & 25.19 & 5908.13805 & 0 & 0 & 27.9 & 300.02 & 1.688 & 0.006 & 42 & 1 & 1.25955 \\
\hline 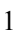 & 5 & 800 & 39.69 & 17.15 & 5991.59681 & 0 & 0 & 27.9 & 200 & 1.149 & 0.006 & 43 & 1 & 0.8574 \\
\hline & 5 & 820 & 19.85 & 8.93 & 6033.47267 & 0 & 0 & 27.9 & 100.02 & 0.598 & 0.006 & 44 & 1 & 0.4466 \\
\hline & 6 & 840 & 17.86 & 8.45 & 6071.15451 & 0 & 0 & 27.9 & 90 & 0.566 & 0.006 & 45 & 1 & 0.42265 \\
\hline
\end{tabular}




\begin{tabular}{|c|c|c|c|c|c|c|c|c|c|c|c|c|c|c|}
\hline 1 & 6 & 860 & 15.88 & 7.75 & 6104.41612 & 0 & 0 & 27.9 & 80.02 & 0.519 & 0.006 & 46 & 1 & 0.3873 \\
\hline & 6 & 880 & 13.89 & 7.28 & 6133.55046 & 0 & 0 & 28 & 69.99 & 0.487 & 0.007 & 47 & 1 & 0.36375 \\
\hline & 6 & 900 & 11.9 & 6.47 & 6158.50649 & 0 & 0 & 28 & 59.96 & 0.433 & 0.007 & 48 & 1 & 0.32325 \\
\hline & 6 & 920 & 9.92 & 5.42 & 6179.30698 & 0 & 0 & 28 & 49.99 & 0.363 & 0.007 & 49 & 1 & 0.2711 \\
\hline & 6 & 940 & 7.93 & 4.7 & 6195.96134 & 0 & 0 & 28 & 39.96 & 0.315 & 0.008 & 50 & 1 & 0.2349 \\
\hline & 6 & 960 & 5.95 & 3.81 & 6208.4531 & 0 & 0 & 28 & 29.98 & 0.255 & 0.009 & 51 & 1 & 0.19025 \\
\hline & 6 & 980 & 3.96 & 3.03 & 6216.78382 & 0 & 0 & 28 & 19.95 & 0.203 & 0.01 & 52 & 1 & 0.1517 \\
\hline & 6 & 1000 & 1.98 & 1.39 & 6220.96371 & 0 & 0 & 28 & 9.98 & 0.093 & 0.009 & 53 & 1 & 0.06945 \\
\hline & 7 & 1020 & 1.79 & 1.51 & 6224.73441 & 0 & 0 & 28 & 9.02 & 0.101 & 0.011 & 54 & 1 & 0.0755 \\
\hline & 7 & 1040 & 1.59 & 1.43 & 6228.06921 & 0 & 0 & 28 & 8.01 & 0.096 & 0.012 & 55 & 1 & 0.07145 \\
\hline & 7 & 1060 & 1.39 & 1.5 & 6230.98932 & 0 & 0 & 28 & 7 & 0.1 & 0.014 & 56 & 1 & 0.07495 \\
\hline & 7 & 1080 & 1.19 & 1.29 & 6233.49238 & 0 & 0 & 28 & 6 & 0.086 & 0.014 & 57 & 1 & 0.06455 \\
\hline & 7 & 1100 & 0.99 & 1.21 & 6235.5784 & 0 & 0 & 28 & 4.99 & 0.081 & 0.016 & 58 & 1 & 0.06045 \\
\hline & 7 & 1120 & 0.8 & 1.19 & 6237.24815 & 0 & 0 & 28 & 4.03 & 0.079 & 0.02 & 59 & 1 & 0.05925 \\
\hline & 7 & 1140 & 0.6 & 0 & 6238.50322 & 0 & 0 & 28 & 3.02 & 0 & 0 & 60 & 1 & 0 \\
\hline & 7 & 1160 & 0.4 & 0 & 6239.34045 & 0 & 0 & 28 & 2.02 & 0 & 0 & 61 & 1 & 0 \\
\hline & 7 & 1180 & 0.2 & 0 & 6239.763 & 0 & 0 & 28 & 1.01 & 0 & 0 & 62 & 1 & 0 \\
\hline
\end{tabular}

2004.02.05 180-5 wt\%-U [sonicated] DG 500-4000 s-1 run 1.txt

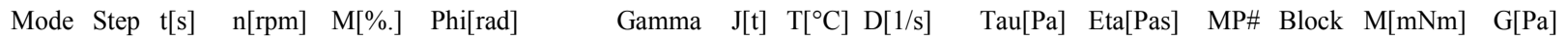

$\begin{array}{rrrrrrrrrrrrrrrr}1 & 1 & 20 & 99.31 & 48.31 & 207.60979 & 0 & 0 & 28 & 500.42 & 3.236 & 0.006 & 1 & 1 & 2.41525 & 0 \\ 1 & 1 & 40 & 198.46 & 86.61 & 621.6937 & 0 & 0 & 28 & 1000.04 & 5.803 & 0.006 & 2 & 1 & 4.33045 & 0 \\ 1 & 1 & 60 & 297.68 & 121.93 & 1244.06284 & 0 & 0 & 28 & 1500.01 & 8.169 & 0.005 & 3 & 1 & 6.09645 & 0 \\ 1 & 1 & 80 & 396.91 & 155.15 & 2073.88783 & 0 & 0 & 28 & 2000.03 & 10.395 & 0.005 & 4 & 1 & 7.7575 & 0 \\ 1 & 1 & 100 & 496.13 & 185.79 & 3110.85453 & 0 & 0 & 28 & 2500 & 12.448 & 0.005 & 5 & 1 & 9.28935 & 0 \\ 1 & 1 & 120 & 595.36 & 215.39 & 4356.72928 & 0 & 0 & 28 & 3000.02 & 14.431 & 0.005 & 6 & 1 & 10.7696 & 0 \\ 1 & 1 & 140 & 694.58 & 244.43 & 5809.69624 & 0 & 0 & 28 & 3499.99 & 16.377 & 0.005 & 7 & 1 & 12.2213 & 0 \\ 1 & 1 & 160 & 793.81 & 277.27 & 7470.11907 & 0 & 0 & 28 & 4000.01 & 18.577 & 0.005 & 8 & 1 & 13.86325 & 0 \\ 1 & 2 & 180 & 793.81 & 276.01 & 9151.14603 & 0 & 0 & 28 & 4000.01 & 18.493 & 0.005 & 9 & 1 & 13.80055 & 0 \\ 1 & 2 & 200 & 793.81 & 275.93 & 10812.03852 & 0 & 0 & 28 & 4000.01 & 18.487 & 0.005 & 10 & 1 & 13.7966 & 0 \\ 1 & 2 & 220 & 793.81 & 274.44 & 12475.83541 & 0 & 0 & 28 & 4000.01 & 18.388 & 0.005 & 11 & 1 & 13.7222 & 0 \\ 1 & 3 & 240 & 793.81 & 272.41 & 14154.59807 & 0 & 0 & 28 & 4000.01 & 18.251 & 0.005 & 12 & 1 & 13.6204 & 0 \\ 1 & 3 & 260 & 694.58 & 228.33 & 15610.99723 & 0 & 0 & 28 & 3499.99 & 15.298 & 0.004 & 13 & 1 & 11.41665 & 0 \\ 1 & 3 & 280 & 595.36 & 195.54 & 16859.88948 & 0 & 0 & 28 & 3000.02 & 13.101 & 0.004 & 14 & 1 & 9.77715 & 0 \\ 1 & 3 & 300 & 496.13 & 162.62 & 17900.8617 & 0 & 0 & 28 & 2500 & 10.896 & 0.004 & 15 & 1 & 8.13105 & 0 \\ 1 & 3 & 320 & 396.91 & 130.21 & 18733.38925 & 0 & 0 & 28 & 2000.03 & 8.724 & 0.004 & 16 & 1 & 6.51065 & 0 \\ 1 & 3 & 340 & 297.68 & 97.92 & 19359.15288 & 0 & 0 & 28 & 1500.01 & 6.561 & 0.004 & 17 & 1 & 4.8962 & 0 \\ 1 & 3 & 360 & 198.46 & 65.44 & 19776.40901 & 0 & 0 & 28 & 1000.04 & 4.384 & 0.004 & 18 & 1 & 3.27195 & 0 \\ 1 & 3 & 380 & 99.23 & 33.55 & 19985.78045 & 0 & 0 & 28 & 500.02 & 2.248 & 0.004 & 19 & 1 & 1.67745 & 0\end{array}$

2004.02.05 180-5 wt\%-U [sonicated] DG 500-4000 s-1 run 2.txt

Mode Step t[s] n[rpm] M[\%.] Phi[rad] Gamma

$\begin{array}{rrrrrrrrrrrrrrrr}1 & 1 & 20 & 99.29 & 34.82 & 207.80222 & 0 & 0 & 28 & 500.32 & 2.333 & 0.005 & 1 & 1 & 1.74105 & 0 \\ 1 & 1 & 40 & 198.46 & 68.12 & 621.62694 & 0 & 0 & 28 & 1000.04 & 4.564 & 0.005 & 2 & 1 & 3.40595 & 0 \\ 1 & 1 & 60 & 297.68 & 100.98 & 1243.63322 & 0 & 0 & 28 & 1500.01 & 6.766 & 0.005 & 3 & 1 & 5.0492 & 0 \\ 1 & 1 & 80 & 396.91 & 133.69 & 2072.93829 & 0 & 0 & 28 & 2000.03 & 8.957 & 0.004 & 4 & 1 & 6.68435 & 0 \\ 1 & 1 & 100 & 496.13 & 165.76 & 3110.11311 & 0 & 0 & 28 & 2500 & 11.106 & 0.004 & 5 & 1 & 8.288 & 0 \\ 1 & 1 & 120 & 595.36 & 197.47 & 4355.31242 & 0 & 0 & 28 & 3000.02 & 13.231 & 0.004 & 6 & 1 & 9.87355 & 0 \\ 1 & 1 & 140 & 694.58 & 228.68 & 5809.78735 & 0 & 0 & 28 & 3499.99 & 15.322 & 0.004 & 7 & 1 & 11.4342 & 0 \\ 1 & 1 & 160 & 793.81 & 267.87 & 7469.79234 & 0 & 0 & 28 & 4000.01 & 17.948 & 0.004 & 8 & 1 & 13.3937 & 0\end{array}$




$\begin{array}{rrrrrrrrrrrrrr}180 & 793.81 & 267.52 & 9150.82323 & 0 & 0 & 28 & 4000.01 & 17.924 & 0.004 & 9 & 1 & 13.37585 & 0 \\ 200 & 793.81 & 267.85 & 10813.7931 & 0 & 0 & 28 & 4000.01 & 17.946 & 0.004 & 10 & 1 & 13.39245 & 0 \\ 220 & 793.81 & 268.82 & 12475.51183 & 0 & 0 & 28 & 4000.01 & 18.011 & 0.005 & 11 & 1 & 13.44115 & 0 \\ 240 & 793.81 & 268.86 & 14155.93796 & 0 & 0 & 28 & 4000.01 & 18.013 & 0.005 & 12 & 1 & 13.4428 & 0 \\ 260 & 694.58 & 220.97 & 15612.75573 & 0 & 0 & 28 & 3499.99 & 14.805 & 0.004 & 13 & 1 & 11.04835 & 0 \\ 280 & 595.36 & 189.57 & 16860.08504 & 0 & 0 & 28 & 3000.02 & 12.701 & 0.004 & 14 & 1 & 9.4786 & 0 \\ 300 & 496.13 & 157.82 & 17901.15936 & 0 & 0 & 28 & 2500 & 10.574 & 0.004 & 15 & 1 & 7.89115 & 0 \\ 320 & 396.91 & 126.49 & 18733.95002 & 0 & 0 & 28 & 2000.03 & 8.475 & 0.004 & 16 & 1 & 6.3244 & 0 \\ 340 & 297.68 & 95.26 & 19359.71444 & 0 & 0 & 28 & 1500.01 & 6.382 & 0.004 & 17 & 1 & 4.763 & 0 \\ 360 & 198.46 & 63.61 & 19776.75223 & 0 & 0 & 28 & 1000.04 & 4.262 & 0.004 & 18 & 1 & 3.1805 & 0 \\ 380 & 99.23 & 32.66 & 19986.28939 & 0 & 0 & 28 & 500.02 & 2.188 & 0.004 & 19 & 1 & 1.63295 & 0\end{array}$
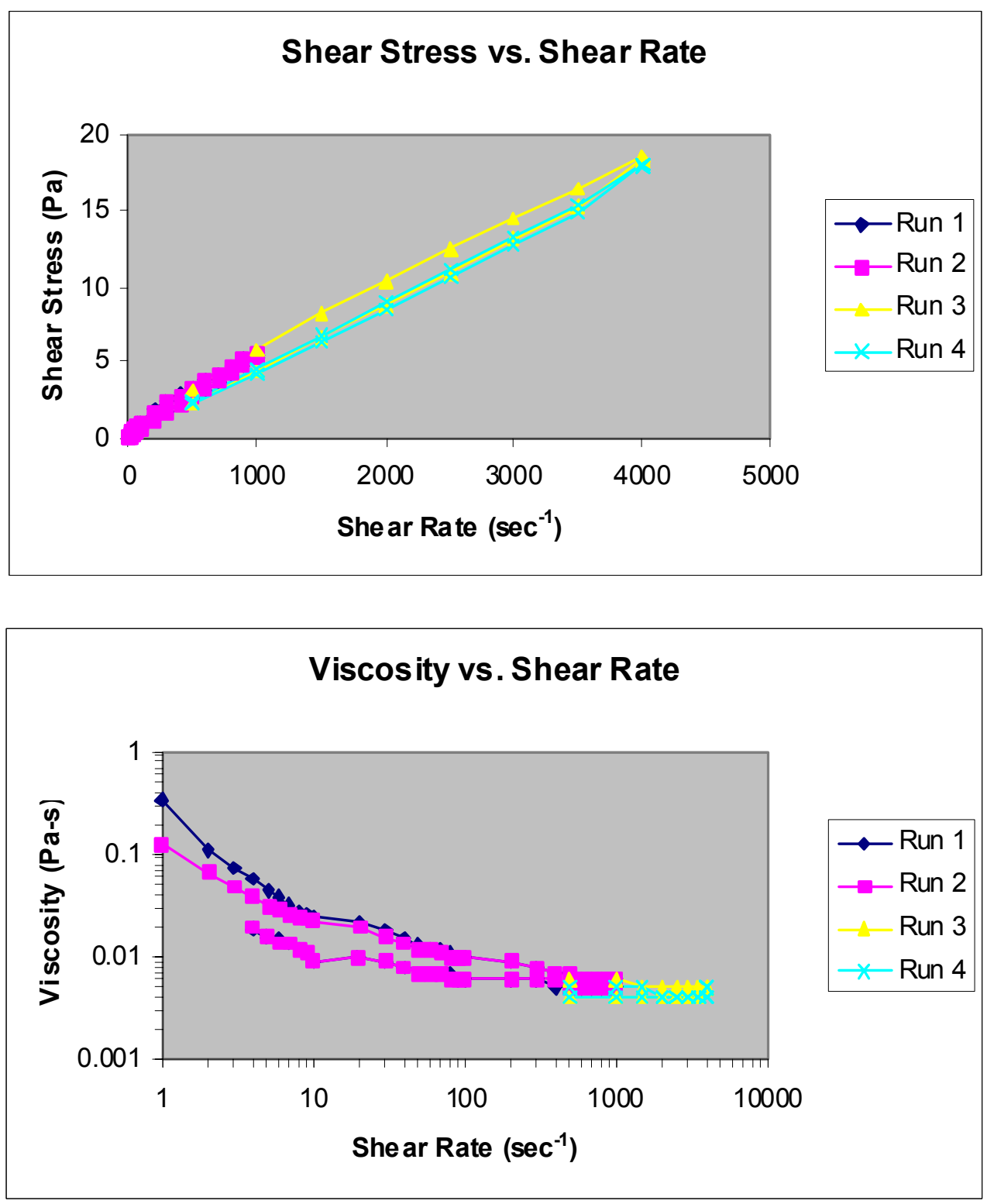


\section{E-2.1.11 189/5wt\%/U/N}

2004.02.06 189-5 wt\%-U DG 1-1000 s-1 run 1.txt

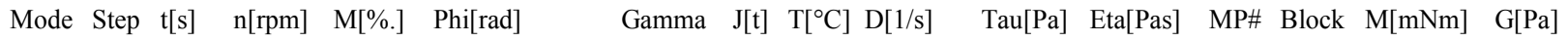

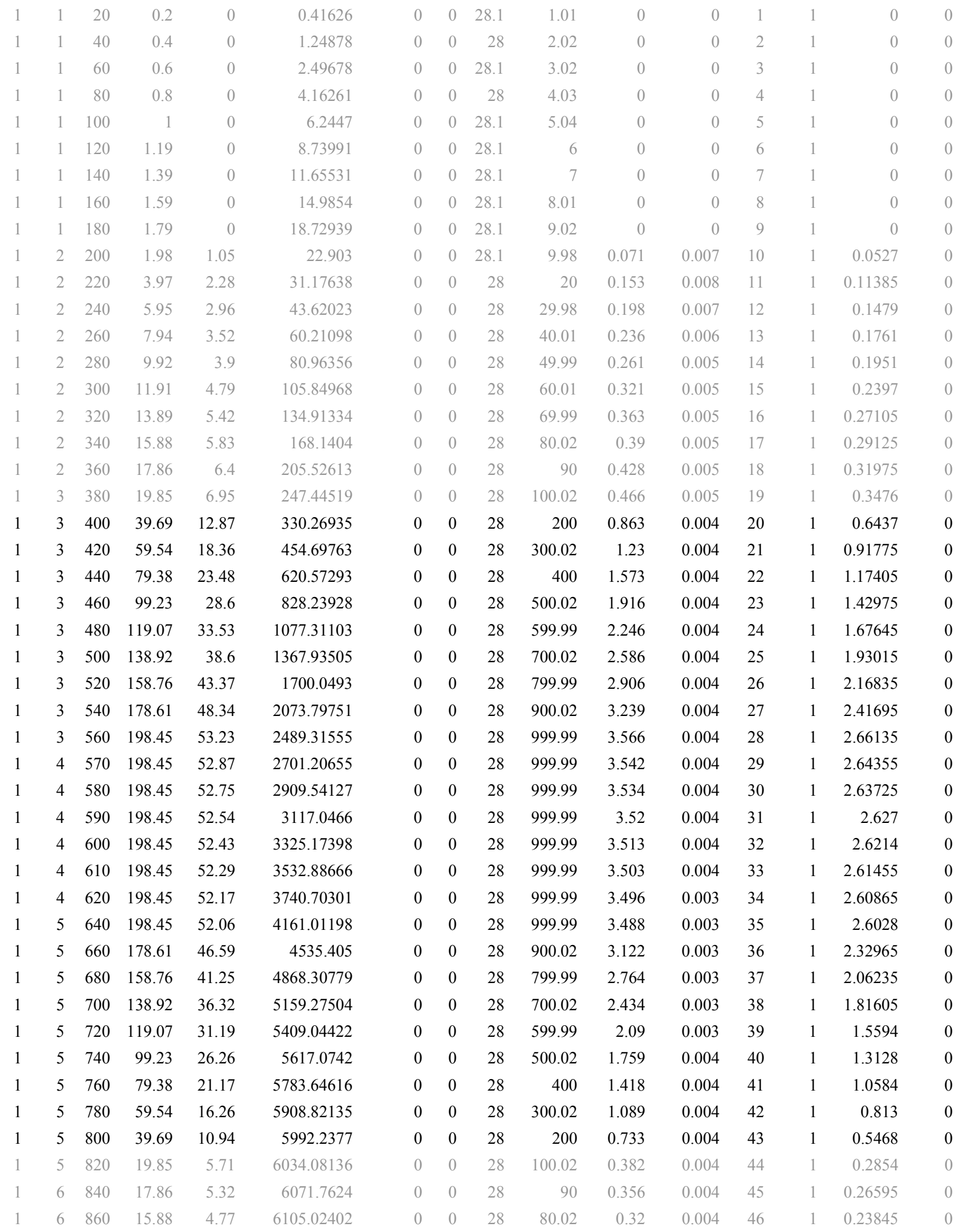




\begin{tabular}{|c|c|c|c|c|c|c|c|c|c|c|c|c|c|c|}
\hline 1 & 6 & 880 & 13.89 & 4.41 & 6134.15051 & 0 & 0 & 28 & 69.99 & 0.295 & 0.004 & 47 & 1 & 0.22045 \\
\hline 1 & 6 & 900 & 11.9 & 3.82 & 6159.11203 & 0 & 0 & 28 & 59.96 & 0.256 & 0.004 & 48 & 1 & 0.19105 \\
\hline 1 & 6 & 920 & 9.92 & 3.07 & 6179.90623 & 0 & 0 & 28 & 49.99 & 0.206 & 0.004 & 49 & 1 & 0.15355 \\
\hline 1 & 6 & 940 & 7.93 & 2.65 & 6196.55275 & 0 & 0 & 28 & 39.96 & 0.177 & 0.004 & 50 & 1 & 0.1324 \\
\hline 1 & 6 & 960 & 5.95 & 2.16 & 6209.05864 & 0 & 0 & 28 & 29.98 & 0.145 & 0.005 & 51 & 1 & 0.10785 \\
\hline 1 & 6 & 980 & 3.96 & 1.73 & 6217.38701 & 0 & 0 & 28 & 19.95 & 0.116 & 0.006 & 52 & 1 & 0.0865 \\
\hline 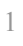 & 6 & 1000 & 1.98 & 0 & 6221.56297 & 0 & 0 & 28 & 9.98 & 0 & 0 & 53 & 1 & 0 \\
\hline 1 & 7 & 1020 & 1.79 & 0 & 6225.33288 & 0 & 0 & 28 & 9.02 & 0 & 0 & 54 & 1 & 0 \\
\hline 1 & 7 & 1040 & 1.59 & 0 & 6228.66689 & 0 & 0 & 28 & 8.01 & 0 & 0 & 55 & 1 & 0 \\
\hline 1 & 7 & 1060 & 1.39 & 0 & 6231.587 & 0 & 0 & 28 & 7 & 0 & 0 & 56 & 1 & 0 \\
\hline 1 & 7 & 1080 & 1.19 & 0 & 6234.08928 & 0 & 0 & 28 & 6 & 0 & 0 & 57 & 1 & 0 \\
\hline 1 & 7 & 1100 & 0.99 & 0 & 6236.17687 & 0 & 0 & 28 & 4.99 & 0 & 0 & 58 & 1 & 0 \\
\hline 1 & 7 & 1120 & 0.8 & 0 & 6237.84741 & 0 & 0 & 28 & 4.03 & 0 & 0 & 59 & 1 & 0 \\
\hline 1 & 7 & 1140 & 0.6 & 0 & 6239.10091 & 0 & 0 & 28 & 3.02 & 0 & 0 & 60 & 1 & 0 \\
\hline 1 & 7 & 1160 & 0.4 & 0 & 6239.93971 & 0 & 0 & 28 & 2.02 & 0 & 0 & 61 & 1 & 0 \\
\hline 1 & 7 & 1180 & 0.2 & 0 & 6240.36069 & 0 & 0 & 28 & 1.01 & 0 & 0 & 62 & 1 & 0 \\
\hline
\end{tabular}

2004.02.06 189-5 wt\%-U DG 1-1000 s-1 run 2.txt

Mode Step $\mathrm{t}[\mathrm{s}] \quad \mathrm{n}[\mathrm{rpm}] \quad \mathrm{M}[\%$. $] \quad$ Phi[rad]

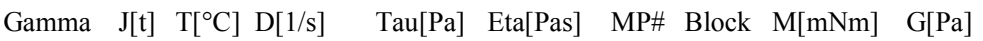

\begin{tabular}{|c|c|c|c|c|c|c|c|c|c|c|c|c|c|c|}
\hline 1 & 1 & 20 & 0.2 & 0 & 0.41705 & 0 & 0 & 28 & 1.01 & 0 & 0 & 1 & 1 & 0 \\
\hline 1 & 1 & 40 & 0.4 & 0 & 1.24878 & 0 & 0 & 28 & 2.02 & 0 & 0 & 2 & 1 & 0 \\
\hline 1 & 1 & 60 & 0.6 & 0 & 2.49757 & 0 & 0 & 28 & 3.02 & 0 & 0 & 3 & 1 & 0 \\
\hline 1 & 1 & 80 & 0.8 & 0 & 4.16261 & 0 & 0 & 28 & 4.03 & 0 & 0 & 4 & 1 & 0 \\
\hline 1 & 1 & 100 & 1 & 0 & 6.24392 & 0 & 0 & 28 & 5.04 & 0 & 0 & 5 & 1 & 0 \\
\hline 1 & 1 & 120 & 1.19 & 0 & 8.73991 & 0 & 0 & 28 & 6 & 0 & 0 & 6 & 1 & 0 \\
\hline 1 & 1 & 140 & 1.39 & 0 & 11.65531 & 0 & 0 & 28 & 7 & 0 & 0 & 7 & 1 & 0 \\
\hline 1 & 1 & 160 & 1.59 & 0 & 14.9854 & 0 & 0 & 28 & 8.01 & 0 & 0 & 8 & 1 & 0 \\
\hline 1 & 1 & 180 & 1.79 & 0 & 18.73018 & 0 & 0 & 28 & 9.02 & 0 & 0 & 9 & 1 & 0 \\
\hline 1 & 2 & 200 & 1.98 & 0 & 22.90221 & 0 & 0 & 28 & 9.98 & 0 & 0 & 10 & 1 & 0 \\
\hline 1 & 2 & 220 & 3.97 & 2.22 & 31.18031 & 0 & 0 & 28 & 20 & 0.149 & 0.007 & 11 & 1 & 0.11085 \\
\hline 1 & 2 & 240 & 5.95 & 2.77 & 43.61237 & 0 & 0 & 28 & 29.98 & 0.186 & 0.006 & 12 & 1 & 0.1385 \\
\hline 1 & 2 & 260 & 7.94 & 3.27 & 60.20705 & 0 & 0 & 28 & 40.01 & 0.219 & 0.005 & 13 & 1 & 0.1633 \\
\hline 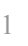 & 2 & 280 & 9.92 & 3.7 & 80.94313 & 0 & 0 & 28 & 49.99 & 0.248 & 0.005 & 14 & 1 & 0.18505 \\
\hline 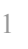 & 2 & 300 & 11.91 & 4.56 & 105.87403 & 0 & 0 & 28 & 60.01 & 0.305 & 0.005 & 15 & 1 & 0.2278 \\
\hline 1 & 2 & 320 & 13.89 & 5.08 & 134.90784 & 0 & 0 & 28 & 69.99 & 0.34 & 0.005 & 16 & 1 & 0.25375 \\
\hline 1 & 2 & 340 & 15.88 & 5.45 & 168.16003 & 0 & 0 & 28 & 80.02 & 0.365 & 0.005 & 17 & 1 & 0.2724 \\
\hline 1 & 2 & 360 & 17.86 & 6.08 & 205.51671 & 0 & 0 & 28 & 90 & 0.407 & 0.005 & 18 & 1 & 0.30405 \\
\hline & 3 & 380 & 19.85 & 6.64 & 247.46639 & 0 & 0 & 28 & 100.02 & 0.445 & 0.004 & 19 & 1 & 0.3318 \\
\hline 1 & 3 & 400 & 39.69 & 12.46 & 330.23872 & 0 & 0 & 28 & 200 & 0.835 & 0.004 & 20 & 1 & 0.62315 \\
\hline 1 & 3 & 420 & 59.54 & 17.77 & 454.656 & 0 & 0 & 28 & 300.02 & 1.191 & 0.004 & 21 & 1 & 0.8887 \\
\hline 1 & 3 & 440 & 79.38 & 22.77 & 620.5211 & 0 & 0 & 28 & 400 & 1.526 & 0.004 & 22 & 1 & 1.1387 \\
\hline & 3 & 460 & 99.23 & 27.73 & 828.18744 & 0 & 0 & 28 & 500.02 & 1.858 & 0.004 & 23 & 1 & 1.3864 \\
\hline & 3 & 480 & 119.07 & 32.57 & 1077.19793 & 0 & 0 & 28 & 599.99 & 2.182 & 0.004 & 24 & 1 & 1.6287 \\
\hline & 3 & 500 & 138.92 & 37.58 & 1367.83295 & 0 & 0 & 28 & 700.02 & 2.518 & 0.004 & 25 & 1 & 1.87905 \\
\hline & 3 & 520 & 158.76 & 42.33 & 1700.11292 & 0 & 0 & 28 & 799.99 & 2.836 & 0.004 & 26 & 1 & 2.11645 \\
\hline 1 & 3 & 540 & 178.61 & 47.41 & 2073.86034 & 0 & 0 & 28 & 900.02 & 3.176 & 0.004 & 27 & 1 & 2.37025 \\
\hline 1 & 3 & 560 & 198.45 & 52.45 & 2489.28492 & 0 & 0 & 28 & 999.99 & 3.514 & 0.004 & 28 & 1 & 2.6223 \\
\hline 1 & 4 & 570 & 198.45 & 52.17 & 2701.07146 & 0 & 0 & 28 & 999.99 & 3.495 & 0.003 & 29 & 1 & 2.60845 \\
\hline 1 & 4 & 580 & 198.45 & 52.01 & 2909.40697 & 0 & 0 & 28 & 999.99 & 3.485 & 0.003 & 30 & 1 & 2.6004 \\
\hline 1 & 4 & 590 & 198.45 & 51.9 & 3116.9123 & 0 & 0 & 28.1 & 999.99 & 3.478 & 0.003 & 31 & 1 & 2.5952 \\
\hline
\end{tabular}




\begin{tabular}{|c|c|c|c|c|c|c|c|c|c|c|c|c|c|c|}
\hline 1 & 4 & 600 & 198.45 & 51.79 & 3324.41685 & 0 & 0 & 28.1 & 999.99 & 3.47 & 0.003 & 32 & 1 & 2.58955 \\
\hline 1 & 4 & 610 & 198.45 & 51.76 & 3532.85603 & 0 & 0 & 28.1 & 999.99 & 3.468 & 0.003 & 33 & 1 & 2.58785 \\
\hline 1 & 4 & 620 & 198.45 & 51.64 & 3740.56871 & 0 & 0 & 28.1 & 999.99 & 3.46 & 0.003 & 34 & 1 & 2.58175 \\
\hline & 5 & 640 & 198.45 & 51.53 & 4160.87689 & 0 & 0 & 28.1 & 999.99 & 3.452 & 0.003 & 35 & 1 & 2.5764 \\
\hline & 5 & 660 & 178.61 & 46.12 & 4535.26991 & 0 & 0 & 28.1 & 900.02 & 3.09 & 0.003 & 36 & 1 & 2.3058 \\
\hline & 5 & 680 & 158.76 & 40.82 & 4867.84127 & 0 & 0 & 28.1 & 799.99 & 2.735 & 0.003 & 37 & 1 & 2.0412 \\
\hline & 5 & 700 & 138.92 & 35.93 & 5159.31745 & 0 & 0 & 28 & 700.02 & 2.408 & 0.003 & 38 & 1 & 1.79665 \\
\hline & 5 & 720 & 119.07 & 30.82 & 5409.01359 & 0 & 0 & 28 & 599.99 & 2.065 & 0.003 & 39 & 1 & 1.54105 \\
\hline & 5 & 740 & 99.23 & 25.92 & 5617.15745 & 0 & 0 & 28 & 500.02 & 1.737 & 0.003 & 40 & 1 & 1.29615 \\
\hline & 5 & 760 & 79.38 & 20.93 & 5783.68778 & 0 & 0 & 28 & 400 & 1.402 & 0.004 & 41 & 1 & 1.04645 \\
\hline & 5 & 780 & 59.54 & 15.92 & 5908.67683 & 0 & 0 & 28 & 300.02 & 1.067 & 0.004 & 42 & 1 & 0.79595 \\
\hline 1 & 5 & 800 & 39.69 & 10.71 & 5992.18665 & 0 & 0 & 28 & 200 & 0.718 & 0.004 & 43 & 1 & 0.5355 \\
\hline 1 & 5 & 820 & 19.85 & 5.48 & 6034.07193 & 0 & 0 & 28 & 100.02 & 0.367 & 0.004 & 44 & 1 & 0.27385 \\
\hline 1 & 6 & 840 & 17.86 & 5.12 & 6071.74277 & 0 & 0 & 28 & 90 & 0.343 & 0.004 & 45 & 1 & 0.256 \\
\hline 1 & 6 & 860 & 15.88 & 4.48 & 6105.05386 & 0 & 0 & 28 & 80.02 & 0.3 & 0.004 & 46 & 1 & 0.22405 \\
\hline 1 & 6 & 880 & 13.89 & 4.22 & 6134.13637 & 0 & 0 & 28.1 & 69.99 & 0.282 & 0.004 & 47 & 1 & 0.21075 \\
\hline 1 & 6 & 900 & 11.9 & 3.73 & 6159.10575 & 0 & 0 & 28.1 & 59.96 & 0.25 & 0.004 & 48 & 1 & 0.18625 \\
\hline 1 & 6 & 920 & 9.92 & 2.95 & 6179.92587 & 0 & 0 & 28.1 & 49.99 & 0.198 & 0.004 & 49 & 1 & 0.14755 \\
\hline 1 & 6 & 940 & 7.93 & 2.59 & 6196.56531 & 0 & 0 & 28 & 39.96 & 0.173 & 0.004 & 50 & 1 & 0.1293 \\
\hline 1 & 6 & 960 & 5.95 & 2.08 & 6209.05707 & 0 & 0 & 28 & 29.98 & 0.139 & 0.005 & 51 & 1 & 0.10375 \\
\hline 1 & 6 & 980 & 3.96 & 1.63 & 6217.38936 & 0 & 0 & 28.1 & 19.95 & 0.109 & 0.005 & 52 & 1 & 0.08145 \\
\hline 1 & 6 & 1000 & 1.98 & 0 & 6221.56689 & 0 & 0 & 28.1 & 9.98 & 0 & 0 & 53 & 1 & 0 \\
\hline 1 & 7 & 1020 & 1.79 & 0 & 6225.33602 & 0 & 0 & 28.1 & 9.02 & 0 & 0 & 54 & 1 & 0 \\
\hline 1 & 7 & 1040 & 1.59 & 0 & 6228.67161 & 0 & 0 & 28.1 & 8.01 & 0 & 0 & 55 & 1 & 0 \\
\hline 1 & 7 & 1060 & 1.39 & 0 & 6231.59172 & 0 & 0 & 28 & 7 & 0 & 0 & 56 & 1 & 0 \\
\hline 1 & 7 & 1080 & 1.19 & 0 & 6234.09399 & 0 & 0 & 28.1 & 6 & 0 & 0 & 57 & 1 & 0 \\
\hline 1 & 7 & 1100 & 0.99 & 0 & 6236.1808 & 0 & 0 & 28.1 & 4.99 & 0 & 0 & 58 & 1 & 0 \\
\hline 1 & 7 & 1120 & 0.8 & 0 & 6237.85134 & 0 & 0 & 28.1 & 4.03 & 0 & 0 & 59 & 1 & 0 \\
\hline 1 & 7 & 1140 & 0.6 & 0 & 6239.10562 & 0 & 0 & 28.1 & 3.02 & 0 & 0 & 60 & 1 & 0 \\
\hline & 7 & 1160 & 0.4 & 0 & 6239.94364 & 0 & 0 & 28.1 & 2.02 & 0 & 0 & 61 & 1 & 0 \\
\hline & 7 & 1180 & 0.2 & 0 & 6240.36461 & 0 & 0 & 28 & 1.01 & 0 & 0 & 62 & 1 & 0 \\
\hline
\end{tabular}

2004.02.06 189-5 wt\%-U DG 500-4000 s-1 run 1.txt Mode Step t[s] n[rpm] M[\%.] Phi[rad]

$\begin{array}{rrrrrrrrrrrrrrrr}1 & 1 & 20 & 99.31 & 26.84 & 207.82656 & 0 & 0 & 28 & 500.42 & 1.798 & 0.004 & 1 & 1 & 1.34195 & 0 \\ 1 & 1 & 40 & 198.46 & 52.32 & 621.44473 & 0 & 0 & 28 & 1000.04 & 3.506 & 0.004 & 2 & 1 & 2.6161 & 0 \\ 1 & 1 & 60 & 297.68 & 77.54 & 1243.91989 & 0 & 0 & 28 & 1500.01 & 5.195 & 0.003 & 3 & 1 & 3.8768 & 0 \\ 1 & 1 & 80 & 396.91 & 102.55 & 2073.58938 & 0 & 0 & 28 & 2000.03 & 6.871 & 0.003 & 4 & 1 & 5.12725 & 0 \\ 1 & 1 & 100 & 496.13 & 126.8 & 3111.07836 & 0 & 0 & 28 & 2500 & 8.496 & 0.003 & 5 & 1 & 6.3402 & 0 \\ 1 & 1 & 120 & 595.36 & 157.23 & 4356.59105 & 0 & 0 & 28 & 3000.02 & 10.534 & 0.004 & 6 & 1 & 7.86125 & 0 \\ 1 & 1 & 140 & 694.58 & 198.59 & 5807.78851 & 0 & 0 & 28 & 3499.99 & 13.305 & 0.004 & 7 & 1 & 9.92945 & 0 \\ 1 & 1 & 160 & 793.81 & 241.74 & 7469.81826 & 0 & 0 & 28 & 4000.01 & 16.196 & 0.004 & 8 & 1 & 12.0869 & 0 \\ 1 & 2 & 180 & 793.81 & 240.85 & 9150.90805 & 0 & 0 & 28 & 4000.01 & 16.137 & 0.004 & 9 & 1 & 12.0423 & 0 \\ 1 & 2 & 200 & 793.81 & 240.18 & 10813.04461 & 0 & 0 & 28 & 4000.01 & 16.092 & 0.004 & 10 & 1 & 12.0088 & 0 \\ 1 & 2 & 220 & 793.81 & 239.33 & 12475.18118 & 0 & 0 & 28 & 4000.01 & 16.035 & 0.004 & 11 & 1 & 11.9663 & 0 \\ 1 & 3 & 240 & 793.81 & 238.23 & 14154.35931 & 0 & 0 & 28 & 4000.01 & 15.961 & 0.004 & 12 & 1 & 11.9114 & 0 \\ 1 & 3 & 260 & 694.58 & 194.08 & 15611.79912 & 0 & 0 & 28 & 3499.99 & 13.004 & 0.004 & 13 & 1 & 9.7042 & 0 \\ 1 & 3 & 280 & 595.36 & 153.01 & 16861.00396 & 0 & 0 & 28 & 3000.02 & 10.251 & 0.003 & 14 & 1 & 7.6503 & 0 \\ 1 & 3 & 300 & 496.13 & 117.2 & 17901.50573 & 0 & 0 & 28 & 2500 & 7.852 & 0.003 & 15 & 1 & 5.85995 & 0 \\ 1 & 3 & 320 & 396.91 & 93.37 & 18734.72835 & 0 & 0 & 28 & 2000.03 & 6.256 & 0.003 & 16 & 1 & 4.66865 & 0\end{array}$




$\begin{array}{rrrrrrrrrrrrrrrr}1 & 3 & 340 & 297.68 & 69.94 & 19359.2754 & 0 & 0 & 28 & 1500.01 & 4.686 & 0.003 & 17 & 1 & 3.49685 & 0 \\ 1 & 3 & 360 & 198.46 & 46.27 & 19776.50718 & 0 & 0 & 28 & 1000.04 & 3.1 & 0.003 & 18 & 1 & 2.3133 & 0 \\ 1 & 3 & 380 & 99.23 & 23.61 & 19986.11503 & 0 & 0 & 28 & 500.02 & 1.582 & 0.003 & 19 & 1 & 1.18035 & 0\end{array}$

2004.02.06 189-5 wt\%-U DG 500-4000 s-1 run 2.txt Mode Step $\mathrm{t}[\mathrm{s}] \quad \mathrm{n}[\mathrm{rpm}] \quad \mathrm{M}[\%$.] $\quad$ Phi[rad]

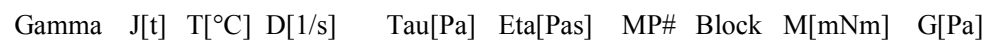

$\begin{array}{rrrrrrrrrrrrrrrr}1 & 1 & 20 & 99.3 & 23.61 & 207.71425 & 0 & 0 & 28 & 500.37 & 1.582 & 0.003 & 1 & 1 & 1.18045 & 0 \\ 1 & 1 & 40 & 198.46 & 47.28 & 621.74868 & 0 & 0 & 28 & 1000.04 & 3.168 & 0.003 & 2 & 1 & 2.36415 & 0 \\ 1 & 1 & 60 & 297.68 & 71.14 & 1243.80837 & 0 & 0 & 28 & 1500.01 & 4.766 & 0.003 & 3 & 1 & 3.55685 & 0 \\ 1 & 1 & 80 & 396.91 & 94.91 & 2073.27051 & 0 & 0 & 28 & 2000.03 & 6.359 & 0.003 & 4 & 1 & 4.7456 & 0 \\ 1 & 1 & 100 & 496.13 & 118.37 & 3111.01789 & 0 & 0 & 28 & 2500 & 7.931 & 0.003 & 5 & 1 & 5.9186 & 0 \\ 1 & 1 & 120 & 595.36 & 153.65 & 4355.59516 & 0 & 0 & 28 & 3000.02 & 10.294 & 0.003 & 6 & 1 & 7.68245 & 0 \\ 1 & 1 & 140 & 694.58 & 193.97 & 5810.01433 & 0 & 0 & 28 & 3499.99 & 12.996 & 0.004 & 7 & 1 & 9.69855 & 0 \\ 1 & 1 & 160 & 793.81 & 238.07 & 7469.96434 & 0 & 0 & 28 & 4000.01 & 15.95 & 0.004 & 8 & 1 & 11.90335 & 0 \\ 1 & 2 & 180 & 793.81 & 237.56 & 9151.06434 & 0 & 0 & 28 & 4000.01 & 15.917 & 0.004 & 9 & 1 & 11.87815 & 0 \\ 1 & 2 & 200 & 793.81 & 236.88 & 10812.78308 & 0 & 0 & 28 & 4000.01 & 15.871 & 0.004 & 10 & 1 & 11.84405 & 0 \\ 1 & 2 & 220 & 793.81 & 236.47 & 12475.33668 & 0 & 0 & 28 & 4000.01 & 15.843 & 0.004 & 11 & 1 & 11.8235 & 0 \\ 1 & 3 & 240 & 793.81 & 236.14 & 14154.09777 & 0 & 0 & 28 & 4000.01 & 15.821 & 0.004 & 12 & 1 & 11.8068 & 0 \\ 1 & 3 & 260 & 694.58 & 192.55 & 15612.36696 & 0 & 0 & 28 & 3499.99 & 12.901 & 0.004 & 13 & 1 & 9.6276 & 0 \\ 1 & 3 & 280 & 595.36 & 152.47 & 16859.70255 & 0 & 0 & 28 & 3000.02 & 10.216 & 0.003 & 14 & 1 & 7.6237 & 0 \\ 1 & 3 & 300 & 496.13 & 115.58 & 17900.46507 & 0 & 0 & 28 & 2500 & 7.744 & 0.003 & 15 & 1 & 5.77905 & 0 \\ 1 & 3 & 320 & 396.91 & 92.05 & 18733.22275 & 0 & 0 & 28 & 2000.03 & 6.167 & 0.003 & 16 & 1 & 4.6025 & 0 \\ 1 & 3 & 340 & 297.68 & 68.93 & 19358.49707 & 0 & 0 & 28 & 1500.01 & 4.618 & 0.003 & 17 & 1 & 3.44645 & 0 \\ 1 & 3 & 360 & 198.46 & 45.58 & 19775.88436 & 0 & 0 & 28 & 1000.04 & 3.054 & 0.003 & 18 & 1 & 2.27875 & 0 \\ 1 & 3 & 380 & 99.23 & 23.29 & 19985.60216 & 0 & 0 & 28 & 500.02 & 1.561 & 0.003 & 19 & 1 & 1.1647 & 0\end{array}$

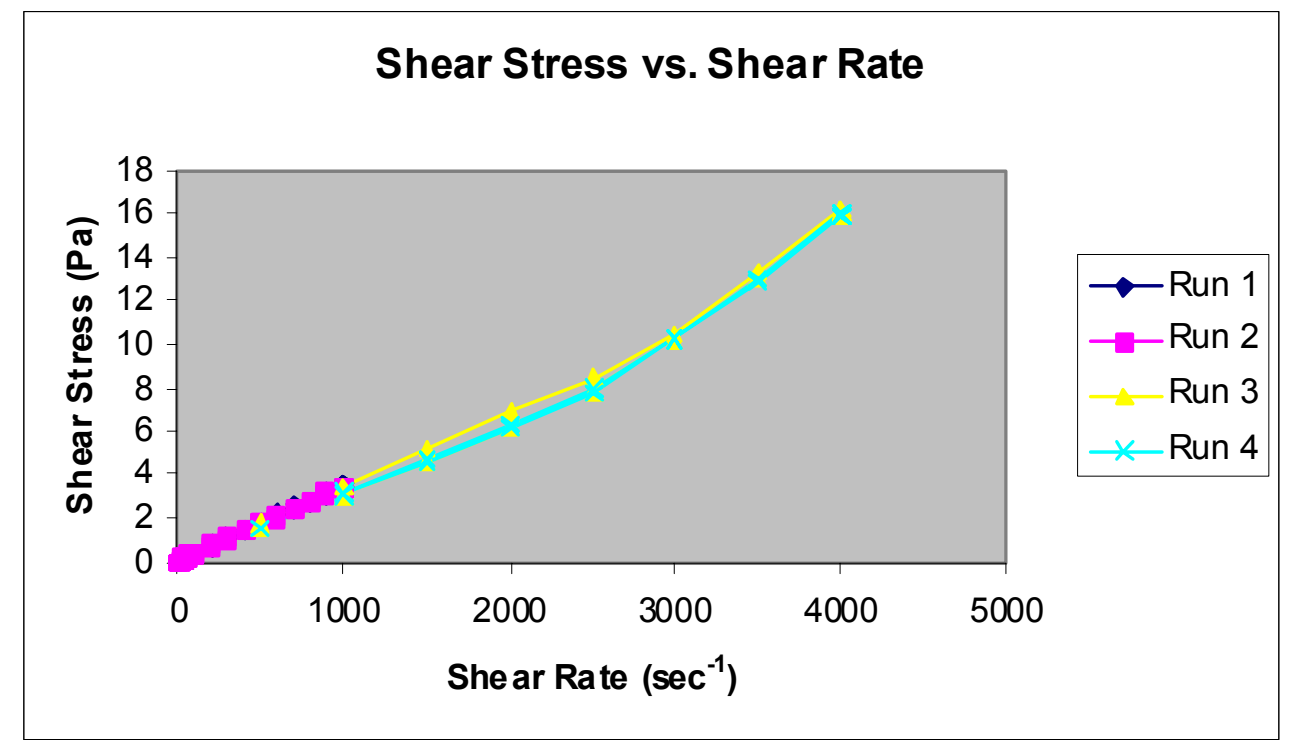




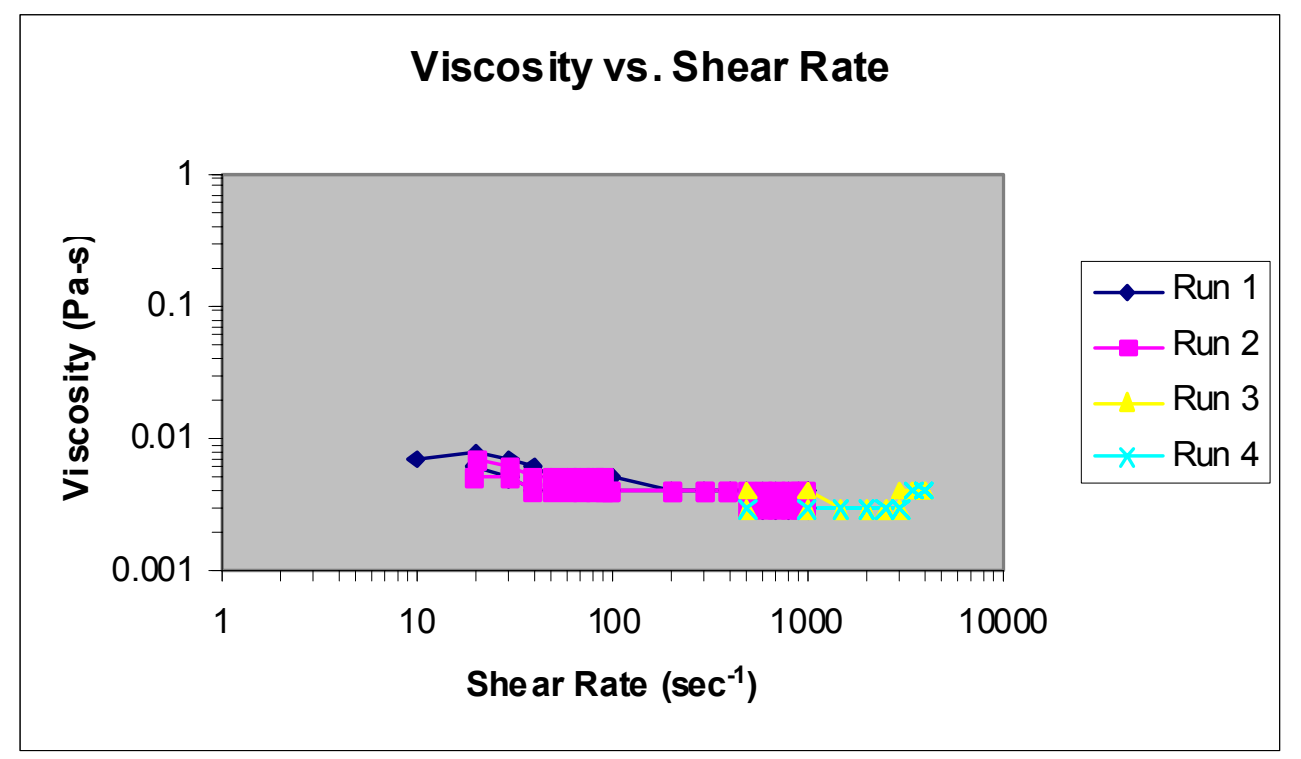

\section{E-2.1.12 189/5wt $\% / U / S$}

2004.02.05 189-5 wt\%-U [sonicated] DG 1-1000 s-1 run 1.txt

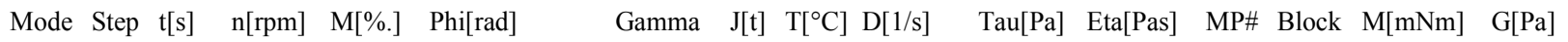

\begin{tabular}{|c|c|c|c|c|c|c|c|c|c|c|c|c|c|c|}
\hline 1 & 1 & 20 & 0.2 & 13.85 & 0.41233 & 0 & 0 & 28 & 1.01 & 0.928 & 0.919 & 1 & 1 & 0.69245 \\
\hline 1 & 1 & 40 & 0.4 & 15.04 & 1.24329 & 0 & 0 & 28 & 2.02 & 1.008 & 0.499 & 2 & 1 & 0.75195 \\
\hline 1 & 1 & 60 & 0.6 & 19.4 & 2.4905 & 0 & 0 & 28 & 3.02 & 1.3 & 0.43 & 3 & 1 & 0.97 \\
\hline 1 & 1 & 80 & 0.8 & 24 & 4.15397 & 0 & 0 & 28 & 4.03 & 1.608 & 0.399 & 4 & 1 & 1.20005 \\
\hline 1 & 1 & 100 & 1 & 25.6 & 6.23606 & 0 & 0 & 28 & 5.04 & 1.715 & 0.34 & 5 & 1 & 1.27995 \\
\hline 1 & 1 & 120 & 1.19 & 27.95 & 8.73049 & 0 & 0 & 28 & 6 & 1.873 & 0.312 & 6 & 1 & 1.3977 \\
\hline 1 & 1 & 140 & 1.39 & 30.16 & 11.64353 & 0 & 0 & 28 & 7 & 2.021 & 0.289 & 7 & 1 & 1.508 \\
\hline 1 & 1 & 160 & 1.59 & 29.24 & 14.97519 & 0 & 0 & 28 & 8.01 & 1.959 & 0.245 & 8 & 1 & 1.4622 \\
\hline 1 & 1 & 180 & 1.79 & 26.19 & 18.72311 & 0 & 0 & 28 & 9.02 & 1.755 & 0.195 & 9 & 1 & 1.30965 \\
\hline 1 & 2 & 200 & 1.98 & 24.34 & 22.89907 & 0 & 0 & 28 & 9.98 & 1.631 & 0.163 & 10 & 1 & 1.217 \\
\hline 1 & 2 & 220 & 3.97 & 27.14 & 31.17717 & 0 & 0 & 28 & 20 & 1.818 & 0.091 & 11 & 1 & 1.3569 \\
\hline 1 & 2 & 240 & 5.95 & 27.15 & 43.60766 & 0 & 0 & 28 & 29.98 & 1.819 & 0.061 & 12 & 1 & 1.3577 \\
\hline 1 & 2 & 260 & 7.94 & 26.61 & 60.20784 & 0 & 0 & 28 & 40.01 & 1.783 & 0.045 & 13 & 1 & 1.33055 \\
\hline 1 & 2 & 280 & 9.92 & 26.67 & 80.93999 & 0 & 0 & 28 & 49.99 & 1.787 & 0.036 & 14 & 1 & 1.33365 \\
\hline 1 & 2 & 300 & 11.91 & 27.41 & 105.85832 & 0 & 0 & 28 & 60.01 & 1.837 & 0.031 & 15 & 1 & 1.3706 \\
\hline 1 & 2 & 320 & 13.89 & 28.16 & 134.90549 & 0 & 0 & 28 & 69.99 & 1.887 & 0.027 & 16 & 1 & 1.408 \\
\hline 1 & 2 & 340 & 15.88 & 28.89 & 168.16553 & 0 & 0 & 28 & 80.02 & 1.936 & 0.024 & 17 & 1 & 1.44455 \\
\hline 1 & 2 & 360 & 17.86 & 29.81 & 205.52378 & 0 & 0 & 28 & 90 & 1.997 & 0.022 & 18 & 1 & 1.4904 \\
\hline 1 & 3 & 380 & 19.85 & 30.57 & 247.43105 & 0 & 0 & 28 & 100.02 & 2.048 & 0.02 & 19 & 1 & 1.52835 \\
\hline 1 & 3 & 400 & 39.69 & 46.15 & 330.3369 & 0 & 0 & 28 & 200 & 3.092 & 0.015 & 20 & 1 & 2.30725 \\
\hline 1 & 3 & 420 & 59.54 & 57.03 & 454.80523 & 0 & 0 & 28 & 300.02 & 3.821 & 0.013 & 21 & 1 & 2.85125 \\
\hline 1 & 3 & 440 & 79.38 & 66.38 & 620.72059 & 0 & 0 & 28 & 400 & 4.447 & 0.011 & 22 & 1 & 3.3189 \\
\hline 1 & 3 & 460 & 99.23 & 74.94 & 828.18901 & 0 & 0 & 28 & 500.02 & 5.021 & 0.01 & 23 & 1 & 3.7468 \\
\hline 1 & 3 & 480 & 119.07 & 83 & 1077.20892 & 0 & 0 & 28 & 599.99 & 5.561 & 0.009 & 24 & 1 & 4.1499 \\
\hline 1 & 3 & 500 & 138.92 & 90.83 & 1367.77012 & 0 & 0 & 28 & 700.02 & 6.086 & 0.009 & 25 & 1 & 4.54145 \\
\hline 1 & 3 & 520 & 158.76 & 97.95 & 1700.03909 & 0 & 0 & 28 & 799.99 & 6.563 & 0.008 & 26 & 1 & 4.89765 \\
\hline 1 & 3 & 540 & 178.61 & 105.42 & 2073.7873 & 0 & 0 & 28 & 900.02 & 7.063 & 0.008 & 27 & 1 & 5.2709 \\
\hline 1 & 3 & 560 & 198.45 & 112.39 & 2488.99275 & 0 & 0 & 28 & 999.99 & 7.53 & 0.008 & 28 & 1 & 5.6194 \\
\hline
\end{tabular}




\begin{tabular}{|c|c|c|c|c|c|c|c|c|c|c|c|c|c|c|}
\hline 1 & 4 & 570 & 198.45 & 109.86 & 2701.19477 & 0 & 0 & 28 & 999.99 & 7.36 & 0.007 & 29 & 1 & 5.4928 \\
\hline 1 & 4 & 580 & 198.45 & 108.78 & 2908.90745 & 0 & 0 & 28 & 999.99 & 7.288 & 0.007 & 30 & 1 & 5.4391 \\
\hline 1 & 4 & 590 & 198.45 & 107.88 & 3116.72381 & 0 & 0 & 28 & 999.99 & 7.228 & 0.007 & 31 & 1 & 5.39375 \\
\hline 1 & 4 & 600 & 198.45 & 107.07 & 3324.33282 & 0 & 0 & 28 & 999.99 & 7.174 & 0.007 & 32 & 1 & 5.3534 \\
\hline 1 & 4 & 610 & 198.45 & 106.36 & 3532.14917 & 0 & 0 & 28 & 999.99 & 7.126 & 0.007 & 33 & 1 & 5.3179 \\
\hline 1 & 4 & 620 & 198.45 & 105.75 & 3740.17287 & 0 & 0 & 28 & 999.99 & 7.085 & 0.007 & 34 & 1 & 5.28725 \\
\hline 1 & 5 & 640 & 198.45 & 105.11 & 4160.68997 & 0 & 0 & 28 & 999.99 & 7.042 & 0.007 & 35 & 1 & 5.2554 \\
\hline 1 & 5 & 660 & 178.61 & 94.6 & 4534.89685 & 0 & 0 & 28 & 900.02 & 6.338 & 0.007 & 36 & 1 & 4.73015 \\
\hline 1 & 5 & 680 & 158.76 & 84.66 & 4867.55146 & 0 & 0 & 28 & 799.99 & 5.672 & 0.007 & 37 & 1 & 4.23285 \\
\hline 1 & 5 & 700 & 138.92 & 75.36 & 5158.89412 & 0 & 0 & 28 & 700.02 & 5.049 & 0.007 & 38 & 1 & 3.7682 \\
\hline 1 & 5 & 720 & 119.07 & 65.84 & 5408.79839 & 0 & 0 & 28 & 599.99 & 4.412 & 0.007 & 39 & 1 & 3.2922 \\
\hline 1 & 5 & 740 & 99.23 & 56.35 & 5616.94383 & 0 & 0 & 28 & 500.02 & 3.775 & 0.008 & 40 & 1 & 2.8175 \\
\hline 1 & 5 & 760 & 79.38 & 46.67 & 5783.4231 & 0 & 0 & 28 & 400 & 3.127 & 0.008 & 41 & 1 & 2.33345 \\
\hline 1 & 5 & 780 & 59.54 & 36.76 & 5908.37053 & 0 & 0 & 28 & 300.02 & 2.463 & 0.008 & 42 & 1 & 1.83775 \\
\hline 1 & 5 & 800 & 39.69 & 25.91 & 5991.88191 & 0 & 0 & 28 & 200 & 1.736 & 0.009 & 43 & 1 & 1.2955 \\
\hline 1 & 5 & 820 & 19.85 & 14.12 & 6033.74756 & 0 & 0 & 28 & 100.02 & 0.946 & 0.009 & 44 & 1 & 0.706 \\
\hline 1 & 6 & 840 & 17.86 & 14.1 & 6071.44118 & 0 & 0 & 28 & 90 & 0.945 & 0.01 & 45 & 1 & 0.70505 \\
\hline 1 & 6 & 860 & 15.88 & 13.6 & 6104.71221 & 0 & 0 & 28 & 80.02 & 0.911 & 0.011 & 46 & 1 & 0.68005 \\
\hline 1 & 6 & 880 & 13.89 & 12.8 & 6133.83164 & 0 & 0 & 28 & 69.99 & 0.858 & 0.012 & 47 & 1 & 0.64005 \\
\hline 1 & 6 & 900 & 11.9 & 11.99 & 6158.79002 & 0 & 0 & 28 & 59.96 & 0.804 & 0.013 & 48 & 1 & 0.5997 \\
\hline 1 & 6 & 920 & 9.92 & 10.95 & 6179.61721 & 0 & 0 & 28 & 49.99 & 0.734 & 0.015 & 49 & 1 & 0.5475 \\
\hline 1 & 6 & 940 & 7.93 & 9.78 & 6196.26451 & 0 & 0 & 28 & 39.96 & 0.655 & 0.016 & 50 & 1 & 0.4888 \\
\hline 1 & 6 & 960 & 5.95 & 8.65 & 6208.7492 & 0 & 0 & 28 & 29.98 & 0.579 & 0.019 & 51 & 1 & 0.4323 \\
\hline 1 & 6 & 980 & 3.96 & 7.6 & 6217.08149 & 0 & 0 & 28 & 19.95 & 0.509 & 0.026 & 52 & 1 & 0.38015 \\
\hline 1 & 6 & 1000 & 1.98 & 5.23 & 6221.25823 & 0 & 0 & 28 & 9.98 & 0.35 & 0.035 & 53 & 1 & 0.26135 \\
\hline 1 & 7 & 1020 & 1.79 & 6.66 & 6225.02893 & 0 & 0 & 28 & 9.02 & 0.446 & 0.049 & 54 & 1 & 0.33295 \\
\hline 1 & 7 & 1040 & 1.59 & 7.67 & 6228.36294 & 0 & 0 & 28 & 8.01 & 0.514 & 0.064 & 55 & 1 & 0.38325 \\
\hline 1 & 7 & 1060 & 1.39 & 8.39 & 6231.28227 & 0 & 0 & 28 & 7 & 0.562 & 0.08 & 56 & 1 & 0.41955 \\
\hline 1 & 7 & 1080 & 1.19 & 9.18 & 6233.78455 & 0 & 0 & 28 & 6 & 0.615 & 0.103 & 57 & 1 & 0.4589 \\
\hline 1 & 7 & 1100 & 0.99 & 9.69 & 6235.87135 & 0 & 0 & 28 & 4.99 & 0.649 & 0.13 & 58 & 1 & 0.48425 \\
\hline 1 & 7 & 1120 & 0.8 & 10.26 & 6237.54111 & 0 & 0 & 28 & 4.03 & 0.687 & 0.17 & 59 & 1 & 0.5129 \\
\hline 1 & 7 & 1140 & 0.6 & 10.83 & 6238.79617 & 0 & 0 & 28 & 3.02 & 0.726 & 0.24 & 60 & 1 & 0.54155 \\
\hline 1 & 7 & 1160 & 0.4 & 11.3 & 6239.63262 & 0 & 0 & 28 & 2.02 & 0.757 & 0.375 & 61 & 1 & 0.56485 \\
\hline 1 & 7 & 1180 & 0.2 & 11.07 & 6240.05438 & 0 & 0 & 28 & 1.01 & 0.742 & 0.735 & 62 & 1 & 0.55345 \\
\hline
\end{tabular}

2004.02.05 189-5 wt\%-U [sonicated] DG 1-1000 s-1 run 2.txt

Mode Step t[s] n[rpm] M[\%.] Phi[rad]

$\begin{array}{rrrrrrrrrrrrrrrr}1 & 1 & 20 & 0.2 & 17.08 & 0.41076 & 0 & 0 & 28 & 1.01 & 1.144 & 1.133 & 1 & 1 & 0.85385 & 0 \\ 1 & 1 & 40 & 0.4 & 18.96 & 1.24171 & 0 & 0 & 28 & 2.02 & 1.27 & 0.629 & 2 & 1 & 0.948 & 0 \\ 1 & 1 & 60 & 0.6 & 19.25 & 2.49207 & 0 & 0 & 28 & 3.02 & 1.289 & 0.427 & 3 & 1 & 0.96225 & 0 \\ 1 & 1 & 80 & 0.8 & 18.86 & 4.15554 & 0 & 0 & 28 & 4.03 & 1.264 & 0.314 & 4 & 1 & 0.94325 & 0 \\ 1 & 1 & 100 & 1 & 18.32 & 6.23685 & 0 & 0 & 28 & 5.04 & 1.228 & 0.244 & 5 & 1 & 0.91605 & 0 \\ 1 & 1 & 120 & 1.19 & 17.63 & 8.73598 & 0 & 0 & 28 & 6 & 1.181 & 0.197 & 6 & 1 & 0.8813 & 0 \\ 1 & 1 & 140 & 1.39 & 17.08 & 11.64824 & 0 & 0 & 28 & 7 & 1.144 & 0.163 & 7 & 1 & 0.8539 & 0 \\ 1 & 1 & 160 & 1.59 & 16.45 & 14.9799 & 0 & 0 & 28 & 8.01 & 1.102 & 0.138 & 8 & 1 & 0.82255 & 0 \\ 1 & 1 & 180 & 1.79 & 16.12 & 18.72468 & 0 & 0 & 28 & 9.02 & 1.08 & 0.12 & 9 & 1 & 0.8059 & 0 \\ 1 & 2 & 200 & 1.98 & 15.77 & 22.89593 & 0 & 0 & 28 & 9.98 & 1.056 & 0.106 & 10 & 1 & 0.78835 & 0 \\ 1 & 2 & 220 & 3.97 & 18.76 & 31.17795 & 0 & 0 & 28 & 20 & 1.257 & 0.063 & 11 & 1 & 0.9382 & 0 \\ 1 & 2 & 240 & 5.95 & 19.77 & 43.6108 & 0 & 0 & 28 & 29.98 & 1.325 & 0.044 & 12 & 1 & 0.9887 & 0 \\ 1 & 2 & 260 & 7.94 & 20.57 & 60.20077 & 0 & 0 & 28 & 40.01 & 1.378 & 0.034 & 13 & 1 & 1.02855 & 0\end{array}$




\begin{tabular}{|c|c|c|c|c|c|c|c|c|c|c|c|c|c|c|}
\hline 1 & 2 & 280 & 9.92 & 21.22 & 80.94942 & 0 & 0 & 28 & 49.99 & 1.422 & 0.028 & 14 & 1 & 1.0612 \\
\hline 1 & 2 & 300 & 11.91 & 22.35 & 105.84261 & 0 & 0 & 28 & 60.01 & 1.498 & 0.025 & 15 & 1 & 1.11755 \\
\hline 1 & 2 & 320 & 13.89 & 23.47 & 134.91805 & 0 & 0 & 28 & 69.99 & 1.572 & 0.022 & 16 & 1 & 1.1733 \\
\hline 1 & 2 & 340 & 15.88 & 24.41 & 168.14668 & 0 & 0 & 28 & 80.02 & 1.636 & 0.02 & 17 & 1 & 1.22065 \\
\hline 1 & 2 & 360 & 17.86 & 25.47 & 205.49393 & 0 & 0 & 28 & 90 & 1.706 & 0.019 & 18 & 1 & 1.2734 \\
\hline 1 & 3 & 380 & 19.85 & 26.54 & 247.41142 & 0 & 0 & 28 & 100.02 & 1.778 & 0.018 & 19 & 1 & 1.32675 \\
\hline 1 & 3 & 400 & 39.69 & 41.64 & 330.30627 & 0 & 0 & 28 & 200 & 2.79 & 0.014 & 20 & 1 & 2.082 \\
\hline 1 & 3 & 420 & 59.54 & 53.42 & 454.57746 & 0 & 0 & 28 & 300.02 & 3.579 & 0.012 & 21 & 1 & 2.67085 \\
\hline 1 & 3 & 440 & 79.38 & 63.31 & 620.57608 & 0 & 0 & 28 & 400 & 4.242 & 0.011 & 22 & 1 & 3.16535 \\
\hline 1 & 3 & 460 & 99.23 & 72.27 & 828.03429 & 0 & 0 & 28 & 500.02 & 4.842 & 0.01 & 23 & 1 & 3.61325 \\
\hline 1 & 3 & 480 & 119.07 & 80.48 & 1077.29375 & 0 & 0 & 28 & 599.99 & 5.392 & 0.009 & 24 & 1 & 4.0239 \\
\hline 1 & 3 & 500 & 138.92 & 88.25 & 1367.9272 & 0 & 0 & 28 & 700.02 & 5.913 & 0.008 & 25 & 1 & 4.4125 \\
\hline 1 & 3 & 520 & 158.76 & 95.5 & 1699.95741 & 0 & 0 & 28 & 799.99 & 6.398 & 0.008 & 26 & 1 & 4.7748 \\
\hline 1 & 3 & 540 & 178.61 & 103.04 & 2073.70562 & 0 & 0 & 28 & 900.02 & 6.903 & 0.008 & 27 & 1 & 5.15185 \\
\hline 1 & 3 & 560 & 198.45 & 110.24 & 2489.09878 & 0 & 0 & 28 & 999.99 & 7.386 & 0.007 & 28 & 1 & 5.51205 \\
\hline 1 & 4 & 570 & 198.45 & 108.03 & 2700.98821 & 0 & 0 & 28 & 999.99 & 7.238 & 0.007 & 29 & 1 & 5.4015 \\
\hline 1 & 4 & 580 & 198.45 & 107.04 & 2908.80456 & 0 & 0 & 28 & 999.99 & 7.171 & 0.007 & 30 & 1 & 5.35185 \\
\hline 1 & 4 & 590 & 198.45 & 106.2 & 3116.62092 & 0 & 0 & 28 & 999.99 & 7.115 & 0.007 & 31 & 1 & 5.30995 \\
\hline 1 & 4 & 600 & 198.45 & 105.44 & 3324.22993 & 0 & 0 & 28 & 999.99 & 7.065 & 0.007 & 32 & 1 & 5.27205 \\
\hline 1 & 4 & 610 & 198.45 & 104.76 & 3532.25441 & 0 & 0 & 28 & 999.99 & 7.019 & 0.007 & 33 & 1 & 5.2379 \\
\hline 1 & 4 & 620 & 198.45 & 104.17 & 3740.07077 & 0 & 0 & 28 & 999.99 & 6.979 & 0.007 & 34 & 1 & 5.2083 \\
\hline 1 & 5 & 640 & 198.45 & 103.52 & 4160.17239 & 0 & 0 & 28 & 999.99 & 6.936 & 0.007 & 35 & 1 & 5.17605 \\
\hline 1 & 5 & 660 & 178.61 & 93.2 & 4534.8458 & 0 & 0 & 28 & 900.02 & 6.245 & 0.007 & 36 & 1 & 4.66015 \\
\hline 1 & 5 & 680 & 158.76 & 83.24 & 4867.51062 & 0 & 0 & 28 & 799.99 & 5.577 & 0.007 & 37 & 1 & 4.1621 \\
\hline 1 & 5 & 700 & 138.92 & 73.95 & 5158.78102 & 0 & 0 & 28 & 700.02 & 4.955 & 0.007 & 38 & 1 & 3.6975 \\
\hline 1 & 5 & 720 & 119.07 & 64.49 & 5408.67508 & 0 & 0 & 28 & 599.99 & 4.321 & 0.007 & 39 & 1 & 3.22455 \\
\hline 1 & 5 & 740 & 99.23 & 55.1 & 5616.70585 & 0 & 0 & 28 & 500.02 & 3.691 & 0.007 & 40 & 1 & 2.75485 \\
\hline 1 & 5 & 760 & 79.38 & 45.52 & 5783.32022 & 0 & 0 & 28 & 400 & 3.05 & 0.008 & 41 & 1 & 2.2761 \\
\hline 1 & 5 & 780 & 59.54 & 35.59 & 5908.33047 & 0 & 0 & 28 & 300.02 & 2.385 & 0.008 & 42 & 1 & 1.7797 \\
\hline 1 & 5 & 800 & 39.69 & 24.99 & 5991.80023 & 0 & 0 & 28 & 200 & 1.674 & 0.008 & 43 & 1 & 1.2496 \\
\hline 1 & 5 & 820 & 19.85 & 13.61 & 6033.66588 & 0 & 0 & 28 & 100.02 & 0.912 & 0.009 & 44 & 1 & 0.68055 \\
\hline 1 & 6 & 840 & 17.86 & 13.33 & 6071.33986 & 0 & 0 & 28 & 90 & 0.893 & 0.01 & 45 & 1 & 0.6665 \\
\hline 1 & 6 & 860 & 15.88 & 12.62 & 6104.61875 & 0 & 0 & 28 & 80.02 & 0.846 & 0.011 & 46 & 1 & 0.6311 \\
\hline 1 & 6 & 880 & 13.89 & 11.73 & 6133.74603 & 0 & 0 & 28 & 69.99 & 0.786 & 0.011 & 47 & 1 & 0.58645 \\
\hline 1 & 6 & 900 & 11.9 & 10.79 & 6158.69734 & 0 & 0 & 28 & 59.96 & 0.723 & 0.012 & 48 & 1 & 0.53925 \\
\hline 1 & 6 & 920 & 9.92 & 9.67 & 6179.52924 & 0 & 0 & 28 & 49.99 & 0.648 & 0.013 & 49 & 1 & 0.48365 \\
\hline 1 & 6 & 940 & 7.93 & 8.49 & 6196.1679 & 0 & 0 & 28 & 39.96 & 0.569 & 0.014 & 50 & 1 & 0.4243 \\
\hline 1 & 6 & 960 & 5.95 & 7.27 & 6208.65416 & 0 & 0 & 28 & 29.98 & 0.487 & 0.016 & 51 & 1 & 0.36365 \\
\hline 1 & 6 & 980 & 3.96 & 6.09 & 6216.98645 & 0 & 0 & 28 & 19.95 & 0.408 & 0.02 & 52 & 1 & 0.3045 \\
\hline 1 & 6 & 1000 & 1.98 & 3.86 & 6221.1632 & 0 & 0 & 28 & 9.98 & 0.258 & 0.026 & 53 & 1 & 0.1929 \\
\hline 1 & 7 & 1020 & 1.79 & 4.49 & 6224.93311 & 0 & 0 & 28 & 9.02 & 0.301 & 0.033 & 54 & 1 & 0.2247 \\
\hline 1 & 7 & 1040 & 1.59 & 5.04 & 6228.2687 & 0 & 0 & 28 & 8.01 & 0.338 & 0.042 & 55 & 1 & 0.2521 \\
\hline 1 & 7 & 1060 & 1.39 & 5.5 & 6231.18724 & 0 & 0 & 28 & 7 & 0.368 & 0.053 & 56 & 1 & 0.2748 \\
\hline 1 & 7 & 1080 & 1.19 & 5.92 & 6233.69109 & 0 & 0 & 28 & 6 & 0.397 & 0.066 & 57 & 1 & 0.29605 \\
\hline 1 & 7 & 1100 & 0.99 & 6.12 & 6235.7771 & 0 & 0 & 28 & 4.99 & 0.41 & 0.082 & 58 & 1 & 0.30585 \\
\hline 1 & 7 & 1120 & 0.8 & 6.41 & 6237.44686 & 0 & 0 & 28 & 4.03 & 0.429 & 0.106 & 59 & 1 & 0.32025 \\
\hline 1 & 7 & 1140 & 0.6 & 6.92 & 6238.70114 & 0 & 0 & 28 & 3.02 & 0.464 & 0.154 & 60 & 1 & 0.3461 \\
\hline 1 & 7 & 1160 & 0.4 & 7.22 & 6239.53916 & 0 & 0 & 28 & 2.02 & 0.484 & 0.24 & 61 & 1 & 0.3612 \\
\hline 1 & 7 & 1180 & 0.2 & 6.94 & 6239.96092 & 0 & 0 & 28 & 1.01 & 0.465 & 0.46 & 62 & 1 & 0.3468 \\
\hline
\end{tabular}


2004.02.05 189-5 wt\%-U [sonicated] DG 500-4000 s-1 run 1.txt Mode Step t[s] n[rpm] M[\%.] Phi[rad] Gamma

\begin{tabular}{|c|c|c|c|c|c|c|c|c|c|c|c|c|c|}
\hline 1 & 20 & 99.28 & 70.84 & 207.66791 & 0 & 0 & 28 & 500.27 & 4.746 & 0.009 & 1 & 1 & 3.54195 \\
\hline 1 & 40 & 198.46 & 112.22 & 621.85313 & 0 & 0 & 28 & 1000.04 & 7.519 & 0.008 & 2 & 1 & 5.61085 \\
\hline 1 & 60 & 297.68 & 153.61 & 1243.54604 & 0 & 0 & 28 & 1500.01 & 10.292 & 0.007 & 3 & 1 & 7.68035 \\
\hline 1 & 80 & 396.91 & 193.24 & 2073.21318 & 0 & 0 & 28 & 2000.03 & 12.947 & 0.006 & 4 & 1 & 9.66175 \\
\hline 1 & 100 & 496.13 & 230.13 & 3111.16711 & 0 & 0 & 28 & 2500 & 15.419 & 0.006 & 5 & 1 & 11.50635 \\
\hline 1 & 120 & 595.36 & 264.28 & 4355.48363 & 0 & 0 & 28 & 3000.02 & 17.707 & 0.006 & 6 & 1 & 13.214 \\
\hline 1 & 140 & 694.58 & 296.6 & 5808.50322 & 0 & 0 & 28 & 3499.99 & 19.872 & 0.006 & 7 & 1 & 14.82985 \\
\hline 1 & 160 & 793.81 & 328.55 & 7468.92291 & 0 & 0 & 28 & 4000.01 & 22.013 & 0.006 & 8 & 1 & 16.4275 \\
\hline 2 & 180 & 793.81 & 322.04 & 9148.26283 & 0 & 0 & 28 & 4000.01 & 21.576 & 0.005 & 9 & 1 & 16.1018 \\
\hline 2 & 200 & 793.81 & 316.62 & 10812.89382 & 0 & 0 & 28 & 4000.01 & 21.214 & 0.005 & 10 & 1 & 15.83105 \\
\hline 2 & 220 & 793.81 & 312.85 & 12475.03273 & 0 & 0 & 28 & 4000.01 & 20.961 & 0.005 & 11 & 1 & 15.64245 \\
\hline 3 & 240 & 793.81 & 310.94 & 14154.2093 & 0 & 0 & 28 & 4000.01 & 20.833 & 0.005 & 12 & 1 & 15.5472 \\
\hline 3 & 260 & 694.58 & 271.71 & 15611.69937 & 0 & 0 & 28 & 3499.99 & 18.204 & 0.005 & 13 & 1 & 13.5854 \\
\hline 3 & 280 & 595.36 & 232.97 & 16860.02142 & 0 & 0 & 28 & 3000.02 & 15.609 & 0.005 & 14 & 1 & 11.6487 \\
\hline 3 & 300 & 496.13 & 194.42 & 17900.835 & 0 & 0 & 28 & 2500 & 13.026 & 0.005 & 15 & 1 & 9.7212 \\
\hline 3 & 320 & 396.91 & 156.38 & 18733.78195 & 0 & 0 & 28 & 2000.03 & 10.478 & 0.005 & 16 & 1 & 7.8191 \\
\hline 3 & 340 & 297.68 & 118.55 & 19359.22121 & 0 & 0 & 28 & 1500.01 & 7.943 & 0.005 & 17 & 1 & 5.92765 \\
\hline 3 & 360 & 198.46 & 80.19 & 19776.29277 & 0 & 0 & 28 & 1000.04 & 5.372 & 0.005 & 18 & 1 & 4.0093 \\
\hline 3 & 380 & 99.23 & 42.15 & 19985.80872 & 0 & 0 & 28 & 500.02 & 2.824 & 0.006 & 19 & 1 & 2.1077 \\
\hline
\end{tabular}

2004.02.05 189-5 wt\%-U [sonicated] DG 500-4000 s-1 run 2.txt

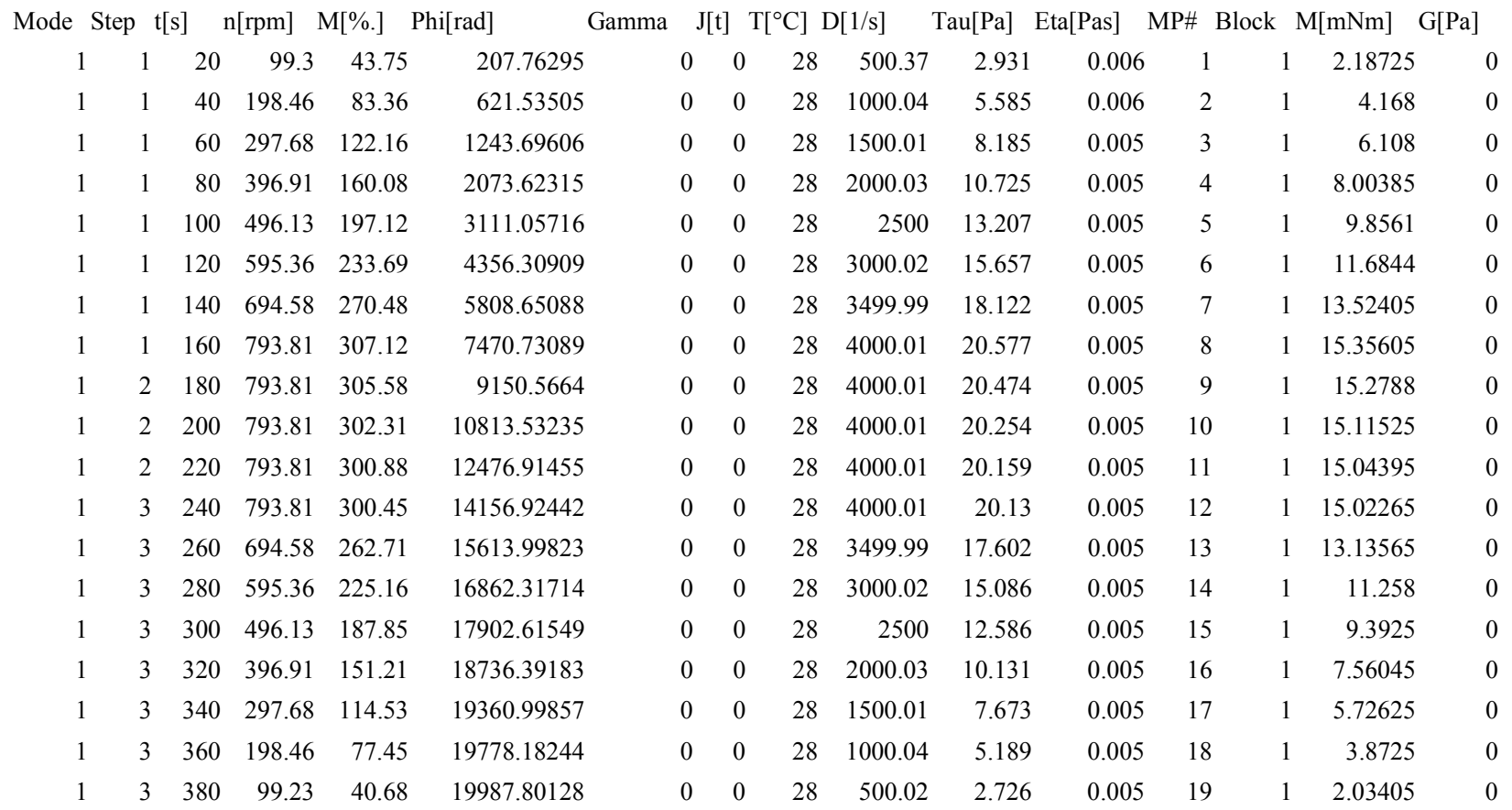



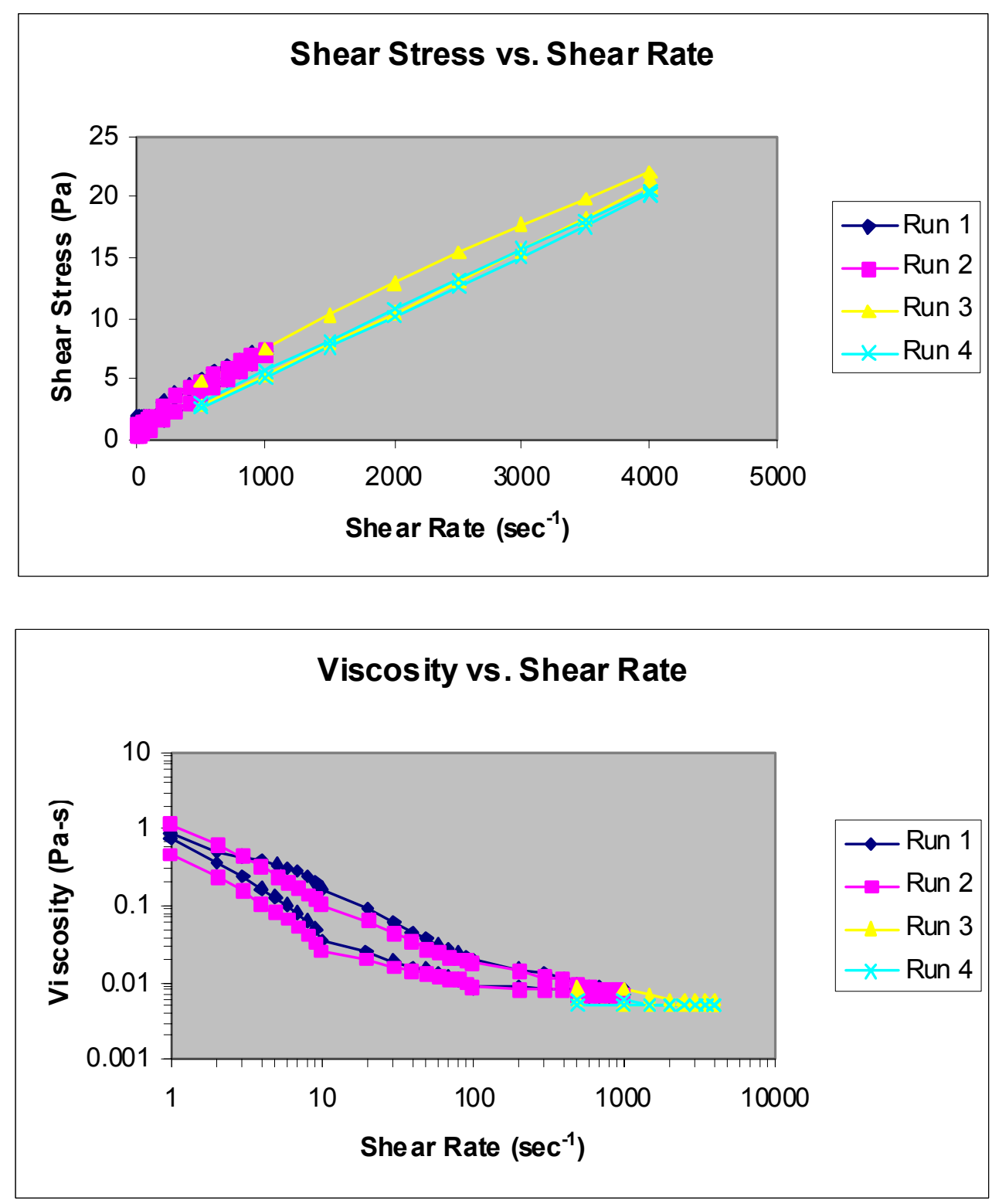

\section{E-2.1.13 META/5wt\%/U/N}

2004.02.06 META-5 wt\%-U DG 1-1000 s-1 run 1.txt

Mode Step t[s] n[rpm] M[\%.] Phi[rad]

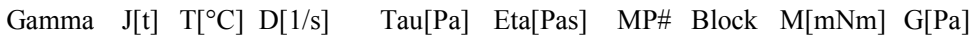

$\begin{array}{rrrrrrrrrrrrrrrr}1 & 1 & 20 & 0.2 & 3.34 & 0.41548 & 0 & 0 & 28 & 1.01 & 0.224 & 0.222 & 1 & 1 & 0.1672 & 0 \\ 1 & 1 & 40 & 0.4 & 8.82 & 1.24564 & 0 & 0 & 28 & 2.02 & 0.591 & 0.293 & 2 & 1 & 0.44125 & 0 \\ 1 & 1 & 60 & 0.6 & 13.73 & 2.48736 & 0 & 0 & 28 & 3.02 & 0.92 & 0.305 & 3 & 1 & 0.68625 & 0 \\ 1 & 1 & 80 & 0.8 & 10.58 & 4.16104 & 0 & 0 & 28 & 4.03 & 0.709 & 0.176 & 4 & 1 & 0.52885 & 0 \\ 1 & 1 & 100 & 1 & 14.41 & 6.23763 & 0 & 0 & 28 & 5.04 & 0.966 & 0.192 & 5 & 1 & 0.7207 & 0 \\ 1 & 1 & 120 & 1.19 & 21.55 & 8.7352 & 0 & 0 & 28 & 6 & 1.444 & 0.241 & 6 & 1 & 1.07725 & 0 \\ 1 & 1 & 140 & 1.39 & 10.17 & 11.6506 & 0 & 0 & 28 & 7 & 0.681 & 0.097 & 7 & 1 & 0.50855 & 0 \\ 1 & 1 & 160 & 1.59 & 10.37 & 14.97833 & 0 & 0 & 28 & 8.01 & 0.695 & 0.087 & 8 & 1 & 0.51835 & 0 \\ 1 & 1 & 180 & 1.79 & 17.71 & 18.7286 & 0 & 0 & 28 & 9.02 & 1.187 & 0.132 & 9 & 1 & 0.8855 & 0 \\ 1 & 2 & 200 & 1.98 & 11.84 & 22.903 & 0 & 0 & 28 & 9.98 & 0.793 & 0.079 & 10 & 1 & 0.59205 & 0\end{array}$




\begin{tabular}{|c|c|c|c|c|c|c|c|c|c|c|c|c|c|c|}
\hline 1 & 2 & 220 & 3.97 & 11.55 & 31.18502 & 0 & 0 & 28 & 20 & 0.774 & 0.039 & 11 & 1 & 0.57755 \\
\hline 1 & 2 & 240 & 5.95 & 11.83 & 43.62258 & 0 & 0 & 28 & 29.98 & 0.792 & 0.026 & 12 & 1 & 0.59125 \\
\hline 1 & 2 & 260 & 7.94 & 9.87 & 60.19763 & 0 & 0 & 28 & 40.01 & 0.661 & 0.017 & 13 & 1 & 0.49365 \\
\hline 1 & 2 & 280 & 9.92 & 10.3 & 80.9667 & 0 & 0 & 28 & 49.99 & 0.69 & 0.014 & 14 & 1 & 0.51485 \\
\hline 1 & 2 & 300 & 11.91 & 8.92 & 105.86617 & 0 & 0 & 28 & 60.01 & 0.598 & 0.01 & 15 & 1 & 0.44615 \\
\hline 1 & 2 & 320 & 13.89 & 7.52 & 134.90784 & 0 & 0 & 28 & 69.99 & 0.504 & 0.007 & 16 & 1 & 0.3758 \\
\hline 1 & 2 & 340 & 15.88 & 7.69 & 168.16867 & 0 & 0 & 28 & 80.02 & 0.515 & 0.006 & 17 & 1 & 0.3843 \\
\hline 1 & 2 & 360 & 17.86 & 7.35 & 205.54498 & 0 & 0 & 28 & 90 & 0.493 & 0.005 & 18 & 1 & 0.36765 \\
\hline 1 & 3 & 380 & 19.85 & 7.57 & 247.47425 & 0 & 0 & 28 & 100.02 & 0.507 & 0.005 & 19 & 1 & 0.3785 \\
\hline 1 & 3 & 400 & 39.69 & 10.82 & 330.37145 & 0 & 0 & 28 & 200 & 0.725 & 0.004 & 20 & 1 & 0.54085 \\
\hline 1 & 3 & 420 & 59.54 & 13.91 & 454.69606 & 0 & 0 & 28 & 300.02 & 0.932 & 0.003 & 21 & 1 & 0.6957 \\
\hline 1 & 3 & 440 & 79.38 & 17.13 & 620.64441 & 0 & 0 & 28 & 400 & 1.148 & 0.003 & 22 & 1 & 0.85655 \\
\hline 1 & 3 & 460 & 99.23 & 20.72 & 828.21729 & 0 & 0 & 28 & 500.02 & 1.388 & 0.003 & 23 & 1 & 1.036 \\
\hline 1 & 3 & 480 & 119.07 & 23.99 & 1077.22777 & 0 & 0 & 28 & 599.99 & 1.607 & 0.003 & 24 & 1 & 1.19925 \\
\hline 1 & 3 & 500 & 138.92 & 27.64 & 1367.70728 & 0 & 0 & 28 & 700.02 & 1.852 & 0.003 & 25 & 1 & 1.38185 \\
\hline 1 & 3 & 520 & 158.76 & 31.07 & 1700.14276 & 0 & 0 & 28 & 799.99 & 2.082 & 0.003 & 26 & 1 & 1.55345 \\
\hline 1 & 3 & 540 & 178.61 & 34.81 & 2073.61059 & 0 & 0 & 28 & 900.02 & 2.333 & 0.003 & 27 & 1 & 1.7407 \\
\hline 1 & 3 & 560 & 198.45 & 38.5 & 2488.80818 & 0 & 0 & 28 & 999.99 & 2.579 & 0.003 & 28 & 1 & 1.92485 \\
\hline 1 & 4 & 570 & 198.45 & 38.26 & 2700.59316 & 0 & 0 & 28 & 999.99 & 2.563 & 0.003 & 29 & 1 & 1.9128 \\
\hline 1 & 4 & 580 & 198.45 & 38.15 & 2908.92866 & 0 & 0 & 28 & 999.99 & 2.556 & 0.003 & 30 & 1 & 1.90745 \\
\hline 1 & 4 & 590 & 198.45 & 38.09 & 3116.43321 & 0 & 0 & 28 & 999.99 & 2.552 & 0.003 & 31 & 1 & 1.90435 \\
\hline 1 & 4 & 600 & 198.45 & 38 & 3323.93855 & 0 & 0 & 28 & 999.99 & 2.546 & 0.003 & 32 & 1 & 1.89985 \\
\hline 1 & 4 & 610 & 198.45 & 37.95 & 3532.27405 & 0 & 0 & 28 & 999.99 & 2.543 & 0.003 & 33 & 1 & 1.89745 \\
\hline 1 & 4 & 620 & 198.45 & 37.92 & 3739.98594 & 0 & 0 & 28 & 999.99 & 2.541 & 0.003 & 34 & 1 & 1.8962 \\
\hline 1 & 5 & 640 & 198.45 & 37.84 & 4159.98311 & 0 & 0 & 28 & 999.99 & 2.535 & 0.003 & 35 & 1 & 1.8921 \\
\hline 1 & 5 & 660 & 178.61 & 33.86 & 4534.83323 & 0 & 0 & 28 & 900.02 & 2.268 & 0.003 & 36 & 1 & 1.69285 \\
\hline 1 & 5 & 680 & 158.76 & 29.94 & 4867.48705 & 0 & 0 & 28 & 799.99 & 2.006 & 0.003 & 37 & 1 & 1.49685 \\
\hline 1 & 5 & 700 & 138.92 & 26.41 & 5158.68206 & 0 & 0 & 28 & 700.02 & 1.769 & 0.003 & 38 & 1 & 1.3203 \\
\hline 1 & 5 & 720 & 119.07 & 22.7 & 5408.38841 & 0 & 0 & 28 & 599.99 & 1.521 & 0.003 & 39 & 1 & 1.13495 \\
\hline 1 & 5 & 740 & 99.23 & 19.22 & 5616.67836 & 0 & 0 & 28 & 500.02 & 1.288 & 0.003 & 40 & 1 & 0.96115 \\
\hline 1 & 5 & 760 & 79.38 & 15.65 & 5783.16628 & 0 & 0 & 28 & 400 & 1.049 & 0.003 & 41 & 1 & 0.78265 \\
\hline 1 & 5 & 780 & 59.54 & 11.92 & 5908.1137 & 0 & 0 & 28 & 300.02 & 0.799 & 0.003 & 42 & 1 & 0.5962 \\
\hline 1 & 5 & 800 & 39.69 & 8.03 & 5991.60231 & 0 & 0 & 28 & 200 & 0.538 & 0.003 & 43 & 1 & 0.4015 \\
\hline 1 & 5 & 820 & 19.85 & 4.47 & 6033.49781 & 0 & 0 & 28 & 100.02 & 0.3 & 0.003 & 44 & 1 & 0.2237 \\
\hline 1 & 6 & 840 & 17.86 & 4.68 & 6071.17021 & 0 & 0 & 28 & 90 & 0.314 & 0.003 & 45 & 1 & 0.23425 \\
\hline 1 & 6 & 860 & 15.88 & 4.48 & 6104.4389 & 0 & 0 & 28 & 80.02 & 0.3 & 0.004 & 46 & 1 & 0.22415 \\
\hline 1 & 6 & 880 & 13.89 & 4.54 & 6133.58109 & 0 & 0 & 28 & 69.99 & 0.304 & 0.004 & 47 & 1 & 0.2272 \\
\hline 1 & 6 & 900 & 11.9 & 4.33 & 6158.53084 & 0 & 0 & 28 & 59.96 & 0.29 & 0.005 & 48 & 1 & 0.21635 \\
\hline 1 & 6 & 920 & 9.92 & 3.84 & 6179.34153 & 0 & 0 & 28 & 49.99 & 0.257 & 0.005 & 49 & 1 & 0.1921 \\
\hline 1 & 6 & 940 & 7.93 & 3.94 & 6195.97705 & 0 & 0 & 28 & 39.96 & 0.264 & 0.007 & 50 & 1 & 0.19705 \\
\hline 1 & 6 & 960 & 5.95 & 3.89 & 6208.46959 & 0 & 0 & 28 & 29.98 & 0.261 & 0.009 & 51 & 1 & 0.19455 \\
\hline 1 & 6 & 980 & 3.96 & 3.95 & 6216.80895 & 0 & 0 & 28 & 19.95 & 0.265 & 0.013 & 52 & 1 & 0.1974 \\
\hline 1 & 6 & 1000 & 1.98 & 3.67 & 6220.9857 & 0 & 0 & 28 & 9.98 & 0.246 & 0.025 & 53 & 1 & 0.1834 \\
\hline 1 & 7 & 1020 & 1.79 & 3.79 & 6224.7564 & 0 & 0 & 28 & 9.02 & 0.254 & 0.028 & 54 & 1 & 0.18945 \\
\hline 1 & 7 & 1040 & 1.59 & 4.87 & 6228.09041 & 0 & 0 & 28 & 8.01 & 0.326 & 0.041 & 55 & 1 & 0.24335 \\
\hline 1 & 7 & 1060 & 1.39 & 4.65 & 6231.00974 & 0 & 0 & 28 & 7 & 0.311 & 0.044 & 56 & 1 & 0.23235 \\
\hline 1 & 7 & 1080 & 1.19 & 5.85 & 6233.51437 & 0 & 0 & 28 & 6 & 0.392 & 0.065 & 57 & 1 & 0.29245 \\
\hline 1 & 7 & 1100 & 0.99 & 5.15 & 6235.60117 & 0 & 0 & 28 & 4.99 & 0.345 & 0.069 & 58 & 1 & 0.25725 \\
\hline 1 & 7 & 1120 & 0.8 & 5.94 & 6237.27015 & 0 & 0 & 28 & 4.03 & 0.398 & 0.099 & 59 & 1 & 0.297 \\
\hline 1 & 7 & 1140 & 0.6 & 6.65 & 6238.52207 & 0 & 0 & 28 & 3.02 & 0.445 & 0.147 & 60 & 1 & 0.33235 \\
\hline
\end{tabular}




$\begin{array}{rrrrrrrrrrrrrrrr}1 & 7 & 1160 & 0.4 & 6.41 & 6239.36088 & 0 & 0 & 28 & 2.02 & 0.43 & 0.213 & 61 & 1 & 0.3206 & 0 \\ 1 & 7 & 1180 & 0.2 & 11.5 & 6239.78185 & 0 & 0 & 28 & 1.01 & 0.771 & 0.763 & 62 & 1 & 0.5752 & 0\end{array}$

2004.02.06 META-5 wt\%-U DG 1-1000 s-1 run 2.txt

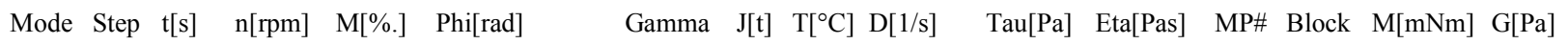

\begin{tabular}{|c|c|c|c|c|c|c|c|c|c|c|c|c|c|c|}
\hline 1 & 1 & 20 & 0.2 & 7.16 & 0.4139 & 0 & 0 & 28 & 1.01 & 0.48 & 0.475 & 1 & 1 & 0.358 \\
\hline 1 & 1 & 40 & 0.4 & 5.49 & 1.24643 & 0 & 0 & 28 & 2.02 & 0.368 & 0.182 & 2 & 1 & 0.27435 \\
\hline 1 & 1 & 60 & 0.6 & 6.03 & 2.496 & 0 & 0 & 28 & 3.02 & 0.404 & 0.134 & 3 & 1 & 0.30165 \\
\hline 1 & 1 & 80 & 0.8 & 5.37 & 4.16104 & 0 & 0 & 28 & 4.03 & 0.36 & 0.089 & 4 & 1 & 0.2685 \\
\hline 1 & 1 & 100 & 1 & 5.96 & 6.24156 & 0 & 0 & 28 & 5.04 & 0.399 & 0.079 & 5 & 1 & 0.29785 \\
\hline 1 & 1 & 120 & 1.19 & 4.91 & 8.73991 & 0 & 0 & 28 & 6 & 0.329 & 0.055 & 6 & 1 & 0.2454 \\
\hline 1 & 1 & 140 & 1.39 & 5.76 & 11.65295 & 0 & 0 & 28 & 7 & 0.386 & 0.055 & 7 & 1 & 0.28775 \\
\hline 1 & 1 & 160 & 1.59 & 4.66 & 14.98461 & 0 & 0 & 28 & 8.01 & 0.312 & 0.039 & 8 & 1 & 0.23295 \\
\hline 1 & 1 & 180 & 1.79 & 4.53 & 18.73175 & 0 & 0 & 28 & 9.02 & 0.304 & 0.034 & 9 & 1 & 0.22655 \\
\hline 1 & 2 & 200 & 1.98 & 4.07 & 22.90614 & 0 & 0 & 28 & 9.98 & 0.272 & 0.027 & 10 & 1 & 0.20325 \\
\hline 1 & 2 & 220 & 3.97 & 4.54 & 31.18345 & 0 & 0 & 28 & 20 & 0.304 & 0.015 & 11 & 1 & 0.2269 \\
\hline 1 & 2 & 240 & 5.95 & 4.36 & 43.61237 & 0 & 0 & 28 & 29.98 & 0.292 & 0.01 & 12 & 1 & 0.21805 \\
\hline 1 & 2 & 260 & 7.94 & 4.42 & 60.20391 & 0 & 0 & 28 & 40.01 & 0.296 & 0.007 & 13 & 1 & 0.22085 \\
\hline 1 & 2 & 280 & 9.92 & 4.63 & 80.95099 & 0 & 0 & 28 & 49.99 & 0.31 & 0.006 & 14 & 1 & 0.23145 \\
\hline 1 & 2 & 300 & 11.91 & 5.26 & 105.86146 & 0 & 0 & 28 & 60.01 & 0.352 & 0.006 & 15 & 1 & 0.26305 \\
\hline 1 & 2 & 320 & 13.89 & 5.38 & 134.93219 & 0 & 0 & 28 & 69.99 & 0.361 & 0.005 & 16 & 1 & 0.26905 \\
\hline 1 & 2 & 340 & 15.88 & 5.47 & 168.16003 & 0 & 0 & 28 & 80.02 & 0.367 & 0.005 & 17 & 1 & 0.2737 \\
\hline 1 & 2 & 360 & 17.86 & 5.83 & 205.50885 & 0 & 0 & 28 & 90 & 0.391 & 0.004 & 18 & 1 & 0.29155 \\
\hline 1 & 3 & 380 & 19.85 & 6.2 & 247.42713 & 0 & 0 & 28 & 100.02 & 0.415 & 0.004 & 19 & 1 & 0.30985 \\
\hline 1 & 3 & 400 & 39.69 & 10.56 & 330.31412 & 0 & 0 & 28 & 200 & 0.708 & 0.004 & 20 & 1 & 0.52815 \\
\hline 1 & 3 & 420 & 59.54 & 14.09 & 454.71098 & 0 & 0 & 28 & 300.02 & 0.944 & 0.003 & 21 & 1 & 0.70445 \\
\hline 1 & 3 & 440 & 79.38 & 17.41 & 620.49361 & 0 & 0 & 28 & 400 & 1.166 & 0.003 & 22 & 1 & 0.8703 \\
\hline 1 & 3 & 460 & 99.23 & 21.06 & 828.16074 & 0 & 0 & 28 & 500.02 & 1.411 & 0.003 & 23 & 1 & 1.0529 \\
\hline 1 & 3 & 480 & 119.07 & 24.42 & 1077.22306 & 0 & 0 & 28 & 599.99 & 1.636 & 0.003 & 24 & 1 & 1.22095 \\
\hline 1 & 3 & 500 & 138.92 & 28.05 & 1367.77483 & 0 & 0 & 28 & 700.02 & 1.88 & 0.003 & 25 & 1 & 1.4027 \\
\hline 1 & 3 & 520 & 158.76 & 31.45 & 1699.79483 & 0 & 0 & 28 & 799.99 & 2.107 & 0.003 & 26 & 1 & 1.57265 \\
\hline 1 & 3 & 540 & 178.61 & 35.23 & 2073.71976 & 0 & 0 & 28 & 900.02 & 2.36 & 0.003 & 27 & 1 & 1.7613 \\
\hline 1 & 3 & 560 & 198.45 & 38.91 & 2488.8341 & 0 & 0 & 28 & 999.99 & 2.607 & 0.003 & 28 & 1 & 1.94555 \\
\hline 1 & 4 & 570 & 198.45 & 38.65 & 2700.72275 & 0 & 0 & 28 & 999.99 & 2.59 & 0.003 & 29 & 1 & 1.9325 \\
\hline 1 & 4 & 580 & 198.45 & 38.56 & 2908.95458 & 0 & 0 & 28 & 999.99 & 2.584 & 0.003 & 30 & 1 & 1.9282 \\
\hline 1 & 4 & 590 & 198.45 & 38.49 & 3116.45913 & 0 & 0 & 28 & 999.99 & 2.579 & 0.003 & 31 & 1 & 1.92465 \\
\hline 1 & 4 & 600 & 198.45 & 38.41 & 3324.69174 & 0 & 0 & 28 & 999.99 & 2.573 & 0.003 & 32 & 1 & 1.92035 \\
\hline 1 & 4 & 610 & 198.45 & 38.34 & 3532.40442 & 0 & 0 & 28 & 999.99 & 2.569 & 0.003 & 33 & 1 & 1.9168 \\
\hline 1 & 4 & 620 & 198.45 & 38.27 & 3740.22078 & 0 & 0 & 28 & 999.99 & 2.564 & 0.003 & 34 & 1 & 1.9137 \\
\hline 1 & 5 & 640 & 198.45 & 38.19 & 4160.63342 & 0 & 0 & 28 & 999.99 & 2.558 & 0.003 & 35 & 1 & 1.90925 \\
\hline 1 & 5 & 660 & 178.61 & 34.16 & 4534.73427 & 0 & 0 & 28 & 900.02 & 2.289 & 0.003 & 36 & 1 & 1.70795 \\
\hline 1 & 5 & 680 & 158.76 & 30.13 & 4867.73131 & 0 & 0 & 28 & 799.99 & 2.019 & 0.003 & 37 & 1 & 1.5064 \\
\hline 1 & 5 & 700 & 138.92 & 26.57 & 5158.92711 & 0 & 0 & 28 & 700.02 & 1.78 & 0.003 & 38 & 1 & 1.3285 \\
\hline 1 & 5 & 720 & 119.07 & 22.77 & 5408.56041 & 0 & 0 & 28 & 599.99 & 1.526 & 0.003 & 39 & 1 & 1.1385 \\
\hline 1 & 5 & 740 & 99.23 & 19.19 & 5616.64145 & 0 & 0 & 28 & 500.02 & 1.286 & 0.003 & 40 & 1 & 0.95945 \\
\hline 1 & 5 & 760 & 79.38 & 15.52 & 5783.29665 & 0 & 0 & 28 & 400 & 1.04 & 0.003 & 41 & 1 & 0.77585 \\
\hline 1 & 5 & 780 & 59.54 & 11.87 & 5908.24329 & 0 & 0 & 28 & 300.02 & 0.795 & 0.003 & 42 & 1 & 0.5935 \\
\hline 1 & 5 & 800 & 39.69 & 7.92 & 5991.7429 & 0 & 0 & 28 & 200 & 0.531 & 0.003 & 43 & 1 & 0.3962 \\
\hline 1 & 5 & 820 & 19.85 & 4.33 & 6033.60698 & 0 & 0 & 28 & 100.02 & 0.29 & 0.003 & 44 & 1 & 0.2163 \\
\hline 1 & 6 & 840 & 17.86 & 4.33 & 6071.27938 & 0 & 0 & 28 & 90 & 0.29 & 0.003 & 45 & 1 & 0.21645 \\
\hline
\end{tabular}




\begin{tabular}{|c|c|c|c|c|c|c|c|c|c|c|c|c|c|c|}
\hline 1 & 6 & 860 & 15.88 & 4.03 & 6104.53943 & 0 & 0 & 28 & 80.02 & 0.27 & 0.003 & 46 & 1 & 0.20165 \\
\hline & 6 & 880 & 13.89 & 3.95 & 6133.65806 & 0 & 0 & 28 & 69.99 & 0.264 & 0.004 & 47 & 1 & 0.19725 \\
\hline & 6 & 900 & 11.9 & 3.69 & 6158.63372 & 0 & 0 & 28 & 59.96 & 0.247 & 0.004 & 48 & 1 & 0.1847 \\
\hline & 6 & 920 & 9.92 & 3.21 & 6179.44913 & 0 & 0 & 28 & 49.99 & 0.215 & 0.004 & 49 & 1 & 0.1603 \\
\hline & 6 & 940 & 7.93 & 3.12 & 6196.08387 & 0 & 0 & 28 & 39.96 & 0.209 & 0.005 & 50 & 1 & 0.15575 \\
\hline & 6 & 960 & 5.95 & 3.05 & 6208.57641 & 0 & 0 & 28 & 29.98 & 0.204 & 0.007 & 51 & 1 & 0.15245 \\
\hline & 6 & 980 & 3.96 & 3.12 & 6216.91498 & 0 & 0 & 28 & 19.95 & 0.209 & 0.01 & 52 & 1 & 0.1559 \\
\hline & 6 & 1000 & 1.98 & 2.57 & 6221.08937 & 0 & 0 & 28 & 9.98 & 0.172 & 0.017 & 53 & 1 & 0.12855 \\
\hline & 7 & 1020 & 1.79 & 2.82 & 6224.85614 & 0 & 0 & 28 & 9.02 & 0.189 & 0.021 & 54 & 1 & 0.14115 \\
\hline & 7 & 1040 & 1.59 & 3.47 & 6228.1933 & 0 & 0 & 28 & 8.01 & 0.233 & 0.029 & 55 & 1 & 0.17355 \\
\hline & 7 & 1060 & 1.39 & 3.82 & 6231.11341 & 0 & 0 & 28.1 & 7 & 0.256 & 0.037 & 56 & 1 & 0.1911 \\
\hline & 7 & 1080 & 1.19 & 4.12 & 6233.61647 & 0 & 0 & 28.1 & 6 & 0.276 & 0.046 & 57 & 1 & 0.2058 \\
\hline 1 & 7 & 1100 & 0.99 & 3.42 & 6235.70171 & 0 & 0 & 28.1 & 4.99 & 0.229 & 0.046 & 58 & 1 & 0.17115 \\
\hline 1 & 7 & 1120 & 0.8 & 4.46 & 6237.37303 & 0 & 0 & 28.1 & 4.03 & 0.299 & 0.074 & 59 & 1 & 0.22295 \\
\hline & 7 & 1140 & 0.6 & 5.13 & 6238.62339 & 0 & 0 & 28.1 & 3.02 & 0.343 & 0.114 & 60 & 1 & 0.2563 \\
\hline & 7 & 1160 & 0.4 & 5.97 & 6239.46376 & 0 & 0 & 28 & 2.02 & 0.4 & 0.198 & 61 & 1 & 0.2984 \\
\hline & 7 & 1180 & 0.2 & 4.99 & 6239.88552 & 0 & 0 & 28 & 1.01 & 0.334 & 0.331 & 62 & 1 & 0.2493 \\
\hline
\end{tabular}

2004.02.06 META-5 wt\%-U DG 500-4000 s-1 run 1.txt

Mode Step t[s] n[rpm] M[\%.] Phi[rad]

$\begin{array}{rrrrrrrrrrrrrrrr}1 & 1 & 20 & 99.23 & 20.96 & 207.67106 & 0 & 0 & 28 & 500.02 & 1.404 & 0.003 & 1 & 1 & 1.04775 & 0 \\ 1 & 1 & 40 & 198.46 & 39.43 & 621.49813 & 0 & 0 & 28 & 1000.04 & 2.642 & 0.003 & 2 & 1 & 1.9716 & 0 \\ 1 & 1 & 60 & 297.68 & 58.18 & 1243.81936 & 0 & 0 & 28 & 1500.01 & 3.898 & 0.003 & 3 & 1 & 2.9092 & 0 \\ 1 & 1 & 80 & 396.91 & 76.09 & 2073.75039 & 0 & 0 & 28 & 2000.03 & 5.098 & 0.003 & 4 & 1 & 3.80445 & 0 \\ 1 & 1 & 100 & 496.13 & 96.25 & 3110.92835 & 0 & 0 & 28 & 2500 & 6.448 & 0.003 & 5 & 1 & 4.8123 & 0 \\ 1 & 1 & 120 & 595.36 & 124.17 & 4355.55746 & 0 & 0 & 28 & 3000.02 & 8.319 & 0.003 & 6 & 1 & 6.20835 & 0 \\ 1 & 1 & 140 & 694.58 & 158.18 & 5808.57627 & 0 & 0 & 28 & 3499.99 & 10.598 & 0.003 & 7 & 1 & 7.9088 & 0 \\ 1 & 1 & 160 & 793.81 & 190.26 & 7469.41221 & 0 & 0 & 28 & 4000.01 & 12.747 & 0.003 & 8 & 1 & 9.5129 & 0 \\ 1 & 2 & 180 & 793.81 & 188.28 & 9148.79611 & 0 & 0 & 28 & 4000.01 & 12.614 & 0.003 & 9 & 1 & 9.41375 & 0 \\ 1 & 2 & 200 & 793.81 & 188.96 & 10812.17989 & 0 & 0 & 28 & 4000.01 & 12.66 & 0.003 & 10 & 1 & 9.44795 & 0 \\ 1 & 2 & 220 & 793.81 & 188.35 & 12474.73271 & 0 & 0 & 28 & 4000.01 & 12.619 & 0.003 & 11 & 1 & 9.4173 & 0 \\ 1 & 3 & 240 & 793.81 & 187.25 & 14155.15413 & 0 & 0 & 28 & 4000.01 & 12.546 & 0.003 & 12 & 1 & 9.36255 & 0 \\ 1 & 3 & 260 & 694.58 & 155.46 & 15610.87942 & 0 & 0 & 28 & 3499.99 & 10.416 & 0.003 & 13 & 1 & 7.77295 & 0 \\ 1 & 3 & 280 & 595.36 & 123.83 & 16859.45829 & 0 & 0 & 28 & 3000.02 & 8.296 & 0.003 & 14 & 1 & 6.1914 & 0 \\ 1 & 3 & 300 & 496.13 & 93.7 & 17900.53262 & 0 & 0 & 28 & 2500 & 6.278 & 0.003 & 15 & 1 & 4.6849 & 0 \\ 1 & 3 & 320 & 396.91 & 68.03 & 18733.10808 & 0 & 0 & 28 & 2000.03 & 4.558 & 0.002 & 16 & 1 & 3.4015 & 0 \\ 1 & 3 & 340 & 297.68 & 50.92 & 19358.92826 & 0 & 0 & 28 & 1500.01 & 3.412 & 0.002 & 17 & 1 & 2.54605 & 0 \\ 1 & 3 & 360 & 198.46 & 33.76 & 19776.13098 & 0 & 0 & 28 & 1000.04 & 2.262 & 0.002 & 18 & 1 & 1.68775 & 0 \\ 1 & 3 & 380 & 99.23 & 17.13 & 19985.54719 & 0 & 0 & 28 & 500.02 & 1.148 & 0.002 & 19 & 1 & 0.85665 & 0\end{array}$

2004.02.06 META-5 wt\%-U DG 500-4000 s-1 run 2.txt

Mode Step t[s] n[rpm] M[\%.] Phi[rad]

Gamma J[t] $\quad \mathrm{T}\left[{ }^{\circ} \mathrm{C}\right] \quad \mathrm{D}[1 / \mathrm{s}] \quad \mathrm{Tau}[\mathrm{Pa}] \quad$ Eta[Pas $] \quad$ MP\# Block $\mathrm{M}[\mathrm{mNm}] \mathrm{G}[\mathrm{Pa}]$

$\begin{array}{rrrrrrrrrrrrrrrr}1 & 1 & 20 & 99.31 & 16.88 & 207.77944 & 0 & 0 & 28 & 500.42 & 1.131 & 0.002 & 1 & 1 & 0.8439 & 0 \\ 1 & 1 & 40 & 198.46 & 34.56 & 621.45179 & 0 & 0 & 28 & 1000.04 & 2.315 & 0.002 & 2 & 1 & 1.7278 & 0 \\ 1 & 1 & 60 & 297.68 & 52.15 & 1243.51227 & 0 & 0 & 28 & 1500.01 & 3.494 & 0.002 & 3 & 1 & 2.6073 & 0 \\ 1 & 1 & 80 & 396.91 & 69.66 & 2073.85956 & 0 & 0 & 28 & 2000.03 & 4.667 & 0.002 & 4 & 1 & 3.48275 & 0 \\ 1 & 1 & 100 & 496.13 & 93.56 & 3110.82782 & 0 & 0 & 28 & 2500 & 6.269 & 0.003 & 5 & 1 & 4.6782 & 0 \\ 1 & 1 & 120 & 595.36 & 123.89 & 4356.75755 & 0 & 0 & 28 & 3000.02 & 8.301 & 0.003 & 6 & 1 & 6.19455 & 0 \\ 1 & 1 & 140 & 694.58 & 156.64 & 5809.77321 & 0 & 0 & 28 & 3499.99 & 10.495 & 0.003 & 7 & 1 & 7.8319 & 0 \\ 1 & 1 & 160 & 793.81 & 187.71 & 7471.02542 & 0 & 0 & 28 & 4000.01 & 12.577 & 0.003 & 8 & 1 & 9.38555 & 0\end{array}$




$\begin{array}{rrrrrrrrrrrrrrrrr}1 & 2 & 180 & 793.81 & 186.56 & 9150.4643 & 0 & 0 & 28 & 4000.01 & 12.499 & 0.003 & 9 & 1 & 9.3278 & 0 \\ 1 & 2 & 200 & 793.81 & 186.87 & 10814.26198 & 0 & 0 & 28 & 4000.01 & 12.52 & 0.003 & 10 & 1 & 9.3434 & 0 \\ 1 & 2 & 220 & 793.81 & 186.71 & 12475.56759 & 0 & 0 & 28 & 4000.01 & 12.51 & 0.003 & 11 & 1 & 9.33545 & 0 \\ 1 & 3 & 240 & 793.81 & 186.27 & 14155.57353 & 0 & 0 & 28 & 4000.01 & 12.48 & 0.003 & 12 & 1 & 9.3136 & 0 \\ 1 & 3 & 260 & 694.58 & 154.75 & 15612.69918 & 0 & 0 & 28 & 3499.99 & 10.369 & 0.003 & 13 & 1 & 7.7377 & 0 \\ 1 & 3 & 280 & 595.36 & 123.46 & 16861.64327 & 0 & 0 & 28 & 3000.02 & 8.272 & 0.003 & 14 & 1 & 6.1728 & 0 \\ 1 & 3 & 300 & 496.13 & 93.4 & 17901.85052 & 0 & 0 & 28 & 2500 & 6.258 & 0.003 & 15 & 1 & 4.6699 & 0 \\ 1 & 3 & 320 & 396.91 & 67.37 & 18734.83046 & 0 & 0 & 28 & 2000.03 & 4.514 & 0.002 & 16 & 1 & 3.3684 & 0 \\ 1 & 3 & 340 & 297.68 & 50.4 & 19359.82283 & 0 & 0 & 28 & 1500.01 & 3.376 & 0.002 & 17 & 1 & 2.51975 & 0 \\ 1 & 3 & 360 & 198.46 & 33.4 & 19777.28551 & 0 & 0 & 28 & 1000.04 & 2.238 & 0.002 & 18 & 1 & 1.6702 & 0 \\ 1 & 3 & 380 & 99.23 & 17.01 & 19986.80932 & 0 & 0 & 28.1 & 500.02 & 1.14 & 0.002 & 19 & 1 & 0.85055 & 0\end{array}$
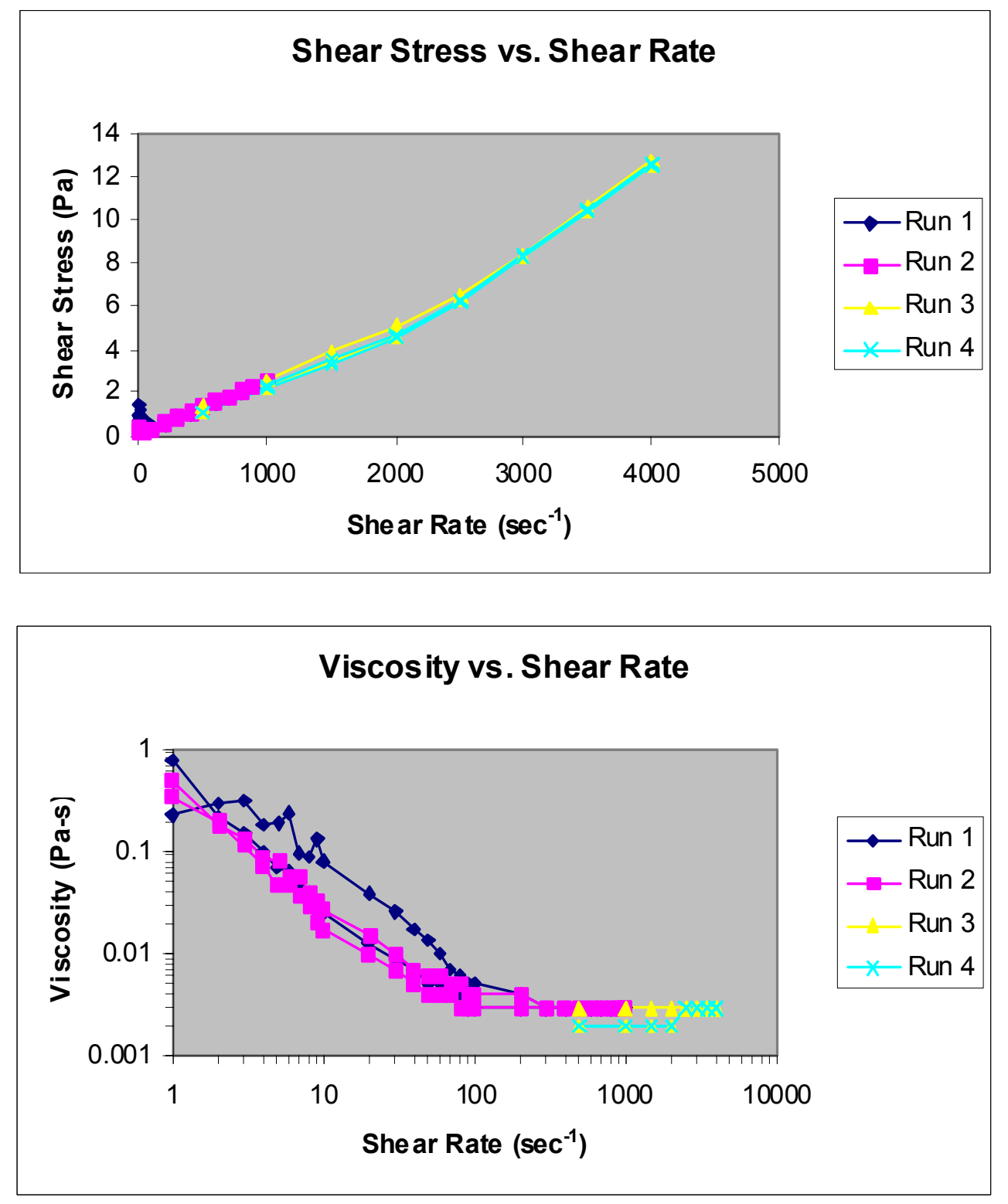


\section{E-2.1.14 META/5wt\%/U/S}

2004.02.04 META-5 wt\%-U [sonicated] DG 1-1000 s-1 run 1.txt

Mode Step $\mathrm{t}[\mathrm{s}] \quad \mathrm{n}[\mathrm{rpm}] \quad \mathrm{M}[\%$.] $\quad$ Phi[rad]

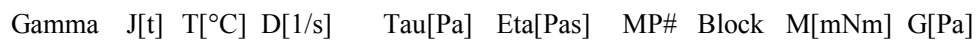

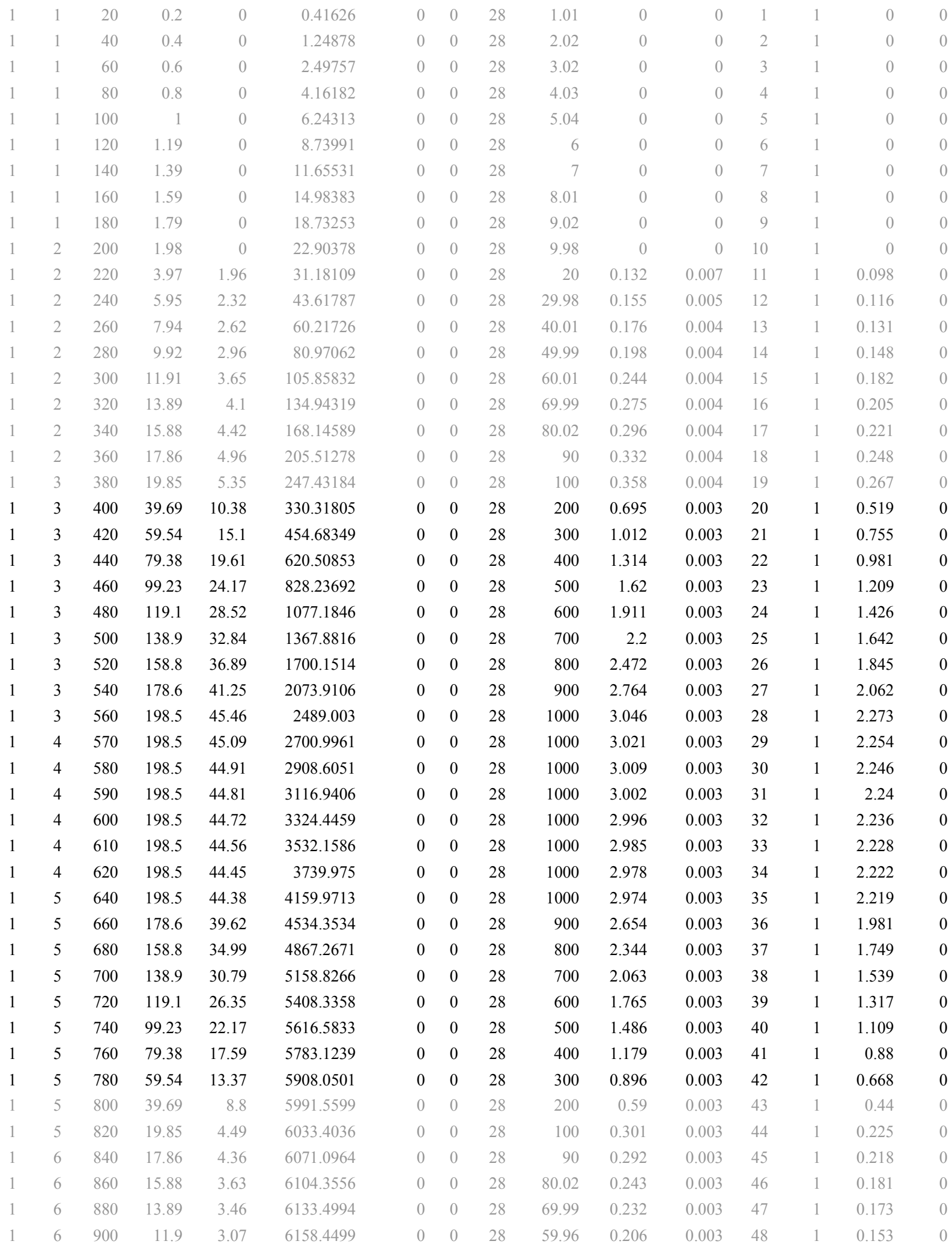




\begin{tabular}{|c|c|c|c|c|c|c|c|c|c|c|c|c|c|c|}
\hline 1 & 6 & 920 & 9.92 & 2.41 & 6179.2449 & 0 & 0 & 28 & 49.99 & 0.161 & 0.003 & 49 & 1 & 0.121 \\
\hline 1 & 6 & 940 & 7.93 & 2.14 & 6195.8922 & 0 & 0 & 28 & 39.96 & 0.143 & 0.004 & 50 & 1 & 0.107 \\
\hline 1 & 6 & 960 & 5.95 & 1.82 & 6208.3824 & 0 & 0 & 28 & 29.98 & 0.122 & 0.004 & 51 & 1 & 0.091 \\
\hline 1 & 6 & 980 & 3.96 & 1.41 & 6216.7249 & 0 & 0 & 28 & 19.95 & 0.094 & 0.005 & 52 & 1 & 0.07 \\
\hline 1 & 6 & 1000 & 1.98 & 0 & 6220.8993 & 0 & 0 & 28 & 9.98 & 0 & 0 & 53 & 1 & 0 \\
\hline 1 & 7 & 1020 & 1.79 & 0 & 6224.6708 & 0 & 0 & 28 & 9.02 & 0 & 0 & 54 & 1 & 0 \\
\hline 1 & 7 & 1040 & 1.59 & 0 & 6228.0056 & 0 & 0 & 28 & 8.01 & 0 & 0 & 55 & 1 & 0 \\
\hline 1 & 7 & 1060 & 1.39 & 0 & 6230.9257 & 0 & 0 & 28 & 7 & 0 & 0 & 56 & 1 & 0 \\
\hline 1 & 7 & 1080 & 1.19 & 0 & 6233.428 & 0 & 0 & 28 & 6 & 0 & 0 & 57 & 1 & 0 \\
\hline 1 & 7 & 1100 & 0.99 & 0 & 6235.5156 & 0 & 0 & 28 & 4.99 & 0 & 0 & 58 & 1 & 0 \\
\hline 1 & 7 & 1120 & 0.8 & 0 & 6237.1853 & 0 & 0 & 28 & 4.03 & 0 & 0 & 59 & 1 & 0 \\
\hline 1 & 7 & 1140 & 0.6 & 0 & 6238.4396 & 0 & 0 & 28 & 3.02 & 0 & 0 & 60 & 1 & 0 \\
\hline 1 & 7 & 1160 & 0.4 & 0 & 6239.2776 & 0 & 0 & 28 & 2.02 & 0 & 0 & 61 & 1 & 0 \\
\hline 1 & 7 & 1180 & 0.2 & 0 & 6239.6986 & 0 & 0 & 28 & 1.01 & 0 & 0 & 62 & 1 & 0 \\
\hline
\end{tabular}

2004.02.04 META-5 wt\%-U [sonicated] DG 1-1000 s-1 run 2.txt

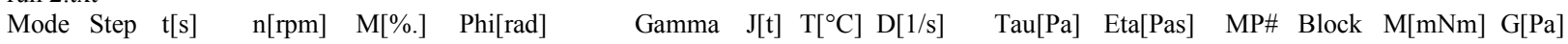

\begin{tabular}{|c|c|c|c|c|c|c|c|c|c|c|c|c|c|c|}
\hline 1 & 1 & 20 & 0.2 & 0 & 0.41626 & 0 & 0 & 28 & 1.01 & 0 & 0 & 1 & 1 & 0 \\
\hline 1 & 1 & 40 & 0.4 & 0 & 1.248 & 0 & 0 & 28 & 2.02 & 0 & 0 & 2 & 1 & 0 \\
\hline 1 & 1 & 60 & 0.6 & 0 & 2.49757 & 0 & 0 & 28 & 3.02 & 0 & 0 & 3 & 1 & 0 \\
\hline 1 & 1 & 80 & 0.8 & 0 & 4.16261 & 0 & 0 & 28 & 4.03 & 0 & 0 & 4 & 1 & 0 \\
\hline 1 & 1 & 100 & 1 & 0 & 6.24234 & 0 & 0 & 28 & 5.04 & 0 & 0 & 5 & 1 & 0 \\
\hline 1 & 1 & 120 & 1.19 & 0 & 8.74227 & 0 & 0 & 28 & 6 & 0 & 0 & 6 & 1 & 0 \\
\hline 1 & 1 & 140 & 1.39 & 0 & 11.65452 & 0 & 0 & 28 & 7 & 0 & 0 & 7 & 1 & 0 \\
\hline 1 & 1 & 160 & 1.59 & 0 & 14.98461 & 0 & 0 & 28 & 8.01 & 0 & 0 & 8 & 1 & 0 \\
\hline 1 & 1 & 180 & 1.79 & 0 & 18.73018 & 0 & 0 & 28 & 9.02 & 0 & 0 & 9 & 1 & 0 \\
\hline 1 & 2 & 200 & 1.98 & 0 & 22.90378 & 0 & 0 & 28 & 9.98 & 0 & 0 & 10 & 1 & 0 \\
\hline 1 & 2 & 220 & 3.97 & 1.66 & 31.18109 & 0 & 0 & 28 & 20 & 0.111 & 0.006 & 11 & 1 & 0.083 \\
\hline 1 & 2 & 240 & 5.95 & 2.16 & 43.6218 & 0 & 0 & 28 & 29.98 & 0.145 & 0.005 & 12 & 1 & 0.108 \\
\hline 1 & 2 & 260 & 7.94 & 2.54 & 60.19684 & 0 & 0 & 28 & 40.01 & 0.17 & 0.004 & 13 & 1 & 0.127 \\
\hline 1 & 2 & 280 & 9.92 & 2.93 & 80.94471 & 0 & 0 & 28 & 49.99 & 0.196 & 0.004 & 14 & 1 & 0.146 \\
\hline 1 & 2 & 300 & 11.91 & 3.64 & 105.86775 & 0 & 0 & 28 & 60.01 & 0.244 & 0.004 & 15 & 1 & 0.182 \\
\hline 1 & 2 & 320 & 13.89 & 4.16 & 134.90784 & 0 & 0 & 28 & 69.99 & 0.279 & 0.004 & 16 & 1 & 0.208 \\
\hline 1 & 2 & 340 & 15.88 & 4.4 & 168.12704 & 0 & 0 & 28 & 80.02 & 0.295 & 0.004 & 17 & 1 & 0.22 \\
\hline 1 & 2 & 360 & 17.86 & 4.97 & 205.50257 & 0 & 0 & 28 & 90 & 0.333 & 0.004 & 18 & 1 & 0.249 \\
\hline 1 & 3 & 380 & 19.85 & 5.38 & 247.42163 & 0 & 0 & 28 & 100 & 0.361 & 0.004 & 19 & 1 & 0.269 \\
\hline 1 & 3 & 400 & 39.69 & 10.37 & 330.30784 & 0 & 0 & 28 & 200 & 0.695 & 0.003 & 20 & 1 & 0.519 \\
\hline 1 & 3 & 420 & 59.54 & 15.28 & 454.7047 & 0 & 0 & 28 & 300 & 1.024 & 0.003 & 21 & 1 & 0.764 \\
\hline 1 & 3 & 440 & 79.38 & 19.76 & 620.62163 & 0 & 0 & 28 & 400 & 1.324 & 0.003 & 22 & 1 & 0.988 \\
\hline 1 & 3 & 460 & 99.23 & 24.32 & 828.01858 & 0 & 0 & 28 & 500 & 1.629 & 0.003 & 23 & 1 & 1.216 \\
\hline 1 & 3 & 480 & 119.1 & 28.64 & 1077.278 & 0 & 0 & 28 & 600 & 1.919 & 0.003 & 24 & 1 & 1.432 \\
\hline 1 & 3 & 500 & 138.9 & 33 & 1367.6005 & 0 & 0 & 28 & 700 & 2.211 & 0.003 & 25 & 1 & 1.65 \\
\hline 1 & 3 & 520 & 158.8 & 37.05 & 1700.12 & 0 & 0 & 28 & 800 & 2.482 & 0.003 & 26 & 1 & 1.853 \\
\hline 1 & 3 & 540 & 178.6 & 41.47 & 2073.5776 & 0 & 0 & 28 & 900 & 2.779 & 0.003 & 27 & 1 & 2.074 \\
\hline 1 & 3 & 560 & 198.5 & 45.68 & 2489.2001 & 0 & 0 & 28 & 1000 & 3.061 & 0.003 & 28 & 1 & 2.284 \\
\hline 1 & 4 & 570 & 198.5 & 45.45 & 2701.0895 & 0 & 0 & 28 & 1000 & 3.045 & 0.003 & 29 & 1 & 2.273 \\
\hline 1 & 4 & 580 & 198.5 & 45.24 & 2909.3221 & 0 & 0 & 28 & 1000 & 3.031 & 0.003 & 30 & 1 & 2.262 \\
\hline 1 & 4 & 590 & 198.5 & 45.05 & 3117.0348 & 0 & 0 & 28 & 1000 & 3.018 & 0.003 & 31 & 1 & 2.253 \\
\hline 1 & 4 & 600 & 198.5 & 44.91 & 3324.5394 & 0 & 0 & 28 & 1000 & 3.009 & 0.003 & 32 & 1 & 2.246 \\
\hline 1 & 4 & 610 & 198.5 & 44.76 & 3532.3557 & 0 & 0 & 28 & 1000 & 2.999 & 0.003 & 33 & 1 & 2.238 \\
\hline 1 & 4 & 620 & 198.5 & 44.67 & 3740.0684 & 0 & 0 & 28 & 1000 & 2.993 & 0.003 & 34 & 1 & 2.234 \\
\hline
\end{tabular}




\begin{tabular}{|c|c|c|c|c|c|c|c|c|c|c|c|c|c|c|}
\hline 1 & 5 & 640 & 198.5 & 44.55 & 4160.5839 & 0 & 0 & 28 & 1000 & 2.985 & 0.003 & 35 & 1 & 2.228 \\
\hline 1 & 5 & 660 & 178.6 & 39.78 & 4534.8733 & 0 & 0 & 28 & 900 & 2.665 & 0.003 & 36 & 1 & 1.989 \\
\hline 1 & 5 & 680 & 158.8 & 35.18 & 4867.5263 & 0 & 0 & 28 & 800 & 2.357 & 0.003 & 37 & 1 & 1.759 \\
\hline 1 & 5 & 700 & 138.9 & 30.97 & 5158.6499 & 0 & 0 & 28 & 700 & 2.075 & 0.003 & 38 & 1 & 1.548 \\
\hline 1 & 5 & 720 & 119.1 & 26.47 & 5408.5942 & 0 & 0 & 28 & 600 & 1.774 & 0.003 & 39 & 1 & 1.324 \\
\hline 1 & 5 & 740 & 99.23 & 22.33 & 5616.5731 & 0 & 0 & 28 & 500 & 1.496 & 0.003 & 40 & 1 & 1.117 \\
\hline 1 & 5 & 760 & 79.38 & 17.77 & 5783.1757 & 0 & 0 & 28 & 400 & 1.191 & 0.003 & 41 & 1 & 0.889 \\
\hline 1 & 5 & 780 & 59.54 & 13.43 & 5908.1333 & 0 & 0 & 28 & 300 & 0.9 & 0.003 & 42 & 1 & 0.672 \\
\hline 1 & 5 & 800 & 39.69 & 8.77 & 5991.6329 & 0 & 0 & 28 & 200 & 0.588 & 0.003 & 43 & 1 & 0.439 \\
\hline 1 & 5 & 820 & 19.85 & 4.46 & 6033.5182 & 0 & 0 & 28 & 100 & 0.299 & 0.003 & 44 & 1 & 0.223 \\
\hline 1 & 6 & 840 & 17.86 & 4.18 & 6071.1899 & 0 & 0 & 28 & 90 & 0.28 & 0.003 & 45 & 1 & 0.209 \\
\hline 1 & 6 & 860 & 15.88 & 3.69 & 6104.4907 & 0 & 0 & 28 & 80.02 & 0.247 & 0.003 & 46 & 1 & 0.184 \\
\hline 1 & 6 & 880 & 13.89 & 3.43 & 6133.5882 & 0 & 0 & 28 & 69.99 & 0.23 & 0.003 & 47 & 1 & 0.172 \\
\hline 1 & 6 & 900 & 11.9 & 3.03 & 6158.5497 & 0 & 0 & 28 & 59.96 & 0.203 & 0.003 & 48 & 1 & 0.151 \\
\hline 1 & 6 & 920 & 9.92 & 2.34 & 6179.3659 & 0 & 0 & 28 & 49.99 & 0.157 & 0.003 & 49 & 1 & 0.117 \\
\hline 1 & 6 & 940 & 7.93 & 2.05 & 6196.0171 & 0 & 0 & 28 & 39.96 & 0.137 & 0.003 & 50 & 1 & 0.102 \\
\hline 1 & 6 & 960 & 5.95 & 1.82 & 6208.5002 & 0 & 0 & 28 & 29.98 & 0.122 & 0.004 & 51 & 1 & 0.091 \\
\hline 1 & 6 & 980 & 3.96 & 1.4 & 6216.8325 & 0 & 0 & 28 & 19.95 & 0.094 & 0.005 & 52 & 1 & 0.07 \\
\hline 1 & 6 & 1000 & 1.98 & 0 & 6221.0085 & 0 & 0 & 28 & 9.98 & 0 & 0 & 53 & 1 & 0 \\
\hline 1 & 7 & 1020 & 1.79 & 0 & 6224.7768 & 0 & 0 & 28 & 9.02 & 0 & 0 & 54 & 1 & 0 \\
\hline 1 & 7 & 1040 & 1.59 & 0 & 6228.114 & 0 & 0 & 28 & 8.01 & 0 & 0 & 55 & 1 & 0 \\
\hline 1 & 7 & 1060 & 1.39 & 0 & 6231.0333 & 0 & 0 & 28 & 7 & 0 & 0 & 56 & 1 & 0 \\
\hline 1 & 7 & 1080 & 1.19 & 0 & 6233.5364 & 0 & 0 & 28 & 6 & 0 & 0 & 57 & 1 & 0 \\
\hline 1 & 7 & 1100 & 0.99 & 0 & 6235.6224 & 0 & 0 & 28 & 4.99 & 0 & 0 & 58 & 1 & 0 \\
\hline 1 & 7 & 1120 & 0.8 & 0 & 6237.2921 & 0 & 0 & 28 & 4.03 & 0 & 0 & 59 & 1 & 0 \\
\hline 1 & 7 & 1140 & 0.6 & 0 & 6238.5472 & 0 & 0 & 28 & 3.02 & 0 & 0 & 60 & 1 & 0 \\
\hline 1 & 7 & 1160 & 0.4 & 0 & 6239.3844 & 0 & 0 & 28 & 2.02 & 0 & 0 & 61 & 1 & 0 \\
\hline 1 & 7 & 1180 & 0.2 & 0 & 6239.807 & 0 & 0 & 28 & 1.01 & 0 & 0 & 62 & 1 & 0 \\
\hline
\end{tabular}

2004.02.04 META-5 wt\%-U [sonicated] DG 500-4000 s-

1 run 1.txt

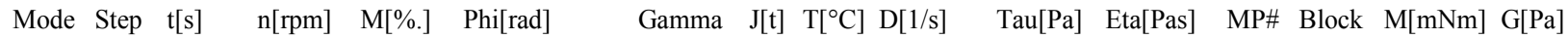

\begin{tabular}{|c|c|c|c|c|c|c|c|c|c|c|c|c|c|c|}
\hline 1 & 1 & 20 & 99.24 & 23.11 & 207.47706 & 0 & 0 & 28 & 500.1 & 1.548 & 0.003 & 1 & 1 & 1.155 \\
\hline 1 & 1 & 40 & 198.5 & 46.16 & 621.66699 & 0 & 0 & 28 & 1000 & 3.092 & 0.003 & 2 & 1 & 2.308 \\
\hline 1 & 1 & 60 & 297.7 & 68.01 & 1243.4157 & 0 & 0 & 28 & 1500 & 4.557 & 0.003 & 3 & 1 & 3.401 \\
\hline 1 & 1 & 80 & 396.9 & 88.87 & 2073.9711 & 0 & 0 & 28 & 2000 & 5.954 & 0.003 & 4 & 1 & 4.444 \\
\hline 1 & 1 & 100 & 496.1 & 108.2 & 3111.4601 & 0 & 0 & 28 & 2500 & 7.247 & 0.003 & 5 & 1 & 5.408 \\
\hline 1 & 1 & 120 & 595.4 & 136.5 & 4356.6625 & 0 & 0 & 28 & 3000 & 9.143 & 0.003 & 6 & 1 & 6.823 \\
\hline 1 & 1 & 140 & 694.6 & 168.9 & 5808.2252 & 0 & 0 & 28 & 3500 & 11.32 & 0.003 & 7 & 1 & 8.444 \\
\hline 1 & 1 & 160 & 793.8 & 205.4 & 7471.1362 & 0 & 0 & 28 & 4000 & 13.76 & 0.003 & 8 & 1 & 10.27 \\
\hline 1 & 2 & 180 & 793.8 & 204.4 & 9152.2377 & 0 & 0 & 28 & 4000 & 13.69 & 0.003 & 9 & 1 & 10.22 \\
\hline 1 & 2 & 200 & 793.8 & 203.4 & 10814.789 & 0 & 0 & 28 & 4000 & 13.63 & 0.003 & 10 & 1 & 10.17 \\
\hline 1 & 2 & 220 & 793.8 & 202.1 & 12476.925 & 0 & 0 & 28 & 4000 & 13.54 & 0.003 & 11 & 1 & 10.1 \\
\hline 1 & 3 & 240 & 793.8 & 200.7 & 14156.101 & 0 & 0 & 28 & 4000 & 13.45 & 0.003 & 12 & 1 & 10.04 \\
\hline 1 & 3 & 260 & 694.6 & 165 & 15613.955 & 0 & 0 & 28 & 3500 & 11.05 & 0.003 & 13 & 1 & 8.25 \\
\hline 1 & 3 & 280 & 595.4 & 131.5 & 16861.913 & 0 & 0 & 28 & 3000 & 8.808 & 0.003 & 14 & 1 & 6.573 \\
\hline 1 & 3 & 300 & 496.1 & 96.93 & 17903.047 & 0 & 0 & 28 & 2500 & 6.494 & 0.003 & 15 & 1 & 4.846 \\
\hline 1 & 3 & 320 & 396.9 & 76.57 & 18735.39 & 0 & 0 & 28 & 2000 & 5.13 & 0.003 & 16 & 1 & 3.828 \\
\hline 1 & 3 & 340 & 297.7 & 57.32 & 19361.365 & 0 & 0 & 28 & 1500 & 3.84 & 0.003 & 17 & 1 & 2.866 \\
\hline 1 & 3 & 360 & 198.5 & 37.73 & 19778.563 & 0 & 0 & 28 & 1000 & 2.528 & 0.003 & 18 & 1 & 1.886 \\
\hline 1 & 3 & 380 & 99.23 & 18.93 & 19988.031 & 0 & 0 & 28 & 500 & 1.268 & 0.003 & 19 & 1 & 0.946 \\
\hline
\end{tabular}


2004.02.04 META-5 wt\%-U [sonicated] DG 500-4000 s-

1 run 2.txt

Mode Step $\mathrm{t}[\mathrm{s}] \quad \mathrm{n}[\mathrm{rpm}] \quad \mathrm{M}[\%$. $] \quad$ Phi[rad] $\quad$ Ga

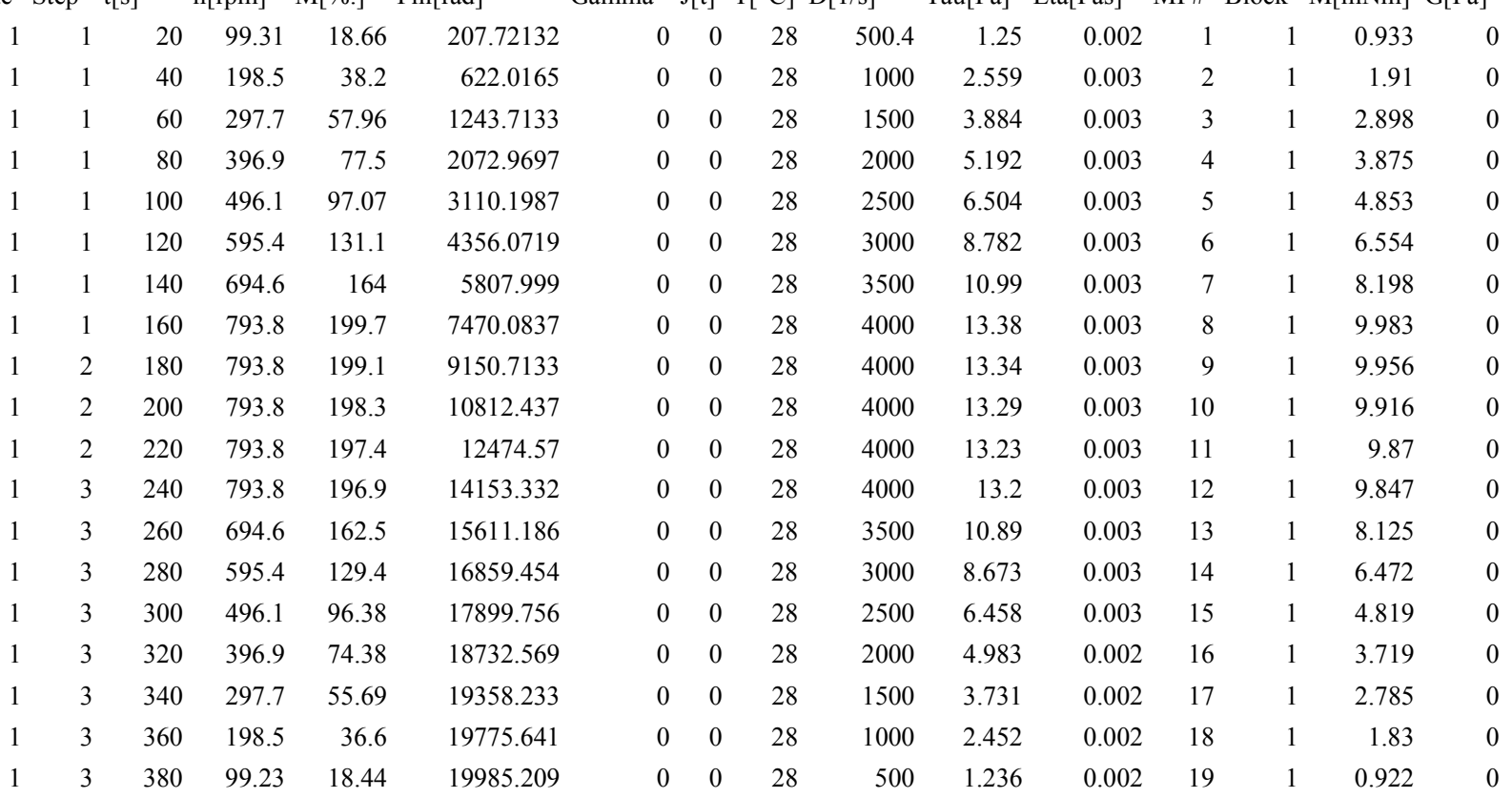

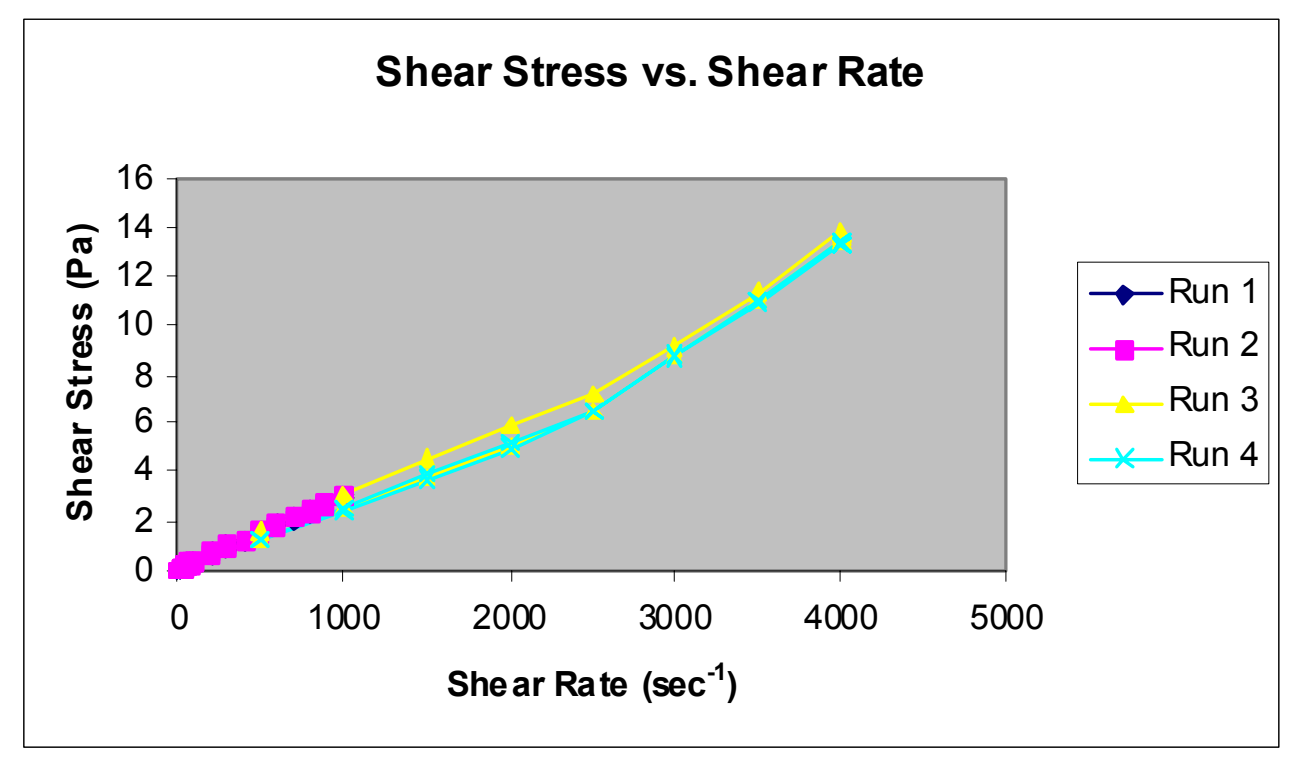




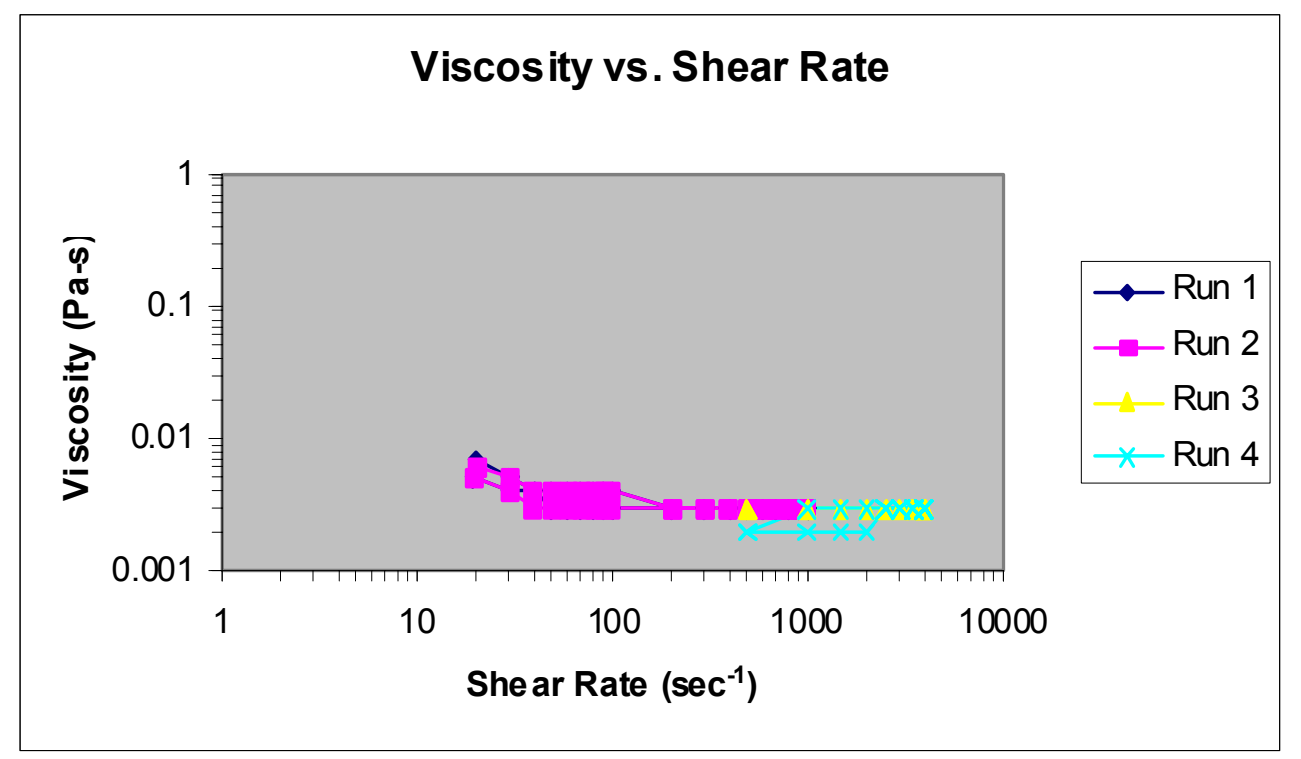

\section{E-2.1.15 META/10wt\%/U/N}

2004.02.09 META-10 wt\%-U DG 1-1000 s-1 run 1.txt

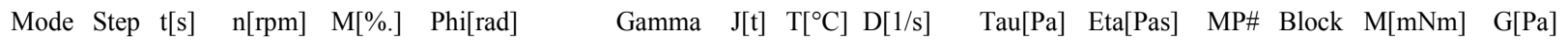

\begin{tabular}{|c|c|c|c|c|c|c|c|c|c|c|c|c|c|c|}
\hline 1 & 1 & 20 & 0.19 & 47.71 & 0.39034 & 0 & 0 & 28 & 0.96 & 3.196 & 3.329 & 1 & 1 & 2.38525 \\
\hline 1 & 1 & 40 & 0.4 & 109.43 & 1.21187 & 0 & 0 & 28 & 2.02 & 7.332 & 3.63 & 2 & 1 & 5.47135 \\
\hline 1 & 1 & 60 & 0.6 & 108.28 & 2.46694 & 0 & 0 & 28 & 3.02 & 7.255 & 2.402 & 3 & 1 & 5.41385 \\
\hline 1 & 1 & 80 & 0.79 & 122.95 & 4.10999 & 0 & 0 & 28 & 3.98 & 8.238 & 2.07 & 4 & 1 & 6.14765 \\
\hline 1 & 1 & 100 & 0.99 & 163.9 & 6.17637 & 0 & 0 & 28 & 4.99 & 10.982 & 2.201 & 5 & 1 & 8.19515 \\
\hline 1 & 1 & 120 & 1.21 & 114.24 & 8.70378 & 0 & 0 & 28 & 6.1 & 7.654 & 1.255 & 6 & 1 & 5.71205 \\
\hline 1 & 1 & 140 & 1.39 & 92.9 & 11.62546 & 0 & 0 & 28.1 & 7 & 6.225 & 0.889 & 7 & 1 & 4.6452 \\
\hline 1 & 1 & 160 & 1.59 & 103.18 & 14.96184 & 0 & 0 & 28.1 & 8.01 & 6.913 & 0.863 & 8 & 1 & 5.1592 \\
\hline 1 & 1 & 180 & 1.78 & 74.04 & 18.69405 & 0 & 0 & 28.1 & 8.97 & 4.961 & 0.553 & 9 & 1 & 3.70205 \\
\hline 1 & 2 & 200 & 1.99 & 68.31 & 22.88964 & 0 & 0 & 28 & 10.03 & 4.577 & 0.456 & 10 & 1 & 3.4153 \\
\hline 1 & 2 & 220 & 3.95 & 86.04 & 31.14339 & 0 & 0 & 28 & 19.9 & 5.764 & 0.29 & 11 & 1 & 4.30185 \\
\hline 1 & 2 & 240 & 5.96 & 75.12 & 43.5786 & 0 & 0 & 28 & 30.03 & 5.033 & 0.168 & 12 & 1 & 3.7562 \\
\hline 1 & 2 & 260 & 7.94 & 57.61 & 60.17642 & 0 & 0 & 28 & 40.01 & 3.86 & 0.096 & 13 & 1 & 2.8803 \\
\hline 1 & 2 & 280 & 9.92 & 45.28 & 80.93292 & 0 & 0 & 28 & 49.99 & 3.034 & 0.061 & 14 & 1 & 2.2641 \\
\hline 1 & 2 & 300 & 11.91 & 39.77 & 105.83476 & 0 & 0 & 28 & 60.01 & 2.665 & 0.044 & 15 & 1 & 1.9885 \\
\hline 1 & 2 & 320 & 13.89 & 35.77 & 134.89135 & 0 & 0 & 28 & 69.99 & 2.397 & 0.034 & 16 & 1 & 1.78845 \\
\hline 1 & 2 & 340 & 15.88 & 34.34 & 168.1349 & 0 & 0 & 28 & 80.02 & 2.301 & 0.029 & 17 & 1 & 1.71715 \\
\hline 1 & 2 & 360 & 17.86 & 23.37 & 205.49393 & 0 & 0 & 28 & 90 & 1.566 & 0.017 & 18 & 1 & 1.16855 \\
\hline 1 & 3 & 380 & 19.85 & 21.5 & 247.42241 & 0 & 0 & 28 & 100.02 & 1.44 & 0.014 & 19 & 1 & 1.0748 \\
\hline 1 & 3 & 400 & 39.69 & 25.9 & 330.24658 & 0 & 0 & 28 & 200 & 1.735 & 0.009 & 20 & 1 & 1.29505 \\
\hline 1 & 3 & 420 & 59.54 & 26.36 & 454.74711 & 0 & 0 & 28 & 300.02 & 1.766 & 0.006 & 21 & 1 & 1.31775 \\
\hline 1 & 3 & 440 & 79.38 & 28.42 & 620.74808 & 0 & 0 & 28 & 400 & 1.904 & 0.005 & 22 & 1 & 1.42105 \\
\hline 1 & 3 & 460 & 99.23 & 31.12 & 828.21729 & 0 & 0 & 28 & 500.02 & 2.085 & 0.004 & 23 & 1 & 1.55575 \\
\hline 1 & 3 & 480 & 119.07 & 35.15 & 1077.17515 & 0 & 0 & 28 & 599.99 & 2.355 & 0.004 & 24 & 1 & 1.75745 \\
\hline 1 & 3 & 500 & 138.92 & 38.82 & 1367.72692 & 0 & 0 & 28 & 700.02 & 2.601 & 0.004 & 25 & 1 & 1.94115 \\
\hline 1 & 3 & 520 & 158.76 & 42.53 & 1700.16319 & 0 & 0 & 28 & 799.99 & 2.849 & 0.004 & 26 & 1 & 2.1264 \\
\hline 1 & 3 & 540 & 178.61 & 46.61 & 2074.00486 & 0 & 0 & 28 & 900.02 & 3.123 & 0.003 & 27 & 1 & 2.33025 \\
\hline 1 & 3 & 560 & 198.45 & 50.65 & 2488.7964 & 0 & 0 & 28 & 999.99 & 3.393 & 0.003 & 28 & 1 & 2.53235 \\
\hline
\end{tabular}




\begin{tabular}{|c|c|c|c|c|c|c|c|c|c|c|c|c|c|c|}
\hline 1 & 4 & 570 & 198.45 & 49.85 & 2700.47849 & 0 & 0 & 28 & 999.99 & 3.34 & 0.003 & 29 & 1 & 2.4923 \\
\hline 1 & 4 & 580 & 198.45 & 49.51 & 2908.60664 & 0 & 0 & 28 & 999.99 & 3.317 & 0.003 & 30 & 1 & 2.47525 \\
\hline 1 & 4 & 590 & 198.45 & 49.28 & 3116.83769 & 0 & 0 & 28 & 999.99 & 3.301 & 0.003 & 31 & 1 & 2.4638 \\
\hline 1 & 4 & 600 & 198.45 & 49.12 & 3324.13568 & 0 & 0 & 28 & 999.99 & 3.291 & 0.003 & 32 & 1 & 2.4562 \\
\hline & 4 & 610 & 198.45 & 49.11 & 3532.36751 & 0 & 0 & 28 & 999.99 & 3.29 & 0.003 & 33 & 1 & 2.45555 \\
\hline & 4 & 620 & 198.45 & 48.8 & 3739.87206 & 0 & 0 & 28 & 999.99 & 3.27 & 0.003 & 34 & 1 & 2.43995 \\
\hline & 5 & 640 & 198.45 & 48.6 & 4160.07657 & 0 & 0 & 28 & 999.99 & 3.256 & 0.003 & 35 & 1 & 2.4301 \\
\hline & 5 & 660 & 178.61 & 43.55 & 4534.65652 & 0 & 0 & 28 & 900.02 & 2.918 & 0.003 & 36 & 1 & 2.1774 \\
\hline & 5 & 680 & 158.76 & 38.67 & 4867.47606 & 0 & 0 & 28 & 799.99 & 2.591 & 0.003 & 37 & 1 & 1.9333 \\
\hline & 5 & 700 & 138.92 & 34.29 & 5158.73468 & 0 & 0 & 28 & 700.02 & 2.298 & 0.003 & 38 & 1 & 1.7146 \\
\hline & 5 & 720 & 119.07 & 29.71 & 5408.3782 & 0 & 0 & 28 & 599.99 & 1.991 & 0.003 & 39 & 1 & 1.48545 \\
\hline & 5 & 740 & 99.23 & 25.25 & 5616.46081 & 0 & 0 & 28 & 500.02 & 1.691 & 0.003 & 40 & 1 & 1.2623 \\
\hline 1 & 5 & 760 & 79.38 & 20.53 & 5783.04219 & 0 & 0 & 28 & 400 & 1.375 & 0.003 & 41 & 1 & 1.02635 \\
\hline 1 & 5 & 780 & 59.54 & 16.11 & 5908.02102 & 0 & 0 & 28 & 300.02 & 1.08 & 0.004 & 42 & 1 & 0.8057 \\
\hline 1 & 5 & 800 & 39.69 & 11.65 & 5991.55126 & 0 & 0 & 28 & 200 & 0.78 & 0.004 & 43 & 1 & 0.58245 \\
\hline 1 & 5 & 820 & 19.85 & 7.83 & 6033.40513 & 0 & 0 & 28 & 100.02 & 0.524 & 0.005 & 44 & 1 & 0.39125 \\
\hline 1 & 6 & 840 & 17.86 & 10.32 & 6071.09717 & 0 & 0 & 28 & 90 & 0.692 & 0.008 & 45 & 1 & 0.5161 \\
\hline 1 & 6 & 860 & 15.88 & 9.83 & 6104.37528 & 0 & 0 & 28 & 80.02 & 0.658 & 0.008 & 46 & 1 & 0.4913 \\
\hline 1 & 6 & 880 & 13.89 & 10.01 & 6133.49627 & 0 & 0 & 28 & 69.99 & 0.67 & 0.01 & 47 & 1 & 0.50035 \\
\hline 1 & 6 & 900 & 11.9 & 9.33 & 6158.47272 & 0 & 0 & 28 & 59.96 & 0.625 & 0.01 & 48 & 1 & 0.46645 \\
\hline 1 & 6 & 920 & 9.92 & 9.22 & 6179.26692 & 0 & 0 & 28 & 49.99 & 0.618 & 0.012 & 49 & 1 & 0.46095 \\
\hline 1 & 6 & 940 & 7.93 & 9.49 & 6195.91736 & 0 & 0 & 28 & 39.96 & 0.636 & 0.016 & 50 & 1 & 0.47445 \\
\hline 1 & 6 & 960 & 5.95 & 8.55 & 6208.40362 & 0 & 0 & 28 & 29.98 & 0.573 & 0.019 & 51 & 1 & 0.42745 \\
\hline 1 & 6 & 980 & 3.96 & 8.57 & 6216.73827 & 0 & 0 & 28 & 19.95 & 0.574 & 0.029 & 52 & 1 & 0.42855 \\
\hline 1 & 6 & 1000 & 1.98 & 8.74 & 6220.90559 & 0 & 0 & 28 & 9.98 & 0.585 & 0.059 & 53 & 1 & 0.4368 \\
\hline 1 & 7 & 1020 & 1.79 & 7.54 & 6224.67943 & 0 & 0 & 28 & 9.02 & 0.505 & 0.056 & 54 & 1 & 0.3771 \\
\hline 1 & 7 & 1040 & 1.59 & 12.25 & 6228.01266 & 0 & 0 & 28 & 8.01 & 0.821 & 0.102 & 55 & 1 & 0.61255 \\
\hline 1 & 7 & 1060 & 1.39 & 7.47 & 6230.93434 & 0 & 0 & 28 & 7 & 0.501 & 0.072 & 56 & 1 & 0.3737 \\
\hline 1 & 7 & 1080 & 1.19 & 12.36 & 6233.43662 & 0 & 0 & 28 & 6 & 0.828 & 0.138 & 57 & 1 & 0.61815 \\
\hline 1 & 7 & 1100 & 0.99 & 8.82 & 6235.52421 & 0 & 0 & 28 & 4.99 & 0.591 & 0.118 & 58 & 1 & 0.44115 \\
\hline 1 & 7 & 1120 & 0.8 & 6.82 & 6237.19475 & 0 & 0 & 28 & 4.03 & 0.457 & 0.113 & 59 & 1 & 0.3408 \\
\hline 1 & 7 & 1140 & 0.6 & 11.15 & 6238.44432 & 0 & 0 & 28 & 3.02 & 0.747 & 0.247 & 60 & 1 & 0.5575 \\
\hline 1 & 7 & 1160 & 0.4 & 18.03 & 6239.28155 & 0 & 0 & 28 & 2.02 & 1.208 & 0.598 & 61 & 1 & 0.90145 \\
\hline & 7 & 1180 & 0.2 & 17.91 & 6239.70409 & 0 & 0 & 28 & 1.01 & 1.2 & 1.188 & 62 & 1 & 0.89555 \\
\hline
\end{tabular}

2004.02.09 META-10 wt\%-U DG 1-1000 s-1 run 2.txt Mode Step t[s] n[rpm] M[\%.] Phi[rad]

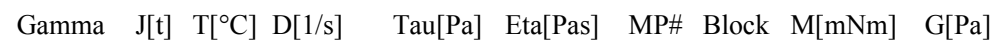

$\begin{array}{rrrrrrrrrrrrrrrr}1 & 1 & 20 & 0.2 & 19.22 & 0.40998 & 0 & 0 & 28 & 1.01 & 1.288 & 1.275 & 1 & 1 & 0.9609 & 0 \\ 1 & 1 & 40 & 0.4 & 13.51 & 1.24486 & 0 & 0 & 28 & 2.02 & 0.905 & 0.448 & 2 & 1 & 0.6754 & 0 \\ 1 & 1 & 60 & 0.6 & 8.62 & 2.49521 & 0 & 0 & 28 & 3.02 & 0.578 & 0.191 & 3 & 1 & 0.43115 & 0 \\ 1 & 1 & 80 & 0.8 & 8.7 & 4.15947 & 0 & 0 & 28 & 4.03 & 0.583 & 0.145 & 4 & 1 & 0.43515 & 0 \\ 1 & 1 & 100 & 1 & 15.65 & 6.23685 & 0 & 0 & 28 & 5.04 & 1.049 & 0.208 & 5 & 1 & 0.7826 & 0 \\ 1 & 1 & 120 & 1.19 & 10.31 & 8.73755 & 0 & 0 & 28 & 6 & 0.691 & 0.115 & 6 & 1 & 0.51545 & 0 \\ 1 & 1 & 140 & 1.39 & 11.28 & 11.64981 & 0 & 0 & 28 & 7 & 0.756 & 0.108 & 7 & 1 & 0.5639 & 0 \\ 1 & 1 & 160 & 1.59 & 11.78 & 14.98226 & 0 & 0 & 28 & 8.01 & 0.789 & 0.099 & 8 & 1 & 0.58895 & 0 \\ 1 & 1 & 180 & 1.79 & 12.2 & 18.72546 & 0 & 0 & 28 & 9.02 & 0.817 & 0.091 & 9 & 1 & 0.60995 & 0 \\ 1 & 2 & 200 & 1.98 & 10.03 & 22.89985 & 0 & 0 & 28 & 9.98 & 0.672 & 0.067 & 10 & 1 & 0.5014 & 0 \\ 1 & 2 & 220 & 3.97 & 13.07 & 31.17167 & 0 & 0 & 28 & 20 & 0.876 & 0.044 & 11 & 1 & 0.65345 & 0 \\ 1 & 2 & 240 & 5.95 & 12.46 & 43.62023 & 0 & 0 & 28 & 29.98 & 0.835 & 0.028 & 12 & 1 & 0.6232 & 0 \\ 1 & 2 & 260 & 7.94 & 11.33 & 60.1992 & 0 & 0 & 28 & 40.01 & 0.759 & 0.019 & 13 & 1 & 0.5666 & 0\end{array}$




\begin{tabular}{|c|c|c|c|c|c|c|c|c|c|c|c|c|c|c|}
\hline 1 & 2 & 280 & 9.92 & 12.05 & 80.93999 & 0 & 0 & 28 & 49.99 & 0.808 & 0.016 & 14 & 1 & 0.60265 \\
\hline 1 & 2 & 300 & 11.91 & 11.59 & 105.84575 & 0 & 0 & 28 & 60.01 & 0.777 & 0.013 & 15 & 1 & 0.5797 \\
\hline 1 & 2 & 320 & 13.89 & 11.77 & 134.93062 & 0 & 0 & 28 & 69.99 & 0.788 & 0.011 & 16 & 1 & 0.58835 \\
\hline 1 & 2 & 340 & 15.88 & 12.17 & 168.1239 & 0 & 0 & 28 & 80.02 & 0.815 & 0.01 & 17 & 1 & 0.6085 \\
\hline 1 & 2 & 360 & 17.86 & 11.94 & 205.50885 & 0 & 0 & 28 & 90 & 0.8 & 0.009 & 18 & 1 & 0.59675 \\
\hline 1 & 3 & 380 & 19.85 & 12.22 & 247.45776 & 0 & 0 & 28 & 100.02 & 0.819 & 0.008 & 19 & 1 & 0.6109 \\
\hline 1 & 3 & 400 & 39.69 & 18.22 & 330.21909 & 0 & 0 & 28 & 200 & 1.221 & 0.006 & 20 & 1 & 0.9109 \\
\hline 1 & 3 & 420 & 59.54 & 22.47 & 454.67799 & 0 & 0 & 28 & 300.02 & 1.505 & 0.005 & 21 & 1 & 1.12345 \\
\hline 1 & 3 & 440 & 79.38 & 25.9 & 620.62634 & 0 & 0 & 28 & 400 & 1.735 & 0.004 & 22 & 1 & 1.29485 \\
\hline 1 & 3 & 460 & 99.23 & 29.44 & 828.20001 & 0 & 0 & 28 & 500.02 & 1.973 & 0.004 & 23 & 1 & 1.47205 \\
\hline 1 & 3 & 480 & 119.07 & 33.44 & 1077.09583 & 0 & 0 & 28 & 599.99 & 2.24 & 0.004 & 24 & 1 & 1.6718 \\
\hline 1 & 3 & 500 & 138.92 & 37.6 & 1367.79289 & 0 & 0 & 28 & 700.02 & 2.519 & 0.004 & 25 & 1 & 1.8802 \\
\hline 1 & 3 & 520 & 158.76 & 41.55 & 1699.9904 & 0 & 0 & 28 & 799.99 & 2.784 & 0.003 & 26 & 1 & 2.07755 \\
\hline 1 & 3 & 540 & 178.61 & 46 & 2073.64514 & 0 & 0 & 28 & 900.02 & 3.082 & 0.003 & 27 & 1 & 2.30015 \\
\hline 1 & 3 & 560 & 198.45 & 50.36 & 2489.16318 & 0 & 0 & 28 & 999.99 & 3.374 & 0.003 & 28 & 1 & 2.5179 \\
\hline 1 & 4 & 570 & 198.45 & 49.89 & 2700.84527 & 0 & 0 & 28 & 999.99 & 3.343 & 0.003 & 29 & 1 & 2.49455 \\
\hline 1 & 4 & 580 & 198.45 & 49.52 & 2909.0771 & 0 & 0 & 28 & 999.99 & 3.318 & 0.003 & 30 & 1 & 2.47615 \\
\hline 1 & 4 & 590 & 198.45 & 49.33 & 3117.20526 & 0 & 0 & 28 & 999.99 & 3.305 & 0.003 & 31 & 1 & 2.46665 \\
\hline 1 & 4 & 600 & 198.45 & 49.15 & 3324.50325 & 0 & 0 & 28 & 999.99 & 3.293 & 0.003 & 32 & 1 & 2.45765 \\
\hline 1 & 4 & 610 & 198.45 & 49.02 & 3532.73429 & 0 & 0 & 28 & 999.99 & 3.284 & 0.003 & 33 & 1 & 2.45095 \\
\hline 1 & 4 & 620 & 198.45 & 48.88 & 3740.3433 & 0 & 0 & 28 & 999.99 & 3.275 & 0.003 & 34 & 1 & 2.44385 \\
\hline 1 & 5 & 640 & 198.45 & 48.72 & 4160.54781 & 0 & 0 & 28 & 999.99 & 3.264 & 0.003 & 35 & 1 & 2.43585 \\
\hline 1 & 5 & 660 & 178.61 & 43.52 & 4535.0233 & 0 & 0 & 28 & 900.02 & 2.916 & 0.003 & 36 & 1 & 2.1762 \\
\hline 1 & 5 & 680 & 158.76 & 38.65 & 4867.59387 & 0 & 0 & 28 & 799.99 & 2.589 & 0.003 & 37 & 1 & 1.9323 \\
\hline 1 & 5 & 700 & 138.92 & 33.98 & 5158.93575 & 0 & 0 & 28 & 700.02 & 2.276 & 0.003 & 38 & 1 & 1.69875 \\
\hline 1 & 5 & 720 & 119.07 & 29.33 & 5408.64131 & 0 & 0 & 28 & 599.99 & 1.965 & 0.003 & 39 & 1 & 1.46645 \\
\hline 1 & 5 & 740 & 99.23 & 24.84 & 5616.73412 & 0 & 0 & 28 & 500.02 & 1.664 & 0.003 & 40 & 1 & 1.2421 \\
\hline 1 & 5 & 760 & 79.38 & 20.07 & 5783.41996 & 0 & 0 & 28 & 400 & 1.345 & 0.003 & 41 & 1 & 1.00345 \\
\hline 1 & 5 & 780 & 59.54 & 15.58 & 5908.33597 & 0 & 0 & 28 & 300.02 & 1.044 & 0.003 & 42 & 1 & 0.77915 \\
\hline 1 & 5 & 800 & 39.69 & 11.03 & 5991.81437 & 0 & 0 & 28 & 200 & 0.739 & 0.004 & 43 & 1 & 0.5514 \\
\hline 1 & 5 & 820 & 19.85 & 6.6 & 6033.72086 & 0 & 0 & 28 & 100.02 & 0.442 & 0.004 & 44 & 1 & 0.33 \\
\hline 1 & 6 & 840 & 17.86 & 7.62 & 6071.42233 & 0 & 0 & 28 & 90 & 0.51 & 0.006 & 45 & 1 & 0.38075 \\
\hline 1 & 6 & 860 & 15.88 & 7.84 & 6104.69886 & 0 & 0 & 28 & 80.02 & 0.525 & 0.007 & 46 & 1 & 0.39215 \\
\hline 1 & 6 & 880 & 13.89 & 7.98 & 6133.81122 & 0 & 0 & 28 & 69.99 & 0.535 & 0.008 & 47 & 1 & 0.3991 \\
\hline 1 & 6 & 900 & 11.9 & 7.68 & 6158.76096 & 0 & 0 & 28 & 59.96 & 0.514 & 0.009 & 48 & 1 & 0.3838 \\
\hline 1 & 6 & 920 & 9.92 & 7.25 & 6179.56537 & 0 & 0 & 28 & 49.99 & 0.485 & 0.01 & 49 & 1 & 0.3623 \\
\hline 1 & 6 & 940 & 7.93 & 7.2 & 6196.2221 & 0 & 0 & 28 & 39.96 & 0.483 & 0.012 & 50 & 1 & 0.3602 \\
\hline 1 & 6 & 960 & 5.95 & 6.55 & 6208.70836 & 0 & 0 & 28 & 29.98 & 0.439 & 0.015 & 51 & 1 & 0.32735 \\
\hline 1 & 6 & 980 & 3.96 & 6.09 & 6217.03986 & 0 & 0 & 28 & 19.95 & 0.408 & 0.02 & 52 & 1 & 0.3044 \\
\hline 1 & 6 & 1000 & 1.98 & 6.85 & 6221.21896 & 0 & 0 & 28 & 9.98 & 0.459 & 0.046 & 53 & 1 & 0.34255 \\
\hline 1 & 7 & 1020 & 1.79 & 5.27 & 6224.99123 & 0 & 0 & 28 & 9.02 & 0.353 & 0.039 & 54 & 1 & 0.26355 \\
\hline 1 & 7 & 1040 & 1.59 & 8.67 & 6228.32603 & 0 & 0 & 28 & 8.01 & 0.581 & 0.073 & 55 & 1 & 0.4335 \\
\hline 1 & 7 & 1060 & 1.39 & 4.82 & 6231.24536 & 0 & 0 & 28 & 7 & 0.323 & 0.046 & 56 & 1 & 0.24105 \\
\hline 1 & 7 & 1080 & 1.19 & 8.57 & 6233.74763 & 0 & 0 & 28 & 6 & 0.574 & 0.096 & 57 & 1 & 0.4283 \\
\hline 1 & 7 & 1100 & 0.99 & 4.92 & 6235.83522 & 0 & 0 & 28 & 4.99 & 0.33 & 0.066 & 58 & 1 & 0.246 \\
\hline 1 & 7 & 1120 & 0.8 & 5.59 & 6237.50419 & 0 & 0 & 28 & 4.03 & 0.375 & 0.093 & 59 & 1 & 0.27965 \\
\hline 1 & 7 & 1140 & 0.6 & 10.03 & 6238.7569 & 0 & 0 & 28.1 & 3.02 & 0.672 & 0.223 & 60 & 1 & 0.5013 \\
\hline 1 & 7 & 1160 & 0.4 & 9.83 & 6239.59649 & 0 & 0 & 28 & 2.02 & 0.658 & 0.326 & 61 & 1 & 0.4914 \\
\hline 1 & 7 & 1180 & 0.2 & 8.73 & 6240.01747 & 0 & 0 & 28.1 & 1.01 & 0.585 & 0.579 & 62 & 1 & 0.4366 \\
\hline
\end{tabular}


2004.02.09 META-10 wt\%-U DG 500-4000 s-1 run 1.txt Mode Step t[s] n[rpm] M[\%.] Phi[rad $] \quad$ Gan

$\begin{array}{rrrrrrrrrrrrrrrr}1 & 1 & 20 & 99.24 & 36.54 & 207.61765 & 0 & 0 & 28.1 & 500.07 & 2.448 & 0.005 & 1 & 1 & 1.8268 & 0 \\ 1 & 1 & 40 & 198.46 & 54.61 & 621.80837 & 0 & 0 & 28.1 & 1000.04 & 3.659 & 0.004 & 2 & 1 & 2.73025 & 0 \\ 1 & 1 & 60 & 297.68 & 75.86 & 1243.24445 & 0 & 0 & 28.1 & 1500.01 & 5.082 & 0.003 & 3 & 1 & 3.79275 & 0 \\ 1 & 1 & 80 & 396.91 & 97.06 & 2073.53912 & 0 & 0 & 28.1 & 2000.03 & 6.503 & 0.003 & 4 & 1 & 4.85295 & 0 \\ 1 & 1 & 100 & 496.13 & 117.26 & 3110.24898 & 0 & 0 & 28.1 & 2500 & 7.856 & 0.003 & 5 & 1 & 5.86305 & 0 \\ 1 & 1 & 120 & 595.36 & 142.49 & 4355.50327 & 0 & 0 & 28.1 & 3000.02 & 9.547 & 0.003 & 6 & 1 & 7.12465 & 0 \\ 1 & 1 & 140 & 694.58 & 173.88 & 5809.97506 & 0 & 0 & 28.1 & 3499.99 & 11.65 & 0.003 & 7 & 1 & 8.6942 & 0 \\ 1 & 1 & 160 & 793.81 & 213.54 & 7470.39239 & 0 & 0 & 28.1 & 4000.01 & 14.307 & 0.004 & 8 & 1 & 10.677 & 0 \\ 1 & 2 & 180 & 793.81 & 214.18 & 9151.07927 & 0 & 0 & 28.1 & 4000.01 & 14.35 & 0.004 & 9 & 1 & 10.709 & 0 \\ 1 & 2 & 200 & 793.81 & 215.93 & 10814.04442 & 0 & 0 & 28 & 4000.01 & 14.468 & 0.004 & 10 & 1 & 10.79665 & 0 \\ 1 & 2 & 220 & 793.81 & 216.05 & 12476.59646 & 0 & 0 & 28 & 4000.01 & 14.476 & 0.004 & 11 & 1 & 10.8027 & 0 \\ 1 & 3 & 240 & 793.81 & 215.07 & 14158.2698 & 0 & 0 & 28 & 4000.01 & 14.41 & 0.004 & 12 & 1 & 10.7537 & 0 \\ 1 & 3 & 260 & 694.58 & 174.92 & 15614.3548 & 0 & 0 & 28 & 3499.99 & 11.72 & 0.003 & 13 & 1 & 8.74615 & 0 \\ 1 & 3 & 280 & 595.36 & 137.51 & 16862.05246 & 0 & 0 & 28 & 3000.02 & 9.213 & 0.003 & 14 & 1 & 6.87545 & 0 \\ 1 & 3 & 300 & 496.13 & 105.12 & 17902.61157 & 0 & 0 & 28 & 2500 & 7.043 & 0.003 & 15 & 1 & 5.25615 & 0 \\ 1 & 3 & 320 & 396.91 & 83.8 & 18736.45544 & 0 & 0 & 28 & 2000.03 & 5.615 & 0.003 & 16 & 1 & 4.19005 & 0 \\ 1 & 3 & 340 & 297.68 & 62.62 & 19361.74312 & 0 & 0 & 28 & 1500.01 & 4.196 & 0.003 & 17 & 1 & 3.131 & 0 \\ 1 & 3 & 360 & 198.46 & 41.51 & 19778.72279 & 0 & 0 & 28 & 1000.04 & 2.781 & 0.003 & 18 & 1 & 2.0757 & 0 \\ 1 & 3 & 380 & 99.23 & 21.08 & 19988.28665 & 0 & 0 & 28 & 500.02 & 1.413 & 0.003 & 19 & 1 & 1.0542 & 0\end{array}$

2004.02.09 META-10 wt\%-U DG 500-4000 s-1 run 2.txt

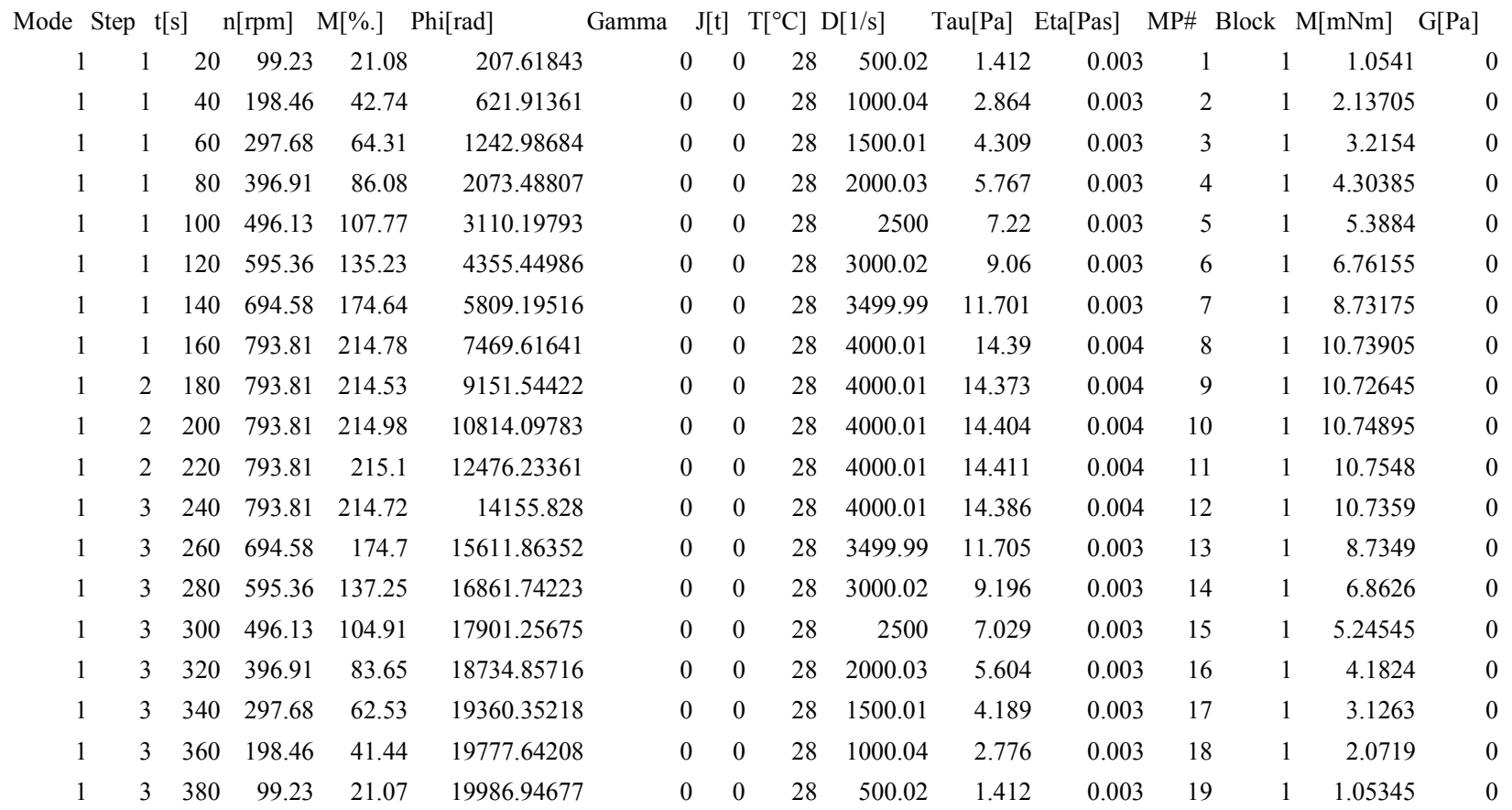



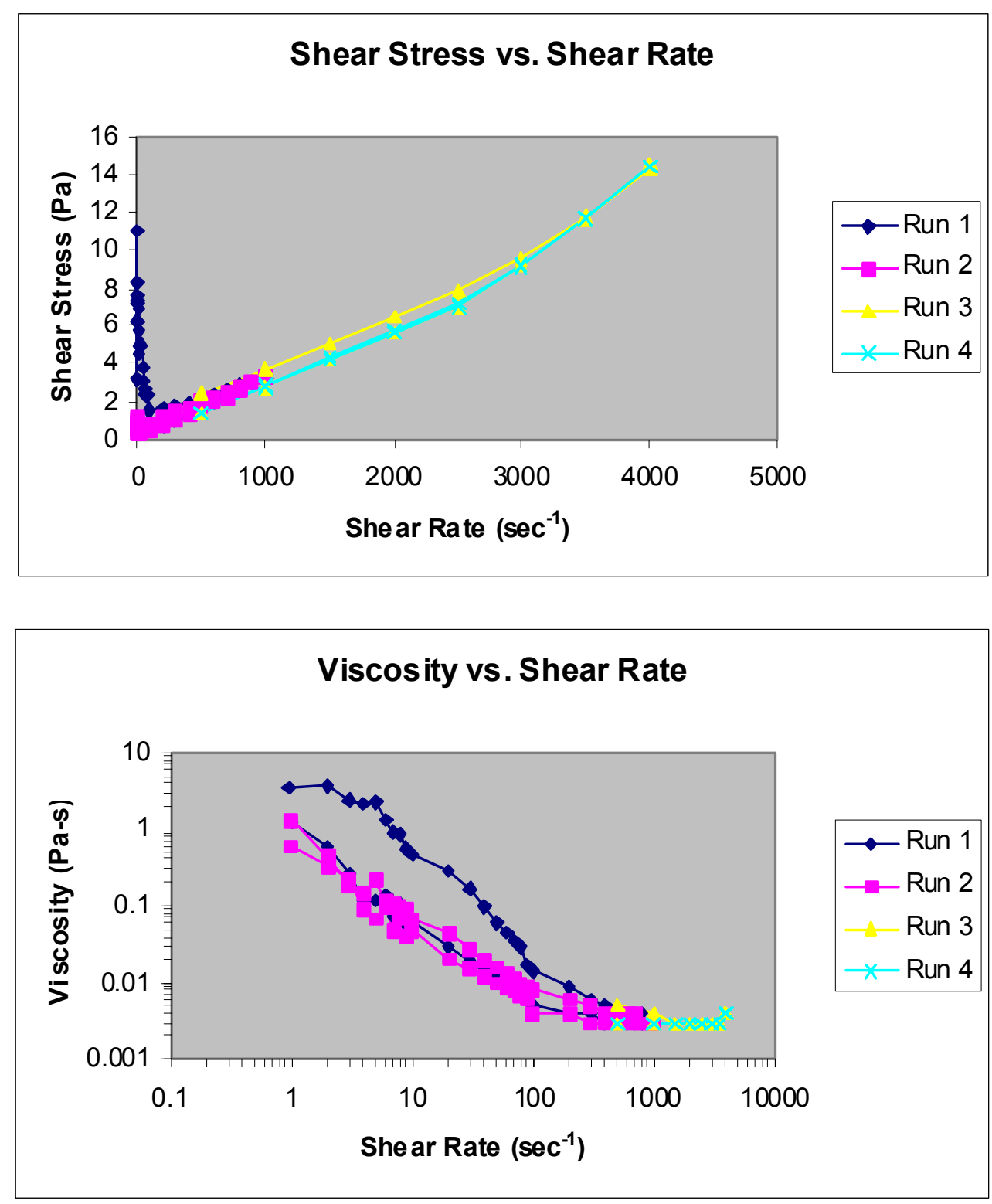

\section{E-2.1.16 META/10wt\%/U/S}

2004.02.14 META-10 wt \%-U [sonicated] DG 0.1-1000 s-1 run 1.txt

Mode Step t[s] n[rpm] M[\%.] Phi[rad] Gamma $\quad J[t] ~ T\left[{ }^{\circ} \mathrm{C}\right] \quad \mathrm{D}[1 / \mathrm{s}] \quad \mathrm{Tau}[\mathrm{Pa}] \quad$ Eta[Pas $] \quad$ MP\# Block $\mathrm{M}[\mathrm{mNm}] \quad \mathrm{G}[\mathrm{Pa}]$

$\begin{array}{rrrrrrrrrrrrrrrr}1 & 1 & 20 & 0.02 & 29.28 & 0.03377 & 0 & 0 & 28 & 0.1 & 1.962 & 19.62 & 1 & 1 & 1.46415 & 0 \\ 1 & 1 & 40 & 0.04 & 18.2 & 0.11702 & 0 & 0 & 28 & 0.2 & 1.22 & 6.1 & 2 & 1 & 0.91025 & 0 \\ 1 & 1 & 60 & 0.06 & 21.42 & 0.24112 & 0 & 0 & 28 & 0.3 & 1.435 & 4.783 & 3 & 1 & 1.07105 & 0 \\ 1 & 1 & 80 & 0.08 & 23.06 & 0.40841 & 0 & 0 & 28 & 0.4 & 1.545 & 3.863 & 4 & 1 & 1.15305 & 0 \\ 1 & 1 & 100 & 0.1 & 25.1 & 0.61654 & 0 & 0 & 28 & 0.5 & 1.682 & 3.364 & 5 & 1 & 1.2551 & 0 \\ 1 & 1 & 120 & 0.12 & 26.81 & 0.86708 & 0 & 0 & 28 & 0.6 & 1.796 & 2.993 & 6 & 1 & 1.3406 & 0 \\ 1 & 1 & 140 & 0.14 & 29.73 & 1.15925 & 0 & 0 & 28 & 0.71 & 1.992 & 2.806 & 7 & 1 & 1.48665 & 0 \\ 1 & 1 & 160 & 0.16 & 32.91 & 1.49226 & 0 & 0 & 28 & 0.81 & 2.205 & 2.722 & 8 & 1 & 1.64545 & 0 \\ 1 & 1 & 180 & 0.18 & 35 & 1.86925 & 0 & 0 & 28 & 0.91 & 2.345 & 2.577 & 9 & 1 & 1.7499 & 0 \\ 1 & 2 & 200 & 0.2 & 37.05 & 2.28944 & 0 & 0 & 28 & 1.01 & 2.483 & 2.458 & 10 & 1 & 1.8527 & 0\end{array}$




\begin{tabular}{|c|c|c|c|c|c|c|c|c|c|c|c|c|c|}
\hline 1 & 220 & 0.4 & 49.72 & 3.11567 & 0 & 0 & 28 & 2.02 & 3.331 & 1.649 & 11 & 1 & 2.48595 \\
\hline 1 & 240 & 0.6 & 69.33 & 4.36053 & 0 & 0 & 28 & 3.02 & 4.645 & 1.538 & 12 & 1 & 3.4667 \\
\hline 1 & 260 & 0.8 & 68.83 & 6.02557 & 0 & 0 & 28 & 4.03 & 4.611 & 1.144 & 13 & 1 & 3.4413 \\
\hline 1 & 280 & 1 & 79.97 & 8.09667 & 0 & 0 & 28 & 5.04 & 5.358 & 1.063 & 14 & 1 & 3.9987 \\
\hline 1 & 300 & 1.2 & 74.7 & 10.61151 & 0 & 0 & 28 & 6.05 & 5.005 & 0.827 & 15 & 1 & 3.73495 \\
\hline 1 & 320 & 1.39 & 44.22 & 13.51984 & 0 & 0 & 28 & 7 & 2.963 & 0.423 & 16 & 1 & 2.2111 \\
\hline 1 & 340 & 1.59 & 66.73 & 16.84993 & 0 & 0 & 28 & 8.01 & 4.471 & 0.558 & 17 & 1 & 3.33665 \\
\hline 1 & 360 & 1.79 & 48.94 & 20.59864 & 0 & 0 & 28 & 9.02 & 3.279 & 0.364 & 18 & 1 & 2.44705 \\
\hline 1 & 380 & 1.98 & 59.65 & 24.77146 & 0 & 0 & 28 & 9.98 & 3.996 & 0.4 & 19 & 1 & 2.98235 \\
\hline 1 & 400 & 3.97 & 73.18 & 33.03699 & 0 & 0 & 28 & 20 & 4.903 & 0.245 & 20 & 1 & 3.65915 \\
\hline 1 & 420 & 5.95 & 84.13 & 45.46749 & 0 & 0 & 28 & 29.98 & 5.636 & 0.188 & 21 & 1 & 4.20625 \\
\hline 1 & 440 & 7.94 & 91.37 & 62.06295 & 0 & 0 & 28 & 40.01 & 6.122 & 0.153 & 22 & 1 & 4.56845 \\
\hline 1 & 460 & 9.92 & 79.21 & 82.81788 & 0 & 0 & 28 & 49.99 & 5.307 & 0.106 & 23 & 1 & 3.96065 \\
\hline 1 & 480 & 11.91 & 67.26 & 107.73071 & 0 & 0 & 28 & 60.01 & 4.507 & 0.075 & 24 & 1 & 3.3631 \\
\hline 1 & 500 & 13.89 & 60.28 & 136.80065 & 0 & 0 & 28 & 69.99 & 4.039 & 0.058 & 25 & 1 & 3.01395 \\
\hline 1 & 520 & 15.88 & 56.82 & 169.99551 & 0 & 0 & 28 & 80.02 & 3.807 & 0.048 & 26 & 1 & 2.8412 \\
\hline 1 & 540 & 17.86 & 55.62 & 207.40952 & 0 & 0 & 28 & 90 & 3.726 & 0.041 & 27 & 1 & 2.7808 \\
\hline 1 & 560 & 19.85 & 56.36 & 249.34899 & 0 & 0 & 28 & 100.02 & 3.776 & 0.038 & 28 & 1 & 2.818 \\
\hline 1 & 580 & 39.69 & 79.47 & 332.14017 & 0 & 0 & 28 & 200 & 5.324 & 0.027 & 29 & 1 & 3.97345 \\
\hline 1 & 600 & 59.54 & 91.17 & 456.55588 & 0 & 0 & 28 & 300.02 & 6.108 & 0.02 & 30 & 1 & 4.55855 \\
\hline 1 & 620 & 79.38 & 99.69 & 622.51365 & 0 & 0 & 28 & 400 & 6.679 & 0.017 & 31 & 1 & 4.98425 \\
\hline 1 & 640 & 99.23 & 107.04 & 830.03391 & 0 & 0 & 28 & 500.02 & 7.172 & 0.014 & 32 & 1 & 5.352 \\
\hline 1 & 660 & 119.07 & 114.07 & 1078.99178 & 0 & 0 & 28 & 599.99 & 7.643 & 0.013 & 33 & 1 & 5.7036 \\
\hline 1 & 680 & 138.92 & 121.08 & 1369.6158 & 0 & 0 & 28 & 700.02 & 8.112 & 0.012 & 34 & 1 & 6.0541 \\
\hline 1 & 700 & 158.76 & 127.53 & 1701.88478 & 0 & 0 & 28 & 799.99 & 8.544 & 0.011 & 35 & 1 & 6.37645 \\
\hline 1 & 720 & 178.61 & 134.94 & 2075.92358 & 0 & 0 & 28 & 900.02 & 9.041 & 0.01 & 36 & 1 & 6.74685 \\
\hline 1 & 740 & 198.45 & 141.52 & 2490.70414 & 0 & 0 & 28 & 999.99 & 9.482 & 0.009 & 37 & 1 & 7.07615 \\
\hline 1 & 750 & 198.45 & 136.97 & 2702.59356 & 0 & 0 & 28 & 999.99 & 9.177 & 0.009 & 38 & 1 & 6.84835 \\
\hline 1 & 760 & 198.45 & 135.31 & 2910.4107 & 0 & 0 & 28 & 999.99 & 9.066 & 0.009 & 39 & 1 & 6.76545 \\
\hline 1 & 770 & 198.45 & 133.93 & 3118.12339 & 0 & 0 & 28 & 999.99 & 8.973 & 0.009 & 40 & 1 & 6.6963 \\
\hline 1 & 780 & 198.45 & 132.74 & 3326.25154 & 0 & 0 & 28 & 999.99 & 8.893 & 0.009 & 41 & 1 & 6.6368 \\
\hline 1 & 790 & 198.45 & 131.64 & 3534.0679 & 0 & 0 & 28 & 999.99 & 8.82 & 0.009 & 42 & 1 & 6.58195 \\
\hline 1 & 800 & 198.45 & 130.66 & 3741.98871 & 0 & 0 & 28 & 999.99 & 8.754 & 0.009 & 43 & 1 & 6.53275 \\
\hline 1 & 820 & 198.45 & 129.61 & 4162.08955 & 0 & 0 & 28 & 999.99 & 8.684 & 0.009 & 44 & 1 & 6.4806 \\
\hline 1 & 840 & 178.61 & 116.6 & 4536.95145 & 0 & 0 & 28 & 900.02 & 7.812 & 0.009 & 45 & 1 & 5.82975 \\
\hline 1 & 860 & 158.76 & 104.47 & 4869.44034 & 0 & 0 & 28 & 799.99 & 6.999 & 0.009 & 46 & 1 & 5.2233 \\
\hline 1 & 880 & 138.92 & 93.04 & 5160.70996 & 0 & 0 & 28 & 700.02 & 6.234 & 0.009 & 47 & 1 & 4.6519 \\
\hline 1 & 900 & 119.07 & 81.39 & 5410.35583 & 0 & 0 & 28 & 599.99 & 5.453 & 0.009 & 48 & 1 & 4.0693 \\
\hline 1 & 920 & 99.23 & 69.72 & 5618.5429 & 0 & 0 & 28 & 500.02 & 4.671 & 0.009 & 49 & 1 & 3.48575 \\
\hline 1 & 940 & 79.38 & 57.65 & 5785.10464 & 0 & 0 & 28 & 400 & 3.863 & 0.01 & 50 & 1 & 2.88255 \\
\hline 1 & 960 & 59.54 & 44.98 & 5910.20993 & 0 & 0 & 28 & 300.02 & 3.014 & 0.01 & 51 & 1 & 2.24895 \\
\hline 1 & 980 & 39.69 & 31.05 & 5993.64906 & 0 & 0 & 28 & 200 & 2.08 & 0.01 & 52 & 1 & 1.55225 \\
\hline 1 & $6 \quad 1000$ & 19.85 & 15.32 & 6035.54691 & 0 & 0 & 28 & 100.02 & 1.027 & 0.01 & 53 & 1 & 0.76615 \\
\hline 1 & $7 \quad 1020$ & 17.86 & 15.3 & 6073.23188 & 0 & 0 & 28 & 90 & 1.025 & 0.011 & 54 & 1 & 0.76505 \\
\hline 1 & $7 \quad 1040$ & 15.88 & 14.77 & 6106.53591 & 0 & 0 & 28 & 80.02 & 0.99 & 0.012 & 55 & 1 & 0.73865 \\
\hline 1 & $7 \quad 1060$ & 13.89 & 13.83 & 6135.65062 & 0 & 0 & 28 & 69.99 & 0.926 & 0.013 & 56 & 1 & 0.69135 \\
\hline 1 & $7 \quad 1080$ & 11.9 & 12.73 & 6160.60036 & 0 & 0 & 28 & 59.96 & 0.853 & 0.014 & 57 & 1 & 0.63665 \\
\hline 1 & $7 \quad 1100$ & 9.92 & 11.39 & 6181.42284 & 0 & 0 & 28 & 49.99 & 0.763 & 0.015 & 58 & 1 & 0.56935 \\
\hline 1 & $7 \quad 1120$ & 7.93 & 9.7 & 6198.05364 & 0 & 0 & 28 & 39.96 & 0.65 & 0.016 & 59 & 1 & 0.48485 \\
\hline 1 & $7 \quad 1140$ & 5.95 & 7.92 & 6210.54305 & 0 & 0 & 28 & 29.98 & 0.53 & 0.018 & 60 & 1 & 0.39585 \\
\hline
\end{tabular}




\begin{tabular}{|c|c|c|c|c|c|c|c|c|c|c|c|c|c|}
\hline 1 & 71160 & 3.96 & 6.06 & 6218.88319 & 0 & 0 & 28 & 19.95 & 0.406 & 0.02 & 61 & 1 & 0.3031 \\
\hline & 71180 & 1.98 & 3.09 & 6223.05994 & 0 & 0 & 28 & 9.98 & 0.207 & 0.021 & 62 & 1 & 0.1544 \\
\hline & $8 \quad 1200$ & 1.79 & 3.95 & 6226.83063 & 0 & 0 & 28 & 9.02 & 0.265 & 0.029 & 63 & 1 & 0.19755 \\
\hline & $8 \quad 1220$ & 1.59 & 4.44 & 6230.16386 & 0 & 0 & 28 & 8.01 & 0.298 & 0.037 & 64 & 1 & 0.22205 \\
\hline & $8 \quad 1240$ & 1.39 & 5.01 & 6233.0824 & 0 & 0 & 28 & 7 & 0.336 & 0.048 & 65 & 1 & 0.2506 \\
\hline & 1260 & 1.19 & 5.39 & 6235.58625 & 0 & 0 & 28 & 6 & 0.361 & 0.06 & 66 & 1 & 0.2694 \\
\hline & 1280 & 0.99 & 5.86 & 6237.67227 & 0 & 0 & 28 & 4.99 & 0.392 & 0.079 & 67 & 1 & 0.29275 \\
\hline & 81300 & 0.8 & 6.15 & 6239.3436 & 0 & 0 & 28 & 4.03 & 0.412 & 0.102 & 68 & 1 & 0.3077 \\
\hline & 81320 & 0.6 & 6.38 & 6240.59631 & 0 & 0 & 28 & 3.02 & 0.427 & 0.141 & 69 & 1 & 0.3189 \\
\hline & $8 \quad 1340$ & 0.4 & 6.64 & 6241.43354 & 0 & 0 & 28 & 2.02 & 0.445 & 0.22 & 70 & 1 & 0.3319 \\
\hline & 81360 & 0.2 & 5.78 & 6241.85687 & 0 & 0 & 28 & 1.01 & 0.387 & 0.383 & 71 & 1 & 0.2889 \\
\hline & 91380 & 0.18 & 8.25 & 6242.23386 & 0 & 0 & 28 & 0.91 & 0.553 & 0.608 & 72 & 1 & 0.41235 \\
\hline & 1400 & 0.16 & 9.86 & 6242.56923 & 0 & 0 & 28 & 0.81 & 0.66 & 0.815 & 73 & 1 & 0.4928 \\
\hline & $9 \quad 1420$ & 0.14 & 10.52 & 6242.86297 & 0 & 0 & 28 & 0.71 & 0.705 & 0.993 & 74 & 1 & 0.52615 \\
\hline & 1440 & 0.12 & 10.57 & 6243.11429 & 0 & 0 & 28 & 0.6 & 0.708 & 1.18 & 75 & 1 & 0.5284 \\
\hline & 1460 & 0.1 & 9.25 & 6243.32399 & 0 & 0 & 28 & 0.5 & 0.62 & 1.24 & 76 & 1 & 0.46255 \\
\hline & 1480 & 0.08 & 7.77 & 6243.49364 & 0 & 0 & 28 & 0.4 & 0.52 & 1.3 & 77 & 1 & 0.38825 \\
\hline & 1500 & 0.06 & 6.55 & 6243.6193 & 0 & 0 & 28 & 0.3 & 0.439 & 1.463 & 78 & 1 & 0.32755 \\
\hline & 1520 & 0.04 & 5.54 & 6243.70413 & 0 & 0 & 28 & 0.2 & 0.371 & 1.855 & 79 & 1 & 0.27715 \\
\hline & 91540 & 0.02 & 4.5 & 6243.74654 & 0 & 0 & 28 & 0.1 & 0.301 & 3.01 & 80 & 1 & 0.22495 \\
\hline
\end{tabular}

2004.02.14 META-10 wt\%-U [sonicated] DG 0.1-1000 s-1 run 2.txt

Mode Step t[s] n[rpm] M[\%.] Phi[rad]

$\begin{array}{rrrrr}1 & 1 & 20 & 0.02 & 4.4 \\ 1 & 1 & 40 & 0.04 & 5.8 \\ 1 & 1 & 60 & 0.06 & 6.3 \\ 1 & 1 & 80 & 0.08 & 6.4 \\ 1 & 1 & 100 & 0.1 & 7.0 \\ 1 & 1 & 120 & 0.12 & 8.1 \\ 1 & 1 & 140 & 0.14 & 9.3 \\ 1 & 1 & 160 & 0.16 & 10.3 \\ 1 & 1 & 180 & 0.18 & 11.7 \\ 1 & 2 & 200 & 0.2 & 12.7 \\ 1 & 2 & 220 & 0.4 & 18.2 \\ 1 & 2 & 240 & 0.6 & 23.83 \\ 1 & 2 & 260 & 0.8 & 29.9 \\ 1 & 2 & 280 & 1 & 33.5 \\ 1 & 2 & 300 & 1.19 & 40.57 \\ 1 & 2 & 320 & 1.39 & 36.67 \\ 1 & 2 & 340 & 1.59 & 24.8 \\ 1 & 2 & 360 & 1.79 & 21.03 \\ 1 & 3 & 380 & 1.98 & 20 \\ 1 & 3 & 400 & 3.97 & 25.95 \\ 1 & 3 & 420 & 5.95 & 27.49 \\ 1 & 3 & 440 & 7.94 & 28.2 \\ 1 & 3 & 460 & 9.92 & 29.4 \\ 1 & 3 & 480 & 11.91 & 30.94 \\ 1 & 3 & 500 & 13.89 & 32.4 \\ 1 & 3 & 520 & 15.88 & 33.9 \\ 1 & 3 & 540 & 17.86 & 35.4 \\ & & & & \end{array}$

$\begin{array}{lllllll}\text { Gamma } & \mathrm{J}[\mathrm{t}] \mathrm{T}\left[{ }^{\circ} \mathrm{C}\right] & \mathrm{D}[1 / \mathrm{s}] \quad \mathrm{Tau}[\mathrm{Pa}] & \mathrm{Eta}[\mathrm{Pas}] & \text { MP\# } & \text { Block } & \mathrm{M}[\mathrm{mNm}] \quad \mathrm{G}[\mathrm{Pa}]\end{array}$

\begin{tabular}{|c|c|c|c|c|c|c|c|c|c|}
\hline 0.03848 & 0 & 0 & 28 & 0.1 & 0.298 & 2.98 & 1 & 2 & 0.2224 \\
\hline 0.12095 & 0 & 0 & 28 & 0.2 & 0.392 & 1.96 & 2 & 2 & 0.2923 \\
\hline 0.24662 & 0 & 0 & 28 & 0.3 & 0.424 & 1.413 & 3 & 2 & 0.3163 \\
\hline 0.4139 & 0 & 0 & 28 & 0.4 & 0.43 & 1.075 & 4 & 2 & 0.32065 \\
\hline 0.62282 & 0 & 0 & 28 & 0.5 & 0.473 & 0.946 & 5 & 2 & 0.3527 \\
\hline 0.87336 & 0 & 0 & 28 & 0.6 & 0.543 & 0.905 & 6 & 2 & 0.40535 \\
\hline 1.16632 & 0 & 0 & 28 & 0.71 & 0.627 & 0.883 & 7 & 2 & 0.4678 \\
\hline 1.5009 & 0 & 0 & 28 & 0.81 & 0.696 & 0.859 & 8 & 2 & 0.51955 \\
\hline 1.8771 & 0 & 0 & 28 & 0.91 & 0.787 & 0.865 & 9 & 2 & 0.58755 \\
\hline 2.29729 & 0 & 0 & 28 & 1.01 & 0.856 & 0.848 & 10 & 2 & 0.63895 \\
\hline 3.12824 & 0 & 0 & 28 & 2.02 & 1.223 & 0.605 & 11 & 2 & 0.9129 \\
\hline 4.37467 & 0 & 0 & 28 & 3.02 & 1.596 & 0.528 & 12 & 2 & 1.19125 \\
\hline 6.03736 & 0 & 0 & 28 & 4.03 & 2.005 & 0.498 & 13 & 2 & 1.49605 \\
\hline 8.11866 & 0 & 0 & 28 & 5.04 & 2.245 & 0.445 & 14 & 2 & 1.67565 \\
\hline 10.6123 & 0 & 0 & 28 & 6 & 2.718 & 0.453 & 15 & 2 & 2.0287 \\
\hline 13.5332 & 0 & 0 & 28 & 7 & 2.457 & 0.351 & 16 & 2 & 1.8336 \\
\hline 16.8625 & 0 & 0 & 28 & 8.01 & 1.668 & 0.208 & 17 & 2 & 1.24445 \\
\hline 20.60963 & 0 & 0 & 28 & 9.02 & 1.409 & 0.156 & 18 & 2 & 1.0513 \\
\hline 24.78245 & 0 & 0 & 28 & 9.98 & 1.34 & 0.134 & 19 & 2 & 0.99995 \\
\hline 33.05741 & 0 & 0 & 28 & 20 & 1.738 & 0.087 & 20 & 2 & 1.29725 \\
\hline 45.49183 & 0 & 0 & 28 & 29.98 & 1.842 & 0.061 & 21 & 2 & 1.3745 \\
\hline 62.09829 & 0 & 0 & 28 & 40.01 & 1.89 & 0.047 & 22 & 2 & 1.41055 \\
\hline 82.83594 & 0 & 0 & 28 & 49.99 & 1.971 & 0.039 & 23 & 2 & 1.4706 \\
\hline 07.73542 & 0 & 0 & 28 & 60.01 & 2.073 & 0.035 & 24 & 2 & 1.5468 \\
\hline 36.80458 & 0 & 0 & 28 & 69.99 & 2.174 & 0.031 & 25 & 2 & 1.62215 \\
\hline 70.01357 & 0 & 0 & 28 & 80.02 & 2.275 & 0.028 & 26 & 2 & 1.69755 \\
\hline 07.40009 & 0 & 0 & 28 & 90 & 2.372 & 0.026 & 27 & 2 & 1.7704 \\
\hline
\end{tabular}




\begin{tabular}{|c|c|c|c|c|c|c|c|c|c|c|c|c|c|c|}
\hline 1 & 4 & 560 & 19.85 & 36.85 & 249.34899 & 0 & 0 & 28 & 100.02 & 2.469 & 0.025 & 28 & 2 & 1.8425 \\
\hline 1 & 4 & 580 & 39.69 & 57.89 & 332.15981 & 0 & 0 & 28 & 200 & 3.878 & 0.019 & 29 & 2 & 2.89435 \\
\hline 1 & 4 & 600 & 59.54 & 70.96 & 456.56609 & 0 & 0 & 28 & 300.02 & 4.754 & 0.016 & 30 & 2 & 3.54795 \\
\hline 1 & 4 & 620 & 79.38 & 82 & 622.59533 & 0 & 0 & 28 & 400 & 5.494 & 0.014 & 31 & 2 & 4.1 \\
\hline 1 & 4 & 640 & 99.23 & 91.69 & 829.85563 & 0 & 0 & 28 & 500.02 & 6.143 & 0.012 & 32 & 2 & 4.5847 \\
\hline 1 & 4 & 660 & 119.07 & 101.04 & 1079.05147 & 0 & 0 & 28 & 599.99 & 6.77 & 0.011 & 33 & 2 & 5.05205 \\
\hline 1 & 4 & 680 & 138.92 & 109.5 & 1369.67471 & 0 & 0 & 28 & 700.02 & 7.337 & 0.01 & 34 & 2 & 5.4751 \\
\hline 1 & 4 & 700 & 158.76 & 117.24 & 1701.77796 & 0 & 0 & 28 & 799.99 & 7.855 & 0.01 & 35 & 2 & 5.86205 \\
\hline 1 & 4 & 720 & 178.61 & 125.47 & 2075.51596 & 0 & 0 & 28 & 900.02 & 8.406 & 0.009 & 36 & 2 & 6.2735 \\
\hline 1 & 4 & 740 & 198.45 & 133.44 & 2491.02301 & 0 & 0 & 28 & 999.99 & 8.94 & 0.009 & 37 & 2 & 6.67185 \\
\hline 1 & 5 & 750 & 198.45 & 130.32 & 2702.91244 & 0 & 0 & 28 & 999.99 & 8.732 & 0.009 & 38 & 2 & 6.5162 \\
\hline 1 & 5 & 760 & 198.45 & 128.99 & 2910.6259 & 0 & 0 & 28 & 999.99 & 8.642 & 0.009 & 39 & 2 & 6.44955 \\
\hline 1 & 5 & 770 & 198.45 & 127.87 & 3118.54672 & 0 & 0 & 28 & 999.99 & 8.567 & 0.009 & 40 & 2 & 6.39325 \\
\hline 1 & 5 & 780 & 198.45 & 126.89 & 3326.46674 & 0 & 0 & 28 & 999.99 & 8.502 & 0.009 & 41 & 2 & 6.3447 \\
\hline 1 & 5 & 790 & 198.45 & 126.02 & 3534.69857 & 0 & 0 & 28 & 999.99 & 8.444 & 0.008 & 42 & 2 & 6.3012 \\
\hline 1 & 5 & 800 & 198.45 & 125.23 & 3742.20391 & 0 & 0 & 28 & 999.99 & 8.39 & 0.008 & 43 & 2 & 6.26125 \\
\hline 1 & 6 & 820 & 198.45 & 124.43 & 4162.40763 & 0 & 0 & 28 & 999.99 & 8.337 & 0.008 & 44 & 2 & 6.2213 \\
\hline 1 & 6 & 840 & 178.61 & 112.09 & 4537.16586 & 0 & 0 & 28 & 900.02 & 7.51 & 0.008 & 45 & 2 & 5.60435 \\
\hline 1 & 6 & 860 & 158.76 & 100.43 & 4869.91472 & 0 & 0 & 28 & 799.99 & 6.729 & 0.008 & 46 & 2 & 5.02155 \\
\hline 1 & 6 & 880 & 138.92 & 89.46 & 5161.12229 & 0 & 0 & 28 & 700.02 & 5.994 & 0.009 & 47 & 2 & 4.47305 \\
\hline 1 & 6 & 900 & 119.07 & 78.1 & 5410.81922 & 0 & 0 & 28 & 599.99 & 5.233 & 0.009 & 48 & 2 & 3.905 \\
\hline 1 & 6 & 920 & 99.23 & 66.72 & 5619.06911 & 0 & 0 & 28 & 500.02 & 4.47 & 0.009 & 49 & 2 & 3.33575 \\
\hline 1 & 6 & 940 & 79.38 & 54.97 & 5785.42351 & 0 & 0 & 28 & 400 & 3.683 & 0.009 & 50 & 2 & 2.7487 \\
\hline 1 & 6 & 960 & 59.54 & 42.69 & 5910.53901 & 0 & 0 & 28 & 300.02 & 2.86 & 0.01 & 51 & 2 & 2.13425 \\
\hline 1 & 6 & 980 & 39.69 & 29.41 & 5993.92473 & 0 & 0 & 28 & 200 & 1.971 & 0.01 & 52 & 2 & 1.47065 \\
\hline 1 & 6 & 1000 & 19.85 & 14.59 & 6035.84301 & 0 & 0 & 28 & 100.02 & 0.977 & 0.01 & 53 & 2 & 0.7293 \\
\hline 1 & 7 & 1020 & 17.86 & 14.41 & 6073.51777 & 0 & 0 & 28 & 90 & 0.965 & 0.011 & 54 & 2 & 0.72035 \\
\hline 1 & 7 & 1040 & 15.88 & 13.69 & 6106.81315 & 0 & 0 & 28 & 80.02 & 0.917 & 0.011 & 55 & 2 & 0.6843 \\
\hline 1 & 7 & 1060 & 13.89 & 12.62 & 6135.92629 & 0 & 0 & 28 & 69.99 & 0.846 & 0.012 & 56 & 2 & 0.63115 \\
\hline 1 & 7 & 1080 & 11.9 & 11.5 & 6160.91374 & 0 & 0 & 28 & 59.96 & 0.771 & 0.013 & 57 & 2 & 0.575 \\
\hline 1 & 7 & 1100 & 9.92 & 10.18 & 6181.69459 & 0 & 0 & 28 & 49.99 & 0.682 & 0.014 & 58 & 2 & 0.50895 \\
\hline 1 & 7 & 1120 & 7.93 & 8.61 & 6198.3411 & 0 & 0 & 28 & 39.96 & 0.577 & 0.014 & 59 & 2 & 0.43065 \\
\hline 1 & 7 & 1140 & 5.95 & 6.98 & 6210.84464 & 0 & 0 & 28 & 29.98 & 0.467 & 0.016 & 60 & 2 & 0.34875 \\
\hline 1 & 7 & 1160 & 3.96 & 5.23 & 6219.17693 & 0 & 0 & 28 & 19.95 & 0.35 & 0.018 & 61 & 2 & 0.2615 \\
\hline 1 & 7 & 1180 & 1.98 & 2.61 & 6223.35289 & 0 & 0 & 28 & 9.98 & 0.175 & 0.018 & 62 & 2 & 0.13025 \\
\hline 1 & 8 & 1200 & 1.79 & 3.01 & 6227.12123 & 0 & 0 & 28 & 9.02 & 0.202 & 0.022 & 63 & 2 & 0.15045 \\
\hline 1 & 8 & 1220 & 1.59 & 3.36 & 6230.4576 & 0 & 0 & 28 & 8.01 & 0.225 & 0.028 & 64 & 2 & 0.16775 \\
\hline 1 & 8 & 1240 & 1.39 & 3.73 & 6233.37693 & 0 & 0 & 28 & 7 & 0.25 & 0.036 & 65 & 2 & 0.18665 \\
\hline 1 & 8 & 1260 & 1.19 & 3.91 & 6235.87921 & 0 & 0 & 28 & 6 & 0.262 & 0.044 & 66 & 2 & 0.19545 \\
\hline 1 & 8 & 1280 & 0.99 & 3.97 & 6237.96601 & 0 & 0 & 28 & 4.99 & 0.266 & 0.053 & 67 & 2 & 0.1984 \\
\hline 1 & 8 & 1300 & 0.8 & 4.13 & 6239.63498 & 0 & 0 & 28 & 4.03 & 0.277 & 0.069 & 68 & 2 & 0.2064 \\
\hline 1 & 8 & 1320 & 0.6 & 4.04 & 6240.89083 & 0 & 0 & 28 & 3.02 & 0.271 & 0.09 & 69 & 2 & 0.2022 \\
\hline 1 & 8 & 1340 & 0.4 & 3.87 & 6241.72807 & 0 & 0 & 28 & 2.02 & 0.259 & 0.128 & 70 & 2 & 0.19345 \\
\hline 1 & 8 & 1360 & 0.2 & 2.99 & 6242.14982 & 0 & 0 & 28 & 1.01 & 0.2 & 0.198 & 71 & 2 & 0.14955 \\
\hline 1 & 9 & 1380 & 0.18 & 4.81 & 6242.52839 & 0 & 0 & 28 & 0.91 & 0.322 & 0.354 & 72 & 2 & 0.2405 \\
\hline 1 & 9 & 1400 & 0.16 & 6.35 & 6242.86297 & 0 & 0 & 28 & 0.81 & 0.425 & 0.525 & 73 & 2 & 0.31725 \\
\hline 1 & 9 & 1420 & 0.14 & 7.54 & 6243.15592 & 0 & 0 & 28 & 0.71 & 0.505 & 0.711 & 74 & 2 & 0.377 \\
\hline 1 & 9 & 1440 & 0.12 & 8 & 6243.40725 & 0 & 0 & 28 & 0.6 & 0.536 & 0.893 & 75 & 2 & 0.3998 \\
\hline 1 & 9 & 1460 & 0.1 & 7.69 & 6243.61773 & 0 & 0 & 28 & 0.5 & 0.515 & 1.03 & 76 & 2 & 0.38425 \\
\hline 1 & 9 & 1480 & 0.08 & 7 & 6243.78581 & 0 & 0 & 28 & 0.4 & 0.469 & 1.172 & 77 & 2 & 0.35 \\
\hline
\end{tabular}




$\begin{array}{rrrrrrrrrrrrrrrr}1 & 9 & 1500 & 0.06 & 6.09 & 6243.91226 & 0 & 0 & 28 & 0.3 & 0.408 & 1.36 & 78 & 2 & 0.3047 & 0 \\ 1 & 9 & 1520 & 0.04 & 4.82 & 6243.9963 & 0 & 0 & 28 & 0.2 & 0.323 & 1.615 & 79 & 2 & 0.2412 & 0 \\ 1 & 9 & 1540 & 0.02 & 3.76 & 6244.03949 & 0 & 0 & 28 & 0.1 & 0.252 & 2.52 & 80 & 2 & 0.188 & 0\end{array}$

2004.02.14 META-10 wt\%-U [sonicated] DG 0.1-1000 s-1 run 3.txt

Mode Step t[s] n[rpm] M[\%.] Phi[rad]

$\begin{array}{lllllll}\text { Gamma } J[t] ~ T\left[{ }^{\circ} \mathrm{C}\right] & \mathrm{D}[1 / \mathrm{s}] \quad \mathrm{Tau}[\mathrm{Pa}] & \mathrm{Eta}[\mathrm{Pas}] & \text { MP\# } & \text { Block } & \mathrm{M}[\mathrm{mNm}] & \mathrm{G}[\mathrm{Pa}\end{array}$

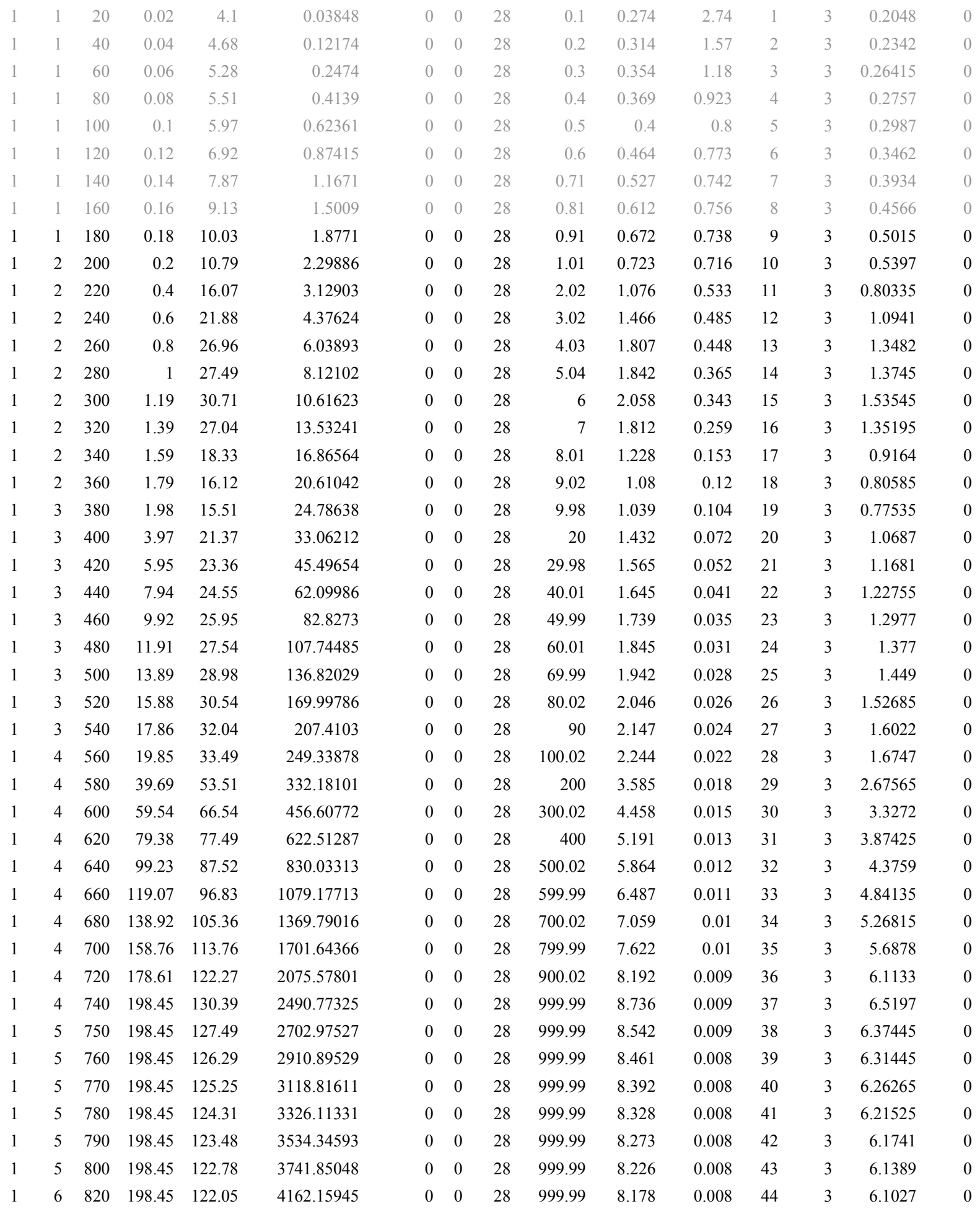




\begin{tabular}{|c|c|c|c|c|c|c|c|c|c|c|c|c|c|c|}
\hline 1 & 6 & 840 & 178.61 & 109.97 & 4536.63572 & 0 & 0 & 28 & 900.02 & 7.368 & 0.008 & 45 & 3 & 5.4986 \\
\hline 1 & 6 & 860 & 158.76 & 98.48 & 4869.37515 & 0 & 0 & 28 & 799.99 & 6.598 & 0.008 & 46 & 3 & 4.92385 \\
\hline 1 & 6 & 880 & 138.92 & 87.59 & 5160.64399 & 0 & 0 & 28 & 700.02 & 5.868 & 0.008 & 47 & 3 & 4.3793 \\
\hline 1 & 6 & 900 & 119.07 & 76.41 & 5410.35191 & 0 & 0 & 28 & 599.99 & 5.12 & 0.009 & 48 & 3 & 3.82065 \\
\hline 1 & 6 & 920 & 99.23 & 65.26 & 5618.49734 & 0 & 0 & 28 & 500.02 & 4.372 & 0.009 & 49 & 3 & 3.26295 \\
\hline 1 & 6 & 940 & 79.38 & 53.76 & 5785.07008 & 0 & 0 & 28 & 400 & 3.602 & 0.009 & 50 & 3 & 2.68795 \\
\hline 1 & 6 & 960 & 59.54 & 41.6 & 5910.15417 & 0 & 0 & 28 & 300.02 & 2.787 & 0.009 & 51 & 3 & 2.0802 \\
\hline 1 & 6 & 980 & 39.69 & 28.7 & 5993.59251 & 0 & 0 & 28 & 200 & 1.923 & 0.01 & 52 & 3 & 1.43515 \\
\hline 1 & 6 & 1000 & 19.85 & 14.33 & 6035.49036 & 0 & 0 & 28 & 100.02 & 0.96 & 0.01 & 53 & 3 & 0.7166 \\
\hline 1 & 7 & 1020 & 17.86 & 14.16 & 6073.19183 & 0 & 0 & 28 & 90 & 0.948 & 0.011 & 54 & 3 & 0.7078 \\
\hline 1 & 7 & 1040 & 15.88 & 13.41 & 6106.44402 & 0 & 0 & 28 & 80.02 & 0.898 & 0.011 & 55 & 3 & 0.6703 \\
\hline 1 & 7 & 1060 & 13.89 & 12.2 & 6135.587 & 0 & 0 & 28 & 69.99 & 0.817 & 0.012 & 56 & 3 & 0.6098 \\
\hline 1 & 7 & 1080 & 11.9 & 11.11 & 6160.53832 & 0 & 0 & 28 & 59.96 & 0.744 & 0.012 & 57 & 3 & 0.5554 \\
\hline 1 & 7 & 1100 & 9.92 & 9.73 & 6181.35922 & 0 & 0 & 28 & 49.99 & 0.652 & 0.013 & 58 & 3 & 0.48645 \\
\hline 1 & 7 & 1120 & 7.93 & 8.21 & 6198.00259 & 0 & 0 & 28 & 39.96 & 0.55 & 0.014 & 59 & 3 & 0.4104 \\
\hline 1 & 7 & 1140 & 5.95 & 6.57 & 6210.48571 & 0 & 0 & 28 & 29.98 & 0.44 & 0.015 & 60 & 3 & 0.3284 \\
\hline 1 & 7 & 1160 & 3.96 & 4.97 & 6218.82664 & 0 & 0 & 28 & 19.95 & 0.333 & 0.017 & 61 & 3 & 0.2487 \\
\hline 1 & 7 & 1180 & 1.98 & 2.45 & 6223.0026 & 0 & 0 & 28 & 9.98 & 0.164 & 0.016 & 62 & 3 & 0.1226 \\
\hline 1 & 8 & 1200 & 1.79 & 2.83 & 6226.77408 & 0 & 0 & 28 & 9.02 & 0.19 & 0.021 & 63 & 3 & 0.14165 \\
\hline 1 & 8 & 1220 & 1.59 & 3.08 & 6230.1081 & 0 & 0 & 28 & 8.01 & 0.206 & 0.026 & 64 & 3 & 0.15395 \\
\hline 1 & 8 & 1240 & 1.39 & 3.27 & 6233.02821 & 0 & 0 & 28 & 7 & 0.219 & 0.031 & 65 & 3 & 0.1634 \\
\hline 1 & 8 & 1260 & 1.19 & 3.46 & 6235.53127 & 0 & 0 & 28 & 6 & 0.232 & 0.039 & 66 & 3 & 0.1732 \\
\hline 1 & 8 & 1280 & 0.99 & 3.28 & 6237.61729 & 0 & 0 & 28 & 4.99 & 0.219 & 0.044 & 67 & 3 & 0.16375 \\
\hline 1 & 8 & 1300 & 0.8 & 3.23 & 6239.28705 & 0 & 0 & 28 & 4.03 & 0.216 & 0.054 & 68 & 3 & 0.1614 \\
\hline 1 & 8 & 1320 & 0.6 & 3.28 & 6240.54133 & 0 & 0 & 28 & 3.02 & 0.22 & 0.073 & 69 & 3 & 0.16415 \\
\hline 1 & 8 & 1340 & 0.4 & 3.02 & 6241.37935 & 0 & 0 & 28 & 2.02 & 0.202 & 0.1 & 70 & 3 & 0.1508 \\
\hline 1 & 8 & 1360 & 0.2 & 2.11 & 6241.80189 & 0 & 0 & 28 & 1.01 & 0.141 & 0.14 & 71 & 3 & 0.1053 \\
\hline 1 & 9 & 1380 & 0.18 & 3.25 & 6242.17967 & 0 & 0 & 28 & 0.91 & 0.218 & 0.24 & 72 & 3 & 0.16235 \\
\hline 1 & 9 & 1400 & 0.16 & 4.62 & 6242.51503 & 0 & 0 & 28 & 0.81 & 0.309 & 0.381 & 73 & 3 & 0.2309 \\
\hline 1 & 9 & 1420 & 0.14 & 5.62 & 6242.80799 & 0 & 0 & 28 & 0.71 & 0.376 & 0.53 & 74 & 3 & 0.2809 \\
\hline 1 & 9 & 1440 & 0.12 & 6.43 & 6243.05932 & 0 & 0 & 28 & 0.6 & 0.431 & 0.718 & 75 & 3 & 0.3214 \\
\hline 1 & 9 & 1460 & 0.1 & 6.29 & 6243.26902 & 0 & 0 & 28 & 0.5 & 0.421 & 0.842 & 76 & 3 & 0.3144 \\
\hline 1 & 9 & 1480 & 0.08 & 6.27 & 6243.43631 & 0 & 0 & 28 & 0.4 & 0.42 & 1.05 & 77 & 3 & 0.3133 \\
\hline 1 & 9 & 1500 & 0.06 & 5.62 & 6243.56276 & 0 & 0 & 28 & 0.3 & 0.377 & 1.257 & 78 & 3 & 0.2811 \\
\hline 1 & 9 & 1520 & 0.04 & 4.64 & 6243.64758 & 0 & 0 & 28 & 0.2 & 0.311 & 1.555 & 79 & 3 & 0.23205 \\
\hline & 9 & 1540 & 0.02 & 3.51 & 6243.68999 & 0 & 0 & 28 & 0.1 & 0.235 & 2.35 & 80 & 3 & 0.1757 \\
\hline
\end{tabular}

2004.02.14 META-10 wt\%-U [sonicated] DG 0.1-1000 s-1 run 4.txt

Mode Step t[s] n[rpm] M[\%.] Phi[rad]

Gamma $\quad J[t] \quad T\left[{ }^{\circ} \mathrm{C}\right] \quad \mathrm{D}[1 / \mathrm{s}] \quad \mathrm{Tau}[\mathrm{Pa}] \quad$ Eta[Pas $] \quad$ MP\# Block $\mathrm{M}[\mathrm{mNm}] \quad \mathrm{G}[\mathrm{Pa}]$

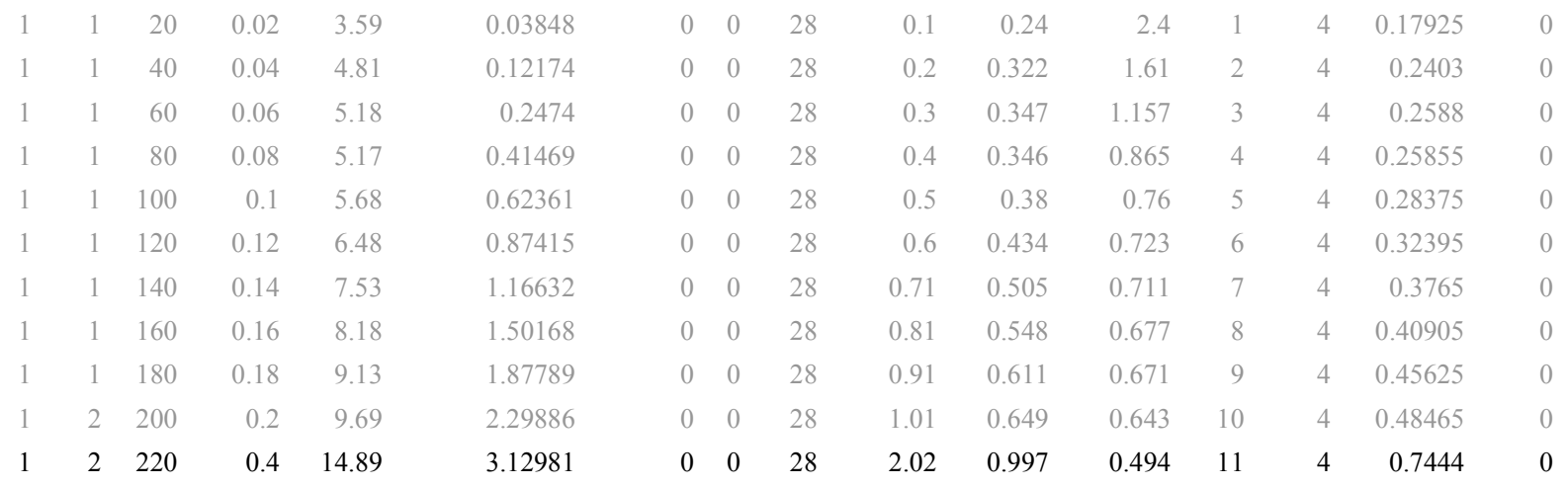




\begin{tabular}{|c|c|c|c|c|c|c|c|c|c|c|c|c|c|}
\hline 1 & 240 & 0.6 & 20.65 & 4.37624 & 0 & 0 & 28 & 3.02 & 1.383 & 0.458 & 12 & 4 & 1.03225 \\
\hline 1 & 260 & 0.8 & 24.81 & 6.0405 & 0 & 0 & 28 & 4.03 & 1.662 & 0.412 & 13 & 4 & 1.2404 \\
\hline 1 & 280 & 1 & 23.24 & 8.12337 & 0 & 0 & 28 & 5.04 & 1.557 & 0.309 & 14 & 4 & 1.1621 \\
\hline 1 & 300 & 1.19 & 24.24 & 10.61937 & 0 & 0 & 28 & 6 & 1.624 & 0.271 & 15 & 4 & 1.21175 \\
\hline 1 & 320 & 1.39 & 21.94 & 13.53555 & 0 & 0 & 28 & 7 & 1.47 & 0.21 & 16 & 4 & 1.09695 \\
\hline 1 & 340 & 1.59 & 15.55 & 16.86485 & 0 & 0 & 28 & 8.01 & 1.042 & 0.13 & 17 & 4 & 0.77755 \\
\hline 1 & 360 & 1.79 & 13.87 & 20.61277 & 0 & 0 & 28 & 9.02 & 0.929 & 0.103 & 18 & 4 & 0.69335 \\
\hline 1 & 380 & 1.98 & 13.5 & 24.78717 & 0 & 0 & 28 & 9.98 & 0.905 & 0.091 & 19 & 4 & 0.6751 \\
\hline 1 & 400 & 3.97 & 19.03 & 33.06683 & 0 & 0 & 28 & 20 & 1.275 & 0.064 & 20 & 4 & 0.95155 \\
\hline 1 & 420 & 5.95 & 21.23 & 45.49733 & 0 & 0 & 28 & 29.98 & 1.422 & 0.047 & 21 & 4 & 1.06155 \\
\hline 1 & 440 & 7.94 & 22.52 & 62.08337 & 0 & 0 & 28 & 40.01 & 1.509 & 0.038 & 22 & 4 & 1.1259 \\
\hline 1 & 460 & 9.92 & 23.83 & 82.84615 & 0 & 0 & 28 & 49.99 & 1.597 & 0.032 & 23 & 4 & 1.19155 \\
\hline 1 & 480 & 11.91 & 25.45 & 107.75663 & 0 & 0 & 28 & 60.01 & 1.705 & 0.028 & 24 & 4 & 1.27265 \\
\hline 1 & 500 & 13.89 & 26.96 & 136.80379 & 0 & 0 & 28 & 69.99 & 1.806 & 0.026 & 25 & 4 & 1.348 \\
\hline 1 & 520 & 15.88 & 28.33 & 170.01357 & 0 & 0 & 28 & 80.02 & 1.898 & 0.024 & 26 & 4 & 1.4164 \\
\hline 1 & 540 & 17.86 & 29.91 & 207.42758 & 0 & 0 & 28 & 90 & 2.004 & 0.022 & 27 & 4 & 1.49535 \\
\hline 1 & 560 & 19.85 & 31.33 & 249.36549 & 0 & 0 & 28 & 100.02 & 2.099 & 0.021 & 28 & 4 & 1.56645 \\
\hline 1 & 580 & 39.69 & 50.66 & 332.2085 & 0 & 0 & 28 & 200 & 3.394 & 0.017 & 29 & 4 & 2.5328 \\
\hline 1 & 600 & 59.54 & 63.46 & 456.64463 & 0 & 0 & 28 & 300.02 & 4.252 & 0.014 & 30 & 4 & 3.1729 \\
\hline 1 & 620 & 79.38 & 74.46 & 622.64403 & 0 & 0 & 28 & 400 & 4.989 & 0.012 & 31 & 4 & 3.7228 \\
\hline 1 & 640 & 99.23 & 84.37 & 830.16429 & 0 & 0 & 28 & 500.02 & 5.653 & 0.011 & 32 & 4 & 4.21855 \\
\hline 1 & 660 & 119.07 & 93.53 & 1079.16299 & 0 & 0 & 28 & 599.99 & 6.267 & 0.01 & 33 & 4 & 4.6767 \\
\hline 1 & 680 & 138.92 & 102.66 & 1369.72419 & 0 & 0 & 28 & 700.02 & 6.878 & 0.01 & 34 & 4 & 5.1328 \\
\hline 1 & 700 & 158.76 & 111.17 & 1702.07563 & 0 & 0 & 28 & 799.99 & 7.448 & 0.009 & 35 & 4 & 5.5585 \\
\hline 1 & 720 & 178.61 & 119.93 & 2075.61571 & 0 & 0 & 28 & 900.02 & 8.035 & 0.009 & 36 & 4 & 5.9964 \\
\hline 1 & 740 & 198.45 & 128.21 & 2490.91541 & 0 & 0 & 28 & 999.99 & 8.59 & 0.009 & 37 & 4 & 6.4105 \\
\hline 1 & 750 & 198.45 & 125.51 & 2703.01375 & 0 & 0 & 28 & 999.99 & 8.409 & 0.008 & 38 & 4 & 6.2753 \\
\hline 1 & 760 & 198.45 & 124.43 & 2910.83011 & 0 & 0 & 28 & 999.99 & 8.337 & 0.008 & 39 & 4 & 6.22135 \\
\hline 1 & 770 & 198.45 & 123.45 & 3118.75013 & 0 & 0 & 28 & 999.99 & 8.271 & 0.008 & 40 & 4 & 6.17225 \\
\hline 1 & 780 & 198.45 & 122.58 & 3326.67016 & 0 & 0 & 28 & 999.99 & 8.213 & 0.008 & 41 & 4 & 6.1292 \\
\hline 1 & 790 & 198.45 & 121.8 & 3534.07104 & 0 & 0 & 28 & 999.99 & 8.161 & 0.008 & 42 & 4 & 6.08995 \\
\hline 1 & 800 & 198.45 & 121.12 & 3742.1992 & 0 & 0 & 28 & 999.99 & 8.115 & 0.008 & 43 & 4 & 6.056 \\
\hline 1 & 820 & 198.45 & 120.34 & 4162.40371 & 0 & 0 & 28 & 999.99 & 8.062 & 0.008 & 44 & 4 & 6.01675 \\
\hline 1 & 840 & 178.61 & 108.36 & 4537.07947 & 0 & 0 & 28 & 900.02 & 7.26 & 0.008 & 45 & 4 & 5.41795 \\
\hline 1 & 860 & 158.76 & 96.85 & 4869.921 & 0 & 0 & 28 & 799.99 & 6.489 & 0.008 & 46 & 4 & 4.84245 \\
\hline 1 & 880 & 138.92 & 86.04 & 5161.05554 & 0 & 0 & 28 & 700.02 & 5.764 & 0.008 & 47 & 4 & 4.3018 \\
\hline 1 & 900 & 119.07 & 74.85 & 5410.69041 & 0 & 0 & 28 & 599.99 & 5.015 & 0.008 & 48 & 4 & 3.74235 \\
\hline 1 & 920 & 99.23 & 63.72 & 5619.03377 & 0 & 0 & 28 & 500.02 & 4.269 & 0.009 & 49 & 4 & 3.1858 \\
\hline 1 & 940 & 79.38 & 52.37 & 5785.42901 & 0 & 0 & 28 & 400 & 3.509 & 0.009 & 50 & 4 & 2.61865 \\
\hline 1 & 960 & 59.54 & 40.51 & 5910.54451 & 0 & 0 & 28 & 300.02 & 2.714 & 0.009 & 51 & 4 & 2.0253 \\
\hline 1 & 980 & 39.69 & 27.85 & 5993.96243 & 0 & 0 & 28 & 200 & 1.866 & 0.009 & 52 & 4 & 1.39235 \\
\hline 1 & $6 \quad 1000$ & 19.85 & 13.9 & 6035.83908 & 0 & 0 & 28 & 100.02 & 0.932 & 0.009 & 53 & 4 & 0.69515 \\
\hline 1 & $7 \quad 1020$ & 17.86 & 13.59 & 6073.53976 & 0 & 0 & 28 & 90 & 0.911 & 0.01 & 54 & 4 & 0.6795 \\
\hline 1 & $7 \quad 1040$ & 15.88 & 12.79 & 6106.81001 & 0 & 0 & 28 & 80.02 & 0.857 & 0.011 & 55 & 4 & 0.6395 \\
\hline 1 & $7 \quad 1060$ & 13.89 & 11.62 & 6135.94671 & 0 & 0 & 28 & 69.99 & 0.779 & 0.011 & 56 & 4 & 0.581 \\
\hline 1 & $7 \quad 1080$ & 11.9 & 10.48 & 6160.91609 & 0 & 0 & 28 & 59.96 & 0.702 & 0.012 & 57 & 4 & 0.52395 \\
\hline 1 & 71100 & 9.92 & 9.15 & 6181.71265 & 0 & 0 & 28 & 49.99 & 0.613 & 0.012 & 58 & 4 & 0.45765 \\
\hline 1 & 71120 & 7.93 & 7.73 & 6198.36073 & 0 & 0 & 28 & 39.96 & 0.518 & 0.013 & 59 & 4 & 0.38625 \\
\hline 1 & $7 \quad 1140$ & 5.95 & 6.08 & 6210.84464 & 0 & 0 & 28 & 29.98 & 0.408 & 0.014 & 60 & 4 & 0.30415 \\
\hline 1 & $7 \quad 1160$ & 3.96 & 4.52 & 6219.18164 & 0 & 0 & 28 & 19.95 & 0.303 & 0.015 & 61 & 4 & 0.22585 \\
\hline
\end{tabular}




\begin{tabular}{|c|c|c|c|c|c|c|c|c|c|c|c|c|c|c|}
\hline & 7 & 1180 & 1.98 & 2.15 & 6223.3576 & 0 & 0 & 28 & 9.98 & 0.144 & 0.014 & 62 & 4 & 0.10775 \\
\hline & 8 & 1200 & 1.79 & 2.41 & 6227.12751 & 0 & 0 & 28 & 9.02 & 0.162 & 0.018 & 63 & 4 & 0.12055 \\
\hline & 8 & 1220 & 1.59 & 2.63 & 6230.46467 & 0 & 0 & 28 & 8.01 & 0.176 & 0.022 & 64 & 4 & 0.1315 \\
\hline & 8 & 1240 & 1.39 & 2.78 & 6233.384 & 0 & 0 & 28 & 7 & 0.186 & 0.027 & 65 & 4 & 0.139 \\
\hline & 8 & 1260 & 1.19 & 2.88 & 6235.88549 & 0 & 0 & 28 & 6 & 0.193 & 0.032 & 66 & 4 & 0.144 \\
\hline & 8 & 1280 & 0.99 & 2.79 & 6237.97229 & 0 & 0 & 28 & 4.99 & 0.187 & 0.037 & 67 & 4 & 0.1395 \\
\hline & 8 & 1300 & 0.8 & 2.67 & 6239.64362 & 0 & 0 & 28 & 4.03 & 0.179 & 0.044 & 68 & 4 & 0.1337 \\
\hline & 8 & 1320 & 0.6 & 2.55 & 6240.89711 & 0 & 0 & 28 & 3.02 & 0.171 & 0.057 & 69 & 4 & 0.12745 \\
\hline & 8 & 1340 & 0.4 & 2.33 & 6241.73513 & 0 & 0 & 28 & 2.02 & 0.156 & 0.077 & 70 & 4 & 0.1167 \\
\hline 1 & 8 & 1360 & 0.2 & 1.6 & 6242.15689 & 0 & 0 & 28 & 1.01 & 0.107 & 0.106 & 71 & 4 & 0.08015 \\
\hline 1 & 9 & 1380 & 0.18 & 2.5 & 6242.53545 & 0 & 0 & 28 & 0.91 & 0.167 & 0.184 & 72 & 4 & 0.1249 \\
\hline 1 & 9 & 1400 & 0.16 & 3.43 & 6242.87082 & 0 & 0 & 28 & 0.81 & 0.23 & 0.284 & 73 & 4 & 0.17155 \\
\hline 1 & 9 & 1420 & 0.14 & 4.24 & 6243.16377 & 0 & 0 & 28 & 0.71 & 0.284 & 0.4 & 74 & 4 & 0.21175 \\
\hline 1 & 9 & 1440 & 0.12 & 4.77 & 6243.4151 & 0 & 0 & 28 & 0.6 & 0.32 & 0.533 & 75 & 4 & 0.2385 \\
\hline 1 & 9 & 1460 & 0.1 & 4.95 & 6243.6248 & 0 & 0 & 28 & 0.5 & 0.331 & 0.662 & 76 & 4 & 0.2473 \\
\hline 1 & 9 & 1480 & 0.08 & 5.02 & 6243.79288 & 0 & 0 & 28 & 0.4 & 0.336 & 0.84 & 77 & 4 & 0.25085 \\
\hline 1 & 9 & 1500 & 0.06 & 5.07 & 6243.91854 & 0 & 0 & 28 & 0.3 & 0.34 & 1.133 & 78 & 4 & 0.25365 \\
\hline 1 & 9 & 1520 & 0.04 & 4.24 & 6244.00336 & 0 & 0 & 28 & 0.2 & 0.284 & 1.42 & 79 & 4 & 0.21175 \\
\hline & 9 & 1540 & 0.02 & 3.19 & 6244.04578 & 0 & 0 & 28 & 0.1 & 0.214 & 2.14 & 80 & 4 & 0.1595 \\
\hline
\end{tabular}

2004.02.14 META-10 wt \%-U [sonicated] DG 0.1-1000 s-1 run 5.txt

Mode Step t[s] n[rpm] $\quad$ M[\%.] Phi[rad]

\begin{tabular}{|c|c|c|c|}
\hline 1 & 1 & 20 & 0.02 \\
\hline & 1 & 40 & 0.04 \\
\hline & 1 & 60 & 0.06 \\
\hline & 1 & 80 & 0.08 \\
\hline & 1 & 100 & 0.1 \\
\hline & 1 & 120 & 0.12 \\
\hline & 1 & 140 & 0.14 \\
\hline & 1 & 160 & 0.16 \\
\hline & 1 & 180 & 0.18 \\
\hline 1 & 2 & 200 & 0.2 \\
\hline & 2 & 220 & 0.4 \\
\hline & 2 & 240 & 0.6 \\
\hline & 2 & 260 & 0.8 \\
\hline & 2 & 280 & 1 \\
\hline & 2 & 300 & 1.19 \\
\hline & 2 & 320 & 1.39 \\
\hline & 2 & 340 & 1.59 \\
\hline & 2 & 360 & 1.79 \\
\hline & 3 & 380 & 1.98 \\
\hline & 3 & 400 & 3.97 \\
\hline & 3 & 420 & 5.95 \\
\hline & 3 & 440 & 7.94 \\
\hline & 3 & 460 & 9.92 \\
\hline 1 & 3 & 480 & 11.91 \\
\hline & 3 & 500 & 13.89 \\
\hline 1 & 3 & 520 & 15.88 \\
\hline & 3 & 540 & 17.86 \\
\hline & 4 & 560 & 19.85 \\
\hline
\end{tabular}

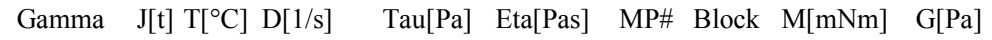

\begin{tabular}{|c|c|c|c|c|c|c|c|c|c|}
\hline 0.03848 & 0 & 0 & 28 & 0.1 & 0.24 & 2.4 & 1 & 4 & 0.17925 \\
\hline 0.12174 & 0 & 0 & 28 & 0.2 & 0.322 & 1.61 & 2 & 4 & 0.2403 \\
\hline 0.2474 & 0 & 0 & 28 & 0.3 & 0.347 & 1.157 & 3 & 4 & 0.2588 \\
\hline 0.41469 & 0 & 0 & 28 & 0.4 & 0.346 & 0.865 & 4 & 4 & 0.25855 \\
\hline 0.62361 & 0 & 0 & 28 & 0.5 & 0.38 & 0.76 & 5 & 4 & 0.28375 \\
\hline 0.87415 & 0 & 0 & 28 & 0.6 & 0.434 & 0.723 & 6 & 4 & 0.32395 \\
\hline 1.16632 & 0 & 0 & 28 & 0.71 & 0.505 & 0.711 & 7 & 4 & 0.3765 \\
\hline 1.50168 & 0 & 0 & 28 & 0.81 & 0.548 & 0.677 & 8 & 4 & 0.40905 \\
\hline 1.87789 & 0 & 0 & 28 & 0.91 & 0.611 & 0.671 & 9 & 4 & 0.45625 \\
\hline 2.29886 & 0 & 0 & 28 & 1.01 & 0.649 & 0.643 & 10 & 4 & 0.48465 \\
\hline 3.12981 & 0 & 0 & 28 & 2.02 & 0.997 & 0.494 & 11 & 4 & 0.7444 \\
\hline 4.37624 & 0 & 0 & 28 & 3.02 & 1.383 & 0.458 & 12 & 4 & 1.03225 \\
\hline 6.0405 & 0 & 0 & 28 & 4.03 & 1.662 & 0.412 & 13 & 4 & 1.2404 \\
\hline 8.12337 & 0 & 0 & 28 & 5.04 & 1.557 & 0.309 & 14 & 4 & 1.1621 \\
\hline 10.61937 & 0 & 0 & 28 & 6 & 1.624 & 0.271 & 15 & 4 & 1.21175 \\
\hline 13.53555 & 0 & 0 & 28 & 7 & 1.47 & 0.21 & 16 & 4 & 1.09695 \\
\hline 16.86485 & 0 & 0 & 28 & 8.01 & 1.042 & 0.13 & 17 & 4 & 0.77755 \\
\hline 20.61277 & 0 & 0 & 28 & 9.02 & 0.929 & 0.103 & 18 & 4 & 0.69335 \\
\hline 24.78717 & 0 & 0 & 28 & 9.98 & 0.905 & 0.091 & 19 & 4 & 0.6751 \\
\hline 33.06683 & 0 & 0 & 28 & 20 & 1.275 & 0.064 & 20 & 4 & 0.95155 \\
\hline 45.49733 & 0 & 0 & 28 & 29.98 & 1.422 & 0.047 & 21 & 4 & 1.06155 \\
\hline 62.08337 & 0 & 0 & 28 & 40.01 & 1.509 & 0.038 & 22 & 4 & 1.1259 \\
\hline 82.84615 & 0 & 0 & 28 & 49.99 & 1.597 & 0.032 & 23 & 4 & 1.19155 \\
\hline 107.75663 & 0 & 0 & 28 & 60.01 & 1.705 & 0.028 & 24 & 4 & 1.27265 \\
\hline 36.80379 & 0 & 0 & 28 & 69.99 & 1.806 & 0.026 & 25 & 4 & 1.348 \\
\hline 70.01357 & 0 & 0 & 28 & 80.02 & 1.898 & 0.024 & 26 & 4 & 1.4164 \\
\hline 07.42758 & 0 & 0 & 28 & 90 & 2.004 & 0.022 & 27 & 4 & 1.49535 \\
\hline 249.36549 & 0 & 0 & 28 & 100.02 & 2.099 & 0.021 & 28 & 4 & 1.56645 \\
\hline
\end{tabular}




\begin{tabular}{|c|c|c|c|c|c|c|c|c|c|c|c|c|c|c|}
\hline 1 & 4 & 580 & 39.69 & 50.66 & 332.2085 & 0 & 0 & 28 & 200 & 3.394 & 0.017 & 29 & 4 & 2.5328 \\
\hline 1 & 4 & 600 & 59.54 & 63.46 & 456.64463 & 0 & 0 & 28 & 300.02 & 4.252 & 0.014 & 30 & 4 & 3.1729 \\
\hline 1 & 4 & 620 & 79.38 & 74.46 & 622.64403 & 0 & 0 & 28 & 400 & 4.989 & 0.012 & 31 & 4 & 3.7228 \\
\hline 1 & 4 & 640 & 99.23 & 84.37 & 830.16429 & 0 & 0 & 28 & 500.02 & 5.653 & 0.011 & 32 & 4 & 4.21855 \\
\hline 1 & 4 & 660 & 119.07 & 93.53 & 1079.16299 & 0 & 0 & 28 & 599.99 & 6.267 & 0.01 & 33 & 4 & 4.6767 \\
\hline 1 & 4 & 680 & 138.92 & 102.66 & 1369.72419 & 0 & 0 & 28 & 700.02 & 6.878 & 0.01 & 34 & 4 & 5.1328 \\
\hline 1 & 4 & 700 & 158.76 & 111.17 & 1702.07563 & 0 & 0 & 28 & 799.99 & 7.448 & 0.009 & 35 & 4 & 5.5585 \\
\hline 1 & 4 & 720 & 178.61 & 119.93 & 2075.61571 & 0 & 0 & 28 & 900.02 & 8.035 & 0.009 & 36 & 4 & 5.9964 \\
\hline 1 & 4 & 740 & 198.45 & 128.21 & 2490.91541 & 0 & 0 & 28 & 999.99 & 8.59 & 0.009 & 37 & 4 & 6.4105 \\
\hline 1 & 5 & 750 & 198.45 & 125.51 & 2703.01375 & 0 & 0 & 28 & 999.99 & 8.409 & 0.008 & 38 & 4 & 6.2753 \\
\hline 1 & 5 & 760 & 198.45 & 124.43 & 2910.83011 & 0 & 0 & 28 & 999.99 & 8.337 & 0.008 & 39 & 4 & 6.22135 \\
\hline 1 & 5 & 770 & 198.45 & 123.45 & 3118.75013 & 0 & 0 & 28 & 999.99 & 8.271 & 0.008 & 40 & 4 & 6.17225 \\
\hline 1 & 5 & 780 & 198.45 & 122.58 & 3326.67016 & 0 & 0 & 28 & 999.99 & 8.213 & 0.008 & 41 & 4 & 6.1292 \\
\hline 1 & 5 & 790 & 198.45 & 121.8 & 3534.07104 & 0 & 0 & 28 & 999.99 & 8.161 & 0.008 & 42 & 4 & 6.08995 \\
\hline 1 & 5 & 800 & 198.45 & 121.12 & 3742.1992 & 0 & 0 & 28 & 999.99 & 8.115 & 0.008 & 43 & 4 & 6.056 \\
\hline 1 & 6 & 820 & 198.45 & 120.34 & 4162.40371 & 0 & 0 & 28 & 999.99 & 8.062 & 0.008 & 44 & 4 & 6.01675 \\
\hline 1 & 6 & 840 & 178.61 & 108.36 & 4537.07947 & 0 & 0 & 28 & 900.02 & 7.26 & 0.008 & 45 & 4 & 5.41795 \\
\hline 1 & 6 & 860 & 158.76 & 96.85 & 4869.921 & 0 & 0 & 28 & 799.99 & 6.489 & 0.008 & 46 & 4 & 4.84245 \\
\hline 1 & 6 & 880 & 138.92 & 86.04 & 5161.05554 & 0 & 0 & 28 & 700.02 & 5.764 & 0.008 & 47 & 4 & 4.3018 \\
\hline 1 & 6 & 900 & 119.07 & 74.85 & 5410.69041 & 0 & 0 & 28 & 599.99 & 5.015 & 0.008 & 48 & 4 & 3.74235 \\
\hline 1 & 6 & 920 & 99.23 & 63.72 & 5619.03377 & 0 & 0 & 28 & 500.02 & 4.269 & 0.009 & 49 & 4 & 3.1858 \\
\hline 1 & 6 & 940 & 79.38 & 52.37 & 5785.42901 & 0 & 0 & 28 & 400 & 3.509 & 0.009 & 50 & 4 & 2.61865 \\
\hline 1 & 6 & 960 & 59.54 & 40.51 & 5910.54451 & 0 & 0 & 28 & 300.02 & 2.714 & 0.009 & 51 & 4 & 2.0253 \\
\hline 1 & 6 & 980 & 39.69 & 27.85 & 5993.96243 & 0 & 0 & 28 & 200 & 1.866 & 0.009 & 52 & 4 & 1.39235 \\
\hline 1 & 6 & 1000 & 19.85 & 13.9 & 6035.83908 & 0 & 0 & 28 & 100.02 & 0.932 & 0.009 & 53 & 4 & 0.69515 \\
\hline 1 & 7 & 1020 & 17.86 & 13.59 & 6073.53976 & 0 & 0 & 28 & 90 & 0.911 & 0.01 & 54 & 4 & 0.6795 \\
\hline 1 & 7 & 1040 & 15.88 & 12.79 & 6106.81001 & 0 & 0 & 28 & 80.02 & 0.857 & 0.011 & 55 & 4 & 0.6395 \\
\hline 1 & 7 & 1060 & 13.89 & 11.62 & 6135.94671 & 0 & 0 & 28 & 69.99 & 0.779 & 0.011 & 56 & 4 & 0.581 \\
\hline 1 & 7 & 1080 & 11.9 & 10.48 & 6160.91609 & 0 & 0 & 28 & 59.96 & 0.702 & 0.012 & 57 & 4 & 0.52395 \\
\hline 1 & 7 & 1100 & 9.92 & 9.15 & 6181.71265 & 0 & 0 & 28 & 49.99 & 0.613 & 0.012 & 58 & 4 & 0.45765 \\
\hline 1 & 7 & 1120 & 7.93 & 7.73 & 6198.36073 & 0 & 0 & 28 & 39.96 & 0.518 & 0.013 & 59 & 4 & 0.38625 \\
\hline 1 & 7 & 1140 & 5.95 & 6.08 & 6210.84464 & 0 & 0 & 28 & 29.98 & 0.408 & 0.014 & 60 & 4 & 0.30415 \\
\hline 1 & 7 & 1160 & 3.96 & 4.52 & 6219.18164 & 0 & 0 & 28 & 19.95 & 0.303 & 0.015 & 61 & 4 & 0.22585 \\
\hline 1 & 7 & 1180 & 1.98 & 2.15 & 6223.3576 & 0 & 0 & 28 & 9.98 & 0.144 & 0.014 & 62 & 4 & 0.10775 \\
\hline 1 & 8 & 1200 & 1.79 & 2.41 & 6227.12751 & 0 & 0 & 28 & 9.02 & 0.162 & 0.018 & 63 & 4 & 0.12055 \\
\hline 1 & 8 & 1220 & 1.59 & 2.63 & 6230.46467 & 0 & 0 & 28 & 8.01 & 0.176 & 0.022 & 64 & 4 & 0.1315 \\
\hline 1 & 8 & 1240 & 1.39 & 2.78 & 6233.384 & 0 & 0 & 28 & 7 & 0.186 & 0.027 & 65 & 4 & 0.139 \\
\hline 1 & 8 & 1260 & 1.19 & 2.88 & 6235.88549 & 0 & 0 & 28 & 6 & 0.193 & 0.032 & 66 & 4 & 0.144 \\
\hline 1 & 8 & 1280 & 0.99 & 2.79 & 6237.97229 & 0 & 0 & 28 & 4.99 & 0.187 & 0.037 & 67 & 4 & 0.1395 \\
\hline 1 & 8 & 1300 & 0.8 & 2.67 & 6239.64362 & 0 & 0 & 28 & 4.03 & 0.179 & 0.044 & 68 & 4 & 0.1337 \\
\hline 1 & 8 & 1320 & 0.6 & 2.55 & 6240.89711 & 0 & 0 & 28 & 3.02 & 0.171 & 0.057 & 69 & 4 & 0.12745 \\
\hline 1 & 8 & 1340 & 0.4 & 2.33 & 6241.73513 & 0 & 0 & 28 & 2.02 & 0.156 & 0.077 & 70 & 4 & 0.1167 \\
\hline 1 & 8 & 1360 & 0.2 & 1.6 & 6242.15689 & 0 & 0 & 28 & 1.01 & 0.107 & 0.106 & 71 & 4 & 0.08015 \\
\hline 1 & 9 & 1380 & 0.18 & 2.5 & 6242.53545 & 0 & 0 & 28 & 0.91 & 0.167 & 0.184 & 72 & 4 & 0.1249 \\
\hline 1 & 9 & 1400 & 0.16 & 3.43 & 6242.87082 & 0 & 0 & 28 & 0.81 & 0.23 & 0.284 & 73 & 4 & 0.17155 \\
\hline 1 & 9 & 1420 & 0.14 & 4.24 & 6243.16377 & 0 & 0 & 28 & 0.71 & 0.284 & 0.4 & 74 & 4 & 0.21175 \\
\hline 1 & 9 & 1440 & 0.12 & 4.77 & 6243.4151 & 0 & 0 & 28 & 0.6 & 0.32 & 0.533 & 75 & 4 & 0.2385 \\
\hline 1 & 9 & 1460 & 0.1 & 4.95 & 6243.6248 & 0 & 0 & 28 & 0.5 & 0.331 & 0.662 & 76 & 4 & 0.2473 \\
\hline 1 & 9 & 1480 & 0.08 & 5.02 & 6243.79288 & 0 & 0 & 28 & 0.4 & 0.336 & 0.84 & 77 & 4 & 0.25085 \\
\hline 1 & 9 & 1500 & 0.06 & 5.07 & 6243.91854 & 0 & 0 & 28 & 0.3 & 0.34 & 1.133 & 78 & 4 & 0.25365 \\
\hline
\end{tabular}




$\begin{array}{rrrrrrrrrrrrrrrr}1 & 9 & 1520 & 0.04 & 4.24 & 6244.00336 & 0 & 0 & 28 & 0.2 & 0.284 & 1.42 & 79 & 4 & 0.21175 & 0 \\ 1 & 9 & 1540 & 0.02 & 3.19 & 6244.04578 & 0 & 0 & 28 & 0.1 & 0.214 & 2.14 & 80 & 4 & 0.1595 & 0\end{array}$

2004.02.14 META-10 wt\%-U [sonicated] DG 0.1-1000 s-1 run 6.txt

Mode Step $\mathrm{t}[\mathrm{s}] \quad \mathrm{n}[\mathrm{rpm}] \quad \mathrm{M}[\%$.] $\quad$ Phi[rad]

Gamma $\quad J[t] T\left[{ }^{\circ} \mathrm{C}\right] \mathrm{D}[1 / \mathrm{s}] \quad \mathrm{Tau}[\mathrm{Pa}] \quad \mathrm{Eta}[\mathrm{Pas}] \quad \mathrm{MP} \#$ Block $\mathrm{M}[\mathrm{mNm}] \quad \mathrm{G}[\mathrm{Pa}]$

\begin{tabular}{|c|c|c|c|c|c|c|c|c|c|c|c|c|c|c|}
\hline 1 & 1 & 20 & 0.02 & 3.28 & 0.03848 & 0 & 0 & 28 & 0.1 & 0.22 & 2.2 & 1 & 6 & 0.16415 \\
\hline 1 & 1 & 40 & 0.04 & 4.58 & 0.12252 & 0 & 0 & 28 & 0.2 & 0.307 & 1.535 & 2 & 6 & 0.22875 \\
\hline 1 & 1 & 60 & 0.06 & 4.82 & 0.2474 & 0 & 0 & 28 & 0.3 & 0.323 & 1.077 & 3 & 6 & 0.2409 \\
\hline 1 & 1 & 80 & 0.08 & 4.79 & 0.41469 & 0 & 0 & 28 & 0.4 & 0.321 & 0.803 & 4 & 6 & 0.2396 \\
\hline 1 & 1 & 100 & 0.1 & 5 & 0.62361 & 0 & 0 & 28 & 0.5 & 0.335 & 0.67 & 5 & 6 & 0.2498 \\
\hline 1 & 1 & 120 & 0.12 & 5.93 & 0.87415 & 0 & 0 & 28 & 0.6 & 0.397 & 0.662 & 6 & 6 & 0.29645 \\
\hline 1 & 1 & 140 & 0.14 & 6.69 & 1.1671 & 0 & 0 & 28 & 0.71 & 0.448 & 0.631 & 7 & 6 & 0.3346 \\
\hline 1 & 1 & 160 & 0.16 & 7.33 & 1.50168 & 0 & 0 & 28 & 0.81 & 0.491 & 0.606 & 8 & 6 & 0.36655 \\
\hline 1 & 1 & 180 & 0.18 & 8.22 & 1.87789 & 0 & 0 & 28 & 0.91 & 0.551 & 0.605 & 9 & 6 & 0.4109 \\
\hline 1 & 2 & 200 & 0.2 & 8.57 & 2.29965 & 0 & 0 & 28 & 1.01 & 0.574 & 0.568 & 10 & 6 & 0.42835 \\
\hline 1 & 2 & 220 & 0.4 & 13.68 & 3.12981 & 0 & 0 & 28 & 2.02 & 0.916 & 0.453 & 11 & 6 & 0.68395 \\
\hline 1 & 2 & 240 & 0.6 & 18.62 & 4.37702 & 0 & 0 & 28 & 3.02 & 1.248 & 0.413 & 12 & 6 & 0.931 \\
\hline 1 & 2 & 260 & 0.8 & 22.56 & 6.04128 & 0 & 0 & 28 & 4.03 & 1.512 & 0.375 & 13 & 6 & 1.128 \\
\hline 1 & 2 & 280 & 1 & 21.61 & 8.12259 & 0 & 0 & 28 & 5.04 & 1.448 & 0.287 & 14 & 6 & 1.0805 \\
\hline 1 & 2 & 300 & 1.19 & 16.68 & 10.62251 & 0 & 0 & 28 & 6 & 1.118 & 0.186 & 15 & 6 & 0.8342 \\
\hline 1 & 2 & 320 & 1.39 & 13.16 & 13.53634 & 0 & 0 & 28 & 7 & 0.882 & 0.126 & 16 & 6 & 0.6581 \\
\hline 1 & 2 & 340 & 1.59 & 11.17 & 16.86643 & 0 & 0 & 28 & 8.01 & 0.748 & 0.093 & 17 & 6 & 0.5583 \\
\hline 1 & 2 & 360 & 1.79 & 10.66 & 20.61435 & 0 & 0 & 28 & 9.02 & 0.714 & 0.079 & 18 & 6 & 0.533 \\
\hline 1 & 3 & 380 & 1.98 & 10.65 & 24.79031 & 0 & 0 & 28 & 9.98 & 0.714 & 0.072 & 19 & 6 & 0.53265 \\
\hline 1 & 3 & 400 & 3.97 & 15.95 & 33.05976 & 0 & 0 & 28 & 20 & 1.069 & 0.053 & 20 & 6 & 0.7977 \\
\hline 1 & 3 & 420 & 5.95 & 18.16 & 45.50675 & 0 & 0 & 28 & 29.98 & 1.217 & 0.041 & 21 & 6 & 0.9082 \\
\hline 1 & 3 & 440 & 7.94 & 19.64 & 62.08337 & 0 & 0 & 28 & 40.01 & 1.316 & 0.033 & 22 & 6 & 0.982 \\
\hline 1 & 3 & 460 & 9.92 & 20.94 & 82.83594 & 0 & 0 & 28 & 49.99 & 1.403 & 0.028 & 23 & 6 & 1.0469 \\
\hline 1 & 3 & 480 & 11.91 & 22.43 & 107.72914 & 0 & 0 & 28 & 60.01 & 1.503 & 0.025 & 24 & 6 & 1.1217 \\
\hline 1 & 3 & 500 & 13.89 & 23.91 & 136.80458 & 0 & 0 & 28 & 69.99 & 1.602 & 0.023 & 25 & 6 & 1.1957 \\
\hline 1 & 3 & 520 & 15.88 & 25.28 & 170.04027 & 0 & 0 & 28 & 80.02 & 1.694 & 0.021 & 26 & 6 & 1.26405 \\
\hline 1 & 3 & 540 & 17.86 & 26.78 & 207.41737 & 0 & 0 & 28 & 90 & 1.794 & 0.02 & 27 & 6 & 1.3391 \\
\hline 1 & 4 & 560 & 19.85 & 28.11 & 249.34507 & 0 & 0 & 28 & 100.02 & 1.883 & 0.019 & 28 & 6 & 1.40545 \\
\hline 1 & 4 & 580 & 39.69 & 46.56 & 332.20772 & 0 & 0 & 28 & 200 & 3.12 & 0.016 & 29 & 6 & 2.328 \\
\hline 1 & 4 & 600 & 59.54 & 59.14 & 456.603 & 0 & 0 & 28 & 300.02 & 3.962 & 0.013 & 30 & 6 & 2.95675 \\
\hline 1 & 4 & 620 & 79.38 & 69.95 & 622.55057 & 0 & 0 & 28 & 400 & 4.686 & 0.012 & 31 & 6 & 3.4973 \\
\hline 1 & 4 & 640 & 99.23 & 80.1 & 830.1635 & 0 & 0 & 28 & 500.02 & 5.367 & 0.011 & 32 & 6 & 4.0052 \\
\hline 1 & 4 & 660 & 119.07 & 89.18 & 1078.99649 & 0 & 0 & 28 & 599.99 & 5.975 & 0.01 & 33 & 6 & 4.45915 \\
\hline 1 & 4 & 680 & 138.92 & 98.3 & 1369.84828 & 0 & 0 & 28 & 700.02 & 6.586 & 0.009 & 34 & 6 & 4.9151 \\
\hline 1 & 4 & 700 & 158.76 & 107.02 & 1702.034 & 0 & 0 & 28 & 799.99 & 7.17 & 0.009 & 35 & 6 & 5.35095 \\
\hline 1 & 4 & 720 & 178.61 & 115.93 & 2075.69896 & 0 & 0 & 28 & 900.02 & 7.767 & 0.009 & 36 & 6 & 5.7963 \\
\hline 1 & 4 & 740 & 198.45 & 124.46 & 2491.00887 & 0 & 0 & 28 & 999.99 & 8.338 & 0.008 & 37 & 6 & 6.22275 \\
\hline 1 & 5 & 750 & 198.45 & 122.16 & 2703.10643 & 0 & 0 & 28 & 999.99 & 8.184 & 0.008 & 38 & 6 & 6.10775 \\
\hline 1 & 5 & 760 & 198.45 & 121.23 & 2911.02724 & 0 & 0 & 28 & 999.99 & 8.123 & 0.008 & 39 & 6 & 6.0617 \\
\hline 1 & 5 & 770 & 198.45 & 120.39 & 3118.8436 & 0 & 0 & 28 & 999.99 & 8.066 & 0.008 & 40 & 6 & 6.0195 \\
\hline 1 & 5 & 780 & 198.45 & 119.64 & 3326.76441 & 0 & 0 & 28 & 999.99 & 8.016 & 0.008 & 41 & 6 & 5.98185 \\
\hline 1 & 5 & 790 & 198.45 & 118.98 & 3534.06083 & 0 & 0 & 28 & 999.99 & 7.971 & 0.008 & 42 & 6 & 5.9488 \\
\hline 1 & 5 & 800 & 198.45 & 118.35 & 3742.29266 & 0 & 0 & 28 & 999.99 & 7.93 & 0.008 & 43 & 6 & 5.91755 \\
\hline 1 & 6 & 820 & 198.45 & 117.71 & 4162.49717 & 0 & 0 & 28 & 999.99 & 7.887 & 0.008 & 44 & 6 & 5.8856 \\
\hline 1 & 6 & 840 & 178.61 & 106.01 & 4536.97501 & 0 & 0 & 28 & 900.02 & 7.103 & 0.008 & 45 & 6 & 5.3005 \\
\hline
\end{tabular}




\begin{tabular}{|c|c|c|c|c|c|c|c|c|c|c|c|c|c|c|}
\hline 1 & 6 & 860 & 158.76 & 94.71 & 4869.71209 & 0 & 0 & 28 & 799.99 & 6.346 & 0.008 & 46 & 6 & 4.73555 \\
\hline 1 & 6 & 880 & 138.92 & 84.02 & 5161.19062 & 0 & 0 & 28 & 700.02 & 5.63 & 0.008 & 47 & 6 & 4.2012 \\
\hline 1 & 6 & 900 & 119.07 & 73.05 & 5410.648 & 0 & 0 & 28 & 599.99 & 4.894 & 0.008 & 48 & 6 & 3.65245 \\
\hline 1 & 6 & 920 & 99.23 & 62.13 & 5619.0432 & 0 & 0 & 28 & 500.02 & 4.163 & 0.008 & 49 & 6 & 3.1067 \\
\hline 1 & 6 & 940 & 79.38 & 50.98 & 5785.45022 & 0 & 0 & 28 & 400 & 3.416 & 0.009 & 50 & 6 & 2.54895 \\
\hline 1 & 6 & 960 & 59.54 & 39.36 & 5910.44005 & 0 & 0 & 28 & 300.02 & 2.637 & 0.009 & 51 & 6 & 1.96815 \\
\hline 1 & 6 & 980 & 39.69 & 27.09 & 5993.97186 & 0 & 0 & 28 & 200 & 1.815 & 0.009 & 52 & 6 & 1.3546 \\
\hline 1 & 6 & 1000 & 19.85 & 13.67 & 6035.81787 & 0 & 0 & 28 & 100.02 & 0.916 & 0.009 & 53 & 6 & 0.6836 \\
\hline 1 & 7 & 1020 & 17.86 & 13.22 & 6073.52877 & 0 & 0 & 28 & 90 & 0.886 & 0.01 & 54 & 6 & 0.66085 \\
\hline 1 & 7 & 1040 & 15.88 & 12.39 & 6106.79823 & 0 & 0 & 28 & 80.02 & 0.83 & 0.01 & 55 & 6 & 0.6195 \\
\hline 1 & 7 & 1060 & 13.89 & 11.21 & 6135.91137 & 0 & 0 & 28 & 69.99 & 0.751 & 0.011 & 56 & 6 & 0.5607 \\
\hline 1 & 7 & 1080 & 11.9 & 10.06 & 6160.87368 & 0 & 0 & 28 & 59.96 & 0.674 & 0.011 & 57 & 6 & 0.50305 \\
\hline 1 & 7 & 1100 & 9.92 & 8.66 & 6181.67966 & 0 & 0 & 28 & 49.99 & 0.58 & 0.012 & 58 & 6 & 0.43285 \\
\hline 1 & 7 & 1120 & 7.93 & 7.27 & 6198.32382 & 0 & 0 & 28 & 39.96 & 0.487 & 0.012 & 59 & 6 & 0.3637 \\
\hline 1 & 7 & 1140 & 5.95 & 5.74 & 6210.82265 & 0 & 0 & 28 & 29.98 & 0.385 & 0.013 & 60 & 6 & 0.28705 \\
\hline 1 & 7 & 1160 & 3.96 & 4.26 & 6219.15415 & 0 & 0 & 28 & 19.95 & 0.286 & 0.014 & 61 & 6 & 0.21315 \\
\hline 1 & 7 & 1180 & 1.98 & 2.01 & 6223.32854 & 0 & 0 & 28 & 9.98 & 0.135 & 0.014 & 62 & 6 & 0.1004 \\
\hline 1 & 8 & 1200 & 1.79 & 2.32 & 6227.0961 & 0 & 0 & 28 & 9.02 & 0.156 & 0.017 & 63 & 6 & 0.1162 \\
\hline 1 & 8 & 1220 & 1.59 & 2.66 & 6230.43168 & 0 & 0 & 28 & 8.01 & 0.178 & 0.022 & 64 & 6 & 0.13285 \\
\hline 1 & 8 & 1240 & 1.39 & 2.74 & 6233.35258 & 0 & 0 & 28 & 7 & 0.184 & 0.026 & 65 & 6 & 0.13705 \\
\hline 1 & 8 & 1260 & 1.19 & 2.49 & 6235.85407 & 0 & 0 & 28 & 6 & 0.167 & 0.028 & 66 & 6 & 0.1246 \\
\hline 1 & 8 & 1280 & 0.99 & 2.35 & 6237.94088 & 0 & 0 & 28 & 4.99 & 0.157 & 0.031 & 67 & 6 & 0.11725 \\
\hline 1 & 8 & 1300 & 0.8 & 2.38 & 6239.61063 & 0 & 0 & 28 & 4.03 & 0.159 & 0.039 & 68 & 6 & 0.11895 \\
\hline 1 & 8 & 1320 & 0.6 & 2.1 & 6240.86648 & 0 & 0 & 28 & 3.02 & 0.141 & 0.047 & 69 & 6 & 0.1052 \\
\hline 1 & 8 & 1340 & 0.4 & 1.87 & 6241.70372 & 0 & 0 & 28 & 2.02 & 0.125 & 0.062 & 70 & 6 & 0.0933 \\
\hline 1 & 8 & 1360 & 0.2 & 1.19 & 6242.12548 & 0 & 0 & 28 & 1.01 & 0.08 & 0.079 & 71 & 6 & 0.0596 \\
\hline 1 & 9 & 1380 & 0.18 & 1.82 & 6242.50404 & 0 & 0 & 28 & 0.91 & 0.122 & 0.134 & 72 & 6 & 0.09095 \\
\hline 1 & 9 & 1400 & 0.16 & 2.33 & 6242.84019 & 0 & 0 & 28 & 0.81 & 0.156 & 0.193 & 73 & 6 & 0.11665 \\
\hline 1 & 9 & 1420 & 0.14 & 2.84 & 6243.13236 & 0 & 0 & 28 & 0.71 & 0.19 & 0.268 & 74 & 6 & 0.1418 \\
\hline 1 & 9 & 1440 & 0.12 & 3.32 & 6243.38368 & 0 & 0 & 28 & 0.6 & 0.222 & 0.37 & 75 & 6 & 0.166 \\
\hline 1 & 9 & 1460 & 0.1 & 3.58 & 6243.59417 & 0 & 0 & 28 & 0.5 & 0.24 & 0.48 & 76 & 6 & 0.17895 \\
\hline 1 & 9 & 1480 & 0.08 & 3.83 & 6243.76146 & 0 & 0 & 28 & 0.4 & 0.256 & 0.64 & 77 & 6 & 0.19135 \\
\hline 1 & 9 & 1500 & 0.06 & 4.16 & 6243.88712 & 0 & 0 & 28 & 0.3 & 0.279 & 0.93 & 78 & 6 & 0.20815 \\
\hline 1 & 9 & 1520 & 0.04 & 4.04 & 6243.97195 & 0 & 0 & 28 & 0.2 & 0.271 & 1.355 & 79 & 6 & 0.20195 \\
\hline 1 & 9 & 1540 & 0.02 & 3.19 & 6244.01436 & 0 & 0 & 28 & 0.1 & 0.214 & 2.14 & 80 & 6 & 0.15965 \\
\hline
\end{tabular}

2004.02.14 META-10 wt\%-U [sonicated] DG 0.1-1000 s-1 run 7.txt
Mode Step t[s] n[rpm] M[\%.] Phi[rad]
Gamma J[t] T[ $\left.{ }^{\circ} \mathrm{C}\right] \mathrm{D}[1 / \mathrm{s}] \quad \mathrm{Tau}[\mathrm{Pa}] \quad \mathrm{Eta}[\mathrm{Pas}] \quad$ MP\# Block $\mathrm{M}[\mathrm{mNm}] \quad \mathrm{G}[\mathrm{Pa}]$

$\begin{array}{rrrrrrrrrrrrrrrrrrrrrr}1 & 1 & 20 & 0.02 & 3.64 & 0.03848 & 0 & 0 & 28 & 0.1 & 0.244 & 2.44 & 1 & 7 & 0.18185 & 0 \\ 1 & 1 & 40 & 0.04 & 4.7 & 0.12252 & 0 & 0 & 28 & 0.2 & 0.315 & 1.575 & 2 & 7 & 0.23515 & 0 \\ 1 & 1 & 60 & 0.06 & 4.79 & 0.2474 & 0 & 0 & 28 & 0.3 & 0.321 & 1.07 & 3 & 7 & 0.2394 & 0 \\ 1 & 1 & 80 & 0.08 & 5.03 & 0.41469 & 0 & 0 & 28 & 0.4 & 0.337 & 0.843 & 4 & 7 & 0.25165 & 0 \\ 1 & 1 & 100 & 0.1 & 5.54 & 0.62361 & 0 & 0 & 28 & 0.5 & 0.371 & 0.742 & 5 & 7 & 0.2769 & 0 \\ 1 & 1 & 120 & 0.12 & 6.18 & 0.87415 & 0 & 0 & 28 & 0.6 & 0.414 & 0.69 & 6 & 7 & 0.30875 & 0 \\ 1 & 1 & 140 & 0.14 & 6.9 & 1.16632 & 0 & 0 & 28 & 0.71 & 0.462 & 0.651 & 7 & 7 & 0.345 & 0 \\ 1 & 1 & 160 & 0.16 & 7.58 & 1.50168 & 0 & 0 & 28 & 0.81 & 0.508 & 0.627 & 8 & 7 & 0.379 \\ 1 & 1 & 180 & 0.18 & 8.13 & 1.87867 & 0 & 0 & 28 & 0.91 & 0.545 & 0.599 & 9 & 7 & 0.40645 & 0 \\ 1 & 2 & 200 & 0.2 & 8.79 & 2.29965 & 0 & 0 & 28 & 1.01 & 0.589 & 0.583 & 10 & 7 & 0.43945 \\ 1 & 2 & 220 & 0.4 & 14.14 & 3.1306 & 0 & 0 & 28 & 2.02 & 0.947 & 0.469 & 11 & 7 & 0.707 & 0 \\ 1 & 2 & 240 & 0.6 & 18.98 & 4.37702 & 0 & 0 & 28 & 3.02 & 1.271 & 0.421 & 12 & 7 & 0.94875 & 0\end{array}$




\begin{tabular}{|c|c|c|c|c|c|c|c|c|c|c|c|c|c|c|}
\hline 1 & 2 & 260 & 0.8 & 20.19 & 6.04207 & 0 & 0 & 28 & 4.03 & 1.353 & 0.336 & 13 & 7 & 1.0095 \\
\hline 1 & 2 & 280 & 1 & 17.6 & 8.12337 & 0 & 0 & 28 & 5.04 & 1.179 & 0.234 & 14 & 7 & 0.88005 \\
\hline 1 & 2 & 300 & 1.19 & 15.77 & 10.6233 & 0 & 0 & 28 & 6 & 1.057 & 0.176 & 15 & 7 & 0.7885 \\
\hline 1 & 2 & 320 & 1.39 & 12.17 & 13.53791 & 0 & 0 & 28 & 7 & 0.816 & 0.117 & 16 & 7 & 0.6087 \\
\hline 1 & 2 & 340 & 1.59 & 10.34 & 16.86878 & 0 & 0 & 28 & 8.01 & 0.693 & 0.087 & 17 & 7 & 0.51715 \\
\hline 1 & 2 & 360 & 1.79 & 10.09 & 20.61277 & 0 & 0 & 28 & 9.02 & 0.676 & 0.075 & 18 & 7 & 0.5047 \\
\hline 1 & 3 & 380 & 1.98 & 9.96 & 24.78795 & 0 & 0 & 28 & 9.98 & 0.668 & 0.067 & 19 & 7 & 0.4982 \\
\hline 1 & 3 & 400 & 3.97 & 15.24 & 33.06448 & 0 & 0 & 28 & 20 & 1.021 & 0.051 & 20 & 7 & 0.76205 \\
\hline 1 & 3 & 420 & 5.95 & 17.37 & 45.49497 & 0 & 0 & 28 & 29.98 & 1.164 & 0.039 & 21 & 7 & 0.8686 \\
\hline 1 & 3 & 440 & 7.94 & 18.86 & 62.08572 & 0 & 0 & 28 & 40.01 & 1.263 & 0.032 & 22 & 7 & 0.94285 \\
\hline 1 & 3 & 460 & 9.92 & 20 & 82.85008 & 0 & 0 & 28 & 49.99 & 1.34 & 0.027 & 23 & 7 & 0.99995 \\
\hline 1 & 3 & 480 & 11.91 & 21.43 & 107.73071 & 0 & 0 & 28 & 60.01 & 1.436 & 0.024 & 24 & 7 & 1.07155 \\
\hline 1 & 3 & 500 & 13.89 & 22.96 & 136.79044 & 0 & 0 & 28 & 69.99 & 1.538 & 0.022 & 25 & 7 & 1.1481 \\
\hline 1 & 3 & 520 & 15.88 & 24.28 & 170.01043 & 0 & 0 & 28 & 80.02 & 1.627 & 0.02 & 26 & 7 & 1.2141 \\
\hline 1 & 3 & 540 & 17.86 & 25.72 & 207.40559 & 0 & 0 & 28 & 90 & 1.723 & 0.019 & 27 & 7 & 1.2858 \\
\hline 1 & 4 & 560 & 19.85 & 27.04 & 249.3647 & 0 & 0 & 28 & 100.02 & 1.812 & 0.018 & 28 & 7 & 1.352 \\
\hline 1 & 4 & 580 & 39.69 & 45.04 & 332.18651 & 0 & 0 & 28 & 200 & 3.018 & 0.015 & 29 & 7 & 2.2521 \\
\hline 1 & 4 & 600 & 59.54 & 57.43 & 456.46791 & 0 & 0 & 28 & 300.02 & 3.847 & 0.013 & 30 & 7 & 2.87125 \\
\hline 1 & 4 & 620 & 79.38 & 68.19 & 622.50737 & 0 & 0 & 28 & 400 & 4.568 & 0.011 & 31 & 7 & 3.40925 \\
\hline 1 & 4 & 640 & 99.23 & 78.22 & 829.97579 & 0 & 0 & 28 & 500.02 & 5.241 & 0.01 & 32 & 7 & 3.9109 \\
\hline 1 & 4 & 660 & 119.07 & 87.39 & 1079.23525 & 0 & 0 & 28 & 599.99 & 5.855 & 0.01 & 33 & 7 & 4.36955 \\
\hline 1 & 4 & 680 & 138.92 & 96.55 & 1369.79644 & 0 & 0 & 28 & 700.02 & 6.469 & 0.009 & 34 & 7 & 4.8275 \\
\hline 1 & 4 & 700 & 158.76 & 105.38 & 1701.89813 & 0 & 0 & 28 & 799.99 & 7.061 & 0.009 & 35 & 7 & 5.26915 \\
\hline 1 & 4 & 720 & 178.61 & 114.32 & 2075.72959 & 0 & 0 & 28 & 900.02 & 7.659 & 0.009 & 36 & 7 & 5.71575 \\
\hline 1 & 4 & 740 & 198.45 & 122.95 & 2491.03872 & 0 & 0 & 28 & 999.99 & 8.238 & 0.008 & 37 & 7 & 6.1476 \\
\hline 1 & 5 & 750 & 198.45 & 120.87 & 2703.34441 & 0 & 0 & 28 & 999.99 & 8.098 & 0.008 & 38 & 7 & 6.0435 \\
\hline 1 & 5 & 760 & 198.45 & 119.97 & 2911.26443 & 0 & 0 & 28 & 999.99 & 8.038 & 0.008 & 39 & 7 & 5.9986 \\
\hline 1 & 5 & 770 & 198.45 & 119.18 & 3119.08079 & 0 & 0 & 28 & 999.99 & 7.985 & 0.008 & 40 & 7 & 5.9589 \\
\hline 1 & 5 & 780 & 198.45 & 118.49 & 3326.89714 & 0 & 0 & 28 & 999.99 & 7.939 & 0.008 & 41 & 7 & 5.9246 \\
\hline 1 & 5 & 790 & 198.45 & 117.83 & 3534.19513 & 0 & 0 & 28 & 999.99 & 7.895 & 0.008 & 42 & 7 & 5.89145 \\
\hline 1 & 5 & 800 & 198.45 & 117.28 & 3742.21883 & 0 & 0 & 28 & 999.99 & 7.858 & 0.008 & 43 & 7 & 5.8639 \\
\hline 1 & 6 & 820 & 198.45 & 116.65 & 4162.5278 & 0 & 0 & 28 & 999.99 & 7.815 & 0.008 & 44 & 7 & 5.8324 \\
\hline 1 & 6 & 840 & 178.61 & 105.1 & 4537.09832 & 0 & 0 & 28 & 900.02 & 7.042 & 0.008 & 45 & 7 & 5.255 \\
\hline 1 & 6 & 860 & 158.76 & 93.9 & 4870.0019 & 0 & 0 & 28 & 799.99 & 6.291 & 0.008 & 46 & 7 & 4.69475 \\
\hline 1 & 6 & 880 & 138.92 & 83.26 & 5161.19926 & 0 & 0 & 28 & 700.02 & 5.579 & 0.008 & 47 & 7 & 4.1632 \\
\hline 1 & 6 & 900 & 119.07 & 72.36 & 5410.96923 & 0 & 0 & 28 & 599.99 & 4.848 & 0.008 & 48 & 7 & 3.61815 \\
\hline 1 & 6 & 920 & 99.23 & 61.54 & 5618.89633 & 0 & 0 & 28 & 500.02 & 4.123 & 0.008 & 49 & 7 & 3.0769 \\
\hline 1 & 6 & 940 & 79.38 & 50.53 & 5785.62536 & 0 & 0 & 28 & 400 & 3.385 & 0.008 & 50 & 7 & 2.5263 \\
\hline 1 & 6 & 960 & 59.54 & 39.06 & 5910.48089 & 0 & 0 & 28 & 300.02 & 2.617 & 0.009 & 51 & 7 & 1.95285 \\
\hline 1 & 6 & 980 & 39.69 & 26.9 & 5994.00249 & 0 & 0 & 28 & 200 & 1.802 & 0.009 & 52 & 7 & 1.3448 \\
\hline 1 & 6 & 1000 & 19.85 & 13.48 & 6035.87913 & 0 & 0 & 28 & 100.02 & 0.903 & 0.009 & 53 & 7 & 0.67375 \\
\hline 1 & 7 & 1020 & 17.86 & 13.03 & 6073.5861 & 0 & 0 & 28 & 90 & 0.873 & 0.01 & 54 & 7 & 0.6517 \\
\hline 1 & 7 & 1040 & 15.88 & 12.2 & 6106.86499 & 0 & 0 & 28 & 80.02 & 0.817 & 0.01 & 55 & 7 & 0.60975 \\
\hline 1 & 7 & 1060 & 13.89 & 11.03 & 6135.95614 & 0 & 0 & 28 & 69.99 & 0.739 & 0.011 & 56 & 7 & 0.55145 \\
\hline 1 & 7 & 1080 & 11.9 & 9.9 & 6160.91374 & 0 & 0 & 28 & 59.96 & 0.663 & 0.011 & 57 & 7 & 0.495 \\
\hline 1 & 7 & 1100 & 9.92 & 8.56 & 6181.73935 & 0 & 0 & 28 & 49.99 & 0.574 & 0.011 & 58 & 7 & 0.42815 \\
\hline 1 & 7 & 1120 & 7.93 & 7.16 & 6198.3788 & 0 & 0 & 28 & 39.96 & 0.48 & 0.012 & 59 & 7 & 0.3582 \\
\hline 1 & 7 & 1140 & 5.95 & 5.63 & 6210.87605 & 0 & 0 & 28 & 29.98 & 0.377 & 0.013 & 60 & 7 & 0.28165 \\
\hline 1 & 7 & 1160 & 3.96 & 4.23 & 6219.20756 & 0 & 0 & 28 & 19.95 & 0.283 & 0.014 & 61 & 7 & 0.21135 \\
\hline 1 & 7 & 1180 & 1.98 & 2.02 & 6223.38352 & 0 & 0 & 28 & 9.98 & 0.135 & 0.014 & 62 & 7 & 0.10095 \\
\hline
\end{tabular}




\begin{tabular}{|c|c|c|c|c|c|c|c|c|c|c|c|c|c|}
\hline 8 & 1200 & 1.79 & 2.15 & 6227.15422 & 0 & 0 & 28 & 9.02 & 0.144 & 0.016 & 63 & 7 & 0.10745 \\
\hline 8 & 1220 & 1.59 & 2.29 & 6230.4898 & 0 & 0 & 28 & 8.01 & 0.153 & 0.019 & 64 & 7 & 0.1144 \\
\hline 8 & 1240 & 1.39 & 2.45 & 6233.40677 & 0 & 0 & 28 & 7 & 0.164 & 0.023 & 65 & 7 & 0.12245 \\
\hline 8 & 1260 & 1.19 & 2.48 & 6235.91141 & 0 & 0 & 28 & 6 & 0.166 & 0.028 & 66 & 7 & 0.124 \\
\hline 8 & 1280 & 0.99 & 2.31 & 6237.99664 & 0 & 0 & 28 & 4.99 & 0.154 & 0.031 & 67 & 7 & 0.11525 \\
\hline 8 & 1300 & 0.8 & 2.19 & 6239.66797 & 0 & 0 & 28 & 4.03 & 0.147 & 0.036 & 68 & 7 & 0.10965 \\
\hline 8 & 1320 & 0.6 & 2.13 & 6240.92225 & 0 & 0 & 28 & 3.02 & 0.142 & 0.047 & 69 & 7 & 0.1063 \\
\hline 8 & 1340 & 0.4 & 1.74 & 6241.75948 & 0 & 0 & 28 & 2.02 & 0.117 & 0.058 & 70 & 7 & 0.08705 \\
\hline 8 & 1360 & 0.2 & 1.12 & 6242.18124 & 0 & 0 & 28 & 1.01 & 0.075 & 0.074 & 71 & 7 & 0.0561 \\
\hline 9 & 1380 & 0.18 & 1.57 & 6242.56059 & 0 & 0 & 28 & 0.91 & 0.105 & 0.115 & 72 & 7 & 0.0785 \\
\hline 9 & 1400 & 0.16 & 2.09 & 6242.89595 & 0 & 0 & 28 & 0.81 & 0.14 & 0.173 & 73 & 7 & 0.1046 \\
\hline 9 & 1420 & 0.14 & 2.63 & 6243.18969 & 0 & 0 & 28 & 0.71 & 0.176 & 0.248 & 74 & 7 & 0.13155 \\
\hline 9 & 1440 & 0.12 & 2.8 & 6243.44023 & 0 & 0 & 28 & 0.6 & 0.188 & 0.313 & 75 & 7 & 0.1401 \\
\hline 9 & 1460 & 0.1 & 3.22 & 6243.64993 & 0 & 0 & 28 & 0.5 & 0.215 & 0.43 & 76 & 7 & 0.16075 \\
\hline 9 & 1480 & 0.08 & 3.34 & 6243.81801 & 0 & 0 & 28 & 0.4 & 0.224 & 0.56 & 77 & 7 & 0.16705 \\
\hline 9 & 1500 & 0.06 & 3.7 & 6243.94367 & 0 & 0 & 28 & 0.3 & 0.248 & 0.827 & 78 & 7 & 0.18515 \\
\hline 9 & 1520 & 0.04 & 3.23 & 6244.02771 & 0 & 0 & 28 & 0.2 & 0.217 & 1.085 & 79 & 7 & 0.1617 \\
\hline 9 & 1540 & 0.02 & 2.57 & 6244.07012 & 0 & 0 & 28 & 0.1 & 0.172 & 1.72 & 80 & 7 & 0.1283 \\
\hline
\end{tabular}

2004.02.14 META-10 wt\%-U [sonicated] DG 0.1-1000 s-1 run 8.txt

Mode Step t[s] n[rpm] M[\%.] Phi[rad]

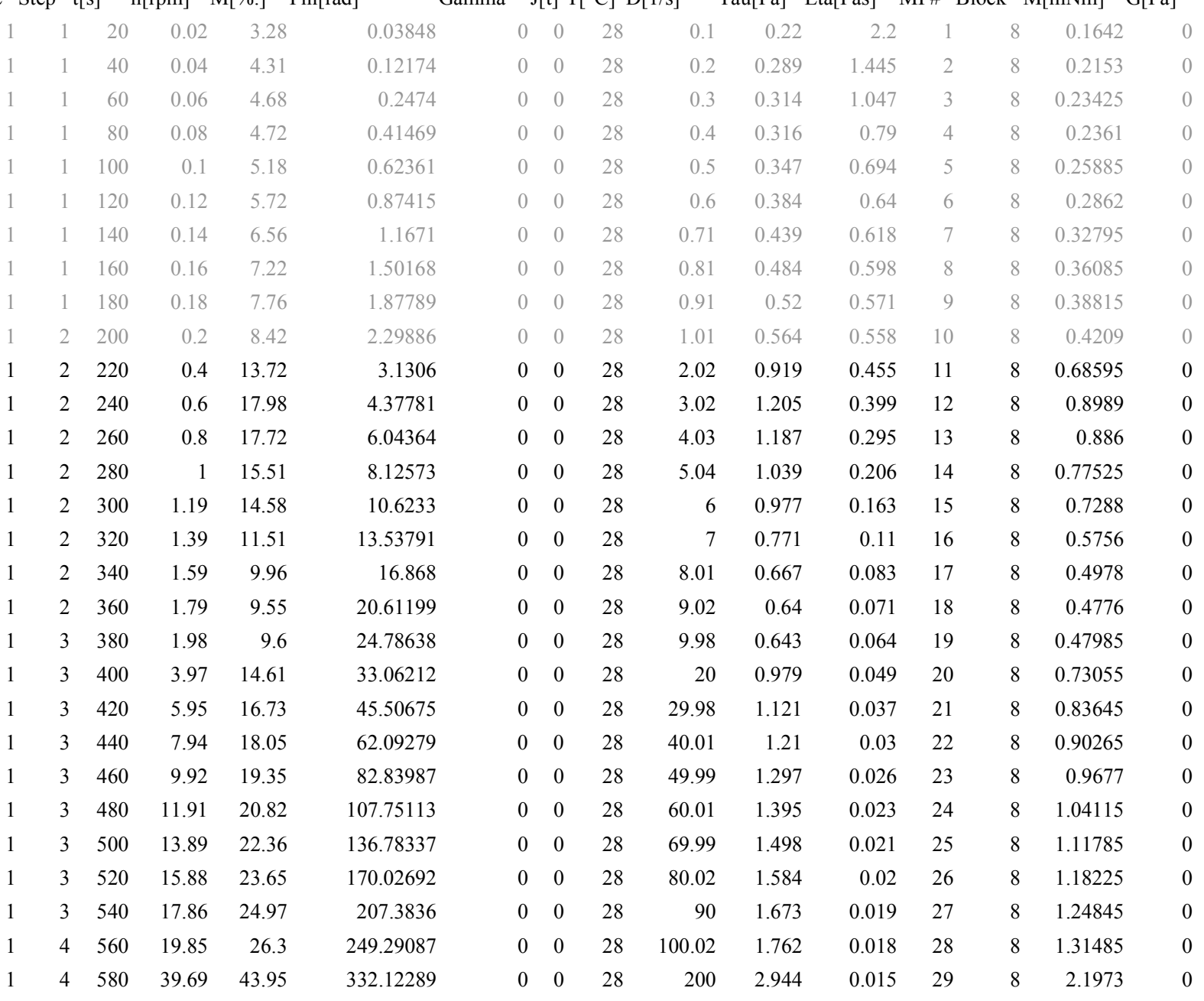




\begin{tabular}{|c|c|c|c|c|c|c|c|c|c|c|c|c|c|c|}
\hline 1 & 4 & 600 & 59.54 & 56.17 & 456.54881 & 0 & 0 & 28 & 300.02 & 3.763 & 0.013 & 30 & 8 & 2.80845 \\
\hline 1 & 4 & 620 & 79.38 & 66.79 & 622.58041 & 0 & 0 & 28 & 400 & 4.475 & 0.011 & 31 & 8 & 3.3397 \\
\hline 1 & 4 & 640 & 99.23 & 76.69 & 830.09989 & 0 & 0 & 28 & 500.02 & 5.139 & 0.01 & 32 & 8 & 3.83475 \\
\hline 1 & 4 & 660 & 119.07 & 86.13 & 1079.1088 & 0 & 0 & 28 & 599.99 & 5.771 & 0.01 & 33 & 8 & 4.3066 \\
\hline 1 & 4 & 680 & 138.92 & 95.5 & 1369.73204 & 0 & 0 & 28 & 700.02 & 6.399 & 0.009 & 34 & 8 & 4.77515 \\
\hline 1 & 4 & 700 & 158.76 & 104.29 & 1702.09448 & 0 & 0 & 28 & 799.99 & 6.987 & 0.009 & 35 & 8 & 5.21435 \\
\hline 1 & 4 & 720 & 178.61 & 113.21 & 2075.56152 & 0 & 0 & 28 & 900.02 & 7.585 & 0.008 & 36 & 8 & 5.66025 \\
\hline 1 & 4 & 740 & 198.45 & 121.8 & 2490.87064 & 0 & 0 & 28 & 999.99 & 8.16 & 0.008 & 37 & 8 & 6.08975 \\
\hline 1 & 5 & 750 & 198.45 & 119.8 & 2702.9792 & 0 & 0 & 28 & 999.99 & 8.026 & 0.008 & 38 & 8 & 5.98975 \\
\hline 1 & 5 & 760 & 198.45 & 119.02 & 2910.89922 & 0 & 0 & 28 & 999.99 & 7.974 & 0.008 & 39 & 8 & 5.95105 \\
\hline 1 & 5 & 770 & 198.45 & 118.22 & 3118.82082 & 0 & 0 & 28 & 999.99 & 7.921 & 0.008 & 40 & 8 & 5.91115 \\
\hline 1 & 5 & 780 & 198.45 & 117.64 & 3326.74085 & 0 & 0 & 28 & 999.99 & 7.882 & 0.008 & 41 & 8 & 5.8819 \\
\hline 1 & 5 & 790 & 198.45 & 117.05 & 3534.14094 & 0 & 0 & 28 & 999.99 & 7.842 & 0.008 & 42 & 8 & 5.8526 \\
\hline 1 & 5 & 800 & 198.45 & 116.5 & 3742.16621 & 0 & 0 & 28 & 999.99 & 7.806 & 0.008 & 43 & 8 & 5.82505 \\
\hline 1 & 6 & 820 & 198.45 & 115.89 & 4162.47439 & 0 & 0 & 28 & 999.99 & 7.764 & 0.008 & 44 & 8 & 5.79435 \\
\hline 1 & 6 & 840 & 178.61 & 104.37 & 4536.96166 & 0 & 0 & 28 & 900.02 & 6.993 & 0.008 & 45 & 8 & 5.21845 \\
\hline 1 & 6 & 860 & 158.76 & 93.28 & 4869.95949 & 0 & 0 & 28 & 799.99 & 6.25 & 0.008 & 46 & 8 & 4.6641 \\
\hline 1 & 6 & 880 & 138.92 & 82.75 & 5161.15607 & 0 & 0 & 28 & 700.02 & 5.545 & 0.008 & 47 & 8 & 4.1377 \\
\hline 1 & 6 & 900 & 119.07 & 71.95 & 5410.80116 & 0 & 0 & 28 & 599.99 & 4.82 & 0.008 & 48 & 8 & 3.59735 \\
\hline 1 & 6 & 920 & 99.23 & 61.13 & 5618.83349 & 0 & 0 & 28 & 500.02 & 4.096 & 0.008 & 49 & 8 & 3.0566 \\
\hline 1 & 6 & 940 & 79.38 & 50.13 & 5785.5209 & 0 & 0 & 28 & 400 & 3.359 & 0.008 & 50 & 8 & 2.5067 \\
\hline 1 & 6 & 960 & 59.54 & 38.68 & 5910.49974 & 0 & 0 & 28 & 300.02 & 2.591 & 0.009 & 51 & 8 & 1.93395 \\
\hline 1 & 6 & 980 & 39.69 & 26.58 & 5993.99071 & 0 & 0 & 28 & 200 & 1.781 & 0.009 & 52 & 8 & 1.3289 \\
\hline 1 & 6 & 1000 & 19.85 & 13.38 & 6035.84615 & 0 & 0 & 28 & 100.02 & 0.896 & 0.009 & 53 & 8 & 0.66885 \\
\hline 1 & 7 & 1020 & 17.86 & 12.99 & 6073.5107 & 0 & 0 & 28 & 90 & 0.87 & 0.01 & 54 & 8 & 0.64935 \\
\hline 1 & 7 & 1040 & 15.88 & 12.16 & 6106.82258 & 0 & 0 & 28 & 80.02 & 0.815 & 0.01 & 55 & 8 & 0.6079 \\
\hline 1 & 7 & 1060 & 13.89 & 10.93 & 6135.91373 & 0 & 0 & 28 & 69.99 & 0.733 & 0.01 & 56 & 8 & 0.54665 \\
\hline 1 & 7 & 1080 & 11.9 & 9.88 & 6160.90274 & 0 & 0 & 28 & 59.96 & 0.662 & 0.011 & 57 & 8 & 0.4939 \\
\hline 1 & 7 & 1100 & 9.92 & 8.53 & 6181.70323 & 0 & 0 & 28 & 49.99 & 0.571 & 0.011 & 58 & 8 & 0.4264 \\
\hline 1 & 7 & 1120 & 7.93 & 7.17 & 6198.35838 & 0 & 0 & 28 & 39.96 & 0.48 & 0.012 & 59 & 8 & 0.35845 \\
\hline 1 & 7 & 1140 & 5.95 & 5.63 & 6210.83678 & 0 & 0 & 28 & 29.98 & 0.377 & 0.013 & 60 & 8 & 0.2813 \\
\hline 1 & 7 & 1160 & 3.96 & 4.15 & 6219.17693 & 0 & 0 & 28 & 19.95 & 0.278 & 0.014 & 61 & 8 & 0.20735 \\
\hline 1 & 7 & 1180 & 1.98 & 2.07 & 6223.35132 & 0 & 0 & 28 & 9.98 & 0.139 & 0.014 & 62 & 8 & 0.1035 \\
\hline 1 & 8 & 1200 & 1.79 & 2.13 & 6227.12123 & 0 & 0 & 28 & 9.02 & 0.143 & 0.016 & 63 & 8 & 0.10655 \\
\hline 1 & 8 & 1220 & 1.59 & 2.28 & 6230.45682 & 0 & 0 & 28 & 8.01 & 0.153 & 0.019 & 64 & 8 & 0.114 \\
\hline 1 & 8 & 1240 & 1.39 & 2.33 & 6233.37614 & 0 & 0 & 28 & 7 & 0.156 & 0.022 & 65 & 8 & 0.1166 \\
\hline 1 & 8 & 1260 & 1.19 & 2.4 & 6235.87999 & 0 & 0 & 28 & 6 & 0.161 & 0.027 & 66 & 8 & 0.1198 \\
\hline 1 & 8 & 1280 & 0.99 & 2.17 & 6237.96679 & 0 & 0 & 28 & 4.99 & 0.146 & 0.029 & 67 & 8 & 0.1087 \\
\hline 1 & 8 & 1300 & 0.8 & 2.08 & 6239.63576 & 0 & 0 & 28 & 4.03 & 0.14 & 0.035 & 68 & 8 & 0.1042 \\
\hline 1 & 8 & 1320 & 0.6 & 2.06 & 6240.89162 & 0 & 0 & 28 & 3.02 & 0.138 & 0.046 & 69 & 8 & 0.10315 \\
\hline 1 & 8 & 1340 & 0.4 & 1.84 & 6241.72807 & 0 & 0 & 28 & 2.02 & 0.124 & 0.061 & 70 & 8 & 0.0922 \\
\hline 1 & 8 & 1360 & 0.2 & 1.21 & 6242.15061 & 0 & 0 & 28 & 1.01 & 0.081 & 0.08 & 71 & 8 & 0.06045 \\
\hline 1 & 9 & 1380 & 0.18 & 1.51 & 6242.52917 & 0 & 0 & 28 & 0.91 & 0.101 & 0.111 & 72 & 8 & 0.0754 \\
\hline 1 & 9 & 1400 & 0.16 & 1.97 & 6242.86454 & 0 & 0 & 28 & 0.81 & 0.132 & 0.163 & 73 & 8 & 0.0984 \\
\hline 1 & 9 & 1420 & 0.14 & 2.33 & 6243.15749 & 0 & 0 & 28 & 0.71 & 0.156 & 0.22 & 74 & 8 & 0.11655 \\
\hline 1 & 9 & 1440 & 0.12 & 2.67 & 6243.40882 & 0 & 0 & 28 & 0.6 & 0.179 & 0.298 & 75 & 8 & 0.13335 \\
\hline 1 & 9 & 1460 & 0.1 & 2.92 & 6243.61852 & 0 & 0 & 28 & 0.5 & 0.196 & 0.392 & 76 & 8 & 0.14605 \\
\hline 1 & 9 & 1480 & 0.08 & 2.92 & 6243.78659 & 0 & 0 & 28 & 0.4 & 0.196 & 0.49 & 77 & 8 & 0.14615 \\
\hline 1 & 9 & 1500 & 0.06 & 3.29 & 6243.91226 & 0 & 0 & 28 & 0.3 & 0.22 & 0.733 & 78 & 8 & 0.16435 \\
\hline 1 & 9 & 1520 & 0.04 & 3.19 & 6243.9963 & 0 & 0 & 28 & 0.2 & 0.214 & 1.07 & 79 & 8 & 0.15965 \\
\hline
\end{tabular}


2004.02.11 META-10 wt\%-U [sonicated] DG 500-4000 s-1 run 1.txt

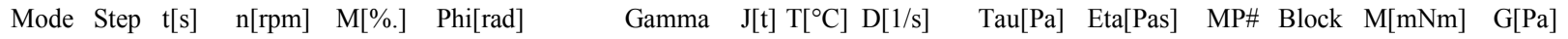

$\begin{array}{rrrrrrrrrrrrrrrr}1 & 1 & 20 & 99.23 & 65.31 & 207.61137 & 0 & 0 & 28.1 & 500.02 & 4.376 & 0.009 & 1 & 1 & 3.2654 & 0 \\ 1 & 1 & 40 & 198.46 & 106.39 & 621.64265 & 0 & 0 & 28.1 & 1000.04 & 7.128 & 0.007 & 2 & 1 & 5.3193 & 0 \\ 1 & 1 & 60 & 297.68 & 143.44 & 1243.33634 & 0 & 0 & 28.1 & 1500.01 & 9.611 & 0.006 & 3 & 1 & 7.1722 & 0 \\ 1 & 1 & 80 & 396.91 & 177.94 & 2073.42052 & 0 & 0 & 28.1 & 2000.03 & 11.922 & 0.006 & 4 & 1 & 8.89695 & 0 \\ 1 & 1 & 100 & 496.13 & 209.04 & 3110.8561 & 0 & 0 & 28.1 & 2500 & 14.006 & 0.006 & 5 & 1 & 10.45195 & 0 \\ 1 & 1 & 120 & 595.36 & 236.55 & 4355.74596 & 0 & 0 & 28.1 & 3000.02 & 15.849 & 0.005 & 6 & 1 & 11.8273 & 0 \\ 1 & 1 & 140 & 694.58 & 262.2 & 5808.4506 & 0 & 0 & 28 & 3499.99 & 17.567 & 0.005 & 7 & 1 & 13.10975 & 0 \\ 1 & 1 & 160 & 793.81 & 288.84 & 7470.53768 & 0 & 0 & 28 & 4000.01 & 19.352 & 0.005 & 8 & 1 & 14.44195 & 0 \\ 1 & 2 & 180 & 793.81 & 280.19 & 9151.15467 & 0 & 0 & 28 & 4000.01 & 18.772 & 0.005 & 9 & 1 & 14.00925 & 0 \\ 1 & 2 & 200 & 793.81 & 274.5 & 10812.0448 & 0 & 0 & 28 & 4000.01 & 18.392 & 0.005 & 10 & 1 & 13.72515 & 0 \\ 1 & 2 & 220 & 793.81 & 270.71 & 12475.427 & 0 & 0 & 28 & 4000.01 & 18.138 & 0.005 & 11 & 1 & 13.53555 & 0 \\ 1 & 3 & 240 & 793.81 & 267.86 & 14156.27097 & 0 & 0 & 28 & 4000.01 & 17.947 & 0.004 & 12 & 1 & 13.39305 & 0 \\ 1 & 3 & 260 & 694.58 & 231.81 & 15613.03219 & 0 & 0 & 28 & 3499.99 & 15.532 & 0.004 & 13 & 1 & 11.5907 & 0 \\ 1 & 3 & 280 & 595.36 & 197.98 & 16861.61107 & 0 & 0 & 27.9 & 3000.02 & 13.265 & 0.004 & 14 & 1 & 9.89895 & 0 \\ 1 & 3 & 300 & 496.13 & 164.87 & 17901.28424 & 0 & 0 & 27.9 & 2500 & 11.046 & 0.004 & 15 & 1 & 8.24355 & 0 \\ 1 & 3 & 320 & 396.91 & 132.28 & 18734.27989 & 0 & 0 & 27.9 & 2000.03 & 8.862 & 0.004 & 16 & 1 & 6.61375 & 0 \\ 1 & 3 & 340 & 297.68 & 99.82 & 19359.41049 & 0 & 0 & 27.9 & 1500.01 & 6.688 & 0.004 & 17 & 1 & 4.991 & 0 \\ 1 & 3 & 360 & 198.46 & 66.93 & 19776.66033 & 0 & 0 & 27.9 & 1000.04 & 4.485 & 0.004 & 18 & 1 & 3.3467 & 0 \\ 1 & 3 & 380 & 99.23 & 34.27 & 19986.18179 & 0 & 0 & 27.9 & 500.02 & 2.296 & 0.005 & 19 & 1 & 1.71345 & 0\end{array}$

2004.02.11 META-10 wt\%-U [sonicated] DG 500-4000 s-1 run 2.txt

Mode Step t[s] n[rpm] M[\%.] Phi[rad]

$\begin{array}{lllllll}\text { Gamma } & \mathrm{J}[\mathrm{t}] \mathrm{T}\left[{ }^{\circ} \mathrm{C}\right] \mathrm{D}[1 / \mathrm{s}] \quad \mathrm{Tau}[\mathrm{Pa}] & \text { Eta[Pas }] & \mathrm{MP} \# & \text { Block } & \mathrm{M}[\mathrm{mNm}] \quad \mathrm{G}[\mathrm{Pa}]\end{array}$

$\begin{array}{rrrrrrrrrrrrrrrr}1 & 1 & 20 & 99.31 & 39.27 & 207.66085 & 0 & 0 & 27.9 & 500.42 & 2.631 & 0.005 & 1 & 1 & 1.96355 & 0 \\ 1 & 1 & 40 & 198.46 & 73.57 & 622.10839 & 0 & 0 & 27.9 & 1000.04 & 4.929 & 0.005 & 2 & 1 & 3.6787 & 0 \\ 1 & 1 & 60 & 297.68 & 107.2 & 1243.54447 & 0 & 0 & 27.9 & 1500.01 & 7.182 & 0.005 & 3 & 1 & 5.36 & 0 \\ 1 & 1 & 80 & 396.91 & 140.11 & 2073.62787 & 0 & 0 & 28 & 2000.03 & 9.388 & 0.005 & 4 & 1 & 7.0056 & 0 \\ 1 & 1 & 100 & 496.13 & 172.33 & 3111.26843 & 0 & 0 & 28 & 2500 & 11.546 & 0.005 & 5 & 1 & 8.6165 & 0 \\ 1 & 1 & 120 & 595.36 & 203.48 & 4355.5339 & 0 & 0 & 28 & 3000.02 & 13.633 & 0.005 & 6 & 1 & 10.17375 & 0 \\ 1 & 1 & 140 & 694.58 & 234.1 & 5808.91713 & 0 & 0 & 28 & 3499.99 & 15.684 & 0.004 & 7 & 1 & 11.70485 & 0 \\ 1 & 1 & 160 & 793.81 & 264.77 & 7470.58638 & 0 & 0 & 28 & 4000.01 & 17.739 & 0.004 & 8 & 1 & 13.23835 & 0 \\ 1 & 2 & 180 & 793.81 & 262.7 & 9150.84129 & 0 & 0 & 28 & 4000.01 & 17.601 & 0.004 & 9 & 1 & 13.1349 & 0 \\ 1 & 2 & 200 & 793.81 & 262.11 & 10813.81038 & 0 & 0 & 28 & 4000.01 & 17.561 & 0.004 & 10 & 1 & 13.1053 & 0 \\ 1 & 2 & 220 & 793.81 & 260.52 & 12476.77868 & 0 & 0 & 28 & 4000.01 & 17.455 & 0.004 & 11 & 1 & 13.0258 & 0 \\ 1 & 3 & 240 & 793.81 & 258.29 & 14156.78619 & 0 & 0 & 28 & 4000.01 & 17.305 & 0.004 & 12 & 1 & 12.9143 & 0 \\ 1 & 3 & 260 & 694.58 & 225.07 & 15612.82249 & 0 & 0 & 28 & 3499.99 & 15.08 & 0.004 & 13 & 1 & 11.2536 & 0 \\ 1 & 3 & 280 & 595.36 & 192.85 & 16861.45242 & 0 & 0 & 28 & 3000.02 & 12.921 & 0.004 & 14 & 1 & 9.64265 & 0 \\ 1 & 3 & 300 & 496.13 & 160.67 & 17902.52517 & 0 & 0 & 28 & 2500 & 10.765 & 0.004 & 15 & 1 & 8.03325 & 0 \\ 1 & 3 & 320 & 396.91 & 129.03 & 18735.52161 & 0 & 0 & 27.9 & 2000.03 & 8.645 & 0.004 & 16 & 1 & 6.4517 & 0 \\ 1 & 3 & 340 & 297.68 & 97.33 & 19360.64906 & 0 & 0 & 27.9 & 1500.01 & 6.521 & 0.004 & 17 & 1 & 4.8665 & 0 \\ 1 & 3 & 360 & 198.46 & 65.25 & 19778.05127 & 0 & 0 & 27.9 & 1000.04 & 4.372 & 0.004 & 18 & 1 & 3.2626 & 0 \\ 1 & 3 & 380 & 99.23 & 33.3 & 19987.37009 & 0 & 0 & 27.9 & 500.02 & 2.231 & 0.004 & 19 & 1 & 1.6649 & 0\end{array}$



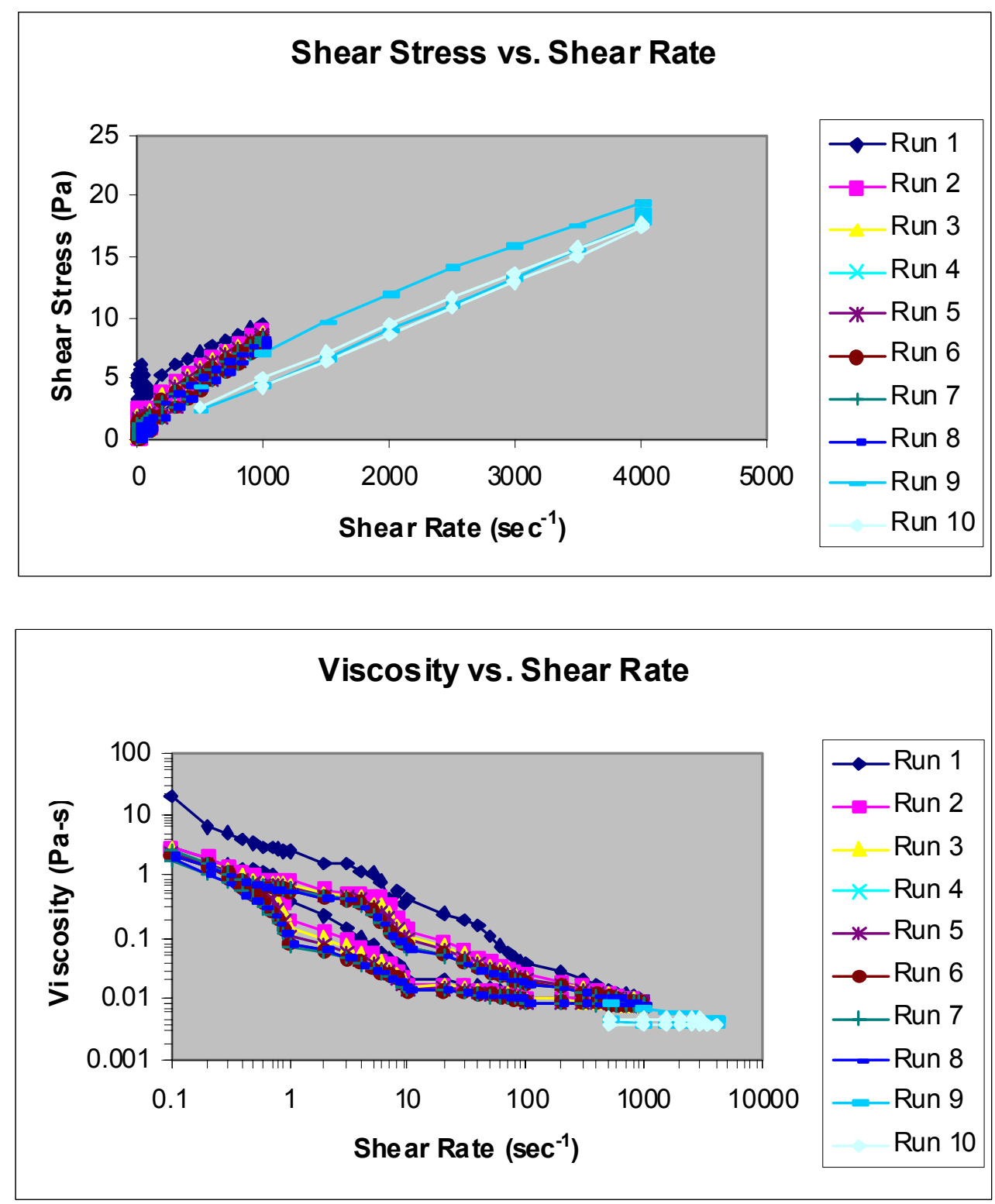

\section{E-2.1.17 META/15wt\%/U/N}

2004.02.09 META-15 wt\%-U DG 1-1000 s-1 run 1.txt Mode Step $\mathrm{t}[\mathrm{s}] \quad \mathrm{n}[\mathrm{rpm}] \quad \mathrm{M}[\%$.] $\quad$ Phi[rad]

\begin{tabular}{rrrrrrrrrrrrrrrr} 
Mode & Step & $\mathrm{t}[\mathrm{s}]$ & \multicolumn{3}{c}{$\mathrm{n}[\mathrm{rpm}]$} & $\mathrm{M}[\%]$. & $\mathrm{Phi}[\mathrm{rad}]$ & \multicolumn{1}{c}{$\mathrm{Gamma}$} & $\mathrm{J}[\mathrm{t}]$ & $\mathrm{T}\left[{ }^{\circ} \mathrm{C}\right]$ & $\mathrm{D}[\mathrm{1} / \mathrm{s}]$ & \multicolumn{5}{c}{$\mathrm{Tau}[\mathrm{Pa}]$} & $\mathrm{Eta}[\mathrm{Pas}]$ & $\mathrm{MP \#}$ & $\mathrm{Block}$ & $\mathrm{M}[\mathrm{mNm}]$ & $\mathrm{G}[\mathrm{Pa}]$ \\
1 & 1 & 20 & 0.17 & 194.7 & 0.33929 & 0 & 0 & 28 & 0.86 & 13.04 & 15.167 & 1 & 1 & 9.7347 & 0 \\
1 & 1 & 40 & 0.4 & 234 & 1.17574 & 0 & 0 & 28 & 2.02 & 15.68 & 7.761 & 2 & 1 & 11.7 & 0 \\
1 & 1 & 60 & 0.6 & 217.4 & 2.42688 & 0 & 0 & 28 & 3.02 & 14.57 & 4.824 & 3 & 1 & 10.871 & 0 \\
1 & 1 & 80 & 0.8 & 200.6 & 4.10135 & 0 & 0 & 28 & 4.03 & 13.44 & 3.335 & 4 & 1 & 10.03 & 0 \\
1 & 1 & 100 & 1 & 229.3 & 6.17323 & 0 & 0 & 28 & 5.04 & 15.36 & 3.048 & 5 & 1 & 11.464 & 0 \\
1 & 1 & 120 & 1.19 & 229.4 & 8.6653 & 0 & 0 & 28 & 6 & 15.37 & 2.561 & 6 & 1 & 11.469 & 0 \\
1 & 1 & 140 & 1.41 & 145.5 & 11.60897 & 0 & 0 & 28 & 7.1 & 9.745 & 1.373 & 7 & 1 & 7.2723 & 0 \\
1 & 1 & 160 & 1.6 & 135.4 & 14.94456 & 0 & 0 & 28 & 8.06 & 9.074 & 1.126 & 8 & 1 & 6.7716 & 0 \\
1 & 1 & 180 & 1.77 & 130.1 & 18.66342 & 0 & 0 & 28 & 8.92 & 8.715 & 0.977 & 9 & 1 & 6.5036 & 0 \\
1 & 2 & 200 & 2.01 & 122.9 & 22.86687 & 0 & 0 & 28 & 10.13 & 8.231 & 0.813 & 10 & 1 & 6.1427 & 0 \\
1 & 2 & 220 & 3.97 & 113.3 & 31.15046 & 0 & 0 & 28 & 20 & 7.588 & 0.379 & 11 & 1 & 5.6628 & 0
\end{tabular}




\begin{tabular}{|c|c|c|c|c|c|c|c|c|c|c|c|c|c|c|}
\hline 1 & 2 & 240 & 5.95 & 102.2 & 43.58803 & 0 & 0 & 28 & 29.98 & 6.847 & 0.228 & 12 & 1 & 5.1094 \\
\hline 1 & 2 & 260 & 7.94 & 80.92 & 60.17721 & 0 & 0 & 28 & 40.01 & 5.421 & 0.135 & 13 & 1 & 4.0458 \\
\hline 1 & 2 & 280 & 9.92 & 72.29 & 80.92821 & 0 & 0 & 28 & 49.99 & 4.844 & 0.097 & 14 & 1 & 3.6147 \\
\hline 1 & 2 & 300 & 11.91 & 66.84 & 105.83476 & 0 & 0 & 28 & 60.01 & 4.478 & 0.075 & 15 & 1 & 3.3419 \\
\hline 1 & 2 & 320 & 13.9 & 56.79 & 134.89449 & 0 & 0 & 28 & 70.04 & 3.805 & 0.054 & 16 & 1 & 2.8397 \\
\hline 1 & 2 & 340 & 15.88 & 49.63 & 168.09798 & 0 & 0 & 28 & 80.02 & 3.325 & 0.042 & 17 & 1 & 2.4815 \\
\hline 1 & 2 & 360 & 17.86 & 48.27 & 205.50964 & 0 & 0 & 28 & 90 & 3.234 & 0.036 & 18 & 1 & 2.4134 \\
\hline 1 & 3 & 380 & 19.85 & 41.05 & 247.42477 & 0 & 0 & 28 & 100 & 2.75 & 0.027 & 19 & 1 & 2.0526 \\
\hline 1 & 3 & 400 & 39.69 & 53.48 & 330.29213 & 0 & 0 & 28 & 200 & 3.583 & 0.018 & 20 & 1 & 2.674 \\
\hline 1 & 3 & 420 & 59.54 & 59.75 & 454.72983 & 0 & 0 & 28 & 300 & 4.003 & 0.013 & 21 & 1 & 2.9873 \\
\hline 1 & 3 & 440 & 79.38 & 63.55 & 620.60514 & 0 & 0 & 28 & 400 & 4.258 & 0.011 & 22 & 1 & 3.1773 \\
\hline 1 & 3 & 460 & 99.23 & 67.98 & 828.26127 & 0 & 0 & 28 & 500 & 4.555 & 0.009 & 23 & 1 & 3.3989 \\
\hline 1 & 3 & 480 & 119.1 & 73.41 & 1077.1453 & 0 & 0 & 28 & 600 & 4.918 & 0.008 & 24 & 1 & 3.6703 \\
\hline 1 & 3 & 500 & 138.9 & 80.34 & 1367.7803 & 0 & 0 & 28 & 700 & 5.383 & 0.008 & 25 & 1 & 4.0169 \\
\hline 1 & 3 & 520 & 158.8 & 86.97 & 1699.893 & 0 & 0 & 28 & 800 & 5.827 & 0.007 & 26 & 1 & 4.3486 \\
\hline 1 & 3 & 540 & 178.6 & 94.39 & 2074.0983 & 0 & 0 & 28 & 900 & 6.324 & 0.007 & 27 & 1 & 4.7197 \\
\hline 1 & 3 & 560 & 198.5 & 101.6 & 2489.3053 & 0 & 0 & 28 & 1000 & 6.805 & 0.007 & 28 & 1 & 5.0782 \\
\hline 1 & 4 & 570 & 198.5 & 99.35 & 2701.2058 & 0 & 0 & 28 & 1000 & 6.656 & 0.007 & 29 & 1 & 4.9675 \\
\hline 1 & 4 & 580 & 198.5 & 98.76 & 2909.6449 & 0 & 0 & 28 & 1000 & 6.617 & 0.007 & 30 & 1 & 4.9378 \\
\hline 1 & 4 & 590 & 198.5 & 97.94 & 3117.2532 & 0 & 0 & 28 & 1000 & 6.562 & 0.007 & 31 & 1 & 4.8968 \\
\hline 1 & 4 & 600 & 198.5 & 97.25 & 3324.7585 & 0 & 0 & 28 & 1000 & 6.516 & 0.007 & 32 & 1 & 4.8625 \\
\hline 1 & 4 & 610 & 198.5 & 96.77 & 3532.4712 & 0 & 0 & 28 & 1000 & 6.484 & 0.006 & 33 & 1 & 4.8386 \\
\hline 1 & 4 & 620 & 198.5 & 96.37 & 3740.9104 & 0 & 0 & 28 & 1000 & 6.457 & 0.006 & 34 & 1 & 4.8185 \\
\hline 1 & 5 & 640 & 198.5 & 95.96 & 4161.3238 & 0 & 0 & 28 & 1000 & 6.429 & 0.006 & 35 & 1 & 4.798 \\
\hline 1 & 5 & 660 & 178.6 & 85.96 & 4535.4364 & 0 & 0 & 28 & 900 & 5.76 & 0.006 & 36 & 1 & 4.2982 \\
\hline 1 & 5 & 680 & 158.8 & 76.49 & 4868.1845 & 0 & 0 & 28 & 800 & 5.125 & 0.006 & 37 & 1 & 3.8243 \\
\hline 1 & 5 & 700 & 138.9 & 68.04 & 5159.3803 & 0 & 0 & 28 & 700 & 4.558 & 0.007 & 38 & 1 & 3.4018 \\
\hline 1 & 5 & 720 & 119.1 & 58.91 & 5409.1503 & 0 & 0 & 28 & 600 & 3.947 & 0.007 & 39 & 1 & 2.9455 \\
\hline 1 & 5 & 740 & 99.23 & 50.19 & 5617.3357 & 0 & 0 & 28 & 500 & 3.362 & 0.007 & 40 & 1 & 2.5093 \\
\hline 1 & 5 & 760 & 79.38 & 41.55 & 5783.826 & 0 & 0 & 28 & 400 & 2.784 & 0.007 & 41 & 1 & 2.0774 \\
\hline 1 & 5 & 780 & 59.54 & 32.65 & 5908.8881 & 0 & 0 & 28 & 300 & 2.187 & 0.007 & 42 & 1 & 1.6323 \\
\hline 1 & 5 & 800 & 39.69 & 27.93 & 5992.3265 & 0 & 0 & 28 & 200 & 1.871 & 0.009 & 43 & 1 & 1.3966 \\
\hline 1 & 5 & 820 & 19.85 & 15.95 & 6034.1913 & 0 & 0 & 28 & 100 & 1.068 & 0.011 & 44 & 1 & 0.7973 \\
\hline 1 & 6 & 840 & 17.86 & 18.35 & 6071.8653 & 0 & 0 & 28 & 90 & 1.23 & 0.014 & 45 & 1 & 0.9177 \\
\hline 1 & 6 & 860 & 15.88 & 20.22 & 6105.134 & 0 & 0 & 28 & 80.02 & 1.355 & 0.017 & 46 & 1 & 1.011 \\
\hline 1 & 6 & 880 & 13.89 & 19.35 & 6134.2644 & 0 & 0 & 28 & 69.99 & 1.296 & 0.019 & 47 & 1 & 0.9674 \\
\hline 1 & 6 & 900 & 11.9 & 16.17 & 6159.222 & 0 & 0 & 28 & 59.96 & 1.083 & 0.018 & 48 & 1 & 0.8084 \\
\hline 1 & 6 & 920 & 9.92 & 16.66 & 6180.0429 & 0 & 0 & 28 & 49.99 & 1.116 & 0.022 & 49 & 1 & 0.8332 \\
\hline 1 & 6 & 940 & 7.93 & 14.65 & 6196.6831 & 0 & 0 & 28 & 39.96 & 0.981 & 0.025 & 50 & 1 & 0.7325 \\
\hline 1 & 6 & 960 & 5.95 & 13.77 & 6209.1835 & 0 & 0 & 28 & 29.98 & 0.923 & 0.031 & 51 & 1 & 0.6886 \\
\hline 1 & 6 & 980 & 3.96 & 13.91 & 6217.5158 & 0 & 0 & 28 & 19.95 & 0.932 & 0.047 & 52 & 1 & 0.6957 \\
\hline 1 & 6 & 1000 & 1.98 & 13.22 & 6221.6894 & 0 & 0 & 28 & 9.98 & 0.886 & 0.089 & 53 & 1 & 0.6611 \\
\hline 1 & 7 & 1020 & 1.79 & 12.74 & 6225.4578 & 0 & 0 & 28 & 9.02 & 0.854 & 0.095 & 54 & 1 & 0.6371 \\
\hline 1 & 7 & 1040 & 1.59 & 16.84 & 6228.7941 & 0 & 0 & 28 & 8.01 & 1.128 & 0.141 & 55 & 1 & 0.8418 \\
\hline 1 & 7 & 1060 & 1.39 & 12.75 & 6231.7111 & 0 & 0 & 28 & 7 & 0.855 & 0.122 & 56 & 1 & 0.6377 \\
\hline 1 & 7 & 1080 & 1.19 & 17.95 & 6234.2165 & 0 & 0 & 28 & 6 & 1.203 & 0.201 & 57 & 1 & 0.8977 \\
\hline 1 & 7 & 1100 & 0.99 & 18.79 & 6236.3057 & 0 & 0 & 28 & 4.99 & 1.259 & 0.252 & 58 & 1 & 0.9394 \\
\hline 1 & 7 & 1120 & 0.8 & 14.04 & 6237.9699 & 0 & 0 & 28 & 4.03 & 0.941 & 0.233 & 59 & 1 & 0.7021 \\
\hline 1 & 7 & 1140 & 0.6 & 18.28 & 6239.2258 & 0 & 0 & 28 & 3.02 & 1.225 & 0.406 & 60 & 1 & 0.914 \\
\hline 1 & 7 & 1160 & 0.4 & 18 & 6240.0638 & 0 & 0 & 28 & 2.02 & 1.206 & 0.597 & 61 & 1 & 0.9 \\
\hline 1 & 7 & 1180 & 0.2 & 15.86 & 6240.4832 & 0 & 0 & 28 & 1.01 & 1.063 & 1.052 & 62 & 1 & 0.7932 \\
\hline
\end{tabular}


2004.02.09 META-15 wt\%-U DG 1-

$1000 \mathrm{~s}-1$ run $2 . t x t$

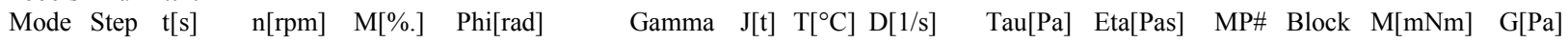

\begin{tabular}{|c|c|c|c|c|c|c|c|c|c|c|c|c|c|c|}
\hline 1 & 1 & 20 & 0.2 & 18.48 & 0.40919 & 0 & 0 & 28 & 1.01 & 1.238 & 1.226 & 1 & 1 & 0.924 \\
\hline 1 & 1 & 40 & 0.4 & 22.9 & 1.24329 & 0 & 0 & 28 & 2.02 & 1.534 & 0.759 & 2 & 1 & 1.1451 \\
\hline 1 & 1 & 60 & 0.6 & 21.89 & 2.4905 & 0 & 0 & 28 & 3.02 & 1.466 & 0.485 & 3 & 1 & 1.0944 \\
\hline 1 & 1 & 80 & 0.8 & 24.01 & 4.15554 & 0 & 0 & 28 & 4.03 & 1.609 & 0.399 & 4 & 1 & 1.2007 \\
\hline 1 & 1 & 100 & 1 & 24.08 & 6.23685 & 0 & 0 & 28 & 5.04 & 1.613 & 0.32 & 5 & 1 & 1.2039 \\
\hline 1 & 1 & 120 & 1.19 & 17.68 & 8.7352 & 0 & 0 & 28 & 6 & 1.185 & 0.198 & 6 & 1 & 0.8842 \\
\hline 1 & 1 & 140 & 1.39 & 20.07 & 11.64903 & 0 & 0 & 28 & 7 & 1.345 & 0.192 & 7 & 1 & 1.0034 \\
\hline 1 & 1 & 160 & 1.59 & 18.18 & 14.9799 & 0 & 0 & 28 & 8.01 & 1.218 & 0.152 & 8 & 1 & 0.9091 \\
\hline 1 & 1 & 180 & 1.79 & 19.54 & 18.72468 & 0 & 0 & 28 & 9.02 & 1.309 & 0.145 & 9 & 1 & 0.9771 \\
\hline 1 & 2 & 200 & 1.98 & 16.19 & 22.90064 & 0 & 0 & 28 & 9.98 & 1.085 & 0.109 & 10 & 1 & 0.8095 \\
\hline 1 & 2 & 220 & 3.97 & 22.49 & 31.17167 & 0 & 0 & 28 & 20 & 1.507 & 0.075 & 11 & 1 & 1.1245 \\
\hline 1 & 2 & 240 & 5.95 & 20.83 & 43.61159 & 0 & 0 & 28 & 29.98 & 1.396 & 0.047 & 12 & 1 & 1.0417 \\
\hline 1 & 2 & 260 & 7.94 & 19.45 & 60.20627 & 0 & 0 & 28 & 40.01 & 1.303 & 0.033 & 13 & 1 & 0.9723 \\
\hline 1 & 2 & 280 & 9.92 & 19.84 & 80.95806 & 0 & 0 & 28 & 49.99 & 1.329 & 0.027 & 14 & 1 & 0.992 \\
\hline 1 & 2 & 300 & 11.91 & 20.16 & 105.86303 & 0 & 0 & 28 & 60.01 & 1.351 & 0.023 & 15 & 1 & 1.008 \\
\hline 1 & 2 & 320 & 13.89 & 21.53 & 134.89528 & 0 & 0 & 28 & 69.99 & 1.443 & 0.021 & 16 & 1 & 1.0766 \\
\hline 1 & 2 & 340 & 15.88 & 21.87 & 168.13097 & 0 & 0 & 28 & 80.02 & 1.465 & 0.018 & 17 & 1 & 1.0935 \\
\hline 1 & 2 & 360 & 17.86 & 22.59 & 205.53556 & 0 & 0 & 28 & 90 & 1.513 & 0.017 & 18 & 1 & 1.1294 \\
\hline 1 & 3 & 380 & 19.85 & 23.45 & 247.48446 & 0 & 0 & 28 & 100 & 1.571 & 0.016 & 19 & 1 & 1.1723 \\
\hline 1 & 3 & 400 & 39.69 & 38.2 & 330.33847 & 0 & 0 & 28 & 200 & 2.559 & 0.013 & 20 & 1 & 1.91 \\
\hline 1 & 3 & 420 & 59.54 & 47.25 & 454.63087 & 0 & 0 & 28 & 300 & 3.166 & 0.011 & 21 & 1 & 2.3624 \\
\hline 1 & 3 & 440 & 79.38 & 55.32 & 620.58864 & 0 & 0 & 28 & 400 & 3.706 & 0.009 & 22 & 1 & 2.7659 \\
\hline 1 & 3 & 460 & 99.23 & 62.5 & 828.10812 & 0 & 0 & 28 & 500 & 4.187 & 0.008 & 23 & 1 & 3.1248 \\
\hline 1 & 3 & 480 & 119.1 & 69.92 & 1077.4304 & 0 & 0 & 28 & 600 & 4.685 & 0.008 & 24 & 1 & 3.496 \\
\hline 1 & 3 & 500 & 138.9 & 77.43 & 1367.8463 & 0 & 0 & 28 & 700 & 5.188 & 0.007 & 25 & 1 & 3.8716 \\
\hline 1 & 3 & 520 & 158.8 & 84.8 & 1700.1977 & 0 & 0 & 28 & 800 & 5.681 & 0.007 & 26 & 1 & 4.2398 \\
\hline 1 & 3 & 540 & 178.6 & 92.56 & 2073.946 & 0 & 0 & 28 & 900 & 6.202 & 0.007 & 27 & 1 & 4.6281 \\
\hline 1 & 3 & 560 & 198.5 & 100.2 & 2489.1522 & 0 & 0 & 28 & 1000 & 6.712 & 0.007 & 28 & 1 & 5.0092 \\
\hline 1 & 4 & 570 & 198.5 & 98.31 & 2701.1461 & 0 & 0 & 28 & 1000 & 6.587 & 0.007 & 29 & 1 & 4.9156 \\
\hline 1 & 4 & 580 & 198.5 & 97.64 & 2908.8588 & 0 & 0 & 28 & 1000 & 6.542 & 0.007 & 30 & 1 & 4.8822 \\
\hline 1 & 4 & 590 & 198.5 & 97.09 & 3117.1943 & 0 & 0 & 28 & 1000 & 6.505 & 0.007 & 31 & 1 & 4.8545 \\
\hline 1 & 4 & 600 & 198.5 & 96.55 & 3324.5951 & 0 & 0 & 28 & 1000 & 6.469 & 0.006 & 32 & 1 & 4.8276 \\
\hline 1 & 4 & 610 & 198.5 & 96.17 & 3532.2042 & 0 & 0 & 28 & 1000 & 6.443 & 0.006 & 33 & 1 & 4.8086 \\
\hline 1 & 4 & 620 & 198.5 & 95.85 & 3740.6433 & 0 & 0 & 28 & 1000 & 6.422 & 0.006 & 34 & 1 & 4.7925 \\
\hline 1 & 5 & 640 & 198.5 & 95.43 & 4160.536 & 0 & 0 & 28 & 1000 & 6.394 & 0.006 & 35 & 1 & 4.7715 \\
\hline 1 & 5 & 660 & 178.6 & 85.53 & 4535.0139 & 0 & 0 & 28 & 900 & 5.73 & 0.006 & 36 & 1 & 4.2763 \\
\hline 1 & 5 & 680 & 158.8 & 76.08 & 4867.8342 & 0 & 0 & 28 & 800 & 5.098 & 0.006 & 37 & 1 & 3.8042 \\
\hline 1 & 5 & 700 & 138.9 & 67.28 & 5159.0206 & 0 & 0 & 28 & 700 & 4.508 & 0.006 & 38 & 1 & 3.3642 \\
\hline 1 & 5 & 720 & 119.1 & 58.37 & 5409.0293 & 0 & 0 & 28 & 600 & 3.911 & 0.007 & 39 & 1 & 2.9184 \\
\hline 1 & 5 & 740 & 99.23 & 49.57 & 5617.0593 & 0 & 0 & 28 & 500 & 3.321 & 0.007 & 40 & 1 & 2.4784 \\
\hline 1 & 5 & 760 & 79.38 & 40.78 & 5783.7153 & 0 & 0 & 28 & 400 & 2.732 & 0.007 & 41 & 1 & 2.0391 \\
\hline 1 & 5 & 780 & 59.54 & 31.72 & 5908.5794 & 0 & 0 & 28 & 300 & 2.126 & 0.007 & 42 & 1 & 1.5862 \\
\hline 1 & 5 & 800 & 39.69 & 22.39 & 5992.112 & 0 & 0 & 28 & 200 & 1.5 & 0.008 & 43 & 1 & 1.1193 \\
\hline 1 & 5 & 820 & 19.85 & 12.43 & 6033.9667 & 0 & 0 & 28 & 100 & 0.833 & 0.008 & 44 & 1 & 0.6215 \\
\hline 1 & 6 & 840 & 17.86 & 13.06 & 6071.6658 & 0 & 0 & 28 & 90 & 0.875 & 0.01 & 45 & 1 & 0.6528 \\
\hline 1 & 6 & 860 & 15.88 & 13.14 & 6104.9282 & 0 & 0 & 28 & 80.02 & 0.881 & 0.011 & 46 & 1 & 0.6571 \\
\hline 1 & 6 & 880 & 13.89 & 12.64 & 6134.0625 & 0 & 0 & 28 & 69.99 & 0.847 & 0.012 & 47 & 1 & 0.6318 \\
\hline 1 & 6 & 900 & 11.9 & 12.41 & 6159.0194 & 0 & 0 & 28 & 59.96 & 0.831 & 0.014 & 48 & 1 & 0.6203 \\
\hline 1 & 6 & 920 & 9.92 & 11.63 & 6179.8301 & 0 & 0 & 28 & 49.99 & 0.779 & 0.016 & 49 & 1 & 0.5813 \\
\hline
\end{tabular}




$\begin{array}{rrrrrrrrrrrrrrrrr}1 & 6 & 940 & 7.93 & 11.61 & 6196.4734 & 0 & 0 & 28 & 39.96 & 0.778 & 0.019 & 50 & 1 & 0.5807 & 0 \\ 1 & 6 & 960 & 5.95 & 10.84 & 6208.9699 & 0 & 0 & 28 & 29.98 & 0.726 & 0.024 & 51 & 1 & 0.5421 & 0 \\ 1 & 6 & 980 & 3.96 & 9.41 & 6217.2943 & 0 & 0 & 28 & 19.95 & 0.631 & 0.032 & 52 & 1 & 0.4706 & 0 \\ 1 & 6 & 1000 & 1.98 & 9 & 6221.4727 & 0 & 0 & 28 & 9.98 & 0.603 & 0.06 & 53 & 1 & 0.4499 & 0 \\ 1 & 7 & 1020 & 1.79 & 9.39 & 6225.2394 & 0 & 0 & 28 & 9.02 & 0.629 & 0.07 & 54 & 1 & 0.4697 & 0 \\ 1 & 7 & 1040 & 1.59 & 11.17 & 6228.5797 & 0 & 0 & 28 & 8.01 & 0.748 & 0.093 & 55 & 1 & 0.5584 & 0 \\ 1 & 7 & 1060 & 1.39 & 11.73 & 6231.4959 & 0 & 0 & 28 & 7 & 0.786 & 0.112 & 56 & 1 & 0.5863 & 0 \\ 1 & 7 & 1080 & 1.19 & 13.47 & 6233.9998 & 0 & 0 & 28 & 6 & 0.902 & 0.15 & 57 & 1 & 0.6733 & 0 \\ 1 & 7 & 1100 & 0.99 & 8.61 & 6236.0858 & 0 & 0 & 28 & 4.99 & 0.577 & 0.116 & 58 & 1 & 0.4305 & 0 \\ 1 & 7 & 1120 & 0.8 & 14.42 & 6237.7555 & 0 & 0 & 28 & 4.03 & 0.966 & 0.24 & 59 & 1 & 0.721 & 0 \\ 1 & 7 & 1140 & 0.6 & 13.74 & 6239.0075 & 0 & 0 & 28 & 3.02 & 0.92 & 0.305 & 60 & 1 & 0.6869 & 0 \\ 1 & 7 & 1160 & 0.4 & 14.32 & 6239.8494 & 0 & 0 & 28 & 2.02 & 0.96 & 0.475 & 61 & 1 & 0.7162 & 0 \\ 1 & 7 & 1180 & 0.2 & 9.23 & 6240.2704 & 0 & 0 & 28 & 1.01 & 0.618 & 0.612 & 62 & 1 & 0.4616 & 0\end{array}$

2004.02.09 META-15 wt\%-U DG 500-

$4000 \mathrm{~s}-1$ run 1.txt

Mode Step $\mathrm{t}[\mathrm{s}] \quad \mathrm{n}[\mathrm{rpm}] \quad \mathrm{M}[\%$. $] \quad$ Phi[rad] Gamma J[t] T[ $\left.{ }^{\circ} \mathrm{C}\right] \quad \mathrm{D}[1 / \mathrm{s}] \quad \mathrm{Tau}[\mathrm{Pa}] \quad \mathrm{Eta}[\mathrm{Pas}] \quad \mathrm{MP} \# \quad \mathrm{Block} \quad \mathrm{M}[\mathrm{mNm}] \quad \mathrm{G}[\mathrm{Pa}]$

\begin{tabular}{|c|c|c|c|c|c|c|c|c|c|c|c|c|c|c|}
\hline 1 & 1 & 20 & 99.3 & 85.6 & 207.56503 & 0 & 0 & 28 & 500.4 & 5.735 & 0.011 & 1 & 1 & 4.28 \\
\hline 1 & 1 & 40 & 198.5 & 111.7 & 621.75182 & 0 & 0 & 28 & 1000 & 7.486 & 0.007 & 2 & 1 & 5.5867 \\
\hline 1 & 1 & 60 & 297.7 & 149 & 1243.4447 & 0 & 0 & 28 & 1500 & 9.985 & 0.007 & 3 & 1 & 7.4517 \\
\hline 1 & 1 & 80 & 396.9 & 186.3 & 2073.9436 & 0 & 0 & 28 & 2000 & 12.48 & 0.006 & 4 & 1 & 9.3151 \\
\hline 1 & 1 & 100 & 496.1 & 221.7 & 3110.3377 & 0 & 0 & 28 & 2500 & 14.85 & 0.006 & 5 & 1 & 11.085 \\
\hline 1 & 1 & 120 & 595.4 & 256 & 4356.5266 & 0 & 0 & 28 & 3000 & 17.15 & 0.006 & 6 & 1 & 12.798 \\
\hline 1 & 1 & 140 & 694.6 & 292.2 & 5808.0885 & 0 & 0 & 28 & 3500 & 19.57 & 0.006 & 7 & 1 & 14.607 \\
\hline 1 & 1 & 160 & 793.8 & 329.3 & 7470.584 & 0 & 0 & 28 & 4000 & 22.06 & 0.006 & 8 & 1 & 16.465 \\
\hline 1 & 2 & 180 & 793.8 & 320 & 9151.2348 & 0 & 0 & 28 & 4000 & 21.44 & 0.005 & 9 & 1 & 15.999 \\
\hline 1 & 2 & 200 & 793.8 & 313.8 & 10812.953 & 0 & 0 & 28 & 4000 & 21.02 & 0.005 & 10 & 1 & 15.689 \\
\hline 1 & 2 & 220 & 793.8 & 316.3 & 12477.167 & 0 & 0 & 28 & 4000 & 21.19 & 0.005 & 11 & 1 & 15.816 \\
\hline 1 & 3 & 240 & 793.8 & 316.7 & 14157.592 & 0 & 0 & 28 & 4000 & 21.22 & 0.005 & 12 & 1 & 15.837 \\
\hline 1 & 3 & 260 & 694.6 & 275.5 & 15613.679 & 0 & 0 & 28 & 3500 & 18.46 & 0.005 & 13 & 1 & 13.773 \\
\hline 1 & 3 & 280 & 595.4 & 235.1 & 16862.313 & 0 & 0 & 28 & 3000 & 15.75 & 0.005 & 14 & 1 & 11.755 \\
\hline 1 & 3 & 300 & 496.1 & 195.7 & 17902.869 & 0 & 0 & 28 & 2500 & 13.11 & 0.005 & 15 & 1 & 9.785 \\
\hline 1 & 3 & 320 & 396.9 & 156.7 & 18735.45 & 0 & 0 & 28 & 2000 & 10.5 & 0.005 & 16 & 1 & 7.8354 \\
\hline 1 & 3 & 340 & 297.7 & 118 & 19361.202 & 0 & 0 & 28 & 1500 & 7.905 & 0.005 & 17 & 1 & 5.8991 \\
\hline 1 & 3 & 360 & 198.5 & 79.02 & 19778.279 & 0 & 0 & 28 & 1000 & 5.295 & 0.005 & 18 & 1 & 3.9512 \\
\hline 1 & 3 & 380 & 99.23 & 40.53 & 19987.798 & 0 & 0 & 28 & 500 & 2.715 & 0.005 & 19 & 1 & 2.0264 \\
\hline
\end{tabular}

2004.02.09 META-15 wt\%-U DG 500-

$4000 \mathrm{~s}-1$ run $2 . \mathrm{txt}$

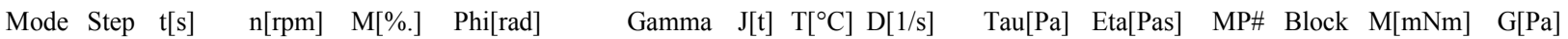

\begin{tabular}{|c|c|c|c|c|c|c|c|c|c|c|c|c|c|c|}
\hline 1 & 1 & 20 & 99.24 & 41.41 & 207.73467 & 0 & 0 & 28 & 500.1 & 2.774 & 0.006 & 1 & 1 & 2.0705 \\
\hline 1 & 1 & 40 & 198.5 & 81.76 & 621.66228 & 0 & 0 & 28 & 1000 & 5.478 & 0.005 & 2 & 1 & 4.0881 \\
\hline 1 & 1 & 60 & 297.7 & 121.6 & 1243.8233 & 0 & 0 & 28 & 1500 & 8.148 & 0.005 & 3 & 1 & 6.0809 \\
\hline 1 & 1 & 80 & 396.9 & 161 & 2073.5423 & 0 & 0 & 28 & 2000 & 10.79 & 0.005 & 4 & 1 & 8.0518 \\
\hline 1 & 1 & 100 & 496.1 & 200.3 & 3109.7283 & 0 & 0 & 28 & 2500 & 13.42 & 0.005 & 5 & 1 & 10.015 \\
\hline 1 & 1 & 120 & 595.4 & 239.4 & 4356.2251 & 0 & 0 & 28 & 3000 & 16.04 & 0.005 & 6 & 1 & 11.97 \\
\hline 1 & 1 & 140 & 694.6 & 278.3 & 5808.1514 & 0 & 0 & 28 & 3500 & 18.64 & 0.005 & 7 & 1 & 13.913 \\
\hline 1 & 1 & 160 & 793.8 & 316.6 & 7469.4028 & 0 & 0 & 28 & 4000 & 21.21 & 0.005 & 8 & 1 & 15.831 \\
\hline 1 & 2 & 180 & 793.8 & 313.6 & 9149.6443 & 0 & 0 & 28 & 4000 & 21.01 & 0.005 & 9 & 1 & 15.68 \\
\hline 1 & 2 & 200 & 793.8 & 313.2 & 10813.444 & 0 & 0 & 28 & 4000 & 20.98 & 0.005 & 10 & 1 & 15.66 \\
\hline 1 & 2 & 220 & 793.8 & 313.7 & 12475.578 & 0 & 0 & 28 & 4000 & 21.02 & 0.005 & 11 & 1 & 15.685 \\
\hline 1 & 3 & 240 & 793.8 & 313.6 & 14156.42 & 0 & 0 & 28 & 4000 & 21.01 & 0.005 & 12 & 1 & 15.678 \\
\hline 1 & 3 & 260 & 694.6 & 273.6 & 15611.414 & 0 & 0 & 28 & 3500 & 18.33 & 0.005 & 13 & 1 & 13.68 \\
\hline
\end{tabular}




$\begin{array}{rrrrrrrrrrrrrrrrr}1 & 3 & 280 & 595.4 & 234.3 & 16861.294 & 0 & 0 & 28 & 3000 & 15.7 & 0.005 & 14 & 1 & 11.715 & 0 \\ 1 & 3 & 300 & 496.1 & 195.3 & 17901.539 & 0 & 0 & 28 & 2500 & 13.09 & 0.005 & 15 & 1 & 9.7656 & 0 \\ 1 & 3 & 320 & 396.9 & 156.5 & 18734.329 & 0 & 0 & 28 & 2000 & 10.49 & 0.005 & 16 & 1 & 7.8253 & 0 \\ 1 & 3 & 340 & 297.7 & 117.7 & 19359.924 & 0 & 0 & 28 & 1500 & 7.888 & 0.005 & 17 & 1 & 5.8865 & 0 \\ 1 & 3 & 360 & 198.5 & 78.78 & 19777.001 & 0 & 0 & 28 & 1000 & 5.278 & 0.005 & 18 & 1 & 3.9388 & 0 \\ 1 & 3 & 380 & 99.23 & 40.26 & 19986.413 & 0 & 0 & 28 & 500 & 2.697 & 0.005 & 19 & 1 & 2.013 & 0\end{array}$
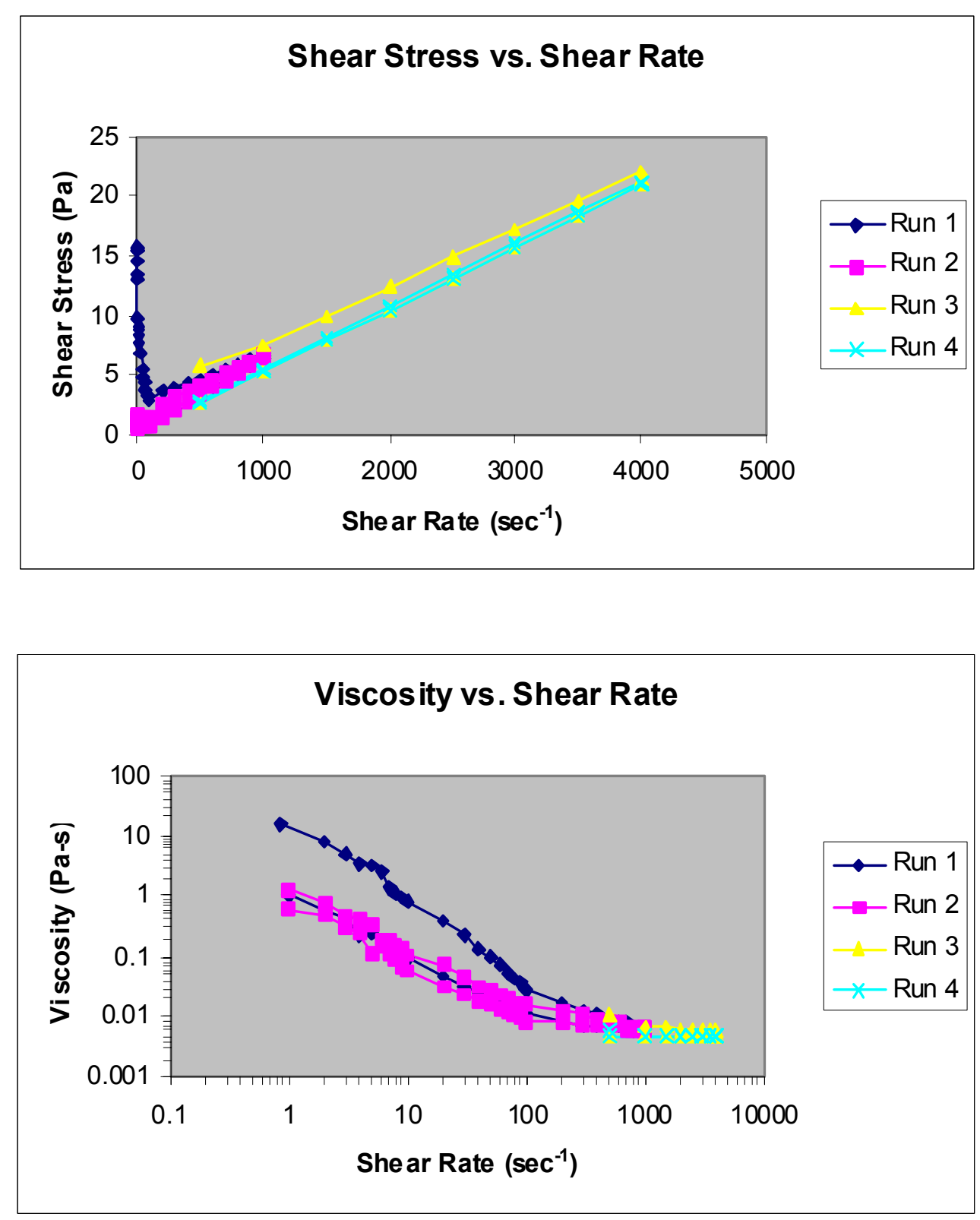

\section{E-2.1.18 META/15wt\%/U/S}

2004.02.13 META-15 wt\%-U [sonicated] DG 0.1-1000 s-1 run 1.txt

Mode Step t[s] n[rpm] M[\%.] Phi[rad] Gamma J[t] T[ $\left.{ }^{\circ} \mathrm{C}\right] \mathrm{D}[1 / \mathrm{s}] \quad \mathrm{Tau}[\mathrm{Pa}] \quad$ Eta[Pas $] \quad$ MP\# Block $\mathrm{M}[\mathrm{mNm}] \quad \mathrm{G}[\mathrm{Pa}]$

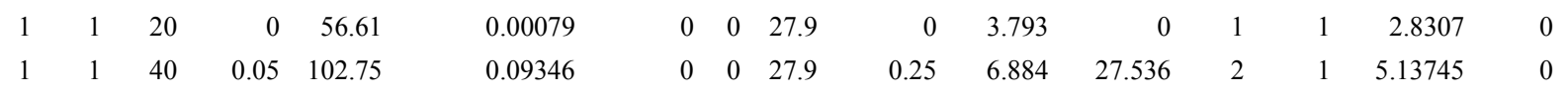




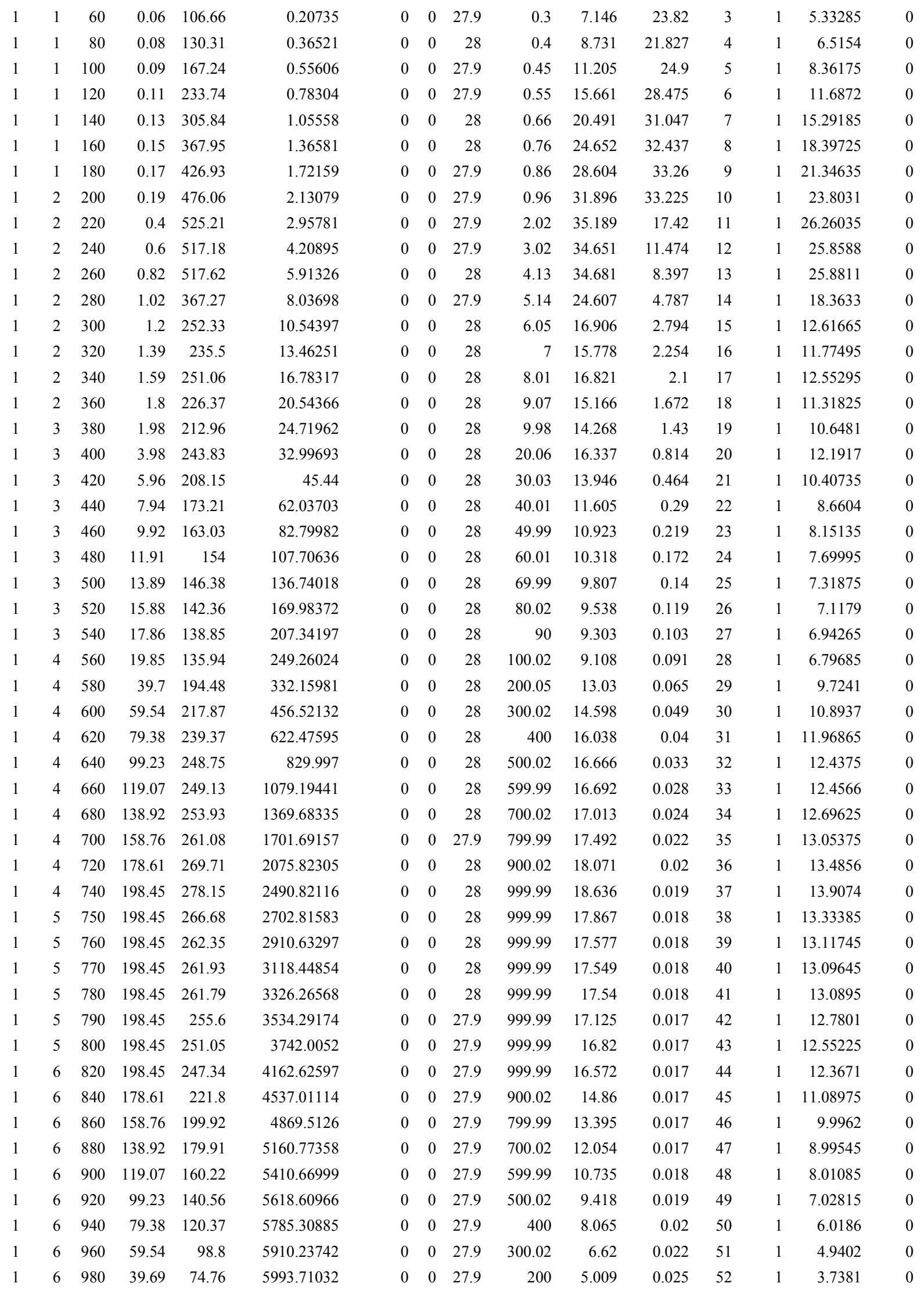




\begin{tabular}{|c|c|c|c|c|c|c|c|c|c|c|c|c|c|c|}
\hline 1 & 6 & 1000 & 19.85 & 45.45 & 6035.60974 & 0 & 0 & 27.9 & 100.02 & 3.045 & 0.03 & 53 & 1 & 2.2725 \\
\hline 1 & 7 & 1020 & 17.86 & 52.84 & 6073.31985 & 0 & 0 & 27.9 & 90 & 3.54 & 0.039 & 54 & 1 & 2.6418 \\
\hline 1 & 7 & 1040 & 15.88 & 55 & 6106.5956 & 0 & 0 & 28 & 80.02 & 3.685 & 0.046 & 55 & 1 & 2.75005 \\
\hline 1 & 7 & 1060 & 13.89 & 55.75 & 6135.68753 & 0 & 0 & 28 & 69.99 & 3.735 & 0.053 & 56 & 1 & 2.78765 \\
\hline 1 & 7 & 1080 & 11.9 & 55.67 & 6160.68047 & 0 & 0 & 28 & 59.96 & 3.73 & 0.062 & 57 & 1 & 2.78365 \\
\hline 1 & 7 & 1100 & 9.92 & 54.99 & 6181.48567 & 0 & 0 & 28 & 49.99 & 3.684 & 0.074 & 58 & 1 & 2.74925 \\
\hline 1 & 7 & 1120 & 7.93 & 54.01 & 6198.12826 & 0 & 0 & 28 & 39.96 & 3.619 & 0.091 & 59 & 1 & 2.7004 \\
\hline 1 & 7 & 1140 & 5.95 & 52.44 & 6210.6098 & 0 & 0 & 28 & 29.98 & 3.513 & 0.117 & 60 & 1 & 2.622 \\
\hline 1 & 7 & 1160 & 3.96 & 53.41 & 6218.94759 & 0 & 0 & 28 & 19.95 & 3.578 & 0.179 & 61 & 1 & 2.6704 \\
\hline 1 & 7 & 1180 & 1.97 & 50.31 & 6223.12198 & 0 & 0 & 28 & 9.93 & 3.371 & 0.339 & 62 & 1 & 2.51535 \\
\hline 1 & 8 & 1200 & 1.79 & 66.79 & 6226.89189 & 0 & 0 & 28 & 9.02 & 4.475 & 0.496 & 63 & 1 & 3.3395 \\
\hline 1 & 8 & 1220 & 1.59 & 71.56 & 6230.22512 & 0 & 0 & 28 & 8.01 & 4.794 & 0.599 & 64 & 1 & 3.5779 \\
\hline 1 & 8 & 1240 & 1.39 & 75 & 6233.14523 & 0 & 0 & 28 & 7 & 5.025 & 0.718 & 65 & 1 & 3.75005 \\
\hline 1 & 8 & 1260 & 1.19 & 78.38 & 6235.64437 & 0 & 0 & 28 & 6 & 5.251 & 0.875 & 66 & 1 & 3.919 \\
\hline 1 & 8 & 1280 & 0.99 & 81.64 & 6237.7296 & 0 & 0 & 28 & 4.99 & 5.47 & 1.096 & 67 & 1 & 4.08175 \\
\hline 1 & 8 & 1300 & 0.8 & 83.39 & 6239.39936 & 0 & 0 & 28 & 4.03 & 5.587 & 1.386 & 68 & 1 & 4.1695 \\
\hline 1 & 8 & 1320 & 0.6 & 80.88 & 6240.65443 & 0 & 0 & 28 & 3.02 & 5.419 & 1.794 & 69 & 1 & 4.0442 \\
\hline 1 & 8 & 1340 & 0.4 & 68.37 & 6241.49951 & 0 & 0 & 28 & 2.02 & 4.581 & 2.268 & 70 & 1 & 3.4186 \\
\hline 1 & 8 & 1360 & 0.2 & 47.31 & 6241.92677 & 0 & 0 & 28 & 1.01 & 3.17 & 3.139 & 71 & 1 & 2.3657 \\
\hline 1 & 9 & 1380 & 0.18 & 46.57 & 6242.3069 & 0 & 0 & 28 & 0.91 & 3.12 & 3.429 & 72 & 1 & 2.32835 \\
\hline 1 & 9 & 1400 & 0.16 & 46.19 & 6242.64305 & 0 & 0 & 28 & 0.81 & 3.094 & 3.82 & 73 & 1 & 2.30925 \\
\hline 1 & 9 & 1420 & 0.14 & 47.08 & 6242.93522 & 0 & 0 & 28 & 0.71 & 3.154 & 4.442 & 74 & 1 & 2.3538 \\
\hline 1 & 9 & 1440 & 0.12 & 48.29 & 6243.18655 & 0 & 0 & 28 & 0.6 & 3.235 & 5.392 & 75 & 1 & 2.4145 \\
\hline 1 & 9 & 1460 & 0.1 & 49.16 & 6243.39625 & 0 & 0 & 28 & 0.5 & 3.294 & 6.588 & 76 & 1 & 2.45805 \\
\hline 1 & 9 & 1480 & 0.08 & 49.41 & 6243.56354 & 0 & 0 & 28 & 0.4 & 3.31 & 8.275 & 77 & 1 & 2.47045 \\
\hline 1 & 9 & 1500 & 0.06 & 48.71 & 6243.6892 & 0 & 0 & 28 & 0.3 & 3.264 & 10.88 & 78 & 1 & 2.4356 \\
\hline 1 & 9 & 1520 & 0.04 & 47.52 & 6243.77481 & 0 & 0 & 28 & 0.2 & 3.184 & 15.92 & 79 & 1 & 2.37605 \\
\hline 1 & 9 & 1540 & 0.02 & 44.46 & 6243.81801 & 0 & 0 & 28 & 0.1 & 2.979 & 29.79 & 80 & 1 & 2.2228 \\
\hline
\end{tabular}

2004.02.13 META-15 wt\%-U [sonicated] DG 0.1-1000 s-1 run 2.txt

Mode Step $\mathrm{t}[\mathrm{s}] \quad \mathrm{n}[\mathrm{rpm}] \quad \mathrm{M}[\%$.] $\quad$ Phi[rad]

\begin{tabular}{|c|c|c|c|c|c|c|c|c|c|c|c|c|c|c|}
\hline 1 & 1 & 20 & 0.01 & 38.42 & 0.02356 & 0 & 0 & 28 & 0.05 & 2.574 & 51.48 & 1 & 2 & 1.9211 \\
\hline 1 & 1 & 40 & 0.04 & 49.12 & 0.10681 & 0 & 0 & 28 & 0.2 & 3.291 & 16.455 & 2 & 2 & 2.45575 \\
\hline 1 & 1 & 60 & 0.06 & 51.94 & 0.23012 & 0 & 0 & 28 & 0.3 & 3.48 & 11.6 & 3 & 2 & 2.5972 \\
\hline 1 & 1 & 80 & 0.08 & 56.88 & 0.39584 & 0 & 0 & 28 & 0.4 & 3.811 & 9.528 & 4 & 2 & 2.8438 \\
\hline 1 & 1 & 100 & 0.1 & 63.15 & 0.6024 & 0 & 0 & 28 & 0.5 & 4.231 & 8.462 & 5 & 2 & 3.1575 \\
\hline 1 & 1 & 120 & 0.12 & 68.95 & 0.85137 & 0 & 0 & 28 & 0.6 & 4.619 & 7.698 & 6 & 2 & 3.44735 \\
\hline 1 & 1 & 140 & 0.14 & 73.61 & 1.14354 & 0 & 0 & 28 & 0.71 & 4.932 & 6.946 & 7 & 2 & 3.6806 \\
\hline 1 & 1 & 160 & 0.16 & 79.06 & 1.47655 & 0 & 0 & 28 & 0.81 & 5.297 & 6.54 & 8 & 2 & 3.95315 \\
\hline 1 & 1 & 180 & 0.18 & 83.76 & 1.85197 & 0 & 0 & 28 & 0.91 & 5.612 & 6.167 & 9 & 2 & 4.18785 \\
\hline 1 & 2 & 200 & 0.2 & 87.25 & 2.27216 & 0 & 0 & 28 & 1.01 & 5.845 & 5.787 & 10 & 2 & 4.36225 \\
\hline 1 & 2 & 220 & 0.4 & 113.44 & 3.09604 & 0 & 0 & 28 & 2.02 & 7.6 & 3.762 & 11 & 2 & 5.672 \\
\hline 1 & 2 & 240 & 0.6 & 137.54 & 4.33854 & 0 & 0 & 28 & 3.02 & 9.215 & 3.051 & 12 & 2 & 6.87675 \\
\hline 1 & 2 & 260 & 0.8 & 137.13 & 6.0028 & 0 & 0 & 28 & 4.03 & 9.187 & 2.28 & 13 & 2 & 6.8563 \\
\hline 1 & 2 & 280 & 1 & 127.56 & 8.08803 & 0 & 0 & 28 & 5.04 & 8.547 & 1.696 & 14 & 2 & 6.3781 \\
\hline 1 & 2 & 300 & 1.19 & 130.12 & 10.58167 & 0 & 0 & 28 & 6 & 8.718 & 1.453 & 15 & 2 & 6.50585 \\
\hline 1 & 2 & 320 & 1.39 & 154.52 & 13.48607 & 0 & 0 & 28 & 7 & 10.353 & 1.479 & 16 & 2 & 7.726 \\
\hline 1 & 2 & 340 & 1.59 & 171.5 & 16.81145 & 0 & 0 & 28 & 8.01 & 11.49 & 1.434 & 17 & 2 & 8.57475 \\
\hline 1 & 2 & 360 & 1.79 & 177.08 & 20.55544 & 0 & 0 & 28 & 9.02 & 11.864 & 1.315 & 18 & 2 & 8.85395 \\
\hline 1 & 3 & 380 & 1.98 & 189.43 & 24.72748 & 0 & 0 & 28 & 9.98 & 12.692 & 1.272 & 19 & 2 & 9.4714 \\
\hline
\end{tabular}




\begin{tabular}{|c|c|c|c|c|c|c|c|c|c|c|c|c|c|}
\hline 1 & 400 & 3.99 & 184.8 & 33.02599 & 0 & 0 & 28 & 20.11 & 12.382 & 0.616 & 20 & 2 & 9.23995 \\
\hline 1 & 420 & 5.96 & 108.96 & 45.47063 & 0 & 0 & 28 & 30.03 & 7.3 & 0.243 & 21 & 2 & 5.4481 \\
\hline 1 & 440 & 7.94 & 86.45 & 62.07394 & 0 & 0 & 28 & 40.01 & 5.792 & 0.145 & 22 & 2 & 4.3224 \\
\hline 1 & 460 & 9.92 & 84.13 & 82.79589 & 0 & 0 & 28 & 49.99 & 5.637 & 0.113 & 23 & 2 & 4.20655 \\
\hline 1 & 480 & 11.91 & 85.19 & 107.71893 & 0 & 0 & 28 & 60.01 & 5.708 & 0.095 & 24 & 2 & 4.25945 \\
\hline & 500 & 13.89 & 87.11 & 136.75824 & 0 & 0 & 28 & 69.99 & 5.837 & 0.083 & 25 & 2 & 4.3556 \\
\hline & 520 & 15.88 & 89.57 & 169.99315 & 0 & 0 & 28 & 80.02 & 6.001 & 0.075 & 26 & 2 & 4.4785 \\
\hline & 540 & 17.86 & 92.11 & 207.36946 & 0 & 0 & 28 & 90 & 6.172 & 0.069 & 27 & 2 & 4.60565 \\
\hline & 560 & 19.85 & 94.55 & 249.31915 & 0 & 0 & 28 & 100.02 & 6.335 & 0.063 & 28 & 2 & 4.72745 \\
\hline & 580 & 39.69 & 139.42 & 332.13703 & 0 & 0 & 28 & 200 & 9.341 & 0.047 & 29 & 2 & 6.97095 \\
\hline 1 & 600 & 59.54 & 163.99 & 456.48834 & 0 & 0 & 28 & 300.02 & 10.987 & 0.037 & 30 & 2 & 8.19955 \\
\hline 1 & 620 & 79.38 & 182.59 & 622.43433 & 0 & 0 & 28 & 400 & 12.233 & 0.031 & 31 & 2 & 9.1294 \\
\hline 1 & 640 & 99.23 & 195.75 & 829.90118 & 0 & 0 & 28 & 500.02 & 13.115 & 0.026 & 32 & 2 & 9.78735 \\
\hline 1 & 660 & 119.07 & 206.42 & 1078.92031 & 0 & 0 & 28 & 599.99 & 13.83 & 0.023 & 33 & 2 & 10.3208 \\
\hline 1 & 680 & 138.92 & 217.74 & 1369.55376 & 0 & 0 & 28 & 700.02 & 14.589 & 0.021 & 34 & 2 & 10.88695 \\
\hline I & 700 & 158.76 & 227.58 & 1701.98767 & 0 & 0 & 28 & 799.99 & 15.248 & 0.019 & 35 & 2 & 11.3788 \\
\hline 1 & 720 & 178.61 & 239.53 & 2075.83876 & 0 & 0 & 28 & 900.02 & 16.048 & 0.018 & 36 & 2 & 11.97635 \\
\hline 1 & 740 & 198.45 & 248.44 & 2490.94054 & 0 & 0 & 28 & 999.99 & 16.646 & 0.017 & 37 & 2 & 12.4221 \\
\hline 1 & 750 & 198.45 & 239.5 & 2702.83154 & 0 & 0 & 28 & 999.99 & 16.046 & 0.016 & 38 & 2 & 11.97475 \\
\hline 1 & 760 & 198.45 & 236.15 & 2910.64789 & 0 & 0 & 28 & 999.99 & 15.822 & 0.016 & 39 & 2 & 11.8074 \\
\hline 1 & 770 & 198.45 & 233.33 & 3118.46503 & 0 & 0 & 28 & 999.99 & 15.633 & 0.016 & 40 & 2 & 11.66655 \\
\hline 1 & 780 & 198.45 & 230.93 & 3326.17772 & 0 & 0 & 28 & 999.99 & 15.472 & 0.015 & 41 & 2 & 11.5465 \\
\hline 1 & 790 & 198.45 & 228.85 & 3534.09853 & 0 & 0 & 28 & 999.99 & 15.333 & 0.015 & 42 & 2 & 11.4427 \\
\hline 1 & 800 & 198.45 & 227.02 & 3742.01934 & 0 & 0 & 28 & 999.99 & 15.21 & 0.015 & 43 & 2 & 11.35095 \\
\hline 1 & 820 & 198.45 & 225.19 & 4162.53565 & 0 & 0 & 28 & 999.99 & 15.088 & 0.015 & 44 & 2 & 11.25965 \\
\hline 1 & 840 & 178.61 & 203.51 & 4536.55797 & 0 & 0 & 28 & 900.02 & 13.635 & 0.015 & 45 & 2 & 10.1755 \\
\hline 1 & 860 & 158.76 & 183.61 & 4869.39164 & 0 & 0 & 28 & 799.99 & 12.302 & 0.015 & 46 & 2 & 9.18065 \\
\hline 1 & 880 & 138.92 & 164.95 & 5160.66205 & 0 & 0 & 28 & 700.02 & 11.051 & 0.016 & 47 & 2 & 8.24725 \\
\hline 1 & 900 & 119.07 & 146.4 & 5410.68256 & 0 & 0 & 28 & 599.99 & 9.809 & 0.016 & 48 & 2 & 7.32 \\
\hline 1 & 920 & 99.23 & 127.88 & 5618.62144 & 0 & 0 & 28 & 500.02 & 8.568 & 0.017 & 49 & 2 & 6.3938 \\
\hline 1 & 940 & 79.38 & 108.77 & 5785.27979 & 0 & 0 & 28 & 400 & 7.287 & 0.018 & 50 & 2 & 5.43825 \\
\hline 1 & 960 & 59.54 & 88.47 & 5910.33324 & 0 & 0 & 28 & 300.02 & 5.928 & 0.02 & 51 & 2 & 4.42365 \\
\hline 1 & 980 & 39.69 & 66.17 & 5993.7221 & 0 & 0 & 28 & 200 & 4.433 & 0.022 & 52 & 2 & 3.3084 \\
\hline 1 & $6 \quad 1000$ & 19.85 & 39.33 & 6035.62152 & 0 & 0 & 28 & 100.02 & 2.635 & 0.026 & 53 & 2 & 1.96645 \\
\hline 1 & $7 \quad 1020$ & 17.86 & 44.26 & 6073.30493 & 0 & 0 & 28 & 90 & 2.965 & 0.033 & 54 & 2 & 2.213 \\
\hline 1 & $7 \quad 1040$ & 15.88 & 45.1 & 6106.60738 & 0 & 0 & 28 & 80.02 & 3.021 & 0.038 & 55 & 2 & 2.25475 \\
\hline 1 & $7 \quad 1060$ & 13.89 & 45.11 & 6135.69853 & 0 & 0 & 28 & 69.99 & 3.022 & 0.043 & 56 & 2 & 2.25555 \\
\hline 1 & $7 \quad 1080$ & 11.9 & 44.5 & 6160.66948 & 0 & 0 & 28 & 59.96 & 2.982 & 0.05 & 57 & 2 & 2.22505 \\
\hline 1 & $7 \quad 1100$ & 9.92 & 43.24 & 6181.46996 & 0 & 0 & 28 & 49.99 & 2.897 & 0.058 & 58 & 2 & 2.16205 \\
\hline 1 & $7 \quad 1120$ & 7.93 & 41.8 & 6198.12276 & 0 & 0 & 28 & 39.96 & 2.801 & 0.07 & 59 & 2 & 2.08995 \\
\hline 1 & $7 \quad 1140$ & 5.95 & 39.92 & 6210.61216 & 0 & 0 & 28 & 29.98 & 2.675 & 0.089 & 60 & 2 & 1.99595 \\
\hline 1 & $7 \quad 1160$ & 3.96 & 39.69 & 6218.94445 & 0 & 0 & 28 & 19.95 & 2.659 & 0.133 & 61 & 2 & 1.9843 \\
\hline 1 & $7 \quad 1180$ & 1.98 & 35.74 & 6223.1212 & 0 & 0 & 28 & 9.98 & 2.395 & 0.24 & 62 & 2 & 1.7872 \\
\hline 1 & $8 \quad 1200$ & 1.79 & 48.25 & 6226.89032 & 0 & 0 & 28 & 9.02 & 3.233 & 0.358 & 63 & 2 & 2.4126 \\
\hline 1 & $8 \quad 1220$ & 1.59 & 52.09 & 6230.22277 & 0 & 0 & 28 & 8.01 & 3.49 & 0.436 & 64 & 2 & 2.60465 \\
\hline 1 & $8 \quad 1240$ & 1.39 & 55.38 & 6233.14052 & 0 & 0 & 28 & 7 & 3.71 & 0.53 & 65 & 2 & 2.76895 \\
\hline 1 & $8 \quad 1260$ & 1.19 & 58.03 & 6235.64359 & 0 & 0 & 28 & 6 & 3.888 & 0.648 & 66 & 2 & 2.90125 \\
\hline 1 & $8 \quad 1280$ & 0.99 & 60.88 & 6237.72882 & 0 & 0 & 28 & 4.99 & 4.079 & 0.817 & 67 & 2 & 3.04405 \\
\hline 1 & $8 \quad 1300$ & 0.8 & 62.69 & 6239.39779 & 0 & 0 & 28 & 4.03 & 4.2 & 1.042 & 68 & 2 & 3.13445 \\
\hline 1 & 1320 & 0.6 & 62.17 & 6240.65128 & 0 & 0 & 28 & 3.02 & 4.165 & 1.379 & 69 & 2 & 3.10835 \\
\hline
\end{tabular}




$\begin{array}{rrrrrrrrrrrrrrrr}1 & 8 & 1340 & 0.4 & 56.32 & 6241.49245 & 0 & 0 & 28 & 2.02 & 3.773 & 1.868 & 70 & 2 & 2.81585 & 0 \\ 1 & 8 & 1360 & 0.2 & 39.8 & 6241.92049 & 0 & 0 & 28 & 1.01 & 2.666 & 2.64 & 71 & 2 & 1.9898 & 0 \\ 1 & 9 & 1380 & 0.18 & 38.87 & 6242.30062 & 0 & 0 & 28 & 0.91 & 2.604 & 2.862 & 72 & 2 & 1.9434 & 0 \\ 1 & 9 & 1400 & 0.16 & 37.69 & 6242.63599 & 0 & 0 & 28 & 0.81 & 2.525 & 3.117 & 73 & 2 & 1.8844 & 0 \\ 1 & 9 & 1420 & 0.14 & 36.96 & 6242.92972 & 0 & 0 & 28 & 0.71 & 2.476 & 3.487 & 74 & 2 & 1.8479 & 0 \\ 1 & 9 & 1440 & 0.12 & 37.33 & 6243.18105 & 0 & 0 & 28 & 0.6 & 2.501 & 4.168 & 75 & 2 & 1.86655 & 0 \\ 1 & 9 & 1460 & 0.1 & 37.81 & 6243.38997 & 0 & 0 & 28 & 0.5 & 2.533 & 5.066 & 76 & 2 & 1.8905 & 0 \\ 1 & 9 & 1480 & 0.08 & 38.17 & 6243.55804 & 0 & 0 & 28 & 0.4 & 2.558 & 6.395 & 77 & 2 & 1.90865 & 0 \\ 1 & 9 & 1500 & 0.06 & 38.38 & 6243.68371 & 0 & 0 & 28 & 0.3 & 2.572 & 8.573 & 78 & 2 & 1.91905 & 0 \\ 1 & 9 & 1520 & 0.04 & 38.49 & 6243.76774 & 0 & 0 & 28 & 0.2 & 2.579 & 12.895 & 79 & 2 & 1.92445 & 0 \\ 1 & 9 & 1540 & 0.02 & 36.53 & 6243.81094 & 0 & 0 & 28 & 0.1 & 2.448 & 24.48 & 80 & 2 & 1.82675 & 0\end{array}$

2004.02.13 META-15 wt\%-U [sonicated] DG 0.1-1000 s-1 run 3.txt

Mode Step t[s] $\mathrm{n}[\mathrm{rpm}] \quad \mathrm{M}[\%$.] $\quad$ Phi[rad $]$

$\begin{array}{rrrrr}1 & 1 & 20 & 0.01 & 31.75 \\ 1 & 1 & 40 & 0.04 & 41.21 \\ 1 & 1 & 60 & 0.06 & 44.1 \\ 1 & 1 & 80 & 0.08 & 48.99 \\ 1 & 1 & 100 & 0.1 & 53.53 \\ 1 & 1 & 120 & 0.12 & 58.45 \\ 1 & 1 & 140 & 0.14 & 62.84 \\ 1 & 1 & 160 & 0.16 & 65.97 \\ 1 & 1 & 180 & 0.18 & 68.62 \\ 1 & 2 & 200 & 0.2 & 70.3 \\ 1 & 2 & 220 & 0.4 & 85.69 \\ 1 & 2 & 240 & 0.6 & 111.55 \\ 1 & 2 & 260 & 0.8 & 120.36 \\ 1 & 2 & 280 & 1 & 129.54 \\ 1 & 2 & 300 & 1.19 & 134.38 \\ 1 & 2 & 320 & 1.39 & 153.72 \\ 1 & 2 & 340 & 1.59 & 161.15 \\ 1 & 2 & 360 & 1.79 & 165.26 \\ 1 & 3 & 380 & 1.98 & 161.06 \\ 1 & 3 & 400 & 3.99 & 143.69 \\ 1 & 3 & 420 & 5.96 & 100.17 \\ 1 & 3 & 440 & 7.94 & 83.84 \\ 1 & 3 & 460 & 9.92 & 78.22 \\ 1 & 3 & 480 & 11.91 & 78.04 \\ 1 & 3 & 500 & 13.89 & 79.79 \\ 1 & 3 & 520 & 15.88 & 82.33 \\ 1 & 3 & 540 & 17.86 & 84.81 \\ 1 & 4 & 560 & 19.85 & 87.15 \\ 1 & 4 & 580 & 39.69 & 128.73 \\ 1 & 4 & 600 & 59.54 & 152.53 \\ 1 & 4 & 620 & 79.38 & 169.65 \\ 1 & 4 & 640 & 99.23 & 184.17 \\ 1 & 4 & 660 & 119.07 & 198.17 \\ 1 & 4 & 680 & 138.92 & 208.9 \\ 1 & 4 & 700 & 158.76 & 219.63 \\ & 4 & 720 & 178.61 & 230.36 \\ & & & & \end{array}$

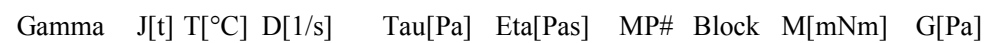

\begin{tabular}{|c|c|c|c|c|c|c|c|c|c|}
\hline 0.02749 & 0 & 0 & 28 & 0.05 & 2.127 & 42.54 & 1 & 3 & 1.58735 \\
\hline 0.10917 & 0 & 0 & 28 & 0.2 & 2.761 & 13.805 & 2 & 3 & 2.0606 \\
\hline 0.23326 & 0 & 0 & 28 & 0.3 & 2.955 & 9.85 & 3 & 3 & 2.20515 \\
\hline 0.39898 & 0 & 0 & 28 & 0.4 & 3.282 & 8.205 & 4 & 3 & 2.4496 \\
\hline 0.60633 & 0 & 0 & 28 & 0.5 & 3.586 & 7.172 & 5 & 3 & 2.6763 \\
\hline 0.8553 & 0 & 0 & 28 & 0.6 & 3.916 & 6.527 & 6 & 3 & 2.92255 \\
\hline 1.14747 & 0 & 0 & 28 & 0.71 & 4.21 & 5.93 & 7 & 3 & 3.14185 \\
\hline 1.48126 & 0 & 0 & 28 & 0.81 & 4.42 & 5.457 & 8 & 3 & 3.29845 \\
\hline 1.85668 & 0 & 0 & 28 & 0.91 & 4.598 & 5.053 & 9 & 3 & 3.431 \\
\hline 2.27765 & 0 & 0 & 28 & 1.01 & 4.71 & 4.663 & 10 & 3 & 3.515 \\
\hline 3.10389 & 0 & 0 & 28 & 2.02 & 5.741 & 2.842 & 11 & 3 & 4.28435 \\
\hline 4.34168 & 0 & 0 & 28 & 3.02 & 7.474 & 2.475 & 12 & 3 & 5.5774 \\
\hline 6.0083 & 0 & 0 & 28 & 4.03 & 8.064 & 2.001 & 13 & 3 & 6.0182 \\
\hline 8.08567 & 0 & 0 & 28 & 5.04 & 8.679 & 1.722 & 14 & 3 & 6.4771 \\
\hline 10.57774 & 0 & 0 & 28 & 6 & 9.003 & 1.501 & 15 & 3 & 6.71895 \\
\hline 13.48607 & 0 & 0 & 28 & 7 & 10.299 & 1.471 & 16 & 3 & 7.68575 \\
\hline 16.81537 & 0 & 0 & 28 & 8.01 & 10.797 & 1.348 & 17 & 3 & 8.05765 \\
\hline 20.56329 & 0 & 0 & 28 & 9.02 & 11.072 & 1.227 & 18 & 3 & 8.263 \\
\hline 24.7369 & 0 & 0 & 28 & 9.98 & 10.791 & 1.081 & 19 & 3 & 8.0529 \\
\hline 33.03071 & 0 & 0 & 28 & 20.11 & 9.627 & 0.479 & 20 & 3 & 7.1847 \\
\hline 45.48241 & 0 & 0 & 28 & 30.03 & 6.711 & 0.223 & 21 & 3 & 5.0084 \\
\hline 62.06452 & 0 & 0 & 28 & 40.01 & 5.617 & 0.14 & 22 & 3 & 4.19215 \\
\hline 82.81867 & 0 & 0 & 28 & 49.99 & 5.24 & 0.105 & 23 & 3 & 3.9108 \\
\hline 107.73542 & 0 & 0 & 28 & 60.01 & 5.229 & 0.087 & 24 & 3 & 3.9022 \\
\hline 136.7606 & 0 & 0 & 28 & 69.99 & 5.346 & 0.076 & 25 & 3 & 3.9897 \\
\hline 169.99551 & 0 & 0 & 28 & 80.02 & 5.516 & 0.069 & 26 & 3 & 4.11655 \\
\hline 207.37103 & 0 & 0 & 28 & 90 & 5.682 & 0.063 & 27 & 3 & 4.2406 \\
\hline 249.28773 & 0 & 0 & 28 & 100.02 & 5.839 & 0.058 & 28 & 3 & 4.35745 \\
\hline 332.18965 & 0 & 0 & 28 & 200 & 8.625 & 0.043 & 29 & 3 & 6.43635 \\
\hline 456.58258 & 0 & 0 & 28 & 300.02 & 10.219 & 0.034 & 30 & 3 & 7.62635 \\
\hline 622.37307 & 0 & 0 & 28 & 400 & 11.366 & 0.028 & 31 & 3 & 8.48235 \\
\hline 830.08889 & 0 & 0 & 28 & 500.02 & 12.339 & 0.025 & 32 & 3 & 9.20855 \\
\hline 1078.9101 & 0 & 0 & 28 & 599.99 & 13.277 & 0.022 & 33 & 3 & 9.90835 \\
\hline 369.53334 & 0 & 0 & 28 & 700.02 & 13.996 & 0.02 & 34 & 3 & 10.44475 \\
\hline 701.80153 & 0 & 0 & 28 & 799.99 & 14.715 & 0.018 & 35 & 3 & 10.9817 \\
\hline 075.55916 & 0 & 0 & 28 & 900.02 & 15.434 & 0.017 & 36 & 3 & 11.51785 \\
\hline
\end{tabular}




\begin{tabular}{|c|c|c|c|c|c|c|c|c|c|c|c|c|c|c|}
\hline 1 & 4 & 740 & 198.45 & 240.57 & 2490.76461 & 0 & 0 & 28 & 999.99 & 16.118 & 0.016 & 37 & 3 & 12.02865 \\
\hline 1 & 5 & 750 & 198.45 & 232.65 & 2702.7585 & 0 & 0 & 28 & 999.99 & 15.587 & 0.016 & 38 & 3 & 11.63225 \\
\hline 1 & 5 & 760 & 198.45 & 229.65 & 2910.47197 & 0 & 0 & 28 & 999.99 & 15.386 & 0.015 & 39 & 3 & 11.48245 \\
\hline 1 & 5 & 770 & 198.45 & 227.09 & 3118.39356 & 0 & 0 & 28 & 999.99 & 15.215 & 0.015 & 40 & 3 & 11.3543 \\
\hline 1 & 5 & 780 & 198.45 & 224.86 & 3326.10624 & 0 & 0 & 28 & 999.99 & 15.065 & 0.015 & 41 & 3 & 11.2428 \\
\hline 1 & 5 & 790 & 198.45 & 222.9 & 3534.02627 & 0 & 0 & 28 & 999.99 & 14.934 & 0.015 & 42 & 3 & 11.14475 \\
\hline 1 & 5 & 800 & 198.45 & 221.17 & 3741.9463 & 0 & 0 & 28 & 999.99 & 14.818 & 0.015 & 43 & 3 & 11.05845 \\
\hline 1 & 6 & 820 & 198.45 & 219.43 & 4162.36051 & 0 & 0 & 28 & 999.99 & 14.702 & 0.015 & 44 & 3 & 10.97165 \\
\hline 1 & 6 & 840 & 178.61 & 198.12 & 4536.84856 & 0 & 0 & 28 & 900.02 & 13.274 & 0.015 & 45 & 3 & 9.9061 \\
\hline 1 & 6 & 860 & 158.76 & 178.65 & 4869.76471 & 0 & 0 & 28 & 799.99 & 11.969 & 0.015 & 46 & 3 & 8.9324 \\
\hline 1 & 6 & 880 & 138.92 & 160.25 & 5161.0359 & 0 & 0 & 28 & 700.02 & 10.736 & 0.015 & 47 & 3 & 8.0123 \\
\hline 1 & 6 & 900 & 119.07 & 141.81 & 5410.56868 & 0 & 0 & 28 & 599.99 & 9.501 & 0.016 & 48 & 3 & 7.0906 \\
\hline 1 & 6 & 920 & 99.23 & 123.48 & 5618.81936 & 0 & 0 & 28 & 500.02 & 8.273 & 0.017 & 49 & 3 & 6.17385 \\
\hline 1 & 6 & 940 & 79.38 & 104.57 & 5785.22716 & 0 & 0 & 28 & 400 & 7.006 & 0.018 & 50 & 3 & 5.22855 \\
\hline 1 & 6 & 960 & 59.54 & 84.68 & 5910.2814 & 0 & 0 & 28 & 300.02 & 5.673 & 0.019 & 51 & 3 & 4.2339 \\
\hline 1 & 6 & 980 & 39.69 & 62.81 & 5993.81556 & 0 & 0 & 28 & 200 & 4.208 & 0.021 & 52 & 3 & 3.1405 \\
\hline 1 & 6 & 1000 & 19.85 & 36.98 & 6035.66315 & 0 & 0 & 28 & 100.02 & 2.477 & 0.025 & 53 & 3 & 1.84875 \\
\hline 1 & 7 & 1020 & 17.86 & 40.69 & 6073.3442 & 0 & 0 & 28 & 90 & 2.726 & 0.03 & 54 & 3 & 2.0347 \\
\hline 1 & 7 & 1040 & 15.88 & 40.89 & 6106.61523 & 0 & 0 & 28 & 80.02 & 2.74 & 0.034 & 55 & 3 & 2.0446 \\
\hline 1 & 7 & 1060 & 13.89 & 40.45 & 6135.75743 & 0 & 0 & 28 & 69.99 & 2.71 & 0.039 & 56 & 3 & 2.0224 \\
\hline 1 & 7 & 1080 & 11.9 & 39.37 & 6160.72131 & 0 & 0 & 28 & 59.96 & 2.638 & 0.044 & 57 & 3 & 1.9686 \\
\hline 1 & 7 & 1100 & 9.92 & 37.89 & 6181.5108 & 0 & 0 & 28 & 49.99 & 2.539 & 0.051 & 58 & 3 & 1.8945 \\
\hline 1 & 7 & 1120 & 7.93 & 36.17 & 6198.17617 & 0 & 0 & 28 & 39.96 & 2.424 & 0.061 & 59 & 3 & 1.8087 \\
\hline 1 & 7 & 1140 & 5.95 & 33.86 & 6210.66007 & 0 & 0 & 28 & 29.98 & 2.269 & 0.076 & 60 & 3 & 1.69295 \\
\hline 1 & 7 & 1160 & 3.96 & 32.75 & 6218.99079 & 0 & 0 & 28 & 19.95 & 2.194 & 0.11 & 61 & 3 & 1.63745 \\
\hline 1 & 7 & 1180 & 1.98 & 28.15 & 6223.16832 & 0 & 0 & 28 & 9.98 & 1.886 & 0.189 & 62 & 3 & 1.40745 \\
\hline 1 & 8 & 1200 & 1.79 & 38.3 & 6226.93745 & 0 & 0 & 28 & 9.02 & 2.566 & 0.284 & 63 & 3 & 1.91485 \\
\hline 1 & 8 & 1220 & 1.59 & 41.47 & 6230.27146 & 0 & 0 & 28 & 8.01 & 2.779 & 0.347 & 64 & 3 & 2.07355 \\
\hline 1 & 8 & 1240 & 1.39 & 44.2 & 6233.19157 & 0 & 0 & 28 & 7 & 2.962 & 0.423 & 65 & 3 & 2.2101 \\
\hline 1 & 8 & 1260 & 1.19 & 46.39 & 6235.69228 & 0 & 0 & 28 & 6 & 3.108 & 0.518 & 66 & 3 & 2.31965 \\
\hline 1 & 8 & 1280 & 0.99 & 48.54 & 6237.77908 & 0 & 0 & 28 & 4.99 & 3.252 & 0.652 & 67 & 3 & 2.42705 \\
\hline 1 & 8 & 1300 & 0.8 & 50.58 & 6239.44805 & 0 & 0 & 28 & 4.03 & 3.389 & 0.841 & 68 & 3 & 2.52895 \\
\hline 1 & 8 & 1320 & 0.6 & 50.41 & 6240.70155 & 0 & 0 & 28 & 3.02 & 3.377 & 1.118 & 69 & 3 & 2.52045 \\
\hline 1 & 8 & 1340 & 0.4 & 46.9 & 6241.54114 & 0 & 0 & 28 & 2.02 & 3.142 & 1.555 & 70 & 3 & 2.34485 \\
\hline 1 & 8 & 1360 & 0.2 & 33.36 & 6241.9684 & 0 & 0 & 28 & 1.01 & 2.235 & 2.213 & 71 & 3 & 1.6678 \\
\hline 1 & 9 & 1380 & 0.18 & 33.44 & 6242.34696 & 0 & 0 & 28 & 0.91 & 2.24 & 2.462 & 72 & 3 & 1.6719 \\
\hline 1 & 9 & 1400 & 0.16 & 32.75 & 6242.68232 & 0 & 0 & 28 & 0.81 & 2.195 & 2.71 & 73 & 3 & 1.6377 \\
\hline 1 & 9 & 1420 & 0.14 & 31.94 & 6242.97685 & 0 & 0 & 28 & 0.71 & 2.14 & 3.014 & 74 & 3 & 1.597 \\
\hline 1 & 9 & 1440 & 0.12 & 31.5 & 6243.22896 & 0 & 0 & 28 & 0.6 & 2.11 & 3.517 & 75 & 3 & 1.57495 \\
\hline 1 & 9 & 1460 & 0.1 & 30.82 & 6243.43866 & 0 & 0 & 28 & 0.5 & 2.065 & 4.13 & 76 & 3 & 1.5412 \\
\hline 1 & 9 & 1480 & 0.08 & 30.43 & 6243.60595 & 0 & 0 & 28 & 0.4 & 2.039 & 5.098 & 77 & 3 & 1.5214 \\
\hline 1 & 9 & 1500 & 0.06 & 29.52 & 6243.7324 & 0 & 0 & 28 & 0.3 & 1.978 & 6.593 & 78 & 3 & 1.47595 \\
\hline 1 & 9 & 1520 & 0.04 & 28.69 & 6243.81644 & 0 & 0 & 28 & 0.2 & 1.922 & 9.61 & 79 & 3 & 1.4343 \\
\hline 1 & 9 & 1540 & 0.02 & 27.09 & 6243.86042 & 0 & 0 & 28 & 0.1 & 1.815 & 18.15 & 80 & 3 & 1.3547 \\
\hline
\end{tabular}

2004.02.13 META-15 wt\%-U [sonicated] DG 0.1-1000 s-1 run 4.txt

Mode Step t[s] n[rpm] M[\%.] Phi[rad]

Gamma J[t $] \mathrm{T}\left[{ }^{\circ} \mathrm{C}\right] \mathrm{D}[1 / \mathrm{s}] \quad \mathrm{Tau}[\mathrm{Pa}] \quad$ Eta[Pas $] \quad$ MP\# Block $\mathrm{M}[\mathrm{mNm}] \quad \mathrm{G}[\mathrm{Pa}]$

$\begin{array}{rrrrrrrrrrrrrrrr}1 & 1 & 20 & 0.02 & 24.8 & 0.03063 & 0 & 0 & 28 & 0.1 & 1.662 & 16.62 & 1 & 4 & 1.2401 & 0 \\ 1 & 1 & 40 & 0.04 & 31.13 & 0.1131 & 0 & 0 & 28 & 0.2 & 2.086 & 10.43 & 2 & 4 & 1.5564 & 0 \\ 1 & 1 & 60 & 0.06 & 33.91 & 0.2364 & 0 & 0 & 28 & 0.3 & 2.272 & 7.573 & 3 & 4 & 1.69545 & 0\end{array}$




\begin{tabular}{|c|c|c|c|c|c|c|c|c|c|c|c|c|c|}
\hline 1 & 80 & 0.08 & 38.13 & 0.40291 & 0 & 0 & 28 & 0.4 & 2.555 & 6.388 & 4 & 4 & 1.9064 \\
\hline 1 & 100 & 0.1 & 41.78 & 0.61104 & 0 & 0 & 28 & 0.5 & 2.799 & 5.598 & 5 & 4 & 2.089 \\
\hline 1 & 120 & 0.12 & 45.66 & 0.86001 & 0 & 0 & 28 & 0.6 & 3.059 & 5.098 & 6 & 4 & 2.2832 \\
\hline 1 & 140 & 0.14 & 49.54 & 1.15218 & 0 & 0 & 28 & 0.71 & 3.319 & 4.675 & 7 & 4 & 2.477 \\
\hline 1 & 160 & 0.16 & 52.93 & 1.48597 & 0 & 0 & 28 & 0.81 & 3.547 & 4.379 & 8 & 4 & 2.64665 \\
\hline 1 & 180 & 0.18 & 55.1 & 1.86139 & 0 & 0 & 28 & 0.91 & 3.692 & 4.057 & 9 & 4 & 2.7552 \\
\hline & 200 & 0.2 & 56.69 & 2.28315 & 0 & 0 & 28 & 1.01 & 3.798 & 3.76 & 10 & 4 & 2.8346 \\
\hline & 220 & 0.4 & 68.18 & 3.11018 & 0 & 0 & 28 & 2.02 & 4.568 & 2.261 & 11 & 4 & 3.4089 \\
\hline & 240 & 0.6 & 83.02 & 4.35346 & 0 & 0 & 28 & 3.02 & 5.562 & 1.842 & 12 & 4 & 4.15075 \\
\hline 1 & 260 & 0.8 & 96.11 & 6.01458 & 0 & 0 & 28 & 4.03 & 6.439 & 1.598 & 13 & 4 & 4.80545 \\
\hline 1 & 280 & 1 & 111.38 & 8.08803 & 0 & 0 & 28 & 5.04 & 7.462 & 1.481 & 14 & 4 & 5.56875 \\
\hline 1 & 300 & 1.19 & 132.97 & 10.58088 & 0 & 0 & 28 & 6 & 8.909 & 1.485 & 15 & 4 & 6.6485 \\
\hline 1 & 320 & 1.39 & 150.32 & 13.48764 & 0 & 0 & 28 & 7 & 10.072 & 1.439 & 16 & 4 & 7.51605 \\
\hline 1 & 340 & 1.59 & 153.4 & 16.82009 & 0 & 0 & 28 & 8.01 & 10.278 & 1.283 & 17 & 4 & 7.6702 \\
\hline 1 & 360 & 1.79 & 161.7 & 20.56172 & 0 & 0 & 28 & 9.02 & 10.834 & 1.201 & 18 & 4 & 8.08495 \\
\hline 1 & 380 & 1.99 & 146.66 & 24.74318 & 0 & 0 & 28 & 10.03 & 9.826 & 0.98 & 19 & 4 & 7.3328 \\
\hline 1 & 400 & 3.98 & 134.45 & 33.03385 & 0 & 0 & 28 & 20.06 & 9.008 & 0.449 & 20 & 4 & 6.7225 \\
\hline 1 & 420 & 5.95 & 105.13 & 45.46906 & 0 & 0 & 28 & 29.98 & 7.044 & 0.235 & 21 & 4 & 5.25655 \\
\hline 1 & 440 & 7.94 & 89.02 & 62.05274 & 0 & 0 & 28 & 40.01 & 5.964 & 0.149 & 22 & 4 & 4.45095 \\
\hline 1 & 460 & 9.92 & 79.46 & 82.8116 & 0 & 0 & 28 & 49.99 & 5.324 & 0.107 & 23 & 4 & 3.9732 \\
\hline 1 & 480 & 11.91 & 74.67 & 107.72914 & 0 & 0 & 28 & 60.01 & 5.003 & 0.083 & 24 & 4 & 3.73325 \\
\hline 1 & 500 & 13.89 & 74.78 & 136.76767 & 0 & 0 & 28 & 69.99 & 5.01 & 0.072 & 25 & 4 & 3.73885 \\
\hline 1 & 520 & 15.88 & 76.57 & 169.98608 & 0 & 0 & 28 & 80.02 & 5.13 & 0.064 & 26 & 4 & 3.82855 \\
\hline 1 & 540 & 17.86 & 79.07 & 207.39067 & 0 & 0 & 28 & 90 & 5.297 & 0.059 & 27 & 4 & 3.95335 \\
\hline 1 & 560 & 19.85 & 81.61 & 249.31758 & 0 & 0 & 28 & 100.02 & 5.468 & 0.055 & 28 & 4 & 4.08045 \\
\hline 1 & 580 & 39.69 & 121.3 & 332.10561 & 0 & 0 & 28 & 200 & 8.127 & 0.041 & 29 & 4 & 6.06505 \\
\hline 1 & 600 & 59.54 & 144.55 & 456.5818 & 0 & 0 & 28 & 300.02 & 9.685 & 0.032 & 30 & 4 & 7.2274 \\
\hline 1 & 620 & 79.38 & 162.56 & 622.57884 & 0 & 0 & 28 & 400 & 10.892 & 0.027 & 31 & 4 & 8.12815 \\
\hline 1 & 640 & 99.23 & 177.07 & 830.03627 & 0 & 0 & 28 & 500.02 & 11.863 & 0.024 & 32 & 4 & 8.85325 \\
\hline 1 & 660 & 119.07 & 190.06 & 1079.16849 & 0 & 0 & 28 & 599.99 & 12.734 & 0.021 & 33 & 4 & 9.5029 \\
\hline 1 & 680 & 138.92 & 203.64 & 1369.86477 & 0 & 0 & 28 & 700.02 & 13.644 & 0.019 & 34 & 4 & 10.1822 \\
\hline 1 & 700 & 158.76 & 215.06 & 1701.63345 & 0 & 0 & 28 & 799.99 & 14.409 & 0.018 & 35 & 4 & 10.75305 \\
\hline 1 & 720 & 178.61 & 225.97 & 2075.38087 & 0 & 0 & 28 & 900.02 & 15.14 & 0.017 & 36 & 4 & 11.2986 \\
\hline 1 & 740 & 198.45 & 236.37 & 2491.09605 & 0 & 0 & 28 & 999.99 & 15.837 & 0.016 & 37 & 4 & 11.8186 \\
\hline 1 & 750 & 198.45 & 229.93 & 2703.08915 & 0 & 0 & 28 & 999.99 & 15.406 & 0.015 & 38 & 4 & 11.4967 \\
\hline 1 & 760 & 198.45 & 227.12 & 2911.42622 & 0 & 0 & 28 & 999.99 & 15.217 & 0.015 & 39 & 4 & 11.35585 \\
\hline 1 & 770 & 198.45 & 224.86 & 3119.13969 & 0 & 0 & 28 & 999.99 & 15.066 & 0.015 & 40 & 4 & 11.24305 \\
\hline 1 & 780 & 198.45 & 222.49 & 3326.85237 & 0 & 0 & 28 & 999.99 & 14.907 & 0.015 & 41 & 4 & 11.12465 \\
\hline 1 & 790 & 198.45 & 220.55 & 3534.7724 & 0 & 0 & 28 & 999.99 & 14.777 & 0.015 & 42 & 4 & 11.02725 \\
\hline 1 & 800 & 198.45 & 218.84 & 3742.58875 & 0 & 0 & 28 & 999.99 & 14.662 & 0.015 & 43 & 4 & 10.9418 \\
\hline 1 & 820 & 198.45 & 217.13 & 4163.00297 & 0 & 0 & 28 & 999.99 & 14.547 & 0.015 & 44 & 4 & 10.85625 \\
\hline 1 & 840 & 178.61 & 196.1 & 4537.49102 & 0 & 0 & 28 & 900.02 & 13.139 & 0.015 & 45 & 4 & 9.80495 \\
\hline 1 & 860 & 158.76 & 176.73 & 4870.08594 & 0 & 0 & 28 & 799.99 & 11.841 & 0.015 & 46 & 4 & 8.8364 \\
\hline 1 & 880 & 138.92 & 158.34 & 5161.57468 & 0 & 0 & 28 & 700.02 & 10.609 & 0.015 & 47 & 4 & 7.917 \\
\hline 1 & 900 & 119.07 & 139.89 & 5411.02421 & 0 & 0 & 28 & 599.99 & 9.372 & 0.016 & 48 & 4 & 6.9944 \\
\hline 1 & 920 & 99.23 & 121.51 & 5619.17122 & 0 & 0 & 28 & 500.02 & 8.141 & 0.016 & 49 & 4 & 6.07565 \\
\hline 1 & 940 & 79.38 & 102.58 & 5785.91203 & 0 & 0 & 28 & 400 & 6.873 & 0.017 & 50 & 4 & 5.12885 \\
\hline 1 & 960 & 59.54 & 82.72 & 5910.89244 & 0 & 0 & 28 & 300.02 & 5.542 & 0.018 & 51 & 4 & 4.136 \\
\hline 1 & 980 & 39.69 & 61.13 & 5994.28131 & 0 & 0 & 28 & 200 & 4.095 & 0.02 & 52 & 4 & 3.0563 \\
\hline 1 & 1000 & 19.85 & 35.6 & 6036.17052 & 0 & 0 & 28 & 100.02 & 2.385 & 0.024 & 53 & 4 & 1.7798 \\
\hline
\end{tabular}




\begin{tabular}{|c|c|c|c|c|c|c|c|c|c|c|c|c|c|c|}
\hline 1 & 7 & 1020 & 17.86 & 38.57 & 6073.84057 & 0 & 0 & 28 & 90 & 2.584 & 0.029 & 54 & 4 & 1.92865 \\
\hline 1 & 7 & 1040 & 15.88 & 38.53 & 6107.13517 & 0 & 0 & 28 & 80.02 & 2.581 & 0.032 & 55 & 4 & 1.92635 \\
\hline 1 & 7 & 1060 & 13.89 & 37.79 & 6136.25616 & 0 & 0 & 28 & 69.99 & 2.532 & 0.036 & 56 & 4 & 1.88955 \\
\hline 1 & 7 & 1080 & 11.9 & 36.48 & 6161.20669 & 0 & 0 & 28 & 59.96 & 2.444 & 0.041 & 57 & 4 & 1.82375 \\
\hline 1 & 7 & 1100 & 9.92 & 34.71 & 6182.00717 & 0 & 0 & 28 & 49.99 & 2.326 & 0.047 & 58 & 4 & 1.7356 \\
\hline 1 & 7 & 1120 & 7.93 & 32.71 & 6198.66704 & 0 & 0 & 28 & 39.96 & 2.192 & 0.055 & 59 & 4 & 1.63565 \\
\hline 1 & 7 & 1140 & 5.95 & 30.29 & 6211.15958 & 0 & 0 & 28 & 29.98 & 2.03 & 0.068 & 60 & 4 & 1.51465 \\
\hline 1 & 7 & 1160 & 3.96 & 28.45 & 6219.4958 & 0 & 0 & 28 & 19.95 & 1.906 & 0.096 & 61 & 4 & 1.4224 \\
\hline 1 & 7 & 1180 & 1.98 & 23.54 & 6223.67333 & 0 & 0 & 28 & 9.98 & 1.577 & 0.158 & 62 & 4 & 1.17695 \\
\hline 1 & 8 & 1200 & 1.79 & 32.18 & 6227.44167 & 0 & 0 & 28 & 9.02 & 2.156 & 0.239 & 63 & 4 & 1.6088 \\
\hline 1 & 8 & 1220 & 1.59 & 34.88 & 6230.77726 & 0 & 0 & 28 & 8.01 & 2.337 & 0.292 & 64 & 4 & 1.74405 \\
\hline 1 & 8 & 1240 & 1.39 & 37.11 & 6233.69266 & 0 & 0 & 28 & 7 & 2.487 & 0.355 & 65 & 4 & 1.85565 \\
\hline 1 & 8 & 1260 & 1.19 & 39.04 & 6236.19651 & 0 & 0 & 28 & 6 & 2.616 & 0.436 & 66 & 4 & 1.9522 \\
\hline 1 & 8 & 1280 & 0.99 & 40.85 & 6238.28174 & 0 & 0 & 28.1 & 4.99 & 2.737 & 0.548 & 67 & 4 & 2.04245 \\
\hline 1 & 8 & 1300 & 0.8 & 42.72 & 6239.95149 & 0 & 0 & 28.1 & 4.03 & 2.862 & 0.71 & 68 & 4 & 2.13595 \\
\hline 1 & 8 & 1320 & 0.6 & 43 & 6241.20656 & 0 & 0 & 28 & 3.02 & 2.881 & 0.954 & 69 & 4 & 2.15005 \\
\hline 1 & 8 & 1340 & 0.4 & 40.59 & 6242.04458 & 0 & 0 & 28.1 & 2.02 & 2.72 & 1.347 & 70 & 4 & 2.0295 \\
\hline 1 & 8 & 1360 & 0.2 & 29.22 & 6242.47105 & 0 & 0 & 28.1 & 1.01 & 1.957 & 1.938 & 71 & 4 & 1.46075 \\
\hline 1 & 9 & 1380 & 0.18 & 28.66 & 6242.8504 & 0 & 0 & 28 & 0.91 & 1.92 & 2.11 & 72 & 4 & 1.4328 \\
\hline 1 & 9 & 1400 & 0.16 & 27.93 & 6243.18576 & 0 & 0 & 28 & 0.81 & 1.871 & 2.31 & 73 & 4 & 1.39655 \\
\hline 1 & 9 & 1420 & 0.14 & 28.36 & 6243.47872 & 0 & 0 & 28 & 0.71 & 1.9 & 2.676 & 74 & 4 & 1.41815 \\
\hline 1 & 9 & 1440 & 0.12 & 28.79 & 6243.73083 & 0 & 0 & 28 & 0.6 & 1.929 & 3.215 & 75 & 4 & 1.43945 \\
\hline 1 & 9 & 1460 & 0.1 & 28.4 & 6243.94053 & 0 & 0 & 28 & 0.5 & 1.903 & 3.806 & 76 & 4 & 1.42015 \\
\hline 1 & 9 & 1480 & 0.08 & 27.48 & 6244.10939 & 0 & 0 & 28 & 0.4 & 1.841 & 4.603 & 77 & 4 & 1.37395 \\
\hline 1 & 9 & 1500 & 0.06 & 26.57 & 6244.23506 & 0 & 0 & 28 & 0.3 & 1.78 & 5.933 & 78 & 4 & 1.3284 \\
\hline 1 & 9 & 1520 & 0.04 & 24.73 & 6244.31988 & 0 & 0 & 28 & 0.2 & 1.657 & 8.285 & 79 & 4 & 1.23655 \\
\hline & 9 & 1540 & 0.02 & 23.71 & 6244.36308 & 0 & 0 & 28 & 0.1 & 1.589 & 15.89 & 80 & 4 & 1.1856 \\
\hline
\end{tabular}

2004.02.13 META-15 wt\%-U [sonicated] DG 0.1-1000 s-1 run 5.txt

Mode Step t[s] n[rpm] M[\%.] Phi[rad]

\begin{tabular}{|c|c|c|c|c|c|c|c|c|c|c|c|c|c|c|}
\hline 1 & 1 & 20 & 0.01 & 30.27 & 0.02985 & 0 & 0 & 28 & 0.05 & 2.028 & 40.56 & 1 & 1 & 1.5135 \\
\hline 1 & 1 & 40 & 0.04 & 29.2 & 0.11388 & 0 & 0 & 28.1 & 0.2 & 1.956 & 9.78 & 2 & 1 & 1.46 \\
\hline 1 & 1 & 60 & 0.06 & 32 & 0.23719 & 0 & 0 & 28.1 & 0.3 & 2.144 & 7.147 & 3 & 1 & 1.59975 \\
\hline 1 & 1 & 80 & 0.08 & 36.87 & 0.40291 & 0 & 0 & 28 & 0.4 & 2.47 & 6.175 & 4 & 1 & 1.8435 \\
\hline 1 & 1 & 100 & 0.1 & 42.92 & 0.60947 & 0 & 0 & 28.1 & 0.5 & 2.876 & 5.752 & 5 & 1 & 2.14605 \\
\hline 1 & 1 & 120 & 0.12 & 49.41 & 0.85923 & 0 & 0 & 28.1 & 0.6 & 3.311 & 5.518 & 6 & 1 & 2.4706 \\
\hline 1 & 1 & 140 & 0.14 & 51.75 & 1.15139 & 0 & 0 & 28.1 & 0.71 & 3.467 & 4.883 & 7 & 1 & 2.58755 \\
\hline 1 & 1 & 160 & 0.16 & 53.99 & 1.48519 & 0 & 0 & 28.1 & 0.81 & 3.617 & 4.465 & 8 & 1 & 2.6994 \\
\hline 1 & 1 & 180 & 0.18 & 56.57 & 1.86061 & 0 & 0 & 28.1 & 0.91 & 3.79 & 4.165 & 9 & 1 & 2.8283 \\
\hline 1 & 2 & 200 & 0.2 & 59.96 & 2.28158 & 0 & 0 & 28.1 & 1.01 & 4.017 & 3.977 & 10 & 1 & 2.9981 \\
\hline 1 & 2 & 220 & 0.4 & 76.72 & 3.10782 & 0 & 0 & 28.1 & 2.02 & 5.14 & 2.545 & 11 & 1 & 3.83585 \\
\hline 1 & 2 & 240 & 0.6 & 88.09 & 4.35425 & 0 & 0 & 28.1 & 3.02 & 5.902 & 1.954 & 12 & 1 & 4.4046 \\
\hline 1 & 2 & 260 & 0.8 & 97.46 & 6.01615 & 0 & 0 & 28.1 & 4.03 & 6.53 & 1.62 & 13 & 1 & 4.873 \\
\hline 1 & 2 & 280 & 1 & 98.59 & 8.09667 & 0 & 0 & 28.1 & 5.04 & 6.606 & 1.311 & 14 & 1 & 4.9295 \\
\hline 1 & 2 & 300 & 1.19 & 117.99 & 10.58245 & 0 & 0 & 28 & 6 & 7.906 & 1.318 & 15 & 1 & 5.8997 \\
\hline 1 & 2 & 320 & 1.39 & 144.29 & 13.49157 & 0 & 0 & 28.1 & 7 & 9.667 & 1.381 & 16 & 1 & 7.21425 \\
\hline 1 & 2 & 340 & 1.59 & 142.73 & 16.82323 & 0 & 0 & 28.1 & 8.01 & 9.563 & 1.194 & 17 & 1 & 7.13665 \\
\hline 1 & 2 & 360 & 1.79 & 148.73 & 20.56251 & 0 & 0 & 28.1 & 9.02 & 9.965 & 1.105 & 18 & 1 & 7.43665 \\
\hline 1 & 3 & 380 & 1.99 & 148.55 & 24.74554 & 0 & 0 & 28.1 & 10.03 & 9.953 & 0.992 & 19 & 1 & 7.42765 \\
\hline 1 & 3 & 400 & 3.98 & 121.33 & 33.04013 & 0 & 0 & 28.1 & 20.06 & 8.129 & 0.405 & 20 & 1 & 6.0665 \\
\hline
\end{tabular}




\begin{tabular}{|c|c|c|c|c|c|c|c|c|c|c|c|c|c|}
\hline 1 & 420 & 5.95 & 87.89 & 45.48476 & 0 & 0 & 28.1 & 29.98 & 5.889 & 0.196 & 21 & 1 & 4.39465 \\
\hline 1 & 440 & 7.94 & 76.44 & 62.0708 & 0 & 0 & 28.1 & 40.01 & 5.121 & 0.128 & 22 & 1 & 3.8218 \\
\hline 1 & 460 & 9.92 & 72.72 & 82.81945 & 0 & 0 & 28.1 & 49.99 & 4.873 & 0.097 & 23 & 1 & 3.6362 \\
\hline 1 & 480 & 11.91 & 72.71 & 107.70636 & 0 & 0 & 28 & 60.01 & 4.872 & 0.081 & 24 & 1 & 3.63555 \\
\hline 1 & 500 & 13.89 & 72.82 & 136.7818 & 0 & 0 & 28 & 69.99 & 4.879 & 0.07 & 25 & 1 & 3.6408 \\
\hline & 520 & 15.88 & 74.14 & 169.98451 & 0 & 0 & 28 & 80.02 & 4.968 & 0.062 & 26 & 1 & 3.7072 \\
\hline & 540 & 17.86 & 76.68 & 207.38831 & 0 & 0 & 28 & 90 & 5.138 & 0.057 & 27 & 1 & 3.83415 \\
\hline & 560 & 19.85 & 79.21 & 249.30501 & 0 & 0 & 28.1 & 100.02 & 5.307 & 0.053 & 28 & 1 & 3.96065 \\
\hline & 580 & 39.69 & 118.71 & 332.21714 & 0 & 0 & 28.1 & 200 & 7.953 & 0.04 & 29 & 1 & 5.9353 \\
\hline 1 & 600 & 59.54 & 141.81 & 456.45535 & 0 & 0 & 28.1 & 300.02 & 9.501 & 0.032 & 30 & 1 & 7.0904 \\
\hline 1 & 620 & 79.38 & 158.72 & 622.6079 & 0 & 0 & 28.1 & 400 & 10.634 & 0.027 & 31 & 1 & 7.93595 \\
\hline 1 & 640 & 99.23 & 173.73 & 830.02292 & 0 & 0 & 28.1 & 500.02 & 11.64 & 0.023 & 32 & 1 & 8.6863 \\
\hline 1 & 660 & 119.07 & 187.31 & 1079.09388 & 0 & 0 & 28.1 & 599.99 & 12.549 & 0.021 & 33 & 1 & 9.36525 \\
\hline 1 & 680 & 138.92 & 200.54 & 1369.86163 & 0 & 0 & 28.1 & 700.02 & 13.436 & 0.019 & 34 & 1 & 10.0269 \\
\hline 1 & 700 & 158.76 & 214.45 & 1701.79681 & 0 & 0 & 28.1 & 799.99 & 14.368 & 0.018 & 35 & 1 & 10.7223 \\
\hline 1 & 720 & 178.61 & 226.21 & 2075.82462 & 0 & 0 & 28.1 & 900.02 & 15.156 & 0.017 & 36 & 1 & 11.31065 \\
\hline 1 & 740 & 198.45 & 238.04 & 2491.02929 & 0 & 0 & 28.1 & 999.99 & 15.949 & 0.016 & 37 & 1 & 11.90205 \\
\hline 1 & 750 & 198.45 & 231.27 & 2702.81583 & 0 & 0 & 28.1 & 999.99 & 15.495 & 0.015 & 38 & 1 & 11.5634 \\
\hline 1 & 760 & 198.45 & 228.51 & 2910.63297 & 0 & 0 & 28.1 & 999.99 & 15.31 & 0.015 & 39 & 1 & 11.4256 \\
\hline 1 & 770 & 198.45 & 226.26 & 3118.96847 & 0 & 0 & 28.1 & 999.99 & 15.159 & 0.015 & 40 & 1 & 11.31305 \\
\hline 1 & 780 & 198.45 & 224.13 & 3326.47381 & 0 & 0 & 28.1 & 999.99 & 15.017 & 0.015 & 41 & 1 & 11.2065 \\
\hline 1 & 790 & 198.45 & 222.17 & 3534.29174 & 0 & 0 & 28.1 & 999.99 & 14.885 & 0.015 & 42 & 1 & 11.10835 \\
\hline 1 & 800 & 198.45 & 220.38 & 3742.21176 & 0 & 0 & 28.1 & 999.99 & 14.765 & 0.015 & 43 & 1 & 11.0189 \\
\hline 1 & 820 & 198.45 & 218.53 & 4162.6244 & 0 & 0 & 28.1 & 999.99 & 14.642 & 0.015 & 44 & 1 & 10.9267 \\
\hline 1 & 840 & 178.61 & 197.23 & 4537.11403 & 0 & 0 & 28.1 & 900.02 & 13.214 & 0.015 & 45 & 1 & 9.8614 \\
\hline 1 & 860 & 158.76 & 177.53 & 4869.93592 & 0 & 0 & 28.1 & 799.99 & 11.895 & 0.015 & 46 & 1 & 8.87665 \\
\hline 1 & 880 & 138.92 & 158.79 & 5161.05239 & 0 & 0 & 28.1 & 700.02 & 10.639 & 0.015 & 47 & 1 & 7.93955 \\
\hline 1 & 900 & 119.07 & 140.01 & 5410.81215 & 0 & 0 & 28.1 & 599.99 & 9.381 & 0.016 & 48 & 1 & 7.0005 \\
\hline 1 & 920 & 99.23 & 121.28 & 5618.95916 & 0 & 0 & 28.1 & 500.02 & 8.125 & 0.016 & 49 & 1 & 6.06375 \\
\hline 1 & 940 & 79.38 & 102.09 & 5785.44079 & 0 & 0 & 28.1 & 400 & 6.84 & 0.017 & 50 & 1 & 5.1044 \\
\hline 1 & 960 & 59.54 & 81.92 & 5910.57828 & 0 & 0 & 28.1 & 300.02 & 5.489 & 0.018 & 51 & 1 & 4.0962 \\
\hline 1 & 980 & 39.69 & 60.08 & 5993.97657 & 0 & 0 & 28.1 & 200 & 4.026 & 0.02 & 52 & 1 & 3.00415 \\
\hline 1 & $6 \quad 1000$ & 19.85 & 34.67 & 6035.85557 & 0 & 0 & 28.1 & 100.02 & 2.323 & 0.023 & 53 & 1 & 1.73365 \\
\hline 1 & $\begin{array}{ll}7 & 1020\end{array}$ & 17.86 & 37.01 & 6073.54683 & 0 & 0 & 28.1 & 90 & 2.479 & 0.028 & 54 & 1 & 1.85025 \\
\hline 1 & $\begin{array}{ll}7 & 1040\end{array}$ & 15.88 & 36.63 & 6106.84143 & 0 & 0 & 28.1 & 80.02 & 2.454 & 0.031 & 55 & 1 & 1.83125 \\
\hline 1 & $\begin{array}{ll}7 & 1060\end{array}$ & 13.89 & 35.76 & 6135.95457 & 0 & 0 & 28.1 & 69.99 & 2.396 & 0.034 & 56 & 1 & 1.7878 \\
\hline 1 & $7 \quad 1080$ & 11.9 & 34.27 & 6160.89881 & 0 & 0 & 28.1 & 59.96 & 2.296 & 0.038 & 57 & 1 & 1.71345 \\
\hline 1 & $7 \quad 1100$ & 9.92 & 32.34 & 6181.7315 & 0 & 0 & 28.1 & 49.99 & 2.167 & 0.043 & 58 & 1 & 1.6171 \\
\hline 1 & $7 \quad 1120$ & 7.93 & 30.27 & 6198.37801 & 0 & 0 & 28.1 & 39.96 & 2.028 & 0.051 & 59 & 1 & 1.5137 \\
\hline 1 & $7 \quad 1140$ & 5.95 & 27.61 & 6210.8627 & 0 & 0 & 28.1 & 29.98 & 1.85 & 0.062 & 60 & 1 & 1.38045 \\
\hline 1 & $7 \quad 1160$ & 3.96 & 25.58 & 6219.19028 & 0 & 0 & 28.1 & 19.95 & 1.714 & 0.086 & 61 & 1 & 1.27915 \\
\hline 1 & $7 \quad 1180$ & 1.98 & 20.27 & 6223.36938 & 0 & 0 & 28.1 & 9.98 & 1.358 & 0.136 & 62 & 1 & 1.0135 \\
\hline 1 & $8 \quad 1200$ & 1.79 & 27.83 & 6227.13772 & 0 & 0 & 28.1 & 9.02 & 1.865 & 0.207 & 63 & 1 & 1.3915 \\
\hline 1 & $8 \quad 1220$ & 1.59 & 30.32 & 6230.47252 & 0 & 0 & 28.1 & 8.01 & 2.032 & 0.254 & 64 & 1 & 1.51615 \\
\hline 1 & $\begin{array}{ll}8 & 1240\end{array}$ & 1.39 & 32.12 & 6233.39263 & 0 & 0 & 28.1 & 7 & 2.152 & 0.307 & 65 & 1 & 1.60595 \\
\hline 1 & $8 \quad 1260$ & 1.19 & 33.85 & 6235.89413 & 0 & 0 & 28.1 & 6 & 2.268 & 0.378 & 66 & 1 & 1.6925 \\
\hline 1 & $8 \quad 1280$ & 0.99 & 35.2 & 6237.98093 & 0 & 0 & 28.1 & 4.99 & 2.359 & 0.473 & 67 & 1 & 1.7601 \\
\hline 1 & $8 \quad 1300$ & 0.8 & 36.63 & 6239.6499 & 0 & 0 & 28.1 & 4.03 & 2.454 & 0.609 & 68 & 1 & 1.83145 \\
\hline 1 & $8 \quad 1320$ & 0.6 & 37.3 & 6240.90497 & 0 & 0 & 28.1 & 3.02 & 2.499 & 0.827 & 69 & 1 & 1.86505 \\
\hline 1 & 1340 & 0.4 & 35.88 & 6241.7422 & 0 & 0 & 28.1 & 2.02 & 2.404 & 1.19 & 70 & 1 & 1.79415 \\
\hline
\end{tabular}




$\begin{array}{rrrrrrrrrrrrrrrr}1 & 8 & 1360 & 0.2 & 26.53 & 6242.16789 & 0 & 0 & 28.1 & 1.01 & 1.778 & 1.76 & 71 & 1 & 1.3265 & 0 \\ 1 & 9 & 1380 & 0.18 & 26.08 & 6242.54802 & 0 & 0 & 28.1 & 0.91 & 1.747 & 1.92 & 72 & 1 & 1.3039 & 0 \\ 1 & 9 & 1400 & 0.16 & 24.42 & 6242.88339 & 0 & 0 & 28.1 & 0.81 & 1.636 & 2.02 & 73 & 1 & 1.2212 & 0 \\ 1 & 9 & 1420 & 0.14 & 24.14 & 6243.17712 & 0 & 0 & 28.1 & 0.71 & 1.617 & 2.277 & 74 & 1 & 1.2068 & 0 \\ 1 & 9 & 1440 & 0.12 & 24.58 & 6243.42845 & 0 & 0 & 28.1 & 0.6 & 1.647 & 2.745 & 75 & 1 & 1.2291 & 0 \\ 1 & 9 & 1460 & 0.1 & 25.26 & 6243.63815 & 0 & 0 & 28.1 & 0.5 & 1.692 & 3.384 & 76 & 1 & 1.2628 & 0 \\ 1 & 9 & 1480 & 0.08 & 25.43 & 6243.80544 & 0 & 0 & 28.1 & 0.4 & 1.704 & 4.26 & 77 & 1 & 1.2715 & 0 \\ 1 & 9 & 1500 & 0.06 & 25.38 & 6243.93189 & 0 & 0 & 28.1 & 0.3 & 1.7 & 5.667 & 78 & 1 & 1.26885 & 0 \\ 1 & 9 & 1520 & 0.04 & 23.72 & 6244.01593 & 0 & 0 & 28.1 & 0.2 & 1.589 & 7.945 & 79 & 1 & 1.18595 & 0 \\ 1 & 9 & 1540 & 0.02 & 22.71 & 6244.05913 & 0 & 0 & 28.1 & 0.1 & 1.522 & 15.22 & 80 & 1 & 1.13555 & 0\end{array}$

2004.02.13 META-15 wt\%-U [sonicated] DG 0.1-1000 s-1 run 6.txt

Mode Step t[s] n[rpm] M[\%.] Phi[rad]
Gamma J $[\mathrm{t}] \mathrm{T}\left[{ }^{\circ} \mathrm{C}\right] \mathrm{D}[1 / \mathrm{s}] \quad \mathrm{Tau}[\mathrm{Pa}] \quad \mathrm{Eta}[\mathrm{Pas}] \quad$ MP\# Block $\mathrm{M}[\mathrm{mNm}] \quad \mathrm{G}[\mathrm{Pa}]$ 0.0322

$\begin{array}{llll}0.0322 & 0 & 0 & 28.1\end{array}$

$\begin{array}{lll}0 & 0 & 28.1\end{array}$

$0.1 \quad 1.458$

$$
14.58
$$$$
14.58
$$$$
\begin{array}{lll}
0 & 0 & 28.1
\end{array}
$$$$
0.23798
$$$$
0.40369
$$$$
0.61104
$$$$
0.8608
$$$$
1.15139
$$$$
1.4844
$$$$
1.86061
$$$$
2.28158
$$$$
3.11096
$$$$
4.35425
$$$$
6.01615
$$$$
8.09746
$$$$
10.59031
$$$$
13.4955
$$$$
16.82401
$$$$
20.56565
$$$$
24.74868
$$$$
33.0417
$$$$
45.48869
$$$$
62.07159
$$$$
\begin{array}{lll}
0 & 0 & 28.1
\end{array}
$$$$
\begin{array}{lll}
0 & 0 & 28.1
\end{array}
$$$$
\begin{array}{lll}
0 & 0 & 28.1
\end{array}
$$$$
\begin{array}{lll}
0 & 0 & 28.1
\end{array}
$$$$
\begin{array}{lll}
0 & 0 & 28.1
\end{array}
$$$$
\begin{array}{lll}
0 & 0 & 28.1
\end{array}
$$$$
\begin{array}{lll}
0 & 0 & 28.1
\end{array}
$$$$
\begin{array}{lll}
0 & 0 & 28.1
\end{array}
$$$$
\begin{array}{lll}
0 & 0 & 28.1
\end{array}
$$$$
\begin{array}{lll}
0 & 0 & 28.1
\end{array}
$$$$
\begin{array}{lll}
0 & 0 & 28.1
\end{array}
$$$$
\begin{array}{lll}
0 & 0 & 28.1
\end{array}
$$$$
\begin{array}{lll}
0 & 0 & 28.1
\end{array}
$$$$
\begin{array}{lll}
0 & 0 & 28.1
\end{array}
$$

$\begin{array}{ll}0.2 & 1.768\end{array}$

$$
\begin{array}{ll}
0.3 & 2.008
\end{array}
$$$$
\begin{array}{ll}
0.4 & 2.258
\end{array}
$$$$
\begin{array}{ll}
0.5 & 2.597
\end{array}
$$$$
0.6 \quad 2.915
$$$$
0.71 \quad 3.262
$$$$
\begin{array}{ll}
0.81 \quad 3.647
\end{array}
$$$$
0.91 \quad 3.885
$$$$
\begin{array}{rr}
1.01 \quad 3.98
\end{array}
$$$$
2.02 \quad 4.925
$$$$
3.02 \quad 5.541
$$$$
4.03 \quad 6.63
$$$$
5.04 \quad 6.69
$$$$
6 \quad 7.174
$$$$
7 \quad 8.546
$$$$
8.01 \quad 9.465
$$$$
\begin{array}{lll}
0 & 0 & 28.1
\end{array}
$$$$
\begin{array}{lll}
0 & 0 & 28.1
\end{array}
$$$$
9.02
$$$$
\begin{array}{ll}
9.02 & 9.525 \\
10.03 & 9.207
\end{array}
$$$$
\begin{array}{lll}
0 & 0 & 28.1
\end{array}
$$$$
\begin{array}{lll}
0 & 0 & 28.1
\end{array}
$$$$
20.06
$$$$
29.98 \quad 5.884
$$$$
\begin{array}{lll}
0 & 0 & 28.1
\end{array}
$$$$
\begin{array}{lll}
0 & 0 & 28.1
\end{array}
$$$$
\begin{array}{lll}
0 & 0 & 28.1
\end{array}
$$$$
\begin{array}{lll}
0 & 0 & 28.1
\end{array}
$$$$
\begin{array}{lll}
0 & 0 & 28.1
\end{array}
$$$$
\begin{array}{lll}
0 & 0 & 28.1
\end{array}
$$$$
\begin{array}{lll}
0 & 0 & 28.1
\end{array}
$$$$
\begin{array}{lll}
0 & 0 & 28.1
\end{array}
$$$$
\begin{array}{lll}
0 & 0 & 28.1
\end{array}
$$$$
\begin{array}{lll}
0 & 0 & 28.1
\end{array}
$$$$
\begin{array}{lll}
0 & 0 & 28.1
\end{array}
$$$$
\begin{array}{lll}
0 & 0 & 28.1
\end{array}
$$$$
\begin{array}{lll}
0 & 0 & 28.1
\end{array}
$$$$
\begin{array}{lll}
0 & 0 & 28.1
\end{array}
$$$$
\begin{array}{lll}
0 & 0 & 28.1
\end{array}
$$$$
\begin{array}{lll}
0 & 0 & 28.1
\end{array}
$$

$40.01 \quad 5.254$

$49.99 \quad 4.945$

$60.01 \quad 4.861$

$69.99 \quad 4.79$

$80.02 \quad 4.779$

$90 \quad 4.936$

$100.02 \quad 5.109$

$200 \quad 7.71$

$300.02 \quad 9.225$

$400 \quad 10.398$

$500.02 \quad 11.422$

$599.99 \quad 12.453$

$700.02 \quad 13.335$

$799.99 \quad 14.094$

$900.02 \quad 14.936$

$999.99 \quad 15.734$

$\begin{array}{rrrrr}14.58 & 1 & 2 & 1.0881 & 0 \\ 8.84 & 2 & 2 & 1.31915 & 0 \\ 6.693 & 3 & 2 & 1.4987 & 0 \\ 5.645 & 4 & 2 & 1.68505 & 0 \\ 5.194 & 5 & 2 & 1.93815 & 0 \\ 4.858 & 6 & 2 & 2.1751 & 0 \\ 4.594 & 7 & 2 & 2.43425 & 0 \\ 4.502 & 8 & 2 & 2.72135 & 0 \\ 4.269 & 9 & 2 & 2.8994 & 0 \\ 3.941 & 10 & 2 & 2.97045 & 0 \\ 2.438 & 11 & 2 & 3.6753 & 0 \\ 1.835 & 12 & 2 & 4.1352 & 0 \\ 1.645 & 13 & 2 & 4.94775 & 0 \\ 1.327 & 14 & 2 & 4.9926 & 0 \\ 1.196 & 15 & 2 & 5.35405 & 0 \\ 1.221 & 16 & 2 & 6.3774 & 0 \\ 1.182 & 17 & 2 & 7.06315 & 0 \\ 1.056 & 18 & 2 & 7.10785 & 0 \\ 0.918 & 19 & 2 & 6.87125 & 0 \\ 0.366 & 20 & 2 & 5.486 & 0 \\ 0.196 & 21 & 2 & 4.39085 & 0 \\ 0.131 & 22 & 2 & 3.9211 & 0 \\ 0.099 & 23 & 2 & 3.69035 & 0 \\ 0.081 & 24 & 2 & 3.62785 & 0 \\ 0.068 & 25 & 2 & 3.57455 & 0 \\ 0.06 & 26 & 2 & 3.5661 & 0 \\ 0.055 & 27 & 2 & 3.6836 & 0 \\ 0.051 & 28 & 2 & 3.81235 & 0 \\ 0.039 & 29 & 2 & 5.7535 & 0 \\ 0.031 & 30 & 2 & 6.8842 & 0 \\ 0.026 & 31 & 2 & 7.75955 & 0 \\ 0.023 & 32 & 2 & 8.5237 & 0 \\ 0.021 & 33 & 2 & 9.29325 & 0 \\ 0.019 & 34 & 2 & 9.95125 & 0 \\ 0.018 & 35 & 2 & 10.5177 & 0 \\ 0.017 & 36 & 2 & 11.14635 & 0 \\ 0.016 & 37 & 2 & 11.74155 & 0\end{array}$




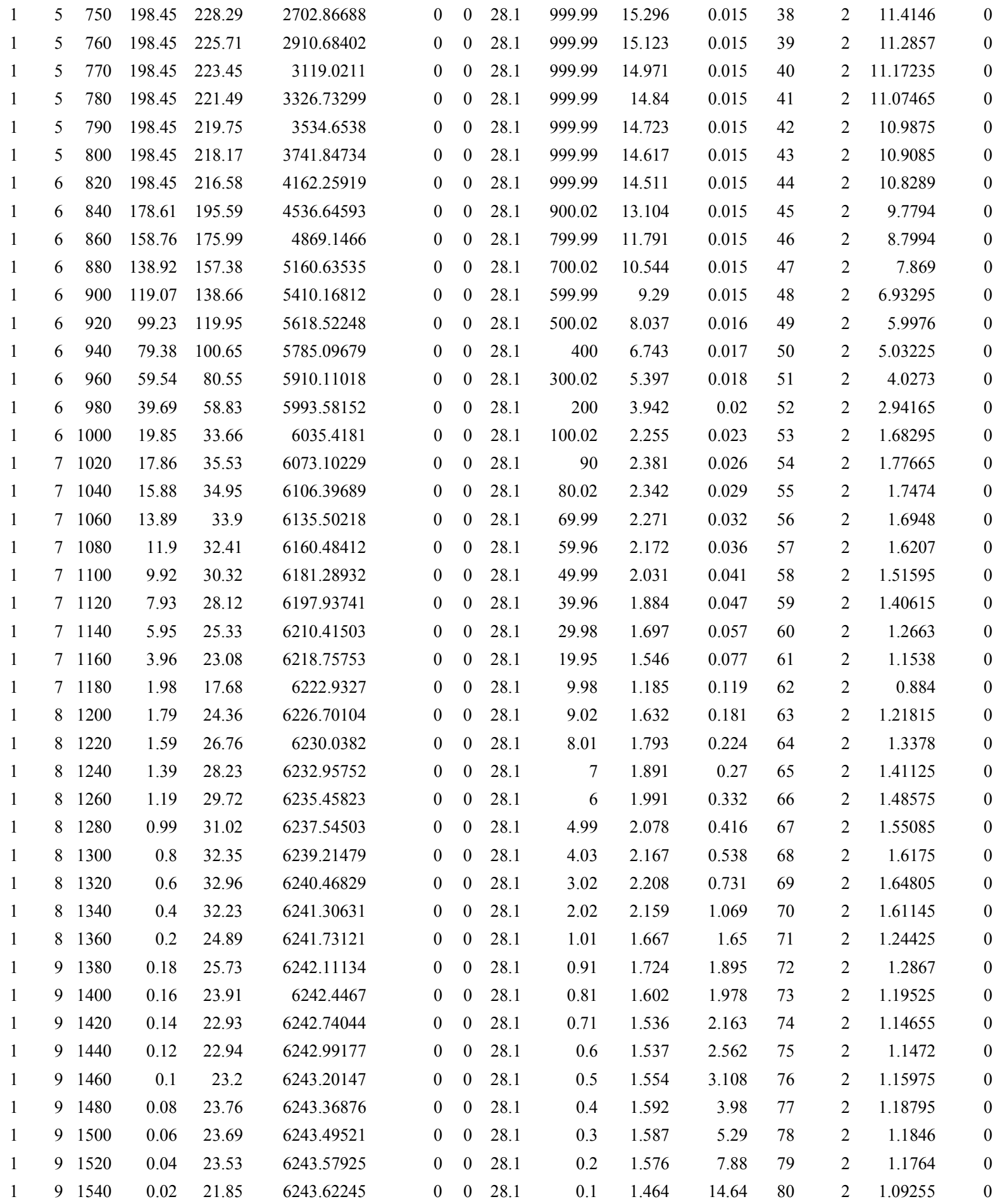

2004.02.13 META-15 wt\%-U [sonicated] DG 0.1-1000 s-1 run 7.txt

Mode Step $\mathrm{t}[\mathrm{s}] \quad \mathrm{n}[\mathrm{rpm}] \quad \mathrm{M}[\%$.] $\quad$ Phi[rad $]$

Gamma J[t] T[ $\left.{ }^{\circ} \mathrm{C}\right] \mathrm{D}[1 / \mathrm{s}] \quad \mathrm{Tau}[\mathrm{Pa}] \quad$ Eta[Pas $] \quad$ MP\# Block $\mathrm{M}[\mathrm{mNm}] \quad \mathrm{G}[\mathrm{Pa}]$

$\begin{array}{rrrrrrrrrrrrrrrr}1 & 1 & 20 & 0.02 & 22.05 & 0.03142 & 0 & 0 & 28.1 & 0.1 & 1.477 & 14.77 & 1 & 3 & 1.1026 & 0 \\ 1 & 1 & 40 & 0.04 & 27.48 & 0.11388 & 0 & 0 & 28.1 & 0.2 & 1.841 & 9.205 & 2 & 3 & 1.3739 & 0 \\ 1 & 1 & 60 & 0.06 & 30.45 & 0.23719 & 0 & 0 & 28.1 & 0.3 & 2.04 & 6.8 & 3 & 3 & 1.5227 & 0 \\ 1 & 1 & 80 & 0.08 & 34.88 & 0.40291 & 0 & 0 & 28.1 & 0.4 & 2.337 & 5.843 & 4 & 3 & 1.74385 & 0\end{array}$




\begin{tabular}{|c|c|c|c|c|c|c|c|c|c|c|c|c|c|c|}
\hline 1 & 1 & 100 & 0.1 & 39.8 & 0.61025 & 0 & 0 & 28.1 & 0.5 & 2.667 & 5.334 & 5 & 3 & 1.99015 \\
\hline 1 & 1 & 120 & 0.12 & 44.42 & 0.86001 & 0 & 0 & 28.1 & 0.6 & 2.976 & 4.96 & 6 & 3 & 2.2211 \\
\hline 1 & 1 & 140 & 0.14 & 48.72 & 1.15218 & 0 & 0 & 28.1 & 0.71 & 3.264 & 4.597 & 7 & 3 & 2.4359 \\
\hline 1 & 1 & 160 & 0.16 & 53.36 & 1.48597 & 0 & 0 & 28.1 & 0.81 & 3.575 & 4.414 & 8 & 3 & 2.6679 \\
\hline 1 & 1 & 180 & 0.18 & 58.09 & 1.86061 & 0 & 0 & 28.1 & 0.91 & 3.892 & 4.277 & 9 & 3 & 2.90435 \\
\hline 1 & 2 & 200 & 0.2 & 62.39 & 2.2808 & 0 & 0 & 28.1 & 1.01 & 4.18 & 4.139 & 10 & 3 & 3.11965 \\
\hline 1 & 2 & 220 & 0.4 & 74.54 & 3.10939 & 0 & 0 & 28.1 & 2.02 & 4.994 & 2.472 & 11 & 3 & 3.7269 \\
\hline 1 & 2 & 240 & 0.6 & 89.69 & 4.35268 & 0 & 0 & 28.1 & 3.02 & 6.009 & 1.99 & 12 & 3 & 4.4846 \\
\hline 1 & 2 & 260 & 0.8 & 98.01 & 6.01458 & 0 & 0 & 28.1 & 4.03 & 6.567 & 1.63 & 13 & 3 & 4.90065 \\
\hline 1 & 2 & 280 & 1 & 105.76 & 8.09667 & 0 & 0 & 28.1 & 5.04 & 7.086 & 1.406 & 14 & 3 & 5.28815 \\
\hline 1 & 2 & 300 & 1.19 & 101.82 & 10.59188 & 0 & 0 & 28.1 & 6 & 6.822 & 1.137 & 15 & 3 & 5.0908 \\
\hline 1 & 2 & 320 & 1.39 & 115.77 & 13.50021 & 0 & 0 & 28.1 & 7 & 7.757 & 1.108 & 16 & 3 & 5.7887 \\
\hline 1 & 2 & 340 & 1.59 & 130.28 & 16.8248 & 0 & 0 & 28.1 & 8.01 & 8.729 & 1.09 & 17 & 3 & 6.51385 \\
\hline 1 & 2 & 360 & 1.79 & 133.45 & 20.57508 & 0 & 0 & 28.1 & 9.02 & 8.941 & 0.991 & 18 & 3 & 6.6725 \\
\hline 1 & 3 & 380 & 1.98 & 122 & 24.75575 & 0 & 0 & 28.1 & 9.98 & 8.174 & 0.819 & 19 & 3 & 6.09985 \\
\hline 1 & 3 & 400 & 3.98 & 104.39 & 33.0362 & 0 & 0 & 28.1 & 20.06 & 6.994 & 0.349 & 20 & 3 & 5.21935 \\
\hline 1 & 3 & 420 & 5.95 & 84.89 & 45.48084 & 0 & 0 & 28.1 & 29.98 & 5.687 & 0.19 & 21 & 3 & 4.24425 \\
\hline 1 & 3 & 440 & 7.94 & 75.88 & 62.08101 & 0 & 0 & 28.1 & 40.01 & 5.084 & 0.127 & 22 & 3 & 3.79405 \\
\hline 1 & 3 & 460 & 9.92 & 72.16 & 82.82495 & 0 & 0 & 28.1 & 49.99 & 4.835 & 0.097 & 23 & 3 & 3.608 \\
\hline 1 & 3 & 480 & 11.91 & 70.66 & 107.73621 & 0 & 0 & 28.1 & 60.01 & 4.734 & 0.079 & 24 & 3 & 3.53295 \\
\hline 1 & 3 & 500 & 13.89 & 68.63 & 136.79123 & 0 & 0 & 28.1 & 69.99 & 4.598 & 0.066 & 25 & 3 & 3.4313 \\
\hline 1 & 3 & 520 & 15.88 & 69.22 & 169.97666 & 0 & 0 & 28.1 & 80.02 & 4.638 & 0.058 & 26 & 3 & 3.4611 \\
\hline 1 & 3 & 540 & 17.86 & 71.67 & 207.37025 & 0 & 0 & 28.1 & 90 & 4.802 & 0.053 & 27 & 3 & 3.5836 \\
\hline 1 & 4 & 560 & 19.85 & 74.26 & 249.27674 & 0 & 0 & 28.1 & 100.02 & 4.975 & 0.05 & 28 & 3 & 3.71285 \\
\hline 1 & 4 & 580 & 39.69 & 112.7 & 332.13781 & 0 & 0 & 28.1 & 200 & 7.551 & 0.038 & 29 & 3 & 5.635 \\
\hline 1 & 4 & 600 & 59.54 & 135.38 & 456.55117 & 0 & 0 & 28.1 & 300.02 & 9.071 & 0.03 & 30 & 3 & 6.7692 \\
\hline 1 & 4 & 620 & 79.38 & 153.09 & 622.53879 & 0 & 0 & 28.1 & 400 & 10.257 & 0.026 & 31 & 3 & 7.65445 \\
\hline 1 & 4 & 640 & 99.23 & 168.06 & 830.00564 & 0 & 0 & 28.1 & 500.02 & 11.26 & 0.023 & 32 & 3 & 8.4028 \\
\hline 1 & 4 & 660 & 119.07 & 182.53 & 1079.01298 & 0 & 0 & 28.1 & 599.99 & 12.229 & 0.02 & 33 & 3 & 9.12645 \\
\hline 1 & 4 & 680 & 138.92 & 196.99 & 1369.71869 & 0 & 0 & 28.1 & 700.02 & 13.199 & 0.019 & 34 & 3 & 9.8497 \\
\hline 1 & 4 & 700 & 158.76 & 210.47 & 1701.73712 & 0 & 0 & 28.1 & 799.99 & 14.101 & 0.018 & 35 & 3 & 10.52335 \\
\hline 1 & 4 & 720 & 178.61 & 222.61 & 2075.67147 & 0 & 0 & 28.1 & 900.02 & 14.915 & 0.017 & 36 & 3 & 11.13025 \\
\hline 1 & 4 & 740 & 198.45 & 234.58 & 2490.77247 & 0 & 0 & 28.1 & 999.99 & 15.717 & 0.016 & 37 & 3 & 11.7292 \\
\hline 1 & 5 & 750 & 198.45 & 228.15 & 2702.76714 & 0 & 0 & 28.1 & 999.99 & 15.286 & 0.015 & 38 & 3 & 11.40765 \\
\hline 1 & 5 & 760 & 198.45 & 225.61 & 2910.58428 & 0 & 0 & 28.1 & 999.99 & 15.116 & 0.015 & 39 & 3 & 11.2807 \\
\hline 1 & 5 & 770 & 198.45 & 223.41 & 3118.29696 & 0 & 0 & 28.1 & 999.99 & 14.969 & 0.015 & 40 & 3 & 11.17055 \\
\hline 1 & 5 & 780 & 198.45 & 221.48 & 3326.73692 & 0 & 0 & 28.1 & 999.99 & 14.839 & 0.015 & 41 & 3 & 11.0738 \\
\hline 1 & 5 & 790 & 198.45 & 219.76 & 3533.93045 & 0 & 0 & 28.1 & 999.99 & 14.724 & 0.015 & 42 & 3 & 10.988 \\
\hline 1 & 5 & 800 & 198.45 & 218.27 & 3741.85048 & 0 & 0 & 28.1 & 999.99 & 14.624 & 0.015 & 43 & 3 & 10.91345 \\
\hline 1 & 6 & 820 & 198.45 & 216.71 & 4162.36758 & 0 & 0 & 28.1 & 999.99 & 14.519 & 0.015 & 44 & 3 & 10.83535 \\
\hline 1 & 6 & 840 & 178.61 & 195.76 & 4536.75275 & 0 & 0 & 28.1 & 900.02 & 13.116 & 0.015 & 45 & 3 & 9.78775 \\
\hline 1 & 6 & 860 & 158.76 & 176.15 & 4869.25341 & 0 & 0 & 28.1 & 799.99 & 11.802 & 0.015 & 46 & 3 & 8.8077 \\
\hline 1 & 6 & 880 & 138.92 & 157.37 & 5160.74216 & 0 & 0 & 28.1 & 700.02 & 10.544 & 0.015 & 47 & 3 & 7.8684 \\
\hline 1 & 6 & 900 & 119.07 & 138.49 & 5410.5137 & 0 & 0 & 28.1 & 599.99 & 9.279 & 0.015 & 48 & 3 & 6.9244 \\
\hline 1 & 6 & 920 & 99.23 & 119.63 & 5618.5052 & 0 & 0 & 28.1 & 500.02 & 8.015 & 0.016 & 49 & 3 & 5.9814 \\
\hline 1 & 6 & 940 & 79.38 & 100.27 & 5785.03788 & 0 & 0 & 28.1 & 400 & 6.718 & 0.017 & 50 & 3 & 5.01345 \\
\hline 1 & 6 & 960 & 59.54 & 80.11 & 5910.17459 & 0 & 0 & 28.1 & 300.02 & 5.367 & 0.018 & 51 & 3 & 4.0054 \\
\hline 1 & 6 & 980 & 39.69 & 58.25 & 5993.62628 & 0 & 0 & 28.1 & 200 & 3.902 & 0.02 & 52 & 3 & 2.91225 \\
\hline 1 & 6 & 1000 & 19.85 & 32.99 & 6035.48408 & 0 & 0 & 28.1 & 100.02 & 2.21 & 0.022 & 53 & 3 & 1.6496 \\
\hline 1 & 7 & 1020 & 17.86 & 34.6 & 6073.16827 & 0 & 0 & 28.1 & 90 & 2.319 & 0.026 & 54 & 3 & 1.73025 \\
\hline
\end{tabular}




\begin{tabular}{|c|c|c|c|c|c|c|c|c|c|c|c|c|c|c|}
\hline 1 & 7 & 1040 & 15.88 & 33.91 & 6106.46208 & 0 & 0 & 28.1 & 80.02 & 2.272 & 0.028 & 55 & 3 & 1.6956 \\
\hline 1 & 7 & 1060 & 13.89 & 32.78 & 6135.56815 & 0 & 0 & 28.1 & 69.99 & 2.196 & 0.031 & 56 & 3 & 1.63905 \\
\hline 1 & 7 & 1080 & 11.9 & 31.16 & 6160.52339 & 0 & 0 & 28.1 & 59.96 & 2.088 & 0.035 & 57 & 3 & 1.55795 \\
\hline 1 & 7 & 1100 & 9.92 & 29.01 & 6181.34037 & 0 & 0 & 28.1 & 49.99 & 1.943 & 0.039 & 58 & 3 & 1.4503 \\
\hline 1 & 7 & 1120 & 7.93 & 26.65 & 6197.98689 & 0 & 0 & 28.1 & 39.96 & 1.785 & 0.045 & 59 & 3 & 1.3324 \\
\hline 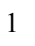 & 7 & 1140 & 5.95 & 23.85 & 6210.47786 & 0 & 0 & 28.1 & 29.98 & 1.598 & 0.053 & 60 & 3 & 1.1925 \\
\hline 1 & 7 & 1160 & 3.96 & 21.49 & 6218.81486 & 0 & 0 & 28.1 & 19.95 & 1.44 & 0.072 & 61 & 3 & 1.0746 \\
\hline 1 & 7 & 1180 & 1.98 & 15.97 & 6222.99004 & 0 & 0 & 28.1 & 9.98 & 1.07 & 0.107 & 62 & 3 & 0.79835 \\
\hline 1 & 8 & 1200 & 1.79 & 22.15 & 6226.75838 & 0 & 0 & 28.1 & 9.02 & 1.484 & 0.165 & 63 & 3 & 1.10735 \\
\hline 1 & 8 & 1220 & 1.59 & 24.17 & 6230.09318 & 0 & 0 & 28.1 & 8.01 & 1.619 & 0.202 & 64 & 3 & 1.20835 \\
\hline 1 & 8 & 1240 & 1.39 & 25.67 & 6233.01172 & 0 & 0 & 28.1 & 7 & 1.72 & 0.246 & 65 & 3 & 1.28325 \\
\hline 1 & 8 & 1260 & 1.19 & 26.84 & 6235.51557 & 0 & 0 & 28.1 & 6 & 1.798 & 0.3 & 66 & 3 & 1.3418 \\
\hline 1 & 8 & 1280 & 0.99 & 28.04 & 6237.60158 & 0 & 0 & 28.1 & 4.99 & 1.879 & 0.377 & 67 & 3 & 1.40215 \\
\hline 1 & 8 & 1300 & 0.8 & 29.18 & 6239.27134 & 0 & 0 & 28.1 & 4.03 & 1.955 & 0.485 & 68 & 3 & 1.4591 \\
\hline 1 & 8 & 1320 & 0.6 & 29.7 & 6240.52484 & 0 & 0 & 28.1 & 3.02 & 1.99 & 0.659 & 69 & 3 & 1.48475 \\
\hline 1 & 8 & 1340 & 0.4 & 29.4 & 6241.36285 & 0 & 0 & 28.1 & 2.02 & 1.97 & 0.975 & 70 & 3 & 1.4701 \\
\hline 1 & 8 & 1360 & 0.2 & 23.42 & 6241.78697 & 0 & 0 & 28.1 & 1.01 & 1.569 & 1.553 & 71 & 3 & 1.17085 \\
\hline 1 & 9 & 1380 & 0.18 & 24.78 & 6242.16632 & 0 & 0 & 28.1 & 0.91 & 1.66 & 1.824 & 72 & 3 & 1.23905 \\
\hline 1 & 9 & 1400 & 0.16 & 23.33 & 6242.50247 & 0 & 0 & 28.1 & 0.81 & 1.563 & 1.93 & 73 & 3 & 1.1663 \\
\hline 1 & 9 & 1420 & 0.14 & 22.38 & 6242.79621 & 0 & 0 & 28.1 & 0.71 & 1.5 & 2.113 & 74 & 3 & 1.11915 \\
\hline 1 & 9 & 1440 & 0.12 & 22.51 & 6243.04753 & 0 & 0 & 28.1 & 0.6 & 1.508 & 2.513 & 75 & 3 & 1.1253 \\
\hline 1 & 9 & 1460 & 0.1 & 22.39 & 6243.25724 & 0 & 0 & 28.1 & 0.5 & 1.5 & 3 & 76 & 3 & 1.1195 \\
\hline 1 & 9 & 1480 & 0.08 & 22.6 & 6243.42531 & 0 & 0 & 28.1 & 0.4 & 1.514 & 3.785 & 77 & 3 & 1.1302 \\
\hline 1 & 9 & 1500 & 0.06 & 22.44 & 6243.55097 & 0 & 0 & 28.1 & 0.3 & 1.503 & 5.01 & 78 & 3 & 1.1219 \\
\hline 1 & 9 & 1520 & 0.04 & 21.53 & 6243.6358 & 0 & 0 & 28.1 & 0.2 & 1.443 & 7.215 & 79 & 3 & 1.0766 \\
\hline & 9 & 1540 & 0.02 & 20.06 & 6243.67742 & 0 & 0 & 28.1 & 0.1 & 1.344 & 13.44 & 80 & 3 & 1.0028 \\
\hline
\end{tabular}

2004.02.13 META-15 wt\%-U [sonicated] DG 0.1-1000 s-1 run 8.txt

Mode Step t[s] n[rpm] M[\%.] Phi[rad]

$\begin{array}{rrrrr}1 & 1 & 20 & 0.02 & 20.69 \\ 1 & 1 & 40 & 0.04 & 25.34 \\ 1 & 1 & 60 & 0.06 & 27.2 \\ 1 & 1 & 80 & 0.08 & 30.65 \\ 1 & 1 & 100 & 0.1 & 34.97 \\ 1 & 1 & 120 & 0.12 & 38.99 \\ 1 & 1 & 140 & 0.14 & 43.02 \\ 1 & 1 & 160 & 0.16 & 47.34 \\ 1 & 1 & 180 & 0.18 & 50.25 \\ 1 & 2 & 200 & 0.2 & 51.95 \\ 1 & 2 & 220 & 0.4 & 62.66 \\ 1 & 2 & 240 & 0.6 & 72.13 \\ 1 & 2 & 260 & 0.8 & 92.54 \\ 1 & 2 & 280 & 1 & 108.97 \\ 1 & 2 & 300 & 1.19 & 115.02 \\ 1 & 2 & 320 & 1.39 & 126.17 \\ 1 & 2 & 340 & 1.59 & 134.4 \\ 1 & 2 & 360 & 1.79 & 122.77 \\ 1 & 3 & 380 & 1.99 & 109.55 \\ 1 & 3 & 400 & 3.98 & 94.89 \\ 1 & 3 & 420 & 5.95 & 85.11\end{array}$

Gamma $\quad J[t] \mathrm{T}\left[{ }^{\circ} \mathrm{C}\right] \quad \mathrm{D}[1 / \mathrm{s}] \quad \mathrm{Tau}[\mathrm{Pa}] \quad$ Eta[Pas $] \quad$ MP\# Block $\mathrm{M}[\mathrm{mNm}] \quad \mathrm{G}[\mathrm{Pa}]$

\begin{tabular}{|c|c|c|c|c|c|c|c|c|c|}
\hline 0.0322 & 0 & 0 & 28.1 & 0.1 & 1.386 & 13.86 & 1 & 4 & 1.0346 \\
\hline 0.11467 & 0 & 0 & 28.1 & 0.2 & 1.698 & 8.49 & 2 & 4 & 1.26715 \\
\hline 0.23955 & 0 & 0 & 28.1 & 0.3 & 1.822 & 6.073 & 3 & 4 & 1.3598 \\
\hline 0.40527 & 0 & 0 & 28.1 & 0.4 & 2.053 & 5.132 & 4 & 4 & 1.53245 \\
\hline 0.61261 & 0 & 0 & 28.1 & 0.5 & 2.343 & 4.686 & 5 & 4 & 1.7483 \\
\hline 0.86237 & 0 & 0 & 28.1 & 0.6 & 2.613 & 4.355 & 6 & 4 & 1.9497 \\
\hline 1.15454 & 0 & 0 & 28.1 & 0.71 & 2.882 & 4.059 & 7 & 4 & 2.15095 \\
\hline 1.48754 & 0 & 0 & 28.1 & 0.81 & 3.172 & 3.916 & 8 & 4 & 2.36685 \\
\hline 1.86375 & 0 & 0 & 28.1 & 0.91 & 3.366 & 3.699 & 9 & 4 & 2.51225 \\
\hline 2.28394 & 0 & 0 & 28.1 & 1.01 & 3.48 & 3.446 & 10 & 4 & 2.59725 \\
\hline 3.11332 & 0 & 0 & 28.1 & 2.02 & 4.198 & 2.078 & 11 & 4 & 3.13295 \\
\hline 4.3566 & 0 & 0 & 28.1 & 3.02 & 4.833 & 1.6 & 12 & 4 & 3.6065 \\
\hline 6.01458 & 0 & 0 & 28.1 & 4.03 & 6.2 & 1.538 & 13 & 4 & 4.6272 \\
\hline 8.09117 & 0 & 0 & 28.1 & 5.04 & 7.301 & 1.449 & 14 & 4 & 5.44845 \\
\hline 10.58952 & 0 & 0 & 28.1 & 6 & 7.706 & 1.284 & 15 & 4 & 5.7509 \\
\hline 13.4955 & 0 & 0 & 28.1 & 7 & 8.454 & 1.208 & 16 & 4 & 6.30865 \\
\hline 16.82637 & 0 & 0 & 28.1 & 8.01 & 9.005 & 1.124 & 17 & 4 & 6.71985 \\
\hline 20.57743 & 0 & 0 & 28.1 & 9.02 & 8.226 & 0.912 & 18 & 4 & 6.13855 \\
\hline 24.75889 & 0 & 0 & 28.1 & 10.03 & 7.34 & 0.732 & 19 & 4 & 5.47735 \\
\hline 33.04563 & 0 & 0 & 28.1 & 20.06 & 6.358 & 0.317 & 20 & 4 & 4.7447 \\
\hline 45.49183 & 0 & 0 & 28.1 & 29.98 & 5.702 & 0.19 & 21 & 4 & 4.25535 \\
\hline
\end{tabular}




\begin{tabular}{|c|c|c|c|c|c|c|c|c|c|c|c|c|c|c|}
\hline 1 & 3 & 440 & 7.94 & 79.27 & 62.07551 & 0 & 0 & 28.1 & 40.01 & 5.311 & 0.133 & 22 & 4 & 3.9634 \\
\hline 1 & 3 & 460 & 9.92 & 74.51 & 82.82338 & 0 & 0 & 28.1 & 49.99 & 4.992 & 0.1 & 23 & 4 & 3.72555 \\
\hline 1 & 3 & 480 & 11.91 & 69.69 & 107.71736 & 0 & 0 & 28.1 & 60.01 & 4.669 & 0.078 & 24 & 4 & 3.4847 \\
\hline 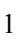 & 3 & 500 & 13.89 & 66.99 & 136.77081 & 0 & 0 & 28.1 & 69.99 & 4.488 & 0.064 & 25 & 4 & 3.34955 \\
\hline 1 & 3 & 520 & 15.88 & 67.57 & 170.0065 & 0 & 0 & 28.1 & 80.02 & 4.527 & 0.057 & 26 & 4 & 3.3783 \\
\hline & 3 & 540 & 17.86 & 69.97 & 207.39145 & 0 & 0 & 28.1 & 90 & 4.688 & 0.052 & 27 & 4 & 3.49845 \\
\hline & 4 & 560 & 19.85 & 72.51 & 249.31836 & 0 & 0 & 28.1 & 100.02 & 4.858 & 0.049 & 28 & 4 & 3.62535 \\
\hline 1 & 4 & 580 & 39.69 & 111.12 & 332.19986 & 0 & 0 & 28.1 & 200 & 7.445 & 0.037 & 29 & 4 & 5.55615 \\
\hline 1 & 4 & 600 & 59.54 & 134.28 & 456.53153 & 0 & 0 & 28.1 & 300.02 & 8.997 & 0.03 & 30 & 4 & 6.71385 \\
\hline & 4 & 620 & 79.38 & 151.65 & 622.60162 & 0 & 0 & 28.1 & 400 & 10.16 & 0.025 & 31 & 4 & 7.58235 \\
\hline & 4 & 640 & 99.23 & 166.98 & 830.11952 & 0 & 0 & 28.1 & 500.02 & 11.187 & 0.022 & 32 & 4 & 8.3488 \\
\hline 1 & 4 & 660 & 119.07 & 181.97 & 1079.07582 & 0 & 0 & 28.1 & 599.99 & 12.192 & 0.02 & 33 & 4 & 9.09865 \\
\hline 1 & 4 & 680 & 138.92 & 195.92 & 1369.76032 & 0 & 0 & 28.1 & 700.02 & 13.127 & 0.019 & 34 & 4 & 9.79595 \\
\hline 1 & 4 & 700 & 158.76 & 209.24 & 1701.61303 & 0 & 0 & 28.1 & 799.99 & 14.019 & 0.018 & 35 & 4 & 10.46205 \\
\hline 1 & 4 & 720 & 178.61 & 222.1 & 2075.37066 & 0 & 0 & 28.1 & 900.02 & 14.881 & 0.017 & 36 & 4 & 11.1049 \\
\hline 1 & 4 & 740 & 198.45 & 234.57 & 2490.9806 & 0 & 0 & 28.1 & 999.99 & 15.716 & 0.016 & 37 & 4 & 11.7285 \\
\hline 1 & 5 & 750 & 198.45 & 228.42 & 2702.87081 & 0 & 0 & 28.1 & 999.99 & 15.304 & 0.015 & 38 & 4 & 11.42095 \\
\hline 1 & 5 & 760 & 198.45 & 226 & 2910.68716 & 0 & 0 & 28.1 & 999.99 & 15.142 & 0.015 & 39 & 4 & 11.30005 \\
\hline 1 & 5 & 770 & 198.45 & 223.85 & 3119.02424 & 0 & 0 & 28.1 & 999.99 & 14.998 & 0.015 & 40 & 4 & 11.1926 \\
\hline 1 & 5 & 780 & 198.45 & 222 & 3326.73692 & 0 & 0 & 28.1 & 999.99 & 14.874 & 0.015 & 41 & 4 & 11.0999 \\
\hline 1 & 5 & 790 & 198.45 & 220.28 & 3534.7614 & 0 & 0 & 28.1 & 999.99 & 14.759 & 0.015 & 42 & 4 & 11.014 \\
\hline 1 & 5 & 800 & 198.45 & 218.8 & 3741.85126 & 0 & 0 & 28.1 & 999.99 & 14.659 & 0.015 & 43 & 4 & 10.9399 \\
\hline 1 & 6 & 820 & 198.45 & 217.27 & 4162.47125 & 0 & 0 & 28.1 & 999.99 & 14.557 & 0.015 & 44 & 4 & 10.86355 \\
\hline 1 & 6 & 840 & 178.61 & 196.27 & 4536.38911 & 0 & 0 & 28.1 & 900.02 & 13.15 & 0.015 & 45 & 4 & 9.81335 \\
\hline 1 & 6 & 860 & 158.76 & 176.51 & 4869.47175 & 0 & 0 & 28.1 & 799.99 & 11.826 & 0.015 & 46 & 4 & 8.8255 \\
\hline 1 & 6 & 880 & 138.92 & 157.68 & 5160.5144 & 0 & 0 & 28.1 & 700.02 & 10.564 & 0.015 & 47 & 4 & 7.8839 \\
\hline 1 & 6 & 900 & 119.07 & 138.75 & 5410.41003 & 0 & 0 & 28.1 & 599.99 & 9.296 & 0.015 & 48 & 4 & 6.93735 \\
\hline 1 & 6 & 920 & 99.23 & 119.83 & 5618.51619 & 0 & 0 & 28.1 & 500.02 & 8.028 & 0.016 & 49 & 4 & 5.9913 \\
\hline 1 & 6 & 940 & 79.38 & 100.29 & 5785.0905 & 0 & 0 & 28.1 & 400 & 6.719 & 0.017 & 50 & 4 & 5.01455 \\
\hline 1 & 6 & 960 & 59.54 & 79.82 & 5910.08191 & 0 & 0 & 28.1 & 300.02 & 5.348 & 0.018 & 51 & 4 & 3.99095 \\
\hline 1 & 6 & 980 & 39.69 & 57.93 & 5993.53204 & 0 & 0 & 28.1 & 200 & 3.881 & 0.019 & 52 & 4 & 2.8963 \\
\hline 1 & 6 & 1000 & 19.85 & 32.76 & 6035.42203 & 0 & 0 & 28.1 & 100.02 & 2.195 & 0.022 & 53 & 4 & 1.6381 \\
\hline 1 & 7 & 1020 & 17.86 & 34.15 & 6073.11486 & 0 & 0 & 28.1 & 90 & 2.288 & 0.025 & 54 & 4 & 1.7074 \\
\hline 1 & 7 & 1040 & 15.88 & 33.33 & 6106.37726 & 0 & 0 & 28.1 & 80.02 & 2.233 & 0.028 & 55 & 4 & 1.66665 \\
\hline 1 & 7 & 1060 & 13.89 & 32.03 & 6135.52653 & 0 & 0 & 28.1 & 69.99 & 2.146 & 0.031 & 56 & 4 & 1.6014 \\
\hline 1 & 7 & 1080 & 11.9 & 30.3 & 6160.46606 & 0 & 0 & 28.1 & 59.96 & 2.03 & 0.034 & 57 & 4 & 1.51485 \\
\hline 1 & 7 & 1100 & 9.92 & 28.12 & 6181.27204 & 0 & 0 & 28.1 & 49.99 & 1.884 & 0.038 & 58 & 4 & 1.4062 \\
\hline 1 & 7 & 1120 & 7.93 & 25.87 & 6197.92877 & 0 & 0 & 28.1 & 39.96 & 1.733 & 0.043 & 59 & 4 & 1.2936 \\
\hline 1 & 7 & 1140 & 5.95 & 23.04 & 6210.42602 & 0 & 0 & 28.1 & 29.98 & 1.544 & 0.052 & 60 & 4 & 1.15205 \\
\hline 1 & 7 & 1160 & 3.96 & 20.31 & 6218.75753 & 0 & 0 & 28.1 & 19.95 & 1.361 & 0.068 & 61 & 4 & 1.01565 \\
\hline 1 & 7 & 1180 & 1.98 & 14.83 & 6222.93663 & 0 & 0 & 28.1 & 9.98 & 0.993 & 0.099 & 62 & 4 & 0.7414 \\
\hline 1 & 8 & 1200 & 1.79 & 20.39 & 6226.70575 & 0 & 0 & 28.1 & 9.02 & 1.366 & 0.151 & 63 & 4 & 1.01965 \\
\hline 1 & 8 & 1220 & 1.59 & 22.22 & 6230.04056 & 0 & 0 & 28.1 & 8.01 & 1.489 & 0.186 & 64 & 4 & 1.11095 \\
\hline 1 & 8 & 1240 & 1.39 & 23.59 & 6232.95909 & 0 & 0 & 28.1 & 7 & 1.581 & 0.226 & 65 & 4 & 1.17965 \\
\hline 1 & 8 & 1260 & 1.19 & 24.68 & 6235.46294 & 0 & 0 & 28.1 & 6 & 1.653 & 0.276 & 66 & 4 & 1.23395 \\
\hline 1 & 8 & 1280 & 0.99 & 25.73 & 6237.54975 & 0 & 0 & 28.1 & 4.99 & 1.724 & 0.345 & 67 & 4 & 1.28625 \\
\hline 1 & 8 & 1300 & 0.8 & 26.75 & 6239.2195 & 0 & 0 & 28.1 & 4.03 & 1.792 & 0.445 & 68 & 4 & 1.33725 \\
\hline 1 & 8 & 1320 & 0.6 & 27.27 & 6240.473 & 0 & 0 & 28.1 & 3.02 & 1.827 & 0.605 & 69 & 4 & 1.3637 \\
\hline 1 & 8 & 1340 & 0.4 & 27.14 & 6241.31023 & 0 & 0 & 28.1 & 2.02 & 1.818 & 0.9 & 70 & 4 & 1.35695 \\
\hline & 8 & 1360 & 0.2 & 22.14 & 6241.73356 & 0 & 0 & 28.1 & 1.01 & 1.484 & 1.469 & 71 & 4 & 1.10715 \\
\hline
\end{tabular}




$\begin{array}{rrrrrrrrrrrrrrrr}1 & 9 & 1380 & 0.18 & 24.3 & 6242.11291 & 0 & 0 & 28.1 & 0.91 & 1.628 & 1.789 & 72 & 4 & 1.21505 & 0 \\ 1 & 9 & 1400 & 0.16 & 22.72 & 6242.44906 & 0 & 0 & 28.1 & 0.81 & 1.522 & 1.879 & 73 & 4 & 1.1361 & 0 \\ 1 & 9 & 1420 & 0.14 & 21.73 & 6242.74201 & 0 & 0 & 28.1 & 0.71 & 1.456 & 2.051 & 74 & 4 & 1.0867 & 0 \\ 1 & 9 & 1440 & 0.12 & 21.92 & 6242.99413 & 0 & 0 & 28.1 & 0.6 & 1.469 & 2.448 & 75 & 4 & 1.0962 & 0 \\ 1 & 9 & 1460 & 0.1 & 22.42 & 6243.20304 & 0 & 0 & 28.1 & 0.5 & 1.502 & 3.004 & 76 & 4 & 1.12095 & 0 \\ 1 & 9 & 1480 & 0.08 & 22.34 & 6243.3719 & 0 & 0 & 28.1 & 0.4 & 1.497 & 3.743 & 77 & 4 & 1.11705 & 0 \\ 1 & 9 & 1500 & 0.06 & 21.93 & 6243.49835 & 0 & 0 & 28.1 & 0.3 & 1.469 & 4.897 & 78 & 4 & 1.0965 & 0 \\ 1 & 9 & 1520 & 0.04 & 21.18 & 6243.58239 & 0 & 0 & 28.1 & 0.2 & 1.419 & 7.095 & 79 & 4 & 1.05895 & 0 \\ 1 & 9 & 1540 & 0.02 & 19.24 & 6243.6248 & 0 & 0 & 28.1 & 0.1 & 1.289 & 12.89 & 80 & 4 & 0.96215 & 0\end{array}$

2004.02.11 META-15 wt\%-U [sonicated] DG 500-4000 s-1 run 1.txt

Mode Step $\mathrm{t}[\mathrm{s}] \quad \mathrm{n}[\mathrm{rpm}] \quad \mathrm{M}[\%$.] $\quad$ Phi[rad]

$\begin{array}{rrrrr}1 & 1 & 20 & 99.26 & 164.86 \\ 1 & 1 & 40 & 198.46 & 215.79 \\ 1 & 1 & 60 & 297.68 & 265.81 \\ 1 & 1 & 80 & 396.91 & 319.41 \\ 1 & 1 & 100 & 496.13 & 354.09 \\ 1 & 1 & 120 & 595.36 & 390.1 \\ 1 & 1 & 140 & 694.58 & 422.82 \\ 1 & 1 & 160 & 793.81 & 455.81 \\ 1 & 2 & 180 & 793.81 & 438.02 \\ 1 & 2 & 200 & 793.81 & 429.17 \\ 1 & 2 & 220 & 793.81 & 423.13 \\ 1 & 3 & 240 & 793.81 & 418.48 \\ 1 & 3 & 260 & 694.58 & 364.94 \\ 1 & 3 & 280 & 595.36 & 314.14 \\ 1 & 3 & 300 & 496.13 & 264.77 \\ 1 & 3 & 320 & 396.91 & 216.71 \\ 1 & 3 & 340 & 297.68 & 168.36 \\ 1 & 3 & 360 & 198.46 & 119.11 \\ 1 & 3 & 380 & 99.23 & 67.61\end{array}$

207.19589
621.6395
1243.79737
2072.6846
3110.37622
4356.30202
5809.31847
7468.41632
9150.18391
10812.73752
12475.28799
14154.46691
15612.74002
16860.12745
17901.98403
18734.87994
19359.69716
19776.958
19986.47945

Gamma J[t $\mathrm{T}\left[{ }^{\circ} \mathrm{C}\right] \mathrm{D}[1 / \mathrm{s}] \quad \mathrm{Tau}[\mathrm{Pa}] \quad$ Eta[Pas $] \quad$ MP\# Block $\mathrm{M}[\mathrm{mNm}] \quad \mathrm{G}[\mathrm{Pa}]$

2004.02.11 META-15 wt\%-U [sonicated] DG 500-4000 s-1 run 2.txt

Mode Step t[s] n[rpm] M[\%.] Phi[rad]

$\begin{array}{rrrrr}1 & 1 & 20 & 99.3 & 83.41 \\ 1 & 1 & 40 & 198.46 & 142.05 \\ 1 & 1 & 60 & 297.68 & 194.92 \\ 1 & 1 & 80 & 396.91 & 245.73 \\ 1 & 1 & 100 & 496.13 & 293.9 \\ 1 & 1 & 120 & 595.36 & 338.69 \\ 1 & 1 & 140 & 694.58 & 381.48 \\ 1 & 1 & 160 & 793.81 & 423.11 \\ 1 & 2 & 180 & 793.81 & 414.93 \\ 1 & 2 & 200 & 793.81 & 411.27 \\ 1 & 2 & 220 & 793.81 & 408.89 \\ 1 & 3 & 240 & 793.81 & 406.81 \\ 1 & 3 & 260 & 694.58 & 356.05 \\ 1 & 3 & 280 & 595.36 & 307.41 \\ 1 & 3 & 300 & 496.13 & 259.79 \\ 1 & 3 & 320 & 396.91 & 212.79\end{array}$

Gamma J[t] T[ $\left.{ }^{\circ} \mathrm{C}\right] \mathrm{D}[1 / \mathrm{s}] \quad \mathrm{Tau}[\mathrm{Pa}] \quad \mathrm{Eta}[\mathrm{Pas}] \quad$ MP\# Block $\mathrm{M}[\mathrm{mNm}] \quad \mathrm{G}[\mathrm{Pa}]$

207.58937
621.82486
1243.77538
2073.64829
3111.39017
4355.70512
5808.35792
7469.17973
9150.54598
10813.10195
12473.98816
14153.99881
15610.34692
16858.98077
17899.54066
18732.74679

$\begin{array}{rrrrrrrrrr}0 & 0 & 28.1 & 500.37 & 5.588 & 0.011 & 1 & 1 & 4.17025 & 0 \\ 0 & 0 & 28 & 1000.04 & 9.517 & 0.01 & 2 & 1 & 7.10255 & 0 \\ 0 & 0 & 28.1 & 1500.01 & 13.06 & 0.009 & 3 & 1 & 9.74605 & 0 \\ 0 & 0 & 28.1 & 2000.03 & 16.464 & 0.008 & 4 & 1 & 12.2867 & 0 \\ 0 & 0 & 28.1 & 2500 & 19.692 & 0.008 & 5 & 1 & 14.69525 & 0 \\ 0 & 0 & 28.1 & 3000.02 & 22.692 & 0.008 & 6 & 1 & 16.93445 & 0 \\ 0 & 0 & 28.1 & 3499.99 & 25.559 & 0.007 & 7 & 1 & 19.07405 & 0 \\ 0 & 0 & 28 & 4000.01 & 28.348 & 0.007 & 8 & 1 & 21.1554 & 0 \\ 0 & 0 & 28 & 4000.01 & 27.8 & 0.007 & 9 & 1 & 20.74655 & 0 \\ 0 & 0 & 28 & 4000.01 & 27.555 & 0.007 & 10 & 1 & 20.5637 & 0 \\ 0 & 0 & 28 & 4000.01 & 27.396 & 0.007 & 11 & 1 & 20.4446 & 0 \\ 0 & 0 & 28 & 4000.01 & 27.256 & 0.007 & 12 & 1 & 20.34025 & 0 \\ 0 & 0 & 28.1 & 3499.99 & 23.855 & 0.007 & 13 & 1 & 17.80245 & 0 \\ 0 & 0 & 28.1 & 3000.02 & 20.597 & 0.007 & 14 & 1 & 15.3707 & 0 \\ 0 & 0 & 28.1 & 2500 & 17.406 & 0.007 & 15 & 1 & 12.9897 & 0 \\ 0 & 0 & 28.1 & 2000.03 & 14.257 & 0.007 & 16 & 1 & 10.63965 & 0\end{array}$

$\begin{array}{rrrrr}0 & 0 & 28 & 500.17 & 11.045 \\ 0 & 0 & 28 & 1000.04 & 14.458 \\ 0 & 0 & 28 & 1500.01 & 17.809 \\ 0 & 0 & 28 & 2000.03 & 21.4 \\ 0 & 0 & 28 & 2500 & 23.724 \\ 0 & 0 & 28 & 3000.02 & 26.137 \\ 0 & 0 & 28 & 3499.99 & 28.329 \\ 0 & 0 & 28.1 & 4000.01 & 30.539 \\ 0 & 0 & 28 & 4000.01 & 29.348 \\ 0 & 0 & 28 & 4000.01 & 28.754 \\ 0 & 0 & 28 & 4000.01 & 28.349 \\ 0 & 0 & 28 & 4000.01 & 28.038 \\ 0 & 0 & 28 & 3499.99 & 24.451 \\ 0 & 0 & 28 & 3000.02 & 21.047 \\ 0 & 0 & 28 & 2500 & 17.739 \\ 0 & 0 & 28 & 2000.03 & 14.52 \\ 0 & 0 & 28 & 1500.01 & 11.28 \\ 0 & 0 & 28.1 & 1000.04 & 7.981 \\ 0 & 0 & 28.1 & 500.02 & 4.53\end{array}$

0.022

0.014

0.012

0.011

$0.009 \quad 5$

$0.009 \quad 6$

$0.008 \quad 7$

$0.008 \quad 8$

$0.007 \quad 9$

$0.007 \quad 10$

$0.007 \quad 11$

$0.007 \quad 12$

$0.007 \quad 13$

$0.007 \quad 14$

$0.007 \quad 15$

$0.007 \quad 16$

0.008

$0.008 \quad 18$

0.009

$\begin{array}{rrr}1 & 8.24275 & 0 \\ 1 & 10.7894 & 0 \\ 1 & 13.29055 & 0 \\ 1 & 15.9705 & 0 \\ 1 & 17.70445 & 0 \\ 1 & 19.5052 & 0 \\ 1 & 21.14085 & 0 \\ 1 & 22.79025 & 0 \\ 1 & 21.90115 & 0 \\ 1 & 21.45855 & 0 \\ 1 & 21.1563 & 0 \\ 1 & 20.92385 & 0 \\ 1 & 18.24675 & 0 \\ 1 & 15.707 & 0 \\ 1 & 13.23825 & 0 \\ 1 & 10.8357 & 0 \\ 1 & 8.41795 & 0 \\ 1 & 5.9556 & 0 \\ 1 & 3.3807 & 0\end{array}$




$\begin{array}{rrrrrrrrrrrrrrrr}1 & 3 & 340 & 297.68 & 165.04 & 19357.61664 & 0 & 0 & 28.1 & 1500.01 & 11.057 & 0.007 & 17 & 1 & 8.25175 & 0 \\ 1 & 3 & 360 & 198.46 & 116.47 & 19775.39584 & 0 & 0 & 28.1 & 1000.04 & 7.803 & 0.008 & 18 & 1 & 5.8234 & 0 \\ 1 & 3 & 380 & 99.23 & 65.91 & 19984.81362 & 0 & 0 & 28.1 & 500.02 & 4.416 & 0.009 & 19 & 1 & 3.29535 & 0\end{array}$
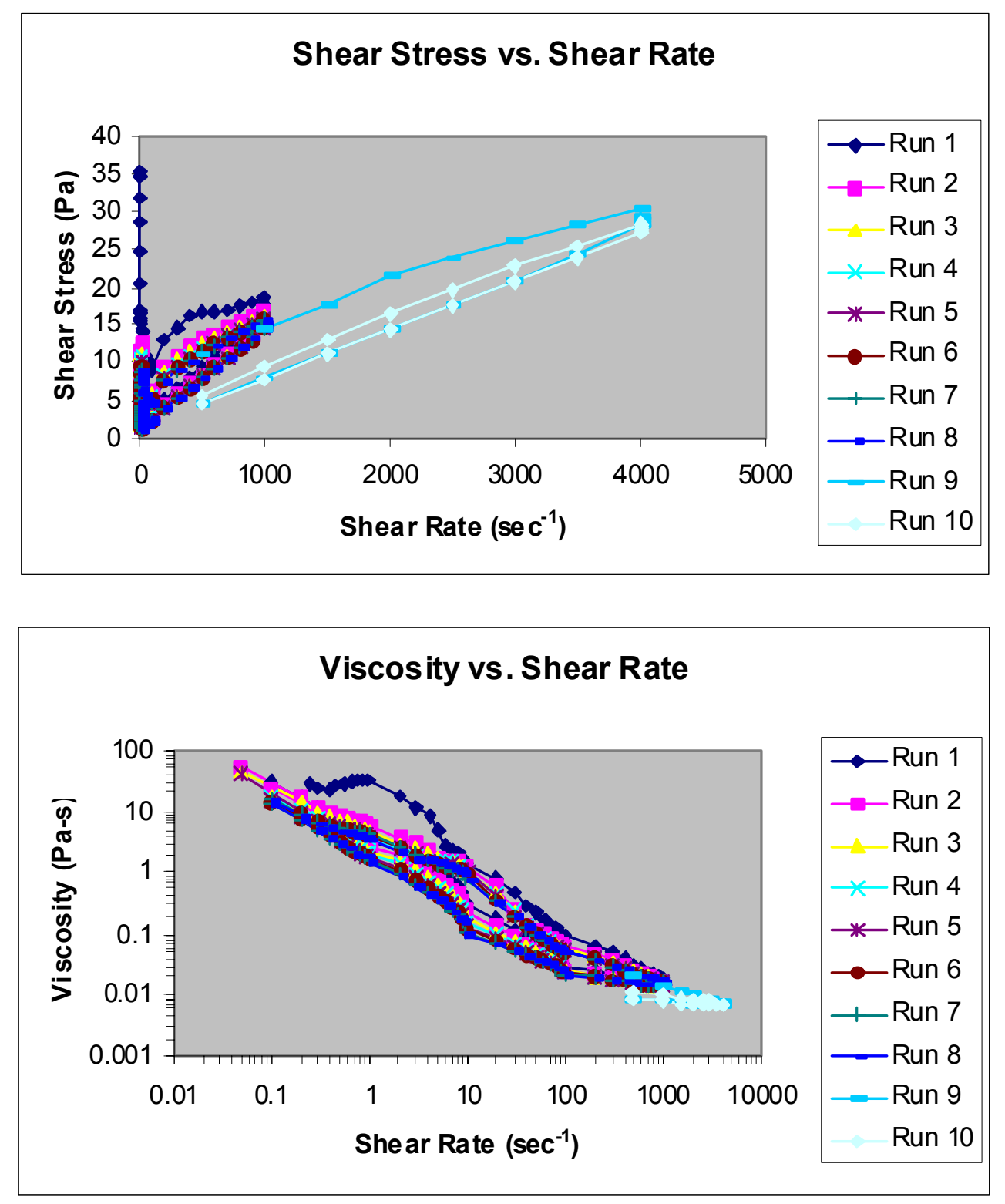

\section{E-2.1.19 187/20wt\%/D/N}

2004.01.21 187-20 wt\%-D [not sonicated] 0.1-1000 s-1 CC-45 run 1.txt Mode Step $\mathrm{t}[\mathrm{s}] \quad \mathrm{n}[\mathrm{rpm}] \quad \mathrm{M}[\%$.] $\quad$ Phi[rad]

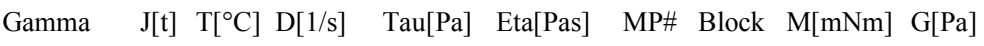




\begin{tabular}{|c|c|c|c|c|c|c|c|c|c|c|c|c|c|c|}
\hline 1 & 1 & 140 & 0.54 & 0 & 4.57416 & 56.391 & 0 & 28 & 0.7 & 0 & 0 & 7 & 1 & 0 \\
\hline 1 & 1 & 160 & 0.62 & 0 & 5.87635 & 72.444 & 0 & 28 & 0.8 & 0 & 0 & 8 & 1 & 0 \\
\hline 1 & 1 & 180 & 0.7 & 0 & 7.34033 & 90.493 & 0 & 28 & 0.9 & 0 & 0 & 9 & 1 & 0 \\
\hline 1 & 2 & 200 & 0.77 & 0 & 8.96453 & 0 & 0 & 28 & 0.99 & 0 & 0 & 10 & 1 & 0 \\
\hline 1 & 2 & 220 & 1.55 & 0 & 12.18938 & 0 & 0 & 28 & 2 & 0 & 0 & 11 & 1 & 0 \\
\hline 1 & 2 & 240 & 2.32 & 0 & 17.03921 & 0 & 0 & 28 & 3 & 0 & 0 & 12 & 1 & 0 \\
\hline 1 & 2 & 260 & 3.09 & 0 & 23.50697 & 0 & 0 & 28 & 3.99 & 0 & 0 & 13 & 1 & 0 \\
\hline 1 & 2 & 280 & 3.87 & 0 & 31.60128 & 0 & 0 & 28 & 5 & 0 & 0 & 14 & 1 & 0 \\
\hline 1 & 2 & 300 & 4.65 & 0 & 41.32294 & 0 & 0 & 28 & 6 & 0 & 0 & 15 & 1 & 0 \\
\hline 1 & 2 & 320 & 5.42 & 0 & 52.67273 & 0 & 0 & 28 & 7 & 0 & 0 & 16 & 1 & 0 \\
\hline 1 & 2 & 340 & 6.2 & 0 & 65.62709 & 0 & 0 & 28 & 8 & 0 & 0 & 17 & 1 & 0 \\
\hline 1 & 2 & 360 & 6.97 & 0 & 80.21036 & 0 & 0 & 28 & 9 & 0 & 0 & 18 & 1 & 0 \\
\hline 1 & 3 & 380 & 7.75 & 0 & 96.57727 & 0 & 0 & 28 & 10.01 & 0 & 0 & 19 & 1 & 0 \\
\hline 1 & 3 & 400 & 15.5 & 0 & 128.92468 & 0 & 0 & 28 & 20.01 & 0 & 0 & 20 & 1 & 0 \\
\hline 1 & 3 & 420 & 23.24 & 0 & 177.48899 & 0 & 0 & 28 & 30 & 0 & 0 & 21 & 1 & 0 \\
\hline 1 & 3 & 440 & 30.99 & 0 & 242.22622 & 0 & 0 & 28 & 40.01 & 0 & 0 & 22 & 1 & 0 \\
\hline 1 & 3 & 460 & 38.73 & 0 & 323.23297 & 0 & 0 & 28 & 50 & 0 & 0 & 23 & 1 & 0 \\
\hline 1 & 3 & 480 & 46.48 & 0 & 420.52574 & 0 & 0 & 28 & 60.01 & 0 & 0 & 24 & 1 & 0 \\
\hline 1 & 3 & 500 & 54.22 & 0 & 533.94273 & 0 & 0 & 28 & 70 & 0 & 0 & 25 & 1 & 0 \\
\hline 1 & 3 & 520 & 61.96 & 0 & 663.66852 & 0 & 0 & 28 & 79.99 & 0 & 0 & 26 & 1 & 0 \\
\hline 1 & 3 & 540 & 69.71 & 0 & 809.5201 & 0 & 0 & 28 & 90 & 0 & 0 & 27 & 1 & 0 \\
\hline 1 & 4 & 560 & 77.46 & 0 & 973.09419 & 0 & 0 & 28 & 100 & 0 & 0 & 28 & 1 & 0 \\
\hline 1 & 4 & 580 & 154.9 & 3.13 & 1296.423 & 0 & 0 & 28 & 200 & 0.613 & 0.003 & 29 & 1 & 0.1565 \\
\hline 1 & 4 & 600 & 232.4 & 6.92 & 1782.1446 & 0 & 0 & 28 & 300 & 1.355 & 0.005 & 30 & 1 & 0.3459 \\
\hline 1 & 4 & 620 & 309.8 & 12.5 & 2429.4855 & 0 & 0 & 28 & 400 & 2.451 & 0.006 & 31 & 1 & 0.6259 \\
\hline 1 & 4 & 640 & 387.3 & 19.4 & 3239.9889 & 0 & 0 & 28 & 500 & 3.788 & 0.008 & 32 & 1 & 0.9674 \\
\hline 1 & 4 & 660 & 464.8 & 27.3 & 4211.3427 & 0 & 0 & 28 & 600 & 5.347 & 0.009 & 33 & 1 & 1.3654 \\
\hline 1 & 4 & 680 & 542.2 & 35.6 & 5346.2249 & 0 & 0 & 28 & 700 & 6.977 & 0.01 & 34 & 1 & 1.7818 \\
\hline 1 & 4 & 700 & 619.7 & 43.3 & 6643.0516 & 0 & 0 & 28 & 800 & 8.48 & 0.011 & 35 & 1 & 2.1656 \\
\hline 1 & 4 & 720 & 697.1 & 50.3 & 8101.8242 & 0 & 0 & 28 & 900 & 9.847 & 0.011 & 36 & 1 & 2.5145 \\
\hline 1 & 4 & 740 & 774.6 & 57.4 & 9722.381 & 0 & 0 & 28 & 1000 & 11.24 & 0.011 & 37 & 1 & 2.8706 \\
\hline 1 & 5 & 750 & 774.6 & 56.6 & 10549.469 & 0 & 0 & 28 & 1000 & 11.08 & 0.011 & 38 & 1 & 2.8285 \\
\hline 1 & 5 & 760 & 774.6 & 56.2 & 11361.834 & 0 & 0 & 28 & 1000 & 10.99 & 0.011 & 39 & 1 & 2.8073 \\
\hline 1 & 5 & 770 & 774.6 & 55.1 & 12172.984 & 0 & 0 & 28 & 1000 & 10.78 & 0.011 & 40 & 1 & 2.7538 \\
\hline 1 & 5 & 780 & 774.6 & 54.2 & 12984.133 & 0 & 0 & 28 & 1000 & 10.61 & 0.011 & 41 & 1 & 2.7104 \\
\hline 1 & 5 & 790 & 774.6 & 54 & 13794.064 & 0 & 0 & 28 & 1000 & 10.57 & 0.011 & 42 & 1 & 2.6984 \\
\hline 1 & 5 & 800 & 774.6 & 54.9 & 14606.025 & 0 & 0 & 28 & 1000 & 10.75 & 0.011 & 43 & 1 & 2.7462 \\
\hline 1 & 6 & 820 & 774.6 & 54.6 & 16247.789 & 0 & 0 & 28 & 1000 & 10.69 & 0.011 & 44 & 1 & 2.7299 \\
\hline 1 & 6 & 840 & 697.1 & 46.7 & 17707.651 & 0 & 0 & 28 & 900 & 9.143 & 0.01 & 45 & 1 & 2.3347 \\
\hline 1 & 6 & 860 & 619.7 & 39.1 & 19006.501 & 0 & 0 & 28 & 800 & 7.656 & 0.01 & 46 & 1 & 1.955 \\
\hline 1 & 6 & 880 & 542.2 & 31.8 & 20143.933 & 0 & 0 & 28 & 700 & 6.223 & 0.009 & 47 & 1 & 1.589 \\
\hline 1 & 6 & 900 & 464.8 & 25.7 & 21118.406 & 0 & 0 & 28 & 600 & 5.034 & 0.008 & 48 & 1 & 1.2855 \\
\hline 1 & 6 & 920 & 387.3 & 19.6 & 21931.066 & 0 & 0 & 28 & 500 & 3.837 & 0.008 & 49 & 1 & 0.98 \\
\hline 1 & 6 & 940 & 309.8 & 13.7 & 22581.422 & 0 & 0 & 28 & 400 & 2.682 & 0.007 & 50 & 1 & 0.6849 \\
\hline 1 & 6 & 960 & 232.4 & 8.29 & 23069.022 & 0 & 0 & 28 & 300 & 1.622 & 0.005 & 51 & 1 & 0.4143 \\
\hline 1 & 6 & 980 & 154.9 & 3.57 & 23394.929 & 0 & 0 & 28 & 200 & 0.699 & 0.003 & 52 & 1 & 0.1785 \\
\hline 1 & 6 & 1000 & 77.46 & 0 & 23558.373 & 0 & 0 & 28 & 100 & 0 & 0 & 53 & 1 & 0 \\
\hline 1 & 7 & 1020 & 69.71 & 0 & 23705.801 & 0 & 0 & 28 & 90 & 0 & 0 & 54 & 1 & 0 \\
\hline 1 & 7 & 1040 & 61.97 & 0 & 23835.661 & 0 & 0 & 28 & 80 & 0 & 0 & 55 & 1 & 0 \\
\hline 1 & 7 & 1060 & 54.22 & 0 & 23949.418 & 0 & 0 & 28 & 70 & 0 & 0 & 56 & 1 & 0 \\
\hline
\end{tabular}




\begin{tabular}{|c|c|c|c|c|c|c|c|c|c|c|c|c|c|c|}
\hline 1 & 7 & 1080 & 46.48 & 0 & 24046.868 & 0 & 0 & 28 & 60.01 & 0 & 0 & 57 & 1 & 0 \\
\hline 1 & 7 & 1100 & 38.73 & 0 & 24128.097 & 0 & 0 & 28 & 50 & 0 & 0 & 58 & 1 & 0 \\
\hline 1 & 7 & 1120 & 30.99 & 0 & 24193.092 & 0 & 0 & 28 & 40.01 & 0 & 0 & 59 & 1 & 0 \\
\hline 1 & 7 & 1140 & 23.24 & 0 & 24241.903 & 0 & 0 & 28 & 30 & 0 & 0 & 60 & 1 & 0 \\
\hline 1 & 7 & 1160 & 15.5 & 0 & 24274.469 & 0 & 0 & 28 & 20.01 & 0 & 0 & 61 & 1 & 0 \\
\hline 1 & 7 & 1180 & 7.75 & 0 & 24290.802 & 0 & 0 & 28 & 10.01 & 0 & 0 & 62 & 1 & 0 \\
\hline 1 & 8 & 1200 & 6.97 & 0 & 24305.5 & 0 & 0 & 28 & 9 & 0 & 0 & 63 & 1 & 0 \\
\hline 1 & 8 & 1220 & 6.2 & 0 & 24318.499 & 0 & 0 & 28 & 8 & 0 & 0 & 64 & 1 & 0 \\
\hline 1 & 8 & 1240 & 5.42 & 0 & 24329.85 & 0 & 0 & 28 & 7 & 0 & 0 & 65 & 1 & 0 \\
\hline 1 & 8 & 1260 & 4.64 & 0 & 24339.593 & 0 & 0 & 28 & 5.99 & 0 & 0 & 66 & 1 & 0 \\
\hline 1 & 8 & 1280 & 3.87 & 0 & 24347.704 & 0 & 0 & 28 & 5 & 0 & 0 & 67 & 1 & 0 \\
\hline 1 & 8 & 1300 & 3.09 & 0 & 24354.202 & 0 & 0 & 28 & 3.99 & 0 & 0 & 68 & 1 & 0 \\
\hline 1 & 8 & 1320 & 2.32 & 0 & 24359.07 & 0 & 0 & 28 & 3 & 0 & 0 & 69 & 1 & 0 \\
\hline 1 & 8 & 1340 & 1.54 & 0 & 24362.318 & 0 & 0 & 28 & 1.99 & 0 & 0 & 70 & 1 & 0 \\
\hline 1 & 8 & 1360 & 0.77 & 0 & 24363.942 & 0 & 0 & 28 & 0.99 & 0 & 0 & 71 & 1 & 0 \\
\hline 1 & 9 & 1380 & 0.7 & 0 & 24365.416 & 18.174 & 0 & 28 & 0.9 & 0 & 0 & 72 & 1 & 0 \\
\hline 1 & 9 & 1400 & 0.62 & 0 & 24366.72 & 34.257 & 0 & 28 & 0.8 & 0 & 0 & 73 & 1 & 0 \\
\hline 1 & 9 & 1420 & 0.55 & 0 & 24367.863 & 48.345 & 0 & 28 & 0.71 & 0 & 0 & 74 & 1 & 0 \\
\hline 1 & 9 & 1440 & 0.47 & 0 & 24368.843 & 60.428 & 0 & 28 & 0.61 & 0 & 0 & 75 & 1 & 0 \\
\hline 1 & 9 & 1460 & 0.39 & 0 & 24369.66 & 70.498 & 0 & 28 & 0.5 & 0 & 0 & 76 & 1 & 0 \\
\hline 1 & 9 & 1480 & 0.31 & 0 & 24370.317 & 78.593 & 0 & 28 & 0.4 & 0 & 0 & 77 & 1 & 0 \\
\hline 1 & 9 & 1500 & 0.24 & 0 & 24370.81 & 84.673 & 0 & 28 & 0.31 & 0 & 0 & 78 & 1 & 0 \\
\hline 1 & 9 & 1520 & 0.16 & 0 & 24371.141 & 88.75 & 0 & 28 & 0.21 & 0 & 0 & 79 & 1 & 0 \\
\hline 1 & 9 & 1540 & 0.08 & 0 & 24371.309 & 90.832 & 0 & 28 & 0.1 & 0 & 0 & 80 & 1 & 0 \\
\hline
\end{tabular}

2004.01.21 187-20 wt\%-D [not sonicated] 0.1-1000 s-1 CC-45 run 2.txt

Mode Step t[s] n[rpm] M[\%.] Phi[rad]

\begin{tabular}{|c|c|c|c|c|c|c|c|c|c|c|c|c|c|c|c|}
\hline 1 & 1 & 20 & 0.08 & 0 & 0.16493 & 2.0333 & 0 & 28 & 0.1 & 0 & 0 & 1 & 1 & 0 & 0 \\
\hline 1 & 1 & 40 & 0.16 & 0 & 0.4948 & 6.1 & 0 & 28 & 0.21 & 0 & 0 & 2 & 1 & 0 & 0 \\
\hline 1 & 1 & 60 & 0.24 & 0 & 0.98489 & 12.142 & 0 & 28 & 0.31 & 0 & 0 & 3 & 1 & 0 & 0 \\
\hline 1 & 1 & 80 & 0.31 & 0 & 1.63834 & 20.198 & 0 & 28 & 0.4 & 0 & 0 & 4 & 1 & 0 & ( \\
\hline 1 & 1 & 100 & 0.39 & 0 & 2.45358 & 30.248 & 0 & 28 & 0.5 & 0 & 0 & 5 & 1 & 0 & \\
\hline 1 & 1 & 120 & 0.47 & 0 & 3.43298 & 42.322 & 0 & 28 & 0.61 & 0 & 0 & 6 & 1 & 0 & \\
\hline 1 & 1 & 140 & 0.54 & 0 & 4.57337 & 56.381 & 0 & 28 & 0.7 & 0 & 0 & 7 & 1 & 0 & \\
\hline 1 & 1 & 160 & 0.62 & 0 & 5.87478 & 72.425 & 0 & 28 & 0.8 & 0 & 0 & 8 & 1 & 0 & \\
\hline 1 & 1 & 180 & 0.7 & 0 & 7.34112 & 90.502 & 0 & 28 & 0.9 & 0 & 0 & 9 & 1 & 0 & \\
\hline 1 & 2 & 200 & 0.77 & 0 & 8.96453 & 0 & 0 & 28 & 0.99 & 0 & 0 & 10 & 1 & 0 & \\
\hline 1 & 2 & 220 & 1.55 & 0 & 12.18859 & 0 & 0 & 28 & 2 & 0 & 0 & 11 & 1 & 0 & 0 \\
\hline 1 & 2 & 240 & 2.32 & 0 & 17.03686 & 0 & 0 & 28 & 3 & 0 & 0 & 12 & 1 & 0 & 0 \\
\hline 1 & 2 & 260 & 3.09 & 0 & 23.51246 & 0 & 0 & 28 & 3.99 & 0 & 0 & 13 & 1 & 0 & 0 \\
\hline 1 & 2 & 280 & 3.87 & 0 & 31.60285 & 0 & 0 & 28 & 5 & 0 & 0 & 14 & 1 & 0 & 0 \\
\hline 1 & 2 & 300 & 4.65 & 0 & 41.3198 & 0 & 0 & 28 & 6 & 0 & 0 & 15 & 1 & 0 & 0 \\
\hline 1 & 2 & 320 & 5.42 & 0 & 52.66959 & 0 & 0 & 28 & 7 & 0 & 0 & 16 & 1 & 0 & 0 \\
\hline 1 & 2 & 340 & 6.2 & 0 & 65.63965 & 0 & 0 & 28 & 8 & 0 & 0 & 17 & 1 & 0 & 0 \\
\hline 1 & 2 & 360 & 6.97 & 0 & 80.22371 & 0 & 0 & 28 & 9 & 0 & 0 & 18 & 1 & 0 & 0 \\
\hline 1 & 3 & 380 & 7.75 & 0 & 96.59376 & 0 & 0 & 28 & 10.01 & 0 & 0 & 19 & 1 & 0 & 0 \\
\hline 1 & 3 & 400 & 15.5 & 0 & 128.9341 & 0 & 0 & 28 & 20.01 & 0 & 0 & 20 & 1 & 0 & ( \\
\hline 1 & 3 & 420 & 23.24 & 0 & 177.49449 & 0 & 0 & 28 & 30 & 0 & 0 & 21 & 1 & 0 & ( \\
\hline 1 & 3 & 440 & 30.99 & 0 & 242.22779 & 0 & 0 & 28 & 40.01 & 0 & 0 & 22 & 1 & 0 & ( \\
\hline 1 & 3 & 460 & 38.73 & 0 & 323.2746 & 0 & 0 & 28 & 50 & 0 & 0 & 23 & 1 & 0 & ( \\
\hline
\end{tabular}




\begin{tabular}{|c|c|c|c|c|c|c|c|c|c|c|c|c|c|c|}
\hline 1 & 3 & 480 & 46.48 & 0 & 420.4739 & 0 & 0 & 28 & 60.01 & 0 & 0 & 24 & 1 & 0 \\
\hline 1 & 3 & 500 & 54.22 & 1.02 & 533.90739 & 0 & 0 & 28 & 70 & 0.2 & 0.003 & 25 & 1 & 0.0511 \\
\hline 1 & 3 & 520 & 61.96 & 1.16 & 663.62925 & 0 & 0 & 28 & 79.99 & 0.227 & 0.003 & 26 & 1 & 0.0579 \\
\hline 1 & 3 & 540 & 69.71 & 1.4 & 809.407 & 0 & 0 & 28 & 90 & 0.274 & 0.003 & 27 & 1 & 0.0699 \\
\hline 1 & 4 & 560 & 77.46 & 1.49 & 972.94103 & 0 & 0 & 28 & 100 & 0.293 & 0.003 & 28 & 1 & 0.0747 \\
\hline 1 & 4 & 580 & 154.9 & 3.34 & 1296.4732 & 0 & 0 & 28 & 200 & 0.654 & 0.003 & 29 & 1 & 0.167 \\
\hline 1 & 4 & 600 & 232.4 & 6.78 & 1782.2349 & 0 & 0 & 28 & 300 & 1.328 & 0.004 & 30 & 1 & 0.3391 \\
\hline 1 & 4 & 620 & 309.8 & 13.9 & 2429.6167 & 0 & 0 & 28 & 400 & 2.715 & 0.007 & 31 & 1 & 0.6933 \\
\hline 1 & 4 & 640 & 387.3 & 20 & 3239.5122 & 0 & 0 & 28 & 500 & 3.916 & 0.008 & 32 & 1 & 1 \\
\hline 1 & 4 & 660 & 464.8 & 27.5 & 4211.0678 & 0 & 0 & 28 & 600 & 5.39 & 0.009 & 33 & 1 & 1.3764 \\
\hline 1 & 4 & 680 & 542.2 & 34.9 & 5346.1935 & 0 & 0 & 28 & 700 & 6.827 & 0.01 & 34 & 1 & 1.7433 \\
\hline 1 & 4 & 700 & 619.7 & 42.2 & 6642.3306 & 0 & 0 & 28 & 800 & 8.254 & 0.01 & 35 & 1 & 2.1077 \\
\hline 1 & 4 & 720 & 697.1 & 49.3 & 8099.9676 & 0 & 0 & 28 & 900 & 9.647 & 0.011 & 36 & 1 & 2.4636 \\
\hline 1 & 4 & 740 & 774.6 & 57.1 & 9721.3349 & 0 & 0 & 28 & 1000 & 11.18 & 0.011 & 37 & 1 & 2.8549 \\
\hline 1 & 5 & 750 & 774.6 & 56.4 & 10548.384 & 0 & 0 & 28 & 1000 & 11.05 & 0.011 & 38 & 1 & 2.8221 \\
\hline 1 & 5 & 760 & 774.6 & 55.5 & 11360.343 & 0 & 0 & 28 & 1000 & 10.87 & 0.011 & 39 & 1 & 2.7749 \\
\hline 1 & 5 & 770 & 774.6 & 54.6 & 12172.303 & 0 & 0 & 28 & 1000 & 10.7 & 0.011 & 40 & 1 & 2.732 \\
\hline 1 & 5 & 780 & 774.6 & 54.5 & 12983.046 & 0 & 0 & 28 & 1000 & 10.68 & 0.011 & 41 & 1 & 2.727 \\
\hline 1 & 5 & 790 & 774.6 & 55.5 & 13795.817 & 0 & 0 & 28 & 1000 & 10.86 & 0.011 & 42 & 1 & 2.7737 \\
\hline 1 & 5 & 800 & 774.6 & 54.9 & 14606.156 & 0 & 0 & 28 & 1000 & 10.75 & 0.011 & 43 & 1 & 2.744 \\
\hline 1 & 6 & 820 & 774.6 & 54.3 & 16245.892 & 0 & 0 & 28 & 1000 & 10.64 & 0.011 & 44 & 1 & 2.7168 \\
\hline 1 & 6 & 840 & 697.1 & 46.2 & 17708.35 & 0 & 0 & 28 & 900 & 9.043 & 0.01 & 45 & 1 & 2.3094 \\
\hline 1 & 6 & 860 & 619.7 & 38.8 & 19006.835 & 0 & 0 & 28 & 800 & 7.595 & 0.009 & 46 & 1 & 1.9395 \\
\hline 1 & 6 & 880 & 542.2 & 33.1 & 20143.456 & 0 & 0 & 28 & 700 & 6.479 & 0.009 & 47 & 1 & 1.6546 \\
\hline 1 & 6 & 900 & 464.8 & 25.4 & 21118.13 & 0 & 0 & 28 & 600 & 4.972 & 0.008 & 48 & 1 & 1.2697 \\
\hline 1 & 6 & 920 & 387.3 & 19 & 21931.188 & 0 & 0 & 28 & 500 & 3.722 & 0.007 & 49 & 1 & 0.9506 \\
\hline 1 & 6 & 940 & 309.8 & 13.4 & 22581.741 & 0 & 0 & 28 & 400 & 2.63 & 0.007 & 50 & 1 & 0.6717 \\
\hline 1 & 6 & 960 & 232.4 & 8.27 & 23069.344 & 0 & 0 & 28 & 300 & 1.618 & 0.005 & 51 & 1 & 0.4133 \\
\hline 1 & 6 & 980 & 154.9 & 3.49 & 23395.129 & 0 & 0 & 28 & 200 & 0.683 & 0.003 & 52 & 1 & 0.1744 \\
\hline 1 & 6 & 1000 & 77.46 & 1.1 & 23558.613 & 0 & 0 & 28 & 100 & 0.214 & 0.002 & 53 & 1 & 0.0548 \\
\hline 1 & 7 & 1020 & 69.71 & 1.03 & 23705.71 & 0 & 0 & 28 & 90 & 0.202 & 0.002 & 54 & 1 & 0.0515 \\
\hline 1 & 7 & 1040 & 61.97 & 0 & 23835.574 & 0 & 0 & 28 & 80 & 0 & 0 & 55 & 1 & 0 \\
\hline 1 & 7 & 1060 & 54.22 & 0 & 23949.245 & 0 & 0 & 28 & 70 & 0 & 0 & 56 & 1 & 0 \\
\hline 1 & 7 & 1080 & 46.48 & 0 & 24046.797 & 0 & 0 & 28 & 60.01 & 0 & 0 & 57 & 1 & 0 \\
\hline 1 & 7 & 1100 & 38.73 & 0 & 24128.01 & 0 & 0 & 28 & 50 & 0 & 0 & 58 & 1 & 0 \\
\hline 1 & 7 & 1120 & 30.99 & 0 & 24193.034 & 0 & 0 & 28 & 40.01 & 0 & 0 & 59 & 1 & 0 \\
\hline 1 & 7 & 1140 & 23.24 & 0 & 24241.833 & 0 & 0 & 28 & 30 & 0 & 0 & 60 & 1 & 0 \\
\hline 1 & 7 & 1160 & 15.5 & 0 & 24274.391 & 0 & 0 & 28 & 20.01 & 0 & 0 & 61 & 1 & 0 \\
\hline 1 & 7 & 1180 & 7.75 & 0 & 24290.719 & 0 & 0 & 28 & 10.01 & 0 & 0 & 62 & 1 & 0 \\
\hline 1 & 8 & 1200 & 6.97 & 0 & 24305.43 & 0 & 0 & 28 & 9 & 0 & 0 & 63 & 1 & 0 \\
\hline 1 & 8 & 1220 & 6.2 & 0 & 24318.418 & 0 & 0 & 28 & 8 & 0 & 0 & 64 & 1 & 0 \\
\hline 1 & 8 & 1240 & 5.42 & 0 & 24329.772 & 0 & 0 & 28 & 7 & 0 & 0 & 65 & 1 & 0 \\
\hline 1 & 8 & 1260 & 4.64 & 0 & 24339.517 & 0 & 0 & 28 & 5.99 & 0 & 0 & 66 & 1 & 0 \\
\hline 1 & 8 & 1280 & 3.87 & 0 & 24347.629 & 0 & 0 & 28 & 5 & 0 & 0 & 67 & 1 & 0 \\
\hline 1 & 8 & 1300 & 3.09 & 0 & 24354.123 & 0 & 0 & 28 & 3.99 & 0 & 0 & 68 & 1 & 0 \\
\hline 1 & 8 & 1320 & 2.32 & 0 & 24358.993 & 0 & 0 & 28 & 3 & 0 & 0 & 69 & 1 & 0 \\
\hline 1 & 8 & 1340 & 1.54 & 0 & 24362.239 & 0 & 0 & 28 & 1.99 & 0 & 0 & 70 & 1 & 0 \\
\hline 1 & 8 & 1360 & 0.77 & 0 & 24363.864 & 0 & 0 & 28 & 0.99 & 0 & 0 & 71 & 1 & 0 \\
\hline 1 & 9 & 1380 & 0.7 & 0 & 24365.339 & 18.184 & 0 & 28 & 0.9 & 0 & 0 & 72 & 1 & 0 \\
\hline 1 & 9 & 1400 & 0.62 & 0 & 24366.643 & 34.266 & 0 & 28 & 0.8 & 0 & 0 & 73 & 1 & 0 \\
\hline
\end{tabular}




$\begin{array}{rrrrrrrrrrrrrrrrr}1 & 9 & 1420 & 0.55 & 0 & 24367.786 & 48.354 & 0 & 28 & 0.71 & 0 & 0 & 74 & 1 & 0 & 0 \\ 1 & 9 & 1440 & 0.47 & 0 & 24368.766 & 60.438 & 0 & 28 & 0.61 & 0 & 0 & 75 & 1 & 0 & 0 \\ 1 & 9 & 1460 & 0.39 & 0 & 24369.584 & 70.518 & 0 & 28 & 0.5 & 0 & 0 & 76 & 1 & 0 & 0 \\ 1 & 9 & 1480 & 0.31 & 0 & 24370.24 & 78.603 & 0 & 28 & 0.4 & 0 & 0 & 77 & 1 & 0 & 0 \\ 1 & 9 & 1500 & 0.24 & 0 & 24370.733 & 84.683 & 0 & 28 & 0.31 & 0 & 0 & 78 & 1 & 0 & 0 \\ 1 & 9 & 1520 & 0.16 & 0 & 24371.064 & 88.759 & 0 & 28 & 0.21 & 0 & 0 & 79 & 1 & 0 & 0 \\ 1 & 9 & 1540 & 0.08 & 0 & 24371.233 & 90.841 & 0 & 28 & 0.1 & 0 & 0 & 80 & 1 & 0 & 0\end{array}$

2004.01.21 187-20 wt\%-D [not sonicated] 0.1-1000 s-1 CC-45 run 3.txt

Mode Step t[s] n[rpm] M[\%.] Phi[rad]

Gamma J[t] $\left.\quad \mathrm{T}^{\circ} \mathrm{C}\right] \quad \mathrm{D}[1 / \mathrm{s}] \quad \mathrm{Tau}[\mathrm{Pa}] \quad \mathrm{Eta}[\mathrm{Pas}] \quad \mathrm{MP} \#$ Block $\mathrm{M}[\mathrm{mNm}] \mathrm{G}[\mathrm{Pa}]$

\begin{tabular}{|c|c|c|c|c|c|c|c|c|c|c|c|c|c|c|}
\hline 1 & 1 & 20 & 0.08 & 0 & 0.16572 & 2.043 & 0 & 28 & 0.1 & 0 & 0 & 1 & 1 & 0 \\
\hline 1 & 1 & 40 & 0.16 & 0 & 0.49402 & 6.0903 & 0 & 28 & 0.21 & 0 & 0 & 2 & 1 & 0 \\
\hline 1 & 1 & 60 & 0.24 & 0 & 0.98567 & 12.152 & 0 & 28 & 0.31 & 0 & 0 & 3 & 1 & 0 \\
\hline 1 & 1 & 80 & 0.31 & 0 & 1.63991 & 20.217 & 0 & 28 & 0.4 & 0 & 0 & 4 & 1 & 0 \\
\hline 1 & 1 & 100 & 0.39 & 0 & 2.45437 & 30.258 & 0 & 28 & 0.5 & 0 & 0 & 5 & 1 & 0 \\
\hline 1 & 1 & 120 & 0.47 & 0 & 3.43298 & 42.322 & 0 & 28 & 0.61 & 0 & 0 & 6 & 1 & 0 \\
\hline 1 & 1 & 140 & 0.54 & 0 & 4.57416 & 56.391 & 0 & 28 & 0.7 & 0 & 0 & 7 & 1 & 0 \\
\hline 1 & 1 & 160 & 0.62 & 0 & 5.87556 & 72.435 & 0 & 28 & 0.8 & 0 & 0 & 8 & 1 & 0 \\
\hline 1 & 1 & 180 & 0.7 & 0 & 7.3419 & 90.512 & 0 & 28 & 0.9 & 0 & 0 & 9 & 1 & 0 \\
\hline 1 & 2 & 200 & 0.77 & 0 & 8.96532 & 0 & 0 & 28 & 0.99 & 0 & 0 & 10 & 1 & 0 \\
\hline 1 & 2 & 220 & 1.55 & 0 & 12.19016 & 0 & 0 & 28 & 2 & 0 & 0 & 11 & 1 & 0 \\
\hline 1 & 2 & 240 & 2.32 & 0 & 17.03686 & 0 & 0 & 28 & 3 & 0 & 0 & 12 & 1 & 0 \\
\hline 1 & 2 & 260 & 3.09 & 0 & 23.50854 & 0 & 0 & 28 & 3.99 & 0 & 0 & 13 & 1 & 0 \\
\hline 1 & 2 & 280 & 3.87 & 0 & 31.60442 & 0 & 0 & 28 & 5 & 0 & 0 & 14 & 1 & 0 \\
\hline 1 & 2 & 300 & 4.65 & 0 & 41.3253 & 0 & 0 & 28 & 6 & 0 & 0 & 15 & 1 & 0 \\
\hline 1 & 2 & 320 & 5.42 & 0 & 52.67351 & 0 & 0 & 28 & 7 & 0 & 0 & 16 & 1 & 0 \\
\hline 1 & 2 & 340 & 6.2 & 0 & 65.63023 & 0 & 0 & 28 & 8 & 0 & 0 & 17 & 1 & 0 \\
\hline 1 & 2 & 360 & 6.97 & 0 & 80.22135 & 0 & 0 & 28 & 9 & 0 & 0 & 18 & 1 & 0 \\
\hline 1 & 3 & 380 & 7.75 & 0 & 96.58748 & 0 & 0 & 28 & 10.01 & 0 & 0 & 19 & 1 & 0 \\
\hline 1 & 3 & 400 & 15.5 & 0 & 128.93175 & 0 & 0 & 28 & 20.01 & 0 & 0 & 20 & 1 & 0 \\
\hline 1 & 3 & 420 & 23.24 & 0 & 177.47956 & 0 & 0 & 28 & 30 & 0 & 0 & 21 & 1 & 0 \\
\hline 1 & 3 & 440 & 30.99 & 0 & 242.22936 & 0 & 0 & 28 & 40.01 & 0 & 0 & 22 & 1 & 0 \\
\hline 1 & 3 & 460 & 38.73 & 0 & 323.33743 & 0 & 0 & 28 & 50 & 0 & 0 & 23 & 1 & 0 \\
\hline 1 & 3 & 480 & 46.48 & 0 & 420.48333 & 0 & 0 & 28 & 60.01 & 0 & 0 & 24 & 1 & 0 \\
\hline 1 & 3 & 500 & 54.22 & 0 & 534.00949 & 0 & 0 & 28 & 70 & 0 & 0 & 25 & 1 & 0 \\
\hline 1 & 3 & 520 & 61.96 & 1.08 & 663.54207 & 0 & 0 & 28 & 79.99 & 0.212 & 0.003 & 26 & 1 & 0.0541 \\
\hline 1 & 3 & 540 & 69.71 & 1.32 & 809.53502 & 0 & 0 & 28 & 90 & 0.258 & 0.003 & 27 & 1 & 0.066 \\
\hline 1 & 4 & 560 & 77.46 & 1.4 & 973.23556 & 0 & 0 & 28 & 100 & 0.273 & 0.003 & 28 & 1 & 0.0698 \\
\hline 1 & 4 & 580 & 154.9 & 3.27 & 1296.3617 & 0 & 0 & 28 & 200 & 0.639 & 0.003 & 29 & 1 & 0.1633 \\
\hline 1 & 4 & 600 & 232.4 & 6.77 & 1782.1226 & 0 & 0 & 28 & 300 & 1.325 & 0.004 & 30 & 1 & 0.3383 \\
\hline 1 & 4 & 620 & 309.8 & 14.4 & 2429.6669 & 0 & 0 & 28 & 400 & 2.82 & 0.007 & 31 & 1 & 0.7201 \\
\hline 1 & 4 & 640 & 387.3 & 19.9 & 3239.1564 & 0 & 0 & 28 & 500 & 3.898 & 0.008 & 32 & 1 & 0.9953 \\
\hline 1 & 4 & 660 & 464.8 & 27.5 & 4211.239 & 0 & 0 & 28 & 600 & 5.381 & 0.009 & 33 & 1 & 1.3741 \\
\hline 1 & 4 & 680 & 542.2 & 34.8 & 5345.2306 & 0 & 0 & 28 & 700 & 6.808 & 0.01 & 34 & 1 & 1.7386 \\
\hline 1 & 4 & 700 & 619.7 & 41.8 & 6641.4093 & 0 & 0 & 28 & 800 & 8.185 & 0.01 & 35 & 1 & 2.0902 \\
\hline 1 & 4 & 720 & 697.1 & 49 & 8100.9108 & 0 & 0 & 28 & 900 & 9.593 & 0.011 & 36 & 1 & 2.4497 \\
\hline 1 & 4 & 740 & 774.6 & 56.9 & 9723.0887 & 0 & 0 & 28 & 1000 & 11.15 & 0.011 & 37 & 1 & 2.8464 \\
\hline 1 & 5 & 750 & 774.6 & 56.3 & 10551.353 & 0 & 0 & 28 & 1000 & 11.02 & 0.011 & 38 & 1 & 2.8131 \\
\hline 1 & 5 & 760 & 774.6 & 55.2 & 11363.313 & 0 & 0 & 28 & 1000 & 10.81 & 0.011 & 39 & 1 & 2.7605 \\
\hline 1 & 5 & 770 & 774.6 & 54.5 & 12174.868 & 0 & 0 & 28 & 1000 & 10.67 & 0.011 & 40 & 1 & 2.7245 \\
\hline
\end{tabular}




\begin{tabular}{|c|c|c|c|c|c|c|c|c|c|c|c|c|c|c|}
\hline 1 & 5 & 780 & 774.6 & 54.1 & 12983.583 & 0 & 0 & 28 & 1000 & 10.58 & 0.011 & 41 & 1 & 2.7024 \\
\hline & 5 & 790 & 774.6 & 54.2 & 13796.354 & 0 & 0 & 28 & 1000 & 10.61 & 0.011 & 42 & 1 & 2.7104 \\
\hline & 5 & 800 & 774.6 & 55.3 & 14606.286 & 0 & 0 & 28 & 1000 & 10.82 & 0.011 & 43 & 1 & 2.7628 \\
\hline & 6 & 820 & 774.6 & 54.2 & 16246.43 & 0 & 0 & 28 & 1000 & 10.62 & 0.011 & 44 & 1 & 2.7107 \\
\hline & 6 & 840 & 697.1 & 46.4 & 17709.252 & 0 & 0 & 28 & 900 & 9.075 & 0.01 & 45 & 1 & 2.3175 \\
\hline & 6 & 860 & 619.7 & 39.2 & 19008.062 & 0 & 0 & 28 & 800 & 7.679 & 0.01 & 46 & 1 & 1.9611 \\
\hline & 6 & 880 & 542.2 & 32.3 & 20144.642 & 0 & 0 & 28 & 700 & 6.326 & 0.009 & 47 & 1 & 1.6155 \\
\hline & 6 & 900 & 464.8 & 25.5 & 21119.6 & 0 & 0 & 28 & 600 & 4.991 & 0.008 & 48 & 1 & 1.2745 \\
\hline & 6 & 920 & 387.3 & 19.5 & 21931.447 & 0 & 0 & 28 & 500 & 3.821 & 0.008 & 49 & 1 & 0.9758 \\
\hline & 6 & 940 & 309.8 & 13.5 & 22582.006 & 0 & 0 & 28 & 400 & 2.634 & 0.007 & 50 & 1 & 0.6726 \\
\hline & 6 & 960 & 232.4 & 8.09 & 23069.771 & 0 & 0 & 28 & 300 & 1.583 & 0.005 & 51 & 1 & 0.4043 \\
\hline & 6 & 980 & 154.9 & 3.44 & 23395.804 & 0 & 0 & 28 & 200 & 0.674 & 0.003 & 52 & 1 & 0.1721 \\
\hline & 6 & 1000 & 77.46 & 0 & 23559.168 & 0 & 0 & 28 & 100 & 0 & 0 & 53 & 1 & 0 \\
\hline & 7 & 1020 & 69.71 & 0 & 23706.6 & 0 & 0 & 28 & 90 & 0 & 0 & 54 & 1 & 0 \\
\hline & 7 & 1040 & 61.97 & 0 & 23836.46 & 0 & 0 & 28 & 80 & 0 & 0 & 55 & 1 & 0 \\
\hline & 7 & 1060 & 54.22 & 0 & 23950.277 & 0 & 0 & 28 & 70 & 0 & 0 & 56 & 1 & 0 \\
\hline & 7 & 1080 & 46.48 & 0 & 24047.63 & 0 & 0 & 28 & 60.01 & 0 & 0 & 57 & 1 & 0 \\
\hline & 7 & 1100 & 38.73 & 0 & 24128.944 & 0 & 0 & 28 & 50 & 0 & 0 & 58 & 1 & 0 \\
\hline & 7 & 1120 & 30.99 & 0 & 24193.887 & 0 & 0 & 28 & 40.01 & 0 & 0 & 59 & 1 & 0 \\
\hline & 7 & 1140 & 23.24 & 0 & 24242.67 & 0 & 0 & 28 & 30 & 0 & 0 & 60 & 1 & 0 \\
\hline & 7 & 1160 & 15.5 & 0 & 24275.236 & 0 & 0 & 28 & 20.01 & 0 & 0 & 61 & 1 & 0 \\
\hline & 7 & 1180 & 7.75 & 0 & 24291.593 & 0 & 0 & 28 & 10.01 & 0 & 0 & 62 & 1 & 0 \\
\hline & 8 & 1200 & 6.97 & 0 & 24306.29 & 0 & 0 & 28 & 9 & 0 & 0 & 63 & 1 & 0 \\
\hline & 8 & 1220 & 6.2 & 0 & 24319.268 & 0 & 0 & 28 & 8 & 0 & 0 & 64 & 1 & 0 \\
\hline & 8 & 1240 & 5.42 & 0 & 24330.634 & 0 & 0 & 28 & 7 & 0 & 0 & 65 & 1 & 0 \\
\hline & 8 & 1260 & 4.64 & 0 & 24340.375 & 0 & 0 & 28 & 5.99 & 0 & 0 & 66 & 1 & 0 \\
\hline & 8 & 1280 & 3.87 & 0 & 24348.495 & 0 & 0 & 28 & 5 & 0 & 0 & 67 & 1 & 0 \\
\hline & 8 & 1300 & 3.09 & 0 & 24354.986 & 0 & 0 & 28 & 3.99 & 0 & 0 & 68 & 1 & 0 \\
\hline & 8 & 1320 & 2.32 & 0 & 24359.858 & 0 & 0 & 28 & 3 & 0 & 0 & 69 & 1 & 0 \\
\hline & 8 & 1340 & 1.54 & 0 & 24363.103 & 0 & 0 & 28 & 1.99 & 0 & 0 & 70 & 1 & 0 \\
\hline & 8 & 1360 & 0.77 & 0 & 24364.727 & 0 & 0 & 28 & 0.99 & 0 & 0 & 71 & 1 & 0 \\
\hline & 9 & 1380 & 0.7 & 0 & 24366.2 & 18.164 & 0 & 28 & 0.9 & 0 & 0 & 72 & 1 & 0 \\
\hline & 9 & 1400 & 0.62 & 0 & 24367.505 & 34.247 & 0 & 28 & 0.8 & 0 & 0 & 73 & 1 & 0 \\
\hline & 9 & 1420 & 0.55 & 0 & 24368.648 & 48.335 & 0 & 28 & 0.71 & 0 & 0 & 74 & 1 & 0 \\
\hline & 9 & 1440 & 0.47 & 0 & 24369.628 & 60.419 & 0 & 28 & 0.61 & 0 & 0 & 75 & 1 & 0 \\
\hline & 9 & 1460 & 0.39 & 0 & 24370.446 & 70.498 & 0 & 28 & 0.5 & 0 & 0 & 76 & 1 & 0 \\
\hline & 9 & 1480 & 0.31 & 0 & 24371.101 & 78.583 & 0 & 28 & 0.4 & 0 & 0 & 77 & 1 & 0 \\
\hline & 9 & 1500 & 0.24 & 0 & 24371.595 & 84.664 & 0 & 28 & 0.31 & 0 & 0 & 78 & 1 & 0 \\
\hline & 9 & 1520 & 0.16 & 0 & 24371.925 & 88.74 & 0 & 28 & 0.21 & 0 & 0 & 79 & 1 & 0 \\
\hline & 9 & 1540 & 0.08 & 0 & 24372.094 & 90.822 & 0 & 28 & 0.1 & 0 & 0 & 80 & 1 & 0 \\
\hline
\end{tabular}



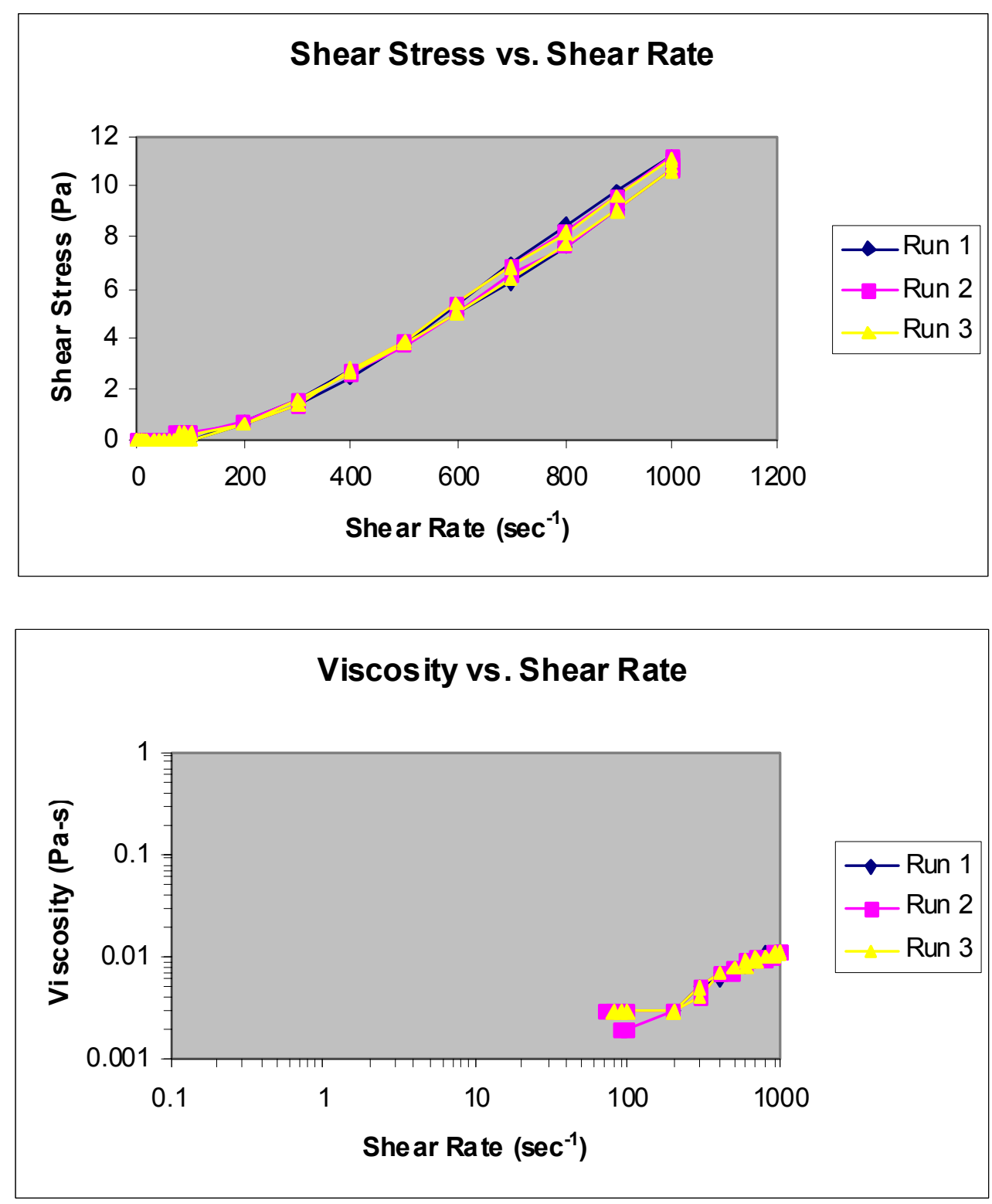

\section{E-2.1.20 $187 / 20 w t \% / D / S$}

2004.02.02 187-20 wt\%-D [sonicated] CC-45 0.1-1000 s-1 run 1.txt

Mode Step t[s] n[rpm] M[\%.] Phi[rad] Gamma J[t]

$\mathrm{T}\left[{ }^{\circ} \mathrm{C}\right] \mathrm{D}[1 / \mathrm{s}] \quad \mathrm{Tau}[\mathrm{Pa}]$ Eta[Pas] MP\# Block M[mNm] G[Pa

\begin{tabular}{|c|c|c|c|c|c|c|c|c|c|c|c|c|c|}
\hline & 20 & 0.08 & 59.57 & 0.16179 & 1.99459 & 0.171 & 28 & 0.1 & 11.664 & 116.64 & 1 & 12.97865 & 5.847818349 \\
\hline & 40 & 0.16 & 10.48 & 0.49009 & 6.04188 & 2.94438 & 28 & 0.21 & 2.052 & 9.771 & 2 & 0.5239 & 0.339629387 \\
\hline & 60 & 0.24 & 12.66 & 0.98018 & 12.08376 & 4.87641 & 28 & 0.31 & 2.478 & 7.994 & 3 & 10.63285 & 0.205068621 \\
\hline & 80 & 0.31 & 15.84 & 1.63284 & 20.12992 & 6.48933 & 28 & 0.4 & 3.102 & 7.755 & 4 & 0.79205 & 0.154098973 \\
\hline & 100 & 0.39 & 18.17 & 2.44887 & 30.19003 & 8.4875 & 28 & 0.5 & 3.557 & 7.114 & 5 & 10.90825 & 0.117820353 \\
\hline & 120 & 0.47 & 19.12 & 3.42669 & 42.24475 & 11.28332 & 28 & 0.61 & 3.744 & 6.138 & 6 & 0.9561 & 0.088626397 \\
\hline & 140 & 0.54 & 18.81 & 4.56709 & 56.30374 & 15.29161 & 28 & 0.7 & 3.682 & 5.26 & 7 & 0.94025 & 0.065395301 \\
\hline & 160 & 0.62 & 16.02 & 5.87085 & 72.37669 & 23.07194 & 28 & 0.8 & 3.137 & 3.921 & 8 & 10.80115 & 0.043342684 \\
\hline & $1 \quad 180$ & 0.7 & 12.43 & 7.33719 & 90.45392 & 37.16265 & 28 & 0.9 & 2.434 & 2.704 & 9 & 10.62165 & 0.026908729 \\
\hline
\end{tabular}




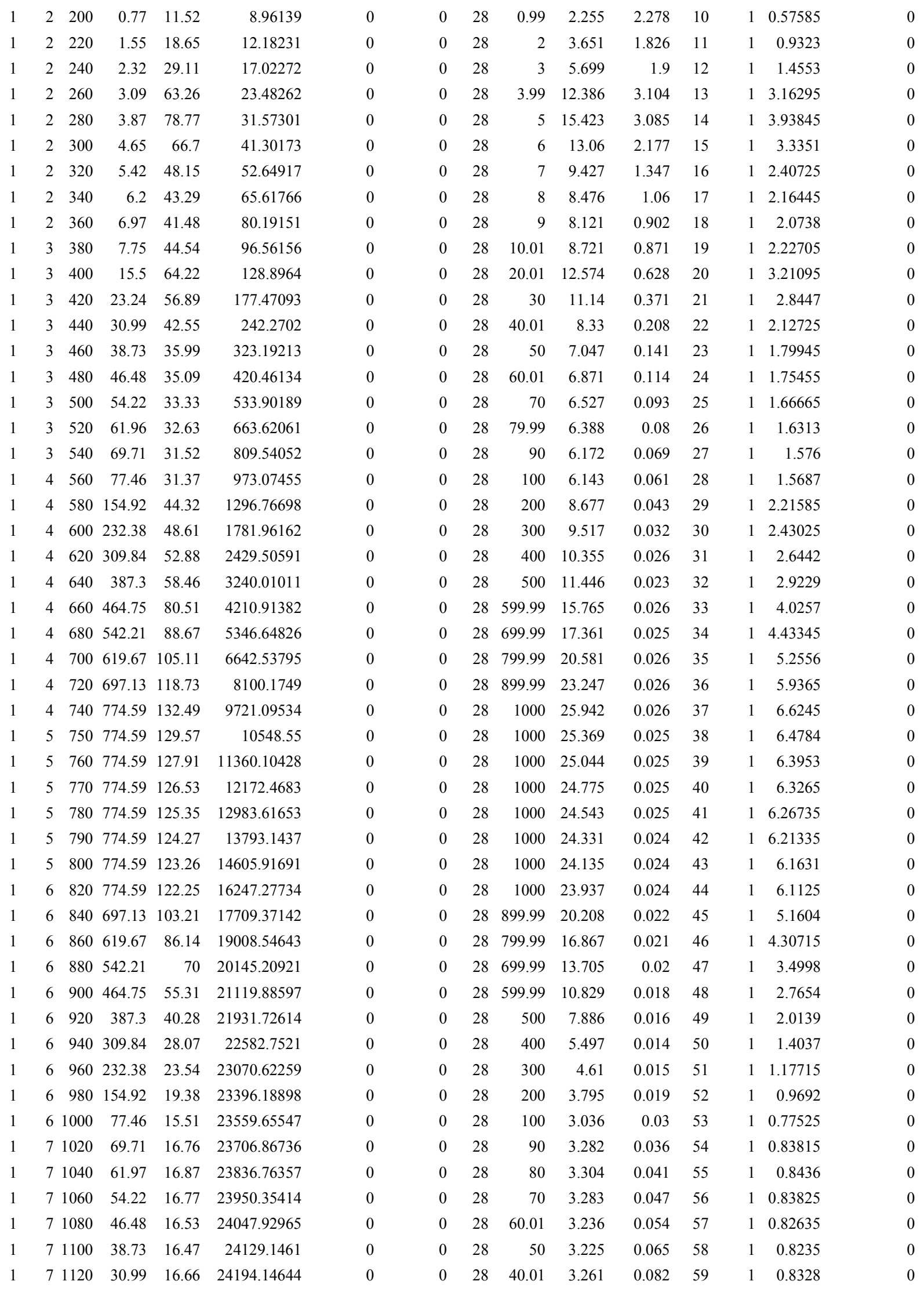




\begin{tabular}{|c|c|c|c|c|c|c|c|c|c|c|c|c|c|}
\hline 71140 & 23.24 & 16.65 & 24242.96208 & 0 & 0 & 28 & 30 & 3.26 & 0.109 & 60 & 1 & 0.8326 & 0 \\
\hline 71160 & 15.5 & 16.51 & 24275.4917 & 0 & 0 & 28 & 20.01 & 3.232 & 0.162 & 61 & 1 & 0.82525 & 0 \\
\hline 71180 & 7.75 & 16.25 & 24291.85626 & 0 & 0 & 28 & 10.01 & 3.182 & 0.318 & 62 & 1 & 0.81265 & \\
\hline 81200 & 6.97 & 17.75 & 24306.56676 & 0 & 0 & 28 & 9 & 3.475 & 0.386 & 63 & 1 & 0.88735 & \\
\hline 81220 & 6.2 & 18.4 & 24319.55803 & 0 & 0 & 28 & 8 & 3.602 & 0.45 & 64 & 1 & 0.9199 & \\
\hline 81240 & 5.42 & 18.48 & 24330.90625 & 0 & 0 & 28 & 7 & 3.618 & 0.517 & 65 & 1 & 0.924 & \\
\hline 81260 & 4.64 & 17.88 & 24340.65147 & 0 & 0 & 28 & 5.99 & 3.5 & 0.584 & 66 & 1 & 0.89375 & \\
\hline 81280 & 3.87 & 17.73 & 24348.76856 & 0 & 0 & 28 & 5 & 3.472 & 0.694 & 67 & 1 & 0.8865 & \\
\hline 81300 & 3.09 & 17.66 & 24355.26224 & 0 & 0 & 28 & 3.99 & 3.458 & 0.867 & 68 & 1 & 0.883 & \\
\hline 81320 & 2.32 & 17.74 & 24360.13092 & 0 & 0 & 28 & 3 & 3.473 & 1.158 & 69 & 1 & 0.88695 & \\
\hline 81340 & 1.54 & 18.03 & 24363.37697 & 0 & 0 & 28 & 1.99 & 3.531 & 1.774 & 70 & 1 & 0.90165 & \\
\hline 81360 & 0.77 & 18.99 & 24365.00039 & 0 & 0 & 28 & 0.99 & 3.719 & 3.757 & 71 & 1 & 0.94965 & 0 \\
\hline 91380 & 0.7 & 21.58 & 24366.47379 & 18.16437 & 4.29824 & 28 & 0.9 & 4.226 & 4.696 & 72 & 1 & 1.07905 & 0.232653266 \\
\hline 91400 & 0.62 & 22.16 & 24367.77834 & 34.247 & 7.89101 & 28 & 0.8 & 4.34 & 5.425 & 73 & 1 & 1.10815 & 0.126726429 \\
\hline 91420 & 0.55 & 21.97 & 24368.92109 & 48.33504 & 11.23809 & 28 & 0.71 & 4.301 & 6.058 & 74 & 1 & 1.0984 & 0.088983065 \\
\hline 91440 & 0.47 & 21.71 & 24369.90127 & 60.4188 & 14.21284 & 27.9 & 0.61 & 4.251 & 6.969 & 75 & 1 & 1.0856 & 0.070358895 \\
\hline 91460 & 0.39 & 20.72 & 24370.72044 & 70.51765 & 17.38172 & 28 & 0.5 & 4.057 & 8.114 & 76 & 1 & 1.03605 & 0.057531696 \\
\hline 91480 & 0.31 & 18.92 & 24371.37625 & 78.60253 & 21.21525 & 28 & 0.4 & 3.705 & 9.263 & 77 & 1 & 0.946 & 0.047135887 \\
\hline 91500 & 0.24 & 16.32 & 24371.87026 & 84.69283 & 26.50792 & 28 & 0.31 & 3.195 & 10.306 & 78 & 1 & 0.8159 & 0.037724563 \\
\hline 91520 & 0.16 & 13.61 & 24372.20249 & 88.78852 & 33.31651 & 27.9 & 0.21 & 2.665 & 12.69 & 79 & 1 & 0.68055 & 0.030015142 \\
\hline 91540 & 0.08 & 10.79 & 24372.37135 & 90.87026 & 43.00531 & 27.8 & 0.1 & 2.113 & 21.13 & 80 & 1 & 0.5395 & 0.023252932 \\
\hline
\end{tabular}

2004.02.02 187-20 wt\%-D [sonicated] CC-45 0.1-1000 s-1 run 2.txt

Mode Step t[s] n[rpm] M[\%.] Phi[rad] Gamma J[t] T[ $\left.{ }^{\circ} \mathrm{C}\right] \mathrm{D}[1 / \mathrm{s}] \quad$ Tau[Pa] Eta[Pas] MP\# Block M[mNm] G[Pa]

\begin{tabular}{|c|c|c|c|c|c|c|c|c|c|c|c|c|c|c|}
\hline & 20 & 0.08 & 10.83 & 0.16101 & 1.98491 & 0.93584 & 27.8 & 0.1 & 2.121 & 21.21 & 1 & 1 & 0.5416 & 1.068562303 \\
\hline & 40 & 0.16 & 13.97 & 0.48852 & 6.02251 & 2.20121 & 27.8 & 0.21 & 2.736 & 13.029 & 2 & 1 & 0.6987 & 0.454295634 \\
\hline & 60 & 0.24 & 16.96 & 0.97939 & 12.07408 & 3.63458 & 27.8 & 0.31 & 3.322 & 10.716 & 3 & 1 & 0.8482 & 0.275134834 \\
\hline & 80 & 0.31 & 19.49 & 1.63127 & 20.11055 & 5.27006 & 27.9 & 0.4 & 3.816 & 9.54 & 4 & 1 & 0.97455 & 0.189751151 \\
\hline & 1100 & 0.39 & 21.69 & 2.4473 & 30.17067 & 7.10399 & 27.9 & 0.5 & 4.247 & 8.494 & 5 & 1 & 1.08455 & 0.14076585 \\
\hline & 1120 & 0.47 & 23.63 & 3.42512 & 42.22538 & 9.12389 & 27.9 & 0.61 & 4.628 & 7.587 & 6 & 1 & 1.1817 & 0.109602329 \\
\hline & $1 \quad 140$ & 0.54 & 25 & 4.56473 & 56.27469 & 11.49636 & 27.9 & 0.7 & 4.895 & 6.993 & 7 & 1 & 1.25005 & 0.086984042 \\
\hline & 1160 & 0.62 & 25.6 & 5.86692 & 72.32827 & 14.43102 & 27.9 & 0.8 & 5.012 & 6.265 & 8 & 1 & 1.27985 & 0.069295173 \\
\hline & 1180 & 0.7 & 27.01 & 7.33012 & 90.36677 & 17.08902 & 27.9 & 0.9 & 5.288 & 5.876 & 9 & 1 & 1.35025 & 0.058517085 \\
\hline & $2 \quad 200$ & 0.77 & 30.51 & 8.95354 & 0 & 0 & 28 & 0.99 & 5.973 & 6.033 & 10 & 1 & 1.52525 & 0 \\
\hline & $2 \quad 220$ & 1.55 & 41.41 & 12.17681 & 0 & 0 & 28 & 2 & 8.109 & 4.055 & 11 & 1 & 2.07065 & 0 \\
\hline & 2240 & 2.32 & 31.95 & 17.02665 & 0 & 0 & 28 & 3 & 6.256 & 2.085 & 12 & 1 & 1.5975 & 0 \\
\hline & 260 & 3.09 & 24.25 & 23.50225 & 0 & 0 & 28 & 3.99 & 4.748 & 1.19 & 13 & 1 & 1.2125 & 0 \\
\hline & 2280 & 3.87 & 24.04 & 31.59578 & 0 & 0 & 28 & 5 & 4.708 & 0.942 & 14 & 1 & 1.2022 & 0 \\
\hline & 2300 & 4.65 & 25.79 & 41.30723 & 0 & 0 & 28 & 6 & 5.05 & 0.842 & 15 & 1 & 1.28955 & 0 \\
\hline & 320 & 5.42 & 28.03 & 52.65938 & 0 & 0 & 28 & 7 & 5.488 & 0.784 & 16 & 1 & 1.4014 & 0 \\
\hline & 2340 & 6.2 & 30.85 & 65.61687 & 0 & 0 & 28 & 8 & 6.041 & 0.755 & 17 & 1 & 1.5427 & 0 \\
\hline & 2360 & 6.97 & 32.78 & 80.20329 & 0 & 0 & 28 & 9 & 6.419 & 0.713 & 18 & 1 & 1.63915 & 0 \\
\hline & 3380 & 7.75 & 33.79 & 96.58198 & 0 & 0 & 28 & 10.01 & 6.616 & 0.661 & 19 & 1 & 1.6895 & 0 \\
\hline & 3400 & 15.5 & 44.15 & 128.91683 & 0 & 0 & 28 & 20.01 & 8.645 & 0.432 & 20 & 1 & 2.2075 & 0 \\
\hline & 3420 & 23.24 & 41.01 & 177.47328 & 0 & 0 & 28 & 30 & 8.029 & 0.268 & 21 & 1 & 2.0503 & 0 \\
\hline & 440 & 30.99 & 38.56 & 242.2058 & 0 & 0 & 28 & 40.01 & 7.551 & 0.189 & 22 & 1 & 1.9282 & 0 \\
\hline & 460 & 38.73 & 37.45 & 323.22904 & 0 & 0 & 28 & 50 & 7.332 & 0.147 & 23 & 1 & 1.8723 & 0 \\
\hline & 480 & 46.48 & 37.44 & 420.47312 & 0 & 0 & 28 & 60.01 & 7.33 & 0.122 & 24 & 1 & 1.8719 & 0 \\
\hline & 500 & 54.22 & 37.55 & 533.96708 & 0 & 0 & 28 & 70 & 7.351 & 0.105 & 25 & 1 & 1.87725 & 0 \\
\hline & 520 & 61.96 & 36.55 & 663.56013 & 0 & 0 & 28 & 79.99 & 7.157 & 0.089 & 26 & 1 & 1.8277 & 0 \\
\hline
\end{tabular}




\begin{tabular}{|c|c|c|c|c|c|c|c|c|c|c|c|c|c|c|}
\hline & 540 & 69.71 & 34.69 & 809.40307 & 0 & 0 & 28 & 90 & 6.792 & 0.075 & 27 & 1 & 1.73435 & 0 \\
\hline & 560 & 77.46 & 33.5 & 973.01408 & 0 & 0 & 28 & 100 & 6.56 & 0.066 & 28 & 1 & 1.67515 & 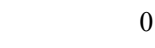 \\
\hline & 580 & 154.92 & 43.2 & 1296.62718 & 0 & 0 & 28 & 200 & 8.459 & 0.042 & 29 & 1 & 2.1601 & \\
\hline & 600 & 232.38 & 38.88 & 1781.94434 & 0 & 0 & 28 & 300 & 7.613 & 0.025 & 30 & 1 & 1.9442 & \\
\hline & 620 & 309.84 & 38 & 2429.44858 & 0 & 0 & 28 & 400 & 7.441 & 0.019 & 31 & 1 & 1.90005 & \\
\hline & 640 & 387.3 & 51.88 & 3239.78706 & 0 & 0 & 28 & 500 & 10.158 & 0.02 & 32 & 1 & 2.5941 & \\
\hline & 660 & 464.75 & 72.48 & 4211.1785 & 0 & 0 & 28 & 599.99 & 14.191 & 0.024 & 33 & 1 & 3.6239 & \\
\hline & 680 & 542.21 & 83.55 & 5345.45524 & 0 & 0 & 28 & 699.99 & 16.359 & 0.023 & 34 & 1 & 4.17735 & \\
\hline & 700 & 619.67 & 93.37 & 6642.60236 & 0 & 0 & 28 & 799.99 & 18.281 & 0.023 & 35 & 1 & 4.66835 & \\
\hline & 720 & 697.13 & 109.47 & 8099.87331 & 0 & 0 & 28 & 899.99 & 21.434 & 0.024 & 36 & 1 & 5.47335 & \\
\hline & 740 & 774.59 & 125.49 & 9722.45486 & 0 & 0 & 28 & 1000 & 24.57 & 0.025 & 37 & 1 & 6.2743 & \\
\hline & 750 & 774.59 & 122.97 & 10551.93507 & 0 & 0 & 28 & 1000 & 24.078 & 0.024 & 38 & 1 & 6.14855 & \\
\hline & 760 & 774.59 & 121.77 & 11361.46223 & 0 & 0 & 28 & 1000 & 23.843 & 0.024 & 39 & 1 & 6.08865 & \\
\hline & 770 & 774.59 & 120.9 & 12173.42335 & 0 & 0 & 28 & 1000 & 23.672 & 0.024 & 40 & 1 & 6.04495 & \\
\hline & 780 & 774.59 & 120.21 & 12982.94894 & 0 & 0 & 28 & 1000 & 23.536 & 0.024 & 41 & 1 & 6.01025 & \\
\hline & 790 & 774.59 & 119.43 & 13796.12899 & 0 & 0 & 28 & 1000 & 23.384 & 0.023 & 42 & 1 & 5.9714 & \\
\hline & 800 & 774.59 & 119.09 & 14606.05985 & 0 & 0 & 28 & 1000 & 23.317 & 0.023 & 43 & 1 & 5.9543 & \\
\hline & 820 & 774.59 & 118.44 & 16246.20291 & 0 & 0 & 28 & 1000 & 23.191 & 0.023 & 44 & 1 & 5.9222 & \\
\hline & 840 & 697.13 & 100.75 & 17709.02741 & 0 & 0 & 28 & 899.99 & 19.728 & 0.022 & 45 & 1 & 5.0377 & \\
\hline & 860 & 619.67 & 84.64 & 19008.52758 & 0 & 0 & 28 & 799.99 & 16.573 & 0.021 & 46 & 1 & 4.23215 & \\
\hline & 880 & 542.21 & 69.17 & 20144.05467 & 0 & 0 & 28 & 699.99 & 13.543 & 0.019 & 47 & 1 & 3.45845 & \\
\hline & 900 & 464.75 & 54.58 & 21118.97412 & 0 & 0 & 28 & 599.99 & 10.687 & 0.018 & 48 & 1 & 2.72905 & \\
\hline & 920 & 387.3 & 38.86 & 21931.21955 & 0 & 0 & 28 & 500 & 7.609 & 0.015 & 49 & 1 & 1.9431 & \\
\hline & 940 & 309.84 & 26.39 & 22581.59756 & 0 & 0 & 28 & 400 & 5.168 & 0.013 & 50 & 1 & 1.31965 & \\
\hline & 960 & 232.38 & 20.96 & 23069.83405 & 0 & 0 & 28 & 300 & 4.104 & 0.014 & 51 & 1 & 1.0481 & \\
\hline & 6980 & 154.92 & 16.92 & 23395.56694 & 0 & 0 & 28 & 200 & 3.314 & 0.017 & 52 & 1 & 0.84615 & \\
\hline & 61000 & 77.46 & 13.46 & 23559.11668 & 0 & 0 & 28 & 100 & 2.636 & 0.026 & 53 & 1 & 0.67315 & \\
\hline & 71020 & 69.71 & 15.33 & 23706.64823 & 0 & 0 & 28 & 90 & 3.002 & 0.033 & 54 & 1 & 0.7667 & \\
\hline & 71040 & 61.97 & 15.24 & 23836.58136 & 0 & 0 & 28 & 80 & 2.984 & 0.037 & 55 & 1 & 0.7619 & \\
\hline & 71060 & 54.22 & 15 & 23950.2002 & 0 & 0 & 28 & 70 & 2.936 & 0.042 & 56 & 1 & 0.74975 & \\
\hline & 71080 & 46.48 & 14.9 & 24047.60921 & 0 & 0 & 28 & 60.01 & 2.916 & 0.049 & 57 & 1 & 0.74475 & 0 \\
\hline & 71100 & 38.73 & 14.76 & 24128.88378 & 0 & 0 & 28 & 50 & 2.89 & 0.058 & 58 & 1 & 0.73795 & 0 \\
\hline & 71120 & 30.99 & 15.08 & 24193.91161 & 0 & 0 & 28 & 40.01 & 2.952 & 0.074 & 59 & 1 & 0.75375 & 0 \\
\hline & 71140 & 23.24 & 15.07 & 24242.66127 & 0 & 0 & 28 & 30 & 2.95 & 0.098 & 60 & 1 & 0.75325 & 0 \\
\hline & 71160 & 15.5 & 14.88 & 24275.23959 & 0 & 0 & 28 & 20.01 & 2.913 & 0.146 & 61 & 1 & 0.7439 & 0 \\
\hline & 71180 & 7.75 & 14.66 & 24291.58058 & 0 & 0 & 28 & 10.01 & 2.87 & 0.287 & 62 & 1 & 0.73295 & 0 \\
\hline & 81200 & 6.97 & 16.01 & 24306.28952 & 0 & 0 & 28 & 9 & 3.135 & 0.348 & 63 & 1 & 0.8005 & 0 \\
\hline & 81220 & 6.2 & 16.61 & 24319.28157 & 0 & 0 & 28 & 8 & 3.253 & 0.407 & 64 & 1 & 0.83065 & 0 \\
\hline & 81240 & 5.42 & 16.94 & 24330.63922 & 0 & 0 & 28 & 7 & 3.316 & 0.474 & 65 & 1 & 0.8468 & 0 \\
\hline & 81260 & 4.64 & 16.67 & 24340.38051 & 0 & 0 & 28 & 5.99 & 3.265 & 0.545 & 66 & 1 & 0.8337 & 0 \\
\hline & 81280 & 3.87 & 16.18 & 24348.49367 & 0 & 0 & 28 & 5 & 3.167 & 0.633 & 67 & 1 & 0.80885 & \\
\hline & 81300 & 3.09 & 16.17 & 24354.99127 & 0 & 0 & 28 & 3.99 & 3.167 & 0.794 & 68 & 1 & 0.8087 & 0 \\
\hline & 81320 & 2.32 & 16.56 & 24359.8576 & 0 & 0 & 28 & 3 & 3.242 & 1.081 & 69 & 1 & 0.82785 & 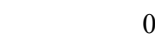 \\
\hline & 81340 & 1.54 & 16.46 & 24363.10365 & 0 & 0 & 28 & 1.99 & 3.222 & 1.619 & 70 & 1 & 0.82285 & \\
\hline & 81360 & 0.77 & 17.66 & 24364.72707 & 0 & 0 & 28 & 0.99 & 3.458 & 3.493 & 71 & 1 & 0.88305 & \\
\hline & 91380 & 0.7 & 20.25 & 24366.20205 & 18.18373 & 4.58722 & 28 & 0.9 & 3.964 & 4.404 & 72 & 1 & 1.01225 & 0.217997078 \\
\hline & 91400 & 0.62 & 21.01 & 24367.50581 & 34.25668 & 8.32685 & 28 & 0.8 & 4.114 & 5.142 & 73 & 1 & 1.05065 & 0.120093366 \\
\hline & 91420 & 0.55 & 20.87 & 24368.64778 & 48.33504 & 11.82942 & 28 & 0.71 & 4.086 & 5.755 & 74 & 1 & 1.0433 & 0.084534946 \\
\hline & 91440 & 0.47 & 20.75 & 24369.62874 & 60.42848 & 14.87287 & 28 & 0.61 & 4.063 & 6.661 & 75 & 1 & 1.03755 & 0.067236508 \\
\hline & 91460 & 0.39 & 20.36 & 24370.44634 & 70.50796 & 17.68446 & 28 & 0.5 & 3.987 & 7.974 & 76 & 1 & 1.01825 & 0.056546807 \\
\hline
\end{tabular}




\begin{tabular}{|c|c|c|c|c|c|c|c|c|c|c|c|c|}
\hline 91480 & 0.31 & 18.93 & 24371.10293 & 78.60253 & 21.20953 & 28 & 0.4 & 3.706 & 9.265 & 77 & 0.9465 & 0.04714861 \\
\hline 91500 & 0.24 & 16.29 & 24371.59695 & 84.69283 & 26.55779 & 28 & 0.31 & 3.189 & 10.287 & 78 & 10.81445 & 0.037653719 \\
\hline 91520 & 0.16 & 13.39 & 24371.92917 & 88.78853 & 33.86289 & 28 & 0.21 & 2.622 & 12.486 & 79 & 0.6696 & 0.029530841 \\
\hline 91540 & 0.08 & 10.45 & 24372.09882 & 90.87995 & 44.44005 & 28 & 0.1 & 2.045 & 20.45 & 80 & 0.52225 & 0.022502213 \\
\hline
\end{tabular}

2004.02.02 187-20 wt\%-D [sonicated] CC-45 0.1-1000 s-1 run 3.txt

Mode Step t[s] n[rpm] M[\%.] Phi[rad] Gamma J[t] T[ $\left[{ }^{\circ} \mathrm{C}\right] \mathrm{D}[1 / \mathrm{s}] \quad$ Tau[Pa $]$ Eta[Pas $]$ MP\# Block M[mNm] G[Pa $]$

\begin{tabular}{|c|c|c|c|c|c|c|c|c|c|c|c|c|c|c|c|}
\hline 1 & 1 & 20 & 0.08 & 10.16 & 0.16179 & 1.99459 & 1.00231 & 28 & 0.1 & 1.99 & 19.9 & 1 & 1 & 0.50805 & 0.997698775 \\
\hline & 1 & 40 & 0.16 & 13.39 & 0.4893 & 6.0322 & 2.30149 & 28 & 0.21 & 2.621 & 12.481 & 2 & 1 & 0.6693 & 0.434501509 \\
\hline & 1 & 60 & 0.24 & 16.31 & 0.97939 & 12.07408 & 3.78142 & 28 & 0.31 & 3.193 & 10.3 & 3 & 1 & 0.8154 & 0.26445079 \\
\hline & 1 & 80 & 0.31 & 18.87 & 1.63206 & 20.12023 & 5.44526 & 28 & 0.4 & 3.695 & 9.237 & 4 & 1 & 0.9435 & 0.183646012 \\
\hline & 1 & 100 & 0.39 & 21.19 & 2.4473 & 30.17067 & 7.27179 & 28 & 0.5 & 4.149 & 8.298 & 5 & 1 & 1.0595 & 0.137517662 \\
\hline & 1 & 120 & 0.47 & 23.12 & 3.42355 & 42.20602 & 9.32317 & 28 & 0.61 & 4.527 & 7.421 & 6 & 1 & 1.15615 & 0.107259581 \\
\hline & 1 & 140 & 0.54 & 24.48 & 4.56395 & 56.26501 & 11.73655 & 28 & 0.7 & 4.794 & 6.849 & 7 & 1 & 1.2242 & 0.08520393 \\
\hline & 1 & 160 & 0.62 & 25.13 & 5.86692 & 72.32827 & 14.69788 & 28 & 0.8 & 4.921 & 6.151 & 8 & 1 & 1.2566 & 0.068037021 \\
\hline & 1 & 180 & 0.7 & 26.98 & 7.33091 & 90.37646 & 17.11027 & 28 & 0.9 & 5.282 & 5.869 & 9 & 1 & 1.34895 & 0.058444422 \\
\hline & 2 & 200 & 0.77 & 31.22 & 8.95275 & 0 & 0 & 28 & 0.99 & 6.113 & 6.175 & 10 & 1 & 1.56095 & 0 \\
\hline & 2 & 220 & 1.55 & 42.89 & 12.17289 & 0 & 0 & 28 & 2 & 8.398 & 4.199 & 11 & 1 & 2.1446 & 0 \\
\hline & 2 & 240 & 2.32 & 32.49 & 17.02979 & 0 & 0 & 28 & 3 & 6.362 & 2.121 & 12 & 1 & 1.6246 & 0 \\
\hline & 2 & 260 & 3.09 & 23.33 & 23.50225 & 0 & 0 & 28 & 3.99 & 4.567 & 1.145 & 13 & 1 & 1.1663 & 0 \\
\hline & 2 & 280 & 3.87 & 22.06 & 31.59657 & 0 & 0 & 28 & 5 & 4.319 & 0.864 & 14 & 1 & 1.10295 & 0 \\
\hline & 2 & 300 & 4.65 & 23.46 & 41.30802 & 0 & 0 & 28 & 6 & 4.594 & 0.766 & 15 & 1 & 1.17315 & 0 \\
\hline & 2 & 320 & 5.42 & 25.24 & 52.64917 & 0 & 0 & 28 & 7 & 4.942 & 0.706 & 16 & 1 & 1.2621 & 0 \\
\hline 1 & 2 & 340 & 6.2 & 27.75 & 65.62866 & 0 & 0 & 28 & 8 & 5.434 & 0.679 & 17 & 1 & 1.3876 & 0 \\
\hline 1 & 2 & 360 & 6.97 & 29.68 & 80.20408 & 0 & 0 & 28 & 9 & 5.81 & 0.646 & 18 & 1 & 1.48375 & 0 \\
\hline 1 & 3 & 380 & 7.75 & 30.53 & 96.57413 & 0 & 0 & 28 & 10.01 & 5.978 & 0.597 & 19 & 1 & 1.52645 & 0 \\
\hline 1 & 3 & 400 & 15.5 & 40.66 & 128.90112 & 0 & 0 & 28 & 20.01 & 7.962 & 0.398 & 20 & 1 & 2.0331 & 0 \\
\hline 1 & 3 & 420 & 23.24 & 41.05 & 177.4615 & 0 & 0 & 28 & 30 & 8.037 & 0.268 & 21 & 1 & 2.05225 & 0 \\
\hline 1 & 3 & 440 & 30.99 & 40.08 & 242.25921 & 0 & 0 & 28 & 40.01 & 7.847 & 0.196 & 22 & 1 & 2.00395 & 0 \\
\hline 1 & 3 & 460 & 38.73 & 38.48 & 323.22512 & 0 & 0 & 28 & 50 & 7.533 & 0.151 & 23 & 1 & 1.92375 & 0 \\
\hline 1 & 3 & 480 & 46.48 & 38.72 & 420.44484 & 0 & 0 & 28 & 60.01 & 7.581 & 0.126 & 24 & 1 & 1.93595 & 0 \\
\hline 1 & 3 & 500 & 54.22 & 37.72 & 533.9388 & 0 & 0 & 28 & 70 & 7.386 & 0.106 & 25 & 1 & 1.8861 & 0 \\
\hline 1 & 3 & 520 & 61.96 & 36.07 & 663.69522 & 0 & 0 & 28 & 79.99 & 7.063 & 0.088 & 26 & 1 & 1.80355 & 0 \\
\hline 1 & 3 & 540 & 69.71 & 34.01 & 809.46905 & 0 & 0 & 28 & 90 & 6.659 & 0.074 & 27 & 1 & 1.70045 & 0 \\
\hline 1 & 4 & 560 & 77.46 & 32.22 & 973.03999 & 0 & 0 & 28 & 100 & 6.308 & 0.063 & 28 & 1 & 1.61075 & 0 \\
\hline$\frac{1}{1}$ & 4 & 580 & 154.92 & 41.42 & 1296.65231 & 0 & 0 & 28 & 200 & 8.11 & 0.041 & 29 & 1 & 2.0711 & 0 \\
\hline 1 & 4 & 600 & 232.38 & 37.29 & 1782.09042 & 0 & 0 & 28 & 300 & 7.301 & 0.024 & 30 & 1 & 1.86445 & 0 \\
\hline 1 & 4 & 620 & 309.84 & 35.57 & 2429.87819 & 0 & 0 & 28 & 400 & 6.965 & 0.017 & 31 & 1 & 1.7785 & 0 \\
\hline 1 & 4 & 640 & 387.3 & 46.99 & 3239.36609 & 0 & 0 & 28 & 500 & 9.201 & 0.018 & 32 & 1 & 2.3496 & 0 \\
\hline 1 & 4 & 660 & 464.75 & 70.22 & 4210.71747 & 0 & 0 & 28 & 599.99 & 13.748 & 0.023 & 33 & 1 & 3.51085 & 0 \\
\hline 1 & 4 & 680 & 542.21 & 78.35 & 5346.12833 & 0 & 0 & 28 & 699.99 & 15.34 & 0.022 & 34 & 1 & 3.9173 & 0 \\
\hline 1 & 4 & 700 & 619.67 & 88.85 & 6641.33158 & 0 & 0 & 28 & 799.99 & 17.398 & 0.022 & 35 & 1 & 4.4427 & 0 \\
\hline 1 & 4 & 720 & 697.13 & 103.01 & 8100.46628 & 0 & 0 & 28 & 899.99 & 20.17 & 0.022 & 36 & 1 & 5.15065 & 0 \\
\hline 1 & 4 & 740 & 774.59 & 120.01 & 9720.97988 & 0 & 0 & 28 & 1000 & 23.498 & 0.023 & 37 & 1 & 6.00045 & 0 \\
\hline 1 & 5 & 750 & 774.59 & 117.78 & 10549.24665 & 0 & 0 & 28 & 1000 & 23.062 & 0.023 & 38 & 1 & 5.88905 & 0 \\
\hline 1 & 5 & 760 & 774.59 & 116.86 & 11359.17829 & 0 & 0 & 28 & 1000 & 22.882 & 0.023 & 39 & 1 & 5.84315 & 0 \\
\hline 1 & 5 & 770 & 774.59 & 116.2 & 12172.7589 & 0 & 0 & 28 & 1000 & 22.753 & 0.023 & 40 & 1 & 5.8102 & 0 \\
\hline 1 & 5 & 780 & 774.59 & 115.54 & 12983.0966 & 0 & 0 & 28 & 1000 & 22.624 & 0.023 & 41 & 1 & 5.7772 & 0 \\
\hline 1 & 5 & 790 & 774.59 & 115.09 & 13793.83956 & 0 & 0 & 28 & 1000 & 22.534 & 0.023 & 42 & 1 & 5.7543 & 0 \\
\hline & 5 & 800 & 774.59 & 114.61 & 14604.58252 & 0 & 0 & 28 & 1000 & 22.441 & 0.022 & 43 & 1 & 5.73065 & 0 \\
\hline
\end{tabular}




\begin{tabular}{|c|c|c|c|c|c|c|c|c|c|c|c|c|c|c|}
\hline & 820 & 774.59 & 114.03 & 16246.75583 & 0 & 0 & 28 & 1000 & 22.327 & 0.022 & 44 & 1 & 5.70145 & 0 \\
\hline & 840 & 697.13 & 97.08 & 17707.71266 & 0 & 0 & 28 & 899.99 & 19.008 & 0.021 & 45 & 1 & 4.85395 & م \\
\hline & 860 & 619.67 & 81.48 & 19006.84997 & 0 & 0 & 28 & 799.99 & 15.953 & 0.02 & 46 & 1 & 4.0738 & 0 \\
\hline & 880 & 542.21 & 66.6 & 20143.22922 & 0 & 0 & 28 & 699.99 & 13.04 & 0.019 & 47 & 1 & 3.32985 & 0 \\
\hline & 900 & 464.75 & 52.52 & 21117.90441 & 0 & 0 & 28 & 599.99 & 10.284 & 0.017 & 48 & 1 & 2.62615 & 0 \\
\hline & 920 & 387.3 & 38.4 & 21931.16379 & 0 & 0 & 28 & 500 & 7.519 & 0.015 & 49 & 1 & 1.92 & 0 \\
\hline & 940 & 309.84 & 26.62 & 22581.33917 & 0 & 0 & 28 & 400 & 5.212 & 0.013 & 50 & 1 & 1.33095 & 0 \\
\hline & 960 & 232.38 & 20.12 & 23068.80675 & 0 & 0 & 28 & 300 & 3.94 & 0.013 & 51 & 1 & 1.0062 & 0 \\
\hline & 6980 & 154.92 & 16.03 & 23394.54042 & 0 & 0 & 28 & 200 & 3.138 & 0.016 & 52 & 1 & 0.80135 & 0 \\
\hline & 61000 & 77.46 & 12.67 & 23558.01006 & 0 & 0 & 28 & 100 & 2.481 & 0.025 & 53 & 1 & 0.6335 & 0 \\
\hline & 71020 & 69.71 & 14.81 & 23705.43086 & 0 & 0 & 28 & 90 & 2.899 & 0.032 & 54 & 1 & 0.74025 & 0 \\
\hline & 71040 & 61.97 & 14.82 & 23835.29566 & 0 & 0 & 28 & 80 & 2.902 & 0.036 & 55 & 1 & 0.741 & 0 \\
\hline & 71060 & 54.22 & 14.53 & 23949.11714 & 0 & 0 & 28 & 70 & 2.846 & 0.041 & 56 & 1 & 0.72665 & 0 \\
\hline & 71080 & 46.48 & 14.33 & 24046.49787 & 0 & 0 & 28 & 60.01 & 2.805 & 0.047 & 57 & 1 & 0.7164 & 0 \\
\hline & 71100 & 38.73 & 14.24 & 24127.71511 & 0 & 0 & 28 & 50 & 2.789 & 0.056 & 58 & 1 & 0.71215 & 0 \\
\hline & 71120 & 30.99 & 14.55 & 24192.73115 & 0 & 0 & 28 & 40.01 & 2.848 & 0.071 & 59 & 1 & 0.72725 & 0 \\
\hline & 71140 & 23.24 & 14.61 & 24241.54208 & 0 & 0 & 28 & 30 & 2.861 & 0.095 & 60 & 1 & 0.7305 & 0 \\
\hline & 71160 & 15.5 & 14.3 & 24274.12511 & 0 & 0 & 28 & 20.01 & 2.801 & 0.14 & 61 & 1 & 0.7152 & 0 \\
\hline & 71180 & 7.75 & 14.04 & 24290.46924 & 0 & 0 & 28 & 10.01 & 2.749 & 0.275 & 62 & 1 & 0.7021 & 0 \\
\hline & 81200 & 6.97 & 15.41 & 24305.17347 & 0 & 0 & 28 & 9 & 3.018 & 0.335 & 63 & 1 & 0.7706 & 0 \\
\hline & 81220 & 6.2 & 15.98 & 24318.16552 & 0 & 0 & 28 & 8 & 3.129 & 0.391 & 64 & 1 & 0.79915 & 0 \\
\hline & 81240 & 5.42 & 16.24 & 24329.53181 & 0 & 0 & 28 & 7 & 3.18 & 0.454 & 65 & 1 & 0.81205 & 0 \\
\hline & 81260 & 4.64 & 16.09 & 24339.25896 & 0 & 0 & 28 & 5.99 & 3.149 & 0.526 & 66 & 1 & 0.80425 & 0 \\
\hline & 81280 & 3.87 & 15.59 & 24347.37919 & 0 & 0 & 28 & 5 & 3.053 & 0.611 & 67 & 1 & 0.77955 & 0 \\
\hline & 81300 & 3.09 & 15.6 & 24353.87287 & 0 & 0 & 28 & 3.99 & 3.054 & 0.765 & 68 & 1 & 0.78 & 0 \\
\hline & 81320 & 2.32 & 15.78 & 24358.73919 & 0 & 0 & 28 & 3 & 3.09 & 1.03 & 69 & 1 & 0.7891 & 0 \\
\hline & 81340 & 1.54 & 15.83 & 24361.9876 & 0 & 0 & 28 & 1.99 & 3.1 & 1.558 & 70 & 1 & 0.7915 & 0 \\
\hline & 81360 & 0.77 & 16.82 & 24363.61102 & 0 & 0 & 28 & 0.99 & 3.294 & 3.327 & 71 & 1 & 0.84115 & 0 \\
\hline & 91380 & 0.7 & 19.12 & 24365.08521 & 18.17405 & 4.85548 & 28 & 0.9 & 3.743 & 4.159 & 72 & 1 & 0.95585 & 0.205952993 \\
\hline & 91400 & 0.62 & 19.82 & 24366.38897 & 34.247 & 8.82427 & 28 & 0.8 & 3.881 & 4.851 & 73 & 1 & 0.99095 & 0.113323795 \\
\hline & 91420 & 0.55 & 19.25 & 24367.53173 & 48.33504 & 12.82096 & 28 & 0.71 & 3.77 & 5.31 & 74 & 1 & 0.96265 & 0.077997246 \\
\hline & 91440 & 0.47 & 18.52 & 24368.51269 & 60.42848 & 16.66532 & 28 & 0.61 & 3.626 & 5.944 & 75 & 1 & 0.92595 & 0.060004819 \\
\hline & 91460 & 0.39 & 17.92 & 24369.33029 & 70.50797 & 20.09346 & 28 & 0.5 & 3.509 & 7.018 & 76 & 1 & 0.896 & 0.049767423 \\
\hline & 91480 & 0.31 & 16.96 & 24369.98609 & 78.59285 & 23.66541 & 28 & 0.4 & 3.321 & 8.303 & 77 & 1 & 0.84815 & 0.042255752 \\
\hline & 91500 & 0.24 & 14.81 & 24370.48011 & 84.68315 & 29.20108 & 28 & 0.31 & 2.9 & 9.355 & 78 & 1 & 0.74065 & 0.034245301 \\
\hline & 91520 & 0.16 & 12.3 & 24370.81233 & 88.77884 & 36.85297 & 28 & 0.21 & 2.409 & 11.471 & 79 & 1 & 0.61505 & 0.027134844 \\
\hline & 91540 & 0.08 & 9.05 & 24370.98198 & 90.87026 & 51.31011 & 28 & 0.1 & 1.771 & 17.71 & 80 & 1 & 0.4523 & 0.019489325 \\
\hline
\end{tabular}

2004.02.02 187-20 wt\%-D [sonicated] CC-45 0.1-1000 s-1 run 4.txt

Mode Step t[s] n[rpm] M[\%.] Phi[rad] Gamma J[t] T $\left[{ }^{\circ} \mathrm{C}\right] \mathrm{D}[1 / \mathrm{s}] \quad$ Tau[Pa] Eta[Pas $]$ MP\# Block M[mNm] G[Pa $]$

\begin{tabular}{|c|c|c|c|c|c|c|c|c|c|c|c|c|c|c|}
\hline & 20 & 0.08 & 9.77 & 0.16179 & 1.99459 & 1.04265 & 28 & 0.1 & 1.913 & 19.13 & 1 & 1 & 0.4886 & 0.95909435 \\
\hline & 40 & 0.16 & 12.48 & 0.4893 & 6.0322 & 2.46918 & 28 & 0.21 & 2.443 & 11.633 & 2 & 1 & 0.62395 & 0.404993203 \\
\hline & 60 & 0.24 & 15.15 & 0.97939 & 12.07408 & 4.07083 & 28 & 0.31 & 2.966 & 9.568 & 3 & 1 & 0.75745 & 0.245650186 \\
\hline & 80 & 0.31 & 18 & 1.63206 & 20.12023 & 5.70787 & 28 & 0.4 & 3.525 & 8.813 & 4 & 1 & 0.90015 & 0.175196804 \\
\hline & 100 & 0.39 & 20.3 & 2.4473 & 30.17067 & 7.59201 & 28 & 0.5 & 3.974 & 7.948 & 5 & 1 & 1.01475 & 0.131717327 \\
\hline & 120 & 0.47 & 21.95 & 3.42512 & 42.22538 & 9.82671 & 28 & 0.61 & 4.297 & 7.044 & 6 & 1 & 1.0974 & 0.101763442 \\
\hline & 140 & 0.54 & 22.79 & 4.56552 & 56.28437 & 12.61133 & 28 & 0.7 & 4.463 & 6.376 & 7 & 1 & 1.13965 & 0.079293772 \\
\hline & 160 & 0.62 & 23.15 & 5.86771 & 72.33796 & 15.96159 & 28 & 0.8 & 4.532 & 5.665 & 8 & 1 & 1.15725 & 0.062650371 \\
\hline & 180 & 0.7 & 23.96 & 7.33169 & 90.38614 & 19.26799 & 28 & 0.9 & 4.691 & 5.212 & 9 & 1 & 1.19785 & 0.05189955 \\
\hline & 200 & 0.77 & 27.07 & 8.95511 & 0 & 0 & 28 & 0.99 & 5.3 & 5.354 & 10 & 1 & 1.35345 & 0 \\
\hline
\end{tabular}




\begin{tabular}{|c|c|c|c|c|c|c|c|c|c|c|c|c|c|c|c|c|c|c|c|c|c|c|c|c|c|}
\hline 1 & 220 & & & & 0 & 0 & 28 & & & & & & & 220 & 1.55 & 38.21 & 12.17603 & & 2 & 7.481 & 3.741 & 11 & 1 & 1.91025 & 0 \\
\hline 1 & 240 & & & & 0 & 0 & 28 & & & & & & & 240 & 2.32 & 30.04 & 17.02822 & & 3 & 5.882 & 1.961 & 12 & 1 & 1.5021 & 0 \\
\hline 1 & 260 & & & & 0 & 0 & 28 & & & & & & & 260 & 3.09 & 23.24 & 23.50304 & & 3.99 & 4.551 & 1.141 & 13 & 1 & 1.1622 & 0 \\
\hline 1 & 280 & & & & 0 & 0 & 28 & & & & & & & 280 & 3.87 & 22.43 & 31.59735 & & 5 & 4.393 & 0.879 & 14 & 1 & 1.1217 & 0 \\
\hline 1 & 300 & & & & 0 & 0 & 28 & & & & & & & 300 & 4.65 & 24.13 & 41.3088 & & 6 & 4.724 & 0.787 & 15 & 1 & 1.2064 & 0 \\
\hline 1 & 320 & & & & 0 & 0 & 28 & & & & & & & 320 & 5.42 & 26.34 & 52.65466 & & 7 & 5.157 & 0.737 & 16 & 1 & 1.31685 & 0 \\
\hline 1 & 340 & & & & 0 & 0 & 28 & & & & & & & 340 & 6.2 & 29.13 & 65.62394 & & 8 & 5.703 & 0.713 & 17 & 1 & 1.45625 & 0 \\
\hline 1 & 360 & & & & 0 & 0 & 28 & & & & & & & 360 & 6.97 & 31 & 80.19701 & & 9 & 6.07 & 0.674 & 18 & 1 & 1.55 & 0 \\
\hline & 380 & & & & 0 & 0 & 28 & & & & & & & 380 & 7.75 & 31.88 & 96.55449 & & 10.01 & 6.242 & 0.624 & 19 & 1 & 1.5939 & 0 \\
\hline & 400 & & & & 0 & 0 & 28 & & & & & & & 400 & 15.5 & 41.54 & 128.89326 & & 20.01 & 8.134 & 0.406 & 20 & 1 & 2.07715 & 0 \\
\hline 1 & 420 & & & & 0 & 0 & 28 & & & & & & & 420 & 23.24 & 41.51 & 177.44972 & & 30 & 8.128 & 0.271 & 21 & 1 & 2.07555 & 0 \\
\hline 1 & 440 & & & & 0 & 0 & 28 & & & & & & & 440 & 30.99 & 39.37 & 242.25999 & & 40.01 & 7.709 & 0.193 & 22 & 1 & 1.9686 & 0 \\
\hline 1 & 460 & & & & 0 & 0 & 28 & & & & & & & 460 & 38.73 & 36.97 & 323.2424 & & 50 & 7.239 & 0.145 & 23 & 1 & 1.8486 & 0 \\
\hline 1 & 480 & & & & 0 & 0 & 28 & & & & & & & 480 & 46.48 & 36.06 & 420.46291 & & 60.01 & 7.06 & 0.118 & 24 & 1 & 1.8029 & 0 \\
\hline 1 & 500 & & & & 0 & 0 & 28 & & & & & & & 500 & 54.22 & 35.23 & 533.87597 & & 70 & 6.898 & 0.099 & 25 & 1 & 1.7615 & 0 \\
\hline 1 & 520 & & & & 0 & 0 & 28 & & & & & & & 520 & 61.96 & 34.67 & 663.5939 & & 79.99 & 6.788 & 0.085 & 26 & 1 & 1.7335 & 0 \\
\hline 1 & 540 & & & & 0 & 0 & 28 & & & & & & & 540 & 69.71 & 33.16 & 809.58214 & & 90 & 6.492 & 0.072 & 27 & 1 & 1.65785 & 0 \\
\hline 1 & 560 & & & & 0 & 0 & 28 & & & & & & & 560 & 77.46 & 31.84 & 973.19393 & & 100 & 6.235 & 0.062 & 28 & 1 & 1.5921 & 0 \\
\hline 1 & 580 & & & & 0 & 0 & 28 & & & & & & & 580 & 154.92 & 42.07 & 1296.48267 & & 200 & 8.237 & 0.041 & 29 & 1 & 2.1033 & 0 \\
\hline 1 & 600 & & & & 0 & 0 & 28 & & & & & & & 600 & 232.38 & 36.78 & 1782.40772 & & 300 & 7.201 & 0.024 & 30 & 1 & 1.8389 & 0 \\
\hline 1 & 620 & & & & 0 & 0 & 28 & & & & & & & 620 & 309.84 & 36.14 & 2429.9528 & & 400 & 7.075 & 0.018 & 31 & 1 & 1.8068 & 0 \\
\hline 1 & 640 & & & & 0 & 0 & 28 & & & & & & & 640 & 387.3 & 52.05 & 3239.68182 & & 500 & 10.192 & 0.02 & 32 & 1 & 2.6026 & 0 \\
\hline 1 & 660 & & & & 0 & 0 & 28 & & & & & & & 660 & 464.75 & 69.71 & 4211.76441 & & 99.99 & 13.649 & 0.023 & 33 & 1 & 3.48535 & 0 \\
\hline 1 & 680 & & & & 0 & 0 & 28 & & & & & & & 680 & 542.21 & 77.71 & 5346.03958 & & 699.99 & 15.215 & 0.022 & 34 & 1 & 3.8853 & 0 \\
\hline 1 & 700 & & & & 0 & 0 & 28 & & & & & & & 700 & 619.67 & 89.05 & 6642.256 & & 99.99 & 17.435 & 0.022 & 35 & 1 & 4.4523 & 0 \\
\hline 1 & 720 & & & & 0 & 0 & 28 & & & & & & & 720 & 697.13 & 106.05 & 8100.98543 & & 999.99 & 20.764 & 0.023 & 36 & 1 & 5.30235 & 0 \\
\hline 1 & 740 & & & & 0 & 0 & 28 & & & & & & & 740 & 774.59 & 121.57 & 9721.09534 & & 1000 & 23.803 & 0.024 & 37 & 1 & 6.07845 & 0 \\
\hline 1 & 750 & & & & 0 & 0 & 28 & & & & & & & 750 & 774.59 & 119.32 & 10548.54921 & & 1000 & 23.363 & 0.023 & 38 & 1 & 5.966 & 0 \\
\hline 1 & 760 & & & & 0 & 0 & 28 & & & & & & & 760 & 774.59 & 118.36 & 11360.91481 & & 1000 & 23.174 & 0.023 & 39 & 1 & 5.9179 & 0 \\
\hline 1 & 770 & & & & 0 & 0 & 28 & & & & & & & 770 & 774.59 & 117.73 & 12173.27805 & & 1000 & 23.052 & 0.023 & 40 & 1 & 5.8867 & 0 \\
\hline 1 & 780 & & & & 0 & 0 & 28 & & & & & & & 780 & 774.59 & 117.07 & 12982.80443 & & 1000 & 22.923 & 0.023 & 41 & 1 & 5.8537 & 0 \\
\hline 1 & 790 & & & & 0 & 0 & 28 & & & & & & & 790 & 774.59 & 116.52 & 13793.54818 & & 1000 & 22.814 & 0.023 & 42 & 1 & 5.82585 & 0 \\
\hline 1 & 800 & & & & 0 & 0 & 28 & & & & & & & 800 & 774.59 & 116.09 & 14606.72587 & & 1000 & 22.73 & 0.023 & 43 & 1 & 5.80435 & 0 \\
\hline 1 & 820 & & & & 0 & 0 & 28 & & & & & & & 820 & 774.59 & 115.57 & 16248.49235 & & 1000 & 22.629 & 0.023 & 44 & 1 & 5.7785 & 0 \\
\hline 1 & 840 & & & & 0 & 0 & 28 & & & & & & & 840 & 697.13 & 98.34 & 17709.85679 & & 999.99 & 19.256 & 0.021 & 45 & 1 & 4.91715 & 0 \\
\hline 1 & 860 & & & & 0 & 0 & 28 & & & & & & & 860 & 619.67 & 82.74 & 19009.31533 & & 99.99 & 16.2 & 0.02 & 46 & 1 & 4.13695 & 0 \\
\hline 1 & 880 & & & & 0 & 0 & 28 & & & & & & & 880 & 542.21 & 67.76 & 20146.50511 & & 699.99 & 13.268 & 0.019 & 47 & 1 & 3.38805 & 0 \\
\hline 1 & 900 & & & & 0 & 0 & 28 & & & & & & & 900 & 464.75 & 53.5 & 21121.18266 & & 99.99 & 10.475 & 0.017 & 48 & 1 & 2.67485 & 0 \\
\hline 1 & 920 & & & & 0 & 0 & 28 & & & & & & & 920 & 387.3 & 38.65 & 21933.46815 & & 500 & 7.568 & 0.015 & 49 & 1 & 1.93255 & 0 \\
\hline 1 & 940 & & & & 0 & 0 & 28 & & & & & & & 940 & 309.84 & 26.72 & 22583.64274 & & 400 & 5.231 & 0.013 & 50 & 1 & 1.33585 & 0 \\
\hline 1 & 960 & & & & 0 & 0 & 28 & & & & & & & 960 & 232.38 & 19.52 & 23071.63418 & & 300 & 3.822 & 0.013 & 51 & 1 & 0.9761 & 0 \\
\hline 1 & 6980 & & & & 0 & 0 & 28 & & & & & & & 980 & 154.92 & 15.67 & 23397.40948 & & 200 & 3.068 & 0.015 & 52 & 1 & 0.78345 & 0 \\
\hline 1 & & & & & 0 & 0 & 28 & & & & & & & 1000 & 77.46 & 12.33 & 23560.79744 & & 100 & 2.414 & 0.024 & 53 & 1 & 0.61645 & 0 \\
\hline 1 & & & & & 0 & 0 & 28 & & & & & & & 1020 & 69.71 & 14.48 & 23707.93471 & & 90 & 2.836 & 0.032 & 54 & 1 & 0.72415 & 0 \\
\hline 1 & & & & & 0 & 0 & 28 & & & & & & & 1040 & 61.97 & 14.47 & 23837.92832 & & 80 & 2.833 & 0.035 & 55 & 1 & 0.72345 & 0 \\
\hline 1 & & & & & 0 & 0 & 28 & & & & & & & 1060 & 54.22 & 14.2 & 23951.52281 & & 70 & 2.78 & 0.04 & 56 & 1 & 0.71 & 0 \\
\hline 1 & 71080 & & & & 0 & 0 & 28 & & & & & & & 1080 & 46.48 & 13.99 & 24049.07869 & & 60.01 & 2.739 & 0.046 & 57 & 1 & 0.69945 & 0 \\
\hline 1 & 71100 & & & & 0 & 0 & 28 & & & & & & & 1100 & 38.73 & 13.92 & 24130.27551 & & 50 & 2.725 & 0.055 & 58 & 1 & 0.6958 & 0 \\
\hline 1 & 71120 & & & & 0 & 0 & 28 & & & & & & & 1120 & 30.99 & 14.13 & 24195.25464 & & 40.01 & 2.767 & 0.069 & 59 & 1 & 0.70655 & 0 \\
\hline & 71140 & & & & 0 & 0 & 28 & & & & & & & 1140 & 23.24 & 14.24 & 24244.07813 & & 30 & 2.789 & 0.093 & 60 & 1 & 0.7122 & 0 \\
\hline
\end{tabular}




$\begin{array}{rrrrrrrrrrrrrrrr}1 & 7 & 1160 & 15.5 & 13.9 & 24276.62346 & 0 & 0 & 28 & 20.01 & 2.722 & 0.136 & 61 & 1 & 0.6952 & 0 \\ 1 & 7 & 1180 & 7.75 & 13.62 & 24292.96445 & 0 & 0 & 28 & 10.01 & 2.667 & 0.266 & 62 & 1 & 0.6811 & 0 \\ 1 & 8 & 1200 & 6.97 & 15 & 24307.66789 & 0 & 0 & 28 & 9 & 2.936 & 0.326 & 63 & 1 & 0.74975 & \\ 1 & 8 & 1220 & 6.2 & 15.59 & 24320.66309 & 0 & 0 & 28 & 8 & 3.052 & 0.382 & 64 & 1 & 0.77945 & 0 \\ 1 & 8 & 1240 & 5.42 & 15.94 & 24332.02073 & 0 & 0 & 28 & 7 & 3.121 & 0.446 & 65 & 1 & 0.79695 & 0 \\ 1 & 8 & 1260 & 4.64 & 15.94 & 24341.75731 & 0 & 0 & 28 & 5.99 & 3.12 & 0.521 & 66 & 1 & 0.79685 & 0 \\ 1 & 8 & 1280 & 3.87 & 15.43 & 24349.8744 & 0 & 0 & 28 & 5 & 3.022 & 0.604 & 67 & 1 & 0.77165 & 0 \\ 1 & 8 & 1300 & 3.09 & 15.45 & 24356.372 & 0 & 0 & 28 & 3.99 & 3.025 & 0.758 & 68 & 1 & 0.7725 & 0 \\ 1 & 8 & 1320 & 2.32 & 15.73 & 24361.23912 & 0 & 0 & 28 & 3 & 3.079 & 1.026 & 69 & 1 & 0.7863 & 0 \\ 1 & 8 & 1340 & 1.54 & 15.68 & 24364.48517 & 0 & 0 & 28 & 1.99 & 3.07 & 1.543 & 70 & 1 & 0.78405 & 0 \\ 1 & 8 & 1360 & 0.77 & 16.79 & 24366.10937 & 0 & 0 & 28 & 0.99 & 3.287 & 3.32 & 71 & 1 & 0.83925 & 0 \\ 1 & 9 & 1380 & 0.7 & 19.19 & 24367.58435 & 18.18373 & 4.83996 & 28 & 0.9 & 3.757 & 4.174 & 72 & 1 & 0.9594 & 0.206613275 \\ 1 & 9 & 1400 & 0.62 & 19.78 & 24368.88889 & 34.26637 & 8.8475 & 28 & 0.8 & 3.873 & 4.841 & 73 & 1 & 0.989 & 0.11302627 \\ 1 & 9 & 1420 & 0.55 & 19.31 & 24370.03165 & 48.3544 & 12.7854 & 28 & 0.71 & 3.782 & 5.327 & 74 & 1 & 0.9657 & 0.078214185 \\ 1 & 9 & 1440 & 0.47 & 18.79 & 24371.01104 & 60.42848 & 16.42524 & 28 & 0.61 & 3.679 & 6.031 & 75 & 1 & 0.9395 & 0.060881889 \\ 1 & 9 & 1460 & 0.39 & 18.19 & 24371.83021 & 70.52733 & 19.79992 & 28 & 0.5 & 3.562 & 7.124 & 76 & 1 & 0.9096 & 0.050505244 \\ 1 & 9 & 1480 & 0.31 & 17.45 & 24372.48602 & 78.61222 & 23.0062 & 28 & 0.4 & 3.417 & 8.542 & 77 & 1 & 0.8726 & 0.043466525 \\ 1 & 9 & 1500 & 0.24 & 16.29 & 24372.97925 & 84.69283 & 26.54947 & 28 & 0.31 & 3.19 & 10.29 & 78 & 1 & 0.8146 & 0.037665526 \\ 1 & 9 & 1520 & 0.16 & 13.84 & 24373.31068 & 88.77884 & 32.7718 & 28 & 0.21 & 2.709 & 12.9 & 79 & 1 & 0.6918 & 0.030514028 \\ 1 & 9 & 1540 & 0.08 & 10.39 & 24373.48112 & 90.87995 & 44.65843 & 28 & 0.1 & 2.035 & 20.35 & 80 & 1 & 0.51955 & 0.022392178\end{array}$

2004.02.02 187-20 wt\%-D [sonicated] CC-45 0.1-1000 s-1 run 5.txt

Mode Step t[s] n[rpm] M[\%.] Phi[rad] Gamma J[t] T $\left[{ }^{\circ} \mathrm{C}\right] \mathrm{D}[1 / \mathrm{s}] \quad$ Tau[Pa] Eta[Pas] MP\# Block M[mNm] G[Pa]

\begin{tabular}{|c|c|c|c|c|c|c|c|c|c|c|c|c|c|c|}
\hline 1 & 20 & 0.08 & 10.89 & 0.16179 & 1.99459 & 0.93555 & 28 & 0.1 & 2.132 & 21.32 & 1 & 1 & 0.54455 & 1.068891351 \\
\hline 1 & 40 & 0.16 & 13.34 & 0.4893 & 6.0322 & 2.30942 & 28 & 0.21 & 2.612 & 12.438 & 2 & 1 & 0.66695 & 0.433009516 \\
\hline 1 & 60 & 0.24 & 16.49 & 0.97939 & 12.07408 & 3.74042 & 28 & 0.31 & 3.228 & 10.413 & 3 & 1 & 0.8243 & 0.267349562 \\
\hline 1 & 80 & 0.31 & 19.83 & 1.63127 & 20.11055 & 5.18046 & 28 & 0.4 & 3.882 & 9.705 & 4 & 1 & 0.9913 & 0.19303301 \\
\hline 1 & 100 & 0.39 & 22.64 & 2.4473 & 30.17067 & 6.80592 & 28 & 0.5 & 4.433 & 8.866 & 5 & 1 & 1.1321 & 0.146930777 \\
\hline 1 & 120 & 0.47 & 24.51 & 3.42434 & 42.2157 & 8.79677 & 28 & 0.61 & 4.799 & 7.867 & 6 & 1 & 1.2254 & 0.113678087 \\
\hline 1 & 140 & 0.54 & 25.31 & 4.56473 & 56.27469 & 11.35486 & 28 & 0.7 & 4.956 & 7.08 & 7 & 1 & 1.26555 & 0.088068011 \\
\hline 1 & 160 & 0.62 & 25.72 & 5.86614 & 72.31859 & 14.36317 & 28 & 0.8 & 5.035 & 6.294 & 8 & 1 & 1.2858 & 0.069622486 \\
\hline 1 & 1180 & 0.7 & 27.23 & 7.33091 & 90.37646 & 16.94982 & 28 & 0.9 & 5.332 & 5.924 & 9 & 1 & 1.36165 & 0.058997664 \\
\hline 2 & 200 & 0.77 & 32.23 & 8.95118 & 0 & 0 & 28 & 0.99 & 6.311 & 6.375 & 10 & 1 & 1.6115 & 0 \\
\hline 2 & 220 & 1.55 & 45.72 & 12.17367 & 0 & 0 & 28 & 2 & 8.952 & 4.476 & 11 & 1 & 2.2861 & 0 \\
\hline 2 & 240 & 2.32 & 35.46 & 17.03057 & 0 & 0 & 28 & 3 & 6.942 & 2.314 & 12 & 1 & 1.77275 & 0 \\
\hline 2 & 260 & 3.09 & 25.88 & 23.50304 & 0 & 0 & 28 & 3.99 & 5.067 & 1.27 & 13 & 1 & 1.29385 & 0 \\
\hline 2 & 280 & 3.87 & 24.68 & 31.59814 & 0 & 0 & 28 & 5 & 4.832 & 0.966 & 14 & 1 & 1.2338 & 0 \\
\hline 2 & 300 & 4.65 & 26.39 & 41.30959 & 0 & 0 & 28 & 6 & 5.166 & 0.861 & 15 & 1 & 1.3193 & 0 \\
\hline 2 & 320 & 5.42 & 29.27 & 52.65231 & 0 & 0 & 28 & 7 & 5.73 & 0.819 & 16 & 1 & 1.4633 & 0 \\
\hline 2 & 340 & 6.2 & 32.55 & 65.62473 & 0 & 0 & 28 & 8 & 6.373 & 0.797 & 17 & 1 & 1.6275 & 0 \\
\hline 2 & 360 & 6.97 & 33.9 & 80.20722 & 0 & 0 & 28 & 9 & 6.638 & 0.738 & 18 & 1 & 1.69515 & 0 \\
\hline 3 & 380 & 7.75 & 33.64 & 96.56549 & 0 & 0 & 28 & 10.01 & 6.587 & 0.658 & 19 & 1 & 1.68205 & 0 \\
\hline 3 & 3400 & 15.5 & 43.74 & 128.9129 & 0 & 0 & 28 & 20.01 & 8.565 & 0.428 & 20 & 1 & 2.18715 & 0 \\
\hline 3 & 3420 & 23.24 & 44.38 & 177.5102 & 0 & 0 & 28 & 30 & 8.689 & 0.29 & 21 & 1 & 2.2189 & 0 \\
\hline 3 & 3440 & 30.99 & 41.84 & 242.29141 & 0 & 0 & 28 & 40.01 & 8.192 & 0.205 & 22 & 1 & 2.09205 & 0 \\
\hline 3 & 460 & 38.73 & 39.92 & 323.2746 & 0 & 0 & 28 & 50 & 7.817 & 0.156 & 23 & 1 & 1.99625 & 0 \\
\hline 3 & 480 & 46.48 & 38.17 & 420.54302 & 0 & 0 & 28 & 60.01 & 7.473 & 0.125 & 24 & 1 & 1.90835 & 0 \\
\hline 3 & 3500 & 54.22 & 37.17 & 533.91838 & 0 & 0 & 28 & 70 & 7.278 & 0.104 & 25 & 1 & 1.8586 & 0 \\
\hline 3 & 3520 & 61.96 & 35.37 & 663.64181 & 0 & 0 & 28 & 79.99 & 6.926 & 0.087 & 26 & 1 & 1.76865 & 0 \\
\hline 3 & 3540 & 69.71 & 33.03 & 809.41642 & 0 & 0 & 28 & 90 & 6.467 & 0.072 & 27 & 1 & 1.65135 & 0 \\
\hline
\end{tabular}




\begin{tabular}{|c|c|c|c|c|c|c|c|c|c|c|c|c|c|c|}
\hline & 560 & 77.46 & 31.58 & 973.02743 & 0 & 0 & 28 & 100 & 6.183 & 0.062 & 28 & 1 & 1.5788 & 0 \\
\hline & 580 & 154.92 & 41.24 & 1296.35622 & 0 & 0 & 28 & 200 & 8.074 & 0.04 & 29 & 1 & 2.06185 & \\
\hline & 600 & 232.38 & 37.68 & 1781.79433 & 0 & 0 & 28 & 300 & 7.378 & 0.025 & 30 & 1 & 1.8841 & \\
\hline & 620 & 309.84 & 36.41 & 2429.62372 & 0 & 0 & 28 & 400 & 7.129 & 0.018 & 31 & 1 & 1.8205 & \\
\hline & 640 & 387.3 & 53.79 & 3239.31346 & 0 & 0 & 28 & 500 & 10.533 & 0.021 & 32 & 1 & 2.6897 & \\
\hline & 660 & 464.75 & 69.99 & 4211.15101 & 0 & 0 & 28 & 599.99 & 13.705 & 0.023 & 33 & 1 & 3.49965 & \\
\hline & 680 & 542.21 & 79.25 & 5345.99481 & 0 & 0 & 28 & 699.99 & 15.518 & 0.022 & 34 & 1 & 3.96265 & \\
\hline & 700 & 619.67 & 88.08 & 6642.1696 & 0 & 0 & 28 & 799.99 & 17.247 & 0.022 & 35 & 1 & 4.4042 & \\
\hline & 720 & 697.13 & 103.49 & 8100.5354 & 0 & 0 & 28 & 899.99 & 20.263 & 0.023 & 36 & 1 & 5.1744 & \\
\hline & 740 & 774.59 & 118.83 & 9722.71326 & 0 & 0 & 28 & 1000 & 23.267 & 0.023 & 37 & 1 & 5.9414 & \\
\hline & 750 & 774.59 & 116.7 & 10551.38136 & 0 & 0 & 28 & 1000 & 22.849 & 0.023 & 38 & 1 & 5.83475 & \\
\hline & 760 & 774.59 & 115.74 & 11363.7501 & 0 & 0 & 28 & 1000 & 22.662 & 0.023 & 39 & 1 & 5.78695 & \\
\hline & 770 & 774.59 & 115.1 & 12173.68096 & 0 & 0 & 28 & 1000 & 22.537 & 0.023 & 40 & 1 & 5.75505 & \\
\hline & 780 & 774.59 & 114.54 & 12986.04812 & 0 & 0 & 28 & 1000 & 22.427 & 0.022 & 41 & 1 & 5.7271 & \\
\hline & 790 & 774.59 & 113.98 & 13797.19635 & 0 & 0 & 28 & 1000 & 22.317 & 0.022 & 42 & 1 & 5.69885 & \\
\hline & 800 & 774.59 & 113.51 & 14607.93931 & 0 & 0 & 28 & 1000 & 22.226 & 0.022 & 43 & 1 & 5.67565 & \\
\hline & 820 & 774.59 & 112.94 & 16249.2966 & 0 & 0 & 28 & 1000 & 22.113 & 0.022 & 44 & 1 & 5.6469 & \\
\hline & 840 & 697.13 & 96.07 & 17710.2597 & 0 & 0 & 28 & 899.99 & 18.811 & 0.021 & 45 & 1 & 4.80365 & \\
\hline & 860 & 619.67 & 80.82 & 19009.35303 & 0 & 0 & 28 & 799.99 & 15.824 & 0.02 & 46 & 1 & 4.0408 & \\
\hline & 880 & 542.21 & 66.05 & 20147.15228 & 0 & 0 & 28 & 699.99 & 12.933 & 0.018 & 47 & 1 & 3.30265 & \\
\hline & 900 & 464.75 & 52.13 & 21120.85515 & 0 & 0 & 28 & 599.99 & 10.207 & 0.017 & 48 & 1 & 2.60655 & \\
\hline & 920 & 387.3 & 38.3 & 21934.15145 & 0 & 0 & 28 & 500 & 7.5 & 0.015 & 49 & 1 & 1.9152 & \\
\hline & 940 & 309.84 & 26.77 & 22584.36923 & 0 & 0 & 28 & 400 & 5.242 & 0.013 & 50 & 1 & 1.3385 & \\
\hline & 960 & 232.38 & 19.43 & 23072.0795 & 0 & 0 & 28 & 300 & 3.805 & 0.013 & 51 & 1 & 0.9717 & \\
\hline & 980 & 154.92 & 15.45 & 23397.77312 & 0 & 0 & 28 & 200 & 3.024 & 0.015 & 52 & 1 & 0.7723 & \\
\hline & 61000 & 77.46 & 12.12 & 23561.36921 & 0 & 0 & 28 & 100 & 2.373 & 0.024 & 53 & 1 & 0.6059 & \\
\hline & 71020 & 69.71 & 14.25 & 23708.61094 & 0 & 0 & 28 & 90 & 2.79 & 0.031 & 54 & 1 & 0.71235 & \\
\hline & 71040 & 61.97 & 14.12 & 23838.37757 & 0 & 0 & 28 & 80 & 2.765 & 0.035 & 55 & 1 & 0.70605 & \\
\hline & 71060 & 54.22 & 13.97 & 23952.11422 & 0 & 0 & 28 & 70 & 2.734 & 0.039 & 56 & 1 & 0.69825 & \\
\hline & 71080 & 46.48 & 13.82 & 24049.49888 & 0 & 0 & 28 & 60.01 & 2.707 & 0.045 & 57 & 1 & 0.6912 & \\
\hline & 71100 & 38.73 & 13.75 & 24130.8135 & 0 & 0 & 28 & 50 & 2.692 & 0.054 & 58 & 1 & 0.68735 & 0 \\
\hline & 71120 & 30.99 & 14.04 & 24195.76043 & 0 & 0 & 28 & 40.01 & 2.749 & 0.069 & 59 & 1 & 0.7019 & 0 \\
\hline & 71140 & 23.24 & 14.11 & 24244.59571 & 0 & 0 & 28 & 30 & 2.763 & 0.092 & 60 & 1 & 0.7055 & 0 \\
\hline & 71160 & 15.5 & 13.88 & 24277.14182 & 0 & 0 & 28 & 20.01 & 2.717 & 0.136 & 61 & 1 & 0.69385 & 0 \\
\hline & 71180 & 7.75 & 13.5 & 24293.48674 & 0 & 0 & 28 & 10.01 & 2.644 & 0.264 & 62 & 1 & 0.6751 & 0 \\
\hline & 81200 & 6.97 & 14.79 & 24308.18625 & 0 & 0 & 28 & 9 & 2.895 & 0.322 & 63 & 1 & 0.7394 & 0 \\
\hline & 81220 & 6.2 & 15.33 & 24321.1791 & 0 & 0 & 28 & 8 & 3.001 & 0.375 & 64 & 1 & 0.76635 & 0 \\
\hline & 81240 & 5.42 & 15.62 & 24332.5391 & 0 & 0 & 28 & 7 & 3.059 & 0.437 & 65 & 1 & 0.78105 & 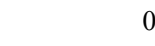 \\
\hline & 81260 & 4.64 & 15.62 & 24342.28039 & 0 & 0 & 28 & 5.99 & 3.058 & 0.511 & 66 & 1 & 0.7808 & 0 \\
\hline & 81280 & 3.87 & 15.11 & 24350.39277 & 0 & 0 & 28 & 5 & 2.959 & 0.592 & 67 & 1 & 0.7557 & 0 \\
\hline & 81300 & 3.09 & 15.06 & 24356.89037 & 0 & 0 & 28 & 3.99 & 2.95 & 0.739 & 68 & 1 & 0.7532 & \\
\hline & 81320 & 2.32 & 15.4 & 24361.75905 & 0 & 0 & 28 & 3 & 3.016 & 1.005 & 69 & 1 & 0.77005 & \\
\hline & 81340 & 1.54 & 15.43 & 24365.00431 & 0 & 0 & 28 & 1.99 & 3.021 & 1.518 & 70 & 1 & 0.77145 & \\
\hline & 81360 & 0.77 & 16.51 & 24366.62616 & 0 & 0 & 28 & 0.99 & 3.232 & 3.265 & 71 & 1 & 0.82535 & \\
\hline & 91380 & 0.7 & 18.3 & 24368.10192 & 18.19342 & 5.07912 & 28 & 0.9 & 3.582 & 3.98 & 72 & 1 & 0.9148 & 0.196884368 \\
\hline & 91400 & 0.62 & 18.97 & 24369.40647 & 34.27605 & 9.22639 & 28 & 0.8 & 3.715 & 4.644 & 73 & 1 & 0.94865 & 0.108384718 \\
\hline & 91420 & 0.55 & 18.66 & 24370.54922 & 48.36409 & 13.23593 & 28 & 0.71 & 3.654 & 5.146 & 74 & 1 & 0.93315 & 0.075551923 \\
\hline & 91440 & 0.47 & 17.84 & 24371.5294 & 60.44785 & 17.30542 & 28 & 0.61 & 3.493 & 5.726 & 75 & 1 & 0.89205 & 0.057785347 \\
\hline & 91460 & 0.39 & 17.32 & 24372.34622 & 70.51765 & 20.79553 & 28 & 0.5 & 3.391 & 6.782 & 76 & 1 & 0.86595 & 0.048087252 \\
\hline & 91480 & 0.31 & 16.33 & 24373.00281 & 78.61222 & 24.58936 & 28 & 0.4 & 3.197 & 7.993 & 77 & 1 & 0.81635 & 0.040667978 \\
\hline
\end{tabular}




\begin{tabular}{|c|c|c|c|c|c|c|c|c|c|c|c|c|c|}
\hline 91500 & 0.24 & 14.67 & 24373.49682 & 84.70251 & 29.48224 & 28 & 0.31 & 2.873 & 9.268 & 78 & 1 & 0.73355 & \\
\hline 91520 & 0.16 & 12.29 & 24373.82905 & 88.79821 & 36.90697 & & 0.21 & 2.406 & 11.457 & 79 & 1 & 0.6145 & 0.027095141 \\
\hline & 0.08 & 9.1 & 24373.99869 & 90.88963 & 51.03289 & & 0.1 & 1.781 & 17.81 & 80 & 1 & 0.4549 & 0.019595195 \\
\hline
\end{tabular}

2004.02.02 187-20 wt\%-D [sonicated] CC-45 0.1-1000 s-1 run 6.txt

Mode Step t[s] n[rpm] M[\%.] Phi[rad] Gamma J[t] T $\left[{ }^{\circ} \mathrm{C}\right] \mathrm{D}[1 / \mathrm{s}] \quad$ Tau[Pa] Eta[Pas $]$ MP\# Block M[mNm] G[Pa $]$

\begin{tabular}{|c|c|c|c|c|c|c|c|c|c|c|c|c|c|c|c|}
\hline & 1 & 20 & 0.08 & 10.33 & 0.16258 & 2.00428 & 0.99124 & 28 & 0.1 & 2.022 & 20.22 & 1 & 1 & 0.51625 & 1.00884108 \\
\hline & 1 & 40 & 0.16 & 12.4 & 0.4893 & 6.0322 & 2.48443 & 28 & 0.21 & 2.428 & 11.562 & 2 & 1 & 0.62 & 0.402506548 \\
\hline & 1 & 60 & 0.24 & 15.42 & 0.97939 & 12.07408 & 4.00069 & 28 & 0.31 & 3.018 & 9.735 & 3 & 1 & 0.77075 & 0.249956933 \\
\hline & 1 & 80 & 0.31 & 18.76 & 1.63206 & 20.12023 & 5.47787 & 28 & 0.4 & 3.673 & 9.183 & 4 & 1 & 0.93805 & 0.182552585 \\
\hline & 1 & 100 & 0.39 & 21.51 & 2.4473 & 30.17067 & 7.16473 & 28 & 0.5 & 4.211 & 8.422 & 5 & 1 & 1.07535 & 0.139572638 \\
\hline & 1 & 120 & 0.47 & 22.87 & 3.42512 & 42.22538 & 9.43162 & 28 & 0.61 & 4.477 & 7.339 & 6 & 1 & 1.14325 & 0.106026281 \\
\hline & 1 & 140 & 0.54 & 22.98 & 4.56473 & 56.27469 & 12.50826 & 28 & 0.7 & 4.499 & 6.427 & 7 & 1 & 1.14885 & 0.079947131 \\
\hline & 1 & 160 & 0.62 & 21.92 & 5.86771 & 72.33796 & 16.85806 & 28 & 0.8 & 4.291 & 5.364 & 8 & 1 & 1.09585 & 0.059318786 \\
\hline & 1 & 180 & 0.7 & 22.65 & 7.33248 & 90.39582 & 20.38237 & 28 & 0.9 & 4.435 & 4.928 & 9 & 1 & 1.1325 & 0.049062003 \\
\hline & 2 & 200 & 0.77 & 26.74 & 8.95275 & 0 & 0 & 28 & 0.99 & 5.235 & 5.288 & 10 & 1 & 1.33685 & 0 \\
\hline & 2 & 220 & 1.55 & 38.61 & 12.1776 & 0 & 0 & 28 & 2 & 7.56 & 3.78 & 11 & 1 & 1.93065 & 0 \\
\hline & 2 & 240 & 2.32 & 31.65 & 17.02665 & 0 & 0 & 28 & 3 & 6.198 & 2.066 & 12 & 1 & 1.58265 & 0 \\
\hline & 2 & 260 & 3.09 & 24.27 & 23.49833 & 0 & 0 & 28 & 3.99 & 4.752 & 1.191 & 13 & 1 & 1.2134 & 0 \\
\hline & 2 & 280 & 3.87 & 23.42 & 31.59814 & 0 & 0 & 28 & 5 & 4.586 & 0.917 & 14 & 1 & 1.171 & 0 \\
\hline & 2 & 300 & 4.65 & 25.02 & 41.3088 & 0 & 0 & 28 & 6 & 4.898 & 0.816 & 15 & 1 & 1.2508 & 0 \\
\hline & 2 & 320 & 5.42 & 27.47 & 52.64838 & 0 & 0 & 28 & 7 & 5.379 & 0.768 & 16 & 1 & 1.3736 & 0 \\
\hline & 2 & 340 & 6.2 & 30.68 & 65.62473 & 0 & 0 & 28 & 8 & 6.006 & 0.751 & 17 & 1 & 1.5338 & 0 \\
\hline & 2 & 360 & 6.97 & 32.86 & 80.20722 & 0 & 0 & 28 & 9 & 6.433 & 0.715 & 18 & 1 & 1.64285 & 0 \\
\hline & 3 & 380 & 7.75 & 33.09 & 96.5702 & 0 & 0 & 28 & 10.01 & 6.479 & 0.647 & 19 & 1 & 1.6545 & 0 \\
\hline & 3 & 400 & 15.5 & 43.08 & 128.90426 & 0 & 0 & 28 & 20.01 & 8.434 & 0.421 & 20 & 1 & 2.15385 & 0 \\
\hline & 3 & 420 & 23.24 & 43.11 & 177.51334 & 0 & 0 & 28 & 30 & 8.441 & 0.281 & 21 & 1 & 2.1556 & 0 \\
\hline & 3 & 440 & 30.99 & 39.86 & 242.24742 & 0 & 0 & 28 & 40.01 & 7.804 & 0.195 & 22 & 1 & 1.99285 & 0 \\
\hline & 3 & 460 & 38.73 & 36.63 & 323.26674 & 0 & 0 & 28 & 50 & 7.172 & 0.143 & 23 & 1 & 1.8314 & 0 \\
\hline & 3 & 480 & 46.48 & 35.61 & 420.45819 & 0 & 0 & 28 & 60.01 & 6.972 & 0.116 & 24 & 1 & 1.78045 & 0 \\
\hline & 3 & 500 & 54.22 & 34.35 & 533.87047 & 0 & 0 & 28 & 70 & 6.727 & 0.096 & 25 & 1 & 1.7177 & 0 \\
\hline & 3 & 520 & 61.96 & 33.15 & 663.68658 & 0 & 0 & 28 & 79.99 & 6.491 & 0.081 & 26 & 1 & 1.65745 & 0 \\
\hline & 3 & 540 & 69.71 & 31.92 & 809.42428 & 0 & 0 & 28 & 90 & 6.249 & 0.069 & 27 & 1 & 1.59585 & 0 \\
\hline & 4 & 560 & 77.46 & 31.64 & 973.03528 & 0 & 0 & 28 & 100 & 6.195 & 0.062 & 28 & 1 & 1.582 & 0 \\
\hline & 4 & 580 & 154.92 & 41.71 & 1296.60833 & 0 & 0 & 28 & 200 & 8.166 & 0.041 & 29 & 1 & 2.0854 & 0 \\
\hline & 4 & 600 & 232.38 & 36.19 & 1781.7205 & 0 & 0 & 28 & 300 & 7.086 & 0.024 & 30 & 1 & 1.80955 & 0 \\
\hline & 4 & 620 & 309.84 & 38.53 & 2429.42737 & 0 & 0 & 28 & 400 & 7.544 & 0.019 & 31 & 1 & 1.92635 & 0 \\
\hline & 4 & 640 & 387.3 & 53.31 & 3239.56401 & 0 & 0 & 28 & 500 & 10.439 & 0.021 & 32 & 1 & 2.6657 & 0 \\
\hline & 4 & 660 & 464.75 & 67.84 & 4212.41943 & 0 & 0 & 28 & 599.99 & 13.283 & 0.022 & 33 & 1 & 3.3919 & 0 \\
\hline & 4 & 680 & 542.21 & 78.11 & 5345.83773 & 0 & 0 & 28 & 699.99 & 15.293 & 0.022 & 34 & 1 & 3.90525 & 0 \\
\hline & 4 & 700 & 619.67 & 88.78 & 6642.05729 & 0 & 0 & 28 & 799.99 & 17.384 & 0.022 & 35 & 1 & 4.43915 & 0 \\
\hline & 4 & 720 & 697.13 & 104.7 & 8101.51871 & 0 & 0 & 28 & 899.99 & 20.499 & 0.023 & 36 & 1 & 5.2348 & 0 \\
\hline & 4 & 740 & 774.59 & 119.82 & 9723.29052 & 0 & 0 & 28 & 1000 & 23.461 & 0.023 & 37 & 1 & 5.99105 & 0 \\
\hline & 5 & 750 & 774.59 & 117.47 & 10552.81157 & 0 & 0 & 28 & 1000 & 23.001 & 0.023 & 38 & 1 & 5.8736 & 0 \\
\hline & 5 & 760 & 774.59 & 114.83 & 11362.33874 & 0 & 0 & 28 & 1000 & 22.484 & 0.022 & 39 & 1 & 5.7416 & 0 \\
\hline & 5 & 770 & 774.59 & 114.92 & 12175.10803 & 0 & 0 & 28 & 1000 & 22.501 & 0.023 & 40 & 1 & 5.74585 & 0 \\
\hline & 5 & 780 & 774.59 & 114.21 & 12984.63598 & 0 & 0 & 28 & 1000 & 22.363 & 0.022 & 41 & 1 & 5.71065 & 0 \\
\hline & 5 & 790 & 774.59 & 113.67 & 13794.97367 & 0 & 0 & 28 & 1000 & 22.257 & 0.022 & 42 & 1 & 5.68355 & 0 \\
\hline & 5 & 800 & 774.59 & 113.25 & 14608.14823 & 0 & 0 & 28 & 1000 & 22.175 & 0.022 & 43 & 1 & 5.66265 & 0 \\
\hline & 6 & 820 & 774.59 & 110.17 & 16249.91549 & 0 & 0 & 28 & 1000 & 21.571 & 0.022 & 44 & 1 & 5.5084 & 0 \\
\hline
\end{tabular}




\begin{tabular}{|c|c|c|c|c|c|c|c|c|c|c|c|c|c|c|}
\hline & 840 & 697.13 & 95.96 & 17710.5503 & 0 & 0 & 28 & 899.99 & 18.79 & 0.021 & 45 & 1 & 4.79815 & 0 \\
\hline & 860 & 619.67 & 80.7 & 19009.64677 & 0 & 0 & 28 & 799.99 & 15.801 & 0.02 & 46 & 1 & 4.03495 & 0 \\
\hline & 880 & 542.21 & 65.98 & 20146.5114 & 0 & 0 & 28 & 699.99 & 12.919 & 0.018 & 47 & 1 & 3.299 & 0 \\
\hline & 6900 & 464.75 & 52.06 & 21121.91544 & 0 & 0 & 28 & 599.99 & 10.193 & 0.017 & 48 & 1 & 2.6029 & 0 \\
\hline & $6 \quad 920$ & 387.3 & 38.04 & 21934.20093 & 0 & 0 & 28 & 500 & 7.447 & 0.015 & 49 & 1 & 1.9018 & \\
\hline & 6940 & 309.84 & 26.71 & 22584.74151 & 0 & 0 & 28 & 400 & 5.229 & 0.013 & 50 & 1 & 1.33535 & \\
\hline & $6 \quad 960$ & 232.38 & 19.43 & 23072.33554 & 0 & 0 & 28 & 300 & 3.804 & 0.013 & 51 & 1 & 0.9714 & \\
\hline & $6 \quad 980$ & 154.92 & 15.39 & 23397.90507 & 0 & 0 & 28 & 200 & 3.014 & 0.015 & 52 & 1 & 0.7697 & \\
\hline & 61000 & 77.46 & 11.99 & 23561.49722 & 0 & 0 & 28 & 100 & 2.347 & 0.023 & 53 & 1 & 0.5994 & \\
\hline & 71020 & 69.71 & 14.13 & 23709.00521 & 0 & 0 & 28 & 90 & 2.766 & 0.031 & 54 & 1 & 0.70635 & \\
\hline & 71040 & 61.97 & 14.09 & 23838.84095 & 0 & 0 & 28 & 80 & 2.758 & 0.034 & 55 & 1 & 0.7044 & \\
\hline & 71060 & 54.22 & 13.82 & 23952.66242 & 0 & 0 & 28 & 70 & 2.707 & 0.039 & 56 & 1 & 0.6912 & 0 \\
\hline & 71080 & 46.48 & 13.62 & 24050.07222 & 0 & 0 & 28 & 60.01 & 2.666 & 0.044 & 57 & 1 & 0.68075 & 0 \\
\hline & 71100 & 38.73 & 13.51 & 24131.34993 & 0 & 0 & 28 & 50 & 2.646 & 0.053 & 58 & 1 & 0.6757 & 0 \\
\hline & 71120 & 30.99 & 13.79 & 24196.29686 & 0 & 0 & 28 & 40.01 & 2.699 & 0.067 & 59 & 1 & 0.68935 & 0 \\
\hline & 71140 & 23.24 & 13.93 & 24245.09601 & 0 & 0 & 28 & 30 & 2.727 & 0.091 & 60 & 1 & 0.6965 & 0 \\
\hline & 71160 & 15.5 & 13.66 & 24277.64526 & 0 & 0 & 28 & 20.01 & 2.674 & 0.134 & 61 & 1 & 0.68275 & 0 \\
\hline & 71180 & 7.75 & 13.4 & 24294.0106 & 0 & 0 & 28 & 10.01 & 2.623 & 0.262 & 62 & 1 & 0.6698 & 0 \\
\hline & 81200 & 6.97 & 14.67 & 24308.70697 & 0 & 0 & 28 & 9 & 2.872 & 0.319 & 63 & 1 & 0.73345 & 0 \\
\hline & 81220 & 6.2 & 15.28 & 24321.70531 & 0 & 0 & 28 & 8 & 2.991 & 0.374 & 64 & 1 & 0.7639 & 0 \\
\hline & 81240 & 5.42 & 15.51 & 24333.05903 & 0 & 0 & 28 & 7 & 3.036 & 0.434 & 65 & 1 & 0.77535 & 0 \\
\hline & 81260 & 4.64 & 15.53 & 24342.79482 & 0 & 0 & 28 & 5.99 & 3.04 & 0.508 & 66 & 1 & 0.77635 & 0 \\
\hline & 81280 & 3.87 & 15.23 & 24350.91034 & 0 & 0 & 28 & 5 & 2.981 & 0.596 & 67 & 1 & 0.76135 & 0 \\
\hline & 81300 & 3.09 & 15.05 & 24357.40559 & 0 & 0 & 28 & 3.99 & 2.946 & 0.738 & 68 & 1 & 0.75225 & 0 \\
\hline & 81320 & 2.32 & 15.33 & 24362.27741 & 0 & 0 & 28 & 3 & 3.002 & 1.001 & 69 & 1 & 0.7666 & 0 \\
\hline & 81340 & 1.54 & 15.44 & 24365.52189 & 0 & 0 & 28 & 1.99 & 3.023 & 1.519 & 70 & 1 & 0.772 & 0 \\
\hline & 81360 & 0.77 & 16.4 & 24367.14452 & 0 & 0 & 28 & 0.99 & 3.211 & 3.243 & 71 & 1 & 0.81985 & 0 \\
\hline & 91380 & 0.7 & 18.75 & 24368.61793 & 18.16437 & 4.94672 & 28 & 0.9 & 3.672 & 4.08 & 72 & 1 & 0.93765 & 0.202153997 \\
\hline & 91400 & 0.62 & 19.18 & 24369.92326 & 34.25668 & 9.12295 & 28 & 0.8 & 3.755 & 4.694 & 73 & 1 & 0.95885 & 0.109613658 \\
\hline & 91420 & 0.55 & 18.59 & 24371.06602 & 48.34472 & 13.28516 & 28 & 0.71 & 3.639 & 5.125 & 74 & 1 & 0.92925 & 0.075271922 \\
\hline & 91440 & 0.47 & 17.3 & 24372.04698 & 60.43816 & 17.84415 & 28 & 0.61 & 3.387 & 5.552 & 75 & 1 & 0.865 & 0.056040753 \\
\hline & 91460 & 0.39 & 16.25 & 24372.86379 & 70.50796 & 22.16534 & 28 & 0.5 & 3.181 & 6.362 & 76 & 1 & 0.8124 & 0.045115473 \\
\hline & 91480 & 0.31 & 15.21 & 24373.52039 & 78.60253 & 26.40326 & 28 & 0.4 & 2.977 & 7.442 & 77 & 1 & 0.76025 & 0.037874099 \\
\hline & 91500 & 0.24 & 13.93 & 24374.01362 & 84.68315 & 31.05358 & 28 & 0.31 & 2.727 & 8.797 & 78 & 1 & 0.69645 & 0.032202392 \\
\hline & 91520 & 0.16 & 11.73 & 24374.34584 & 88.77884 & 38.66673 & 28 & 0.21 & 2.296 & 10.933 & 79 & 1 & 0.58625 & 0.025862018 \\
\hline & 91540 & 0.08 & 8.61 & 24374.51549 & 90.87026 & 53.92891 & 28 & 0.1 & 1.685 & 16.85 & 80 & 1 & 0.4303 & 0.01854292 \\
\hline
\end{tabular}



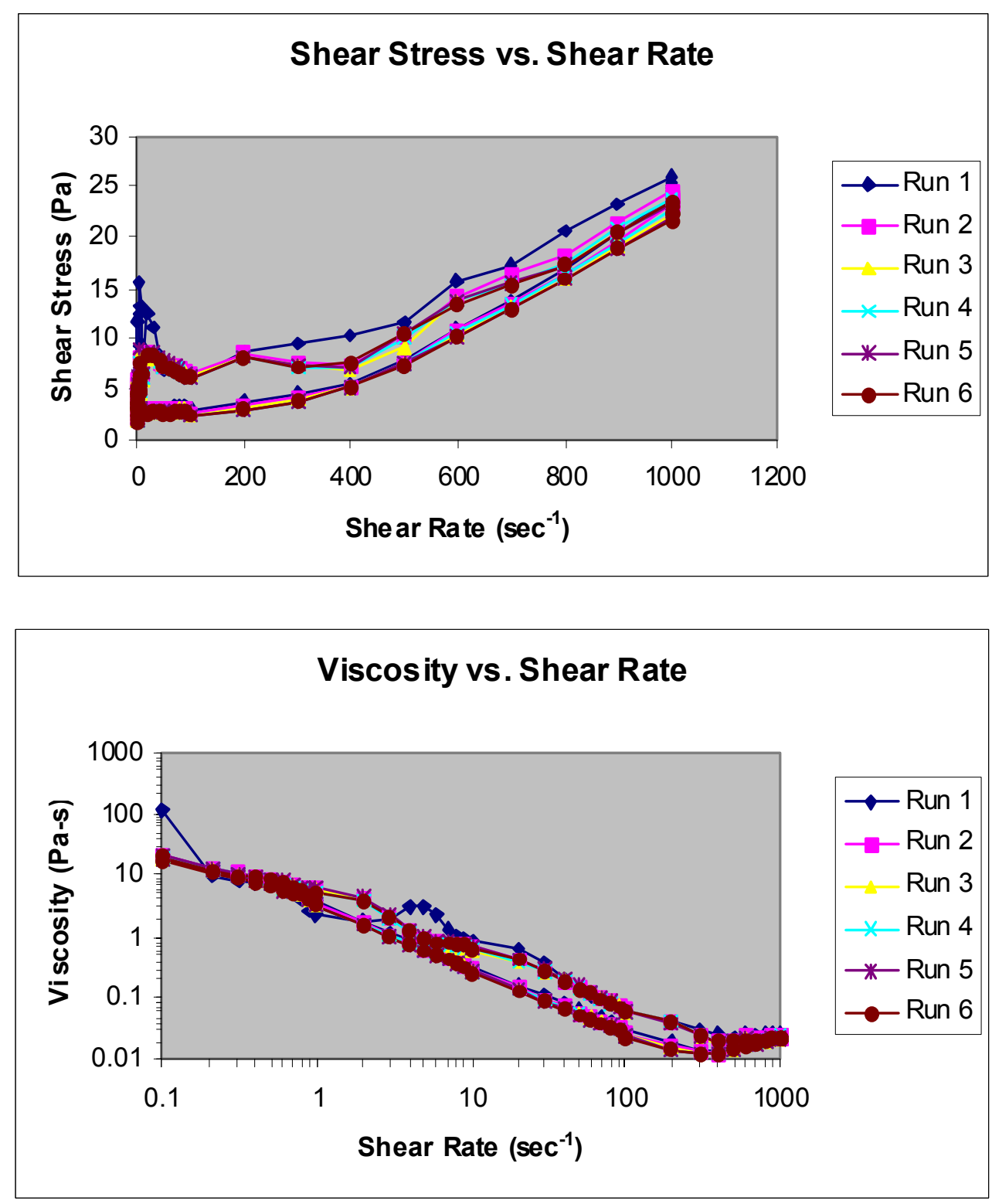

\section{E-2.1.21 META/20wt\%/U/N}

2004.01.22 META-20 wt\%-U [not sonicated] 0.1-1000 s-1 CC-45 run 1.txt

Mode Step t[s] n[rpm] M[\%.] Phi[rad]

Gamma J[t]

$\left.\mathrm{T}\left[{ }^{\circ} \mathrm{C}\right] \mathrm{D}[1 / \mathrm{s}] \quad \mathrm{Tau}[\mathrm{Pa}] \mathrm{Eta}[\mathrm{Pas}] \mathrm{MP \#} \mathrm{Block} \mathrm{M[mNm]} \mathrm{G[Pa}\right]$

\begin{tabular}{|c|c|c|c|c|c|c|c|c|c|c|c|c|c|c|}
\hline 1 & 20 & 0.08 & 5.47 & 0.16336 & 2.01396 & 1.8822 & 28 & 0.1 & 1.07 & 10.7 & 1 & 1 & 0.27335 & 0.531291585 \\
\hline 1 & 40 & 0.16 & 7 & 0.49166 & 6.06125 & 4.42427 & 28 & 0.21 & 1.37 & 6.524 & 2 & 1 & 0.34995 & 0.226025985 \\
\hline 1 & 60 & 0.24 & 15.86 & 0.97389 & 12.0063 & 3.86552 & 28 & 0.31 & 3.106 & 10.019 & 3 & 1 & 0.79315 & 0.258697517 \\
\hline 1 & 80 & 0.31 & 35.7 & 1.62185 & 19.99436 & 2.86083 & 28 & 0.4 & 6.989 & 17.473 & 4 & 1 & 1.78485 & 0.349548573 \\
\hline 1 & 100 & 0.39 & 59.09 & 2.43159 & 29.97702 & 2.59115 & 28 & 0.5 & 11.569 & 23.138 & 5 & 1 & 2.95425 & 0.385928955 \\
\hline 1 & 120 & 0.47 & 75.15 & 3.40549 & 41.98332 & 2.8531 & 28 & 0.61 & 14.715 & 24.123 & 6 & 1 & 3.75755 & 0.35049634 \\
\hline 1 & 140 & 0.54 & 72.03 & 4.55138 & 56.11009 & 3.97831 & 28 & 0.7 & 14.104 & 20.149 & 7 & 1 & 3.60165 & 0.25136299 \\
\hline 1 & 160 & 0.62 & 48.22 & 5.86221 & 72.27018 & 7.65412 & 27.9 & 0.8 & 9.442 & 11.803 & 8 & 1 & 2.41115 & 0.13064863 \\
\hline 1 & 180 & 0.7 & 32.74 & 7.33248 & 90.39582 & 14.10231 & 28 & 0.9 & 6.41 & 7.122 & 9 & 1 & 1.6369 & 0.070910358 \\
\hline
\end{tabular}




\begin{tabular}{|c|c|c|c|c|}
\hline & 2200 & 0.77 & 25.6 & 8.9559 \\
\hline & 2220 & 1.55 & 39.81 & 12.17446 \\
\hline & 2240 & 2.32 & 48.36 & 17.02115 \\
\hline & 2260 & 3.09 & 84.34 & 23.47005 \\
\hline & 2280 & 3.84 & 227.19 & 31.49761 \\
\hline & 2300 & 4.66 & 304.03 & 41.22791 \\
\hline & 2320 & 5.43 & 268.03 & 52.5879 \\
\hline & 2340 & 6.2 & 225.77 & 65.5509 \\
\hline & 2360 & 6.97 & 204.9 & 80.13496 \\
\hline & $3 \quad 380$ & 7.75 & 189.56 & 96.51522 \\
\hline & 400 & 15.5 & 234.62 & 128.81944 \\
\hline & 420 & 23.24 & 217.72 & 177.37511 \\
\hline & 440 & 30.99 & 212.91 & 242.15161 \\
\hline & 460 & 38.73 & 218.06 & 323.25103 \\
\hline & 480 & 46.48 & 223.12 & 420.3718 \\
\hline & 500 & 54.22 & 227.64 & 533.86026 \\
\hline & 520 & 61.96 & 232.27 & 663.58134 \\
\hline & 540 & 69.71 & 238.67 & 809.45962 \\
\hline & 560 & 77.46 & 242.07 & 973.06984 \\
\hline & 580 & 154.92 & 333.73 & 1296.62561 \\
\hline & 600 & 232.38 & 387.29 & 1781.68987 \\
\hline & 620 & 309.84 & 416.76 & 2429.55461 \\
\hline & 640 & 387.3 & 430.61 & 3239.28597 \\
\hline & 660 & 464.75 & 434.9 & 4212.09899 \\
\hline & 680 & 542.21 & 435.22 & 5346.33567 \\
\hline & 700 & 619.67 & 436.15 & 6642.51361 \\
\hline & 720 & 697.13 & 437.54 & 8101.2454 \\
\hline & 740 & 774.59 & 452.23 & 9720.98459 \\
\hline & 750 & 774.59 & 447.98 & 10550.44045 \\
\hline & 760 & 774.59 & 434.27 & 11361.18577 \\
\hline & 770 & 774.59 & 424.91 & 12172.33557 \\
\hline & 780 & 774.59 & 417.96 & 12983.0801 \\
\hline & 790 & 774.59 & 411.61 & 13794.63517 \\
\hline & 800 & 774.59 & 403.03 & 14604.16312 \\
\hline & 820 & 774.59 & 395.06 & 16245.93116 \\
\hline & 840 & 697.13 & 351.67 & 17706.93668 \\
\hline & 860 & 619.67 & 313.24 & 19005.10481 \\
\hline & 880 & 542.21 & 277.7 & 20143.15068 \\
\hline & 900 & 464.75 & 244.17 & 21117.62717 \\
\hline & 920 & 387.3 & 212.21 & 21929.46812 \\
\hline & 940 & 309.84 & 180.57 & 22579.85162 \\
\hline & 960 & 232.38 & 148.21 & 23068.20749 \\
\hline & $6 \quad 980$ & 154.92 & 113.29 & 23393.76681 \\
\hline & 61000 & 77.46 & 73.32 & 23557.30084 \\
\hline & 71020 & 69.71 & 68.87 & 23704.74835 \\
\hline & 71040 & 61.97 & 64.26 & 23834.67834 \\
\hline & 71060 & 54.22 & 58.98 & 23948.32546 \\
\hline & 71080 & 46.48 & 53.38 & 24045.73211 \\
\hline & 71100 & 38.73 & 47.19 & 24127.02788 \\
\hline & 71120 & 30.99 & 41.32 & 24192.05728 \\
\hline
\end{tabular}

\begin{tabular}{|c|c|c|c|c|c|c|}
\hline 0 & 28 & 0.99 & 5.012 & 5.063 & 10 & 1.2799 \\
\hline 0 & 28 & 2 & 7.795 & 3.898 & 11 & 1.99045 \\
\hline 0 & 28 & 3 & 9.47 & 3.157 & 12 & 2.4182 \\
\hline 0 & 28 & 3.99 & 16.514 & 4.139 & 13 & 4.217 \\
\hline 0 & 27.9 & 4.96 & 44.484 & 8.969 & 14 & 111.35955 \\
\hline 0 & 28 & 6.02 & 59.528 & 9.888 & 15 & 115.20135 \\
\hline 0 & 28 & 7.01 & 52.48 & 7.486 & 16 & $1 \quad 13.4014$ \\
\hline 0 & 28 & 8 & 44.205 & 5.526 & 17 & 111.28835 \\
\hline 0 & 28 & 9 & 40.12 & 4.458 & 18 & $1 \quad 10.2451$ \\
\hline 0 & 28 & 10.01 & 37.116 & 3.708 & 19 & 9.47795 \\
\hline 0 & 28 & 20.01 & 45.939 & 2.296 & 20 & 11.7311 \\
\hline 0 & 28 & 30 & 42.63 & 1.421 & 21 & $1 \quad 10.8861$ \\
\hline 0 & 28 & 40.01 & 41.688 & 1.042 & 22 & 110.64545 \\
\hline 0 & 27.9 & 50 & 42.696 & 0.854 & 23 & 110.9029 \\
\hline 0 & 27.9 & 60.01 & 43.686 & 0.728 & 24 & 111.1558 \\
\hline 0 & 27.9 & 70 & 44.572 & 0.637 & 25 & 111.38205 \\
\hline 0 & 27.9 & 79.99 & 45.478 & 0.569 & 26 & 111.6135 \\
\hline 0 & 27.9 & 90 & 46.731 & 0.519 & 27 & 111.93325 \\
\hline 0 & 28 & 100 & 47.398 & 0.474 & 28 & $1 \quad 12.1037$ \\
\hline 0 & 27.9 & 200 & 65.343 & 0.327 & 29 & 116.68625 \\
\hline 0 & 28 & 300 & 75.832 & 0.253 & 30 & 119.36465 \\
\hline 0 & 28 & 400 & 81.602 & 0.204 & 31 & 120.83805 \\
\hline 0 & 28 & 500 & 84.314 & 0.169 & 32 & 121.53065 \\
\hline 0 & 28 & 599.99 & 85.153 & 0.142 & 33 & 121.7448 \\
\hline 0 & 28 & 699.99 & 85.216 & 0.122 & 34 & 21.7611 \\
\hline 0 & 28 & 799.99 & 85.397 & 0.107 & 35 & 21.8073 \\
\hline 0 & 28 & 899.99 & 85.671 & 0.095 & 36 & 121.8772 \\
\hline 0 & 28 & 1000 & 88.546 & 0.089 & 37 & 122.61145 \\
\hline 0 & 28 & 1000 & 87.715 & 0.088 & 38 & $1 \quad 22.3992$ \\
\hline 0 & 28 & 1000 & 85.03 & 0.085 & 39 & 121.71355 \\
\hline 0 & 28 & 1000 & 83.197 & 0.083 & 40 & $1 \quad 21.2453$ \\
\hline 0 & 28 & 1000 & 81.837 & 0.082 & 41 & 20.8981 \\
\hline 0 & 28 & 1000 & 80.592 & 0.081 & 42 & 120.58025 \\
\hline 0 & 28 & 1000 & 78.914 & 0.079 & 43 & 120.1517 \\
\hline 0 & 28 & 1000 & 77.353 & 0.077 & 44 & 119.75295 \\
\hline 0 & 28 & 899.99 & 68.856 & 0.077 & 45 & 117.58325 \\
\hline 0 & 28 & 799.99 & 61.332 & 0.077 & 46 & 115.66195 \\
\hline 0 & 28 & 699.99 & 54.374 & 0.078 & 47 & 13.885 \\
\hline 0 & 28 & 599.99 & 47.809 & 0.08 & 48 & $1 \quad 12.2086$ \\
\hline 0 & 28 & 500 & 41.551 & 0.083 & 49 & 110.61065 \\
\hline 0 & 28 & 400 & 35.356 & 0.088 & 50 & 9.0285 \\
\hline 0 & 28 & 300 & 29.02 & 0.097 & 51 & 7.4106 \\
\hline 0 & 28 & 200 & 22.181 & 0.111 & 52 & 5.66425 \\
\hline 0 & 28 & 100 & 14.356 & 0.144 & 53 & 3.6659 \\
\hline 0 & 28 & 90 & 13.484 & 0.15 & 54 & 3.4434 \\
\hline 0 & 28 & 80 & 12.583 & 0.157 & 55 & 13.21315 \\
\hline 0 & 28 & 70 & 11.549 & 0.165 & 56 & 2.9491 \\
\hline 0 & 28 & 60.01 & 10.451 & 0.174 & 57 & 2.6688 \\
\hline 0 & 28 & 50 & 9.239 & 0.185 & 58 & 12.35935 \\
\hline 0 & 28 & 40.01 & 8.091 & 0.202 & 59 & 12.06605 \\
\hline
\end{tabular}




$\begin{array}{rrrrrrrrrrrrrrrr}1 & 7 & 1140 & 23.24 & 33.51 & 24240.78495 & 0 & 0 & 28 & 30 & 6.562 & 0.219 & 60 & 1 & 1.6757 & 0 \\ 1 & 7 & 1160 & 15.5 & 24.99 & 24273.37191 & 0 & 0 & 28 & 20.01 & 4.893 & 0.245 & 61 & 1 & 1.2494 & 0 \\ 1 & 7 & 1180 & 7.75 & 14.02 & 24289.73175 & 0 & 0 & 28 & 10.01 & 2.746 & 0.274 & 62 & 1 & 0.7011 & 0 \\ 1 & 8 & 1200 & 6.97 & 13.63 & 24304.43991 & 0 & 0 & 28 & 9 & 2.669 & 0.297 & 63 & 1 & 0.68145 & 0 \\ 1 & 8 & 1220 & 6.2 & 12.69 & 24317.4194 & 0 & 0 & 28 & 8 & 2.484 & 0.311 & 64 & 1 & 0.6344 & 0 \\ 1 & 8 & 1240 & 5.42 & 11.61 & 24328.78803 & 0 & 0 & 28 & 7 & 2.273 & 0.325 & 65 & 1 & 0.5805 & 0 \\ 1 & 8 & 1260 & 4.64 & 10.6 & 24338.52461 & 0 & 0 & 28 & 5.99 & 2.075 & 0.346 & 66 & 1 & 0.52985 & 0 \\ 1 & 8 & 1280 & 3.87 & 9.23 & 24346.64563 & 0 & 0 & 28 & 5 & 1.807 & 0.361 & 67 & 1 & 0.46155 & 0 \\ 1 & 8 & 1300 & 3.09 & 8.1 & 24353.14009 & 0 & 0 & 28 & 3.99 & 1.586 & 0.397 & 68 & 1 & 0.4049 & 0 \\ 1 & 8 & 1320 & 2.32 & 6.43 & 24358.00799 & 0 & 0 & 28 & 3 & 1.258 & 0.419 & 69 & 1 & 0.3213 & 0 \\ 1 & 8 & 1340 & 1.54 & 4.1 & 24361.25718 & 0 & 0 & 28 & 1.99 & 0.803 & 0.404 & 70 & 1 & 0.205 & 0 \\ 1 & 8 & 1360 & 0.77 & 2.66 & 24362.88138 & 0 & 0 & 28 & 0.99 & 0.521 & 0.526 & 71 & 1 & 0.133 & 0 \\ 1 & 9 & 1380 & 0.7 & 3.61 & 24364.35557 & 18.17405 & 25.70583 & 28 & 0.9 & 0.707 & 0.786 & 72 & 1 & 0.1805 & 0.038901621 \\ 1 & 9 & 1400 & 0.62 & 4.01 & 24365.66012 & 34.25668 & 43.63903 & 28 & 0.8 & 0.785 & 0.981 & 73 & 1 & 0.20055 & 0.022915239 \\ 1 & 9 & 1420 & 0.55 & 4.32 & 24366.80209 & 48.33504 & 57.13355 & 28 & 0.71 & 0.846 & 1.192 & 74 & 1 & 0.21595 & 0.01750283 \\ 1 & 9 & 1440 & 0.47 & 4.7 & 24367.78227 & 60.4188 & 65.67254 & 28 & 0.61 & 0.92 & 1.508 & 75 & 1 & 0.23495 & 0.015227049 \\ 1 & 9 & 1460 & 0.39 & 5.83 & 24368.60065 & 70.50796 & 61.79483 & 28 & 0.5 & 1.141 & 2.282 & 76 & 1 & 0.2914 & 0.01618257 \\ 1 & 9 & 1480 & 0.31 & 4.36 & 24369.25646 & 78.59285 & 92.13688 & 28 & 0.4 & 0.853 & 2.133 & 77 & 1 & 0.21795 & 0.010853405 \\ 1 & 9 & 1500 & 0.24 & 4.11 & 24369.7489 & 84.66378 & 105.17227 & 28 & 0.31 & 0.805 & 2.597 & 78 & 1 & 0.20555 & 0.009508198 \\ 1 & 9 & 1520 & 0.16 & 3.63 & 24370.08034 & 88.74979 & 124.82372 & 28 & 0.21 & 0.711 & 3.386 & 79 & 1 & 0.1815 & 0.008011287 \\ 1 & 9 & 1540 & 0.08 & 1.85 & 24370.2492 & 90.83153 & 250.91514 & 28 & 0.1 & 0.362 & 3.62 & 80 & 1 & 0.0924 & 0.0039854\end{array}$

2004.01.22 META-20 wt\%-U [not sonicated] 0.1-1000 s-1 CC-45 run 2.txt Mode Step t[s] n[rpm] M[\%.] Phi[rad

Gamma J[t]

\begin{tabular}{|c|c|c|c|c|c|c|c|c|c|c|c|c|c|c|}
\hline 1 & 20 & 0.08 & 3.97 & 0.16415 & 2.02364 & 2.60442 & 28 & 0.1 & 0.777 & 7.77 & 1 & 1 & 0.1985 & 0.383961574 \\
\hline 1 & 40 & 0.16 & 6.66 & 0.49166 & 6.06125 & 4.64819 & 28 & 0.21 & 1.304 & 6.21 & 2 & 1 & 0.33295 & 0.215137142 \\
\hline 1 & 60 & 0.24 & 8.69 & 0.98253 & 12.11281 & 7.11681 & 28 & 0.31 & 1.702 & 5.49 & 3 & 1 & 0.4347 & 0.1405124 \\
\hline 1 & 80 & 0.31 & 10.78 & 1.6352 & 20.15896 & 9.554 & 28 & 0.4 & 2.11 & 5.275 & 4 & 1 & 0.5388 & 0.104668098 \\
\hline 1 & 100 & 0.39 & 12.62 & 2.45044 & 30.2094 & 12.22557 & 28 & 0.5 & 2.471 & 4.942 & 5 & 1 & 0.63095 & 0.081795732 \\
\hline 1 & 120 & 0.47 & 14.5 & 3.42748 & 42.25443 & 14.88356 & 28 & 0.61 & 2.839 & 4.654 & 6 & 1 & 0.72485 & 0.067188221 \\
\hline 1 & 140 & 0.54 & 15.95 & 4.56788 & 56.31342 & 18.02606 & 28 & 0.7 & 3.124 & 4.463 & 7 & 1 & 0.79765 & 0.055475231 \\
\hline 1 & 160 & 0.62 & 17.17 & 5.87007 & 72.367 & 21.52498 & 27.9 & 0.8 & 3.362 & 4.203 & 8 & 1 & 0.85845 & 0.04645764 \\
\hline 1 & 180 & 0.7 & 18.75 & 7.33483 & 90.42487 & 24.63892 & 28 & 0.9 & 3.67 & 4.078 & 9 & 1 & 0.9373 & 0.040586179 \\
\hline 2 & 200 & 0.77 & 19 & 8.95825 & 0 & 0 & 28 & 0.99 & 3.72 & 3.758 & 10 & 1 & 0.95005 & \\
\hline 2 & 220 & 1.55 & 30.37 & 12.18074 & 0 & 0 & 28 & 2 & 5.947 & 2.974 & 11 & 1 & 1.5187 & \\
\hline 2 & 240 & 2.32 & 31.73 & 17.02665 & 0 & 0 & 28 & 3 & 6.213 & 2.071 & 12 & 1 & 1.5866 & \\
\hline 2 & 260 & 3.09 & 29.38 & 23.50147 & 0 & 0 & 28 & 3.99 & 5.752 & 1.442 & 13 & 1 & 1.46885 & \\
\hline 2 & 280 & 3.87 & 27.99 & 31.59657 & 0 & 0 & 28 & 5 & 5.48 & 1.096 & 14 & 1 & 1.3995 & \\
\hline 2 & 300 & 4.65 & 27.55 & 41.30802 & 0 & 0 & 28 & 6 & 5.394 & 0.899 & 15 & 1 & 1.37745 & \\
\hline 2 & 320 & 5.42 & 27.77 & 52.64995 & 0 & 0 & 28 & 7 & 5.438 & 0.777 & 16 & 1 & 1.3886 & \\
\hline 2 & 340 & 6.2 & 28.36 & 65.61687 & 0 & 0 & 28 & 8 & 5.554 & 0.694 & 17 & 1 & 1.4182 & \\
\hline 2 & 360 & 6.97 & 29.28 & 80.20722 & 0 & 0 & 28 & 9 & 5.732 & 0.637 & 18 & 1 & 1.46375 & \\
\hline 3 & 380 & 7.75 & 29.74 & 96.5812 & 0 & 0 & 28 & 10.01 & 5.822 & 0.582 & 19 & 1 & 1.4868 & \\
\hline 3 & 400 & 15.5 & 43.52 & 128.89091 & 0 & 0 & 28 & 20.01 & 8.521 & 0.426 & 20 & 1 & 2.17605 & \\
\hline 3 & 420 & 23.24 & 50.43 & 177.4505 & 0 & 0 & 28 & 30 & 9.875 & 0.329 & 21 & 1 & 2.5217 & \\
\hline 3 & 440 & 30.99 & 52.14 & 242.2435 & 0 & 0 & 28 & 40.01 & 10.21 & 0.255 & 22 & 1 & 2.60715 & \\
\hline 3 & 460 & 38.73 & 54 & 323.26203 & 0 & 0 & 28 & 50 & 10.573 & 0.211 & 23 & 1 & 2.69995 & \\
\hline 3 & 480 & 46.48 & 58.47 & 420.42756 & 0 & 0 & 28 & 60.01 & 11.448 & 0.191 & 24 & 1 & 2.9234 & \\
\hline 3 & 500 & 54.22 & 63.26 & 533.95294 & 0 & 0 & 28 & 70 & 12.386 & 0.177 & 25 & 1 & 3.16295 & \\
\hline 3 & 520 & 61.96 & 68.34 & 663.6426 & 0 & 0 & 28 & 79.99 & 13.38 & 0.167 & 26 & 1 & 3.4168 & \\
\hline
\end{tabular}




\begin{tabular}{|c|c|c|c|c|c|c|c|c|c|c|c|c|c|c|}
\hline & 540 & 69.71 & 71.97 & 809.4879 & 0 & 0 & 28 & 90 & 14.091 & 0.157 & 27 & 1 & 3.5984 & 0 \\
\hline & 560 & 77.46 & 76.44 & 973.18294 & 0 & 0 & 28 & 100 & 14.967 & 0.15 & 28 & 1 & 3.8221 & \\
\hline & 580 & 154.92 & 118.51 & 1296.09861 & 0 & 0 & 28 & 200 & 23.203 & 0.116 & 29 & 1 & 5.9253 & \\
\hline & 600 & 232.38 & 155.09 & 1781.52886 & 0 & 0 & 28 & 300 & 30.367 & 0.101 & 30 & 1 & 7.75455 & \\
\hline & 620 & 309.84 & 186.32 & 2429.83813 & 0 & 0 & 28 & 400 & 36.48 & 0.091 & 31 & 1 & 9.31575 & \\
\hline & 640 & 387.3 & 215.19 & 3239.93157 & 0 & 0 & 28 & 500 & 42.134 & 0.084 & 32 & 1 & 10.75955 & \\
\hline & 660 & 464.75 & 243.16 & 4211.52643 & 0 & 0 & 28 & 599.99 & 47.611 & 0.079 & 33 & 1 & 12.1581 & \\
\hline & 680 & 542.21 & 273.01 & 5346.07885 & 0 & 0 & 28 & 699.99 & 53.456 & 0.076 & 34 & 1 & 13.65065 & \\
\hline & 700 & 619.67 & 297.3 & 6642.25521 & 0 & 0 & 28 & 799.99 & 58.211 & 0.073 & 35 & 1 & 14.86485 & \\
\hline & 720 & 697.13 & 321.56 & 8100.61865 & 0 & 0 & 28 & 899.99 & 62.961 & 0.07 & 36 & 1 & 16.0778 & \\
\hline & 740 & 774.59 & 344.75 & 9721.57757 & 0 & 0 & 28 & 1000 & 67.502 & 0.068 & 37 & 1 & 17.2375 & \\
\hline & 750 & 774.59 & 337.82 & 10549.02909 & 0 & 0 & 28 & 1000 & 66.145 & 0.066 & 38 & 1 & 16.8909 & \\
\hline & 760 & 774.59 & 334.63 & 11360.17889 & 0 & 0 & 28 & 1000 & 65.52 & 0.066 & 39 & 1 & 16.73145 & \\
\hline & 770 & 774.59 & 331.62 & 12171.73474 & 0 & 0 & 28 & 1000 & 64.931 & 0.065 & 40 & 1 & 16.58085 & \\
\hline & 780 & 774.59 & 328.77 & 12982.47927 & 0 & 0 & 28 & 1000 & 64.374 & 0.064 & 41 & 1 & 16.4386 & \\
\hline & 790 & 774.59 & 326.11 & 13793.62829 & 0 & 0 & 28 & 1000 & 63.853 & 0.064 & 42 & 1 & 16.3056 & \\
\hline & 800 & 774.59 & 323.65 & 14604.77651 & 0 & 0 & 28 & 1000 & 63.37 & 0.063 & 43 & 1 & 16.1824 & \\
\hline & 820 & 774.59 & 321.13 & 16247.35352 & 0 & 0 & 28 & 1000 & 62.877 & 0.063 & 44 & 1 & 16.0565 & \\
\hline & 840 & 697.13 & 288.66 & 17708.35747 & 0 & 0 & 28 & 899.99 & 56.519 & 0.063 & 45 & 1 & 14.4328 & \\
\hline & 860 & 619.67 & 258.46 & 19006.56251 & 0 & 0 & 28 & 799.99 & 50.606 & 0.063 & 46 & 1 & 12.923 & \\
\hline & 880 & 542.21 & 229.5 & 20143.79549 & 0 & 0 & 28 & 699.99 & 44.935 & 0.064 & 47 & 1 & 11.47475 & \\
\hline & 900 & 464.75 & 201.22 & 21119.20503 & 0 & 0 & 28 & 599.99 & 39.398 & 0.066 & 48 & 1 & 10.0609 & 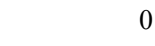 \\
\hline & 920 & 387.3 & 174.04 & 21930.88183 & 0 & 0 & 28 & 500 & 34.076 & 0.068 & 49 & 1 & 8.7018 & \\
\hline & 940 & 309.84 & 146.95 & 22581.83318 & 0 & 0 & 28 & 400 & 28.774 & 0.072 & 50 & 1 & 7.3477 & \\
\hline & 960 & 232.38 & 119.3 & 23069.21437 & 0 & 0 & 28 & 300 & 23.358 & 0.078 & 51 & 1 & 5.96485 & \\
\hline & 980 & 154.92 & 89.69 & 23395.09727 & 0 & 0 & 28 & 200 & 17.561 & 0.088 & 52 & 1 & 4.4844 & \\
\hline & 61000 & 77.46 & 56.43 & 23558.50878 & 0 & 0 & 28 & 100 & 11.049 & 0.11 & 53 & 1 & 2.82155 & \\
\hline & 71020 & 69.71 & 52.95 & 23705.67983 & 0 & 0 & 28 & 90 & 10.367 & 0.115 & 54 & 1 & 2.64735 & \\
\hline & 71040 & 61.97 & 49.09 & 23835.54385 & 0 & 0 & 28 & 80 & 9.612 & 0.12 & 55 & 1 & 2.45465 & \\
\hline & 71060 & 54.22 & 44.87 & 23949.16348 & 0 & 0 & 28 & 70 & 8.786 & 0.126 & 56 & 1 & 2.24365 & \\
\hline & 71080 & 46.48 & 40.11 & 24046.61882 & 0 & 0 & 28 & 60.01 & 7.853 & 0.131 & 57 & 1 & 2.0053 & 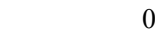 \\
\hline & 71100 & 38.73 & 35.24 & 24127.94287 & 0 & 0 & 28 & 50 & 6.899 & 0.138 & 58 & 1 & 1.76185 & 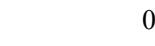 \\
\hline & 71120 & 30.99 & 30.19 & 24192.90708 & 0 & 0 & 28 & 40.01 & 5.91 & 0.148 & 59 & 1 & 1.5093 & 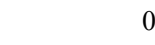 \\
\hline & 71140 & 23.24 & 24.41 & 24241.73136 & 0 & 0 & 28 & 30 & 4.779 & 0.159 & 60 & 1 & 1.2205 & 0 \\
\hline & 71160 & 15.5 & 17.9 & 24274.31125 & 0 & 0 & 28 & 20.01 & 3.505 & 0.175 & 61 & 1 & 0.89515 & 0 \\
\hline & 71180 & 7.75 & 9.48 & 24290.65303 & 0 & 0 & 28 & 10.01 & 1.857 & 0.186 & 62 & 1 & 0.4742 & 0 \\
\hline & 81200 & 6.97 & 9.65 & 24305.36432 & 0 & 0 & 28 & 9 & 1.89 & 0.21 & 63 & 1 & 0.48265 & 0 \\
\hline & 81220 & 6.2 & 8.8 & 24318.34302 & 0 & 0 & 28 & 8 & 1.723 & 0.215 & 64 & 1 & 0.43995 & 0 \\
\hline & 81240 & 5.42 & 8.11 & 24329.70852 & 0 & 0 & 28 & 7 & 1.588 & 0.227 & 65 & 1 & 0.4056 & 0 \\
\hline & 81260 & 4.64 & 7.41 & 24339.44824 & 0 & 0 & 28 & 5.99 & 1.451 & 0.242 & 66 & 1 & 0.3706 & 0 \\
\hline & 81280 & 3.87 & 6.57 & 24347.56455 & 0 & 0 & 28 & 5 & 1.286 & 0.257 & 67 & 1 & 0.32845 & 0 \\
\hline & 81300 & 3.09 & 5.66 & 24354.05822 & 0 & 0 & 28 & 3.99 & 1.109 & 0.278 & 68 & 1 & 0.2832 & 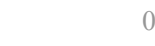 \\
\hline & 81320 & 2.32 & 5.62 & 24358.92533 & 0 & 0 & 28 & 3 & 1.1 & 0.367 & 69 & 1 & 0.281 & 0 \\
\hline & 81340 & 1.54 & 3.05 & 24362.17295 & 0 & 0 & 28 & 1.99 & 0.598 & 0.301 & 70 & 1 & 0.1526 & 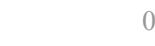 \\
\hline & 81360 & 0.77 & 2.02 & 24363.79716 & 0 & 0 & 28 & 0.99 & 0.395 & 0.399 & 71 & 1 & 0.10085 & \\
\hline & 91380 & 0.7 & 2.96 & 24365.27213 & 18.18373 & 31.3512 & 28 & 0.9 & 0.58 & 0.644 & 72 & 1 & 0.148 & 0.031896646 \\
\hline & 91400 & 0.62 & 3.31 & 24366.5759 & 34.25668 & 52.86517 & 28 & 0.8 & 0.648 & 0.81 & 73 & 1 & 0.1655 & 0.018916019 \\
\hline & 91420 & 0.55 & 3.58 & 24367.71865 & 48.34472 & 69.06379 & 28 & 0.71 & 0.7 & 0.986 & 74 & 1 & 0.17885 & 0.014479347 \\
\hline & 91440 & 0.47 & 4.11 & 24368.69883 & 60.42848 & 75.06634 & 28 & 0.61 & 0.805 & 1.32 & 75 & 1 & 0.2056 & 0.013321533 \\
\hline & 91460 & 0.39 & 4.01 & 24369.51643 & 70.50796 & 89.70467 & 28 & 0.5 & 0.786 & 1.572 & 76 & 1 & 0.20065 & 0.011147678 \\
\hline
\end{tabular}




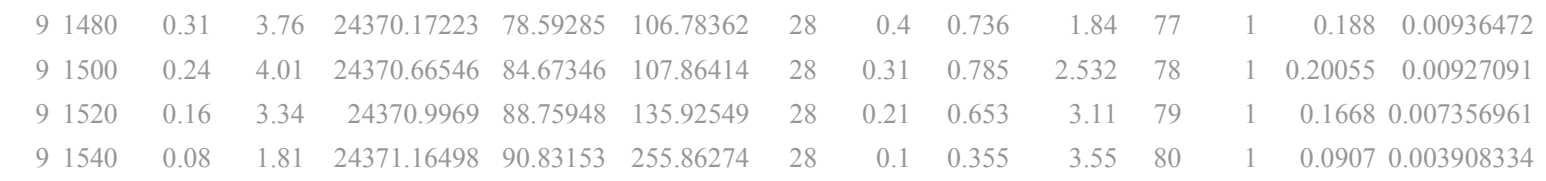

2004.01.22 META-20 wt\%-U [not sonicated] 0.1-1000 s-1 CC-45 run 3.txt Mode Step t[s] n[rpm] M[\%.] Phi[rad]

Gamma J[t]

\begin{tabular}{|c|c|c|c|c|c|c|c|c|c|c|c|c|c|c|}
\hline 1 & 20 & 0.08 & 6.11 & 0.16415 & 2.02364 & 1.69059 & 28 & 0.1 & 1.197 & 11.97 & 1 & 1 & 0.3057 & 0.591508371 \\
\hline 1 & 40 & 0.16 & 7.67 & 0.49166 & 6.06125 & 4.03814 & 28 & 0.21 & 1.501 & 7.148 & 2 & 1 & 0.38335 & 0.247638688 \\
\hline 1 & 60 & 0.24 & 10.27 & 0.98175 & 12.10312 & 6.01846 & 28 & 0.31 & 2.011 & 6.487 & 3 & 1 & 0.5135 & 0.166155504 \\
\hline 1 & 80 & 0.31 & 13.62 & 1.63441 & 20.14928 & 7.5522 & 28 & 0.4 & 2.668 & 6.67 & 4 & 1 & 0.6812 & 0.132411679 \\
\hline 1 & 100 & 0.39 & 16.66 & 2.44887 & 30.19003 & 9.25506 & 28 & 0.5 & 3.262 & 6.524 & 5 & 1 & 0.83305 & 0.108048915 \\
\hline 1 & 120 & 0.47 & 18.35 & 3.42591 & 42.23506 & 11.75481 & 28 & 0.61 & 3.593 & 5.89 & 6 & 1 & 0.9176 & 0.085071502 \\
\hline 1 & 140 & 0.54 & 19.1 & 4.56709 & 56.30374 & 15.0585 & 28 & 0.7 & 3.739 & 5.341 & 7 & 1 & 0.9549 & 0.066407667 \\
\hline 1 & 160 & 0.62 & 19.16 & 5.87007 & 72.367 & 19.28757 & 28 & 0.8 & 3.752 & 4.69 & 8 & 1 & 0.958 & 0.051846836 \\
\hline 1 & 180 & 0.7 & 19.28 & 7.33405 & 90.41519 & 23.95104 & 28 & 0.9 & 3.775 & 4.194 & 9 & 1 & 0.964 & 0.041751834 \\
\hline 2 & 200 & 0.77 & 19.35 & 8.95825 & 0 & 0 & 28 & 0.99 & 3.788 & 3.826 & 10 & 1 & 0.9673 & 0 \\
\hline 2 & 220 & 1.55 & 28.85 & 12.18153 & 0 & 0 & 28 & 2 & 5.648 & 2.824 & 11 & 1 & 1.4424 & 0 \\
\hline 2 & 240 & 2.32 & 30.15 & 17.02979 & 0 & 0 & 28 & 3 & 5.904 & 1.968 & 12 & 1 & 1.5077 & 0 \\
\hline 2 & 260 & 3.09 & 27.97 & 23.50147 & 0 & 0 & 28 & 3.99 & 5.477 & 1.373 & 13 & 1 & 1.39855 & 0 \\
\hline 2 & 280 & 3.87 & 26.13 & 31.59264 & 0 & 0 & 28 & 5 & 5.117 & 1.023 & 14 & 1 & 1.30665 & 0 \\
\hline 2 & 300 & 4.65 & 25.48 & 41.3088 & 0 & 0 & 28 & 6 & 4.989 & 0.832 & 15 & 1 & 1.2741 & 0 \\
\hline 2 & 320 & 5.42 & 25.53 & 52.66252 & 0 & 0 & 28 & 7 & 4.998 & 0.714 & 16 & 1 & 1.2763 & 0 \\
\hline 2 & 340 & 6.2 & 25.95 & 65.61923 & 0 & 0 & 28 & 8 & 5.08 & 0.635 & 17 & 1 & 1.2973 & 0 \\
\hline 2 & 360 & 6.97 & 25.62 & 80.2135 & 0 & 0 & 28 & 9 & 5.017 & 0.557 & 18 & 1 & 1.2812 & 0 \\
\hline 3 & 380 & 7.75 & 26.01 & 96.5757 & 0 & 0 & 28 & 10.01 & 5.092 & 0.509 & 19 & 1 & 1.30025 & 0 \\
\hline 3 & 400 & 15.5 & 39.32 & 128.94589 & 0 & 0 & 28 & 20.01 & 7.699 & 0.385 & 20 & 1 & 1.96595 & 0 \\
\hline 3 & 420 & 23.24 & 45.49 & 177.5047 & 0 & 0 & 28 & 30 & 8.907 & 0.297 & 21 & 1 & 2.2744 & 0 \\
\hline 3 & 440 & 30.99 & 48.27 & 242.22072 & 0 & 0 & 28 & 40.01 & 9.45 & 0.236 & 22 & 1 & 2.41325 & 0 \\
\hline 3 & 460 & 38.73 & 48.46 & 323.21962 & 0 & 0 & 28 & 50 & 9.488 & 0.19 & 23 & 1 & 2.42295 & 0 \\
\hline 3 & 480 & 46.48 & 48.91 & 420.51239 & 0 & 0 & 28 & 60.01 & 9.577 & 0.16 & 24 & 1 & 2.44565 & 0 \\
\hline 3 & 500 & 54.22 & 51.01 & 533.83042 & 0 & 0 & 28 & 70 & 9.988 & 0.143 & 25 & 1 & 2.5505 & 0 \\
\hline 3 & 520 & 61.96 & 54.69 & 663.52008 & 0 & 0 & 28 & 79.99 & 10.708 & 0.134 & 26 & 1 & 2.73455 & 0 \\
\hline 3 & 540 & 69.71 & 57.25 & 809.4714 & 0 & 0 & 28 & 90 & 11.21 & 0.125 & 27 & 1 & 2.86265 & 0 \\
\hline 4 & 560 & 77.46 & 60.49 & 973.12639 & 0 & 0 & 28 & 100 & 11.843 & 0.118 & 28 & 1 & 3.0243 & 0 \\
\hline 4 & 580 & 154.92 & 95.67 & 1296.2871 & 0 & 0 & 28 & 200 & 18.732 & 0.094 & 29 & 1 & 4.78335 & 0 \\
\hline 4 & 600 & 232.38 & 126 & 1781.71736 & 0 & 0 & 28 & 300 & 24.671 & 0.082 & 30 & 1 & 6.30005 & 0 \\
\hline 4 & 620 & 309.84 & 152.79 & 2429.90646 & 0 & 0 & 28 & 400 & 29.916 & 0.075 & 31 & 1 & 7.63935 & 0 \\
\hline 4 & 640 & 387.3 & 178.19 & 3239.39279 & 0 & 0 & 28 & 500 & 34.89 & 0.07 & 32 & 1 & 8.90965 & 0 \\
\hline 4 & 660 & 464.75 & 203.41 & 4211.87986 & 0 & 0 & 28 & 599.99 & 39.828 & 0.066 & 33 & 1 & 10.1706 & 0 \\
\hline 4 & 680 & 542.21 & 229.48 & 5346.22886 & 0 & 0 & 28 & 699.99 & 44.932 & 0.064 & 34 & 1 & 11.47395 & 0 \\
\hline 4 & 700 & 619.67 & 255.64 & 6641.43133 & 0 & 0 & 28 & 799.99 & 50.055 & 0.063 & 35 & 1 & 12.78205 & 0 \\
\hline 4 & 720 & 697.13 & 280.79 & 8100.52519 & 0 & 0 & 28 & 899.99 & 54.979 & 0.061 & 36 & 1 & 14.03955 & 0 \\
\hline 4 & 740 & 774.59 & 304.52 & 9721.88937 & 0 & 0 & 28 & 1000 & 59.625 & 0.06 & 37 & 1 & 15.2259 & 0 \\
\hline 5 & 750 & 774.59 & 300.47 & 10548.93563 & 0 & 0 & 28 & 1000 & 58.831 & 0.059 & 38 & 1 & 15.0233 & 0 \\
\hline 5 & 760 & 774.59 & 298.4 & 11360.08543 & 0 & 0 & 28 & 1000 & 58.427 & 0.058 & 39 & 1 & 14.92015 & 0 \\
\hline 5 & 770 & 774.59 & 296.54 & 12171.23523 & 0 & 0 & 28 & 1000 & 58.062 & 0.058 & 40 & 1 & 14.8268 & 0 \\
\hline 5 & 780 & 774.59 & 294.94 & 12982.38424 & 0 & 0 & 28 & 1000 & 57.749 & 0.058 & 41 & 1 & 14.74685 & 0 \\
\hline 5 & 790 & 774.59 & 293.44 & 13794.75062 & 0 & 0 & 28 & 1000 & 57.455 & 0.057 & 42 & 1 & 14.6719 & 0 \\
\hline 5 & 800 & 774.59 & 292 & 14604.68305 & 0 & 0 & 28 & 1000 & 57.174 & 0.057 & 43 & 1 & 14.6 & 0 \\
\hline
\end{tabular}




\begin{tabular}{|c|c|c|c|c|c|c|c|c|c|c|c|c|c|}
\hline & 820 & 774.59 & 290.39 & 16246.85244 & 0 & 0 & 28 & 1000 & 56.857 & 0.057 & 44 & 114.51925 & 0 \\
\hline & 840 & 697.13 & 261.36 & 17706.71913 & 0 & 0 & 28 & 899.99 & 51.174 & 0.057 & 45 & 113.06805 & \\
\hline & 860 & 619.67 & 234.08 & 19006.54759 & 0 & 0 & 28 & 799.99 & 45.833 & 0.057 & 46 & 111.70415 & \\
\hline & 880 & 542.21 & 207.58 & 20144.0641 & 0 & 0 & 28 & 699.99 & 40.643 & 0.058 & 47 & 10.3788 & \\
\hline & 900 & 464.75 & 181.47 & 21117.56669 & 0 & 0 & 28 & 599.99 & 35.533 & 0.059 & 48 & 9.0737 & \\
\hline & 920 & 387.3 & 156.27 & 21931.07033 & 0 & 0 & 28 & 500 & 30.598 & 0.061 & 49 & 7.81355 & \\
\hline & 940 & 309.84 & 131.28 & 22580.51764 & 0 & 0 & 28 & 400 & 25.705 & 0.064 & 50 & 6.5641 & \\
\hline & 960 & 232.38 & 105.84 & 23069.07614 & 0 & 0 & 28 & 300 & 20.724 & 0.069 & 51 & 5.2921 & , \\
\hline & 980 & 154.92 & 78.66 & 23394.83731 & 0 & 0 & 28 & 200 & 15.402 & 0.077 & 52 & 3.93305 & \\
\hline & 61000 & 77.46 & 48.78 & 23558.16635 & 0 & 0 & 28 & 100 & 9.551 & 0.096 & 53 & 2.43895 & \\
\hline & 71020 & 69.71 & 45.58 & 23705.64763 & 0 & 0 & 28 & 90 & 8.925 & 0.099 & 54 & 2.2791 & م \\
\hline & 71040 & 61.97 & 42.29 & 23835.58076 & 0 & 0 & 28 & 80 & 8.281 & 0.104 & 55 & 2.11455 & \\
\hline & 71060 & 54.22 & 38.51 & 23949.20432 & 0 & 0 & 28 & 70 & 7.54 & 0.108 & 56 & 1.92555 & م \\
\hline & 71080 & 46.48 & 34.31 & 24046.68401 & 0 & 0 & 28 & 60.01 & 6.718 & 0.112 & 57 & 1.7156 & م \\
\hline & 71100 & 38.73 & 30.09 & 24127.88161 & 0 & 0 & 28 & 50 & 5.891 & 0.118 & 58 & 1.50425 & 0 \\
\hline & 71120 & 30.99 & 25.54 & 24192.94321 & 0 & 0 & 28 & 40.01 & 5 & 0.125 & 59 & 1.2768 & 0 \\
\hline & 71140 & 23.24 & 20.52 & 24241.71879 & 0 & 0 & 28 & 30 & 4.017 & 0.134 & 60 & 1.02585 & 0 \\
\hline & 71160 & 15.5 & 14.73 & 24274.25705 & 0 & 0 & 28 & 20.01 & 2.885 & 0.144 & 61 & 0.7367 & 0 \\
\hline & 71180 & 7.75 & 7.71 & 24290.60747 & 0 & 0 & 28 & 10.01 & 1.51 & 0.151 & 62 & 0.3855 & 0 \\
\hline & 81200 & 6.97 & 7.85 & 24305.32505 & 0 & 0 & 28 & 9 & 1.536 & 0.171 & 63 & 0.3923 & 0 \\
\hline & 81220 & 6.2 & 7.35 & 24318.30454 & 0 & 0 & 28 & 8 & 1.44 & 0.18 & 64 & 0.3676 & 0 \\
\hline & 81240 & 5.42 & 6.76 & 24329.66454 & 0 & 0 & 28 & 7 & 1.324 & 0.189 & 65 & 0.3382 & 0 \\
\hline & 81260 & 4.64 & 6.28 & 24339.40505 & 0 & 0 & 28 & 5.99 & 1.229 & 0.205 & 66 & 0.31385 & 0 \\
\hline & 81280 & 3.87 & 5.56 & 24347.52135 & 0 & 0 & 28 & 5 & 1.089 & 0.218 & 67 & 0.2781 & 0 \\
\hline & 81300 & 3.09 & 4.93 & 24354.01188 & 0 & 0 & 28 & 3.99 & 0.966 & 0.242 & 68 & 0.24675 & 0 \\
\hline & 81320 & 2.32 & 4.13 & 24358.88214 & 0 & 0 & 28 & 3 & 0.808 & 0.269 & 69 & 0.2063 & 0 \\
\hline & 81340 & 1.54 & 2.86 & 24362.13133 & 0 & 0 & 28 & 1.99 & 0.559 & 0.281 & 70 & 0.1428 & 0 \\
\hline & 81360 & 0.77 & 1.77 & 24363.75317 & 0 & 0 & 28 & 0.99 & 0.347 & 0.351 & 71 & 0.0887 & 0 \\
\hline & 91380 & 0.7 & 3.02 & 24365.22815 & 18.18373 & 30.76768 & 28 & 0.9 & 0.591 & 0.657 & 72 & 0.15085 & 0.032501582 \\
\hline & 91400 & 0.62 & 3.18 & 24366.53191 & 34.25668 & 55.07496 & 28 & 0.8 & 0.622 & 0.778 & 73 & 0.1588 & 0.018157043 \\
\hline & 91420 & 0.55 & 3.38 & 24367.67388 & 48.33504 & 73.124 & 28 & 0.71 & 0.661 & 0.931 & 74 & 0.16885 & 0.013675379 \\
\hline & 91440 & 0.47 & 3.49 & 24368.65406 & 60.4188 & 88.46078 & 28 & 0.61 & 0.683 & 1.12 & 75 & 0.1743 & 0.011304428 \\
\hline & 91460 & 0.39 & 3.48 & 24369.47323 & 70.51765 & 103.39816 & 28 & 0.5 & 0.682 & 1.364 & 76 & 0.1742 & 0.009671338 \\
\hline & 91480 & 0.31 & 3.49 & 24370.12747 & 78.58317 & 115.05572 & 28 & 0.4 & 0.683 & 1.708 & 77 & 0.17445 & 0.008691428 \\
\hline & 91500 & 0.24 & 3.5 & 24370.62148 & 84.67346 & 123.61071 & 28 & 0.31 & 0.685 & 2.21 & 78 & 0.1748 & 0.008089902 \\
\hline & 91520 & 0.16 & 3.32 & 24370.95213 & 88.74979 & 136.74831 & 28 & 0.21 & 0.649 & 3.09 & 79 & 0.16585 & 0.007312693 \\
\hline & 91540 & 0.08 & 1.95 & 24371.12099 & 90.83153 & 238.40234 & 28 & 0.1 & 0.381 & 3.81 & 80 & 0.09725 & 0.004194579 \\
\hline
\end{tabular}

2004.01.22 META-20 wt\%-U [not sonicated] 0.1-1000 s-1 CC-45 run 4.txt

Mode Step $\mathrm{t}[\mathrm{s}] \quad \mathrm{n}[\mathrm{rpm}] \quad \mathrm{M}[\%$.] $\quad$ Phi[rad] Gamma J $[\mathrm{t}]$

$\begin{array}{rrrrrrrrrrrrrrrrr}1 & 1 & 20 & 0.08 & 5.48 & 0.16336 & 2.01396 & 1.87694 & 28 & 0.1 & 1.073 & 10.73 & 1 & 1 & 0.274 & 0.532781187 \\ 1 & 1 & 40 & 0.16 & 7.22 & 0.49166 & 6.06125 & 4.28963 & 28 & 0.21 & 1.413 & 6.729 & 2 & 1 & 0.36085 & 0.233120231 \\ 1 & 1 & 60 & 0.24 & 9.5 & 0.98175 & 12.10312 & 6.50355 & 28 & 0.31 & 1.861 & 6.003 & 3 & 1 & 0.4752 & 0.153762005 \\ 1 & 1 & 80 & 0.31 & 12.17 & 1.63441 & 20.14928 & 8.45542 & 28 & 0.4 & 2.383 & 5.958 & 4 & 1 & 0.60855 & 0.118267253 \\ 1 & 1 & 100 & 0.39 & 14.56 & 2.44966 & 30.19972 & 10.59267 & 28 & 0.5 & 2.851 & 5.702 & 5 & 1 & 0.72795 & 0.094404849 \\ 1 & 1 & 120 & 0.47 & 16.26 & 3.42748 & 42.25443 & 13.27086 & 28 & 0.61 & 3.184 & 5.22 & 6 & 1 & 0.8132 & 0.075353046 \\ 1 & 1 & 140 & 0.54 & 16.7 & 4.56709 & 56.30374 & 17.21826 & 28 & 0.7 & 3.27 & 4.671 & 7 & 1 & 0.83495 & 0.058077847 \\ 1 & 1 & 160 & 0.62 & 16.23 & 5.87085 & 72.37669 & 22.78145 & 28 & 0.8 & 3.177 & 3.971 & 8 & 1 & 0.81135 & 0.043895348 \\ 1 & 1 & 180 & 0.7 & 15.57 & 7.33562 & 90.43455 & 29.67012 & 28 & 0.9 & 3.048 & 3.387 & 9 & 1 & 0.77825 & 0.033703933 \\ 1 & 2 & 200 & 0.77 & 15.21 & 8.95747 & 0 & 0 & 28 & 0.99 & 2.978 & 3.008 & 10 & 1 & 0.7604 & 0\end{array}$




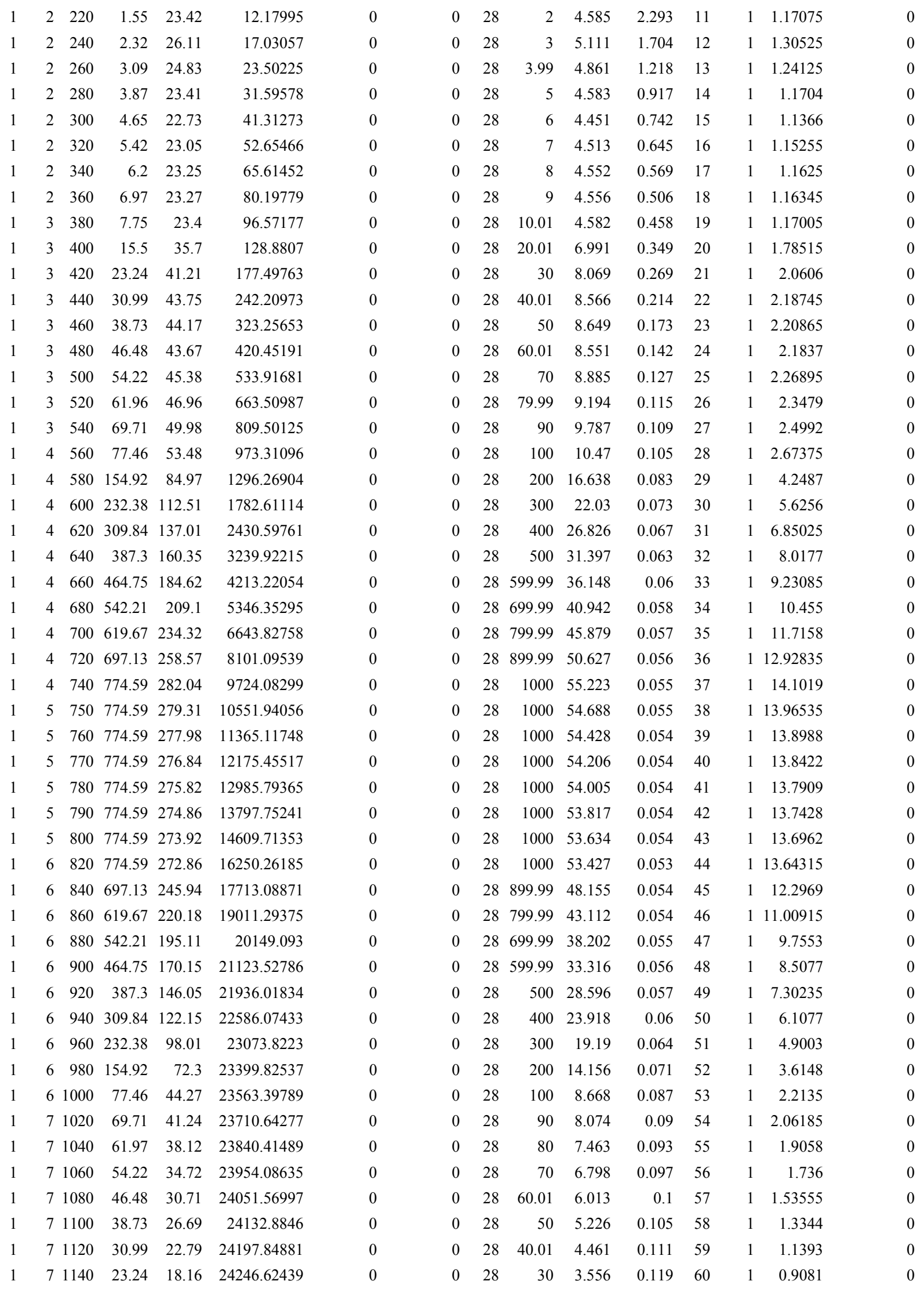




\begin{tabular}{|c|c|c|c|c|c|c|c|c|c|c|c|c|c|c|}
\hline & 71160 & 15.5 & 12.81 & 24279.19564 & 0 & 0 & 28 & 20.01 & 2.508 & 0.125 & 61 & 1 & 0.64055 & 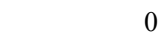 \\
\hline & 71180 & 7.75 & 6.51 & 24295.56176 & 0 & 0 & 28 & 10.01 & 1.274 & 0.127 & 62 & 1 & 0.3253 & \\
\hline & 81200 & 6.97 & 6.68 & 24310.26285 & 0 & 0 & 28 & 9 & 1.307 & 0.145 & 63 & 1 & 0.33375 & \\
\hline & 81220 & 6.2 & 6.37 & 24323.24862 & 0 & 0 & 28 & 8 & 1.246 & 0.156 & 64 & 1 & 0.31825 & \\
\hline & 81240 & 5.42 & 5.81 & 24334.62276 & 0 & 0 & 28 & 7 & 1.138 & 0.163 & 65 & 1 & 0.29065 & \\
\hline & 81260 & 4.64 & 5.46 & 24344.35541 & 0 & 0 & 28 & 5.99 & 1.068 & 0.178 & 66 & 1 & 0.27285 & \\
\hline & 81280 & 3.87 & 5.14 & 24352.47564 & 0 & 0 & 28 & 5 & 1.006 & 0.201 & 67 & 1 & 0.25685 & \\
\hline & 81300 & 3.09 & 4.32 & 24358.96931 & 0 & 0 & 28 & 3.99 & 0.845 & 0.212 & 68 & 1 & 0.2159 & \\
\hline & 81320 & 2.32 & 3.66 & 24363.83957 & 0 & 0 & 28 & 3 & 0.716 & 0.239 & 69 & 1 & 0.1829 & \\
\hline & 81340 & 1.54 & 2.43 & 24367.08405 & 0 & 0 & 28 & 1.99 & 0.476 & 0.239 & 70 & 1 & 0.1216 & \\
\hline & 81360 & 0.77 & 1.43 & 24368.70825 & 0 & 0 & 28 & 0.99 & 0.279 & 0.282 & 71 & 1 & 0.07125 & \\
\hline & 91380 & 0.7 & 2.46 & 24370.18166 & 18.16437 & 37.76368 & 28 & 0.9 & 0.481 & 0.534 & 72 & 1 & 0.1228 & 0.026480412 \\
\hline & 91400 & 0.62 & 2.91 & 24371.4862 & 34.247 & 60.08235 & 28 & 0.8 & 0.57 & 0.712 & 73 & 1 & 0.14555 & 0.016643794 \\
\hline & 91420 & 0.55 & 3.06 & 24372.62896 & 48.33504 & 80.55827 & 28 & 0.71 & 0.6 & 0.845 & 74 & 1 & 0.1531 & 0.012413355 \\
\hline & 91440 & 0.47 & 3.21 & 24373.60914 & 60.4188 & 96.20813 & 28 & 0.61 & 0.628 & 1.03 & 75 & 1 & 0.16035 & 0.010394116 \\
\hline & 91460 & 0.39 & 3.17 & 24374.42674 & 70.49828 & 113.52362 & 28 & 0.5 & 0.621 & 1.242 & 76 & 1 & 0.15855 & 0.008808726 \\
\hline & 91480 & 0.31 & 3.01 & 24375.08254 & 78.58317 & 133.41772 & 28 & 0.4 & 0.589 & 1.473 & 77 & 1 & 0.1503 & 0.007495244 \\
\hline & 91500 & 0.24 & 2.85 & 24375.57577 & 84.66378 & 151.72693 & 28 & 0.31 & 0.558 & 1.8 & 78 & 1 & 0.1426 & 0.006590776 \\
\hline & 91520 & 0.16 & 2.69 & 24375.90643 & 88.74011 & 168.70711 & 28 & 0.21 & 0.526 & 2.505 & 79 & 1 & 0.1343 & 0.005927421 \\
\hline & 91540 & 0.08 & 1.49 & 24376.07607 & 90.83153 & 312.13477 & 28 & 0.1 & 0.291 & 2.91 & 80 & 1 & 0.07435 & 0.003203733 \\
\hline
\end{tabular}

2004.01.22 META-20 wt\%-U [not sonicated] 0.1-1000 s-1 CC-45 run 5.txt

Mode Step t[s] n[rpm] M[\%.] Phi[rad] Gamma J[t]

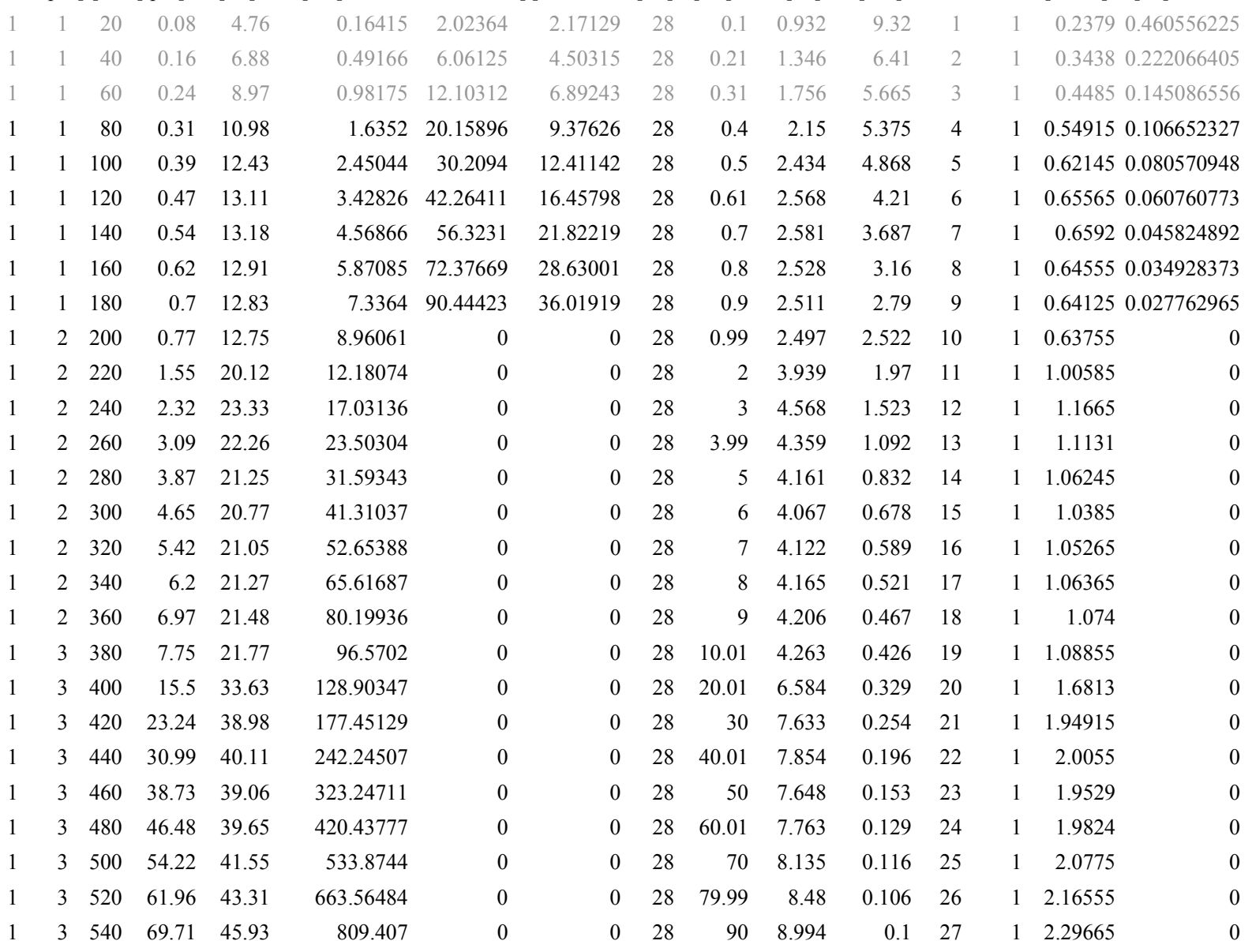




\begin{tabular}{|c|c|c|c|c|c|c|c|c|c|c|c|c|c|c|}
\hline 1 & 560 & 77.46 & 48.86 & 973.09811 & 0 & 0 & 28 & 100 & 9.566 & 0.096 & 28 & 1 & 2.44285 & 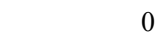 \\
\hline 1 & 580 & 154.92 & 78.57 & 1296.34051 & 0 & 0 & 28 & 200 & 15.383 & 0.077 & 29 & 1 & 3.92835 & 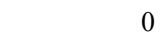 \\
\hline 1 & 600 & 232.38 & 104.49 & 1782.0166 & 0 & 0 & 28 & 300 & 20.458 & 0.068 & 30 & 1 & 5.2243 & 0 \\
\hline 1 & 620 & 309.84 & 127.63 & 2429.31427 & 0 & 0 & 28 & 400 & 24.99 & 0.062 & 31 & 1 & 6.3816 & 0 \\
\hline 1 & 640 & 387.3 & 149.85 & 3238.96239 & 0 & 0 & 28 & 500 & 29.34 & 0.059 & 32 & 1 & 7.49225 & 0 \\
\hline 1 & 660 & 464.75 & 173.59 & 4211.04263 & 0 & 0 & 28 & 599.99 & 33.99 & 0.057 & 33 & 1 & 8.67965 & 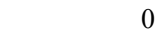 \\
\hline 1 & 680 & 542.21 & 197.03 & 5345.63903 & 0 & 0 & 28 & 699.99 & 38.577 & 0.055 & 34 & 1 & 9.85125 & 0 \\
\hline 1 & 700 & 619.67 & 221.68 & 6642.74608 & 0 & 0 & 28 & 799.99 & 43.404 & 0.054 & 35 & 1 & 11.08375 & 0 \\
\hline$x$ & 720 & 697.13 & 244.95 & 8101.47316 & 0 & 0 & 28 & 899.99 & 47.961 & 0.053 & 36 & 1 & 12.24745 & 0 \\
\hline & 740 & 774.59 & 267.88 & 9722.43208 & 0 & 0 & 28 & 1000 & 52.45 & 0.052 & 37 & 1 & 13.39375 & 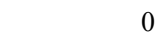 \\
\hline 1 & 750 & 774.59 & 266.22 & 10551.50781 & 0 & 0 & 28 & 1000 & 52.126 & 0.052 & 38 & 1 & 13.3111 & 0 \\
\hline & 760 & 774.59 & 265.23 & 11361.44024 & 0 & 0 & 28 & 1000 & 51.931 & 0.052 & 39 & 1 & 13.26135 & 0 \\
\hline 1 & 770 & 774.59 & 264.4 & 12174.21189 & 0 & 0 & 28 & 1000 & 51.77 & 0.052 & 40 & 1 & 13.22005 & 0 \\
\hline 1 & 780 & 774.59 & 263.62 & 12984.14353 & 0 & 0 & 28 & 1000 & 51.616 & 0.052 & 41 & 1 & 13.18075 & 0 \\
\hline 1 & 790 & 774.59 & 262.88 & 13794.88571 & 0 & 0 & 28 & 1000 & 51.471 & 0.051 & 42 & 1 & 13.14375 & 0 \\
\hline 1 & 800 & 774.59 & 262.1 & 14605.63024 & 0 & 0 & 28 & 1000 & 51.319 & 0.051 & 43 & 1 & 13.105 & 0 \\
\hline 1 & 820 & 774.59 & 261.3 & 16247.80277 & 0 & 0 & 28 & 1000 & 51.162 & 0.051 & 44 & 1 & 13.0649 & 0 \\
\hline 1 & 840 & 697.13 & 235.57 & 17708.43836 & 0 & 0 & 28 & 899.99 & 46.124 & 0.051 & 45 & 1 & 11.77845 & 0 \\
\hline 1 & 860 & 619.67 & 210.96 & 19008.22441 & 0 & 0 & 28 & 799.99 & 41.305 & 0.052 & 46 & 1 & 10.5478 & 0 \\
\hline 1 & 880 & 542.21 & 186.73 & 20144.32171 & 0 & 0 & 28 & 699.99 & 36.561 & 0.052 & 47 & 1 & 9.33625 & 0 \\
\hline 1 & 900 & 464.75 & 162.58 & 21118.79662 & 0 & 0 & 28 & 599.99 & 31.833 & 0.053 & 48 & 1 & 8.12885 & 0 \\
\hline 1 & 920 & 387.3 & 139.18 & 21931.69236 & 0 & 0 & 28 & 500 & 27.251 & 0.055 & 49 & 1 & 6.9588 & 0 \\
\hline 1 & 940 & 309.84 & 115.97 & 22581.91015 & 0 & 0 & 28 & 400 & 22.707 & 0.057 & 50 & 1 & 5.79845 & 0 \\
\hline 1 & 960 & 232.38 & 92.55 & 23069.61649 & 0 & 0 & 28 & 300 & 18.122 & 0.06 & 51 & 1 & 4.6277 & 0 \\
\hline 1 & 980 & 154.92 & 67.86 & 23395.41771 & 0 & 0 & 28 & 200 & 13.287 & 0.066 & 52 & 1 & 3.39305 & 0 \\
\hline 1 & 61000 & 77.46 & 41.13 & 23558.86693 & 0 & 0 & 28 & 100 & 8.054 & 0.081 & 53 & 1 & 2.0567 & 0 \\
\hline 1 & 71020 & 69.71 & 38.35 & 23706.07646 & 0 & 0 & 28 & 90 & 7.509 & 0.083 & 54 & 1 & 1.9174 & 0 \\
\hline 1 & 71040 & 61.97 & 35.3 & 23836.00959 & 0 & 0 & 28 & 80 & 6.912 & 0.086 & 55 & 1 & 1.76515 & 0 \\
\hline 1 & 71060 & 54.22 & 32.13 & 23949.71875 & 0 & 0 & 28 & 70 & 6.29 & 0.09 & 56 & 1 & 1.60635 & 0 \\
\hline 1 & 71080 & 46.48 & 28.44 & 24047.17802 & 0 & 0 & 28 & 60.01 & 5.568 & 0.093 & 57 & 1 & 1.4218 & 0 \\
\hline 1 & 71100 & 38.73 & 24.61 & 24128.3505 & 0 & 0 & 28 & 50 & 4.818 & 0.096 & 58 & 1 & 1.23045 & 0 \\
\hline 1 & 71120 & 30.99 & 20.93 & 24193.40031 & 0 & 0 & 28 & 40.01 & 4.099 & 0.102 & 59 & 1 & 1.04665 & 0 \\
\hline 1 & 71140 & 23.24 & 16.58 & 24242.16804 & 0 & 0 & 28 & 30 & 3.247 & 0.108 & 60 & 1 & 0.82915 & 0 \\
\hline 1 & 71160 & 15.5 & 11.57 & 24274.74636 & 0 & 0 & 28 & 20.01 & 2.265 & 0.113 & 61 & 1 & 0.57835 & 0 \\
\hline 1 & 71180 & 7.75 & 5.88 & 24291.1007 & 0 & 0 & 28 & 10.01 & 1.15 & 0.115 & 62 & 1 & 0.29375 & 0 \\
\hline 1 & 81200 & 6.97 & 6.13 & 24305.81121 & 0 & 0 & 28 & 9 & 1.201 & 0.133 & 63 & 1 & 0.3066 & 0 \\
\hline 1 & 81220 & 6.2 & 5.77 & 24318.80091 & 0 & 0 & 28 & 8 & 1.13 & 0.141 & 64 & 1 & 0.2885 & 0 \\
\hline 1 & 81240 & 5.42 & 5.2 & 24330.15777 & 0 & 0 & 28 & 7 & 1.019 & 0.146 & 65 & 1 & 0.2601 & 0 \\
\hline 1 & 81260 & 4.64 & 4.96 & 24339.89513 & 0 & 0 & 28 & 5.99 & 0.971 & 0.162 & 66 & 1 & 0.2479 & 0 \\
\hline 1 & 81280 & 3.87 & 4.52 & 24348.01537 & 0 & 0 & 28 & 5 & 0.884 & 0.177 & 67 & 1 & 0.22585 & 0 \\
\hline 1 & 81300 & 3.09 & 3.96 & 24354.50668 & 0 & 0 & 28 & 3.99 & 0.775 & 0.194 & 68 & 1 & 0.1978 & 0 \\
\hline 1 & 81320 & 2.32 & 3.46 & 24359.37851 & 0 & 0 & 28 & 3 & 0.676 & 0.225 & 69 & 1 & 0.17275 & 0 \\
\hline 1 & 81340 & 1.54 & 2.27 & 24362.62691 & 0 & 0 & 28 & 1.99 & 0.444 & 0.223 & 70 & 1 & 0.11345 & 0 \\
\hline 1 & 81360 & 0.77 & 1.36 & 24364.24876 & 0 & 0 & 28 & 0.99 & 0.266 & 0.269 & 71 & 1 & 0.0678 & 0 \\
\hline 1 & 91380 & 0.7 & 2.34 & 24365.72452 & 18.19342 & 39.72354 & 28 & 0.9 & 0.458 & 0.509 & 72 & 1 & 0.11705 & 0.025173937 \\
\hline 1 & 91400 & 0.62 & 2.69 & 24367.02828 & 34.26637 & 65.02145 & 28 & 0.8 & 0.527 & 0.659 & 73 & 1 & 0.1347 & 0.015379511 \\
\hline 1 & 91420 & 0.55 & 2.98 & 24368.17104 & 48.35441 & 82.94053 & 28 & 0.71 & 0.583 & 0.821 & 74 & 1 & 0.14875 & 0.012056811 \\
\hline 1 & 91440 & 0.47 & 2.93 & 24369.15122 & 60.43817 & 105.47655 & 28 & 0.61 & 0.573 & 0.939 & 75 & 1 & 0.1464 & 0.009480764 \\
\hline 1 & 91460 & 0.39 & 2.94 & 24369.96882 & 70.51765 & 122.63918 & 28 & 0.5 & 0.575 & 1.15 & 76 & 1 & 0.1468 & 0.008153987 \\
\hline & 91480 & 0.31 & 2.9 & 24370.62462 & 78.60253 & 138.62856 & 28 & 0.4 & 0.567 & 1.417 & 77 & 1 & 0.14485 & 0.007213508 \\
\hline
\end{tabular}




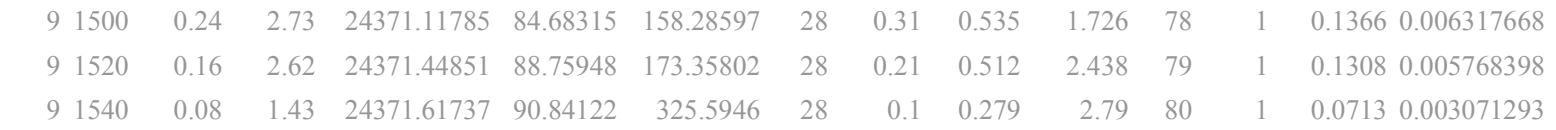

2004.01.22 META-20 wt\%-U [not sonicated] 0.1-1000 s-1 CC-45 run 6.txt

Mode Step t[s] n[rpm] M[\%.] Phi[rad] Gamma J[t] T[ $\left.\quad{ }^{\circ} \mathrm{C}\right] \mathrm{D}[1 / \mathrm{s}] \quad \mathrm{Tau}[\mathrm{Pa}] \mathrm{Eta}[\mathrm{Pas}] \mathrm{MP \#} \mathrm{Block} \mathrm{M[mNm]} \mathrm{G[Pa]}$

\begin{tabular}{|c|c|c|c|c|c|c|c|c|c|c|c|c|c|c|c|}
\hline 1 & 1 & 20 & 0.08 & 5.12 & 0.16415 & 2.02364 & 2.0196 & 28 & 0.1 & 1.002 & 10.02 & 1 & 1 & 0.256 & 0.495147358 \\
\hline & 1 & 40 & 0.16 & 7.01 & 0.49166 & 6.06125 & 4.4146 & 28 & 0.21 & 1.373 & 6.538 & 2 & 1 & 0.35065 & 0.226520932 \\
\hline & 1 & 60 & 0.24 & 9.03 & 0.98253 & 12.11281 & 6.84726 & 28 & 0.31 & 1.769 & 5.706 & 3 & 1 & 0.45165 & 0.146043734 \\
\hline & 1 & 80 & 0.31 & 11.12 & 1.6352 & 20.15896 & 9.25572 & 28 & 0.4 & 2.178 & 5.445 & 4 & 1 & 0.5562 & 0.108041288 \\
\hline & 1 & 100 & 0.39 & 12.67 & 2.45044 & 30.2094 & 12.1812 & 28 & 0.5 & 2.48 & 4.96 & 5 & 1 & 0.63335 & 0.082093653 \\
\hline & 1 & 120 & 0.47 & 13.14 & 3.42826 & 42.26411 & 16.426 & 28 & 0.61 & 2.573 & 4.218 & 6 & 1 & 0.65695 & 0.060879077 \\
\hline & 1 & 140 & 0.54 & 12.81 & 4.56788 & 56.31342 & 22.46246 & 28 & 0.7 & 2.507 & 3.581 & 7 & 1 & 0.6403 & 0.044518696 \\
\hline & 1 & 160 & 0.62 & 12.79 & 5.87164 & 72.38637 & 28.91983 & 28 & 0.8 & 2.503 & 3.129 & 8 & 1 & 0.6393 & 0.034578333 \\
\hline & 1 & 180 & 0.7 & 12.83 & 7.33562 & 90.43455 & 35.98667 & 28 & 0.9 & 2.513 & 2.792 & 9 & 1 & 0.6416 & 0.027788052 \\
\hline & 2 & 200 & 0.77 & 12.71 & 8.95982 & 0 & 0 & 28 & 0.99 & 2.488 & 2.513 & 10 & 1 & 0.63545 & \\
\hline & 2 & 220 & 1.55 & 19.72 & 12.1831 & 0 & 0 & 28 & 2 & 3.86 & 1.93 & 11 & 1 & 0.98575 & \\
\hline & 2 & 240 & 2.32 & 23 & 17.02743 & 0 & 0 & 28 & 3 & 4.502 & 1.501 & 12 & 1 & 1.14975 & 0 \\
\hline & 2 & 260 & 3.09 & 21.92 & 23.50147 & 0 & 0 & 28 & 3.99 & 4.291 & 1.075 & 13 & 1 & 1.0958 & 0 \\
\hline & 2 & 280 & 3.87 & 20.93 & 31.59186 & 0 & 0 & 28 & 5 & 4.098 & 0.82 & 14 & 1 & 1.0464 & 0 \\
\hline & 2 & 300 & 4.65 & 20.47 & 41.30959 & 0 & 0 & 28 & 6 & 4.008 & 0.668 & 15 & 1 & 1.0236 & 0 \\
\hline & 2 & 320 & 5.42 & 20.67 & 52.64995 & 0 & 0 & 28 & 7 & 4.047 & 0.578 & 16 & 1 & 1.0335 & 0 \\
\hline & 2 & 340 & 6.2 & 20.86 & 65.6263 & 0 & 0 & 28 & 8 & 4.084 & 0.51 & 17 & 1 & 1.04285 & 0 \\
\hline & 2 & 360 & 6.97 & 20.92 & 80.21664 & 0 & 0 & 28 & 9 & 4.096 & 0.455 & 18 & 1 & 1.04585 & 0 \\
\hline & 3 & 380 & 7.75 & 21.25 & 96.57884 & 0 & 0 & 28 & 10.01 & 4.161 & 0.416 & 19 & 1 & 1.06255 & 0 \\
\hline & 3 & 400 & 15.5 & 32.79 & 128.92939 & 0 & 0 & 28 & 20.01 & 6.421 & 0.321 & 20 & 1 & 1.6397 & 0 \\
\hline & 3 & 420 & 23.24 & 37.53 & 177.47642 & 0 & 0 & 28 & 30 & 7.349 & 0.245 & 21 & 1 & 1.87675 & 0 \\
\hline & 3 & 440 & 30.99 & 38.04 & 242.30319 & 0 & 0 & 28 & 40.01 & 7.449 & 0.186 & 22 & 1 & 1.9021 & 0 \\
\hline & 3 & 460 & 38.73 & 36.84 & 323.2047 & 0 & 0 & 28 & 50 & 7.213 & 0.144 & 23 & 1 & 1.842 & 0 \\
\hline & 3 & 480 & 46.48 & 38.01 & 420.49668 & 0 & 0 & 28 & 60.01 & 7.441 & 0.124 & 24 & 1 & 1.90025 & 0 \\
\hline & 3 & 500 & 54.22 & 39.81 & 533.95765 & 0 & 0 & 28 & 70 & 7.795 & 0.111 & 25 & 1 & 1.99065 & 0 \\
\hline & 3 & 520 & 61.96 & 41.44 & 663.64731 & 0 & 0 & 28 & 79.99 & 8.114 & 0.101 & 26 & 1 & 2.0719 & 0 \\
\hline & 3 & 540 & 69.71 & 43.43 & 809.48947 & 0 & 0 & 28 & 90 & 8.504 & 0.094 & 27 & 1 & 2.1715 & 0 \\
\hline & 4 & 560 & 77.46 & 46.06 & 973.26226 & 0 & 0 & 28 & 100 & 9.018 & 0.09 & 28 & 1 & 2.3029 & 0 \\
\hline & 4 & 580 & 154.92 & 74.43 & 1296.4646 & 0 & 0 & 28 & 200 & 14.573 & 0.073 & 29 & 1 & 3.72135 & 0 \\
\hline & 4 & 600 & 232.38 & 99.13 & 1781.93806 & 0 & 0 & 28 & 300 & 19.409 & 0.065 & 30 & 1 & 4.9563 & 0 \\
\hline & 4 & 620 & 309.84 & 121.44 & 2429.47921 & 0 & 0 & 28 & 400 & 23.778 & 0.059 & 31 & 1 & 6.07195 & 0 \\
\hline & 4 & 640 & 387.3 & 142.88 & 3239.41164 & 0 & 0 & 28 & 500 & 27.976 & 0.056 & 32 & 1 & 7.14405 & 0 \\
\hline & 4 & 660 & 464.75 & 165.8 & 4211.73456 & 0 & 0 & 28 & 599.99 & 32.464 & 0.054 & 33 & 1 & 8.29005 & 0 \\
\hline & 4 & 680 & 542.21 & 188.59 & 5345.15365 & 0 & 0 & 28 & 699.99 & 36.926 & 0.053 & 34 & 1 & 9.4295 & 0 \\
\hline & 4 & 700 & 619.67 & 212.82 & 6642.58665 & 0 & 0 & 28 & 799.99 & 41.67 & 0.052 & 35 & 1 & 10.64085 & 0 \\
\hline & 4 & 720 & 697.13 & 235.53 & 8100.95009 & 0 & 0 & 28 & 899.99 & 46.117 & 0.051 & 36 & 1 & 11.7765 & 0 \\
\hline & 4 & 740 & 774.59 & 258.39 & 9721.90901 & 0 & 0 & 28 & 1000 & 50.592 & 0.051 & 37 & 1 & 12.9193 & 0 \\
\hline & 5 & 750 & 774.59 & 256.94 & 10548.99768 & 0 & 0 & 28 & 1000 & 50.308 & 0.05 & 38 & 1 & 12.8468 & 0 \\
\hline & 5 & 760 & 774.59 & 256.15 & 11360.14669 & 0 & 0 & 28 & 1000 & 50.155 & 0.05 & 39 & 1 & 12.80775 & 0 \\
\hline & 5 & 770 & 774.59 & 255.42 & 12171.2957 & 0 & 0 & 28 & 1000 & 50.011 & 0.05 & 40 & 1 & 12.77085 & 0 \\
\hline & 5 & 780 & 774.59 & 254.9 & 12982.44393 & 0 & 0 & 28 & 1000 & 49.91 & 0.05 & 41 & 1 & 12.74515 & 0 \\
\hline & 5 & 790 & 774.59 & 254.42 & 13794.80795 & 0 & 0 & 28 & 1000 & 49.815 & 0.05 & 42 & 1 & 12.72095 & 0 \\
\hline & 5 & 800 & 774.59 & 253.9 & 14606.76907 & 0 & 0 & 28 & 1000 & 49.713 & 0.05 & 43 & 1 & 12.6949 & 0 \\
\hline & 6 & 820 & 774.59 & 253.58 & 16249.34608 & 0 & 0 & 28 & 1000 & 49.651 & 0.05 & 44 & 1 & 12.6791 & 0 \\
\hline
\end{tabular}




\begin{tabular}{|c|c|c|c|c|c|c|c|c|c|c|c|c|c|c|}
\hline & 840 & 697.13 & 229.25 & 17709.61725 & 0 & 0 & 28 & 899.99 & 44.887 & 0.05 & 45 & & 11.46245 & 0 \\
\hline & 860 & 619.67 & 205.09 & 19009.07814 & 0 & 0 & 28 & 799.99 & 40.156 & 0.05 & 46 & 1 & 10.25435 & \\
\hline & 880 & 542.21 & 181.36 & 20145.45896 & 0 & 0 & 28 & 699.99 & 35.51 & 0.051 & 47 & 1 & 9.0679 & \\
\hline & 900 & 464.75 & 157.69 & 21120.1373 & 0 & 0 & 28 & 599.99 & 30.876 & 0.051 & 48 & 1 & 7.88465 & \\
\hline & 920 & 387.3 & 134.81 & 21933.19483 & 0 & 0 & 28 & 500 & 26.395 & 0.053 & 49 & 1 & 6.7403 & \\
\hline & 940 & 309.84 & 111.99 & 22583.45346 & 0 & 0 & 28 & 400 & 21.927 & 0.055 & 50 & 1 & 5.59945 & \\
\hline & 960 & 232.38 & 89.11 & 23070.91633 & 0 & 0 & 28 & 300 & 17.447 & 0.058 & 51 & 1 & 4.45525 & \\
\hline & 980 & 154.92 & 64.98 & 23396.9194 & 0 & 0 & 28 & 200 & 12.723 & 0.064 & 52 & 1 & 3.249 & \\
\hline & 61000 & 77.46 & 39.13 & 23560.37018 & 0 & 0 & 28 & 100 & 7.661 & 0.077 & 53 & 1 & 1.9563 & \\
\hline & 71020 & 69.71 & 36.4 & 23707.61663 & 0 & 0 & 28 & 90 & 7.126 & 0.079 & 54 & 1 & 1.8198 & \\
\hline & 71040 & 61.97 & 33.54 & 23837.38325 & 0 & 0 & 28 & 80 & 6.568 & 0.082 & 55 & 1 & 1.6771 & \\
\hline & 71060 & 54.22 & 30.41 & 23951.06414 & 0 & 0 & 28 & 70 & 5.954 & 0.085 & 56 & 1 & 1.5205 & \\
\hline & 71080 & 46.48 & 26.87 & 24048.52263 & 0 & 0 & 28 & 60.01 & 5.261 & 0.088 & 57 & 1 & 1.34335 & \\
\hline & 71100 & 38.73 & 23.17 & 24129.75636 & 0 & 0 & 28 & 50 & 4.537 & 0.091 & 58 & 1 & 1.1586 & 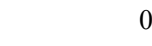 \\
\hline & 71120 & 30.99 & 19.68 & 24194.83366 & 0 & 0 & 28 & 40.01 & 3.852 & 0.096 & 59 & 1 & 0.98375 & \\
\hline & 71140 & 23.24 & 15.63 & 24243.60139 & 0 & 0 & 28 & 30 & 3.061 & 0.102 & 60 & 1 & 0.78165 & 0 \\
\hline & 71160 & 15.5 & 10.83 & 24276.18364 & 0 & 0 & 28 & 20.01 & 2.121 & 0.106 & 61 & 1 & 0.5415 & 0 \\
\hline & 71180 & 7.75 & 5.48 & 24292.5262 & 0 & 0 & 28 & 10.01 & 1.073 & 0.107 & 62 & 1 & 0.2741 & 0 \\
\hline & 81200 & 6.97 & 5.69 & 24307.22179 & 0 & 0 & 28 & 9 & 1.113 & 0.124 & 63 & 1 & 0.28425 & 0 \\
\hline & 81220 & 6.2 & 5.45 & 24320.2107 & 0 & 0 & 28 & 8 & 1.068 & 0.134 & 64 & 1 & 0.2727 & 0 \\
\hline & 81240 & 5.42 & 5.02 & 24331.5707 & 0 & 0 & 28 & 7 & 0.983 & 0.14 & 65 & 1 & 0.25105 & 0 \\
\hline & 81260 & 4.64 & 4.81 & 24341.32063 & 0 & 0 & 28 & 5.99 & 0.941 & 0.157 & 66 & 1 & 0.24025 & 0 \\
\hline & 81280 & 3.87 & 4.37 & 24349.43694 & 0 & 0 & 28 & 5 & 0.856 & 0.171 & 67 & 1 & 0.2186 & 0 \\
\hline & 81300 & 3.09 & 3.91 & 24355.93061 & 0 & 0 & 28 & 3.99 & 0.766 & 0.192 & 68 & 1 & 0.1956 & 0 \\
\hline & 81320 & 2.32 & 3.32 & 24360.79851 & 0 & 0 & 28 & 3 & 0.649 & 0.216 & 69 & 1 & 0.1658 & 0 \\
\hline & 81340 & 1.54 & 2.18 & 24364.0477 & 0 & 0 & 28 & 1.99 & 0.427 & 0.215 & 70 & 1 & 0.10905 & 0 \\
\hline & 81360 & 0.77 & 1.39 & 24365.67033 & 0 & 0 & 28 & 0.99 & 0.273 & 0.276 & 71 & 1 & 0.0696 & 0 \\
\hline & 91380 & 0.7 & 2.4 & 24367.14374 & 18.16437 & 38.64751 & 28 & 0.9 & 0.47 & 0.522 & 72 & 1 & 0.1199 & 0.025874831 \\
\hline & 91400 & 0.62 & 3.08 & 24368.44986 & 34.26637 & 56.92079 & 28 & 0.8 & 0.602 & 0.753 & 73 & 1 & 0.15375 & 0.017568245 \\
\hline & 91420 & 0.55 & 9.53 & 24369.5934 & 48.36409 & 25.91858 & 28 & 0.71 & 1.866 & 2.628 & 74 & 1 & 0.47655 & 0.038582345 \\
\hline & 91440 & 0.47 & 2.76 & 24370.572 & 60.42848 & 111.69754 & 28 & 0.61 & 0.541 & 0.887 & 75 & 1 & 0.13815 & 0.008952732 \\
\hline & 91460 & 0.39 & 3.52 & 24371.39039 & 70.51765 & 102.49644 & 28 & 0.5 & 0.688 & 1.376 & 76 & 1 & 0.1758 & 0.009756423 \\
\hline & 91480 & 0.31 & 2.96 & 24372.04541 & 78.59285 & 135.50468 & 28 & 0.4 & 0.58 & 1.45 & 77 & 1 & 0.14815 & 0.007379806 \\
\hline & 91500 & 0.24 & 2.67 & 24372.53942 & 84.68315 & 162.22795 & 28 & 0.31 & 0.522 & 1.684 & 78 & 1 & 0.1333 & 0.006164154 \\
\hline & 91520 & 0.16 & 2.38 & 24372.87008 & 88.75948 & 190.06272 & 28 & 0.21 & 0.467 & 2.224 & 79 & 1 & 0.11915 & 0.00526141 \\
\hline & 91540 & 0.08 & 1.65 & 24373.03894 & 90.84122 & 282.11471 & 28 & 0.1 & 0.322 & 3.22 & 80 & 1 & 0.08235 & 0.003544646 \\
\hline
\end{tabular}

2004.01.22 META-20 wt\%-U [not sonicated] 0.1-1000 s-1 CC-45 run 7.txt

Mode Step $\mathrm{t}[\mathrm{s}] \quad \mathrm{n}[\mathrm{rpm}] \quad \mathrm{M}[\%$. $] \quad$ Phi[rad $] \quad$ Gamma $\mathrm{J}[\mathrm{t}]$
$\mathrm{T}\left[{ }^{\circ} \mathrm{C}\right] \mathrm{D}[1 / \mathrm{s}] \quad \mathrm{Tau}[\mathrm{Pa}]$ Eta[Pas] MP\# Block M[mNm] G[Pa]

$\begin{array}{rrrrrrrrrrr}0.16415 & 2.02364 & 1.72078 & 28 & 0.1 & 1.176 & 11.76 & 1 & 1 & 0.3003 & 0.581131031 \\ 0.49166 & 6.06125 & 4.36689 & 28 & 0.21 & 1.388 & 6.61 & 2 & 1 & 0.35445 & 0.228995669 \\ 0.98253 & 12.11281 & 6.44984 & 28 & 0.31 & 1.878 & 6.058 & 3 & 1 & 0.47945 & 0.155042472 \\ 1.63441 & 20.14928 & 8.64776 & 28 & 0.4 & 2.33 & 5.825 & 4 & 1 & 0.595 & 0.115636886 \\ 2.45044 & 30.2094 & 11.42564 & 28 & 0.5 & 2.644 & 5.288 & 5 & 1 & 0.6753 & 0.087522427 \\ 3.42826 & 42.26411 & 15.13757 & 28 & 0.61 & 2.792 & 4.577 & 6 & 1 & 0.7129 & 0.066060778 \\ 4.56866 & 56.3231 & 20.54088 & 28 & 0.7 & 2.742 & 3.917 & 7 & 1 & 0.70015 & 0.0486834 \\ 5.87164 & 72.38637 & 26.93947 & 28 & 0.8 & 2.687 & 3.359 & 8 & 1 & 0.68625 & 0.037120248 \\ 7.3364 & 90.44423 & 33.79828 & 28 & 0.9 & 2.676 & 2.973 & 9 & 1 & 0.68345 & 0.029587294 \\ 8.95982 & 0 & 0 & 28 & 0.99 & 2.586 & 2.612 & 10 & 1 & 0.6603 & 0 \\ 2.18231 & 0 & 0 & 28 & 2 & 4.02 & 2.01 & 11 & 1 & 1.02645 & 0\end{array}$




\begin{tabular}{|c|c|c|c|c|c|c|c|c|c|c|c|c|c|c|}
\hline & 2240 & 2.32 & 23.66 & 17.02665 & 0 & 0 & 28 & 3 & 4.633 & 1.544 & 12 & 1 & 1.18305 & 0 \\
\hline 1 & 260 & 3.09 & 22.96 & 23.49833 & 0 & 0 & 28 & 3.99 & 4.496 & 1.127 & 13 & 1 & 1.1482 & 0 \\
\hline & 280 & 3.87 & 21.27 & 31.59657 & 0 & 0 & 28 & 5 & 4.165 & 0.833 & 14 & 1 & 1.0636 & 0 \\
\hline & 300 & 4.65 & 23.11 & 41.3088 & 0 & 0 & 28 & 6 & 4.524 & 0.754 & 15 & 1 & 1.15525 & 0 \\
\hline & 320 & 5.42 & 21.64 & 52.65938 & 0 & 0 & 28 & 7 & 4.237 & 0.605 & 16 & 1 & 1.082 & 0 \\
\hline & 340 & 6.2 & 21.28 & 65.6263 & 0 & 0 & 28 & 8 & 4.166 & 0.521 & 17 & 1 & 1.0638 & 0 \\
\hline & 360 & 6.97 & 21.43 & 80.20486 & 0 & 0 & 28 & 9 & 4.195 & 0.466 & 18 & 1 & 1.07135 & 0 \\
\hline & 380 & 7.75 & 21.5 & 96.57649 & 0 & 0 & 28 & 10.01 & 4.209 & 0.42 & 19 & 1 & 1.07475 & 0 \\
\hline & 400 & 15.5 & 32.39 & 128.89798 & 0 & 0 & 28 & 20.01 & 6.343 & 0.317 & 20 & 1 & 1.6197 & 0 \\
\hline & 420 & 23.24 & 36.08 & 177.44187 & 0 & 0 & 28 & 30 & 7.064 & 0.235 & 21 & 1 & 1.804 & 0 \\
\hline & 440 & 30.99 & 35.78 & 242.25528 & 0 & 0 & 28 & 40.01 & 7.006 & 0.175 & 22 & 1 & 1.78915 & 0 \\
\hline & 460 & 38.73 & 35.51 & 323.2369 & 0 & 0 & 28 & 50 & 6.952 & 0.139 & 23 & 1 & 1.7754 & 0 \\
\hline & 480 & 46.48 & 36.54 & 420.53045 & 0 & 0 & 28 & 60.01 & 7.155 & 0.119 & 24 & 1 & 1.8271 & 0 \\
\hline & 500 & 54.22 & 38.17 & 533.94273 & 0 & 0 & 28 & 70 & 7.473 & 0.107 & 25 & 1 & 1.9083 & 0 \\
\hline & 520 & 61.96 & 39.21 & 663.535 & 0 & 0 & 28 & 79.99 & 7.678 & 0.096 & 26 & 1 & 1.9606 & 0 \\
\hline & 540 & 69.71 & 41.36 & 809.34495 & 0 & 0 & 28 & 90 & 8.099 & 0.09 & 27 & 1 & 2.06815 & 0 \\
\hline & 560 & 77.46 & 44.03 & 972.99523 & 0 & 0 & 28 & 100 & 8.621 & 0.086 & 28 & 1 & 2.2016 & 0 \\
\hline & 580 & 154.92 & 71.65 & 1296.07662 & 0 & 0 & 28 & 200 & 14.029 & 0.07 & 29 & 1 & 3.5824 & 0 \\
\hline & 600 & 232.38 & 95.85 & 1782.15875 & 0 & 0 & 28 & 300 & 18.766 & 0.063 & 30 & 1 & 4.79225 & 0 \\
\hline & 620 & 309.84 & 117.24 & 2429.65906 & 0 & 0 & 28 & 400 & 22.955 & 0.057 & 31 & 1 & 5.86195 & 0 \\
\hline & 640 & 387.3 & 137.98 & 3238.9027 & 0 & 0 & 28 & 500 & 27.017 & 0.054 & 32 & 1 & 6.8991 & 0 \\
\hline & 660 & 464.75 & 159.51 & 4210.98215 & 0 & 0 & 28 & 599.99 & 31.232 & 0.052 & 33 & 1 & 7.9754 & 0 \\
\hline & 680 & 542.21 & 182.99 & 5345.57855 & 0 & 0 & 28 & 699.99 & 35.83 & 0.051 & 34 & 1 & 9.14965 & 0 \\
\hline & 700 & 619.67 & 206.81 & 6641.71329 & 0 & 0 & 28 & 799.99 & 40.494 & 0.051 & 35 & 1 & 10.34055 & 0 \\
\hline & 720 & 697.13 & 228.67 & 8101.94204 & 0 & 0 & 28 & 899.99 & 44.773 & 0.05 & 36 & 1 & 11.4334 & 0 \\
\hline & 740 & 774.59 & 250.91 & 9722.08886 & 0 & 0 & 28 & 1000 & 49.128 & 0.049 & 37 & 1 & 12.5455 & 0 \\
\hline & 750 & 774.59 & 250.23 & 10551.20465 & 0 & 0 & 28 & 1000 & 48.996 & 0.049 & 38 & 1 & 12.51165 & 0 \\
\hline & 760 & 774.59 & 249.9 & 11361.94839 & 0 & 0 & 28 & 1000 & 48.931 & 0.049 & 39 & 1 & 12.4952 & 0 \\
\hline & 770 & 774.59 & 249.87 & 12172.69136 & 0 & 0 & 28 & 1000 & 48.925 & 0.049 & 40 & 1 & 12.49355 & 0 \\
\hline & 780 & 774.59 & 249.91 & 12983.02905 & 0 & 0 & 28 & 1000 & 48.932 & 0.049 & 41 & 1 & 12.4953 & 0 \\
\hline & 790 & 774.59 & 249.44 & 13794.17807 & 0 & 0 & 28 & 1000 & 48.841 & 0.049 & 42 & 1 & 12.4722 & 0 \\
\hline & 800 & 774.59 & 248.99 & 14606.54445 & 0 & 0 & 28 & 1000 & 48.753 & 0.049 & 43 & 1 & 12.4497 & 0 \\
\hline & 820 & 774.59 & 248.54 & 16247.49882 & 0 & 0 & 28 & 1000 & 48.664 & 0.049 & 44 & 1 & 12.42685 & 0 \\
\hline & 840 & 697.13 & 224.22 & 17709.59526 & 0 & 0 & 28 & 899.99 & 43.902 & 0.049 & 45 & 1 & 11.2109 & 0 \\
\hline & 860 & 619.67 & 200.52 & 19008.40663 & 0 & 0 & 28 & 799.99 & 39.261 & 0.049 & 46 & 1 & 10.0259 & 0 \\
\hline & 880 & 542.21 & 177.16 & 20146.20666 & 0 & 0 & 28 & 699.99 & 34.687 & 0.05 & 47 & 1 & 8.85775 & 0 \\
\hline & 900 & 464.75 & 153.86 & 21119.91189 & 0 & 0 & 28 & 599.99 & 30.126 & 0.05 & 48 & 1 & 7.69305 & 0 \\
\hline & 920 & 387.3 & 131.35 & 21932.56416 & 0 & 0 & 28 & 500 & 25.718 & 0.051 & 49 & 1 & 6.5675 & 0 \\
\hline & 940 & 309.84 & 108.89 & 22582.822 & 0 & 0 & 28 & 400 & 21.32 & 0.053 & 50 & 1 & 5.4443 & 0 \\
\hline & 960 & 232.38 & 86.3 & 23070.89276 & 0 & 0 & 28 & 300 & 16.898 & 0.056 & 51 & 1 & 4.315 & 0 \\
\hline & 980 & 154.92 & 62.67 & 23396.6932 & 0 & 0 & 28 & 200 & 12.27 & 0.061 & 52 & 1 & 3.1333 & 0 \\
\hline & 61000 & 77.46 & 37.54 & 23560.06309 & 0 & 0 & 28 & 100 & 7.35 & 0.074 & 53 & 1 & 1.87695 & 0 \\
\hline & 71020 & 69.71 & 34.9 & 23707.26712 & 0 & 0 & 28 & 90 & 6.833 & 0.076 & 54 & 1 & 1.7448 & 0 \\
\hline & 71040 & 61.97 & 32.06 & 23837.06674 & 0 & 0 & 28 & 80 & 6.278 & 0.078 & 55 & 1 & 1.6031 & 0 \\
\hline & 71060 & 54.22 & 28.99 & 23950.71385 & 0 & 0 & 28 & 70 & 5.676 & 0.081 & 56 & 1 & 1.44935 & 0 \\
\hline & 71080 & 46.48 & 25.54 & 24048.19354 & 0 & 0 & 28 & 60.01 & 5 & 0.083 & 57 & 1 & 1.27685 & 0 \\
\hline & 71100 & 38.73 & 21.99 & 24129.40764 & 0 & 0 & 28 & 50 & 4.306 & 0.086 & 58 & 1 & 1.09955 & 0 \\
\hline & 71120 & 30.99 & 18.7 & 24194.44018 & 0 & 0 & 28 & 40.01 & 3.662 & 0.092 & 59 & 1 & 0.9352 & 0 \\
\hline & 71140 & 23.24 & 14.82 & 24243.25189 & 0 & 0 & 28 & 30 & 2.902 & 0.097 & 60 & 1 & 0.74105 & 0 \\
\hline & 71160 & 15.5 & 10.18 & 24275.81528 & 0 & 0 & 28 & 20.01 & 1.992 & 0.1 & 61 & 1 & 0.5088 & 0 \\
\hline
\end{tabular}




\begin{tabular}{|c|c|c|c|c|c|c|c|c|c|c|c|c|c|}
\hline 71180 & 7.75 & 4.87 & 24292.17277 & 0 & 0 & 28 & 10.01 & 0.954 & 0.095 & 62 & 1 & 0.24355 & 0 \\
\hline 81200 & 6.97 & 5.14 & 24306.88171 & 0 & 0 & 28 & 9 & 1.006 & 0.112 & 63 & 1 & 0.2569 & 0 \\
\hline 81220 & 6.2 & 4.99 & 24319.86748 & 0 & 0 & 28 & 8 & 0.978 & 0.122 & 64 & 1 & 0.24965 & \\
\hline 81240 & 5.42 & 4.61 & 24331.22984 & 0 & 0 & 28 & 7 & 0.902 & 0.129 & 65 & 1 & 0.2304 & \\
\hline 81260 & 4.64 & 4.39 & 24340.96328 & 0 & 0 & 28 & 5.99 & 0.859 & 0.143 & 66 & 1 & 0.2194 & \\
\hline 81280 & 3.87 & 4.04 & 24349.08429 & 0 & 0 & 28 & 5 & 0.792 & 0.158 & 67 & 1 & 0.2022 & \\
\hline 81300 & 3.09 & 3.54 & 24355.57561 & 0 & 0 & & 3.99 & 0.694 & 0.174 & 68 & 1 & 0.17715 & \\
\hline 81320 & 2.32 & 3.22 & 24360.44508 & 0 & 0 & & 3 & 0.629 & 0.21 & 69 & 1 & 0.16075 & \\
\hline 81340 & 1.54 & 1.93 & 24363.69191 & 0 & 0 & 28 & 1.99 & 0.378 & 0.19 & 70 & 1 & 0.09665 & \\
\hline 81360 & 0.77 & 1.33 & 24365.3169 & 0 & 0 & 28 & 0.99 & 0.26 & 0.263 & 71 & 1 & 0.06645 & \\
\hline 91380 & 0.7 & 2.72 & 24366.79267 & 18.19342 & 34.13393 & 28 & 0.9 & 0.533 & 0.592 & 72 & 1 & 0.136 & 0.029296306 \\
\hline 91400 & 0.62 & 2.61 & 24368.09643 & 34.26637 & 67.05734 & 28 & 0.8 & 0.511 & 0.639 & 73 & 1 & 0.1305 & 0.01491258 \\
\hline 91420 & 0.55 & 2.49 & 24369.23997 & 48.36409 & 99.10654 & 28 & 0.71 & 0.488 & 0.687 & 74 & 1 & 0.1245 & 0.010090131 \\
\hline 91440 & 0.47 & 2.59 & 24370.21936 & 60.43817 & 119.2072 & 28 & 0.61 & 0.507 & 0.831 & 75 & 1 & 0.1295 & 0.008388738 \\
\hline 91460 & 0.39 & 2.65 & 24371.03696 & 70.51765 & 136.1342 & 28 & 0.5 & 0.518 & 1.036 & 76 & 1 & 0.13235 & 0.007345679 \\
\hline 91480 & 0.31 & 2.55 & 24371.69355 & 78.61222 & 157.22413 & 28 & 0.4 & 0.5 & 1.25 & 77 & 1 & 0.1276 & 0.006360334 \\
\hline 91500 & 0.24 & 2.36 & 24372.18599 & 84.68315 & 183.29646 & 28 & 0.31 & 0.462 & 1.49 & 78 & 1 & 0.11805 & 0.005455631 \\
\hline 91520 & 0.16 & 2.25 & 24372.51665 & 88.75948 & 201.26821 & & 0.21 & 0.441 & 2.1 & 79 & 1 & 0.11265 & 0.004968483 \\
\hline 91540 & 0.08 & 1.41 & 24372.68551 & 90.84122 & 330.33051 & & 0.1 & 0.275 & 2.75 & 80 & 1 & 0.07025 & 0.00302726 \\
\hline
\end{tabular}

2004.01.22 META-20 wt\%-U [not sonicated] 0.1-1000 s-1 CC-45 run 8.txt

Mode Step t[s] n[rpm] M[\%.] Phi[rad]

Gamma J[t]

$\begin{array}{rrrrr}1 & 1 & 20 & 0.08 & 5.66 \\ 1 & 1 & 40 & 0.16 & 7.14 \\ 1 & 1 & 60 & 0.24 & 9.04 \\ 1 & 1 & 80 & 0.31 & 10.86 \\ 1 & 1 & 100 & 0.39 & 12.3 \\ 1 & 1 & 120 & 0.47 & 12.54 \\ 1 & 1 & 140 & 0.54 & 12.15 \\ 1 & 1 & 160 & 0.62 & 12.13 \\ 1 & 1 & 180 & 0.7 & 12.34 \\ 1 & 2 & 200 & 0.77 & 12.03 \\ 1 & 2 & 220 & 1.55 & 19.4 \\ 1 & 2 & 240 & 2.32 & 22.06 \\ 1 & 2 & 260 & 3.09 & 21.08 \\ 1 & 2 & 280 & 3.87 & 20.21 \\ 1 & 2 & 300 & 4.65 & 20.09 \\ 1 & 2 & 320 & 5.42 & 20.31 \\ 1 & 2 & 340 & 6.2 & 20.46 \\ 1 & 2 & 360 & 6.97 & 20.51 \\ 1 & 3 & 380 & 7.75 & 20.79 \\ 1 & 3 & 400 & 15.5 & 31.88 \\ 1 & 3 & 420 & 23.27 & 36.78 \\ 1 & 3 & 440 & 30.99 & 34.71 \\ 1 & 3 & 460 & 38.73 & 34.49 \\ 1 & 3 & 480 & 46.48 & 44.5 \\ 1 & 3 & 500 & 54.22 & 37.03 \\ 1 & 3 & 520 & 61.96 & 37.56 \\ 1 & 3 & 540 & 69.71 & 39.74 \\ 1 & 4 & 560 & 77.46 & 42.25 \\ & & & & \end{array}$

$0.16336 \quad 2.01396$

$\begin{array}{ll}0.49166 & 6.06125\end{array}$

$\begin{array}{ll}0.98175 & 12.10312\end{array}$

$\begin{array}{ll}1.6352 & 20.15896\end{array}$

$\begin{array}{ll}2.45044 & 30.2094\end{array}$

$\begin{array}{ll}3.42905 & 42.27379\end{array}$

$\begin{array}{ll}4.56866 & 56.3231\end{array}$

$\begin{array}{ll}5.87085 & 72.37669\end{array}$

$7.33562 \quad 90.43455$

8.95904

12.17995

17.02743

23.50225

31.59735

41.3088

52.65231

65.61923

80.20329

96.57727

128.85556

177.47171

242.21601

323.27538

420.47076

533.93959

663.49966

809.49104

973.06199
$\left.\mathrm{T}\left[{ }^{\circ} \mathrm{C}\right] \mathrm{D}[1 / \mathrm{s}] \quad \mathrm{Tau}[\mathrm{Pa}] \mathrm{Eta}[\mathrm{Pas}] \mathrm{MP \#} \mathrm{Block} \mathrm{M[mNm]} \mathrm{G[Pa}\right]$

$\begin{array}{rrrrrrrrr}1.81765 & 28 & 0.1 & 1.108 & 11.08 & 1 & 1 & 0.28295 & 0.550159884 \\ 4.33876 & 28 & 0.21 & 1.397 & 6.652 & 2 & 1 & 0.35675 & 0.230480511 \\ 6.83792 & 28 & 0.31 & 1.77 & 5.71 & 3 & 1 & 0.45195 & 0.146243283 \\ 9.48656 & 28 & 0.4 & 2.125 & 5.313 & 4 & 1 & 0.54275 & 0.105412184 \\ 12.54543 & 28 & 0.5 & 2.408 & 4.816 & 5 & 1 & 0.61485 & 0.079710289 \\ 17.21245 & 28 & 0.61 & 2.456 & 4.026 & 6 & 1 & 0.6272 & 0.058097464 \\ 23.67511 & 28 & 0.7 & 2.379 & 3.399 & 7 & 1 & 0.6076 & 0.042238442 \\ 30.46156 & 28 & 0.8 & 2.376 & 2.97 & 8 & 1 & 0.60665 & 0.032828249 \\ 37.447 & 28 & 0.9 & 2.415 & 2.683 & 9 & 1 & 0.61675 & 0.026704396 \\ 0 & 28 & 0.99 & 2.356 & 2.38 & 10 & 1 & 0.60155 & 0 \\ 0 & 28 & 2 & 3.798 & 1.899 & 11 & 1 & 0.9699 & 0 \\ 0 & 28 & 3 & 4.319 & 1.44 & 12 & 1 & 1.103 & 0 \\ 0 & 28 & 3.99 & 4.126 & 1.034 & 13 & 1 & 1.05375 & 0 \\ 0 & 28 & 5 & 3.957 & 0.791 & 14 & 1 & 1.0105 & 0 \\ 0 & 28 & 6 & 3.934 & 0.656 & 15 & 1 & 1.00455 & 0 \\ 0 & 28 & 7 & 3.977 & 0.568 & 16 & 1 & 1.0155 & 0 \\ 0 & 28 & 8 & 4.005 & 0.501 & 17 & 1 & 1.0228 & 0 \\ 0 & 28 & 9 & 4.015 & 0.446 & 18 & 1 & 1.0253 & 0 \\ 0 & 28 & 10.01 & 4.071 & 0.407 & 19 & 1 & 1.0395 & 0 \\ 0 & 28 & 20.01 & 6.242 & 0.312 & 20 & 1 & 1.5941 & 0 \\ 0 & 28 & 30.04 & 7.202 & 0.24 & 21 & 1 & 1.8392 & 0 \\ 0 & 28 & 40.01 & 6.796 & 0.17 & 22 & 1 & 1.73545 & 0 \\ 0 & 28 & 50 & 6.754 & 0.135 & 23 & 1 & 1.7246 & 0 \\ 0 & 28 & 60.01 & 8.713 & 0.145 & 24 & 1 & 2.22505 & 0 \\ 0 & 28 & 70 & 7.25 & 0.104 & 25 & 1 & 1.85135 & 0 \\ 0 & 28 & 79.99 & 7.353 & 0.092 & 26 & 1 & 1.87775 & 0 \\ 0 & 28 & 90 & 7.781 & 0.086 & 27 & 1 & 1.9871 & 0 \\ 0 & 28 & 100 & 8.273 & 0.083 & 28 & 1 & 2.1126 & 0 \\ & & & & & & & & 0 \\ 0\end{array}$




\begin{tabular}{|c|c|c|c|c|c|c|c|c|c|c|c|c|c|c|}
\hline & 580 & 154.92 & 69.12 & 1296.58948 & 0 & 0 & 28 & 200 & 13.533 & 0.068 & 29 & 1 & 3.4559 & 0 \\
\hline & 600 & 232.38 & 92.66 & 1781.94041 & 0 & 0 & 28 & 300 & 18.143 & 0.06 & 30 & 1 & 4.63315 & . \\
\hline & 620 & 309.84 & 113.78 & 2430.13109 & 0 & 0 & 28 & 400 & 22.278 & 0.056 & 31 & 1 & 5.6889 & \\
\hline & 640 & 387.3 & 134.25 & 3239.41557 & 0 & 0 & 28 & 500 & 26.285 & 0.053 & 32 & 1 & 6.7123 & \\
\hline & 660 & 464.75 & 155.62 & 4211.25155 & 0 & 0 & 28 & 599.99 & 30.469 & 0.051 & 33 & 1 & 7.78075 & \\
\hline & 680 & 542.21 & 179.22 & 5346.415 & 0 & 0 & 28 & 699.99 & 35.092 & 0.05 & 34 & 1 & 8.9612 & \\
\hline & 700 & 619.67 & 201.14 & 6641.61668 & 0 & 0 & 28 & 799.99 & 39.383 & 0.049 & 35 & 1 & 10.0569 & \\
\hline & 720 & 697.13 & 223.01 & 8101.80617 & 0 & 0 & 28 & 899.99 & 43.665 & 0.049 & 36 & 1 & 11.15035 & \\
\hline & 740 & 774.59 & 245.55 & 9723.17036 & 0 & 0 & 28 & 1000 & 48.078 & 0.048 & 37 & 1 & 12.27725 & \\
\hline & 750 & 774.59 & 245.06 & 10550.62267 & 0 & 0 & 28 & 1000 & 47.982 & 0.048 & 38 & 1 & 12.2528 & \\
\hline & 760 & 774.59 & 245.12 & 11361.36563 & 0 & 0 & 28 & 1000 & 47.995 & 0.048 & 39 & 1 & 12.25605 & \\
\hline & 770 & 774.59 & 245.05 & 12172.51464 & 0 & 0 & 28 & 1000 & 47.981 & 0.048 & 40 & 1 & 12.25245 & \\
\hline & 780 & 774.59 & 244.77 & 12986.09917 & 0 & 0 & 28 & 1000 & 47.927 & 0.048 & 41 & 1 & 12.23865 & \\
\hline & 790 & 774.59 & 244.64 & 13795.21793 & 0 & 0 & 28 & 1000 & 47.9 & 0.048 & 42 & 1 & 12.2319 & \\
\hline & 800 & 774.59 & 244.26 & 14606.77378 & 0 & 0 & 28 & 1000 & 47.827 & 0.048 & 43 & 1 & 12.21315 & \\
\hline & 820 & 774.59 & 243.89 & 16247.3221 & 0 & 0 & 28 & 1000 & 47.754 & 0.048 & 44 & 1 & 12.1946 & C \\
\hline & 840 & 697.13 & 220.17 & 17709.05333 & 0 & 0 & 28 & 899.99 & 43.109 & 0.048 & 45 & 1 & 11.0083 & . \\
\hline & 860 & 619.67 & 196.71 & 19008.55507 & 0 & 0 & 28 & 799.99 & 38.516 & 0.048 & 46 & 1 & 9.83555 & . \\
\hline & 880 & 542.21 & 173.66 & 20145.21863 & 0 & 0 & 28 & 699.99 & 34.003 & 0.049 & 47 & 1 & 8.6831 & 0 \\
\hline & 900 & 464.75 & 150.6 & 21119.93859 & 0 & 0 & 28 & 599.99 & 29.488 & 0.049 & 48 & 1 & 7.53015 & . \\
\hline & 920 & 387.3 & 128.34 & 21932.59007 & 0 & 0 & 28 & 500 & 25.129 & 0.05 & 49 & 1 & 6.41695 & 0 \\
\hline & 940 & 309.84 & 106.2 & 22583.29481 & 0 & 0 & 28 & 400 & 20.793 & 0.052 & 50 & 1 & 5.30975 & 0 \\
\hline & 960 & 232.38 & 83.96 & 23070.75611 & 0 & 0 & 28 & 300 & 16.439 & 0.055 & 51 & 1 & 4.198 & 0 \\
\hline & 980 & 154.92 & 60.74 & 23396.8008 & 0 & 0 & 28 & 200 & 11.892 & 0.059 & 52 & 1 & 3.03685 & 0 \\
\hline & 61000 & 77.46 & 36.13 & 23560.20996 & 0 & 0 & 28 & 100 & 7.074 & 0.071 & 53 & 1 & 1.8064 & 0 \\
\hline & 71020 & 69.71 & 33.55 & 23707.45562 & 0 & 0 & 28 & 90 & 6.569 & 0.073 & 54 & 1 & 1.6774 & 0 \\
\hline & 71040 & 61.97 & 30.78 & 23837.25523 & 0 & 0 & 28 & 80 & 6.027 & 0.075 & 55 & 1 & 1.53915 & 0 \\
\hline & 71060 & 54.22 & 27.92 & 23951.04372 & 0 & 0 & 28 & 70 & 5.467 & 0.078 & 56 & 1 & 1.39615 & 0 \\
\hline & 71080 & 46.48 & 24.52 & 24048.50299 & 0 & 0 & 28 & 60.01 & 4.801 & 0.08 & 57 & 1 & 1.226 & 0 \\
\hline & 71100 & 38.73 & 21.12 & 24129.68017 & 0 & 0 & 28 & 50 & 4.136 & 0.083 & 58 & 1 & 1.05615 & 0 \\
\hline & 71120 & 30.99 & 17.83 & 24194.6813 & 0 & 0 & 28 & 40.01 & 3.492 & 0.087 & 59 & 1 & 0.8917 & 0 \\
\hline & 71140 & 23.24 & 14.1 & 24243.49693 & 0 & 0 & 28 & 30 & 2.76 & 0.092 & 60 & 1 & 0.7049 & 0 \\
\hline & 71160 & 15.5 & 9.63 & 24276.0674 & 0 & 0 & 28 & 20.01 & 1.885 & 0.094 & 61 & 1 & 0.4813 & 0 \\
\hline & 71180 & 7.75 & 4.74 & 24292.41782 & 0 & 0 & 28 & 10.01 & 0.928 & 0.093 & 62 & 1 & 0.2369 & 0 \\
\hline & 81200 & 6.97 & 5.03 & 24307.13618 & 0 & 0 & 28 & 9 & 0.984 & 0.109 & 63 & 1 & 0.25125 & 0 \\
\hline & 81220 & 6.2 & 8.24 & 24320.10781 & 0 & 0 & 28 & 8 & 1.613 & 0.202 & 64 & 1 & 0.41195 & 0 \\
\hline & 81240 & 5.42 & 4.21 & 24331.46781 & 0 & 0 & 28 & 7 & 0.824 & 0.118 & 65 & 1 & 0.21035 & 0 \\
\hline & 81260 & 4.64 & 4.04 & 24341.20989 & 0 & 0 & 28 & 5.99 & 0.791 & 0.132 & 66 & 1 & 0.2019 & 0 \\
\hline & 81280 & 3.87 & 3.66 & 24349.3262 & 0 & 0 & 28 & 5 & 0.717 & 0.143 & 67 & 1 & 0.1831 & 0 \\
\hline & 81300 & 3.09 & 3.29 & 24355.82222 & 0 & 0 & 28 & 3.99 & 0.644 & 0.161 & 68 & 1 & 0.16435 & 0 \\
\hline & 81320 & 2.32 & 3.08 & 24360.69012 & 0 & 0 & 28 & 3 & 0.603 & 0.201 & 69 & 1 & 0.1539 & 0 \\
\hline & 81340 & 1.54 & 1.78 & 24363.93617 & 0 & 0 & 28 & 1.99 & 0.349 & 0.175 & 70 & 1 & 0.08905 & 0 \\
\hline & 81360 & 0.77 & 0 & 24365.56038 & 0 & 0 & 28 & 0.99 & 0 & 0 & 71 & 1 & 0 & 0 \\
\hline & 91380 & 0.7 & 1.99 & 24367.03614 & 18.19342 & 46.7696 & 28 & 0.9 & 0.389 & 0.432 & 72 & 1 & 0.09935 & 0.021381357 \\
\hline & 91400 & 0.62 & 2.15 & 24368.3399 & 34.26637 & 81.19974 & 28 & 0.8 & 0.422 & 0.528 & 73 & 1 & 0.10775 & 0.012315282 \\
\hline & 91420 & 0.55 & 2.34 & 24369.48344 & 48.36409 & 105.36816 & 28 & 0.71 & 0.459 & 0.646 & 74 & 1 & 0.1172 & 0.009490512 \\
\hline & 91440 & 0.47 & 2.5 & 24370.46283 & 60.43817 & 123.59519 & 28 & 0.61 & 0.489 & 0.802 & 75 & 1 & 0.12495 & 0.008090913 \\
\hline & 91460 & 0.39 & 2.48 & 24371.28043 & 70.51765 & 145.39692 & 28 & 0.5 & 0.485 & 0.97 & 76 & 1 & 0.12375 & 0.006877711 \\
\hline & 91480 & 0.31 & 2.44 & 24371.93702 & 78.61222 & 164.80515 & 28 & 0.4 & 0.477 & 1.193 & 77 & 1 & 0.1218 & 0.006067759 \\
\hline & 91500 & 0.24 & 2.51 & 24372.42947 & 84.68315 & 172.47042 & 28 & 0.31 & 0.491 & 1.584 & 78 & 1 & 0.1255 & 0.005798084 \\
\hline
\end{tabular}



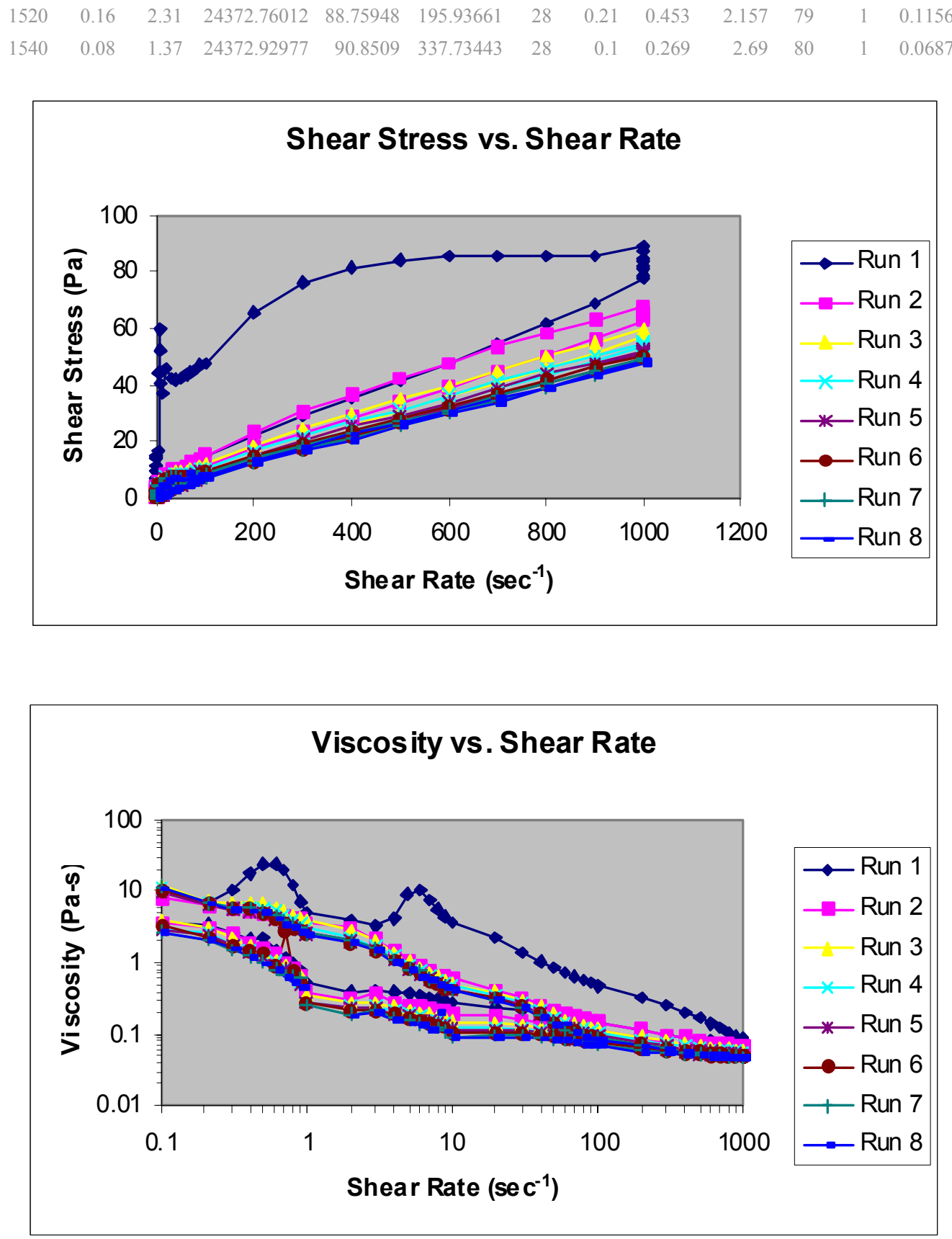

\section{E-2.1.22 META/20wt\%/U/S}

2004.02.12 META-20 wt\%-U [sonicated] CC-45 0.1-1000 s-1 run 1.txt Mode Step t[s] n[rpm] M[\%.] Phi[rad] Gamma J[t]

$\mathrm{T}\left[{ }^{\circ} \mathrm{C}\right] \mathrm{D}[1 / \mathrm{s}]$ Tau[Pa] Eta[Pas $]$ MP\# Block M[mNm] G[Pa]

\begin{tabular}{|c|c|c|c|c|c|c|c|c|c|c|c|c|c|c|c|}
\hline de & Step & $\mathrm{t}[\mathrm{s}]$ & $\mathrm{n}[\mathrm{rpm}]$ & $\mathrm{M}[\%]$. & Phi[rad] & Gamma & $\mathrm{J}[\mathrm{t}]$ & $\mathrm{T}\left[{ }^{\circ} \mathrm{C}\right]$ & $\mathrm{D}[1 / \mathrm{s}]$ & & & {$[\mathrm{P} \#$} & & & \\
\hline 1 & 1 & 20 & 0.08 & 27.31 & 0.16179 & 1.9946 & 0.373 & 28 & 0.1 & 5.348 & 53.48 & 1 & 1 & 1.366 & 2.6812528 \\
\hline 1 & 1 & 40 & 0.16 & 12.85 & 0.4893 & 6.0322 & 2.3975 & 28 & 0.21 & 2.516 & 11.981 & 2 & 1 & 0.642 & 0.4170949 \\
\hline 1 & 1 & 60 & 0.24 & 19.14 & 0.97704 & 12.045 & 3.2146 & 28 & 0.31 & 3.747 & 12.087 & 3 & 1 & 0.957 & 0.3110827 \\
\hline 1 & 1 & 80 & 0.31 & 29.38 & 1.62734 & 20.062 & 3.4873 & 28 & 0.4 & 5.753 & 14.383 & 4 & 1 & 1.469 & 0.286759 \\
\hline 1 & 1 & 100 & 0.39 & 41.17 & 2.43866 & 30.064 & 3.73 & 28 & 0.5 & 8.06 & 16.12 & 5 & 1 & 2.058 & 0.2680933 \\
\hline 1 & 1 & 120 & 0.47 & 51.68 & 3.41413 & 42.09 & 4.1595 & 28 & 0.61 & 10.12 & 16.589 & 6 & 1 & 2.584 & 0.2404144 \\
\hline
\end{tabular}




\begin{tabular}{|c|c|c|c|c|c|c|c|c|c|c|c|c|c|c|c|}
\hline 1 & 1 & 140 & 0.54 & 52.07 & 4.55609 & 56.168 & 5.5099 & 28 & 0.7 & 10.19 & 14.563 & 7 & 1 & 2.603 & 0.1814907 \\
\hline & 1 & 160 & 0.62 & 43.68 & 5.86221 & 72.27 & 8.4507 & 28 & 0.8 & 8.552 & 10.69 & 8 & 1 & 2.184 & 0.1183337 \\
\hline & 1 & 180 & 0.7 & 28.81 & 7.33248 & 90.396 & 16.025 & 28 & 0.9 & 5.641 & 6.268 & 9 & 1 & 1.441 & 0.0624033 \\
\hline & 2 & 200 & 0.77 & 34.59 & 8.94804 & 0 & 0 & 28 & 0.99 & 6.773 & 6.841 & 10 & 1 & 1.73 & 0 \\
\hline & 2 & 220 & 1.55 & 77.14 & 12.16739 & 0 & 0 & 28 & 2 & 15.1 & 7.552 & 11 & 1 & 3.857 & 0 \\
\hline & 2 & 240 & 2.31 & 117.2 & 16.98502 & 0 & 0 & 28 & 2.98 & 22.94 & 7.698 & 12 & 1 & 5.858 & 0 \\
\hline & 2 & 260 & 3.09 & 186.5 & 23.44571 & 0 & 0 & 28 & 3.99 & 36.53 & 9.154 & 13 & 1 & 9.327 & 0 \\
\hline & 2 & 280 & 3.87 & 190.9 & 31.53609 & 0 & 0 & 28 & 5 & 37.38 & 7.475 & 14 & 1 & 9.545 & 0 \\
\hline & 2 & 300 & 4.64 & 256.5 & 41.22398 & 0 & 0 & 28 & 5.99 & 50.21 & 8.383 & 15 & 1 & 12.82 & 0 \\
\hline & 2 & 320 & 5.42 & 271.7 & 52.57141 & 0 & 0 & 28 & 7 & 53.2 & 7.599 & 16 & 1 & 13.58 & 0 \\
\hline & 2 & 340 & 6.2 & 234.5 & 65.54619 & 0 & 0 & 28 & 8 & 45.92 & 5.74 & 17 & 1 & 11.73 & 0 \\
\hline & 2 & 360 & 6.97 & 196.9 & 80.14674 & 0 & 0 & 28 & 9 & 38.55 & 4.283 & 18 & 1 & 9.843 & 0 \\
\hline & 3 & 380 & 7.75 & 176.5 & 96.53329 & 0 & 0 & 28 & 10.01 & 34.57 & 3.453 & 19 & 1 & 8.827 & 0 \\
\hline & 3 & 400 & 15.51 & 186.7 & 128.8697 & 0 & 0 & 28 & 20.02 & 36.56 & 1.826 & 20 & 1 & 9.336 & 0 \\
\hline & 3 & 420 & 23.25 & 142.3 & 177.40652 & 0 & 0 & 28 & 30.02 & 27.86 & 0.928 & 21 & 1 & 7.115 & 0 \\
\hline & 3 & 440 & 30.99 & 116.6 & 242.23564 & 0 & 0 & 28 & 40.01 & 22.82 & 0.57 & 22 & 1 & 5.828 & 0 \\
\hline & 3 & 460 & 38.73 & 105.2 & 323.17564 & 0 & 0 & 28 & 50 & 20.6 & 0.412 & 23 & 1 & 5.262 & 0 \\
\hline & 3 & 480 & 46.48 & 96.08 & 420.39772 & 0 & 0 & 28 & 60.01 & 18.81 & 0.313 & 24 & 1 & 4.804 & 0 \\
\hline & 3 & 500 & 54.22 & 81.6 & 533.86419 & 0 & 0 & 28 & 70 & 15.98 & 0.228 & 25 & 1 & 4.08 & 0 \\
\hline & 3 & 520 & 61.96 & 74.66 & 663.52243 & 0 & 0 & 28 & 79.99 & 14.62 & 0.183 & 26 & 1 & 3.733 & 0 \\
\hline & 3 & 540 & 69.71 & 71.45 & 809.29155 & 0 & 0 & 28 & 90 & 13.99 & 0.155 & 27 & 1 & 3.573 & 0 \\
\hline & 4 & 560 & 77.46 & 70.98 & 972.98423 & 0 & 0 & 28 & 100 & 13.9 & 0.139 & 28 & 1 & 3.549 & 0 \\
\hline & 4 & 580 & 154.9 & 117.6 & 1296.1858 & 0 & 0 & 28 & 200 & 23.02 & 0.115 & 29 & 1 & 5.877 & 0 \\
\hline & 4 & 600 & 232.4 & 133.1 & 1781.8234 & 0 & 0 & 28 & 300 & 26.06 & 0.087 & 30 & 1 & 6.654 & 0 \\
\hline & 4 & 620 & 309.8 & 136.5 & 2428.8807 & 0 & 0 & 28 & 400 & 26.72 & 0.067 & 31 & 1 & 6.823 & 0 \\
\hline & 4 & 640 & 387.3 & 142.2 & 3239.1807 & 0 & 0 & 28 & 500 & 27.84 & 0.056 & 32 & 1 & 7.108 & 0 \\
\hline & 4 & 660 & 464.8 & 150.7 & 4211.4652 & 0 & 0 & 28 & 600 & 29.5 & 0.049 & 33 & 1 & 7.533 & 0 \\
\hline & 4 & 680 & 542.2 & 173.1 & 5344.9275 & 0 & 0 & 28 & 700 & 33.89 & 0.048 & 34 & 1 & 8.655 & 0 \\
\hline & 4 & 700 & 619.7 & 170.4 & 6642.0793 & 0 & 0 & 28 & 800 & 33.36 & 0.042 & 35 & 1 & 8.518 & 0 \\
\hline 1 & 4 & 720 & 697.1 & 181.2 & 8100.1231 & 0 & 0 & 28 & 900 & 35.48 & 0.039 & 36 & 1 & 9.061 & 0 \\
\hline I & 4 & 740 & 774.6 & 190.9 & 9721.8925 & 0 & 0 & 28 & 1000 & 37.37 & 0.037 & 37 & 1 & 9.542 & 0 \\
\hline 1 & 5 & 750 & 774.6 & 183.3 & 10550.564 & 0 & 0 & 28 & 1000 & 35.88 & 0.036 & 38 & 1 & 9.164 & 0 \\
\hline 1 & 5 & 760 & 774.6 & 180.4 & 11361.307 & 0 & 0 & 28 & 1000 & 35.32 & 0.035 & 39 & 1 & 9.02 & 0 \\
\hline 1 & 5 & 770 & 774.6 & 182.4 & 12171.644 & 0 & 0 & 28 & 1000 & 35.71 & 0.036 & 40 & 1 & 9.12 & 0 \\
\hline 1 & 5 & 780 & 774.6 & 178.7 & 12984.415 & 0 & 0 & 28 & 1000 & 34.99 & 0.035 & 41 & 1 & 8.934 & 0 \\
\hline 1 & 5 & 790 & 774.6 & 177.2 & 13795.565 & 0 & 0 & 28 & 1000 & 34.69 & 0.035 & 42 & 1 & 8.859 & 0 \\
\hline 1 & 5 & 800 & 774.6 & 180.7 & 14606.713 & 0 & 0 & 28 & 1000 & 35.39 & 0.035 & 43 & 1 & 9.036 & 0 \\
\hline 1 & 6 & 820 & 774.6 & 183.9 & 16247.668 & 0 & 0 & 28 & 1000 & 36 & 0.036 & 44 & 1 & 9.193 & 0 \\
\hline 1 & 6 & 840 & 697.1 & 151.4 & 17709.441 & 0 & 0 & 28 & 900 & 29.65 & 0.033 & 45 & 1 & 7.572 & 0 \\
\hline 1 & 6 & 860 & 619.7 & 120.8 & 19009.267 & 0 & 0 & 28 & 800 & 23.65 & 0.03 & 46 & 1 & 6.038 & 0 \\
\hline 1 & 6 & 880 & 542.2 & 106.3 & 20145.362 & 0 & 0 & 28 & 700 & 20.82 & 0.03 & 47 & 1 & 5.317 & 0 \\
\hline 1 & 6 & 900 & 464.8 & 89.23 & 21120.81 & 0 & 0 & 28 & 600 & 17.47 & 0.029 & 48 & 1 & 4.461 & 0 \\
\hline 1 & 6 & 920 & 387.3 & 73.54 & 21932.245 & 0 & 0 & 28 & 500 & 14.4 & 0.029 & 49 & 1 & 3.677 & 0 \\
\hline 1 & 6 & 940 & 309.8 & 58.69 & 22582.623 & 0 & 0 & 28 & 400 & 11.49 & 0.029 & 50 & 1 & 2.934 & 0 \\
\hline 1 & 6 & 960 & 232.4 & 44.39 & 23070.935 & 0 & 0 & 28 & 300 & 8.691 & 0.029 & 51 & 1 & 2.219 & 0 \\
\hline 1 & 6 & 980 & 154.9 & 30.09 & 23396.45 & 0 & 0 & 28 & 200 & 5.891 & 0.029 & 52 & 1 & 1.504 & 0 \\
\hline 1 & 6 & 1000 & 77.46 & 16.79 & 23560.069 & 0 & 0 & 28 & 100 & 3.288 & 0.033 & 53 & 1 & 0.84 & 0 \\
\hline 1 & 7 & 1020 & 69.71 & 17.94 & 23707.589 & 0 & 0 & 28 & 90 & 3.513 & 0.039 & 54 & 1 & 0.897 & 0 \\
\hline 1 & 7 & 1040 & 61.97 & 17.7 & 23837.454 & 0 & 0 & 28 & 80 & 3.466 & 0.043 & 55 & 1 & 0.885 & 0 \\
\hline 1 & 7 & 1060 & 54.22 & 16.83 & 23951.162 & 0 & 0 & 28 & 70 & 3.295 & 0.047 & 56 & 1 & 0.842 & \\
\hline
\end{tabular}




\begin{tabular}{|c|c|c|c|c|c|c|c|c|c|c|c|c|c|c|}
\hline 7 & 1080 & 46.48 & 15.74 & 24048.616 & 0 & 0 & 28 & 60.01 & 3.081 & 0.051 & 57 & 1 & 0.787 & 0 \\
\hline 7 & 1100 & 38.73 & 14.66 & 24129.817 & 0 & 0 & 28 & 50 & 2.87 & 0.057 & 58 & 1 & 0.733 & 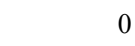 \\
\hline 7 & 1120 & 30.99 & 13.75 & 24194.866 & 0 & 0 & 28 & 40.01 & 2.692 & 0.067 & 59 & 1 & 0.688 & \\
\hline 7 & 1140 & 23.24 & 12.45 & 24243.616 & 0 & 0 & 28 & 30 & 2.438 & 0.081 & 60 & 1 & 0.623 & \\
\hline 7 & 1160 & 15.5 & 10.6 & 24276.202 & 0 & 0 & 28 & 20.01 & 2.075 & 0.104 & 61 & 1 & 0.53 & \\
\hline 7 & 1180 & 7.75 & 8.29 & 24292.536 & 0 & 0 & 28 & 10.01 & 1.623 & 0.162 & 62 & 1 & 0.414 & \\
\hline 8 & 1200 & 6.97 & 10.82 & 24307.238 & 0 & 0 & 28 & 9 & 2.118 & 0.235 & 63 & 1 & 0.541 & \\
\hline 8 & 1220 & 6.2 & 11.9 & 24320.23 & 0 & 0 & 28 & 8 & 2.33 & 0.291 & 64 & 1 & 0.595 & \\
\hline 8 & 1240 & 5.42 & 12.69 & 24331.582 & 0 & 0 & 28 & 7 & 2.485 & 0.355 & 65 & 1 & 0.635 & \\
\hline 8 & 1260 & 4.64 & 13.19 & 24341.323 & 0 & 0 & 28 & 5.99 & 2.583 & 0.431 & 66 & 1 & 0.66 & \\
\hline 8 & 1280 & 3.87 & 13.31 & 24349.44 & 0 & 0 & 28 & 5 & 2.606 & 0.521 & 67 & 1 & 0.665 & \\
\hline 8 & 1300 & 3.09 & 13.22 & 24355.935 & 0 & 0 & 28 & 3.99 & 2.588 & 0.649 & 68 & 1 & 0.661 & \\
\hline 8 & 1320 & 2.32 & 13.06 & 24360.807 & 0 & 0 & 28 & 3 & 2.557 & 0.852 & 69 & 1 & 0.653 & \\
\hline 8 & 1340 & 1.54 & 11.96 & 24364.052 & 0 & 0 & 28 & 1.99 & 2.342 & 1.177 & 70 & 1 & 0.598 & \\
\hline 8 & 1360 & 0.77 & 10.74 & 24365.675 & 0 & 0 & 28 & 0.99 & 2.103 & 2.124 & 71 & 1 & 0.537 & 0 \\
\hline 9 & 1380 & 0.7 & 13.75 & 24367.15 & 18.184 & 6.7547 & 28 & 0.9 & 2.692 & 2.991 & 72 & 1 & 0.687 & 0.1480444 \\
\hline 9 & 1400 & 0.62 & 14.37 & 24368.454 & 34.257 & 12.174 & 28 & 0.8 & 2.814 & 3.518 & 73 & 1 & 0.719 & 0.0821446 \\
\hline 9 & 1420 & 0.55 & 14.3 & 24369.597 & 48.354 & 17.269 & 28 & 0.71 & 2.8 & 3.944 & 74 & 1 & 0.715 & 0.0579058 \\
\hline 9 & 1440 & 0.47 & 14.59 & 24370.577 & 60.428 & 21.158 & 28 & 0.61 & 2.856 & 4.682 & 75 & 1 & 0.729 & 0.0472625 \\
\hline 9 & 1460 & 0.39 & 15.01 & 24371.394 & 70.508 & 23.99 & 28 & 0.5 & 2.939 & 5.878 & 76 & 1 & 0.75 & 0.0416832 \\
\hline 9 & 1480 & 0.31 & 15.23 & 24372.049 & 78.583 & 26.361 & 28 & 0.4 & 2.981 & 7.453 & 77 & 1 & 0.761 & 0.0379343 \\
\hline 9 & 1500 & 0.24 & 15.73 & 24372.543 & 84.664 & 27.488 & 28 & 0.31 & 3.08 & 9.935 & 78 & 1 & 0.787 & 0.0363792 \\
\hline 9 & 1520 & 0.16 & 16.02 & 24372.873 & 88.74 & 28.288 & 28 & 0.21 & 3.137 & 14.938 & 79 & 1 & 0.801 & 0.0353504 \\
\hline 9 & 1540 & 0.08 & 13.6 & 24373.042 & 90.822 & 34.118 & 28 & 0.1 & 2.662 & 26.62 & 80 & 1 & 0.68 & 0.0293101 \\
\hline
\end{tabular}

2004.02.12 META-20 wt\%-U [sonicated] CC-45

$0.1-1000 \mathrm{~s}-1$ run 2.txt

\begin{tabular}{|c|c|c|c|c|c|c|c|c|c|c|c|c|c|c|c|}
\hline 1 & 1 & 20 & 0.08 & 17.18 & 0.15944 & 1.9656 & 0.5845 & 28 & 0.1 & 3.363 & 33.63 & 1 & 2 & 0.859 & 1.7109715 \\
\hline 1 & 1 & 40 & 0.16 & 25.46 & 0.48538 & 5.9838 & 1.2001 & 28 & 0.21 & 4.986 & 23.743 & 2 & 2 & 1.273 & 0.8332526 \\
\hline & 1 & 60 & 0.24 & 30.46 & 0.97468 & 12.016 & 2.0144 & 28 & 0.31 & 5.965 & 19.242 & 3 & 2 & 1.523 & 0.4964223 \\
\hline & 1 & 80 & 0.31 & 32.53 & 1.62813 & 20.072 & 3.151 & 28 & 0.4 & 6.37 & 15.925 & 4 & 2 & 1.627 & 0.3173604 \\
\hline & 1 & 100 & 0.39 & 32.68 & 2.44416 & 30.132 & 4.7096 & 28 & 0.5 & 6.398 & 12.796 & 5 & 2 & 1.634 & 0.2123328 \\
\hline & 1 & 120 & 0.47 & 31.18 & 3.42277 & 42.196 & 6.9118 & 28 & 0.61 & 6.105 & 10.008 & 6 & 2 & 1.559 & 0.1446808 \\
\hline & 1 & 140 & 0.54 & 29.25 & 4.56395 & 56.265 & 9.8245 & 28 & 0.7 & 5.727 & 8.181 & 7 & 2 & 1.462 & 0.1017862 \\
\hline & 1 & 160 & 0.62 & 27.41 & 5.86614 & 72.319 & 13.475 & 28 & 0.8 & 5.367 & 6.709 & 8 & 2 & 1.371 & 0.0742133 \\
\hline & 1 & 180 & 0.7 & 26.67 & 7.33248 & 90.396 & 17.307 & 28 & 0.9 & 5.223 & 5.803 & 9 & 2 & 1.334 & 0.0577792 \\
\hline & 2 & 200 & 0.77 & 26.24 & 8.95511 & 0 & 0 & 28 & 0.99 & 5.138 & 5.19 & 10 & 2 & 1.312 & 0 \\
\hline & 2 & 220 & 1.55 & 37.68 & 12.1776 & 0 & 0 & 28 & 2 & 7.378 & 3.689 & 11 & 2 & 1.884 & 0 \\
\hline & 2 & 240 & 2.32 & 41.46 & 17.02743 & 0 & 0 & 28 & 3 & 8.118 & 2.706 & 12 & 2 & 2.073 & 0 \\
\hline & 2 & 260 & 3.09 & 43.41 & 23.49362 & 0 & 0 & 28 & 3.99 & 8.5 & 2.13 & 13 & 2 & 2.171 & 0 \\
\hline & 2 & 280 & 3.87 & 45.93 & 31.59107 & 0 & 0 & 28 & 5 & 8.993 & 1.799 & 14 & 2 & 2.296 & 0 \\
\hline 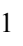 & 2 & 300 & 4.65 & 48.95 & 41.30252 & 0 & 0 & 28 & 6 & 9.584 & 1.597 & 15 & 2 & 2.447 & 0 \\
\hline 1 & 2 & 320 & 5.42 & 52.95 & 52.65623 & 0 & 0 & 28 & 7 & 10.37 & 1.481 & 16 & 2 & 2.648 & 0 \\
\hline 1 & 2 & 340 & 6.2 & 56.01 & 65.61609 & 0 & 0 & 28 & 8 & 10.97 & 1.371 & 17 & 2 & 2.801 & 0 \\
\hline 1 & 2 & 360 & 6.97 & 58.82 & 80.19465 & 0 & 0 & 28 & 9 & 11.52 & 1.28 & 18 & 2 & 2.941 & 0 \\
\hline 1 & 3 & 380 & 7.75 & 61.25 & 96.55999 & 0 & 0 & 28 & 10.01 & 11.99 & 1.198 & 19 & 2 & 3.062 & 0 \\
\hline 1 & 3 & 400 & 15.5 & 98.29 & 128.89562 & 0 & 0 & 28 & 20.01 & 19.25 & 0.962 & 20 & 2 & 4.915 & 0 \\
\hline 1 & 3 & 420 & 23.25 & 99.67 & 177.44422 & 0 & 0 & 28 & 30.02 & 19.52 & 0.65 & 21 & 2 & 4.984 & 0 \\
\hline & 3 & 440 & 30.99 & 94.44 & 242.27413 & 0 & 0 & 28 & 40.01 & 18.49 & 0.462 & 22 & 2 & 4.722 & 0 \\
\hline & 3 & 460 & 38.73 & 89.55 & 323.25653 & 0 & 0 & 28 & 50 & 17.53 & 0.351 & 23 & 2 & 4.478 & 0 \\
\hline
\end{tabular}




\begin{tabular}{|c|c|c|c|c|c|c|c|c|c|c|c|c|c|c|c|}
\hline 1 & 3 & 480 & 46.48 & 89.68 & 420.50061 & 0 & 0 & 28 & 60.01 & 17.56 & 0.293 & 24 & 2 & 4.484 & 0 \\
\hline 1 & 3 & 500 & 54.22 & 89.39 & 533.90896 & 0 & 0 & 28 & 70 & 17.5 & 0.25 & 25 & 2 & 4.47 & 0 \\
\hline 1 & 3 & 520 & 61.96 & 90.46 & 663.53814 & 0 & 0 & 28 & 79.99 & 17.71 & 0.221 & 26 & 2 & 4.523 & 0 \\
\hline 1 & 3 & 540 & 69.71 & 91.85 & 809.49339 & 0 & 0 & 28 & 90 & 17.98 & 0.2 & 27 & 2 & 4.592 & 0 \\
\hline 1 & 4 & 560 & 77.46 & 94.39 & 973.06513 & 0 & 0 & 28 & 100 & 18.48 & 0.185 & 28 & 2 & 4.719 & \\
\hline 1 & 4 & 580 & 154.9 & 126.2 & 1296.6335 & 0 & 0 & 28 & 200 & 24.72 & 0.124 & 29 & 2 & 6.312 & \\
\hline 1 & 4 & 600 & 232.4 & 136.3 & 1781.8257 & 0 & 0 & 28 & 300 & 26.68 & 0.089 & 30 & 2 & 6.814 & \\
\hline 1 & 4 & 620 & 309.8 & 140.4 & 2429.6528 & 0 & 0 & 28 & 400 & 27.48 & 0.069 & 31 & 2 & 7.018 & \\
\hline & 4 & 640 & 387.3 & 149.6 & 3239.9952 & 0 & 0 & 28 & 500 & 29.3 & 0.059 & 32 & 2 & 7.481 & \\
\hline 1 & 4 & 660 & 464.8 & 141.1 & 4211.799 & 0 & 0 & 28 & 600 & 27.62 & 0.046 & 33 & 2 & 7.054 & \\
\hline 1 & 4 & 680 & 542.2 & 151.1 & 5345.8236 & 0 & 0 & 28 & 700 & 29.58 & 0.042 & 34 & 2 & 7.553 & 0 \\
\hline 1 & 4 & 700 & 619.7 & 180.4 & 6642 & 0 & 0 & 28 & 800 & 35.31 & 0.044 & 35 & 2 & 9.017 & 0 \\
\hline 1 & 4 & 720 & 697.1 & 180.5 & 8102.1541 & 0 & 0 & 28 & 900 & 35.33 & 0.039 & 36 & 2 & 9.022 & 0 \\
\hline 1 & 4 & 740 & 774.6 & 187.6 & 9721.8964 & 0 & 0 & 28 & 1000 & 36.73 & 0.037 & 37 & 2 & 9.378 & 0 \\
\hline 1 & 5 & 750 & 774.6 & 176.6 & 10551.378 & 0 & 0 & 28 & 1000 & 34.57 & 0.035 & 38 & 2 & 8.829 & 0 \\
\hline 1 & 5 & 760 & 774.6 & 174.5 & 11361.311 & 0 & 0 & 28 & 1000 & 34.17 & 0.034 & 39 & 2 & 8.726 & 0 \\
\hline 1 & 5 & 770 & 774.6 & 173.3 & 12171.648 & 0 & 0 & 28 & 1000 & 33.93 & 0.034 & 40 & 2 & 8.665 & 0 \\
\hline 1 & 5 & 780 & 774.6 & 172.9 & 12984.826 & 0 & 0 & 28 & 1000 & 33.85 & 0.034 & 41 & 2 & 8.643 & 0 \\
\hline 1 & 5 & 790 & 774.6 & 172.1 & 13796.381 & 0 & 0 & 28 & 1000 & 33.7 & 0.034 & 42 & 2 & 8.605 & 0 \\
\hline 1 & 5 & 800 & 774.6 & 171.7 & 14604.69 & 0 & 0 & 28 & 1000 & 33.62 & 0.034 & 43 & 2 & 8.585 & 0 \\
\hline 1 & 6 & 820 & 774.6 & 170.6 & 16247.268 & 0 & 0 & 28 & 1000 & 33.4 & 0.033 & 44 & 2 & 8.528 & 0 \\
\hline 1 & 6 & 840 & 697.1 & 142.4 & 17707.497 & 0 & 0 & 28 & 900 & 27.88 & 0.031 & 45 & 2 & 7.119 & 0 \\
\hline 1 & 6 & 860 & 619.7 & 111.2 & 19007.326 & 0 & 0 & 28 & 800 & 21.76 & 0.027 & 46 & 2 & 5.557 & 0 \\
\hline 1 & 6 & 880 & 542.2 & 95.72 & 20143.989 & 0 & 0 & 28 & 700 & 18.74 & 0.027 & 47 & 2 & 4.786 & 0 \\
\hline 1 & 6 & 900 & 464.8 & 81.18 & 21119.152 & 0 & 0 & 28 & 600 & 15.89 & 0.026 & 48 & 2 & 4.059 & 0 \\
\hline 1 & 6 & 920 & 387.3 & 67.16 & 21931.234 & 0 & 0 & 28 & 500 & 13.15 & 0.026 & 49 & 2 & 3.358 & 0 \\
\hline 1 & 6 & 940 & 309.8 & 53.71 & 22581.126 & 0 & 0 & 28 & 400 & 10.52 & 0.026 & 50 & 2 & 2.685 & 0 \\
\hline 1 & 6 & 960 & 232.4 & 40.56 & 23069.316 & 0 & 0 & 28 & 300 & 7.942 & 0.026 & 51 & 2 & 2.028 & 0 \\
\hline 1 & 6 & 980 & 154.9 & 27.46 & 23394.79 & 0 & 0 & 28 & 200 & 5.377 & 0.027 & 52 & 2 & 1.373 & 0 \\
\hline 1 & 6 & 1000 & 77.46 & 15.4 & 23558.495 & 0 & 0 & 28 & 100 & 3.015 & 0.03 & 53 & 2 & 0.77 & 0 \\
\hline 1 & 7 & 1020 & 69.71 & 16.42 & 23705.593 & 0 & 0 & 28 & 90 & 3.215 & 0.036 & 54 & 2 & 0.821 & 0 \\
\hline 1 & 7 & 1040 & 61.97 & 16.11 & 23835.554 & 0 & 0 & 28 & 80 & 3.155 & 0.039 & 55 & 2 & 0.806 & 0 \\
\hline 1 & 7 & 1060 & 54.22 & 15.38 & 23949.177 & 0 & 0 & 28 & 70 & 3.01 & 0.043 & 56 & 2 & 0.769 & 0 \\
\hline 1 & 7 & 1080 & 46.48 & 14.33 & 24046.729 & 0 & 0 & 28 & 60.01 & 2.805 & 0.047 & 57 & 2 & 0.716 & 0 \\
\hline 1 & 7 & 1100 & 38.73 & 13.23 & 24127.885 & 0 & 0 & 28 & 50 & 2.59 & 0.052 & 58 & 2 & 0.661 & 0 \\
\hline 1 & 7 & 1120 & 30.99 & 12.36 & 24192.897 & 0 & 0 & 28 & 40.01 & 2.421 & 0.061 & 59 & 2 & 0.618 & 0 \\
\hline 1 & 7 & 1140 & 23.24 & 11.06 & 24241.725 & 0 & 0 & 28 & 30 & 2.165 & 0.072 & 60 & 2 & 0.553 & 0 \\
\hline 1 & 7 & 1160 & 15.5 & 9.45 & 24274.287 & 0 & 0 & 28 & 20.01 & 1.851 & 0.093 & 61 & 2 & 0.473 & م \\
\hline 1 & 7 & 1180 & 7.75 & 7.56 & 24290.62 & 0 & 0 & 28 & 10.01 & 1.48 & 0.148 & 62 & 2 & 0.378 & 0 \\
\hline 1 & 8 & 1200 & 6.97 & 9.48 & 24305.331 & 0 & 0 & 28 & 9 & 1.855 & 0.206 & 63 & 2 & 0.474 & 0 \\
\hline 1 & 8 & 1220 & 6.2 & 10 & 24318.312 & 0 & 0 & 28 & 8 & 1.958 & 0.245 & 64 & 2 & 0.5 & 0 \\
\hline 1 & 8 & 1240 & 5.42 & 10.24 & 24329.677 & 0 & 0 & 28 & 7 & 2.005 & 0.286 & 65 & 2 & 0.512 & 0 \\
\hline 1 & 8 & 1260 & 4.64 & 10.72 & 24339.412 & 0 & 0 & 28 & 5.99 & 2.099 & 0.35 & 66 & 2 & 0.536 & 0 \\
\hline 1 & 8 & 1280 & 3.87 & 10.79 & 24347.528 & 0 & 0 & 28 & 5 & 2.112 & 0.422 & 67 & 2 & 0.539 & 0 \\
\hline 1 & 8 & 1300 & 3.09 & 11.03 & 24354.019 & 0 & 0 & 28 & 3.99 & 2.16 & 0.541 & 68 & 2 & 0.552 & 0 \\
\hline 1 & 8 & 1320 & 2.32 & 11.08 & 24358.888 & 0 & 0 & 28 & 3 & 2.17 & 0.723 & 69 & 2 & 0.554 & 0 \\
\hline 1 & 8 & 1340 & 1.54 & 10.46 & 24362.137 & 0 & 0 & 28 & 1.99 & 2.047 & 1.029 & 70 & 2 & 0.523 & 0 \\
\hline 1 & 8 & 1360 & 0.77 & 9.49 & 24363.759 & 0 & 0 & 28 & 0.99 & 1.859 & 1.878 & 71 & 2 & 0.475 & 0 \\
\hline 1 & 9 & 1380 & 0.7 & 12.24 & 24365.234 & 18.184 & 7.586 & 28 & 0.9 & 2.397 & 2.663 & 72 & 2 & 0.612 & 0.1318211 \\
\hline 1 & 9 & 1400 & 0.62 & 12.68 & 24366.538 & 34.266 & 13.806 & 28 & 0.8 & 2.482 & 3.103 & 73 & 2 & 0.634 & 0.0724325 \\
\hline
\end{tabular}




$\begin{array}{rrrrrrrrrrrrrrrr}1 & 9 & 1420 & 0.55 & 12.73 & 24367.679 & 48.335 & 19.396 & 28 & 0.71 & 2.492 & 3.51 & 74 & 2 & 0.636 & 0.0515568 \\ 1 & 9 & 1440 & 0.47 & 12.9 & 24368.66 & 60.428 & 23.932 & 28 & 0.61 & 2.525 & 4.139 & 75 & 2 & 0.645 & 0.0417849 \\ 1 & 9 & 1460 & 0.39 & 13.11 & 24369.478 & 70.508 & 27.467 & 28 & 0.5 & 2.567 & 5.134 & 76 & 2 & 0.655 & 0.0364072 \\ 1 & 9 & 1480 & 0.31 & 13.36 & 24370.134 & 78.593 & 30.043 & 28 & 0.4 & 2.616 & 6.54 & 77 & 2 & 0.668 & 0.0332855 \\ 1 & 9 & 1500 & 0.24 & 13.91 & 24370.626 & 84.664 & 31.081 & 28 & 0.31 & 2.724 & 8.787 & 78 & 2 & 0.696 & 0.0321743 \\ 1 & 9 & 1520 & 0.16 & 14.01 & 24370.957 & 88.74 & 32.363 & 28 & 0.21 & 2.742 & 13.057 & 79 & 2 & 0.7 & 0.0308992 \\ 1 & 9 & 1540 & 0.08 & 12.26 & 24371.126 & 90.822 & 37.842 & 28 & 0.1 & 2.4 & 24 & 80 & 2 & 0.613 & 0.0264254\end{array}$

2004.02.12 META-20 wt\%-U [sonicated] CC-45

$0.1-1000 \mathrm{~s}-1$ run 3.txt

Mode Step t[s] n[rpm] M[\%.] Phi[rad] Gamma J[t]

\begin{tabular}{|c|c|c|c|c|c|c|c|c|c|c|c|c|c|c|c|}
\hline & 1 & 20 & 0.08 & 16.21 & 0.15944 & 1.9656 & 0.6191 & 28 & 0.1 & 3.175 & 31.75 & 1 & 3 & 0.811 & 1.615324 \\
\hline & 1 & 40 & 0.16 & 24 & 0.48616 & 5.9935 & 1.2752 & 28 & 0.21 & 4.7 & 22.381 & 2 & 3 & 1.2 & 0.7841868 \\
\hline & 1 & 60 & 0.24 & 28.16 & 0.97625 & 12.035 & 2.1831 & 28 & 0.31 & 5.513 & 17.784 & 3 & 3 & 1.408 & 0.4580673 \\
\hline & 1 & 80 & 0.31 & 29.64 & 1.6297 & 20.091 & 3.4622 & 28 & 0.4 & 5.803 & 14.507 & 4 & 3 & 1.482 & 0.2888331 \\
\hline & 1 & 100 & 0.39 & 28.51 & 2.44573 & 30.151 & 5.4015 & 28 & 0.5 & 5.582 & 11.164 & 5 & 3 & 1.426 & 0.185133 \\
\hline & 1 & 120 & 0.47 & 26.22 & 3.42434 & 42.216 & 8.2244 & 28 & 0.61 & 5.133 & 8.415 & 6 & 3 & 1.311 & 0.1215898 \\
\hline & 1 & 140 & 0.54 & 24.2 & 4.56552 & 56.284 & 11.879 & 28 & 0.7 & 4.738 & 6.769 & 7 & 3 & 1.21 & 0.0841797 \\
\hline & 1 & 160 & 0.62 & 22.93 & 5.8685 & 72.348 & 16.117 & 28 & 0.8 & 4.489 & 5.611 & 8 & 3 & 1.146 & 0.0620476 \\
\hline & 1 & 180 & 0.7 & 22.66 & 7.33405 & 90.415 & 20.382 & 28 & 0.9 & 4.436 & 4.929 & 9 & 3 & 1.133 & 0.0490626 \\
\hline & 2 & 200 & 0.77 & 22.62 & 8.95668 & 0 & 0 & 28 & 0.99 & 4.43 & 4.475 & 10 & 3 & 1.131 & 0 \\
\hline & 2 & 220 & 1.55 & 32.7 & 12.17995 & 0 & 0 & 28 & 2 & 6.402 & 3.201 & 11 & 3 & 1.635 & 0 \\
\hline & 2 & 240 & 2.32 & 36.42 & 17.02508 & 0 & 0 & 28 & 3 & 7.13 & 2.377 & 12 & 3 & 1.821 & 0 \\
\hline & 2 & 260 & 3.09 & 39.14 & 23.49833 & 0 & 0 & 28 & 3.99 & 7.663 & 1.921 & 13 & 3 & 1.957 & 0 \\
\hline & 2 & 280 & 3.87 & 41.93 & 31.59028 & 0 & 0 & 28 & 5 & 8.21 & 1.642 & 14 & 3 & 2.097 & 0 \\
\hline & 2 & 300 & 4.65 & 44.92 & 41.30645 & 0 & 0 & 28 & 6 & 8.796 & 1.466 & 15 & 3 & 2.246 & 0 \\
\hline & 2 & 320 & 5.42 & 48.64 & 52.64681 & 0 & 0 & 28 & 7 & 9.524 & 1.361 & 16 & 3 & 2.432 & 0 \\
\hline & 2 & 340 & 6.2 & 51.51 & 65.61609 & 0 & 0 & 28 & 8 & 10.09 & 1.261 & 17 & 3 & 2.576 & 0 \\
\hline & 2 & 360 & 6.97 & 54.3 & 80.19544 & 0 & 0 & 28 & 9 & 10.63 & 1.181 & 18 & 3 & 2.715 & 0 \\
\hline & 3 & 380 & 7.75 & 57.15 & 96.55685 & 0 & 0 & 28 & 10.01 & 11.19 & 1.118 & 19 & 3 & 2.858 & 0 \\
\hline & 3 & 400 & 15.5 & 92.82 & 128.9129 & 0 & 0 & 28 & 20.01 & 18.17 & 0.908 & 20 & 3 & 4.641 & 0 \\
\hline & 3 & 420 & 23.24 & 95.59 & 177.47328 & 0 & 0 & 28 & 30 & 18.72 & 0.624 & 21 & 3 & 4.78 & 0 \\
\hline & 3 & 440 & 30.99 & 91.27 & 242.2058 & 0 & 0 & 28 & 40.01 & 17.87 & 0.447 & 22 & 3 & 4.564 & 0 \\
\hline & 3 & 460 & 38.73 & 88.95 & 323.22904 & 0 & 0 & 28 & 50 & 17.42 & 0.348 & 23 & 3 & 4.448 & 0 \\
\hline & 3 & 480 & 46.48 & 87.52 & 420.44877 & 0 & 0 & 28 & 60.01 & 17.14 & 0.286 & 24 & 3 & 4.376 & 0 \\
\hline & 3 & 500 & 54.22 & 87.98 & 533.85634 & 0 & 0 & 28 & 70 & 17.23 & 0.246 & 25 & 3 & 4.399 & 0 \\
\hline 1 & 3 & 520 & 61.96 & 86.7 & 663.64967 & 0 & 0 & 28 & 79.99 & 16.98 & 0.212 & 26 & 3 & 4.335 & 0 \\
\hline & 3 & 540 & 69.71 & 74.97 & 809.38815 & 0 & 0 & 28 & 90 & 14.68 & 0.163 & 27 & 3 & 3.748 & 0 \\
\hline & 4 & 560 & 77.46 & 72.41 & 973.08005 & 0 & 0 & 28 & 100 & 14.18 & 0.142 & 28 & 3 & 3.62 & 0 \\
\hline & 4 & 580 & 154.9 & 117.3 & 1296.3225 & 0 & 0 & 28 & 200 & 22.96 & 0.115 & 29 & 3 & 5.863 & 0 \\
\hline & 4 & 600 & 232.4 & 128.2 & 1781.9208 & 0 & 0 & 28 & 300 & 25.1 & 0.084 & 30 & 3 & 6.41 & 0 \\
\hline & 4 & 620 & 309.8 & 132.4 & 2428.8957 & 0 & 0 & 28 & 400 & 25.92 & 0.065 & 31 & 3 & 6.619 & 0 \\
\hline & 4 & 640 & 387.3 & 151.3 & 3239.2357 & 0 & 0 & 28 & 500 & 29.62 & 0.059 & 32 & 3 & 7.564 & 0 \\
\hline & 4 & 660 & 464.8 & 136.9 & 4211.0819 & 0 & 0 & 28 & 600 & 26.8 & 0.045 & 33 & 3 & 6.843 & 0 \\
\hline & 4 & 680 & 542.2 & 141.6 & 5345.6359 & 0 & 0 & 28 & 700 & 27.73 & 0.04 & 34 & 3 & 7.082 & 0 \\
\hline & 4 & 700 & 619.7 & 155.4 & 6642.7461 & 0 & 0 & 28 & 800 & 30.42 & 0.038 & 35 & 3 & 7.768 & 0 \\
\hline & 4 & 720 & 697.1 & 170.9 & 8100.7475 & 0 & 0 & 28 & 900 & 33.47 & 0.037 & 36 & 3 & 8.547 & 0 \\
\hline & 4 & 740 & 774.6 & 185.8 & 9720.8982 & 0 & 0 & 28 & 1000 & 36.39 & 0.036 & 37 & 3 & 9.292 & 0 \\
\hline & 5 & 750 & 774.6 & 173 & 10549.569 & 0 & 0 & 28 & 1000 & 33.88 & 0.034 & 38 & 3 & 8.651 & 0 \\
\hline & 5 & 760 & 774.6 & 171.5 & 11359.906 & 0 & 0 & 28 & 1000 & 33.57 & 0.034 & 39 & 3 & 8.573 & 0 \\
\hline & 5 & 770 & 774.6 & 170.3 & 12170.649 & 0 & 0 & 28 & 1000 & 33.35 & 0.033 & 40 & 3 & 8.516 & 0 \\
\hline
\end{tabular}




\begin{tabular}{|c|c|c|c|c|c|c|c|c|c|c|c|c|c|c|}
\hline 5 & 780 & 774.6 & 170.9 & 12983.013 & 0 & 0 & 28 & 1000 & 33.47 & 0.033 & 41 & 3 & 8.546 & 0 \\
\hline 5 & 790 & 774.6 & 169.8 & 13794.162 & 0 & 0 & 28 & 1000 & 33.24 & 0.033 & 42 & 3 & 8.487 & 0 \\
\hline 5 & 800 & 774.6 & 169.1 & 14605.717 & 0 & 0 & 28 & 1000 & 33.11 & 0.033 & 43 & 3 & 8.454 & 0 \\
\hline 6 & 820 & 774.6 & 168.6 & 16248.294 & 0 & 0 & 28 & 1000 & 33.02 & 0.033 & 44 & 3 & 8.432 & 0 \\
\hline 6 & 840 & 697.1 & 139.3 & 17708.16 & 0 & 0 & 28 & 900 & 27.27 & 0.03 & 45 & 3 & 6.962 & 0 \\
\hline 6 & 860 & 619.7 & 108.5 & 19007.624 & 0 & 0 & 28 & 800 & 21.24 & 0.027 & 46 & 3 & 5.423 & 0 \\
\hline 6 & 880 & 542.2 & 92.97 & 20144.004 & 0 & 0 & 28 & 700 & 18.2 & 0.026 & 47 & 3 & 4.649 & 0 \\
\hline 6 & 900 & 464.8 & 78.73 & 21118.718 & 0 & 0 & 28 & 600 & 15.42 & 0.026 & 48 & 3 & 3.936 & 0 \\
\hline 6 & 920 & 387.3 & 65.04 & 21931.368 & 0 & 0 & 28 & 500 & 12.74 & 0.025 & 49 & 3 & 3.252 & 0 \\
\hline 6 & 940 & 309.8 & 51.92 & 22582.031 & 0 & 0 & 28 & 400 & 10.17 & 0.025 & 50 & 3 & 2.596 & 0 \\
\hline 6 & 960 & 232.4 & 39.17 & 23069.979 & 0 & 0 & 28 & 300 & 7.67 & 0.026 & 51 & 3 & 1.959 & 0 \\
\hline 6 & 980 & 154.9 & 26.45 & 23395.451 & 0 & 0 & 28 & 200 & 5.178 & 0.026 & 52 & 3 & 1.322 & 0 \\
\hline 6 & 1000 & 77.46 & 14.81 & 23559.039 & 0 & 0 & 28 & 100 & 2.901 & 0.029 & 53 & 3 & 0.741 & 0 \\
\hline 7 & 1020 & 69.71 & 15.88 & 23706.248 & 0 & 0 & 28 & 90 & 3.109 & 0.035 & 54 & 3 & 0.794 & 0 \\
\hline 7 & 1040 & 61.97 & 15.58 & 23836.178 & 0 & 0 & 28 & 80 & 3.051 & 0.038 & 55 & 3 & 0.779 & 0 \\
\hline 7 & 1060 & 54.22 & 14.7 & 23949.829 & 0 & 0 & 28 & 70 & 2.879 & 0.041 & 56 & 3 & 0.735 & 0 \\
\hline 7 & 1080 & 46.48 & 13.76 & 24047.239 & 0 & 0 & 28 & 60.01 & 2.695 & 0.045 & 57 & 3 & 0.688 & 0 \\
\hline 7 & 1100 & 38.73 & 12.68 & 24128.497 & 0 & 0 & 28 & 50 & 2.483 & 0.05 & 58 & 3 & 0.634 & 0 \\
\hline 7 & 1120 & 30.99 & 11.94 & 24193.542 & 0 & 0 & 28 & 40.01 & 2.338 & 0.058 & 59 & 3 & 0.597 & 0 \\
\hline 7 & 1140 & 23.24 & 10.6 & 24242.341 & 0 & 0 & 28 & 30 & 2.076 & 0.069 & 60 & 3 & 0.53 & 0 \\
\hline 7 & 1160 & 15.5 & 8.91 & 24274.887 & 0 & 0 & 28 & 20.01 & 1.746 & 0.087 & 61 & 3 & 0.446 & 0 \\
\hline 7 & 1180 & 7.75 & 7.17 & 24291.227 & 0 & 0 & 28 & 10.01 & 1.404 & 0.14 & 62 & 3 & 0.358 & 0 \\
\hline 8 & 1200 & 6.97 & 8.97 & 24305.935 & 0 & 0 & 28 & 9 & 1.757 & 0.195 & 63 & 3 & 0.449 & 0 \\
\hline 8 & 1220 & 6.2 & 9.41 & 24318.931 & 0 & 0 & 28 & 8 & 1.842 & 0.23 & 64 & 3 & 0.47 & 0 \\
\hline 8 & 1240 & 5.42 & 9.56 & 24330.284 & 0 & 0 & 28 & 7 & 1.872 & 0.267 & 65 & 3 & 0.478 & 0 \\
\hline 8 & 1260 & 4.64 & 9.95 & 24340.028 & 0 & 0 & 28 & 5.99 & 1.948 & 0.325 & 66 & 3 & 0.497 & 0 \\
\hline 8 & 1280 & 3.87 & 10.13 & 24348.144 & 0 & 0 & 28 & 5 & 1.983 & 0.397 & 67 & 3 & 0.506 & 0 \\
\hline 8 & 1300 & 3.09 & 10.24 & 24354.635 & 0 & 0 & 28 & 3.99 & 2.005 & 0.503 & 68 & 3 & 0.512 & 0 \\
\hline 8 & 1320 & 2.32 & 10.24 & 24359.507 & 0 & 0 & 28 & 3 & 2.005 & 0.668 & 69 & 3 & 0.512 & 0 \\
\hline 8 & 1340 & 1.54 & 9.62 & 24362.753 & 0 & 0 & 28 & 1.99 & 1.883 & 0.946 & 70 & 3 & 0.481 & 0 \\
\hline 8 & 1360 & 0.77 & 8.94 & 24364.377 & 0 & 0 & 28 & 0.99 & 1.751 & 1.769 & 71 & 3 & 0.447 & 0 \\
\hline 9 & 1380 & 0.7 & 11.39 & 24365.851 & 18.174 & 8.1461 & 28 & 0.9 & 2.231 & 2.479 & 72 & 3 & 0.57 & 0.1227574 \\
\hline 9 & 1400 & 0.62 & 11.84 & 24367.156 & 34.257 & 14.785 & 28 & 0.8 & 2.317 & 2.896 & 73 & 3 & 0.592 & 0.0676364 \\
\hline 9 & 1420 & 0.55 & 11.98 & 24368.297 & 48.335 & 20.612 & 28 & 0.71 & 2.345 & 3.303 & 74 & 3 & 0.599 & 0.0485155 \\
\hline 9 & 1440 & 0.47 & 11.97 & 24369.278 & 60.419 & 25.776 & 28 & 0.61 & 2.344 & 3.843 & 75 & 3 & 0.599 & 0.0387959 \\
\hline 9 & 1460 & 0.39 & 12.33 & 24370.095 & 70.498 & 29.192 & 28 & 0.5 & 2.415 & 4.83 & 76 & 3 & 0.617 & 0.0342562 \\
\hline 9 & 1480 & 0.31 & 12.55 & 24370.751 & 78.583 & 31.97 & 28 & 0.4 & 2.458 & 6.145 & 77 & 3 & 0.628 & 0.031279 \\
\hline 9 & 1500 & 0.24 & 13.05 & 24371.244 & 84.664 & 33.149 & 28 & 0.31 & 2.554 & 8.239 & 78 & 3 & 0.652 & 0.0301664 \\
\hline 9 & 1520 & 0.16 & 13.23 & 24371.574 & 88.73 & 34.246 & 28 & 0.21 & 2.591 & 12.338 & 79 & 3 & 0.662 & 0.0292008 \\
\hline 9 & 1540 & 0.08 & 11.54 & 24371.744 & 90.822 & 40.187 & 28 & 0.1 & 2.26 & 22.6 & 80 & 3 & 0.577 & 0.0248839 \\
\hline
\end{tabular}

2004.02.12 META-20 wt\%-U [sonicated] CC-45

0.1-1000 s-1 run 4.txt

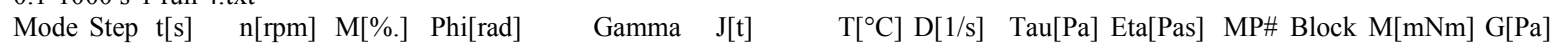

\begin{tabular}{|c|c|c|c|c|c|c|c|c|c|c|c|c|c|c|c|}
\hline 1 & 1 & 20 & 0.08 & 15.57 & 0.15944 & 1.9656 & 0.6447 & 28 & 0.1 & 3.049 & 30.49 & 1 & 4 & 0.779 & 1.5512198 \\
\hline 1 & 1 & 40 & 0.16 & 23.16 & 0.48616 & 5.9935 & 1.3219 & 28 & 0.21 & 4.534 & 21.59 & 2 & 4 & 1.158 & 0.75649 \\
\hline 1 & 1 & 60 & 0.24 & 26.74 & 0.97625 & 12.035 & 2.299 & 28 & 0.31 & 5.235 & 16.887 & 3 & 4 & 1.337 & 0.4349687 \\
\hline 1 & 1 & 80 & 0.31 & 26.69 & 1.63049 & 20.101 & 3.8456 & 28 & 0.4 & 5.227 & 13.068 & 4 & 4 & 1.335 & 0.2600385 \\
\hline 1 & 1 & 100 & 0.39 & 25.17 & 2.44652 & 30.161 & 6.1203 & 28 & 0.5 & 4.928 & 9.856 & 5 & 4 & 1.258 & 0.1633899 \\
\hline 1 & 1 & 120 & 0.47 & 23.35 & 3.42512 & 42.225 & 9.2336 & 28 & 0.61 & 4.573 & 7.497 & 6 & 4 & 1.168 & 0.1082998 \\
\hline & 1 & 140 & 0.54 & 22.09 & 4.56709 & 56.304 & 13.018 & 28 & 0.7 & 4.325 & 6.179 & 7 & 4 & 1.104 & 0.0768155 \\
\hline
\end{tabular}




\begin{tabular}{|c|c|c|c|c|c|c|c|c|c|c|c|c|c|c|c|}
\hline 1 & 1 & 160 & 0.62 & 20.98 & 5.86928 & 72.357 & 17.614 & 28 & 0.8 & 4.108 & 5.135 & 8 & 4 & 1.049 & 0.0567738 \\
\hline 1 & 1 & 180 & 0.7 & 20.82 & 7.33326 & 90.406 & 22.18 & 28 & 0.9 & 4.076 & 4.529 & 9 & 4 & 1.041 & 0.0450858 \\
\hline & 2 & 200 & 0.77 & 21.07 & 8.9559 & 0 & 0 & 28 & 0.99 & 4.125 & 4.167 & 10 & 4 & 1.053 & 0 \\
\hline & 2 & 220 & 1.55 & 30.32 & 12.17917 & 0 & 0 & 28 & 2 & 5.936 & 2.968 & 11 & 4 & 1.516 & 0 \\
\hline & 2 & 240 & 2.32 & 33.98 & 17.02586 & 0 & 0 & 28 & 3 & 6.652 & 2.217 & 12 & 4 & 1.699 & 0 \\
\hline & 2 & 260 & 3.09 & 36.72 & 23.49911 & 0 & 0 & 28 & 3.99 & 7.19 & 1.802 & 13 & 4 & 1.836 & 0 \\
\hline & 2 & 280 & 3.87 & 39.47 & 31.59264 & 0 & 0 & 28 & 5 & 7.727 & 1.545 & 14 & 4 & 1.973 & 0 \\
\hline & 2 & 300 & 4.65 & 42.22 & 41.30802 & 0 & 0 & 28 & 6 & 8.266 & 1.378 & 15 & 4 & 2.111 & 0 \\
\hline & 2 & 320 & 5.42 & 45.89 & 52.65466 & 0 & 0 & 28 & 7 & 8.986 & 1.284 & 16 & 4 & 2.295 & 0 \\
\hline & 2 & 340 & 6.2 & 49.21 & 65.6208 & 0 & 0 & 28 & 8 & 9.636 & 1.205 & 17 & 4 & 2.461 & 0 \\
\hline & 2 & 360 & 6.97 & 52.47 & 80.18837 & 0 & 0 & 28 & 9 & 10.27 & 1.142 & 18 & 4 & 2.624 & 0 \\
\hline & 3 & 380 & 7.75 & 55.23 & 96.56156 & 0 & 0 & 28 & 10.01 & 10.81 & 1.08 & 19 & 4 & 2.761 & 0 \\
\hline & 3 & 400 & 15.5 & 90.59 & 128.86892 & 0 & 0 & 28 & 20.01 & 17.74 & 0.886 & 20 & 4 & 4.529 & 0 \\
\hline & 3 & 420 & 23.24 & 93.82 & 177.45286 & 0 & 0 & 28 & 30 & 18.37 & 0.612 & 21 & 4 & 4.691 & 0 \\
\hline & 3 & 440 & 30.99 & 89.19 & 242.28434 & 0 & 0 & 28 & 40.01 & 17.46 & 0.436 & 22 & 4 & 4.46 & 0 \\
\hline & 3 & 460 & 38.73 & 87.24 & 323.20548 & 0 & 0 & 28 & 50 & 17.08 & 0.342 & 23 & 4 & 4.362 & 0 \\
\hline & 3 & 480 & 46.48 & 87.71 & 420.46998 & 0 & 0 & 28 & 60.01 & 17.17 & 0.286 & 24 & 4 & 4.386 & 0 \\
\hline & 3 & 500 & 54.22 & 86.27 & 533.87833 & 0 & 0 & 28 & 70 & 16.89 & 0.241 & 25 & 4 & 4.314 & 0 \\
\hline 1 & 3 & 520 & 61.96 & 85.81 & 663.50358 & 0 & 0 & 28 & 79.99 & 16.8 & 0.21 & 26 & 4 & 4.29 & 0 \\
\hline 1 & 3 & 540 & 69.71 & 83.74 & 809.49182 & 0 & 0 & 28 & 90 & 16.4 & 0.182 & 27 & 4 & 4.187 & 0 \\
\hline 1 & 4 & 560 & 77.46 & 82.4 & 973.14367 & 0 & 0 & 28 & 100 & 16.14 & 0.161 & 28 & 4 & 4.12 & 0 \\
\hline 1 & 4 & 580 & 154.9 & 132.2 & 1296.4269 & 0 & 0 & 28 & 200 & 25.88 & 0.129 & 29 & 4 & 6.608 & 0 \\
\hline 1 & 4 & 600 & 232.4 & 149.7 & 1781.7386 & 0 & 0 & 28 & 300 & 29.31 & 0.098 & 30 & 4 & 7.484 & 0 \\
\hline 1 & 4 & 620 & 309.8 & 159.6 & 2429.8908 & 0 & 0 & 28 & 400 & 31.25 & 0.078 & 31 & 4 & 7.979 & 0 \\
\hline 1 & 4 & 640 & 387.3 & 154.9 & 3239.3842 & 0 & 0 & 28 & 500 & 30.33 & 0.061 & 32 & 4 & 7.746 & 0 \\
\hline 1 & 4 & 660 & 464.8 & 121 & 4211.2311 & 0 & 0 & 28 & 600 & 23.69 & 0.039 & 33 & 4 & 6.051 & 0 \\
\hline 1 & 4 & 680 & 542.2 & 134.2 & 5346.0686 & 0 & 0 & 28 & 700 & 26.27 & 0.038 & 34 & 4 & 6.708 & 0 \\
\hline 1 & 4 & 700 & 619.7 & 149.1 & 6642.5694 & 0 & 0 & 28 & 800 & 29.2 & 0.036 & 35 & 4 & 7.455 & 0 \\
\hline 1 & 4 & 720 & 697.1 & 166.6 & 8101.9892 & 0 & 0 & 28 & 900 & 32.63 & 0.036 & 36 & 4 & 8.332 & 0 \\
\hline 1 & 4 & 740 & 774.6 & 185.7 & 9722.1383 & 0 & 0 & 28 & 1000 & 36.35 & 0.036 & 37 & 4 & 9.283 & 0 \\
\hline 1 & 5 & 750 & 774.6 & 171.8 & 10551.214 & 0 & 0 & 28 & 1000 & 33.63 & 0.034 & 38 & 4 & 8.588 & 0 \\
\hline 1 & 5 & 760 & 774.6 & 170.5 & 11361.957 & 0 & 0 & 28 & 1000 & 33.38 & 0.033 & 39 & 4 & 8.524 & 0 \\
\hline 1 & 5 & 770 & 774.6 & 169.7 & 12172.7 & 0 & 0 & 28 & 1000 & 33.22 & 0.033 & 40 & 4 & 8.482 & 0 \\
\hline 1 & 5 & 780 & 774.6 & 168.3 & 12983.444 & 0 & 0 & 28 & 1000 & 32.95 & 0.033 & 41 & 4 & 8.413 & 0 \\
\hline 1 & 5 & 790 & 774.6 & 168.1 & 13795.404 & 0 & 0 & 28 & 1000 & 32.91 & 0.033 & 42 & 4 & 8.403 & 0 \\
\hline 1 & 5 & 800 & 774.6 & 167.5 & 14606.552 & 0 & 0 & 28 & 1000 & 32.8 & 0.033 & 43 & 4 & 8.377 & 0 \\
\hline 1 & 6 & 820 & 774.6 & 168.2 & 16247.101 & 0 & 0 & 28 & 1000 & 32.93 & 0.033 & 44 & 4 & 8.408 & 0 \\
\hline 1 & 6 & 840 & 697.1 & 138.8 & 17709.564 & 0 & 0 & 28 & 900 & 27.18 & 0.03 & 45 & 4 & 6.941 & 0 \\
\hline 1 & 6 & 860 & 619.7 & 107.8 & 19007.73 & 0 & 0 & 28 & 800 & 21.11 & 0.026 & 46 & 4 & 5.391 & 0 \\
\hline 1 & 6 & 880 & 542.2 & 91.52 & 20144.717 & 0 & 0 & 28 & 700 & 17.92 & 0.026 & 47 & 4 & 4.576 & 0 \\
\hline 1 & 6 & 900 & 464.8 & 77.55 & 21120.122 & 0 & 0 & 28 & 600 & 15.18 & 0.025 & 48 & 4 & 3.877 & 0 \\
\hline 1 & 6 & 920 & 387.3 & 64.03 & 21933.017 & 0 & 0 & 28 & 500 & 12.54 & 0.025 & 49 & 4 & 3.202 & 0 \\
\hline 1 & 6 & 940 & 309.8 & 50.97 & 22582.908 & 0 & 0 & 28 & 400 & 9.979 & 0.025 & 50 & 4 & 2.548 & 0 \\
\hline 1 & 6 & 960 & 232.4 & 38.44 & 23070.733 & 0 & 0 & 28 & 300 & 7.527 & 0.025 & 51 & 4 & 1.922 & 0 \\
\hline 1 & 6 & 980 & 154.9 & 25.94 & 23396.657 & 0 & 0 & 28 & 200 & 5.078 & 0.025 & 52 & 4 & 1.297 & 0 \\
\hline 1 & 6 & 1000 & 77.46 & 14.57 & 23560.238 & 0 & 0 & 28 & 100 & 2.853 & 0.029 & 53 & 4 & 0.729 & 0 \\
\hline 1 & 7 & 1020 & 69.71 & 15.42 & 23707.444 & 0 & 0 & 28 & 90 & 3.018 & 0.034 & 54 & 4 & 0.771 & 0 \\
\hline 1 & 7 & 1040 & 61.97 & 15.15 & 23837.378 & 0 & 0 & 28 & 80 & 2.966 & 0.037 & 55 & 4 & 0.757 & 0 \\
\hline 1 & 7 & 1060 & 54.22 & 14.43 & 23951.001 & 0 & 0 & 28 & 70 & 2.825 & 0.04 & 56 & 4 & 0.721 & 0 \\
\hline 1 & 7 & 1080 & 46.48 & 13.5 & 24048.479 & 0 & 0 & 28 & 60.01 & 2.642 & 0.044 & 57 & 4 & 0.675 & 0 \\
\hline
\end{tabular}




\begin{tabular}{|c|c|c|c|c|c|c|c|c|c|c|c|c|c|c|}
\hline 7 & 1100 & 38.73 & 12.41 & 24129.737 & 0 & 0 & 28 & 50 & 2.43 & 0.049 & 58 & 4 & 0.621 & 0 \\
\hline 7 & 1120 & 30.99 & 11.61 & 24194.668 & 0 & 0 & 28 & 40.01 & 2.273 & 0.057 & 59 & 4 & 0.58 & \\
\hline 7 & 1140 & 23.24 & 10.27 & 24243.459 & 0 & 0 & 28 & 30 & 2.011 & 0.067 & 60 & 4 & 0.513 & \\
\hline 7 & 1160 & 15.5 & 8.7 & 24276.07 & 0 & 0 & 28 & 20.01 & 1.704 & 0.085 & 61 & 4 & 0.435 & o \\
\hline 7 & 1180 & 7.75 & 6.87 & 24292.398 & 0 & 0 & 28 & 10.01 & 1.345 & 0.134 & 62 & 4 & 0.344 & 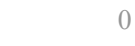 \\
\hline 8 & 1200 & 6.97 & 8.64 & 24307.108 & 0 & 0 & 28 & 9 & 1.692 & 0.188 & 63 & 4 & 0.432 & 0 \\
\hline 8 & 1220 & 6.2 & 9.14 & 24320.088 & 0 & 0 & 28 & 8 & 1.789 & 0.224 & 64 & 4 & 0.457 & 0 \\
\hline 8 & 1240 & 5.42 & 9.36 & 24331.459 & 0 & 0 & 28 & 7 & 1.832 & 0.262 & 65 & 4 & 0.468 & 0 \\
\hline 8 & 1260 & 4.64 & 9.73 & 24341.191 & 0 & 0 & 28 & 5.99 & 1.905 & 0.318 & 66 & 4 & 0.486 & 0 \\
\hline 8 & 1280 & 3.87 & 9.72 & 24349.311 & 0 & 0 & 28 & 5 & 1.902 & 0.38 & 67 & 4 & 0.486 & 0 \\
\hline 8 & 1300 & 3.09 & 9.85 & 24355.802 & 0 & 0 & 28 & 3.99 & 1.928 & 0.483 & 68 & 4 & 0.492 & 0 \\
\hline 8 & 1320 & 2.32 & 9.77 & 24360.671 & 0 & 0 & 28 & 3 & 1.913 & 0.638 & 69 & 4 & 0.488 & 0 \\
\hline 8 & 1340 & 1.54 & 9.24 & 24363.919 & 0 & 0 & 28 & 1.99 & 1.81 & 0.91 & 70 & 4 & 0.462 & 0 \\
\hline 8 & 1360 & 0.77 & 8.44 & 24365.542 & 0 & 0 & 28 & 0.99 & 1.652 & 1.669 & 71 & 4 & 0.422 & 0 \\
\hline 9 & 1380 & 0.7 & 10.73 & 24367.017 & 18.184 & 8.6507 & 28 & 0.9 & 2.102 & 2.336 & 72 & 4 & 0.537 & 0.1155978 \\
\hline 9 & 1400 & 0.62 & 11.18 & 24368.321 & 34.266 & 15.654 & 28 & 0.8 & 2.189 & 2.736 & 73 & 4 & 0.559 & 0.0638819 \\
\hline 9 & 1420 & 0.55 & 11.27 & 24369.463 & 48.345 & 21.905 & 28 & 0.71 & 2.207 & 3.108 & 74 & 4 & 0.564 & 0.0456513 \\
\hline 9 & 1440 & 0.47 & 11.69 & 24370.443 & 60.428 & 26.4 & 28 & 0.61 & 2.289 & 3.752 & 75 & 4 & 0.585 & 0.0378795 \\
\hline 9 & 1460 & 0.39 & 11.67 & 24371.261 & 70.508 & 30.857 & 28 & 0.5 & 2.285 & 4.57 & 76 & 4 & 0.584 & 0.0324077 \\
\hline 9 & 1480 & 0.31 & 11.93 & 24371.917 & 78.593 & 33.659 & 28 & 0.4 & 2.335 & 5.837 & 77 & 4 & 0.596 & 0.0297101 \\
\hline 9 & 1500 & 0.24 & 12.05 & 24372.41 & 84.673 & 35.894 & 28 & 0.31 & 2.359 & 7.61 & 78 & 4 & 0.602 & 0.02786 \\
\hline 9 & 1520 & 0.16 & 12.34 & 24372.74 & 88.75 & 36.734 & 28 & 0.21 & 2.416 & 11.505 & 79 & 4 & 0.617 & 0.0272226 \\
\hline 9 & 1540 & 0.08 & 11.05 & 24372.909 & 90.822 & 41.989 & 28 & 0.1 & 2.163 & 21.63 & 80 & 4 & 0.552 & 0.0238159 \\
\hline
\end{tabular}

2004.02.12 META-20 wt\%-U [sonicated] CC-45

0.1-1000 s-1 run 5.txt

Mode Step t[s] n[rpm] M[\%.] Phi[rad $\quad$ Gamma J[t] $\quad T\left[{ }^{\circ} \mathrm{C}\right] \mathrm{D}[1 / \mathrm{s}] \quad$ Tau[Pa] Eta[Pas] MP\# Block M[mNm] G[Pa]

\begin{tabular}{|c|c|c|c|c|c|c|c|c|c|c|c|c|c|c|}
\hline & 20 & 0.08 & 15.57 & 0.15944 & 1.9656 & 0.6447 & 28 & 0.1 & 3.049 & 30.49 & 1 & 4 & 0.779 & 1.5512198 \\
\hline & 40 & 0.16 & 23.16 & 0.48616 & 5.9935 & 1.3219 & 28 & 0.21 & 4.534 & 21.59 & 2 & 4 & 1.158 & 0.75649 \\
\hline & 60 & 0.24 & 26.74 & 0.97625 & 12.035 & 2.299 & 28 & 0.31 & 5.235 & 16.887 & 3 & 4 & 1.337 & 0.4349687 \\
\hline & 80 & 0.31 & 26.69 & 1.63049 & 20.101 & 3.8456 & 28 & 0.4 & 5.227 & 13.068 & 4 & 4 & 1.335 & 0.2600385 \\
\hline & 100 & 0.39 & 25.17 & 2.44652 & 30.161 & 6.1203 & 28 & 0.5 & 4.928 & 9.856 & 5 & 4 & 1.258 & 0.1633899 \\
\hline & 120 & 0.47 & 23.35 & 3.42512 & 42.225 & 9.2336 & 28 & 0.61 & 4.573 & 7.497 & 6 & 4 & 1.168 & 0.1082998 \\
\hline & 140 & 0.54 & 22.09 & 4.56709 & 56.304 & 13.018 & 28 & 0.7 & 4.325 & 6.179 & 7 & 4 & 1.104 & 0.0768155 \\
\hline & 160 & 0.62 & 20.98 & 5.86928 & 72.357 & 17.614 & 28 & 0.8 & 4.108 & 5.135 & 8 & 4 & 1.049 & 0.0567738 \\
\hline & 180 & 0.7 & 20.82 & 7.33326 & 90.406 & 22.18 & 28 & 0.9 & 4.076 & 4.529 & 9 & 4 & 1.041 & 0.0450858 \\
\hline & 200 & 0.77 & 21.07 & 8.9559 & 0 & 0 & 28 & 0.99 & 4.125 & 4.167 & 10 & 4 & 1.053 & 0 \\
\hline & 220 & 1.55 & 30.32 & 12.17917 & 0 & 0 & 28 & 2 & 5.936 & 2.968 & 11 & 4 & 1.516 & 0 \\
\hline & 240 & 2.32 & 33.98 & 17.02586 & 0 & 0 & 28 & 3 & 6.652 & 2.217 & 12 & 4 & 1.699 & 0 \\
\hline & 260 & 3.09 & 36.72 & 23.49911 & 0 & 0 & 28 & 3.99 & 7.19 & 1.802 & 13 & 4 & 1.836 & 0 \\
\hline & 280 & 3.87 & 39.47 & 31.59264 & 0 & 0 & 28 & 5 & 7.727 & 1.545 & 14 & 4 & 1.973 & 0 \\
\hline & 300 & 4.65 & 42.22 & 41.30802 & 0 & 0 & 28 & 6 & 8.266 & 1.378 & 15 & 4 & 2.111 & 0 \\
\hline & 320 & 5.42 & 45.89 & 52.65466 & 0 & 0 & 28 & 7 & 8.986 & 1.284 & 16 & 4 & 2.295 & 0 \\
\hline & 340 & 6.2 & 49.21 & 65.6208 & 0 & 0 & 28 & 8 & 9.636 & 1.205 & 17 & 4 & 2.461 & 0 \\
\hline & 360 & 6.97 & 52.47 & 80.18837 & 0 & 0 & 28 & 9 & 10.27 & 1.142 & 18 & 4 & 2.624 & 0 \\
\hline & 380 & 7.75 & 55.23 & 96.56156 & 0 & 0 & 28 & 10.01 & 10.81 & 1.08 & 19 & 4 & 2.761 & 0 \\
\hline & 400 & 15.5 & 90.59 & 128.86892 & 0 & 0 & 28 & 20.01 & 17.74 & 0.886 & 20 & 4 & 4.529 & 0 \\
\hline & 420 & 23.24 & 93.82 & 177.45286 & 0 & 0 & 28 & 30 & 18.37 & 0.612 & 21 & 4 & 4.691 & 0 \\
\hline & 440 & 30.99 & 89.19 & 242.28434 & 0 & 0 & 28 & 40.01 & 17.46 & 0.436 & 22 & 4 & 4.46 & 0 \\
\hline & 460 & 38.73 & 87.24 & 323.20548 & 0 & 0 & 28 & 50 & 17.08 & 0.342 & 23 & 4 & 4.362 & 0 \\
\hline & 480 & 46.48 & 87.71 & 420.46998 & 0 & 0 & 28 & 60.01 & 17.17 & 0.286 & 24 & 4 & 4.386 & 0 \\
\hline
\end{tabular}




\begin{tabular}{|c|c|c|c|c|c|c|c|c|c|c|c|c|c|c|c|}
\hline 1 & 3 & 500 & 54.22 & 86.27 & 533.87833 & 0 & 0 & 28 & 70 & 16.89 & 0.241 & 25 & 4 & 4.314 & 0 \\
\hline 1 & 3 & 520 & 61.96 & 85.81 & 663.50358 & 0 & 0 & 28 & 79.99 & 16.8 & 0.21 & 26 & 4 & 4.29 & 0 \\
\hline 1 & 3 & 540 & 69.71 & 83.74 & 809.49182 & 0 & 0 & 28 & 90 & 16.4 & 0.182 & 27 & 4 & 4.187 & 0 \\
\hline 1 & 4 & 560 & 77.46 & 82.4 & 973.14367 & 0 & 0 & 28 & 100 & 16.14 & 0.161 & 28 & 4 & 4.12 & 0 \\
\hline 1 & 4 & 580 & 154.9 & 132.2 & 1296.4269 & 0 & 0 & 28 & 200 & 25.88 & 0.129 & 29 & 4 & 6.608 & 0 \\
\hline l & 4 & 600 & 232.4 & 149.7 & 1781.7386 & 0 & 0 & 28 & 300 & 29.31 & 0.098 & 30 & 4 & 7.484 & 0 \\
\hline 1 & 4 & 620 & 309.8 & 159.6 & 2429.8908 & 0 & 0 & 28 & 400 & 31.25 & 0.078 & 31 & 4 & 7.979 & 0 \\
\hline 1 & 4 & 640 & 387.3 & 154.9 & 3239.3842 & 0 & 0 & 28 & 500 & 30.33 & 0.061 & 32 & 4 & 7.746 & 0 \\
\hline 1 & 4 & 660 & 464.8 & 121 & 4211.2311 & 0 & 0 & 28 & 600 & 23.69 & 0.039 & 33 & 4 & 6.051 & 0 \\
\hline 1 & 4 & 680 & 542.2 & 134.2 & 5346.0686 & 0 & 0 & 28 & 700 & 26.27 & 0.038 & 34 & 4 & 6.708 & \\
\hline 1 & 4 & 700 & 619.7 & 149.1 & 6642.5694 & 0 & 0 & 28 & 800 & 29.2 & 0.036 & 35 & 4 & 7.455 & \\
\hline 1 & 4 & 720 & 697.1 & 166.6 & 8101.9892 & 0 & 0 & 28 & 900 & 32.63 & 0.036 & 36 & 4 & 8.332 & \\
\hline 1 & 4 & 740 & 774.6 & 185.7 & 9722.1383 & 0 & 0 & 28 & 1000 & 36.35 & 0.036 & 37 & 4 & 9.283 & \\
\hline 1 & 5 & 750 & 774.6 & 171.8 & 10551.214 & 0 & 0 & 28 & 1000 & 33.63 & 0.034 & 38 & 4 & 8.588 & \\
\hline 1 & 5 & 760 & 774.6 & 170.5 & 11361.957 & 0 & 0 & 28 & 1000 & 33.38 & 0.033 & 39 & 4 & 8.524 & \\
\hline 1 & 5 & 770 & 774.6 & 169.7 & 12172.7 & 0 & 0 & 28 & 1000 & 33.22 & 0.033 & 40 & 4 & 8.482 & \\
\hline 1 & 5 & 780 & 774.6 & 168.3 & 12983.444 & 0 & 0 & 28 & 1000 & 32.95 & 0.033 & 41 & 4 & 8.413 & \\
\hline 1 & 5 & 790 & 774.6 & 168.1 & 13795.404 & 0 & 0 & 28 & 1000 & 32.91 & 0.033 & 42 & 4 & 8.403 & \\
\hline 1 & 5 & 800 & 774.6 & 167.5 & 14606.552 & 0 & 0 & 28 & 1000 & 32.8 & 0.033 & 43 & 4 & 8.377 & 0 \\
\hline 1 & 6 & 820 & 774.6 & 168.2 & 16247.101 & 0 & 0 & 28 & 1000 & 32.93 & 0.033 & 44 & 4 & 8.408 & 0 \\
\hline 1 & 6 & 840 & 697.1 & 138.8 & 17709.564 & 0 & 0 & 28 & 900 & 27.18 & 0.03 & 45 & 4 & 6.941 & 0 \\
\hline 1 & 6 & 860 & 619.7 & 107.8 & 19007.73 & 0 & 0 & 28 & 800 & 21.11 & 0.026 & 46 & 4 & 5.391 & 0 \\
\hline 1 & 6 & 880 & 542.2 & 91.52 & 20144.717 & 0 & 0 & 28 & 700 & 17.92 & 0.026 & 47 & 4 & 4.576 & 0 \\
\hline 1 & 6 & 900 & 464.8 & 77.55 & 21120.122 & 0 & 0 & 28 & 600 & 15.18 & 0.025 & 48 & 4 & 3.877 & 0 \\
\hline 1 & 6 & 920 & 387.3 & 64.03 & 21933.017 & 0 & 0 & 28 & 500 & 12.54 & 0.025 & 49 & 4 & 3.202 & 0 \\
\hline 1 & 6 & 940 & 309.8 & 50.97 & 22582.908 & 0 & 0 & 28 & 400 & 9.979 & 0.025 & 50 & 4 & 2.548 & 0 \\
\hline 1 & 6 & 960 & 232.4 & 38.44 & 23070.733 & 0 & 0 & 28 & 300 & 7.527 & 0.025 & 51 & 4 & 1.922 & 0 \\
\hline 1 & 6 & 980 & 154.9 & 25.94 & 23396.657 & 0 & 0 & 28 & 200 & 5.078 & 0.025 & 52 & 4 & 1.297 & 0 \\
\hline 1 & 6 & 1000 & 77.46 & 14.57 & 23560.238 & 0 & 0 & 28 & 100 & 2.853 & 0.029 & 53 & 4 & 0.729 & 0 \\
\hline 1 & 7 & 1020 & 69.71 & 15.42 & 23707.444 & 0 & 0 & 28 & 90 & 3.018 & 0.034 & 54 & 4 & 0.771 & 0 \\
\hline 1 & 7 & 1040 & 61.97 & 15.15 & 23837.378 & 0 & 0 & 28 & 80 & 2.966 & 0.037 & 55 & 4 & 0.757 & 0 \\
\hline 1 & 7 & 1060 & 54.22 & 14.43 & 23951.001 & 0 & 0 & 28 & 70 & 2.825 & 0.04 & 56 & 4 & 0.721 & 0 \\
\hline 1 & 7 & 1080 & 46.48 & 13.5 & 24048.479 & 0 & 0 & 28 & 60.01 & 2.642 & 0.044 & 57 & 4 & 0.675 & 0 \\
\hline 1 & 7 & 1100 & 38.73 & 12.41 & 24129.737 & 0 & 0 & 28 & 50 & 2.43 & 0.049 & 58 & 4 & 0.621 & 0 \\
\hline 1 & 7 & 1120 & 30.99 & 11.61 & 24194.668 & 0 & 0 & 28 & 40.01 & 2.273 & 0.057 & 59 & 4 & 0.58 & 0 \\
\hline 1 & 7 & 1140 & 23.24 & 10.27 & 24243.459 & 0 & 0 & 28 & 30 & 2.011 & 0.067 & 60 & 4 & 0.513 & 0 \\
\hline 1 & 7 & 1160 & 15.5 & 8.7 & 24276.07 & 0 & 0 & 28 & 20.01 & 1.704 & 0.085 & 61 & 4 & 0.435 & 0 \\
\hline 1 & 7 & 1180 & 7.75 & 6.87 & 24292.398 & 0 & 0 & 28 & 10.01 & 1.345 & 0.134 & 62 & 4 & 0.344 & 0 \\
\hline 1 & 8 & 1200 & 6.97 & 8.64 & 24307.108 & 0 & 0 & 28 & 9 & 1.692 & 0.188 & 63 & 4 & 0.432 & 0 \\
\hline 1 & 8 & 1220 & 6.2 & 9.14 & 24320.088 & 0 & 0 & 28 & 8 & 1.789 & 0.224 & 64 & 4 & 0.457 & 0 \\
\hline 1 & 8 & 1240 & 5.42 & 9.36 & 24331.459 & 0 & 0 & 28 & 7 & 1.832 & 0.262 & 65 & 4 & 0.468 & 0 \\
\hline 1 & 8 & 1260 & 4.64 & 9.73 & 24341.191 & 0 & 0 & 28 & 5.99 & 1.905 & 0.318 & 66 & 4 & 0.486 & 0 \\
\hline 1 & 8 & 1280 & 3.87 & 9.72 & 24349.311 & 0 & 0 & 28 & 5 & 1.902 & 0.38 & 67 & 4 & 0.486 & 0 \\
\hline 1 & 8 & 1300 & 3.09 & 9.85 & 24355.802 & 0 & 0 & 28 & 3.99 & 1.928 & 0.483 & 68 & 4 & 0.492 & 0 \\
\hline 1 & 8 & 1320 & 2.32 & 9.77 & 24360.671 & 0 & 0 & 28 & 3 & 1.913 & 0.638 & 69 & 4 & 0.488 & 0 \\
\hline 1 & 8 & 1340 & 1.54 & 9.24 & 24363.919 & 0 & 0 & 28 & 1.99 & 1.81 & 0.91 & 70 & 4 & 0.462 & 0 \\
\hline 1 & 8 & 1360 & 0.77 & 8.44 & 24365.542 & 0 & 0 & 28 & 0.99 & 1.652 & 1.669 & 71 & 4 & 0.422 & 0 \\
\hline 1 & 9 & 1380 & 0.7 & 10.73 & 24367.017 & 18.184 & 8.6507 & 28 & 0.9 & 2.102 & 2.336 & 72 & 4 & 0.537 & 0.1155978 \\
\hline 1 & 9 & 1400 & 0.62 & 11.18 & 24368.321 & 34.266 & 15.654 & 28 & 0.8 & 2.189 & 2.736 & 73 & 4 & 0.559 & 0.0638819 \\
\hline 1 & 9 & 1420 & 0.55 & 11.27 & 24369.463 & 48.345 & 21.905 & 28 & 0.71 & 2.207 & 3.108 & 74 & 4 & 0.564 & 0.0456513 \\
\hline
\end{tabular}




$\begin{array}{rrrrrrrrrrrrrrrr}1 & 9 & 1440 & 0.47 & 11.69 & 24370.443 & 60.428 & 26.4 & 28 & 0.61 & 2.289 & 3.752 & 75 & 4 & 0.585 & 0.0378795 \\ 1 & 9 & 1460 & 0.39 & 11.67 & 24371.261 & 70.508 & 30.857 & 28 & 0.5 & 2.285 & 4.57 & 76 & 4 & 0.584 & 0.0324077 \\ 1 & 9 & 1480 & 0.31 & 11.93 & 24371.917 & 78.593 & 33.659 & 28 & 0.4 & 2.335 & 5.837 & 77 & 4 & 0.596 & 0.0297101 \\ 1 & 9 & 1500 & 0.24 & 12.05 & 24372.41 & 84.673 & 35.894 & 28 & 0.31 & 2.359 & 7.61 & 78 & 4 & 0.602 & 0.02786 \\ 1 & 9 & 1520 & 0.16 & 12.34 & 24372.74 & 88.75 & 36.734 & 28 & 0.21 & 2.416 & 11.505 & 79 & 4 & 0.617 & 0.0272226 \\ 1 & 9 & 1540 & 0.08 & 11.05 & 24372.909 & 90.822 & 41.989 & 28 & 0.1 & 2.163 & 21.63 & 80 & 4 & 0.552 & 0.0238159\end{array}$

2004.02.12 META-20 wt\%-U [sonicated] CC-45

$0.1-1000 \mathrm{~s}-1$ run $6 . t x t$

Mode Step t[s] n[rpm] M[\%.] Phi[rad $\quad$ Gamma J[t] $\quad T\left[{ }^{\circ} \mathrm{C}\right] \quad \mathrm{D}[1 / \mathrm{s}] \quad$ Tau[Pa] Eta[Pas] MP\# Block M[mNm] G[Pa]

\begin{tabular}{|c|c|c|c|c|c|c|c|c|c|c|c|c|c|c|}
\hline & 20 & 0.08 & 14.91 & 0.16022 & 1.9752 & 0.6767 & 28 & 0.1 & 2.919 & 29.19 & 1 & 6 & 0.745 & 1.4778026 \\
\hline & 40 & 0.16 & 22.32 & 0.48695 & 6.0032 & 1.3737 & 28 & 0.21 & 4.37 & 20.81 & 2 & 6 & 1.116 & 0.7279512 \\
\hline & 60 & 0.24 & 24.43 & 0.97782 & 12.055 & 2.5203 & 28 & 0.31 & 4.783 & 15.429 & 3 & 6 & 1.221 & 0.3967744 \\
\hline & 80 & 0.31 & 23.67 & 1.63206 & 20.12 & 4.3409 & 28 & 0.4 & 4.635 & 11.587 & 4 & 6 & 1.184 & 0.2303652 \\
\hline & 100 & 0.39 & 22.52 & 2.4473 & 30.171 & 6.8414 & 28 & 0.5 & 4.41 & 8.82 & 5 & 6 & 1.126 & 0.1461684 \\
\hline & 120 & 0.47 & 21.9 & 3.42669 & 42.245 & 9.8519 & 28 & 0.61 & 4.288 & 7.03 & 6 & 6 & 1.095 & 0.1015037 \\
\hline & 140 & 0.54 & 20.75 & 4.5663 & 56.294 & 13.855 & 28 & 0.7 & 4.063 & 5.804 & 7 & 6 & 1.038 & 0.0721746 \\
\hline & 160 & 0.62 & 19.31 & 5.8685 & 72.348 & 19.14 & 28 & 0.8 & 3.78 & 4.725 & 8 & 6 & 0.965 & 0.0522477 \\
\hline & 180 & 0.7 & 19.05 & 7.33326 & 90.406 & 24.237 & 28 & 0.9 & 3.73 & 4.144 & 9 & 6 & 0.953 & 0.0412586 \\
\hline & 200 & 0.77 & 19.1 & 8.95668 & 0 & 0 & 28 & 0.99 & 3.739 & 3.777 & 10 & 6 & 0.955 & 0 \\
\hline & 220 & 1.55 & 27.94 & 12.17995 & 0 & 0 & 28 & 2 & 5.47 & 2.735 & 11 & 6 & 1.397 & 0 \\
\hline & 240 & 2.32 & 31.58 & 17.02743 & 0 & 0 & 28 & 3 & 6.183 & 2.061 & 12 & 6 & 1.579 & 0 \\
\hline & 260 & 3.09 & 34.36 & 23.50068 & 0 & 0 & 28 & 3.99 & 6.728 & 1.686 & 13 & 6 & 1.718 & 0 \\
\hline & 280 & 3.87 & 37 & 31.5895 & 0 & 0 & 28 & 5 & 7.245 & 1.449 & 14 & 6 & 1.85 & 0 \\
\hline & 300 & 4.65 & 39.8 & 41.30566 & 0 & 0 & 28 & 6 & 7.794 & 1.299 & 15 & 6 & 1.99 & 0 \\
\hline & 320 & 5.42 & 43.39 & 52.64602 & 0 & 0 & 28 & 7 & 8.496 & 1.214 & 16 & 6 & 2.17 & 0 \\
\hline & 340 & 6.2 & 46.59 & 65.61216 & 0 & 0 & 28 & 8 & 9.123 & 1.14 & 17 & 6 & 2.33 & 0 \\
\hline & 360 & 6.97 & 49.96 & 80.21271 & 0 & 0 & 28 & 9 & 9.782 & 1.087 & 18 & 6 & 2.498 & 0 \\
\hline & 380 & 7.75 & 52.93 & 96.58277 & 0 & 0 & 28 & 10.01 & 10.36 & 1.035 & 19 & 6 & 2.646 & 0 \\
\hline & 400 & 15.5 & 88.25 & 128.93018 & 0 & 0 & 28 & 20.01 & 17.28 & 0.864 & 20 & 6 & 4.412 & 0 \\
\hline & 420 & 23.25 & 94.14 & 177.42851 & 0 & 0 & 28 & 30.02 & 18.43 & 0.614 & 21 & 6 & 4.707 & 0 \\
\hline & 440 & 30.99 & 93.22 & 242.24271 & 0 & 0 & 28 & 40.01 & 18.25 & 0.456 & 22 & 6 & 4.661 & 0 \\
\hline & 460 & 38.73 & 92.68 & 323.28559 & 0 & 0 & 28 & 50 & 18.15 & 0.363 & 23 & 6 & 4.634 & 0 \\
\hline & 480 & 46.48 & 92.12 & 420.53045 & 0 & 0 & 28 & 60.01 & 18.04 & 0.301 & 24 & 6 & 4.606 & 0 \\
\hline & 500 & 54.22 & 88.09 & 533.91446 & 0 & 0 & 28 & 70 & 17.25 & 0.246 & 25 & 6 & 4.404 & 0 \\
\hline & 520 & 61.96 & 85.17 & 663.5727 & 0 & 0 & 28 & 79.99 & 16.68 & 0.208 & 26 & 6 & 4.258 & 0 \\
\hline & 540 & 69.71 & 78.67 & 809.48633 & 0 & 0 & 28 & 90 & 15.4 & 0.171 & 27 & 6 & 3.934 & 0 \\
\hline & 560 & 77.46 & 69.79 & 973.21985 & 0 & 0 & 28 & 100 & 13.66 & 0.137 & 28 & 6 & 3.489 & 0 \\
\hline & 580 & 154.9 & 111.5 & 1296.2596 & 0 & 0 & 28 & 200 & 21.83 & 0.109 & 29 & 6 & 5.575 & 0 \\
\hline & 600 & 232.4 & 124.8 & 1782.1807 & 0 & 0 & 28 & 300 & 24.44 & 0.081 & 30 & 6 & 6.24 & 0 \\
\hline & 620 & 309.8 & 131.4 & 2429.0347 & 0 & 0 & 28 & 400 & 25.73 & 0.064 & 31 & 6 & 6.569 & 0 \\
\hline & 640 & 387.3 & 138.4 & 3239.3386 & 0 & 0 & 28 & 500 & 27.1 & 0.054 & 32 & 6 & 6.921 & 0 \\
\hline & 660 & 464.8 & 120.8 & 4210.9774 & 0 & 0 & 28 & 600 & 23.66 & 0.039 & 33 & 6 & 6.041 & 0 \\
\hline & 680 & 542.2 & 136.1 & 5346.1016 & 0 & 0 & 28 & 700 & 26.64 & 0.038 & 34 & 6 & 6.803 & 0 \\
\hline & 700 & 619.7 & 146.9 & 6642.8859 & 0 & 0 & 28 & 800 & 28.77 & 0.036 & 35 & 6 & 7.346 & 0 \\
\hline & 720 & 697.1 & 160.2 & 8100.1568 & 0 & 0 & 28 & 900 & 31.37 & 0.035 & 36 & 6 & 8.01 & 0 \\
\hline & 740 & 774.6 & 178.8 & 9723.1444 & 0 & 0 & 28 & 1000 & 35.01 & 0.035 & 37 & 6 & 8.939 & 0 \\
\hline & 750 & 774.6 & 170.7 & 10553.031 & 0 & 0 & 28 & 1000 & 33.43 & 0.033 & 38 & 6 & 8.536 & 0 \\
\hline & 760 & 774.6 & 169.3 & 11364.181 & 0 & 0 & 28 & 1000 & 33.15 & 0.033 & 39 & 6 & 8.464 & 0 \\
\hline & 770 & 774.6 & 168.9 & 12174.925 & 0 & 0 & 28 & 1000 & 33.07 & 0.033 & 40 & 6 & 8.446 & 0 \\
\hline & 780 & 774.6 & 168.6 & 12985.262 & 0 & 0 & 28 & 1000 & 33 & 0.033 & 41 & 6 & 8.428 & 0 \\
\hline
\end{tabular}




\begin{tabular}{|c|c|c|c|c|c|c|c|c|c|c|c|c|c|c|}
\hline 5 & 790 & 774.6 & 168.2 & 13796.411 & 0 & 0 & 28 & 1000 & 32.94 & 0.033 & 42 & 6 & 8.411 & 0 \\
\hline 5 & 800 & 774.6 & 167.8 & 14607.966 & 0 & 0 & 28 & 1000 & 32.85 & 0.033 & 43 & 6 & 8.387 & 0 \\
\hline 6 & 820 & 774.6 & 167.3 & 16250.135 & 0 & 0 & 28 & 1000 & 32.76 & 0.033 & 44 & 6 & 8.367 & 0 \\
\hline 6 & 840 & 697.1 & 138 & 17710.409 & 0 & 0 & 28 & 900 & 27.01 & 0.03 & 45 & 6 & 6.898 & 0 \\
\hline 6 & 860 & 619.7 & 107.7 & 19010.521 & 0 & 0 & 28 & 800 & 21.1 & 0.026 & 46 & 6 & 5.387 & 0 \\
\hline 6 & 880 & 542.2 & 91.05 & 20146.331 & 0 & 0 & 28 & 700 & 17.83 & 0.025 & 47 & 6 & 4.553 & 0 \\
\hline 6 & 900 & 464.8 & 76.87 & 21121.941 & 0 & 0 & 28 & 600 & 15.05 & 0.025 & 48 & 6 & 3.844 & 0 \\
\hline 6 & 920 & 387.3 & 63.37 & 21933.819 & 0 & 0 & 28 & 500 & 12.41 & 0.025 & 49 & 6 & 3.168 & 0 \\
\hline 6 & 940 & 309.8 & 50.45 & 22584.157 & 0 & 0 & 28 & 400 & 9.878 & 0.025 & 50 & 6 & 2.523 & 0 \\
\hline 6 & 960 & 232.4 & 38.06 & 23072.469 & 0 & 0 & 28 & 300 & 7.452 & 0.025 & 51 & 6 & 1.903 & 0 \\
\hline 6 & 980 & 154.9 & 25.55 & 23397.861 & 0 & 0 & 28 & 200 & 5.003 & 0.025 & 52 & 6 & 1.278 & 0 \\
\hline 6 & 1000 & 77.46 & 14.24 & 23561.363 & 0 & 0 & 28 & 100 & 2.789 & 0.028 & 53 & 6 & 0.712 & 0 \\
\hline 7 & 1020 & 69.71 & 15.13 & 23708.532 & 0 & 0 & 28 & 90 & 2.963 & 0.033 & 54 & 6 & 0.757 & 0 \\
\hline 7 & 1040 & 61.97 & 14.76 & 23838.396 & 0 & 0 & 28 & 80 & 2.89 & 0.036 & 55 & 6 & 0.738 & 0 \\
\hline 7 & 1060 & 54.22 & 14.02 & 23952.071 & 0 & 0 & 28 & 70 & 2.744 & 0.039 & 56 & 6 & 0.701 & 0 \\
\hline 7 & 1080 & 46.48 & 13.03 & 24049.554 & 0 & 0 & 28 & 60.01 & 2.551 & 0.043 & 57 & 6 & 0.651 & 0 \\
\hline 7 & 1100 & 38.73 & 11.95 & 24130.792 & 0 & 0 & 28 & 50 & 2.339 & 0.047 & 58 & 6 & 0.597 & 0 \\
\hline 7 & 1120 & 30.99 & 11.18 & 24195.804 & 0 & 0 & 28 & 40.01 & 2.19 & 0.055 & 59 & 6 & 0.559 & 0 \\
\hline 7 & 1140 & 23.24 & 9.84 & 24244.603 & 0 & 0 & 28 & 30 & 1.927 & 0.064 & 60 & 6 & 0.492 & 0 \\
\hline 7 & 1160 & 15.5 & 8.4 & 24277.154 & 0 & 0 & 28 & 20.01 & 1.645 & 0.082 & 61 & 6 & 0.42 & 0 \\
\hline 7 & 1180 & 7.75 & 6.47 & 24293.498 & 0 & 0 & 28 & 10.01 & 1.267 & 0.127 & 62 & 6 & 0.324 & 0 \\
\hline 8 & 1200 & 6.97 & 8.16 & 24308.193 & 0 & 0 & 28 & 9 & 1.597 & 0.177 & 63 & 6 & 0.408 & 0 \\
\hline 8 & 1220 & 6.2 & 8.57 & 24321.185 & 0 & 0 & 28 & 8 & 1.677 & 0.21 & 64 & 6 & 0.428 & 0 \\
\hline 8 & 1240 & 5.42 & 8.67 & 24332.545 & 0 & 0 & 28 & 7 & 1.697 & 0.242 & 65 & 6 & 0.433 & 0 \\
\hline 8 & 1260 & 4.64 & 9.11 & 24342.288 & 0 & 0 & 28 & 5.99 & 1.783 & 0.298 & 66 & 6 & 0.455 & 0 \\
\hline 8 & 1280 & 3.87 & 9.21 & 24350.401 & 0 & 0 & 28 & 5 & 1.803 & 0.361 & 67 & 6 & 0.46 & 0 \\
\hline 8 & 1300 & 3.09 & 9.32 & 24356.898 & 0 & 0 & 28 & 3.99 & 1.826 & 0.458 & 68 & 6 & 0.466 & 0 \\
\hline 8 & 1320 & 2.32 & 9.34 & 24361.768 & 0 & 0 & 28 & 3 & 1.829 & 0.61 & 69 & 6 & 0.467 & 0 \\
\hline 8 & 1340 & 1.54 & 8.73 & 24365.013 & 0 & 0 & 28 & 1.99 & 1.71 & 0.859 & 70 & 6 & 0.437 & 0 \\
\hline 8 & 1360 & 0.77 & 8.06 & 24366.636 & 0 & 0 & 28 & 0.99 & 1.579 & 1.595 & 71 & 6 & 0.403 & 0 \\
\hline 9 & 1380 & 0.7 & 9.96 & 24368.11 & 18.164 & 9.3151 & 28 & 0.9 & 1.95 & 2.167 & 72 & 6 & 0.498 & 0.107353 \\
\hline 9 & 1400 & 0.62 & 10.24 & 24369.415 & 34.257 & 17.077 & 28 & 0.8 & 2.006 & 2.507 & 73 & 6 & 0.512 & 0.0585579 \\
\hline 9 & 1420 & 0.55 & 10.52 & 24370.558 & 48.345 & 23.48 & 28 & 0.71 & 2.059 & 2.9 & 74 & 6 & 0.526 & 0.04259 \\
\hline 9 & 1440 & 0.47 & 10.76 & 24371.538 & 60.428 & 28.693 & 28 & 0.61 & 2.106 & 3.452 & 75 & 6 & 0.538 & 0.0348511 \\
\hline 9 & 1460 & 0.39 & 11.08 & 24372.356 & 70.508 & 32.507 & 28 & 0.5 & 2.169 & 4.338 & 76 & 6 & 0.554 & 0.0307625 \\
\hline 9 & 1480 & 0.31 & 11.39 & 24373.011 & 78.593 & 35.243 & 28 & 0.4 & 2.23 & 5.575 & 77 & 6 & 0.57 & 0.0283741 \\
\hline 9 & 1500 & 0.24 & 11.75 & 24373.503 & 84.654 & 36.806 & 28 & 0.31 & 2.3 & 7.419 & 78 & 6 & 0.587 & 0.0271694 \\
\hline 9 & 1520 & 0.16 & 11.8 & 24373.835 & 88.74 & 38.399 & 28 & 0.21 & 2.311 & 11.005 & 79 & 6 & 0.59 & 0.0260423 \\
\hline 9 & 1540 & 0.08 & 10.54 & 24374.003 & 90.822 & 44.003 & 28 & 0.1 & 2.064 & 20.64 & 80 & 6 & 0.527 & 0.0227258 \\
\hline
\end{tabular}

2004.02.12 META-20 wt\%-U [sonicated] CC-45 $0.1-1000 \mathrm{~s}-1$ run 7.txt

Mode Step t[s] n[rpm] M[\%.] Phi[rad $\quad$ Gamma J[t] T[ $\left.{ }^{\circ} \mathrm{C}\right] \mathrm{D}[1 / \mathrm{s}] \quad$ Tau[Pa] Eta[Pas] MP\# Block M[mNm] G[Pa]

\begin{tabular}{|c|c|c|c|c|c|c|c|c|c|c|c|c|c|c|}
\hline 1 & 20 & 0.08 & 14.65 & 0.16022 & 1.9752 & 0.6887 & 28 & 0.1 & 2.868 & 28.68 & 1 & 7 & 0.732 & 1.4519828 \\
\hline 1 & 40 & 0.16 & 21.5 & 0.48773 & 6.0128 & 1.4282 & 28 & 0.21 & 4.21 & 20.048 & 2 & 7 & 1.075 & 0.7001695 \\
\hline 1 & 60 & 0.24 & 23.45 & 0.97782 & 12.055 & 2.6252 & 28 & 0.31 & 4.592 & 14.813 & 3 & 7 & 1.173 & 0.3809299 \\
\hline 1 & 80 & 0.31 & 22.65 & 1.63206 & 20.12 & 4.5377 & 28 & 0.4 & 4.434 & 11.085 & 4 & 7 & 1.132 & 0.2203752 \\
\hline 1 & 100 & 0.39 & 21.66 & 2.44809 & 30.18 & 7.118 & 28 & 0.5 & 4.24 & 8.48 & 5 & 7 & 1.083 & 0.1404888 \\
\hline 1 & 120 & 0.47 & 20.75 & 3.42591 & 42.235 & 10.395 & 28 & 0.61 & 4.063 & 6.661 & 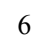 & 7 & 1.038 & 0.0961997 \\
\hline 1 & 140 & 0.54 & 20.06 & 4.5663 & 56.294 & 14.335 & 28 & 0.7 & 3.927 & 5.61 & t & 7 & 1.003 & 0.0697587 \\
\hline 1 & 160 & 0.62 & 19.41 & 5.86928 & 72.357 & 19.046 & 28 & 0.8 & 3.799 & 4.749 & 8 & 7 & 0.97 & 0.0525033 \\
\hline
\end{tabular}




\begin{tabular}{|c|c|c|c|c|c|c|c|c|c|c|c|c|c|c|c|}
\hline 1 & 1 & 180 & 0.7 & 18.73 & 7.33405 & 90.415 & 24.663 & 28 & 0.9 & 3.666 & 4.073 & 9 & 7 & 0.936 & 0.0405463 \\
\hline 1 & 2 & 200 & 0.77 & 18.71 & 8.95747 & 0 & 0 & 28 & 0.99 & 3.663 & 3.7 & 10 & 7 & 0.935 & 0 \\
\hline 1 & 2 & 220 & 1.55 & 27.18 & 12.18231 & 0 & 0 & 28 & 2 & 5.322 & 2.661 & 11 & 7 & 1.359 & 0 \\
\hline 1 & 2 & 240 & 2.32 & 31.01 & 17.02665 & 0 & 0 & 28 & 3 & 6.071 & 2.024 & 12 & 7 & 1.55 & 0 \\
\hline 1 & 2 & 260 & 3.09 & 33.75 & 23.4999 & 0 & 0 & 28 & 3.99 & 6.609 & 1.656 & 13 & 7 & 1.688 & 0 \\
\hline I & 2 & 280 & 3.87 & 36.55 & 31.59421 & 0 & 0 & 28 & 5 & 7.156 & 1.431 & 14 & 7 & 1.827 & 0 \\
\hline 1 & 2 & 300 & 4.65 & 39.6 & 41.30959 & 0 & 0 & 28 & 6 & 7.754 & 1.292 & 15 & 7 & 1.98 & 0 \\
\hline ] & 2 & 320 & 5.42 & 43.36 & 52.64995 & 0 & 0 & 28 & 7 & 8.49 & 1.213 & 16 & 7 & 2.168 & 0 \\
\hline & 2 & 340 & 6.2 & 46.66 & 65.61687 & 0 & 0 & 28 & 8 & 9.136 & 1.142 & 17 & 7 & 2.333 & 0 \\
\hline & 2 & 360 & 6.97 & 49.99 & 80.19229 & 0 & 0 & 28 & 9 & 9.787 & 1.087 & 18 & 7 & 2.499 & 0 \\
\hline & 3 & 380 & 7.75 & 52.95 & 96.55371 & 0 & 0 & 28 & 10.01 & 10.37 & 1.036 & 19 & 7 & 2.647 & 0 \\
\hline & 3 & 400 & 15.5 & 88.2 & 128.9019 & 0 & 0 & 28 & 20.01 & 17.27 & 0.863 & 20 & 7 & 4.41 & 0 \\
\hline I & 3 & 420 & 23.24 & 94.24 & 177.44815 & 0 & 0 & 28 & 30 & 18.45 & 0.615 & 21 & 7 & 4.712 & 0 \\
\hline 1 & 3 & 440 & 30.99 & 91.82 & 242.27805 & 0 & 0 & 28 & 40.01 & 17.98 & 0.449 & 22 & 7 & 4.591 & 0 \\
\hline 1 & 3 & 460 & 38.73 & 92.42 & 323.19998 & 0 & 0 & 28 & 50 & 18.1 & 0.362 & 23 & 7 & 4.621 & 0 \\
\hline 1 & 3 & 480 & 46.48 & 91.07 & 420.41892 & 0 & 0 & 28 & 60.01 & 17.83 & 0.297 & 24 & 7 & 4.553 & 0 \\
\hline 1 & 3 & 500 & 54.22 & 91.44 & 533.91603 & 0 & 0 & 28 & 70 & 17.9 & 0.256 & 25 & 7 & 4.572 & 0 \\
\hline 1 & 3 & 520 & 61.96 & 88.22 & 663.54442 & 0 & 0 & 28 & 79.99 & 17.27 & 0.216 & 26 & 7 & 4.411 & 0 \\
\hline 1 & 3 & 540 & 69.71 & 75.43 & 809.49732 & 0 & 0 & 28 & 90 & 14.77 & 0.164 & 27 & 7 & 3.772 & 0 \\
\hline 1 & 4 & 560 & 77.46 & 73.5 & 973.23006 & 0 & 0 & 28 & 100 & 14.39 & 0.144 & 28 & 7 & 3.675 & 0 \\
\hline 1 & 4 & 580 & 154.9 & 115.6 & 1296.3924 & 0 & 0 & 28 & 200 & 22.63 & 0.113 & 29 & 7 & 5.78 & 0 \\
\hline 1 & 4 & 600 & 232.4 & 124.8 & 1782.2318 & 0 & 0 & 28 & 300 & 24.44 & 0.081 & 30 & 7 & 6.24 & 0 \\
\hline 1 & 4 & 620 & 309.8 & 131.5 & 2429.4502 & 0 & 0 & 28 & 400 & 25.74 & 0.064 & 31 & 7 & 6.572 & 0 \\
\hline 1 & 4 & 640 & 387.3 & 128.5 & 3239.392 & 0 & 0 & 28 & 500 & 25.16 & 0.05 & 32 & 7 & 6.425 & 0 \\
\hline 1 & 4 & 660 & 464.8 & 120.7 & 4211.4715 & 0 & 0 & 28 & 600 & 23.63 & 0.039 & 33 & 7 & 6.034 & 0 \\
\hline 1 & 4 & 680 & 542.2 & 136.9 & 5346.5972 & 0 & 0 & 28 & 700 & 26.81 & 0.038 & 34 & 7 & 6.845 & 0 \\
\hline 1 & 4 & 700 & 619.7 & 145.9 & 6641.7628 & 0 & 0 & 28 & 800 & 28.56 & 0.036 & 35 & 7 & 7.293 & 0 \\
\hline 1 & 4 & 720 & 697.1 & 158.6 & 8100.1686 & 0 & 0 & 28 & 900 & 31.05 & 0.035 & 36 & 7 & 7.93 & 0 \\
\hline 1 & 4 & 740 & 774.6 & 173.5 & 9723.1578 & 0 & 0 & 28 & 1000 & 33.97 & 0.034 & 37 & 7 & 8.676 & 0 \\
\hline 1 & 5 & 750 & 774.6 & 170.1 & 10553.084 & 0 & 0 & 28 & 1000 & 33.31 & 0.033 & 38 & 7 & 8.507 & 0 \\
\hline 1 & 5 & 760 & 774.6 & 169.6 & 11364.233 & 0 & 0 & 28 & 1000 & 33.21 & 0.033 & 39 & 7 & 8.479 & 0 \\
\hline 1 & 5 & 770 & 774.6 & 168.8 & 12174.571 & 0 & 0 & 28 & 1000 & 33.06 & 0.033 & 40 & 7 & 8.441 & 0 \\
\hline 1 & 5 & 780 & 774.6 & 168.9 & 12984.909 & 0 & 0 & 28 & 1000 & 33.07 & 0.033 & 41 & 7 & 8.444 & 0 \\
\hline 1 & 5 & 790 & 774.6 & 168.2 & 13796.057 & 0 & 0 & 28 & 1000 & 32.93 & 0.033 & 42 & 7 & 8.409 & 0 \\
\hline 1 & 5 & 800 & 774.6 & 167.9 & 14608.016 & 0 & 0 & 28 & 1000 & 32.88 & 0.033 & 43 & 7 & 8.395 & 0 \\
\hline 1 & 6 & 820 & 774.6 & 167.7 & 16249.782 & 0 & 0 & 28 & 1000 & 32.83 & 0.033 & 44 & 7 & 8.384 & 0 \\
\hline 1 & 6 & 840 & 697.1 & 138.4 & 17710.096 & 0 & 0 & 28 & 900 & 27.09 & 0.03 & 45 & 7 & 6.919 & 0 \\
\hline 1 & 6 & 860 & 619.7 & 107.8 & 19009.275 & 0 & 0 & 28 & 800 & 21.1 & 0.026 & 46 & 7 & 5.389 & 0 \\
\hline 1 & 6 & 880 & 542.2 & 91.22 & 20146.465 & 0 & 0 & 28 & 700 & 17.86 & 0.026 & 47 & 7 & 4.561 & 0 \\
\hline 1 & 6 & 900 & 464.8 & 77.12 & 21121.911 & 0 & 0 & 28 & 600 & 15.1 & 0.025 & 48 & 7 & 3.856 & 0 \\
\hline 1 & 6 & 920 & 387.3 & 63.64 & 21933.748 & 0 & 0 & 28 & 500 & 12.46 & 0.025 & 49 & 7 & 3.182 & 0 \\
\hline 1 & 6 & 940 & 309.8 & 50.66 & 22584.129 & 0 & 0 & 28 & 400 & 9.919 & 0.025 & 50 & 7 & 2.533 & 0 \\
\hline 1 & 6 & 960 & 232.4 & 38.09 & 23072.197 & 0 & 0 & 28 & 300 & 7.458 & 0.025 & 51 & 7 & 1.904 & 0 \\
\hline 1 & 6 & 980 & 154.9 & 25.56 & 23397.914 & 0 & 0 & 28 & 200 & 5.004 & 0.025 & 52 & 7 & 1.278 & 0 \\
\hline 1 & 6 & 1000 & 77.46 & 14.17 & 23561.379 & 0 & 0 & 28 & 100 & 2.775 & 0.028 & 53 & 7 & 0.709 & 0 \\
\hline 1 & 7 & 1020 & 69.71 & 14.99 & 23708.513 & 0 & 0 & 28 & 90 & 2.935 & 0.033 & 54 & 7 & 0.75 & 0 \\
\hline 1 & 7 & 1040 & 61.97 & 14.76 & 23838.413 & 0 & 0 & 28 & 80 & 2.889 & 0.036 & 55 & 7 & 0.738 & 0 \\
\hline 1 & 7 & 1060 & 54.22 & 13.97 & 23952.117 & 0 & 0 & 28 & 70 & 2.735 & 0.039 & 56 & 7 & 0.698 & 0 \\
\hline 1 & 7 & 1080 & 46.48 & 12.97 & 24049.6 & 0 & 0 & 28 & 60.01 & 2.54 & 0.042 & 57 & 7 & 0.649 & \\
\hline 1 & 7 & 1100 & 38.73 & 11.87 & 24130.817 & 0 & 0 & 28 & 50 & 2.325 & 0.047 & 58 & 7 & 0.594 & \\
\hline
\end{tabular}




$\begin{array}{rrrrrrrrrrrrrrrr}1 & 7 & 1120 & 30.99 & 11.1 & 24195.833 & 0 & 0 & 28 & 40.01 & 2.174 & 0.054 & 59 & 7 & 0.555 & 0 \\ 1 & 7 & 1140 & 23.24 & 9.82 & 24244.621 & 0 & 0 & 28 & 30 & 1.922 & 0.064 & 60 & 7 & 0.491 & 0 \\ 1 & 7 & 1160 & 15.5 & 8.23 & 24277.171 & 0 & 0 & 28 & 20.01 & 1.611 & 0.081 & 61 & 7 & 0.412 & 0 \\ 1 & 7 & 1180 & 7.75 & 6.49 & 24293.532 & 0 & 0 & 28 & 10.01 & 1.27 & 0.127 & 62 & 7 & 0.324 & 0 \\ 1 & 8 & 1200 & 6.97 & 8.16 & 24308.238 & 0 & 0 & 28 & 9 & 1.598 & 0.178 & 63 & 7 & 0.408 & 0 \\ 1 & 8 & 1220 & 6.2 & 8.58 & 24321.224 & 0 & 0 & 28 & 8 & 1.679 & 0.21 & 64 & 7 & 0.429 & 0 \\ 1 & 8 & 1240 & 5.42 & 8.66 & 24332.581 & 0 & 0 & 28 & 7 & 1.697 & 0.242 & 65 & 7 & 0.433 & 0 \\ 1 & 8 & 1260 & 4.64 & 9.02 & 24342.327 & 0 & 0 & 28 & 5.99 & 1.766 & 0.295 & 66 & 7 & 0.451 & 0 \\ 1 & 8 & 1280 & 3.87 & 9.11 & 24350.44 & 0 & 0 & 28 & 5 & 1.783 & 0.357 & 67 & 7 & 0.455 & \\ 1 & 8 & 1300 & 3.09 & 9.18 & 24356.934 & 0 & 0 & 28 & 3.99 & 1.798 & 0.451 & 68 & 7 & 0.459 & 0 \\ 1 & 8 & 1320 & 2.32 & 9.04 & 24361.805 & 0 & 0 & 28 & 3 & 1.769 & 0.59 & 69 & 7 & 0.452 & \\ 1 & 8 & 1340 & 1.54 & 8.46 & 24365.051 & 0 & 0 & 28 & 1.99 & 1.656 & 0.832 & 70 & 7 & 0.423 & 0 \\ 1 & 8 & 1360 & 0.77 & 7.74 & 24366.673 & 0 & 0 & 28 & 0.99 & 1.515 & 1.53 & 71 & 7 & 0.387 & 0 \\ 1 & 9 & 1380 & 0.7 & 9.77 & 24368.147 & 18.164 & 9.5002 & 28 & 0.9 & 1.912 & 2.124 & 72 & 7 & 0.488 & 0.105261 \\ 1 & 9 & 1400 & 0.62 & 10.12 & 24369.453 & 34.266 & 17.289 & 28 & 0.8 & 1.982 & 2.478 & 73 & 7 & 0.506 & 0.057841 \\ 1 & 9 & 1420 & 0.55 & 10.41 & 24370.594 & 48.335 & 23.717 & 28 & 0.71 & 2.038 & 2.87 & 74 & 7 & 0.52 & 0.042164 \\ 1 & 9 & 1440 & 0.47 & 10.63 & 24371.574 & 60.419 & 29.034 & 28 & 0.61 & 2.081 & 3.411 & 75 & 7 & 0.531 & 0.0344429 \\ 1 & 9 & 1460 & 0.39 & 10.65 & 24372.392 & 70.498 & 33.812 & 28 & 0.5 & 2.085 & 4.17 & 76 & 7 & 0.532 & 0.0295752 \\ 1 & 9 & 1480 & 0.31 & 11.01 & 24373.048 & 78.583 & 36.465 & 28 & 0.4 & 2.155 & 5.387 & 77 & 7 & 0.55 & 0.0274232 \\ 1 & 9 & 1500 & 0.24 & 11.3 & 24373.541 & 84.664 & 38.257 & 28 & 0.31 & 2.213 & 7.139 & 78 & 7 & 0.565 & 0.0261387 \\ 1 & 9 & 1520 & 0.16 & 11.54 & 24373.871 & 88.73 & 39.261 & 28 & 0.21 & 2.26 & 10.762 & 79 & 7 & 0.577 & 0.0254704 \\ 1 & 9 & 1540 & 0.08 & 10.08 & 24374.04 & 90.822 & 46.032 & 28 & 0.1 & 1.973 & 19.73 & 80 & 7 & 0.504 & 0.0217238\end{array}$

2004.02.12 META-20 wt\%-U [sonicated] CC-45

0.1-1000 s-1 run 8.txt

\begin{tabular}{|c|c|c|c|c|c|c|c|c|c|c|c|c|c|c|c|}
\hline & & & & {$[\%]$.} & & Gamma & [t] & $\mathrm{T}\left[{ }^{\circ} \mathrm{C}\right.$ & {$[1 / \mathrm{s}]$} & $\mathrm{Tau}[\mathrm{Pa}$ & Eta[Pas] & MP\# & ock & $\mathrm{M}[\mathrm{ml}$ & a] \\
\hline 1 & 1 & 20 & 0.08 & 13.99 & 0.16022 & 1.9752 & 0.7209 & 28 & 0.1 & 2.74 & 27.4 & 1 & 8 & 0.7 & 1.3871802 \\
\hline 1 & 1 & 40 & 0.16 & 20.9 & 0.48773 & 6.0128 & 1.4691 & 28 & 0.21 & 4.093 & 19.49 & 2 & 8 & 1.045 & 0.6807111 \\
\hline 1 & 1 & 60 & 0.24 & 22.59 & 0.97861 & 12.064 & 2.7277 & 28 & 0.31 & 4.423 & 14.268 & 3 & 8 & 1.129 & 0.3666161 \\
\hline 1 & 1 & 80 & 0.31 & 21.72 & 1.63284 & 20.13 & 4.7342 & 28 & 0.4 & 4.252 & 10.63 & 4 & 8 & 1.086 & 0.2112279 \\
\hline 1 & 1 & 100 & 0.39 & 20.71 & 2.44887 & 30.19 & 7.4451 & 28 & 0.5 & 4.055 & 8.11 & 5 & 8 & 1.036 & 0.1343159 \\
\hline 1 & 1 & 120 & 0.47 & 20.38 & 3.42669 & 42.245 & 10.588 & 28 & 0.61 & 3.99 & 6.541 & 6 & 8 & 1.019 & 0.0944496 \\
\hline 1 & 1 & 140 & 0.54 & 19.44 & 4.56709 & 56.304 & 14.79 & 28 & 0.7 & 3.807 & 5.439 & 7 & 8 & 0.972 & 0.0676154 \\
\hline 1 & 1 & 160 & 0.62 & 18.38 & 5.86928 & 72.357 & 20.099 & 28 & 0.8 & 3.6 & 4.5 & 8 & 8 & 0.919 & 0.0497531 \\
\hline 1 & 1 & 180 & 0.7 & 17.95 & 7.33405 & 90.415 & 25.723 & 28 & 0.9 & 3.515 & 3.906 & 9 & 8 & 0.898 & 0.0388762 \\
\hline 1 & 2 & 200 & 0.77 & 18.08 & 8.95668 & 0 & 0 & 28 & 0.99 & 3.54 & 3.576 & 10 & 8 & 0.904 & 0 \\
\hline 1 & 2 & 220 & 1.55 & 26.48 & 12.17995 & 0 & 0 & 28 & 2 & 5.184 & 2.592 & 11 & 8 & 1.324 & 0 \\
\hline 1 & 2 & 240 & 2.32 & 30.54 & 17.02665 & 0 & 0 & 28 & 3 & 5.981 & 1.994 & 12 & 8 & 1.527 & 0 \\
\hline 1 & 2 & 260 & 3.09 & 33.39 & 23.4999 & 0 & 0 & 28 & 3.99 & 6.538 & 1.639 & 13 & 8 & 1.67 & 0 \\
\hline 1 & 2 & 280 & 3.87 & 36.07 & 31.59421 & 0 & 0 & 28 & 5 & 7.063 & 1.413 & 14 & 8 & 1.804 & 0 \\
\hline 1 & 2 & 300 & 4.65 & 39.16 & 41.3088 & 0 & 0 & 28 & 6 & 7.668 & 1.278 & 15 & 8 & 1.958 & 0 \\
\hline 1 & 2 & 320 & 5.42 & 42.67 & 52.65466 & 0 & 0 & 28 & 7 & 8.354 & 1.193 & 16 & 8 & 2.133 & 0 \\
\hline 1 & 2 & 340 & 6.2 & 46.04 & 65.62002 & 0 & 0 & 28 & 8 & 9.015 & 1.127 & 17 & 8 & 2.302 & 0 \\
\hline 1 & 2 & 360 & 6.97 & 49.17 & 80.19544 & 0 & 0 & 28 & 9 & 9.627 & 1.07 & 18 & 8 & 2.458 & 0 \\
\hline 1 & 3 & 380 & 7.75 & 51.94 & 96.55292 & 0 & 0 & 28 & 10.01 & 10.17 & 1.016 & 19 & 8 & 2.597 & 0 \\
\hline 1 & 3 & 400 & 15.5 & 87.31 & 128.90033 & 0 & 0 & 28 & 20.01 & 17.1 & 0.854 & 20 & 8 & 4.365 & 0 \\
\hline 1 & 3 & 420 & 23.24 & 94.71 & 177.44736 & 0 & 0 & 28 & 30 & 18.55 & 0.618 & 21 & 8 & 4.736 & 0 \\
\hline 1 & 3 & 440 & 30.99 & 93.85 & 242.27334 & 0 & 0 & 28 & 40.01 & 18.38 & 0.459 & 22 & 8 & 4.692 & 0 \\
\hline 1 & 3 & 460 & 38.73 & 93.27 & 323.21491 & 0 & 0 & 28 & 50 & 18.26 & 0.365 & 23 & 8 & 4.663 & 0 \\
\hline 1 & 3 & 480 & 46.48 & 91.08 & 420.43149 & 0 & 0 & 28 & 60.01 & 17.83 & 0.297 & 24 & 8 & 4.554 & 0 \\
\hline 1 & 3 & 500 & 54.22 & 90.52 & 533.95294 & 0 & 0 & 28 & 70 & 17.72 & 0.253 & 25 & 8 & 4.526 & 0 \\
\hline
\end{tabular}




\begin{tabular}{|c|c|c|c|c|c|c|c|c|c|c|c|c|c|c|c|}
\hline 1 & 3 & 520 & 61.96 & 83.41 & 663.64574 & 0 & 0 & 28 & 79.99 & 16.33 & 0.204 & 26 & 8 & 4.171 & 0 \\
\hline 1 & 3 & 540 & 69.71 & 74.02 & 809.56172 & 0 & 0 & 28 & 90 & 14.49 & 0.161 & 27 & 8 & 3.701 & 0 \\
\hline 1 & 4 & 560 & 77.46 & 72.08 & 973.29446 & 0 & 0 & 28 & 100 & 14.11 & 0.141 & 28 & 8 & 3.604 & 0 \\
\hline 1 & 4 & 580 & 154.9 & 112.4 & 1296.4568 & 0 & 0 & 28 & 200 & 22 & 0.11 & 29 & 8 & 5.617 & 0 \\
\hline 1 & 4 & 600 & 232.4 & 120.7 & 1781.6482 & 0 & 0 & 28 & 300 & 23.64 & 0.079 & 30 & 8 & 6.036 & \\
\hline & 4 & 620 & 309.8 & 126.4 & 2429.4792 & 0 & 0 & 28 & 400 & 24.76 & 0.062 & 31 & 8 & 6.322 & \\
\hline & 4 & 640 & 387.3 & 116.2 & 3239.8193 & 0 & 0 & 28 & 500 & 22.74 & 0.045 & 32 & 8 & 5.808 & \\
\hline 1 & 4 & 660 & 464.8 & 130.5 & 4211.1322 & 0 & 0 & 28 & 600 & 25.55 & 0.043 & 33 & 8 & 6.524 & \\
\hline & 4 & 680 & 542.2 & 135.3 & 5346.8258 & 0 & 0 & 28 & 700 & 26.49 & 0.038 & 34 & 8 & 6.764 & \\
\hline 1 & 4 & 700 & 619.7 & 144.6 & 6641.7062 & 0 & 0 & 28 & 800 & 28.31 & 0.035 & 35 & 8 & 7.229 & \\
\hline 1 & 4 & 720 & 697.1 & 156.3 & 8101.5313 & 0 & 0 & 28 & 900 & 30.6 & 0.034 & 36 & 8 & 7.813 & \\
\hline 1 & 4 & 740 & 774.6 & 180 & 9721.2752 & 0 & 0 & 28 & 1000 & 35.25 & 0.035 & 37 & 8 & 9.001 & \\
\hline 1 & 5 & 750 & 774.6 & 170.2 & 10550.755 & 0 & 0 & 28 & 1000 & 33.33 & 0.033 & 38 & 8 & 8.51 & 0 \\
\hline 1 & 5 & 760 & 774.6 & 169.2 & 11359.47 & 0 & 0 & 28 & 1000 & 33.14 & 0.033 & 39 & 8 & 8.462 & 0 \\
\hline 1 & 5 & 770 & 774.6 & 168.7 & 12173.459 & 0 & 0 & 28 & 1000 & 33.03 & 0.033 & 40 & 8 & 8.435 & 0 \\
\hline 1 & 5 & 780 & 774.6 & 167.7 & 12983.391 & 0 & 0 & 28 & 1000 & 32.83 & 0.033 & 41 & 8 & 8.382 & 0 \\
\hline 1 & 5 & 790 & 774.6 & 168.2 & 13794.134 & 0 & 0 & 28 & 1000 & 32.93 & 0.033 & 42 & 8 & 8.409 & 0 \\
\hline 1 & 5 & 800 & 774.6 & 167.7 & 14606.498 & 0 & 0 & 28 & 1000 & 32.83 & 0.033 & 43 & 8 & 8.384 & 0 \\
\hline 1 & 6 & 820 & 774.6 & 167.7 & 16247.453 & 0 & 0 & 28 & 1000 & 32.84 & 0.033 & 44 & 8 & 8.386 & 0 \\
\hline 1 & 6 & 840 & 697.1 & 137.9 & 17709.51 & 0 & 0 & 28 & 900 & 27.01 & 0.03 & 45 & 8 & 6.897 & 0 \\
\hline 1 & 6 & 860 & 619.7 & 107.7 & 19008.73 & 0 & 0 & 28 & 800 & 21.08 & 0.026 & 46 & 8 & 5.384 & 0 \\
\hline 1 & 6 & 880 & 542.2 & 91.94 & 20145.636 & 0 & 0 & 28 & 700 & 18 & 0.026 & 47 & 8 & 4.597 & 0 \\
\hline 1 & 6 & 900 & 464.8 & 77.82 & 21119.542 & 0 & 0 & 28 & 600 & 15.24 & 0.025 & 48 & 8 & 3.891 & 0 \\
\hline 1 & 6 & 920 & 387.3 & 64.17 & 21932.19 & 0 & 0 & 28 & 500 & 12.56 & 0.025 & 49 & 8 & 3.208 & 0 \\
\hline 1 & 6 & 940 & 309.8 & 50.92 & 22582.368 & 0 & 0 & 28 & 400 & 9.97 & 0.025 & 50 & 8 & 2.546 & 0 \\
\hline 1 & 6 & 960 & 232.4 & 38.2 & 23070.557 & 0 & 0 & 28 & 300 & 7.48 & 0.025 & 51 & 8 & 1.91 & 0 \\
\hline 1 & 6 & 980 & 154.9 & 25.52 & 23396.275 & 0 & 0 & 28 & 200 & 4.996 & 0.025 & 52 & 8 & 1.276 & 0 \\
\hline 1 & 6 & 1000 & 77.46 & 14.07 & 23559.782 & 0 & 0 & 28 & 100 & 2.754 & 0.028 & 53 & 8 & 0.703 & 0 \\
\hline 1 & 7 & 1020 & 69.71 & 14.95 & 23707.24 & 0 & 0 & 28 & 90 & 2.927 & 0.033 & 54 & 8 & 0.747 & 0 \\
\hline 1 & 7 & 1040 & 61.97 & 14.44 & 23837.233 & 0 & 0 & 28 & 80 & 2.827 & 0.035 & 55 & 8 & 0.722 & 0 \\
\hline 1 & 7 & 1060 & 54.22 & 13.69 & 23950.909 & 0 & 0 & 28 & 70 & 2.681 & 0.038 & 56 & 8 & 0.685 & 0 \\
\hline 1 & 7 & 1080 & 46.48 & 12.73 & 24048.295 & 0 & 0 & 28 & 60.01 & 2.492 & 0.042 & 57 & 8 & 0.636 & 0 \\
\hline 1 & 7 & 1100 & 38.73 & 11.61 & 24129.533 & 0 & 0 & 28 & 50 & 2.274 & 0.045 & 58 & 8 & 0.581 & 0 \\
\hline 1 & 7 & 1120 & 30.99 & 10.85 & 24194.549 & 0 & 0 & 28 & 40.01 & 2.125 & 0.053 & 59 & 8 & 0.543 & 0 \\
\hline 1 & 7 & 1140 & 23.24 & 9.53 & 24243.34 & 0 & 0 & 28 & 30 & 1.865 & 0.062 & 60 & 8 & 0.476 & 0 \\
\hline 1 & 7 & 1160 & 15.5 & 8 & 24275.89 & 0 & 0 & 28 & 20.01 & 1.566 & 0.078 & 61 & 8 & 0.4 & 0 \\
\hline 1 & 7 & 1180 & 7.75 & 6.16 & 24292.247 & 0 & 0 & 28 & 10.01 & 1.206 & 0.12 & 62 & 8 & 0.308 & 0 \\
\hline 1 & 8 & 1200 & 6.97 & 7.95 & 24306.944 & 0 & 0 & 28 & 9 & 1.557 & 0.173 & 63 & 8 & 0.398 & 0 \\
\hline 1 & 8 & 1220 & 6.2 & 8.23 & 24319.932 & 0 & 0 & 28 & 8 & 1.611 & 0.201 & 64 & 8 & 0.411 & 0 \\
\hline 1 & 8 & 1240 & 5.42 & 8.33 & 24331.306 & 0 & 0 & 28 & 7 & 1.631 & 0.233 & 65 & 8 & 0.417 & 0 \\
\hline 1 & 8 & 1260 & 4.64 & 8.76 & 24341.036 & 0 & 0 & 28 & 5.99 & 1.715 & 0.286 & 66 & 8 & 0.438 & 0 \\
\hline 1 & 8 & 1280 & 3.87 & 8.86 & 24349.152 & 0 & 0 & 28 & 5 & 1.734 & 0.347 & 67 & 8 & 0.443 & 0 \\
\hline 1 & 8 & 1300 & 3.09 & 9.09 & 24355.649 & 0 & 0 & 28 & 3.99 & 1.779 & 0.446 & 68 & 8 & 0.454 & 0 \\
\hline 1 & 8 & 1320 & 2.32 & 8.94 & 24360.516 & 0 & 0 & 28 & 3 & 1.75 & 0.583 & 69 & 8 & 0.447 & 0 \\
\hline 1 & 8 & 1340 & 1.54 & 8.28 & 24363.765 & 0 & 0 & 28 & 1.99 & 1.621 & 0.815 & 70 & 8 & 0.414 & 0 \\
\hline 1 & 8 & 1360 & 0.77 & 7.5 & 24365.389 & 0 & 0 & 28 & 0.99 & 1.469 & 1.484 & 71 & 8 & 0.375 & 0 \\
\hline 1 & 9 & 1380 & 0.7 & 9.6 & 24366.863 & 18.174 & 9.6722 & 28 & 0.9 & 1.879 & 2.088 & 72 & 8 & 0.48 & 0.1033892 \\
\hline 1 & 9 & 1400 & 0.62 & 9.89 & 24368.168 & 34.257 & 17.695 & 28 & 0.8 & 1.936 & 2.42 & 73 & 8 & 0.494 & 0.0565145 \\
\hline 1 & 9 & 1420 & 0.55 & 10.24 & 24369.311 & 48.345 & 24.1 & 28 & 0.71 & 2.006 & 2.825 & 74 & 8 & 0.512 & 0.0414937 \\
\hline 1 & 9 & 1440 & 0.47 & 10.32 & 24370.291 & 60.428 & 29.9 & 28 & 0.61 & 2.021 & 3.313 & 75 & 8 & 0.516 & 0.0334445 \\
\hline
\end{tabular}




$\begin{array}{rrrrrrrrrrrrrrrr}1 & 9 & 1460 & 0.39 & 10.48 & 24371.108 & 70.508 & 34.361 & 28 & 0.5 & 2.052 & 4.104 & 76 & 8 & 0.524 & 0.0291031 \\ 1 & 9 & 1480 & 0.31 & 10.75 & 24371.763 & 78.583 & 37.349 & 28 & 0.4 & 2.104 & 5.26 & 77 & 8 & 0.537 & 0.0267742 \\ 1 & 9 & 1500 & 0.24 & 11.05 & 24372.257 & 84.664 & 39.142 & 28 & 0.31 & 2.163 & 6.977 & 78 & 8 & 0.552 & 0.0255481 \\ 1 & 9 & 1520 & 0.16 & 11.48 & 24372.587 & 88.74 & 39.475 & 28 & 0.21 & 2.248 & 10.705 & 79 & 8 & 0.574 & 0.0253324 \\ 1 & 9 & 1540 & 0.08 & 10.32 & 24372.755 & 90.812 & 44.957 & 28 & 0.1 & 2.02 & 20.2 & 80 & 8 & 0.516 & 0.0222437\end{array}$
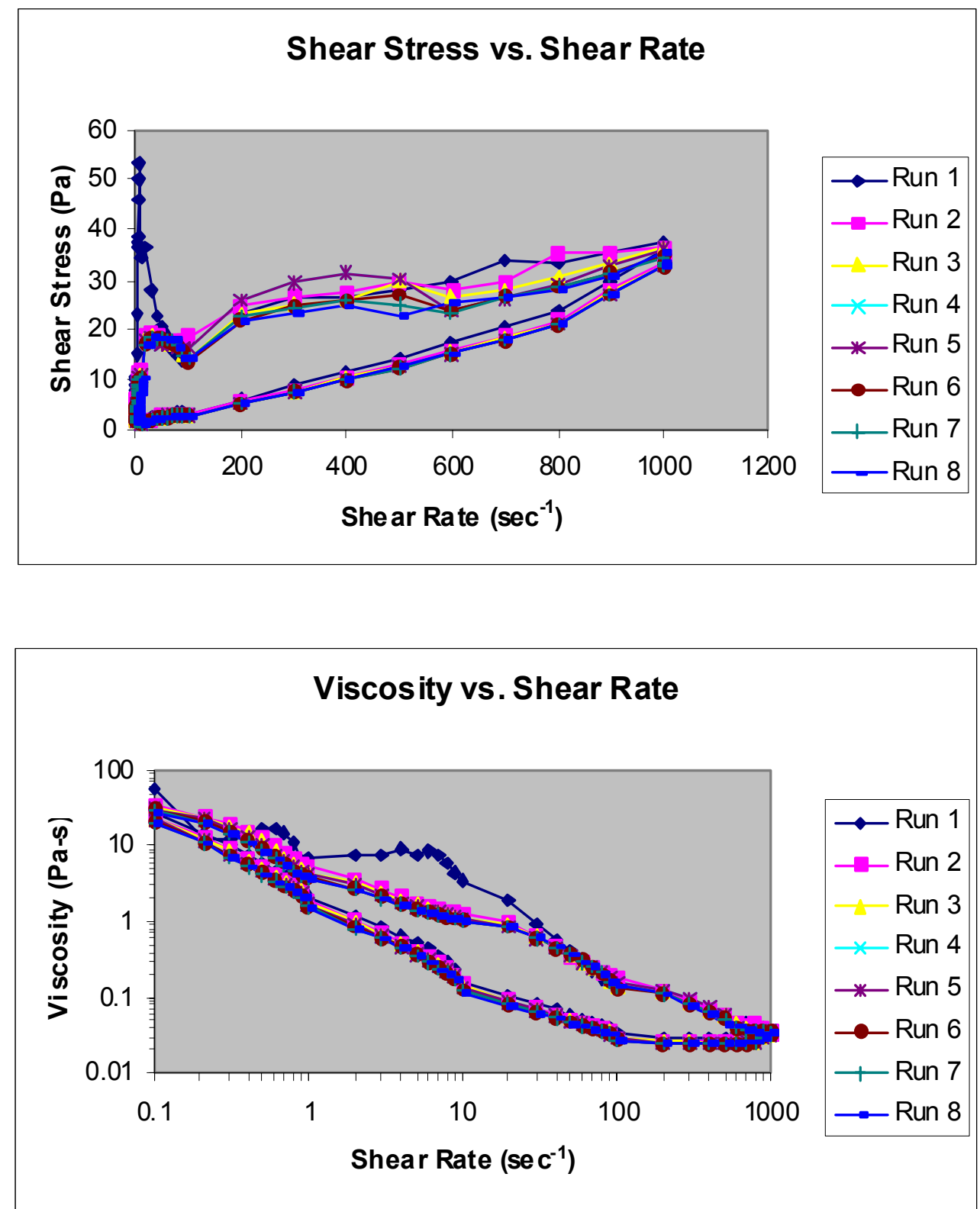

\section{E-2.1.23 META/20wt $\% / \mathrm{U} / \mathrm{N} 10^{\circ} \mathrm{C}$}

2004.02.10 META-20 wt\%-U CC-45 0.1-1000 s-1 run 1.txt Mode Step t[s] n[rpm] M[\%.] Phi[rad] Gamma $\mathrm{J}[\mathrm{t}] \quad \mathrm{T}\left[{ }^{\circ} \mathrm{C}\right] \mathrm{D}[1 / \mathrm{s}] \quad \mathrm{Tau}[\mathrm{Pa}]$ Eta[Pas $]$ MP\# Block M[mNm] G[Pa]

$\begin{array}{lllllll}1 & 1 & 20 & 0.06 & 183.37 & 0.12331 & 1.52015\end{array}$ $\begin{array}{lllllllll}0.04234 & 10 & 0.08 & 35.903 & 448.787 & 1 & 1 & 9.16835 & 23.61806401\end{array}$ 


\begin{tabular}{|c|c|c|c|c|c|c|c|c|c|c|c|c|c|c|c|}
\hline & 1 & 40 & 0.15 & 172.63 & 0.42804 & 5.27696 & 0.15612 & 10 & 0.19 & 33.8 & 177.895 & 2 & 1 & 8.6313 & 6.405202996 \\
\hline & 1 & 60 & 0.25 & 180.4 & 0.95819 & 11.81265 & 0.33443 & 10 & 0.32 & 35.322 & 110.381 & 3 & 1 & 9.0199 & 2.990184252 \\
\hline & 1 & 80 & 0.31 & 106.23 & 1.59671 & 19.68452 & 0.94637 & 10 & 0.4 & 20.8 & 52 & 4 & 1 & 5.31155 & 1.056667879 \\
\hline & 1 & 100 & 0.38 & 151.41 & 2.39861 & 29.57036 & 0.99745 & 10 & 0.49 & 29.646 & 60.502 & 5 & 1 & 7.57045 & 1.002557967 \\
\hline & 1 & 120 & 0.46 & 186.99 & 3.36229 & 41.45078 & 1.13216 & 10 & 0.59 & 36.612 & 62.054 & 6 & 1 & 9.34945 & 0.88326444 \\
\hline & 1 & 140 & 0.54 & 228.61 & 4.49248 & 55.3839 & 1.2373 & 10 & 0.7 & 44.762 & 63.946 & 7 & 1 & 11.43045 & 0.808213217 \\
\hline & 1 & 160 & 0.64 & 199.39 & 5.8253 & 71.8151 & 1.83953 & 10 & 0.83 & 39.04 & 47.036 & 8 & 1 & 9.9693 & 0.543618264 \\
\hline & 1 & 180 & 0.7 & 161.75 & 7.283 & 89.78582 & 2.83495 & 10 & 0.9 & 31.671 & 35.19 & 9 & 1 & 8.08755 & 0.352739441 \\
\hline & 2 & 200 & 0.77 & 169.34 & 8.90327 & 0 & 0 & 10 & 0.99 & 33.156 & 33.491 & 10 & 1 & 8.4669 & 0 \\
\hline & 2 & 220 & 1.55 & 342.17 & 12.09592 & 0 & 0 & 10 & 2 & 66.998 & 33.499 & 11 & 1 & 17.1087 & 0 \\
\hline & 2 & 240 & 2.33 & 281.68 & 16.94497 & 0 & 0 & 10 & 3.01 & 55.153 & 18.323 & 12 & 1 & 14.0841 & 0 \\
\hline & 2 & 260 & 3.09 & 288.89 & 23.3923 & 0 & 0 & 10 & 3.99 & 56.565 & 14.177 & 13 & 1 & 14.44455 & 0 \\
\hline & 2 & 280 & 3.89 & 317.54 & 31.50153 & 0 & 0 & 10 & 5.02 & 62.174 & 12.385 & 14 & 1 & 15.877 & 0 \\
\hline & 2 & 300 & 4.67 & 265.46 & 41.25225 & 0 & 0 & 10 & 6.03 & 51.976 & 8.62 & 15 & 1 & 13.27285 & 0 \\
\hline & 2 & 320 & 5.42 & 196.13 & 52.59497 & 0 & 0 & 10 & 7 & 38.402 & 5.486 & 16 & 1 & 9.80655 & 0 \\
\hline & 2 & 340 & 6.19 & 207.38 & 65.56033 & 0 & 0 & 10 & 7.99 & 40.604 & 5.082 & 17 & 1 & 10.36875 & 0 \\
\hline & 2 & 360 & 6.98 & 203.54 & 80.16402 & 0 & 0 & 10 & 9.01 & 39.853 & 4.423 & 18 & 1 & 10.177 & 0 \\
\hline & 3 & 380 & 7.75 & 176.13 & 96.52936 & 0 & 0 & 10.1 & 10.01 & 34.486 & 3.445 & 19 & 1 & 8.80635 & 0 \\
\hline & 3 & 400 & 15.51 & 216.3 & 128.86813 & 0 & 0 & 10 & 20.02 & 42.352 & 2.115 & 20 & 1 & 10.81505 & 0 \\
\hline & 3 & 420 & 23.24 & 224.83 & 177.39788 & 0 & 0 & 10 & 30 & 44.022 & 1.467 & 21 & 1 & 11.2416 & 0 \\
\hline & 3 & 440 & 30.99 & 263.7 & 242.14218 & 0 & 0 & 10 & 40.01 & 51.631 & 1.29 & 22 & 1 & 13.18475 & 0 \\
\hline & 3 & 460 & 38.73 & 258.9 & 323.16228 & 0 & 0 & 10 & 50 & 50.693 & 1.014 & 23 & 1 & 12.945 & 0 \\
\hline & 3 & 480 & 46.48 & 265.79 & 420.38358 & 0 & 0 & 10 & 60.01 & 52.042 & 0.867 & 24 & 1 & 13.28955 & 0 \\
\hline & 3 & 500 & 54.22 & 272.4 & 533.91995 & 0 & 0 & 10 & 70 & 53.336 & 0.762 & 25 & 1 & 13.6199 & 0 \\
\hline & 3 & 520 & 61.96 & 276.33 & 663.48238 & 0 & 0 & 10.1 & 79.99 & 54.104 & 0.676 & 26 & 1 & 13.81625 & 0 \\
\hline & 3 & 540 & 69.71 & 287.93 & 809.35752 & 0 & 0 & 10 & 90 & 56.376 & 0.626 & 27 & 1 & 14.3963 & 0 \\
\hline & 4 & 560 & 77.46 & 295.41 & 973.12639 & 0 & 0 & 10.1 & 100 & 57.841 & 0.578 & 28 & 1 & 14.77045 & 0 \\
\hline & 4 & 580 & 154.92 & 434.29 & 1296.43162 & 0 & 0 & 10.1 & 200 & 85.033 & 0.425 & 29 & 1 & 21.7143 & 0 \\
\hline & 4 & 600 & 232.38 & 514.85 & 1781.89486 & 0 & 0 & 10.1 & 300 & 100.81 & 0.336 & 30 & 1 & 25.74245 & 0 \\
\hline & 4 & 620 & 309.84 & 554.87 & 2429.10928 & 0 & 0 & 10.1 & 400 & 108.64 & 0.272 & 31 & 1 & 27.7435 & 0 \\
\hline & 4 & 640 & 387.3 & 574.29 & 3239.00245 & 0 & 0 & 10.1 & 500 & 112.45 & 0.225 & 32 & 1 & 28.71425 & 0 \\
\hline & 4 & 660 & 464.76 & 581.64 & 4211.0764 & 0 & 0 & 10.1 & 600.01 & 113.89 & 0.19 & 33 & 1 & 29.0819 & 0 \\
\hline & 4 & 680 & 542.22 & 582.22 & 5345.91156 & 0 & 0 & 10.1 & 700.01 & 114 & 0.163 & 34 & 1 & 29.1108 & 0 \\
\hline & 4 & 700 & 619.68 & 600.12 & 6641.11089 & 0 & 0 & 10.1 & 800.01 & 117.5 & 0.147 & 35 & 1 & 30.00575 & 0 \\
\hline & 4 & 720 & 697.13 & 587.78 & 8101.28781 & 0 & 0 & 10.1 & 899.99 & 115.09 & 0.128 & 36 & 1 & 29.38915 & 0 \\
\hline & 4 & 740 & 774.59 & 637.22 & 9721.3176 & 0 & 0 & 10.1 & 1000 & 124.77 & 0.125 & 37 & 1 & 31.86115 & 0 \\
\hline & 5 & 750 & 774.59 & 593.12 & 10549.87968 & 0 & 0 & 10.1 & 1000 & 116.13 & 0.116 & 38 & 1 & 29.65595 & 0 \\
\hline & 5 & 760 & 774.59 & 575.53 & 11360.21973 & 0 & 0 & 10.1 & 1000 & 112.69 & 0.113 & 39 & 1 & 28.77625 & 0 \\
\hline & 5 & 770 & 774.59 & 560.1 & 12172.18399 & 0 & 0 & 10.1 & 1000 & 109.67 & 0.11 & 40 & 1 & 28.005 & 0 \\
\hline & 5 & 780 & 774.59 & 546.58 & 12981.71194 & 0 & 0 & 10.1 & 1000 & 107.02 & 0.107 & 41 & 1 & 27.3288 & 0 \\
\hline & 5 & 790 & 774.59 & 534.5 & 13793.27015 & 0 & 0 & 10.1 & 1000 & 104.65 & 0.105 & 42 & 1 & 26.7248 & 0 \\
\hline & 5 & 800 & 774.59 & 523.78 & 14604.42151 & 0 & 0 & 10.1 & 1000 & 102.56 & 0.103 & 43 & 1 & 26.1891 & 0 \\
\hline & 6 & 820 & 774.59 & 512.62 & 16244.5685 & 0 & 0 & 10.1 & 1000 & 100.37 & 0.1 & 44 & 1 & 25.63105 & 0 \\
\hline & 6 & 840 & 697.13 & 452.74 & 17706.30287 & 0 & 0 & 10.1 & 899.99 & 88.646 & 0.098 & 45 & 1 & 22.63675 & 0 \\
\hline & 6 & 860 & 619.67 & 400.18 & 19005.12131 & 0 & 0 & 10.1 & 799.99 & 78.355 & 0.098 & 46 & 1 & 20.009 & 0 \\
\hline & 6 & 880 & 542.21 & 351.75 & 20142.64096 & 0 & 0 & 10.1 & 699.99 & 68.872 & 0.098 & 47 & 1 & 17.58735 & 0 \\
\hline & 6 & 900 & 464.75 & 306.37 & 21117.6091 & 0 & 0 & 10.1 & 599.99 & 59.988 & 0.1 & 48 & 1 & 15.3186 & 0 \\
\hline & 6 & 920 & 387.3 & 262.56 & 21930.46793 & 0 & 0 & 10.1 & 500 & 51.409 & 0.103 & 49 & 1 & 13.1279 & 0 \\
\hline & 6 & 940 & 309.84 & 219.32 & 22580.2027 & 0 & 0 & 10.1 & 400 & 42.944 & 0.107 & 50 & 1 & 10.9662 & 0 \\
\hline & 6 & 960 & 232.38 & 175.7 & 23068.19649 & 0 & 0 & 10.1 & 300 & 34.403 & 0.115 & 51 & 1 & 8.78515 & 0 \\
\hline
\end{tabular}




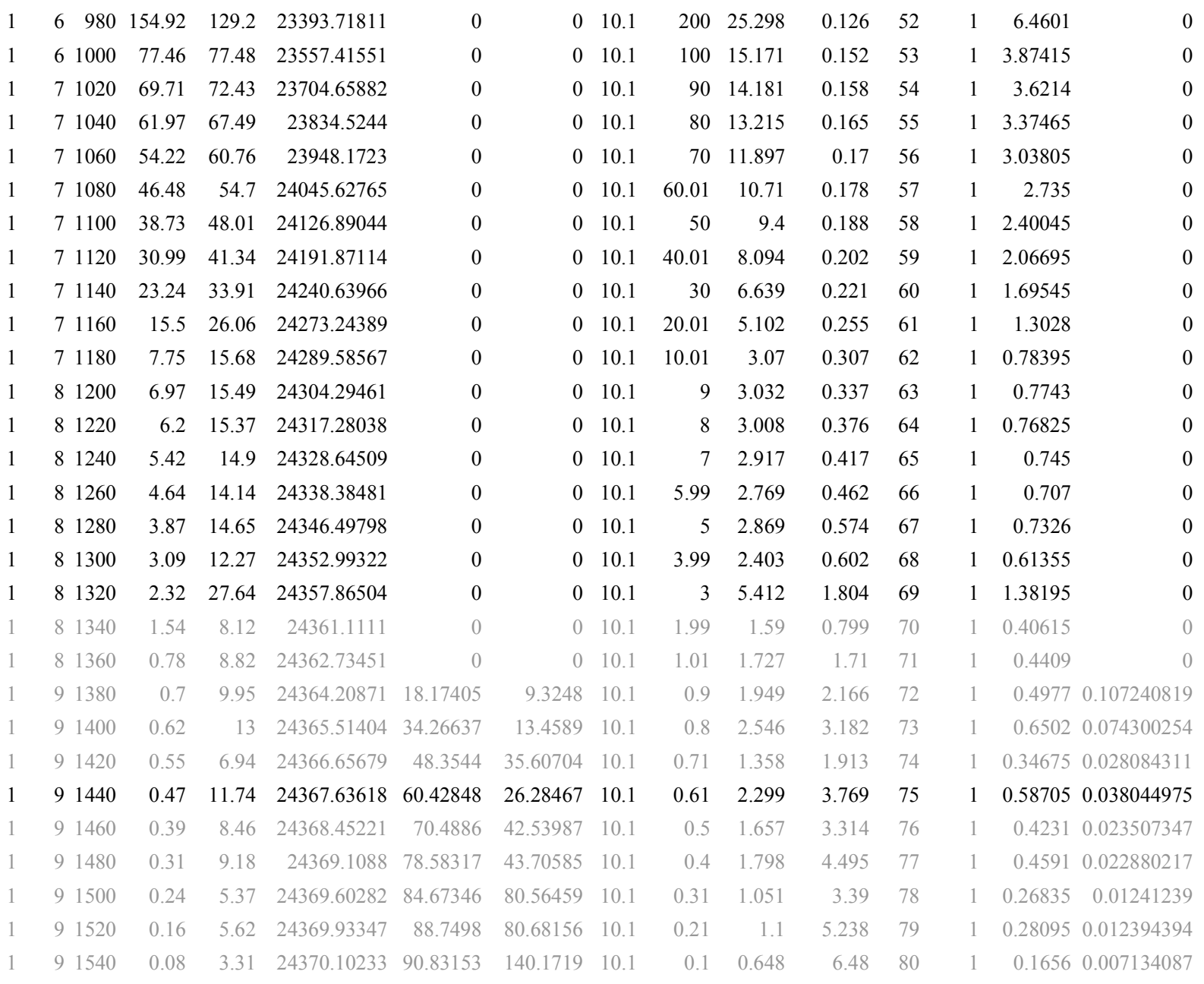

2004.02.10 META-20 wt\%-U CC-45 0.1-1000 s-1 run 2.txt

Mode Step t[s] n[rpm] M[\%.] Phi[rad] Gamma

$\begin{array}{rrrrr}1 & 1 & 20 & 0.08 & 6.92 \\ 1 & 1 & 40 & 0.16 & 9.81 \\ 1 & 1 & 60 & 0.24 & 13.07 \\ 1 & 1 & 80 & 0.31 & 12.55 \\ 1 & 1 & 100 & 0.39 & 18.39 \\ 1 & 1 & 120 & 0.47 & 13.65 \\ 1 & 1 & 140 & 0.54 & 14.09 \\ 1 & 1 & 160 & 0.62 & 14.01 \\ 1 & 1 & 180 & 0.7 & 14.66 \\ 1 & 2 & 200 & 0.77 & 14.51 \\ 1 & 2 & 220 & 1.55 & 22.51 \\ 1 & 2 & 240 & 2.32 & 25.06 \\ 1 & 2 & 260 & 3.09 & 39.28 \\ 1 & 2 & 280 & 3.87 & 34.64 \\ 1 & 2 & 300 & 4.65 & 35.48 \\ 1 & 2 & 320 & 5.42 & 31.18 \\ 1 & 2 & 340 & 6.2 & 34.77 \\ 1 & 2 & 360 & 6.97 & 27.64\end{array}$

$0.16336 \quad 2.01396$

$0.49087 \quad 6.05156$

$0.98175 \quad 12.10312$

$1.6352 \quad 20.15896$

$2.45044 \quad 30.2094$

$3.42748 \quad 42.25443$

4.5670956 .30374

$5.87164 \quad 72.38637$

7.336490 .44423

8.95982

12.1831

17.02743

23.48969

31.59343

41.3143

52.66016

65.6263

80.19858
$\mathrm{J}[\mathrm{t}] \quad \mathrm{T}\left[{ }^{\circ} \mathrm{C}\right] \mathrm{D}[1 / \mathrm{s}] \quad \mathrm{Tau}[\mathrm{Pa}] \mathrm{Eta}[\mathrm{Pas}] \mathrm{MP} \#$ Block M[mNm] G[Pa]

$\begin{array}{rrrrrrrrr}1.48632 & 10.1 & 0.1 & 1.355 & 13.55 & 1 & 1 & 0.3459 & 0.672803829 \\ 3.15021 & 10.1 & 0.21 & 1.921 & 9.148 & 2 & 1 & 0.49045 & 0.317438809 \\ 4.72963 & 10.1 & 0.31 & 2.559 & 8.255 & 3 & 1 & 0.65345 & 0.211433085 \\ 8.20136 & 10.1 & 0.4 & 2.458 & 6.145 & 4 & 1 & 0.62765 & 0.121930893 \\ 8.3915 & 10.1 & 0.5 & 3.6 & 7.2 & 5 & 1 & 0.9193 & 0.119168206 \\ 15.80786 & 10.1 & 0.61 & 2.673 & 4.382 & 6 & 1 & 0.6825 & 0.063259639 \\ 20.40729 & 10.1 & 0.7 & 2.759 & 3.941 & 7 & 1 & 0.7045 & 0.049002073 \\ 26.38948 & 10.1 & 0.8 & 2.743 & 3.429 & 8 & 1 & 0.70055 & 0.037893874 \\ 31.50268 & 10.1 & 0.9 & 2.871 & 3.19 & 9 & 1 & 0.7331 & 0.031743319 \\ 0 & 10.1 & 0.99 & 2.842 & 2.871 & 10 & 1 & 0.72565 & 0 \\ 0 & 10.1 & 2 & 4.407 & 2.204 & 11 & 1 & 1.12545 & 0 \\ 0 & 10.1 & 3 & 4.906 & 1.635 & 12 & 1 & 1.2528 & 0 \\ 0 & 10.1 & 3.99 & 7.691 & 1.928 & 13 & 1 & 1.96405 & 0 \\ 0 & 10.1 & 5 & 6.783 & 1.357 & 14 & 1 & 1.73205 & 0 \\ 0 & 10.1 & 6 & 6.948 & 1.158 & 15 & 1 & 1.77415 & 0 \\ 0 & 10.1 & 7 & 6.104 & 0.872 & 16 & 1 & 1.55885 & 0 \\ 0 & 10.1 & 8 & 6.808 & 0.851 & 17 & 1 & 1.73845 & 0 \\ 0 & 10.1 & 9 & 5.412 & 0.601 & 18 & 1 & 1.382 & 0\end{array}$




\begin{tabular}{|c|c|c|c|c|c|c|c|c|c|c|c|c|c|c|}
\hline & $3 \quad 380$ & 7.75 & 41.23 & 96.56863 & 0 & 0 & 10.1 & 10.01 & 8.073 & 0.806 & 19 & 1 & 2.06155 & 0 \\
\hline 1 & 3400 & 15.5 & 40.95 & 128.91997 & 0 & 0 & 10.1 & 20.01 & 8.018 & 0.401 & 20 & 1 & 2.04755 & 0 \\
\hline & 3420 & 23.24 & 46.72 & 177.46621 & 0 & 0 & 10.1 & 30 & 9.147 & 0.305 & 21 & 1 & 2.33585 & 0 \\
\hline & 3440 & 30.99 & 49.9 & 242.24664 & 0 & 0 & 10.1 & 40.01 & 9.77 & 0.244 & 22 & 1 & 2.49485 & 0 \\
\hline & 3460 & 38.73 & 54.6 & 323.26831 & 0 & 0 & 10.1 & 50 & 10.691 & 0.214 & 23 & 1 & 2.73 & 0 \\
\hline & 480 & 46.48 & 60.5 & 420.44249 & 0 & 0 & 10.1 & 60.01 & 11.845 & 0.197 & 24 & 1 & 3.02475 & 0 \\
\hline & 500 & 54.22 & 66.08 & 533.84927 & 0 & 0 & 10.1 & 70 & 12.939 & 0.185 & 25 & 1 & 3.30405 & 0 \\
\hline & 3520 & 61.96 & 74.83 & 663.60254 & 0 & 0 & 10.1 & 79.99 & 14.652 & 0.183 & 26 & 1 & 3.74165 & 0 \\
\hline & 540 & 69.71 & 76.89 & 809.33553 & 0 & 0 & 10.1 & 90 & 15.054 & 0.167 & 27 & 1 & 3.8443 & 0 \\
\hline & 4560 & 77.46 & 82.31 & 972.98737 & 0 & 0 & 10.1 & 100 & 16.116 & 0.161 & 28 & 1 & 4.11545 & 0 \\
\hline & 4580 & 154.92 & 138.1 & 1296.26433 & 0 & 0 & 10.1 & 200 & 27.041 & 0.135 & 29 & 1 & 6.9052 & 0 \\
\hline & 4600 & 232.38 & 186.98 & 1781.89643 & 0 & 0 & 10.1 & 300 & 36.611 & 0.122 & 30 & 1 & 9.3491 & 0 \\
\hline & 620 & 309.84 & 229.35 & 2429.39281 & 0 & 0 & 10.1 & 400 & 44.907 & 0.112 & 31 & 1 & 11.46745 & 0 \\
\hline & 640 & 387.3 & 268.92 & 3239.68731 & 0 & 0 & 10.1 & 500 & 52.654 & 0.105 & 32 & 1 & 13.4458 & 0 \\
\hline & 660 & 464.75 & 307.33 & 4211.27589 & 0 & 0 & 10.1 & 599.99 & 60.175 & 0.1 & 33 & 1 & 15.36635 & 0 \\
\hline & 680 & 542.21 & 345.24 & 5345.30288 & 0 & 0 & 10.1 & 699.99 & 67.597 & 0.097 & 34 & 1 & 17.26185 & 0 \\
\hline & 700 & 619.67 & 385.29 & 6642.77122 & 0 & 0 & 10.1 & 799.99 & 75.439 & 0.094 & 35 & 1 & 19.2644 & 0 \\
\hline & 720 & 697.13 & 417.74 & 8100.40266 & 0 & 0 & 10.1 & 899.99 & 81.793 & 0.091 & 36 & 1 & 20.88695 & 0 \\
\hline & 740 & 774.59 & 447.74 & 9722.17133 & 0 & 0 & 10.1 & 1000 & 87.667 & 0.088 & 37 & 1 & 22.387 & 0 \\
\hline & 750 & 774.59 & 436.79 & 10550.42632 & 0 & 0 & 10.1 & 1000 & 85.523 & 0.086 & 38 & 1 & 21.83945 & 0 \\
\hline & 760 & 774.59 & 431.86 & 11363.20032 & 0 & 0 & 10.1 & 1000 & 84.559 & 0.085 & 39 & 1 & 21.5931 & 0 \\
\hline & 770 & 774.59 & 427.58 & 12172.31986 & 0 & 0 & 10.1 & 1000 & 83.721 & 0.084 & 40 & 1 & 21.3792 & 0 \\
\hline & 780 & 774.59 & 423.47 & 12984.28176 & 0 & 0 & 10.1 & 1000 & 82.916 & 0.083 & 41 & 1 & 21.1736 & 0 \\
\hline & 790 & 774.59 & 419.44 & 13795.02551 & 0 & 0 & 10.1 & 1000 & 82.126 & 0.082 & 42 & 1 & 20.972 & 0 \\
\hline & 800 & 774.59 & 415.78 & 14605.36399 & 0 & 0 & 10.1 & 1000 & 81.41 & 0.081 & 43 & 1 & 20.78915 & 0 \\
\hline & 820 & 774.59 & 411.8 & 16245.50862 & 0 & 0 & 10.1 & 1000 & 80.631 & 0.081 & 44 & 1 & 20.5901 & 0 \\
\hline & 840 & 697.13 & 367.75 & 17708.70304 & 0 & 0 & 10.1 & 899.99 & 72.005 & 0.08 & 45 & 1 & 18.3873 & 0 \\
\hline & 860 & 619.67 & 326.69 & 19007.23481 & 0 & 0 & 10.1 & 799.99 & 63.966 & 0.08 & 46 & 1 & 16.33445 & 0 \\
\hline & 880 & 542.21 & 287.42 & 20144.14499 & 0 & 0 & 10.1 & 699.99 & 56.277 & 0.08 & 47 & 1 & 14.3711 & 0 \\
\hline & 900 & 464.75 & 249.81 & 21119.11 & 0 & 0 & 10.1 & 599.99 & 48.912 & 0.082 & 48 & 1 & 12.49025 & 0 \\
\hline & 920 & 387.3 & 213.05 & 21930.99493 & 0 & 0 & 10.1 & 500 & 41.715 & 0.083 & 49 & 1 & 10.65255 & 0 \\
\hline & 940 & 309.84 & 176.53 & 22581.21586 & 0 & 0 & 10.1 & 400 & 34.564 & 0.086 & 50 & 1 & 8.82645 & 0 \\
\hline & 960 & 232.38 & 139.85 & 23069.65419 & 0 & 0 & 10.1 & 300 & 27.384 & 0.091 & 51 & 1 & 6.99275 & 0 \\
\hline & 980 & 154.92 & 101.16 & 23395.2143 & 0 & 0 & 10.1 & 200 & 19.806 & 0.099 & 52 & 1 & 5.0578 & 0 \\
\hline & 61000 & 77.46 & 59.26 & 23558.74912 & 0 & 0 & 10.1 & 100 & 11.603 & 0.116 & 53 & 1 & 2.9631 & 0 \\
\hline & 71020 & 69.71 & 55.04 & 23705.92566 & 0 & 0 & 10.1 & 90 & 10.776 & 0.12 & 54 & 1 & 2.75175 & 0 \\
\hline & 71040 & 61.97 & 50.69 & 23835.85958 & 0 & 0 & 10.1 & 80 & 9.925 & 0.124 & 55 & 1 & 2.53445 & 0 \\
\hline & 71060 & 54.22 & 45.96 & 23949.51219 & 0 & 0 & 10.1 & 70 & 8.998 & 0.129 & 56 & 1 & 2.29785 & 0 \\
\hline & 71080 & 46.48 & 40.73 & 24046.94712 & 0 & 0 & 10.1 & 60.01 & 7.975 & 0.133 & 57 & 1 & 2.0365 & 0 \\
\hline & 71100 & 38.73 & 36.53 & 24128.22562 & 0 & 0 & 10.1 & 50 & 7.153 & 0.143 & 58 & 1 & 1.8265 & 0 \\
\hline & 71120 & 30.99 & 30.5 & 24193.27072 & 0 & 0 & 10.1 & 40.01 & 5.972 & 0.149 & 59 & 1 & 1.525 & 0 \\
\hline & 71140 & 23.24 & 25.04 & 24241.99761 & 0 & 0 & 10.1 & 30 & 4.903 & 0.163 & 60 & 1 & 1.252 & 0 \\
\hline & 71160 & 15.5 & 19 & 24274.59399 & 0 & 0 & 10.1 & 20.01 & 3.721 & 0.186 & 61 & 1 & 0.9502 & 0 \\
\hline & 71180 & 7.75 & 11.33 & 24290.93106 & 0 & 0 & 10.1 & 10.01 & 2.219 & 0.222 & 62 & 1 & 0.56665 & 0 \\
\hline & 81200 & 6.97 & 12.43 & 24305.64628 & 0 & 0 & 10.1 & 9 & 2.434 & 0.27 & 63 & 1 & 0.6215 & 0 \\
\hline & 81220 & 6.2 & 13.06 & 24318.6187 & 0 & 0 & 10.1 & 8 & 2.557 & 0.32 & 64 & 1 & 0.65305 & 0 \\
\hline & 81240 & 5.42 & 15.31 & 24329.98341 & 0 & 0 & 10.1 & 7 & 2.997 & 0.428 & 65 & 1 & 0.76535 & 0 \\
\hline & 81260 & 4.65 & 56.18 & 24339.73648 & 0 & 0 & 10.1 & 6 & 10.999 & 1.833 & 66 & 1 & 2.80885 & 0 \\
\hline & 81280 & 3.87 & 12.56 & 24347.85043 & 0 & 0 & 10.1 & 5 & 2.458 & 0.492 & 67 & 1 & 0.62775 & 0 \\
\hline & 81300 & 3.09 & 9.49 & 24354.3496 & 0 & 0 & 10.1 & 3.99 & 1.858 & 0.466 & 68 & 1 & 0.47435 & 0 \\
\hline
\end{tabular}




$\begin{array}{rrrrrrrrrrrrrrrr}1 & 8 & 1320 & 2.32 & 11.92 & 24359.2175 & 0 & 0 & 10.1 & 3 & 2.333 & 0.778 & 69 & 1 & 0.59575 & 0 \\ 1 & 8 & 1340 & 1.54 & 7.28 & 24362.46512 & 0 & 0 & 10.1 & 1.99 & 1.425 & 0.716 & 70 & 1 & 0.3639 & 0 \\ 1 & 8 & 1360 & 0.77 & 3.29 & 24364.09011 & 0 & 0 & 10.1 & 0.99 & 0.645 & 0.652 & 71 & 1 & 0.16465 & 0 \\ 1 & 9 & 1380 & 0.7 & 7.61 & 24365.56352 & 18.16437 & 12.19084 & 10.1 & 0.9 & 1.49 & 1.656 & 72 & 1 & 0.3805 & 0.082028719 \\ 1 & 9 & 1400 & 0.62 & 15.82 & 24366.86806 & 34.247 & 11.05455 & 10.1 & 0.8 & 3.098 & 3.872 & 73 & 1 & 0.79115 & 0.090460478 \\ 1 & 9 & 1420 & 0.55 & 12.99 & 24368.0116 & 48.34472 & 19.00342 & 10.1 & 0.71 & 2.544 & 3.583 & 74 & 1 & 0.6497 & 0.052622086 \\ 1 & 9 & 1440 & 0.47 & 12.15 & 24368.99099 & 60.4188 & 25.39671 & 10.1 & 0.61 & 2.379 & 3.9 & 75 & 1 & 0.6076 & 0.039375161 \\ 1 & 9 & 1460 & 0.39 & 7.81 & 24369.80781 & 70.4886 & 46.07095 & 10.1 & 0.5 & 1.53 & 3.06 & 76 & 1 & 0.39065 & 0.021705638 \\ 1 & 9 & 1480 & 0.31 & 8.9 & 24370.46047 & 78.53476 & 45.08307 & 10.1 & 0.4 & 1.742 & 4.355 & 77 & 1 & 0.44495 & 0.022181261 \\ 1 & 9 & 1500 & 0.24 & 20.05 & 24370.95449 & 84.62505 & 21.54954 & 10.1 & 0.31 & 3.927 & 12.668 & 78 & 1 & 1.0027 & 0.046404699 \\ 1 & 9 & 1520 & 0.16 & 12.97 & 24371.2875 & 88.73043 & 34.93323 & 10.1 & 0.21 & 2.54 & 12.095 & 79 & 1 & 0.64855 & 0.028626031 \\ 1 & 9 & 1540 & 0.08 & 9.96 & 24371.45636 & 90.81217 & 46.54645 & 10.1 & 0.1 & 1.951 & 19.51 & 80 & 1 & 0.49815 & 0.021483905\end{array}$

2004.02.10 META-20 wt\%-U CC-45 0.1-1000 s-1 run 3.txt

Mode Step t[s] n[rpm] M[\%] _ Phi[rad] Gamm

$\begin{array}{rrrrrrr}1 & 1 & 20 & 0.08 & 8.48 & 0.16101 & 1.98491 \\ 1 & 1 & 40 & 0.16 & 16.84 & 0.49087 & 6.05156 \\ 1 & 1 & 60 & 0.24 & 20.23 & 0.98096 & 12.09344 \\ 1 & 1 & 80 & 0.31 & 22.88 & 1.63206 & 20.12023 \\ 1 & 1 & 100 & 0.39 & 15.55 & 2.44652 & 30.16099 \\ 1 & 1 & 120 & 0.47 & 24.78 & 3.42591 & 42.23507 \\ 1 & 1 & 140 & 0.54 & 15.61 & 4.57023 & 56.34247 \\ 1 & 1 & 160 & 0.62 & 13.24 & 5.8685 & 72.34764 \\ 1 & 1 & 180 & 0.7 & 19.77 & 7.3364 & 90.44423 \\ 1 & 2 & 200 & 0.77 & 29.14 & 8.95354 & 0 \\ 1 & 2 & 220 & 1.55 & 26.82 & 12.18153 & 0 \\ 1 & 2 & 240 & 2.31 & 28.69 & 17.01722 & 0 \\ 1 & 2 & 260 & 3.09 & 88.57 & 23.47712 & 0 \\ 1 & 2 & 280 & 3.88 & 78.07 & 31.59421 & 0 \\ 1 & 2 & 300 & 4.65 & 39.89 & 41.31194 & 0 \\ 1 & 2 & 320 & 5.41 & 36.74 & 52.65938 & 0 \\ 1 & 2 & 340 & 6.2 & 42.31 & 65.62237 & 0 \\ 1 & 2 & 360 & 6.97 & 33.99 & 80.19544 & 0 \\ 1 & 3 & 380 & 7.75 & 34.37 & 96.55606 & 0 \\ 1 & 3 & 400 & 15.5 & 35.33 & 128.93175 & 0 \\ 1 & 3 & 420 & 23.24 & 44.42 & 177.47878 & 0 \\ 1 & 3 & 440 & 30.99 & 39.55 & 242.22308 & 0 \\ 1 & 3 & 460 & 38.73 & 43.45 & 323.28167 & 0 \\ 1 & 3 & 480 & 46.48 & 46.1 & 420.404 & 0 \\ 1 & 3 & 500 & 54.22 & 50.6 & 533.86341 & 0 \\ 1 & 3 & 520 & 61.96 & 54.64 & 663.54914 & 0 \\ 1 & 3 & 540 & 69.71 & 59.09 & 809.46748 & 0 \\ 1 & 4 & 560 & 77.46 & 63.67 & 973.07298 & 0 \\ 1 & 4 & 580 & 154.92 & 108.51 & 1296.71907 & 0 \\ 1 & 4 & 600 & 232.38 & 147.45 & 1782.27107 & 0 \\ 1 & 4 & 620 & 309.84 & 182.88 & 2429.48549 & 0 \\ 1 & 4 & 640 & 387.3 & 216.84 & 3239.37551 & 0 \\ 1 & 4 & 660 & 464.75 & 250.35 & 4211.49266 & 0 \\ 1 & 4 & 680 & 542.21 & 284.94 & 5346.04743 & 0 \\ 1 & 4 & 700 & 619.67 & 321.63 & 6642.50104 & 0\end{array}$

$\mathrm{J}[\mathrm{t}] \quad \mathrm{T}\left[{ }^{\circ} \mathrm{C}\right] \quad \mathrm{D}[1 / \mathrm{s}] \quad \mathrm{Tau}[\mathrm{Pa}] \mathrm{Eta}[\mathrm{Pas}] \mathrm{MP} \#$ Block M[mNm] G[Pa]

$\begin{array}{lllllllll}1.19501 & 10.1 & 0.1 & 1.661 & 16.61 & 1 & 1 & 0.4241 & 0.83681376\end{array}$

$\begin{array}{lllllllll}1.83547 & 10.1 & 0.21 & 3.297 & 15.7 & 2 & 1 & 0.842 & 0.544818196\end{array}$

$\begin{array}{lllllllll}3.05236 & 10.1 & 0.31 & 3.962 & 12.781 & 3 & 1 & 1.01165 & 0.327615633\end{array}$

$\begin{array}{lllllllll}4.49112 & 10.1 & 0.4 & 4.48 & 11.2 & 4 & 1 & 1.14395 & 0.222661471\end{array}$

$\begin{array}{lllllllll}9.90834 & 10.1 & 0.5 & 3.044 & 6.088 & 5 & 1 & 0.77735 & 0.100925069\end{array}$

$\begin{array}{lllllllll}8.70646 & 10.1 & 0.61 & 4.851 & 7.952 & 6 & 1 & 1.2388 & 0.114857155\end{array}$

$\begin{array}{llllllllll}18.43064 & 10.1 & 0.7 & 3.057 & 4.367 & 7 & 1 & 0.78055 & 0.054257472\end{array}$

$\begin{array}{lllllllll}27.92266 & 10.1 & 0.8 & 2.591 & 3.239 & 8 & 1 & 0.66175 & 0.035813193\end{array}$

$\begin{array}{lllllllll}23.36456 & 10.1 & 0.9 & 3.871 & 4.301 & 9 & 1 & 0.9885 & 0.042799856\end{array}$

$\begin{array}{llllll}0.99 & 5.706 & 5.764 & 10 & 1 & 1.4572\end{array}$

$\begin{array}{llllll}2 & 5.252 & 2.626 & 11 & 1 & 1.3411\end{array}$

$\begin{array}{ll}0 & 10.1\end{array}$

$\begin{array}{llll}0 & 10.1 & 2.98 & 5.617\end{array}$

$\begin{array}{llll}0 & 10.1 & 3.99 & 17.341\end{array}$

$\begin{array}{llll}0 & 10.1 & 5.01 & 15.286\end{array}$

$\begin{array}{llll}1.885 & 12 & 1 & 1.43445\end{array}$

$\begin{array}{llll}4.346 & 13 & 1 & 4.42835\end{array}$

$\begin{array}{ll}0 & 10.1\end{array}$

$6 \quad 7.811$

$\begin{array}{llll}3.051 & 14 & 1 & 3.90345\end{array}$

$\begin{array}{ll}0 & 10.1\end{array}$

$6.98 \quad 7.193$

$1.302 \quad 15$

$\begin{array}{ll}0 & 10.1\end{array}$

$8 \quad 8.285$

$1.031 \quad 16$

$1 \quad 1.9946$

$1 \quad 1.83675$

$\begin{array}{ll}0 & 10.1\end{array}$

$9 \quad 6.655$

$1.036 \quad 17$

$1 \quad 2.11565$

$\begin{array}{llll}0 & 10.1 & 10.01 & 6.729\end{array}$

$0.739 \quad 18$

$1 \quad 1.69955$

$1 \quad 1.71845$

$\begin{array}{llllllll}0 & 10.1 & 20.01 & 6.917 & 0.346 & 20 & 1 & 1.7663\end{array}$

$\begin{array}{llllllll}0 & 10.1 & 30 & 8.698 & 0.29 & 21 & 1 & 2.2212\end{array}$

$\begin{array}{llllll}0 & 10.1 & 40.01 & 7.743 & 0.194 & 22\end{array}$

$\begin{array}{llllll}0 & 10.1 & 50 & 8.508 & 0.17 & 23\end{array}$

$\begin{array}{llll}0 & 10.1 & 60.01 & 9.025\end{array}$

$0.15 \quad 24$

$0.142 \quad 25$

$\begin{array}{llll}0 & 10.1 & 70 & 9.908\end{array}$

$0.134 \quad 26$

$0.129 \quad 27$

$0.125 \quad 28$

$0.106 \quad 29$

$0.096 \quad 30$

$0.09 \quad 31$

$0.085 \quad 32$

$0.082 \quad 33$

$0.08 \quad 34$

$0.079 \quad 35$
$1 \quad 1.97725$

$1 \quad 2.1726$

12.30475

$1 \quad 2.5301$

$1 \quad 2.73215$

12.95465

$1 \quad 3.1834$

$1 \quad 5.42565$

17.3725

$1 \quad 9.1439$

$1 \quad 10.84175$

$1 \quad 12.51765$

$1 \quad 14.2471$

116.08155

\section{0

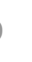




\begin{tabular}{|c|c|c|c|c|c|c|c|c|c|c|c|c|c|c|}
\hline & 4720 & 697.13 & 357.9 & 8100.13327 & 0 & 0 & 10.1 & 899.99 & 70.077 & 0.078 & 36 & 1 & 17.89505 & 0 \\
\hline & 740 & 774.59 & 388.83 & 9722.71169 & 0 & 0 & 10.1 & 1000 & 76.133 & 0.076 & 37 & 1 & 19.4416 & 0 \\
\hline & 750 & 774.59 & 382.17 & 10550.97688 & 0 & 0 & 10.1 & 1000 & 74.829 & 0.075 & 38 & 1 & 19.10845 & 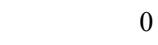 \\
\hline & 760 & 774.59 & 379.17 & 11363.34405 & 0 & 0 & 10.1 & 1000 & 74.242 & 0.074 & 39 & 1 & 18.95855 & \\
\hline & 5770 & 774.59 & 376.65 & 12172.86964 & 0 & 0 & 10.1 & 1000 & 73.749 & 0.074 & 40 & 1 & 18.8327 & \\
\hline & 5780 & 774.59 & 374.34 & 12985.23838 & 0 & 0 & 10.1 & 1000 & 73.295 & 0.073 & 41 & 1 & 18.7168 & \\
\hline & 5790 & 774.59 & 372.17 & 13795.16845 & 0 & 0 & 10.1 & 1000 & 72.87 & 0.073 & 42 & 1 & 18.6084 & \\
\hline & 5800 & 774.59 & 369.88 & 14605.50693 & 0 & 0 & 10.1 & 1000 & 72.422 & 0.072 & 43 & 1 & 18.49375 & \\
\hline & 6820 & 774.59 & 367.45 & 16246.46366 & 0 & 0 & 10.1 & 1000 & 71.947 & 0.072 & 44 & 1 & 18.3726 & \\
\hline & 6840 & 697.13 & 329.22 & 17707.46526 & 0 & 0 & 10.1 & 899.99 & 64.461 & 0.072 & 45 & 1 & 16.4609 & \\
\hline & 6860 & 619.67 & 292.87 & 19007.94403 & 0 & 0 & 10.1 & 799.99 & 57.344 & 0.072 & 46 & 1 & 14.64355 & \\
\hline & 6880 & 542.21 & 257.66 & 20143.72009 & 0 & 0 & 10.1 & 699.99 & 50.45 & 0.072 & 47 & 1 & 12.88295 & \\
\hline & 6900 & 464.75 & 223.53 & 21118.1958 & 0 & 0 & 10.1 & 599.99 & 43.768 & 0.073 & 48 & 1 & 11.17665 & 0 \\
\hline & $6 \quad 920$ & 387.3 & 189.98 & 21931.29652 & 0 & 0 & 10.1 & 500 & 37.199 & 0.074 & 49 & 1 & 9.4992 & 0 \\
\hline & 6940 & 309.84 & 156.63 & 22581.1923 & 0 & 0 & 10.1 & 400 & 30.668 & 0.077 & 50 & 1 & 7.8314 & 0 \\
\hline & 6960 & 232.38 & 123.22 & 23069.62984 & 0 & 0 & 10.1 & 300 & 24.126 & 0.08 & 51 & 1 & 6.1609 & 0 \\
\hline & 980 & 154.92 & 88.14 & 23395.18916 & 0 & 0 & 10.1 & 200 & 17.258 & 0.086 & 52 & 1 & 4.4071 & 0 \\
\hline & 61000 & 77.46 & 50.88 & 23558.64152 & 0 & 0 & 10.1 & 100 & 9.963 & 0.1 & 53 & 1 & 2.5441 & r \\
\hline & 71020 & 69.71 & 46.92 & 23705.81492 & 0 & 0 & 10.1 & 90 & 9.186 & 0.102 & 54 & 1 & 2.34585 & 0 \\
\hline & 71040 & 61.97 & 42.82 & 23835.67972 & 0 & 0 & 10.1 & 80 & 8.384 & 0.105 & 55 & 1 & 2.14105 & 0 \\
\hline & 71060 & 54.22 & 38.77 & 23949.30328 & 0 & 0 & 10.1 & 70 & 7.591 & 0.108 & 56 & 1 & 1.93855 & 0 \\
\hline & 71080 & 46.48 & 34.41 & 24046.83166 & 0 & 0 & 10.1 & 60.01 & 6.737 & 0.112 & 57 & 1 & 1.7205 & 0 \\
\hline & 71100 & 38.73 & 30.12 & 24128.06932 & 0 & 0 & 10.1 & 50 & 5.897 & 0.118 & 58 & 1 & 1.50585 & 0 \\
\hline & 71120 & 30.99 & 26.95 & 24193.05395 & 0 & 0 & 10.1 & 40.01 & 5.277 & 0.132 & 59 & 1 & 1.3476 & 0 \\
\hline & 71140 & 23.24 & 20.75 & 24241.86566 & 0 & 0 & 10.1 & 30 & 4.063 & 0.135 & 60 & 1 & 1.0376 & 0 \\
\hline & 71160 & 15.5 & 15.46 & 24274.43691 & 0 & 0 & 10.1 & 20.01 & 3.027 & 0.151 & 61 & 1 & 0.7729 & 0 \\
\hline & 71180 & 7.75 & 9.77 & 24290.77947 & 0 & 0 & 10.1 & 10.01 & 1.913 & 0.191 & 62 & 1 & 0.48855 & 0 \\
\hline & 81200 & 6.97 & 9.66 & 24305.48213 & 0 & 0 & 10.1 & 9 & 1.891 & 0.21 & 63 & 1 & 0.48285 & 0 \\
\hline & 81220 & 6.2 & 8.64 & 24318.4679 & 0 & 0 & 10.1 & 8 & 1.692 & 0.212 & 64 & 1 & 0.43205 & 0 \\
\hline & 81240 & 5.42 & 9.49 & 24329.83811 & 0 & 0 & 10.1 & 7 & 1.858 & 0.265 & 65 & 1 & 0.47455 & 0 \\
\hline & 81260 & 4.64 & 11.64 & 24339.57705 & 0 & 0 & 10.1 & 5.99 & 2.279 & 0.38 & 66 & 1 & 0.58205 & 0 \\
\hline & 81280 & 3.87 & 8.97 & 24347.69257 & 0 & 0 & 10.1 & 5 & 1.757 & 0.351 & 67 & 1 & 0.4486 & 0 \\
\hline & 81300 & 3.09 & 11.71 & 24354.18388 & 0 & 0 & 10.1 & 3.99 & 2.293 & 0.575 & 68 & 1 & 0.5856 & 0 \\
\hline & 81320 & 2.32 & 7.59 & 24359.05728 & 0 & 0 & 10.1 & 3 & 1.487 & 0.496 & 69 & 1 & 0.37965 & 0 \\
\hline & 81340 & 1.54 & 6.3 & 24362.30254 & 0 & 0 & 10.1 & 1.99 & 1.233 & 0.62 & 70 & 1 & 0.3148 & 0 \\
\hline & 81360 & 0.76 & 6.74 & 24363.91418 & 0 & 0 & 10.1 & 0.98 & 1.319 & 1.346 & 71 & 1 & 0.33675 & 0 \\
\hline & 91380 & 0.7 & 6.55 & 24365.39387 & 18.24183 & 14.22919 & 10.1 & 0.9 & 1.282 & 1.424 & 72 & 1 & 0.3274 & 0.070278037 \\
\hline & 91400 & 0.61 & 10.49 & 24366.67878 & 34.0824 & 16.59318 & 10.1 & 0.79 & 2.054 & 2.6 & 73 & 1 & 0.5245 & 0.060265709 \\
\hline & 91420 & 0.55 & 6.02 & 24367.84746 & 48.48996 & 41.12801 & 10.1 & 0.71 & 1.179 & 1.661 & 74 & 1 & 0.301 & 0.024314312 \\
\hline & 91440 & 0.47 & 4.59 & 24368.82763 & 60.57372 & 67.45396 & 10.1 & 0.61 & 0.898 & 1.472 & 75 & 1 & 0.22935 & 0.014824911 \\
\hline & 91460 & 0.39 & 7.18 & 24369.64445 & 70.64352 & 50.24429 & 10.1 & 0.5 & 1.406 & 2.812 & 76 & 1 & 0.35895 & 0.019902746 \\
\hline & 91480 & 0.31 & 7.65 & 24370.30025 & 78.72841 & 52.55565 & 10.1 & 0.4 & 1.498 & 3.745 & 77 & 1 & 0.3826 & 0.019027439 \\
\hline & 91500 & 0.24 & 4.29 & 24370.79427 & 84.8187 & 100.97452 & 10.1 & 0.31 & 0.84 & 2.71 & 78 & 1 & 0.2146 & 0.009903476 \\
\hline & 91520 & 0.16 & 7.2 & 24371.12099 & 88.84662 & 63.05646 & 10.1 & 0.21 & 1.409 & 6.71 & 79 & 1 & 0.35975 & 0.015858791 \\
\hline & 91540 & 0.08 & 2.45 & 24371.29378 & 90.97677 & 189.53454 & 10.1 & 0.1 & 0.48 & 4.8 & 80 & 1 & 0.12245 & 0.005276072 \\
\hline
\end{tabular}

2004.02.10 META-20 wt\%-U CC-45 0.1-1000 s-1 run 4.txt Mode Step t[s] n[rpm] M[\%.] Phi[rad] Gamma
$\mathrm{J}[\mathrm{t}] \quad \mathrm{T}\left[{ }^{\circ} \mathrm{C}\right] \mathrm{D}[1 / \mathrm{s}] \quad \mathrm{Tau}[\mathrm{Pa}] \mathrm{Eta}[\mathrm{Pas}] \mathrm{MP} \#$ Block M[mNm] G[Pa $]$

$\begin{array}{lllllllllll}0.16415 & 2.02364 & 1.99964 & 10.1 & 0.1 & 1.012 & 10.12 & 1 & 1 & 0.2584 & 0.500088949\end{array}$ $\begin{array}{lllllllllll}0.49166 & 6.06125 & 3.74845 & 10.1 & 0.21 & 1.617 & 7.7 & 2 & 1 & 0.4129 & 0.266776655\end{array}$ 


\begin{tabular}{|c|c|c|c|c|c|c|c|c|c|c|c|c|c|c|}
\hline & 60 & 0.24 & 9.35 & 0.98253 & 12.11281 & 6.61902 & 10.1 & 0.31 & 1.83 & 5.903 & 3 & 1 & 0.4674 & 0.151079725 \\
\hline & 80 & 0.31 & 9.35 & 1.63598 & 20.16865 & 11.0151 & 10.1 & 0.4 & 1.831 & 4.578 & 4 & 1 & 0.4676 & 0.09078446 \\
\hline & $1 \quad 100$ & 0.39 & 9.57 & 2.45201 & 30.22876 & 16.13921 & 10.1 & 0.5 & 1.873 & 3.746 & 5 & 1 & 0.47835 & 0.061960861 \\
\hline & $1 \quad 120$ & 0.47 & 9.64 & 3.42905 & 42.27379 & 22.40263 & 10.1 & 0.61 & 1.887 & 3.093 & 6 & 1 & 0.48175 & 0.044637587 \\
\hline & 140 & 0.54 & 10.7 & 4.57023 & 56.34247 & 26.89377 & 10.1 & 0.7 & 2.095 & 2.993 & 7 & 1 & 0.5351 & 0.037183318 \\
\hline & 160 & 0.62 & 14.44 & 5.87242 & 72.39605 & 25.60878 & 10.1 & 0.8 & 2.827 & 3.534 & 8 & 1 & 0.7218 & 0.039049092 \\
\hline & $1 \quad 180$ & 0.7 & 11.99 & 7.33719 & 90.45392 & 38.54021 & 10.1 & 0.9 & 2.347 & 2.608 & 9 & 1 & 0.5993 & 0.025946913 \\
\hline & 2200 & 0.77 & 10.81 & 8.95904 & 0 & 0 & 10.1 & 0.99 & 2.117 & 2.138 & 10 & 1 & 0.5407 & 0 \\
\hline & 220 & 1.55 & 15.87 & 12.18467 & 0 & 0 & 10.1 & 2 & 3.107 & 1.554 & 11 & 1 & 0.7934 & 0 \\
\hline & $2 \quad 240$ & 2.32 & 18.72 & 17.03057 & 0 & 0 & 10.1 & 3 & 3.666 & 1.222 & 12 & 1 & 0.9362 & 0 \\
\hline & $2 \quad 260$ & 3.09 & 23.77 & 23.50225 & 0 & 0 & 10.1 & 3.99 & 4.655 & 1.167 & 13 & 1 & 1.18865 & 0 \\
\hline & 2280 & 3.87 & 28.3 & 31.59657 & 0 & 0 & 10.1 & 5 & 5.542 & 1.108 & 14 & 1 & 1.4152 & 0 \\
\hline & 2300 & 4.65 & 20.66 & 41.31666 & 0 & 0 & 10.1 & 6 & 4.045 & 0.674 & 15 & 1 & 1.03305 & 0 \\
\hline & 2320 & 5.42 & 19.7 & 52.65466 & 0 & 0 & 10.1 & 7 & 3.857 & 0.551 & 16 & 1 & 0.9849 & 0 \\
\hline & 2340 & 6.2 & 23.38 & 65.62002 & 0 & 0 & 10.1 & 8 & 4.578 & 0.572 & 17 & 1 & 1.169 & 0 \\
\hline & 2360 & 6.97 & 22.49 & 80.21507 & 0 & 0 & 10.1 & 9 & 4.404 & 0.489 & 18 & 1 & 1.1247 & 0 \\
\hline & $3 \quad 380$ & 7.75 & 20.75 & 96.58198 & 0 & 0 & 10.1 & 10.01 & 4.063 & 0.406 & 19 & 1 & 1.03765 & 0 \\
\hline & 3400 & 15.5 & 35.18 & 128.91211 & 0 & 0 & 10.1 & 20.01 & 6.889 & 0.344 & 20 & 1 & 1.75915 & 0 \\
\hline & 3420 & 23.24 & 31.36 & 177.50391 & 0 & 0 & 10.1 & 30 & 6.14 & 0.205 & 21 & 1 & 1.56805 & 0 \\
\hline & 3440 & 30.99 & 34.49 & 242.28984 & 0 & 0 & 10.1 & 40.01 & 6.753 & 0.169 & 22 & 1 & 1.7244 & 0 \\
\hline & 3460 & 38.73 & 37.35 & 323.27145 & 0 & 0 & 10.1 & 50 & 7.314 & 0.146 & 23 & 1 & 1.86765 & 0 \\
\hline & 3480 & 46.48 & 40.03 & 420.48647 & 0 & 0 & 10.1 & 60.01 & 7.838 & 0.131 & 24 & 1 & 2.00145 & 0 \\
\hline & 3500 & 54.22 & 43.85 & 533.86969 & 0 & 0 & 10.1 & 70 & 8.587 & 0.123 & 25 & 1 & 2.1927 & 0 \\
\hline & 3520 & 61.96 & 47.48 & 663.55542 & 0 & 0 & 10.1 & 79.99 & 9.296 & 0.116 & 26 & 1 & 2.37375 & 0 \\
\hline & 3540 & 69.71 & 51.35 & 809.46983 & 0 & 0 & 10.1 & 90 & 10.055 & 0.112 & 27 & 1 & 2.5677 & 0 \\
\hline & $4 \quad 560$ & 77.46 & 55.06 & 973.12089 & 0 & 0 & 10.1 & 100 & 10.78 & 0.108 & 28 & 1 & 2.75285 & 0 \\
\hline & 580 & 154.92 & 94.21 & 1296.56435 & 0 & 0 & 10.1 & 200 & 18.446 & 0.092 & 29 & 1 & 4.71045 & 0 \\
\hline & 4600 & 232.38 & 128.78 & 1781.83281 & 0 & 0 & 10.1 & 300 & 25.215 & 0.084 & 30 & 1 & 6.43885 & 0 \\
\hline & 620 & 309.84 & 160.33 & 2429.04802 & 0 & 0 & 10.1 & 400 & 31.393 & 0.078 & 31 & 1 & 8.01655 & 0 \\
\hline & $4 \quad 640$ & 387.3 & 191.34 & 3238.93726 & 0 & 0 & 10.1 & 500 & 37.464 & 0.075 & 32 & 1 & 9.567 & 0 \\
\hline & $4 \quad 660$ & 464.75 & 222.77 & 4211.22013 & 0 & 0 & 10.1 & 599.99 & 43.618 & 0.073 & 33 & 1 & 11.1385 & 0 \\
\hline & $4 \quad 680$ & 542.21 & 255.05 & 5346.05686 & 0 & 0 & 10.1 & 699.99 & 49.938 & 0.071 & 34 & 1 & 12.75235 & 0 \\
\hline & 4700 & 619.67 & 287.68 & 6641.86644 & 0 & 0 & 10.1 & 799.99 & 56.327 & 0.07 & 35 & 1 & 14.38375 & 0 \\
\hline & 4720 & 697.13 & 323.84 & 8100.59116 & 0 & 0 & 10.1 & 899.99 & 63.409 & 0.07 & 36 & 1 & 16.1922 & 0 \\
\hline & 4740 & 774.59 & 357.72 & 9722.76352 & 0 & 0 & 10.1 & 1000 & 70.041 & 0.07 & 37 & 1 & 17.8858 & 0 \\
\hline & 5750 & 774.59 & 353.75 & 10551.43398 & 0 & 0 & 10.1 & 1000 & 69.264 & 0.069 & 38 & 1 & 17.68745 & 0 \\
\hline & 5760 & 774.59 & 352.06 & 11362.98826 & 0 & 0 & 10.1 & 1000 & 68.934 & 0.069 & 39 & 1 & 17.60315 & 0 \\
\hline & 5770 & 774.59 & 350.42 & 12174.94859 & 0 & 0 & 10.1 & 1000 & 68.612 & 0.069 & 40 & 1 & 17.521 & 0 \\
\hline & 780 & 774.59 & 348.91 & 12983.66365 & 0 & 0 & 10.1 & 1000 & 68.316 & 0.068 & 41 & 1 & 17.44545 & 0 \\
\hline & 5790 & 774.59 & 347.39 & 13796.43687 & 0 & 0 & 10.1 & 1000 & 68.019 & 0.068 & 42 & 1 & 17.36955 & 0 \\
\hline 1 & 5800 & 774.59 & 345.94 & 14606.77457 & 0 & 0 & 10.1 & 1000 & 67.735 & 0.068 & 43 & 1 & 17.2971 & 0 \\
\hline 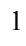 & $6 \quad 820$ & 774.59 & 344.18 & 16247.32368 & 0 & 0 & 10.1 & 1000 & 67.391 & 0.067 & 44 & 1 & 17.2091 & 0 \\
\hline 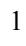 & 840 & 697.13 & 308.89 & 17708.32605 & 0 & 0 & 10.1 & 899.99 & 60.48 & 0.067 & 45 & 1 & 15.44425 & 0 \\
\hline$x$ & 860 & 619.67 & 275.21 & 19008.80404 & 0 & 0 & 10.1 & 799.99 & 53.886 & 0.067 & 46 & 1 & 13.76055 & 0 \\
\hline 1 & 880 & 542.21 & 242.23 & 20145.18643 & 0 & 0 & 10.1 & 699.99 & 47.428 & 0.068 & 47 & 1 & 12.11125 & 0 \\
\hline$t$ & 6900 & 464.75 & 210.04 & 21120.59519 & 0 & 0 & 10.1 & 599.99 & 41.126 & 0.069 & 48 & 1 & 10.5021 & 0 \\
\hline 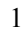 & $\begin{array}{ll}6 & 920\end{array}$ & 387.3 & 178.29 & 21933.04796 & 0 & 0 & 10.1 & 500 & 34.909 & 0.07 & 49 & 1 & 8.91435 & 0 \\
\hline & 6940 & 309.84 & 146.54 & 22583.10474 & 0 & 0 & 10.1 & 400 & 28.694 & 0.072 & 50 & 1 & 7.32725 & 0 \\
\hline & 6960 & 232.38 & 114.82 & 23070.56918 & 0 & 0 & 10.1 & 300 & 22.482 & 0.075 & 51 & 1 & 5.74095 & 0 \\
\hline & 980 & 154.92 & 81.61 & 23396.57461 & 0 & 0 & 10.1 & 200 & 15.979 & 0.08 & 52 & 1 & 4.08045 & 0 \\
\hline
\end{tabular}




\begin{tabular}{|c|c|c|c|c|c|c|c|c|c|c|c|c|c|}
\hline 61000 & 77.46 & 46.49 & 23560.10707 & 0 & 0 & 10.1 & 100 & 9.104 & 0.091 & 53 & 1 & 2.3247 & 0 \\
\hline 71020 & 69.71 & 42.87 & 23707.36137 & 0 & 0 & 10.1 & 90 & 8.395 & 0.093 & 54 & 1 & 2.1437 & 0 \\
\hline 71040 & 61.97 & 39.11 & 23837.22617 & 0 & 0 & 10.1 & 80 & 7.658 & 0.096 & 55 & 1 & 1.95545 & \\
\hline 71060 & 54.22 & 35.31 & 23950.90235 & 0 & 0 & 10.1 & 70 & 6.913 & 0.099 & 56 & 1 & 1.7654 & \\
\hline 71080 & 46.48 & 31.13 & 24048.38204 & 0 & 0 & 10.1 & 60.01 & 6.095 & 0.102 & 57 & 1 & 1.55645 & \\
\hline 71100 & 38.73 & 26.97 & 24129.6197 & 0 & 0 & 10.1 & 50 & 5.28 & 0.106 & 58 & 1 & 1.3484 & \\
\hline 71120 & 30.99 & 23.02 & 24194.58391 & 0 & 0 & 10.1 & 40.01 & 4.507 & 0.113 & 59 & 1 & 1.15095 & \\
\hline 71140 & 23.24 & 18.86 & 24243.39955 & 0 & 0 & 10.1 & 30 & 3.692 & 0.123 & 60 & 1 & 0.94275 & \\
\hline 71160 & 15.5 & 13.38 & 24275.96215 & 0 & 0 & 10.1 & 20.01 & 2.62 & 0.131 & 61 & 1 & 0.6691 & \\
\hline 71180 & 7.75 & 7.72 & 24292.31257 & 0 & 0 & 10.1 & 10.01 & 1.512 & 0.151 & 62 & 1 & 0.38615 & \\
\hline 81200 & 6.97 & 9.5 & 24307.01915 & 0 & 0 & 10.1 & 9 & 1.86 & 0.207 & 63 & 1 & 0.47495 & 0 \\
\hline 81220 & 6.2 & 8.15 & 24320.001 & 0 & 0 & 10.1 & 8 & 1.597 & 0.2 & 64 & 1 & 0.4077 & 0 \\
\hline 81240 & 5.42 & 7.2 & 24331.37042 & 0 & 0 & 10.1 & 7 & 1.41 & 0.201 & 65 & 1 & 0.3601 & 0 \\
\hline 81260 & 4.64 & 9.48 & 24341.10779 & 0 & 0 & 10.1 & 5.99 & 1.857 & 0.31 & 66 & 1 & 0.47415 & 0 \\
\hline 81280 & 3.87 & 9.16 & 24349.22881 & 0 & 0 & 10.1 & 5 & 1.794 & 0.359 & 67 & 1 & 0.458 & 0 \\
\hline 81300 & 3.09 & 6.26 & 24355.72169 & 0 & 0 & 10.1 & 3.99 & 1.226 & 0.307 & 68 & 1 & 0.31295 & 0 \\
\hline 81320 & 2.32 & 5.29 & 24360.59195 & 0 & 0 & 10.1 & 3 & 1.035 & 0.345 & 69 & 1 & 0.26425 & 0 \\
\hline 81340 & 1.54 & 5.88 & 24363.838 & 0 & 0 & 10.1 & 1.99 & 1.151 & 0.578 & 70 & 1 & 0.29385 & 0 \\
\hline 81360 & 0.77 & 2.71 & 24365.4622 & 0 & 0 & 10.1 & 0.99 & 0.531 & 0.536 & 71 & 1 & 0.13565 & 0 \\
\hline 91380 & 0.7 & 5.21 & 24366.93718 & 18.18373 & 17.80971 & 10.1 & 0.9 & 1.021 & 1.134 & 72 & 1 & 0.26065 & 0.056149096 \\
\hline 91400 & 0.62 & 5.04 & 24368.24173 & 34.26637 & 34.75288 & 10.1 & 0.8 & 0.986 & 1.233 & 73 & 1 & 0.25175 & 0.028774568 \\
\hline 91420 & 0.55 & 15.45 & 24369.37663 & 48.25758 & 15.95291 & 10.1 & 0.71 & 3.025 & 4.261 & 74 & 1 & 0.7724 & 0.062684453 \\
\hline 91440 & 0.47 & 16.7 & 24370.36151 & 60.39943 & 18.47642 & 10.1 & 0.61 & 3.269 & 5.359 & 75 & 1 & 0.83475 & 0.054123027 \\
\hline 91460 & 0.39 & 7.1 & 24371.17911 & 70.47892 & 50.74073 & 10.1 & 0.5 & 1.389 & 2.778 & 76 & 1 & 0.35475 & 0.01970802 \\
\hline 91480 & 0.31 & 6.93 & 24371.83806 & 78.60253 & 57.96642 & 10.1 & 0.4 & 1.356 & 3.39 & 77 & 1 & 0.34635 & 0.017251353 \\
\hline 91500 & 0.24 & 3.78 & 24372.33051 & 84.67346 & 114.42344 & 10.1 & 0.31 & 0.74 & 2.387 & 78 & 1 & 0.1889 & 0.008739456 \\
\hline 91520 & 0.16 & 4.38 & 24372.66116 & 88.74979 & 103.43786 & 10.1 & 0.21 & 0.858 & 4.086 & 79 & 1 & 0.2191 & 0.009667629 \\
\hline 91540 & 0.08 & 2.36 & 24372.83081 & 90.84121 & 196.62557 & 10.1 & 0.1 & 0.462 & 4.62 & 80 & 1 & 0.11795 & 0.005085798 \\
\hline
\end{tabular}

2004.02.10 META-20 wt\%-U CC-45 0.1-1000 s-1 run 5.txt Mode Step t[s] n[rpm] M[\%.]

$\begin{array}{rrrrr}1 & 1 & 20 & 0.08 & 4.21 \\ 1 & 1 & 40 & 0.16 & 7.51 \\ 1 & 1 & 60 & 0.24 & 8.52 \\ 1 & 1 & 80 & 0.31 & 25.38 \\ 1 & 1 & 100 & 0.4 & 8.61 \\ 1 & 1 & 120 & 0.47 & 8.1 \\ 1 & 1 & 140 & 0.54 & 8.3 \\ 1 & 1 & 160 & 0.62 & 8.55 \\ 1 & 1 & 180 & 0.7 & 8.71 \\ 1 & 2 & 200 & 0.77 & 11.15 \\ 1 & 2 & 220 & 1.55 & 56.19 \\ 1 & 2 & 240 & 2.31 & 41.96 \\ 1 & 2 & 260 & 3.09 & 58.82 \\ 1 & 2 & 280 & 3.88 & 47.76 \\ 1 & 2 & 300 & 4.65 & 37.14 \\ 1 & 2 & 320 & 5.42 & 37.18 \\ 1 & 2 & 340 & 6.19 & 29.53 \\ 1 & 2 & 360 & 6.97 & 39.31 \\ 1 & 3 & 380 & 7.75 & 17.46\end{array}$

Phi[rad] Gamma

$\begin{array}{rr}0.16336 & 2.01396 \\ 0.49166 & 6.06125 \\ 0.98332 & 12.12249 \\ 1.62734 & 20.06214 \\ 2.4528 & 30.23845 \\ 3.43062 & 42.29316 \\ 4.57102 & 56.35215 \\ 5.87321 & 72.40573 \\ 7.33876 & 90.47328 \\ 8.95668 & 0 \\ 12.18545 & 0 \\ 17.00308 & 0 \\ 23.47398 & 0 \\ 31.59186 & 0 \\ 41.32058 & 0 \\ 52.66566 & 0 \\ 65.62002 & 0 \\ 80.21429 & 0 \\ 96.58434 & 0\end{array}$

$\mathrm{J}[\mathrm{t}] \quad \mathrm{T}\left[{ }^{\circ} \mathrm{C}\right] \mathrm{D}[1 / \mathrm{s}] \quad \mathrm{Tau}[\mathrm{Pa}]$ Eta[Pas] MP\# Block M[mNm] G[Pa]

$\begin{array}{rrrrrrrrr}2.44116 & 10.1 & 0.1 & 0.825 & 8.25 & 1 & 1 & 0.2107 & 0.409640708 \\ 4.12049 & 10.1 & 0.21 & 1.471 & 7.005 & 2 & 1 & 0.3757 & 0.242689214 \\ 7.26768 & 10.1 & 0.31 & 1.668 & 5.381 & 3 & 1 & 0.426 & 0.137595494 \\ 4.03746 & 10.1 & 0.4 & 4.969 & 12.423 & 4 & 1 & 1.2689 & 0.247680457 \\ 17.94566 & 10.1 & 0.52 & 1.685 & 3.24 & 5 & 1 & 0.4303 & 0.055723756 \\ 26.68336 & 10.1 & 0.61 & 1.585 & 2.598 & 6 & 1 & 0.40475 & 0.037476509 \\ 34.67822 & 10.1 & 0.7 & 1.625 & 2.321 & 7 & 1 & 0.415 & 0.028836522 \\ 43.2531 & 10.1 & 0.8 & 1.674 & 2.093 & 8 & 1 & 0.4276 & 0.023119717 \\ 53.03237 & 10.1 & 0.9 & 1.706 & 1.896 & 9 & 1 & 0.4357 & 0.018856396 \\ 0 & 10.1 & 0.99 & 2.183 & 2.205 & 10 & 1 & 0.55735 & 0 \\ 0 & 10.1 & 2 & 11.001 & 5.501 & 11 & 1 & 2.80925 & 0 \\ 0 & 10.1 & 2.98 & 8.216 & 2.757 & 12 & 1 & 2.09815 & 0 \\ 0 & 10.1 & 3.99 & 11.518 & 2.887 & 13 & 1 & 2.9412 & 0 \\ 0 & 10.1 & 5.01 & 9.352 & 1.867 & 14 & 1 & 2.38805 & 0 \\ 0 & 10.1 & 6 & 7.271 & 1.212 & 15 & 1 & 1.85685 & 0 \\ 0 & 10.1 & 7 & 7.28 & 1.04 & 16 & 1 & 1.85895 & 0 \\ 0 & 10.1 & 7.99 & 5.782 & 0.724 & 17 & 1 & 1.4765 & 0 \\ 0 & 10.1 & 9 & 7.697 & 0.855 & 18 & 1 & 1.9654 & 0 \\ 0 & 10.1 & 10.01 & 3.419 & 0.342 & 19 & 1 & 0.8732 & 0\end{array}$




\begin{tabular}{|c|c|c|c|c|c|c|c|c|c|c|c|c|c|c|}
\hline & 3400 & 15.5 & 25.89 & 128.91525 & 0 & 0 & 10.1 & 20.01 & 5.069 & 0.253 & 20 & 1 & 1.29455 & 0 \\
\hline 1 & 3420 & 23.24 & 28.74 & 177.4992 & 0 & 0 & 10.1 & 30 & 5.627 & 0.188 & 21 & 1 & 1.4369 & 0 \\
\hline & 3440 & 30.99 & 30.3 & 242.28434 & 0 & 0 & 10.1 & 40.01 & 5.933 & 0.148 & 22 & 1 & 1.51515 & 0 \\
\hline & 3460 & 38.73 & 33.47 & 323.24161 & 0 & 0 & 10.1 & 50 & 6.553 & 0.131 & 23 & 1 & 1.6734 & 0 \\
\hline & 3480 & 46.48 & 37.07 & 420.50846 & 0 & 0 & 10.1 & 60.01 & 7.258 & 0.121 & 24 & 1 & 1.8534 & 0 \\
\hline & 500 & 54.22 & 40.65 & 533.89247 & 0 & 0 & 10.1 & 70 & 7.958 & 0.114 & 25 & 1 & 2.0323 & 0 \\
\hline & 520 & 61.96 & 44.21 & 663.61432 & 0 & 0 & 10.1 & 79.99 & 8.657 & 0.108 & 26 & 1 & 2.21055 & 0 \\
\hline & 540 & 69.71 & 47.43 & 809.45648 & 0 & 0 & 10.1 & 90 & 9.288 & 0.103 & 27 & 1 & 2.3717 & 0 \\
\hline & 560 & 77.46 & 50.68 & 973.22928 & 0 & 0 & 10.1 & 100 & 9.924 & 0.099 & 28 & 1 & 2.53415 & 0 \\
\hline & 580 & 154.92 & 86.28 & 1296.10568 & 0 & 0 & 10.1 & 200 & 16.893 & 0.084 & 29 & 1 & 4.31385 & 0 \\
\hline & 600 & 232.38 & 118.14 & 1782.06451 & 0 & 0 & 10.1 & 300 & 23.131 & 0.077 & 30 & 1 & 5.9068 & 0 \\
\hline & 620 & 309.84 & 147.38 & 2429.07708 & 0 & 0 & 10.1 & 400 & 28.857 & 0.072 & 31 & 1 & 7.36895 & 0 \\
\hline & 640 & 387.3 & 176.85 & 3239.57657 & 0 & 0 & 10.1 & 500 & 34.628 & 0.069 & 32 & 1 & 8.8426 & 0 \\
\hline & 660 & 464.75 & 206.49 & 4211.16751 & 0 & 0 & 10.1 & 599.99 & 40.43 & 0.067 & 33 & 1 & 10.32435 & 0 \\
\hline & 680 & 542.21 & 236.99 & 5346.33175 & 0 & 0 & 10.1 & 699.99 & 46.403 & 0.066 & 34 & 1 & 11.84955 & 0 \\
\hline & 700 & 619.67 & 266.66 & 6642.17981 & 0 & 0 & 10.1 & 799.99 & 52.213 & 0.065 & 35 & 1 & 13.33315 & 0 \\
\hline & 720 & 697.13 & 297.98 & 8100.17647 & 0 & 0 & 10.1 & 899.99 & 58.345 & 0.065 & 36 & 1 & 14.89905 & 0 \\
\hline & 740 & 774.59 & 333.99 & 9721.53673 & 0 & 0 & 10.1 & 1000 & 65.396 & 0.065 & 37 & 1 & 16.6996 & 0 \\
\hline & 750 & 774.59 & 335 & 10548.58691 & 0 & 0 & 10.1 & 1000 & 65.593 & 0.066 & 38 & 1 & 16.7499 & 0 \\
\hline & 760 & 774.59 & 333.69 & 11360.14119 & 0 & 0 & 10.1 & 1000 & 65.337 & 0.065 & 39 & 1 & 16.68455 & 0 \\
\hline & 770 & 774.59 & 332.43 & 12171.69469 & 0 & 0 & 10.1 & 1000 & 65.09 & 0.065 & 40 & 1 & 16.6215 & 0 \\
\hline & 780 & 774.59 & 331.25 & 12982.43765 & 0 & 0 & 10.1 & 1000 & 64.859 & 0.065 & 41 & 1 & 16.56265 & 0 \\
\hline & 790 & 774.59 & 330.12 & 13794.80246 & 0 & 0 & 10.1 & 1000 & 64.638 & 0.065 & 42 & 1 & 16.5062 & 0 \\
\hline & 800 & 774.59 & 328.96 & 14604.73489 & 0 & 0 & 10.1 & 1000 & 64.41 & 0.064 & 43 & 1 & 16.4478 & 0 \\
\hline & 820 & 774.59 & 327.76 & 16246.9082 & 0 & 0 & 10.1 & 1000 & 64.176 & 0.064 & 44 & 1 & 16.38805 & 0 \\
\hline & 840 & 697.13 & 294.47 & 17707.17937 & 0 & 0 & 10.1 & 899.99 & 57.657 & 0.064 & 45 & 1 & 14.7235 & 0 \\
\hline & 860 & 619.67 & 262.32 & 19007.33299 & 0 & 0 & 10.1 & 799.99 & 51.363 & 0.064 & 46 & 1 & 13.1162 & 0 \\
\hline & 880 & 542.21 & 230.71 & 20143.99969 & 0 & 0 & 10.1 & 699.99 & 45.174 & 0.065 & 47 & 1 & 11.53565 & 0 \\
\hline & 900 & 464.75 & 199.79 & 21118.19187 & 0 & 0 & 10.1 & 599.99 & 39.119 & 0.065 & 48 & 1 & 9.98965 & 0 \\
\hline & 920 & 387.3 & 169.29 & 21930.51976 & 0 & 0 & 10.1 & 500 & 33.147 & 0.066 & 49 & 1 & 8.4645 & 0 \\
\hline & 940 & 309.84 & 138.73 & 22580.78153 & 0 & 0 & 10.1 & 400 & 27.164 & 0.068 & 50 & 1 & 6.9366 & 0 \\
\hline & 960 & 232.38 & 108.33 & 23069.13661 & 0 & 0 & 10.1 & 300 & 21.211 & 0.071 & 51 & 1 & 5.4164 & 0 \\
\hline & 980 & 154.92 & 76.53 & 23394.73599 & 0 & 0 & 10.1 & 200 & 14.985 & 0.075 & 52 & 1 & 3.8267 & 0 \\
\hline & 61000 & 77.46 & 43.27 & 23558.22761 & 0 & 0 & 10.1 & 100 & 8.473 & 0.085 & 53 & 1 & 2.1637 & 0 \\
\hline & 71020 & 69.71 & 39.85 & 23705.44264 & 0 & 0 & 10.1 & 90 & 7.802 & 0.087 & 54 & 1 & 1.99235 & 0 \\
\hline & 71040 & 61.97 & 36.33 & 23835.34357 & 0 & 0 & 10.1 & 80 & 7.114 & 0.089 & 55 & 1 & 1.81655 & 0 \\
\hline & 71060 & 54.22 & 32.55 & 23948.96241 & 0 & 0 & 10.1 & 70 & 6.374 & 0.091 & 56 & 1 & 1.6276 & 0 \\
\hline & 71080 & 46.48 & 28.77 & 24046.44211 & 0 & 0 & 10.1 & 60.01 & 5.634 & 0.094 & 57 & 1 & 1.4387 & 0 \\
\hline & 71100 & 38.73 & 24.68 & 24127.67977 & 0 & 0 & 10.1 & 50 & 4.832 & 0.097 & 58 & 1 & 1.2339 & 0 \\
\hline & 71120 & 30.99 & 21.09 & 24192.72958 & 0 & 0 & 10.1 & 40.01 & 4.129 & 0.103 & 59 & 1 & 1.0545 & 0 \\
\hline & 71140 & 23.24 & 17.05 & 24241.51695 & 0 & 0 & 10.1 & 30 & 3.339 & 0.111 & 60 & 1 & 0.85255 & 0 \\
\hline & 71160 & 15.5 & 12.37 & 24274.08034 & 0 & 0 & 10.1 & 20.01 & 2.422 & 0.121 & 61 & 1 & 0.6186 & 0 \\
\hline & 71180 & 7.75 & 7.63 & 24290.42133 & 0 & 0 & 10.1 & 10.01 & 1.493 & 0.149 & 62 & 1 & 0.38135 & 0 \\
\hline & 81200 & 6.97 & 7.61 & 24305.13734 & 0 & 0 & 10.1 & 9 & 1.49 & 0.166 & 63 & 1 & 0.3806 & 0 \\
\hline & 81220 & 6.2 & 9.8 & 24318.11761 & 0 & 0 & 10.1 & 8 & 1.919 & 0.24 & 64 & 1 & 0.49 & 0 \\
\hline & 81240 & 5.42 & 6.74 & 24329.47683 & 0 & 0 & 10.1 & 7 & 1.319 & 0.188 & 65 & 1 & 0.33695 & 0 \\
\hline & 81260 & 4.64 & 9.23 & 24339.21341 & 0 & 0 & 10.1 & 5.99 & 1.806 & 0.302 & 66 & 1 & 0.4613 & 0 \\
\hline & 81280 & 3.87 & 8.91 & 24347.32971 & 0 & 0 & 10.1 & 5 & 1.744 & 0.349 & 67 & 1 & 0.4453 & 0 \\
\hline & 81300 & 3.09 & 6.07 & 24353.82339 & 0 & 0 & 10.1 & 3.99 & 1.189 & 0.298 & 68 & 1 & 0.3037 & 0 \\
\hline & 81320 & 2.32 & 5.59 & 24358.69364 & 0 & 0 & 10.1 & 3 & 1.094 & 0.365 & 69 & 1 & 0.27935 & 0 \\
\hline
\end{tabular}




$\begin{array}{rrrrrrrrrrrrrrr}8 & 1340 & 1.54 & 5.3 & 24361.94126 & 0 & 0 & 10.1 & 1.99 & 1.038 & 0.522 & 70 & 1 & 0.2651 & 0 \\ 8 & 1360 & 0.77 & 5.93 & 24363.56546 & 0 & 0 & 10.1 & 0.99 & 1.161 & 1.173 & 71 & 1 & 0.2965 & 0 \\ 9 & 1380 & 0.7 & 4.07 & 24365.04044 & 18.18373 & 22.81519 & 10.1 & 0.9 & 0.797 & 0.886 & 72 & 1 & 0.20365 & 0.043830391 \\ 9 & 1400 & 0.62 & 4.41 & 24366.34577 & 34.27605 & 39.67131 & 10.1 & 0.8 & 0.864 & 1.08 & 73 & 1 & 0.22055 & 0.025207105 \\ 9 & 1420 & 0.55 & 5.01 & 24367.48696 & 48.34472 & 49.28101 & 10.1 & 0.71 & 0.981 & 1.382 & 74 & 1 & 0.25055 & 0.020291771 \\ 9 & 1440 & 0.47 & 5.93 & 24368.46792 & 60.43816 & 52.10182 & 10.1 & 0.61 & 1.16 & 1.902 & 75 & 1 & 0.2963 & 0.019193172 \\ 9 & 1460 & 0.39 & 11.44 & 24369.28552 & 70.51765 & 31.48108 & 10.1 & 0.5 & 2.24 & 4.48 & 76 & 1 & 0.5721 & 0.031765097 \\ 9 & 1480 & 0.31 & 4.14 & 24369.94133 & 78.60253 & 96.92039 & 10.1 & 0.4 & 0.811 & 2.027 & 77 & 1 & 0.2072 & 0.010317734 \\ 9 & 1500 & 0.24 & 3.59 & 24370.43456 & 84.68315 & 120.4595 & 10.1 & 0.31 & 0.703 & 2.268 & 78 & 1 & 0.1795 & 0.008301533 \\ 9 & 1520 & 0.16 & 8.83 & 24370.76521 & 88.75948 & 51.30603 & 10.1 & 0.21 & 1.73 & 8.238 & 79 & 1 & 0.4417 & 0.019490876 \\ 9 & 1540 & 0.08 & 5.03 & 24370.93407 & 90.84122 & 92.13096 & 10.1 & 0.1 & 0.986 & 9.86 & 80 & 1 & 0.2517 & 0.010854103\end{array}$

2004.02.10 META-20 wt\%-U CC-45 0.1-1000 s-1 run 6.txt

Mode Step t[s] n[rpm] M[\%.] Phi[rad] Gamma

$\begin{array}{rrrrrrr}1 & 1 & 20 & 0.08 & 5.61 & 0.16336 & 2.0139 \\ 1 & 1 & 40 & 0.16 & 8.2 & 0.49166 & 6.06125 \\ 1 & 1 & 60 & 0.24 & 9.59 & 0.98332 & 12.122 \\ 1 & 1 & 80 & 0.31 & 8.5 & 1.63598 & 20.16865 \\ 1 & 1 & 100 & 0.39 & 8.39 & 2.4528 & 30.2384 \\ 1 & 1 & 120 & 0.47 & 10.45 & 3.43062 & 42.29316 \\ 1 & 1 & 140 & 0.54 & 8.63 & 4.57102 & 56.3521 \\ 1 & 1 & 160 & 0.62 & 8.89 & 5.87242 & 72.39605 \\ 1 & 1 & 180 & 0.7 & 9.12 & 7.33876 & 90.47328 \\ 1 & 2 & 200 & 0.77 & 9.18 & 8.96296 & \\ 1 & 2 & 220 & 1.55 & 14.11 & 12.18467 & \\ 1 & 2 & 240 & 2.32 & 16.24 & 17.03372 & \\ 1 & 2 & 260 & 3.09 & 16.95 & 23.5054 & \\ 1 & 2 & 280 & 3.87 & 17.34 & 31.59578 & \\ 1 & 2 & 300 & 4.65 & 17.7 & 41.31744 & \\ 1 & 2 & 320 & 5.42 & 17.92 & 52.65545 & \\ 1 & 2 & 340 & 6.2 & 17.92 & 65.62002 & \\ 1 & 2 & 360 & 6.97 & 19.46 & 80.21978 & \\ 1 & 3 & 380 & 7.75 & 18.34 & 96.59376 & \\ 1 & 3 & 400 & 15.5 & 28.1 & 128.90033 & \\ 1 & 3 & 420 & 23.24 & 28.38 & 177.49763 & \\ 1 & 3 & 440 & 30.99 & 28.48 & 242.29455 & \\ 1 & 3 & 460 & 38.73 & 31.28 & 323.23611 & \\ 1 & 3 & 480 & 46.48 & 34.47 & 420.4794 & \\ 1 & 3 & 500 & 54.22 & 37.72 & 533.91524 & \\ 1 & 3 & 520 & 61.96 & 40.87 & 663.54442 & \\ 1 & 3 & 540 & 69.71 & 43.95 & 809.53581 & \\ 1 & 4 & 560 & 77.46 & 47.13 & 973.18765 & \\ 1 & 4 & 580 & 154.92 & 81.32 & 1296.46931 & \\ 1 & 4 & 600 & 232.38 & 111.19 & 1781.78019 & \\ 1 & 4 & 620 & 309.84 & 138.67 & 2429.76509 & \\ 1 & 4 & 640 & 387.3 & 166.81 & 3239.6559 & \\ 1 & 4 & 660 & 464.75 & 195.4 & 4212.2223 & \\ 1 & 4 & 680 & 542.21 & 224.69 & 5346.25007 & \\ 1 & 4 & 700 & 619.67 & 254.12 & 6643.07202 & \\ & 4 & 720 & 697.13 & 285.18 & 8100.33826 & \end{array}$

$\mathrm{J}[\mathrm{t}] \quad \mathrm{T}\left[{ }^{\circ} \mathrm{C}\right] \mathrm{D}[1 / \mathrm{s}] \quad \mathrm{Tau}[\mathrm{Pa}] \mathrm{Eta}[\mathrm{Pas}] \mathrm{MP} \#$ Block M[mNm] G[Pa]

\begin{tabular}{|c|c|c|c|c|c|c|c|c|}
\hline 1.83421 & 10.1 & 0.1 & 1.098 & 10.98 & 1 & 1 & 0.2804 & 0.545194542 \\
\hline 3.77413 & 10.1 & 0.21 & 1.606 & 7.648 & 2 & 1 & 0.41015 & 0.264961848 \\
\hline 6.45844 & 10.1 & 0.31 & 1.877 & 6.055 & 3 & 1 & 0.4794 & 0.154836176 \\
\hline .12058 & 10.1 & 0.4 & 1.664 & 4.16 & 4 & 1 & 0.4249 & 0.082504283 \\
\hline 41561 & 10.1 & 0.5 & 1.642 & 3.284 & 5 & 1 & 0.4193 & 0.054301725 \\
\hline .66104 & 10.1 & 0.61 & 2.047 & 3.356 & 6 & 1 & 0.5227 & 0.048400261 \\
\hline 36419 & 10.1 & 0.7 & 1.689 & 2.413 & 7 & 1 & 0.43135 & 0.029972237 \\
\hline 41.583 & 10.1 & 0.8 & 1.741 & 2.176 & 8 & 1 & 0.4446 & 0.024048273 \\
\hline 68528 & 10 & 0.9 & 1.785 & 1.983 & 9 & 1 & 0.4559 & 0.019729582 \\
\hline 0 & 10 & 0.99 & 1.797 & 1.815 & 10 & 1 & 0.4588 & 0 \\
\hline 0 & 10 & 2 & 2.762 & 1.381 & 11 & 1 & 0.70525 & 0 \\
\hline 0 & 10 & 3 & 3.18 & 1.06 & 12 & 1 & 0.812 & 0 \\
\hline 0 & 10 & 3.99 & 3.32 & 0.832 & 13 & 1 & 0.84775 & 0 \\
\hline 0 & 10 & 5 & 3.395 & 0.679 & 14 & 1 & 0.867 & 0 \\
\hline 0 & 10 & 6 & 3.466 & 0.578 & 15 & 1 & 0.885 & 0 \\
\hline 0 & 10 & 7 & 3.508 & 0.501 & 16 & 1 & 0.8959 & 0 \\
\hline 0 & 10 & 8 & 3.509 & 0.439 & 17 & 1 & 0.896 & 0 \\
\hline 0 & 10 & 9 & 3.81 & 0.423 & 18 & 1 & 0.97285 & 0 \\
\hline 0 & 10 & 10.01 & 3.592 & 0.359 & 19 & 1 & 0.91715 & 0 \\
\hline 0 & 10 & 20.01 & 5.502 & 0.275 & 20 & 1 & 1.40505 & 0 \\
\hline 0 & 10 & 30 & 5.556 & 0.185 & 21 & 1 & 1.4188 & 0 \\
\hline 0 & 10 & 40.01 & 5.576 & 0.139 & 22 & 1 & 1.42385 & 0 \\
\hline 0 & 10 & 50 & 6.125 & 0.123 & 23 & 1 & 1.56405 & 0 \\
\hline 0 & 10 & 60.01 & 6.748 & 0.112 & 24 & 1 & 1.7233 & 0 \\
\hline 0 & 10 & 70 & 7.386 & 0.106 & 25 & 1 & 1.88615 & 0 \\
\hline 0 & 10 & 79.99 & 8.003 & 0.1 & 26 & 1 & 2.0436 & 0 \\
\hline 0 & 10 & 90 & 8.606 & 0.096 & 27 & 1 & 2.19755 & 0 \\
\hline 0 & 10 & 100 & 9.228 & 0.092 & 28 & 1 & 2.3565 & 0 \\
\hline 0 & 10 & 200 & 15.923 & 0.08 & 29 & 1 & 4.0661 & 0 \\
\hline 0 & 10 & 300 & 21.771 & 0.073 & 30 & 1 & 5.5595 & 0 \\
\hline 0 & 10 & 400 & 27.151 & 0.068 & 31 & 1 & 6.93325 & 0 \\
\hline 0 & 10 & 500 & 32.661 & 0.065 & 32 & 1 & 8.3403 & 0 \\
\hline 0 & 10 & 599.99 & 38.258 & 0.064 & 33 & 1 & 9.76975 & 0 \\
\hline 0 & 10 & 699.99 & 43.994 & 0.063 & 34 & 1 & 11.2345 & 0 \\
\hline 0 & 10 & 799.99 & 49.756 & 0.062 & 35 & 1 & 12.70575 & 0 \\
\hline 0 & 10 & 899.99 & 55.838 & 0.062 & 36 & 1 & 14.25895 & 0 \\
\hline
\end{tabular}




\begin{tabular}{|c|c|c|c|c|c|c|c|c|c|c|c|c|c|c|}
\hline & 4740 & 774.59 & 322.83 & 9720.89113 & 0 & 0 & 10 & 1000 & 63.211 & 0.063 & 37 & 1 & 16.14165 & 0 \\
\hline & 5750 & 774.59 & 321.44 & 10547.52977 & 0 & 0 & 10 & 1000 & 62.939 & 0.063 & 38 & 1 & 16.07215 & 0 \\
\hline & 5760 & 774.59 & 320.14 & 11359.8985 & 0 & 0 & 10 & 1000 & 62.683 & 0.063 & 39 & 1 & 16.00695 & \\
\hline & 5770 & 774.59 & 319.24 & 12169.4241 & 0 & 0 & 10 & 1000 & 62.507 & 0.063 & 40 & 1 & 15.96195 & \\
\hline & 5780 & 774.59 & 318.31 & 12983.00628 & 0 & 0 & 10 & 1000 & 62.325 & 0.062 & 41 & 1 & 15.9154 & \\
\hline & 5790 & 774.59 & 317.59 & 13794.15372 & 0 & 0 & 10 & 1000 & 62.184 & 0.062 & 42 & 1 & 15.8794 & \\
\hline & 5800 & 774.59 & 316.66 & 14602.87192 & 0 & 0 & 10 & 1000 & 62.001 & 0.062 & 43 & 1 & 15.83285 & \\
\hline & 6820 & 774.59 & 315.61 & 16244.63526 & 0 & 0 & 10 & 1000 & 61.797 & 0.062 & 44 & 1 & 15.78065 & \\
\hline & 6840 & 697.13 & 283.37 & 17705.27478 & 0 & 0 & 10 & 899.99 & 55.484 & 0.062 & 45 & 1 & 14.1686 & \\
\hline & 6860 & 619.67 & 252.39 & 19003.76178 & 0 & 0 & 10 & 799.99 & 49.418 & 0.062 & 46 & 1 & 12.6195 & \\
\hline & 6880 & 542.21 & 221.82 & 20140.71202 & 0 & 0 & 10 & 699.99 & 43.433 & 0.062 & 47 & 1 & 11.0911 & \\
\hline & 6900 & 464.75 & 191.9 & 21116.32655 & 0 & 0 & 10 & 599.99 & 37.574 & 0.063 & 48 & 1 & 9.59505 & 0 \\
\hline & $6 \quad 920$ & 387.3 & 162.26 & 21928.81702 & 0 & 0 & 10 & 500 & 31.77 & 0.064 & 49 & 1 & 8.11275 & 0 \\
\hline & 6940 & 309.84 & 132.73 & 22578.87302 & 0 & 0 & 10 & 400 & 25.989 & 0.065 & 50 & 1 & 6.6365 & 0 \\
\hline & 6960 & 232.38 & 103.31 & 23067.10715 & 0 & 0 & 10 & 300 & 20.227 & 0.067 & 51 & 1 & 5.16525 & 0 \\
\hline & 980 & 154.92 & 72.71 & 23392.54473 & 0 & 0 & 10 & 200 & 14.237 & 0.071 & 52 & 1 & 3.6356 & 0 \\
\hline & 61000 & 77.46 & 40.82 & 23555.95467 & 0 & 0 & 10 & 100 & 7.992 & 0.08 & 53 & 1 & 2.0408 & 0 \\
\hline & 71020 & 69.71 & 37.48 & 23703.20504 & 0 & 0 & 10 & 90 & 7.339 & 0.082 & 54 & 1 & 1.8742 & r \\
\hline & 71040 & 61.97 & 34.11 & 23833.10205 & 0 & 0 & 10 & 80 & 6.679 & 0.083 & 55 & 1 & 1.7056 & 0 \\
\hline & 71060 & 54.22 & 30.56 & 23946.66355 & 0 & 0 & 10 & 70 & 5.983 & 0.085 & 56 & 1 & 1.52775 & 0 \\
\hline & 71080 & 46.48 & 26.96 & 24044.21707 & 0 & 0 & 10 & 60.01 & 5.278 & 0.088 & 57 & 1 & 1.3478 & 0 \\
\hline & 71100 & 38.73 & 23.09 & 24125.38954 & 0 & 0 & 10 & 50 & 4.521 & 0.09 & 58 & 1 & 1.15455 & 0 \\
\hline & 71120 & 30.99 & 19.64 & 24190.40245 & 0 & 0 & 10 & 40.01 & 3.846 & 0.096 & 59 & 1 & 0.98215 & 0 \\
\hline & 71140 & 23.24 & 15.69 & 24239.19374 & 0 & 0 & 10 & 30 & 3.072 & 0.102 & 60 & 1 & 0.7844 & 0 \\
\hline & 71160 & 15.5 & 11.38 & 24271.75713 & 0 & 0 & 10 & 20.01 & 2.229 & 0.111 & 61 & 1 & 0.5691 & 0 \\
\hline & 71180 & 7.75 & 6.27 & 24288.10598 & 0 & 0 & 10 & 10.01 & 1.228 & 0.123 & 62 & 1 & 0.3135 & 0 \\
\hline & 81200 & 6.97 & 6.65 & 24302.81727 & 0 & 0 & 10 & 9 & 1.302 & 0.145 & 63 & 1 & 0.33255 & 0 \\
\hline & 81220 & 6.2 & 6.42 & 24315.79676 & 0 & 0 & 10 & 8 & 1.258 & 0.157 & 64 & 1 & 0.3212 & 0 \\
\hline & 81240 & 5.42 & 5.79 & 24327.15833 & 0 & 0 & 10 & 7 & 1.133 & 0.162 & 65 & 1 & 0.28935 & 0 \\
\hline & 81260 & 4.64 & 6.07 & 24336.90355 & 0 & 0 & 10 & 5.99 & 1.188 & 0.198 & 66 & 1 & 0.30325 & 0 \\
\hline & 81280 & 3.87 & 6.99 & 24345.02064 & 0 & 0 & 10 & 5 & 1.368 & 0.274 & 67 & 1 & 0.3494 & 0 \\
\hline & 81300 & 3.09 & 5.97 & 24351.5096 & 0 & 0 & 10 & 3.99 & 1.169 & 0.293 & 68 & 1 & 0.2985 & 0 \\
\hline & 81320 & 2.3 & 5.47 & 24356.3453 & 0 & 0 & 10 & 2.97 & 1.072 & 0.361 & 69 & 1 & 0.2737 & 0 \\
\hline & 81340 & 1.54 & 3.48 & 24359.62748 & 0 & 0 & 10 & 1.99 & 0.682 & 0.343 & 70 & 1 & 0.1742 & 0 \\
\hline & 81360 & 0.77 & 4.97 & 24361.25247 & 0 & 0 & 10 & 0.99 & 0.972 & 0.982 & 71 & 1 & 0.24825 & 0 \\
\hline & 91380 & 0.7 & 3.02 & 24362.72587 & 18.16437 & 30.73492 & 10 & 0.9 & 0.591 & 0.657 & 72 & 1 & 0.1509 & 0.032536223 \\
\hline & 91400 & 0.62 & 3.38 & 24364.03042 & 34.247 & 51.81081 & 10 & 0.8 & 0.661 & 0.826 & 73 & 1 & 0.1689 & 0.019300961 \\
\hline & 91420 & 0.55 & 3.46 & 24365.17317 & 48.33504 & 71.29051 & 10 & 0.71 & 0.678 & 0.955 & 74 & 1 & 0.1732 & 0.014027091 \\
\hline & 91440 & 0.47 & 3.39 & 24366.15335 & 60.4188 & 91.12927 & 10 & 0.61 & 0.663 & 1.087 & 75 & 1 & 0.1694 & 0.010973406 \\
\hline & 91460 & 0.39 & 3.44 & 24366.97095 & 70.49828 & 104.75212 & 10 & 0.5 & 0.673 & 1.346 & 76 & 1 & 0.17185 & 0.009546332 \\
\hline & 91480 & 0.31 & 3.39 & 24367.62676 & 78.58317 & 118.34797 & 10 & 0.4 & 0.664 & 1.66 & 77 & 1 & 0.16965 & 0.008449646 \\
\hline & 91500 & 0.24 & 3.29 & 24368.11999 & 84.66378 & 131.46529 & 10 & 0.31 & 0.644 & 2.077 & 78 & 1 & 0.1645 & 0.007606559 \\
\hline & 91520 & 0.16 & 3.12 & 24368.45064 & 88.74011 & 145.23726 & 10 & 0.21 & 0.611 & 2.91 & 79 & 1 & 0.15605 & 0.006885274 \\
\hline & 91540 & 0.08 & 2.12 & 24368.6195 & 90.82185 & 218.8473 & 10 & 0.1 & 0.415 & 4.15 & 80 & 1 & 0.1061 & 0.004569385 \\
\hline
\end{tabular}

2004.02.10 META-20 wt\%-U CC-45 0.1-1000 s-1 run 7.txt Mode Step t[s] n[rpm] M[\%.] Phi[rad] Gamma

\begin{tabular}{|c|c|c|c|c|c|c|c|c|c|c|c|c|c|}
\hline 1 & 20 & 0.08 & 3.32 & 0.16415 & 2.02364 & 3.11808 & 10 & 0.1 & 0.649 & 6.49 & 1 & 0.16575 & 0.320709217 \\
\hline 1 & 40 & 0.16 & 6.01 & 0.49244 & 6.07093 & 5.15359 & 10 & 0.21 & 1.178 & 5.61 & 2 & 0.3007 & 0.194039463 \\
\hline 1 & 60 & 0.24 & 6.37 & 0.98332 & 12.12249 & 9.72912 & 10 & 0.31 & 1.246 & 4.019 & 3 & 0.3183 & 0.102784164 \\
\hline
\end{tabular}

$\mathrm{J}[\mathrm{t}] \quad \mathrm{T}\left[{ }^{\circ} \mathrm{C}\right] \mathrm{D}[1 / \mathrm{s}] \quad \mathrm{Tau}[\mathrm{Pa}]$ Eta[Pas $]$ MP\# Block M[mNm] G[Pa] 


\begin{tabular}{|c|c|c|c|c|c|c|c|c|c|c|c|c|c|c|}
\hline & 80 & 0.31 & 6.46 & 1.63677 & 20.17833 & 15.96386 & 10 & 0.4 & 1.264 & 3.16 & 4 & 2 & 0.3228 & 0.062641457 \\
\hline & 1100 & 0.39 & 6.83 & 2.4528 & 30.23845 & 22.61662 & 10 & 0.5 & 1.337 & 2.674 & 5 & 2 & 0.34135 & 0.044215229 \\
\hline & $1 \quad 120$ & 0.47 & 6.86 & 3.43062 & 42.29316 & 31.49153 & 10 & 0.61 & 1.343 & 2.202 & 6 & 2 & 0.343 & 0.031754544 \\
\hline & $1 \quad 140$ & 0.54 & 7.07 & 4.57102 & 56.35215 & 40.71684 & 10 & 0.7 & 1.384 & 1.977 & 7 & 2 & 0.3533 & 0.024559844 \\
\hline & 1160 & 0.62 & 7.31 & 5.87399 & 72.41542 & 50.56939 & 10 & 0.8 & 1.432 & 1.79 & 8 & 2 & 0.36565 & 0.019774794 \\
\hline & 1180 & 0.7 & 7.45 & 7.33876 & 90.47328 & 62.05296 & 10 & 0.9 & 1.458 & 1.62 & 9 & 2 & 0.37235 & 0.016115255 \\
\hline & 2200 & 0.77 & 7.36 & 8.96139 & 0 & 0 & 10 & 0.99 & 1.442 & 1.457 & 10 & 2 & 0.36815 & 0 \\
\hline & 220 & 1.55 & 11.24 & 12.18467 & 0 & 0 & 10 & 2 & 2.201 & 1.101 & 11 & 2 & 0.5621 & 0 \\
\hline & 240 & 2.32 & 13.51 & 17.03607 & 0 & 0 & 10 & 3 & 2.645 & 0.882 & 12 & 2 & 0.67545 & 0 \\
\hline & 2260 & 3.09 & 14.01 & 23.50383 & 0 & 0 & 10 & 3.99 & 2.744 & 0.688 & 13 & 2 & 0.70065 & 0 \\
\hline & 2280 & 3.87 & 14.56 & 31.59814 & 0 & 0 & 10 & 5 & 2.85 & 0.57 & 14 & 2 & 0.72785 & 0 \\
\hline & 2300 & 4.65 & 15.11 & 41.31823 & 0 & 0 & 10 & 6 & 2.958 & 0.493 & 15 & 2 & 0.7554 & 0 \\
\hline & 2320 & 5.42 & 15.69 & 52.65938 & 0 & 0 & 10 & 7 & 3.073 & 0.439 & 16 & 2 & 0.7847 & 0 \\
\hline & 2340 & 6.2 & 16.15 & 65.6153 & 0 & 0 & 10 & 8 & 3.163 & 0.395 & 17 & 2 & 0.8076 & 0 \\
\hline & 2360 & 6.97 & 16.35 & 80.20565 & 0 & 0 & 10 & 9 & 3.202 & 0.356 & 18 & 2 & 0.8177 & 0 \\
\hline & 380 & 7.75 & 16.5 & 96.5757 & 0 & 0 & 10 & 10.01 & 3.231 & 0.323 & 19 & 2 & 0.82505 & 0 \\
\hline & 400 & 15.5 & 25.81 & 128.90583 & 0 & 0 & 10 & 20.01 & 5.054 & 0.253 & 20 & 2 & 1.2905 & 0 \\
\hline & 420 & 23.24 & 28.95 & 177.44108 & 0 & 0 & 10 & 30 & 5.668 & 0.189 & 21 & 2 & 1.4473 & 0 \\
\hline & 440 & 30.99 & 28.65 & 242.30083 & 0 & 0 & 10 & 40.01 & 5.609 & 0.14 & 22 & 2 & 1.4324 & 0 \\
\hline & 460 & 38.73 & 29.54 & 323.24554 & 0 & 0 & 10 & 50 & 5.783 & 0.116 & 23 & 2 & 1.47685 & 0 \\
\hline & 480 & 46.48 & 32.65 & 420.4684 & 0 & 0 & 10 & 60.01 & 6.393 & 0.107 & 24 & 2 & 1.6326 & 0 \\
\hline & 500 & 54.22 & 35.72 & 533.8477 & 0 & 0 & 10 & 70 & 6.994 & 0.1 & 25 & 2 & 1.786 & 0 \\
\hline & 520 & 61.96 & 38.71 & 663.53814 & 0 & 0 & 10 & 79.99 & 7.58 & 0.095 & 26 & 2 & 1.9356 & 0 \\
\hline & 3540 & 69.71 & 41.67 & 809.45255 & 0 & 0 & 10 & 90 & 8.158 & 0.091 & 27 & 2 & 2.0833 & 0 \\
\hline & $4 \quad 560$ & 77.46 & 44.65 & 972.98659 & 0 & 0 & 10 & 100 & 8.742 & 0.087 & 28 & 2 & 2.2323 & 0 \\
\hline & 580 & 154.92 & 76.56 & 1296.26982 & 0 & 0 & 10 & 200 & 14.99 & 0.075 & 29 & 2 & 3.82785 & 0 \\
\hline & 600 & 232.38 & 104.86 & 1782.1886 & 0 & 0 & 10 & 300 & 20.531 & 0.068 & 30 & 2 & 5.24275 & 0 \\
\hline & $4 \quad 620$ & 309.84 & 131.86 & 2430.01171 & 0 & 0 & 10 & 400 & 25.818 & 0.065 & 31 & 2 & 6.5929 & 0 \\
\hline & 640 & 387.3 & 159.37 & 3239.13361 & 0 & 0 & 10 & 500 & 31.205 & 0.062 & 32 & 2 & 7.96855 & 0 \\
\hline & $4 \quad 660$ & 464.75 & 187.29 & 4212.22701 & 0 & 0 & 10 & 599.99 & 36.672 & 0.061 & 33 & 2 & 9.3647 & 0 \\
\hline & $4 \quad 680$ & 542.21 & 215.57 & 5345.68458 & 0 & 0 & 10 & 699.99 & 42.208 & 0.06 & 34 & 2 & 10.77825 & 0 \\
\hline & 4700 & 619.67 & 243.07 & 6642.83169 & 0 & 0 & 10 & 799.99 & 47.593 & 0.059 & 35 & 2 & 12.1535 & 0 \\
\hline & 4720 & 697.13 & 271.26 & 8100.82914 & 0 & 0 & 10 & 899.99 & 53.113 & 0.059 & 36 & 2 & 13.5631 & 0 \\
\hline & 4740 & 774.59 & 301.68 & 9721.33881 & 0 & 0 & 10 & 1000 & 59.068 & 0.059 & 37 & 2 & 15.08385 & 0 \\
\hline & 5750 & 774.59 & 309.17 & 10548.79269 & 0 & 0 & 10 & 1000 & 60.535 & 0.061 & 38 & 2 & 15.45835 & 0 \\
\hline & 5760 & 774.59 & 309.21 & 11360.3454 & 0 & 0 & 10 & 1000 & 60.544 & 0.061 & 39 & 2 & 15.4606 & 0 \\
\hline & 770 & 774.59 & 308.47 & 12173.11783 & 0 & 0 & 10 & 1000 & 60.398 & 0.06 & 40 & 2 & 15.4235 & 0 \\
\hline & 780 & 774.59 & 307.74 & 12984.67368 & 0 & 0 & 10 & 1000 & 60.256 & 0.06 & 41 & 2 & 15.38715 & 0 \\
\hline & 790 & 774.59 & 307.06 & 13795.01059 & 0 & 0 & 10 & 1000 & 60.121 & 0.06 & 42 & 2 & 15.35275 & 0 \\
\hline 1 & 800 & 774.59 & 306.41 & 14606.16039 & 0 & 0 & 10 & 1000 & 59.994 & 0.06 & 43 & 2 & 15.32035 & 0 \\
\hline 1 & 820 & 774.59 & 305.63 & 16247.11397 & 0 & 0 & 10 & 1000 & 59.843 & 0.06 & 44 & 2 & 15.28155 & 0 \\
\hline 1 & 840 & 697.13 & 274.45 & 17708.48235 & 0 & 0 & 10 & 899.99 & 53.737 & 0.06 & 45 & 2 & 13.72235 & 0 \\
\hline 1 & 860 & 619.67 & 244.34 & 19007.61887 & 0 & 0 & 10 & 799.99 & 47.842 & 0.06 & 46 & 2 & 12.21715 & 0 \\
\hline 1 & 880 & 542.21 & 214.71 & 20145.09611 & 0 & 0 & 10 & 699.99 & 42.04 & 0.06 & 47 & 2 & 10.7355 & 0 \\
\hline 1 & 900 & 464.75 & 185.59 & 21119.5726 & 0 & 0 & 10 & 599.99 & 36.339 & 0.061 & 48 & 2 & 9.27955 & 0 \\
\hline 1 & $6 \quad 920$ & 387.3 & 156.79 & 21931.61697 & 0 & 0 & 10 & 500 & 30.699 & 0.061 & 49 & 2 & 7.8395 & 0 \\
\hline 1 & 940 & 309.84 & 128.08 & 22581.67296 & 0 & 0 & 10 & 400 & 25.077 & 0.063 & 50 & 2 & 6.40385 & 0 \\
\hline 1 & 6960 & 232.38 & 99.41 & 23069.94872 & 0 & 0 & 10 & 300 & 19.465 & 0.065 & 51 & 2 & 4.9706 & 0 \\
\hline & 6980 & 154.92 & 69.65 & 23395.58893 & 0 & 0 & 10 & 200 & 13.637 & 0.068 & 52 & 2 & 3.4823 & 0 \\
\hline & 61000 & 77.46 & 38.84 & 23558.99809 & 0 & 0 & 10 & 100 & 7.606 & 0.076 & 53 & 2 & 1.9422 & 0 \\
\hline
\end{tabular}




\begin{tabular}{|c|c|c|c|c|c|c|c|c|c|c|c|c|c|}
\hline 71020 & 69.71 & 35.71 & 23706.20527 & 0 & 0 & 10 & 90 & 6.992 & 0.078 & 54 & 2 & 1.7854 & 4 \\
\hline 71040 & 61.97 & 32.46 & 23836.03708 & 0 & 0 & 10 & 80 & 6.355 & 0.079 & 55 & 2 & 1.6229 & ) \\
\hline 71060 & 54.22 & 29.01 & 23949.63236 & 0 & 0 & 10 & 70 & 5.679 & 0.081 & 56 & 2 & 1.4503 & \\
\hline 71080 & 46.48 & 25.53 & 24047.13954 & 0 & 0 & 10 & 60.01 & 4.999 & 0.083 & 57 & 2 & 1.27645 & \\
\hline 71100 & 38.73 & 21.82 & 24128.33636 & 0 & 0 & 10 & 50 & 4.272 & 0.085 & 58 & 2 & 1.09085 & \\
\hline 71120 & 30.99 & 18.56 & 24193.43487 & 0 & 0 & 10 & 40.01 & 3.635 & 0.091 & 59 & 2 & 0.9282 & \\
\hline 71140 & 23.24 & 14.94 & 24242.19789 & 0 & 0 & 10 & 30 & 2.924 & 0.097 & 60 & 2 & 0.74675 & \\
\hline 71160 & 15.5 & 10.51 & 24274.78563 & 0 & 0 & 10 & 20.01 & 2.057 & 0.103 & 61 & 2 & 0.5254 & \\
\hline 71180 & 7.75 & 5.96 & 24291.12269 & 0 & 0 & 10 & 10.01 & 1.167 & 0.117 & 62 & 2 & 0.29805 & \\
\hline 81200 & 6.97 & 6.19 & 24305.82378 & 0 & 0 & 10 & 9 & 1.213 & 0.135 & 63 & 2 & 0.30965 & \\
\hline 81220 & 6.2 & 6.49 & 24318.81976 & 0 & 0 & 10 & 8 & 1.27 & 0.159 & 64 & 2 & 0.3243 & \\
\hline 81240 & 5.42 & 5.82 & 24330.18447 & 0 & 0 & 10 & 7 & 1.139 & 0.163 & 65 & 2 & 0.2908 & 3 \\
\hline 81260 & 4.64 & 5.26 & 24339.91398 & 0 & 0 & 10 & 5.99 & 1.03 & 0.172 & 66 & 2 & 0.26295 & 5 \\
\hline 81280 & 3.87 & 4.82 & 24348.03107 & 0 & 0 & 10 & 5 & 0.944 & 0.189 & 67 & 2 & 0.24105 & 5 \\
\hline 81300 & 3.09 & 4.56 & 24354.52789 & 0 & 0 & 10 & 3.99 & 0.892 & 0.224 & 68 & 2 & 0.2278 & 0 \\
\hline 81320 & 2.32 & 4.21 & 24359.39579 & 0 & 0 & 10 & 3 & 0.824 & 0.275 & 69 & 2 & 0.2105 & 0 \\
\hline 81340 & 1.54 & 3.48 & 24362.64184 & 0 & 0 & 10 & 1.99 & 0.681 & 0.342 & 70 & 2 & 0.17385 & 0 \\
\hline 81360 & 0.77 & 1.97 & 24364.26525 & 0 & 0 & 10 & 0.99 & 0.385 & 0.389 & 71 & 2 & 0.0984 & 0 \\
\hline 91380 & 0.7 & 2.8 & 24365.73788 & 18.15469 & 33.1895 & 10 & 0.9 & 0.547 & 0.608 & 72 & 2 & 0.13975 & 0.030129955 \\
\hline 91400 & 0.62 & 3.1 & 24367.04321 & 34.247 & 56.42001 & 10 & 0.8 & 0.607 & 0.759 & 73 & 2 & 0.1549 & 0.01772418 \\
\hline 91420 & 0.55 & 3.23 & 24368.18596 & 48.33504 & 76.35855 & 10 & 0.71 & 0.633 & 0.892 & 74 & 2 & 0.16155 & 0.013096089 \\
\hline 91440 & 0.47 & 3.36 & 24369.16614 & 60.4188 & 91.82174 & 10 & 0.61 & 0.658 & 1.079 & 75 & 2 & 0.16815 & 0.01089065 \\
\hline 91460 & 0.39 & 3.4 & 24369.98374 & 70.49828 & 105.69441 & 10 & 0.5 & 0.667 & 1.334 & 76 & 2 & 0.1702 & 0.009461224 \\
\hline 91480 & 0.31 & 3.29 & 24370.63955 & 78.58317 & 121.83418 & 10 & 0.4 & 0.645 & 1.613 & 77 & 2 & 0.1647 & 0.008207864 \\
\hline 91500 & 0.24 & 3.06 & 24371.13278 & 84.66378 & 141.34163 & 10 & 0.31 & 0.599 & 1.932 & 78 & 2 & 0.153 & 0.007075044 \\
\hline 91520 & 0.16 & 3.02 & 24371.46343 & 88.74011 & 149.89858 & 10 & 0.21 & 0.592 & 2.819 & 79 & 2 & 0.1512 & 0.006671166 \\
\hline 91540 & 0.08 & 2.2 & 24371.63229 & 90.82185 & 210.23528 & 10 & 0.1 & 0.432 & 4.32 & 80 & 2 & 0.1102 & 0.004756565 \\
\hline
\end{tabular}

2004.02.10 META-20 wt\%-U CC-45 0.1-1000 s-1 run 8.txt Mode Step $\mathrm{t}[\mathrm{s}] \mathrm{n}[\mathrm{rpm}] \mathrm{M}[\%$.]

$\begin{array}{rrrrr}1 & 1 & 20 & 0.08 & 3.39 \\ 1 & 1 & 40 & 0.16 & 5.88 \\ 1 & 1 & 60 & 0.24 & 6.23 \\ 1 & 1 & 80 & 0.31 & 6.21 \\ 1 & 1 & 100 & 0.39 & 6.25 \\ 1 & 1 & 120 & 0.47 & 6.49 \\ 1 & 1 & 140 & 0.54 & 6.67 \\ 1 & 1 & 160 & 0.62 & 6.76 \\ 1 & 1 & 180 & 0.7 & 7.33 \\ 1 & 2 & 200 & 0.77 & 7.16 \\ 1 & 2 & 220 & 1.55 & 10.45 \\ 1 & 2 & 240 & 2.32 & 12.38 \\ 1 & 2 & 260 & 3.09 & 13.24 \\ 1 & 2 & 280 & 3.87 & 13.98 \\ 1 & 2 & 300 & 4.65 & 14.67 \\ 1 & 2 & 320 & 5.42 & 15.26 \\ 1 & 2 & 340 & 6.2 & 15.71 \\ 1 & 2 & 360 & 6.97 & 16.2 \\ 1 & 3 & 380 & 7.75 & 16.11 \\ 1 & 3 & 400 & 15.5 & 25.46\end{array}$

Phi[rad] Gamma

$0.16415 \quad 2.02364$

$0.49244 \quad 6.07093$

$0.98332 \quad 12.12249$

$1.63677 \quad 20.17833$

$2.4528 \quad 30.23845$

$3.43062 \quad 42.29316$

4.5710256 .35215

$5.87399 \quad 72.41542$

$7.33798 \quad 90.4636$

8.96061

12.18545

17.03057

23.50147

31.59657

41.31744

52.66566

65.61373

80.20329

96.56942

128.92861
$\mathrm{J}[\mathrm{t}] \quad \mathrm{T}\left[{ }^{\circ} \mathrm{C}\right] \mathrm{D}[1 / \mathrm{s}] \quad \mathrm{Tau}[\mathrm{Pa}]$ Eta[Pas] MP\# Block M[mNm] G[Pa]

$\begin{array}{rrrrrrrrr}3.05224 & 10 & 0.1 & 0.663 & 6.63 & 1 & 3 & 0.1693 & 0.327627444 \\ 5.27448 & 10 & 0.21 & 1.151 & 5.481 & 2 & 3 & 0.294 & 0.189592039 \\ 9.93646 & 10 & 0.31 & 1.22 & 3.935 & 3 & 3 & 0.31165 & 0.10063939 \\ 16.60767 & 10 & 0.4 & 1.215 & 3.038 & 4 & 3 & 0.3103 & 0.06021311 \\ 24.7046 & 10 & 0.5 & 1.224 & 2.448 & 5 & 3 & 0.3125 & 0.040478265 \\ 33.27547 & 10 & 0.61 & 1.271 & 2.084 & 6 & 3 & 0.32455 & 0.030052141 \\ 43.11561 & 10 & 0.7 & 1.307 & 1.867 & 7 & 3 & 0.3337 & 0.023193436 \\ 54.73573 & 10 & 0.8 & 1.323 & 1.654 & 8 & 3 & 0.3379 & 0.01826959 \\ 63.04079 & 10 & 0.9 & 1.435 & 1.594 & 9 & 3 & 0.3664 & 0.015862734 \\ 0 & 10 & 0.99 & 1.402 & 1.416 & 10 & 3 & 0.3581 & 0 \\ 0 & 10 & 2 & 2.047 & 1.024 & 11 & 3 & 0.52265 & 0 \\ 0 & 10 & 3 & 2.423 & 0.808 & 12 & 3 & 0.61885 & 0 \\ 0 & 10 & 3.99 & 2.592 & 0.65 & 13 & 3 & 0.662 & 0 \\ 0 & 10 & 5 & 2.737 & 0.547 & 14 & 3 & 0.69895 & 0 \\ 0 & 10 & 6 & 2.872 & 0.479 & 15 & 3 & 0.7333 & 0 \\ 0 & 10 & 7 & 2.988 & 0.427 & 16 & 3 & 0.763 & 0 \\ 0 & 10 & 8 & 3.075 & 0.384 & 17 & 3 & 0.78525 & 0 \\ 0 & 10 & 9 & 3.172 & 0.352 & 18 & 3 & 0.8099 & 0 \\ 0 & 10 & 10.01 & 3.155 & 0.315 & 19 & 3 & 0.80565 & 0 \\ 0 & 10 & 20.01 & 4.985 & 0.249 & 20 & 3 & 1.27305 & 0\end{array}$




\begin{tabular}{|c|c|c|c|c|c|c|c|c|c|c|c|c|c|c|}
\hline & 3420 & 23.24 & 29.23 & 177.45129 & 0 & 0 & 10 & 30 & 5.723 & 0.191 & 21 & 3 & 1.4615 & 0 \\
\hline 1 & 3440 & 30.99 & 28.46 & 242.23329 & 0 & 0 & 10 & 40.01 & 5.573 & 0.139 & 22 & 3 & 1.4232 & 0 \\
\hline & 3460 & 38.73 & 28.13 & 323.25575 & 0 & 0 & 10 & 50 & 5.508 & 0.11 & 23 & 3 & 1.4065 & 0 \\
\hline & 3480 & 46.48 & 31.11 & 420.44641 & 0 & 0 & 10 & 60.01 & 6.091 & 0.101 & 24 & 3 & 1.5554 & 0 \\
\hline & 3500 & 54.22 & 34.08 & 533.93566 & 0 & 0 & 10 & 70 & 6.672 & 0.095 & 25 & 3 & 1.70385 & 0 \\
\hline & 3520 & 61.96 & 37.18 & 663.62532 & 0 & 0 & 10 & 79.99 & 7.28 & 0.091 & 26 & 3 & 1.85915 & 0 \\
\hline & 3540 & 69.71 & 40.01 & 809.35752 & 0 & 0 & 10 & 90 & 7.833 & 0.087 & 27 & 3 & 2.00035 & 0 \\
\hline & $4 \quad 560$ & 77.46 & 43.09 & 972.96852 & 0 & 0 & 10 & 100 & 8.437 & 0.084 & 28 & 3 & 2.1545 & 0 \\
\hline & $4 \quad 580$ & 154.92 & 73.7 & 1296.57613 & 0 & 0 & 10 & 200 & 14.431 & 0.072 & 29 & 3 & 3.6852 & 0 \\
\hline & 4600 & 232.38 & 100.7 & 1781.80611 & 0 & 0 & 10 & 300 & 19.716 & 0.066 & 30 & 3 & 5.0348 & 0 \\
\hline & $4 \quad 620$ & 309.84 & 126.82 & 2429.1839 & 0 & 0 & 10 & 400 & 24.832 & 0.062 & 31 & 3 & 6.34115 & 0 \\
\hline & 640 & 387.3 & 153.62 & 3239.31896 & 0 & 0 & 10 & 500 & 30.078 & 0.06 & 32 & 3 & 7.6809 & 0 \\
\hline & 660 & 464.75 & 180.8 & 4211.88536 & 0 & 0 & 10 & 599.99 & 35.4 & 0.059 & 33 & 3 & 9.0399 & 0 \\
\hline & 680 & 542.21 & 208.48 & 5345.3445 & 0 & 0 & 10 & 699.99 & 40.821 & 0.058 & 34 & 3 & 10.42415 & 0 \\
\hline & 700 & 619.67 & 235.32 & 6642.81441 & 0 & 0 & 10 & 799.99 & 46.076 & 0.058 & 35 & 3 & 11.76615 & 0 \\
\hline & 720 & 697.13 & 262.38 & 8100.48827 & 0 & 0 & 10 & 899.99 & 51.374 & 0.057 & 36 & 3 & 13.1191 & 0 \\
\hline & 740 & 774.59 & 290.77 & 9721.03957 & 0 & 0 & 10 & 1000 & 56.932 & 0.057 & 37 & 3 & 14.5384 & 0 \\
\hline & 750 & 774.59 & 290.38 & 10549.71003 & 0 & 0 & 10 & 1000 & 56.856 & 0.057 & 38 & 3 & 14.51895 & 0 \\
\hline & 760 & 774.59 & 290.03 & 11360.45221 & 0 & 0 & 10 & 1000 & 56.788 & 0.057 & 39 & 3 & 14.50145 & 0 \\
\hline & 770 & 774.59 & 289.72 & 12170.79069 & 0 & 0 & 10 & 1000 & 56.726 & 0.057 & 40 & 3 & 14.4858 & 0 \\
\hline & 780 & 774.59 & 289.34 & 12981.93813 & 0 & 0 & 10 & 1000 & 56.653 & 0.057 & 41 & 3 & 14.467 & 0 \\
\hline & 790 & 774.59 & 289.06 & 13793.08793 & 0 & 0 & 10 & 1000 & 56.597 & 0.057 & 42 & 3 & 14.45275 & 0 \\
\hline & 800 & 774.59 & 288.68 & 14605.04905 & 0 & 0 & 10 & 1000 & 56.523 & 0.057 & 43 & 3 & 14.4339 & 0 \\
\hline & 820 & 774.59 & 288.3 & 16246.41104 & 0 & 0 & 10 & 1000 & 56.449 & 0.056 & 44 & 3 & 14.41485 & 0 \\
\hline & 840 & 697.13 & 259.51 & 17708.10064 & 0 & 0 & 10 & 899.99 & 50.812 & 0.056 & 45 & 3 & 12.97555 & 0 \\
\hline & 860 & 619.67 & 231.21 & 19007.60238 & 0 & 0 & 10 & 799.99 & 45.272 & 0.057 & 46 & 3 & 11.56065 & 0 \\
\hline & 880 & 542.21 & 203.19 & 20143.37609 & 0 & 0 & 10 & 699.99 & 39.784 & 0.057 & 47 & 3 & 10.15925 & 0 \\
\hline & 900 & 464.75 & 175.55 & 21118.78484 & 0 & 0 & 10 & 599.99 & 34.372 & 0.057 & 48 & 3 & 8.77725 & 0 \\
\hline & 920 & 387.3 & 148.16 & 21931.03263 & 0 & 0 & 10 & 500 & 29.01 & 0.058 & 49 & 3 & 7.40795 & 0 \\
\hline & 940 & 309.84 & 120.79 & 22581.2512 & 0 & 0 & 10 & 400 & 23.651 & 0.059 & 50 & 3 & 6.0396 & 0 \\
\hline & 960 & 232.38 & 93.65 & 23068.87743 & 0 & 0 & 10 & 300 & 18.337 & 0.061 & 51 & 3 & 4.6825 & 0 \\
\hline & 6980 & 154.92 & 65.51 & 23394.55692 & 0 & 0 & 10 & 200 & 12.826 & 0.064 & 52 & 3 & 3.2754 & 0 \\
\hline & 61000 & 77.46 & 36.54 & 23558.04854 & 0 & 0 & 10 & 100 & 7.155 & 0.072 & 53 & 3 & 1.8272 & 0 \\
\hline & 71020 & 69.71 & 33.77 & 23705.22509 & 0 & 0 & 10 & 90 & 6.611 & 0.073 & 54 & 3 & 1.6883 & 0 \\
\hline & 71040 & 61.97 & 30.93 & 23835.12602 & 0 & 0 & 10 & 80 & 6.056 & 0.076 & 55 & 3 & 1.54635 & 0 \\
\hline & 71060 & 54.22 & 27.64 & 23948.74879 & 0 & 0 & 10 & 70 & 5.413 & 0.077 & 56 & 3 & 1.3822 & 0 \\
\hline & 71080 & 46.48 & 24.29 & 24046.20335 & 0 & 0 & 10 & 60.01 & 4.756 & 0.079 & 57 & 3 & 1.21455 & 0 \\
\hline & 71100 & 38.73 & 21.52 & 24127.441 & 0 & 0 & 10 & 50 & 4.214 & 0.084 & 58 & 3 & 1.0762 & 0 \\
\hline & 71120 & 30.99 & 17.88 & 24192.48297 & 0 & 0 & 10 & 40.01 & 3.501 & 0.088 & 59 & 3 & 0.894 & 0 \\
\hline & 71140 & 23.24 & 14.22 & 24241.2342 & 0 & 0 & 10 & 30 & 2.783 & 0.093 & 60 & 3 & 0.71075 & 0 \\
\hline & 71160 & 15.5 & 10.06 & 24273.82116 & 0 & 0 & 10 & 20.01 & 1.97 & 0.098 & 61 & 3 & 0.5031 & 0 \\
\hline & 71180 & 7.75 & 5.83 & 24290.17001 & 0 & 0 & 10 & 10.01 & 1.141 & 0.114 & 62 & 3 & 0.2913 & 0 \\
\hline & 81200 & 6.97 & 6.67 & 24304.88444 & 0 & 0 & 10 & 9 & 1.306 & 0.145 & 63 & 3 & 0.33345 & 0 \\
\hline 1 & 81220 & 6.2 & 5.67 & 24317.86707 & 0 & 0 & 10 & 8 & 1.109 & 0.139 & 64 & 3 & 0.2833 & 0 \\
\hline 1 & 81240 & 5.42 & 7.34 & 24329.22079 & 0 & 0 & 10 & 7 & 1.437 & 0.205 & 65 & 3 & 0.367 & 0 \\
\hline 1 & 81260 & 4.64 & 5.56 & 24338.96758 & 0 & 0 & 10 & 5.99 & 1.089 & 0.182 & 66 & 3 & 0.278 & 0 \\
\hline 1 & 81280 & 3.87 & 4.6 & 24347.08388 & 0 & 0 & 10 & 5 & 0.901 & 0.18 & 67 & 3 & 0.2301 & 0 \\
\hline 1 & 81300 & 3.09 & 4.14 & 24353.57363 & 0 & 0 & 10 & 3.99 & 0.811 & 0.203 & 68 & 3 & 0.20705 & 0 \\
\hline 1 & 81320 & 2.32 & 5.35 & 24358.44545 & 0 & 0 & 10 & 3 & 1.048 & 0.349 & 69 & 3 & 0.2676 & 0 \\
\hline & 81340 & 1.54 & 2.67 & 24361.6915 & 0 & 0 & 10 & 1.99 & 0.523 & 0.263 & 70 & 3 & 0.13345 & 0 \\
\hline
\end{tabular}




$\begin{array}{rrrrrrrrrrrrrrr}8 & 1360 & 0.77 & 2.52 & 24363.31571 & 0 & 0 & 10 & 0.99 & 0.493 & 0.498 & 71 & 3 & 0.1258 & 0 \\ 9 & 1380 & 0.7 & 2.58 & 24364.78833 & 18.15469 & 35.87876 & 10 & 0.9 & 0.506 & 0.562 & 72 & 3 & 0.1291 & 0.027871586 \\ 9 & 1400 & 0.62 & 2.93 & 24366.09445 & 34.25668 & 59.78468 & 10 & 0.8 & 0.573 & 0.716 & 73 & 3 & 0.14625 & 0.016726665 \\ 9 & 1420 & 0.55 & 3.12 & 24367.2372 & 48.34472 & 79.1238 & 10 & 0.71 & 0.611 & 0.861 & 74 & 3 & 0.1559 & 0.012638402 \\ 9 & 1440 & 0.47 & 3.11 & 24368.21659 & 60.4188 & 99.04705 & 10 & 0.61 & 0.61 & 1 & 75 & 3 & 0.1557 & 0.010096195 \\ 9 & 1460 & 0.39 & 3.18 & 24369.03419 & 70.49828 & 113.3411 & 10 & 0.5 & 0.622 & 1.244 & 76 & 3 & 0.15895 & 0.00882291 \\ 9 & 1480 & 0.31 & 3.41 & 24369.69 & 78.58317 & 117.81567 & 10 & 0.4 & 0.667 & 1.668 & 77 & 3 & 0.17045 & 0.008487823 \\ 9 & 1500 & 0.24 & 3.09 & 24370.18323 & 84.66378 & 139.9399 & 10 & 0.31 & 0.605 & 1.952 & 78 & 3 & 0.1544 & 0.007145913 \\ 9 & 1520 & 0.16 & 2.93 & 24370.51388 & 88.74011 & 154.59923 & 10 & 0.21 & 0.574 & 2.733 & 79 & 3 & 0.14665 & 0.006468326 \\ 9 & 1540 & 0.08 & 2.19 & 24370.68274 & 90.82185 & 211.70545 & 10 & 0.1 & 0.429 & 4.29 & 80 & 3 & 0.10945 & 0.004723533\end{array}$
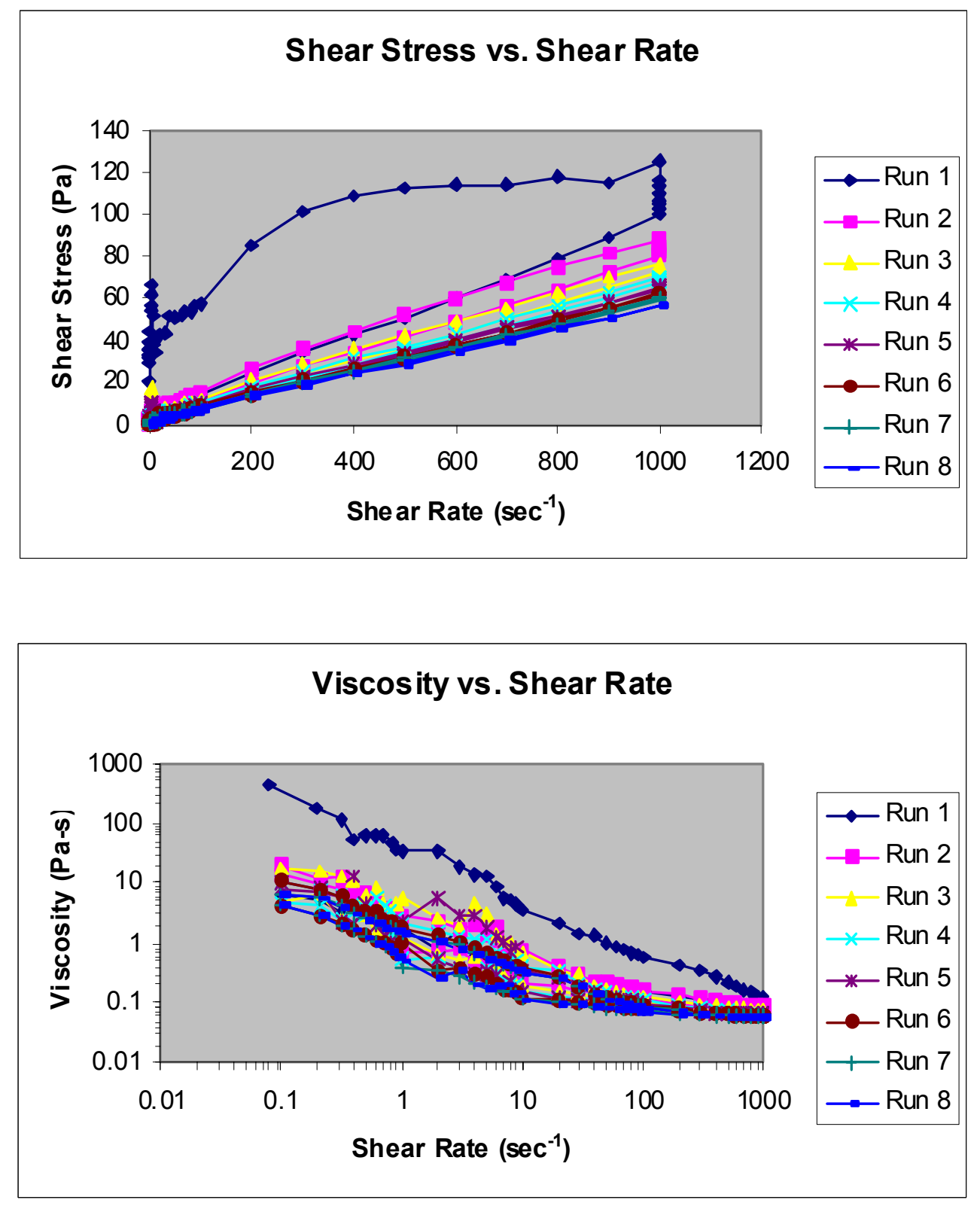


\section{E-2.1.24 META/20wt\%/U/S $10^{\circ} \mathrm{C}$}

\begin{tabular}{|c|c|c|c|c|c|c|c|c|c|c|c|c|c|c|c|}
\hline Mode St & & $\mathrm{t}[\mathrm{s}]$ & $\mathrm{n}[\mathrm{rpm}]$ & $\mathrm{M}[\%]$. & Phi[rad] & Gamma & $J[t]$ & $\mathrm{T}\left[{ }^{\circ} \mathrm{C}\right]$ & $\mathrm{D}[1 / \mathrm{s}]$ & $\mathrm{Tau}[\mathrm{Pa}]$ & $\mathrm{Eta}[\mathrm{Pas}]$ & MP\# & Block & $\mathrm{M}[\mathrm{mNm}]$ & $\mathrm{G}[\mathrm{Pa}]$ \\
\hline 1 & 1 & 20 & 0.03 & 229.38 & 0.05341 & 0.65841 & 0.01466 & 10 & 0.04 & 44.912 & 1122.8 & 1 & 1 & 111.46875 & 68.21281572 \\
\hline 1 & 1 & 40 & 0.17 & 81.45 & 0.46967 & 5.79013 & 0.36306 & 10 & 0.22 & 15.948 & 72.491 & 2 & 1 & 4.0725 & 2.754342303 \\
\hline 1 & 1 & 60 & 0.24 & 77.44 & 0.95819 & 11.81265 & 0.77904 & 10 & 0.31 & 15.163 & 48.913 & 3 & 1 & 3.87195 & 1.283623912 \\
\hline 1 & 1 & 80 & 0.31 & 86.99 & 1.6085 & 19.82976 & 1.16426 & 10 & 0.4 & 17.032 & 42.58 & 4 & 1 & 4.34945 & 0.858911051 \\
\hline 1 & 1 & 100 & 0.39 & 101.52 & 2.41903 & 29.8221 & 1.50033 & 10 & 0.5 & 19.877 & 39.754 & 5 & 1 & 5.07585 & 0.666519125 \\
\hline 1 & 1 & 120 & 0.47 & 108.72 & 3.39606 & 41.86713 & 1.96679 & 10 & 0.61 & 21.287 & 34.897 & 6 & 1 & 5.43595 & 0.508441825 \\
\hline 1 & 1 & 140 & 0.54 & 100.77 & 4.54117 & 55.98421 & 2.83737 & 10 & 0.7 & 19.731 & 28.187 & 7 & 1 & 5.03845 & 0.352438661 \\
\hline 1 & 1 & 160 & 0.62 & 85.01 & 5.84965 & 72.11526 & 4.33255 & 10 & 0.8 & 16.645 & 20.806 & 8 & 1 & 4.25055 & 0.230811066 \\
\hline 1 & 1 & 180 & 0.7 & 74.02 & 7.31677 & 90.20217 & 6.22427 & 10 & 0.9 & 14.492 & 16.102 & 9 & 1 & 3.7008 & 0.160661323 \\
\hline 1 & 2 & 200 & 0.77 & 84.21 & 8.93233 & 0 & 0 & 10 & 0.99 & 16.487 & 16.654 & 10 & 1 & 4.21025 & 0 \\
\hline 1 & 2 & 220 & 1.55 & 140.08 & 12.14226 & 0 & 0 & 10 & 2 & 27.428 & 13.714 & 11 & 1 & 7.0041 & 0 \\
\hline 1 & 2 & 240 & 2.3 & 256.27 & 16.94339 & 0 & 0 & 10 & 2.97 & 50.177 & 16.895 & 12 & 1 & 12.81345 & 0 \\
\hline 1 & 2 & 260 & 3.1 & 293.31 & 23.41508 & 0 & 0 & 10 & 4 & 57.43 & 14.358 & 13 & 1 & 114.66535 & 0 \\
\hline 1 & 2 & 280 & 3.88 & 277.78 & 31.51803 & 0 & 0 & 10 & 5.01 & 54.39 & 10.856 & 14 & 1 & 13.88915 & 0 \\
\hline 1 & 2 & 300 & 4.66 & 237.62 & 41.2444 & 0 & 0 & 10 & 6.02 & 46.527 & 7.729 & 15 & 1 & 111.88115 & 0 \\
\hline 1 & 2 & 320 & 5.42 & 221.72 & 52.58476 & 0 & 0 & 10 & 7 & 43.413 & 6.202 & 16 & 1 & 111.08605 & 0 \\
\hline 1 & 2 & 340 & 6.2 & 208.77 & 65.56504 & 0 & 0 & 10 & 8 & 40.878 & 5.11 & 17 & 1 & 10.4387 & 0 \\
\hline 1 & 2 & 360 & 6.97 & 191.17 & 80.15459 & 0 & 0 & 10 & 9 & 37.43 & 4.159 & 18 & 1 & 9.55825 & 0 \\
\hline 1 & 3 & 380 & 7.75 & 183.59 & 96.53486 & 0 & 0 & 10 & 10.01 & 35.946 & 3.591 & 19 & 1 & 9.17935 & 0 \\
\hline 1 & 3 & 400 & 15.51 & 230.31 & 128.86813 & 0 & 0 & 10 & 20.02 & 45.095 & 2.252 & 20 & 1 & 11.5155 & 0 \\
\hline 1 & 3 & 420 & 23.25 & 220.29 & 177.43008 & 0 & 0 & 10 & 30.02 & 43.132 & 1.437 & 21 & 1 & 111.01435 & 0 \\
\hline 1 & 3 & 440 & 30.99 & 200.02 & 242.16731 & 0 & 0 & 10 & 40.01 & 39.164 & 0.979 & 22 & 1 & 10.001 & 0 \\
\hline 1 & 3 & 460 & 38.74 & 167.22 & 323.17407 & 0 & 0 & 10 & 50.01 & 32.742 & 0.655 & 23 & 1 & 8.36115 & 0 \\
\hline 1 & 3 & 480 & 46.48 & 158.19 & 420.44484 & 0 & 0 & 10 & 60.01 & 30.973 & 0.516 & 24 & 1 & 7.9094 & 0 \\
\hline 1 & 3 & 500 & 54.22 & 138.81 & 533.85005 & 0 & 0 & 10 & 70 & 27.178 & 0.388 & 25 & 1 & 6.94035 & 0 \\
\hline 1 & 3 & 520 & 61.96 & 131.76 & 663.44546 & 0 & 0 & 10 & 79.99 & 25.798 & 0.323 & 26 & 1 & 6.58775 & 0 \\
\hline 1 & 3 & 540 & 69.71 & 124.67 & 809.36145 & 0 & 0 & 10 & 90 & 24.411 & 0.271 & 27 & 1 & 6.2337 & 0 \\
\hline 1 & 4 & 560 & 77.46 & 121.51 & 973.09419 & 0 & 0 & 10 & 100 & 23.791 & 0.238 & 28 & 1 & 6.07545 & 0 \\
\hline 1 & 4 & 580 & 154.93 & 207.24 & 1296.4112 & 0 & 0 & 10 & 200.01 & 40.577 & 0.203 & 29 & 1 & 10.36175 & 0 \\
\hline 1 & 4 & 600 & 232.39 & 239.4 & 1781.48017 & 0 & 0 & 10 & 300.02 & 46.874 & 0.156 & 30 & 1 & 111.96975 & 0 \\
\hline 1 & 4 & 620 & 309.84 & 251.18 & 2429.30406 & 0 & 0 & 10 & 400 & 49.181 & 0.123 & 31 & 1 & 12.5589 & 0 \\
\hline 1 & 4 & 640 & 387.3 & 265.73 & 3239.43991 & 0 & 0 & 10 & 500 & 52.031 & 0.104 & 32 & 1 & 13.28665 & 0 \\
\hline 1 & 4 & 660 & 464.75 & 269.42 & 4211.76598 & 0 & 0 & 10 & 599.99 & 52.752 & 0.088 & 33 & 1 & 113.47095 & 0 \\
\hline 1 & 4 & 680 & 542.21 & 285.69 & 5346.03251 & 0 & 0 & 10 & 699.99 & 55.937 & 0.08 & 34 & 1 & 14.2843 & 0 \\
\hline 1 & 4 & 700 & 619.68 & 315.05 & 6642.1751 & 0 & 0 & 10 & 800.01 & 61.687 & 0.077 & 35 & 1 & 15.7525 & 0 \\
\hline 1 & 4 & 720 & 697.13 & 307.44 & 8100.1749 & 0 & 0 & 10 & 899.99 & 60.197 & 0.067 & 36 & 1 & $1 \quad 15.37215$ & 0 \\
\hline 1 & 4 & 740 & 774.6 & 320.33 & 9721.5438 & 0 & 0 & 10 & 1000 & 62.721 & 0.063 & 37 & 1 & 16.0165 & 0 \\
\hline 1 & 5 & 750 & 774.59 & 292.85 & 10550.21662 & 0 & 0 & 10 & 1000 & 57.34 & 0.057 & 38 & 1 & 114.64245 & 0 \\
\hline 1 & 5 & 760 & 774.59 & 283.9 & 11359.74535 & 0 & 0 & 10 & 1000 & 55.587 & 0.056 & 39 & 1 & 14.1949 & 0 \\
\hline 1 & 5 & 770 & 774.59 & 276.23 & 12171.30042 & 0 & 0 & 10 & 1000 & 54.086 & 0.054 & 40 & 1 & 113.81145 & 0 \\
\hline 1 & 5 & 780 & 774.59 & 270.1 & 12983.25917 & 0 & 0 & 10 & 1000 & 52.886 & 0.053 & 41 & 1 & 13.505 & 0 \\
\hline 1 & 5 & 790 & 774.59 & 265.01 & 13793.59844 & 0 & 0 & 10 & 1000 & 51.888 & 0.052 & 42 & 1 & 13.25025 & 0 \\
\hline 1 & 5 & 800 & 774.59 & 260.73 & 14604.34219 & 0 & 0 & 10 & 1000 & 51.05 & 0.051 & 43 & 1 & 113.03635 & 0 \\
\hline 1 & 6 & 820 & 774.59 & 257.16 & 16245.29735 & 0 & 0 & 10 & 1000 & 50.353 & 0.05 & 44 & 1 & 12.8582 & 0 \\
\hline 1 & 6 & 840 & 697.13 & 223.62 & 17705.93844 & 0 & 0 & 10.1 & 899.99 & 43.785 & 0.049 & 45 & 1 & 111.18105 & 0 \\
\hline 1 & 6 & 860 & 619.67 & 193.12 & 19005.07654 & 0 & 0 & 10.1 & 799.99 & 37.814 & 0.047 & 46 & 1 & 9.6562 & 0 \\
\hline
\end{tabular}




\begin{tabular}{|c|c|c|c|c|c|c|c|c|c|c|c|c|c|c|}
\hline & 880 & 542.21 & 164.87 & 20143.12083 & 0 & 0 & 10.1 & 699.99 & 32.281 & 0.046 & 47 & 1 & 8.2433 & 0 \\
\hline & 900 & 464.75 & 138.23 & 21118.08113 & 0 & 0 & 10.1 & 599.99 & 27.065 & 0.045 & 48 & 1 & 6.91145 & 0 \\
\hline & 920 & 387.3 & 112.49 & 21929.51603 & 0 & 0 & 10.1 & 500 & 22.025 & 0.044 & 49 & 1 & 5.62435 & 0 \\
\hline & 940 & 309.84 & 87.42 & 22580.58833 & 0 & 0 & 10.1 & 400 & 17.117 & 0.043 & 50 & 1 & 4.37095 & \\
\hline & 960 & 232.38 & 63.2 & 23068.04962 & 0 & 0 & 10.1 & 300 & 12.375 & 0.041 & 51 & 1 & 3.16 & \\
\hline & 980 & 154.92 & 39.75 & 23393.76838 & 0 & 0 & 10 & 200 & 7.783 & 0.039 & 52 & 1 & 1.9876 & \\
\hline & 61000 & 77.46 & 18.86 & 23557.38017 & 0 & 0 & 10 & 100 & 3.693 & 0.037 & 53 & 1 & 0.94295 & \\
\hline & 71020 & 69.71 & 18.18 & 23704.51273 & 0 & 0 & 10 & 90 & 3.559 & 0.04 & 54 & 1 & 0.90875 & \\
\hline & 71040 & 61.97 & 16.86 & 23834.44194 & 0 & 0 & 10 & 80 & 3.301 & 0.041 & 55 & 1 & 0.843 & \\
\hline & 71060 & 54.22 & 15.11 & 23948.17466 & 0 & 0 & 10 & 70 & 2.958 & 0.042 & 56 & 1 & 0.75525 & \\
\hline & 71080 & 46.48 & 13.41 & 24045.56403 & 0 & 0 & 10 & 60.01 & 2.625 & 0.044 & 57 & 1 & 0.67035 & \\
\hline & 71100 & 38.73 & 11.48 & 24126.8174 & 0 & 0 & 10 & 50 & 2.247 & 0.045 & 58 & 1 & 0.5739 & م \\
\hline & 71120 & 30.99 & 10.06 & 24191.7981 & 0 & 0 & 10 & 40.01 & 1.969 & 0.049 & 59 & 1 & 0.5028 & 0 \\
\hline & 71140 & 23.24 & 8.11 & 24240.62552 & 0 & 0 & 10 & 30 & 1.587 & 0.053 & 60 & 1 & 0.40535 & 0 \\
\hline & 71160 & 15.5 & 6.11 & 24273.18341 & 0 & 0 & 10 & 20.01 & 1.197 & 0.06 & 61 & 1 & 0.30565 & 0 \\
\hline & 71180 & 7.75 & 3.84 & 24289.51655 & 0 & 0 & 10 & 10.01 & 0.751 & 0.075 & 62 & 1 & 0.19175 & 0 \\
\hline & 81200 & 6.97 & 4.56 & 24304.22628 & 0 & 0 & 10 & 9 & 0.892 & 0.099 & 63 & 1 & 0.22775 & 0 \\
\hline & 81220 & 6.2 & 4.64 & 24317.20577 & 0 & 0 & 10 & 8 & 0.908 & 0.114 & 64 & 1 & 0.2319 & 0 \\
\hline & 81240 & 5.42 & 4.45 & 24328.57441 & 0 & 0 & 10 & 7 & 0.87 & 0.124 & 65 & 1 & 0.22225 & 0 \\
\hline & 81260 & 4.64 & 4.57 & 24338.31334 & 0 & 0 & 10 & 5.99 & 0.894 & 0.149 & 66 & 1 & 0.2284 & 0 \\
\hline & 81280 & 3.87 & 4.38 & 24346.42886 & 0 & 0 & 10 & 5 & 0.858 & 0.172 & 67 & 1 & 0.21915 & 0 \\
\hline & 81300 & 3.09 & 4.25 & 24352.92253 & 0 & 0 & 10 & 3.99 & 0.833 & 0.209 & 68 & 1 & 0.21265 & 0 \\
\hline & 81320 & 2.32 & 3.98 & 24357.79279 & 0 & 0 & 10 & 3 & 0.779 & 0.26 & 69 & 1 & 0.19905 & 0 \\
\hline & 81340 & 1.54 & 3.15 & 24361.04041 & 0 & 0 & 10 & 1.99 & 0.616 & 0.31 & 70 & 1 & 0.15735 & 0 \\
\hline & 81360 & 0.77 & 2.44 & 24362.66226 & 0 & 0 & 10 & 0.99 & 0.479 & 0.484 & 71 & 1 & 0.1222 & 0 \\
\hline & 91380 & 0.7 & 3.11 & 24364.13802 & 18.19342 & 29.92334 & 10 & 0.9 & 0.608 & 0.676 & 72 & 1 & 0.15535 & 0.033418676 \\
\hline & 91400 & 0.62 & 3.47 & 24365.44178 & 34.26637 & 50.46586 & 10 & 0.8 & 0.679 & 0.849 & 73 & 1 & 0.17335 & 0.019815347 \\
\hline & 91420 & 0.55 & 3.48 & 24366.58532 & 48.36409 & 71.01912 & 10 & 0.71 & 0.681 & 0.959 & 74 & 1 & 0.1739 & 0.014080695 \\
\hline & 91440 & 0.47 & 3.75 & 24367.56471 & 60.43817 & 82.45305 & 10 & 0.61 & 0.733 & 1.202 & 75 & 1 & 0.18725 & 0.012128097 \\
\hline & 91460 & 0.39 & 3.64 & 24368.38231 & 70.51765 & 98.9026 & 10 & 0.5 & 0.713 & 1.426 & 76 & 1 & 0.1821 & 0.010110944 \\
\hline & 91480 & 0.31 & 3.61 & 24369.03812 & 78.60253 & 111.33487 & 10 & 0.4 & 0.706 & 1.765 & 77 & 1 & 0.18025 & 0.008981899 \\
\hline & 91500 & 0.24 & 3.44 & 24369.53135 & 84.68315 & 125.82916 & 10 & 0.31 & 0.673 & 2.171 & 78 & 1 & 0.1719 & 0.007947272 \\
\hline & 91520 & 0.16 & 3.39 & 24369.86122 & 88.7498 & 133.45815 & 10 & 0.21 & 0.665 & 3.167 & 79 & 1 & 0.1697 & 0.007492975 \\
\hline & 91540 & 0.08 & 2.56 & 24370.03086 & 90.84122 & 181.31944 & 10 & 0.1 & 0.501 & 5.01 & 80 & 1 & 0.12795 & 0.005515117 \\
\hline
\end{tabular}

2004.02.15 META-20 wt\%-U [sonicated] CC-45 0.1-1000 s-1 run 2.txt

\begin{tabular}{crrrrrrrrrrrrrrr} 
Mode Step & $\mathrm{t}[\mathrm{s}]$ & $\mathrm{n}[\mathrm{rpm}]$ & $\mathrm{M}[\%]$. & $\mathrm{Phi}[\mathrm{rad}]$ & \multicolumn{1}{c}{$\mathrm{Gamma}$} & \multicolumn{1}{c}{$\mathrm{J}[\mathrm{t}]$} & \multicolumn{3}{c}{$\mathrm{T}\left[{ }^{\circ} \mathrm{C}\right]$} & $\mathrm{D}[1 / \mathrm{s}]$ & $\mathrm{Tau}[\mathrm{Pa}]$ & $\mathrm{Eta}[\mathrm{Pas}]$ & $\mathrm{MP} \#$ Block $\mathrm{M}[\mathrm{mNm}]$ & $\mathrm{G}[\mathrm{Pa}]$ \\
1 & 1 & 20 & 0.08 & 3.96 & 0.16336 & 2.01396 & 2.59531 & 10 & 0.1 & 0.776 & 7.76 & 1 & 2 & 0.19805 & 0.385310532 \\
1 & 1 & 40 & 0.16 & 5.81 & 0.49244 & 6.07093 & 5.33942 & 10 & 0.21 & 1.137 & 5.414 & 2 & 2 & 0.2903 & 0.187285968 \\
1 & 1 & 60 & 0.24 & 5.61 & 0.9841 & 12.13217 & 11.04933 & 10 & 0.31 & 1.098 & 3.542 & 3 & 2 & 0.2804 & 0.090503183 \\
1 & 1 & 80 & 0.31 & 5.65 & 1.63677 & 20.17833 & 18.26092 & 10 & 0.4 & 1.105 & 2.762 & 4 & 2 & 0.28225 & 0.054761717 \\
1 & 1 & 100 & 0.39 & 5.79 & 2.45358 & 30.24813 & 26.69736 & 10 & 0.5 & 1.133 & 2.266 & 5 & 2 & 0.28925 & 0.037456861 \\
1 & 1 & 120 & 0.47 & 5.93 & 3.43062 & 42.29316 & 36.42819 & 10 & 0.61 & 1.161 & 1.903 & 6 & 2 & 0.2965 & 0.027451247 \\
1 & 1 & 140 & 0.54 & 6.04 & 4.57102 & 56.35215 & 47.63491 & 10 & 0.7 & 1.183 & 1.69 & 7 & 2 & 0.30215 & 0.020992988 \\
1 & 1 & 160 & 0.62 & 6.15 & 5.87399 & 72.41542 & 60.09573 & 10 & 0.8 & 1.205 & 1.506 & 8 & 2 & 0.3076 & 0.016640102 \\
1 & 1 & 180 & 0.7 & 6.43 & 7.33876 & 90.47328 & 71.86117 & 10 & 0.9 & 1.259 & 1.399 & 9 & 2 & 0.32155 & 0.013915711 \\
1 & 2 & 200 & 0.77 & 6.29 & 8.96139 & 0 & 0 & 10 & 0.99 & 1.232 & 1.244 & 10 & 2 & 0.3147 & 0 \\
1 & 2 & 220 & 1.55 & 9.51 & 12.18781 & 0 & 0 & 10 & 2 & 1.863 & 0.932 & 11 & 2 & 0.4757 & 0 \\
1 & 2 & 240 & 2.32 & 10.91 & 17.03607 & 0 & 0 & 10 & 3 & 2.137 & 0.712 & 12 & 2 & 0.5456 & 0 \\
1 & 2 & 260 & 3.09 & 11.77 & 23.50775 & 0 & 0 & 10 & 3.99 & 2.304 & 0.577 & 13 & 2 & 0.5884
\end{tabular}




\begin{tabular}{|c|c|c|c|c|c|c|c|c|c|c|c|c|c|c|}
\hline & 2280 & 3.87 & 12.54 & 31.59814 & 0 & 0 & 10 & 5 & 2.455 & 0.491 & 14 & 2 & 0.627 & 0 \\
\hline 1 & 2300 & 4.65 & 13.2 & 41.31508 & 0 & 0 & 10 & 6 & 2.584 & 0.431 & 15 & 2 & 0.65985 & 0 \\
\hline & 2320 & 5.42 & 14.06 & 52.66173 & 0 & 0 & 10 & 7 & 2.752 & 0.393 & 16 & 2 & 0.70275 & 0 \\
\hline & 2340 & 6.2 & 14.65 & 65.62709 & 0 & 0 & 10 & 8 & 2.868 & 0.359 & 17 & 2 & 0.73235 & 0 \\
\hline & 2360 & 6.97 & 15.21 & 80.20722 & 0 & 0 & 10 & 9 & 2.978 & 0.331 & 18 & 2 & 0.7605 & 0 \\
\hline & 3380 & 7.75 & 15.73 & 96.58434 & 0 & 0 & 10.1 & 10.01 & 3.079 & 0.308 & 19 & 2 & 0.78625 & 0 \\
\hline & 400 & 15.5 & 29.44 & 128.89405 & 0 & 0 & 10 & 20.01 & 5.764 & 0.288 & 20 & 2 & 1.47185 & 0 \\
\hline & 420 & 23.24 & 35.65 & 177.50234 & 0 & 0 & 10 & 30 & 6.98 & 0.233 & 21 & 2 & 1.7824 & 0 \\
\hline & 440 & 30.99 & 37.89 & 242.25135 & 0 & 0 & 10 & 40.01 & 7.418 & 0.185 & 22 & 2 & 1.89435 & 0 \\
\hline & 460 & 38.73 & 38.82 & 323.27381 & 0 & 0 & 10 & 50 & 7.602 & 0.152 & 23 & 2 & 1.9412 & 0 \\
\hline & 480 & 46.48 & 37.92 & 420.39615 & 0 & 0 & 10 & 60.01 & 7.425 & 0.124 & 24 & 2 & 1.8961 & 0 \\
\hline & 500 & 54.22 & 36.53 & 533.91838 & 0 & 0 & 10 & 70 & 7.152 & 0.102 & 25 & 2 & 1.82635 & 0 \\
\hline & 520 & 61.96 & 33.8 & 663.54442 & 0 & 0 & 10 & 79.99 & 6.618 & 0.083 & 26 & 2 & 1.6901 & 0 \\
\hline & 540 & 69.71 & 34.73 & 809.42271 & 0 & 0 & 10 & 90 & 6.801 & 0.076 & 27 & 2 & 1.7366 & 0 \\
\hline & 560 & 77.46 & 36.72 & 973.11461 & 0 & 0 & 10 & 100 & 7.19 & 0.072 & 28 & 2 & 1.83605 & 0 \\
\hline & 580 & 154.92 & 72.81 & 1296.19521 & 0 & 0 & 10 & 200 & 14.256 & 0.071 & 29 & 2 & 3.6405 & 0 \\
\hline & 600 & 232.38 & 96.14 & 1782.11556 & 0 & 0 & 10 & 300 & 18.823 & 0.063 & 30 & 2 & 4.80675 & 0 \\
\hline & 620 & 309.84 & 111.16 & 2429.00718 & 0 & 0 & 10 & 400 & 21.764 & 0.054 & 31 & 2 & 5.5578 & 0 \\
\hline & 640 & 387.3 & 132.55 & 3239.14225 & 0 & 0 & 10 & 500 & 25.953 & 0.052 & 32 & 2 & 6.62745 & 0 \\
\hline & 660 & 464.75 & 159.1 & 4210.94053 & 0 & 0 & 10 & 599.99 & 31.151 & 0.052 & 33 & 2 & 7.95485 & 0 \\
\hline & 680 & 542.21 & 179.93 & 5345.77726 & 0 & 0 & 10 & 699.99 & 35.23 & 0.05 & 34 & 2 & 8.9963 & 0 \\
\hline & 700 & 619.67 & 203.04 & 6642.56309 & 0 & 0 & 10 & 799.99 & 39.755 & 0.05 & 35 & 2 & 10.1519 & 0 \\
\hline & 720 & 697.13 & 226.46 & 8100.19689 & 0 & 0 & 10 & 899.99 & 44.34 & 0.049 & 36 & 2 & 11.32275 & 0 \\
\hline & 740 & 774.59 & 249.07 & 9721.96713 & 0 & 0 & 10 & 1000 & 48.768 & 0.049 & 37 & 2 & 12.45355 & 0 \\
\hline & 750 & 774.59 & 243.24 & 10549.0126 & 0 & 0 & 10 & 1000 & 47.625 & 0.048 & 38 & 2 & 12.16175 & 0 \\
\hline & 760 & 774.59 & 241.27 & 11361.7866 & 0 & 0 & 10 & 1000 & 47.24 & 0.047 & 39 & 2 & 12.06335 & 0 \\
\hline & 770 & 774.59 & 239.26 & 12171.3122 & 0 & 0 & 10 & 1000 & 46.847 & 0.047 & 40 & 2 & 11.9631 & 0 \\
\hline & 780 & 774.59 & 237.41 & 12984.08306 & 0 & 0 & 10 & 1000 & 46.485 & 0.046 & 41 & 2 & 11.87055 & 0 \\
\hline & 790 & 774.59 & 235.75 & 13794.01627 & 0 & 0 & 10 & 1000 & 46.159 & 0.046 & 42 & 2 & 11.7874 & 0 \\
\hline & 800 & 774.59 & 234.13 & 14604.75924 & 0 & 0 & 10 & 1000 & 45.842 & 0.046 & 43 & 2 & 11.70645 & 0 \\
\hline & 820 & 774.59 & 232.52 & 16246.1173 & 0 & 0 & 10 & 1000 & 45.526 & 0.046 & 44 & 2 & 11.62575 & 0 \\
\hline & 840 & 697.13 & 203.83 & 17708.54204 & 0 & 0 & 10 & 899.99 & 39.91 & 0.044 & 45 & 2 & 10.1916 & 0 \\
\hline & 860 & 619.67 & 177.24 & 19007.35498 & 0 & 0 & 10 & 799.99 & 34.703 & 0.043 & 46 & 2 & 8.86185 & 0 \\
\hline & 880 & 542.21 & 151.54 & 20143.12633 & 0 & 0 & 10 & 699.99 & 29.672 & 0.042 & 47 & 2 & 7.57705 & 0 \\
\hline & 900 & 464.75 & 126.79 & 21118.08898 & 0 & 0 & 10 & 599.99 & 24.826 & 0.041 & 48 & 2 & 6.3396 & 0 \\
\hline & 920 & 387.3 & 103.05 & 21930.5771 & 0 & 0 & 10 & 500 & 20.177 & 0.04 & 49 & 2 & 5.15245 & 0 \\
\hline & 940 & 309.84 & 80.08 & 22581.40514 & 0 & 0 & 10 & 400 & 15.679 & 0.039 & 50 & 2 & 4.00395 & 0 \\
\hline & 960 & 232.38 & 58.05 & 23068.98817 & 0 & 0 & 10 & 300 & 11.365 & 0.038 & 51 & 2 & 2.90225 & 0 \\
\hline & 6980 & 154.92 & 36.69 & 23394.78861 & 0 & 0 & 10 & 200 & 7.185 & 0.036 & 52 & 2 & 1.8347 & 0 \\
\hline & 61000 & 77.46 & 17.79 & 23558.1585 & 0 & 0 & 10 & 100 & 3.484 & 0.035 & 53 & 2 & 0.8896 & 0 \\
\hline & 71020 & 69.71 & 17.24 & 23705.21645 & 0 & 0 & 10 & 90 & 3.375 & 0.038 & 54 & 2 & 0.86175 & 0 \\
\hline & 71040 & 61.97 & 16 & 23835.24226 & 0 & 0 & 10 & 80 & 3.132 & 0.039 & 55 & 2 & 0.79975 & 0 \\
\hline & 71060 & 54.22 & 14.64 & 23948.94671 & 0 & 0 & 10 & 70 & 2.867 & 0.041 & 56 & 2 & 0.7321 & 0 \\
\hline & 71080 & 46.48 & 12.89 & 24046.27953 & 0 & 0 & 10 & 60.01 & 2.524 & 0.042 & 57 & 2 & 0.64465 & 0 \\
\hline & 71100 & 38.73 & 10.99 & 24127.5541 & 0 & 0 & 10 & 50 & 2.152 & 0.043 & 58 & 2 & 0.54965 & 0 \\
\hline & 71120 & 30.99 & 9.68 & 24192.57093 & 0 & 0 & 10 & 40.01 & 1.894 & 0.047 & 59 & 2 & 0.48375 & 0 \\
\hline & 71140 & 23.24 & 7.89 & 24241.33238 & 0 & 0 & 10 & 30 & 1.544 & 0.051 & 60 & 2 & 0.3943 & 0 \\
\hline & 71160 & 15.5 & 6.01 & 24273.93111 & 0 & 0 & 10 & 20.01 & 1.177 & 0.059 & 61 & 2 & 0.3005 & 0 \\
\hline & 71180 & 7.75 & 3.77 & 24290.28075 & 0 & 0 & 10 & 10.01 & 0.737 & 0.074 & 62 & 2 & 0.1883 & 0 \\
\hline & 81200 & 6.97 & 4.39 & 24304.99361 & 0 & 0 & 10 & 9 & 0.86 & 0.096 & 63 & 2 & 0.2196 & 0 \\
\hline
\end{tabular}




\begin{tabular}{|c|c|c|c|c|c|c|c|c|c|c|c|c|c|c|}
\hline & 81220 & 6.2 & 4.56 & 24317.97546 & 0 & 0 & 10 & 8 & 0.894 & 0.112 & 64 & 2 & 0.2282 & 0 \\
\hline & 81240 & 5.42 & 4.41 & 24329.33231 & 0 & 0 & 10 & 7 & 0.862 & 0.123 & 65 & 2 & 0.22025 & \\
\hline & 81260 & 4.64 & 4.56 & 24339.07675 & 0 & 0 & 10 & 5.99 & 0.892 & 0.149 & 66 & 2 & 0.22785 & \\
\hline & 81280 & 3.87 & 4.41 & 24347.19227 & 0 & 0 & 10 & 5 & 0.863 & 0.173 & 67 & 2 & 0.2204 & \\
\hline & 81300 & 3.09 & 4.24 & 24353.68594 & 0 & 0 & 10 & 3.99 & 0.829 & 0.208 & 68 & 2 & 0.21175 & \\
\hline & 81320 & 2.32 & 3.93 & 24358.55384 & 0 & 0 & 10 & 3 & 0.769 & 0.256 & 69 & 2 & 0.19625 & \\
\hline & 81340 & 1.54 & 3.04 & 24361.80067 & 0 & 0 & 10 & 1.99 & 0.594 & 0.298 & 70 & 2 & 0.1518 & \\
\hline & 81360 & 0.77 & 2.54 & 24363.42566 & 0 & 0 & 10 & 0.99 & 0.498 & 0.503 & 71 & 2 & 0.1272 & \\
\hline & 91380 & 0.7 & 3.14 & 24364.90064 & 18.18373 & 29.61515 & 10 & 0.9 & 0.614 & 0.682 & 72 & 2 & 0.15675 & 0.033766449 \\
\hline & 91400 & 0.62 & 3.32 & 24366.20519 & 34.26637 & 52.71741 & 10 & 0.8 & 0.65 & 0.813 & 73 & 2 & 0.16605 & 0.018969036 \\
\hline & 91420 & 0.55 & 3.35 & 24367.34794 & 48.3544 & 73.71086 & 10 & 0.71 & 0.656 & 0.924 & 74 & 2 & 0.16755 & 0.013566501 \\
\hline & 91440 & 0.47 & 3.41 & 24368.32812 & 60.43816 & 90.6118 & 10 & 0.61 & 0.667 & 1.093 & 75 & 2 & 0.1703 & 0.011036074 \\
\hline & 91460 & 0.39 & 3.48 & 24369.14493 & 70.50796 & 103.53577 & 10 & 0.5 & 0.681 & 1.362 & 76 & 2 & 0.17385 & 0.009658484 \\
\hline & 91480 & 0.31 & 3.45 & 24369.80153 & 78.60253 & 116.44802 & 10 & 0.4 & 0.675 & 1.688 & 77 & 2 & 0.1724 & 0.00858751 \\
\hline & 91500 & 0.24 & 3.24 & 24370.29397 & 84.67346 & 133.55414 & 10 & 0.31 & 0.634 & 2.045 & 78 & 2 & 0.16185 & 0.007487588 \\
\hline & 91520 & 0.16 & 3.13 & 24370.62462 & 88.74979 & 144.5434 & 10 & 0.21 & 0.614 & 2.924 & 79 & 2 & 0.1567 & 0.006918326 \\
\hline & 91540 & 0.08 & 2.51 & 24370.79348 & 90.83153 & 185.37009 & 10 & 0.1 & 0.49 & 4.9 & 80 & 2 & 0.12525 & 0.005394603 \\
\hline
\end{tabular}

2004.02.15 META-20 wt\%-U [sonicated] CC-45 0.1-1000 s-1 run 3.txt

Mode Step t[s] n[rpm] M[\%.]

$\begin{array}{rrrrr}1 & 1 & 20 & 0.08 & 3.59 \\ 1 & 1 & 40 & 0.16 & 5.44 \\ 1 & 1 & 60 & 0.24 & 5.21 \\ 1 & 1 & 80 & 0.31 & 5.37 \\ 1 & 1 & 100 & 0.39 & 5.61 \\ 1 & 1 & 120 & 0.47 & 5.67 \\ 1 & 1 & 140 & 0.54 & 5.73 \\ 1 & 1 & 160 & 0.62 & 5.72 \\ 1 & 1 & 180 & 0.7 & 5.85 \\ 1 & 2 & 200 & 0.77 & 6.01 \\ 1 & 2 & 220 & 1.55 & 8.79 \\ 1 & 2 & 240 & 2.32 & 9.98 \\ 1 & 2 & 260 & 3.09 & 10.69 \\ 1 & 2 & 280 & 3.87 & 11.36 \\ 1 & 2 & 300 & 4.65 & 12.01 \\ 1 & 2 & 320 & 5.42 & 12.86 \\ 1 & 2 & 340 & 6.2 & 13.35 \\ 1 & 2 & 360 & 6.97 & 13.88 \\ 1 & 3 & 380 & 7.75 & 14.28 \\ 1 & 3 & 400 & 15.5 & 26.19 \\ 1 & 3 & 420 & 23.24 & 32.4 \\ 1 & 3 & 440 & 30.99 & 35.55 \\ 1 & 3 & 460 & 38.73 & 36.65 \\ 1 & 3 & 480 & 46.48 & 35.22 \\ 1 & 3 & 500 & 54.22 & 35.89 \\ 1 & 3 & 520 & 61.96 & 36.9 \\ 1 & 3 & 540 & 69.71 & 35.81 \\ 1 & 4 & 560 & 77.46 & 34.33 \\ 1 & 4 & 580 & 154.92 & 66.78 \\ 1 & 4 & 600 & 232.38 & 87.96 \\ & & & & \\ 1 & & \\ 1 & & \\ 1 & & \end{array}$

Phi[rad] Gamma J[t]

$0.49323-6.08061$

$0.9841 \quad 12.13217$

1.6375620 .18801

$2.4528 \quad 30.23845$

$3.4314 \quad 42.30284$

$4.57102 \quad 56.35215$

5.8739972 .41542

$7.33876 \quad 90.47328$

8.96218

12.18702

17.03843

23.50932

31.59892

41.31508

52.66252

65.62237

80.20957

96.58041

128.89719

177.50156

242.28198

323.28952

420.41186

533.96158

663.58762

809.43449

973.08555

1296.24705

1781.56028 $\begin{array}{lrrr}2.87448 & 10 & 0.1 & 0.704\end{array}$

$\begin{array}{llll}5.70949 & 10 & 0.21 & 1.065\end{array}$

$\begin{array}{llll}11.88262 & 10 & 0.31 & 1.021\end{array}$

$\begin{array}{llll}19.19011 & 10 & 0.4 & 1.052\end{array}$

$\begin{array}{llll}27.53955 & 10 & 0.5 & 1.098\end{array}$

38.07633

$50.22469 \quad 10$

$64.65657 \quad 10$

$79.0159 \quad 10$

$0 \quad 10$

$0 \quad 10$

$0 \quad 10$

$0 \quad 10$

$0.61 \quad 1.111$

$0.7 \quad 1.122$

$0.8 \quad 1.12$
0.9

$0.9 \quad 1.145$

$0.99 \quad 1.178$

$2 \quad 1.72$

31.955

$3.99 \quad 2.094$

$5 \quad 2.224$

$6 \quad 2.352$

$7 \quad 2.518$

$8 \quad 2.613$

$9 \quad 2.718$

2.797

$\begin{array}{ll}10.01 & 2.797 \\ 20.01 & 5.129\end{array}$

$\begin{array}{rrrr}10 & 30 & 6.344\end{array}$

$\begin{array}{llll}0 & 10 & 40.01 & 6.961\end{array}$

$\begin{array}{llll}0 & 10 & 50 & 7.176\end{array}$

$\begin{array}{llll}0 & 10 & 60.01 & 6.897\end{array}$

$\begin{array}{llll}0 & 10 & 70 & 7.028\end{array}$

$\begin{array}{llll}0 & 10 & 79.99 & 7.226\end{array}$

$\begin{array}{llll}0 & 10 & 90 & 7.012\end{array}$

$\begin{array}{llll}0 & 10 & 100 & 6.722\end{array}$

$\begin{array}{llll}0 & 10 & 200 & 13.075\end{array}$

$\begin{array}{llll}0 & 10 & 300 & 17.223\end{array}$
a[Pas] MP\# Block M[mNm] G[Pa]

$\begin{array}{llllll}7.04 & 1 & 3 & 0.1797 & 0.347887964\end{array}$

$\begin{array}{llllll}5.071 & 2 & 3 & 0.27185 & 0.175146901\end{array}$

$\begin{array}{lllll}3.294 & 3 & 3 & 0.2607 & 0.08415642\end{array}$

$\begin{array}{lllll}2.63 & 4 & 3 & 0.2686 & 0.052110139\end{array}$

$\begin{array}{lllll}2.196 & 5 & 3 & 0.2803 & 0.036311385\end{array}$

$\begin{array}{lllll}1.821 & 6 & 3 & 0.2836 & 0.026263012\end{array}$

$\begin{array}{lllll}1.603 & 7 & 3 & 0.2865 & 0.019910509\end{array}$

$\begin{array}{lllll}1.4 & 8 & 3 & 0.2861 & 0.015466319\end{array}$

$\begin{array}{lllll}1.272 & 9 & 3 & 0.29235 & 0.01265567\end{array}$

$\begin{array}{llll}1.19 & 10 & 3 & 0.3007\end{array}$

$\begin{array}{llll}0.86 & 11 & 3 & 0.4393\end{array}$

$\begin{array}{llll}0.652 & 12 & 3 & 0.4992\end{array}$

$\begin{array}{llll}0.525 & 13 & 3 & 0.5347\end{array}$

$\begin{array}{llll}0.445 & 14 & 3 & 0.568\end{array}$

$\begin{array}{llll}0.392 & 15 & 3 & 0.6005\end{array}$

$\begin{array}{llll}0.36 & 16 & 3 & 0.64295\end{array}$

$\begin{array}{llll}0.327 & 17 & 3 & 0.66735\end{array}$

$\begin{array}{llll}0.302 & 18 & 3 & 0.69415\end{array}$

$\begin{array}{llll}0.279 & 19 & 3 & 0.71415\end{array}$

$\begin{array}{llll}0.256 & 20 & 3 & 1.30965\end{array}$

$\begin{array}{llll}0.211 & 21 & 3 & 1.62\end{array}$

$\begin{array}{llll}0.174 & 22 & 3 & 1.7775\end{array}$

$\begin{array}{llll}0.144 & 23 & 3 & 1.83245\end{array}$

$\begin{array}{llll}0.115 & 24 & 3 & 1.76115\end{array}$

$\begin{array}{llll}0.1 & 25 & 3 & 1.7946\end{array}$

$\begin{array}{llll}0.09 & 26 & 3 & 1.84515\end{array}$

$\begin{array}{llll}0.078 & 27 & 3 & 1.7905\end{array}$

$\begin{array}{llll}0.067 & 28 & 3 & 1.71645\end{array}$

$\begin{array}{llll}0.065 & 29 & 3 & 3.3389\end{array}$

$\begin{array}{llll}0.057 & 30 & 3 & 4.39805\end{array}$ 


\begin{tabular}{|c|c|c|c|c|c|c|c|c|c|c|c|c|c|c|}
\hline & 620 & 309.84 & 98.45 & 2429.38496 & 0 & 0 & 10 & 400 & 19.276 & 0.048 & 31 & 3 & 4.92225 & \\
\hline & 640 & 387.3 & 122.64 & 3239.47997 & 0 & 0 & 10 & 500 & 24.013 & 0.048 & 32 & 3 & 6.132 & \\
\hline & 660 & 464.75 & 145.26 & 4211.56256 & 0 & 0 & 10 & 599.99 & 28.441 & 0.047 & 33 & 3 & 7.2628 & \\
\hline & 680 & 542.21 & 165.49 & 5345.50708 & 0 & 0 & 10 & 699.99 & 32.402 & 0.046 & 34 & 3 & 8.2743 & \\
\hline & 700 & 619.67 & 188.02 & 6642.00702 & 0 & 0 & 10 & 799.99 & 36.814 & 0.046 & 35 & 3 & 9.40095 & \\
\hline & 720 & 697.13 & 211.64 & 8101.10245 & 0 & 0 & 10 & 899.99 & 41.44 & 0.046 & 36 & 3 & 10.5821 & \\
\hline & 740 & 774.59 & 234.52 & 9722.83264 & 0 & 0 & 10 & 1000 & 45.919 & 0.046 & 37 & 3 & 11.726 & \\
\hline & 750 & 774.59 & 230.01 & 10551.09548 & 0 & 0 & 10 & 1000 & 45.036 & 0.045 & 38 & 3 & 11.5005 & \\
\hline & 760 & 774.59 & 227.88 & 11363.05738 & 0 & 0 & 10 & 1000 & 44.619 & 0.045 & 39 & 3 & 11.39405 & \\
\hline & 770 & 774.59 & 226.27 & 12172.58376 & 0 & 0 & 10 & 1000 & 44.304 & 0.044 & 40 & 3 & 11.3137 & \\
\hline & 780 & 774.59 & 224.81 & 12985.35383 & 0 & 0 & 10 & 1000 & 44.018 & 0.044 & 41 & 3 & 11.24065 & \\
\hline & 790 & 774.59 & 223.67 & 13795.28626 & 0 & 0 & 10 & 1000 & 43.795 & 0.044 & 42 & 3 & 11.1837 & \\
\hline & 800 & 774.59 & 222.5 & 14606.03001 & 0 & 0 & 10 & 1000 & 43.565 & 0.044 & 43 & 3 & 11.12495 & \\
\hline & 820 & 774.59 & 221.18 & 16246.98281 & 0 & 0 & 10 & 1000 & 43.307 & 0.043 & 44 & 3 & 11.0591 & \\
\hline & 840 & 697.13 & 193.68 & 17708.67634 & 0 & 0 & 10 & 899.99 & 37.922 & 0.042 & 45 & 3 & 9.6839 & \\
\hline & 860 & 619.67 & 168.36 & 19007.4885 & 0 & 0 & 10 & 799.99 & 32.964 & 0.041 & 46 & 3 & 8.41785 & \\
\hline & 880 & 542.21 & 143.93 & 20143.82769 & 0 & 0 & 10 & 699.99 & 28.181 & 0.04 & 47 & 3 & 7.1965 & \\
\hline & 900 & 464.75 & 120.5 & 21119.0346 & 0 & 0 & 10 & 599.99 & 23.594 & 0.039 & 48 & 3 & 6.0251 & \\
\hline & 920 & 387.3 & 97.84 & 21931.52193 & 0 & 0 & 10 & 500 & 19.156 & 0.038 & 49 & 3 & 4.89175 & \\
\hline & 940 & 309.84 & 75.92 & 22581.70045 & 0 & 0 & 10 & 400 & 14.865 & 0.037 & 50 & 3 & 3.79585 & \\
\hline & 960 & 232.38 & 55.04 & 23069.40522 & 0 & 0 & 10 & 300 & 10.777 & 0.036 & 51 & 3 & 2.7521 & \\
\hline & 980 & 154.92 & 34.84 & 23395.16482 & 0 & 0 & 10 & 200 & 6.821 & 0.034 & 52 & 3 & 1.74175 & \\
\hline & 61000 & 77.46 & 17.02 & 23558.74283 & 0 & 0 & 10 & 100 & 3.333 & 0.033 & 53 & 3 & 0.8511 & \\
\hline & 71020 & 69.71 & 16.58 & 23705.88011 & 0 & 0 & 10 & 90 & 3.245 & 0.036 & 54 & 3 & 0.82875 & \\
\hline & 71040 & 61.97 & 15.43 & 23835.84152 & 0 & 0 & 10 & 80 & 3.021 & 0.038 & 55 & 3 & 0.7714 & \\
\hline & 71060 & 54.22 & 13.94 & 23949.52162 & 0 & 0 & 10 & 70 & 2.73 & 0.039 & 56 & 3 & 0.69705 & \\
\hline & 71080 & 46.48 & 12.38 & 24046.93141 & 0 & 0 & 10 & 60.01 & 2.424 & 0.04 & 57 & 3 & 0.6189 & \\
\hline & 71100 & 38.73 & 10.7 & 24128.20598 & 0 & 0 & 10 & 50 & 2.094 & 0.042 & 58 & 3 & 0.53475 & 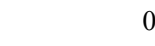 \\
\hline & 71120 & 30.99 & 9.42 & 24193.1859 & 0 & 0 & 10 & 40.01 & 1.844 & 0.046 & 59 & 3 & 0.4709 & 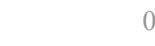 \\
\hline & 71140 & 23.24 & 7.71 & 24241.98112 & 0 & 0 & 10 & 30 & 1.51 & 0.05 & 60 & 3 & 0.38565 & 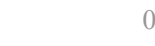 \\
\hline & 71160 & 15.5 & 6.01 & 24274.53037 & 0 & 0 & 10 & 20.01 & 1.177 & 0.059 & 61 & 3 & 0.30065 & 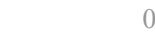 \\
\hline & 71180 & 7.75 & 3.87 & 24290.87922 & 0 & 0 & 10 & 10.01 & 0.758 & 0.076 & 62 & 3 & 0.1935 & 0 \\
\hline & 81200 & 6.97 & 4.39 & 24305.57716 & 0 & 0 & 10 & 9 & 0.859 & 0.095 & 63 & 3 & 0.2193 & 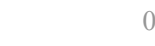 \\
\hline & 81220 & 6.2 & 4.59 & 24318.57943 & 0 & 0 & 10 & 8 & 0.898 & 0.112 & 64 & 3 & 0.22935 & 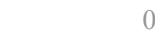 \\
\hline & 81240 & 5.42 & 4.39 & 24329.92765 & 0 & 0 & 10 & 7 & 0.86 & 0.123 & 65 & 3 & 0.2197 & 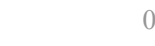 \\
\hline & 81260 & 4.64 & 4.68 & 24339.66894 & 0 & 0 & 10 & 5.99 & 0.917 & 0.153 & 66 & 3 & 0.23415 & 0 \\
\hline & 81280 & 3.87 & 4.39 & 24347.78603 & 0 & 0 & 10 & 5 & 0.86 & 0.172 & 67 & 3 & 0.2196 & 0 \\
\hline & 81300 & 3.09 & 4.48 & 24354.28284 & 0 & 0 & 10 & 3.99 & 0.878 & 0.22 & 68 & 3 & 0.2241 & 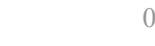 \\
\hline & 81320 & 2.32 & 4.14 & 24359.15074 & 0 & 0 & 10 & 3 & 0.81 & 0.27 & 69 & 3 & 0.20695 & 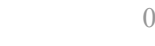 \\
\hline & 81340 & 1.54 & 3.2 & 24362.39915 & 0 & 0 & 10 & 1.99 & 0.626 & 0.315 & 70 & 3 & 0.1599 & \\
\hline & 81360 & 0.77 & 2.41 & 24364.02335 & 0 & 0 & 10 & 0.99 & 0.472 & 0.477 & 71 & 3 & 0.12055 & \\
\hline & 91380 & 0.7 & 3.03 & 24365.49676 & 18.16437 & 30.63126 & 10 & 0.9 & 0.593 & 0.659 & 72 & 3 & 0.15155 & 0.032646329 \\
\hline & 91400 & 0.62 & 3.36 & 24366.8013 & 34.247 & 51.96805 & 10 & 0.8 & 0.659 & 0.824 & 73 & 3 & 0.1682 & 0.019242561 \\
\hline & 91420 & 0.55 & 3.69 & 24367.94406 & 48.33504 & 66.94595 & 10 & 0.71 & 0.722 & 1.017 & 74 & 3 & 0.18445 & 0.014937404 \\
\hline & 91440 & 0.47 & 3.71 & 24368.92424 & 60.4188 & 83.1069 & 10 & 0.61 & 0.727 & 1.192 & 75 & 3 & 0.1857 & 0.012032679 \\
\hline & 91460 & 0.39 & 3.62 & 24369.74262 & 70.50796 & 99.58737 & 10 & 0.5 & 0.708 & 1.416 & 76 & 3 & 0.18085 & 0.010041419 \\
\hline & 91480 & 0.31 & 3.52 & 24370.39764 & 78.58317 & 114.21956 & 10 & 0.4 & 0.688 & 1.72 & 77 & 3 & 0.1758 & 0.008755055 \\
\hline & 91500 & 0.24 & 3.35 & 24370.89087 & 84.66378 & 128.864 & 10 & 0.31 & 0.657 & 2.119 & 78 & 3 & 0.16765 & 0.007760107 \\
\hline & 91520 & 0.16 & 3.2 & 24371.22231 & 88.74979 & 141.7726 & 10 & 0.21 & 0.626 & 2.981 & 79 & 3 & 0.1599 & 0.007053538 \\
\hline & 91540 & 0.08 & 2.38 & 24371.39039 & 90.82185 & 194.89626 & 10 & 0.1 & 0.466 & 4.66 & 80 & 3 & 0.11895 & 0.005130924 \\
\hline
\end{tabular}


2004.02.15 META-20 wt\%-U [sonicated] CC-45 0.1-1000 s-1 run 4.txt

Mode Step t[s] n[rpm] M[\%.]

\begin{tabular}{|c|c|c|c|c|c|c|}
\hline & 1 & 20 & 0.08 & 3.63 & 0.16415 & 2.023 \\
\hline & 1 & 40 & 0.16 & 5.37 & 0.49244 & 6.070 \\
\hline & 1 & 60 & 0.24 & 5.05 & 0.9841 & 12 \\
\hline & 1 & 80 & 0.31 & 5.33 & 1.63756 & \\
\hline & 1 & 100 & 0.39 & 5.42 & 2.4528 & 30.238 \\
\hline & 1 & 120 & 0.47 & 5.53 & 3.43219 & \\
\hline & 1 & 140 & 0.54 & 5.64 & 4.57102 & \\
\hline & 1 & 160 & 0.62 & 5.79 & 5.87399 & \\
\hline & 1 & 180 & 0.7 & 5.86 & 7.33798 & \\
\hline & 2 & 200 & 0.77 & 5.92 & 8.96218 & \\
\hline & 2 & 220 & 1.55 & 8.71 & 12.18467 & \\
\hline & 2 & 240 & 2.32 & 9.97 & 17.03214 & \\
\hline & 2 & 260 & 3.09 & 10.64 & 23.50775 & \\
\hline & 2 & 280 & 3.87 & 11.3 & 31.59735 & \\
\hline & 2 & 300 & 4.65 & 11.9 & 41.31351 & \\
\hline & 2 & 320 & 5.42 & 12.68 & 52.65702 & \\
\hline & 2 & 340 & 6.2 & 13.28 & 65.6208 & \\
\hline & 2 & 360 & 6.97 & 13.78 & 80.208 & \\
\hline & 3 & 380 & 7.75 & 14.19 & 96.57727 & \\
\hline & 3 & 400 & 15.5 & 25.53 & 128.93567 & \\
\hline & 3 & 420 & 23.24 & 31.15 & 177.47093 & \\
\hline & 3 & 440 & 30.99 & 33.77 & 242.20344 & \\
\hline & 3 & 460 & 38.73 & 33.77 & 323.24711 & \\
\hline & 3 & 480 & 46.48 & 34.6 & 420.46291 & \\
\hline & 3 & 500 & 54.22 & 34.32 & 533.98122 & \\
\hline & 3 & 520 & 61.96 & 30.2 & 663.60647 & \\
\hline & 3 & 540 & 69.71 & 30.55 & 809.45255 & \\
\hline & 4 & 560 & 77.46 & 32.21 & 973.14445 & \\
\hline & 4 & 580 & 154.92 & 63.1 & 1296.22584 & \\
\hline & 4 & 600 & 232.38 & 80.39 & 1781.82339 & \\
\hline & 4 & 620 & 309.84 & 95.17 & 2429.68812 & \\
\hline & 4 & 640 & 387.3 & 116.73 & 3239.01344 & \\
\hline & 4 & 660 & 464.75 & 136.1 & 4211.54136 & \\
\hline & 4 & 680 & 542.21 & 156.7 & 5345.48744 & \\
\hline & 4 & 700 & 619.67 & 178.98 & 6642.27013 & \\
\hline & 4 & 720 & 697.13 & 201.65 & 8101.36478 & \\
\hline & 4 & 740 & 774.59 & 224.91 & 9721.10633 & \\
\hline & 5 & 750 & 774.59 & 221.25 & 10549.77522 & \\
\hline & 5 & 760 & 774.59 & 219.64 & 11361.73712 & \\
\hline & 5 & 770 & 774.59 & 218.43 & 12170.85667 & \\
\hline & 5 & 780 & 774.59 & 217.3 & 12984.03672 & \\
\hline & 5 & 790 & 774.59 & 216.3 & 13794.7789 & \\
\hline & 5 & 800 & 774.59 & 215.12 & 14604.71133 & \\
\hline & 6 & 820 & 774.59 & 213.94 & 16246.07175 & \\
\hline & 6 & 840 & 697.13 & 188.42 & 17708.12656 & \\
\hline & 6 & 860 & 619.67 & 164.25 & 19006.69446 & \\
\hline & 6 & 880 & 542.21 & 140.4 & 20143.8866 & \\
\hline
\end{tabular}

$\mathrm{J}[\mathrm{t}] \quad \mathrm{T}\left[{ }^{\circ} \mathrm{C}\right] \mathrm{D}[1 / \mathrm{s}] \mathrm{Tau}[\mathrm{Pa}]$ Eta[Pas $]$ MP\# Block M[mNm] G[Pa]

$\begin{array}{lllllllll}2.84618 & 10 & 0.1 & 0.711 & 7.11 & 1 & 4 & 0.1815 & 0.351347078\end{array}$

$\begin{array}{lllllllll}5.77084 & 10 & 0.21 & 1.052 & 5.01 & 2 & 4 & 0.26865 & 0.173284818\end{array}$

$\begin{array}{lllllllll}12.27951 & 10 & 0.31 & 0.988 & 3.187 & 3 & 4 & 0.25225 & 0.081436379\end{array}$

$\begin{array}{lllllllll}19.3557 & 10 & 0.4 & 1.043 & 2.607 & 4 & 4 & 0.26635 & 0.051664329\end{array}$

$\begin{array}{lllllllll}28.49993 & 10 & 0.5 & 1.061 & 2.122 & 5 & 4 & 0.27105 & 0.035087777\end{array}$

$\begin{array}{lllllllll}39.06971 & 10 & 0.61 & 1.083 & 1.775 & 6 & 4 & 0.27655 & 0.025595255\end{array}$

$\begin{array}{lllllllll}50.99737 & 10 & 0.7 & 1.105 & 1.579 & 7 & 4 & 0.2822 & 0.019608835\end{array}$

$\begin{array}{lllllllll}63.9147 & 10 & 0.8 & 1.133 & 1.416 & 8 & 4 & 0.2893 & 0.015645839\end{array}$

$\begin{array}{lllllllll}78.86968 & 10 & 0.9 & 1.147 & 1.274 & 9 & 4 & 0.2929 & 0.012679133\end{array}$

0.2959

$4 \quad 0.43525$

40.4986

$4 \quad 0.5321$

$4 \quad 0.56515$

$4 \quad 0.59505$

$4 \quad 0.634$

$\begin{array}{ll}4 & 0.66415\end{array}$

010

$8 \quad 2.601$

$0.325 \quad 17$

$0.3 \quad 18$

$4 \quad 0.6892$

$\begin{array}{llll}0 & 10 & 10.01 & 2.778\end{array}$

$0.278 \quad 19$

$\begin{array}{ll}4 & 0.70945\end{array}$

$\begin{array}{llll}0.25 & 20 & 4 & 1.27655\end{array}$

$\begin{array}{llll}0.203 & 21 & 4 & 1.55725\end{array}$

$\begin{array}{llll}0.165 & 22 & 4 & 1.6885\end{array}$

$\begin{array}{llll}0.132 & 23 & 4 & 1.68835\end{array}$

$\begin{array}{llll}0.113 & 24 & 4 & 1.72995\end{array}$

$\begin{array}{llll}0.096 & 25 & 4 & 1.7158\end{array}$

$\begin{array}{llll}0.074 & 26 & 4 & 1.50975\end{array}$

$\begin{array}{llll}0.066 & 27 & 4 & 1.52765\end{array}$

$\begin{array}{llll}0.063 & 28 & 4 & 1.6103\end{array}$

$\begin{array}{llll}0.062 & 29 & 4 & 3.1551\end{array}$

$\begin{array}{llll}0.052 & 30 & 4 & 4.01965\end{array}$

$\begin{array}{llll}0.047 & 31 & 4 & 4.75835\end{array}$

$\begin{array}{llll}0.046 & 32 & 4 & 5.83635\end{array}$

$4 \quad 6.8051$

$4 \quad 7.8348$

$4 \quad 8.94875$

410.08265

$4 \quad 11.2455$

411.06255

$4 \quad 10.98185$

$4 \quad 10.9216$

$0.043 \quad 40$

$0.043 \quad 41$

$0.042 \quad 42$

$0.042 \quad 43$

$0.042 \quad 44$

$0.041 \quad 45$

$\begin{array}{llllllllll}0 & 10 & 799.99 & 32.161 & 0.04 & 46 & 4 & 8.21265 & 0\end{array}$

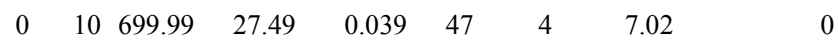

$4 \quad 10.8652$

$4 \quad 10.815$

$4 \quad 10.75595$

$\begin{array}{ll}4 & 10.6972\end{array}$

$\begin{array}{ll}4 & 9.42095\end{array}$ $\begin{array}{llllllllll}0 & 10 & 799.99 & 32.161 & 0.04 & 46 & 4 & 8.21265 & 0\end{array}$

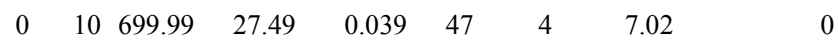

\section{0}

0

0

0

0

0

0

0

0

0

0

0

0

0

0

0

0

0

0

0

0

0

0

0

0

0

0

0

0

0

0

0

0

0

0

0 


\begin{tabular}{|c|c|c|c|c|c|c|c|c|c|c|c|c|c|c|}
\hline & 900 & 464.75 & 117.35 & 21118.32224 & 0 & 0 & 10 & 599.99 & 22.977 & 0.038 & 48 & 4 & 5.86755 & 0 \\
\hline & 920 & 387.3 & 95.19 & 21930.6093 & 0 & 0 & 10 & 500 & 18.639 & 0.037 & 49 & 4 & 4.7596 & \\
\hline & 940 & 309.84 & 73.84 & 22581.3533 & 0 & 0 & 10 & 400 & 14.458 & 0.036 & 50 & 4 & 3.692 & \\
\hline & 960 & 232.38 & 53.58 & 23068.81617 & 0 & 0 & 10 & 300 & 10.491 & 0.035 & 51 & 4 & 2.679 & \\
\hline & 980 & 154.92 & 33.97 & 23394.65509 & 0 & 0 & 10 & 200 & 6.652 & 0.033 & 52 & 4 & 1.69855 & \\
\hline & 61000 & 77.46 & 16.66 & 23558.18756 & 0 & 0 & 10 & 100 & 3.261 & 0.033 & 53 & 4 & 0.8328 & \\
\hline & 71020 & 69.71 & 16.27 & 23705.40495 & 0 & 0 & 10 & 90 & 3.185 & 0.035 & 54 & 4 & 0.81325 & \\
\hline & 71040 & 61.97 & 15.06 & 23835.33493 & 0 & 0 & 10 & 80 & 2.948 & 0.037 & 55 & 4 & 0.7528 & \\
\hline & 71060 & 54.22 & 13.67 & 23948.95377 & 0 & 0 & 10 & 70 & 2.676 & 0.038 & 56 & 4 & 0.68325 & \\
\hline & 71080 & 46.48 & 12.11 & 24046.48059 & 0 & 0 & 10 & 60.01 & 2.37 & 0.039 & 57 & 4 & 0.60525 & \\
\hline & 71100 & 38.73 & 10.46 & 24127.63735 & 0 & 0 & 10 & 50 & 2.047 & 0.041 & 58 & 4 & 0.5228 & \\
\hline & 71120 & 30.99 & 9.24 & 24192.63298 & 0 & 0 & 10 & 40.01 & 1.809 & 0.045 & 59 & 4 & 0.46195 & \\
\hline & 71140 & 23.24 & 7.47 & 24241.46904 & 0 & 0 & 10 & 30 & 1.462 & 0.049 & 60 & 4 & 0.37345 & \\
\hline & 71160 & 15.5 & 5.77 & 24274.01515 & 0 & 0 & 10 & 20.01 & 1.13 & 0.056 & 61 & 4 & 0.28865 & \\
\hline & 71180 & 7.75 & 3.57 & 24290.36871 & 0 & 0 & 10 & 10.01 & 0.698 & 0.07 & 62 & 4 & 0.17835 & \\
\hline & 81200 & 6.97 & 4.12 & 24305.08079 & 0 & 0 & 10 & 9 & 0.807 & 0.09 & 63 & 4 & 0.2061 & \\
\hline & 81220 & 6.2 & 4.38 & 24318.05792 & 0 & 0 & 10 & 8 & 0.857 & 0.107 & 64 & 4 & 0.21895 & \\
\hline & 81240 & 5.42 & 4.27 & 24329.42264 & 0 & 0 & 10 & 7 & 0.835 & 0.119 & 65 & 4 & 0.2133 & \\
\hline & 81260 & 4.64 & 4.44 & 24339.16 & 0 & 0 & 10 & 5.99 & 0.87 & 0.145 & 66 & 4 & 0.22205 & \\
\hline & 81280 & 3.87 & 4.28 & 24347.28102 & 0 & 0 & 10 & 5 & 0.837 & 0.167 & 67 & 4 & 0.21375 & \\
\hline & 81300 & 3.09 & 4.18 & 24353.77155 & 0 & 0 & 10 & 3.99 & 0.819 & 0.205 & 68 & 4 & 0.20915 & \\
\hline & 81320 & 2.32 & 3.89 & 24358.64416 & 0 & 0 & 10 & 3 & 0.762 & 0.254 & 69 & 4 & 0.19465 & \\
\hline & 81340 & 1.54 & 2.98 & 24361.88942 & 0 & 0 & 10 & 1.99 & 0.584 & 0.293 & 70 & 4 & 0.14905 & \\
\hline & 81360 & 0.77 & 2.35 & 24363.51363 & 0 & 0 & 10 & 0.99 & 0.459 & 0.464 & 71 & 4 & 0.11725 & \\
\hline & 91380 & 0.7 & 3.03 & 24364.98704 & 18.16437 & 30.68301 & 10 & 0.9 & 0.592 & 0.658 & 72 & 4 & 0.15125 & 0.032591276 \\
\hline & 91400 & 0.62 & 3.24 & 24366.2908 & 34.23732 & 53.91695 & 10 & 0.8 & 0.635 & 0.794 & 73 & 4 & 0.1622 & 0.018547012 \\
\hline & 91420 & 0.55 & 3.33 & 24367.43434 & 48.33504 & 74.13338 & 10 & 0.71 & 0.652 & 0.918 & 74 & 4 & 0.1664 & 0.013489179 \\
\hline & 91440 & 0.47 & 3.43 & 24368.41373 & 60.40912 & 90.02836 & 10 & 0.61 & 0.671 & 1.1 & 75 & 4 & 0.1714 & 0.011107594 \\
\hline & 91460 & 0.39 & 3.65 & 24369.23133 & 70.4886 & 98.58532 & 10 & 0.5 & 0.715 & 1.43 & 76 & 4 & 0.18255 & 0.010143484 \\
\hline & 91480 & 0.31 & 3.48 & 24369.88792 & 78.58317 & 115.22442 & 10 & 0.4 & 0.682 & 1.705 & 77 & 4 & 0.17405 & 0.008678703 \\
\hline & 91500 & 0.24 & 3.46 & 24370.38115 & 84.66378 & 124.87265 & 10 & 0.31 & 0.678 & 2.187 & 78 & 4 & 0.17305 & 0.008008147 \\
\hline & 91520 & 0.16 & 2.95 & 24370.71102 & 88.73043 & 153.51259 & 10 & 0.21 & 0.578 & 2.752 & 79 & 4 & 0.1476 & 0.006514112 \\
\hline & 91540 & 0.08 & 2.7 & 24370.87988 & 90.81217 & 171.66729 & 10 & 0.1 & 0.529 & 5.29 & 80 & 4 & 0.135 & 0.00582521 \\
\hline
\end{tabular}

2004.02.15 META-20 wt\%-U [sonicated] CC-45 0.1-1000 s-1 run 5.txt

\begin{tabular}{|c|c|c|c|c|c|c|c|c|c|c|c|c|c|c|c|}
\hline Mode $S$ & Step & $\mathrm{t}[\mathrm{s}]$ & {$[\mathrm{rpm}]$} & $\mathrm{M}[\%]$. & Phi[rad] & Gamma & $J[t]$ & $\mathrm{T}\left[{ }^{\circ} \mathrm{C}\right]$ & $\mathrm{D}[1 / \mathrm{s}]$ & $\mathrm{Tau}[\mathrm{Pa}]$ & $\mathrm{Eta}[\mathrm{Pas}]$ & MP\# & Block & $\mathrm{M}[\mathrm{mNm}]$ & $\mathrm{G}[\mathrm{Pa}]$ \\
\hline 1 & 1 & 20 & 0.08 & 3.63 & 0.16415 & 2.02364 & 2.84618 & 10 & 0.1 & 0.711 & 7.11 & 1 & 4 & 0.1815 & 0.351347078 \\
\hline 1 & 1 & 40 & 0.16 & 5.37 & 0.49244 & 6.07093 & 5.77084 & 10 & 0.21 & 1.052 & 5.01 & 2 & 4 & 0.26865 & 0.173284818 \\
\hline 1 & 1 & 60 & 0.24 & 5.05 & 0.9841 & 12.13217 & 12.27951 & 10 & 0.31 & 0.988 & 3.187 & 3 & 4 & 0.25225 & 0.081436379 \\
\hline 1 & 1 & 80 & 0.31 & 5.33 & 1.63756 & 20.18801 & 19.3557 & 10 & 0.4 & 1.043 & 2.607 & 4 & 4 & 0.26635 & 0.051664329 \\
\hline 1 & 1 & 100 & 0.39 & 5.42 & 2.4528 & 30.23845 & 28.49993 & 10 & 0.5 & 1.061 & 2.122 & 5 & 4 & 0.27105 & 0.035087777 \\
\hline 1 & 1 & 120 & 0.47 & 5.53 & 3.43219 & 42.31253 & 39.06971 & 10 & 0.61 & 1.083 & 1.775 & 6 & 4 & 0.27655 & 0.025595255 \\
\hline 1 & 1 & 140 & 0.54 & 5.64 & 4.57102 & 56.35215 & 50.99737 & 10 & 0.7 & 1.105 & 1.579 & 7 & 4 & 0.2822 & 0.019608835 \\
\hline 1 & 1 & 160 & 0.62 & 5.79 & 5.87399 & 72.41542 & 63.9147 & 10 & 0.8 & 1.133 & 1.416 & 8 & 4 & 0.2893 & 0.015645839 \\
\hline 1 & 1 & 180 & 0.7 & 5.86 & 7.33798 & 90.4636 & 78.86968 & 10 & 0.9 & 1.147 & 1.274 & 9 & 4 & 0.2929 & 0.012679133 \\
\hline 1 & 2 & 200 & 0.77 & 5.92 & 8.96218 & 0 & 0 & 10 & 0.99 & 1.159 & 1.171 & 10 & 4 & 0.2959 & 0 \\
\hline 1 & 2 & 220 & 1.55 & 8.71 & 12.18467 & 0 & 0 & 10 & 2 & 1.704 & 0.852 & 11 & 4 & 0.43525 & \\
\hline 1 & 2 & 240 & 2.32 & 9.97 & 17.03214 & 0 & 0 & 10 & 3 & 1.953 & 0.651 & 12 & 4 & 0.4986 & \\
\hline 1 & 2 & 260 & 3.09 & 10.64 & 23.50775 & 0 & 0 & 10 & 3.99 & 2.084 & 0.522 & 13 & 4 & 0.5321 & \\
\hline 1 & 2 & 280 & 3.87 & 11.3 & 31.59735 & 0 & 0 & 10 & 5 & 2.213 & 0.443 & 14 & 4 & 0.56515 & \\
\hline
\end{tabular}




\begin{tabular}{|c|c|c|c|c|c|c|c|c|c|c|c|c|c|c|}
\hline & 2300 & 4.65 & 11.9 & 41.31351 & 0 & 0 & 10 & 6 & 2.33 & 0.388 & 15 & 4 & 0.59505 & 0 \\
\hline 1 & 2320 & 5.42 & 12.68 & 52.65702 & 0 & 0 & 10 & 7 & 2.483 & 0.355 & 16 & 4 & 0.634 & 0 \\
\hline & 2340 & 6.2 & 13.28 & 65.6208 & 0 & 0 & 10 & 8 & 2.601 & 0.325 & 17 & 4 & 0.66415 & 0 \\
\hline & 2360 & 6.97 & 13.78 & 80.208 & 0 & 0 & 10 & 9 & 2.699 & 0.3 & 18 & 4 & 0.6892 & 0 \\
\hline & $3 \quad 380$ & 7.75 & 14.19 & 96.57727 & 0 & 0 & 10 & 10.01 & 2.778 & 0.278 & 19 & 4 & 0.70945 & 0 \\
\hline & 3400 & 15.5 & 25.53 & 128.93567 & 0 & 0 & 10 & 20.01 & 4.999 & 0.25 & 20 & 4 & 1.27655 & 0 \\
\hline & 3420 & 23.24 & 31.15 & 177.47093 & 0 & 0 & 10 & 30 & 6.098 & 0.203 & 21 & 4 & 1.55725 & 0 \\
\hline & 3440 & 30.99 & 33.77 & 242.20344 & 0 & 0 & 10 & 40.01 & 6.612 & 0.165 & 22 & 4 & 1.6885 & 0 \\
\hline & 3460 & 38.73 & 33.77 & 323.24711 & 0 & 0 & 10 & 50 & 6.612 & 0.132 & 23 & 4 & 1.68835 & 0 \\
\hline & 3480 & 46.48 & 34.6 & 420.46291 & 0 & 0 & 10 & 60.01 & 6.774 & 0.113 & 24 & 4 & 1.72995 & 0 \\
\hline & 3500 & 54.22 & 34.32 & 533.98122 & 0 & 0 & 10 & 70 & 6.719 & 0.096 & 25 & 4 & 1.7158 & 0 \\
\hline & 3520 & 61.96 & 30.2 & 663.60647 & 0 & 0 & 10 & 79.99 & 5.912 & 0.074 & 26 & 4 & 1.50975 & 0 \\
\hline & 3540 & 69.71 & 30.55 & 809.45255 & 0 & 0 & 10 & 90 & 5.982 & 0.066 & 27 & 4 & 1.52765 & 0 \\
\hline & $4 \quad 560$ & 77.46 & 32.21 & 973.14445 & 0 & 0 & 10 & 100 & 6.306 & 0.063 & 28 & 4 & 1.6103 & 0 \\
\hline & $4 \quad 580$ & 154.92 & 63.1 & 1296.22584 & 0 & 0 & 10 & 200 & 12.355 & 0.062 & 29 & 4 & 3.1551 & 0 \\
\hline & 4600 & 232.38 & 80.39 & 1781.82339 & 0 & 0 & 10 & 300 & 15.741 & 0.052 & 30 & 4 & 4.01965 & 0 \\
\hline & $4 \quad 620$ & 309.84 & 95.17 & 2429.68812 & 0 & 0 & 10 & 400 & 18.634 & 0.047 & 31 & 4 & 4.75835 & 0 \\
\hline & $4 \quad 640$ & 387.3 & 116.73 & 3239.01344 & 0 & 0 & 10 & 500 & 22.855 & 0.046 & 32 & 4 & 5.83635 & 0 \\
\hline & $4 \quad 660$ & 464.75 & 136.1 & 4211.54136 & 0 & 0 & 10 & 599.99 & 26.649 & 0.044 & 33 & 4 & 6.8051 & 0 \\
\hline & 680 & 542.21 & 156.7 & 5345.48744 & 0 & 0 & 10 & 699.99 & 30.681 & 0.044 & 34 & 4 & 7.8348 & 0 \\
\hline & 700 & 619.67 & 178.98 & 6642.27013 & 0 & 0 & 10 & 799.99 & 35.043 & 0.044 & 35 & 4 & 8.94875 & 0 \\
\hline & 720 & 697.13 & 201.65 & 8101.36478 & 0 & 0 & 10 & 899.99 & 39.484 & 0.044 & 36 & 4 & 10.08265 & 0 \\
\hline & 4740 & 774.59 & 224.91 & 9721.10633 & 0 & 0 & 10 & 1000 & 44.037 & 0.044 & 37 & 4 & 11.2455 & 0 \\
\hline & 5750 & 774.59 & 221.25 & 10549.77522 & 0 & 0 & 10 & 1000 & 43.321 & 0.043 & 38 & 4 & 11.06255 & 0 \\
\hline & 5760 & 774.59 & 219.64 & 11361.73712 & 0 & 0 & 10 & 1000 & 43.005 & 0.043 & 39 & 4 & 10.98185 & 0 \\
\hline & $5 \quad 770$ & 774.59 & 218.43 & 12170.85667 & 0 & 0 & 10 & 1000 & 42.769 & 0.043 & 40 & 4 & 10.9216 & 0 \\
\hline & $5 \quad 780$ & 774.59 & 217.3 & 12984.03672 & 0 & 0 & 10 & 1000 & 42.548 & 0.043 & 41 & 4 & 10.8652 & 0 \\
\hline & 5790 & 774.59 & 216.3 & 13794.7789 & 0 & 0 & 10 & 1000 & 42.352 & 0.042 & 42 & 4 & 10.815 & 0 \\
\hline & 800 & 774.59 & 215.12 & 14604.71133 & 0 & 0 & 10 & 1000 & 42.12 & 0.042 & 43 & 4 & 10.75595 & 0 \\
\hline & $6 \quad 820$ & 774.59 & 213.94 & 16246.07175 & 0 & 0 & 10 & 1000 & 41.89 & 0.042 & 44 & 4 & 10.6972 & 0 \\
\hline & 840 & 697.13 & 188.42 & 17708.12656 & 0 & 0 & 10 & 899.99 & 36.892 & 0.041 & 45 & 4 & 9.42095 & 0 \\
\hline & 6860 & 619.67 & 164.25 & 19006.69446 & 0 & 0 & 10 & 799.99 & 32.161 & 0.04 & 46 & 4 & 8.21265 & 0 \\
\hline & 6880 & 542.21 & 140.4 & 20143.8866 & 0 & 0 & 10 & 699.99 & 27.49 & 0.039 & 47 & 4 & 7.02 & 0 \\
\hline & 6900 & 464.75 & 117.35 & 21118.32224 & 0 & 0 & 10 & 599.99 & 22.977 & 0.038 & 48 & 4 & 5.86755 & 0 \\
\hline & $6 \quad 920$ & 387.3 & 95.19 & 21930.6093 & 0 & 0 & 10 & 500 & 18.639 & 0.037 & 49 & 4 & 4.7596 & 0 \\
\hline & 6940 & 309.84 & 73.84 & 22581.3533 & 0 & 0 & 10 & 400 & 14.458 & 0.036 & 50 & 4 & 3.692 & 0 \\
\hline 1 & 960 & 232.38 & 53.58 & 23068.81617 & 0 & 0 & 10 & 300 & 10.491 & 0.035 & 51 & 4 & 2.679 & 0 \\
\hline 1 & 6980 & 154.92 & 33.97 & 23394.65509 & 0 & 0 & 10 & 200 & 6.652 & 0.033 & 52 & 4 & 1.69855 & 0 \\
\hline & 61000 & 77.46 & 16.66 & 23558.18756 & 0 & 0 & 10 & 100 & 3.261 & 0.033 & 53 & 4 & 0.8328 & 0 \\
\hline & 71020 & 69.71 & 16.27 & 23705.40495 & 0 & 0 & 10 & 90 & 3.185 & 0.035 & 54 & 4 & 0.81325 & 0 \\
\hline & 71040 & 61.97 & 15.06 & 23835.33493 & 0 & 0 & 10 & 80 & 2.948 & 0.037 & 55 & 4 & 0.7528 & 0 \\
\hline & 71060 & 54.22 & 13.67 & 23948.95377 & 0 & 0 & 10 & 70 & 2.676 & 0.038 & 56 & 4 & 0.68325 & 0 \\
\hline & 71080 & 46.48 & 12.11 & 24046.48059 & 0 & 0 & 10 & 60.01 & 2.37 & 0.039 & 57 & 4 & 0.60525 & 0 \\
\hline & 71100 & 38.73 & 10.46 & 24127.63735 & 0 & 0 & 10 & 50 & 2.047 & 0.041 & 58 & 4 & 0.5228 & 0 \\
\hline 1 & 71120 & 30.99 & 9.24 & 24192.63298 & 0 & 0 & 10 & 40.01 & 1.809 & 0.045 & 59 & 4 & 0.46195 & 0 \\
\hline 1 & 71140 & 23.24 & 7.47 & 24241.46904 & 0 & 0 & 10 & 30 & 1.462 & 0.049 & 60 & 4 & 0.37345 & 0 \\
\hline 1 & 71160 & 15.5 & 5.77 & 24274.01515 & 0 & 0 & 10 & 20.01 & 1.13 & 0.056 & 61 & 4 & 0.28865 & 0 \\
\hline 1 & 71180 & 7.75 & 3.57 & 24290.36871 & 0 & 0 & 10 & 10.01 & 0.698 & 0.07 & 62 & 4 & 0.17835 & 0 \\
\hline 1 & 81200 & 6.97 & 4.12 & 24305.08079 & 0 & 0 & 10 & 9 & 0.807 & 0.09 & 63 & 4 & 0.2061 & 0 \\
\hline & 81220 & 6.2 & 4.38 & 24318.05792 & 0 & 0 & 10 & 8 & 0.857 & 0.107 & 64 & 4 & 0.21895 & 0 \\
\hline
\end{tabular}




\begin{tabular}{|c|c|c|c|c|c|c|c|c|c|c|c|c|c|c|}
\hline 1 & 81240 & 5.42 & 4.27 & 24329.42264 & 0 & 0 & 10 & 7 & 0.835 & 0.119 & 65 & 4 & 0.2133 & 0 \\
\hline & 81260 & 4.64 & 4.44 & 24339.16 & 0 & 0 & 10 & 5.99 & 0.87 & 0.145 & 66 & 4 & 0.22205 & \\
\hline & 81280 & 3.87 & 4.28 & 24347.28102 & 0 & 0 & 10 & 5 & 0.837 & 0.167 & 67 & 4 & 0.21375 & \\
\hline & 81300 & 3.09 & 4.18 & 24353.77155 & 0 & 0 & 10 & 3.99 & 0.819 & 0.205 & 68 & 4 & 0.20915 & \\
\hline & 81320 & 2.32 & 3.89 & 24358.64416 & 0 & 0 & 10 & 3 & 0.762 & 0.254 & 69 & 4 & 0.19465 & \\
\hline & 81340 & 1.54 & 2.98 & 24361.88942 & 0 & 0 & 10 & 1.99 & 0.584 & 0.293 & 70 & 4 & 0.14905 & \\
\hline & 81360 & 0.77 & 2.35 & 24363.51363 & 0 & 0 & 10 & 0.99 & 0.459 & 0.464 & 71 & 4 & 0.11725 & \\
\hline & 91380 & 0.7 & 3.03 & 24364.98704 & 18.16437 & 30.68301 & 10 & 0.9 & 0.592 & 0.658 & 72 & 4 & 0.15125 & 0.032591276 \\
\hline & 91400 & 0.62 & 3.24 & 24366.2908 & 34.23732 & 53.91695 & 10 & 0.8 & 0.635 & 0.794 & 73 & 4 & 0.1622 & 0.018547012 \\
\hline & 91420 & 0.55 & 3.33 & 24367.43434 & 48.33504 & 74.13338 & 10 & 0.71 & 0.652 & 0.918 & 74 & 4 & 0.1664 & 0.013489179 \\
\hline & 91440 & 0.47 & 3.43 & 24368.41373 & 60.40912 & 90.02836 & 10 & 0.61 & 0.671 & 1.1 & 75 & 4 & 0.1714 & 0.011107594 \\
\hline & 91460 & 0.39 & 3.65 & 24369.23133 & 70.4886 & 98.58532 & 10 & 0.5 & 0.715 & 1.43 & 76 & 4 & 0.18255 & 0.010143484 \\
\hline & 91480 & 0.31 & 3.48 & 24369.88792 & 78.58317 & 115.22442 & 10 & 0.4 & 0.682 & 1.705 & 77 & 4 & 0.17405 & 0.008678703 \\
\hline & 91500 & 0.24 & 3.46 & 24370.38115 & 84.66378 & 124.87265 & 10 & 0.31 & 0.678 & 2.187 & 78 & 4 & 0.17305 & 0.008008147 \\
\hline & 91520 & 0.16 & 2.95 & 24370.71102 & 88.73043 & 153.51259 & 10 & 0.21 & 0.578 & 2.752 & 79 & 4 & 0.1476 & 0.006514112 \\
\hline & & 0.08 & 2.7 & 24370.87988 & 90.81217 & 171.66729 & 10 & 0.1 & 0.529 & 5.29 & 80 & 4 & 0.135 & 0.00582521 \\
\hline
\end{tabular}

2004.02.15 META-20 wt\%-U [sonicated] CC-45 0.1-1000 s-1 run 6.txt

Mode Step t[s] n[rpm] M[\%.]

\begin{tabular}{|c|c|c|c|c|}
\hline 1 & 1 & 20 & 0.08 & 3.47 \\
\hline 1 & 1 & 40 & 0.16 & 5.07 \\
\hline 1 & 1 & 60 & 0.24 & 4.84 \\
\hline 1 & 1 & 80 & 0.31 & 4.98 \\
\hline 1 & 1 & 100 & 0.39 & 5.28 \\
\hline 1 & 1 & 120 & 0.47 & 5.31 \\
\hline 1 & 1 & 140 & 0.54 & 5.37 \\
\hline 1 & 1 & 160 & 0.62 & 5.43 \\
\hline 1 & 1 & 180 & 0.7 & 5.58 \\
\hline 1 & 2 & 200 & 0.77 & 5.68 \\
\hline 1 & 2 & 220 & 1.55 & 8.15 \\
\hline 1 & 2 & 240 & 2.32 & 9.48 \\
\hline 1 & 2 & 260 & 3.09 & 10.12 \\
\hline 1 & 2 & 280 & 3.87 & 10.72 \\
\hline 1 & 2 & 300 & 4.65 & 11.31 \\
\hline 1 & 2 & 320 & 5.42 & 12.07 \\
\hline 1 & 2 & 340 & 6.2 & 12.65 \\
\hline 1 & 2 & 360 & 6.97 & 13.1 \\
\hline 1 & 3 & 380 & 7.75 & 13.52 \\
\hline 1 & 3 & 400 & 15.5 & 24.09 \\
\hline 1 & 3 & 420 & 23.24 & 29.36 \\
\hline 1 & 3 & 440 & 30.99 & 30.53 \\
\hline 1 & 3 & 460 & 38.73 & 30.78 \\
\hline 1 & 3 & 480 & 46.48 & 32.09 \\
\hline 1 & 3 & 500 & 54.22 & 32.37 \\
\hline 1 & 3 & 520 & 61.96 & 31.35 \\
\hline 1 & 3 & 540 & 69.71 & 29.81 \\
\hline 1 & 4 & 560 & 77.46 & 29.92 \\
\hline 1 & 4 & 580 & 154.92 & 57.4 \\
\hline 1 & 4 & 600 & 232.38 & 74.66 \\
\hline 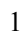 & 4 & 620 & 309.84 & 90.62 \\
\hline
\end{tabular}

Phi[rad]

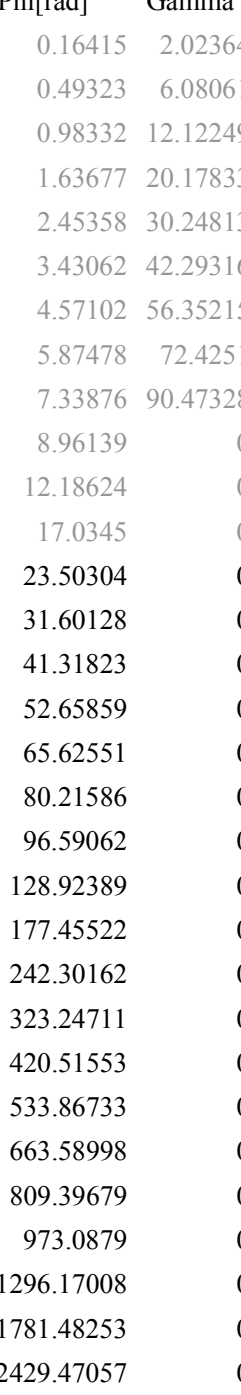

$\mathrm{J}[\mathrm{t}] \quad \mathrm{T}\left[{ }^{\circ} \mathrm{C}\right] \mathrm{D}[1 / \mathrm{s}] \mathrm{Tau}[\mathrm{Pa}] \mathrm{Eta}[\mathrm{Pas}] \mathrm{MP} \#$ Block M[mNm] G[Pa]

$\begin{array}{llllllllll}2.97594 & 10 & 0.1 & 0.68 & 6.8 & 1 & 6 & 0.1737 & 0.336028147\end{array}$

$\begin{array}{lllllllll}6.12347 & 10 & 0.21 & 0.993 & 4.729 & 2 & 6 & 0.2535 & 0.163305984\end{array}$

$\begin{array}{lllllllll}12.78742 & 10 & 0.31 & 0.948 & 3.058 & 3 & 6 & 0.24205 & 0.078201756\end{array}$

$\begin{array}{lllllllll}20.6745 & 10 & 0.4 & 0.976 & 2.44 & 4 & 6 & 0.2492 & 0.04836872\end{array}$

$\begin{array}{lllllllll}29.25348 & 10 & 0.5 & 1.034 & 2.068 & 5 & 6 & 0.2641 & 0.034183931\end{array}$

$\begin{array}{lllllllll}40.66646 & 10 & 0.61 & 1.04 & 1.705 & 6 & 6 & 0.26565 & 0.024590265\end{array}$

$\begin{array}{lllllllll}53.56663 & 10 & 0.7 & 1.052 & 1.503 & 7 & 6 & 0.2687 & 0.018668321\end{array}$

$\begin{array}{lllllllll}68.13267 & 10 & 0.8 & 1.063 & 1.329 & 8 & 6 & 0.2714 & 0.014677232\end{array}$

$\begin{array}{lllllllll}82.77511 & 10 & 0.9 & 1.093 & 1.214 & 9 & 6 & 0.27905 & 0.012080915\end{array}$

$\begin{array}{lllllllll}0 & 10 & 0.99 & 1.112 & 1.123 & 10 & 6 & 0.28385 & 0\end{array}$

$\begin{array}{lllllllll}0 & 10 & 2 & 1.595 & 0.798 & 11 & 6 & 0.40725 & 0\end{array}$

$\begin{array}{lllllllll}0 & 10 & 3 & 1.856 & 0.619 & 12 & 6 & 0.47385 & 0\end{array}$

$\begin{array}{lllllllll}0 & 10 & 3.99 & 1.981 & 0.496 & 13 & 6 & 0.5059 & 0\end{array}$

$\begin{array}{lllllllll}0 & 10 & 5 & 2.099 & 0.42 & 14 & 6 & 0.5359 & 0\end{array}$

$\begin{array}{lllllllll}0 & 10 & 6 & 2.215 & 0.369 & 15 & 6 & 0.5656 & 0\end{array}$

$\begin{array}{lllllllll}0 & 10 & 7 & 2.364 & 0.338 & 16 & 6 & 0.60355 & 0\end{array}$

$\begin{array}{lllllllll}0 & 10 & 8 & 2.477 & 0.31 & 17 & 6 & 0.6325 & 0\end{array}$

$\begin{array}{lllllllll}0 & 10 & 9 & 2.564 & 0.285 & 18 & 6 & 0.65475 & 0\end{array}$

$\begin{array}{lllllllll}0 & 10 & 10.01 & 2.647 & 0.264 & 19 & 6 & 0.67595 & 0\end{array}$

$\begin{array}{lllllllll}0 & 10 & 20.01 & 4.717 & 0.236 & 20 & 6 & 1.20455 & 0\end{array}$

$\begin{array}{lllllllll}0 & 10 & 30 & 5.748 & 0.192 & 21 & 6 & 1.4678 & 0\end{array}$

$\begin{array}{lllllllll}0 & 10 & 40.01 & 5.978 & 0.149 & 22 & 6 & 1.52655 & 0\end{array}$

$\begin{array}{lllllllll}0 & 10 & 50 & 6.027 & 0.121 & 23 & 6 & 1.53915 & 0\end{array}$

$\begin{array}{lllllllll}0 & 10 & 60.01 & 6.283 & 0.105 & 24 & 6 & 1.60455 & 0\end{array}$

$\begin{array}{lllllllll}0 & 10 & 70 & 6.337 & 0.091 & 25 & 6 & 1.6183 & 0\end{array}$

$\begin{array}{lllllllll}0 & 10 & 79.99 & 6.138 & 0.077 & 26 & 6 & 1.5674 & 0\end{array}$

$\begin{array}{lllllllll}0 & 10 & 90 & 5.837 & 0.065 & 27 & 6 & 1.4905 & 0\end{array}$

$\begin{array}{lllllllll}0 & 10 & 100 & 5.858 & 0.059 & 28 & 6 & 1.496 & 0\end{array}$

$\begin{array}{lllllllll}0 & 10 & 200 & 11.239 & 0.056 & 29 & 6 & 2.8699 & 0\end{array}$

$\begin{array}{lllllllll}0 & 10 & 300 & 14.619 & 0.049 & 30 & 6 & 3.73315 & 0\end{array}$

$\begin{array}{lllllllll}0 & 10 & 400 & 17.743 & 0.044 & 31 & 6 & 4.531 & 0\end{array}$ 


\begin{tabular}{|c|c|c|c|c|c|c|c|c|c|c|c|c|c|c|}
\hline & 640 & 387.3 & 110.77 & 3238.95611 & 0 & 0 & 10 & 500 & 21.688 & 0.043 & 32 & 6 & 5.5383 & \\
\hline & 660 & 464.75 & 130.19 & 4212.01103 & 0 & 0 & 10 & 599.99 & 25.491 & 0.042 & 33 & 6 & 6.50945 & \\
\hline & 680 & 542.21 & 149.96 & 5345.4309 & 0 & 0 & 10 & 699.99 & 29.361 & 0.042 & 34 & 6 & 7.49775 & \\
\hline & 700 & 619.67 & 170.83 & 6642.25757 & 0 & 0 & 10 & 799.99 & 33.448 & 0.042 & 35 & 6 & 8.5414 & \\
\hline & 720 & 697.13 & 192.73 & 8100.98543 & 0 & 0 & 10 & 899.99 & 37.736 & 0.042 & 36 & 6 & 9.63635 & \\
\hline & 740 & 774.59 & 214.22 & 9721.53987 & 0 & 0 & 10 & 1000 & 41.945 & 0.042 & 37 & 6 & 10.71115 & \\
\hline & 750 & 774.59 & 210.43 & 10550.6156 & 0 & 0 & 10 & 1000 & 41.202 & 0.041 & 38 & 6 & 10.5214 & \\
\hline & 760 & 774.59 & 209.14 & 11362.57357 & 0 & 0 & 10 & 1000 & 40.95 & 0.041 & 39 & 6 & 10.4571 & \\
\hline & 770 & 774.59 & 208.15 & 12172.50679 & 0 & 0 & 10 & 1000 & 40.756 & 0.041 & 40 & 6 & 10.4076 & \\
\hline & 780 & 774.59 & 207.4 & 12984.87081 & 0 & 0 & 10 & 1000 & 40.61 & 0.041 & 41 & 6 & 10.3702 & \\
\hline & 790 & 774.59 & 206.6 & 13794.80246 & 0 & 0 & 10 & 1000 & 40.452 & 0.04 & 42 & 6 & 10.3299 & \\
\hline & 800 & 774.59 & 205.88 & 14605.5462 & 0 & 0 & 10 & 1000 & 40.31 & 0.04 & 43 & 6 & 10.29375 & \\
\hline & 820 & 774.59 & 205.15 & 16246.09531 & 0 & 0 & 10 & 1000 & 40.168 & 0.04 & 44 & 6 & 10.2573 & \\
\hline & 840 & 697.13 & 180.51 & 17708.15248 & 0 & 0 & 10 & 899.99 & 35.344 & 0.039 & 45 & 6 & 9.0255 & \\
\hline & 860 & 619.67 & 157.34 & 19006.68189 & 0 & 0 & 10 & 799.99 & 30.806 & 0.039 & 46 & 6 & 7.8668 & \\
\hline & 880 & 542.21 & 134.8 & 20144.19761 & 0 & 0 & 10 & 699.99 & 26.394 & 0.038 & 47 & 6 & 6.73995 & \\
\hline & 900 & 464.75 & 112.97 & 21119.36133 & 0 & 0 & 10 & 599.99 & 22.119 & 0.037 & 48 & 6 & 5.64825 & \\
\hline & 920 & 387.3 & 91.75 & 21931.64681 & 0 & 0 & 10 & 500 & 17.965 & 0.036 & 49 & 6 & 4.58755 & \\
\hline & 940 & 309.84 & 71.07 & 22581.37922 & 0 & 0 & 10 & 400 & 13.916 & 0.035 & 50 & 6 & 3.55355 & \\
\hline & 960 & 232.38 & 51.48 & 23069.4492 & 0 & 0 & 10 & 300 & 10.079 & 0.034 & 51 & 6 & 2.5738 & \\
\hline & 980 & 154.92 & 32.66 & 23395.24807 & 0 & 0 & 10 & 200 & 6.395 & 0.032 & 52 & 6 & 1.633 & \\
\hline & 61000 & 77.46 & 16.18 & 23558.7821 & 0 & 0 & 10 & 100 & 3.169 & 0.032 & 53 & 6 & 0.8092 & \\
\hline & 71020 & 69.71 & 15.66 & 23705.95394 & 0 & 0 & 10 & 90 & 3.065 & 0.034 & 54 & 6 & 0.7828 & \\
\hline & 71040 & 61.97 & 14.54 & 23835.78968 & 0 & 0 & 10 & 80 & 2.846 & 0.036 & 55 & 6 & 0.7268 & \\
\hline & 71060 & 54.22 & 13.22 & 23949.46507 & 0 & 0 & 10 & 70 & 2.589 & 0.037 & 56 & 6 & 0.66115 & \\
\hline & 71080 & 46.48 & 11.76 & 24046.91963 & 0 & 0 & 10 & 60.01 & 2.302 & 0.038 & 57 & 6 & 0.5879 & \\
\hline & 71100 & 38.73 & 10.2 & 24128.24211 & 0 & 0 & 10 & 50 & 1.997 & 0.04 & 58 & 6 & 0.51005 & \\
\hline & 71120 & 30.99 & 9.09 & 24193.2181 & 0 & 0 & 10 & 40.01 & 1.781 & 0.045 & 59 & 6 & 0.4547 & \\
\hline & 71140 & 23.24 & 7.35 & 24242.00546 & 0 & 0 & 10 & 30 & 1.439 & 0.048 & 60 & 6 & 0.36755 & \\
\hline & 71160 & 15.5 & 5.67 & 24274.54687 & 0 & 0 & 10 & 20.01 & 1.11 & 0.055 & 61 & 6 & 0.28335 & \\
\hline & 71180 & 7.75 & 3.63 & 24290.8965 & 0 & 0 & 10 & 10.01 & 0.71 & 0.071 & 62 & 6 & 0.18135 & 0 \\
\hline & 81200 & 6.97 & 4.2 & 24305.60544 & 0 & 0 & 10 & 9 & 0.822 & 0.091 & 63 & 6 & 0.2098 & 0 \\
\hline & 81220 & 6.2 & 4.39 & 24318.581 & 0 & 0 & 10 & 8 & 0.861 & 0.108 & 64 & 6 & 0.21975 & 0 \\
\hline & 81240 & 5.42 & 4.41 & 24329.94414 & 0 & 0 & 10 & 7 & 0.862 & 0.123 & 65 & 6 & 0.22025 & 0 \\
\hline & 81260 & 4.64 & 4.51 & 24339.68936 & 0 & 0 & 10 & 5.99 & 0.883 & 0.147 & 66 & 6 & 0.22555 & 0 \\
\hline & 81280 & 3.87 & 4.24 & 24347.80174 & 0 & 0 & 10 & 5 & 0.83 & 0.166 & 67 & 6 & 0.2119 & 0 \\
\hline & 81300 & 3.09 & 4.16 & 24354.29541 & 0 & 0 & 10 & 3.99 & 0.814 & 0.204 & 68 & 6 & 0.20785 & 0 \\
\hline & 81320 & 2.32 & 3.78 & 24359.16645 & 0 & 0 & 10 & 3 & 0.74 & 0.247 & 69 & 6 & 0.18905 & 0 \\
\hline & 81340 & 1.54 & 3 & 24362.41329 & 0 & 0 & 10 & 1.99 & 0.587 & 0.295 & 70 & 6 & 0.14995 & 0 \\
\hline & 81360 & 0.77 & 2.33 & 24364.0367 & 0 & 0 & 10 & 0.99 & 0.456 & 0.461 & 71 & 6 & 0.11655 & 0 \\
\hline & 91380 & 0.7 & 2.86 & 24365.5109 & 18.17405 & 32.51166 & 10 & 0.9 & 0.559 & 0.621 & 72 & 6 & 0.14285 & 0.030758141 \\
\hline & 91400 & 0.62 & 3.07 & 24366.81701 & 34.27605 & 57.0316 & 10 & 0.8 & 0.601 & 0.751 & 73 & 6 & 0.15355 & 0.017534109 \\
\hline & 91420 & 0.55 & 3.38 & 24367.9582 & 48.34472 & 73.02817 & 10 & 0.71 & 0.662 & 0.932 & 74 & 6 & 0.169 & 0.013693326 \\
\hline & 91440 & 0.47 & 3.66 & 24368.93837 & 60.42848 & 84.27949 & 10 & 0.61 & 0.717 & 1.175 & 75 & 6 & 0.1832 & 0.011865266 \\
\hline & 91460 & 0.39 & 3.72 & 24369.75676 & 70.51765 & 96.7319 & 10 & 0.5 & 0.729 & 1.458 & 76 & 6 & 0.18615 & 0.010337837 \\
\hline & 91480 & 0.31 & 3.53 & 24370.41178 & 78.59285 & 113.57332 & 10 & 0.4 & 0.692 & 1.73 & 77 & 6 & 0.1766 & 0.008804872 \\
\hline & 91500 & 0.24 & 3.28 & 24370.9058 & 84.68315 & 131.90501 & 10 & 0.31 & 0.642 & 2.071 & 78 & 6 & 0.16395 & 0.007581201 \\
\hline & 91520 & 0.16 & 3.08 & 24371.23645 & 88.75948 & 147.19624 & 10 & 0.21 & 0.603 & 2.871 & 79 & 6 & 0.1541 & 0.006793641 \\
\hline & 91540 & 0.08 & 2.31 & 24371.40531 & 90.84122 & 201.42133 & 10 & 0.1 & 0.451 & 4.51 & 80 & 6 & 0.11525 & 0.004964707 \\
\hline
\end{tabular}


2004.02.15 META-20 wt\%-U [sonicated] CC-45 0.1-1000 s-1 run 7.txt

Mode Step t[s] n[rpm] M[\%.]

\begin{tabular}{|c|c|c|c|c|c|c|}
\hline & 1 & 20 & 0.08 & 3.51 & 0.16415 & 2.023 \\
\hline & 1 & 40 & 0.16 & 5.2 & 0.49323 & 6.080 \\
\hline & 1 & 60 & 0.24 & 4.93 & 0.9841 & 12.132 \\
\hline & 1 & 80 & 0.31 & 5.1 & 1.63756 & 520.188 \\
\hline & 1 & 100 & 0.39 & 5.24 & 2.4528 & \\
\hline & 1 & 120 & 0.47 & 5.63 & 3.4314 & $\begin{array}{l}+42.302 \\
\end{array}$ \\
\hline & 1 & 140 & 0.54 & 5.62 & 4.5718 & 36 \\
\hline & 1 & 160 & 0.62 & 5.68 & 5.87321 & \\
\hline & 1 & 180 & 0.7 & 5.65 & 7.33955 & \\
\hline & 2 & 200 & 0.77 & 5.65 & 8.96139 & \\
\hline & 2 & 220 & 1.55 & 8.22 & 12.18624 & \\
\hline & 2 & 240 & 2.32 & 9.52 & 17.03686 & \\
\hline & 2 & 260 & 3.09 & 10.03 & 23.5054 & \\
\hline & 2 & 280 & 3.87 & 10.64 & 31.60207 & \\
\hline & 2 & 300 & 4.65 & 11.21 & 41.31901 & \\
\hline & 2 & 320 & 5.42 & 12.11 & 52.65623 & \\
\hline & 2 & 340 & 6.2 & 12.47 & 65.62316 & \\
\hline & 2 & 360 & 6.97 & 12.97 & 80.21664 & \\
\hline & 3 & 380 & 7.75 & 13.24 & 96.57884 & \\
\hline & 3 & 400 & 15.5 & 23.71 & 128.9129 & \\
\hline & 3 & 420 & 23.24 & 28.96 & 177.49606 & \\
\hline & 3 & 440 & 30.99 & 31.45 & 242.28984 & \\
\hline & 3 & 460 & 38.73 & 30.99 & 323.28873 & \\
\hline & 3 & 480 & 46.48 & 31.39 & 420.43934 & \\
\hline & 3 & 500 & 54.22 & 28.86 & 533.93409 & \\
\hline & 3 & 520 & 61.96 & 26.68 & 663.65595 & \\
\hline & 3 & 540 & 69.71 & 27.63 & 809.42585 & \\
\hline & 4 & 560 & 77.46 & 29.09 & 973.04078 & \\
\hline & 4 & 580 & 154.92 & 56.28 & 1296.48738 & \\
\hline & 4 & 600 & 232.38 & 74.33 & 1781.55635 & \\
\hline & 4 & 620 & 309.84 & 87.63 & 2429.90882 & \\
\hline & 4 & 640 & 387.3 & 106.95 & 3239.1933 & \\
\hline & 4 & 660 & 464.75 & 125.91 & 4210.78973 & \\
\hline & 4 & 680 & 542.21 & 147.72 & 5345.6673 & \\
\hline & 4 & 700 & 619.67 & 167.05 & 6642.77829 & \\
\hline & 4 & 720 & 697.13 & 188.91 & 8100.04688 & \\
\hline & 4 & 740 & 774.59 & 209.4 & 9722.18311 & \\
\hline & 5 & 750 & 774.59 & 206.01 & 10549.63542 & \\
\hline & 5 & 760 & 774.59 & 204.71 & 11361.59575 & \\
\hline & 5 & 770 & 774.59 & 203.83 & 12173.55372 & \\
\hline & 5 & 780 & 774.59 & 203.17 & 12985.108 & \\
\hline & 5 & 790 & 774.59 & 202.75 & 13795.44491 & \\
\hline & 5 & 800 & 774.59 & 202.34 & 14605.37813 & \\
\hline & 6 & 820 & 774.59 & 201.97 & 16246.33172 & \\
\hline & 6 & 840 & 697.13 & 177.8 & 17708.42972 & \\
\hline & 6 & 860 & 619.67 & 154.94 & 19007.60866 & \\
\hline & 6 & 880 & 542.21 & 132.76 & 20143.42007 & \\
\hline & 6 & 900 & 464.75 & 111.37 & 21118.62541 & \\
\hline
\end{tabular}

$\mathrm{J}[\mathrm{t}] \quad \mathrm{T}\left[{ }^{\circ} \mathrm{C}\right] \mathrm{D}[1 / \mathrm{s}] \mathrm{Tau}[\mathrm{Pa}]$ Eta[Pas $]$ MP\# Block M[mNm] G[Pa]

\begin{tabular}{|c|c|c|c|c|c|c|c|c|}
\hline 2.94133 & 10 & 0.1 & 0.688 & 6.88 & 1 & 7 & 0.1757 & 0.33998142 \\
\hline 5.97309 & 10 & 0.21 & 1.018 & 4.848 & 2 & 7 & 0.2599 & 0.167417414 \\
\hline 12.57218 & 10 & 0.31 & 0.965 & 3.113 & 3 & 7 & 0.24635 & 0.079540593 \\
\hline 20.2082 & 10 & 0.4 & 0.999 & 2.498 & 4 & 7 & 0.2551 & 0.049484818 \\
\hline .47214 & 10 & 0.5 & 1.026 & 2.052 & 5 & 7 & 0.26205 & 0.033930311 \\
\hline .35249 & 10 & 0.61 & 1.103 & 1.808 & 6 & 7 & 0.2817 & 0.0260739 \\
\hline 23798 & 10 & 0.7 & 1.1 & 1.571 & 7 & 7 & 0.2809 & 0.019516755 \\
\hline 11301 & 10 & 0.8 & 1.112 & 1.39 & 8 & 7 & 0.2839 & 0.015357901 \\
\hline 81092 & 10 & 0.9 & 1.106 & 1.229 & 9 & 7 & 0.28245 & 0.012223296 \\
\hline 0 & 10 & 0.99 & 1.106 & 1.117 & 10 & 7 & 0.2824 & 0 \\
\hline 0 & 10 & 2 & 1.609 & 0.805 & 11 & 7 & 0.4108 & 0 \\
\hline 0 & 10 & 3 & 1.864 & 0.621 & 12 & 7 & 0.47605 & 0 \\
\hline 0 & 10 & 3.99 & 1.964 & 0.492 & 13 & 7 & 0.5016 & 0 \\
\hline 0 & 10 & 5 & 2.083 & 0.417 & 14 & 7 & 0.5318 & 0 \\
\hline 0 & 10 & 6 & 2.195 & 0.366 & 15 & 7 & 0.56055 & 0 \\
\hline 0 & 10 & 7 & 2.371 & 0.339 & 16 & 7 & 0.60555 & 0 \\
\hline 0 & 10 & 8 & 2.441 & 0.305 & 17 & 7 & 0.62325 & 0 \\
\hline 0 & 10 & 9 & 2.54 & 0.282 & 18 & 7 & 0.6487 & 0 \\
\hline 0 & 10 & 10.01 & 2.591 & 0.259 & 19 & 7 & 0.66175 & 0 \\
\hline 0 & 10 & 20.01 & 4.643 & 0.232 & 20 & 7 & 1.1857 & 0 \\
\hline 0 & 10 & 30 & 5.671 & 0.189 & 21 & 7 & 1.44815 & 0 \\
\hline 0 & 10 & 40.01 & 6.158 & 0.154 & 22 & 7 & 1.57255 & 0 \\
\hline 0 & 10 & 50 & 6.068 & 0.121 & 23 & 7 & 1.5496 & 0 \\
\hline 0 & 10 & 60.01 & 6.145 & 0.102 & 24 & 7 & 1.5693 & 0 \\
\hline 0 & 10 & 70 & 5.65 & 0.081 & 25 & 7 & 1.44275 & 0 \\
\hline 0 & 10 & 79.99 & 5.224 & 0.065 & 26 & 7 & 1.33405 & 0 \\
\hline 0 & 10 & 90 & 5.41 & 0.06 & 27 & 7 & 1.38155 & 0 \\
\hline 0 & 10 & 100 & 5.696 & 0.057 & 28 & 7 & 1.45445 & 0 \\
\hline 0 & 10 & 200 & 11.02 & 0.055 & 29 & 7 & 2.8141 & 0 \\
\hline 0 & 10 & 300 & 14.554 & 0.049 & 30 & 7 & 3.7165 & 0 \\
\hline 0 & 10 & 400 & 17.157 & 0.043 & 31 & 7 & 4.3813 & 0 \\
\hline 0 & 10 & 500 & 20.941 & 0.042 & 32 & 7 & 5.34765 & 0 \\
\hline 0 & 10 & 599.99 & 24.652 & 0.041 & 33 & 7 & 6.2953 & 0 \\
\hline 0 & 10 & 699.99 & 28.924 & 0.041 & 34 & 7 & 7.386 & 0 \\
\hline 0 & 10 & 799.99 & 32.708 & 0.041 & 35 & 7 & 8.3525 & 0 \\
\hline 0 & 10 & 899.99 & 36.989 & 0.041 & 36 & 7 & 9.4457 & 0 \\
\hline 0 & 10 & 1000 & 41.001 & 0.041 & 37 & 7 & 10.47 & 0 \\
\hline 0 & 10 & 1000 & 40.338 & 0.04 & 38 & 7 & 10.3007 & 0 \\
\hline 0 & 10 & 1000 & 40.083 & 0.04 & 39 & 7 & 10.2357 & 0 \\
\hline 0 & 10 & 1000 & 39.909 & 0.04 & 40 & 7 & 10.19135 & 0 \\
\hline 0 & 10 & 1000 & 39.781 & 0.04 & 41 & 7 & 10.1585 & 0 \\
\hline 0 & 10 & 1000 & 39.699 & 0.04 & 42 & 7 & 10.13755 & 0 \\
\hline 0 & 10 & 1000 & 39.619 & 0.04 & 43 & 7 & 10.11715 & 0 \\
\hline 0 & 10 & 1000 & 39.546 & 0.04 & 44 & 7 & 10.09855 & 0 \\
\hline 0 & 10 & 899.99 & 34.814 & 0.039 & 45 & 7 & 8.89015 & 0 \\
\hline 0 & 10 & 799.99 & 30.338 & 0.038 & 46 & 7 & 7.7472 & 0 \\
\hline 0 & 10 & 699.99 & 25.994 & 0.037 & 47 & 7 & 6.63785 & 0 \\
\hline 0 & 10 & 599.99 & 21.806 & 0.036 & 48 & 7 & 5.5684 & 0 \\
\hline
\end{tabular}




\begin{tabular}{|c|c|c|c|c|c|c|c|c|c|c|c|c|c|c|}
\hline & 920 & 387.3 & 90.48 & 21930.90932 & 0 & 0 & 10 & 500 & 17.717 & 0.035 & 49 & 7 & 4.5242 & 0 \\
\hline & 940 & 309.84 & 70.23 & 22581.12868 & 0 & 0 & 10 & 400 & 13.75 & 0.034 & 50 & 7 & 3.51135 & \\
\hline & 960 & 232.38 & 50.97 & 23069.31883 & 0 & 0 & 10 & 300 & 9.979 & 0.033 & 51 & 7 & 2.54835 & \\
\hline & 980 & 154.92 & 32.4 & 23394.91585 & 0 & 0 & 10 & 200 & 6.344 & 0.032 & 52 & 7 & 1.6201 & \\
\hline & 61000 & 77.46 & 16.04 & 23558.44988 & 0 & 0 & 10 & 100 & 3.14 & 0.031 & 53 & 7 & 0.8018 & \\
\hline & 71020 & 69.71 & 15.6 & 23705.59108 & 0 & 0 & 10 & 90 & 3.054 & 0.034 & 54 & 7 & 0.77985 & \\
\hline & 71040 & 61.97 & 14.42 & 23835.4551 & 0 & 0 & 10 & 80 & 2.824 & 0.035 & 55 & 7 & 0.72105 & \\
\hline & 71060 & 54.22 & 13.15 & 23949.13049 & 0 & 0 & 10 & 70 & 2.576 & 0.037 & 56 & 7 & 0.6577 & \\
\hline & 71080 & 46.48 & 11.65 & 24046.6416 & 0 & 0 & 10 & 60.01 & 2.281 & 0.038 & 57 & 7 & 0.58245 & \\
\hline & 71100 & 38.73 & 10.08 & 24127.86276 & 0 & 0 & 10 & 50 & 1.974 & 0.039 & 58 & 7 & 0.5041 & \\
\hline & 71120 & 30.99 & 8.9 & 24192.87959 & 0 & 0 & 10 & 40.01 & 1.743 & 0.044 & 59 & 7 & 0.4451 & \\
\hline & 71140 & 23.24 & 7.34 & 24241.65518 & 0 & 0 & 10 & 30 & 1.437 & 0.048 & 60 & 7 & 0.36685 & \\
\hline & 71160 & 15.5 & 5.7 & 24274.24527 & 0 & 0 & 10 & 20.01 & 1.117 & 0.056 & 61 & 7 & 0.2852 & \\
\hline & 71180 & 7.75 & 3.68 & 24290.58234 & 0 & 0 & 10 & 10.01 & 0.72 & 0.072 & 62 & 7 & 0.1838 & \\
\hline & 81200 & 6.97 & 4.16 & 24305.28499 & 0 & 0 & 10 & 9 & 0.815 & 0.091 & 63 & 7 & 0.208 & \\
\hline & 81220 & 6.2 & 4.34 & 24318.27784 & 0 & 0 & 10 & 8 & 0.849 & 0.106 & 64 & 7 & 0.2169 & \\
\hline & 81240 & 5.42 & 4.18 & 24329.64098 & 0 & 0 & 10 & 7 & 0.819 & 0.117 & 65 & 7 & 0.20915 & \\
\hline & 81260 & 4.64 & 4.4 & 24339.37677 & 0 & 0 & 10 & 5.99 & 0.861 & 0.144 & 66 & 7 & 0.21995 & \\
\hline & 81280 & 3.87 & 4.26 & 24347.49386 & 0 & 0 & 10 & 5 & 0.833 & 0.167 & 67 & 7 & 0.2128 & \\
\hline & 81300 & 3.09 & 4.17 & 24353.98361 & 0 & 0 & 10 & 3.99 & 0.816 & 0.205 & 68 & 7 & 0.20835 & \\
\hline & 81320 & 2.32 & 3.78 & 24358.85386 & 0 & 0 & 10 & 3 & 0.74 & 0.247 & 69 & 7 & 0.18905 & \\
\hline & 81340 & 1.54 & 3.01 & 24362.10148 & 0 & 0 & 10 & 1.99 & 0.589 & 0.296 & 70 & 7 & 0.1504 & \\
\hline & 81360 & 0.77 & 2.39 & 24363.72569 & 0 & 0 & 10 & 0.99 & 0.468 & 0.473 & 71 & 7 & 0.1196 & \\
\hline & 91380 & 0.7 & 3.13 & 24365.20066 & 18.18373 & 29.71193 & 10 & 0.9 & 0.612 & 0.68 & 72 & 7 & 0.15625 & 0.033656461 \\
\hline & 91400 & 0.62 & 3.39 & 24366.506 & 34.27605 & 51.62048 & 10 & 0.8 & 0.664 & 0.83 & 73 & 7 & 0.1696 & 0.019372127 \\
\hline & 91420 & 0.55 & 3.34 & 24367.64718 & 48.34472 & 74.03468 & 10 & 0.71 & 0.653 & 0.92 & 74 & 7 & 0.1668 & 0.013507163 \\
\hline & 91440 & 0.47 & 3.46 & 24368.62736 & 60.42848 & 89.25907 & 10 & 0.61 & 0.677 & 1.11 & 75 & 7 & 0.173 & 0.011203327 \\
\hline & 91460 & 0.39 & 3.46 & 24369.44653 & 70.52733 & 104.17611 & 10 & 0.5 & 0.677 & 1.354 & 76 & 7 & 0.17285 & 0.009599116 \\
\hline & 91480 & 0.31 & 3.35 & 24370.10076 & 78.59285 & 119.62364 & 10 & 0.4 & 0.657 & 1.643 & 77 & 7 & 0.16765 & 0.008359539 \\
\hline & 91500 & 0.24 & 3.14 & 24370.59399 & 84.67346 & 137.6802 & 10 & 0.31 & 0.615 & 1.984 & 78 & 7 & 0.1571 & 0.007263197 \\
\hline & 91520 & 0.16 & 3.04 & 24370.92543 & 88.75948 & 149.17535 & 10 & 0.21 & 0.595 & 2.833 & 79 & 7 & 0.15195 & 0.006703509 \\
\hline & 91540 & 0.08 & 2.47 & 24371.09429 & 90.84121 & 188.07665 & 10 & 0.1 & 0.483 & 4.83 & 80 & 7 & 0.12335 & 0.00531697 \\
\hline
\end{tabular}

2004.02.15 META-20 wt\%-U [sonicated] CC-45 0.1-1000 s-1 run 8.txt

\begin{tabular}{|c|c|c|c|c|c|c|c|c|c|c|c|c|c|c|c|}
\hline Mode $S$ & Step & $\mathrm{t}[\mathrm{s}]$ & [rpm] & $\mathrm{M}[\%]$. & $\mathrm{Phi}[\mathrm{rad}]$ & Gamma & $J[t]$ & $\mathrm{T}\left[{ }^{\circ} \mathrm{C}\right]$ & $\mathrm{D}[1 / \mathrm{s}]$ & $\mathrm{Tau}[\mathrm{Pa}]$ & $\mathrm{Eta}[\mathrm{Pas}]$ & MP\# & Block & $\mathrm{M}[\mathrm{mNm}]$ & $\mathrm{G}[\mathrm{Pa}]$ \\
\hline 1 & 1 & 20 & 0.08 & 3.37 & 0.16415 & 2.02364 & 3.06148 & 10 & 0.1 & 0.661 & 6.61 & 1 & 8 & 0.1687 & 0.326639126 \\
\hline 1 & 1 & 40 & 0.16 & 5.13 & 0.49323 & 6.08061 & 6.05035 & 10 & 0.21 & 1.005 & 4.786 & 2 & 8 & 0.2566 & 0.16527947 \\
\hline 1 & 1 & 60 & 0.24 & 4.87 & 0.9841 & 12.13217 & 12.71715 & 10 & 0.31 & 0.954 & 3.077 & 3 & 8 & 0.2437 & 0.078633913 \\
\hline 1 & 1 & 80 & 0.31 & 5.12 & 1.63834 & 20.19769 & 20.15736 & 10 & 0.4 & 1.002 & 2.505 & 4 & 8 & 0.2558 & 0.049609634 \\
\hline 1 & 1 & 100 & 0.39 & 5.22 & 2.45201 & 30.22876 & 29.57801 & 10 & 0.5 & 1.022 & 2.044 & 5 & 8 & 0.26095 & 0.033808863 \\
\hline 1 & 1 & 120 & 0.47 & 5.35 & 3.4314 & 42.30284 & 40.40382 & 10 & 0.61 & 1.047 & 1.716 & 6 & 8 & 0.26725 & 0.024750111 \\
\hline 1 & 1 & 140 & 0.54 & 5.49 & 4.57259 & 56.37151 & 52.48739 & 10 & 0.7 & 1.074 & 1.534 & 7 & 8 & 0.27435 & 0.019052177 \\
\hline 1 & 1 & 160 & 0.62 & 5.47 & 5.87399 & 72.41542 & 67.55163 & 10 & 0.8 & 1.072 & 1.34 & 8 & 8 & 0.2737 & 0.014803477 \\
\hline 1 & 1 & 180 & 0.7 & 5.53 & 7.33876 & 90.47328 & 83.53942 & 10 & 0.9 & 1.083 & 1.203 & 9 & 8 & 0.2765 & 0.011970385 \\
\hline 1 & 2 & 200 & 0.77 & 5.61 & 8.96139 & 0 & 0 & 10 & 0.99 & 1.099 & 1.11 & 10 & 8 & 0.2807 & 0 \\
\hline 1 & 2 & 220 & 1.55 & 8.54 & 12.18702 & 0 & 0 & 10 & 2 & 1.672 & 0.836 & 11 & 8 & 0.427 & 0 \\
\hline 1 & 2 & 240 & 2.32 & 9.43 & 17.0345 & 0 & 0 & 10 & 3 & 1.846 & 0.615 & 12 & 8 & 0.4714 & \\
\hline 1 & 2 & 260 & 3.09 & 10.23 & 23.50304 & 0 & 0 & 10 & 3.99 & 2.004 & 0.502 & 13 & 8 & 0.51165 & \\
\hline 1 & 2 & 280 & 3.87 & 11.02 & 31.6005 & 0 & 0 & 10 & 5 & 2.157 & 0.431 & 14 & 8 & 0.5509 & \\
\hline 1 & 2 & 300 & 4.65 & 11.18 & 41.31273 & 0 & 0 & 10 & 6 & 2.189 & 0.365 & 15 & 8 & 0.55895 & \\
\hline
\end{tabular}




\begin{tabular}{|c|c|c|c|c|c|c|c|c|c|c|c|c|c|c|}
\hline & 2320 & 5.42 & 11.86 & 52.6633 & 0 & 0 & 10 & 7 & 2.322 & 0.332 & 16 & 8 & 0.5929 & 0 \\
\hline 1 & 2340 & 6.2 & 12.33 & 65.6263 & 0 & 0 & 10 & 8 & 2.414 & 0.302 & 17 & 8 & 0.61655 & 0 \\
\hline & 2360 & 6.97 & 12.9 & 80.22057 & 0 & 0 & 10 & 9 & 2.526 & 0.281 & 18 & 8 & 0.64505 & 0 \\
\hline & $3 \quad 380$ & 7.75 & 13.29 & 96.57413 & 0 & 0 & 10 & 10.01 & 2.603 & 0.26 & 19 & 8 & 0.6647 & 0 \\
\hline & 3400 & 15.5 & 23.61 & 128.94039 & 0 & 0 & 10 & 20.01 & 4.623 & 0.231 & 20 & 8 & 1.1805 & 0 \\
\hline & 3420 & 23.24 & 28.74 & 177.48349 & 0 & 0 & 10 & 30 & 5.627 & 0.188 & 21 & 8 & 1.4368 & 0 \\
\hline & 3440 & 30.99 & 29.21 & 242.29376 & 0 & 0 & 10 & 40.01 & 5.719 & 0.143 & 22 & 8 & 1.4605 & 0 \\
\hline & 3460 & 38.73 & 29.82 & 323.23533 & 0 & 0 & 10 & 50 & 5.838 & 0.117 & 23 & 8 & 1.49085 & 0 \\
\hline & 3480 & 46.48 & 31.52 & 420.50375 & 0 & 0 & 10 & 60.01 & 6.172 & 0.103 & 24 & 8 & 1.576 & 0 \\
\hline & 3500 & 54.22 & 31.51 & 533.99771 & 0 & 0 & 10 & 70 & 6.17 & 0.088 & 25 & 8 & 1.5756 & 0 \\
\hline & 3520 & 61.96 & 30.78 & 663.65516 & 0 & 0 & 10 & 79.99 & 6.027 & 0.075 & 26 & 8 & 1.5391 & 0 \\
\hline & 3540 & 69.71 & 30.79 & 809.50203 & 0 & 0 & 10 & 90 & 6.028 & 0.067 & 27 & 8 & 1.53945 & 0 \\
\hline & 4560 & 77.46 & 30.28 & 973.15309 & 0 & 0 & 10 & 100 & 5.93 & 0.059 & 28 & 8 & 1.5142 & 0 \\
\hline & $4 \quad 580$ & 154.92 & 56.34 & 1296.60048 & 0 & 0 & 10 & 200 & 11.031 & 0.055 & 29 & 8 & 2.8169 & 0 \\
\hline & 4600 & 232.38 & 71.62 & 1782.19724 & 0 & 0 & 10 & 300 & 14.023 & 0.047 & 30 & 8 & 3.5809 & 0 \\
\hline & $4 \quad 620$ & 309.84 & 87.39 & 2429.25144 & 0 & 0 & 10 & 400 & 17.111 & 0.043 & 31 & 8 & 4.3694 & 0 \\
\hline & $4 \quad 640$ & 387.3 & 104.82 & 3239.95592 & 0 & 0 & 10 & 500 & 20.525 & 0.041 & 32 & 8 & 5.2412 & 0 \\
\hline & $4 \quad 660$ & 464.75 & 124.2 & 4211.34972 & 0 & 0 & 10 & 599.99 & 24.318 & 0.041 & 33 & 8 & 6.21 & 0 \\
\hline & 680 & 542.21 & 144.06 & 5346.14561 & 0 & 0 & 10 & 699.99 & 28.208 & 0.04 & 34 & 8 & 7.2032 & 0 \\
\hline & 700 & 619.67 & 163.98 & 6641.67323 & 0 & 0 & 10 & 799.99 & 32.107 & 0.04 & 35 & 8 & 8.19885 & 0 \\
\hline & 720 & 697.13 & 184.79 & 8100.44508 & 0 & 0 & 10 & 899.99 & 36.181 & 0.04 & 36 & 8 & 9.2393 & 0 \\
\hline & 4740 & 774.59 & 205.49 & 9722.62136 & 0 & 0 & 10 & 1000 & 40.236 & 0.04 & 37 & 8 & 10.27465 & 0 \\
\hline & 750 & 774.59 & 202.41 & 10550.88499 & 0 & 0 & 10 & 1000 & 39.632 & 0.04 & 38 & 8 & 10.1206 & 0 \\
\hline & 5760 & 774.59 & 201.19 & 11363.65506 & 0 & 0 & 10 & 1000 & 39.394 & 0.039 & 39 & 8 & 10.0597 & 0 \\
\hline & $5 \quad 770$ & 774.59 & 200.14 & 12173.58828 & 0 & 0 & 10 & 1000 & 39.188 & 0.039 & 40 & 8 & 10.00715 & 0 \\
\hline & $5 \quad 780$ & 774.59 & 199.34 & 12985.14177 & 0 & 0 & 10 & 1000 & 39.031 & 0.039 & 41 & 8 & 9.9671 & 0 \\
\hline & 790 & 774.59 & 198.66 & 13795.47947 & 0 & 0 & 10 & 1000 & 38.897 & 0.039 & 42 & 8 & 9.9328 & 0 \\
\hline & 800 & 774.59 & 198.23 & 14608.25033 & 0 & 0 & 10 & 1000 & 38.813 & 0.039 & 43 & 8 & 9.91145 & 0 \\
\hline & 820 & 774.59 & 197.87 & 16249.20549 & 0 & 0 & 10 & 1000 & 38.742 & 0.039 & 44 & 8 & 9.89335 & 0 \\
\hline & 840 & 697.13 & 174.38 & 17710.20551 & 0 & 0 & 10 & 899.99 & 34.144 & 0.038 & 45 & 8 & 8.719 & 0 \\
\hline & 860 & 619.67 & 152.1 & 19010.63951 & 0 & 0 & 10 & 799.99 & 29.781 & 0.037 & 46 & 8 & 7.60505 & 0 \\
\hline & 6880 & 542.21 & 130.4 & 20147.30465 & 0 & 0 & 10 & 699.99 & 25.533 & 0.036 & 47 & 8 & 6.5201 & 0 \\
\hline & 6900 & 464.75 & 109.43 & 21121.29183 & 0 & 0 & 10 & 599.99 & 21.427 & 0.036 & 48 & 8 & 5.4716 & 0 \\
\hline & $6 \quad 920$ & 387.3 & 89 & 21934.38942 & 0 & 0 & 10 & 500 & 17.426 & 0.035 & 49 & 8 & 4.44995 & 0 \\
\hline 1 & 6940 & 309.84 & 69.05 & 22584.08021 & 0 & 0 & 10 & 400 & 13.52 & 0.034 & 50 & 8 & 3.4525 & 0 \\
\hline 1 & 6960 & 232.38 & 50.13 & 23071.9075 & 0 & 0 & 10 & 300 & 9.816 & 0.033 & 51 & 8 & 2.50655 & 0 \\
\hline 1 & 6980 & 154.92 & 31.84 & 23397.8281 & 0 & 0 & 10 & 200 & 6.234 & 0.031 & 52 & 8 & 1.592 & 0 \\
\hline 1 & 61000 & 77.46 & 15.71 & 23561.24511 & 0 & 0 & 10 & 100 & 3.076 & 0.031 & 53 & 8 & 0.78555 & 0 \\
\hline 1 & 71020 & 69.71 & 15.43 & 23708.42323 & 0 & 0 & 10 & 90 & 3.021 & 0.034 & 54 & 8 & 0.77155 & 0 \\
\hline & 71040 & 61.97 & 14.3 & 23838.35243 & 0 & 0 & 10 & 80 & 2.8 & 0.035 & 55 & 8 & 0.7149 & 0 \\
\hline & 71060 & 54.22 & 13.06 & 23952.0561 & 0 & 0 & 10 & 70 & 2.557 & 0.037 & 56 & 8 & 0.65285 & 0 \\
\hline & 71080 & 46.48 & 11.65 & 24049.44154 & 0 & 0 & 10 & 60.01 & 2.281 & 0.038 & 57 & 8 & 0.5826 & 0 \\
\hline 1 & 71100 & 38.73 & 10.09 & 24130.76481 & 0 & 0 & 10 & 50 & 1.975 & 0.04 & 58 & 8 & 0.50435 & 0 \\
\hline 1 & 71120 & 30.99 & 8.91 & 24195.76122 & 0 & 0 & 10 & 40.01 & 1.745 & 0.044 & 59 & 8 & 0.4456 & 0 \\
\hline 1 & 71140 & 23.24 & 7.21 & 24244.52345 & 0 & 0 & 10 & 30 & 1.411 & 0.047 & 60 & 8 & 0.36035 & 0 \\
\hline 1 & 71160 & 15.5 & 5.57 & 24277.11433 & 0 & 0 & 10 & 20.01 & 1.09 & 0.054 & 61 & 8 & 0.2783 & 0 \\
\hline 1 & 71180 & 7.75 & 3.54 & 24293.44276 & 0 & 0 & 10 & 10.01 & 0.693 & 0.069 & 62 & 8 & 0.1769 & 0 \\
\hline 1 & 81200 & 6.97 & 4.06 & 24308.1407 & 0 & 0 & 10 & 9 & 0.795 & 0.088 & 63 & 8 & 0.20295 & 0 \\
\hline 1 & 81220 & 6.2 & 4.26 & 24321.12883 & 0 & 0 & 10 & 8 & 0.834 & 0.104 & 64 & 8 & 0.2131 & 0 \\
\hline & 81240 & 5.42 & 4.08 & 24332.49983 & 0 & 0 & 10 & 7 & 0.798 & 0.114 & 65 & 8 & 0.20385 & 0 \\
\hline
\end{tabular}




\begin{tabular}{|c|c|c|c|c|c|c|c|c|c|c|c|c|c|}
\hline 81260 & 4.64 & 4.23 & 24342.22855 & 0 & 0 & 10 & 5.99 & 0.829 & 0.138 & 66 & 8 & 0.2116 & 0 \\
\hline 81280 & 3.87 & 4.07 & 24350.34486 & 0 & 0 & 10 & 5 & 0.798 & 0.16 & 67 & 8 & 0.2037 & 0 \\
\hline 81300 & 3.09 & 4.02 & 24356.83853 & 0 & 0 & 10 & 3.99 & 0.788 & 0.197 & 68 & 8 & 0.20115 & \\
\hline 81320 & 2.32 & 3.7 & 24361.708 & 0 & 0 & 10 & 3 & 0.725 & 0.242 & 69 & 8 & 0.1852 & \\
\hline 81340 & 1.54 & 2.95 & 24364.95405 & 0 & 0 & 10 & 1.99 & 0.577 & 0.29 & 70 & 8 & 0.1474 & \\
\hline 81360 & 0.77 & 2.25 & 24366.57904 & 0 & 0 & 10 & 0.99 & 0.44 & 0.444 & 71 & 8 & 0.11235 & \\
\hline 91380 & 0.7 & 2.78 & 24368.05323 & 18.17405 & 33.46964 & 10 & 0.9 & 0.543 & 0.603 & 72 & 8 & 0.13875 & 0.029877765 \\
\hline 91400 & 0.62 & 3.31 & 24369.35699 & 34.247 & 52.85023 & 10 & 0.8 & 0.648 & 0.81 & 73 & 8 & 0.16535 & 0.018921365 \\
\hline 91420 & 0.55 & 3.72 & 24370.50053 & 48.34472 & 66.40749 & 10 & 0.71 & 0.728 & 1.025 & 74 & 8 & 0.186 & 0.015058521 \\
\hline 91440 & 0.47 & 3.3 & 24371.48071 & 60.42848 & 93.54239 & 10 & 0.61 & 0.646 & 1.059 & 75 & 8 & 0.165 & 0.010690324 \\
\hline 91460 & 0.39 & 3.3 & 24372.29752 & 70.49828 & 108.96162 & 10 & 0.5 & 0.647 & 1.294 & 76 & 8 & 0.1652 & 0.009177529 \\
\hline 91480 & 0.31 & 3.33 & 24372.95411 & 78.59285 & 120.72616 & 10 & 0.4 & 0.651 & 1.628 & 77 & 8 & 0.16635 & 0.008283196 \\
\hline 91500 & 0.24 & 3.16 & 24373.44734 & 84.67346 & 136.79051 & 10 & 0.31 & 0.619 & 1.997 & 78 & 8 & 0.158 & 0.007310437 \\
\hline 91520 & 0.16 & 3.03 & 24373.77721 & 88.74011 & 149.6458 & 10 & 0.21 & 0.593 & 2.824 & 79 & 8 & 0.15145 & 0.006682435 \\
\hline 91540 & 0.08 & 2.48 & 24373.94686 & 90.83153 & 187.28112 & 10 & 0.1 & 0.485 & 4.85 & 80 & 8 & 0.12385 & 0.00533955 \\
\hline
\end{tabular}

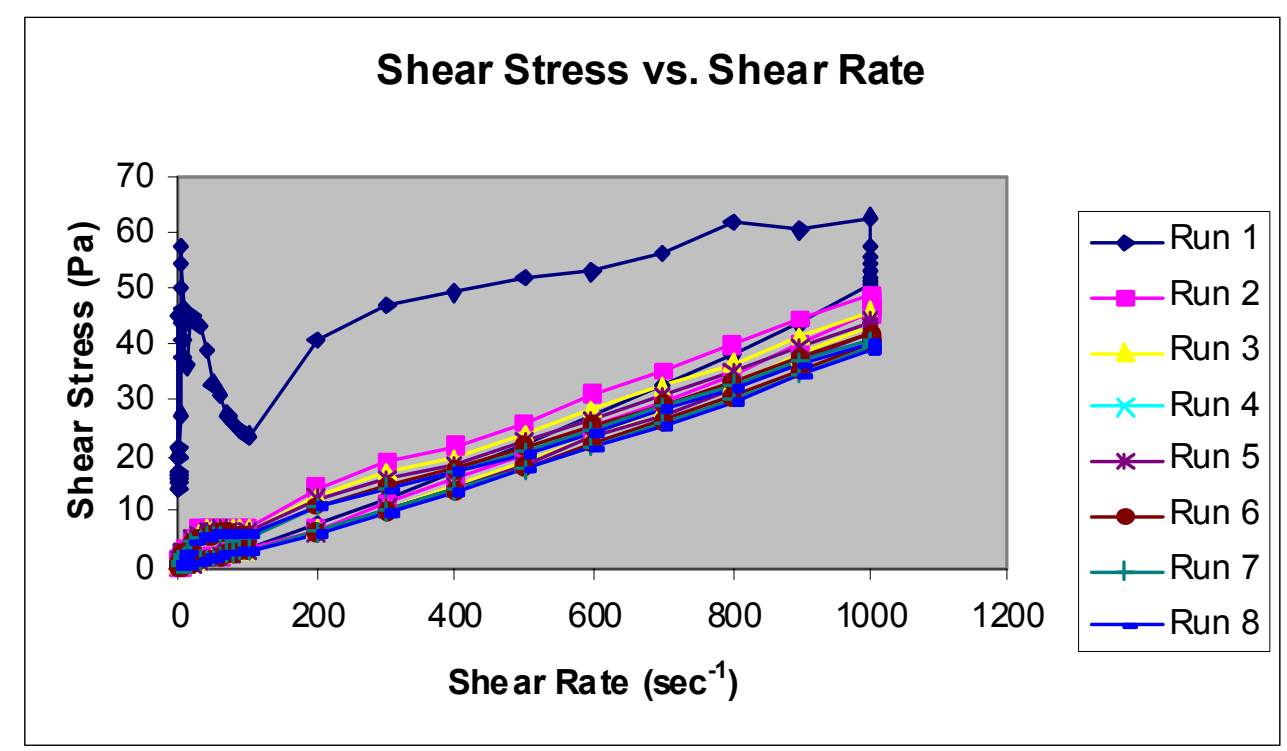




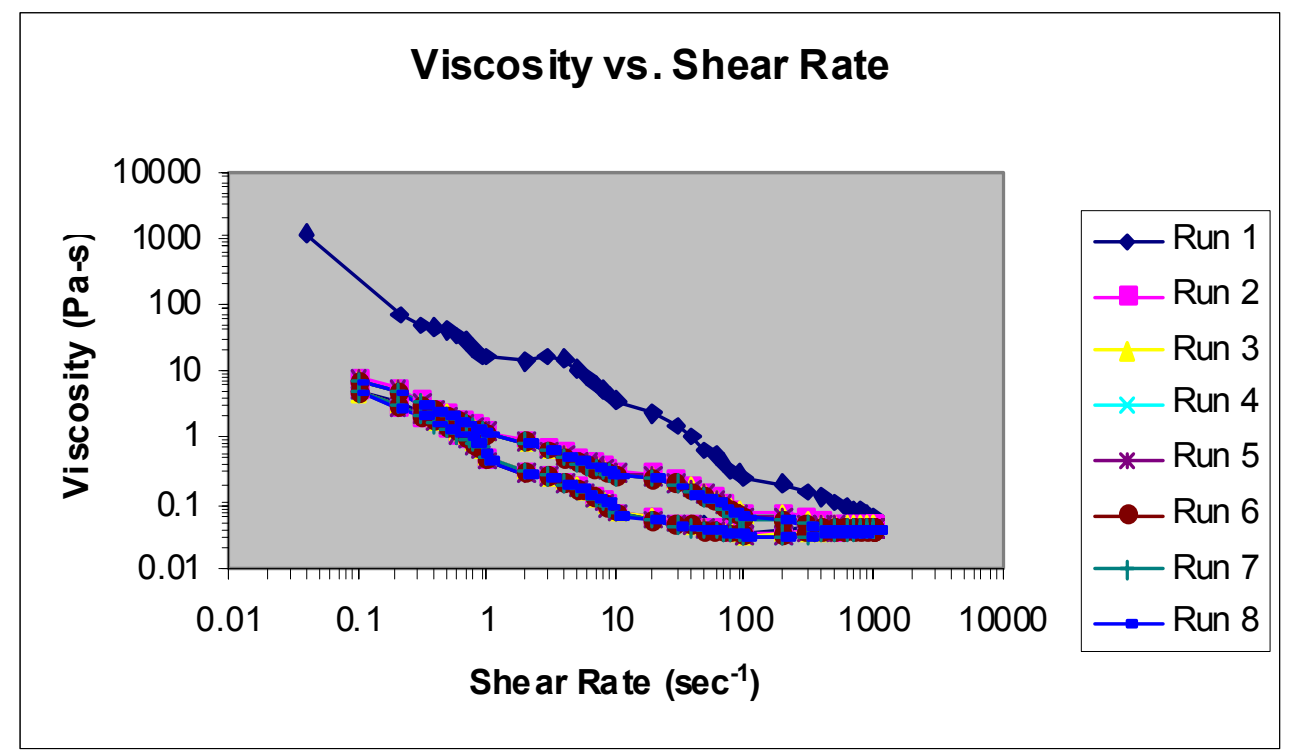

\section{E-2.2 WM-187 Tank Farm Waste Samples}

\section{E-2.2.1 Supernate}

04.01.04 WM-187 supernate DG run 01.txt

Mode Step $\mathrm{t}[\mathrm{s}] \quad \mathrm{n}[\mathrm{rpm}] \quad \mathrm{M}[\%$.]

$\begin{array}{rrrrr}1 & 1 & 20 & 99.26 & 7.4 \\ 1 & 1 & 40 & 198.5 & 16.55 \\ 1 & 1 & 60 & 297.7 & 28.46 \\ 1 & 1 & 80 & 396.9 & 41.91 \\ 1 & 1 & 100 & 496.1 & 57.19 \\ 1 & 1 & 120 & 595.4 & 74.4 \\ 1 & 1 & 140 & 694.6 & 92.69 \\ 1 & 1 & 160 & 793.8 & 112.4 \\ 1 & 2 & 180 & 793.8 & 112.1 \\ 1 & 2 & 200 & 793.8 & 111.7 \\ 1 & 2 & 220 & 793.8 & 111.5 \\ 1 & 3 & 240 & 793.8 & 111.5 \\ 1 & 3 & 260 & 694.6 & 92.22 \\ 1 & 3 & 280 & 595.4 & 73.91 \\ 1 & 3 & 300 & 496.1 & 56.74 \\ 1 & 3 & 320 & 396.9 & 41.18 \\ 1 & 3 & 340 & 297.7 & 27.29 \\ 1 & 3 & 360 & 198.5 & 15.61 \\ 1 & 3 & 380 & 99.23 & 7.83\end{array}$

Phi[rad]

207.61372

621.70391

1243.9223

2073.6475

3111.3972

4355.7169

5809.8274

7471.0835

9151.7586

10813.896

12476.445

14156.871

15612.615

16861.18

17902.069

18735.717

19361.131

19778.479

19988.03
Gamma $J[t] \quad T\left[{ }^{\circ} \mathrm{C}\right]$

$\begin{array}{lll}0 & 0 & 30 \\ 0 & 0 & 30\end{array}$

$\mathrm{D}[1 / \mathrm{s}] \quad \mathrm{Tau}[\mathrm{Pa}] \quad \mathrm{E}$

$500.2 \quad 0.496$

$1000 \quad 1.109$

$1500 \quad 1.907$

$2000 \quad 2.808$

$2500 \quad 3.832$

$3000 \quad 4.985$

$3500 \quad 6.21$

$4000 \quad 7.53$

$4000 \quad 7.513$

$4000 \quad 7.482$

$4000 \quad 7.469$

$4000 \quad 7.469$

$3500 \quad 6.179$

$3000 \quad 4.952$

$2500 \quad 3.801$

$2000 \quad 2.759$

$1500 \quad 1.828$

$1000 \quad 1.046$

$500 \quad 0.524$
ta[Pas] MP\# Block M[mNm] G[Pa]

$\begin{array}{lllll}0.001 & 1 & 1 & 0.3701 & 0\end{array}$

$\begin{array}{lllll}0.001 & 2 & 1 & 0.8276 & 0\end{array}$

$\begin{array}{lllll}0.001 & 3 & 1 & 1.4229 & 0\end{array}$

$\begin{array}{lllll}0.001 & 4 & 1 & 2.0954 & 0\end{array}$

$\begin{array}{lllll}0.002 & 5 & 1 & 2.8595 & 0\end{array}$

$\begin{array}{lllll}0.002 & 6 & 1 & 3.7199 & 0\end{array}$

$\begin{array}{lllll}0.002 & 7 & 1 & 4.6344 & 0\end{array}$

$\begin{array}{lllll}0.002 & 8 & 1 & 5.6197 & 0\end{array}$

$\begin{array}{lllll}0.002 & 9 & 1 & 5.6068 & 0\end{array}$

$\begin{array}{lllll}0.002 & 10 & 1 & 5.5838 & 0\end{array}$

$\begin{array}{lllll}0.002 & 11 & 1 & 5.574 & 0\end{array}$

$\begin{array}{lllll}0.002 & 12 & 1 & 5.5736 & 0\end{array}$

$\begin{array}{lllll}0.002 & 13 & 1 & 4.6109 & 0\end{array}$

$\begin{array}{lllll}0.002 & 14 & 1 & 3.6955 & 0\end{array}$

$\begin{array}{lllll}0.002 & 15 & 1 & 2.8368 & 0\end{array}$

$\begin{array}{lllll}0.001 & 16 & 1 & 2.0592 & 0\end{array}$

$\begin{array}{lllll}0.001 & 17 & 1 & 1.3644 & 0\end{array}$

$\begin{array}{lllll}0.001 & 18 & 1 & 0.7805 & 0\end{array}$

$\begin{array}{lllll}0.001 & 19 & 1 & 0.3913 & 0\end{array}$

04.01.04 WM-187 supernate

DG run 02.txt

Mode Step t[s] $\quad \mathrm{n}[\mathrm{rpm}] \quad \mathrm{M}[\%$.]

$\begin{array}{rrrrr}1 & 1 & 20 & 99.28 & 7.12 \\ 1 & 1 & 40 & 198.5 & 15.58 \\ 1 & 1 & 60 & 297.7 & 27.16 \\ 1 & 1 & 80 & 396.9 & 41.05\end{array}$

Phi[rad] Gamma J[t] $\mathrm{T}\left[{ }^{\circ} \mathrm{C}\right]$

207.68205
621.82329
1243.3662
2072.8315

$\begin{array}{lll}0 & 0 & 30 \\ 0 & 0 & 30 \\ 0 & 0 & 30 \\ 0 & 0 & 30\end{array}$

$\mathrm{D}[1 / \mathrm{s}] \quad \mathrm{Tau}[\mathrm{Pa}] \quad$ Eta[Pas $] \quad$ MP\# Block $\mathrm{M}[\mathrm{mNm}] \quad \mathrm{G}[\mathrm{Pa}]$

$\begin{array}{rrrrrrr}500.3 & 0.477 & 0.001 & 1 & 1 & 0.3558 & 0 \\ 1000 & 1.044 & 0.001 & 2 & 1 & 0.7789 & 0 \\ 1500 & 1.82 & 0.001 & 3 & 1 & 1.358 & 0 \\ 2000 & 2.75 & 0.001 & 4 & 1 & 2.0523 & 0\end{array}$




$\begin{array}{rrrrrrrrrrrrrrrrrrr}1 & 1 & 100 & 496.1 & 56.61 & 3111.3069 & 0 & 0 & 30 & 2500 & 3.793 & 0.002 & 5 & 1 & 2.8307 & 0 \\ 1 & 1 & 120 & 595.4 & 73.77 & 4356.5636 & 0 & 0 & 30 & 3000 & 4.943 & 0.002 & 6 & 1 & 3.6887 & 0 \\ 1 & 1 & 140 & 694.6 & 92.21 & 5808.9093 & 0 & 0 & 30 & 3500 & 6.178 & 0.002 & 7 & 1 & 4.6106 & 0 \\ 1 & 1 & 160 & 793.8 & 111.7 & 7469.3337 & 0 & 0 & 30 & 4000 & 7.481 & 0.002 & 8 & 1 & 5.583 & 0 \\ 1 & 2 & 180 & 793.8 & 111.7 & 9148.7576 & 0 & 0 & 30 & 4000 & 7.481 & 0.002 & 9 & 1 & 5.5831 & 0 \\ 1 & 2 & 200 & 793.8 & 111.4 & 10810.893 & 0 & 0 & 30 & 4000 & 7.464 & 0.002 & 10 & 1 & 5.5699 & 0 \\ 1 & 2 & 220 & 793.8 & 111.3 & 12474.691 & 0 & 0 & 30 & 4000 & 7.457 & 0.002 & 11 & 1 & 5.5648 & 0 \\ 1 & 3 & 240 & 793.8 & 111.3 & 14155.117 & 0 & 0 & 30 & 4000 & 7.456 & 0.002 & 12 & 1 & 5.564 \\ 1 & 3 & 260 & 694.6 & 91.88 & 15610.494 & 0 & 0 & 30 & 3500 & 6.156 & 0.002 & 13 & 1 & 4.594 & 0 \\ 1 & 3 & 280 & 595.4 & 73.48 & 16860.051 & 0 & 0 & 30 & 3000 & 4.923 & 0.002 & 14 & 1 & 3.6742 & 0 \\ 1 & 3 & 300 & 496.1 & 56.32 & 17900.361 & 0 & 0 & 30 & 2500 & 3.774 & 0.002 & 15 & 1 & 2.8161 \\ 1 & 3 & 320 & 396.9 & 40.85 & 18734.076 & 0 & 0 & 30 & 2000 & 2.737 & 0.001 & 16 \\ 1 & 3 & 340 & 297.7 & 27.09 & 19358.807 & 0 & 0 & 30 & 1500 & 1.815 & 0.001 & 17 & 1 & 1 \\ 1 & 3 & 360 & 198.5 & 15.61 & 19776.256 & 0 & 0 & 30 & 1000 & 1.046 & 0.001 & 18 & 1 & 0.7807 & 0 \\ 1 & 3 & 380 & 99.23 & 7.73 & 19985.862 & 0 & 0 & 30 & 500 & 0.518 & 0.001 & 19 & 1 & 0.3863 & 0\end{array}$

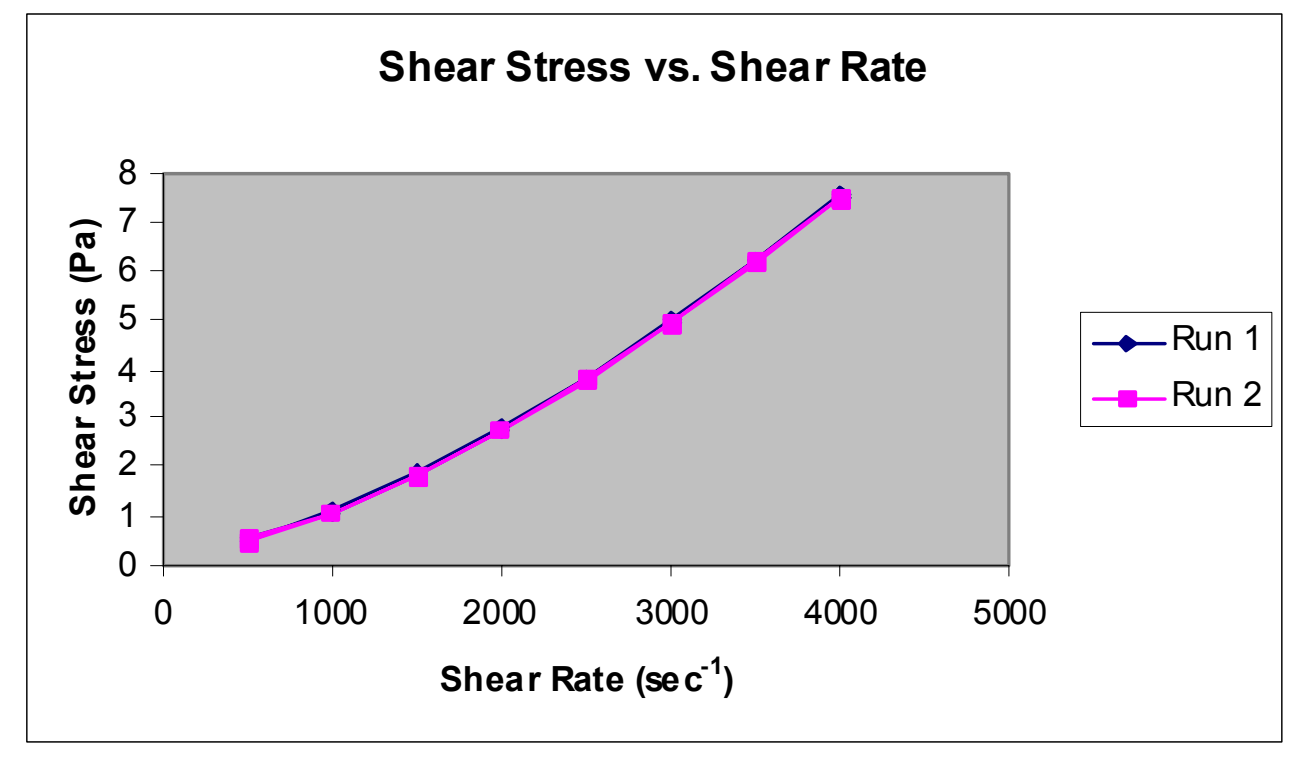




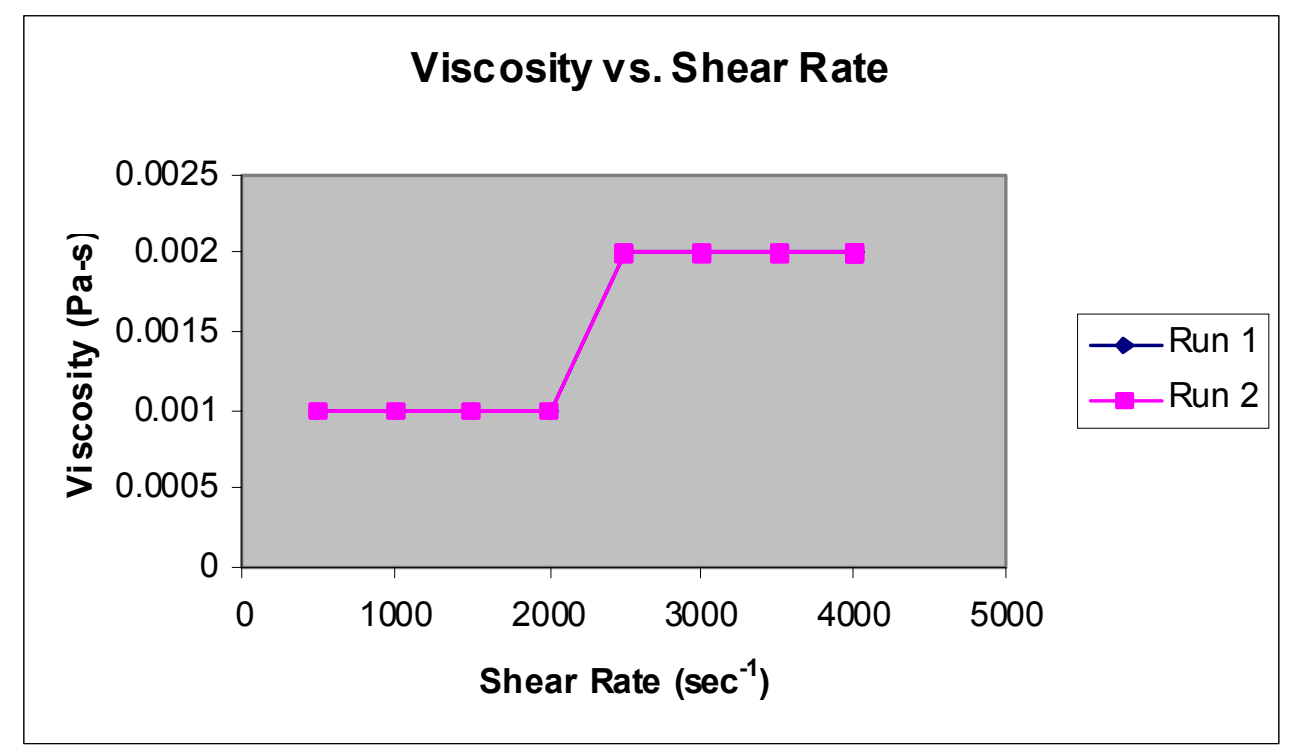

\section{E-2.2.2 As Received}

04.01.04 WM-187 as received CC-48 run 01.txt

Mode Step $\mathrm{t}[\mathrm{s}] \quad \mathrm{n}[\mathrm{rpm}] \quad \mathrm{M}[\%$.] $\quad$ Phi[rad

\begin{tabular}{|c|c|c|c|c|c|c|}
\hline 1 & 1 & 20 & 0.02 & 0 & 0.04006 & 1.9668 \\
\hline 1 & 1 & 40 & 0.04 & 0 & 0.12331 & 6.0547 \\
\hline 1 & 1 & 60 & 0.06 & 0 & 0.24897 & 12.225 \\
\hline 1 & 1 & 80 & 0.08 & 0 & 0.41626 & 20.439 \\
\hline 1 & 1 & 100 & 0.1 & 0 & 0.62518 & 30.698 \\
\hline 1 & 1 & 120 & 0.12 & 0 & 0.87572 & 43 \\
\hline 1 & 1 & 140 & 0.14 & 0 & 1.17024 & 57.462 \\
\hline 1 & 1 & 160 & 0.16 & 0 & 1.50404 & 73.852 \\
\hline 1 & 1 & 180 & 0.18 & 0 & 1.88103 & 92.363 \\
\hline 1 & 2 & 200 & 0.19 & 0 & 2.28158 & 112.03 \\
\hline 1 & 2 & 220 & 0.39 & 0 & 3.08426 & 151.44 \\
\hline 1 & 2 & 240 & 0.58 & 0 & 4.29691 & 210.99 \\
\hline 1 & 2 & 260 & 0.78 & 0 & 5.91719 & 0 \\
\hline 1 & 2 & 280 & 0.97 & 0 & 7.94587 & 0 \\
\hline 1 & 2 & 300 & 1.17 & 0 & 10.38296 & 0 \\
\hline 1 & 2 & 320 & 1.36 & 0 & 13.23082 & 0 \\
\hline 1 & 2 & 340 & 1.55 & 0 & 16.48394 & 0 \\
\hline 1 & 2 & 360 & 1.75 & 0 & 20.14703 & 0 \\
\hline 1 & 3 & 380 & 1.94 & 0 & 24.23739 & 0 \\
\hline 1 & 3 & 400 & 3.89 & 1.3 & 32.34427 & 0 \\
\hline 1 & 3 & 420 & 5.83 & 1.43 & 44.52893 & 0 \\
\hline 1 & 3 & 440 & 7.77 & 1.4 & 60.8016 & 0 \\
\hline 1 & 3 & 460 & 9.72 & 1.56 & 81.13163 & 0 \\
\hline 1 & 3 & 480 & 11.66 & 1.67 & 105.51746 & 0 \\
\hline 1 & 3 & 500 & 13.61 & 1.7 & 133.99285 & 0 \\
\hline 1 & 3 & 520 & 15.55 & 1.83 & 166.56724 & 0 \\
\hline 1 & 3 & 540 & 17.5 & 1.95 & 203.16444 & 0 \\
\hline
\end{tabular}

$\mathrm{J}[\mathrm{t}] \quad \mathrm{T}\left[{ }^{\circ} \mathrm{C}\right] \mathrm{D}[1 / \mathrm{s}] \quad \mathrm{Tau}[\mathrm{Pa}] \mathrm{Eta}[\mathrm{Pas}] \mathrm{MP \#}$ Block $\mathrm{M}[\mathrm{mNm}] \mathrm{G}[\mathrm{Pa}]$

\begin{tabular}{|c|c|c|c|c|c|c|}
\hline 30.3 & 0.1 & 0 & 0 & 1 & 1 & 0 \\
\hline 30.3 & 0.21 & 0 & 0 & 2 & 1 & 0 \\
\hline 30.3 & 0.31 & 0 & 0 & 3 & 1 & 0 \\
\hline 30.3 & 0.41 & 0 & 0 & 4 & 1 & 0 \\
\hline 30.3 & 0.51 & 0 & 0 & 5 & 1 & 0 \\
\hline 30.3 & 0.62 & 0 & 0 & 6 & 1 & 0 \\
\hline 30.3 & 0.72 & 0 & 0 & 7 & 1 & 0 \\
\hline 30.3 & 0.82 & 0 & 0 & 8 & 1 & 0 \\
\hline 30.3 & 0.93 & 0 & 0 & 9 & 1 & 0 \\
\hline 30.3 & 0.98 & 0 & 0 & 10 & 1 & 0 \\
\hline 30.3 & 2.01 & 0 & 0 & 11 & 1 & 0 \\
\hline 30.3 & 2.98 & 0 & 0 & 12 & 1 & 0 \\
\hline 30.3 & 4.01 & 0 & 0 & 13 & 1 & 0 \\
\hline 30.3 & 4.99 & 0 & 0 & 14 & 1 & 0 \\
\hline 30.3 & 6.02 & 0 & 0 & 15 & 1 & 0 \\
\hline 30.3 & 6.99 & 0 & 0 & 16 & 1 & 0 \\
\hline 30.3 & 7.97 & 0 & 0 & 17 & 1 & 0 \\
\hline 30.3 & 9 & 0 & 0 & 18 & 1 & 0 \\
\hline 30.3 & 9.98 & 0 & 0 & 19 & 1 & 0 \\
\hline 30.3 & 20 & 0.247 & 0.012 & 20 & 1 & 0.0649 \\
\hline 30.3 & 29.98 & 0.272 & 0.009 & 21 & 1 & 0.07155 \\
\hline 30.3 & 39.95 & 0.266 & 0.007 & 22 & 1 & 0.0701 \\
\hline 30.3 & 49.98 & 0.297 & 0.006 & 23 & 1 & 0.0781 \\
\hline 30.3 & 59.96 & 0.317 & 0.005 & 24 & 1 & 0.0835 \\
\hline 30.3 & 69.98 & 0.322 & 0.005 & 25 & 1 & 0.08485 \\
\hline 30.3 & 79.96 & 0.348 & 0.004 & 26 & 1 & 0.0917 \\
\hline 30.3 & 89.99 & 0.37 & 0.004 & 27 & 1 & 0.0974 \\
\hline
\end{tabular}




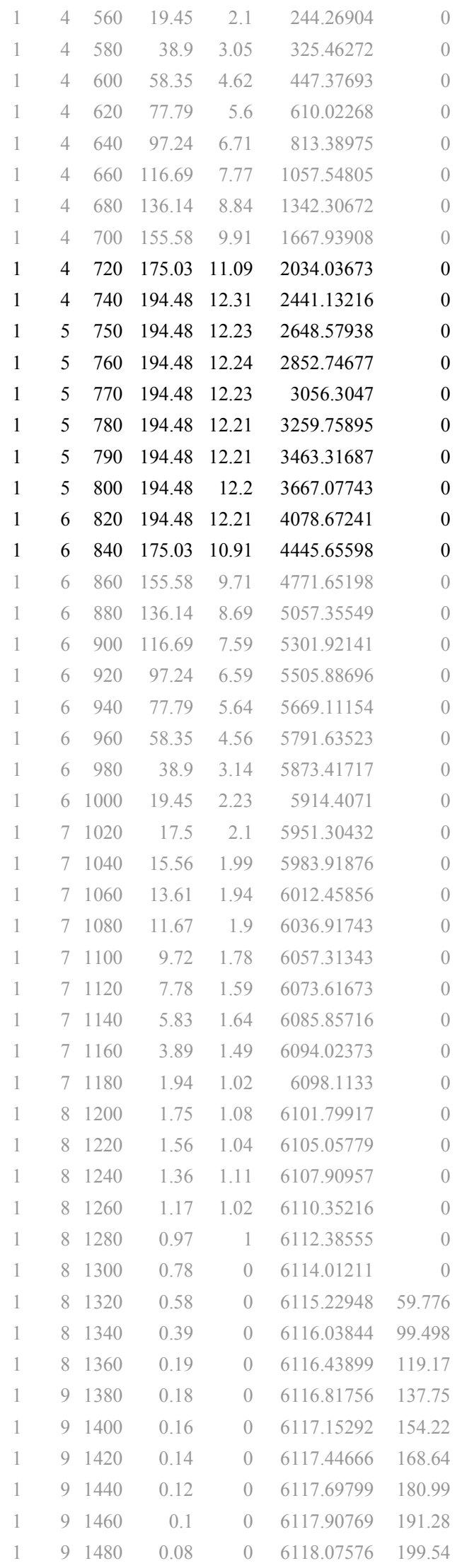

\begin{tabular}{|c|c|c|c|c|c|c|c|}
\hline 0 & 30.3 & 100.01 & 0.399 & 0.004 & 28 & 1 & 0.1049 \\
\hline 0 & 30.3 & 200.02 & 0.579 & 0.003 & 29 & 1 & 0.15235 \\
\hline 0 & 30.3 & 300.04 & 0.877 & 0.003 & 30 & 1 & 0.2308 \\
\hline 0 & 30.3 & 400 & 1.064 & 0.003 & 31 & 1 & 0.28005 \\
\hline 0 & 30.3 & 500.01 & 1.275 & 0.003 & 32 & 1 & 0.3356 \\
\hline 0 & 30.3 & 600.02 & 1.476 & 0.002 & 33 & 1 & 0.38855 \\
\hline 0 & 30.3 & 700.03 & 1.679 & 0.002 & 34 & 1 & 0.44195 \\
\hline 0 & 30.3 & 799.99 & 1.884 & 0.002 & 35 & 1 & 0.4957 \\
\hline 0 & 30.4 & 900 & 2.107 & 0.002 & 36 & 1 & 0.5545 \\
\hline 0 & 30.4 & 1000 & 2.338 & 0.002 & 37 & 1 & 0.61535 \\
\hline 0 & 30.4 & 1000 & 2.324 & 0.002 & 38 & 1 & 0.6117 \\
\hline 0 & 30.4 & 1000 & 2.326 & 0.002 & 39 & 1 & 0.6121 \\
\hline 0 & 30.4 & 1000 & 2.324 & 0.002 & 40 & 1 & 0.6115 \\
\hline 0 & 30.4 & 1000 & 2.32 & 0.002 & 41 & 1 & 0.61045 \\
\hline 0 & 30.4 & 1000 & 2.32 & 0.002 & 42 & 1 & 0.6105 \\
\hline 0 & 30.4 & 1000 & 2.318 & 0.002 & 43 & 1 & 0.60995 \\
\hline 0 & 30.4 & 1000 & 2.32 & 0.002 & 44 & 1 & 0.6104 \\
\hline 0 & 30.4 & 900 & 2.073 & 0.002 & 45 & 1 & 0.5455 \\
\hline 0 & 30.4 & 799.99 & 1.844 & 0.002 & 46 & 1 & 0.48535 \\
\hline 0 & 30.4 & 700.03 & 1.651 & 0.002 & 47 & 1 & 0.43445 \\
\hline 0 & 30.4 & 600.02 & 1.443 & 0.002 & 48 & 1 & 0.3797 \\
\hline 0 & 30.4 & 500.01 & 1.252 & 0.003 & 49 & 1 & 0.32955 \\
\hline 0 & 30.4 & 400 & 1.071 & 0.003 & 50 & 1 & 0.2818 \\
\hline 0 & 30.4 & 300.04 & 0.866 & 0.003 & 51 & 1 & 0.22795 \\
\hline 0 & 30.4 & 200.02 & 0.597 & 0.003 & 52 & 1 & 0.15705 \\
\hline 0 & 30.4 & 100.01 & 0.424 & 0.004 & 53 & 1 & 0.11165 \\
\hline 0 & 30.4 & 89.99 & 0.399 & 0.004 & 54 & 1 & 0.105 \\
\hline 0 & 30.4 & 80.01 & 0.379 & 0.005 & 55 & 1 & 0.0997 \\
\hline 0 & 30.4 & 69.98 & 0.368 & 0.005 & 56 & 1 & 0.09675 \\
\hline 0 & 30.4 & 60.01 & 0.36 & 0.006 & 57 & 1 & 0.09475 \\
\hline 0 & 30.4 & 49.98 & 0.337 & 0.007 & 58 & 1 & 0.08875 \\
\hline 0 & 30.4 & 40 & 0.301 & 0.008 & 59 & 1 & 0.07925 \\
\hline 0 & 30.4 & 29.98 & 0.312 & 0.01 & 60 & 1 & 0.08215 \\
\hline 0 & 30.4 & 20 & 0.283 & 0.014 & 61 & 1 & 0.07445 \\
\hline 0 & 30.4 & 9.98 & 0.195 & 0.02 & 62 & 1 & 0.05125 \\
\hline 0 & 30.4 & 9 & 0.205 & 0.023 & 63 & 1 & 0.054 \\
\hline 0 & 30.4 & 8.02 & 0.197 & 0.025 & 64 & 1 & 0.05195 \\
\hline 0 & 30.4 & 6.99 & 0.211 & 0.03 & 65 & 1 & 0.0556 \\
\hline 0 & 30.4 & 6.02 & 0.194 & 0.032 & 66 & 1 & 0.0511 \\
\hline 0 & 30.3 & 4.99 & 0.19 & 0.038 & 67 & 1 & 0.05005 \\
\hline 0 & 30.3 & 4.01 & 0 & 0 & 68 & 1 & 0 \\
\hline 0 & 30.3 & 2.98 & 0 & 0 & 69 & 1 & 0 \\
\hline 0 & 30.3 & 2.01 & 0 & 0 & 70 & 1 & 0 \\
\hline 0 & 30.3 & 0.98 & 0 & 0 & 71 & 1 & 0 \\
\hline 0 & 30.3 & 0.93 & 0 & 0 & 72 & 1 & 0 \\
\hline 0 & 30.3 & 0.82 & 0 & 0 & 73 & 1 & 0 \\
\hline 0 & 30.3 & 0.72 & 0 & 0 & 74 & 1 & 0 \\
\hline 0 & 30.3 & 0.62 & 0 & 0 & 75 & 1 & 0 \\
\hline 0 & 30.3 & 0.51 & 0 & 0 & 76 & 1 & 0 \\
\hline 0 & 30.3 & 0.41 & 0 & 0 & 77 & 1 & 0 \\
\hline
\end{tabular}




$\begin{array}{lllllll}1 & 9 & 1500 & 0.06 & 0 & 6118.20143 & 205.71 \\ 1 & 9 & 1520 & 0.04 & 0 & 6118.28546 & 209.83 \\ 1 & 9 & 1540 & 0.02 & 0 & 6118.32788 & 211.91\end{array}$

04.01.04 WM-187 as received CC-48 run 02.txt

\begin{tabular}{|c|c|c|c|c|c|c|}
\hline Mode & Step & $\mathrm{t}[\mathrm{s}]$ & $\mathrm{n}[\mathrm{rpm}]$ & $\mathrm{M}[\%]$. & Phi[rad] & Gamma \\
\hline 1 & 1 & 20 & 0.02 & 0 & 0.04006 & 1.9668 \\
\hline 1 & 1 & 40 & 0.04 & 0 & 0.12331 & 6.0547 \\
\hline 1 & 1 & 60 & 0.06 & 0 & 0.24819 & 12.187 \\
\hline 1 & 1 & 80 & 0.08 & 0 & 0.41626 & 20.439 \\
\hline 1 & 1 & 100 & 0.1 & 0 & 0.62518 & 30.698 \\
\hline 1 & 1 & 120 & 0.12 & 0 & 0.8765 & 43.039 \\
\hline 1 & 1 & 140 & 0.14 & 0 & 1.16867 & 57.385 \\
\hline 1 & 1 & 160 & 0.16 & 0 & 1.50404 & 73.852 \\
\hline 1 & 1 & 180 & 0.18 & 0 & 1.88103 & 92.363 \\
\hline 1 & 2 & 200 & 0.19 & 0 & 2.2808 & 111.99 \\
\hline 1 & 2 & 220 & 0.39 & 1.03 & 3.08504 & 151.48 \\
\hline 1 & 2 & 240 & 0.58 & 0 & 4.29691 & 210.99 \\
\hline 1 & 2 & 260 & 0.78 & 1.09 & 5.91798 & 0 \\
\hline 1 & 2 & 280 & 0.97 & 0 & 7.94666 & 0 \\
\hline 1 & 2 & 300 & 1.17 & 0 & 10.38296 & 0 \\
\hline 1 & 2 & 320 & 1.36 & 0 & 13.22846 & 0 \\
\hline 1 & 2 & 340 & 1.55 & 0 & 16.48237 & 0 \\
\hline 1 & 2 & 360 & 1.75 & 0 & 20.14468 & 0 \\
\hline 1 & 3 & 380 & 1.94 & 0 & 24.23346 & 0 \\
\hline 1 & 3 & 400 & 3.89 & 1.45 & 32.34819 & 0 \\
\hline 1 & 3 & 420 & 5.83 & 1.53 & 44.53757 & 0 \\
\hline 1 & 3 & 440 & 7.77 & 1.42 & 60.78982 & 0 \\
\hline 1 & 3 & 460 & 9.72 & 1.65 & 81.12927 & 0 \\
\hline 1 & 3 & 480 & 11.66 & 1.75 & 105.50332 & 0 \\
\hline 1 & 3 & 500 & 13.61 & 1.77 & 134.00856 & 0 \\
\hline 1 & 3 & 520 & 15.55 & 1.83 & 166.53347 & 0 \\
\hline 1 & 3 & 540 & 17.5 & 1.92 & 203.14873 & 0 \\
\hline 1 & 4 & 560 & 19.45 & 2.14 & 244.25255 & 0 \\
\hline 1 & 4 & 580 & 38.9 & 3.01 & 325.43601 & 0 \\
\hline 1 & 4 & 600 & 58.35 & 4.46 & 447.2175 & 0 \\
\hline 1 & 4 & 620 & 77.79 & 5.56 & 609.90409 & 0 \\
\hline 1 & 4 & 640 & 97.24 & 6.63 & 813.33163 & 0 \\
\hline 1 & 4 & 660 & 116.69 & 7.57 & 1057.30772 & 0 \\
\hline 1 & 4 & 680 & 136.14 & 8.71 & 1342.41275 & 0 \\
\hline 1 & 4 & 700 & 155.58 & 9.73 & 1668.0349 & 0 \\
\hline 1 & 4 & 720 & 175.03 & 10.89 & 2034.13255 & 0 \\
\hline 1 & 4 & 740 & 194.48 & 12.09 & 2441.0442 & 0 \\
\hline 1 & 5 & 750 & 194.48 & 12.05 & 2648.49141 & 0 \\
\hline 1 & 5 & 760 & 194.48 & 12.01 & 2852.55749 & 0 \\
\hline 1 & 5 & 770 & 194.48 & 11.96 & 3055.80911 & 0 \\
\hline 1 & 5 & 780 & 194.48 & 12.03 & 3259.87519 & 0 \\
\hline 1 & 5 & 790 & 194.48 & 12.02 & 3463.53443 & 0 \\
\hline 1 & 5 & 800 & 194.48 & 12.01 & 3667.19367 & 0 \\
\hline 1 & 6 & 820 & 194.48 & 11.96 & 4078.58444 & 0 \\
\hline
\end{tabular}

$\begin{array}{rrrrrrrrr}0 & 30.3 & 0.31 & 0 & 0 & 78 & 1 & 0 & 0 \\ 0 & 30.3 & 0.21 & 0 & 0 & 79 & 1 & 0 & 0 \\ 0 & 30.3 & 0.1 & 0 & 0 & 80 & 1 & 0 & 0\end{array}$

$\mathrm{J}[\mathrm{t}] \quad \mathrm{T}\left[{ }^{\circ} \mathrm{C}\right] \quad \mathrm{D}[1 / \mathrm{s}] \quad \mathrm{Tau}[\mathrm{Pa}] \mathrm{Eta}[\mathrm{Pas}] \mathrm{MP \#}$ Block $\mathrm{M}[\mathrm{mNm}] \mathrm{G}[\mathrm{Pa}]$

$\begin{array}{lllllllll}0 & 30.3 & 0.1 & 0 & 0 & 1 & 1 & 0 & 0 \\ 0 & 30.3 & 0.21 & 0 & 0 & 2 & 1 & 0 & 0 \\ 0 & 30.3 & 0.31 & 0 & 0 & 3 & 1 & 0 & 0 \\ 0 & 30.3 & 0.41 & 0 & 0 & 4 & 1 & 0 & 0 \\ 0 & 30.3 & 0.51 & 0 & 0 & 5 & 1 & 0 & 0 \\ 0 & 30.3 & 0.62 & 0 & 0 & 6 & 1 & 0 & 0 \\ 0 & 30.3 & 0.72 & 0 & 0 & 7 & 1 & 0 & 0 \\ 0 & 30.3 & 0.82 & 0 & 0 & 8 & 1 & 0 & 0 \\ 0 & 30.3 & 0.93 & 0 & 0 & 9 & 1 & 0 & 0 \\ 0 & 30.3 & 0.98 & 0 & 0 & 10 & 1 & 0 & 0\end{array}$

$\begin{array}{lllllllll}772.87014 & 30.3 & 2.01 & 0.196 & 0.098 & 11 & 1 & 0.0516 & 0.001\end{array}$

$\begin{array}{lllllllll}0 & 30.2 & 2.98 & 0 & 0 & 12 & 1 & 0 & 0\end{array}$

$\begin{array}{lllllllll}0 & 30.2 & 4.01 & 0.207 & 0.052 & 13 & 1 & 0.0544 & 0\end{array}$

$\begin{array}{lllllllll}0 & 30.2 & 4.99 & 0 & 0 & 14 & 1 & 0 & 0\end{array}$

$\begin{array}{lllllllll}0 & 30.2 & 6.02 & 0 & 0 & 15 & 1 & 0 & 0\end{array}$

$\begin{array}{lllllllll}0 & 30.2 & 6.99 & 0 & 0 & 16 & 1 & 0 & 0\end{array}$

$\begin{array}{lllllllll}0 & 30.2 & 7.97 & 0 & 0 & 17 & 1 & 0 & 0\end{array}$

$\begin{array}{lllllllll}0 & 30.2 & 9 & 0 & 0 & 18 & 1 & 0 & 0\end{array}$

$\begin{array}{lllllllll}0 & 30.2 & 9.98 & 0 & 0 & 19 & 1 & 0 & 0\end{array}$

$\begin{array}{lllllllll}0 & 30.2 & 20 & 0.276 & 0.014 & 20 & 1 & 0.07255 & 0\end{array}$

$\begin{array}{lllllllll}0 & 30.2 & 29.98 & 0.291 & 0.01 & 21 & 1 & 0.07665 & 0\end{array}$

$\begin{array}{lllllllll}0 & 30.2 & 39.95 & 0.269 & 0.007 & 22 & 1 & 0.07085 & 0\end{array}$

$\begin{array}{lllllllll}0 & 30.2 & 49.98 & 0.314 & 0.006 & 23 & 1 & 0.0825 & 0\end{array}$

$\begin{array}{lllllllll}0 & 30.2 & 59.96 & 0.332 & 0.006 & 24 & 1 & 0.0874 & 0\end{array}$

$\begin{array}{lllllllll}0 & 30.2 & 69.98 & 0.336 & 0.005 & 25 & 1 & 0.08845 & 0\end{array}$

$\begin{array}{lllllllll}0 & 30.2 & 79.96 & 0.347 & 0.004 & 26 & 1 & 0.09125 & 0\end{array}$

$\begin{array}{lllllllll}0 & 30.2 & 89.99 & 0.365 & 0.004 & 27 & 1 & 0.096 & 0\end{array}$

$\begin{array}{lllllllll}0 & 30.2 & 100.01 & 0.407 & 0.004 & 28 & 1 & 0.107 & 0\end{array}$

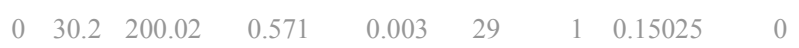

$\begin{array}{lllllllll}0 & 30.2 & 300.04 & 0.847 & 0.003 & 30 & 1 & 0.22295 & 0\end{array}$

$\begin{array}{lllllllll}0 & 30.2 & 400 & 1.057 & 0.003 & 31 & 1 & 0.2782 & 0\end{array}$

$\begin{array}{lllllllll}0 & 30.2 & 500.01 & 1.259 & 0.003 & 32 & 1 & 0.3313 & 0\end{array}$

$\begin{array}{lllllllll}0 & 30.2 & 600.02 & 1.438 & 0.002 & 33 & 1 & 0.37835 & 0\end{array}$

$\begin{array}{lllllllll}0 & 30.2 & 700.03 & 1.654 & 0.002 & 34 & 1 & 0.4353 & 0\end{array}$

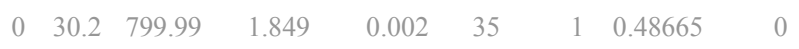

$\begin{array}{lllllllll}0 & 30.2 & 900 & 2.07 & 0.002 & 36 & 1 & 0.5447 & 0\end{array}$

$\begin{array}{lllllllll}0 & 30.2 & 1000 & 2.298 & 0.002 & 37 & 1 & 0.6047 & 0\end{array}$

$\begin{array}{lllllllll}0 & 30.2 & 1000 & 2.29 & 0.002 & 38 & 1 & 0.60265 & 0\end{array}$

$\begin{array}{lllllllll}0 & 30.2 & 1000 & 2.282 & 0.002 & 39 & 1 & 0.6005 & 0\end{array}$

$\begin{array}{lllllllll}0 & 30.2 & 1000 & 2.273 & 0.002 & 40 & 1 & 0.5982 & 0\end{array}$

$\begin{array}{lllllllll}0 & 30.2 & 1000 & 2.286 & 0.002 & 41 & 1 & 0.60155 & 0\end{array}$

$\begin{array}{lllllllll}0 & 30.2 & 1000 & 2.284 & 0.002 & 42 & 1 & 0.601 & 0\end{array}$

$\begin{array}{lllllllll}0 & 30.2 & 1000 & 2.281 & 0.002 & 43 & 1 & 0.60035 & 0\end{array}$

$\begin{array}{lllllllll}0 & 30.2 & 1000 & 2.272 & 0.002 & 44 & 1 & 0.59785 & 0\end{array}$ 


\begin{tabular}{|c|c|c|c|c|c|}
\hline 6 & 840 & 175.03 & 10.74 & 4445.84291 & 0 \\
\hline 6 & 860 & 155.58 & 9.59 & 4772.00227 & 0 \\
\hline 6 & 880 & 136.14 & 8.56 & 5057.22747 & 0 \\
\hline 6 & 900 & 116.69 & 7.47 & 5301.98581 & 0 \\
\hline 6 & 920 & 97.24 & 6.52 & 5505.96235 & 0 \\
\hline 6 & 940 & 77.79 & 5.48 & 5669.1461 & 0 \\
\hline 6 & 960 & 58.35 & 4.5 & 5791.70042 & 0 \\
\hline 6 & 980 & 38.9 & 3.03 & 5873.4313 & 0 \\
\hline 6 & 1000 & 19.45 & 2.15 & 5914.46208 & 0 \\
\hline 7 & 1020 & 17.5 & 2.02 & 5951.38521 & 0 \\
\hline 7 & 1040 & 15.56 & 1.91 & 5984.00044 & 0 \\
\hline 7 & 1060 & 13.61 & 1.86 & 6012.51118 & 0 \\
\hline 7 & 1080 & 11.67 & 1.84 & 6036.98183 & 0 \\
\hline 7 & 1100 & 9.72 & 1.69 & 6057.37313 & 0 \\
\hline 7 & 1120 & 7.78 & 1.52 & 6073.67721 & 0 \\
\hline 7 & 1140 & 5.83 & 1.57 & 6085.9247 & 0 \\
\hline 7 & 1160 & 3.89 & 1.51 & 6094.08813 & 0 \\
\hline 7 & 1180 & 1.94 & 1.08 & 6098.18006 & 0 \\
\hline 8 & 1200 & 1.75 & 1.09 & 6101.86436 & 0 \\
\hline 8 & 1220 & 1.56 & 1.07 & 6105.12533 & 0 \\
\hline 8 & 1240 & 1.36 & 1.07 & 6107.97476 & 0 \\
\hline 8 & 1260 & 1.17 & 1.04 & 6110.41892 & 0 \\
\hline 8 & 1280 & 0.97 & 0 & 6112.4531 & 0 \\
\hline 8 & 1300 & 0.78 & 0 & 6114.07809 & 0 \\
\hline 8 & 1320 & 0.58 & 0 & 6115.29467 & 59.737 \\
\hline 8 & 1340 & 0.39 & 0 & 6116.10441 & 99.498 \\
\hline 8 & 1360 & 0.19 & 0 & 6116.50575 & 119.2 \\
\hline 9 & 1380 & 0.18 & 0 & 6116.88353 & 137.75 \\
\hline 9 & 1400 & 0.16 & 0 & 6117.21889 & 154.22 \\
\hline 9 & 1420 & 0.14 & 0 & 6117.51263 & 168.64 \\
\hline 9 & 1440 & 0.12 & 0 & 6117.76475 & 181.02 \\
\hline 9 & 1460 & 0.1 & 0 & 6117.97366 & 191.28 \\
\hline 9 & 1480 & 0.08 & 0 & 6118.14174 & 199.54 \\
\hline 9 & 1500 & 0.06 & 0 & 6118.26819 & 205.74 \\
\hline 9 & 1520 & 0.04 & 0 & 6118.35222 & 209.87 \\
\hline 9 & 1540 & 0.02 & 0 & 6118.39385 & 211.91 \\
\hline
\end{tabular}

04.01.04 WM-187 as received DG run 01.txt

Mode Step $\mathrm{t}[\mathrm{s}] \quad \mathrm{n}[\mathrm{rpm}] \quad \mathrm{M}[\%$. $] \quad$ Phi[rad] Gamma

$\begin{array}{rrrrrrr}1 & 1 & 20 & 0.02 & 0 & 0.04006 & 0 \\ 1 & 1 & 40 & 0.04 & 0 & 0.12331 & 0 \\ 1 & 1 & 60 & 0.06 & 0 & 0.24897 & 0 \\ 1 & 1 & 80 & 0.08 & 0 & 0.41626 & 0 \\ 1 & 1 & 100 & 0.1 & 0 & 0.62518 & 0 \\ 1 & 1 & 120 & 0.12 & 0 & 0.8765 & 0 \\ 1 & 1 & 140 & 0.14 & 0 & 1.16946 & 0 \\ 1 & 1 & 160 & 0.16 & 0 & 1.50404 & 0 \\ 1 & 1 & 180 & 0.18 & 0 & 1.88103 & 0 \\ 1 & 2 & 200 & 0.2 & 0 & 2.302 & 0 \\ 1 & 2 & 220 & 0.4 & 0 & 3.13531 & 0\end{array}$

\begin{tabular}{|c|c|c|c|c|c|c|c|}
\hline 0 & 30.2 & 900 & 2.04 & 0.002 & 45 & 1 & 0.5369 \\
\hline 0 & 30.2 & 799.99 & 1.822 & 0.002 & 46 & 1 & 0.4794 \\
\hline 0 & 30.2 & 700.03 & 1.627 & 0.002 & 47 & 1 & 0.42815 \\
\hline 0 & 30.2 & 600.02 & 1.42 & 0.002 & 48 & 1 & 0.3736 \\
\hline c & 30.2 & 500.01 & 1.239 & 0.002 & 49 & 1 & 0.32605 \\
\hline c & 30.2 & 400 & 1.041 & 0.003 & 50 & 1 & 0.27405 \\
\hline c & 30.2 & 300.04 & 0.854 & 0.003 & 51 & 1 & 0.2248 \\
\hline c & 30.2 & 200.02 & 0.576 & 0.003 & 52 & 1 & 0.1517 \\
\hline 0 & 30.2 & 100.01 & 0.409 & 0.004 & 53 & 1 & 0.1077 \\
\hline 0 & 30.2 & 89.99 & 0.385 & 0.004 & 54 & 1 & 0.1012 \\
\hline 0 & 30.2 & 80.01 & 0.363 & 0.005 & 55 & 1 & 0.09555 \\
\hline 0 & 30.2 & 69.98 & 0.353 & 0.005 & 56 & 1 & 0.09295 \\
\hline 0 & 30.2 & 60.01 & 0.349 & 0.006 & 57 & 1 & 0.09175 \\
\hline 0 & 30.2 & 49.98 & 0.322 & 0.006 & 58 & 1 & 0.08465 \\
\hline 0 & 30.2 & 40 & 0.288 & 0.007 & 59 & 1 & 0.0758 \\
\hline 0 & 30.2 & 29.98 & 0.298 & 0.01 & 60 & 1 & 0.07835 \\
\hline 0 & 30.2 & 20 & 0.287 & 0.014 & 61 & 1 & 0.07565 \\
\hline 0 & 30.2 & 9.98 & 0.205 & 0.021 & 62 & 1 & 0.05395 \\
\hline 0 & 30.2 & 9 & 0.207 & 0.023 & 63 & 1 & 0.05435 \\
\hline 0 & 30.2 & 8.02 & 0.203 & 0.025 & 64 & 1 & 0.05345 \\
\hline 0 & 30.2 & 6.99 & 0.203 & 0.029 & 65 & 1 & 0.05355 \\
\hline 0 & 30.2 & 6.02 & 0.198 & 0.033 & 66 & 1 & 0.0521 \\
\hline 0 & 30.2 & 4.99 & 0 & 0 & 67 & 1 & 0 \\
\hline 0 & 30.2 & 4.01 & 0 & 0 & 68 & 1 & 0 \\
\hline 0 & 30.2 & 2.98 & 0 & 0 & 69 & 1 & 0 \\
\hline 0 & 30.2 & 2.01 & 0 & 0 & 70 & 1 & 0 \\
\hline 0 & 30.2 & 0.98 & 0 & 0 & 71 & 1 & 0 \\
\hline 0 & 30.2 & 0.93 & 0 & 0 & 72 & 1 & 0 \\
\hline 0 & 30.2 & 0.82 & 0 & 0 & 73 & 1 & 0 \\
\hline 0 & 30.2 & 0.72 & 0 & 0 & 74 & 1 & 0 \\
\hline 0 & 30.2 & 0.62 & 0 & 0 & 75 & 1 & 0 \\
\hline 0 & 30.2 & 0.51 & 0 & 0 & 76 & 1 & 0 \\
\hline 0 & 30.2 & 0.41 & 0 & 0 & 77 & 1 & 0 \\
\hline 0 & 30.2 & 0.31 & 0 & 0 & 78 & 1 & 0 \\
\hline 0 & 30.2 & 0.21 & 0 & 0 & 79 & 1 & 0 \\
\hline & 30.2 & 0.1 & 0 & 0 & 80 & 1 & 0 \\
\hline
\end{tabular}

$\mathrm{J}[\mathrm{t}] \quad \mathrm{T}\left[{ }^{\circ} \mathrm{C}\right] \quad \mathrm{D}[1 / \mathrm{s}] \quad \mathrm{Tau}[\mathrm{Pa}] \quad \mathrm{Eta}[\mathrm{Pas}] \quad \mathrm{MP} \#$ Block $\mathrm{M}[\mathrm{mNm}] \mathrm{G}[\mathrm{Pa}]$

$\begin{array}{lllllllll}0 & 30.3 & 0.1 & 0 & 0 & 1 & 1 & 0 & 0 \\ 0 & 30.3 & 0.2 & 0 & 0 & 2 & 1 & 0 & 0 \\ 0 & 30.3 & 0.3 & 0 & 0 & 3 & 1 & 0 & 0 \\ 0 & 30.3 & 0.4 & 0 & 0 & 4 & 1 & 0 & 0 \\ 0 & 30.3 & 0.5 & 0 & 0 & 5 & 1 & 0 & 0 \\ 0 & 30.3 & 0.6 & 0 & 0 & 6 & 1 & 0 & 0 \\ 0 & 30.3 & 0.71 & 0 & 0 & 7 & 1 & 0 & 0 \\ 0 & 30.3 & 0.81 & 0 & 0 & 8 & 1 & 0 & 0 \\ 0 & 30.3 & 0.91 & 0 & 0 & 9 & 1 & 0 & 0 \\ 0 & 30.3 & 1.01 & 0 & 0 & 10 & 1 & 0 & 0 \\ 0 & 30.3 & 2.02 & 0 & 0 & 11 & 1 & 0 & 0\end{array}$




\begin{tabular}{|c|c|c|c|c|c|}
\hline 1 & 2 & 240 & 0.6 & 1.23 & 4.38252 \\
\hline & 2 & 260 & 0.8 & 1.23 & 6.04835 \\
\hline & 2 & 280 & 1 & 1.56 & 8.12966 \\
\hline & 2 & 300 & 1.19 & 1.85 & 10.62644 \\
\hline & 2 & 320 & 1.39 & 1.68 & 13.54105 \\
\hline & 2 & 340 & 1.59 & 1.76 & 16.86957 \\
\hline & 2 & 360 & 1.79 & 1.82 & 20.61513 \\
\hline & 3 & 380 & 1.98 & 1.92 & 24.78717 \\
\hline & 3 & 400 & 3.97 & 2.65 & 33.06448 \\
\hline & 3 & 420 & 5.95 & 3.09 & 45.51147 \\
\hline & 3 & 440 & 7.94 & 3.4 & 62.08965 \\
\hline & 3 & 460 & 9.92 & 3.69 & 82.85322 \\
\hline & 3 & 480 & 11.91 & 4.19 & 107.73935 \\
\hline & 3 & 500 & 13.89 & 4.66 & 136.81636 \\
\hline & 3 & 520 & 15.88 & 4.93 & 170.02692 \\
\hline & 3 & 540 & 17.86 & 5.34 & 207.43151 \\
\hline & 4 & 560 & 19.85 & 5.66 & 249.33014 \\
\hline 1 & 4 & 580 & 39.69 & 9.13 & 332.23756 \\
\hline 1 & 4 & 600 & 59.54 & 12.5 & 456.64541 \\
\hline 1 & 4 & 620 & 79.38 & 15.34 & 622.55292 \\
\hline I & 4 & 640 & 99.23 & 18.06 & 830.21927 \\
\hline 1 & 4 & 660 & 119.07 & 20.77 & 1079.16771 \\
\hline 1 & 4 & 680 & 138.92 & 23.51 & 1369.87498 \\
\hline 1 & 4 & 700 & 158.76 & 26.13 & 1701.98924 \\
\hline 1 & 4 & 720 & 178.61 & 28.86 & 2075.92673 \\
\hline & 4 & 740 & 198.45 & 31.45 & 2491.23664 \\
\hline 1 & 5 & 750 & 198.45 & 31.4 & 2703.43787 \\
\hline 1 & 5 & 760 & 198.45 & 31.36 & 2911.46235 \\
\hline 1 & 5 & 770 & 198.45 & 31.3 & 3118.86323 \\
\hline 1 & 5 & 780 & 198.45 & 31.3 & 3326.9906 \\
\hline 1 & 5 & 790 & 198.45 & 31.24 & 3534.59961 \\
\hline 1 & 5 & 800 & 198.45 & 31.23 & 3742.31229 \\
\hline & 6 & 820 & 198.45 & 31.23 & 4162.51602 \\
\hline & 6 & 840 & 178.61 & 28.44 & 4536.9813 \\
\hline 1 & 6 & 860 & 158.76 & 25.61 & 4869.46861 \\
\hline 1 & 6 & 880 & 138.92 & 23 & 5160.87175 \\
\hline 1 & 6 & 900 & 119.07 & 20.21 & 5410.45322 \\
\hline 1 & 6 & 920 & 99.23 & 17.45 & 5618.80443 \\
\hline 1 & 6 & 940 & 79.38 & 14.78 & 5785.2091 \\
\hline 1 & 6 & 960 & 59.54 & 11.96 & 5910.39607 \\
\hline 1 & 6 & 980 & 39.69 & 8.59 & 5993.79043 \\
\hline 1 & 6 & 1000 & 19.85 & 5.39 & 6035.67493 \\
\hline 1 & 7 & 1020 & 17.86 & 5.22 & 6073.37011 \\
\hline 1 & 7 & 1040 & 15.88 & 4.84 & 6106.63801 \\
\hline 1 & 7 & 1060 & 13.89 & 4.71 & 6135.78021 \\
\hline & 7 & 1080 & 11.9 & 4.33 & 6160.72367 \\
\hline & 7 & 1100 & 9.92 & 3.84 & 6181.53279 \\
\hline 1 & 7 & 1120 & 7.93 & 3.61 & 6198.18481 \\
\hline 1 & 7 & 1140 & 5.95 & 3.28 & 6210.67813 \\
\hline 1 & 7 & 1160 & 3.96 & 2.94 & 6219.00571 \\
\hline
\end{tabular}

\begin{tabular}{|c|c|c|c|c|c|c|c|}
\hline 0 & 30.3 & 3.02 & 0.082 & 0.027 & 12 & 1 & 0.06125 \\
\hline 0 & 30.3 & 4.03 & 0.083 & 0.021 & 13 & 1 & 0.0617 \\
\hline 0 & 30.3 & 5.04 & 0.105 & 0.021 & 14 & 1 & 0.07815 \\
\hline 0 & 30.3 & 6 & 0.124 & 0.021 & 15 & 1 & 0.0927 \\
\hline 0 & 30.3 & 7 & 0.112 & 0.016 & 16 & 1 & 0.0839 \\
\hline 0 & 30.3 & 8.01 & 0.118 & 0.015 & 17 & 1 & 0.0879 \\
\hline 0 & 30.3 & 9.02 & 0.122 & 0.014 & 18 & 1 & 0.091 \\
\hline 0 & 30.3 & 9.98 & 0.129 & 0.013 & 19 & 1 & 0.09605 \\
\hline 0 & 30.3 & 20 & 0.177 & 0.009 & 20 & 1 & 0.13245 \\
\hline 0 & 30.3 & 29.98 & 0.207 & 0.007 & 21 & 1 & 0.1544 \\
\hline c & 30.3 & 40.01 & 0.228 & 0.006 & 22 & 1 & 0.1699 \\
\hline c & 30.3 & 49.99 & 0.247 & 0.005 & 23 & 1 & 0.1843 \\
\hline c & 30.3 & 60.01 & 0.281 & 0.005 & 24 & 1 & 0.2095 \\
\hline 0 & 30.3 & 69.99 & 0.312 & 0.004 & 25 & 1 & 0.23275 \\
\hline 0 & 30.3 & 80.02 & 0.33 & 0.004 & 26 & 1 & 0.2466 \\
\hline 0 & 30.3 & 90 & 0.358 & 0.004 & 27 & 1 & 0.26685 \\
\hline 0 & 30.3 & 100.02 & 0.379 & 0.004 & 28 & 1 & 0.28295 \\
\hline 0 & 30.3 & 200 & 0.612 & 0.003 & 29 & 1 & 0.4567 \\
\hline 0 & 30.3 & 300.02 & 0.838 & 0.003 & 30 & 1 & 0.6252 \\
\hline 0 & 30.3 & 400 & 1.028 & 0.003 & 31 & 1 & 0.76705 \\
\hline 0 & 30.3 & 500.02 & 1.21 & 0.002 & 32 & 1 & 0.90285 \\
\hline 0 & 30.3 & 599.99 & 1.392 & 0.002 & 33 & 1 & 1.03845 \\
\hline 0 & 30.3 & 700.02 & 1.575 & 0.002 & 34 & 1 & 1.17555 \\
\hline 0 & 30.3 & 799.99 & 1.75 & 0.002 & 35 & 1 & 1.30625 \\
\hline 0 & 30.3 & 900.02 & 1.934 & 0.002 & 36 & 1 & 1.44315 \\
\hline 0 & 30.3 & 999.99 & 2.107 & 0.002 & 37 & 1 & 1.57245 \\
\hline 0 & 30.3 & 999.99 & 2.104 & 0.002 & 38 & 1 & 1.5702 \\
\hline 0 & 30.3 & 999.99 & 2.101 & 0.002 & 39 & 1 & 1.56815 \\
\hline 0 & 30.3 & 999.99 & 2.097 & 0.002 & 40 & 1 & 1.565 \\
\hline 0 & 30.3 & 999.99 & 2.097 & 0.002 & 41 & 1 & 1.5649 \\
\hline 0 & 30.3 & 999.99 & 2.093 & 0.002 & 42 & 1 & 1.56195 \\
\hline 0 & 30.3 & 999.99 & 2.092 & 0.002 & 43 & 1 & 1.56125 \\
\hline 0 & 30.3 & 999.99 & 2.092 & 0.002 & 44 & 1 & 1.56125 \\
\hline 0 & 30.3 & 900.02 & 1.906 & 0.002 & 45 & 1 & 1.4221 \\
\hline 0 & 30.3 & 799.99 & 1.716 & 0.002 & 46 & 1 & 1.28045 \\
\hline 0 & 30.3 & 700.02 & 1.541 & 0.002 & 47 & 1 & 1.1501 \\
\hline 0 & 30.3 & 599.99 & 1.354 & 0.002 & 48 & 1 & 1.0107 \\
\hline 0 & 30.3 & 500.02 & 1.169 & 0.002 & 49 & 1 & 0.87265 \\
\hline 0 & 30.3 & 400 & 0.99 & 0.002 & 50 & 1 & 0.7389 \\
\hline 0 & 30.3 & 300.02 & 0.801 & 0.003 & 51 & 1 & 0.59805 \\
\hline 0 & 30.3 & 200 & 0.576 & 0.003 & 52 & 1 & 0.4296 \\
\hline 0 & 30.3 & 100.02 & 0.361 & 0.004 & 53 & 1 & 0.2693 \\
\hline 0 & 30.3 & 90 & 0.349 & 0.004 & 54 & 1 & 0.2608 \\
\hline 0 & 30.3 & 80.02 & 0.324 & 0.004 & 55 & 1 & 0.24215 \\
\hline 0 & 30.3 & 69.99 & 0.316 & 0.005 & 56 & 1 & 0.2355 \\
\hline 0 & 30.3 & 59.96 & 0.29 & 0.005 & 57 & 1 & 0.2167 \\
\hline 0 & 30.3 & 49.99 & 0.257 & 0.005 & 58 & 1 & 0.19185 \\
\hline 0 & 30.3 & 39.96 & 0.242 & 0.006 & 59 & 1 & 0.1806 \\
\hline 0 & 30.3 & 29.98 & 0.22 & 0.007 & 60 & 1 & 0.1642 \\
\hline 0 & 30.3 & 19.95 & 0.197 & 0.01 & 61 & 1 & 0.1472 \\
\hline
\end{tabular}




$\begin{array}{rrrrrrl}1 & 7 & 1180 & 1.98 & 2.07 & 6223.17932 & 0 \\ 1 & 8 & 1200 & 1.79 & 2.1 & 6226.94766 & 0 \\ 1 & 8 & 1220 & 1.59 & 2.01 & 6230.28403 & 0 \\ 1 & 8 & 1240 & 1.39 & 2.04 & 6233.20414 & 0 \\ 1 & 8 & 1260 & 1.19 & 1.84 & 6235.70642 & 0 \\ 1 & 8 & 1280 & 0.99 & 1.92 & 6237.79322 & 0 \\ 1 & 8 & 1300 & 0.8 & 1.83 & 6239.46376 & 0 \\ 1 & 8 & 1320 & 0.6 & 1.7 & 6240.71883 & 0 \\ 1 & 8 & 1340 & 0.4 & 1.48 & 6241.55528 & 0 \\ 1 & 8 & 1360 & 0.2 & 1.49 & 6241.97704 & 0 \\ 1 & 9 & 1380 & 0.18 & 1.63 & 6242.35638 & 0 \\ 1 & 9 & 1400 & 0.16 & 1.62 & 6242.69175 & 0 \\ 1 & 9 & 1420 & 0.14 & 1.42 & 6242.98549 & 0 \\ 1 & 9 & 1440 & 0.12 & 1.4 & 6243.23681 & 0 \\ 1 & 9 & 1460 & 0.1 & 0 & 6243.44652 & 0 \\ 1 & 9 & 1480 & 0.08 & 0 & 6243.61459 & 0 \\ 1 & 9 & 1500 & 0.06 & 0 & 6243.74104 & 0 \\ 1 & 9 & 1520 & 0.04 & 1.1 & 6243.82429 & 0 \\ 1 & 9 & 1540 & 0.02 & 0 & 6243.8667 & 0\end{array}$

04.01.04 WM-187 as received DG run 02.txt

\begin{tabular}{|c|c|c|c|c|c|c|}
\hline Mode & Step & $\mathrm{t}[\mathrm{s}]$ & $\mathrm{n}[\mathrm{rpm}]$ & $\mathrm{M}[\%]$. & Phi[rad] & Gamma \\
\hline 1 & 1 & 20 & 0.02 & 1.04 & 0.03927 & 0 \\
\hline 1 & 1 & 40 & 0.04 & 1.28 & 0.12331 & 0 \\
\hline 1 & 1 & 60 & 0.06 & 0 & 0.24897 & 0 \\
\hline 1 & 1 & 80 & 0.08 & 0 & 0.41548 & 0 \\
\hline 1 & 1 & 100 & 0.1 & 0 & 0.62518 & 0 \\
\hline 1 & 1 & 120 & 0.12 & 1.01 & 0.87572 & 0 \\
\hline 1 & 1 & 140 & 0.14 & 0 & 1.16867 & 0 \\
\hline 1 & 1 & 160 & 0.16 & 0 & 1.50404 & 0 \\
\hline 1 & 1 & 180 & 0.18 & 0 & 1.88024 & 0 \\
\hline 1 & 2 & 200 & 0.2 & 0 & 2.30122 & 0 \\
\hline 1 & 2 & 220 & 0.4 & 1.5 & 3.13374 & 0 \\
\hline 1 & 2 & 240 & 0.6 & 1.51 & 4.38331 & 0 \\
\hline 1 & 2 & 260 & 0.8 & 2.09 & 6.04678 & 0 \\
\hline 1 & 2 & 280 & 1 & 2.02 & 8.12966 & 0 \\
\hline 1 & 2 & 300 & 1.19 & 1.9 & 10.62644 & 0 \\
\hline 1 & 2 & 320 & 1.39 & 2.15 & 13.53869 & 0 \\
\hline 1 & 2 & 340 & 1.59 & 1.97 & 16.86878 & 0 \\
\hline 1 & 2 & 360 & 1.79 & 2.14 & 20.61513 & 0 \\
\hline 1 & 3 & 380 & 1.98 & 2.09 & 24.78874 & 0 \\
\hline 1 & 3 & 400 & 3.97 & 2.92 & 33.06919 & 0 \\
\hline 1 & 3 & 420 & 5.95 & 3.24 & 45.50754 & 0 \\
\hline 1 & 3 & 440 & 7.94 & 3.61 & 62.08494 & 0 \\
\hline 1 & 3 & 460 & 9.92 & 3.84 & 82.85951 & 0 \\
\hline 1 & 3 & 480 & 11.91 & 4.35 & 107.7527 & 0 \\
\hline 1 & 3 & 500 & 13.89 & 4.75 & 136.81479 & 0 \\
\hline 1 & 3 & 520 & 15.88 & 4.98 & 170.05048 & 0 \\
\hline 1 & 3 & 540 & 17.86 & 5.41 & 207.42758 & 0 \\
\hline 1 & 4 & 560 & 19.85 & 5.7 & 249.31601 & 0 \\
\hline
\end{tabular}

$\begin{array}{rrrrrrrrr}0 & 30.3 & 9.98 & 0.139 & 0.014 & 62 & 1 & 0.10345 & 0 \\ 0 & 30.3 & 9.02 & 0.14 & 0.016 & 63 & 1 & 0.10475 & 0 \\ 0 & 30.3 & 8.01 & 0.135 & 0.017 & 64 & 1 & 0.10065 & 0 \\ 0 & 30.4 & 7 & 0.137 & 0.02 & 65 & 1 & 0.10195 & 0 \\ 0 & 30.3 & 6 & 0.123 & 0.021 & 66 & 1 & 0.092 & 0 \\ 0 & 30.3 & 4.99 & 0.129 & 0.026 & 67 & 1 & 0.0959 & 0 \\ 0 & 30.3 & 4.03 & 0.123 & 0.031 & 68 & 1 & 0.09155 & 0 \\ 0 & 30.3 & 3.02 & 0.114 & 0.038 & 69 & 1 & 0.0852 & 0 \\ 0 & 30.3 & 2.02 & 0.099 & 0.049 & 70 & 1 & 0.0739 & 0 \\ 0 & 30.3 & 1.01 & 0.1 & 0.099 & 71 & 1 & 0.07455 & 0 \\ 0 & 30.3 & 0.91 & 0.109 & 0.12 & 72 & 1 & 0.0816 & 0 \\ 0 & 30.3 & 0.81 & 0.109 & 0.135 & 73 & 1 & 0.0811 & 0 \\ 0 & 30.4 & 0.71 & 0.095 & 0.134 & 74 & 1 & 0.07085 & 0 \\ 0 & 30.3 & 0.6 & 0.094 & 0.157 & 75 & 1 & 0.0702 & 0 \\ 0 & 30.4 & 0.5 & 0 & 0 & 76 & 1 & 0 & 0 \\ 0 & 30.3 & 0.4 & 0 & 0 & 77 & 1 & 0 & 0 \\ 0 & 30.3 & 0.3 & 0 & 0 & 78 & 1 & 0 & 0 \\ 0 & 30.3 & 0.2 & 0.074 & 0.37 & 79 & 1 & 0.05495 & 0 \\ 0 & 30.4 & 0.1 & 0 & 0 & 80 & 1 & 0 & 0\end{array}$

$\mathrm{J}[\mathrm{t}] \quad \mathrm{T}\left[{ }^{\circ} \mathrm{C}\right] \quad \mathrm{D}[1 / \mathrm{s}] \quad \mathrm{Tau}[\mathrm{Pa}] \mathrm{Eta}[\mathrm{Pas}] \mathrm{MP \#}$ Block $\mathrm{M}[\mathrm{mNm}] \mathrm{G}[\mathrm{Pa}]$

$\begin{array}{rrrrrrrrr}0 & 30.3 & 0.1 & 0.07 & 0.7 & 1 & 1 & 0.0521 & 0 \\ 0 & 30.3 & 0.2 & 0.086 & 0.43 & 2 & 1 & 0.06405 & 0 \\ 0 & 30.3 & 0.3 & 0 & 0 & 3 & 1 & 0 & 0 \\ 0 & 30.3 & 0.4 & 0 & 0 & 4 & 1 & 0 & 0 \\ 0 & 30.4 & 0.5 & 0 & 0 & 5 & 1 & 0 & 0 \\ 0 & 30.4 & 0.6 & 0.068 & 0.113 & 6 & 1 & 0.05065 & 0 \\ 0 & 30.3 & 0.71 & 0 & 0 & 7 & 1 & 0 & 0 \\ 0 & 30.3 & 0.81 & 0 & 0 & 8 & 1 & 0 & 0 \\ 0 & 30.4 & 0.91 & 0 & 0 & 9 & 1 & 0 & 0 \\ 0 & 30.4 & 1.01 & 0 & 0 & 10 & 1 & 0 & 0 \\ 0 & 30.4 & 2.02 & 0.101 & 0.05 & 11 & 1 & 0.075 & 0 \\ 0 & 30.4 & 3.02 & 0.101 & 0.033 & 12 & 1 & 0.07535 & 0 \\ 0 & 30.4 & 4.03 & 0.14 & 0.035 & 13 & 1 & 0.10455 & 0 \\ 0 & 30.4 & 5.04 & 0.135 & 0.027 & 14 & 1 & 0.10075 & 0 \\ 0 & 30.4 & 6 & 0.127 & 0.021 & 15 & 1 & 0.09505 & 0 \\ 0 & 30.4 & 7 & 0.144 & 0.021 & 16 & 1 & 0.10745 & 0 \\ 0 & 30.4 & 8.01 & 0.132 & 0.016 & 17 & 1 & 0.09845 & 0 \\ 0 & 30.3 & 9.02 & 0.144 & 0.016 & 18 & 1 & 0.1071 & 0 \\ 0 & 30.3 & 9.98 & 0.14 & 0.014 & 19 & 1 & 0.10445 & 0 \\ 0 & 30.3 & 20 & 0.196 & 0.01 & 20 & 1 & 0.146 & 0 \\ 0 & 30.4 & 29.98 & 0.217 & 0.007 & 21 & 1 & 0.1618 & 0 \\ 0 & 30.4 & 40.01 & 0.242 & 0.006 & 22 & 1 & 0.1803 & 0 \\ 0 & 30.4 & 49.99 & 0.257 & 0.005 & 23 & 1 & 0.19185 & 0 \\ 0 & 30.4 & 60.01 & 0.292 & 0.005 & 24 & 1 & 0.21755 & 0 \\ 0 & 30.4 & 69.99 & 0.318 & 0.005 & 25 & 1 & 0.2374 & 0 \\ 0 & 30.4 & 80.02 & 0.334 & 0.004 & 26 & 1 & 0.2489 & 0 \\ 0 & 30.3 & 90 & 0.363 & 0.004 & 27 & 1 & 0.27055 & 0 \\ 0 & 100.02 & 0.382 & 0.004 & 28 & 1 & 0.2851 & 0\end{array}$




\begin{tabular}{|c|c|c|c|c|c|}
\hline & 4 & 580 & 39.69 & 9.23 & 332.17237 \\
\hline & 4 & 600 & 59.54 & 12.58 & 456.57866 \\
\hline & 4 & 620 & 79.38 & 15.45 & 622.62125 \\
\hline & 4 & 640 & 99.23 & 18.1 & 830.13209 \\
\hline & 4 & 660 & 119.07 & 20.85 & 1079.1952 \\
\hline & 4 & 680 & 138.92 & 23.57 & 1369.83022 \\
\hline & 4 & 700 & 158.76 & 26.18 & 1702.01751 \\
\hline & 4 & 720 & 178.61 & 28.94 & 2076.0571 \\
\hline & 4 & 740 & 198.45 & 31.48 & 2490.95311 \\
\hline & 5 & 750 & 198.45 & 31.38 & 2703.0491 \\
\hline & 5 & 760 & 198.45 & 31.29 & 2911.07358 \\
\hline & 5 & 770 & 198.45 & 31.28 & 3118.47446 \\
\hline & 5 & 780 & 198.45 & 31.23 & 3326.60262 \\
\hline & 5 & 790 & 198.45 & 31.22 & 3534.3153 \\
\hline & 5 & 800 & 198.45 & 31.18 & 3742.13165 \\
\hline & 6 & 820 & 198.45 & 31.18 & 4162.44062 \\
\hline & 6 & 840 & 178.61 & 28.38 & 4536.64515 \\
\hline & 6 & 860 & 158.76 & 25.53 & 4869.55893 \\
\hline & 6 & 880 & 138.92 & 22.9 & 5160.60786 \\
\hline & 6 & 900 & 119.07 & 20.13 & 5410.31421 \\
\hline & 6 & 920 & 99.23 & 17.37 & 5618.60337 \\
\hline & 6 & 940 & 79.38 & 14.62 & 5785.04966 \\
\hline & 6 & 960 & 59.54 & 11.78 & 5910.16359 \\
\hline & 6 & 980 & 39.69 & 8.43 & 5993.55874 \\
\hline & 6 & 1000 & 19.85 & 5.31 & 6035.44324 \\
\hline & 7 & 1020 & 17.86 & 5.13 & 6073.12507 \\
\hline & 7 & 1040 & 15.88 & 4.72 & 6106.41731 \\
\hline & 7 & 1060 & 13.89 & 4.58 & 6135.5226 \\
\hline & 7 & 1080 & 11.9 & 4.25 & 6160.47313 \\
\hline & 7 & 1100 & 9.92 & 3.71 & 6181.27754 \\
\hline & 7 & 1120 & 7.93 & 3.54 & 6197.92877 \\
\hline & 7 & 1140 & 5.95 & 3.14 & 6210.41267 \\
\hline & 7 & 1160 & 3.96 & 2.78 & 6218.7481 \\
\hline & 7 & 1180 & 1.98 & 2.03 & 6222.92563 \\
\hline & 8 & 1200 & 1.79 & 2.06 & 6226.6979 \\
\hline & 8 & 1220 & 1.59 & 1.95 & 6230.03349 \\
\hline & 8 & 1240 & 1.39 & 2 & 6232.95281 \\
\hline 1 & 8 & 1260 & 1.19 & 1.97 & 6235.4543 \\
\hline 1 & 8 & 1280 & 0.99 & 1.72 & 6237.54111 \\
\hline 1 & 8 & 1300 & 0.8 & 1.86 & 6239.21165 \\
\hline 1 & 8 & 1320 & 0.6 & 1.54 & 6240.46593 \\
\hline 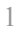 & 8 & 1340 & 0.4 & 1.63 & 6241.30474 \\
\hline 1 & 8 & 1360 & 0.2 & 1.49 & 6241.72571 \\
\hline 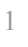 & 9 & 1380 & 0.18 & 1.44 & 6242.10506 \\
\hline 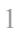 & 9 & 1400 & 0.16 & 1.41 & 6242.44042 \\
\hline & 9 & 1420 & 0.14 & 1.1 & 6242.73416 \\
\hline & 9 & 1440 & 0.12 & 1.12 & 6242.98549 \\
\hline 1 & 9 & 1460 & 0.1 & 1.2 & 6243.19519 \\
\hline & 9 & 1480 & 0.08 & 0 & 6243.36248 \\
\hline 1 & 9 & 1500 & 0.06 & 1.05 & 6243.48893 \\
\hline
\end{tabular}

\begin{tabular}{|c|c|c|c|c|c|c|c|}
\hline 0 & 30.3 & 200 & 0.618 & 0.003 & 29 & 1 & 0.46135 \\
\hline 0 & 30.4 & 300.02 & 0.843 & 0.003 & 30 & 1 & 0.62875 \\
\hline 0 & 30.3 & 400 & 1.035 & 0.003 & 31 & 1 & 0.7723 \\
\hline 0 & 30.4 & 500.02 & 1.212 & 0.002 & 32 & 1 & 0.90475 \\
\hline 0 & 30.3 & 599.99 & 1.397 & 0.002 & 33 & 1 & 1.0424 \\
\hline 0 & 30.4 & 700.02 & 1.579 & 0.002 & 34 & 1 & 1.1787 \\
\hline 0 & 30.4 & 799.99 & 1.754 & 0.002 & 35 & 1 & 1.30875 \\
\hline 0 & 30.4 & 900.02 & 1.939 & 0.002 & 36 & 1 & 1.4472 \\
\hline 0 & 30.3 & 999.99 & 2.109 & 0.002 & 37 & 1 & 1.57395 \\
\hline 0 & 30.3 & 999.99 & 2.102 & 0.002 & 38 & 1 & 1.5688 \\
\hline 0 & 30.3 & 999.99 & 2.096 & 0.002 & 39 & 1 & 1.56445 \\
\hline 0 & 30.3 & 999.99 & 2.096 & 0.002 & 40 & 1 & 1.56405 \\
\hline 0 & 30.4 & 999.99 & 2.092 & 0.002 & 41 & 1 & 1.56155 \\
\hline 0 & 30.4 & 999.99 & 2.091 & 0.002 & 42 & 1 & 1.5608 \\
\hline 0 & 30.4 & 999.99 & 2.089 & 0.002 & 43 & 1 & 1.5589 \\
\hline 0 & 30.3 & 999.99 & 2.089 & 0.002 & 44 & 1 & 1.55895 \\
\hline 0 & 30.4 & 900.02 & 1.902 & 0.002 & 45 & 1 & 1.41915 \\
\hline 0 & 30.4 & 799.99 & 1.711 & 0.002 & 46 & 1 & 1.2766 \\
\hline 0 & 30.4 & 700.02 & 1.534 & 0.002 & 47 & 1 & 1.14505 \\
\hline 0 & 30.4 & 599.99 & 1.349 & 0.002 & 48 & 1 & 1.0064 \\
\hline 0 & 30.4 & 500.02 & 1.164 & 0.002 & 49 & 1 & 0.8684 \\
\hline 0 & 30.4 & 400 & 0.98 & 0.002 & 50 & 1 & 0.7311 \\
\hline 0 & 30.4 & 300.02 & 0.789 & 0.003 & 51 & 1 & 0.5891 \\
\hline 0 & 30.3 & 200 & 0.565 & 0.003 & 52 & 1 & 0.4216 \\
\hline 0 & 30.3 & 100.02 & 0.356 & 0.004 & 53 & 1 & 0.26555 \\
\hline 0 & 30.4 & 90 & 0.344 & 0.004 & 54 & 1 & 0.25635 \\
\hline 0 & 30.4 & 80.02 & 0.316 & 0.004 & 55 & 1 & 0.2361 \\
\hline 0 & 30.4 & 69.99 & 0.307 & 0.004 & 56 & 1 & 0.22905 \\
\hline 0 & 30.4 & 59.96 & 0.285 & 0.005 & 57 & 1 & 0.2125 \\
\hline 0 & 30.4 & 49.99 & 0.249 & 0.005 & 58 & 1 & 0.18545 \\
\hline 0 & 30.4 & 39.96 & 0.237 & 0.006 & 59 & 1 & 0.17685 \\
\hline 0 & 30.4 & 29.98 & 0.211 & 0.007 & 60 & 1 & 0.1571 \\
\hline 0 & 30.4 & 19.95 & 0.186 & 0.009 & 61 & 1 & 0.13895 \\
\hline 0 & 30.4 & 9.98 & 0.136 & 0.014 & 62 & 1 & 0.10165 \\
\hline 0 & 30.4 & 9.02 & 0.138 & 0.015 & 63 & 1 & 0.10285 \\
\hline 0 & 30.4 & 8.01 & 0.13 & 0.016 & 64 & 1 & 0.0973 \\
\hline 0 & 30.4 & 7 & 0.134 & 0.019 & 65 & 1 & 0.1002 \\
\hline 0 & 30.4 & 6 & 0.132 & 0.022 & 66 & 1 & 0.0983 \\
\hline 0 & 30.3 & 4.99 & 0.115 & 0.023 & 67 & 1 & 0.08575 \\
\hline 0 & 30.4 & 4.03 & 0.124 & 0.031 & 68 & 1 & 0.0929 \\
\hline 0 & 30.4 & 3.02 & 0.103 & 0.034 & 69 & 1 & 0.077 \\
\hline 0 & 30.4 & 2.02 & 0.109 & 0.054 & 70 & 1 & 0.08125 \\
\hline 0 & 30.4 & 1.01 & 0.1 & 0.099 & 71 & 1 & 0.0744 \\
\hline 0 & 30.4 & 0.91 & 0.096 & 0.105 & 72 & 1 & 0.0719 \\
\hline 0 & 30.4 & 0.81 & 0.094 & 0.116 & 73 & 1 & 0.07025 \\
\hline 0 & 30.4 & 0.71 & 0.074 & 0.104 & 74 & 1 & 0.05495 \\
\hline 0 & 30.4 & 0.6 & 0.075 & 0.125 & 75 & 1 & 0.0559 \\
\hline 0 & 30.4 & 0.5 & 0.08 & 0.16 & 76 & 1 & 0.0598 \\
\hline 0 & 30.3 & 0.4 & 0 & 0 & 77 & 1 & 0 \\
\hline & 30.3 & 0.3 & 0.07 & 0.233 & 78 & 1 & 0.0525 \\
\hline
\end{tabular}




\begin{tabular}{|c|c|c|c|c|c|c|c|c|c|c|c|c|}
\hline 1520 & 0.04 & 0 & 6243.57297 & 0 & 0 & 30.3 & 0.2 & 0 & 0 & 79 & & 0 \\
\hline 1540 & 0.02 & 1.27 & 6243.61459 & 0 & 0 & 30.4 & 0.1 & 0.085 & 0.85 & 80 & & 0.0633 \\
\hline
\end{tabular}
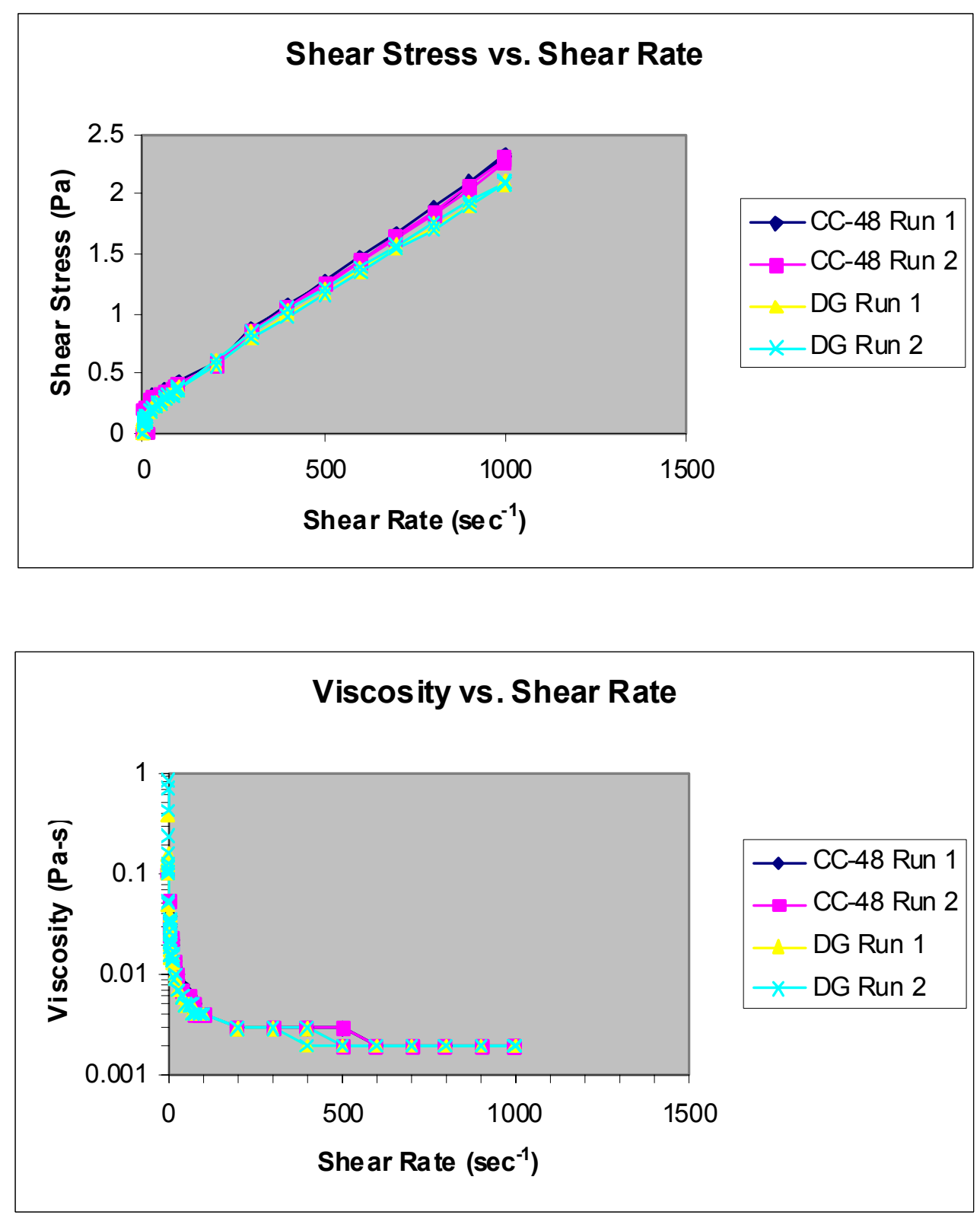

\section{E-2.2.3 Gravity Settled}

03.31.04 WM-187 gravity settled slurry CC-45 run 01.txt

Mode Step t[s] n[rpm] M[\%.] Phi[rad] Gamma

$\begin{array}{rrrrr}20 & 0.08 & 4.28 & 0.16415 & 2.0236 \\ 40 & 0.16 & 4.59 & 0.49244 & 6.0709 \\ 60 & 0.24 & 5.32 & 0.98332 & 12.122 \\ 80 & 0.31 & 5.7 & 1.63598 & 20.169\end{array}$

$\mathrm{J}[\mathrm{t}] \quad \mathrm{T}\left[{ }^{\circ} \mathrm{C}\right] \quad \mathrm{D}[1 / \mathrm{s}] \quad \mathrm{Tau}[\mathrm{Pa}] \quad$ Eta[Pas $] \quad$ MP\# Block M[mNm] G[Pa $]$

$\begin{array}{lllllllll}2.41773 & 30.2 & 0.1 & 0.837 & 8.37 & 1 & 1 & 0.21375 & 0.414\end{array}$

$\begin{array}{lllllllll}6.75297 & 30.2 & 0.21 & 0.899 & 4.281 & 2 & 1 & 0.22965 & 0.148\end{array}$

$\begin{array}{lllllllll}11.64503 & 30.2 & 0.31 & 1.041 & 3.358 & 3 & 1 & 0.26585 & 0.086\end{array}$

$\begin{array}{lllllllll}18.07225 & 30.2 & 0.4 & 1.116 & 2.79 & 4 & 1 & 0.285 & 0.055\end{array}$ 


\begin{tabular}{|c|c|c|c|c|c|c|c|c|c|c|c|c|c|c|c|}
\hline 1 & 1 & 100 & 0.39 & 5.96 & 2.4528 & 30.238 & 25.93347 & 30.2 & 0.5 & 1.166 & 2.332 & 5 & 1 & 0.29785 & 0.039 \\
\hline & 1 & 120 & 0.47 & 6.2 & 3.42983 & 42.283 & 34.85857 & 30.2 & 0.61 & 1.213 & 1.989 & 6 & 1 & 0.30975 & 0.029 \\
\hline & 1 & 140 & 0.54 & 6.31 & 4.57023 & 56.342 & 45.58449 & 30.2 & 0.7 & 1.236 & 1.766 & 7 & 1 & 0.31565 & 0.022 \\
\hline & 1 & 160 & 0.62 & 6.76 & 5.87399 & 72.415 & 54.73573 & 30.2 & 0.8 & 1.323 & 1.654 & 8 & 1 & 0.33775 & 0.018 \\
\hline & 1 & 180 & 0.7 & 7.45 & 7.33798 & 90.464 & 62.0038 & 30.2 & 0.9 & 1.459 & 1.621 & 9 & 1 & 0.37255 & 0.016 \\
\hline & 2 & 200 & 0.77 & 8.1 & 8.96061 & 0 & 0 & 30.2 & 0.99 & 1.586 & 1.602 & 10 & 1 & 0.40505 & 0 \\
\hline & 2 & 220 & 1.55 & 9.71 & 12.18545 & 0 & 0 & 30.2 & 2 & 1.902 & 0.951 & 11 & 1 & 0.4857 & 0 \\
\hline & 2 & 240 & 2.32 & 10.71 & 17.03607 & 0 & 0 & 30.2 & 3 & 2.096 & 0.699 & 12 & 1 & 0.5353 & 0 \\
\hline & 2 & 260 & 3.09 & 10.58 & 23.50775 & 0 & 0 & 30.3 & 3.99 & 2.072 & 0.519 & 13 & 1 & 0.5291 & 0 \\
\hline & 2 & 280 & 3.87 & 10.61 & 31.60207 & 0 & 0 & 30.3 & 5 & 2.077 & 0.415 & 14 & 1 & 0.5305 & 0 \\
\hline & 2 & 300 & 4.65 & 10.69 & 41.3143 & 0 & 0 & 30.3 & 6 & 2.093 & 0.349 & 15 & 1 & 0.5345 & 0 \\
\hline & 2 & 320 & 5.42 & 10.67 & 52.66644 & 0 & 0 & 30.3 & 7 & 2.09 & 0.299 & 16 & 1 & 0.5336 & 0 \\
\hline & 2 & 340 & 6.2 & 10.69 & 65.6263 & 0 & 0 & 30.3 & 8 & 2.093 & 0.262 & 17 & 1 & 0.5344 & 0 \\
\hline & 2 & 360 & 6.97 & 10.7 & 80.2135 & 0 & 0 & 30.3 & 9 & 2.095 & 0.233 & 18 & 1 & 0.53505 & 0 \\
\hline & 3 & 380 & 7.75 & 10.57 & 96.5757 & 0 & 0 & 30.3 & 10.01 & 2.069 & 0.207 & 19 & 1 & 0.52825 & 0 \\
\hline & 3 & 400 & 15.5 & 11.18 & 128.92704 & 0 & 0 & 30.3 & 20.01 & 2.188 & 0.109 & 20 & 1 & 0.5588 & 0 \\
\hline & 3 & 420 & 23.24 & 11.84 & 177.50784 & 0 & 0 & 30.3 & 30 & 2.319 & 0.077 & 21 & 1 & 0.59215 & 0 \\
\hline & 3 & 440 & 30.99 & 12.3 & 242.23643 & 0 & 0 & 30.3 & 40.01 & 2.408 & 0.06 & 22 & 1 & 0.6149 & 0 \\
\hline & 3 & 460 & 38.73 & 12.42 & 323.21962 & 0 & 0 & 30.3 & 50 & 2.431 & 0.049 & 23 & 1 & 0.6209 & 0 \\
\hline & 3 & 480 & 46.48 & 12.94 & 420.45898 & 0 & 0 & 30.3 & 60.01 & 2.533 & 0.042 & 24 & 1 & 0.6468 & 0 \\
\hline & 3 & 500 & 54.22 & 13.51 & 533.89561 & 0 & 0 & 30.3 & 70 & 2.646 & 0.038 & 25 & 1 & 0.6756 & 0 \\
\hline & 3 & 520 & 61.96 & 13.83 & 663.65124 & 0 & 0 & 30.3 & 79.99 & 2.709 & 0.034 & 26 & 1 & 0.6917 & 0 \\
\hline & 3 & 540 & 69.71 & 14.14 & 809.42035 & 0 & 0 & 30.3 & 90 & 2.768 & 0.031 & 27 & 1 & 0.70685 & 0 \\
\hline & 4 & 560 & 77.46 & 14.28 & 973.11304 & 0 & 0 & 30.3 & 100 & 2.795 & 0.028 & 28 & 1 & 0.71385 & 0 \\
\hline & 4 & 580 & 154.92 & 18.18 & 1296.32009 & 0 & 0 & 30.3 & 200 & 3.56 & 0.018 & 29 & 1 & 0.90915 & 0 \\
\hline & 4 & 600 & 232.38 & 22.39 & 1782.04094 & 0 & 0 & 30.3 & 300 & 4.385 & 0.015 & 30 & 1 & 1.11965 & 0 \\
\hline & 4 & 620 & 309.84 & 28.99 & 2429.5444 & 0 & 0 & 30.3 & 400 & 5.676 & 0.014 & 31 & 1 & 1.4494 & 0 \\
\hline & 4 & 640 & 387.3 & 37.59 & 3239.47918 & 0 & 0 & 30.3 & 500 & 7.361 & 0.015 & 32 & 1 & 1.8797 & 0 \\
\hline & 4 & 660 & 464.75 & 45.56 & 4211.60497 & 0 & 0 & 30.3 & 599.99 & 8.921 & 0.015 & 33 & 1 & 2.278 & 0 \\
\hline & 4 & 680 & 542.21 & 55.47 & 5346.48647 & 0 & 0 & 30.3 & 699.99 & 10.861 & 0.016 & 34 & 1 & 2.77355 & 0 \\
\hline & 4 & 700 & 619.67 & 66.75 & 6641.69051 & 0 & 0 & 30.3 & 799.99 & 13.069 & 0.016 & 35 & 1 & 3.3373 & 0 \\
\hline & 4 & 720 & 697.13 & 75.09 & 8100.78829 & 0 & 0 & 30.3 & 899.99 & 14.702 & 0.016 & 36 & 1 & 3.75445 & 0 \\
\hline & 4 & 740 & 774.59 & 86.52 & 9722.15641 & 0 & 0 & 30.3 & 1000 & 16.94 & 0.017 & 37 & 1 & 4.3259 & 0 \\
\hline & 5 & 750 & 774.59 & 83.68 & 10551.2306 & 0 & 0 & 30.3 & 1000 & 16.385 & 0.016 & 38 & 1 & 4.1841 & 0 \\
\hline 1 & 5 & 760 & 774.59 & 83.32 & 11360.3509 & 0 & 0 & 30.3 & 1000 & 16.314 & 0.016 & 39 & 1 & 4.16605 & 0 \\
\hline & 5 & 770 & 774.59 & 83.09 & 12171.9067 & 0 & 0 & 30.3 & 1000 & 16.268 & 0.016 & 40 & 1 & 4.15435 & 0 \\
\hline & 5 & 780 & 774.59 & 82.98 & 12984.27 & 0 & 0 & 30.3 & 1000 & 16.248 & 0.016 & 41 & 1 & 4.1492 & 0 \\
\hline & 5 & 790 & 774.59 & 83.16 & 13793.7979 & 0 & 0 & 30.3 & 1000 & 16.284 & 0.016 & 42 & 1 & 4.1582 & 0 \\
\hline & 5 & 800 & 774.59 & 82.38 & 14606.1612 & 0 & 0 & 30.3 & 1000 & 16.129 & 0.016 & 43 & 1 & 4.11875 & 0 \\
\hline 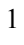 & 6 & 820 & 774.59 & 81.98 & 16246.7103 & 0 & 0 & 30.3 & 1000 & 16.051 & 0.016 & 44 & 1 & 4.0989 & 0 \\
\hline & 6 & 840 & 697.13 & 71.18 & 17707.0207 & 0 & 0 & 30.3 & 899.99 & 13.938 & 0.015 & 45 & 1 & 3.5592 & 0 \\
\hline & 6 & 860 & 619.67 & 61.23 & 19006.8044 & 0 & 0 & 30.3 & 799.99 & 11.988 & 0.015 & 46 & 1 & 3.0613 & 0 \\
\hline 1 & 6 & 880 & 542.21 & 51.19 & 20143.7091 & 0 & 0 & 30.3 & 699.99 & 10.023 & 0.014 & 47 & 1 & 2.55955 & 0 \\
\hline 1 & 6 & 900 & 464.75 & 41.13 & 21117.9382 & 0 & 0 & 30.3 & 599.99 & 8.054 & 0.013 & 48 & 1 & 2.0566 & 0 \\
\hline 1 & 6 & 920 & 387.3 & 33.34 & 21930.5449 & 0 & 0 & 30.3 & 500 & 6.528 & 0.013 & 49 & 1 & 1.66695 & 0 \\
\hline 1 & 6 & 940 & 309.84 & 26.01 & 22581.0863 & 0 & 0 & 30.3 & 400 & 5.093 & 0.013 & 50 & 1 & 1.30055 & 0 \\
\hline 1 & 6 & 960 & 232.38 & 18.45 & 23069.0879 & 0 & 0 & 30.3 & 300 & 3.612 & 0.012 & 51 & 1 & 0.9223 & 0 \\
\hline 1 & 6 & 980 & 154.92 & 14.71 & 23394.6253 & 0 & 0 & 30.3 & 200 & 2.879 & 0.014 & 52 & 1 & 0.73525 & 0 \\
\hline ] & 6 & 1000 & 77.46 & 11.82 & 23558.1397 & 0 & 0 & 30.3 & 100 & 2.314 & 0.023 & 53 & 1 & 0.5908 & 0 \\
\hline & 7 & 1020 & 69.71 & 11.74 & 23705.284 & 0 & 0 & 30.3 & 90 & 2.298 & 0.026 & 54 & 1 & 0.58675 & 0 \\
\hline
\end{tabular}




\begin{tabular}{|c|c|c|c|c|c|c|c|c|c|c|c|c|c|c|}
\hline 1 & $7 \quad 1040$ & 61.97 & 11.37 & 23835.2776 & 0 & 0 & 30.3 & 80 & 2.227 & 0.028 & 55 & 1 & 0.56865 & 0 \\
\hline 1 & $7 \quad 1060$ & 54.22 & 11.13 & 23948.8391 & 0 & 0 & 30.3 & 70 & 2.178 & 0.031 & 56 & 1 & 0.5563 & 0 \\
\hline & 71080 & 46.48 & 10.42 & 24046.2937 & 0 & 0 & 30.3 & 60.01 & 2.039 & 0.034 & 57 & 1 & 0.5208 & 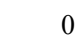 \\
\hline & 71100 & 38.73 & 9.95 & 24127.5675 & 0 & 0 & 30.3 & 50 & 1.948 & 0.039 & 58 & 1 & 0.4975 & \\
\hline & 71120 & 30.99 & 9.85 & 24192.5921 & 0 & 0 & 30.3 & 40.01 & 1.929 & 0.048 & 59 & 1 & 0.49265 & \\
\hline & 71140 & 23.24 & 9.29 & 24241.3748 & 0 & 0 & 30.3 & 30 & 1.819 & 0.061 & 60 & 1 & 0.4646 & \\
\hline & 71160 & 15.5 & 8.77 & 24273.9492 & 0 & 0 & 30.3 & 20.01 & 1.718 & 0.086 & 61 & 1 & 0.43865 & \\
\hline & 71180 & 7.75 & 8.52 & 24290.2855 & 0 & 0 & 30.3 & 10.01 & 1.669 & 0.167 & 62 & 1 & 0.42615 & \\
\hline & $8 \quad 1200$ & 6.97 & 8.7 & 24304.9975 & 0 & 0 & 30.3 & 9 & 1.703 & 0.189 & 63 & 1 & 0.43495 & \\
\hline & $8 \quad 1220$ & 6.2 & 8.64 & 24317.9833 & 0 & 0 & 30.3 & 8 & 1.692 & 0.212 & 64 & 1 & 0.43205 & \\
\hline & $8 \quad 1240$ & 5.42 & 8.58 & 24329.3347 & 0 & 0 & 30.3 & 7 & 1.679 & 0.24 & 65 & 1 & 0.4288 & \\
\hline & $8 \quad 1260$ & 4.64 & 8.65 & 24339.0713 & 0 & 0 & 30.3 & 5.99 & 1.693 & 0.283 & 66 & 1 & 0.43225 & \\
\hline & $8 \quad 1280$ & 3.87 & 8.51 & 24347.1915 & 0 & 0 & 30.3 & 5 & 1.666 & 0.333 & 67 & 1 & 0.42545 & 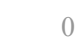 \\
\hline & $8 \quad 1300$ & 3.09 & 8.64 & 24353.6859 & 0 & 0 & 30.3 & 3.99 & 1.692 & 0.424 & 68 & 1 & 0.43215 & 0 \\
\hline & $8 \quad 1320$ & 2.32 & 8.52 & 24358.5531 & 0 & 0 & 30.3 & 3 & 1.668 & 0.556 & 69 & 1 & 0.4259 & 0 \\
\hline & $8 \quad 1340$ & 1.54 & 7.96 & 24361.8007 & 0 & 0 & 30.3 & 1.99 & 1.559 & 0.783 & 70 & 1 & 0.39805 & 0 \\
\hline & $8 \quad 1360$ & 0.77 & 7.44 & 24363.4233 & 0 & 0 & 30.3 & 0.99 & 1.457 & 1.472 & 71 & 1 & 0.37205 & 0 \\
\hline & 91380 & 0.7 & 7.18 & 24364.8967 & 18.164 & 12.92837 & 30.3 & 0.9 & 1.405 & 1.561 & 72 & 1 & 0.3588 & 0.077 \\
\hline & 91400 & 0.62 & 6.88 & 24366.2021 & 34.257 & 25.45071 & 30.3 & 0.8 & 1.346 & 1.683 & 73 & 1 & 0.34375 & 0.039 \\
\hline & $9 \quad 1420$ & 0.55 & 6.43 & 24367.344 & 48.335 & 38.39158 & 30.3 & 0.71 & 1.259 & 1.773 & 74 & 1 & 0.3215 & 0.026 \\
\hline & 91440 & 0.47 & 6.25 & 24368.3242 & 60.419 & 49.36172 & 30.3 & 0.61 & 1.224 & 2.007 & 75 & 1 & 0.3126 & 0.02 \\
\hline & 91460 & 0.39 & 5.84 & 24369.1434 & 70.518 & 61.64125 & 30.3 & 0.5 & 1.144 & 2.288 & 76 & 1 & 0.29205 & 0.016 \\
\hline & 91480 & 0.31 & 5.03 & 24369.7984 & 78.593 & 79.70869 & 30.3 & 0.4 & 0.986 & 2.465 & 77 & 1 & 0.2517 & 0.013 \\
\hline & $9 \quad 1500$ & 0.24 & 4.46 & 24370.2916 & 84.673 & 96.88028 & 30.3 & 0.31 & 0.874 & 2.819 & 78 & 1 & 0.22315 & 0.01 \\
\hline & $9 \quad 1520$ & 0.16 & 3.86 & 24370.6231 & 88.759 & 117.56207 & 30.3 & 0.21 & 0.755 & 3.595 & 79 & 1 & 0.1929 & 0.009 \\
\hline & 91540 & 0.08 & 2.87 & 24370.7919 & 90.841 & 161.927 & 30.3 & 0.1 & 0.561 & 5.61 & 80 & 1 & 0.1433 & 0.006 \\
\hline
\end{tabular}

03.31.04 WM-187 gravity settled slurry CC-45 run 02.txt

\begin{tabular}{rrrrrrrrrrrrrrrrrrr} 
Mode & Step & $\mathrm{t}[\mathrm{s}]$ & $\mathrm{n}[\mathrm{rpm}]$ & $\mathrm{M}[\%]$. & $\mathrm{Phi}[\mathrm{rad}]$ & $\mathrm{Gamma}$ & $\mathrm{J}[\mathrm{t}]$ & \multicolumn{2}{c}{$\mathrm{T}\left[{ }^{\circ} \mathrm{C}\right]$} & $\mathrm{D}[1 / \mathrm{s}]$ & $\mathrm{Tau}[\mathrm{Pa}]$ & $\mathrm{Eta}[\mathrm{Pas}]$ & $\mathrm{MP} \#$ & $\mathrm{Block}$ & $\mathrm{M}[\mathrm{mNm}]$ & $\mathrm{G}[\mathrm{Pa}]$ \\
1 & 1 & 20 & 0.08 & 2.74 & 0.16493 & 2.0333 & 3.7935 & 30.3 & 0.1 & 0.536 & 5.36 & 1 & 1 & 0.1369 & 0.264 \\
1 & 1 & 40 & 0.16 & 2.96 & 0.49323 & 6.0806 & 10.5019 & 30.3 & 0.21 & 0.579 & 2.757 & 2 & 1 & 0.14785 & 0.095 \\
1 & 1 & 60 & 0.24 & 3.57 & 0.98489 & 12.142 & 17.3703 & 30.3 & 0.31 & 0.699 & 2.255 & 3 & 1 & 0.1786 & 0.058 \\
1 & 1 & 80 & 0.31 & 4 & 1.63677 & 20.178 & 25.80346 & 30.3 & 0.4 & 0.782 & 1.955 & 4 & 1 & 0.19975 & 0.039 \\
1 & 1 & 100 & 0.39 & 4.07 & 2.45358 & 30.248 & 37.95244 & 30.3 & 0.5 & 0.797 & 1.594 & 5 & 1 & 0.2034 & 0.026 \\
1 & 1 & 120 & 0.47 & 4.66 & 3.43062 & 42.293 & 46.37403 & 30.3 & 0.61 & 0.912 & 1.495 & 6 & 1 & 0.23295 & 0.022 \\
1 & 1 & 140 & 0.54 & 5.22 & 4.57102 & 56.352 & 55.13904 & 30.3 & 0.7 & 1.022 & 1.46 & 7 & 1 & 0.26105 & 0.018 \\
1 & 1 & 160 & 0.62 & 5.41 & 5.87399 & 72.415 & 68.38088 & 30.3 & 0.8 & 1.059 & 1.324 & 8 & 1 & 0.27055 & 0.015 \\
1 & 1 & 180 & 0.7 & 5.69 & 7.33955 & 90.483 & 81.22341 & 30.3 & 0.9 & 1.114 & 1.238 & 9 & 1 & 0.2844 & 0.012 \\
1 & 2 & 200 & 0.77 & 5.69 & 8.96296 & 0 & 0 & 30.3 & 0.99 & 1.115 & 1.126 & 10 & 1 & 0.2847 & 0 \\
1 & 2 & 220 & 1.55 & 7.8 & 12.18702 & 0 & 0 & 30.3 & 2 & 1.528 & 0.764 & 11 & 1 & 0.39015 & 0 \\
1 & 2 & 240 & 2.32 & 9.35 & 17.03607 & 0 & 0 & 30.3 & 3 & 1.831 & 0.61 & 12 & 1 & 0.4675 & 0 \\
1 & 2 & 260 & 3.09 & 8.76 & 23.50461 & 0 & 0 & 30.3 & 3.99 & 1.716 & 0.43 & 13 & 1 & 0.4382 & 0 \\
1 & 2 & 280 & 3.87 & 8.36 & 31.60364 & 0 & 0 & 30.3 & 5 & 1.637 & 0.327 & 14 & 1 & 0.4181 & 0 \\
1 & 2 & 300 & 4.65 & 8.35 & 41.32137 & 0 & 0 & 30.3 & 6 & 1.634 & 0.272 & 15 & 1 & 0.41735 & 0 \\
1 & 2 & 320 & 5.42 & 8.27 & 52.65859 & 0 & 0 & 30.3 & 7 & 1.619 & 0.231 & 16 & 1 & 0.41345 & 0 \\
1 & 2 & 340 & 6.2 & 8.4 & 65.62316 & 0 & 0 & 30.3 & 8 & 1.645 & 0.206 & 17 & 1 & 0.41995 & 0 \\
1 & 2 & 360 & 6.97 & 8.35 & 80.21271 & 0 & 0 & 30.3 & 9 & 1.635 & 0.182 & 18 & 1 & 0.41745 & 0 \\
1 & 3 & 380 & 7.75 & 8.32 & 96.57099 & 0 & 0 & 30.3 & 10.01 & 1.629 & 0.163 & 19 & 1 & 0.41595 & 0 \\
1 & 3 & 400 & 15.5 & 8.6 & 128.94274 & 0 & 0 & 30.3 & 20.01 & 1.685 & 0.084 & 20 & 1 & 0.4302 & 0 \\
1 & 3 & 420 & 23.24 & 9.04 & 177.51569 & 0 & 0 & 30.3 & 30 & 1.77 & 0.059 & 21 & 1 & 0.452 & 0
\end{tabular}




\begin{tabular}{|c|c|c|c|c|c|c|c|c|c|c|c|c|c|c|c|c|c|c|c|c|c|c|c|c|c|c|}
\hline 1 & 3 & & 30.99 & & 242.2969 & 0 & 0 & & & & & & & & 440 & 30.99 & 9.6 & 242.2909 & 0.3 & 40.01 & 1.884 & 0.047 & 22 & 1 & 0.4812 & 0 \\
\hline 1 & 3 & & & & & 0 & 0 & & & & & & & & 460 & 38.73 & 9.7 & 323.25889 & 0.3 & 50 & 1.909 & 0.038 & 23 & 1 & 0.48755 & 0 \\
\hline & 3 & & & & & 0 & 0 & & & & & & & & 480 & 46.48 & 10.1 & 420.42992 & 0.3 & 60.01 & 1.994 & 0.033 & 24 & 1 & 0.50925 & 0 \\
\hline & 3 & & & & & 0 & 0 & & & & & & & & 500 & 54.22 & 10.9 & 533.98043 & 0.3 & 70 & 2.149 & 0.031 & 25 & 1 & 0.54875 & 0 \\
\hline & 3 & & & & & 0 & 0 & & & & & & & & 520 & 61.96 & 11.1 & 663.53657 & 0.3 & 79.99 & 2.191 & 0.027 & 26 & 1 & 0.5594 & 0 \\
\hline & 3 & & & & & 0 & 0 & & & & & & & & 540 & 69.71 & 11.5 & 809.37951 & 0.3 & 90 & 2.253 & 0.025 & 27 & 1 & 0.57545 & 0 \\
\hline & 4 & & & & & 0 & 0 & & & & & & & & 560 & 77.46 & 11.8 & 973.11225 & 0.3 & 100 & 2.317 & 0.023 & 28 & 1 & 0.5917 & 0 \\
\hline & 4 & & & & & 0 & 0 & & & & & & & & 580 & 154.92 & 15.0 & 1296.11667 & 0.3 & 200 & 2.941 & 0.015 & 29 & 1 & 0.7509 & 0 \\
\hline & 4 & & & & & 0 & 0 & & & & & & & & 600 & 232.38 & 18.9 & 1781.715 & 0.3 & 300 & 3.714 & 0.012 & 30 & 1 & 0.94845 & 0 \\
\hline & 4 & & & & & 0 & 0 & & & & & & & & 620 & 309.84 & 26.6 & 2429.50198 & 0.3 & 400 & 5.226 & 0.013 & 31 & 1 & 1.3346 & 0 \\
\hline & 4 & & & & & 0 & 0 & & & & & & & & 640 & 387.3 & 33.8 & 3239.23571 & 0.3 & 500 & 6.628 & 0.013 & 32 & 1 & 1.69265 & 0 \\
\hline & 4 & & & & & 0 & 0 & & & & & & & & 660 & 464.75 & 42.2 & 4211.3615 & 0.3 & 599.99 & 8.264 & 0.014 & 33 & 1 & 2.11025 & 0 \\
\hline & 4 & & & & & 0 & 0 & & & & & & & & 680 & 542.21 & 52.7 & 5346.80927 & 30.3 & 699.99 & 10.332 & 0.015 & 34 & 1 & 2.63845 & 0 \\
\hline & 4 & & & & & 0 & 0 & & & & & & & & 700 & 619.67 & 63 & 6642.98799 & 30.3 & 799.99 & 12.355 & 0.015 & 35 & 1 & 3.1551 & 0 \\
\hline & 4 & & & & & 0 & 0 & & & & & & & & 720 & 697.13 & 73.2 & 8101.35614 & 30.3 & 899.99 & 14.349 & 0.016 & 36 & 1 & 3.6642 & 0 \\
\hline & 4 & & & & & 0 & 0 & & & & & & & & 740 & 774.59 & 82.2 & 9722.31663 & 30.3 & 1000 & 16.109 & 0.016 & 37 & 1 & 4.11375 & 0 \\
\hline & 5 & & & & & 0 & 0 & & & & & & & & 750 & 774.59 & 81.9 & 10550.9855 & 30.3 & 1000 & 16.049 & 0.016 & 38 & 1 & 4.0984 & 0 \\
\hline & 5 & & & & & 0 & 0 & & & & & & & & 760 & 774.59 & 80.0 & 11360.5111 & 30.3 & 1000 & 15.678 & 0.016 & 39 & 1 & 4.0035 & 0 \\
\hline & 5 & & & & & 0 & 0 & & & & & & & & 770 & 774.59 & 80.2 & 12172.067 & 30.3 & 1000 & 15.708 & 0.016 & 40 & 1 & 4.01135 & 0 \\
\hline & 5 & & & & & 0 & 0 & & & & & & & & 780 & 774.59 & 80.4 & 12983.622 & 30.3 & 1000 & 15.76 & 0.016 & 41 & 1 & 4.0245 & 0 \\
\hline & 5 & & & & & 0 & 0 & & & & & & & & 790 & 774.59 & 80.7 & 13796.3913 & 30.3 & 1000 & 15.803 & 0.016 & 42 & 1 & 4.03555 & 0 \\
\hline & 5 & & & & & 0 & 0 & & & & & & & & 800 & 774.59 & 80.5 & 14606.729 & 30.3 & 1000 & 15.764 & 0.016 & 43 & 1 & 4.02565 & 0 \\
\hline & 6 & & & & & 0 & 0 & & & & & & & & 820 & 774.59 & 80.5 & 16246.0615 & 30.3 & 1000 & 15.78 & 0.016 & 44 & 1 & 4.0295 & 0 \\
\hline & 6 & & & & & 0 & 0 & & & & & & & & 840 & 697.13 & 69.9 & 17708.1556 & 30.3 & 899.99 & 13.704 & 0.015 & 45 & 1 & 3.49945 & 0 \\
\hline & 6 & & & & & 0 & 0 & & & & & & & & 860 & 619.67 & 60.0 & 19007.6142 & 30.3 & 799.99 & 11.762 & 0.015 & 46 & 1 & 3.0037 & 0 \\
\hline & 6 & & & & & 0 & 0 & & & & & & & & 880 & 542.21 & 50.3 & 20145.0867 & 30.3 & 699.99 & 9.853 & 0.014 & 47 & 1 & 2.516 & 0 \\
\hline & 6 & & & & & 0 & 0 & & & & & & & & 900 & 464.75 & 40.3 & 21119.4791 & 30.3 & 599.99 & 7.905 & 0.013 & 48 & 1 & 2.01875 & 0 \\
\hline & 6 & & & & & 0 & 0 & & & & & & & & 920 & 387.3 & 32.4 & 21931.5172 & 30.3 & 500 & 6.35 & 0.013 & 49 & 1 & 1.6215 & 0 \\
\hline & 6 & & & & & 0 & 0 & & & & & & & & 940 & 309.84 & 25.1 & 22581.5716 & 30.3 & 400 & 4.931 & 0.012 & 50 & 1 & 1.2591 & 0 \\
\hline & 6 & & & & & 0 & 0 & & & & & & & & 960 & 232.38 & 17.6 & 23069.4131 & 30.3 & 300 & 3.454 & 0.012 & 51 & 1 & 0.88195 & 0 \\
\hline 1 & 6 & & & & & 0 & 0 & & & & & & & & 980 & 154.92 & 14.0 & 23395.5638 & 30.3 & 200 & 2.743 & 0.014 & 52 & 1 & 0.70055 & 0 \\
\hline & 6 & & & & & 0 & 0 & & & & & & & & 1000 & 77.46 & 11.2 & 23558.9612 & 30.3 & 100 & 2.198 & 0.022 & 53 & 1 & 0.5614 & 0 \\
\hline 1 & 7 & & & & & 0 & 0 & & & & & & & & 1020 & 69.71 & 11.0 & 23706.1 & 30.3 & 90 & 2.16 & 0.024 & 54 & 1 & 0.5517 & 0 \\
\hline 1 & 7 & & & & & 0 & 0 & & & & & & & & 1040 & 61.97 & 10. & 23836.0622 & 30.3 & 80 & 2.096 & 0.026 & 55 & 1 & 0.5352 & 0 \\
\hline 1 & 7 & & & & & 0 & 0 & & & & & & & & 1060 & 54.22 & 10.5 & 23949.7164 & 30.3 & 70 & 2.061 & 0.029 & 56 & 1 & 0.52635 & 0 \\
\hline 1 & 7 & & & 9.8 & & 0 & 0 & & & & & & & & 1080 & 46.48 & 9. & 24047.2236 & 30.3 & 60.01 & 1.918 & 0.032 & 57 & 1 & 0.4899 & 0 \\
\hline 1 & 7 & & & & & 0 & 0 & & & & & & & & 1100 & 38.73 & 9.4 & 24128.3717 & 30.3 & 50 & 1.846 & 0.037 & 58 & 1 & 0.4714 & 0 \\
\hline 1 & 7 & & & & & 0 & 0 & & & & & & & & 1120 & 30.99 & 9.3 & 24193.4317 & 30.3 & 40.01 & 1.836 & 0.046 & 59 & 1 & 0.46885 & 0 \\
\hline & 7 & & & & & 0 & 0 & & & & & & & & 1140 & 23.24 & 8.8 & 24242.1665 & 30.3 & 30 & 1.735 & 0.058 & 60 & 1 & 0.44295 & 0 \\
\hline & 7 & & & & & 0 & 0 & & & & & & & & 1160 & 15.5 & 8.3 & 24274.7448 & 30.3 & 20.01 & 1.641 & 0.082 & 61 & 1 & 0.4191 & 0 \\
\hline & 7 & & & & & 0 & 0 & & & & & & & & 1180 & 7.75 & 8.1 & 24291.0889 & 30.3 & 10.01 & 1.588 & 0.159 & 62 & 1 & 0.40545 & 0 \\
\hline & 8 & & & & & 0 & 0 & & & & & & & & 1200 & 6.97 & 8.2 & 24305.7892 & 30.3 & 9 & 1.624 & 0.18 & 63 & 1 & 0.4146 & 0 \\
\hline & 8 & & & & & 0 & 0 & & & & & & & & 1220 & 6.2 & 8.3 & 24318.7852 & 30.3 & 8 & 1.628 & 0.204 & 64 & 1 & 0.41575 & 0 \\
\hline & 8 & & & & & 0 & 0 & & & & & & & & 1240 & 5.42 & 8.1 & 24330.1413 & 30.3 & 7 & 1.602 & 0.229 & 65 & 1 & 0.40905 & 0 \\
\hline & 8 & & & & & 0 & 0 & & & & & & & & 1260 & 4.64 & 8.2 & 24339.8857 & 30.3 & 5.99 & 1.616 & 0.27 & 66 & 1 & 0.4126 & 0 \\
\hline & 8 & & & 8.2 & & 0 & 0 & & & & & & & & 1280 & 3.87 & 8 & 24347.9981 & 30.3 & 5 & 1.606 & 0.321 & 67 & 1 & 0.41005 & 0 \\
\hline & 8 & & & & & 0 & 0 & & & & & & & & 1300 & 3.09 & 8.1 & 24354.4941 & 30.3 & 3.99 & 1.6 & 0.401 & 68 & 1 & 0.4087 & 0 \\
\hline & 8 & & & 8.2 & & 0 & 0 & & & & & & & & 1320 & 2.32 & 8 & 24359.362 & 30.3 & 3 & 1.605 & 0.535 & 69 & 1 & 0.40995 & 0 \\
\hline & 8 & & & & & 0 & 0 & & & & & & & & 1340 & 1.54 & 7.6 & 24362.6112 & 30.3 & 1.99 & 1.503 & 0.755 & 70 & 1 & 0.3839 & 0 \\
\hline & 8 & & & & & 0 & 0 & & & & & & & & 1360 & 0.77 & 7.2 & 24364.2338 & 30.3 & 0.99 & 1.413 & 1.427 & 71 & 1 & 0.3607 & 0 \\
\hline
\end{tabular}




$\begin{array}{rrrrrrrrrrrrrrrrr}1 & 9 & 1380 & 0.7 & 6.94 & 24365.708 & 18.174 & 13.38294 & 30.3 & 0.9 & 1.358 & 1.509 & 72 & 1 & 0.34675 & 0.075 \\ 1 & 9 & 1400 & 0.62 & 6.58 & 24367.0126 & 34.257 & 26.59678 & 30.3 & 0.8 & 1.288 & 1.61 & 73 & 1 & 0.3288 & 0.038 \\ 1 & 9 & 1420 & 0.55 & 6.23 & 24368.1553 & 48.345 & 39.65929 & 30.3 & 0.71 & 1.219 & 1.717 & 74 & 1 & 0.31125 & 0.025 \\ 1 & 9 & 1440 & 0.47 & 5.83 & 24369.1363 & 60.438 & 52.96943 & 30.3 & 0.61 & 1.141 & 1.87 & 75 & 1 & 0.29135 & 0.019 \\ 1 & 9 & 1460 & 0.39 & 5.43 & 24369.9539 & 70.518 & 66.33827 & 30.3 & 0.5 & 1.063 & 2.126 & 76 & 1 & 0.27135 & 0.015 \\ 1 & 9 & 1480 & 0.31 & 5 & 24370.6097 & 78.603 & 80.3706 & 30.3 & 0.4 & 0.978 & 2.445 & 77 & 1 & 0.24975 & 0.012 \\ 1 & 9 & 1500 & 0.24 & 4.25 & 24371.1029 & 84.683 & 101.90499 & 30.3 & 0.31 & 0.831 & 2.681 & 78 & 1 & 0.2123 & 0.01 \\ 1 & 9 & 1520 & 0.16 & 3.74 & 24371.4344 & 88.769 & 121.43507 & 30.3 & 0.21 & 0.731 & 3.481 & 79 & 1 & 0.18675 & 0.008 \\ 1 & 9 & 1540 & 0.08 & 2.56 & 24371.6032 & 90.851 & 181.33876 & 30.3 & 0.1 & 0.501 & 5.01 & 80 & 1 & 0.1279 & 0.006\end{array}$

03.31.04 WM-187 gravity settled slurry CC-48 run 01.txt

\begin{tabular}{|c|c|c|c|c|c|c|c|c|c|c|c|c|c|c|c|}
\hline Mode & Step & $\mathrm{t}[\mathrm{s}]$ & $\mathrm{n}[\mathrm{rpm}] \mathrm{I}$ & $\mathrm{M}[\%]$. & Phi[rad] & Gamma & $J[t]$ & $\mathrm{T}\left[{ }^{\circ} \mathrm{C}\right]$ & $\mathrm{D}[1 / \mathrm{s}]$ & $\mathrm{Tau}[\mathrm{Pa}]$ & $\mathrm{Eta}[\mathrm{Pas}]$ & MP\# & Block & $\mathrm{M}[\mathrm{mNm}]$ & $\mathrm{G}[\mathrm{Pa}]$ \\
\hline 1 & 1 & 20 & 0.02 & 2.2 & 0.03927 & 1.9283 & 4.60202 & 30.3 & 0.1 & 0.419 & 4.19 & 1 & 1 & 0.11015 & 0.217 \\
\hline 1 & 1 & 40 & 0.04 & 2.55 & 0.12252 & 6.0161 & 12.40439 & 30.3 & 0.21 & 0.485 & 2.31 & 2 & 1 & 0.1275 & 0.081 \\
\hline 1 & 1 & 60 & 0.06 & 2.28 & 0.24819 & 12.187 & 28.14437 & 30.3 & 0.31 & 0.433 & 1.397 & 3 & 1 & 0.11405 & 0.036 \\
\hline 1 & 1 & 80 & 0.08 & 1.98 & 0.41548 & 20.401 & 54.40223 & 30.3 & 0.41 & 0.375 & 0.915 & 4 & 1 & 0.09875 & 0.018 \\
\hline 1 & 1 & 100 & 0.1 & 2.33 & 0.62439 & 30.659 & 69.20793 & 30.3 & 0.51 & 0.443 & 0.869 & 5 & 1 & 0.1166 & 0.014 \\
\hline 1 & 1 & 120 & 0.12 & 2.48 & 0.87572 & 43 & 91.10146 & 30.3 & 0.62 & 0.472 & 0.761 & 6 & 1 & 0.1242 & 0.011 \\
\hline 1 & 1 & 140 & 0.14 & 2.57 & 1.16867 & 57.385 & 117.5914 & 30.3 & 0.72 & 0.488 & 0.678 & 7 & 1 & 0.12835 & 0.009 \\
\hline 1 & 1 & 160 & 0.16 & 2.62 & 1.50247 & 73.775 & 148.44004 & 30.3 & 0.82 & 0.497 & 0.606 & 8 & 1 & 0.13085 & 0.007 \\
\hline 1 & 1 & 180 & 0.18 & 3.04 & 1.87946 & 92.286 & 159.66417 & 30.3 & 0.93 & 0.578 & 0.622 & 9 & 1 & 0.1521 & 0.006 \\
\hline 1 & 2 & 200 & 0.19 & 3.1 & 2.28001 & 111.95 & 190.07472 & 30.3 & 0.98 & 0.589 & 0.601 & 10 & 1 & 0.15505 & 0.005 \\
\hline 1 & 2 & 220 & 0.39 & 4.46 & 3.08347 & 151.41 & 178.54482 & 30.3 & 2.01 & 0.848 & 0.422 & 11 & 1 & 0.2231 & 0.006 \\
\hline 1 & 2 & 240 & 0.58 & 5.32 & 4.29534 & 210.91 & 208.61699 & 30.3 & 2.98 & 1.011 & 0.339 & 12 & 1 & 0.2661 & 0.005 \\
\hline 1 & 2 & 260 & 0.78 & 5.8 & 5.91562 & 0 & 0 & 30.3 & 4.01 & 1.102 & 0.275 & 13 & 1 & 0.29 & 0 \\
\hline 1 & 2 & 280 & 0.97 & 7.2 & 7.9443 & 0 & 0 & 30.3 & 4.99 & 1.368 & 0.274 & 14 & 1 & 0.36005 & 0 \\
\hline 1 & 2 & 300 & 1.17 & 9.39 & 10.38139 & 0 & 0 & 30.3 & 6.02 & 1.784 & 0.296 & 15 & 1 & 0.46935 & 0 \\
\hline 1 & 2 & 320 & 1.36 & 9.6 & 13.22689 & 0 & 0 & 30.3 & 6.99 & 1.825 & 0.261 & 16 & 1 & 0.4802 & 0 \\
\hline 1 & 2 & 340 & 1.55 & 10.05 & 16.4808 & 0 & 0 & 30.3 & 7.97 & 1.91 & 0.24 & 17 & 1 & 0.50255 & 0 \\
\hline 1 & 2 & 360 & 1.75 & 10 & 20.14389 & 0 & 0 & 30.3 & 9 & 1.901 & 0.211 & 18 & 1 & 0.50015 & 0 \\
\hline 1 & 3 & 380 & 1.94 & 10.29 & 24.23267 & 0 & 0 & 30.3 & 9.98 & 1.955 & 0.196 & 19 & 1 & 0.51455 & 0 \\
\hline 1 & 3 & 400 & 3.89 & 11.27 & 32.34034 & 0 & 0 & 30.3 & 20 & 2.141 & 0.107 & 20 & 1 & 0.5634 & 0 \\
\hline 1 & 3 & 420 & 5.83 & 11.73 & 44.52422 & 0 & 0 & 30.3 & 29.98 & 2.229 & 0.074 & 21 & 1 & 0.58645 & 0 \\
\hline 1 & 3 & 440 & 7.77 & 12.08 & 60.79296 & 0 & 0 & 30.3 & 39.95 & 2.296 & 0.057 & 22 & 1 & 0.60415 & 0 \\
\hline 1 & 3 & 460 & 9.72 & 12.52 & 81.11671 & 0 & 0 & 30.3 & 49.98 & 2.379 & 0.048 & 23 & 1 & 0.62605 & 0 \\
\hline 1 & 3 & 480 & 11.66 & 12.92 & 105.5261 & 0 & 0 & 30.3 & 59.96 & 2.456 & 0.041 & 24 & 1 & 0.6462 & 0 \\
\hline 1 & 3 & 500 & 13.61 & 13.4 & 133.99442 & 0 & 0 & 30.3 & 69.98 & 2.546 & 0.036 & 25 & 1 & 0.67005 & 0 \\
\hline 1 & 3 & 520 & 15.55 & 13.55 & 166.52719 & 0 & 0 & 30.3 & 79.96 & 2.574 & 0.032 & 26 & 1 & 0.6773 & 0 \\
\hline 1 & 3 & 540 & 17.5 & 14.06 & 203.16837 & 0 & 0 & 30.3 & 89.99 & 2.672 & 0.03 & 27 & 1 & 0.70315 & 0 \\
\hline 1 & 4 & 560 & 19.45 & 14.3 & 244.25097 & 0 & 0 & 30.3 & 100.01 & 2.717 & 0.027 & 28 & 1 & 0.71505 & 0 \\
\hline 1 & 4 & 580 & 38.9 & 17.67 & 325.51534 & 0 & 0 & 30.3 & 200.02 & 3.357 & 0.017 & 29 & 1 & 0.88345 & 0 \\
\hline 1 & 4 & 600 & 58.35 & 21.04 & 447.29682 & 0 & 0 & 30.3 & 300.04 & 3.998 & 0.013 & 30 & 1 & 1.0522 & 0 \\
\hline 1 & 4 & 620 & 77.79 & 23.67 & 610.0541 & 0 & 0 & 30.3 & 400 & 4.497 & 0.011 & 31 & 1 & 1.1835 & 0 \\
\hline 1 & 4 & 640 & 97.24 & 26.12 & 813.26802 & 0 & 0 & 30.3 & 500.01 & 4.963 & 0.01 & 32 & 1 & 1.306 & 0 \\
\hline 1 & 4 & 660 & 116.69 & 28.36 & 1057.54884 & 0 & 0 & 30.3 & 600.02 & 5.389 & 0.009 & 33 & 1 & 1.4182 & 0 \\
\hline 1 & 4 & 680 & 136.14 & 30.94 & 1342.27609 & 0 & 0 & 30.3 & 700.03 & 5.878 & 0.008 & 34 & 1 & 1.54675 & 0 \\
\hline 1 & 4 & 700 & 155.58 & 33.24 & 1667.97992 & 0 & 0 & 30.3 & 799.99 & 6.316 & 0.008 & 35 & 1 & 1.66205 & 0 \\
\hline 1 & 4 & 720 & 175.03 & 35.66 & 2034.07836 & 0 & 0 & 30.3 & 900 & 6.776 & 0.008 & 36 & 1 & 1.78305 & 0 \\
\hline 1 & 4 & 740 & 194.48 & 38.28 & 2440.8667 & 0 & 0 & 30.3 & 1000 & 7.274 & 0.007 & 37 & 1 & 1.91425 & 0 \\
\hline 1 & 5 & 750 & 194.48 & 37.74 & 2648.82285 & 0 & 0 & 30.3 & 1000 & 7.17 & 0.007 & 38 & 1 & 1.8868 & 0 \\
\hline
\end{tabular}




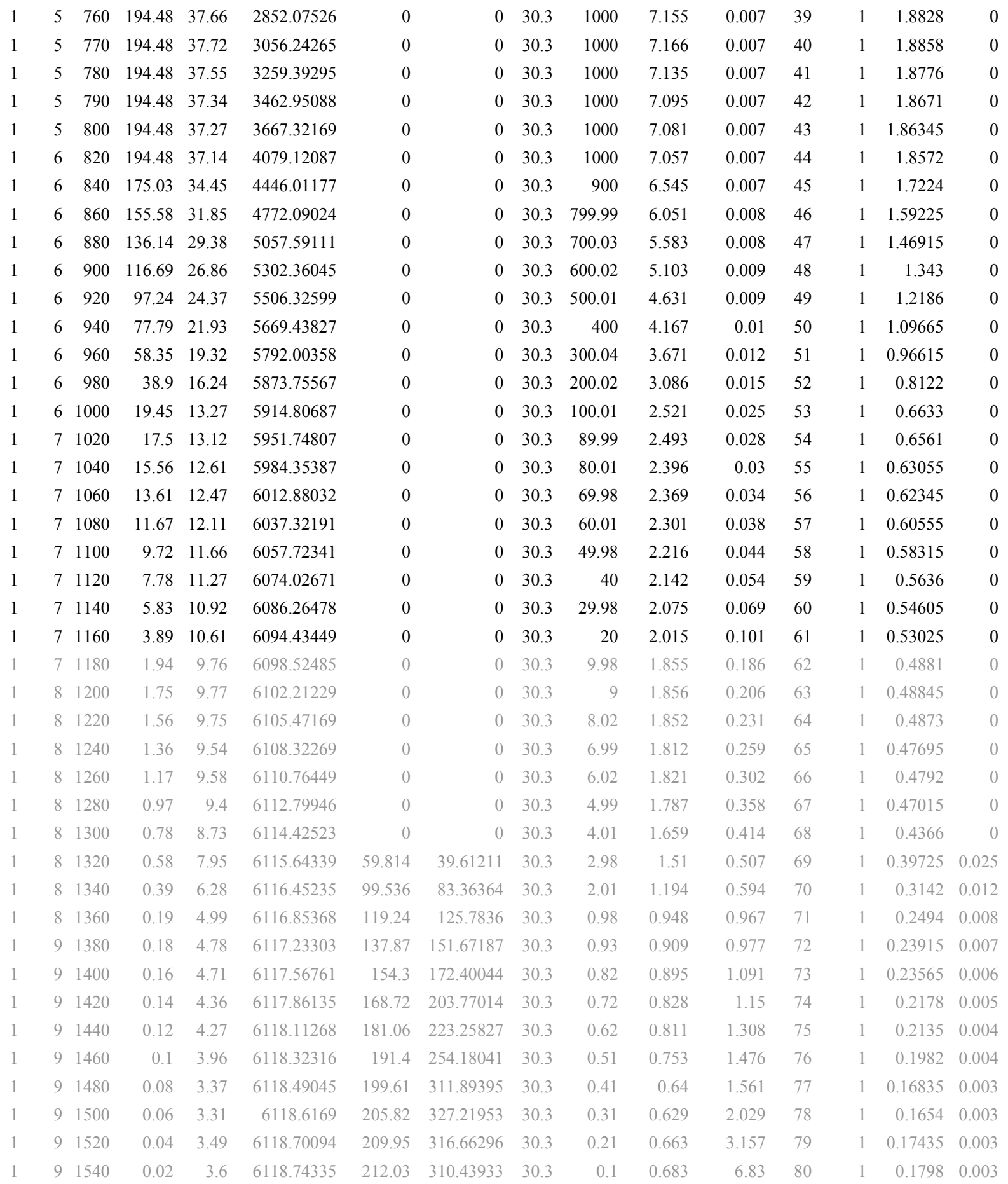

03.31.04 WM-187 gravity settled slurry CC-48 run 02.txt

Mode Step t[s] n[rpm] M[\%.] Phi[rad] Gamma

$\begin{array}{rrrrr}1 & 1 & 20 & 0.02 & 3.47 \\ 1 & 1 & 40 & 0.04 & 3.2 \\ 1 & 1 & 60 & 0.06 & 3.01 \\ 1 & 1 & 80 & 0.08 & 3.5 \\ 1 & 1 & 100 & 0.1 & 3.41\end{array}$

$0.03848 \quad 1.889$

$0.12252 \quad 6.0161$

$0.2474 \quad 12.148$

$0.41469 \quad 20.362$

$0.62439 \quad 30.659$
$\mathrm{J}[\mathrm{t}] \quad \mathrm{T}\left[{ }^{\circ} \mathrm{C}\right] \quad \mathrm{D}[1 / \mathrm{s}] \quad \mathrm{Tau}[\mathrm{Pa}] \quad \mathrm{Eta}[\mathrm{Pas}] \quad \mathrm{MP} \#$ Block $\mathrm{M}[\mathrm{mNm}] \mathrm{G}[\mathrm{Pa}]$

$\begin{array}{lllllllll}2.86751 & 30.3 & 0.1 & 0.659 & 6.59 & 1 & 1 & 0.17345 & 0.349\end{array}$

$\begin{array}{llll}9.8787 & 30.3 & 0.21 & 0.609\end{array}$

2.9

0.16020 .101

$\begin{array}{llll}21.23769 & 30.3 & 0.31 & 0.572\end{array}$

$\begin{array}{llll}30.6661 & 30.3 & 0.41 & 0.664\end{array}$

$47.3866 \quad 30.3$

$0.51 \quad 0.647$

$\begin{array}{lllll}1.62 & 4 & 1 & 0.1748 & 0.033\end{array}$

$\begin{array}{lllll}1.269 & 5 & 1 & 0.1703 & 0.021\end{array}$ 


\begin{tabular}{|c|c|c|c|c|c|c|c|c|c|c|c|c|c|c|c|}
\hline 1 & 1 & 120 & 0.12 & 3.64 & 0.87493 & 42.961 & 62.08287 & 30.3 & 0.62 & 0.692 & 1.116 & 6 & 1 & 0.182 & 0.016 \\
\hline & 1 & 140 & 0.14 & 3.68 & 1.16867 & 57.385 & 82.09533 & 30.3 & 0.72 & 0.699 & 0.971 & 7 & 1 & 0.18395 & 0.012 \\
\hline & 1 & 160 & 0.16 & 3.6 & 1.50247 & 73.775 & 107.85781 & 30.3 & 0.82 & 0.684 & 0.834 & 8 & 1 & 0.17995 & 0.009 \\
\hline & 1 & 180 & 0.18 & 3.39 & 1.87946 & 92.286 & 143.3011 & 30.3 & 0.93 & 0.644 & 0.692 & 9 & 1 & 0.1695 & 0.007 \\
\hline & 2 & 200 & 0.19 & 3.14 & 2.2808 & 111.99 & 187.59225 & 30.3 & 0.98 & 0.597 & 0.609 & 10 & 1 & 0.1571 & 0.005 \\
\hline & 2 & 220 & 0.39 & 3.81 & 3.08269 & 151.37 & 209.07102 & 30.3 & 2.01 & 0.724 & 0.36 & 11 & 1 & 0.1906 & 0.005 \\
\hline & 2 & 240 & 0.58 & 5.36 & 4.29534 & 210.91 & 207.1825 & 30.3 & 2.98 & 1.018 & 0.342 & 12 & 1 & 0.26785 & 0.005 \\
\hline & 2 & 260 & 0.78 & 7.14 & 5.91562 & 0 & 0 & 30.3 & 4.01 & 1.357 & 0.338 & 13 & 1 & 0.3571 & 0 \\
\hline & 2 & 280 & 0.97 & 8.49 & 7.94273 & 0 & 0 & 30.3 & 4.99 & 1.613 & 0.323 & 14 & 1 & 0.4244 & 0 \\
\hline & 2 & 300 & 1.17 & 9.5 & 10.38061 & 0 & 0 & 30.3 & 6.02 & 1.806 & 0.3 & 15 & 1 & 0.4752 & 0 \\
\hline & 2 & 320 & 1.36 & 9.28 & 13.22611 & 0 & 0 & 30.3 & 6.99 & 1.763 & 0.252 & 16 & 1 & 0.46405 & 0 \\
\hline & 2 & 340 & 1.55 & 9.34 & 16.48001 & 0 & 0 & 30.2 & 7.97 & 1.774 & 0.223 & 17 & 1 & 0.4669 & 0 \\
\hline & 2 & 360 & 1.75 & 9.44 & 20.14389 & 0 & 0 & 30.2 & 9 & 1.793 & 0.199 & 18 & 1 & 0.47185 & 0 \\
\hline & 3 & 380 & 1.94 & 9.46 & 24.23267 & 0 & 0 & 30.2 & 9.98 & 1.797 & 0.18 & 19 & 1 & 0.473 & 0 \\
\hline & 3 & 400 & 3.89 & 10.41 & 32.34662 & 0 & 0 & 30.2 & 20 & 1.978 & 0.099 & 20 & 1 & 0.5205 & 0 \\
\hline & 3 & 420 & 5.83 & 10.77 & 44.52501 & 0 & 0 & 30.2 & 29.98 & 2.046 & 0.068 & 21 & 1 & 0.53845 & 0 \\
\hline & 3 & 440 & 7.77 & 11.03 & 60.78511 & 0 & 0 & 30.2 & 39.95 & 2.096 & 0.052 & 22 & 1 & 0.5517 & 0 \\
\hline & 3 & 460 & 9.72 & 11.46 & 81.12456 & 0 & 0 & 30.2 & 49.98 & 2.178 & 0.044 & 23 & 1 & 0.5731 & 0 \\
\hline & 3 & 480 & 11.66 & 11.89 & 105.51589 & 0 & 0 & 30.2 & 59.96 & 2.258 & 0.038 & 24 & 1 & 0.59425 & 0 \\
\hline & 3 & 500 & 13.61 & 12.22 & 133.99914 & 0 & 0 & 30.2 & 69.98 & 2.322 & 0.033 & 25 & 1 & 0.61115 & 0 \\
\hline & 3 & 520 & 15.55 & 12.4 & 166.54918 & 0 & 0 & 30.2 & 79.96 & 2.356 & 0.029 & 26 & 1 & 0.6199 & 0 \\
\hline & 3 & 540 & 17.5 & 12.94 & 203.19114 & 0 & 0 & 30.2 & 89.99 & 2.459 & 0.027 & 27 & 1 & 0.647 & 0 \\
\hline & 4 & 560 & 19.45 & 13.14 & 244.27532 & 0 & 0 & 30.2 & 100.01 & 2.496 & 0.025 & 28 & 1 & 0.6568 & 0 \\
\hline & 4 & 580 & 38.9 & 16.3 & 325.51848 & 0 & 0 & 30.2 & 200.02 & 3.097 & 0.015 & 29 & 1 & 0.81495 & 0 \\
\hline & 4 & 600 & 58.35 & 19.4 & 447.27012 & 0 & 0 & 30.2 & 300.04 & 3.685 & 0.012 & 30 & 1 & 0.9698 & 0 \\
\hline & 4 & 620 & 77.79 & 21.9 & 609.98655 & 0 & 0 & 30.2 & 400 & 4.16 & 0.01 & 31 & 1 & 1.09485 & 0 \\
\hline & 4 & 640 & 97.24 & 24.14 & 813.45494 & 0 & 0 & 30.2 & 500.01 & 4.586 & 0.009 & 32 & 1 & 1.2068 & 0 \\
\hline & 4 & 660 & 116.69 & 26.43 & 1057.35956 & 0 & 0 & 30.2 & 600.02 & 5.022 & 0.008 & 33 & 1 & 1.32165 & 0 \\
\hline & 4 & 680 & 136.14 & 29.09 & 1342.23918 & 0 & 0 & 30.2 & 700.03 & 5.527 & 0.008 & 34 & 1 & 1.45435 & 0 \\
\hline & 4 & 700 & 155.58 & 31.38 & 1667.94223 & 0 & 0 & 30.2 & 799.99 & 5.961 & 0.007 & 35 & 1 & 1.5688 & 0 \\
\hline & 4 & 720 & 175.03 & 34.01 & 2034.04066 & 0 & 0 & 30.2 & 900 & 6.463 & 0.007 & 36 & 1 & 1.7007 & 0 \\
\hline & 4 & 740 & 194.48 & 36.42 & 2441.24761 & 0 & 0 & 30.2 & 1000 & 6.921 & 0.007 & 37 & 1 & 1.8212 & 0 \\
\hline & 5 & 750 & 194.48 & 36.24 & 2649.20298 & 0 & 0 & 30.2 & 1000 & 6.885 & 0.007 & 38 & 1 & 1.81185 & 0 \\
\hline & 5 & 760 & 194.48 & 36.18 & 2852.65881 & 0 & 0 & 30.2 & 1000 & 6.873 & 0.007 & 39 & 1 & 1.8088 & 0 \\
\hline & 5 & 770 & 194.48 & 36.19 & 3056.62357 & 0 & 0 & 30.2 & 1000 & 6.876 & 0.007 & 40 & 1 & 1.8094 & 0 \\
\hline & 5 & 780 & 194.48 & 36.2 & 3259.9765 & 0 & 0 & 30.2 & 1000 & 6.878 & 0.007 & 41 & 1 & 1.8099 & 0 \\
\hline & 5 & 790 & 194.48 & 36.41 & 3463.43233 & 0 & 0 & 30.2 & 1000 & 6.917 & 0.007 & 42 & 1 & 1.8203 & 0 \\
\hline & 5 & 800 & 194.48 & 36.37 & 3667.60051 & 0 & 0 & 30.2 & 1000 & 6.911 & 0.007 & 43 & 1 & 1.81865 & 0 \\
\hline & 6 & 820 & 194.48 & 36.08 & 4079.60232 & 0 & 0 & 30.2 & 1000 & 6.856 & 0.007 & 44 & 1 & 1.8041 & 0 \\
\hline & 6 & 840 & 175.03 & 33.38 & 4446.03533 & 0 & 0 & 30.2 & 900 & 6.342 & 0.007 & 45 & 1 & 1.66905 & 0 \\
\hline & 6 & 860 & 155.58 & 30.85 & 4772.28737 & 0 & 0 & 30.2 & 799.99 & 5.862 & 0.007 & 46 & 1 & 1.5425 & 0 \\
\hline & 6 & 880 & 136.14 & 28.41 & 5057.94061 & 0 & 0 & 30.2 & 700.03 & 5.397 & 0.008 & 47 & 1 & 1.4203 & 0 \\
\hline & 6 & 900 & 116.69 & 25.9 & 5302.39422 & 0 & 0 & 30.2 & 600.02 & 4.92 & 0.008 & 48 & 1 & 1.2948 & 0 \\
\hline & 6 & 920 & 97.24 & 23.49 & 5506.47286 & 0 & 0 & 30.2 & 500.01 & 4.464 & 0.009 & 49 & 1 & 1.1747 & 0 \\
\hline & 6 & 940 & 77.79 & 21.08 & 5669.77835 & 0 & 0 & 30.2 & 400 & 4.006 & 0.01 & 50 & 1 & 1.0541 & 0 \\
\hline & 6 & 960 & 58.35 & 18.56 & 5792.17008 & 0 & 0 & 30.2 & 300.04 & 3.526 & 0.012 & 51 & 1 & 0.9278 & 0 \\
\hline & 6 & 980 & 38.9 & 15.51 & 5873.94181 & 0 & 0 & 30.2 & 200.02 & 2.946 & 0.015 & 52 & 1 & 0.77535 & 0 \\
\hline & 6 & 1000 & 19.45 & 12.6 & 5915.03385 & 0 & 0 & 30.2 & 100.01 & 2.393 & 0.024 & 53 & 1 & 0.62985 & 0 \\
\hline & 7 & 1020 & 17.5 & 12.48 & 5951.9397 & 0 & 0 & 30.2 & 89.99 & 2.371 & 0.026 & 54 & 1 & 0.62405 & 0 \\
\hline & 7 & 1040 & 15.56 & 11.95 & 5984.57143 & 0 & 0 & 30.2 & 80.01 & 2.27 & 0.028 & 55 & 1 & 0.5974 & 0 \\
\hline
\end{tabular}




\begin{tabular}{|c|c|c|c|c|c|c|c|c|c|c|c|c|c|c|}
\hline 1 & $7 \quad 1060$ & 13.61 & 11.87 & 6013.10416 & 0 & 0 & 30.2 & 69.98 & 2.255 & 0.032 & 56 & 1 & 0.59335 & 0 \\
\hline 1 & $7 \quad 1080$ & 11.67 & 11.5 & 6037.5701 & 0 & 0 & 30.2 & 60.01 & 2.186 & 0.036 & 57 & 1 & 0.5752 & 0 \\
\hline 1 & $7 \quad 1100$ & 9.72 & 11.1 & 6057.94018 & 0 & 0 & 30.2 & 49.98 & 2.108 & 0.042 & 58 & 1 & 0.55475 & 0 \\
\hline & $7 \quad 1120$ & 7.78 & 10.72 & 6074.2694 & 0 & 0 & 30.2 & 40 & 2.037 & 0.051 & 59 & 1 & 0.53615 & 0 \\
\hline 1 & 71140 & 5.83 & 10.43 & 6086.49176 & 0 & 0 & 30.2 & 29.98 & 1.982 & 0.066 & 60 & 1 & 0.5217 & \\
\hline 1 & 71160 & 3.89 & 10.15 & 6094.6599 & 0 & 0 & 30.2 & 20 & 1.928 & 0.096 & 61 & 1 & 0.5073 & \\
\hline 1 & 71180 & 1.94 & 9.16 & 6098.75104 & 0 & 0 & 30.2 & 9.98 & 1.74 & 0.174 & 62 & 1 & 0.458 & \\
\hline & $8 \quad 1200$ & 1.75 & 9.29 & 6102.43692 & 0 & 0 & 30.2 & 9 & 1.765 & 0.196 & 63 & 1 & 0.4646 & 0 \\
\hline 1 & $8 \quad 1220$ & 1.56 & 9.26 & 6105.69475 & 0 & 0 & 30.2 & 8.02 & 1.76 & 0.219 & 64 & 1 & 0.46305 & \\
\hline 1 & $8 \quad 1240$ & 1.36 & 9.21 & 6108.54574 & 0 & 0 & 30.2 & 6.99 & 1.75 & 0.25 & 65 & 1 & 0.46055 & 0 \\
\hline 1 & $8 \quad 1260$ & 1.17 & 9.09 & 6110.9899 & 0 & 0 & 30.2 & 6.02 & 1.726 & 0.287 & 66 & 1 & 0.4543 & 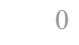 \\
\hline 1 & $8 \quad 1280$ & 0.97 & 8.91 & 6113.0233 & 0 & 0 & 30.2 & 4.99 & 1.693 & 0.339 & 67 & 1 & 0.4455 & 0 \\
\hline & $8 \quad 1300$ & 0.78 & 8.46 & 6114.64986 & 0 & 0 & 30.2 & 4.01 & 1.608 & 0.401 & 68 & 1 & 0.4231 & 0 \\
\hline 1 & $8 \quad 1320$ & 0.58 & 7.5 & 6115.86801 & 59.814 & 41.97493 & 30.2 & 2.98 & 1.425 & 0.478 & 69 & 1 & 0.3749 & 0.024 \\
\hline 1 & $8 \quad 1340$ & 0.39 & 6.29 & 6116.67697 & 99.536 & 83.22424 & 30.2 & 2.01 & 1.196 & 0.595 & 70 & 1 & 0.31465 & 0.012 \\
\hline 1 & $8 \quad 1360$ & 0.19 & 4.77 & 6117.07831 & 119.24 & 131.61462 & 30.2 & 0.98 & 0.906 & 0.924 & 71 & 1 & 0.23845 & 0.008 \\
\hline 1 & 91380 & 0.18 & 4.27 & 6117.45687 & 137.83 & 169.95208 & 30.2 & 0.93 & 0.811 & 0.872 & 72 & 1 & 0.21355 & 0.006 \\
\hline 1 & $9 \quad 1400$ & 0.16 & 4.19 & 6117.79223 & 154.3 & 193.84218 & 30.2 & 0.82 & 0.796 & 0.971 & 73 & 1 & 0.2094 & 0.005 \\
\hline 1 & $9 \quad 1420$ & 0.14 & 4.01 & 6118.08519 & 168.68 & 221.36888 & 30.2 & 0.72 & 0.762 & 1.058 & 74 & 1 & 0.2006 & 0.005 \\
\hline 1 & $9 \quad 1440$ & 0.12 & 4.26 & 6118.3373 & 181.06 & 223.5339 & 30.2 & 0.62 & 0.81 & 1.306 & 75 & 1 & 0.21315 & 0.004 \\
\hline 1 & $9 \quad 1460$ & 0.1 & 4.08 & 6118.547 & 191.36 & 246.59701 & 30.2 & 0.51 & 0.776 & 1.522 & 76 & 1 & 0.20415 & 0.004 \\
\hline 1 & $9 \quad 1480$ & 0.08 & 3.54 & 6118.71508 & 199.61 & 296.60051 & 30.2 & 0.41 & 0.673 & 1.641 & 77 & 1 & 0.177 & 0.003 \\
\hline & 91500 & 0.06 & 3.34 & 6118.84074 & 205.78 & 324.06695 & 30.2 & 0.31 & 0.635 & 2.048 & 78 & 1 & 0.1672 & 0.003 \\
\hline & $9 \quad 1520$ & 0.04 & 3.35 & 6118.92478 & 209.91 & 329.52743 & 30.2 & 0.21 & 0.637 & 3.033 & 79 & 1 & 0.1675 & 0.003 \\
\hline & $9 \quad 1540$ & 0.02 & 3.91 & 6118.96719 & 211.99 & 285.70286 & 30.2 & 0.1 & 0.742 & 7.42 & 80 & 1 & 0.19535 & 0.004 \\
\hline
\end{tabular}

04.01.04 WM-187 gravity settled CC-48 run 01.txt

Mode Step t[s] n[rpm] M[\%.]

$\begin{array}{rrrrr}1 & 1 & 20 & 0.02 & 7.62 \\ 1 & 1 & 40 & 0.04 & 6.55 \\ 1 & 1 & 60 & 0.06 & 7.59 \\ 1 & 1 & 80 & 0.08 & 7.9 \\ 1 & 1 & 100 & 0.1 & 8.47 \\ 1 & 1 & 120 & 0.12 & 8.86 \\ 1 & 1 & 140 & 0.14 & \\ 1 & 1 & 160 & 0.16 & 8.58 \\ 1 & 1 & 180 & 0.18 & 8 . \\ 1 & 2 & 200 & 0.19 & 8.0 \\ 1 & 2 & 220 & 0.39 & 8.16 \\ 1 & 2 & 240 & 0.58 & 8.2 \\ 1 & 2 & 260 & 0.78 & 9.7 \\ 1 & 2 & 280 & 0.97 & 11.28 \\ 1 & 2 & 300 & 1.17 & 12.22 \\ 1 & 2 & 320 & 1.36 & 12.6 \\ 1 & 2 & 340 & 1.55 & 11.57 \\ 1 & 2 & 360 & 1.75 & 10.8 \\ 1 & 3 & 380 & 1.94 & 10.23 \\ 1 & 3 & 400 & 3.89 & 10.47 \\ 1 & 3 & 420 & 5.83 & 9.82 \\ 1 & 3 & 440 & 7.77 & 9.89\end{array}$

Phi[rad]

$\begin{array}{rrrrrrrrrrr}0.0377 & 1.8511 & 1.27928 & 30 & 0.1 & 1.447 & 14.47 & 1 & 1 & 0.3809 & 0.782 \\ 0.12095 & 5.939 & 4.77412 & 30 & 0.21 & 1.244 & 5.924 & 2 & 1 & 0.32745 & 0.209 \\ 0.24583 & 12.071 & 8.3651 & 29.9 & 0.31 & 1.443 & 4.655 & 3 & 1 & 0.3797 & 0.12 \\ 0.41312 & 20.285 & 13.3984 & 29.9 & 0.41 & 1.514 & 3.693 & 4 & 1 & 0.3985 & 0.075 \\ 0.62204 & 30.543 & 18.97109 & 29.9 & 0.51 & 1.61 & 3.157 & 5 & 1 & 0.4237 & 0.053 \\ 0.87336 & 42.884 & 25.46571 & 30 & 0.62 & 1.684 & 2.716 & 6 & 1 & 0.44315 & 0.039 \\ 1.16632 & 57.269 & 33.49064 & 30 & 0.72 & 1.71 & 2.375 & 7 & 1 & 0.45 & 0.03 \\ 1.50168 & 73.736 & 45.23695 & 30 & 0.82 & 1.63 & 1.988 & 8 & 1 & 0.42905 & 0.022 \\ 1.87789 & 92.209 & 57.77498 & 30 & 0.93 & 1.596 & 1.716 & 9 & 1 & 0.42005 & 0.017 \\ 2.27844 & 111.88 & 73.5549 & 30 & 0.98 & 1.521 & 1.552 & 10 & 1 & 0.40035 & 0.014 \\ 3.0819 & 151.33 & 97.63159 & 29.9 & 2.01 & 1.55 & 0.771 & 11 & 1 & 0.40795 & 0.01 \\ 4.29456 & 210.87 & 135.34871 & 30 & 2.98 & 1.558 & 0.523 & 12 & 1 & 0.4099 & 0.007 \\ 5.91483 & 0 & 0 & 30 & 4.01 & 1.845 & 0.46 & 13 & 1 & 0.48555 & 0 \\ 7.94195 & 0 & 0 & 30 & 4.99 & 2.144 & 0.43 & 14 & 1 & 0.5642 & 0 \\ 10.37904 & 0 & 0 & 30 & 6.02 & 2.321 & 0.386 & 15 & 1 & 0.61075 & 0 \\ 13.22611 & 0 & 0 & 30 & 6.99 & 2.393 & 0.342 & 16 & 1 & 0.62975 & 0 \\ 16.48001 & 0 & 0 & 29.9 & 7.97 & 2.199 & 0.276 & 17 & 1 & 0.57865 & 0 \\ 20.14311 & 0 & 0 & 29.9 & 9 & 2.051 & 0.228 & 18 & 1 & 0.53975 & 0 \\ 24.23189 & 0 & 0 & 29.9 & 9.98 & 1.943 & 0.195 & 19 & 1 & 0.51135 & 0 \\ 32.34034 & 0 & 0 & 30 & 20 & 1.989 & 0.099 & 20 & 1 & 0.5234 & 0 \\ 44.53443 & 0 & 0 & 30 & 29.98 & 1.867 & 0.062 & 21 & 1 & 0.4912 & 0 \\ 60.78668 & 0 & 0 & 30 & 39.95 & 1.879 & 0.047 & 22 & 1 & 0.49455 & 0\end{array}$




\begin{tabular}{|c|c|c|c|c|c|c|c|c|c|c|c|c|c|c|c|}
\hline 1 & 3 & 460 & 9.72 & 10.13 & 81.12692 & 0 & 0 & 29.9 & 49.98 & 1.925 & 0.039 & 23 & 1 & 0.50645 & 0 \\
\hline 1 & 3 & 480 & 11.66 & 10.67 & 105.51196 & 0 & 0 & 29.9 & 59.96 & 2.027 & 0.034 & 24 & 1 & 0.53335 & 0 \\
\hline & 3 & 500 & 13.61 & 11.04 & 134.00935 & 0 & 0 & 30 & 69.98 & 2.097 & 0.03 & 25 & 1 & 0.55185 & 0 \\
\hline & 3 & 520 & 15.55 & 11.24 & 166.55782 & 0 & 0 & 29.9 & 79.96 & 2.136 & 0.027 & 26 & 1 & 0.56205 & \\
\hline & 3 & 540 & 17.5 & 11.84 & 203.15266 & 0 & 0 & 29.9 & 89.99 & 2.249 & 0.025 & 27 & 1 & 0.59185 & \\
\hline & 4 & 560 & 19.45 & 12.03 & 244.26747 & 0 & 0 & 29.9 & 100.01 & 2.285 & 0.023 & 28 & 1 & 0.6014 & \\
\hline & 4 & 580 & 38.9 & 15.23 & 325.45015 & 0 & 0 & 29.9 & 200.02 & 2.894 & 0.014 & 29 & 1 & 0.76165 & \\
\hline & 4 & 600 & 58.35 & 18.35 & 447.201 & 0 & 0 & 29.9 & 300.04 & 3.486 & 0.012 & 30 & 1 & 0.91725 & \\
\hline & 4 & 620 & 77.79 & 20.9 & 609.95828 & 0 & 0 & 29.9 & 400 & 3.971 & 0.01 & 31 & 1 & 1.04495 & \\
\hline & 4 & 640 & 97.24 & 23.52 & 813.32457 & 0 & 0 & 29.9 & 500.01 & 4.469 & 0.009 & 32 & 1 & 1.17605 & \\
\hline & 4 & 660 & 116.69 & 26.31 & 1057.22918 & 0 & 0 & 29.9 & 600.02 & 4.999 & 0.008 & 33 & 1 & 1.3154 & \\
\hline & 4 & 680 & 136.14 & 29.35 & 1342.18027 & 0 & 0 & 30 & 700.03 & 5.576 & 0.008 & 34 & 1 & 1.4674 & \\
\hline & 4 & 700 & 155.58 & 32.03 & 1667.79221 & 0 & 0 & 30 & 799.99 & 6.086 & 0.008 & 35 & 1 & 1.6017 & 0 \\
\hline & 4 & 720 & 175.03 & 34.67 & 2033.96212 & 0 & 0 & 30 & 900 & 6.588 & 0.007 & 36 & 1 & 1.73375 & 0 \\
\hline & 4 & 740 & 194.48 & 37.33 & 2441.16829 & 0 & 0 & 30 & 1000 & 7.092 & 0.007 & 37 & 1 & 1.86625 & 0 \\
\hline & 5 & 750 & 194.48 & 37.47 & 2649.22576 & 0 & 0 & 30 & 1000 & 7.12 & 0.007 & 38 & 1 & 1.8736 & 0 \\
\hline & 5 & 760 & 194.48 & 37.56 & 2852.47738 & 0 & 0 & 30 & 1000 & 7.137 & 0.007 & 39 & 1 & 1.87805 & 0 \\
\hline & 5 & 770 & 194.48 & 37.62 & 3056.54424 & 0 & 0 & 30 & 1000 & 7.148 & 0.007 & 40 & 1 & 1.88115 & 0 \\
\hline & 5 & 780 & 194.48 & 37.65 & 3260.1006 & 0 & 0 & 30 & 1000 & 7.154 & 0.007 & 41 & 1 & 1.8827 & 0 \\
\hline & 5 & 790 & 194.48 & 37.73 & 3463.76062 & 0 & 0 & 30 & 1000 & 7.169 & 0.007 & 42 & 1 & 1.8866 & 0 \\
\hline & 5 & 800 & 194.48 & 37.88 & 3667.41829 & 0 & 0 & 30 & 1000 & 7.196 & 0.007 & 43 & 1 & 1.89375 & 0 \\
\hline & 6 & 820 & 194.48 & 37.95 & 4079.01327 & 0 & 0 & 30 & 1000 & 7.21 & 0.007 & 44 & 1 & 1.89735 & 0 \\
\hline & 6 & 840 & 175.03 & 35.55 & 4446.09895 & 0 & 0 & 30 & 900 & 6.754 & 0.008 & 45 & 1 & 1.7773 & 0 \\
\hline & 6 & 860 & 155.58 & 32.99 & 4772.2591 & 0 & 0 & 29.9 & 799.99 & 6.268 & 0.008 & 46 & 1 & 1.64935 & 0 \\
\hline & 6 & 880 & 136.14 & 30.57 & 5057.4733 & 0 & 0 & 29.9 & 700.03 & 5.809 & 0.008 & 47 & 1 & 1.52865 & 0 \\
\hline & 6 & 900 & 116.69 & 28.03 & 5302.42642 & 0 & 0 & 29.9 & 600.02 & 5.326 & 0.009 & 48 & 1 & 1.40155 & 0 \\
\hline & 6 & 920 & 97.24 & 25.56 & 5506.2608 & 0 & 0 & 30 & 500.01 & 4.857 & 0.01 & 49 & 1 & 1.2781 & 0 \\
\hline & 6 & 940 & 77.79 & 23.01 & 5669.61734 & 0 & 0 & 30 & 400 & 4.372 & 0.011 & 50 & 1 & 1.15055 & 0 \\
\hline & 6 & 960 & 58.35 & 20.38 & 5792.02871 & 0 & 0 & 30 & 300.04 & 3.872 & 0.013 & 51 & 1 & 1.0189 & 0 \\
\hline & 6 & 980 & 38.9 & 17.2 & 5873.86249 & 0 & 0 & 30 & 200.02 & 3.269 & 0.016 & 52 & 1 & 0.8602 & 0 \\
\hline & 6 & 1000 & 19.45 & 14.18 & 5914.8532 & 0 & 0 & 29.9 & 100.01 & 2.694 & 0.027 & 53 & 1 & 0.70885 & 0 \\
\hline & 7 & 1020 & 17.5 & 14.01 & 5951.75749 & 0 & 0 & 29.9 & 89.99 & 2.661 & 0.03 & 54 & 1 & 0.70025 & 0 \\
\hline & 7 & 1040 & 15.56 & 13.51 & 5984.36408 & 0 & 0 & 29.9 & 80.01 & 2.567 & 0.032 & 55 & 1 & 0.6756 & 0 \\
\hline & 7 & 1060 & 13.61 & 13.31 & 6012.89838 & 0 & 0 & 30 & 69.98 & 2.529 & 0.036 & 56 & 1 & 0.66545 & 0 \\
\hline & 7 & 1080 & 11.67 & 12.95 & 6037.37767 & 0 & 0 & 30 & 60.01 & 2.46 & 0.041 & 57 & 1 & 0.64745 & 0 \\
\hline & 7 & 1100 & 9.72 & 12.57 & 6057.75404 & 0 & 0 & 30 & 49.98 & 2.389 & 0.048 & 58 & 1 & 0.62865 & 0 \\
\hline & 7 & 1120 & 7.78 & 12.07 & 6074.06676 & 0 & 0 & 29.9 & 40 & 2.293 & 0.057 & 59 & 1 & 0.60345 & 0 \\
\hline & 7 & 1140 & 5.83 & 11.76 & 6086.31033 & 0 & 0 & 30 & 29.98 & 2.234 & 0.075 & 60 & 1 & 0.58785 & 0 \\
\hline & 7 & 1160 & 3.89 & 11.4 & 6094.47769 & 0 & 0 & 30 & 20 & 2.167 & 0.108 & 61 & 1 & 0.5702 & 0 \\
\hline & 7 & 1180 & 1.94 & 10.32 & 6098.56804 & 0 & 0 & 30 & 9.98 & 1.961 & 0.196 & 62 & 1 & 0.516 & 0 \\
\hline & 8 & 1200 & 1.75 & 10.36 & 6102.25313 & 0 & 0 & 29.9 & 9 & 1.968 & 0.219 & 63 & 1 & 0.518 & 0 \\
\hline & 8 & 1220 & 1.56 & 10.18 & 6105.51411 & 0 & 0 & 30 & 8.02 & 1.934 & 0.241 & 64 & 1 & 0.50885 & 0 \\
\hline & 8 & 1240 & 1.36 & 10.12 & 6108.3651 & 0 & 0 & 30 & 6.99 & 1.923 & 0.275 & 65 & 1 & 0.506 & 0 \\
\hline & 8 & 1260 & 1.17 & 9.69 & 6110.80769 & 0 & 0 & 30 & 6.02 & 1.842 & 0.306 & 66 & 1 & 0.4847 & 0 \\
\hline & 8 & 1280 & 0.97 & 9.34 & 6112.84344 & 0 & 0 & 30 & 4.99 & 1.775 & 0.356 & 67 & 1 & 0.46705 & 0 \\
\hline & 8 & 1300 & 0.78 & 8.84 & 6114.46764 & 0 & 0 & 30 & 4.01 & 1.68 & 0.419 & 68 & 1 & 0.44205 & 0 \\
\hline & 8 & 1320 & 0.58 & 7.77 & 6115.68658 & 59.853 & 40.5507 & 29.9 & 2.98 & 1.476 & 0.495 & 69 & 1 & 0.3883 & 0.025 \\
\hline & 8 & 1340 & 0.39 & 6.65 & 6116.49633 & 99.613 & 78.8704 & 29.9 & 2.01 & 1.263 & 0.628 & 70 & 1 & 0.3324 & 0.013 \\
\hline & 8 & 1360 & 0.19 & 4.96 & 6116.8961 & 119.24 & 126.58477 & 29.9 & 0.98 & 0.942 & 0.961 & 71 & 1 & 0.248 & 0.008 \\
\hline & 9 & 1380 & 0.18 & 4.34 & 6117.27623 & 137.91 & 167.16154 & 30 & 0.93 & 0.825 & 0.887 & 72 & 1 & 0.2171 & 0.006 \\
\hline
\end{tabular}




$\begin{array}{rrrrrrrrrrrrrrrrr}1 & 9 & 1400 & 0.16 & 4.59 & 6117.61159 & 154.38 & 177.03615 & 30 & 0.82 & 0.872 & 1.063 & 73 & 1 & 0.22955 & 0.006 \\ 1 & 9 & 1420 & 0.14 & 4.85 & 6117.90455 & 168.76 & 183.03716 & 29.9 & 0.72 & 0.922 & 1.281 & 74 & 1 & 0.24265 & 0.005 \\ 1 & 9 & 1440 & 0.12 & 4.68 & 6118.15587 & 181.1 & 203.48431 & 30 & 0.62 & 0.89 & 1.435 & 75 & 1 & 0.2342 & 0.005 \\ 1 & 9 & 1460 & 0.1 & 4.49 & 6118.36558 & 191.4 & 224.38203 & 30 & 0.51 & 0.853 & 1.673 & 76 & 1 & 0.2245 & 0.004 \\ 1 & 9 & 1480 & 0.08 & 4.1 & 6118.53365 & 199.65 & 256.29108 & 30 & 0.41 & 0.779 & 1.9 & 77 & 1 & 0.20495 & 0.004 \\ 1 & 9 & 1500 & 0.06 & 3.7 & 6118.65931 & 205.82 & 292.77542 & 29.9 & 0.31 & 0.703 & 2.268 & 78 & 1 & 0.185 & 0.003 \\ 1 & 9 & 1520 & 0.04 & 3.88 & 6118.74335 & 209.95 & 284.86781 & 29.9 & 0.21 & 0.737 & 3.51 & 79 & 1 & 0.19385 & 0.004 \\ 1 & 9 & 1540 & 0.02 & 3.89 & 6118.78576 & 212.03 & 287.30364 & 29.9 & 0.1 & 0.738 & 7.38 & 80 & 1 & 0.19425 & 0.003\end{array}$

04.01.04 WM-187 gravity settled CC-48 run 02.txt

Mode Step

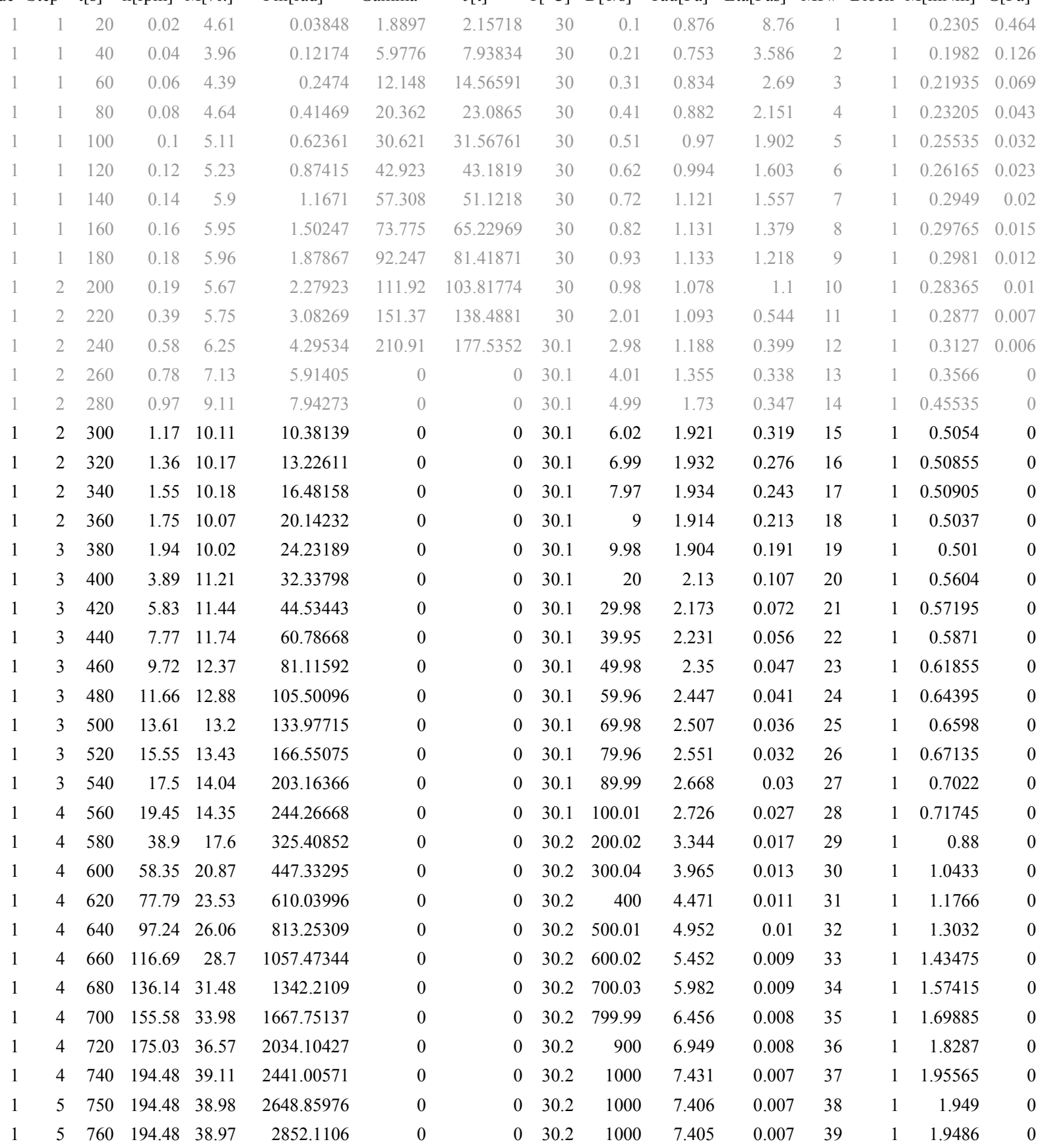




\begin{tabular}{|c|c|c|c|c|c|c|c|c|c|c|c|c|c|c|c|}
\hline 1 & 5 & 770 & 194.48 & 38.99 & 3055.87194 & 0 & 0 & 30.2 & 1000 & 7.409 & 0.007 & 40 & 1 & 1.94965 & 0 \\
\hline 1 & 5 & 780 & 194.48 & 38.95 & 3259.7346 & 0 & 0 & 30.2 & 1000 & 7.4 & 0.007 & 41 & 1 & 1.94725 & 0 \\
\hline & 5 & 790 & 194.48 & 38.94 & 3463.18964 & 0 & 0 & 30.2 & 1000 & 7.399 & 0.007 & 42 & 1 & 1.94715 & 0 \\
\hline & 5 & 800 & 194.48 & 38.93 & 3667.25572 & 0 & 0 & 30.2 & 1000 & 7.396 & 0.007 & 43 & 1 & 1.94635 & \\
\hline & 6 & 820 & 194.48 & 38.95 & 4078.85069 & 0 & 0 & 30.2 & 1000 & 7.4 & 0.007 & 44 & 1 & 1.9473 & \\
\hline & 6 & 840 & 175.03 & 36.21 & 4445.74238 & 0 & 0 & 30.2 & 900 & 6.88 & 0.008 & 45 & 1 & 1.8106 & \\
\hline & 6 & 860 & 155.58 & 33.65 & 4771.82163 & 0 & 0 & 30.2 & 799.99 & 6.393 & 0.008 & 46 & 1 & 1.6824 & \\
\hline & 6 & 880 & 136.14 & 31.27 & 5057.33193 & 0 & 0 & 30.2 & 700.03 & 5.942 & 0.008 & 47 & 1 & 1.56365 & \\
\hline & 6 & 900 & 116.69 & 28.68 & 5302.21279 & 0 & 0 & 30.2 & 600.02 & 5.45 & 0.009 & 48 & 1 & 1.4341 & \\
\hline & 6 & 920 & 97.24 & 26.19 & 5506.07702 & 0 & 0 & 30.2 & 500.01 & 4.976 & 0.01 & 49 & 1 & 1.30955 & \\
\hline & 6 & 940 & 77.79 & 23.68 & 5669.23014 & 0 & 0 & 30.2 & 400 & 4.5 & 0.011 & 50 & 1 & 1.18415 & \\
\hline & 6 & 960 & 58.35 & 20.96 & 5791.76482 & 0 & 0 & 30.2 & 300.04 & 3.982 & 0.013 & 51 & 1 & 1.04785 & \\
\hline & 6 & 980 & 38.9 & 17.72 & 5873.56796 & 0 & 0 & 30.2 & 200.02 & 3.367 & 0.017 & 52 & 1 & 0.88595 & \\
\hline & 6 & 1000 & 19.45 & 14.61 & 5914.62858 & 0 & 0 & 30.2 & 100.01 & 2.776 & 0.028 & 53 & 1 & 0.73065 & O \\
\hline & 7 & 1020 & 17.5 & 14.46 & 5951.58156 & 0 & 0 & 30.2 & 89.99 & 2.748 & 0.031 & 54 & 1 & 0.72315 & 0 \\
\hline & 7 & 1040 & 15.56 & 13.96 & 5984.18815 & 0 & 0 & 30.2 & 80.01 & 2.653 & 0.033 & 55 & 1 & 0.69815 & 0 \\
\hline & 7 & 1060 & 13.61 & 13.8 & 6012.71381 & 0 & 0 & 30.2 & 69.98 & 2.622 & 0.037 & 56 & 1 & 0.69005 & 0 \\
\hline & 7 & 1080 & 11.67 & 13.45 & 6037.16797 & 0 & 0 & 30.2 & 60.01 & 2.556 & 0.043 & 57 & 1 & 0.6726 & 0 \\
\hline & 7 & 1100 & 9.72 & 13.05 & 6057.54905 & 0 & 0 & 30.2 & 49.98 & 2.479 & 0.05 & 58 & 1 & 0.6524 & 0 \\
\hline & 7 & 1120 & 7.78 & 12.48 & 6073.88141 & 0 & 0 & 30.2 & 40 & 2.372 & 0.059 & 59 & 1 & 0.62415 & 0 \\
\hline & 7 & 1140 & 5.83 & 12.3 & 6086.1132 & 0 & 0 & 30.2 & 29.98 & 2.337 & 0.078 & 60 & 1 & 0.61505 & 0 \\
\hline & 7 & 1160 & 3.89 & 11.88 & 6094.27977 & 0 & 0 & 30.2 & 20 & 2.257 & 0.113 & 61 & 1 & 0.59395 & 0 \\
\hline & 7 & 1180 & 1.94 & 10.8 & 6098.37169 & 0 & 0 & 30.2 & 9.98 & 2.052 & 0.206 & 62 & 1 & 0.54005 & 0 \\
\hline & 8 & 1200 & 1.75 & 10.69 & 6102.05992 & 0 & 0 & 30.2 & 9 & 2.031 & 0.226 & 63 & 1 & 0.53435 & 0 \\
\hline & 8 & 1220 & 1.56 & 10.48 & 6105.31697 & 0 & 0 & 30.2 & 8.02 & 1.991 & 0.248 & 64 & 1 & 0.52395 & 0 \\
\hline & 8 & 1240 & 1.36 & 10.54 & 6108.16875 & 0 & 0 & 30.2 & 6.99 & 2.003 & 0.287 & 65 & 1 & 0.52705 & 0 \\
\hline & 8 & 1260 & 1.17 & 10.1 & 6110.61291 & 0 & 0 & 30.2 & 6.02 & 1.918 & 0.319 & 66 & 1 & 0.50485 & 0 \\
\hline & 8 & 1280 & 0.97 & 9.52 & 6112.64631 & 0 & 0 & 30.2 & 4.99 & 1.808 & 0.362 & 67 & 1 & 0.4759 & 0 \\
\hline & 8 & 1300 & 0.78 & 9.22 & 6114.27208 & 0 & 0 & 30.2 & 4.01 & 1.752 & 0.437 & 68 & 1 & 0.46095 & 0 \\
\hline & 8 & 1320 & 0.58 & 8.08 & 6115.48945 & 59.776 & 38.94183 & 30.2 & 2.98 & 1.535 & 0.515 & 69 & 1 & 0.4039 & 0.026 \\
\hline & 8 & 1340 & 0.39 & 6.67 & 6116.29919 & 99.536 & 78.49857 & 30.2 & 2.01 & 1.268 & 0.631 & 70 & 1 & 0.33365 & 0.013 \\
\hline & 8 & 1360 & 0.19 & 4.88 & 6116.70053 & 119.24 & 128.77198 & 30.3 & 0.98 & 0.926 & 0.945 & 71 & 1 & 0.24375 & 0.008 \\
\hline & 9 & 1380 & 0.18 & 4.45 & 6117.07909 & 137.83 & 162.92098 & 30.3 & 0.93 & 0.846 & 0.91 & 72 & 1 & 0.2225 & 0.006 \\
\hline & 9 & 1400 & 0.16 & 4.12 & 6117.41524 & 154.34 & 196.85833 & 30.3 & 0.82 & 0.784 & 0.956 & 73 & 1 & 0.2062 & 0.005 \\
\hline & 9 & 1420 & 0.14 & 4.14 & 6117.7082 & 168.72 & 214.38585 & 30.3 & 0.72 & 0.787 & 1.093 & 74 & 1 & 0.20705 & 0.005 \\
\hline & 9 & 1440 & 0.12 & 4.2 & 6117.95952 & 181.06 & 226.8953 & 30.3 & 0.62 & 0.798 & 1.287 & 75 & 1 & 0.21 & 0.004 \\
\hline & 9 & 1460 & 0.1 & 4 & 6118.16923 & 191.36 & 251.78852 & 30.3 & 0.51 & 0.76 & 1.49 & 76 & 1 & 0.20005 & 0.004 \\
\hline & 9 & 1480 & 0.08 & 3.68 & 6118.33809 & 199.65 & 286.03256 & 30.3 & 0.41 & 0.698 & 1.702 & 77 & 1 & 0.18375 & 0.003 \\
\hline & 9 & 1500 & 0.06 & 4.1 & 6118.46375 & 205.82 & 264.5516 & 30.3 & 0.31 & 0.778 & 2.51 & 78 & 1 & 0.2048 & 0.004 \\
\hline & 9 & 1520 & 0.04 & 4 & 6118.547 & 209.91 & 276.55998 & 30.3 & 0.21 & 0.759 & 3.614 & 79 & 1 & 0.1998 & 0.004 \\
\hline & 9 & 1540 & 0.02 & 4.07 & 6118.58941 & 211.99 & 274.2452 & 30.3 & 0.1 & 0.773 & 7.73 & 80 & 1 & 0.20335 & 0.004 \\
\hline
\end{tabular}

04.01.04 WM-187 gravity settled DG run $01 . t x t$

Mode Step $\mathrm{t}[\mathrm{s}] \quad \mathrm{n}[\mathrm{rpm}] \quad \mathrm{M}[\%$.] $\quad$ Phi[rad]

$\begin{array}{rrrrrrr}1 & 1 & 20 & 0.02 & 1.63 & 0.03927 & 0 \\ 1 & 1 & 40 & 0.04 & 1.83 & 0.12252 & 0 \\ 1 & 1 & 60 & 0.06 & 2.16 & 0.24819 & 0 \\ 1 & 1 & 80 & 0.08 & 2.84 & 0.41469 & 0 \\ 1 & 1 & 100 & 0.1 & 3.73 & 0.62439 & 0 \\ 1 & 1 & 120 & 0.12 & 4.89 & 0.87493 & 0\end{array}$

$\mathrm{J}[\mathrm{t}] \quad \mathrm{T}\left[{ }^{\circ} \mathrm{C}\right] \quad \mathrm{D}[\mathrm{1} / \mathrm{s}] \quad \mathrm{Tau}[\mathrm{Pa}] \quad \mathrm{Eta}[\mathrm{Pas}] \quad$ MP\# Block M[mNm] G[Pa $]$

$\begin{array}{rrrrrrrrr}0 & 30.4 & 0.1 & 0.109 & 1.09 & 1 & 1 & 0.0814 & 0 \\ 0 & 30.4 & 0.2 & 0.123 & 0.615 & 2 & 1 & 0.0915 & 0 \\ 0 & 30.4 & 0.3 & 0.145 & 0.483 & 3 & 1 & 0.10795 & 0 \\ 0 & 30.4 & 0.4 & 0.19 & 0.475 & 4 & 1 & 0.142 & 0 \\ 0 & 30.4 & 0.5 & 0.25 & 0.5 & 5 & 1 & 0.1865 & 0 \\ 0 & 30.4 & 0.6 & 0.328 & 0.547 & 6 & 1 & 0.2447 & 0\end{array}$




\begin{tabular}{|c|c|c|c|c|c|c|c|c|c|c|c|c|c|c|c|c|c|c|c|c|c|c|c|c|c|}
\hline 1 & 1 & & & & & 0 & 0 & 30.4 & & & & & & & 140 & 0.14 & 6.28 & 1.1671 & 0.4 & 0.71 & 0.42 & 0.592 & 7 & 1 & 0.3138 \\
\hline 1 & 1 & & & & & 0 & 0 & 30.4 & & & & & & & 160 & 0.16 & 9.18 & 1.50168 & 0.4 & 0.81 & 0.615 & 0.759 & 8 & 1 & 0.45915 \\
\hline 1 & 1 & & & & & 0 & 0 & 30.4 & & & & & & & 180 & 0.18 & 7.35 & 1.87867 & .4 & 0.91 & 0.492 & 0.541 & 9 & 1 & 0.36745 \\
\hline 1 & 2 & & & & & 0 & 0 & 30.4 & & & & & & & 200 & 0.2 & 7.55 & 2.29965 & 0.4 & 1.01 & 0.506 & 0.501 & 10 & 1 & 0.37725 \\
\hline 1 & 2 & & & & & 0 & 0 & 30.3 & & & & & & & 220 & 0.4 & 8.68 & 3.13138 & 0.3 & 2.02 & 0.581 & 0.288 & 11 & 1 & 0.4338 \\
\hline 1 & 2 & & & & & 0 & 0 & 30.3 & & & & & & & 240 & 0.6 & 9.87 & 4.37938 & 0.3 & 3.02 & 0.661 & 0.219 & 12 & 1 & 0.4935 \\
\hline 1 & 2 & & & & & 0 & 0 & 30.3 & & & & & & & 260 & 0.8 & 11.86 & 6.04442 & 0.3 & 4.03 & 0.795 & 0.197 & 13 & 1 & 0.5932 \\
\hline 1 & 2 & & & & & 0 & 0 & 30.4 & & & & & & & 280 & 1 & 18.77 & 8.1218 & 0.4 & 5.04 & 1.258 & 0.25 & 14 & 1 & 0.9387 \\
\hline 1 & 2 & & & & & 0 & 0 & 30.4 & & & & & & & 300 & 1.19 & 22.81 & 10.61937 & 0.4 & 6 & 1.528 & 0.255 & 15 & 1 & 1.1404 \\
\hline 1 & 2 & & & & & 0 & 0 & 30.4 & & & & & & & 320 & 1.39 & 24.61 & 13.53162 & 0.4 & 7 & 1.649 & 0.236 & 16 & 1 & 1.2305 \\
\hline 1 & 2 & & & & & 0 & 0 & 30.3 & & & & & & & 340 & 1.59 & 25.92 & 16.8625 & 0.3 & 8.01 & 1.736 & 0.217 & 17 & 1 & 1.2958 \\
\hline 1 & 2 & & & & & 0 & 0 & 30.3 & & & & & & & 360 & 1.79 & 26.12 & 20.60885 & 0.3 & 9.02 & 1.75 & 0.194 & 18 & 1 & 1.3061 \\
\hline 1 & 3 & & & & & 0 & 0 & 30.3 & & & & & & & 380 & 1.98 & 25.55 & 24.78088 & 0.3 & 9.98 & 1.712 & 0.172 & 19 & 1 & 1.27755 \\
\hline 1 & 3 & & & & & 0 & 0 & 30.3 & & & & & & & 400 & 3.97 & 28.01 & 33.06212 & 0.3 & 20 & 1.877 & 0.094 & 20 & 1 & 1.4005 \\
\hline 1 & 3 & & & & & 0 & 0 & 30.3 & & & & & & & 420 & 5.95 & 29.33 & 45.49969 & 0.3 & 29.98 & 1.965 & 0.066 & 21 & 1 & 1.46635 \\
\hline 1 & 3 & & & & & 0 & 0 & 30.3 & & & & & & & 440 & 7.94 & 30.8 & 62.07787 & 0.3 & 40.01 & 2.064 & 0.052 & 22 & 1 & 1.53995 \\
\hline 1 & 3 & & & & & 0 & 0 & 30.3 & & & & & & & 460 & 9.92 & 32.83 & 82.82652 & 0.3 & 49.99 & 2.2 & 0.044 & 23 & 1 & 1.64145 \\
\hline 1 & 3 & & & & & 0 & 0 & 30.3 & & & & & & & 480 & 11.91 & 33.56 & 107.73778 & 0.3 & 60.01 & 2.249 & 0.037 & 24 & 1 & 1.6782 \\
\hline 1 & 3 & & & & & 0 & 0 & 30.3 & & & & & & & 500 & 13.89 & 34.51 & 136.80615 & 0.3 & 69.99 & 2.312 & 0.033 & 25 & 1 & 1.7255 \\
\hline 1 & 3 & & & & & 0 & 0 & 30.3 & & & & & & & 520 & 15.88 & 36.23 & 170.04106 & 0.3 & 80.02 & 2.427 & 0.03 & 26 & 1 & 1.81155 \\
\hline 1 & 3 & & & & & 0 & 0 & 30.3 & & & & & & & 540 & 17.86 & 36.51 & 207.38046 & 0.3 & 90 & 2.446 & 0.027 & 27 & 1 & 1.82525 \\
\hline 1 & 4 & & & & & 0 & 0 & 30.3 & & & & & & & 560 & 19.85 & 37.9 & 249.31993 & 0.3 & 100.02 & 2.54 & 0.025 & 28 & 1 & 1.8952 \\
\hline 1 & 4 & & & & & 0 & 0 & 30.3 & & & & & & & 580 & 39.69 & 47.74 & 332.12211 & 0.3 & 200 & 3.199 & 0.016 & 29 & 1 & 2.387 \\
\hline 1 & 4 & & & & & 0 & 0 & 30.3 & & & & & & & 600 & 59.54 & 56.45 & 456.58101 & 0.3 & 300.02 & 3.782 & 0.013 & 30 & 1 & 2.82255 \\
\hline 1 & 4 & & & & & 0 & 0 & 30.3 & & & & & & & 620 & 79.38 & 64 & 622.45632 & 0.3 & 400 & 4.288 & 0.011 & 31 & 1 & 3.20015 \\
\hline 1 & 4 & & & & & 0 & 0 & 30.3 & & & & & & & 640 & 99.23 & 71 & 829.97658 & 0.3 & 500.02 & 4.757 & 0.01 & 32 & 1 & 3.54985 \\
\hline 1 & 4 & & & & & 0 & 0 & 30.3 & & & & & & & 660 & 119.07 & 77.47 & 1078.99728 & 0.3 & 599.99 & 5.191 & 0.009 & 33 & 1 & 3.8737 \\
\hline 1 & 4 & & & & & 0 & 0 & 30.3 & & & & & & & 680 & 138.92 & 83.76 & 1369.84907 & 0.3 & 700.02 & 5.612 & 0.008 & 34 & 1 & 4.18815 \\
\hline 1 & 4 & & & & & 0 & 0 & 30.3 & & & & & & & 700 & 158.76 & 89.61 & 1701.95311 & 0.3 & 799.99 & 6.004 & 0.008 & 35 & 1 & 4.48035 \\
\hline 1 & 4 & & & & & 0 & 0 & 30.3 & & & & & & & 720 & 178.61 & 95.57 & 2075.70053 & 0.3 & 900.02 & 6.403 & 0.007 & 36 & 1 & 4.7784 \\
\hline 1 & 4 & & & & & 0 & 0 & 30.3 & & & & & & & 740 & 198.45 & 101.1 & 2491.11568 & 0.3 & 999.99 & 6.775 & 0.007 & 37 & 1 & 5.05565 \\
\hline 1 & 5 & & & & & 0 & 0 & 30.3 & & & & & & & 750 & 198.45 & 100.2 & 2703.108 & 0.3 & 999.99 & 6.711 & 0.007 & 38 & 1 & 5.00805 \\
\hline 1 & 5 & & & & & 0 & 0 & 30.3 & & & & & & & 760 & 198.45 & 99.76 & 2911.02881 & 0.3 & 999.99 & 6.684 & 0.007 & 39 & 1 & 4.988 \\
\hline 1 & 5 & & & & & 0 & 0 & 30.3 & & & & & & & 770 & 198.45 & 99.43 & 3118.94962 & 0.3 & 999.99 & 6.662 & 0.007 & 40 & 1 & 4.97145 \\
\hline 1 & 5 & & & & & 0 & 0 & 30.3 & & & & & & & 780 & 198.45 & 99.09 & 3326.76598 & 0.3 & 999.99 & 6.639 & 0.007 & 41 & 1 & 4.9547 \\
\hline 1 & 5 & & & & & 0 & 0 & 30.3 & & & & & & & 790 & 198.45 & 98.83 & 3534.0624 & 0.3 & 999.99 & 6.621 & 0.007 & 42 & 1 & 4.94135 \\
\hline 1 & 5 & & & & & 0 & 0 & 30.3 & & & & & & & 800 & 198.45 & 98.53 & 3742.29501 & 0.3 & 999.99 & 6.601 & 0.007 & 43 & 1 & 4.92645 \\
\hline 1 & 6 & & & & & 0 & 0 & 30.3 & & & & & & & 820 & 198.45 & 98.24 & 4162.70766 & 0.3 & 999.99 & 6.582 & 0.007 & 44 & 1 & 4.91215 \\
\hline 1 & 6 & & & & & 0 & 0 & 30.3 & & & & & & & 840 & 178.61 & 91.28 & 4537.00643 & 0.3 & 900.02 & 6.116 & 0.007 & 45 & 1 & 4.5639 \\
\hline 1 & 6 & & & & & 0 & 0 & 30.2 & & & & & & & 860 & 158.76 & 84.46 & 4869.7545 & 0.2 & 799.99 & 5.658 & 0.007 & 46 & 1 & 4.22275 \\
\hline 1 & 6 & & & & & 0 & 0 & 30.2 & & & & & & & 880 & 138.92 & 77.92 & 5161.09638 & 0.2 & 700.02 & 5.221 & 0.007 & 47 & 1 & 3.8961 \\
\hline 1 & 6 & & & & & 0 & 0 & 30.2 & & & & & & & 900 & 119.07 & 71.19 & 5410.67785 & 0.2 & 599.99 & 4.77 & 0.008 & 48 & 1 & 3.55945 \\
\hline 1 & 6 & & & & & 0 & 0 & 30.2 & & & & & & & 920 & 99.23 & 64.52 & 5618.8225 & 0.2 & 500.02 & 4.323 & 0.009 & 49 & 1 & 3.22595 \\
\hline 1 & 6 & & & & & 0 & 0 & 30.2 & & & & & & & 940 & 79.38 & 57.59 & 5785.43608 & .2 & 400 & 3.858 & 0.01 & 50 & 1 & 2.87925 \\
\hline 1 & 6 & & & & & 0 & 0 & 30.2 & & & & & & & 960 & 59.54 & 50.37 & 5910.50917 & 0.2 & 300.02 & 3.375 & 0.011 & 51 & 1 & 2.5183 \\
\hline 1 & 6 & & & & & 0 & 0 & 30.2 & & & & & & & 980 & 39.69 & 42.23 & 5993.8941 & 0.2 & 200 & 2.829 & 0.014 & 52 & 1 & 2.11145 \\
\hline 1 & 6 & & & & & 0 & 0 & 30.2 & & & & & & & 1000 & 19.85 & 33.35 & 6035.78017 & 0.2 & 100.02 & 2.234 & 0.022 & 53 & 1 & 1.6674 \\
\hline 1 & 7 & & & & & 0 & 0 & 30.2 & & & & & & & 1020 & 17.86 & 32.73 & 6073.44237 & 0.2 & 90 & 2.193 & 0.024 & 54 & 1 & 1.63645 \\
\hline 1 & 7 & & & & & 0 & 0 & 30.2 & & & & & & & 1040 & 15.88 & 31.74 & 6106.7621 & 0.2 & 80.02 & 2.127 & 0.027 & 55 & 1 & 1.58705 \\
\hline 1 & 7 & & & & & 0 & 0 & 30.2 & & & & & & & 1060 & 13.89 & 30.84 & 6135.86111 & 0.2 & 69.99 & 2.066 & 0.03 & 56 & 1 & 1.54185 \\
\hline
\end{tabular}




\begin{tabular}{|c|c|c|c|c|c|c|c|c|c|c|c|c|c|}
\hline 1 & 71080 & 11.9 & 29.83 & 6160.81242 & 0 & 0 & 30.2 & 59.96 & 1.999 & 0.033 & 57 & 1 & 1.49145 \\
\hline 1 & 71100 & 9.92 & 28.6 & 6181.63332 & 0 & 0 & 30.1 & 49.99 & 1.916 & 0.038 & 58 & 1 & 1.43005 \\
\hline & $7 \quad 1120$ & 7.93 & 27.55 & 6198.28612 & 0 & 0 & 30.1 & 39.96 & 1.846 & 0.046 & 59 & 1 & 1.3776 \\
\hline & 71140 & 5.95 & 26.36 & 6210.77395 & 0 & 0 & 30.1 & 29.98 & 1.766 & 0.059 & 60 & 1 & 1.31815 \\
\hline & 71160 & 3.96 & 25.3 & 6219.10624 & 0 & 0 & 30.1 & 19.95 & 1.695 & 0.085 & 61 & 1 & 1.2649 \\
\hline & 71180 & 1.98 & 23.83 & 6223.28063 & 0 & 0 & 30.1 & 9.98 & 1.596 & 0.16 & 62 & 1 & 1.1913 \\
\hline & $8 \quad 1200$ & 1.79 & 23.69 & 6227.05133 & 0 & 0 & 30.1 & 9.02 & 1.587 & 0.176 & 63 & 1 & 1.18425 \\
\hline & $8 \quad 1220$ & 1.59 & 23.53 & 6230.38692 & 0 & 0 & 30.1 & 8.01 & 1.577 & 0.197 & 64 & 1 & 1.1767 \\
\hline & $8 \quad 1240$ & 1.39 & 23.45 & 6233.30546 & 0 & 0 & 30.1 & 7 & 1.571 & 0.224 & 65 & 1 & 1.1727 \\
\hline & $8 \quad 1260$ & 1.19 & 23.02 & 6235.80773 & 0 & 0 & 30.1 & 6 & 1.542 & 0.257 & 66 & 1 & 1.151 \\
\hline & $\begin{array}{ll}8 & 1280\end{array}$ & 0.99 & 22.47 & 6237.89454 & 0 & 0 & 30.1 & 4.99 & 1.505 & 0.302 & 67 & 1 & 1.1234 \\
\hline & $8 \quad 1300$ & 0.8 & 21.84 & 6239.56665 & 0 & 0 & 30.1 & 4.03 & 1.463 & 0.363 & 68 & 1 & 1.09195 \\
\hline & $8 \quad 1320$ & 0.6 & 19.93 & 6240.82014 & 0 & 0 & 30.1 & 3.02 & 1.335 & 0.442 & 69 & 1 & 0.99645 \\
\hline & $8 \quad 1340$ & 0.4 & 16.2 & 6241.66052 & 0 & 0 & 30.1 & 2.02 & 1.086 & 0.538 & 70 & 1 & 0.8102 \\
\hline & $8 \quad 1360$ & 0.2 & 12.36 & 6242.08307 & 0 & 0 & 30.1 & 1.01 & 0.828 & 0.82 & 71 & 1 & 0.61785 \\
\hline & 91380 & 0.18 & 11.15 & 6242.46241 & 0 & 0 & 30.1 & 0.91 & 0.747 & 0.821 & 72 & 1 & 0.5576 \\
\hline & 91400 & 0.16 & 10.42 & 6242.79778 & 0 & 0 & 30.1 & 0.81 & 0.698 & 0.862 & 73 & 1 & 0.521 \\
\hline & 91420 & 0.14 & 9.72 & 6243.09152 & 0 & 0 & 30.1 & 0.71 & 0.651 & 0.917 & 74 & 1 & 0.48585 \\
\hline & 91440 & 0.12 & 8.96 & 6243.34284 & 0 & 0 & 30.1 & 0.6 & 0.601 & 1.002 & 75 & 1 & 0.44815 \\
\hline & 91460 & 0.1 & 8.61 & 6243.55333 & 0 & 0 & 30.1 & 0.5 & 0.577 & 1.154 & 76 & 1 & 0.4303 \\
\hline & 91480 & 0.08 & 8.4 & 6243.72062 & 0 & 0 & 30.1 & 0.4 & 0.563 & 1.407 & 77 & 1 & 0.42015 \\
\hline & 91500 & 0.06 & 8.07 & 6243.84707 & 0 & 0 & 30.1 & 0.3 & 0.541 & 1.803 & 78 & 1 & 0.40345 \\
\hline & 91520 & 0.04 & 8.14 & 6243.93111 & 0 & 0 & 30.1 & 0.2 & 0.545 & 2.725 & 79 & 1 & 0.40675 \\
\hline & 91540 & 0.02 & 8.03 & 6243.97352 & 0 & 0 & 30.2 & 0.1 & 0.538 & 5.38 & 80 & 1 & 0.4015 \\
\hline
\end{tabular}

04.01.04 WM-187 gravity settled DG run 02.txt

$\begin{array}{rrrrrrr}\text { Mode } & \text { Step } & \mathrm{t}[\mathrm{s}] & \mathrm{n}[\mathrm{rpm}] & \mathrm{M}[\% .] & \mathrm{Phi}[\mathrm{rad}] & \text { Gamma } \\ 1 & 1 & 20 & 0.02 & 8.46 & 0.03691 & 0 \\ 1 & 1 & 40 & 0.04 & 7.67 & 0.12017 & 0 \\ 1 & 1 & 60 & 0.06 & 8.19 & 0.24583 & 0 \\ 1 & 1 & 80 & 0.08 & 8.56 & 0.41312 & 0 \\ 1 & 1 & 100 & 0.1 & 9.48 & 0.62204 & 0 \\ 1 & 1 & 120 & 0.12 & 10.19 & 0.87258 & 0 \\ 1 & 1 & 140 & 0.14 & 11.12 & 1.16553 & 0 \\ 1 & 1 & 160 & 0.16 & 11.54 & 1.50011 & 0 \\ 1 & 1 & 180 & 0.18 & 11.9 & 1.87632 & 0 \\ 1 & 2 & 200 & 0.2 & 12.28 & 2.29808 & 0 \\ 1 & 2 & 220 & 0.4 & 14.19 & 3.12981 & 0 \\ 1 & 2 & 240 & 0.6 & 16.01 & 4.37781 & 0 \\ 1 & 2 & 260 & 0.8 & 17.7 & 6.04207 & 0 \\ 1 & 2 & 280 & 1 & 22.28 & 8.12102 & 0 \\ 1 & 2 & 300 & 1.19 & 23.78 & 10.61937 & 0 \\ 1 & 2 & 320 & 1.39 & 23.03 & 13.5332 & 0 \\ 1 & 2 & 340 & 1.59 & 23.16 & 16.86328 & 0 \\ 1 & 2 & 360 & 1.79 & 23.19 & 20.60728 & 0 \\ 1 & 3 & 380 & 1.98 & 23.36 & 24.78088 & 0 \\ 1 & 3 & 400 & 3.97 & 25.16 & 33.05741 & 0 \\ 1 & 3 & 420 & 5.95 & 26.27 & 45.4989 & 0 \\ 1 & 3 & 440 & 7.94 & 27.3 & 62.08494 & 0 \\ 1 & 3 & 460 & 9.92 & 28.39 & 82.82181 & 0\end{array}$

$\mathrm{J}[\mathrm{t}] \quad \mathrm{T}\left[{ }^{\circ} \mathrm{C}\right] \quad \mathrm{D}[1 / \mathrm{s}] \quad \mathrm{Tau}[\mathrm{Pa}] \quad$ Eta[Pas $]$ MP\# Block M[mNm] G[Pa]

$\begin{array}{rrrrrrrrr}0 & 30.2 & 0.1 & 0.567 & 5.67 & 1 & 1 & 0.42315 & 0 \\ 0 & 30.2 & 0.2 & 0.514 & 2.57 & 2 & 1 & 0.38345 & 0 \\ 0 & 30.2 & 0.3 & 0.549 & 1.83 & 3 & 1 & 0.4097 & 0 \\ 0 & 30.2 & 0.4 & 0.573 & 1.432 & 4 & 1 & 0.4279 & 0 \\ 0 & 30.2 & 0.5 & 0.635 & 1.27 & 5 & 1 & 0.47405 & 0 \\ 0 & 30.2 & 0.6 & 0.683 & 1.138 & 6 & 1 & 0.50955 & 0 \\ 0 & 30.2 & 0.71 & 0.745 & 1.049 & 7 & 1 & 0.5558 & 0 \\ 0 & 30.2 & 0.81 & 0.773 & 0.954 & 8 & 1 & 0.5768 & 0 \\ 0 & 30.2 & 0.91 & 0.797 & 0.876 & 9 & 1 & 0.59475 & 0 \\ 0 & 30.2 & 1.01 & 0.823 & 0.815 & 10 & 1 & 0.6142 & 0 \\ 0 & 30.2 & 2.02 & 0.951 & 0.471 & 11 & 1 & 0.70945 & 0 \\ 0 & 30.2 & 3.02 & 1.073 & 0.355 & 12 & 1 & 0.80065 & 0 \\ 0 & 30.2 & 4.03 & 1.186 & 0.294 & 13 & 1 & 0.88525 & 0 \\ 0 & 30.2 & 5.04 & 1.493 & 0.296 & 14 & 1 & 1.11385 & 0 \\ 0 & 30.2 & 6 & 1.593 & 0.266 & 15 & 1 & 1.18915 & 0 \\ 0 & 30.2 & 7 & 1.543 & 0.22 & 16 & 1 & 1.1517 & 0 \\ 0 & 30.2 & 8.01 & 1.551 & 0.194 & 17 & 1 & 1.1578 & 0 \\ 0 & 30.2 & 9.02 & 1.554 & 0.172 & 18 & 1 & 1.15945 & 0 \\ 0 & 30.2 & 9.98 & 1.565 & 0.157 & 19 & 1 & 1.16795 & 0 \\ 0 & 30.2 & 20 & 1.686 & 0.084 & 20 & 1 & 1.25795 & 0 \\ 0 & 30.2 & 29.98 & 1.76 & 0.059 & 21 & 1 & 1.31325 & 0 \\ 0 & 30.2 & 40.01 & 1.829 & 0.046 & 22 & 1 & 1.3652 & 0 \\ 0 & 30.2 & 49.99 & 1.902 & 0.038 & 23 & 1 & 1.41955 & 0\end{array}$




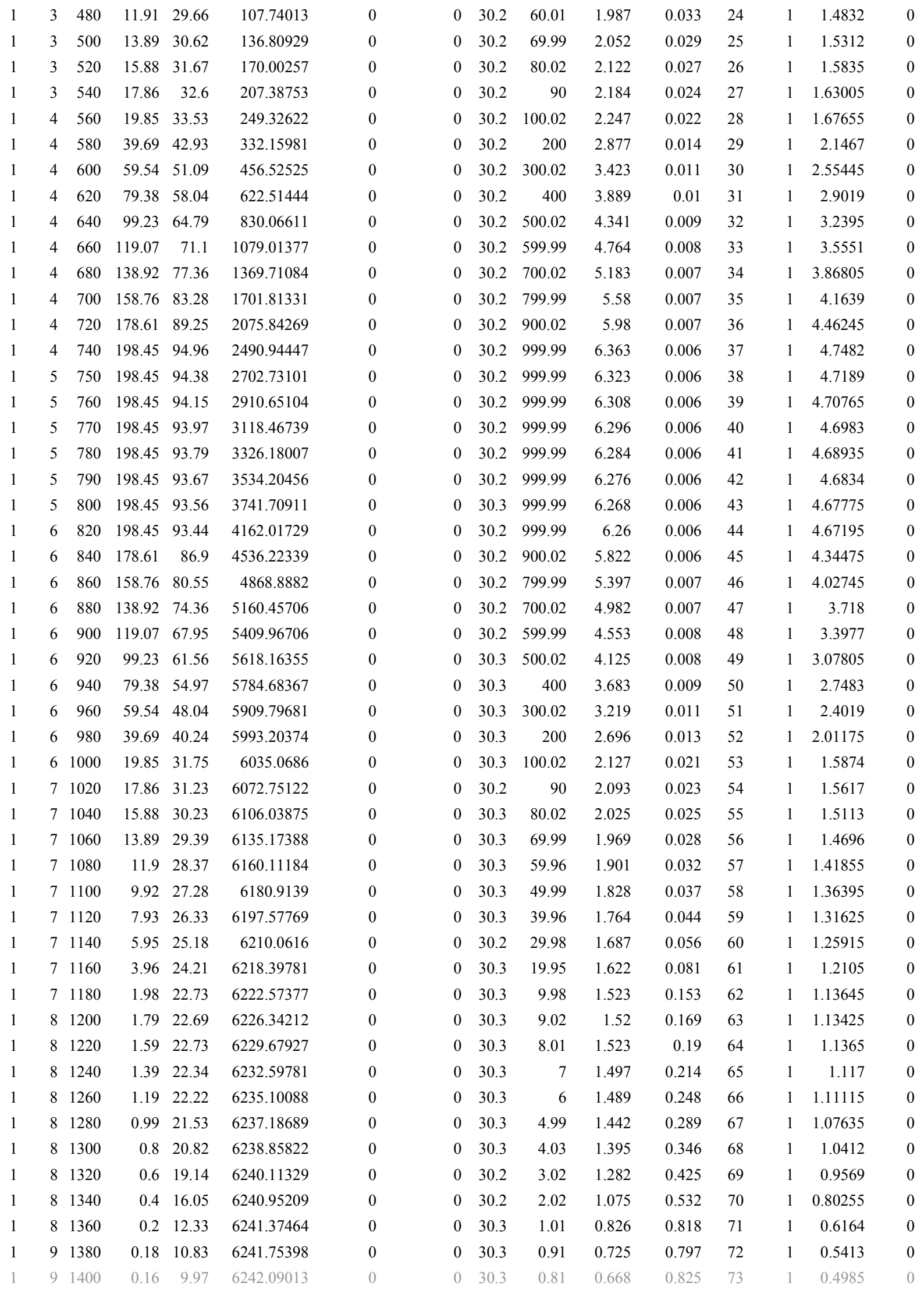




$\begin{array}{rrrrrrrrrrrrrrr}9 & 1420 & 0.14 & 9.3 & 6242.38309 & 0 & 0 & 30.2 & 0.71 & 0.623 & 0.877 & 74 & 1 & 0.4648 & 0 \\ 9 & 1440 & 0.12 & 8.75 & 6242.63441 & 0 & 0 & 30.2 & 0.6 & 0.586 & 0.977 & 75 & 1 & 0.4375 & 0 \\ 9 & 1460 & 0.1 & 8.28 & 6242.8449 & 0 & 0 & 30.3 & 0.5 & 0.555 & 1.11 & 76 & 1 & 0.41405 & 0 \\ 9 & 1480 & 0.08 & 7.52 & 6243.01298 & 0 & 0 & 30.2 & 0.4 & 0.504 & 1.26 & 77 & 1 & 0.3762 & 0 \\ 9 & 1500 & 0.06 & 7.31 & 6243.13864 & 0 & 0 & 30.3 & 0.3 & 0.49 & 1.633 & 78 & 1 & 0.36545 & 0 \\ 9 & 1520 & 0.04 & 7.47 & 6243.22346 & 0 & 0 & 30.2 & 0.2 & 0.5 & 2.5 & 79 & 1 & 0.3733 & 0 \\ 9 & 1540 & 0.02 & 7.3 & 6243.26509 & 0 & 0 & 30.2 & 0.1 & 0.489 & 4.89 & 80 & 1 & 0.3649 & 0\end{array}$
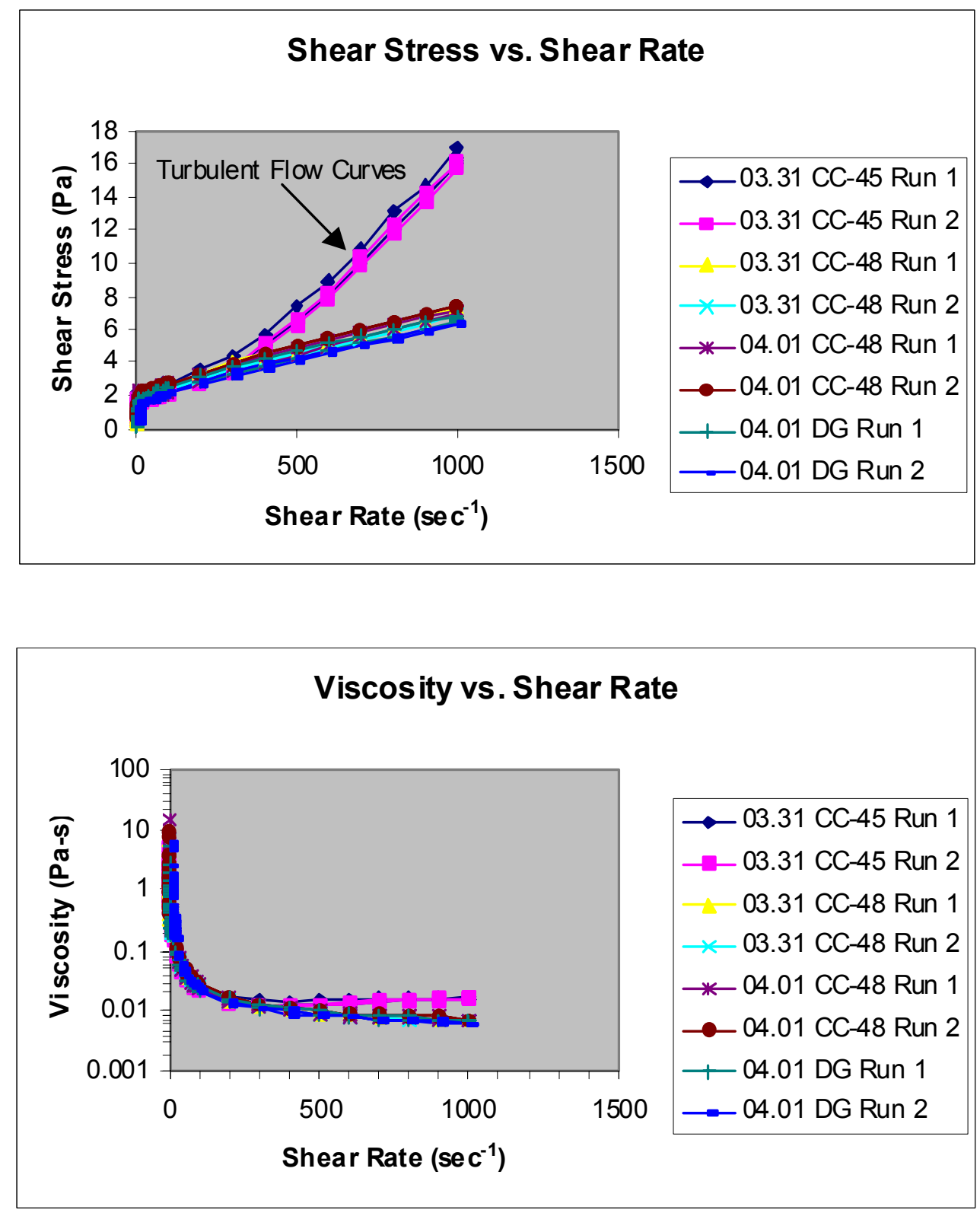


\section{E-2.2.4 Centrifuged}

04.01.04 WM-187 centrifuged cc-45 run 01.txt

\begin{tabular}{|c|c|c|c|c|c|c|c|c|c|c|c|c|c|c|c|}
\hline Mode & Step & $\mathrm{t}[\mathrm{s}]$ & $\mathrm{n}[\mathrm{rpm}]$ & $\mathrm{M}[\%]$. & Phi[rad] & Gamma & $J[t]$ & $\mathrm{T}\left[{ }^{\circ} \mathrm{C}\right]$ & $\mathrm{D}[1 / \mathrm{s}]$ & $\mathrm{Tau}[\mathrm{Pa}]$ & $\mathrm{Eta}[\mathrm{Pas}]$ & MP\# & Block $\mathrm{N}$ & $\mathrm{M}[\mathrm{mNm}]$ & $\mathrm{G}[\mathrm{Pa}]$ \\
\hline 1 & 1 & 20 & 0.08 & 25.37 & 0.15708 & 1.9365 & 0.38979 & 30.3 & 0.1 & 4.968 & 49.68 & 1 & 1 & 1.26865 & 2.565453137 \\
\hline 1 & 1 & 40 & 0.16 & 25.85 & 0.48616 & 5.99347 & 1.18401 & 30.3 & 0.21 & 5.062 & 24.105 & 2 & 1 & 1.29265 & 0.844585858 \\
\hline 1 & 1 & 60 & 0.24 & 24.46 & 0.97625 & 12.03535 & 2.51365 & $5 \quad 30.3$ & 0.31 & 4.788 & 15.445 & 3 & 1 & 1.22275 & 0.397828065 \\
\hline 1 & 1 & 80 & 0.31 & 26.97 & 1.62892 & 20.0815 & 3.80331 & 30.3 & 0.4 & 5.28 & 13.2 & 4 & 1 & 1.3484 & 0.262928566 \\
\hline 1 & 1 & 100 & 0.39 & 29.81 & 2.44416 & 30.13194 & 5.16223 & $3 \quad 30.3$ & 0.5 & 5.837 & 11.674 & 5 & 1 & 1.49065 & 0.193714709 \\
\hline 1 & 1 & 120 & 0.47 & 32.21 & 3.42041 & 42.16729 & 6.68579 & 9 30.3 & 0.61 & 6.307 & 10.339 & 6 & 1 & 1.6105 & 0.149570911 \\
\hline 1 & 1 & 140 & 0.54 & 35.68 & 4.56081 & 56.22628 & 8.04727 & $7 \quad 30.3$ & 0.7 & 6.987 & 9.981 & 7 & 1 & 1.78415 & 0.124265735 \\
\hline 1 & 1 & 160 & 0.62 & 35.18 & 5.86378 & 72.28954 & 10.49347 & $7 \quad 30.3$ & 0.8 & 6.889 & 8.611 & 8 & 1 & 1.7592 & 0.095297328 \\
\hline 1 & 1 & 180 & 0.7 & 34.35 & 7.32934 & 90.35709 & 13.434 & $4 \quad 30.3$ & 0.9 & 6.726 & 7.473 & 9 & 1 & 1.71765 & 0.074437988 \\
\hline 1 & 2 & 200 & 0.77 & 34.43 & 8.95197 & 0 & 0 & 30.3 & 0.99 & 6.742 & 6.81 & 10 & 1 & 1.7216 & 0 \\
\hline 1 & 2 & 220 & 1.55 & 44.37 & 12.17289 & 0 & 0 & 30.3 & 2 & 8.687 & 4.344 & 11 & 1 & 2.21835 & 0 \\
\hline 1 & 2 & 240 & 2.32 & 58.06 & 17.01722 & 0 & 0 & 30.3 & 3 & 11.367 & 3.789 & 12 & 1 & 2.9028 & 0 \\
\hline 1 & 2 & 260 & 3.09 & 60.46 & 23.4889 & 0 & 0 & 30.3 & 3.99 & 11.839 & 2.967 & 13 & 1 & 3.02315 & 0 \\
\hline 1 & 2 & 280 & 3.87 & 58.62 & 31.58793 & 0 & 0 & 30.3 & 5 & 11.479 & 2.296 & 14 & 1 & 2.9312 & 0 \\
\hline 1 & 2 & 300 & 4.65 & 57.86 & 41.29938 & 0 & 0 & 30.3 & 6 & 11.328 & 1.888 & 15 & 1 & 2.8928 & 0 \\
\hline 1 & 2 & 320 & 5.42 & 57.61 & 52.65152 & 0 & 0 & 30.3 & 7 & 11.281 & 1.612 & 16 & 1 & 2.88065 & 0 \\
\hline 1 & 2 & 340 & 6.2 & 57.97 & 65.61609 & 0 & 0 & 30.3 & 8 & 11.35 & 1.419 & 17 & 1 & 2.89835 & 0 \\
\hline 1 & 2 & 360 & 6.97 & 57.61 & 80.19151 & 0 & 0 & 30.3 & 9 & 11.279 & 1.253 & 18 & 1 & 2.8803 & 0 \\
\hline 1 & 3 & 380 & 7.75 & 57.35 & 96.5702 & 0 & 0 & 30.3 & 10.01 & 11.228 & 1.122 & 19 & 1 & 2.8673 & 0 \\
\hline 1 & 3 & 400 & 15.5 & 60.34 & 128.8807 & 0 & 0 & 30.3 & 20.01 & 11.815 & 0.59 & 20 & 1 & 3.017 & 0 \\
\hline 1 & 3 & 420 & 23.24 & 61.53 & 177.46543 & 0 & 0 & 30.3 & 30 & 12.048 & 0.402 & 21 & 1 & 3.0765 & 0 \\
\hline 1 & 3 & 440 & 30.99 & 61.76 & 242.21444 & 0 & 0 & 30.3 & 40.01 & 12.092 & 0.302 & 22 & 1 & 3.08775 & 0 \\
\hline 1 & 3 & 460 & 38.73 & 61.26 & 323.20155 & 0 & 0 & 30.3 & 50 & 11.994 & 0.24 & 23 & 1 & 3.06275 & 0 \\
\hline 1 & 3 & 480 & 46.48 & 61.35 & 420.51396 & 0 & 0 & 30.3 & 60.01 & 12.013 & 0.2 & 24 & 1 & 3.0676 & 0 \\
\hline 1 & 3 & 500 & 54.22 & 61.76 & 533.9176 & 0 & 0 & 30.3 & 70 & 12.093 & 0.173 & 25 & 1 & 3.08815 & 0 \\
\hline 1 & 3 & 520 & 61.96 & 61.8 & 663.51144 & 0 & 0 & 30.3 & 79.99 & 12.101 & 0.151 & 26 & 1 & 3.09005 & 0 \\
\hline 1 & 3 & 540 & 69.71 & 62.25 & 809.43135 & 0 & 0 & 30.3 & 90 & 12.189 & 0.135 & 27 & 1 & 3.1127 & 0 \\
\hline 1 & 4 & 560 & 77.46 & 61.91 & 973.08241 & 0 & 0 & 30.3 & 100 & 12.122 & 0.121 & 28 & 1 & 3.0954 & 0 \\
\hline 1 & 4 & 580 & 154.92 & 67.85 & 1296.20856 & 0 & 0 & 30.3 & 200 & 13.285 & 0.066 & 29 & 1 & 3.39255 & 0 \\
\hline 1 & 4 & 600 & 232.38 & 72.35 & 1781.80768 & 0 & 0 & 30.3 & 300 & 14.166 & 0.047 & 30 & 1 & 3.61755 & 0 \\
\hline 1 & 4 & 620 & 309.84 & 76.88 & 2429.67713 & 0 & 0 & 30.3 & 400 & 15.053 & 0.038 & 31 & 1 & 3.844 & 0 \\
\hline 1 & 4 & 640 & 387.3 & 81.45 & 3240.14049 & 0 & 0 & 30.3 & 500 & 15.947 & 0.032 & 32 & 1 & 4.07235 & 0 \\
\hline 1 & 4 & 660 & 464.75 & 86.38 & 4211.98118 & 0 & 0 & 30.3 & 599.99 & 16.913 & 0.028 & 33 & 1 & 4.31895 & 0 \\
\hline 1 & 4 & 680 & 542.21 & 96.96 & 5346.53909 & 0 & 0 & 30.3 & 699.99 & 18.984 & 0.027 & 34 & 1 & 4.8479 & 0 \\
\hline 1 & 4 & 700 & 619.67 & 112.8 & 6641.41719 & 0 & 0 & 30.3 & 799.99 & 22.087 & 0.028 & 35 & 1 & 5.6401 & 0 \\
\hline 1 & 4 & 720 & 697.13 & 123.21 & 8100.51341 & 0 & 0 & 30.3 & 899.99 & 24.124 & 0.027 & 36 & 1 & 6.16035 & 0 \\
\hline 1 & 4 & 740 & 774.59 & 136.82 & 9722.68969 & 0 & 0 & 30.3 & 1000 & 26.789 & 0.027 & 37 & 1 & 6.84085 & 0 \\
\hline 1 & 5 & 750 & 774.59 & 134.7 & 10551.35937 & 0 & 0 & 30.3 & 1000 & 26.375 & 0.026 & 38 & 1 & 6.7351 & 0 \\
\hline 1 & 5 & 760 & 774.59 & 134.32 & 11362.91443 & 0 & 0 & 30.3 & 1000 & 26.299 & 0.026 & 39 & 1 & 6.71575 & 0 \\
\hline 1 & 5 & 770 & 774.59 & 133.71 & 12174.4695 & 0 & 0 & 30.3 & 1000 & 26.18 & 0.026 & 40 & 1 & 6.6855 & 0 \\
\hline 1 & 5 & 780 & 774.59 & 132.7 & 12985.21324 & 0 & 0 & 30.3 & 1000 & 25.983 & 0.026 & 41 & 1 & 16.63515 & 0 \\
\hline 1 & 5 & 790 & 774.59 & 131.45 & 13795.14411 & 0 & 0 & 30.3 & 1000 & 25.738 & 0.026 & 42 & 1 & 1 6.57255 & 0 \\
\hline 1 & 5 & 800 & 774.59 & 130.59 & 14607.91889 & 0 & 0 & 30.3 & 1000 & 25.57 & 0.026 & 43 & 1 & 6.5296 & 0 \\
\hline 1 & 6 & 820 & 774.59 & 130.89 & 16248.06038 & 0 & 0 & 30.3 & 1000 & 25.628 & 0.026 & 44 & 1 & 16.54435 & 0 \\
\hline 1 & 6 & 840 & 697.13 & 116.16 & 17710.84482 & 0 & 0 & 30.3 & 899.99 & 22.744 & 0.025 & 45 & 1 & 5.8079 & 0 \\
\hline 1 & 6 & 860 & 619.67 & 103.59 & 19009.69389 & 0 & 0 & 30.3 & 799.99 & 20.283 & 0.025 & 46 & 1 & 15.17945 & 0 \\
\hline
\end{tabular}




\begin{tabular}{|c|c|c|c|c|c|c|c|c|c|c|c|c|c|c|}
\hline & 880 & 542.21 & 89.34 & 20147.16878 & 0 & 0 & 30.3 & 699.99 & 17.493 & 0.025 & 47 & 1 & 4.46695 & 0 \\
\hline & 900 & 464.75 & 74.32 & 21120.58733 & 0 & 0 & 30.3 & 599.99 & 14.551 & 0.024 & 48 & 1 & 3.71575 & \\
\hline & 920 & 387.3 & 67.37 & 21933.03304 & 0 & 0 & 30.3 & 500 & 13.192 & 0.026 & 49 & 1 & 3.3687 & 0 \\
\hline & 940 & 309.84 & 60.72 & 22583.57205 & 0 & 0 & 30.3 & 400 & 11.889 & 0.03 & 50 & 1 & 3.03605 & \\
\hline & 960 & 232.38 & 54.83 & 23071.80147 & 0 & 0 & 30.3 & 300 & 10.736 & 0.036 & 51 & 1 & 2.7416 & 0 \\
\hline & 980 & 154.92 & 49.17 & 23397.15266 & 0 & 0 & 30.3 & 200 & 9.628 & 0.048 & 52 & 1 & 2.4587 & 0 \\
\hline & 61000 & 77.46 & 44.09 & 23560.67884 & 0 & 0 & 30.3 & 100 & 8.633 & 0.086 & 53 & 1 & 2.2046 & 0 \\
\hline & 71020 & 69.71 & 43.47 & 23707.82004 & 0 & 0 & 30.3 & 90 & 8.511 & 0.095 & 54 & 1 & 2.17345 & 0 \\
\hline & 71040 & 61.97 & 42.76 & 23837.75239 & 0 & 0 & 30.3 & 80 & 8.373 & 0.105 & 55 & 1 & 2.1381 & 0 \\
\hline & 71060 & 54.22 & 42.03 & 23951.43171 & 0 & 0 & 30.3 & 70 & 8.23 & 0.118 & 56 & 1 & 2.10155 & 0 \\
\hline & 71080 & 46.48 & 40.93 & 24048.86663 & 0 & 0 & 30.3 & 60.01 & 8.014 & 0.134 & 57 & 1 & 2.0465 & 0 \\
\hline & 71100 & 38.73 & 40.07 & 24130.14042 & 0 & 0 & 30.3 & 50 & 7.846 & 0.157 & 58 & 1 & 2.00365 & 0 \\
\hline & 71120 & 30.99 & 39.49 & 24195.11955 & 0 & 0 & 30.3 & 40.01 & 7.732 & 0.193 & 59 & 1 & 1.9745 & 0 \\
\hline & 71140 & 23.24 & 38.62 & 24243.90691 & 0 & 0 & 30.3 & 30 & 7.562 & 0.252 & 60 & 1 & 1.9311 & 0 \\
\hline & 71160 & 15.5 & 37.64 & 24276.4813 & 0 & 0 & 30.3 & 20.01 & 7.37 & 0.368 & 61 & 1 & 1.882 & v \\
\hline & 71180 & 7.75 & 36.53 & 24292.82622 & 0 & 0 & 30.3 & 10.01 & 7.153 & 0.715 & 62 & 1 & 1.82655 & 0 \\
\hline & 81200 & 6.97 & 36.1 & 24307.53516 & 0 & 0 & 30.2 & 9 & 7.068 & 0.785 & 63 & 1 & 1.8048 & 0 \\
\hline & 81220 & 6.2 & 35.66 & 24320.52093 & 0 & 0 & 30.3 & 8 & 6.982 & 0.873 & 64 & 1 & 1.783 & 0 \\
\hline & 81240 & 5.42 & 34.93 & 24331.88015 & 0 & 0 & 30.3 & 7 & 6.84 & 0.977 & 65 & 1 & 1.74665 & 0 \\
\hline & 81260 & 4.64 & 34.46 & 24341.62065 & 0 & 0 & 30.3 & 5.99 & 6.747 & 1.126 & 66 & 1 & 1.723 & 0 \\
\hline & 81280 & 3.87 & 33.64 & 24349.74167 & 0 & 0 & 30.3 & 5 & 6.587 & 1.317 & 67 & 1 & 1.6822 & 0 \\
\hline & 81300 & 3.09 & 32.53 & 24356.23456 & 0 & 0 & 30.3 & 3.99 & 6.37 & 1.596 & 68 & 1 & 1.62665 & 0 \\
\hline & 81320 & 2.32 & 30.31 & 24361.10324 & 0 & 0 & 30.3 & 3 & 5.934 & 1.978 & 69 & 1 & 1.51535 & 0 \\
\hline & 81340 & 1.54 & 25.48 & 24364.35165 & 0 & 0 & 30.3 & 1.99 & 4.988 & 2.507 & 70 & 1 & 1.2738 & 0 \\
\hline & 81360 & 0.77 & 19.91 & 24365.97742 & 0 & 0 & 30.3 & 0.99 & 3.899 & 3.938 & 71 & 1 & 0.9957 & 0 \\
\hline & 91380 & 0.7 & 19.37 & 24367.45161 & 18.17405 & 4.79147 & 30.3 & 0.9 & 3.793 & 4.214 & 72 & 1 & 0.9686 & 0.208704169 \\
\hline & 91400 & 0.62 & 17.54 & 24368.75852 & 34.28573 & 9.98129 & 30.3 & 0.8 & 3.435 & 4.294 & 73 & 1 & 0.87705 & 0.100187454 \\
\hline & 91420 & 0.55 & 15.96 & 24369.90127 & 48.37377 & 15.4796 & 30.3 & 0.71 & 3.125 & 4.401 & 74 & 1 & 0.79795 & 0.064601126 \\
\hline & 91440 & 0.47 & 15.17 & 24370.88145 & 60.45753 & 20.35606 & 30.3 & 0.61 & 2.97 & 4.869 & 75 & 1 & 0.75845 & 0.049125394 \\
\hline & 91460 & 0.39 & 14.35 & 24371.69905 & 70.53701 & 25.10213 & 30.3 & 0.5 & 2.81 & 5.62 & 76 & 1 & 0.71765 & 0.039837243 \\
\hline & 91480 & 0.31 & 14.06 & 24372.35486 & 78.6219 & 28.55862 & 30.3 & 0.4 & 2.753 & 6.883 & 77 & 1 & 0.7031 & 0.035015689 \\
\hline & 91500 & 0.24 & 13.38 & 24372.84887 & 84.71219 & 32.33288 & 30.3 & 0.31 & 2.62 & 8.452 & 78 & 1 & 0.66915 & 0.030928252 \\
\hline & 91520 & 0.16 & 12.29 & 24373.18031 & 88.79821 & 36.90697 & 30.2 & 0.21 & 2.406 & 11.457 & 79 & 1 & 0.6143 & 0.027095141 \\
\hline & 91540 & 0.08 & 10.88 & 24373.34917 & 90.87995 & 42.6466 & 30.3 & 0.1 & 2.131 & 21.31 & 80 & 1 & 0.5442 & 0.023448516 \\
\hline
\end{tabular}

04.01.04 WM-187 centrifuged cc-45 run 02.txt

\begin{tabular}{rrrrrrrrrrrrrrrr} 
Mode Step & \multicolumn{1}{c}{$\mathrm{t}[\mathrm{s}]$} & $\mathrm{n}[\mathrm{rpm}]$ & $\mathrm{M}[\%]$. & \multicolumn{1}{c}{$\mathrm{Phi}[\mathrm{rad}]$} & \multicolumn{1}{c}{$\mathrm{Gamma}$} & \multicolumn{1}{c}{$\mathrm{J}[\mathrm{t}]$} & \multicolumn{3}{c}{$\mathrm{T}\left[{ }^{\circ} \mathrm{C}\right]$} & $\mathrm{D}[1 / \mathrm{s}]$ & $\mathrm{Tau}[\mathrm{Pa}]$ & $\mathrm{Eta}[\mathrm{Pas}]$ & $\mathrm{MP} \# \mathrm{Block} \mathrm{M}[\mathrm{mNm}]$ & $\mathrm{G}[\mathrm{Pa}]$ \\
1 & 1 & 20 & 0.08 & 11.35 & 0.16179 & 1.99459 & 0.89765 & 30.3 & 0.1 & 2.222 & 22.22 & 1 & 1 & 0.56735 & 1.114013406 \\
1 & 1 & 40 & 0.16 & 10.24 & 0.49009 & 6.04188 & 3.0134 & 30.3 & 0.21 & 2.005 & 9.548 & 2 & 1 & 0.5119 & 0.331850351 \\
1 & 1 & 60 & 0.24 & 11.13 & 0.98175 & 12.10312 & 5.55189 & 30.3 & 0.31 & 2.18 & 7.032 & 3 & 1 & 0.5567 & 0.180118845 \\
1 & 1 & 80 & 0.31 & 11.9 & 1.63441 & 20.14928 & 8.65147 & 30.3 & 0.4 & 2.329 & 5.823 & 4 & 1 & 0.59485 & 0.115587257 \\
1 & 1 & 100 & 0.39 & 12.32 & 2.45044 & 30.2094 & 12.51943 & 30.3 & 0.5 & 2.413 & 4.826 & 5 & 1 & 0.61615 & 0.0798758 \\
1 & 1 & 120 & 0.47 & 11.2 & 3.42826 & 42.26411 & 19.27227 & 30.3 & 0.61 & 2.193 & 3.595 & 6 & 1 & 0.56 & 0.051887997 \\
1 & 1 & 140 & 0.54 & 12.48 & 4.56866 & 56.3231 & 23.05488 & 30.3 & 0.7 & 2.443 & 3.49 & 7 & 1 & 0.6239 & 0.043374743 \\
1 & 1 & 160 & 0.62 & 14.29 & 5.87007 & 72.367 & 25.86382 & 30.3 & 0.8 & 2.798 & 3.498 & 8 & 1 & 0.71455 & 0.038664032 \\
1 & 1 & 180 & 0.7 & 15.18 & 7.33562 & 90.43455 & 30.41861 & 30.3 & 0.9 & 2.973 & 3.303 & 9 & 1 & 0.7592 & 0.032874604 \\
1 & 2 & 200 & 0.77 & 14.99 & 8.95825 & 0 & 0 & 30.3 & 0.99 & 2.934 & 2.964 & 10 & 1 & 0.74935 & 0 \\
1 & 2 & 220 & 1.55 & 18.9 & 12.18153 & 0 & 0 & 30.3 & 2 & 3.7 & 1.85 & 11 & 1 & 0.94495 & 0 \\
1 & 2 & 240 & 2.32 & 26.6 & 17.03136 & 0 & 0 & 30.3 & 3 & 5.209 & 1.736 & 12 & 1 & 1.33015 & 0 \\
1 & 2 & 260 & 3.09 & 30.79 & 23.50068 & 0 & 0 & 30.3 & 3.99 & 6.029 & 1.511 & 13 & 1 & 1.5397
\end{tabular}




\begin{tabular}{|c|c|c|c|c|c|c|c|c|c|c|c|c|c|c|}
\hline . & 280 & 3.87 & 35.01 & 31.59028 & 0 & 0 & 30.3 & 5 & 6.856 & 1.371 & 14 & 1 & 1.75065 & 0 \\
\hline 1 & 300 & 4.65 & 36.8 & 41.30645 & 0 & 0 & 30.3 & 6 & 7.206 & 1.201 & 15 & 1 & 1.8402 & 0 \\
\hline & 320 & 5.42 & 35.68 & 52.65623 & 0 & 0 & 30.3 & 7 & 6.987 & 0.998 & 16 & 1 & 1.7841 & 0 \\
\hline & 340 & 6.2 & 34.8 & 65.61609 & 0 & 0 & 30.3 & 8 & 6.813 & 0.852 & 17 & 1 & 1.7398 & 0 \\
\hline & 360 & 6.97 & 34.38 & 80.21429 & 0 & 0 & 30.3 & 9 & 6.732 & 0.748 & 18 & 1 & 1.719 & 0 \\
\hline & 380 & 7.75 & 34.24 & 96.5812 & 0 & 0 & 30.3 & 10.01 & 6.703 & 0.67 & 19 & 1 & 1.7118 & 0 \\
\hline & 400 & 15.5 & 35.33 & 128.91211 & 0 & 0 & 30.3 & 20.01 & 6.917 & 0.346 & 20 & 1 & 1.7663 & 0 \\
\hline & 420 & 23.24 & 35.86 & 177.44422 & 0 & 0 & 30.3 & 30 & 7.021 & 0.234 & 21 & 1 & 1.793 & 0 \\
\hline & 440 & 30.99 & 36.69 & 242.24271 & 0 & 0 & 30.3 & 40.01 & 7.183 & 0.18 & 22 & 1 & 1.83435 & 0 \\
\hline & 460 & 38.73 & 37.22 & 323.2691 & 0 & 0 & 30.3 & 50 & 7.287 & 0.146 & 23 & 1 & 1.8608 & 0 \\
\hline & 480 & 46.48 & 37.89 & 420.4362 & 0 & 0 & 30.3 & 60.01 & 7.42 & 0.124 & 24 & 1 & 1.8947 & 0 \\
\hline & 500 & 54.22 & 38.95 & 533.92938 & 0 & 0 & 30.3 & 70 & 7.626 & 0.109 & 25 & 1 & 1.9474 & 0 \\
\hline & 520 & 61.96 & 39.63 & 663.58684 & 0 & 0 & 30.3 & 79.99 & 7.76 & 0.097 & 26 & 1 & 1.98165 & 0 \\
\hline & 540 & 69.71 & 40.16 & 809.47062 & 0 & 0 & 30.3 & 90 & 7.862 & 0.087 & 27 & 1 & 2.00775 & 0 \\
\hline & 560 & 77.46 & 40.72 & 973.08555 & 0 & 0 & 30.3 & 100 & 7.972 & 0.08 & 28 & 1 & 2.03585 & 0 \\
\hline & 580 & 154.92 & 46.08 & 1296.33266 & 0 & 0 & 30.3 & 200 & 9.023 & 0.045 & 29 & 1 & 2.30415 & 0 \\
\hline & 600 & 232.38 & 51.43 & 1781.6883 & 0 & 0 & 30.3 & 300 & 10.071 & 0.034 & 30 & 1 & 2.5717 & 0 \\
\hline & 620 & 309.84 & 56.91 & 2429.88133 & 0 & 0 & 30.3 & 400 & 11.143 & 0.028 & 31 & 1 & 2.8456 & 0 \\
\hline & 640 & 387.3 & 62.86 & 3239.98027 & 0 & 0 & 30.3 & 500 & 12.307 & 0.025 & 32 & 1 & 3.1428 & 0 \\
\hline & 660 & 464.75 & 70.52 & 4211.61833 & 0 & 0 & 30.3 & 599.99 & 13.808 & 0.023 & 33 & 1 & 3.52615 & 0 \\
\hline & 680 & 542.21 & 86.53 & 5346.49747 & 0 & 0 & 30.3 & 699.99 & 16.942 & 0.024 & 34 & 1 & 4.32625 & 0 \\
\hline & 700 & 619.67 & 97.95 & 6642.99977 & 0 & 0 & 30.3 & 799.99 & 19.179 & 0.024 & 35 & 1 & 4.89765 & 0 \\
\hline & 720 & 697.13 & 112.16 & 8099.98719 & 0 & 0 & 30.3 & 899.99 & 21.962 & 0.024 & 36 & 1 & 5.6082 & 0 \\
\hline & 740 & 774.59 & 124.13 & 9722.52869 & 0 & 0 & 30.3 & 1000 & 24.304 & 0.024 & 37 & 1 & 6.20635 & 0 \\
\hline & 750 & 774.59 & 124.51 & 10551.19758 & 0 & 0 & 30.3 & 1000 & 24.378 & 0.024 & 38 & 1 & 6.22535 & 0 \\
\hline & 760 & 774.59 & 124.07 & 11362.34738 & 0 & 0 & 30.3 & 1000 & 24.293 & 0.024 & 39 & 1 & 6.20345 & 0 \\
\hline & 770 & 774.59 & 123.37 & 12174.31006 & 0 & 0 & 30.3 & 1000 & 24.156 & 0.024 & 40 & 1 & 6.16845 & 0 \\
\hline & 780 & 774.59 & 123.5 & 12982.61593 & 0 & 0 & 30.3 & 1000 & 24.181 & 0.024 & 41 & 1 & 6.17505 & 0 \\
\hline & 790 & 774.59 & 123.75 & 13794.9831 & 0 & 0 & 30.3 & 1000 & 24.231 & 0.024 & 42 & 1 & 6.1876 & 0 \\
\hline & 800 & 774.59 & 122.22 & 14607.35026 & 0 & 0 & 30.3 & 1000 & 23.93 & 0.024 & 43 & 1 & 6.1108 & 0 \\
\hline & 820 & 774.59 & 122.72 & 16248.30228 & 0 & 0 & 30.3 & 1000 & 24.029 & 0.024 & 44 & 1 & 6.1362 & 0 \\
\hline & 840 & 697.13 & 109.64 & 17710.03429 & 0 & 0 & 30.3 & 899.99 & 21.467 & 0.024 & 45 & 1 & 5.48175 & 0 \\
\hline & 860 & 619.67 & 95.82 & 19008.84331 & 0 & 0 & 30.3 & 799.99 & 18.762 & 0.023 & 46 & 1 & 4.79105 & 0 \\
\hline & 880 & 542.21 & 84.96 & 20146.88446 & 0 & 0 & 30.3 & 699.99 & 16.635 & 0.024 & 47 & 1 & 4.248 & 0 \\
\hline & 900 & 464.75 & 69.69 & 21120.58733 & 0 & 0 & 30.3 & 599.99 & 13.646 & 0.023 & 48 & 1 & 3.48465 & 0 \\
\hline & 920 & 387.3 & 61.52 & 21933.43673 & 0 & 0 & 30.3 & 500 & 12.046 & 0.024 & 49 & 1 & 3.07615 & 0 \\
\hline & 940 & 309.84 & 55.45 & 22583.532 & 0 & 0 & 30.3 & 400 & 10.856 & 0.027 & 50 & 1 & 2.7723 & 0 \\
\hline & 960 & 232.38 & 49.9 & 23071.31374 & 0 & 0 & 30.3 & 300 & 9.77 & 0.033 & 51 & 1 & 2.49485 & 0 \\
\hline & 980 & 154.92 & 44.54 & 23397.07019 & 0 & 0 & 30.3 & 200 & 8.72 & 0.044 & 52 & 1 & 2.22685 & 0 \\
\hline & 61000 & 77.46 & 39.93 & 23560.638 & 0 & 0 & 30.3 & 100 & 7.818 & 0.078 & 53 & 1 & 1.9965 & 0 \\
\hline & 71020 & 69.71 & 39.44 & 23707.78156 & 0 & 0 & 30.3 & 90 & 7.723 & 0.086 & 54 & 1 & 1.97205 & 0 \\
\hline & 71040 & 61.97 & 38.92 & 23837.77909 & 0 & 0 & 30.3 & 80 & 7.62 & 0.095 & 55 & 1 & 1.9459 & 0 \\
\hline & 71060 & 54.22 & 38.31 & 23951.48276 & 0 & 0 & 30.3 & 70 & 7.501 & 0.107 & 56 & 1 & 1.9155 & 0 \\
\hline & 71080 & 46.48 & 37.34 & 24048.91297 & 0 & 0 & 30.3 & 60.01 & 7.312 & 0.122 & 57 & 1 & 1.86715 & 0 \\
\hline & 71100 & 38.73 & 36.59 & 24130.08544 & 0 & 0 & 30.3 & 50 & 7.165 & 0.143 & 58 & 1 & 1.82965 & 0 \\
\hline & 71120 & 30.99 & 36.04 & 24195.16275 & 0 & 0 & 30.3 & 40.01 & 7.056 & 0.176 & 59 & 1 & 1.8018 & 0 \\
\hline & 71140 & 23.24 & 35.28 & 24243.93362 & 0 & 0 & 30.3 & 30 & 6.907 & 0.23 & 60 & 1 & 1.7638 & 0 \\
\hline & 71160 & 15.5 & 34.43 & 24276.47895 & 0 & 0 & 30.3 & 20.01 & 6.742 & 0.337 & 61 & 1 & 1.7217 & 0 \\
\hline & 71180 & 7.75 & 33.57 & 24292.82858 & 0 & 0 & 30.3 & 10.01 & 6.573 & 0.657 & 62 & 1 & 1.6786 & 0 \\
\hline & $8 \quad 1200$ & 6.97 & 33.36 & 24307.54458 & 0 & 0 & 30.3 & 9 & 6.531 & 0.726 & 63 & 1 & 1.66785 & 0 \\
\hline
\end{tabular}




$\begin{array}{rrrrrrrrrrrrrrrr}1 & 8 & 1220 & 6.2 & 32.94 & 24320.52722 & 0 & 0 & 30.3 & 8 & 6.449 & 0.806 & 64 & 1 & 1.6468 & 0 \\ 1 & 8 & 1240 & 5.42 & 32.35 & 24331.88407 & 0 & 0 & 30.3 & 7 & 6.335 & 0.905 & 65 & 1 & 1.6176 & 0 \\ 1 & 8 & 1260 & 4.64 & 31.92 & 24341.62615 & 0 & 0 & 30.3 & 5.99 & 6.249 & 1.043 & 66 & 1 & 1.59575 & 0 \\ 1 & 8 & 1280 & 3.87 & 31.25 & 24349.73932 & 0 & 0 & 30.3 & 5 & 6.119 & 1.224 & 67 & 1 & 1.5626 & 0 \\ 1 & 8 & 1300 & 3.09 & 30.36 & 24356.23534 & 0 & 0 & 30.3 & 3.99 & 5.944 & 1.49 & 68 & 1 & 1.51775 & 0 \\ 1 & 8 & 1320 & 2.32 & 28.26 & 24361.10638 & 0 & 0 & 30.3 & 3 & 5.533 & 1.844 & 69 & 1 & 1.4128 & 0 \\ 1 & 8 & 1340 & 1.54 & 24.28 & 24364.35479 & 0 & 0 & 30.3 & 1.99 & 4.753 & 2.388 & 70 & 1 & 1.21385 & 0 \\ 1 & 8 & 1360 & 0.77 & 18.96 & 24365.97899 & 0 & 0 & 30.3 & 0.99 & 3.713 & 3.751 & 71 & 1 & 0.9481 & 0 \\ 1 & 9 & 1380 & 0.7 & 17.4 & 24367.4524 & 18.16437 & 5.33148 & 30.3 & 0.9 & 3.407 & 3.786 & 72 & 1 & 0.87 & 0.187564997 \\ 1 & 9 & 1400 & 0.62 & 16.37 & 24368.7593 & 34.27605 & 10.69122 & 30.3 & 0.8 & 3.206 & 4.007 & 73 & 1 & 0.81865 & 0.093534698 \\ 1 & 9 & 1420 & 0.55 & 15.39 & 24369.90049 & 48.34472 & 16.04005 & 30.3 & 0.71 & 3.014 & 4.245 & 74 & 1 & 0.7696 & 0.062343933 \\ 1 & 9 & 1440 & 0.47 & 14.97 & 24370.88066 & 60.42848 & 20.61701 & 30.3 & 0.61 & 2.931 & 4.805 & 75 & 1 & 0.7485 & 0.048503619 \\ 1 & 9 & 1460 & 0.39 & 14.52 & 24371.69905 & 70.51765 & 24.80395 & 30.3 & 0.5 & 2.843 & 5.686 & 76 & 1 & 0.7259 & 0.040316148 \\ 1 & 9 & 1480 & 0.31 & 13.2 & 24372.35564 & 78.61222 & 30.42267 & 30.3 & 0.4 & 2.584 & 6.46 & 77 & 1 & 0.65975 & 0.032870208 \\ 1 & 9 & 1500 & 0.24 & 11.73 & 24372.84887 & 84.69283 & 36.87105 & 30.3 & 0.31 & 2.297 & 7.41 & 78 & 1 & 0.58645 & 0.02712154 \\ 1 & 9 & 1520 & 0.16 & 10.6 & 24373.18031 & 88.77884 & 42.78496 & 30.3 & 0.21 & 2.075 & 9.881 & 79 & 1 & 0.52985 & 0.023372687 \\ 1 & 9 & 1540 & 0.08 & 9.22 & 24373.34917 & 90.86058 & 50.33824 & 30.3 & 0.1 & 1.805 & 18.05 & 80 & 1 & 0.4609 & 0.019865601\end{array}$

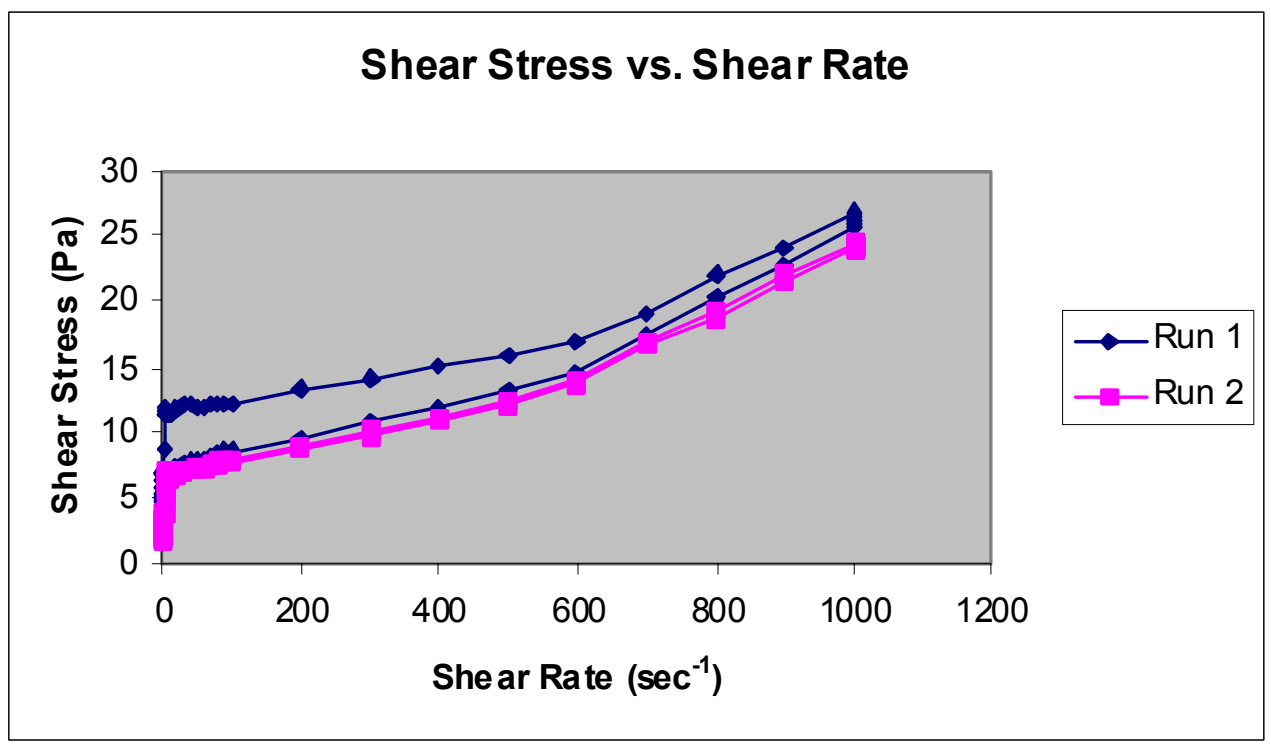




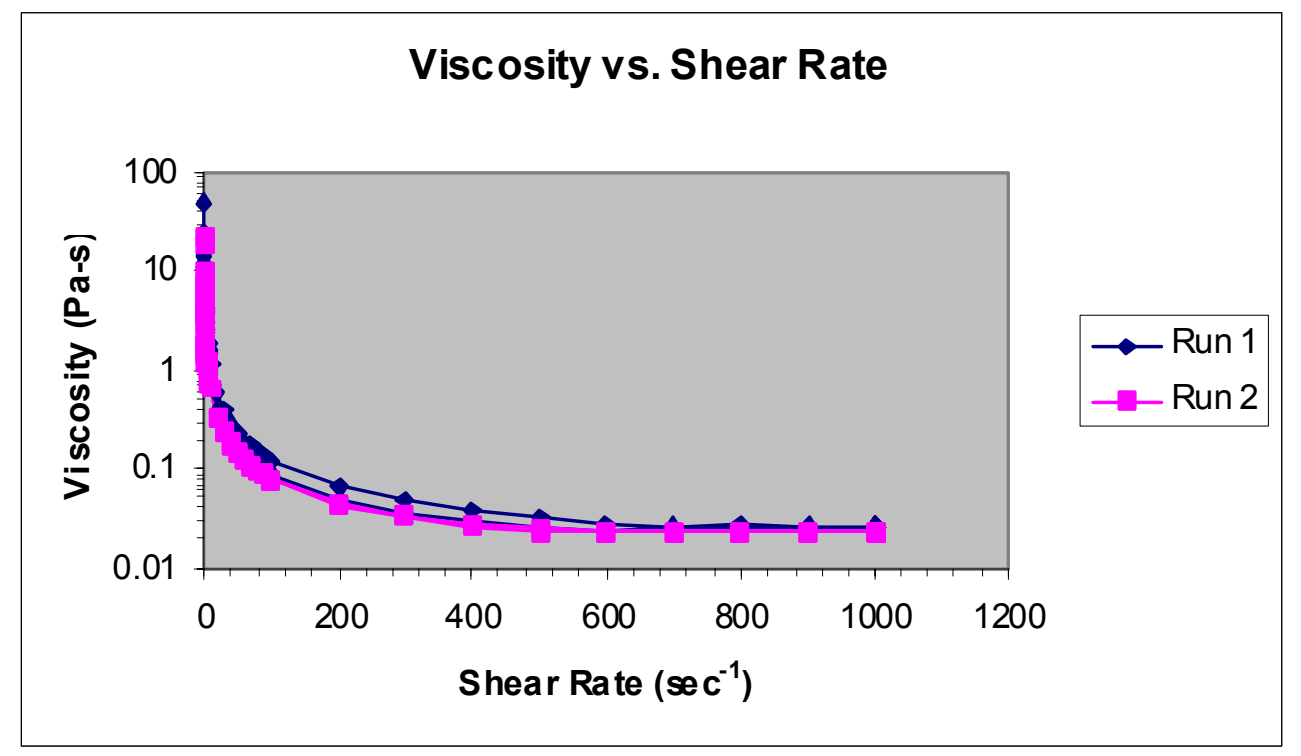

\section{E-2.2.5 Aged Centrifuged}

04.20.04 WM-187 centrifuged CC-45 run 01.txt

Mode Step t[s] n[rpm] M[\%.] Phi[rad]

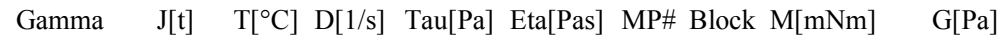

\begin{tabular}{|c|c|c|c|c|c|c|c|c|c|c|c|c|c|c|}
\hline 1 & 20 & 0.08 & 31.15 & 0.16336 & 2.01396 & 0.33021 & 28.7 & 0.1 & 6.099 & 60.99 & 1 & 1 & 1.5575 & 3.028362033 \\
\hline 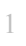 & 40 & 0.16 & 4.13 & 0.49244 & 6.07093 & 7.51352 & 28.8 & 0.21 & 0.808 & 3.848 & 2 & 1 & 0.2064 & 0.133093282 \\
\hline & 60 & 0.24 & 5.72 & 0.98332 & 12.12249 & 10.8236 & 28.8 & 0.31 & 1.12 & 3.613 & 3 & 1 & 0.28595 & 0.09239026 \\
\hline & 80 & 0.31 & 8.14 & 1.63677 & 20.17833 & 12.6669 & 28.8 & 0.4 & 1.593 & 3.983 & 4 & 1 & 0.40675 & 0.078946077 \\
\hline & 100 & 0.39 & 21.24 & 2.45201 & 30.22876 & 7.27002 & 28.8 & 0.5 & 4.158 & 8.316 & 5 & 1 & 1.06175 & 0.137551127 \\
\hline & 120 & 0.47 & 13.24 & 3.42905 & 42.27379 & 16.303 & 28.8 & 0.61 & 2.593 & 4.251 & 0 & 1 & 0.6621 & 0.061338243 \\
\hline & 140 & 0.54 & 24.06 & 4.56945 & 56.33278 & 11.9603 & 28.9 & 0.7 & 4.71 & 6.729 & 7 & 1 & 1.20275 & 0.083610289 \\
\hline & 160 & 0.62 & 22.27 & 5.85593 & 72.19272 & 16.5542 & 28.8 & 0.8 & 4.361 & 5.451 & 8 & 1 & 1.11355 & 0.060407753 \\
\hline & 180 & 0.71 & 29.36 & 7.33483 & 90.42487 & 15.7288 & 28.8 & 0.92 & 5.749 & 6.249 & 9 & 1 & 1.4681 & 0.063577642 \\
\hline & 200 & 0.77 & 20.84 & 8.95825 & 0 & 0 & 28.8 & 0.99 & 4.081 & 4.122 & 10 & 1 & 1.0422 & 0 \\
\hline & 220 & 1.55 & 33.83 & 12.17838 & 0 & 0 & 28.8 & 2 & 6.625 & 3.313 & 11 & 1 & 1.6917 & 0 \\
\hline & 240 & 2.33 & 59.26 & 17.01801 & 0 & 0 & 28.8 & 3.01 & 11.602 & 3.854 & 12 & 1 & 2.96275 & 0 \\
\hline & 260 & 3.1 & 19.45 & 23.50697 & 0 & 0 & 28.8 & 4 & 3.809 & 0.952 & 13 & 1 & 0.97265 & 0 \\
\hline & 280 & 3.87 & 8.08 & 31.59735 & 0 & 0 & 28.7 & 5 & 1.583 & 0.317 & 14 & 1 & 0.40415 & 0 \\
\hline & 300 & 4.65 & 18.85 & 41.31587 & 0 & 0 & 28.7 & 6 & 3.691 & 0.615 & 15 & 1 & 0.94255 & 0 \\
\hline & 320 & 5.42 & 16.91 & 52.65152 & 0 & 0 & 28.8 & 7 & 3.311 & 0.473 & 16 & 1 & 0.84555 & 0 \\
\hline & 340 & 6.2 & 7.41 & 65.62237 & 0 & 0 & 28.7 & 8 & 1.451 & 0.181 & 17 & 1 & 0.3706 & 0 \\
\hline & 360 & 6.97 & 7.34 & 80.22057 & 0 & 0 & 28.8 & 9 & 1.436 & 0.16 & 18 & 1 & 0.36675 & 0 \\
\hline & 380 & 7.75 & 7.61 & 96.5867 & 0 & 0 & 28.8 & 10.01 & 1.49 & 0.149 & 19 & 1 & 0.3804 & 0 \\
\hline & 400 & 15.5 & 9.75 & 128.90662 & 0 & 0 & 28.8 & 20.01 & 1.91 & 0.095 & 20 & 1 & 0.48765 & 0 \\
\hline & 420 & 23.24 & 9.13 & 177.51883 & 0 & 0 & 28.7 & 30 & 1.787 & 0.06 & 21 & 1 & 0.45625 & 0 \\
\hline & 440 & 30.99 & 12.58 & 242.25371 & 0 & 0 & 28.7 & 40.01 & 2.464 & 0.062 & 22 & 1 & 0.6291 & 0 \\
\hline & 460 & 38.72 & 10.66 & 323.23454 & 0 & 0 & 28.7 & 49.99 & 2.088 & 0.042 & 23 & 1 & 0.53315 & 0 \\
\hline & 480 & 46.48 & 10.35 & 420.52731 & 0 & 0 & 28.7 & 60.01 & 2.026 & 0.034 & 24 & 1 & 0.5174 & 0 \\
\hline & 500 & 54.22 & 9.61 & 533.93959 & 0 & 0 & 28.8 & 70 & 1.881 & 0.027 & 25 & 1 & 0.4804 & 0 \\
\hline & 520 & 61.96 & 9.81 & 663.56563 & 0 & 0 & 28.7 & 79.99 & 1.921 & 0.024 & 26 & 1 & 0.4905 & 0 \\
\hline & 540 & 69.71 & 10.01 & 809.44863 & 0 & 0 & 28.7 & 90 & 1.96 & 0.022 & 27 & 1 & 0.50055 & 0 \\
\hline
\end{tabular}




\begin{tabular}{|c|c|c|c|c|c|c|c|c|c|c|c|c|c|c|}
\hline & 560 & 77.46 & 10.35 & 973.21749 & 0 & 0 & 28.7 & 100 & 2.027 & 0.02 & 28 & 1 & 0.5176 & 0 \\
\hline & 580 & 154.92 & 13.72 & 1296.46539 & 0 & 0 & 28.7 & 200 & 2.686 & 0.013 & 29 & 1 & 0.6859 & 0 \\
\hline & 600 & 232.38 & 17.74 & 1781.94277 & 0 & 0 & 28.7 & 300 & 3.474 & 0.012 & 30 & 1 & 0.88715 & 0 \\
\hline & 620 & 309.84 & 25.56 & 2428.91922 & 0 & 0 & 28.7 & 400 & 5.004 & 0.013 & 31 & 1 & 1.27775 & \\
\hline & 640 & 387.3 & 33.05 & 3239.21843 & 0 & 0 & 28.7 & 500 & 6.47 & 0.013 & 32 & 1 & 1.6523 & \\
\hline & 660 & 464.75 & 43.11 & 4211.58691 & 0 & 0 & 28.7 & 599.99 & 8.441 & 0.014 & 33 & 1 & 2.1554 & \\
\hline & 680 & 542.21 & 55.08 & 5346.46919 & 0 & 0 & 28.7 & 699.99 & 10.785 & 0.015 & 34 & 1 & 2.75415 & \\
\hline & 700 & 619.67 & 65.44 & 6642.97149 & 0 & 0 & 28.7 & 799.99 & 12.813 & 0.016 & 35 & 1 & 3.2719 & \\
\hline & 720 & 697.13 & 75.42 & 8100.60844 & 0 & 0 & 28.7 & 899.99 & 14.767 & 0.016 & 36 & 1 & 3.77085 & \\
\hline & 740 & 774.59 & 83.61 & 9722.01661 & 0 & 0 & 28.7 & 1000 & 16.37 & 0.016 & 37 & 1 & 4.18035 & \\
\hline & 750 & 774.59 & 82.52 & 10550.68628 & 0 & 0 & 28.7 & 1000 & 16.157 & 0.016 & 38 & 1 & 4.1258 & \\
\hline & 760 & 774.59 & 82.35 & 11361.43003 & 0 & 0 & 28.7 & 1000 & 16.124 & 0.016 & 39 & 1 & 4.11735 & \\
\hline & 770 & 774.59 & 82.06 & 12172.17221 & 0 & 0 & 28.7 & 1000 & 16.067 & 0.016 & 40 & 1 & 4.1029 & \\
\hline & 780 & 774.59 & 81.95 & 12983.72492 & 0 & 0 & 28.7 & 1000 & 16.047 & 0.016 & 41 & 1 & 4.0977 & 0 \\
\hline & 790 & 774.59 & 81.73 & 13795.68367 & 0 & 0 & 28.7 & 1000 & 16.003 & 0.016 & 42 & 1 & 4.08645 & 0 \\
\hline & 800 & 774.59 & 81.52 & 14605.61689 & 0 & 0 & 28.7 & 1000 & 15.962 & 0.016 & 43 & 1 & 4.07615 & 0 \\
\hline & 820 & 774.59 & 81.42 & 16246.16521 & 0 & 0 & 28.7 & 1000 & 15.942 & 0.016 & 44 & 1 & 4.0711 & 0 \\
\hline & 840 & 697.13 & 70.43 & 17706.75997 & 0 & 0 & 28.7 & 899.99 & 13.79 & 0.015 & 45 & 1 & 3.52155 & 0 \\
\hline & 860 & 619.67 & 60.16 & 19007.47672 & 0 & 0 & 28.7 & 799.99 & 11.779 & 0.015 & 46 & 1 & 3.008 & 0 \\
\hline & 880 & 542.21 & 50.52 & 20143.81356 & 0 & 0 & 28.7 & 699.99 & 9.891 & 0.014 & 47 & 1 & 2.52575 & 0 \\
\hline & 900 & 464.75 & 40.93 & 21118.48875 & 0 & 0 & 28.8 & 599.99 & 8.013 & 0.013 & 48 & 1 & 2.0463 & 0 \\
\hline & 920 & 387.3 & 31.68 & 21930.56767 & 0 & 0 & 28.7 & 500 & 6.202 & 0.012 & 49 & 1 & 1.58375 & 0 \\
\hline & 940 & 309.84 & 23.53 & 22580.94804 & 0 & 0 & 28.7 & 400 & 4.606 & 0.012 & 50 & 1 & 1.1763 & 0 \\
\hline & 960 & 232.38 & 16.12 & 23069.15311 & 0 & 0 & 28.7 & 300 & 3.156 & 0.011 & 51 & 1 & 0.8059 & 0 \\
\hline & 980 & 154.92 & 12.18 & 23394.9292 & 0 & 0 & 28.6 & 200 & 2.384 & 0.012 & 52 & 1 & 0.60885 & 0 \\
\hline & 61000 & 77.46 & 16.56 & 23558.49151 & 0 & 0 & 28.7 & 100 & 3.243 & 0.032 & 53 & 1 & 0.82805 & 0 \\
\hline & 71020 & 69.71 & 9.12 & 23705.7034 & 0 & 0 & 28.7 & 90 & 1.786 & 0.02 & 54 & 1 & 0.456 & 0 \\
\hline & 71040 & 61.97 & 8.81 & 23835.59647 & 0 & 0 & 28.7 & 80 & 1.726 & 0.022 & 55 & 1 & 0.4407 & 0 \\
\hline & 71060 & 54.22 & 8.68 & 23949.2428 & 0 & 0 & 28.7 & 70 & 1.7 & 0.024 & 56 & 1 & 0.43405 & 0 \\
\hline & 71080 & 46.48 & 8.02 & 24046.67301 & 0 & 0 & 28.7 & 60.01 & 1.57 & 0.026 & 57 & 1 & 0.401 & 0 \\
\hline & 71100 & 38.73 & 7.68 & 24127.95073 & 0 & 0 & 28.7 & 50 & 1.504 & 0.03 & 58 & 1 & 0.38415 & 0 \\
\hline & 71120 & 30.99 & 7.57 & 24192.96285 & 0 & 0 & 28.7 & 40.01 & 1.481 & 0.037 & 59 & 1 & 0.37825 & 0 \\
\hline & 71140 & 23.24 & 6.97 & 24241.72115 & 0 & 0 & 28.7 & 30 & 1.365 & 0.046 & 60 & 1 & 0.34855 & 0 \\
\hline & 71160 & 15.5 & 6.67 & 24274.30418 & 0 & 0 & 28.7 & 20.01 & 1.306 & 0.065 & 61 & 1 & 0.33355 & 0 \\
\hline & 71180 & 7.75 & 6.4 & 24290.63653 & 0 & 0 & 28.7 & 10.01 & 1.254 & 0.125 & 62 & 1 & 0.32015 & 0 \\
\hline & 81200 & 6.97 & 6.56 & 24305.33919 & 0 & 0 & 28.7 & 9 & 1.285 & 0.143 & 63 & 1 & 0.32805 & 0 \\
\hline & 81220 & 6.2 & 6.59 & 24318.32574 & 0 & 0 & 28.7 & 8 & 1.291 & 0.161 & 64 & 1 & 0.3296 & 0 \\
\hline & 81240 & 5.42 & 6.47 & 24329.69046 & 0 & 0 & 28.7 & 7 & 1.267 & 0.181 & 65 & 1 & 0.32345 & 0 \\
\hline & 81260 & 4.64 & 6.57 & 24339.42547 & 0 & 0 & 28.7 & 5.99 & 1.285 & 0.215 & 66 & 1 & 0.32825 & 0 \\
\hline & 81280 & 3.87 & 6.59 & 24347.54256 & 0 & 0 & 28.7 & 5 & 1.291 & 0.258 & 67 & 1 & 0.3297 & 0 \\
\hline & 81300 & 3.09 & 6.59 & 24354.03544 & 0 & 0 & 28.7 & 3.99 & 1.291 & 0.324 & 68 & 1 & 0.3296 & 0 \\
\hline & 81320 & 2.32 & 6.59 & 24358.90805 & 0 & 0 & 28.7 & 3 & 1.29 & 0.43 & 69 & 1 & 0.3295 & 0 \\
\hline & 81340 & 1.54 & 6.21 & 24362.15253 & 0 & 0 & 28.8 & 1.99 & 1.216 & 0.611 & 70 & 1 & 0.31055 & 0 \\
\hline & 81360 & 0.77 & 5.9 & 24363.77752 & 0 & 0 & 28.8 & 0.99 & 1.156 & 1.168 & 71 & 1 & 0.2952 & 0 \\
\hline & 91380 & 0.7 & 5.93 & 24365.2525 & 18.18373 & 3 15.6756 & 28.7 & 0.9 & 1.16 & 1.289 & 72 & 1 & 0.2963 & 0.063793292 \\
\hline & 91400 & 0.62 & 5.92 & 24366.55783 & 34.27605 & 29.5738 & 28.8 & 0.8 & 1.159 & 1.449 & 73 & 1 & 0.296 & 0.033813698 \\
\hline & 91420 & 0.55 & 5.8 & 24367.69901 & 48.34472 & 242.5944 & 28.7 & 0.71 & 1.135 & 1.599 & 74 & 1 & 0.28995 & 0.023477228 \\
\hline & 91440 & 0.47 & 5.7 & 24368.67919 & 60.42848 & 8 54.0989 & 28.6 & 0.61 & 1.117 & 1.831 & 75 & 1 & 0.28515 & 0.018484662 \\
\hline & 91460 & 0.39 & 5.31 & 24369.49836 & 70.52733 & 3 67.8799 & 28.6 & 0.5 & 1.039 & 2.078 & 76 & 1 & 0.2653 & 0.014731878 \\
\hline & 91480 & 0.31 & 4.74 & 24370.1526 & 78.59285 & 584.6905 & 28.7 & 0.4 & 0.928 & 2.32 & 77 & 1 & 0.23695 & 0.01180769 \\
\hline
\end{tabular}




$\begin{array}{rrrrrrrrrrrrrrrr}9 & 1500 & 0.24 & 3.97 & 24370.64661 & 84.68314 & 109.128 & 28.6 & 0.31 & 0.776 & 2.503 & 78 & 1 & 0.19825 & 0.009163571 \\ 9 & 1520 & 0.16 & 3.48 & 24370.97805 & 88.76916 & 130.351 & 28.7 & 0.21 & 0.681 & 3.243 & 79 & 1 & 0.17385 & 0.007671583 \\ 9 & 1540 & 0.08 & 2.56 & 24371.14691 & 90.8509 & 181.339 & 28.7 & 0.1 & 0.501 & 5.01 & 80 & 1 & 0.12795 & 0.00551453\end{array}$

04.21.04 WM-187 centrifuged CC-45 run 01.txt

Mode Step t[s] n[rpm] M[\%.] Phi[rad]

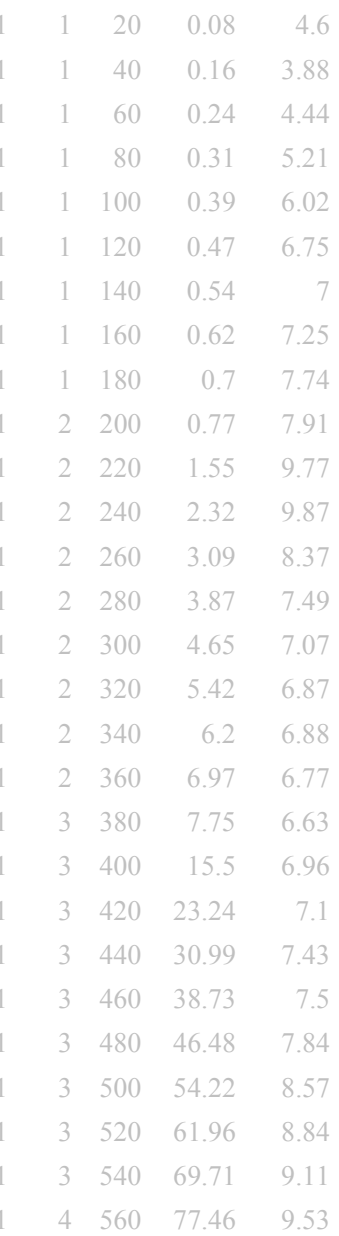

$\begin{array}{lllll}1 & 4 & 580 & 154.92 & 12.88\end{array}$

$\begin{array}{lllll}1 & 4 & 600 & 232.38 & 17.14\end{array}$

$\begin{array}{lllll}1 & 4 & 620 & 309.84 & 24.84\end{array}$

$\begin{array}{lllll}1 & 4 & 640 & 387.3 & 32.81\end{array}$

$\begin{array}{lllll}1 & 4 & 660 & 464.75 & 43.93\end{array}$

$\begin{array}{lllll}1 & 4 & 680 & 542.21 & 55.62\end{array}$

$\begin{array}{lllll}1 & 4 & 700 & 619.67 & 67.09\end{array}$

$\begin{array}{lllll}1 & 4 & 720 & 697.13 & 78.05\end{array}$

$\begin{array}{lllll}1 & 4 & 740 & 774.59 & 89.35\end{array}$

$\begin{array}{lllll}1 & 5 & 750 & 774.59 & 89.25\end{array}$

$\begin{array}{lllll}1 & 5 & 760 & 774.59 & 88.83\end{array}$

$\begin{array}{lllll}1 & 5 & 770 & 774.59 & 88.6\end{array}$

$\begin{array}{lllll}1 & 5 & 780 & 774.59 & 88.43\end{array}$

$\begin{array}{lllll}1 & 5 & 790 & 774.59 & 88.35\end{array}$

$\begin{array}{llllll}1 & 5 & 800 & 774.59 & 88.29 & 14605.52186\end{array}$

$\begin{array}{llllll}1 & 6 & 820 & 774.59 & 88.2 & 16246.47545\end{array}$ 8.96061 12.18624 17.03293 23.5054

31.60364

41.32058

52.65545

65.62473

80.208

96.57491

128.94274

177.44579

242.2647

323.29109

420.4904

533.96001

663.55699

809.51303

973.12403

1296.41277

1782.13362

2430.08239

3239.40928

4212.02124

5345.76469

6642.26935

8102.13604

9721.51552

10549.37231

11360.11606

12171.26586

12982.41487

3793.96993

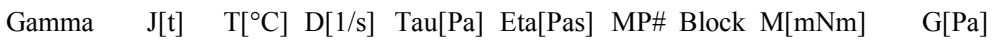

$\begin{array}{llllll}0.16415 & 2.02364 & 2.24849 & 28.6 & 0.1 & 0.9\end{array}$ $\begin{array}{lllll}0.49323 & 6.08061 & 8.01133 & 28.6\end{array}$ $\begin{array}{llll}0.98332 & 12.12249 & 13.9339 & 28.6\end{array}$ $\begin{array}{lllll}1.63677 & 20.17833 & 19.7827 & 28.6\end{array}$ $\begin{array}{lllll}2.45201 & 30.22876 & 25.6393 & 28.6\end{array}$ $\begin{array}{llll}3.43062 & 42.29316 & 32.016 & 28.6\end{array}$ $\begin{array}{lllll}4.57023 & 56.34247 & 41.1259 & 28.6\end{array}$ $\begin{array}{lllll}5.87321 & 72.40573 & 50.9899 & 28.6\end{array}$ $\begin{array}{llll}7.33876 & 90.47328 & 59.6789 & 28.6\end{array}$
$0.21 \quad 0.759$

$0.31 \quad 0.87$

$0.4 \quad 1.02$

$0.5 \quad 1.179$

$0.61 \quad 1.321$

$0.7 \quad 1.37$

$0.8 \quad 1.42$

$0.9 \quad 1.516$

$0 \quad 28.6$

$0 \quad 28.6$

$0 \quad 28.6$

$0 \quad 28.6$

$0 \quad 28.6$

$0 \quad 28.6$

$0 \quad 28.6$

$0 \quad 28.6$

$0 \quad 28.6$

$0 \quad 28.6$

$0 \quad 28.6$

$0 \quad 28.6$

$0 \quad 28.6$

$0 \quad 28.6$

$0 \quad 28.6$

$0 \quad 28.6$

$0 \quad 28.6$

$0 \quad 28.6$

(0) 28.6

$\begin{array}{ll}0 & 28.6\end{array}$

$\begin{array}{ll}0 & 28.6\end{array}$

$\begin{array}{ll}0 & 28.6\end{array}$

$0 \quad 28.6$

$\begin{array}{llll}0 & 28.6 & 599.99 & 8.601\end{array}$

$\begin{array}{lllll}0 & 28.6 & 699.99 & 10.891\end{array}$

$\begin{array}{llll}0 & 28.6 & 799.99 & 13.136\end{array}$

$\begin{array}{llll}0 & 28.6 & 899.99 & 15.282\end{array}$

$\begin{array}{llll}0 & 28.6 & 1000 & 17.495\end{array}$

$\begin{array}{llll}0 & 28.6 & 1000 & 17.475\end{array}$

$\begin{array}{llll}0 & 28.6 & 1000 & 17.393\end{array}$

$\begin{array}{llll}0 & 28.6 & 1000 & 17.349\end{array}$

$0 \quad 28.6$

$\begin{array}{ll}0 & 28.6\end{array}$

$\begin{array}{ll}0 & 28.6\end{array}$

$1000 \quad 17.315$

$1000 \quad 17.298$

$1000 \quad 17.288$

$\begin{array}{llll}0 & 28.6 & 1000 & 17.27\end{array}$

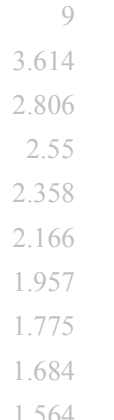

0.957

0.644

0.411

0.293

0.231

0.192

0.168

0.147

0.13

0.068

0.046

0.036

0.029

$0.026 \quad 24$

$0.024 \quad 25$

$0.022 \quad 26$

$0.02 \quad 27$

$0.019 \quad 2$

$0.013 \quad 29$

$0.011 \quad 30$

$0.012 \quad 31$

$0.013 \quad 32$

$0.014 \quad 33$

$0.016 \quad 34$

$0.016 \quad 35$

$0.017 \quad 36$

$0.017 \quad 37$

$0.017 \quad 38$

$0.017 \quad 39$

$0.017 \quad 40$

$0.017 \quad 4$

$0.017 \quad 42$

$0.017 \quad 43$

$0.017 \quad 4$
$0.2298 \quad 0.444743136$ 0.19380 .124823003 $\begin{array}{ll}0.2222 & 0.071767434\end{array}$ $\begin{array}{lll}0.26055 & 0.050549277\end{array}$ $\begin{array}{lll}0.3012 & 0.039002592\end{array}$ $\begin{array}{ll}0.3373 & 0.031234365\end{array}$ $\begin{array}{lll}0.3498 & 0.024315583\end{array}$ $\begin{array}{lll}0.36265 & 0.019611708\end{array}$ $\begin{array}{lll}0.38715 & 0.016756328\end{array}$ 0.3954

0.4887

0.4937

0.41835

0.3744

0.35325

0.34345

0.3441

0.33865

0.3315

0.348

0.3548

0.37165

0.37495

0.392

0.4286

0.442

0.45545

0.47645

0.64385

0.85695

1.24215

1.64055

2.19625

2.7811

3.3545

3.9024

4.4676

14.46245

14.44145

4.4302

14.4217

14.41725

14.41465

14.41015 


\begin{tabular}{|c|c|c|c|c|c|c|c|c|c|c|c|c|c|c|}
\hline & 840 & 697.13 & 76.52 & 17706.74583 & 0 & 0 & 28.6 & 899.99 & 14.983 & 0.017 & 45 & 1 & 3.8262 & 0 \\
\hline & 860 & 619.67 & 65.72 & 19006.20437 & 0 & 0 & 28.6 & 799.99 & 12.868 & 0.016 & 46 & 1 & 3.2859 & \\
\hline & 880 & 542.21 & 54.92 & 20143.10905 & 0 & 0 & 28.6 & 699.99 & 10.752 & 0.015 & 47 & 1 & 2.74575 & \\
\hline & 900 & 464.75 & 44.02 & 21117.78582 & 0 & 0 & 28.6 & 599.99 & 8.619 & 0.014 & 48 & 1 & 2.20095 & \\
\hline & 920 & 387.3 & 34.51 & 21930.59595 & 0 & 0 & 28.6 & 500 & 6.757 & 0.014 & 49 & 1 & 1.72555 & \\
\hline & 940 & 309.84 & 25.76 & 22580.48779 & 0 & 0 & 28.6 & 400 & 5.044 & 0.013 & 50 & 1 & 1.2881 & \\
\hline & 960 & 232.38 & 18.03 & 23068.84837 & 0 & 0 & 28.6 & 300 & 3.531 & 0.012 & 51 & 1 & 0.90165 & \\
\hline & 980 & 154.92 & 14.17 & 23394.46346 & 0 & 0 & 28.6 & 200 & 2.774 & 0.014 & 52 & 1 & 0.7084 & \\
\hline & 61000 & 77.46 & 11.13 & 23557.93859 & 0 & 0 & 28.6 & 100 & 2.178 & 0.022 & 53 & 1 & 0.5563 & \\
\hline & 71020 & 69.71 & 10.91 & 23705.04837 & 0 & 0 & 28.6 & 90 & 2.136 & 0.024 & 54 & 1 & 0.5454 & \\
\hline & 71040 & 61.97 & 10.63 & 23835.01292 & 0 & 0 & 28.6 & 80 & 2.082 & 0.026 & 55 & 1 & 0.53155 & \\
\hline & 71060 & 54.22 & 10.49 & 23948.71737 & 0 & 0 & 28.6 & 70 & 2.054 & 0.029 & 56 & 1 & 0.5245 & \\
\hline & 71080 & 46.48 & 9.78 & 24046.13109 & 0 & 0 & 28.6 & 60.01 & 1.915 & 0.032 & 57 & 1 & 0.489 & \\
\hline & 71100 & 38.73 & 9.33 & 24127.42058 & 0 & 0 & 28.6 & 50 & 1.827 & 0.037 & 58 & 1 & 0.4666 & \\
\hline & 71120 & 30.99 & 9.29 & 24192.39579 & 0 & 0 & 28.6 & 40.01 & 1.819 & 0.045 & 59 & 1 & 0.46455 & \\
\hline & 71140 & 23.24 & 8.75 & 24241.17922 & 0 & 0 & 28.6 & 30 & 1.712 & 0.057 & 60 & 1 & 0.4373 & \\
\hline & 71160 & 15.5 & 8.29 & 24273.73712 & 0 & 0 & 28.6 & 20.01 & 1.623 & 0.081 & 61 & 1 & 0.4144 & \\
\hline & 71180 & 7.75 & 8.04 & 24290.09461 & 0 & 0 & 28.6 & 10.01 & 1.575 & 0.157 & 62 & 1 & 0.40215 & \\
\hline & 81200 & 6.97 & 8.21 & 24304.8059 & 0 & 0 & 28.6 & 9 & 1.608 & 0.179 & 63 & 1 & 0.41065 & \\
\hline & 81220 & 6.2 & 8.19 & 24317.77518 & 0 & 0 & 28.6 & 8 & 1.604 & 0.201 & 64 & 1 & 0.4096 & \\
\hline & 81240 & 5.42 & 8.13 & 24329.14853 & 0 & 0 & 28.6 & 7 & 1.592 & 0.227 & 65 & 1 & 0.4066 & \\
\hline & 81260 & 4.64 & 8.2 & 24338.88668 & 0 & 0 & 28.6 & 5.99 & 1.605 & 0.268 & 66 & 1 & 0.4098 & \\
\hline & 81280 & 3.87 & 8.11 & 24346.99828 & 0 & 0 & 28.6 & 5 & 1.588 & 0.318 & 67 & 1 & 0.40555 & \\
\hline & 81300 & 3.09 & 8.15 & 24353.49273 & 0 & 0 & 28.6 & 3.99 & 1.596 & 0.4 & 68 & 1 & 0.40765 & 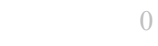 \\
\hline & 81320 & 2.32 & 8.17 & 24358.36142 & 0 & 0 & 28.6 & 3 & 1.599 & 0.533 & 69 & 1 & 0.40835 & 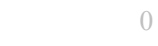 \\
\hline & 81340 & 1.54 & 7.62 & 24361.60904 & 0 & 0 & 28.6 & 1.99 & 1.492 & 0.75 & 70 & 1 & 0.38105 & 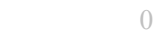 \\
\hline & 81360 & 0.77 & 7.26 & 24363.23324 & 0 & 0 & 28.6 & 0.99 & 1.421 & 1.435 & 71 & 1 & 0.36275 & \\
\hline & 91380 & 0.7 & 7.17 & 24364.70822 & 18.18373 & 12.9514 & 28.6 & 0.9 & 1.404 & 1.56 & 72 & 1 & 0.35865 & 0.077211881 \\
\hline & 91400 & 0.62 & 6.86 & 24366.01277 & 34.26637 & 25.5148 & 28.5 & 0.8 & 1.343 & 1.679 & 73 & 1 & 0.343 & 0.039192946 \\
\hline & 91420 & 0.55 & 6.65 & 24367.15552 & 48.3544 & 37.11 & 28.5 & 0.71 & 1.303 & 1.835 & 74 & 1 & 0.33265 & 0.026946876 \\
\hline & 91440 & 0.47 & 6 & 24368.13648 & 60.44785 & 51.4888 & 28.6 & 0.61 & 1.174 & 1.925 & 75 & 1 & 0.29975 & 0.0194217 \\
\hline & 91460 & 0.39 & 5.46 & 24368.9533 & 70.51765 & 66.0277 & 28.6 & 0.5 & 1.068 & 2.136 & 76 & 1 & 0.2728 & 0.015145145 \\
\hline & 91480 & 0.31 & 5.01 & 24369.6091 & 78.60253 & 80.1248 & 28.6 & 0.4 & 0.981 & 2.452 & 77 & 1 & 0.2504 & 0.012480514 \\
\hline & 91500 & 0.24 & 4.53 & 24370.10312 & 84.69283 & 95.59 & 28.6 & 0.31 & 0.886 & 2.858 & 78 & 1 & 0.22635 & 0.010461334 \\
\hline & 91520 & 0.16 & 3.71 & 24370.43377 & 88.76916 & 122.44 & 28.6 & 0.21 & 0.725 & 3.452 & 79 & 1 & 0.18525 & 0.008167251 \\
\hline & 91540 & 0.08 & 2.57 & 24370.60263 & 90.8509 & 180.259 & 28.6 & 0.1 & 0.504 & 5.04 & 80 & 1 & 0.1287 & 0.005547551 \\
\hline
\end{tabular}

04.27.04 WM-187 centrifuged CC-45 run 01.txt

Mode Step t[s] n[rpm] M[\%.]

$\begin{array}{rrrrr}1 & 1 & 20 & 0 & 243.19 \\ 1 & 1 & 40 & 0.09 & 677.05 \\ 1 & 1 & 60 & 0.21 & 972.2 \\ 1 & 1 & 80 & 0.33 & 1019.9 \\ 1 & 1 & 100 & 0.39 & 923.08 \\ 1 & 1 & 120 & 0.47 & 890.98 \\ 1 & 1 & 140 & 0.54 & 871.65 \\ 1 & 1 & 160 & 0.62 & 860.92 \\ 1 & 1 & 180 & 0.71 & 871.68 \\ 1 & 2 & 200 & 0.77 & 846.05 \\ 1 & 2 & 220 & 1.55 & 866.94\end{array}$

Phi[rad $\quad$ Gamma $\quad J[\mathrm{t}] \quad \mathrm{T}\left[{ }^{\circ} \mathrm{C}\right] \quad \mathrm{D}[1 / \mathrm{s}] \quad \mathrm{Tau}[\mathrm{Pa}] \quad$ Eta[Pas $] \quad$ MP\# Block M[mNm] G[Pa]

$\begin{array}{rrrrrrrrrrr}0.00942 & 0.11619 & 0.00244 & 31.1 & 0 & 47.617 & 0 & 1 & 1 & 12.1595 & 409.8201222 \\ 0.19242 & 2.37221 & 0.01789 & 31.1 & 0.12 & 132.57 & 1104.73 & 2 & 1 & 33.85255 & 55.88333242 \\ 0.63303 & 7.80409 & 0.041 & 31.1 & 0.27 & 190.36 & 705.026 & 3 & 1 & 48.61005 & 24.39195345 \\ 1.32261 & 16.30533 & 0.08165 & 31.1 & 0.43 & 199.69 & 464.405 & 4 & 1 & 50.99435 & 12.2471609 \\ 2.14492 & 26.44291 & 0.14631 & 31.1 & 0.5 & 180.74 & 361.476 & 5 & 1 & 46.15375 & 6.835026856 \\ 3.13295 & 38.62349 & 0.2214 & 31.2 & 0.61 & 174.45 & 285.99 & 6 & 1 & 44.549 & 4.516784993 \\ 4.27728 & 52.7309 & 0.30897 & 31.2 & 0.7 & 170.67 & 243.811 & 7 & 1 & 43.5823 & 3.236584242 \\ 5.57554 & 68.73607 & 0.40776 & 31.2 & 0.8 & 168.57 & 210.71 & 8 & 1 & 43.04595 & 2.452395082 \\ 7.05523 & 86.9779 & 0.50961 & 31.2 & 0.92 & 170.68 & 185.516 & 9 & 1 & 43.584 & 1.962280073 \\ 8.67865 & 0 & 0 & 31.2 & 0.99 & 165.66 & 167.33 & 10 & 1 & 42.30265 & 0 \\ 11.89328 & 0 & 0 & 31.2 & 2 & 169.75 & 84.874 & 11 & 1 & 43.347 & 0\end{array}$




\begin{tabular}{|c|c|c|c|c|c|c|c|c|c|c|c|c|c|c|}
\hline & 240 & 2.33 & 886.09 & 16.7439 & 0 & 0 & 31.2 & 3.01 & 173.5 & 57.64 & 12 & 1 & 44.30425 & 0 \\
\hline 1 & 260 & 3.09 & 896.95 & 23.21244 & 0 & 0 & 31.2 & 3.99 & 175.62 & 44.016 & 13 & 1 & 44.8475 & 0 \\
\hline & 280 & 3.87 & 873.66 & 31.31147 & 0 & 0 & 31.2 & 5 & 171.06 & 34.213 & 14 & 1 & 43.68305 & 0 \\
\hline & 300 & 4.65 & 863.68 & 41.0347 & 0 & 0 & 31.2 & 6 & 169.11 & 28.185 & 15 & 1 & 43.1841 & 0 \\
\hline & 320 & 5.42 & 858.55 & 52.37428 & 0 & 0 & 31.2 & 7 & 168.1 & 24.015 & 16 & 1 & 42.92755 & 0 \\
\hline & 340 & 6.2 & 857.82 & 65.33492 & 0 & 0 & 31.2 & 8 & 167.96 & 20.995 & 17 & 1 & 42.89105 & 0 \\
\hline & 360 & 6.97 & 858.5 & 79.92604 & 0 & 0 & 31.2 & 9 & 168.09 & 18.677 & 18 & 1 & 42.925 & 0 \\
\hline & 380 & 7.75 & 870.38 & 96.3016 & 0 & 0 & 31.2 & 10.01 & 170.42 & 17.025 & 19 & 1 & 43.5188 & 0 \\
\hline & 400 & 15.52 & 906.22 & 128.65686 & 0 & 0 & 31.2 & 20.04 & 177.44 & 8.854 & 20 & 1 & 45.31095 & 0 \\
\hline & 420 & 23.25 & 776.16 & 177.2141 & 0 & 0 & 31.2 & 30.02 & 151.97 & 5.062 & 21 & 1 & 38.80815 & 0 \\
\hline & 440 & 31.01 & 597.34 & 242.01809 & 0 & 0 & 31.2 & 40.03 & 116.96 & 2.922 & 22 & 1 & 29.86695 & 0 \\
\hline & 460 & 38.75 & 466.71 & 323.04447 & 0 & 0 & 31.2 & 50.03 & 91.382 & 1.827 & 23 & 1 & 23.3355 & 0 \\
\hline & 480 & 46.48 & 411.5 & 420.24535 & 0 & 0 & 31.2 & 60.01 & 80.572 & 1.343 & 24 & 1 & 20.5751 & 0 \\
\hline & 500 & 54.22 & 386.5 & 533.73382 & 0 & 0 & 31.2 & 70 & 75.677 & 1.081 & 25 & 1 & 19.32515 & 0 \\
\hline & 520 & 61.96 & 383.21 & 663.32765 & 0 & 0 & 31.2 & 79.99 & 75.032 & 0.938 & 26 & 1 & 19.16035 & 0 \\
\hline & 540 & 69.72 & 378.77 & 809.17609 & 0 & 0 & 31.3 & 90.01 & 74.163 & 0.824 & 27 & 1 & 18.93835 & 0 \\
\hline & 560 & 77.46 & 362.9 & 972.7494 & 0 & 0 & 31.3 & 100 & 71.056 & 0.711 & 28 & 1 & 18.14515 & 0 \\
\hline & 580 & 154.93 & 370.36 & 1296.35543 & 0 & 0 & 31.3 & 200.01 & 72.517 & 0.363 & 29 & 1 & 18.51805 & 0 \\
\hline & 600 & 232.4 & 333.43 & 1781.4409 & 0 & 0 & 31.3 & 300.03 & 65.285 & 0.218 & 30 & 1 & 16.67125 & 0 \\
\hline & 620 & 309.84 & 322.88 & 2429.47292 & 0 & 0 & 31.3 & 400 & 63.219 & 0.158 & 31 & 1 & 16.14385 & 0 \\
\hline & 640 & 387.3 & 304.44 & 3239.00794 & 0 & 0 & 31.3 & 500 & 59.608 & 0.119 & 32 & 1 & 15.22175 & 0 \\
\hline & 660 & 464.76 & 297.2 & 4211.58927 & 0 & 0 & 31.3 & 600.01 & 58.192 & 0.097 & 33 & 1 & 14.86 & 0 \\
\hline & 680 & 542.21 & 264.07 & 5345.02092 & 0 & 0 & 31.3 & 699.99 & 51.704 & 0.074 & 34 & 1 & 13.20325 & 0 \\
\hline & 700 & 619.68 & 175.87 & 6641.86251 & 0 & 0 & 31.3 & 800.01 & 34.436 & 0.043 & 35 & 1 & 8.79355 & 0 \\
\hline & 720 & 697.13 & 164.55 & 8102.05357 & 0 & 0 & 31.3 & 899.99 & 32.218 & 0.036 & 36 & 1 & 8.22725 & 0 \\
\hline & 740 & 774.6 & 127.13 & 9723.0227 & 0 & 0 & 31.3 & 1000 & 24.892 & 0.025 & 37 & 1 & 6.3564 & 0 \\
\hline & 750 & 774.6 & 97.23 & 10551.29183 & 0 & 0 & 31.3 & 1000 & 19.037 & 0.019 & 38 & 1 & 4.86125 & 0 \\
\hline & 760 & 774.59 & 80.93 & 11361.62952 & 0 & 0 & 31.3 & 1000 & 15.847 & 0.016 & 39 & 1 & 4.0467 & 0 \\
\hline & 770 & 774.59 & 79.96 & 12172.77853 & 0 & 0 & 31.3 & 1000 & 15.656 & 0.016 & 40 & 1 & 3.99805 & 0 \\
\hline & 780 & 774.59 & 78.65 & 12983.52071 & 0 & 0 & 31.3 & 1000 & 15.399 & 0.015 & 41 & 1 & 3.93225 & 0 \\
\hline & 790 & 774.58 & 92.1 & 13794.6658 & 0 & 0 & 31.3 & 999.98 & 18.033 & 0.018 & 42 & 1 & 4.60505 & 0 \\
\hline & 800 & 774.59 & 87.58 & 14606.63084 & 0 & 0 & 31.3 & 1000 & 17.148 & 0.017 & 43 & 1 & 4.379 & 0 \\
\hline & 820 & 774.59 & 84.92 & 16245.96337 & 0 & 0 & 31.3 & 1000 & 16.628 & 0.017 & 44 & 1 & 4.24615 & 0 \\
\hline & 840 & 697.13 & 75.82 & 17709.10988 & 0 & 0 & 31.3 & 899.99 & 14.845 & 0.016 & 45 & 1 & 3.79095 & 0 \\
\hline & 860 & 619.67 & 66.67 & 19007.59374 & 0 & 0 & 31.3 & 799.99 & 13.054 & 0.016 & 46 & 1 & 3.3334 & 0 \\
\hline & 880 & 542.21 & 70.09 & 20143.64784 & 0 & 0 & 31.3 & 699.99 & 13.724 & 0.02 & 47 & 1 & 3.5045 & 0 \\
\hline & 900 & 464.75 & 52.56 & 21119.57653 & 0 & 0 & 31.3 & 599.99 & 10.291 & 0.017 & 48 & 1 & 2.628 & 0 \\
\hline & 920 & 387.27 & 128.56 & 21931.78583 & 0 & 0 & 31.3 & 499.97 & 25.172 & 0.05 & 49 & 1 & 6.4279 & 0 \\
\hline & 940 & 309.84 & 222.1 & 22581.52059 & 0 & 0 & 31.3 & 400 & 43.486 & 0.109 & 50 & 1 & 11.10475 & 0 \\
\hline & 960 & 232.38 & 180.32 & 23069.49947 & 0 & 0 & 31.3 & 300 & 35.306 & 0.118 & 51 & 1 & 9.01595 & 0 \\
\hline & 6980 & 154.92 & 239.94 & 23395.21194 & 0 & 0 & 31.3 & 200 & 46.979 & 0.235 & 52 & 1 & 11.9968 & 0 \\
\hline & 61000 & 77.46 & 223.24 & 23558.70121 & 0 & 0 & 31.3 & 100 & 43.71 & 0.437 & 53 & 1 & 11.1619 & 0 \\
\hline & 71020 & 69.71 & 221.66 & 23705.93273 & 0 & 0 & 31.3 & 90 & 43.401 & 0.482 & 54 & 1 & 11.08295 & 0 \\
\hline & 71040 & 61.97 & 225.26 & 23835.7991 & 0 & 0 & 31.3 & 80 & 44.106 & 0.551 & 55 & 1 & 11.26315 & 0 \\
\hline & 71060 & 54.22 & 235.18 & 23949.44386 & 0 & 0 & 31.3 & 70 & 46.048 & 0.658 & 56 & 1 & 11.75905 & 0 \\
\hline & 71080 & 46.48 & 234.8 & 24046.90156 & 0 & 0 & 31.3 & 60.01 & 45.973 & 0.766 & 57 & 1 & 11.73975 & 0 \\
\hline & 71100 & 38.73 & 242.86 & 24128.17692 & 0 & 0 & 31.3 & 50 & 47.552 & 0.951 & 58 & 1 & 12.14295 & 0 \\
\hline & 71120 & 30.99 & 246.7 & 24193.12699 & 0 & 0 & 31.3 & 40.01 & 48.304 & 1.207 & 59 & 1 & 12.33505 & 0 \\
\hline & 71140 & 23.24 & 251.98 & 24241.93478 & 0 & 0 & 31.3 & 30 & 49.338 & 1.645 & 60 & 1 & 12.5991 & 0 \\
\hline & 71160 & 15.5 & 277.51 & 24274.48875 & 0 & 0 & 31.3 & 20.01 & 54.337 & 2.715 & 61 & 1 & 13.8756 & 0 \\
\hline
\end{tabular}




$\begin{array}{rrrrrrrrrrrrrrrr}1 & 7 & 1180 & 7.74 & 285.53 & 24290.82424 & 0 & 0 & 31.3 & 9.99 & 55.907 & 5.596 & 62 & 1 & 14.2765 & 0 \\ 1 & 8 & 1200 & 6.97 & 307.71 & 24305.5214 & 0 & 0 & 31.3 & 9 & 60.249 & 6.694 & 63 & 1 & 15.3853 & 0 \\ 1 & 8 & 1220 & 6.2 & 323.95 & 24318.51581 & 0 & 0 & 31.3 & 8 & 63.429 & 7.929 & 64 & 1 & 16.19735 & 0 \\ 1 & 8 & 1240 & 5.42 & 314.65 & 24329.88759 & 0 & 0 & 31.3 & 7 & 61.607 & 8.801 & 65 & 1 & 15.73225 & 0 \\ 1 & 8 & 1260 & 4.64 & 303.04 & 24339.62653 & 0 & 0 & 31.3 & 5.99 & 59.336 & 9.906 & 66 & 1 & 15.1522 & 0 \\ 1 & 8 & 1280 & 3.87 & 293.43 & 24347.74755 & 0 & 0 & 31.3 & 5 & 57.453 & 11.491 & 67 & 1 & 14.6713 & 0 \\ 1 & 8 & 1300 & 3.09 & 282.94 & 24354.24593 & 0 & 0 & 31.3 & 3.99 & 55.399 & 13.884 & 68 & 1 & 14.1469 & 0 \\ 1 & 8 & 1320 & 2.32 & 268.97 & 24359.11775 & 0 & 0 & 31.3 & 3 & 52.665 & 17.555 & 69 & 1 & 13.4486 & 0 \\ 1 & 8 & 1340 & 1.54 & 252.17 & 24362.37009 & 0 & 0 & 31.3 & 1.99 & 49.376 & 24.812 & 70 & 1 & 12.6087 & 0 \\ 1 & 8 & 1360 & 0.77 & 233.6 & 24363.99979 & 0 & 0 & 31.3 & 0.99 & 45.738 & 46.2 & 71 & 1 & 11.67985 & 0 \\ 1 & 9 & 1380 & 0.7 & 236.05 & 24365.47398 & 18.17405 & 0.39322 & 31.3 & 0.9 & 46.218 & 51.353 & 72 & 1 & 11.8023 & 2.543076529 \\ 1 & 9 & 1400 & 0.62 & 237.64 & 24366.77853 & 34.25668 & 0.73621 & 31.3 & 0.8 & 46.531 & 58.164 & 73 & 1 & 11.8822 & 1.358304424 \\ 1 & 9 & 1420 & 0.55 & 242.97 & 24367.91893 & 48.31568 & 1.01559 & 31.3 & 0.71 & 47.574 & 67.006 & 74 & 1 & 12.14865 & 0.98464929 \\ 1 & 9 & 1440 & 0.47 & 239.29 & 24368.90146 & 60.42848 & 1.28975 & 31.3 & 0.61 & 46.853 & 76.808 & 75 & 1 & 11.9646 & 0.775346327 \\ 1 & 9 & 1460 & 0.39 & 236.48 & 24369.71906 & 70.50797 & 1.52278 & 31.3 & 0.5 & 46.302 & 92.604 & 76 & 1 & 11.8238 & 0.656691719 \\ 1 & 9 & 1480 & 0.31 & 234.04 & 24370.37722 & 78.6219 & 1.71566 & 31.3 & 0.4 & 45.826 & 114.565 & 77 & 1 & 11.7022 & 0.582865588 \\ 1 & 9 & 1500 & 0.24 & 233.39 & 24370.86888 & 84.68315 & 1.85314 & 31.3 & 0.31 & 45.697 & 147.41 & 78 & 1 & 11.66935 & 0.53962329 \\ 1 & 9 & 1520 & 0.16 & 228.48 & 24371.20032 & 88.76916 & 1.98429 & 31.3 & 0.21 & 44.736 & 213.029 & 79 & 1 & 11.424 & 0.503958807 \\ 1 & 9 & 1540 & 0.08 & 220.76 & 24371.37232 & 90.88963 & 2.10276 & 31.3 & 0.1 & 43.224 & 432.24 & 80 & 1 & 11.03775 & 0.475565804\end{array}$

04.27.04 WM-187 centrifuged CC-45 run 02.txt

\begin{tabular}{|c|c|c|c|c|c|c|c|c|c|c|c|c|c|c|c|}
\hline Mode & Step & $\mathrm{t}[\mathrm{s}]$ & $\mathrm{n}[\mathrm{rpm}]$ & $\mathrm{M}[\%]$. & Phi[rad] & Gamma & $J[t]$ & $\mathrm{T}\left[{ }^{\circ} \mathrm{C}\right]$ & $\mathrm{D}[1 / \mathrm{s}]$ & $\mathrm{Tau}[\mathrm{Pa}]$ & Eta[Pas] & MP\# & Block & $\mathrm{M}[\mathrm{mNm}]$ & $\mathrm{G}[\mathrm{Pa}]$ \\
\hline 1 & 1 & 20 & 0.04 & 176.3 & 0.08875 & 1.09412 & 0.0317 & 31.3 & 0.05 & 34.519 & 690.38 & 1 & 2 & 8.8148 & 31.54955581 \\
\hline 1 & 1 & 40 & 0.16 & 234.99 & 0.41155 & 5.07363 & 0.11027 & 31.3 & 0.21 & 46.011 & 219.1 & 2 & 2 & 11.74955 & 9.068654987 \\
\hline 1 & 1 & 60 & 0.24 & 245.8 & 0.90242 & 11.12519 & 0.23116 & 31.3 & 0.31 & 48.128 & 155.252 & 3 & 2 & 12.29 & 4.326038477 \\
\hline 1 & 1 & 80 & 0.31 & 255.33 & 1.54959 & 19.10357 & 0.38212 & 31.3 & 0.4 & 49.994 & 124.985 & 4 & 2 & 12.7665 & 2.616997765 \\
\hline 1 & 1 & 100 & 0.39 & 264.88 & 2.36326 & 29.13464 & 0.56176 & 31.3 & 0.5 & 51.863 & 103.726 & 5 & 2 & 13.24395 & 1.780114668 \\
\hline 1 & 1 & 120 & 0.47 & 270.04 & 3.3403 & 41.17967 & 0.77884 & 31.3 & 0.61 & 52.873 & 86.677 & 6 & 2 & 13.50175 & 1.283958808 \\
\hline 1 & 1 & 140 & 0.54 & 271.51 & 4.4807 & 55.23866 & 1.03908 & 31.3 & 0.7 & 53.161 & 75.944 & 7 & 2 & 13.57525 & 0.962387574 \\
\hline 1 & 1 & 160 & 0.62 & 275.87 & 5.78053 & 71.2632 & 1.3193 & 31.3 & 0.8 & 54.016 & 67.52 & 8 & 2 & 13.79365 & 0.757978873 \\
\hline 1 & 1 & 180 & 0.7 & 282.01 & 7.24451 & 89.31138 & 1.61746 & 31.3 & 0.9 & 55.217 & 61.352 & 9 & 2 & 14.1004 & 0.61825268 \\
\hline 1 & 2 & 200 & 0.77 & 289.64 & 8.86322 & 0 & 0 & 31.3 & 0.99 & 56.712 & 57.285 & 10 & 2 & 14.48215 & 0 \\
\hline 1 & 2 & 220 & 1.55 & 314.9 & 12.08099 & 0 & 0 & 31.3 & 2 & 61.657 & 30.829 & 11 & 2 & 15.745 & 0 \\
\hline 1 & 2 & 240 & 2.32 & 332.73 & 16.92926 & 0 & 0 & 31.3 & 3 & 65.148 & 21.716 & 12 & 2 & 16.6363 & 0 \\
\hline 1 & 2 & 260 & 3.09 & 341.63 & 23.39387 & 0 & 0 & 31.3 & 3.99 & 66.891 & 16.765 & 13 & 2 & 17.08135 & 0 \\
\hline 1 & 2 & 280 & 3.87 & 350.46 & 31.48583 & 0 & 0 & 31.3 & 5 & 68.619 & 13.724 & 14 & 2 & 17.5228 & 0 \\
\hline 1 & 2 & 300 & 4.65 & 354.81 & 41.1957 & 0 & 0 & 31.3 & 6 & 69.473 & 11.579 & 15 & 2 & 17.7407 & 0 \\
\hline 1 & 2 & 320 & 5.42 & 362.75 & 52.54471 & 0 & 0 & 31.3 & 7 & 71.027 & 10.147 & 16 & 2 & 18.13755 & 0 \\
\hline 1 & 2 & 340 & 6.2 & 375.58 & 65.50613 & 0 & 0 & 31.4 & 8 & 73.539 & 9.192 & 17 & 2 & 18.7792 & 0 \\
\hline 1 & 2 & 360 & 6.97 & 391.35 & 80.09019 & 0 & 0 & 31.4 & 9 & 76.626 & 8.514 & 18 & 2 & 19.5675 & 0 \\
\hline 1 & 3 & 380 & 7.75 & 388.03 & 96.45004 & 0 & 0 & 31.4 & 10.01 & 75.976 & 7.59 & 19 & 2 & 19.4014 & 0 \\
\hline 1 & 3 & 400 & 15.51 & 363.78 & 128.81001 & 0 & 0 & 31.4 & 20.02 & 71.229 & 3.558 & 20 & 2 & 18.1891 & 0 \\
\hline 1 & 3 & 420 & 23.25 & 308.83 & 177.34997 & 0 & 0 & 31.4 & 30.02 & 60.468 & 2.014 & 21 & 2 & 15.44135 & 0 \\
\hline 1 & 3 & 440 & 30.99 & 313.62 & 242.19323 & 0 & 0 & 31.4 & 40.01 & 61.406 & 1.535 & 22 & 2 & 15.68075 & 0 \\
\hline 1 & 3 & 460 & 38.73 & 304.85 & 323.11595 & 0 & 0 & 31.4 & 50 & 59.69 & 1.194 & 23 & 2 & 15.2425 & 0 \\
\hline 1 & 3 & 480 & 46.48 & 292.44 & 420.33567 & 0 & 0 & 31.4 & 60.01 & 57.261 & 0.954 & 24 & 2 & 14.6222 & 0 \\
\hline 1 & 3 & 500 & 54.22 & 289.17 & 533.799 & 0 & 0 & 31.4 & 70 & 56.62 & 0.809 & 25 & 2 & 14.4586 & 0 \\
\hline 1 & 3 & 520 & 61.96 & 285.46 & 663.49259 & 0 & 0 & 31.4 & 79.99 & 55.894 & 0.699 & 26 & 2 & 14.2732 & 0 \\
\hline 1 & 3 & 540 & 69.71 & 271.71 & 809.30019 & 0 & 0 & 31.4 & 90 & 53.201 & 0.591 & 27 & 2 & 13.58555 & 0 \\
\hline 1 & 4 & 560 & 77.46 & 276.76 & 973.03528 & 0 & 0 & 31.4 & 100 & 54.189 & 0.542 & 28 & 2 & 13.83775 & 0 \\
\hline
\end{tabular}




\begin{tabular}{|c|c|c|c|c|c|c|c|c|c|c|c|c|c|c|}
\hline & 580 & 154.93 & 307.15 & 1296.31616 & 0 & 0 & 31.4 & 200.01 & 60.141 & 0.301 & 29 & 2 & 15.3577 & 0 \\
\hline & 600 & 232.38 & 292.63 & 1781.31445 & 0 & 0 & 31.4 & 300 & 57.296 & 0.191 & 30 & 2 & 14.63125 & 0 \\
\hline & 620 & 309.85 & 251.48 & 2429.35747 & 0 & 0 & 31.4 & 400.02 & 49.24 & 0.123 & 31 & 2 & 12.574 & 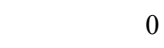 \\
\hline & 640 & 387.3 & 205.42 & 3238.89092 & 0 & 0 & 31.4 & 500 & 40.221 & 0.08 & 32 & 2 & 10.271 & \\
\hline & 660 & 464.76 & 186.09 & 4211.47853 & 0 & 0 & 31.4 & 600.01 & 36.436 & 0.061 & 33 & 2 & 9.3045 & \\
\hline & 680 & 542.21 & 134.69 & 5345.18585 & 0 & 0 & 31.4 & 699.99 & 26.372 & 0.038 & 34 & 2 & 6.73455 & \\
\hline & 700 & 619.67 & 131.61 & 6642.34082 & 0 & 0 & 31.4 & 799.99 & 25.77 & 0.032 & 35 & 2 & 6.5807 & \\
\hline & 720 & 697.13 & 105.63 & 8101.76533 & 0 & 0 & 31.4 & 899.99 & 20.682 & 0.023 & 36 & 2 & 5.28145 & \\
\hline & 740 & 774.59 & 96.26 & 9722.286 & 0 & 0 & 31.4 & 1000 & 18.847 & 0.019 & 37 & 2 & 4.8129 & \\
\hline & 750 & 774.59 & 74.77 & 10549.33461 & 0 & 0 & 31.4 & 1000 & 14.64 & 0.015 & 38 & 2 & 3.73845 & \\
\hline & 760 & 774.59 & 74.56 & 11360.07758 & 0 & 0 & 31.4 & 1000 & 14.598 & 0.015 & 39 & 2 & 3.7279 & \\
\hline & 770 & 774.59 & 72.42 & 12172.03948 & 0 & 0 & 31.4 & 1000 & 14.179 & 0.014 & 40 & 2 & 3.62085 & 0 \\
\hline & 780 & 774.59 & 71.52 & 12983.5914 & 0 & 0 & 31.4 & 1000 & 14.003 & 0.014 & 41 & 2 & 3.57575 & 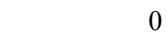 \\
\hline & 790 & 774.59 & 76.33 & 13795.55016 & 0 & 0 & 31.4 & 1000 & 14.946 & 0.015 & 42 & 2 & 3.81675 & 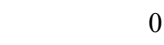 \\
\hline & 800 & 774.59 & 72.32 & 14605.48259 & 0 & 0 & 31.4 & 1000 & 14.16 & 0.014 & 43 & 2 & 3.61605 & 0 \\
\hline & 820 & 774.59 & 71.91 & 16246.02934 & 0 & 0 & 31.4 & 1000 & 14.08 & 0.014 & 44 & 2 & 3.5955 & 0 \\
\hline & 840 & 697.13 & 73.03 & 17707.76135 & 0 & 0 & 31.4 & 899.99 & 14.299 & 0.016 & 45 & 2 & 3.65145 & 0 \\
\hline & 860 & 619.67 & 77.49 & 19006.60257 & 0 & 0 & 31.4 & 799.99 & 15.172 & 0.019 & 46 & 2 & 3.87435 & 0 \\
\hline & 880 & 542.21 & 128.42 & 20142.68886 & 0 & 0 & 31.4 & 699.99 & 25.145 & 0.036 & 47 & 2 & 6.4211 & 0 \\
\hline & 900 & 464.75 & 111.2 & 21118.10547 & 0 & 0 & 31.4 & 599.99 & 21.773 & 0.036 & 48 & 2 & 5.56 & 0 \\
\hline & 920 & 387.3 & 58.71 & 21930.58181 & 0 & 0 & 31.4 & 500 & 11.495 & 0.023 & 49 & 2 & 2.9354 & 0 \\
\hline & 940 & 309.82 & 149.2 & 22580.42261 & 0 & 0 & 31.4 & 399.98 & 29.213 & 0.073 & 50 & 2 & 7.45995 & 0 \\
\hline & 960 & 232.36 & 196.68 & 23068.23733 & 0 & 0 & 31.4 & 299.98 & 38.511 & 0.128 & 51 & 2 & 9.83415 & 0 \\
\hline & 980 & 154.91 & 206.54 & 23394.19014 & 0 & 0 & 31.4 & 199.99 & 40.44 & 0.202 & 52 & 2 & 10.32675 & 0 \\
\hline & 61000 & 77.46 & 186.6 & 23557.5938 & 0 & 0 & 31.4 & 100 & 36.537 & 0.365 & 53 & 2 & 9.33015 & 0 \\
\hline & 71020 & 69.71 & 197.75 & 23704.69023 & 0 & 0 & 31.4 & 90 & 38.72 & 0.43 & 54 & 2 & 9.88765 & 0 \\
\hline & 71040 & 61.97 & 216.43 & 23834.65321 & 0 & 0 & 31.4 & 80 & 42.378 & 0.53 & 55 & 2 & 10.8217 & 0 \\
\hline & 71060 & 54.22 & 232.83 & 23948.32703 & 0 & 0 & 31.4 & 70 & 45.587 & 0.651 & 56 & 2 & 11.64125 & 0 \\
\hline & 71080 & 46.48 & 233.51 & 24045.83107 & 0 & 0 & 31.4 & 60.01 & 45.721 & 0.762 & 57 & 2 & 11.67555 & 0 \\
\hline & 71100 & 38.73 & 215.28 & 24127.01061 & 0 & 0 & 31.4 & 50 & 42.151 & 0.843 & 58 & 2 & 10.7639 & 0 \\
\hline & 71120 & 30.99 & 206.07 & 24192.0243 & 0 & 0 & 31.4 & 40.01 & 40.349 & 1.008 & 59 & 2 & 10.30365 & 0 \\
\hline & 71140 & 23.24 & 205.05 & 24240.81166 & 0 & 0 & 31.4 & 30 & 40.148 & 1.338 & 60 & 2 & 10.25235 & 0 \\
\hline & 71160 & 15.5 & 207.04 & 24273.4049 & 0 & 0 & 31.4 & 20.01 & 40.538 & 2.026 & 61 & 2 & 10.3519 & 0 \\
\hline & 71180 & 7.74 & 279.27 & 24289.73097 & 0 & 0 & 31.4 & 9.99 & 54.682 & 5.474 & 62 & 2 & 13.96365 & 0 \\
\hline & 81200 & 6.97 & 278.46 & 24304.42734 & 0 & 0 & 31.4 & 9 & 54.523 & 6.058 & 63 & 2 & 13.92325 & 0 \\
\hline & 81220 & 6.2 & 277.54 & 24317.42332 & 0 & 0 & 31.4 & 8 & 54.342 & 6.793 & 64 & 2 & 13.877 & 0 \\
\hline & 81240 & 5.42 & 277.53 & 24328.79275 & 0 & 0 & 31.4 & 7 & 54.341 & 7.763 & 65 & 2 & 13.87665 & 0 \\
\hline & 81260 & 4.64 & 267.96 & 24338.52697 & 0 & 0 & 31.4 & 5.99 & 52.467 & 8.759 & 66 & 2 & 13.398 & 0 \\
\hline & 81280 & 3.87 & 255.29 & 24346.65113 & 0 & 0 & 31.4 & 5 & 49.986 & 9.997 & 67 & 2 & 12.76455 & 0 \\
\hline & 81300 & 3.09 & 241.33 & 24353.14637 & 0 & 0 & 31.4 & 3.99 & 47.251 & 11.842 & 68 & 2 & 12.06625 & 0 \\
\hline & 81320 & 2.32 & 225.92 & 24358.02684 & 0 & 0 & 31.4 & 3 & 44.235 & 14.745 & 69 & 2 & 11.29605 & 0 \\
\hline & 81340 & 1.54 & 211.91 & 24361.27367 & 0 & 0 & 31.4 & 1.99 & 41.493 & 20.851 & 70 & 2 & 10.5957 & 0 \\
\hline & 81360 & 0.77 & 196.71 & 24362.9073 & 0 & 0 & 31.4 & 0.99 & 38.515 & 38.904 & 71 & 2 & 9.83525 & 0 \\
\hline & 91380 & 0.7 & 191.49 & 24364.38071 & 18.16437 & 0.48446 & 31.4 & 0.9 & 37.494 & 41.66 & 72 & 2 & 9.5746 & 2.064150862 \\
\hline & 91400 & 0.62 & 194.72 & 24365.68368 & 34.22764 & 0.89773 & 31.4 & 0.8 & 38.127 & 47.659 & 73 & 2 & 9.7362 & 1.113924302 \\
\hline & 91420 & 0.55 & 202.08 & 24366.82408 & 48.28663 & 1.22041 & 31.4 & 0.71 & 39.566 & 55.727 & 74 & 2 & 10.1038 & 0.819398662 \\
\hline & 91440 & 0.47 & 201.47 & 24367.80426 & 60.37039 & 1.53038 & 31.4 & 0.61 & 39.448 & 64.669 & 75 & 2 & 10.07345 & 0.653432916 \\
\hline & 91460 & 0.39 & 199.82 & 24368.62578 & 70.49828 & 1.80187 & 31.4 & 0.5 & 39.125 & 78.25 & 76 & 2 & 9.9911 & 0.554978079 \\
\hline & 91480 & 0.31 & 192.69 & 24369.28081 & 78.57349 & 2.08263 & 31.4 & 0.4 & 37.728 & 94.32 & 77 & 2 & 9.63435 & 0.480161948 \\
\hline & 91500 & 0.24 & 186.31 & 24369.77953 & 84.72188 & 2.32248 & 31.4 & 0.31 & 36.479 & 117.674 & 78 & 2 & 9.31545 & 0.430573543 \\
\hline
\end{tabular}


$\begin{array}{lllllllll}1 & 9 & 1520 & 0.16 & 182.8 & 24370.1094 & 88.78853 & 2.48061 & 31.4\end{array}$

$\begin{array}{lllllllll}1 & 9 & 1540 & 0.08 & 175.97 & 24370.27983 & 90.88963 & 2.63792 & 31.4\end{array}$ $\begin{array}{lllllll}0.21 & 35.793 & 170.443 & 79 & 2 & 9.14015 & 0.403126395\end{array}$

$\begin{array}{lllllll}0.1 & 34.455 & 344.55 & 80 & 2 & 8.7986 & 0.379086151\end{array}$

04.27.04 WM-187 centrifuged CC-45 run 03.txt

Mode Step t[s] n[rpm] M[\%.] Phi[rad]

\begin{tabular}{|c|c|c|c|c|}
\hline & 1 & 20 & 0.05 & 146.2 \\
\hline & 1 & 40 & 0.16 & 184.82 \\
\hline & 1 & 60 & 0.24 & 196.23 \\
\hline & 1 & 80 & 0.31 & 207.08 \\
\hline & 1 & 100 & 0.39 & 218 \\
\hline & 1 & 120 & 0.47 & 225.05 \\
\hline & 1 & 140 & 0.54 & 229.33 \\
\hline & 1 & 160 & 0.62 & 226.32 \\
\hline & 1 & 180 & 0.7 & 226.32 \\
\hline & 2 & 200 & 0.77 & 239.66 \\
\hline & 2 & 220 & 1.55 & 261.3 \\
\hline & 2 & 240 & 2.32 & 275.75 \\
\hline & 2 & 260 & 3.09 & 284.16 \\
\hline & 2 & 280 & 3.87 & 291.06 \\
\hline & 2 & 300 & 4.65 & 303.86 \\
\hline & 2 & 320 & 5.42 & 315.56 \\
\hline & 2 & 340 & 6.2 & 323.14 \\
\hline & 2 & 360 & 6.97 & 338.56 \\
\hline & 3 & 380 & 7.75 & 334.7 \\
\hline & 3 & 400 & 15.51 & 308.75 \\
\hline & 3 & 420 & 23.24 & 301.28 \\
\hline & 3 & 440 & 30.99 & 296.49 \\
\hline & 3 & 460 & 38.73 & 300.12 \\
\hline & 3 & 480 & 46.48 & 313.92 \\
\hline & 3 & 500 & 54.22 & 294.1 \\
\hline & 3 & 520 & 61.96 & 269.25 \\
\hline & 3 & 540 & 69.71 & 273.77 \\
\hline & 4 & 560 & 77.46 & 273.39 \\
\hline & 4 & 580 & 154.92 & 268.46 \\
\hline & 4 & 600 & 232.38 & 283.17 \\
\hline & 4 & 620 & 309.84 & 224.83 \\
\hline & 4 & 640 & 387.3 & 170.73 \\
\hline & 4 & 660 & 464.75 & 153.41 \\
\hline & 4 & 680 & 542.2 & 130.15 \\
\hline & 4 & 700 & 619.68 & 160.92 \\
\hline & 4 & 720 & 697.14 & 130.34 \\
\hline & 4 & 740 & 774.6 & 85.7 \\
\hline & 5 & 750 & 774.58 & 103.56 \\
\hline & 5 & 760 & 774.59 & 110.84 \\
\hline & 5 & 770 & 774.56 & 142.93 \\
\hline & 5 & 780 & 774.6 & 203.78 \\
\hline & 5 & 790 & 774.58 & 229.48 \\
\hline & 5 & 800 & 774.6 & 220.19 \\
\hline & 6 & 820 & 774.59 & 240.38 \\
\hline & 6 & 840 & 697.13 & 187.34 \\
\hline
\end{tabular}

\begin{tabular}{|c|c|c|c|c|c|c|c|c|c|c|}
\hline 0.10603 & 1.30714 & 0.04566 & 31.4 & 0.06 & 28.625 & 477.083 & 1 & 3 & 7.30975 & 21.89895497 \\
\hline 0.43197 & 5.32537 & 0.14716 & 31.4 & 0.21 & 36.187 & 172.319 & 2 & 3 & 9.2409 & 6.795208596 \\
\hline 0.91656 & 11.29948 & 0.29409 & 31.4 & 0.31 & 38.422 & 123.942 & 3 & 3 & 9.81165 & 3.400333467 \\
\hline 1.56844 & 19.33595 & 0.47688 & 31.4 & 0.4 & 40.547 & 101.367 & 4 & 3 & 10.3542 & 2.096974806 \\
\hline 2.38054 & 29.34766 & 0.68754 & 31.4 & 0.5 & 42.685 & 85.37 & 5 & 3 & 10.90015 & 1.454460083 \\
\hline 3.35522 & 41.36364 & 0.93872 & 31.4 & 0.61 & 44.064 & 72.236 & 6 & 3 & 11.25225 & 1.065283423 \\
\hline 4.49483 & 55.41295 & 1.23406 & 31.4 & 0.7 & 44.903 & 64.147 & 7 & 3 & 11.4666 & 0.810334046 \\
\hline 5.80095 & 71.51495 & 1.61382 & 31.4 & 0.8 & 44.314 & 55.393 & 8 & 3 & 11.31605 & 0.619646661 \\
\hline 7.26179 & 89.5244 & 2.02023 & 31.4 & 0.9 & 44.314 & 49.238 & 9 & 3 & 11.3161 & 0.494993544 \\
\hline 8.88207 & 0 & 0 & 31.4 & 0.99 & 46.925 & 47.399 & 10 & 3 & 11.98295 & 0 \\
\hline 12.10377 & 0 & 0 & 31.4 & 2 & 51.162 & 25.581 & 11 & 3 & 13.06495 & 0 \\
\hline 16.94339 & 0 & 0 & 31.4 & 3 & 53.992 & 17.997 & 12 & 3 & 13.78745 & 0 \\
\hline 23.41193 & 0 & 0 & 31.4 & 3.99 & 55.639 & 13.945 & 13 & 3 & 14.20815 & 0 \\
\hline 31.50468 & 0 & 0 & 31.4 & 5 & 56.99 & 11.398 & 14 & 3 & 14.5531 & 0 \\
\hline 41.2122 & 0 & 0 & 31.4 & 6 & 59.495 & 9.916 & 15 & 3 & 15.1929 & 0 \\
\hline 52.56277 & 0 & 0 & 31.4 & 7 & 61.786 & 8.827 & 16 & 3 & 15.77795 & 0 \\
\hline 65.52106 & 0 & 0 & 31.4 & 8 & 63.27 & 7.909 & 17 & 3 & 16.15685 & 0 \\
\hline 80.09883 & 0 & 0 & 31.4 & 9 & 66.289 & 7.365 & 18 & 3 & 16.9278 & 0 \\
\hline 96.46889 & 0 & 0 & 31.4 & 10.01 & 65.534 & 6.547 & 19 & 3 & 16.73505 & 0 \\
\hline 128.82258 & 0 & 0 & 31.4 & 20.02 & 60.452 & 3.02 & 20 & 3 & 15.43725 & 0 \\
\hline 177.34448 & 0 & 0 & 31.4 & 30 & 58.99 & 1.966 & 21 & 3 & 15.0638 & 0 \\
\hline 242.19088 & 0 & 0 & 31.4 & 40.01 & 58.052 & 1.451 & 22 & 3 & 14.8244 & 0 \\
\hline 323.16464 & 0 & 0 & 31.4 & 50 & 58.763 & 1.175 & 23 & 3 & 15.00595 & 0 \\
\hline 420.38515 & 0 & 0 & 31.4 & 60.01 & 61.466 & 1.024 & 24 & 3 & 15.69605 & 0 \\
\hline 533.82885 & 0 & 0 & 31.4 & 70 & 57.584 & 0.823 & 25 & 3 & 14.70485 & 0 \\
\hline 663.51929 & 0 & 0 & 31.4 & 79.99 & 52.72 & 0.659 & 26 & 3 & 13.4626 & 0 \\
\hline 809.39836 & 0 & 0 & 31.4 & 90 & 53.603 & 0.596 & 27 & 3 & 13.68825 & 0 \\
\hline 973.09419 & 0 & 0 & 31.4 & 100 & 53.53 & 0.535 & 28 & 3 & 13.6695 & 0 \\
\hline 296.09939 & 0 & 0 & 31.4 & 200 & 52.563 & 0.263 & 29 & 3 & 13.42275 & 0 \\
\hline 781.54222 & 0 & 0 & 31.4 & 300 & 55.445 & 0.185 & 30 & 3 & 14.1586 & 0 \\
\hline 429.82243 & 0 & 0 & 31.4 & 400 & 44.021 & 0.11 & 31 & 3 & 11.24135 & 0 \\
\hline 239.52552 & 0 & 0 & 31.4 & 500 & 33.429 & 0.067 & 32 & 3 & 8.5364 & 0 \\
\hline 211.86573 & 0 & 0 & 31.4 & 599.99 & 30.037 & 0.05 & 33 & 3 & 7.67035 & 0 \\
\hline 345.51022 & 0 & 0 & 31.4 & 699.98 & 25.484 & 0.036 & 34 & 3 & 6.50755 & 0 \\
\hline 6641.69758 & 0 & 0 & 31.4 & 800.01 & 31.509 & 0.039 & 35 & 3 & 8.0462 & 0 \\
\hline 8100.8472 & 0 & 0 & 31.4 & 900.01 & 25.521 & 0.028 & 36 & 3 & 6.51705 & 0 \\
\hline 9722.22238 & 0 & 0 & 31.4 & 1000 & 16.779 & 0.017 & 37 & 3 & 4.2848 & 0 \\
\hline 549.66448 & 0 & 0 & 31.4 & 999.98 & 20.278 & 0.02 & 38 & 3 & 5.17815 & 0 \\
\hline 1361.22347 & 0 & 0 & 31.4 & 1000 & 21.703 & 0.022 & 39 & 3 & 5.5422 & 0 \\
\hline 2170.72236 & 0 & 0 & 31.4 & 999.96 & 27.986 & 0.028 & 40 & 3 & 7.14665 & 0 \\
\hline 12984.7208 & 0 & 0 & 31.4 & 1000 & 39.9 & 0.04 & 41 & 3 & 10.18905 & 0 \\
\hline 3795.45826 & 0 & 0 & 31.4 & 999.98 & 44.932 & 0.045 & 42 & 3 & 11.474 & 0 \\
\hline 4605.80146 & 0 & 0 & 31.4 & 1000 & 43.113 & 0.043 & 43 & 3 & 11.0095 & 0 \\
\hline 6245.94295 & 0 & 0 & 31.4 & 1000 & 47.067 & 0.047 & 44 & 3 & 12.01915 & 0 \\
\hline 7707.27519 & 0 & 0 & 31.4 & 899.99 & 36.68 & 0.041 & 45 & 3 & 9.36675 & 0 \\
\hline
\end{tabular}




\begin{tabular}{|c|c|c|c|c|c|c|c|c|c|c|c|c|c|c|}
\hline & 860 & 619.67 & 148.22 & 19007.37226 & 0 & 0 & 31.4 & 799.99 & 29.02 & 0.036 & 46 & 3 & 7.41075 & 0 \\
\hline & 880 & 542.23 & 156.08 & 20144.32485 & 0 & 0 & 31.4 & 700.02 & 30.56 & 0.044 & 47 & 3 & 7.8038 & \\
\hline & 900 & 464.74 & 150.07 & 21119.04167 & 0 & 0 & 31.4 & 599.98 & 29.384 & 0.049 & 48 & 3 & 7.50345 & \\
\hline & 920 & 387.3 & 115.22 & 21930.91875 & 0 & 0 & 31.4 & 500 & 22.56 & 0.045 & 49 & 3 & 5.76105 & \\
\hline & 940 & 309.83 & 112.29 & 22581.64861 & 0 & 0 & 31.4 & 399.99 & 21.987 & 0.055 & 50 & 3 & 5.6146 & 0 \\
\hline & 960 & 232.38 & 155.6 & 23062.90527 & 0 & 0 & 31.4 & 300 & 30.466 & 0.102 & 51 & 3 & 7.77985 & 0 \\
\hline & 980 & 154.92 & 148.31 & 23393.00104 & 0 & 0 & 31.4 & 200 & 29.039 & 0.145 & 52 & 3 & 7.4156 & 0 \\
\hline & 61000 & 77.46 & 144.19 & 23556.35679 & 0 & 0 & 31.4 & 100 & 28.233 & 0.282 & 53 & 3 & 7.2097 & 0 \\
\hline & 71020 & 69.71 & 193.27 & 23703.59146 & 0 & 0 & 31.4 & 90 & 37.841 & 0.42 & 54 & 3 & 9.6633 & 0 \\
\hline & 71040 & 61.97 & 189.73 & 23833.36358 & 0 & 0 & 31.4 & 80 & 37.148 & 0.464 & 55 & 3 & 9.4863 & 0 \\
\hline & 71060 & 54.22 & 189.75 & 23947.00991 & 0 & 0 & 31.4 & 70 & 37.153 & 0.531 & 56 & 3 & 9.48755 & 0 \\
\hline & 71080 & 46.48 & 189.36 & 24044.46526 & 0 & 0 & 31.4 & 60.01 & 37.076 & 0.618 & 57 & 3 & 9.46795 & 0 \\
\hline & 71100 & 38.73 & 193.98 & 24125.76182 & 0 & 0 & 31.4 & 50 & 37.982 & 0.76 & 58 & 3 & 9.69915 & 0 \\
\hline & 71120 & 30.99 & 191.78 & 24190.77316 & 0 & 0 & 31.4 & 40.01 & 37.551 & 0.939 & 59 & 3 & 9.589 & م \\
\hline & 71140 & 23.24 & 192.23 & 24239.57623 & 0 & 0 & 31.4 & 30 & 37.638 & 1.255 & 60 & 3 & 9.6113 & 0 \\
\hline & 71160 & 15.5 & 202.48 & 24272.15062 & 0 & 0 & 31.4 & 20.01 & 39.646 & 1.981 & 61 & 3 & 10.12405 & 0 \\
\hline & 71180 & 7.74 & 224.06 & 24288.48533 & 0 & 0 & 31.4 & 9.99 & 43.871 & 4.391 & 62 & 3 & 11.20305 & 0 \\
\hline & 81200 & 6.97 & 241.46 & 24303.19505 & 0 & 0 & 31.4 & 9 & 47.278 & 5.253 & 63 & 3 & 12.073 & 0 \\
\hline & 81220 & 6.2 & 239.34 & 24316.18868 & 0 & 0 & 31.4 & 8 & 46.863 & 5.858 & 64 & 3 & 11.967 & 0 \\
\hline & 81240 & 5.42 & 234.4 & 24327.54004 & 0 & 0 & 31.4 & 7 & 45.896 & 6.557 & 65 & 3 & 11.72005 & 0 \\
\hline & 81260 & 4.64 & 233.21 & 24337.28918 & 0 & 0 & 31.4 & 5.99 & 45.663 & 7.623 & 66 & 3 & 11.6605 & 0 \\
\hline & 81280 & 3.87 & 222.63 & 24345.4102 & 0 & 0 & 31.4 & 5 & 43.591 & 8.718 & 67 & 3 & 11.1314 & 0 \\
\hline & 81300 & 3.09 & 214.38 & 24351.90544 & 0 & 0 & 31.4 & 3.99 & 41.976 & 10.52 & 68 & 3 & 10.719 & 0 \\
\hline & 81320 & 2.32 & 200.7 & 24356.7812 & 0 & 0 & 31.4 & 3 & 39.297 & 13.099 & 69 & 3 & 10.0351 & 0 \\
\hline & 81340 & 1.54 & 174.38 & 24360.03353 & 0 & 0 & 31.4 & 1.99 & 34.143 & 17.157 & 70 & 3 & 8.71875 & 0 \\
\hline & 81360 & 0.77 & 160.66 & 24361.66402 & 0 & 0 & 31.4 & 0.99 & 31.458 & 31.776 & 71 & 3 & 8.0332 & 0 \\
\hline & 91380 & 0.7 & 157.94 & 24363.14056 & 18.2031 & 0.58862 & 31.4 & 0.9 & 30.925 & 34.361 & 72 & 3 & 7.8972 & 1.698886453 \\
\hline & 91400 & 0.62 & 152.29 & 24364.44747 & 34.31478 & 1.15085 & 31.4 & 0.8 & 29.817 & 37.271 & 73 & 3 & 7.61425 & 0.868925868 \\
\hline & 91420 & 0.55 & 147.08 & 24365.59022 & 48.40282 & 1.68071 & 31.4 & 0.71 & 28.799 & 40.562 & 74 & 3 & 7.3542 & 0.594985995 \\
\hline & 91440 & 0.47 & 149.79 & 24366.56961 & 60.47689 & 2.06209 & 31.4 & 0.61 & 29.328 & 48.079 & 75 & 3 & 7.4894 & 0.484945572 \\
\hline & 91460 & 0.39 & 154.14 & 24367.38721 & 70.55638 & 2.33785 & 31.4 & 0.5 & 30.18 & 60.36 & 76 & 3 & 7.7068 & 0.427743033 \\
\hline & 91480 & 0.31 & 154.87 & 24368.04066 & 78.61222 & 2.59241 & 31.4 & 0.4 & 30.324 & 75.81 & 77 & 3 & 7.74365 & 0.385741555 \\
\hline & 91500 & 0.24 & 152.47 & 24368.53703 & 84.73156 & 2.83829 & 31.4 & 0.31 & 29.853 & 96.3 & 78 & 3 & 7.6234 & 0.352324447 \\
\hline & 91520 & 0.16 & 147.37 & 24368.86926 & 88.82726 & 3.0784 & 31.4 & 0.21 & 28.855 & 137.405 & 79 & 3 & 7.36845 & 0.324843972 \\
\hline & 91540 & 0.08 & 139.9 & 24369.03969 & 90.92836 & 3.31952 & 31.4 & 0.1 & 27.392 & 273.92 & 80 & 3 & 6.995 & 0.301248147 \\
\hline
\end{tabular}

04.27.04 WM-187 centrifuged CC-45 run 04.txt

\begin{tabular}{rrrrrrrrrrrrrrrr} 
Mode Step & $\mathrm{t}[\mathrm{s}]$ & $\mathrm{n}[\mathrm{rpm}]$ & $\mathrm{M}[\%]$. & \multicolumn{1}{c}{$\mathrm{Ph}[\mathrm{rad}]$} & \multicolumn{1}{c}{$\mathrm{Gamma}$} & \multicolumn{1}{c}{$\mathrm{J}[\mathrm{t}]$} & \multicolumn{1}{c}{$\mathrm{T}\left[{ }^{\circ} \mathrm{C}\right]$} & $\mathrm{D}[1 / \mathrm{s}]$ & $\mathrm{Tau}[\mathrm{Pa}]$ & $\mathrm{Eta}[\mathrm{Pas}]$ & $\mathrm{MP} \#$ Block $\mathrm{M}[\mathrm{mNm}]$ & $\mathrm{G}[\mathrm{Pa}]$ \\
1 & 1 & 20 & 0.06 & 121.97 & 0.11781 & 1.45237 & 0.06082 & 31.4 & 0.08 & 23.881 & 298.513 & 1 & 4 & 6.09825 & 16.44277973 \\
1 & 1 & 40 & 0.16 & 148.76 & 0.44454 & 5.48029 & 0.18815 & 31.4 & 0.21 & 29.127 & 138.7 & 2 & 4 & 7.43785 & 5.314864724 \\
1 & 1 & 60 & 0.24 & 154.17 & 0.93227 & 11.49313 & 0.38073 & 31.4 & 0.31 & 30.187 & 97.377 & 3 & 4 & 7.7086 & 2.626525585 \\
1 & 1 & 80 & 0.31 & 155.76 & 1.58808 & 19.57801 & 0.64192 & 31.4 & 0.4 & 30.499 & 76.247 & 4 & 4 & 7.78825 & 1.557819206 \\
1 & 1 & 100 & 0.39 & 155.17 & 2.40175 & 29.60908 & 0.97456 & 31.4 & 0.5 & 30.382 & 60.764 & 5 & 4 & 7.75835 & 1.026104155 \\
1 & 1 & 120 & 0.47 & 159.3 & 3.37643 & 41.62507 & 1.33452 & 31.4 & 0.61 & 31.191 & 51.133 & 6 & 4 & 7.9649 & 0.749332073 \\
1 & 1 & 140 & 0.54 & 170.21 & 4.5129 & 55.63565 & 1.66939 & 31.4 & 0.7 & 33.327 & 47.61 & 7 & 4 & 8.5105 & 0.599022389 \\
1 & 1 & 160 & 0.62 & 176.61 & 5.81666 & 71.7086 & 2.07376 & 31.4 & 0.8 & 34.579 & 43.224 & 8 & 4 & 8.83025 & 0.482215522 \\
1 & 1 & 180 & 0.7 & 178.77 & 7.28064 & 89.75678 & 2.56419 & 31.4 & 0.9 & 35.004 & 38.893 & 9 & 4 & 8.93865 & 0.389987252 \\
1 & 2 & 200 & 0.77 & 176.17 & 8.90249 & 0 & 0 & 31.4 & 0.99 & 34.493 & 34.841 & 10 & 4 & 8.80835 & 0 \\
1 & 2 & 220 & 1.55 & 204.27 & 12.11948 & 0 & 0 & 31.4 & 2 & 39.995 & 19.998 & 11 & 4 & 10.2133 & 0 \\
1 & 2 & 240 & 2.32 & 219.42 & 16.95989 & 0 & 0 & 31.4 & 3 & 42.963 & 14.321 & 12 & 4 & 10.97105
\end{tabular}




\begin{tabular}{|c|c|c|c|c|c|}
\hline & 2 & 260 & 3.09 & 237.26 & 23.4245 \\
\hline & 2 & 280 & 3.87 & 254.39 & 31.52117 \\
\hline & 2 & 300 & 4.65 & 254.42 & 41.23262 \\
\hline & 2 & 320 & 5.42 & 256.75 & 52.57927 \\
\hline & 2 & 340 & 6.2 & 265.43 & 65.53676 \\
\hline & 2 & 360 & 6.97 & 272.65 & 80.12396 \\
\hline & 3 & 380 & 7.75 & 272.82 & 96.48852 \\
\hline & 3 & 400 & 15.51 & 235.14 & 128.85792 \\
\hline & 3 & 420 & 23.24 & 229.28 & 177.41752 \\
\hline & 3 & 440 & 30.99 & 239.27 & 242.17752 \\
\hline & 3 & 460 & 38.73 & 246.42 & 323.19763 \\
\hline & 3 & 480 & 46.48 & 246.69 & 420.38515 \\
\hline & 3 & 500 & 54.22 & 252.65 & 533.76209 \\
\hline & 3 & 520 & 61.96 & 255.34 & 663.49023 \\
\hline & 3 & 540 & 69.71 & 253.04 & 809.33474 \\
\hline & 4 & 560 & 77.46 & 241.93 & 973.09654 \\
\hline & 4 & 580 & 154.93 & 267.17 & 1296.39156 \\
\hline & 4 & 600 & 232.39 & 256.08 & 1781.86894 \\
\hline & 4 & 620 & 309.84 & 222.77 & 2429.38653 \\
\hline & 4 & 640 & 387.3 & 188.96 & 3239.69045 \\
\hline & 4 & 660 & 464.75 & 161.99 & 4211.29081 \\
\hline & 4 & 680 & 542.21 & 224.62 & 5345.05076 \\
\hline & 4 & 700 & 619.66 & 171.44 & 6642.50575 \\
\hline & 4 & 720 & 697.14 & 170.49 & 8100.52754 \\
\hline & 4 & 740 & 774.59 & 126.87 & 9721.04664 \\
\hline & 5 & 750 & 774.59 & 111.39 & 10550.12315 \\
\hline & 5 & 760 & 774.59 & 109.2 & 11359.64796 \\
\hline & 5 & 770 & 774.59 & 85.64 & 12171.61457 \\
\hline & 5 & 780 & 774.59 & 78.55 & 12982.76202 \\
\hline & 5 & 790 & 774.59 & 76.66 & 13795.12761 \\
\hline & 5 & 800 & 774.59 & 73.21 & 14605.06083 \\
\hline & 6 & 820 & 774.59 & 72.23 & 16245.20389 \\
\hline & 6 & 840 & 697.13 & 72.76 & 17707.98676 \\
\hline & 6 & 860 & 619.67 & 77.47 & 19006.14704 \\
\hline & 6 & 880 & 542.21 & 62.18 & 20143.94393 \\
\hline & 6 & 900 & 464.75 & 49.79 & 21117.44417 \\
\hline & 6 & 920 & 387.3 & 37.63 & 21930.73968 \\
\hline & 6 & 940 & 309.84 & 32.34 & 22580.59304 \\
\hline & 6 & 960 & 232.38 & 27.41 & 23068.79025 \\
\hline & 6 & 980 & 154.92 & 25.22 & 23394.51686 \\
\hline & 6 & 1000 & 77.46 & 22.06 & 23557.89696 \\
\hline & 7 & 1020 & 69.71 & 23.14 & 23705.11356 \\
\hline & 7 & 1040 & 61.97 & 26.27 & 23834.94852 \\
\hline & 7 & 1060 & 54.22 & 31.91 & 23948.59485 \\
\hline & 7 & 1080 & 46.48 & 31.38 & 24046.09889 \\
\hline & 7 & 1100 & 38.73 & 26.1 & 24127.29178 \\
\hline & 7 & 1120 & 30.99 & 29.18 & 24192.35573 \\
\hline & 7 & 1140 & 23.24 & 30.47 & 24241.16666 \\
\hline & 7 & 1160 & 15.5 & 34.97 & 24273.70256 \\
\hline & 7 & 1180 & 7.75 & 50.11 & 24290.04277 \\
\hline
\end{tabular}

\begin{tabular}{|c|c|c|c|c|c|c|c|}
\hline 0 & 31.4 & 3.99 & 46.456 & 11.643 & 13 & 4 & 11.8631 \\
\hline 0 & 31.4 & 5 & 49.809 & 9.962 & 14 & 4 & 12.7193 \\
\hline 0 & 31.4 & 6 & 49.815 & 8.303 & 15 & 4 & 12.7209 \\
\hline 0 & 31.4 & 7 & 50.271 & 7.182 & 16 & 4 & 12.8374 \\
\hline 0 & 31.4 & 8 & 51.972 & 6.497 & 17 & 4 & 13.27165 \\
\hline 0 & 31.4 & 9 & 53.385 & 5.932 & 18 & 4 & 13.63245 \\
\hline 0 & 31.4 & 10.01 & 53.418 & 5.336 & 19 & 4 & 13.641 \\
\hline 0 & 31.4 & 20.02 & 46.041 & 2.3 & 20 & 4 & 11.75705 \\
\hline 0 & 31.4 & 30 & 44.892 & 1.496 & 21 & 4 & 11.4638 \\
\hline 0 & 31.4 & 40.01 & 46.85 & 1.171 & 22 & 4 & 11.9637 \\
\hline 0 & 31.4 & 50 & 48.249 & 0.965 & 23 & 4 & 12.321 \\
\hline 0 & 31.4 & 60.01 & 48.302 & 0.805 & 24 & 4 & 12.3345 \\
\hline 0 & 31.4 & 70 & 49.47 & 0.707 & 25 & 4 & 12.6327 \\
\hline 0 & 31.4 & 79.99 & 49.996 & 0.625 & 26 & 4 & 12.76715 \\
\hline 0 & 31.4 & 90 & 49.545 & 0.551 & 27 & 4 & 12.65205 \\
\hline 0 & 31.4 & 100 & 47.369 & 0.474 & 28 & 4 & 12.0963 \\
\hline 0 & 31.4 & 200.01 & 52.312 & 0.262 & 29 & 4 & 13.35865 \\
\hline 0 & 31.4 & 300.02 & 50.14 & 0.167 & 30 & 4 & 12.8038 \\
\hline 0 & 31.4 & 400 & 43.618 & 0.109 & 31 & 4 & 11.13845 \\
\hline 0 & 31.4 & 500 & 36.999 & 0.074 & 32 & 4 & 9.44805 \\
\hline 0 & 31.4 & 599.99 & 31.717 & 0.053 & 33 & 4 & 8.0994 \\
\hline 0 & 31.4 & 699.99 & 43.981 & 0.063 & 34 & 4 & 11.23105 \\
\hline 0 & 31.4 & 799.98 & 33.567 & 0.042 & 35 & 4 & 8.57175 \\
\hline 0 & 31.4 & 900.01 & 33.381 & 0.037 & 36 & 4 & 8.5243 \\
\hline 0 & 31.4 & 1000 & 24.841 & 0.025 & 37 & 4 & 6.34355 \\
\hline 0 & 31.4 & 1000 & 21.809 & 0.022 & 38 & 4 & 5.56925 \\
\hline 0 & 31.4 & 1000 & 21.382 & 0.021 & 39 & 4 & 5.4602 \\
\hline 0 & 31.4 & 1000 & 16.768 & 0.017 & 40 & 4 & 4.28185 \\
\hline 0 & 31.4 & 1000 & 15.38 & 0.015 & 41 & 4 & 3.92745 \\
\hline 0 & 31.4 & 1000 & 15.011 & 0.015 & 42 & 4 & 3.83315 \\
\hline 0 & 31.4 & 1000 & 14.334 & 0.014 & 43 & 4 & 3.66035 \\
\hline 0 & 31.4 & 1000 & 14.143 & 0.014 & 44 & 4 & 3.6115 \\
\hline 0 & 31.4 & 899.99 & 14.246 & 0.016 & 45 & 4 & 3.63785 \\
\hline 0 & 31.4 & 799.99 & 15.17 & 0.019 & 46 & 4 & 3.87375 \\
\hline 0 & 31.4 & 699.99 & 12.175 & 0.017 & 47 & 4 & 3.1091 \\
\hline 0 & 31.4 & 599.99 & 9.748 & 0.016 & 48 & 4 & 2.4893 \\
\hline 0 & 31.4 & 500 & 7.369 & 0.015 & 49 & 4 & 1.88165 \\
\hline 0 & 31.4 & 400 & 6.333 & 0.016 & 50 & 4 & 1.61715 \\
\hline 0 & 31.4 & 300 & 5.367 & 0.018 & 51 & 4 & 1.37065 \\
\hline 0 & 31.4 & 200 & 4.938 & 0.025 & 52 & 4 & 1.26105 \\
\hline 0 & 31.4 & 100 & 4.319 & 0.043 & 53 & 4 & 1.1028 \\
\hline 0 & 31.4 & 90 & 4.53 & 0.05 & 54 & 4 & 1.1569 \\
\hline 0 & 31.4 & 80 & 5.144 & 0.064 & 55 & 4 & 1.3136 \\
\hline 0 & 31.4 & 70 & 6.248 & 0.089 & 56 & 4 & 1.59555 \\
\hline 0 & 31.4 & 60.01 & 6.145 & 0.102 & 57 & 4 & 1.5692 \\
\hline 0 & 31.4 & 50 & 5.11 & 0.102 & 58 & 4 & 1.3049 \\
\hline 0 & 31.4 & 40.01 & 5.714 & 0.143 & 59 & 4 & 1.45905 \\
\hline 0 & 31.4 & 30 & 5.966 & 0.199 & 60 & 4 & 1.5234 \\
\hline 0 & 31.4 & 20.01 & 6.848 & 0.342 & 61 & 4 & 1.74865 \\
\hline 0 & 31.4 & 10.01 & 9.811 & 0.98 & 62 & 4 & 2.50525 \\
\hline
\end{tabular}




\begin{tabular}{|c|c|c|c|c|c|c|c|c|c|c|c|c|c|c|}
\hline & 81200 & 6.97 & 73 & 24304.75564 & 0 & 0 & 31.4 & 9 & 14.294 & 1.588 & 63 & 4 & 3.65015 & 0 \\
\hline & 81220 & 6.2 & 91.55 & 24317.74062 & 0 & 0 & 31.4 & 8 & 17.925 & 2.241 & 64 & 4 & 4.57725 & \\
\hline & 81240 & 5.42 & 87.8 & 24329.09591 & 0 & 0 & 31.4 & 7 & 17.19 & 2.456 & 65 & 4 & 4.3898 & \\
\hline & 81260 & 4.64 & 80.42 & 24338.83485 & 0 & 0 & 31.4 & 5.99 & 15.745 & 2.629 & 66 & 4 & 4.02075 & \\
\hline & 81280 & 3.87 & 77.16 & 24346.95508 & 0 & 0 & 31.4 & 5 & 15.109 & 3.022 & 67 & 4 & 3.85815 & \\
\hline & 81300 & 3.09 & 78.11 & 24353.45032 & 0 & 0 & 31.4 & 3.99 & 15.294 & 3.833 & 68 & 4 & 3.9055 & \\
\hline & 81320 & 2.32 & 74.81 & 24358.32058 & 0 & 0 & 31.4 & 3 & 14.648 & 4.883 & 69 & 4 & 3.74045 & \\
\hline & 81340 & 1.54 & 67.75 & 24361.56663 & 0 & 0 & 31.4 & 1.99 & 13.266 & 6.666 & 70 & 4 & 3.38755 & \\
\hline & 81360 & 0.77 & 67.05 & 24363.19083 & 0 & 0 & 31.4 & 0.99 & 13.129 & 13.262 & 71 & 4 & 3.35265 & \\
\hline & 91380 & 0.7 & 64.13 & 24364.66738 & 18.2031 & 1.44975 & 31.4 & 0.9 & 12.556 & 13.951 & 72 & 4 & 3.2063 & 0.689772621 \\
\hline & 91400 & 0.62 & 60.96 & 24365.97114 & 34.27605 & 2.87165 & 31.4 & 0.8 & 11.936 & 14.92 & 73 & 4 & 3.04795 & 0.348231491 \\
\hline & 91420 & 0.55 & 65.76 & 24367.11154 & 48.33504 & 3.75389 & 31.4 & 0.71 & 12.876 & 18.135 & 74 & 4 & 3.28815 & 0.266390594 \\
\hline & 91440 & 0.47 & 69.54 & 24368.09171 & 60.4188 & 4.43734 & 31.4 & 0.61 & 13.616 & 22.321 & 75 & 4 & 3.4771 & 0.225360318 \\
\hline & 91460 & 0.39 & 70.87 & 24368.9101 & 70.50797 & 5.08092 & 31.4 & 0.5 & 13.877 & 27.754 & 76 & 4 & 3.5437 & 0.19681463 \\
\hline & 91480 & 0.31 & 69.44 & 24369.56591 & 78.59285 & 5.78016 & 31.4 & 0.4 & 13.597 & 33.993 & 77 & 4 & 3.47215 & 0.17300556 \\
\hline & 91500 & 0.24 & 68.19 & 24370.05992 & 84.68315 & 6.34236 & 31.4 & 0.31 & 13.352 & 43.071 & 78 & 4 & 3.4097 & 0.157670091 \\
\hline & 91520 & 0.16 & 65.32 & 24370.39136 & 88.76916 & 6.94051 & 31.4 & 0.21 & 12.79 & 60.905 & 79 & 4 & 3.2662 & 0.144081571 \\
\hline & 91540 & 0.08 & 60.93 & 24370.56258 & 90.87995 & 7.6184 & 31.4 & 0.1 & 11.929 & 119.29 & 80 & 4 & 3.0463 & 0.131261076 \\
\hline
\end{tabular}



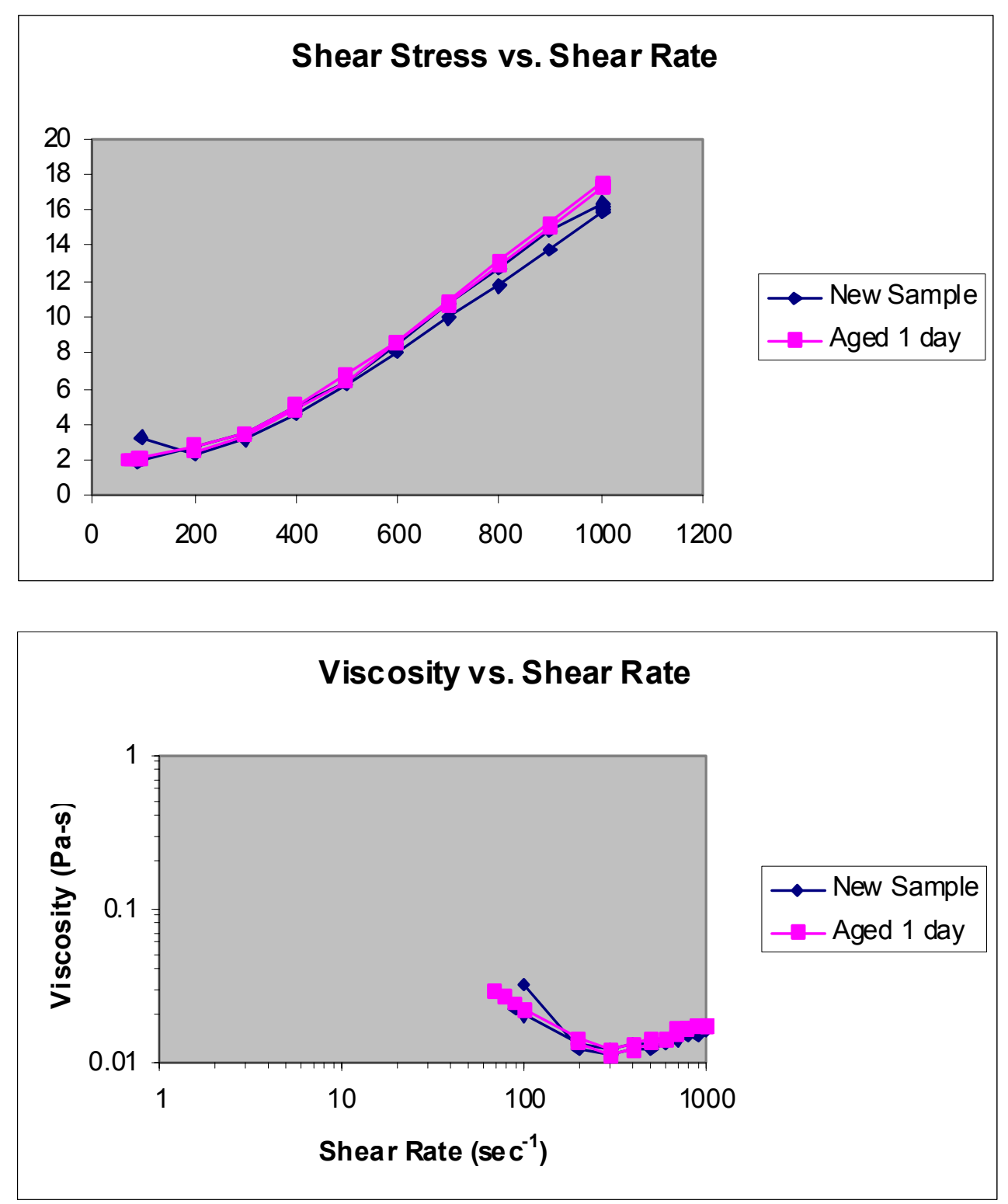

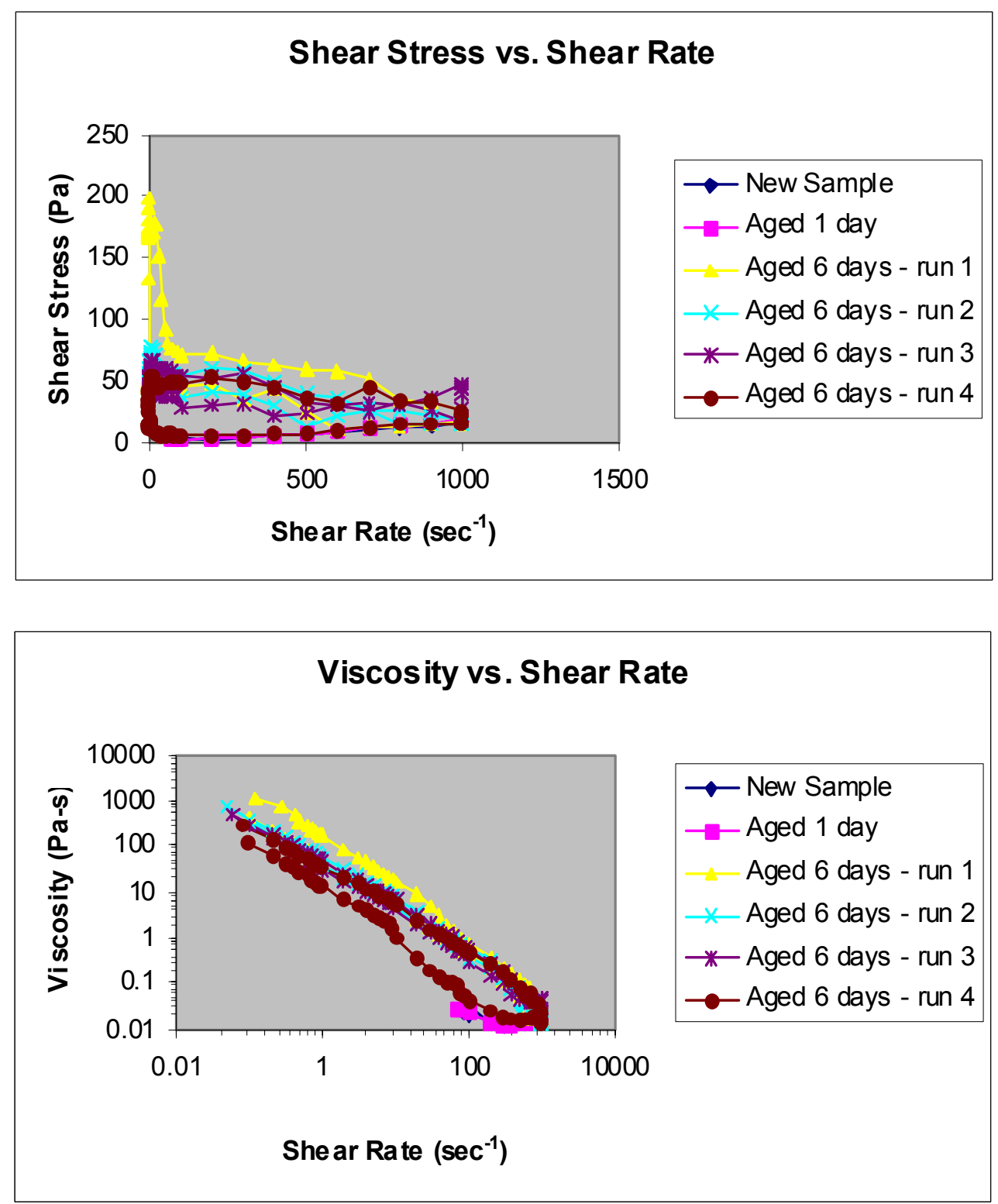


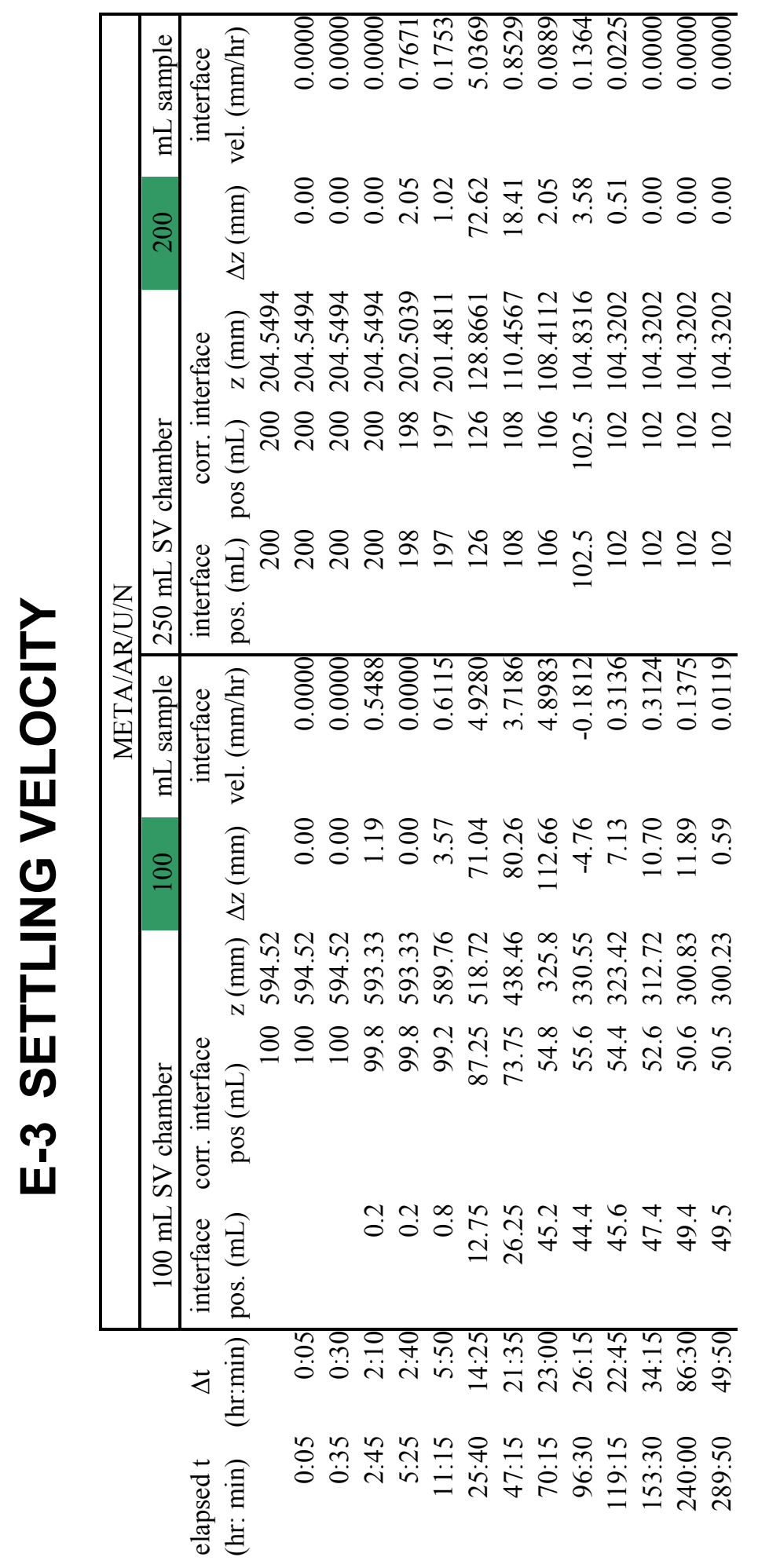

กิ

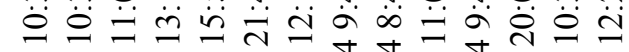

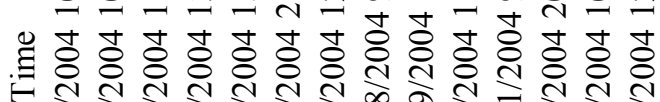

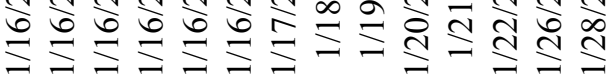




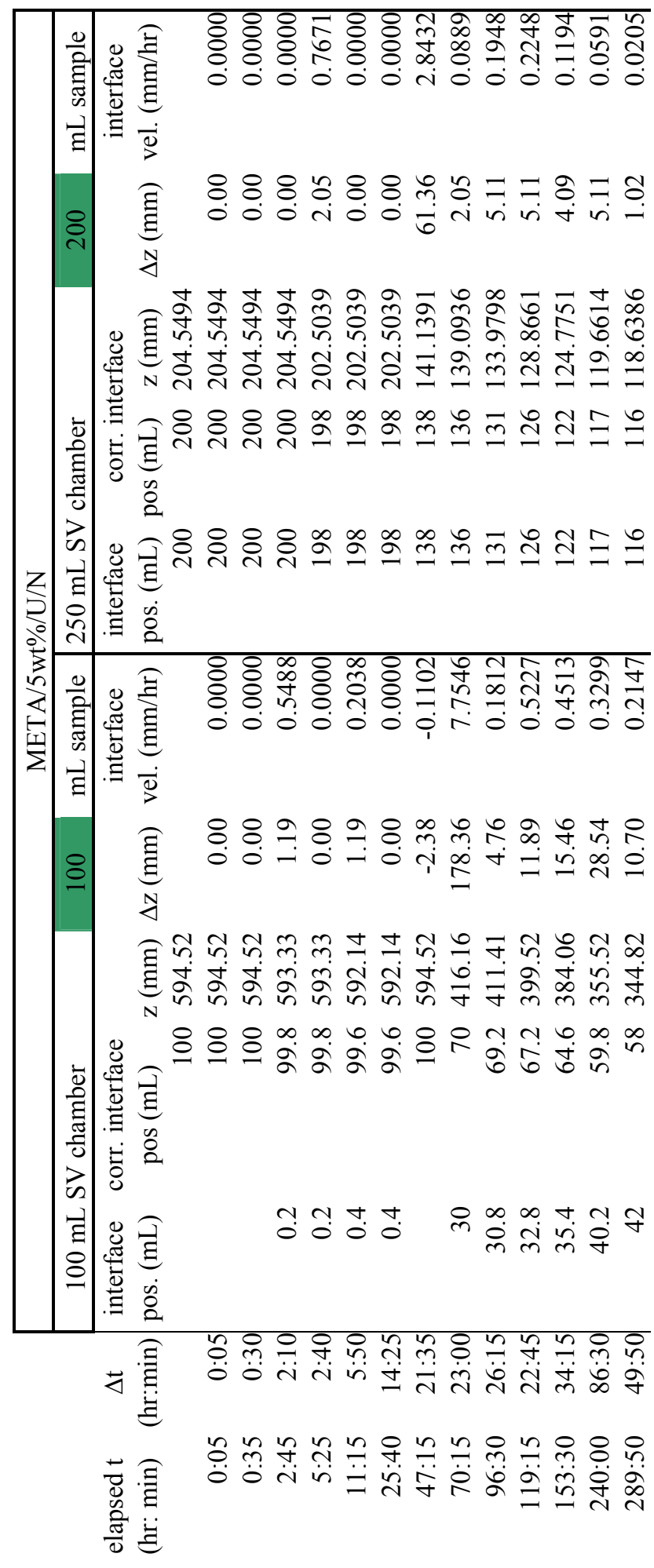

ㅇำำำ

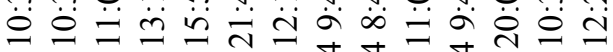

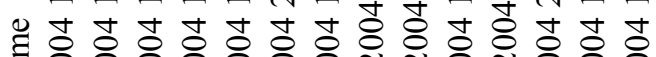

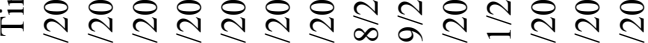
은

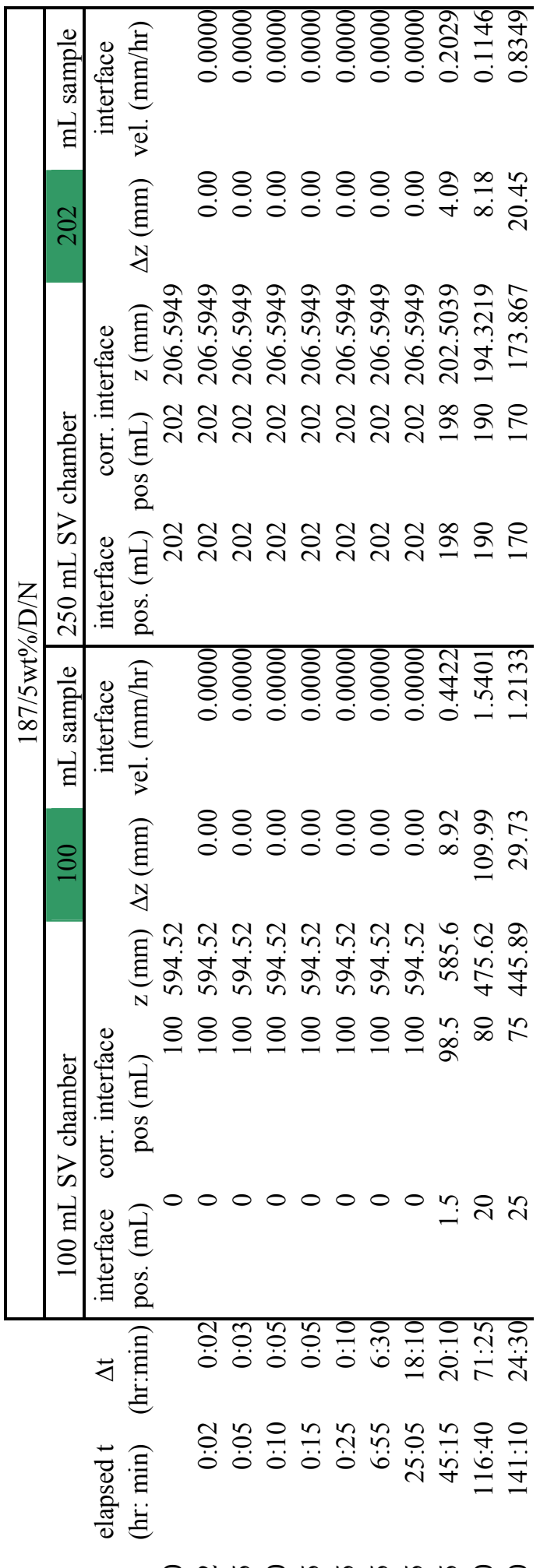

ิㅗㅊำ

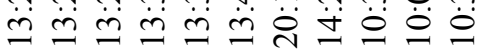

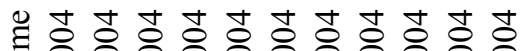

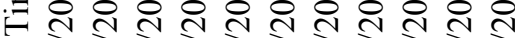
苮 


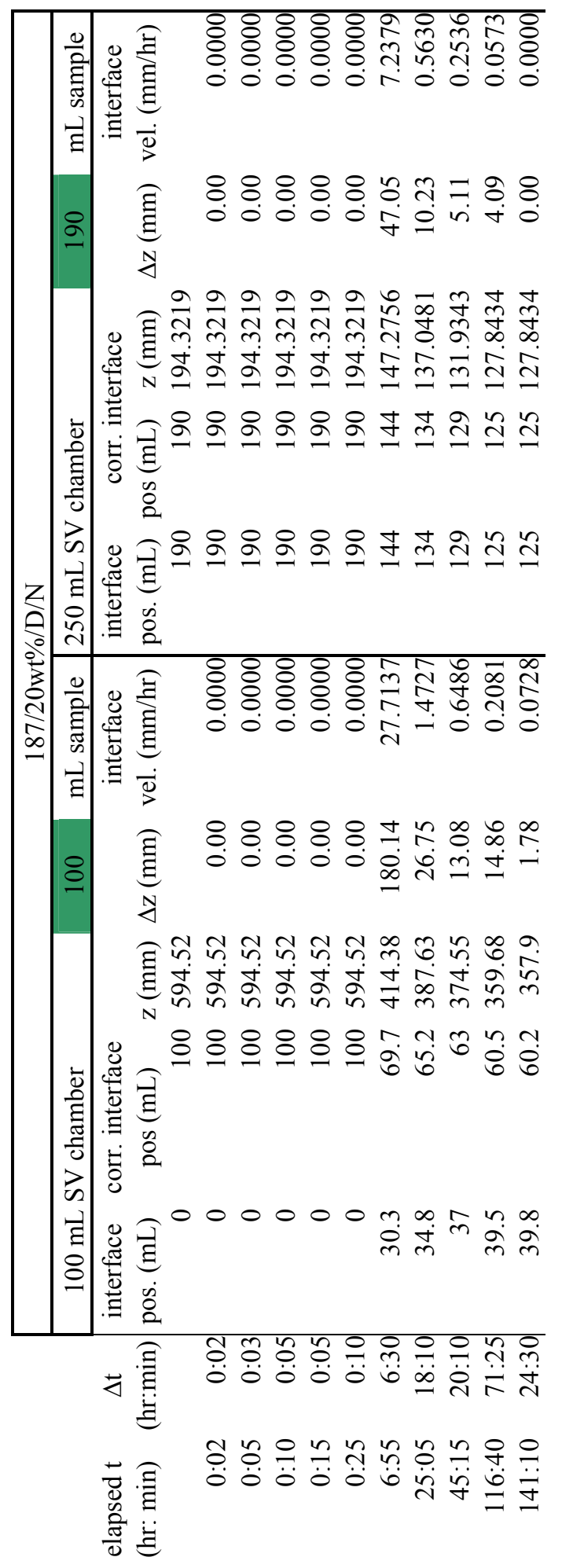

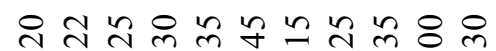
$\ddot{n} \ddot{n} \ddot{n} \ddot{n} \ddot{n} \ddot{\dot{U}} \ddot{\dot{O}} \dot{0}$

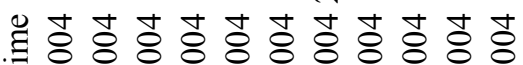

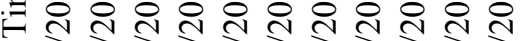
苮

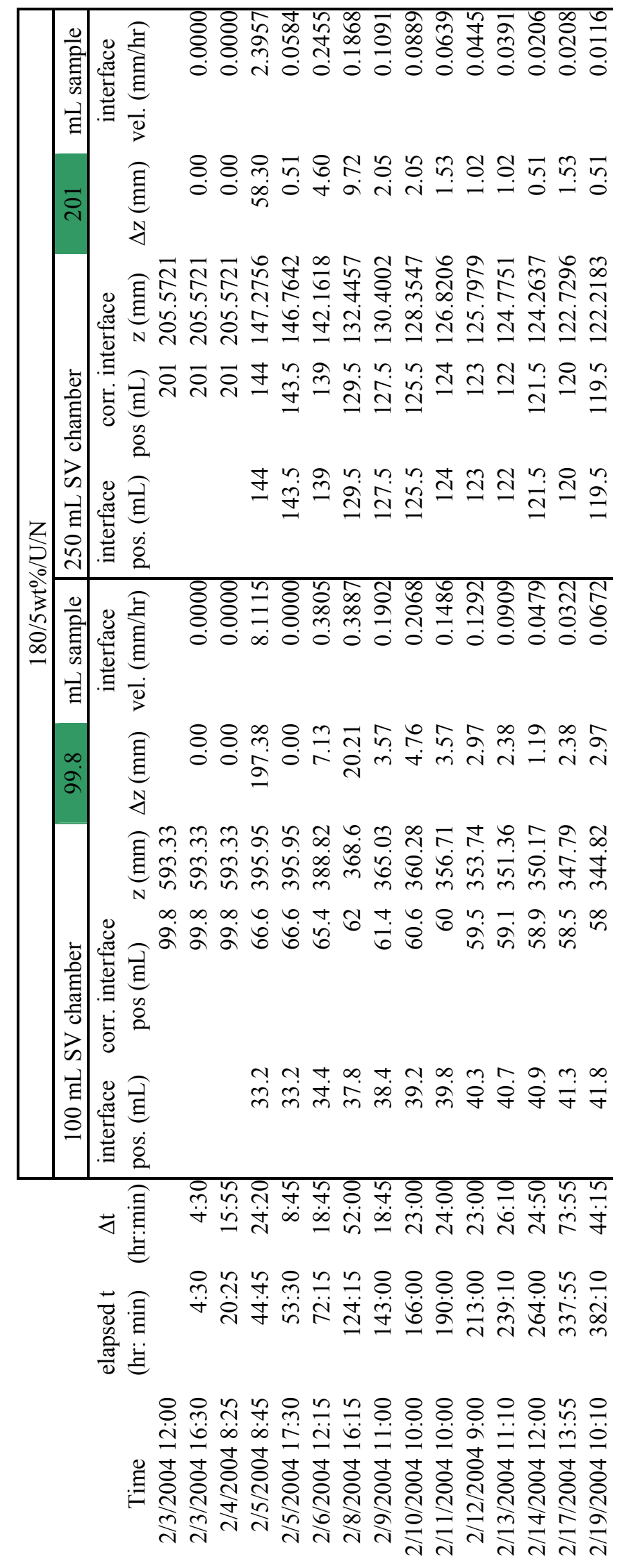




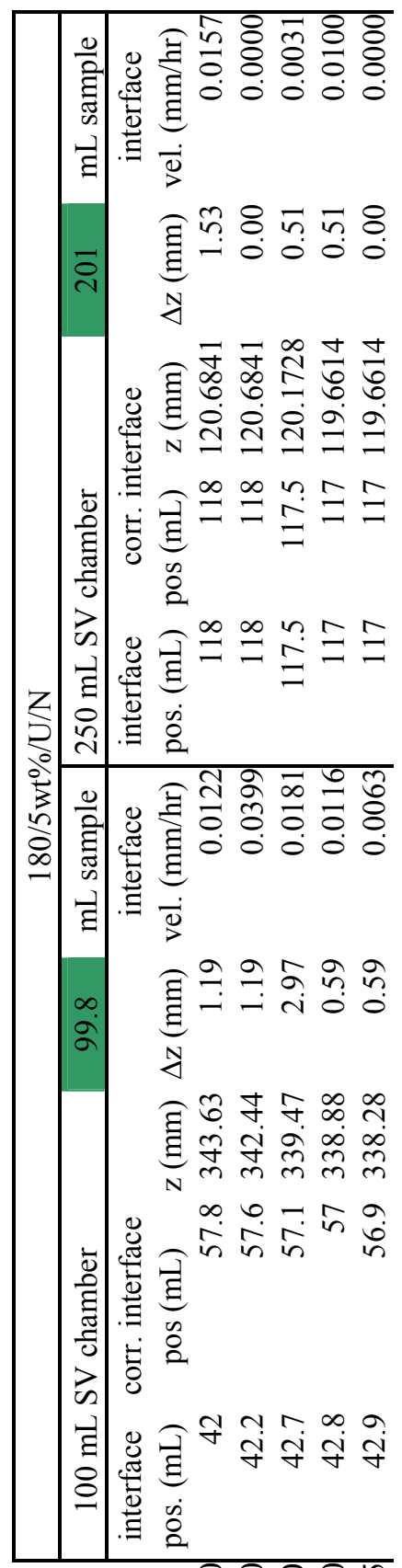

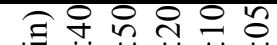

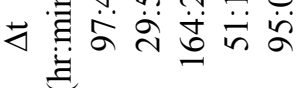

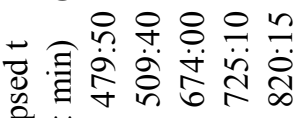
要这

응ㅇ $8 ㅇ ㅡ$ $\ddot{\exists} \ddot{\Xi} \dot{\Xi} \ddot{\Xi}$ 艺苍苍苍苍

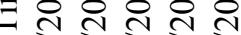

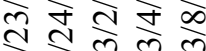
ते $\frac{\text { n }}{m}$

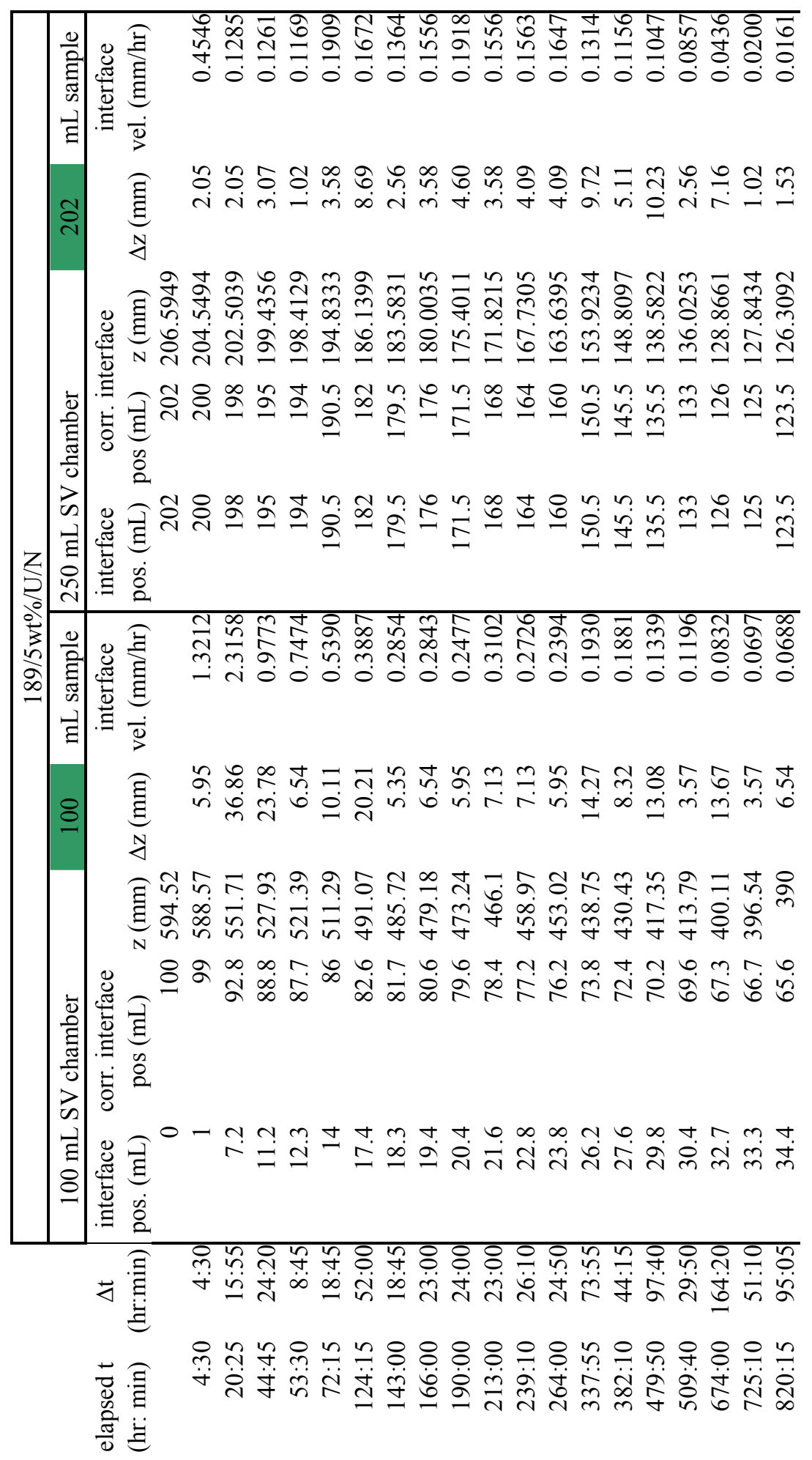

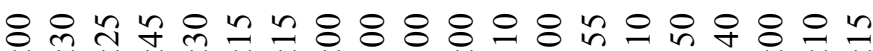

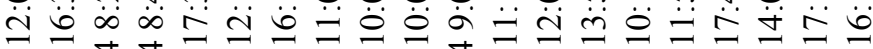

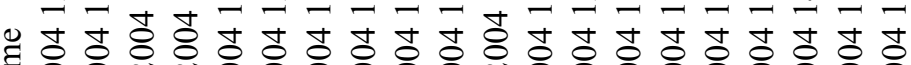

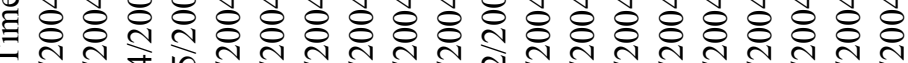

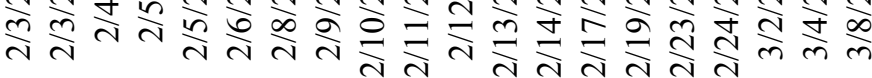




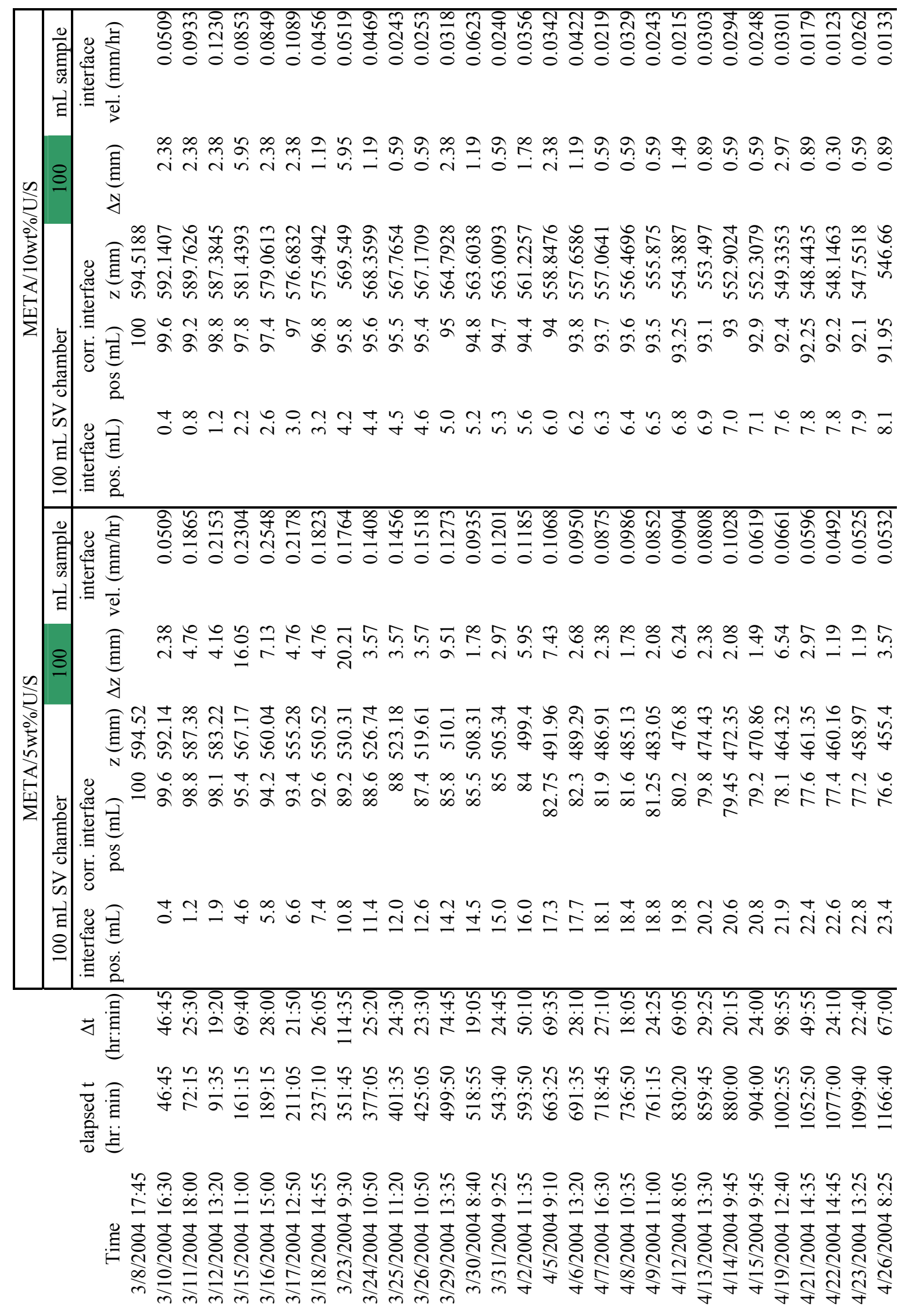




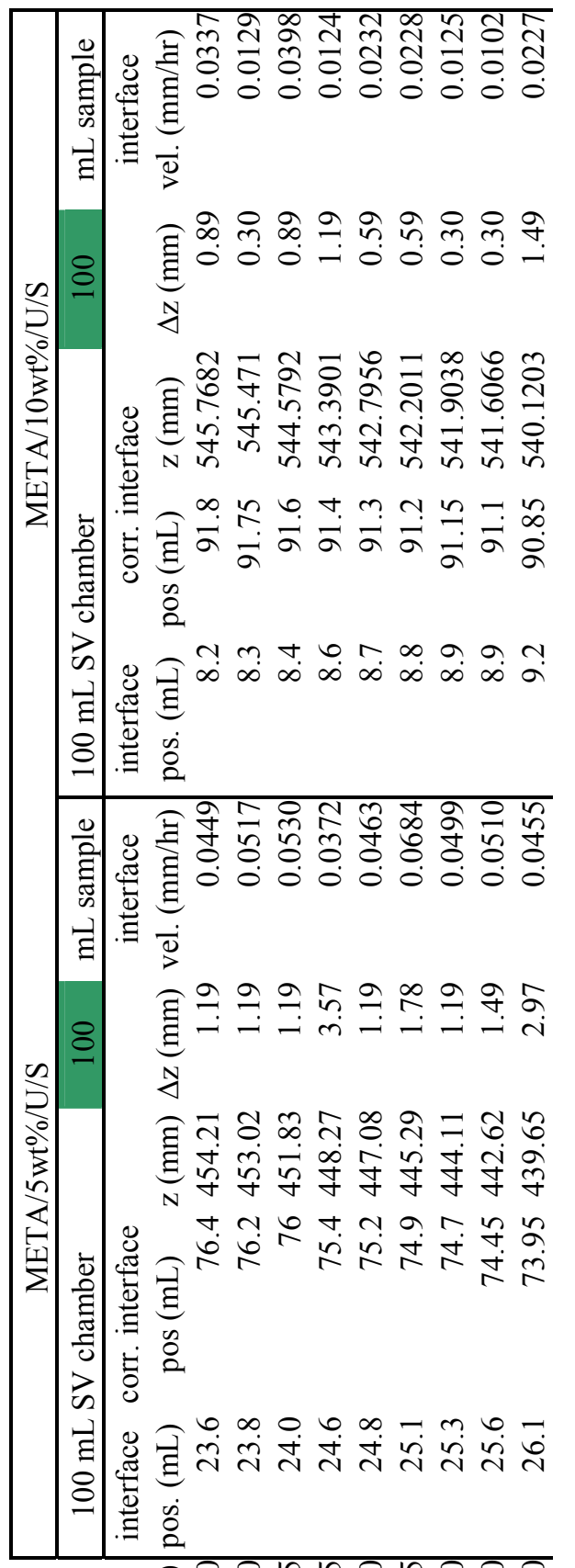

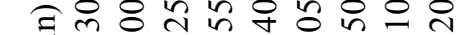

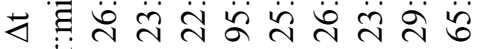
ق

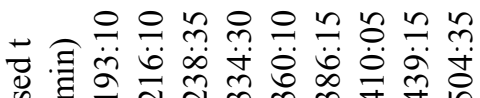
政 胥

ำ 유는

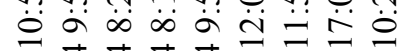

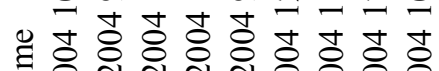

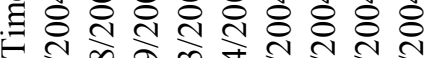

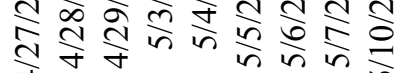

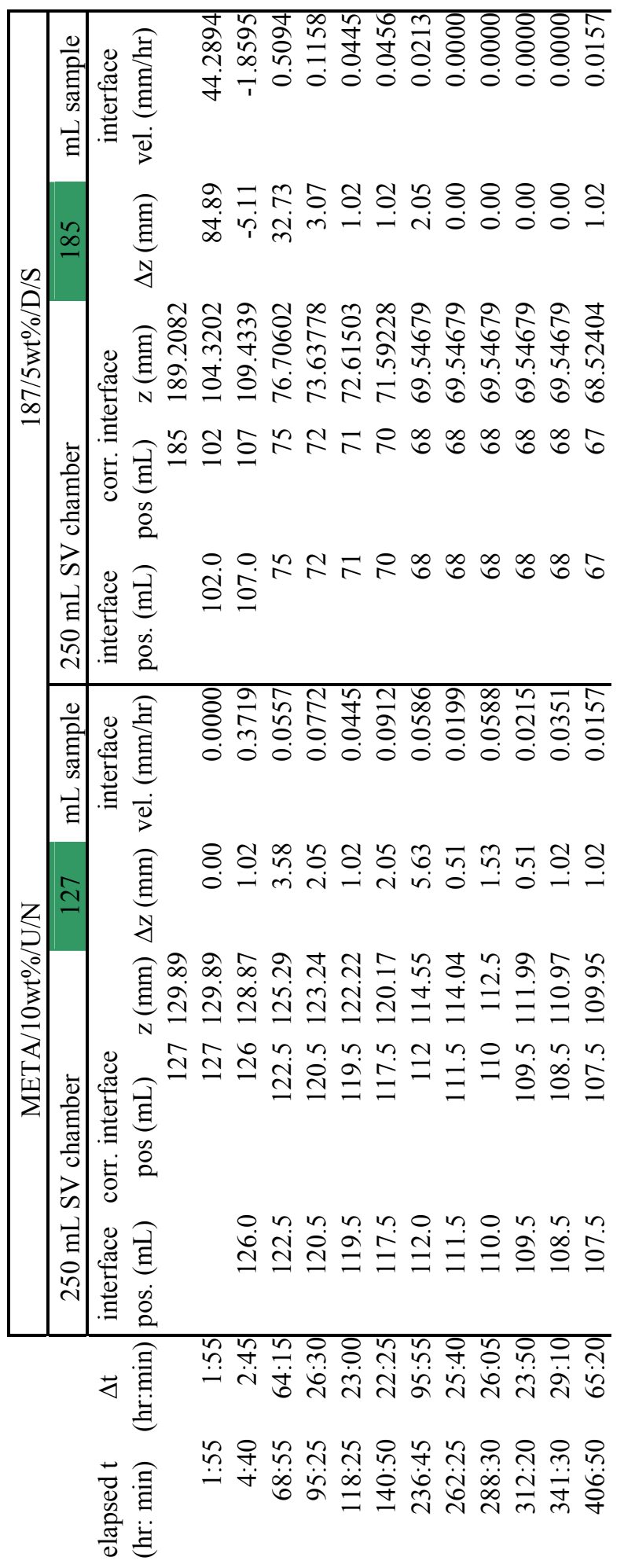

으읏ำ

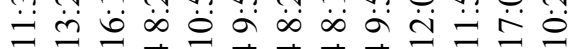

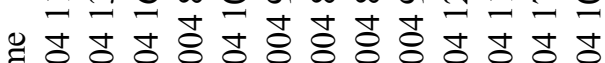

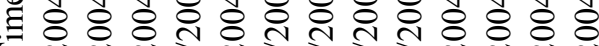

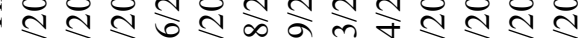

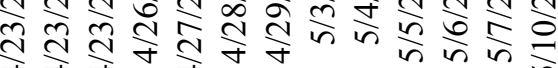


$\overline{\bar{o}}$

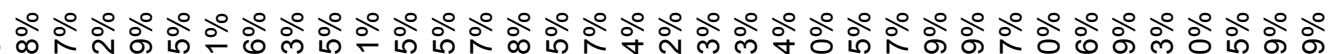

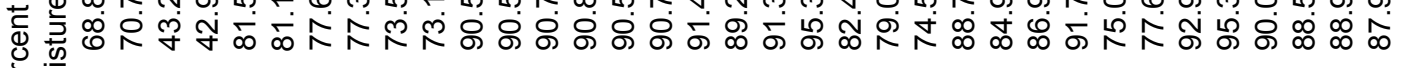
¿

๑

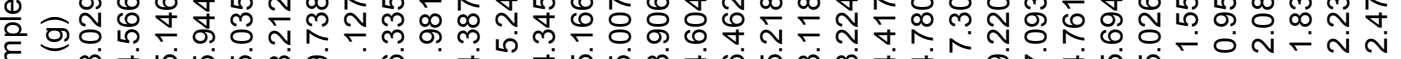

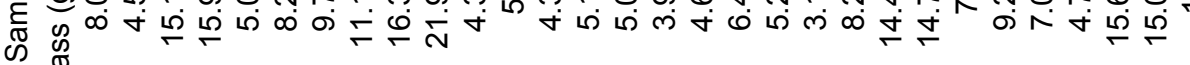

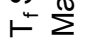

สิ น

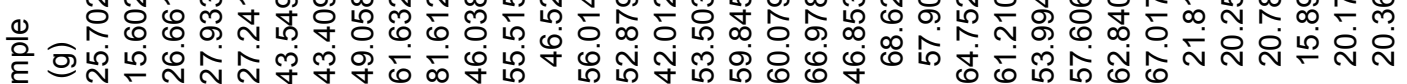
în

山 $\quad \vdash \sum^{\infty}$

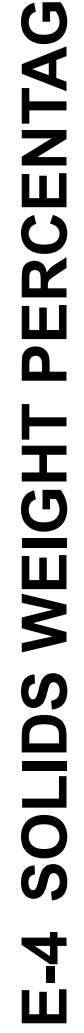

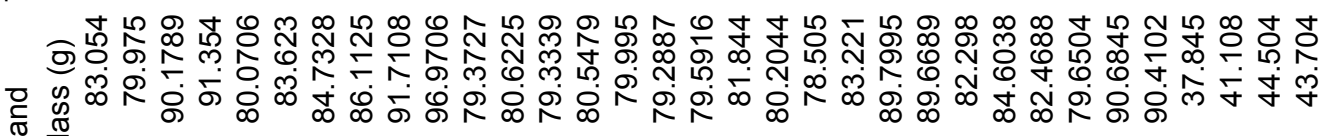
$\frac{\sigma}{\mathbb{\sigma}} \sum^{\pi}$ Ф) $>$

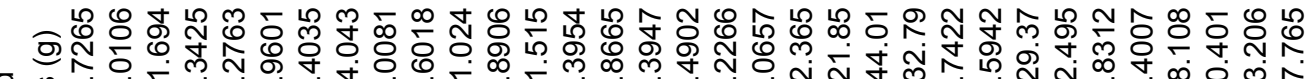

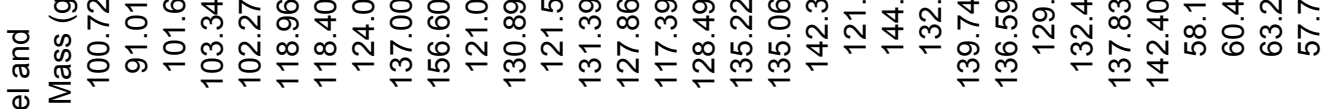
( $\stackrel{\infty}{\infty} \frac{0}{\circ}$ $\vdash \infty$

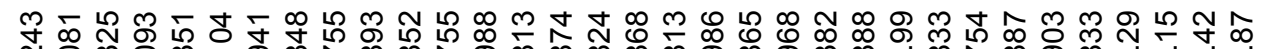

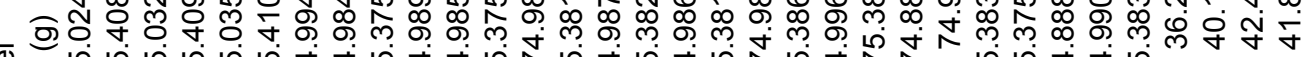

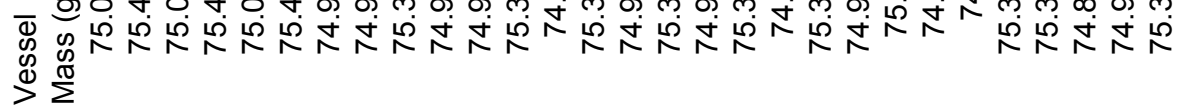

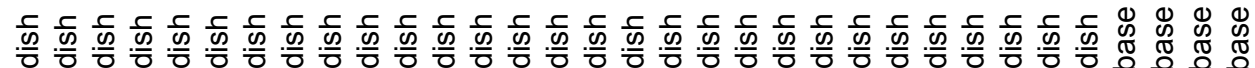

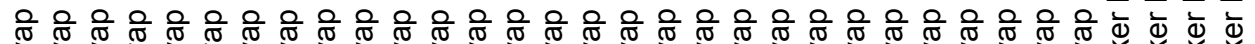

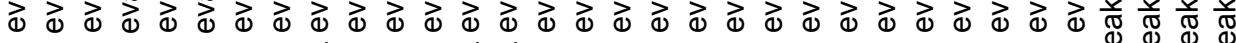

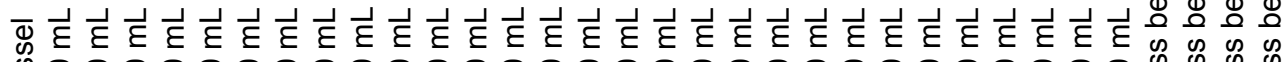

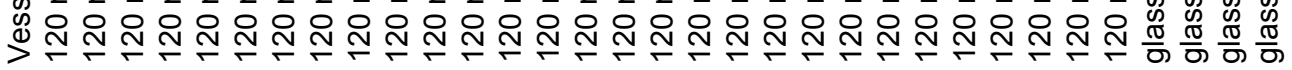

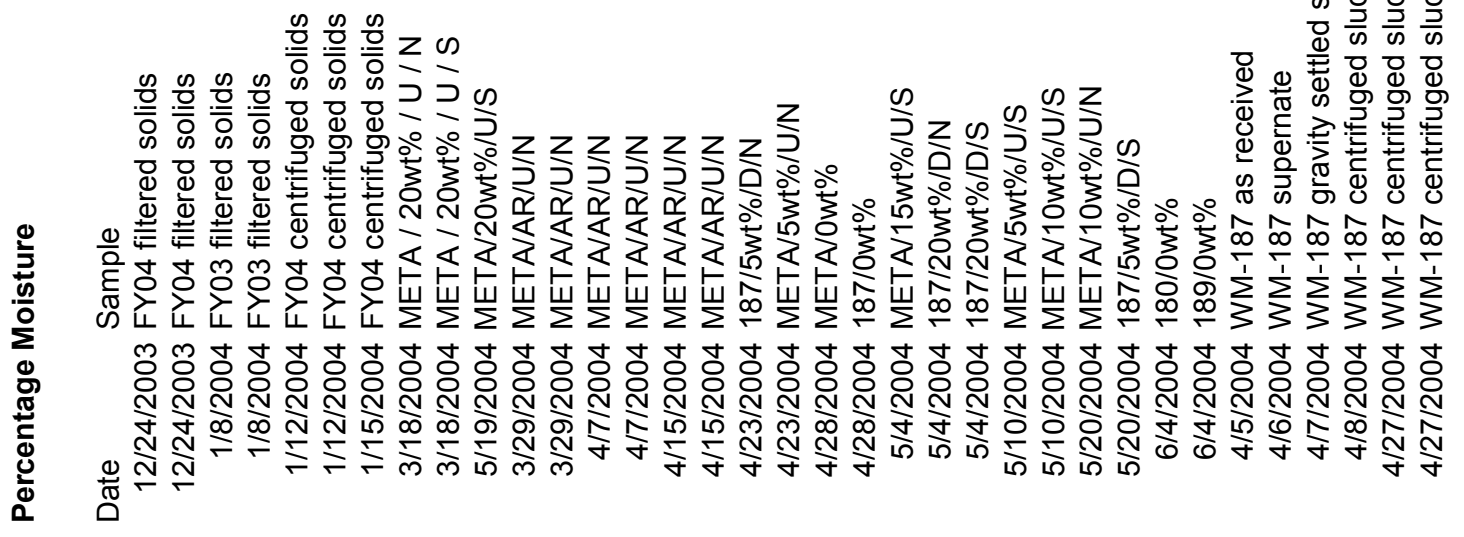




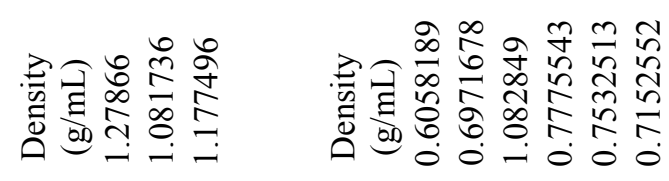

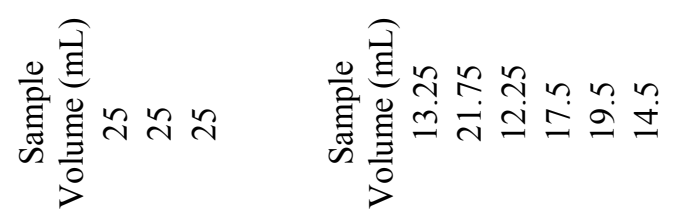
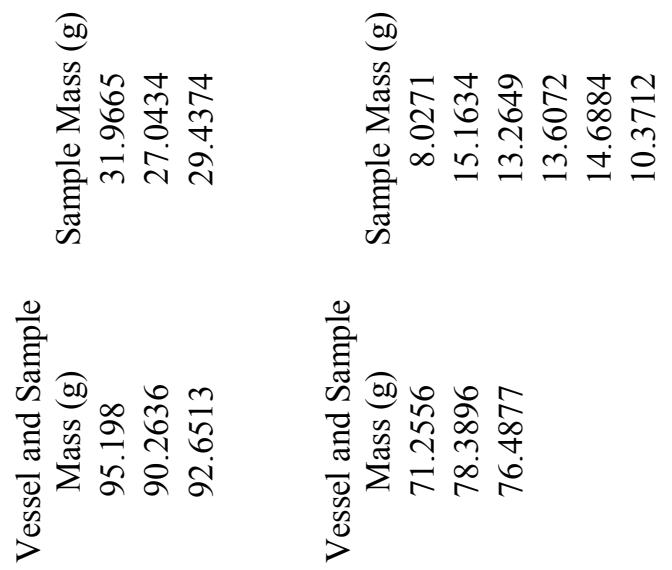

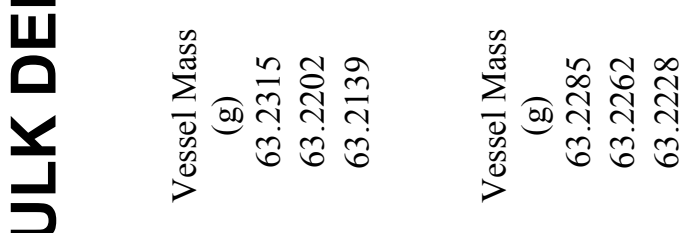

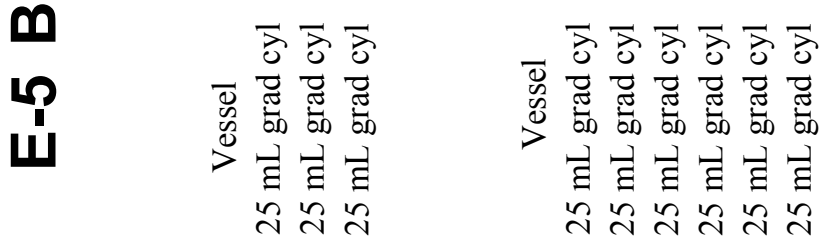

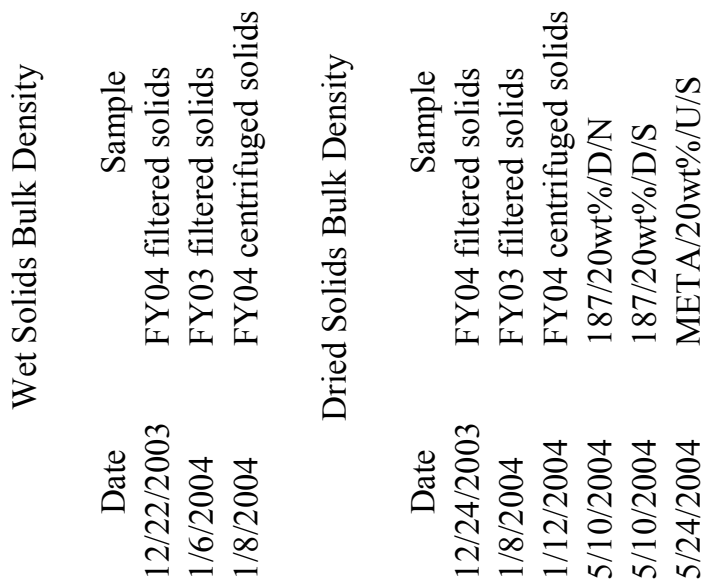




\section{Sample Bulk Density}

Date
$1 / 14 / 2004$
$1 / 14 / 2004$
$1 / 14 / 2004$
$1 / 14 / 2004$
$1 / 14 / 2004$
$1 / 14 / 2004$
$1 / 14 / 2004$
$1 / 14 / 2004$
$1 / 14 / 2004$
$1 / 14 / 2004$
$1 / 14 / 2004$
$4 / 23 / 2004$
$4 / 23 / 2004$
$4 / 23 / 2004$
$4 / 23 / 2004$
$4 / 23 / 2004$
$5 / 10 / 2004$
$5 / 10 / 2004$

Sample

META / AR / U

187 / 5wt $\%$ / D

META / 5wt\% / U

$180 / 5 \mathrm{wt} \% / \mathrm{U}$

$189 / 5 \mathrm{wt} \% / \mathrm{U}$

187 / 20wt $\%$ / D

META / 20wt \% / U

META / 0wt $\%$

$187 / 0 \mathrm{wt} \%$

$180 / 0 \mathrm{wt} \%$

$189 / 0 \mathrm{wt} \%$

$187 / 5 \mathrm{wt} \% / \mathrm{D} / \mathrm{S}$

META $/ 10 \mathrm{wt} \% / \mathrm{U} / \mathrm{N}$

META $/ 15 \mathrm{wt} \% / \mathrm{U} / \mathrm{S}$

$187 / 20 \mathrm{wt} \% / \mathrm{D} / \mathrm{S}$

META/20wt\%/U/S

META $/ 5 \mathrm{wt} \% / \mathrm{U} / \mathrm{S}$

META $/ 10 \mathrm{wt} \% / \mathrm{U} / \mathrm{S}$
Sample Vol.

$\begin{array}{ccc}\text { Sample Mass }(\mathrm{g}) & (\mathrm{mL}) & \text { Density }(\mathrm{g} / \mathrm{mL}) \\ 72.7350 & 65.0 & 1.12 \\ 70.4022 & 66.0 & 1.07 \\ 75.3557 & 66.5 & 1.13 \\ 69.0007 & 56.0 & 1.23 \\ 69.9303 & 55.0 & 1.27 \\ 71.0840 & 61.0 & 1.17 \\ 73.1548 & 59.5 & 1.23 \\ 69.5804 & 62.0 & 1.12 \\ 73.2010 & 69.5 & 1.05 \\ 76.8701 & 61.5 & 1.25 \\ 79.0573 & 60.5 & 1.31 \\ 71.5106 & 67.0 & 1.07 \\ 75.6030 & 66.0 & 1.15 \\ 73.7372 & 63.0 & 1.17 \\ 78.9818 & 66.0 & 1.20 \\ 83.2490 & 67.0 & 1.24 \\ 68.4386 & 60.5 & 1.13 \\ 76.4387 & 66.0 & 1.16\end{array}$




\section{E-6 pH}

Sample pH

\begin{tabular}{clcc} 
Date & \multicolumn{1}{c}{ Sample } & $\mathrm{pH}$ indicator strip & $\mathrm{pH}$ probe \\
$1 / 15 / 2004$ & META / 0wt $\%$ & 0 & 0.77 \\
$1 / 15 / 2004$ & $187 / 0 \mathrm{wt} \%$ & 0 & 1.01 \\
$1 / 15 / 2004$ & $180 / 0 \mathrm{wt} \%$ & 0 & 0.44 \\
$1 / 15 / 2004$ & $189 / 0 \mathrm{wt} \%$ & 0 & 0.24
\end{tabular}

